
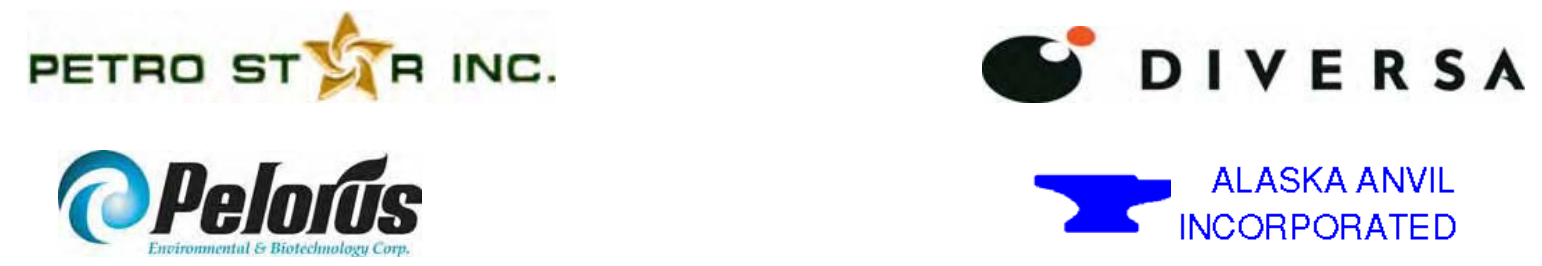

\title{
The Biocatalytic Desulfurization Project
}

\author{
Final Technical \\ Progress Report \\ January 2002-December 2006 \\ Prepared for \\ U.S. Department of Energy \\ National Energy Technology Laboratory \\ One West Third Street \\ Suite 1400 \\ Tulsa, OK 74103-3519 \\ DOE Award Number: DE-FC26-02NT15340 \\ Prepared by

$\begin{array}{cc}\text { David Nunn } & \text { James Boltz } \\ \text { Diversa } & \text { Petro Star } \\ 4955 \text { Directors Place } & 3900 \text { C Street } \\ \text { San Diego, CA } & \text { Suite 401 } \\ & \text { Anchorage, AK 99503 } \\ \text { Philip M. DiGrazia } & \text { Larry Nace } \\ \text { Pelorus } & \text { Anvil } \\ 3528 \text { Evergreen Parkway } & \text { 1675 West Bakerview Rd } \\ \text { Evergreen, CO 80439 } & \text { Bellingham, WA 98226 }\end{array}$

March 3, 2006 


\section{DISCLAIMER}

This report was prepared as an account of work sponsored by an agency of the United States Government. Neither the United States Government nor any agency thereof, nor any of their employees, makes any warranty, express or implied, or assumes any legal liability or responsibility for the accuracy, completeness, or usefulness of any information, apparatus, product, or process disclosed, or represents that its use would not infringe privately owned rights. Reference herein to any specific commercial product, process, or service by trade name, trademark, manufacturer, or otherwise does not necessarily constitute or imply its endorsement, recommendation, or favoring by the United States Government or any agency thereof. These views and opinions of authors expressed herein do not necessarily state or reflect those of the United States Government or any agency thereof. 


\section{ABSTRACT}

The material in this report summarizes the Diversa technical effort in development of a biocatalyst for the biodesulfurization of Petro Star diesel as well as an economic report of standalone and combined desulfurization options, prepared by Pelorus and Anvil, to support and inform the development of a commercially viable process. We will discuss goals of the projected as originally stated and their modification as guided by parallel efforts to evaluate commercialization economics and process parameters. We describe efforts to identify novel genes and hosts for the generation of an optimal biocatalyst, analysis of diesel fuels (untreated, chemically oxidized and hydrotreated) for organosulfur compound composition and directed evolution of enzymes central to the biodesulfurization pathway to optimize properties important for their use in a biocatalyst. Finally we will summarize the challenges and issues that are central to successful development of a viable biodesulfurization process. 


\section{TABLE OF CONTENTS}

Section $\quad \underline{\text { Page }}$

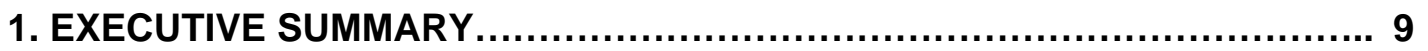

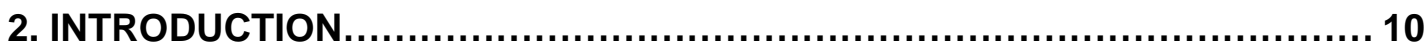

2.1 Regulations ....................................................... 10

2.2 Alternative desulfurization technologies................................. 10

2.3 Biodesulfurization.................................................. 11

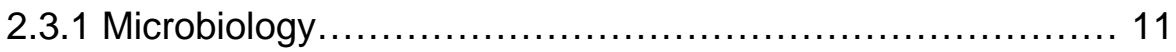

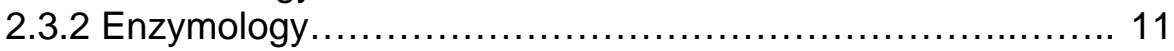

2.3.3 Issues and challenges to commercialization................... 13

2.4 Original focus of research plan.......................................... 17

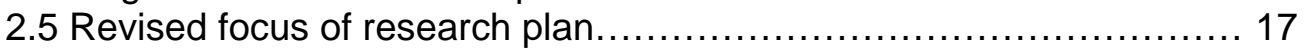

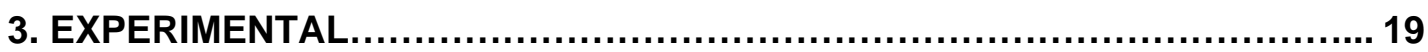

3.1 Chemical analysis......................................................... 19

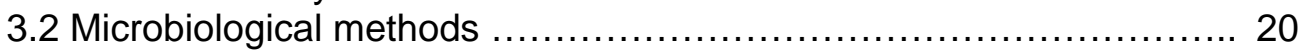

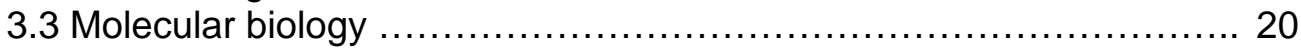

3.4 Expression and purification of enzymes.............................. 21

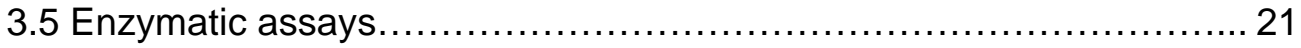

3.6 Whole cell biocatalysis assays ....................................... 22

4. RESULTS AND DISCUSSION.................................................... 23

4.1 Analysis of organosulfur compounds in diesel fuel..................... 23

4.1.1 Determination of organosulfur compounds in chemically oxidized Petro Star diesel................................... 23

4.1.2 Determination of organosulfur compounds in partially hydrotreated Petro Star diesel................................ 23

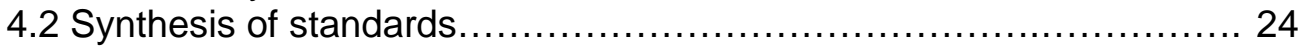

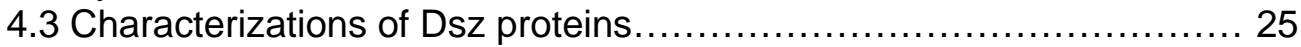

4.3.1 Cloning and expression........................................ 25

4.3.2 Characterization of flavin reductase activities................. 25

4.3.3 Characterization of DszA activities........................... 26

4.3.4 Characterization of DszC activities............................ 27

4.4 Construction of DszA-reductase fusions............................ 28

4.5 Discovery of new dsz genes......................................... 29

4.5.1 Sequence-based discovery ............................... 29

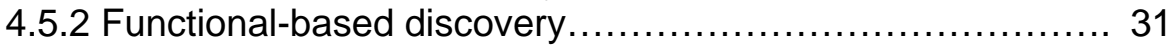

4.6 Directed evolution of DszA ......................................... 34

4.6.1 GSSM $^{\mathrm{TM}}$ mutagenesis........................................ 35

4.6.2 Assay development ......................................... 35

4.6.3 Primary screening of $d S Z A$ GSSM library ..................... 38

4.6.4 Secondary screening of $d s z A$ GSSM library ................. 42

4.6.5 Tertiary screening of dszA GSSM library ..................... 42

4.6.6 Bioinformatic analysis of DszA GSSM mutants................ 47

4.6.7 GeneReassembly mutagenesis of DszA GSSM mutants...... 49

4.6.8 Characterization of DszA GSSM mutants and reassembly variants........................................................ 51

4.6.9 Expression of selected variants in Rhodococcus............. 52 


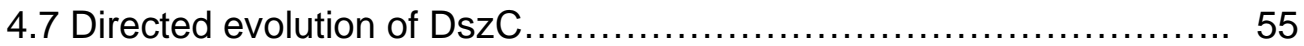

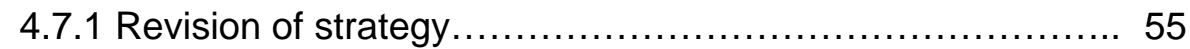

4.7.2 Optimization of high throughput assay..................... 55

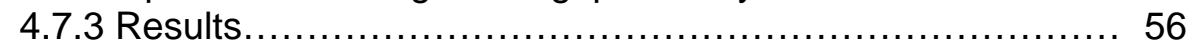

4.7.4 Bioinformatic analyses of DszC GSSM mutations............ 58

4.8 Identification of alternate hosts ...................................... 59

4.8.1 Growth characteristics...................................... 59

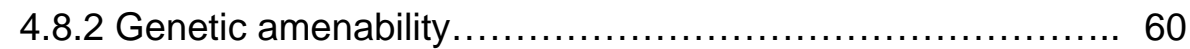

4.8 .3 In vivo biodesulfurization activity .......................... 61

4.8.4 Survival on exposure to hexadecane and diesel............. 61

4.8.5 Whole cell two-phase BDS activity ....................... 63

4.8.6 Contribution of pSOX genes to BDS activity................ 64

4.8.7 Introduction of pBKO53A into Rhodococcus opacus...........65 65

4.8.8 Characterization of biocatalyst activities on partially

hydrotreated diesel...................................... 65

4.8.9 Stability of pBKO53A in host strains....................... 70

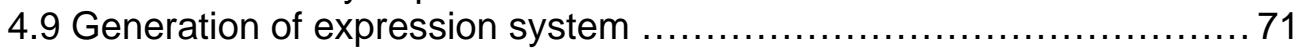

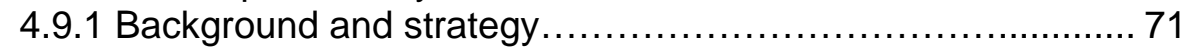

4.9.2 Generation of thyA deletion strain ......................... 72

4.9.3 Complementation and construction of $d s z$ gene cassette.....75

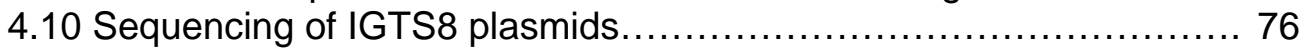

4.11 Economic study of BDS standalone and combined processes.......... 79

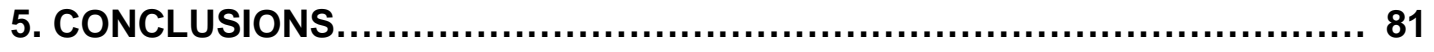

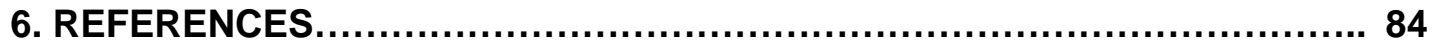

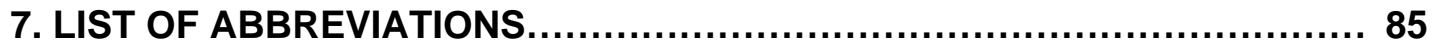

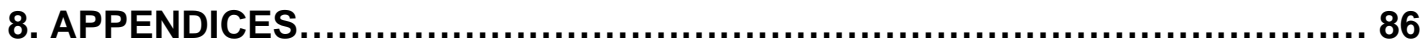

8.1 Chemical analysis of oxidized Petro Star diesel...................... 86

8.2 Chemical analysis of hydrotreated Petro Star diesel.................. 99

8.3 Nucleotide sequence of IGTS8 plasmids............................ 106

8.4 BDS/HDS combination cases report and cost estimates............... 152 


\section{List of Figures and Tables}

Figure

Page

1. Dsz enzymes and the 4 S pathway of DBT oxidation .......................... 12

2. Physiology of DBT oxidation from an oil matrix............................... 14

3. Gradient conditions and parent ion/fragment ion relationships for analysis of BT and DBT compounds....................................... 19

4. Scheme for the synthesis of the 4, 6-dimethylbenzothiophene sultine derivative ............................................................... 24

5. Reductase activity of fusion proteins and comparison to native Fre.............. 28

6. Phylogenetic tree of known sulfone/sulfan monoxygenases, nitriloacetate monooxygenases and hypothetical flavin-dependent

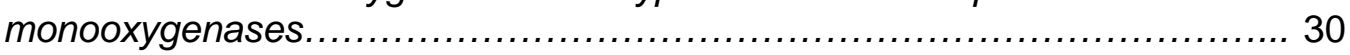

7. Sequence alignment of DszA and ToeA proteins........................... 30

8. Diagram of hydroxybiphenyl detection reporter plasmid..................... 32

9. Map of bioreporter plasmid showing sites of IS insertions.................... 33

10. Protocol for analysis of DszA GSSM library............................. 36

11. Photograph of robotic platform used for DSZA GSSM assay................ 37

12. Example of DszA test plate data....................................... 37

13. Example of DszA GSSM data of mutations at residue 112 (leucine)........... 38

14. Results from sequencing and enzymatic assay for an alanine residue........ 39

15. Results from sequencing and enzymatic assay for an aspartate residue..... 40

16. Results from sequencing and enzymatic assay of a non-permissive residue (Arg)

17. Results from sequencing and enzymatic assay of a permissive residue (Glu)........................................................ 41

18. Results from primary and secondary screen for a selected mutant.......... 42

19. Primary screening data from GSSM optimization of DszA residue A......... 43

20. Secondary screening data for two DszA residue $A$ variants................... 43

21. Tertiary screening data from two $D$ szA residue $A$ variants .................... 44

22. Primary screening data from GSSM optimization of DszA residue B......... 44

23. Secondary screening data for two DszA residue $B$ variants.................. 45

24. Tertiary screening data from two DszA residue $B$ variants................... 45

25. Primary screening data from GSSM optimization of DszA residue C......... 45

26. Secondary screening data for two DszA residue $C$ variants................... 46

27. Tertiary screening data from two DszA residue $C$ variants.................... 46

28. Effect of varying the amount of flavin reductase on ratio of
diMetDBTO $\mathrm{BO}_{2}$ to $\mathrm{DBTO} \mathrm{O}_{2}$ activity of DszA mutants........................... 47

29. Ribbon model of DszA derived from threading of E. coli SsuD protein........ 48

30. GSSM data superimposed onto DszA space-filled model..................... 48

31. Hypothetical active site glutamate active residue, indicated by asterisk, as determined by homology and GSSM data...................... 49

32. GSSM variants selected for GeneReassembly and activity on substrates relative to wild-type parent.................................... 50

33. Parental contribution to each of the 142 randomly selected and sequenced GeneReassembly mutants.

34. Distribution of the number of parental contributions to each of the 142 sequenced GeneReassembly mutants... 
35. Relative contribution of each of the parents to the final library as seen in the 142 sequenced GeneReassembly mutants.............. 51

36. Plasmid pEB1104 and strategy for cloning DszA variants for expression in Rhodococcus CPE648.

37. Purification of DszA and DszC proteins expressed in Rhodococcus IGTS8 (pEBC1104).

38. Kinetic analysis of wild-type DszA and variant activities on $\mathrm{DBTO}_{2}$ and 4, 6-dimethyl $\mathrm{DBTO} \mathrm{H}_{2}$

39. Result of DszC GSSM variant activity screening for a "permissive" residue on DBT and 4, 6-dimethyIDBT.

40. Result of DszC GSSM variant activity screening for a "restrictive" residue on DBT and 4, 6-dimethyIDBT.

41. Example of a DszC residue showing substitutions leading to a selective reduction in activity on 4, 6-dimethyl DBT.....

42. Structural model of DszC derived from threading to the known structure of an acyl CoA dehydrogenase.

43. Mapping of DszC GSSM residue substitutions to a predicted structure of DszC...

44. Electron micrograph of Rhodococcus opacus DSM 43943 and accumulated droplets of triacylglycerols....

45. Electroporation efficiency of representative Rhodococcus opacus strains

46. Utilization of organosulfur compounds by R. opacus strains as sole source of sulfur in strains containing pEBC1100.

47. Transient survivability of strains with hexadecane....

48. Continuous survivability of strains with hexadecane.

49. Whole cell BDS activity of R. opacus strains containing pEBC1104.

50. Whole cell BDS activity of $R$. opacus strains DSM 43943 and DSM 44311 containing pEBC1104.

51. Activity of pEBC1104-containing strains in DBT-hexadecane shake flask assay....

52. Whole cell BDS activity of BKO53A compared to two independent isolates of $R$. opacus DSM43943 containing pBKO53A plasmid.

53. Time course of biocatalyst activity on partially hydrotreated diesel fuel (418ppm) showing reduction of CX-DBT compounds.

54. Time course of IGTS8-BKO53A biocatalyst activity on partially hydrotreated diesel fuel (418ppm)....

55. Expanded chromatogram of 22hour time point

56. Time course of IGTS8-BKO53A biocatalyst activity on partially hydrotreated diesel fuel (43ppm).

57. Activity of biocatalysts on partially hydrotreated diesel fuels with and without the addition of glucose as reductant and the effect on total sulfur.....

58. Activity of biocatalysts on partially hydrotreated diesel fuels with and without the addition of glucose as reductant and the effect on $C_{x}-D B T$ compounds. 
60. Phenotypes of Rhodococcus hosts on BSM-glucose and $B S M-g l u c o s e$ overlaid with diesel.

61. Role of thymidylate synthase (ThyA) in cellular metabolism and thymidine salvage pathway.

62. Diagram of the strategy for generating an antibiotic markerless selection system for the introduction and maintenance of modified dsz genes.

63. Generation of primary cointegrate for construction of JB55 thyA deletion mutant.

64. Generation of second crossover and creation of JB55 thyA deletion mutant

65. Growth phenotype of thyA mutant in the presence and absence of added thymidine.

66. Diagram of dsz gene cassettes for integration into JB55 thyA deletion mutant.....

67. Whole cell BDS activity of BKO53A vs. JB55 $\triangle$ thy $A-d s z$ integrants..... 76

68. Map of the $90 \mathrm{~kb}(85,328 \mathrm{bp})$ IGTS8 plasmid............................ 77

69. Maps of the two largest IGTS8 pSOX contigs............................ 78

70. Maps of the two smallest IGTS8 pSOX contigs............................ 79

\section{$\underline{\text { Table }}$}

1. Cloning and expression of Dsz genes from various strains.................. 25

2. Subcloning and activity of flavin reductases............................... 26

3. Assay of Rhodococcus IGTS8 DszA using different flavin reductases.......... 26

4. Activity of DszA and ToeA proteins with different benzothiophene

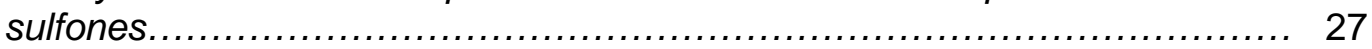

5. Activity of DszC from Rhodococcus IGTS8............................... 27

6. In vitro activities of reassembled DSZA GSSM variants on $\mathrm{DBTO}_{2}$ and 4,6-dimethylDBTO $\mathrm{Iin}_{2}$ bold) (uM product/endpoint)............ 52

7. Specific activities of wild-type DszA and variants on $\mathrm{DBTO}_{2}$ and 4, 6-dimethyIDBTO 


\subsection{EXECUTIVE SUMMARY}

In this report we present a summary and overview of the studies carried out to evaluate and develop a biocatalyst for the removal of organosulfur compounds from diesel fuel.

We will describe our original proposed goals to develop a biocatalyst for standalone use or in combination with Conversion-Extraction Desulfurization technology and the reasons for revision to, instead examine the possibilities of using a biocatalyst in combination with established hydrodesulfurization technologies.

Analytical studies to determine the organosulfur composition of chemically oxidized, as well as partially hydrotreated, diesel fuel are presented. This work defines the extreme heterogeneity of organosulfur compounds found in diesel fuel and highlights the challenges of achieving EPA ULSD targets with a biocatalyst.

Efforts to characterize known biodesulfurization enzymes and discover new enzymes to address the challenge of the highly heterogeneous substrates that must be acted upon are described. These include both sequence-based and functional discovery efforts and the development of new screening strains for this purpose.

Directed evolution of the DszA and DszC proteins of IGTS8 to meet the challenges of a combined BDS/HDS process are described. This effort resulted in the identification of novel DszA variants with improved activity on relevant substrates as well as a more thorough understanding of the structure-function of both of these important enzymes.

The importance of the biocatalyst host is demonstrated by a number of different studies presented here and we describe our efforts to identify new potential hosts for the expression of the biodesulfurization phenotype. Strains with improved growth characteristics and biocatalytic properties have been identified and might serve as the basis for further development of a host for whole cell oxidations of organosulfur compounds as well as other economically important biotransformations.

Because of the need for a robust recombinant biodesulfurization biocatalyst that is able to maintain its desirable properties without the need for the addition of antibiotics to maintain stability, we describe our efforts to generate an antibiotic-free selection system and demonstration of its viability for the introduction,expression and maintenance of improved dsz genes.

Finally, we present a comprehensive economic study of the cost of biodesulfurization as a standalone process as well as in combination with hydrotreatment and compare the costs to the current hydrotreatment process. This work serves as a basis for any additional studies in this area and describes the performance and economic challenges to successful commercial implementation of this novel technology. 


\title{
2.0 INTRODUCTION
}

\author{
Overview \\ This proposal responds to the U.S. Department of Energy's solicitation \\ announcement "Development of Technologies and Capabilities of Fossil Energy-wide \\ Coal, Natural Gas, and Oil R\&D Programs", Area of Interest \#15: Oil Technology - \\ Emerging Process Technology. Petro Star Inc. is proposing a project to improve existing \\ biodesulfurization (BDS) technology for commercially desulfurizing diesel fuel.
}

\subsection{Regulations}

Sulfur in diesel fuel is not only a source of air pollution, but also plays a significant role in determining the tailpipe emissions of other pollutants, such as nitrogen oxides, carbon monoxide, and particulate matter because if its inhibitory effect on current catalytic systems. The Environmental Protection Agency and associated government agencies have mandated the reduction of sulfur in on-road diesel fuels to $15 \mathrm{ppm}$ by June, 2006 and 10ppm by 2010. As most of the current and known reserves of petroleum in the world have increasingly higher levels of organosulfur compounds (sour vs. sweet), this will require greater cost and infrastructure to meet these new ultra-low sulfur in diesel (ULSD) requirements using exist hydro-desulfurization technology.

\subsection{Alternative Desulfurization technologies}

\section{Hydrotreatment}

Hydrodesulfurization (HDS) is the current refinery standard for removing sulfur from diesel fuel. HDS is an expensive and energy-intensive process, requiring high temperature and pressure. It also requires expensive collateral processes to generate hydrogen for the HDS process and to convert the hydrogen sulfide by-product from a poisonous, odorous gas into elemental sulfur. For small refiners HDS is not costeffective because of the high capital and energy costs.

\section{Adsorption}

Another method reported for removal of sulfur compounds from fuel is the use of a catalyst/adsorbent primarily as developed by Phillips Petroleum (S-Zorb). A proprietary adsorbent material is used to bind the reduced sulfur compounds resulting from a mild hydrotreatment process. Originally promoted for the removal of sulfur from gasoline, it has also been proposed for use with diesel, although capital costs appear to be prohibitive and plans for a demonstration unit appear to be on hold.

\section{Chemical Oxidation/Extraction}

In contrast to the previous methods, chemical oxidation of sulfur-containing diesel fuels through the action of peroxides results in the production of sulfones that can be selectively removed by extraction due to their reduced hydrophobicity. Of particular interest is the ability of the process to act on the highly alkylated dibenzothiophenes that 
are particularly difficult for removal by current hydrotreatment catalysts. This method however suffers from a major disadvantage of yield losses due to the removal of carboncontaining sulfur compounds as well as some proportion of the fuel because of incomplete selectivity in the extraction process. As of yet, pilot scale performance of the process has yet to be convincingly demonstrated.

\subsection{Biodesulfurization}

Similarly to chemical oxidation, the biological removal of sulfur from organic sulfur compounds in diesel fuel involves its oxidation to more hydrophilic, water soluble species that can be separated from the oil phase.

\subsubsection{Microbiology}

The ability to oxidize organic sulfur compounds from fossil fuels such as coal and petroleum in order to utilize the sulfur as a nutrient is widespread amongst fungi and bacterial species. Many bacterial have been reported to oxidize organic sulfur compounds including Sphingomonas, Tsukamarella, Bacillus, Mycobacterium, Nocardia, and Rhodococcus sp..

The first organism with this ability was identified and characterized by the Institute of Gas Technology and was called Rhodococcus sp. IGTS8 (1). Rhodococci are members of the Actinomycetes and are characterized by their high $\mathrm{G}+\mathrm{C}$ content and their relatively hydrophobic cell was, consisting of wax-like mycolate esters. Other members of the Actinomycetes include Mycobacterium and Streptomyces. This hydrophobic nature of the cells enables their association with hydrophobic oil and organic compounds and utilization of these compounds as growth substrates.

\subsubsection{Enzymology}

Two main enzymatic pathways have been described for the oxidation of organic sulfur compounds in fossil fuels.

1) The so-called destructive pathway results in ring-opening reactions of the aromatic sulfur-containing compounds and the subsequent utilization of both the carbon skeleton and sulfur. Since this pathway would result in the degradation of the hydrocarbon components of fuel it is not a major focus of research in the industrial use of biodesulfurization.

2) The non-destructive, or 4S oxidation pathway (Figure 1), in contrast, results in the specific removal of the sulfur without degradation of the hydrocarbon skeleton and is therefore the preferred pathway for commercial biodesulfurization technology.

Three enzymes are directly involved in the four oxidation steps (hence 4S pathway) and conversion of insoluble sulfur compounds in diesel fuel, mainly dibenzothiophenes and benzothiophenes, to water-soluble species. 
The first enzyme in the 4S pathway in DszC, dibenzothiophene monooxygenase, which carries out two sequential oxidation steps, converting the model compound dibenzothiophene to the transient intermediate DBT sulfoxide and finally to DBT sulfone. The oxidation requires two mole equivalents of the reduced flavin $\mathrm{FMNH}_{2}$, regenerated by the flavin reductase DszD from the cellular reductant NADH.

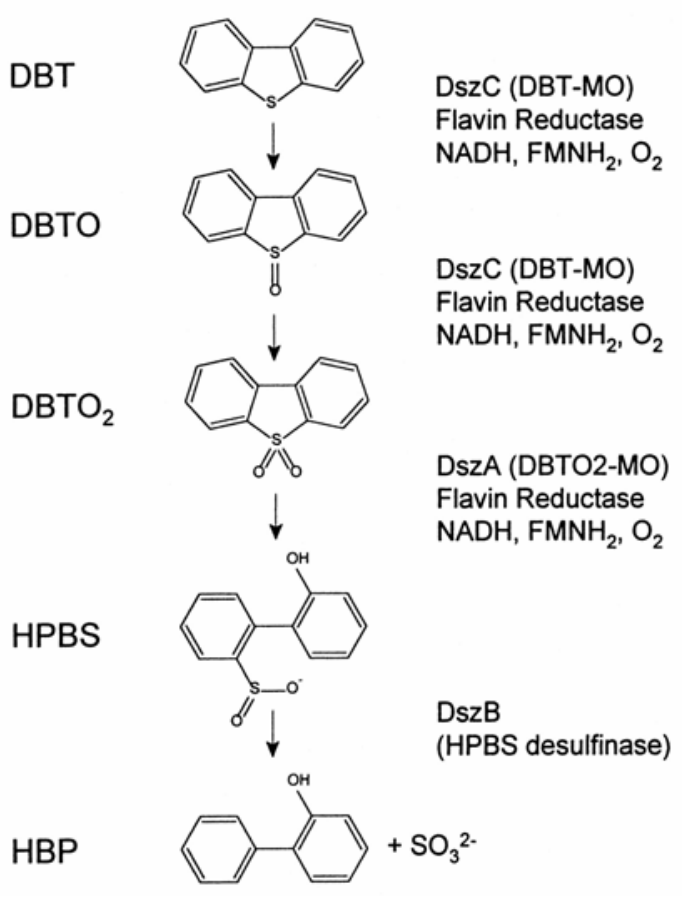

Figure 1. Dsz enzymes and the $4 S$ pathway of DBT oxidation.

The second enzyme in the pathway, DszA (DBT sulfone monooxygenase), carries out the oxidation of DBT sulfone to hydroxy-biphenyl sulfinate (HBPS), the first water soluble organosulfur compound generated. DszA also requires reduced $\mathrm{FMNH}_{2}$ to affect the oxidation step.

The final enzyme in the complete $4 \mathrm{~S}$ pathway is DszB, HBPS sulfinolyase, which converts HBPS to sulfite and hydroxybiphenyl. The sulfite is then assimilated by the microorganism, releasing the hydroxybiphenyl hydrocarbon skeleton to repartition into the oil phase. Although required for organisms to use the sulfite as a source of sulfur, DszB is dispensable to a commercial BDS process as the HBPS is sufficiently water soluble to affect its removal from the oil matrix. It does however lead to a loss of the hydroxybiphenyl skeleton and its contribution to the total hydrocarbon pool available.

As mentioned above, another enzyme, DszD (or equivalent) flavin reductase is required for recycling reduced $\mathrm{FMNH}_{2}$ as cofactor for DszC and DszD but is not directly involved in oxidation of the organosulfur compounds.

Among those organisms characterized at the molecular level for biodesulfurization ability, the amino acid sequences of their Dsz proteins are reasonably well conserved. A 
number of diverse genera possess Dsz proteins whose sequences are very similar to those of the prototype organism Rhodococcus sp. IGTS8, suggesting a potential common origin. In addition, the majority of $d s z$ genes (with the exception of the flavin reductase component) have been found on large extrachromosomal plasmids, in a similar gene order, further suggesting the possibility of their horizontal transmission.

The range of thiophenic substrates that are oxidized by various bacteria is often diverse and is, at least in some measure, determined by the diversity of Dsz sequences. Recent evidence, however, has suggested that the host (and additional genes) contributes significantly to the overall substrate range as well as other phenotypic differences including temperature optima and stability. For example, it has recently been shown that the introduction of a defined $d s z A B C D$ operon (within an artificially constructed transposon) into a number of Rhodococcus and Mycobacterium strains results in a variety of substrate ranges (2). In another example of the contribution of the host to phenotype, it has been shown that the dsz genes of Mycobacterium phlei, while identical to those of Rhodococcus erythropolis IGTS8, display a moderate thermostability (active at $55^{\circ} \mathrm{C}$ ) not seen in IGTS8 (3). Clearly, the contribution of the host physiology and morphology are important to biological removal of sulfur as well as the desulfurization enzymes themselves

\subsubsection{Issues and Challenges to Commercialization}

Although organisms that can oxidize petroleum organosulfur compound naturally do so at an efficiency required to support their biological sulfur demand, their effectiveness at removing sulfur compounds at a commercially relevant rate falls far short (estimated to require $>1 \mathrm{mM}$ DBT/gram of biocatalyst DCW/hour). A number of limitations and issues must be overcome in order to develop a viable biocatalyst and are described briefly here.

\section{Mass transfer}

Because of the relatively insoluble nature of the organosulfur compounds in petroleum and the high molecular weights of their alkylated structures, access of the desulfurization enzymes to their substrates is limited. This requires both the intimate interaction of the organism to oil droplets containing the substrates as well as intracellular solubilization of the substrates into aqueous solvent in order to be acted upon by the DszC enzyme, the first step in the conversion of the very hydrophobic BT and DBT substrates to more water-soluble oxidized molecules (Figure 2).

This is a significant issue for the development of a commercial biodesulfurization process and must be dealt with through a combination of process engineering, to generate efficient oil/water emulsions, and appropriate choice of biocatalyst organism. A number of different organisms have been considered as biocatalysts but vary greatly in their ability to interact with relevant substrates dissolved in an oil matrix. For instance, although Pseudomonas sp. have been investigated as biodesulfurization hosts (by introduction of non-native $d s z$ genes) and shown very high levels of activity on substrates presented in aqueous phases, they fall short in their ability to oxidize the same substrates directly from the oil matrix. This is likely due to the relatively hydrophilic nature of these organisms and their tendency to partition to the water phase in separated oil/water emulsions. In contrast, Rhodococcus and Mycobacterium sp., which 
have a relatively hydrophobic, waxy cell wall associate more readily with the oil phase and have better activity on substrates dissolved in the oil.

\section{Substrate range and extent}

Although dibenzothiophene and benzothiophene are typically used as model compounds in the development of biodesulfurization strains, the true composition of organosulfur compounds in petroleum feedstocks is much more diverse. In addition to the highly alkylated derivatives of these compounds there are also a broad range of minor alkyl and aryl sulfide species that must be removed in order to meet EPA specification for ultra-low sulfur products. This requires not only a broad substrate range for each of the three Dsz enzymes as individuals but their coordinated ability to completely oxidize organosulfur compounds to avoid bottlenecks and accumulation of intermediate compounds. Although the contribution of any given organosulfur species to the total sulfur content may be small, the ability of the biocatalyst to completely remove all species is essential to commercial viability.

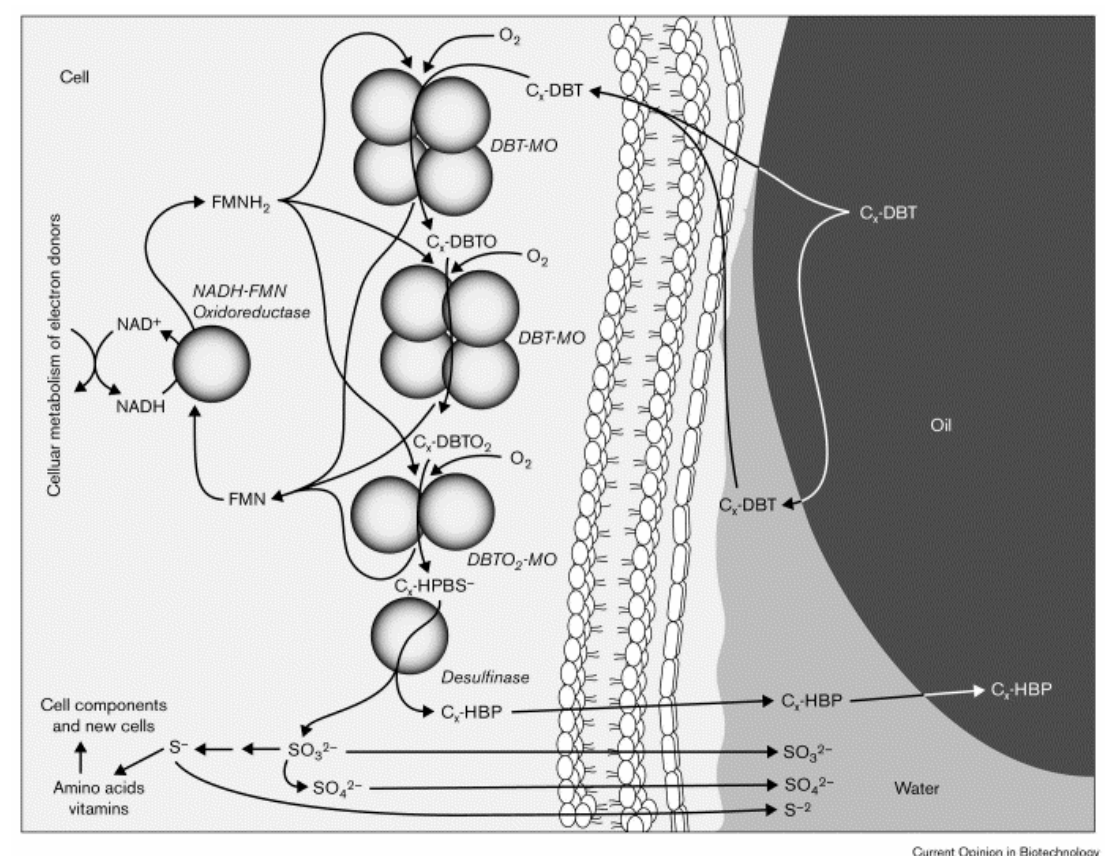

Figure 2. Physiology of DBT oxidation from an oil matrix. This figure was taken from the following reference: Monticello, D,J. (2000). Biodesulfurization and the upgrading of petroleum distillates. Curr. Opin. Biotechnol., 11:540-546

\section{Enzyme rates and affinities}

In order to meet the requirements for a viable commercial process, with limited residence times for the biocatalyst and petroleum feedstock to act, it is essential to oxidize the substrates as quickly as possible for their removal from the oil phase. Additionally, as mentioned above, it is important for the activities of the three enzymes to be as closely coordinated as possible, both in terms of substrate affinity and relative abundance, in order to avoid bottlenecks in the conversion of substrates to a watersoluble form that can be separated and removed. Finally, because of the highly diverse organosulfur compounds and their relatively small individual contribution to the total 
sulfur content, the affinity of the enzymes must be extremely high in order to act on the substrates without extended periods of time being required for their complete conversion.

\section{Reductant supply}

The biological oxidation of organosulfur compounds by DszC and DszA requires reducing equivalents in the form of the reduced flavin $\mathrm{FMNH}_{2}$, via the action of the flavin reductase DszD, at the expense of $\mathrm{NADH}$. It is this requirement that precludes the development of an in vitro process due to the extremely high expense of redox recycling and impracticalities at the scale required for commercial biodesulfurization.

Even an in vivo system requires input in the form of a co-oxidizable substrate to provide reducing equivalents. A number of different substrates have been proposed but their utility is a function of their economic and metabolic costs. For instance, glucose is a common substrate for commercial fermentations but its cost is prohibitive in a commercial biodesulfurization process. Cheaper substrates like acetate, ethanol or methanol have been proposed but their energetic value to the biocatalyst is somewhat limited and significantly more must be used to provide sufficient reducing equivalents to the process. Alkanes such as octane or hexadecane have also been suggested as they have a relatively high energy value and could be provided as a side stream in a petroleum refining operation but an organism's ability to metabolize these compounds would have implications for the fate of the bulk alkane phase of the petroleum feedstock to be desulfurized.

The intracellular machinery providing reductant to Dsz enzymes is also critical in order to maintain a proper redox balance in the cell as a whole. Properly coordinated expression and activity of the flavin reductase component with the Dsz enzymes will be essential in order to avoid either an undersupply or oversupply of reduced flavin. Reduced flavins, in particular, $\mathrm{FMNH}_{2}$, can auto-oxidize and produce oxygen free radicals that can poison the cell by attacking sensitive enzymes and metabolic pathways, reducing the stability and longevity of a viable biocatalyst.

\section{Extent}

Directly related to the issues described above, the extent of biodesulfurization and removal of organosulfur compounds is critical to the commercial viability of the process. In order to fully remove these compounds will require a biocatalyst with sufficient rates and affinities for the diverse substrates present in the feedstock. As the majority of the sulfur compounds are removed, it will take increasingly longer periods of time for their full removal, particularly as the $\mathrm{K}_{\mathrm{m}}$ for the individual species is approached. In addition, the organism must retain viability during the process and be able to efficiently carry out the oxidation of organosulfur compounds and metabolism of substrates to provide the necessary for extended periods of time and cycles before the need to regenerate the biocatalyst by fermentation. 
Recycling, robustness and biocatalyst supply

In order to achieve the economics of a biocatalyst for desulfurization it will be necessary to not only prepare large enough quantities of cells for initial but also to recharge the biocatalyst between rounds of feedstock treatment. This is required to allow the cell to rebuild the cellular enzymes and structure to carry out additional rounds of biodesulfurization. For this to be an effective process will require that the biocatalyst be very robust, not only to constituents of the petroleum feedstock that might otherwise be inhibitory or toxic to growth but be relatively robust to contaminants that often plague bacterial fermentations at industrial scale.

Waste disposal and value-added products

As with any desulfurization process, there is a need for the economic disposal of the sulfur that results, in whatever form it may take. Since the goal of biodesulfurization is to convert sulfur compounds to a water-soluble form that can be separated from the bulk oil, the combined actions of DszC and DszA are enough to convert hydrophobic organosulfur compounds to the corresponding water-soluble organosulfinates. This class of compounds can be chemically converted to potential value-added surfactants for use in the detergent and structural materials industries although significant effort to create commercial interest by Energy Biosystems was unsuccessful. Another issue with terminating the process at this point is the loss of the hydrocarbon backbone which will affect the overall yield of the treated petroleum. The addition of DszB to the biocatalyst results in the conversion of the organosulfinates to sulfite and the corresponding hydrocarbon backbone. The hydrocarbon backbone can then repartition back to the bulk oil phase and reduce the overall yield loss. The sulfite that results however has no valueadded properties and must somehow be economically disposed of.

\section{Project Engineering}

Critical to the success of a commercially viable biodesulfurization process is the design and implementation of the process engineering and supporting infrastructure. As the ultimate design parameters are highly dependent on biocatalyst performance and properties, mutually inclusive assumptions about each must be made during the course of development of the overall process. Although described in more detail in the Economic Report in Appendix 4, many processes must be developed and interconnected to create an operational unit. These include fermentation facilities, oil/water emulsion generation, separation methods, product and waste-stream management, cooling, heating and recirculation equipment and sterilization. 


\subsection{Original focus of research plan}

The original objective of this proposal consisted of two distinct overlapping efforts:

The first was to develop a standalone biocatalyst that could effectively remove a large portion of sulfur from diesel fuel at commercially acceptable rates. The original performance requirements were to achieve reduction of sulfur levels from $\sim 3500 \mathrm{ppm}$ in

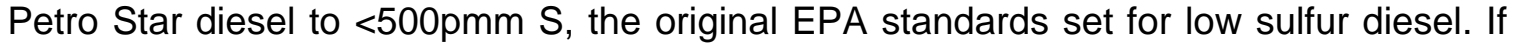
successful, the team, consisting of Petro Star, Pelorus and Anvil were to develop design parameters and cost estimates for a biocatalytic desulfurization demonstration unit to produce about 5,000 barrels per day (bpd) of low-sulfur diesel.

The second effort, to be carried out in parallel to the first, was to develop a biocatalyst for use in a combined process with a Conversion Extraction Desulfurization (CED) technology being developed by Petro Star in collaboration with Degussa. The CED process was designed to produce an ultra-low diesel fuel by converting organosulfur compounds in the diesel to their sulfone derivatives using a chemical oxidation procedure. The more hydrophobic sulfones could then be selectively extracted to remove from the diesel bulk phase. An issue with the process was the loss of fuel to the sulfur-concentrated extract in both the form of the lost hydrocarbon backbone and entrained alkane bulk phase. It was proposed that a biocatalyst could work synergistically with the CED process, as it would require only the activity of the DszA and $\mathrm{B}$ enzymes since the chemical conversion process generated the sulfone derivatives normally created by DszC. In addition, as the CED-derived sulfones were more hydrophilic, mass transfer issues of the biocatalyst might be more efficiently addressed. If successful, this would increase the yield of marketable fuel and raise the economical viability of the CED process.

\subsection{Revised focus of research plan}

In the course of carrying out the original objectives of the proposal, work on the CED process by Petro Star and Degussa was coming to completion. A conclusion of this work was that the economics of the process at scale would prove cost-prohibitive either as a standalone process or in combination with BDS. Only incremental gains in cost-savings would be achieved in the combined process and would require the introduction of two untested novel technologies simultaneously.

In addition, new EPA requirements for ULSD were introduced during the course of our initial work that precluded the use of BDS as a standalone process. Although the original goal of achieving reductions of sulfur from $3500 \mathrm{ppm}$ to $<500 \mathrm{ppm}$ were challenging in itself, the ability of a biocatalyst to reduce sulfur to $<15 \mathrm{ppm}$ were felt to be unattainable within reasonable economic and performance parameter.

For this reason, we revised our goals to develop a biocatalyst that could be used in combination with conventional hydrotreatment to help reduce the high capital and recurrent costs that would be incurred by the industry with new ULSD requirements.

With this mind, we set out to develop a biocatalyst that could act on organosulfur substrates that are known to be problematic for HDS, namely 4-methyl and 4, 6- 
Final Technical Progress Report DOE Award No. DE-FC26-02NT15340

dimethyl DBT. If these compounds could be reduced by a biocatalyst, either before or after hydrotreatment, lesser amounts of hydrogen, temperature and pressure could be used to finally produce an ULSD that would meet EPA requirements at reduced cost. 


\subsection{EXPERIMENTAL}

\subsection{Chemical analysis}

Analyses of benzothiophenes and dibenzothiophene sulfone derivatives, either synthesized as standards or experimentally determined from chemically or biologically oxidized diesel were carried out using HPLC-MS-MS and GC-MS. HPLC-MS-MS for the determination BT and DBT sulfone substrates and products was carried out by injecting samples from 96-well plates using a CTCPAL autosampler (Leap Technologies, Carrboro, N.C.) into an LC mobile phase of various mixtures of $\mathrm{H}_{2} \mathrm{O} / \mathrm{ACN}(0.1 \%$ formic acid) provided by LC-10ADvp pumps (Shimadzu, Kyoto, Japan) at $1.0 \mathrm{~mL} / \mathrm{min}$ through an Agilent Zorbax C8 column (4.6 x $150 \mathrm{~mm}$ ) (Agilent Technologies, Palo Alto, CA) to an API 4000 Turbolon spray triple-quadrupole mass spectrometer (Applied Biosystems, Foster City, CA). Ion spray and Multiple Reaction Monitoring (MRM) were performed for analytes in the positive ion mode. Initial ionization produces parent ions, fragments of which are observed and quantified. Instrumentation control and data generation was accomplished using Analyst 1.2 software (Applied Biosystems, Foster City, CA). A detailed description of the gradient conditions and parent/fragment ion relationships for MS/MS are described in Figure 3.

\begin{tabular}{|c|c|c|}
\hline \multicolumn{2}{|c|}{ Substrate: } & Parent ion/fragment ion \\
\hline \multicolumn{2}{|c|}{$\mathrm{DBTO}_{2}:$} & 217.269/152.104 \\
\hline \multicolumn{2}{|c|}{ HBPS: } & $217.265 / 168.097$ \\
\hline 0.0 & $50 \% \mathrm{~A}$ & $\mathrm{H}_{2} \mathrm{O} / \mathrm{ACN}$ gradient $@ 1200$ uL/min for 3 minutes \\
\hline 2.0 & $10 \% \mathrm{~A}$ & \\
\hline 2.1 & $50 \% \mathrm{~A}$ & \\
\hline 3.0 & $50 \% \mathrm{~A}$ & \\
\hline \multicolumn{2}{|c|}{ 4-MeDBBTO ${ }_{2}:$} & 231.155/152.138 \\
\hline \multicolumn{2}{|c|}{ 4,6-DiMeDBTO ${ }_{2}:$} & $245.194 / 165.235$ \\
\hline \multicolumn{2}{|c|}{ 4-EtDBTO } & 245.207/165.091 \\
\hline \multirow{2}{*}{\multicolumn{2}{|c|}{$\begin{array}{l}\text { 4,6-DiEtDBTO } \\
\text { 2,8-DiMeDBTO }\end{array}$}} & 273.224/181.004 \\
\hline & & $245.057 / 165.255$ \\
\hline \multicolumn{2}{|c|}{$\mathrm{H}_{2} \mathrm{O} / \mathrm{ACN}$} & $25 / 75$ isocratic mixture @ 1200 uL/min for 3 minutes \\
\hline \multicolumn{2}{|c|}{ 2,4,6 TriMeDBTO $_{2}:$} & 259.032/165.082 \\
\hline \multirow{2}{*}{\multicolumn{2}{|c|}{$\begin{array}{l}\mathrm{BTO}_{2} \text { : } \\
2-\mathrm{MeBTO}_{2}\end{array}$}} & $167.127 / 103.149$ \\
\hline \multirow{2}{*}{\multicolumn{2}{|c|}{ 2-MeBTO ${ }_{2}:$}} & 181.145/91.035 \\
\hline & & 181.108/91.051 \\
\hline \multicolumn{2}{|c|}{ 5-MeBTO } & 181.119/91.133 \\
\hline \multicolumn{2}{|c|}{ 7-MeBTO } & $181.152 / 91.025$ \\
\hline \multicolumn{2}{|c|}{$\mathrm{H}_{2} \mathrm{O} / \mathrm{ACN}$} & $20 / 80$ isocratic mixture @ 1000 uL/min for 3 minutes \\
\hline \multicolumn{2}{|c|}{ DBT: } & 184.085/152.107 \\
\hline \multicolumn{2}{|c|}{ DBTO: } & 201.116/183.971 \\
\hline \multicolumn{2}{|c|}{ 4,6-DiMeDBT: } & 213.163/184.153 \\
\hline \multicolumn{2}{|c|}{ 4-MeDBT: } & $198.137 / 165.170$ \\
\hline \multicolumn{2}{|c|}{ ACN } & $100 \% @ 1000$ uL/min for 3 minutes \\
\hline
\end{tabular}


For high-throughput screening of DszA GSSM variants, activity was determined on $\mathrm{DBTO}_{2}$ and 4, 6-dimethylDBTO${ }_{2}$, analyzing resultant HBPS derivatives. For highthroughput screening of DszC GSSM variants, activity on DBT and 4, 6-dimethyIDBT was determined, analyzing resultant sulfone derivatives.

For analyses of single- and two-phase biodesulfurization assays, hexadecane/diesel and/or water samples for the biocatalytic reactions were initially diluted 10-fold into ethanol, and then further diluted 10-fold in acetonitrile before separation and analysis by LC/MS/MS. Cells from the reactions were isolated from the oil and water phase by centrifugation, extracted with methanol and diluted into acetonitrile for analyses. In the above examples, all relevant compounds (dibenzothiophenes, -sulfoxides, -sulfones and -sulfinates), with the exception of hydroxybiphenyl derivatives, could be determined in a single run. Hydroxybiphenyls were determined by using the negative ion mode in a separate separation. For analysis of total sulfur, samples from the initial 10-fold dilution in ethanol were further diluted in ethanol before analysis on the Antek 9000 sulfur analyzer.

For GC separations, 1ul samples were injected onto a DB-1 column (30m x $0.25 \mathrm{~mm} \times$ $0.25 \mathrm{um}$ ) with helium carrier gas at a flow rate of $1.5 \mathrm{ml} / \mathrm{min}$. Oven temperature ranged from $100^{\circ} \mathrm{C}$ to $240^{\circ} \mathrm{C}$ with a gradient of $4^{\circ} \mathrm{C} / \mathrm{min}$ (injection/detection temp. $290^{\circ} \mathrm{C} / 320^{\circ} \mathrm{C}$ ) and a run time of 35 minutes. Sample streams were split into two for detection by either MS for total ion composition or SCD for sulfur-containing ion determination.

\subsection{Microbiological methods}

Microbiological culture and media. Standard microbiological procedures were used for the isolation and cultivation of bacterial strains. All strains were cultivated on either nutrient or Luria broth (4), with appropriate additions of vitamin supplements and antibiotics when necessary. A basal salts medium was used for preparation and testing of biocatalyst strains, using DMSO as sole source of sulfur when preparing cells for in vivo activity determination. Basal salts media was composed of the following per liter: $\mathrm{Na}_{2} \mathrm{HPO4}$, 5.11g; $\mathrm{KH}_{2} \mathrm{P04}, 1.90 \mathrm{~g} ; \mathrm{NH}_{4} \mathrm{Cl}, 1 \mathrm{~g} ; \mathrm{MgCl}_{2}{ }^{*} 6 \mathrm{H}_{2} \mathrm{O}$ ), $0.2 \mathrm{~g}$, Hutner's minerals, $5 \mathrm{ml}$; $50 \%$ glucose, $10 \mathrm{ml} ; \mathrm{pH} 7.2$.

\subsection{Molecular biology}

Molecular biology. Generation of PCR products was carried out using established procedures with varying incubation temperatures and cycling conditions according to experimental requirements. Recombinant DNA procedures were carried out according to methods previously described (4). Nucleotide sequencing was carried out using the method of Sanger and dye termination chemistry and analysis by the ABI 3700 DNA Analyzer.

For cloning of Dsz and Dsz-homologues for expression and characterization, PCR products of the coding regions were amplified and cloned into pASK5 plasmid between $\mathrm{Ncol}$ and Bg/ll sites to create a plasmid that expresses native or carboxy-terminal hexahistidine-tagged oxidoreductases. For amino-terminal hexahistidine-tagged proteins, the PCR product was cloned into PASK1 between Bg/II and HindIII sites 
For preparation of the Rhodococcus IGTS8 plasmids for sequence characterization, a procedure for the isolation of plasmids from this organism was developed. A single colony was inoculated into $200 \mathrm{mls}$ of $2 x Y T$ medium in a $2 \mathrm{~L}$ flask and incubated for 48 hours at $30^{\circ} \mathrm{C}, 250$ RPM. Fifty mls of culture was then inoculated into each of four flasks containing $400 \mathrm{ml} 2 \mathrm{xYT}$ with $2 \%$ glycine and further incubated for 8 hours. Cells were harvested, washed with $0.5 \mathrm{X}$ volume of $50 \mathrm{mM}$ EDTA, pH8.0, and the cell pellet resuspended in $80 \mathrm{ml}$ Qiagen solution I (Qiagen, Valencia, CA). Mutanolysin (100U/ml) and lysozyme $(2 \mathrm{mg} / \mathrm{ml})$ was then added and the cells incubated for 1 hour at $37^{\circ} \mathrm{C}$. From this point, directions for the Qiagen Large Construct Kit were followed.

To construct an ordered cosmid library of the IGTS8 plasmids, DNA was randomly sheared to obtain $30-45 \mathrm{~kb}$ fragments. After treating digested DNA to repair ends, these fragments were then ligated into Eco72I-digested pCC1FOS (Epicentre, Madison, WI). Ligated DNA was packaged and transfected into E. coli cells. Recombinants were selected by plating transfected cells on L-agar with chloramphenicol $(12.5 \mathrm{ug} / \mathrm{ml})$. Plasmid DNA was prepared and clones characterized by restriction digest.

To construct a small insert library for sequencing, DNA was randomly sheared and end repaired followed by ligation into pCR4TopoBlunt (Invitrogen, Carlsbad, CA). Individual clones were grown in 96-well microtiter plates and plasmid DNA was prepared for nucleotide sequencing with dye termination chemistry. Nucleotide sequences obtained in this manner were assembled using Sequencher ${ }^{\mathrm{TM}}$ software (Gene Codes Corp., Ann Arbor, MI).

\subsection{Expression and purification of enzymes}

Expression and Purification. E. coli TOP10 (Invitrogen, Carlsbad, CA) were used as host for the expression of recombinant oxidoreductases. Freshly transformed colonies were inoculated into LB medium with kanamycin $(50 \mathrm{ug} / \mathrm{ml})$. $1 \mathrm{~L}$ cultures were grown until $\mathrm{OD}_{600} \sim 1$ and expression was induced with anhydrotetracycline $(10-100 \mu \mathrm{g} / \mathrm{l})$ and carried on overnight at $30^{\circ} \mathrm{C}$. Cells were lysed in buffer I $(50 \mathrm{mM}$ sodium phosphate; $100 \mathrm{mM} \mathrm{NaCl}$ supplemented with lysozyme (EpiCentre, Madison, WI) by Frenchpressure treatment. Cell lysates were clarified by centrifugation at $15,000 \times \mathrm{g}$ for $30 \mathrm{~min}$ and recombinant protein was bound to pre-equilibrated $\mathrm{Ni}^{+2}-\mathrm{NTA}$ resins at $4^{\circ} \mathrm{C}$ for $1 \mathrm{hr}$. Resins were washed with buffer I containing imidazole (20 to $80 \mathrm{mM}$ ) and eluted with 0.5 $\mathrm{M}$ imidazole in buffer I. The eluted protein was dialyzed against buffer II (50 mM Tris pH 7.5, $100 \mathrm{mM} \mathrm{NaCl}$ ) and than against buffer II containing 40\% glycerol. Protein was stored at $-20^{\circ} \mathrm{C}$ in buffer II with $40 \%$ glycerol and $1 \mathrm{mM}$ DTT.

\subsection{Enzymatic assays}

In vitro DszA, DszC assays were carried out as described previously (5) with slight modifications. Purified proteins (200pmols of protein and 450pmols of reductase) as well as crude lysates were incubated with substrates in the presence of $10 \mu \mathrm{M} F M N$, $4 \mathrm{mM} \mathrm{NADH}, 100 \mathrm{mM} \mathrm{NaCl}$, and $25 \mathrm{mM}$ sodium phosphate buffer $(\mathrm{pH} 7.5)$. For DszA assays, $\mathrm{DBTO}_{2}, 4-\mathrm{MeDBTO}_{2}, \mathrm{BTO}_{2}, 3-\mathrm{MeBTO}_{2}, 5-\mathrm{MeBTO}_{2} ; 7-\mathrm{MeBTO}_{2}$ were used at $100 \mu \mathrm{M}$ concentration; 4, 6-diMeDBTO ${ }_{2}$ was used at $30 \mu \mathrm{M}$ concentration, and 2, 4, 8triMeDBTO ${ }_{2}$ was used at 50uM concentration. For DszC assays DBTs and BTs were 
used at $100 \mu \mathrm{M}$ concentration. The reaction mixtures were shaken at $230 \mathrm{rpm}$ at $30^{\circ} \mathrm{C}$. At designated time points, reactions were quenched with equal volume of acetonitrile. Substrate and product concentrations were determined by LC/MS analysis.

In vitro reductase assays were carried out as described previously (5). The reductase activity was measured by the FMN-dependent oxidation of NADH monitored at 340nm. Reactions were carried out at $30^{\circ} \mathrm{C}$ in $50 \mathrm{mM}$ phosphate buffer, in the presence of $0.1 \mathrm{mM}$ $\mathrm{NADH}$ and $20 \mu \mathrm{M} F \mathrm{FN}$. Five to twenty pmols of enzyme was used.

High throughput screening of the DszA and DszC GSSM and GeneReassembly libraries and variants was carried out using modifications of the assays as described above, described in more detail in the respective sections below.

\subsection{Whole cell biocatalysis assays}

To test whole cell activity of biocatalyst strains on either model substrates or diesel fuel, strains were streaked out on BSM-glucose minimal agar to obtain single colonies. A single colony was then inoculated into $1 \mathrm{ml}$ of BSM-glucose liquid media containing appropriate antibiotics, incubated overnight at $30^{\circ} \mathrm{C}$, and then used to inoculate $50 \mathrm{ml}$ of BSMS-glucose-DMSO and further incubated for 2 days at $30^{\circ} \mathrm{C}$. This culture was then used to inoculate an $800 \mathrm{ml}$ culture of BSMS-glucose-DMSO which was grown to $\mathrm{OD}_{600}$ of $\sim 10$ before harvesting. Pelleted cells were then resuspended in $15-20 \mathrm{ml}$ of phosphate buffer and an aliquot removed to determine dry cell weight/ml. To carry out the reaction for model compounds, a 3:1 phosphate buffer-hexadecane emulsion was prepared to which substrate, previously dissolved in hexadecane, was added at the appropriate concentration to be tested. For assays on diesel fuel (either straight-run or partially hydrotreated), 3:1 phosphate buffer-diesel emulsions were directly prepared. Following generation of an emulsion, a source of co-oxidizable substrate, such as glucose, ethanol or acetate, was added to provide cellular reductant. The reaction was incubated by shaking in a baffled flask at $200 \mathrm{rpm}$ and aliquots were removed at various time points. Reactions were terminated and analyzed for reaction products and total sulfur as described above. 


\subsection{RESULTS AND DISCUSSION}

\subsection{Analysis of organosulfur compounds in diesel fuel}

Two analytical efforts were carried out in the course of this work; 1 ) the determination of organosulfur compounds in chemically oxidized Petro Star diesel and 2) the determination of organosulfur compound in partially hydrotreated Petro Star diesel.

\subsubsection{Determination of organosulfur compounds in chemically oxidized Petro Star diesel}

Since the objective of the original research proposal was to develop a biocatalyst that would work in combination with a chemical oxidation/extraction step, it was imperative to determine the composition of the sulfur-containing chemical oxidation products that would need to be acted upon by the biocatalyst for their removal.

Petro Star middle distillate diesel was received and characterized initially for total sulfur content, and determined to be $0.339 \%$ sulfur, in close agreement with results provided by Petro Star $(0.342 \%$ sulfur). A strategy for analyzing Petro Star diesel was developed which incorporates a fractionation step of diesel polar compounds on reverse phaseHPLC (RP-HPLC) before analysis and ion assignments by GC-MS-SCD. The basic assumption of the separation strategy is that sulfur-containing compounds are most likely present in diesel as series of homologues. As each series is separated on RPHPLC, less alkylated (and thus more polar) representatives are eluted first, followed by more highly alkylated homologues. Subsequent GC analysis of these fractions should result in less alkylated homologues appearing at lower retention times. Better-separated peaks should also result from fractionation due to the smaller number of possible isomers in each GC analysis.

Appendix 1 describes in detail the results of these analyses. The origin and nature of the samples, their handling, methodology of analysis and detailed procedures are explained. Qualitative and quantitative results of analysis are discussed.

4.1.2 Determination of organosulfur compounds in partially hydrotreated Petro Star diesel.

As described above, during the course of this work our objectives were modified to develop a biocatalyst to be used in combination with HDS. To determine the nature of the organosulfur compounds that remain after partial hydrotreatment that must be acted upon by the BDS biocatalyst, we obtained partially hydrotreated diesel fuel from Petro Star (provided by a separate subcontractor).

Three samples of diesel fuels with varying degrees of hydrotreatment (1746ppm, $418 \mathrm{ppm}$ and 43ppm residual sulfur) were analyzed, in addition to the a new batch of straight-run fuel (4130ppm). A GC/MS method was developed to identify organosulfur compounds that remained following hydrotreatment. Appendix 2 details these analyses and concludes that, as expected, the majority of identifiable organosulfur remaining after hydrotreatment is 4-methyl and 4, 6-dimethyl DBT but a substantial amount of extremely heterogeneous unidentified organosulfur compounds remain that contribute significantly 
to the total sulfur composition of hydrotreated diesel. It is this "hump" of diverse organosulfur compounds that represents the major challenge to development a biocatalyst that can achieve the ULSD mandated by the EPA

\subsection{Synthesis of standards}

To provide as many standards for subsequent analysis of the biological oxidation products of Petro Star diesel, a number of methods were used for the synthesis of alkylated benzothiophenes and dibenzothiophenes as well as sulfone and sulfinate derivatives. Products were evaluated for structure and purity using nuclear magnetic resonance procedures.

We have acquired parent benzothiophene and three monomethyl derivatives (2-, 3-, and 5 -MeBTs, $\mathrm{C}_{1} \mathrm{BTs}$ ) commercially (various sources). From these we have produced three pure sulfones by standard oxidation with peracetic acid. The 2-methylbenzothiophene sulfone, however, proved to be unstable and was not prepared.

A number of DBT derivatives were synthesized, including 4-MeDBT $\left(\mathrm{C}_{1} \mathrm{DBT}\right)$, 4,6diMeDBT $\left(\mathrm{C}_{2} \mathrm{DBT}\right)$, 4-EtDBT $\left(\mathrm{C}_{2} \mathrm{DBT}\right), 4,6$-diEtDBT $\left(\mathrm{C}_{4} \mathrm{DBT}\right)$, 4-PrDBT $\left(\mathrm{C}_{3} \mathrm{DBT}\right), 4,6-$ diPrDBT $\left(\mathrm{C}_{4} \mathrm{DBT}\right)$, 2,8-diMeDBT $\left(\mathrm{C}_{2} \mathrm{DBT}\right)$, 3,7-diMeDBT $\left(\mathrm{C}_{2} \mathrm{DBT}\right)$, and 2,4,8-triMeDBT $\left(\mathrm{C}_{3} \mathrm{DBT}\right)$. All these DBT compounds were then converted to the corresponding sulfones. In addition, several precursors for alkylated DBTs and a mixture of monoethyl DBTs have been prepared. Precursors include a mixture of monoacylated 4-MeDBT $\left(\mathrm{C}_{3} \mathrm{DBT}\right)$, mixture of bisacylated 4-MeDBT ( $\left.\mathrm{C}_{5} \mathrm{DBT}\right), 2$-acyl-3,7-diMeDBT $\left(\mathrm{C}_{4} \mathrm{DBT}\right)$, mixture of bisacylated 3,7-diMeDBT ( $\left.\mathrm{C}_{6} \mathrm{DBT}\right)$, a mixture of monoacylated 4,6-diMeDBT $\left(\mathrm{C}_{4} \mathrm{DBT}\right)$, and mixture of bisacylated 4,6-diMeDBT $\left(\mathrm{C}_{6} \mathrm{DBT}\right)$.

In addition, a synthetic reaction for the formation of a sultine derivative of 4, 6-dimethyl $\mathrm{DBTO}_{2}$ was devised and carried out (Figure 4). This compound is to be used as a molecular product standard for the quantification of DszA activity on dimethyl $\mathrm{DBTO}_{2}$. The two-step synthesis resulted in the generation of a mixture of two isomers of the sultine. The mixture was analyzed by LC/MS/MS and the fragmentation ions of both isomers were confirmed to be identical to those of the DszA-generated product. As each component can be separated from the other by LC, purification was carried out in order to use the correct single isomer as a quantitative standard.

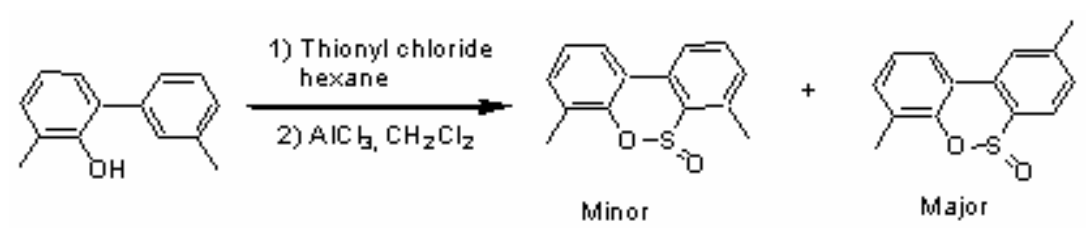

Figure 4. Scheme for the synthesis of the 4, 6-dimethylbenzothiophene sultine derivative.

We had also acquired seven commercially available symmetric dialkyl sulfides (butyl through decyl) and prepared a series of corresponding sulfones. Analysis of GC-MS chromatograms of the sulfones confirmed our initial observation that these compounds are not present in CED of Petro Star diesel. Currently, we use the dialkyl sulfones as internal standards for quantitative analysis of CED fractions. 
4.3 Cloning, expression and characterization of DszA, -B, -C and -D

\subsubsection{Cloning and expression}

In order to most effectively characterize the enzymatic properties of various Dsz enzymes for their substrate specificities and to generate DNA constructions amenable to directed evolution, $d s z$ genes from a variety of sources were subcloned for expression in E. coli. In those cases where DNA sequence was not already available, available genomic clones from the Energy Biosystems strain collection were prepared for DNA sequence analysis. We also cloned and expressed three ToeA proteins, which are close homologues to DszA and have been proposed to have significant activity on benzothiophenes compounds.

From these subclones, we generated expression clones that enabled us to overexpress and purify active proteins for enzymatic characterization. In all cases, with the exception of the DszB proteins, native and His-tagged (both at the $\mathrm{N}$ - and $\mathrm{C}$-termini) derivatives were generated. In addition, we generated subclones of three different flavin reductase accessory proteins, DszD (from Rhodococcus IGTS8), Fre (from E. coli K12) and HpaC (from E. coli W), which can each be used for the generation of $\mathrm{FMNH}_{2}$ reducing equivalents for the DszA and C monooxygenases (summary of constructs in Table 1).

\begin{tabular}{|c|c|c|c|c|}
\hline Enzyme & Organism & Native & $\mathrm{N}-\mathrm{His}$ & C-His \\
\hline \multicolumn{5}{|c|}{$\mathrm{DBT} / \mathrm{BTO}_{2}$ monooxygenase } \\
\hline DszA & Rhodococcus IGTS8 & + & + & + \\
\hline DszA & Nocardia A3HI & + & + & + \\
\hline DszA & Sphingomonas AD109 & + & + & + \\
\hline ToeA & Tsukamarella 670-1 & + & + & + \\
\hline ToeA & Tsukamarella EMT4 & + & + & + \\
\hline ToeA & Nocardia KGB1 & + & + & + \\
\hline \multicolumn{5}{|c|}{ Desulfinase } \\
\hline DszB & Rhodococcus IGTS8 & + & n.t & n.t \\
\hline DszB & Nocardia A3HI & + & n.t & n.t \\
\hline DszB & Sphingomonas AD109 & + & n.t & n.t \\
\hline \multicolumn{5}{|c|}{ DBT/BT monooxygenase } \\
\hline DszC & Rhodococcus IGTS8 & + & + & + \\
\hline DszC & Nocardia A3HI & + & + & + \\
\hline DszC & Sphingomonas AD109 & + & + & + \\
\hline
\end{tabular}

Table 1. Cloning and expression of Dsz genes from various strains.

\subsubsection{Characterization of flavin reductase activities}

In order to evaluate the flavin-dependent monooxygenase in vitro, it requires the addition of a flavin reductase to regenerate the reduced $\mathrm{FMNH}_{2}$ from $\mathrm{NADH}$. Purified DszD, Fre and $\mathrm{HpaC}$ proteins were each evaluated for flavin reductase activity using standard 
assay conditions. From these results it was seen that purified DszD appeared to have more activity than the others (Table 2).

\begin{tabular}{|c|c|c|c|c|}
\hline \multicolumn{2}{|c|}{ Reductase } & Subcloning & Purification & $\begin{array}{c}\text { Specific } \\
\text { activity: } \\
(\mu \mathrm{M} / \mathrm{min} / \mathrm{mg})\end{array}$ \\
\hline \multirow{3}{*}{ IGTS8 DszD } & Native & + & - & $N D^{*}$ \\
\hline & \multirow{2}{*}{$\begin{array}{l}\mathrm{N} \text {-His } \\
\mathrm{C} \text {-His }\end{array}$} & BD10606 & + & 42.9 \\
\hline & & BD10607 & + & 25.0 \\
\hline \multirow{3}{*}{ E. coli Fre } & Native & + & - & ND \\
\hline & \multirow{2}{*}{$\begin{array}{l}\mathrm{N} \text {-His } \\
\mathrm{C} \text {-His }\end{array}$} & + & + & 19.6 \\
\hline & & + & + & 9.8 \\
\hline \multirow{3}{*}{ E. coli $\mathrm{HpaC}$} & Native & + & - & ND \\
\hline & \multirow{2}{*}{$\begin{array}{l}\mathrm{N} \text {-His } \\
\mathrm{C} \text {-His }\end{array}$} & BD10465 & + & 23.3 \\
\hline & & BD10466 & + & 13.7 \\
\hline
\end{tabular}

Table 2. Subcloning and activity of flavin reductases.

To evaluate an optimum combination and concentration together with DszA, each were tested using DBT sulfone as substrate together with IGTS8 DszA. As shown in Table 3, most demonstrated nearly equivalent activity. As the Fre-NHis fusion protein proved easiest to express and purify in large quantities and was the most stable to long term storage (data not shown), it was selected for all further evaluations of in vitro monooxygenase activity.

\begin{tabular}{|c|c|c|}
\hline Flavin Reductase & $\begin{array}{c}\mathrm{uM} \mathrm{DBTO}_{2} \\
\text { conversion/min/200pmole }\end{array}$ & $\begin{array}{c}\mathrm{uM} \mathrm{DBTO}_{2} \\
\text { conversion/min/1nmole }\end{array}$ \\
\hline HpaC-N & $6.5+/-1.3$ & $29.3+/-1.9$ \\
\hline HpaC-C & $7.6+/-1.1$ & $38.0+/-5.7$ \\
\hline Fre-N & $6.1+/-1.4$ & $30.7+/-7.3$ \\
\hline Fre-C & $3.6+/-0.9$ & $17.9+/-4.4$ \\
\hline DszD-N & $5.3+/-1.8$ & $26.4+/-8.8$ \\
\hline
\end{tabular}

Table 3. Assay of Rhodococcus IGTS8 DszA using different flavin reductases.

\subsubsection{Characterization of DszA activities}

Once cloned and expressed we then set out to determine the specific activity of the different DszA proteins with substrates likely to be relevant to biodesulfurization. We focused on the following substrates: $\mathrm{DBTO}_{2}$; 4-methylDBTO ${ }_{2}, 4$, 6-dimethylDBTO $\mathrm{IDT}_{2}, 2$, 4, 8-trimethylDBTO$, \mathrm{BTO}_{2}, 3$-methylBTO $\mathrm{I}_{2} ; 5$-methylBTO${ }_{2}$ and 7-methylBTO $\mathrm{I}_{2}$. In initial experiments, we used crude lysates of $E$. coli expressing the genes of interest, with the addition of purified Fre protein. These initial experiments allow us chose the right conditions for the experiments to be performed with purified proteins. Since the majority of substrates are poorly soluble in water the specific activity was determined at close to their saturation concentration. 
A summary of rates observed is shown in Table 4. From these data, certain characteristics of the enzymes could be seen. For instance, whereas the Rhodococcus and Nocardia DszA proteins demonstrated higher activity towards $\mathrm{DBTO}_{2}$ and derivatives, the Tsukamarella ToeA proteins displayed a preference for $\mathrm{BTO}_{2}$ compounds (unfortunately we were not able to observe activity from the Gordonia KGB ToeA). Sphingomonas DszA, unlike IGTS8 DszA, was most active at pH 8.2 and also required more Fre reductase for optimal activity. The highest specific activity for $\mathrm{DBTO}_{2}$ was obtained with 1:10 DszA:Fre ratio comparing to 1:2 that was optimal for IGTS8 DszA. Under these conditions, specific activity of Sphingomonas DszA with $\mathrm{DBTO}_{2}$ was determined to be $\sim 22 \mathrm{nmols} / \mathrm{min} / \mathrm{nmol}$ of enzyme. In addition, the Sphingomonas DszA appears to have a higher level of activity on unsubstituted benzothiophene sulfone than the IGTS8 enzyme and was less affected by methyl substitution on the dibenzothiophene sulfones.

\begin{tabular}{|c|c|c|c|c|c|c|c|c|}
\hline & DBTO2 & $\begin{array}{c}4- \\
\mathrm{MeDBTO}_{2}\end{array}$ & $\begin{array}{c}4,6- \\
\text { diMeDBTO }_{2}\end{array}$ & $\begin{array}{c}2,4,8- \\
\text { trMeDBTO }_{2}\end{array}$ & $\mathrm{BTO}_{2}$ & $\begin{array}{l}3 \mathrm{Me}_{-} \\
\mathrm{BTO}_{2}\end{array}$ & $\begin{array}{l}5 \mathrm{Me}^{-} \\
\mathrm{BTO}_{2}\end{array}$ & $\begin{array}{l}7 \mathrm{Me}- \\
\mathrm{BTO}_{2}\end{array}$ \\
\hline $\begin{array}{l}\text { Rhodococcus } \\
\text { IGTS8 DszA }\end{array}$ & $\begin{array}{c}1 \\
(23.9+/-6.0) \\
\end{array}$ & $\begin{array}{c}0.4 \\
(9.0+/-1.7) \\
\end{array}$ & $\begin{array}{c}0.2 \\
(4.1+/-1.3) \\
\end{array}$ & $\begin{array}{c}0.5 \\
(11.2+/-5.5) \\
\end{array}$ & $\begin{array}{c}0.11 \\
(2.64)\end{array}$ & $\begin{array}{r}0.21 \\
(5.24) \\
\end{array}$ & $\begin{array}{r}0.18 \\
(4.32) \\
\end{array}$ & $\begin{array}{c}0.26 \\
(6.16) \\
\end{array}$ \\
\hline $\begin{array}{l}\text { Sphingomona } \\
\text { s AD109 DszA }\end{array}$ & 1 & 1.6 & 0.7 & 0.9 & 1.8 & 0.26 & 0.21 & 0.14 \\
\hline $\begin{array}{c}\text { Nocardia } \\
\text { A3H1DszA }\end{array}$ & 1 & 0.1 & 0.7 & 2.6 & 0.01 & 0.03 & 0.03 & 0.9 \\
\hline $\begin{array}{c}\text { Tsukamarella } \\
\text { EMT4 ToeA }\end{array}$ & 1 & 0.11 & 0.07 & 0.1 & 2.1 & 2.0 & 1.9 & 1.9 \\
\hline $\begin{array}{c}\text { Tsukamarella } \\
670-1\end{array}$ & 1 & 0.5 & 0.01 & 0.16 & 0.9 & 1.25 & 0.28 & 1.1 \\
\hline $\begin{array}{c}\text { Gordonia KGB } \\
\text { ToeA }\end{array}$ & n.d. & n.d. & n.d. & n.d. & n.d. & n.d. & n.d. & n.d. \\
\hline
\end{tabular}

Table 4. Activity of DszA and ToeA proteins with different benzothiophene sulfones. The activity is expressed as the fraction of activity with $\mathrm{DBTO}_{2}$ (expressed as 1 ). In those cases where the specific activity was determined using purified proteins, the values are given in brackets (nmols of substrate/min/nmol of enzyme). n.d., not detected.

\subsubsection{Characterization of DszC activities}

In contrast to DszA, we were only able to clone and stably express the DszC from Rhodococcus IGTS8. While we could detect activity from recombinant $E$. coli clones expressing the Sphingomonas AD109 DszC, it proved unstable and we were unable to obtain specific activity data. As in the case of the DszA, DszC of IGTS8 demonstrated higher activities towards the dibenzothiophenes and derivatives as compared to the benzothiophenes (Table 5). Activity of DszC also appeared less affected by substitutions on DBT compared to the influence of $\mathrm{DBTO}_{2}$ modifications on DszA activity.

\begin{tabular}{|c|c|c|c|c|c|c|c|c|}
\hline & DBT & $\begin{array}{c}4 \mathrm{Me} \\
\mathrm{DBT}\end{array}$ & $\begin{array}{c}4,6 \mathrm{diMe} \\
\mathrm{DBT}\end{array}$ & $\begin{array}{c}2,4,8 \mathrm{triMe} \\
\mathrm{DBT}\end{array}$ & $\mathrm{BT}$ & $\begin{array}{c}3 \mathrm{Me} \\
\mathrm{BT}\end{array}$ & $\begin{array}{c}5 \mathrm{Me} \\
\mathrm{BT}\end{array}$ & $\begin{array}{c}7 \mathrm{Me} \\
\mathrm{BT}\end{array}$ \\
\hline $\begin{array}{c}\text { Rhodococcus } \\
\text { IGTS8 }\end{array}$ & 1 & $\begin{array}{c}1 \\
(2.6 \pm 0.5)\end{array}$ & $\begin{array}{c}0.6 \\
(2.6 \pm 0.8)\end{array}$ & $\begin{array}{c}0.5 \\
(1.6 \pm 0.4)\end{array}$ & $\begin{array}{c}(1.4 \pm 0.67) \\
(0.016 \pm 0 . \\
0007)\end{array}$ & $\begin{array}{c}0.05 \\
(0.12 \pm 0 . \\
04)\end{array}$ & $\begin{array}{c}0.005 \\
(0.013 \pm 0.0 \\
009)\end{array}$ & $\begin{array}{c}0.02 \\
(0.06 \pm \\
0.01)\end{array}$ \\
\hline $\begin{array}{c}\text { Sphingomonas } \\
\text { AD109 }\end{array}$ & + & n.t. & n.t & n.t & n.t & n.t & n.t & n.t \\
\hline
\end{tabular}

Table 5. Activity of DszC from Rhodococcus IGTS8. The activity is expressed as the fraction of activity with DBT (expressed as 1). Specific activity was determined with purified protein; the values are given in brackets (nmols of substrate $/ \mathrm{min} / \mathrm{nmol}$ of enzyme). 


\subsection{Construction of DszA-reductase fusions}

One of the challenges for improved biocatalyst is the coordinated expression and activity of flavin reductase component with the DszA and DszC monooxygenases. A possible strategy for addressing this challenge is the construction a translational fusion of each the monooxygenases with a flavin reductase component. Examples of naturally occurring fusion proteins are the sterol $\alpha$-demethylase/ferredoxin fusion protein from Methylococcus capsulatus (6) and the $\mathrm{P}_{450_{\mathrm{BM}-3}}$ a Bacillus megaterium flavocytochrome, in which the fatty acid hydroxylase component is fused to a diflavin NADPH reductase and has the highest catalytic activity of any known cytochrome P450 (7).

To investigate this possibility, we constructed translational fusions between the IGTS8 DszA and either one of two flavin reductases, DszD and Fre. The fusions were constructed to join each of the proteins by a flexible alanine-rich linker region and in both possible orientations DszA-linker DszD (11902), DszA-linker-Fre (11903), DszD-linkerDszA (11904), and Fre-linker-DszA (11901).

All proteins were expressed and purified to determine both their respective reductase and oxygenase activities. As can be seen in Figure 5, all constructs except one (DszAlinker-Fre) had comparable activities to the Fre reductase itself. Unfortunately, under assay conditions tested, no oxygenase activities could be seen for any of the fusion proteins.

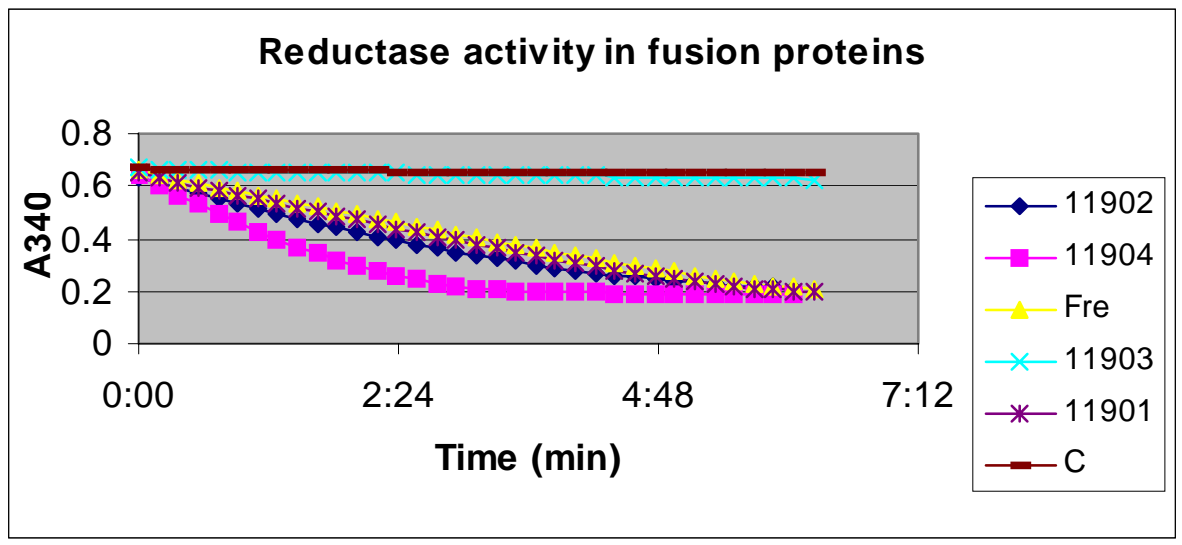

Figure 5. Reductase activity of fusion proteins and comparison to native Fre. " $\mathrm{C}$ " = no enzyme control.

Although we investigated a number of possible reasons for the lack of monooxygenase activity, including subunit structure and aggregation state, no suitable explanations were found. Work in this area was discontinued. 


\subsection{Discovery of new $d s z$ genes}

As one of the challenges of biodesulfurization is the highly complex composition of the organosulfur compounds in petroleum feedstocks, the ability to access Dsz monooxygenases with a wide range of substrate specificities was felt to be important. Although we had already cloned, expressed and characterized a number of DszA and Toe A proteins, we investigated a number of methods to identify additional $d s z$ homologues from the genetic diversity that was available to Diversa in the form of metagenomic environmental libraries and microbial strains. Both sequence-based and functional screening strategies were employed as described.

\subsubsection{Sequence-based discovery}

Sequenced-based discovery relies on the bioinformatic analysis of previously discovered genes of a particular class to discern regions of sequence homology that can be used to design oligonucleotide primers for PCR amplification or hybridization of novel genes. As the nucleotide sequences of a number of $d s z A$ genes were either available to us from prior sequencing efforts or had been described in the literature, we decided to focus our discovery efforts on this gene. Because of the observation that all previously described $d s z$ genes were found in an operon along with $d s z B$ and $-C$, this strategy would also potentially allow us to obtain these genes from individual strains or Diversa environmental libraries.

Dsz sequences that were already on hand, as well as ToeA sequences were used to search the GenBank database and alignments were constructed. As shown in Figure 6, these sequences could be placed in a phylogenic tree and families of relatedness determined. This analysis resulted in the identification of three main groups of sequences, the bona fide DszA-like monoxygenases, a larger family of nitriloacetate monooxygenase-like proteins and a family of hypothetical flavin-dependent monooxygenases (no activities described in literature).

Using this information, we designed degenerate oligonucleotides to specifically amplify sequences belonging to the bona fide DszA-like family. Correct PCR products (Figure 7) that result should be -350 base pairs in length or about $25 \%$ of the hypothesized full length genes. To first validate these primers, test PCR reactions were carried out on genomic DNA prepared from in-house BDS strains known to encode DszA homologues. PCR products of the predicted size were obtained in these test reactions and were then cloned for subsequent DNA sequencing to confirm their identities. 


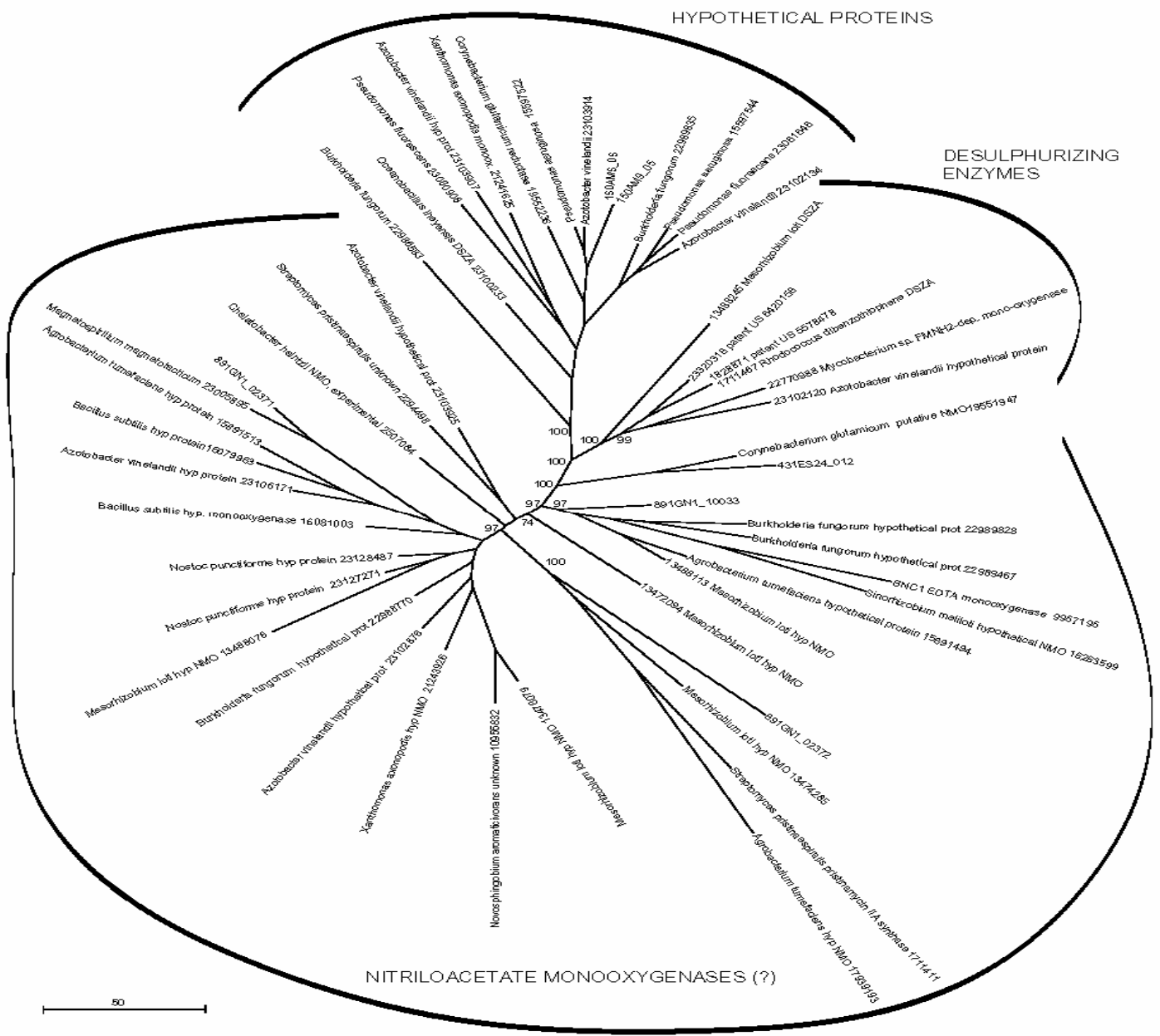

Figure 6. Phylogenetic tree of known sulfone/sulfan monoxygenases, nitriloacetate monooxygenases and hypothetical flavin-dependent monooxygenases.

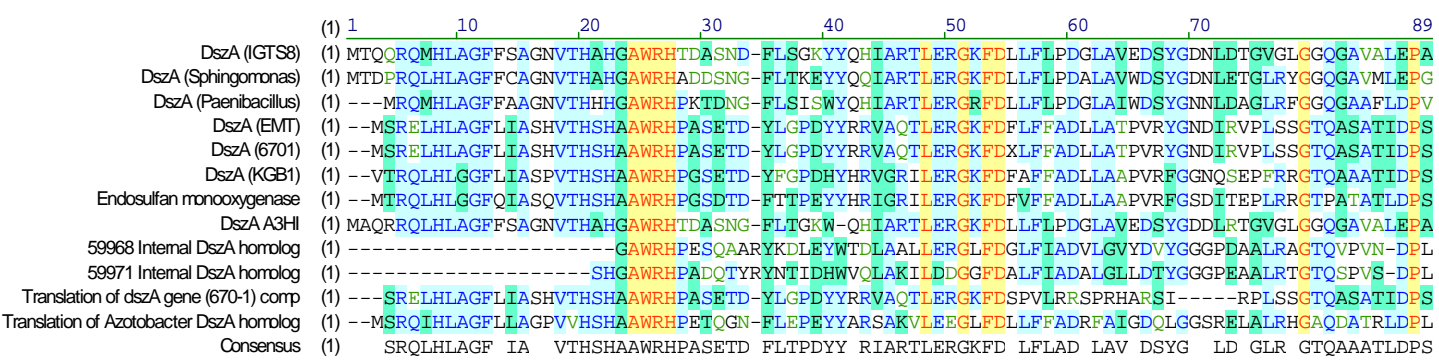

5' SCCAYGSSGCSTGGCGGCA 3'

\section{$\mathrm{M}=\mathrm{A}$ or $\mathrm{C}$ \\ $S=G$ or $C$ \\ $R=G$ or $A$ \\ $\mathrm{Y}=\mathrm{T}$ or $\mathrm{C}$}

Figure 7. Sequence alignment of DszA and ToeA proteins. PCR primers used to amplify products from Diversa environmental libraries and strains are indicated below. 
Surprisingly, for many of the BDS strain isolates, at least two different products were obtained. In addition to the predicted $d s z A$ or toe $A$ product, additional sequences encoding products closely related to DszA were found. Phylogenetic comparisons of these sequences, however, suggested that they cluster closest to the hypothetical flavindependent monooxygenases.

Comparisons of the sequences amplified from the microbial isolates suggested that the designed PCR primers were specifically amplifying dszA or dszA-related sequences. Amplification of products from three environmental DNA libraries was been carried out and products were cloned and sequenced. Sequence analyses and alignment showed that $d s z A$-related sequences were also very prevalent in these environmental libraries. Although we have obtained a few products highly related to the dszA gene of IGTS8, the vast majority of products, however, clustered within the hypothetical flavin-dependent monooxygenases described above.

Because of the observation that the hypothetical flavin-dependent monooxygenase were abundant, not only in the environmental libraries but within individual organisms, we felt it would have required considerable work with no guarantee that the encoded proteins would have activity on relevant sulfur-containing compounds. For this reason, work in this area was discontinued.

\subsubsection{Functional-based screening for new dsz genes.}

Another, more direct approach for discovering novel BDS enzymes from environmental libraries is the use of functional-based expression screening. By developing an appropriate $E$. coli host it is possible to select directly or screen for genes that express functional enzymes, in this case that catalyze the oxidation of sulfur compounds. To this end, we constructed two host systems for the selection/screening of environmental clones that express the DszB-dependent conversion of hydroxybiphenylsulfinate to hydroxybiphenyl and sulfite. As described above, because of the operon organization of known $d s z$ genes, it is likely that clones containing $d s z B$ would also contain $d s z A$ and $-C$ sequences.

The first system was designed to specifically select for metagenomic clones expressing DszB activity by linking production of hydroxybiphenyl and its detection by the regulatory protein $\mathrm{HbpR}$ to the expression of a reporter gene (diagrammed in Figure 8). HbpR is a positive regulator of Pseudomonas azelaica HBP1 that, in the presence of hydroxybiphenyl, activates the transcription of the hbpCA operon, which encodes enzymes for the oxidation of hydroxybiphenyl in its native host (8). By cloning the gene for $\mathrm{HbpR}$ and the regulatory sequences upstream of the $h b p C A$ operon into a transcriptional reporter plasmid, the presence or production of HBP could be detected by expression of reporter genes. 


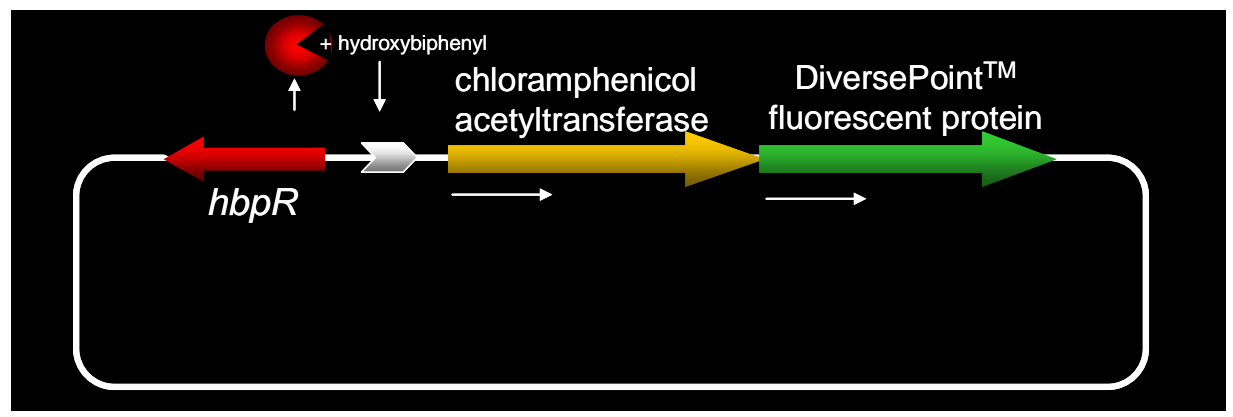

Figure 8. Diagram of hydroxybiphenyl detection reporter plasmid.

The reporters selected for this experiment were the chloramphenicol acetyltransferase, which allows for the direct selection of expressing clones by conferring chloramphenicol resistance and the DiversaPoint ${ }^{\mathrm{TM}}$ green fluorescent protein, which allows for the detection of expression by FACS or direct screening of fluorescent clones.

The reporter construct was tested initially for its response to exogenously added HBP and the ability to confer chloramphenicol resistance. As predicted, the addition of exogenous HBP at nanomolar levels resulted in increased levels of chloramphenicol resistance and the ability to grow on normally inhibitory levels of the antibiotic.

Unfortunately, the control experiment where HBP was not added resulted in spontaneous chloramphenicol-resistant clones that arose at a frequency of $10 \mathrm{e}^{-5}-10 \mathrm{e}^{-6}$. Upon further investigation it was shown that the reporter plasmid alone, without the hbpRC sequences that respond to HBP, gives a similar level of spontaneous resistant colonies. Cells without any added plasmid, however, did not demonstrate detectable levels of resistant colonies.

To determine the cause of the resistant clones, a number of chloramphenicol-resistant clones from the reporter plasmid alone (without the $h b p R C$ sequences) were selected and the nucleotide sequence of the reporter plasmid was determined. As can be seen in Figure 9, IS sequences (insertion elements) of both the IS3 and IS10 family have appeared to transpose upstream of the chloramphenicol acetyltransferase gene, within or immediately downstream of the resident terminator sequences. Insertion elements are found scattered throughout the sequence of most bacterial strains and can transpose into multiple DNA sites, including foreign plasmids. Many IS sequences contain active promoters and can induce expression of genes that normally would not be expressed. This observation suggests that the low level of false positives seen with the bioreporter is unavoidable in normal strains of $E$. coli. Although this work was discontinued for this reason, Kolisnychnko et al (9) have reported the construction of $E$. coli strains that are devoid of IS sequences and may be useful as host if these studies were to continue. 


\section{IS3 Insertion}

97381 IS3 Insertion

Terminator 4

97369 IS10 Insertion

97375 IS3 Insertion

97370,73,76,82,83,84 IS3 Insertion

97372 IS10 Insertion

97371,74,78,80 IS3 Insertion

cat gene

Terminator_3

RBS_1

Terminator_2

sequencing primer

rep Terminator_1

gfp Terminator_5npt

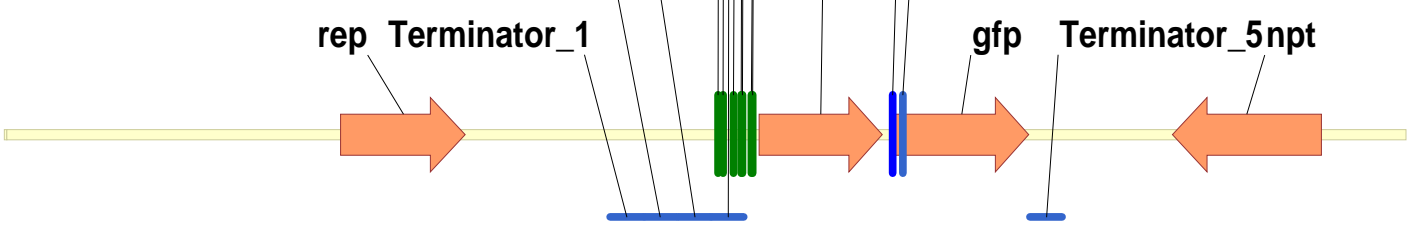

Figure 9. Map of bioreporter plasmid showing sites of IS insertions. The IS insertions leading to false-positive expression of cat gene (encoding chloramphenicol resistance). gfp = Diversa green fluorescent protein

The second system was designed as a direct selection for $E$. coli clones from environmental libraries having DszB activity will be based on the generation of intracellular sulfite to provide sulfur for microbial growth from the conversion of hydroxybiphenyl sulfinate. As mentioned above, all known $d s z B$ genes are directly linked to $d s z A$ and $d s z C$, suggesting such a strategy should yield these genes if the DNA insert is of adequate size as isolated from the environmental library. This system could also be used for the selection of evolved variants of DszB that have higher rates of activity or expanded substrate specificity provided they result in increased levels of sulfite production.

A difficulty in using this type of selection arises from the observation that very small amounts of sulfur are need for cellular metabolic requirements. Traces of sulfate ions in even highly purified microbiological media can provide sufficient sulfur for interfering levels of bacterial growth. In addition, cross-feeding of cells in a population precludes the use of this method in liquid cultures or at high cell densities. With this in mind, we attempted to construct an E. coli strain that is unable to transport extracellular sulfate, thiosulfate or taurine derivatives and required cysteine for growth in minimal medium. By 
introducing environmental libraries into this strain and selecting for growth in the absence of added cysteine, clones expressing DszA-like activities and able to oxidize sulfones to sulfinate derivatives (which are hydrolyzed by co-expressed DszB to sulfite) could be isolated. This system may also be used for the selection of evolved variants of DszA with high rates of activity or expanded substrate specificity.

To construct the cysP operon (encoding sulfate/thiosulfate permease) deletion in $E$. coli, a composite PCR fragment containing $\sim 1 \mathrm{~kb}$ on either side of the cysP operon was generated by overlap PCR and introduced into the EcoRI site of pST98-AS, a vector for the markerless replacement of genes in the $E$. coli chromosome. This construct was then used to generate the chromosomal deletion of the cysP operon and the mutation was confirmed by chromosomal PCR.

The cys $P$ operon deletion mutant was then tested and found to be unable to utilize sulfate in both liquid and on solid media. In addition, its ability to utilize the residual sulfur present in solid agar or agarose media was almost completely eliminated as compared to the wild-type strain. It was still able to grow as well as wild-type on rich media or minimal media containing sulfite or thiosulfate as sole source of sulfur. This latter observation is surprising, as it has been reported that the cysP operon encodes a thiosulfate transporter and its elimination should abolish the ability to utilize thiosulfate (10). This result remains to be resolved but did not directly affect the use of this strain for sulfur selection in a BDS screen.

The dszB genes from Sphingomonas AD109 and A3HI were then cloned and introduced into both the cysP mutant and the wild-type strain parent. HBPS was then tested for its ability to support the growth of the strains in the absence of any other sulfur source and was found to support growth when only when DszB was expressed (vector only control was unable to significantly grow although a noticeable was seen with the wild-type strain). There was, however, a significant delay in the growth of the cysP mutant as compared to the wild-type strain that is not readily explained. This is also apparent when comparing the utilization of HBPS on solid medium, with growth of the mutant being much slower and less vigorous than wild-type. This suggested that the deletion of the cys $P$ operon has some additional unexplained effect on HBPS or sulfite utilization that remains to be resolved. No further work was carried out to investigate these possibilities.

\subsection{Directed evolution of DszA}

Because of the critical need for the DszA protein in any commercial biodesulfurization process, whether it is BDS-standalone or in combination with CED or HDS, efforts to directly evolve and expand the substrate specificity of the BDS system began with this protein. From our previous work and subsequent experience with purification and stability, we chose the Rhodococcus IGTS8 DszA protein as a starting point for directed evolution using Diversa Gene Site Saturation Mutagenesis ${ }^{\mathrm{TM}}$ and GeneReassembly ${ }^{\mathrm{TM}}$ technology.

As the goals of the project changed from the development of a BDS standalone, or BDS biocatalyst to be combined with CED, to a BDS biocatalyst to be used in combination with HDS, it was decided to direct the evolution of DszA substrate specificity to improve activity on the HDS-recalcitrant substrate 4, 6-dimethyl DBT, relative to DBT. 


\subsubsection{GSSM mutagenesis}

For construction of the dszA GSSM library, the dszA gene, cloned into pASK5, was mutagenized at every position in the protein using degenerate oligonucleotides where the residue codon to be modified was represented by the sequence NNK ( $N=G, A, T$ or $\mathrm{C} ; \mathrm{K}=\mathrm{G}$ or $\mathrm{C}$ ) and was carried out by Diversa proprietary GSSM technology. Quality control of the GSSM library was carried out by the random selection of codon mutants and nucleotide sequencing to determine the extent of the substitutions obtained. This strategy, using 32-fold codon degeneracy (NNK) and $3 \mathrm{X}$ oversampling, results in a library of $\sim 50,000$ variants to be screened. This requires the need for a high throughput screen for DszA activity on substrates of interest.

\subsubsection{Assay development}

Optimal reaction and assay conditions for carrying out an in vitro high throughput characterization of mutant libraries were developed, investigating a variety of reaction conditions and compositions that could be adapted to robotic analysis.

\section{Growth and induction of DszA activity.}

An important first step in the robotic automation of the DszA GSSM assay was to determine growth characteristics of cells that were inoculated into 96-well microtiter plates using a high throughput colony picker. Test samples of the GSSM library as well as vector only controls were picked into microtiter plates and grown under a variety of conditions to obtain maximum reproducible growth in this format.

Further investigations were carried out to determine the optimal timing and concentration of anhydrotetracycline (AHT) added to induce DszA gene expression (pASK5 contains a tet element responsive promoter for gene expression). The time of induction was determined to be optimal when the cells had reached an absorbance of 1 at 600nm with 50ng/ml AHT added.

\section{Determination of optimal assay conditions.}

To investigate optimal conditions for assaying DszA activity, time of harvesting cells post-induction was determined and found to vary little between 7 hours and 18 hours, suggesting that DszA activity was stable in the induced cells. To obtain maximum and reproducible activity it was also determined that cells needed to be concentrated to $1 / 5$ the original culture volume by centrifugation before lysis and activity determination.

In early investigations of in vitro DszA activity, oxidation of the substrate was found to reach a maximum level of conversion within 15 minutes but before complete utilization of substrate. These results suggested that the uncoupled oxidation of NADH by the Fre reductase used in the assay was problematic. To address this problem, a number of Fre concentrations were tested and it was found that linear DszA activity could be increased to a period of thirty minutes, which was deemed adequate for the robotic screen, by using a concentration of $0.5 \mu \mathrm{g} / \mathrm{ml}$ in the assay mixture. 
As a minimum number of additions are important for robotic automation of the assay a number of conditions were investigated to determine the optimal order and minimum number of additions that could be easily implemented. The result of these experiments and the protocol that was established for transfer to the robotic platform is described in Figure 10.

\section{Growth and induction of cells}

1) Colonies from plated GSSM library picked into $1.2 \mathrm{ml} \mathrm{LB}$ (w/carbenicillin at $100 \mu \mathrm{g} / \mathrm{ml}$ ) in deep well plates and incubated at $30^{\circ} \mathrm{C}, 220 \mathrm{rpm}$.

2) When cell reached an O.D. 600 of 1 cells were induced with $50 \mathrm{ng} / \mathrm{ml} \mathrm{AHT} \mathrm{(a} \mathrm{daughter}$ plate is prepared by pin-tool prior to addition of AHT for long-term storage)

3) Cells are harvest by centrifugation after 7 hours of induction. Plates are stored at $-20^{\circ} \mathrm{C}$ overnight before assay.

\section{Assay of DszA activity}

4) 200ul of reaction mixture (see below) is added to each well and incubated for 15 minutes, with mixing to lyse cells

5) 150ul of lysed cells are transferred to shallow well 96-well microtiter plate

6) Reaction is started by addition of $\mathrm{NADH}$ (to $5 \mathrm{mM}$ )* and shaken.

7) Reaction is stopped after 15 minutes by addition of 150 ul of acetonitrile.

8) Mixture is centrifuged for ten minutes and diluted with 3 volumes of $\mathrm{dH}_{2} \mathrm{O}$ before analysis by LC/MS/MS.

Reaction mixture (final concentration):

10\% (v/v) B-PER (lysis reagent, Pierce Biotechnology, Rockford, IL)

$0.1 \%$ Triton $\mathrm{X}-100$

Potassium phosphate buffer ( $\mathrm{pH} 7.5,25 \mathrm{mM})$

$\mathrm{NaCl}(100 \mathrm{mM})$

FMN $(10 \mu \mathrm{M})$

$\mathrm{DBTO}_{2}$ (50uM)

4,6-methylDBTO $2(50 \mu \mathrm{M})$

Reductase (Fre) $(0.5 \mu \mathrm{g} / \mathrm{ml})$

*NADH is prepared as $50 \mathrm{mM}$ solution in alkaline buffer ( $\mathrm{pH} 10)$.

Figure 10. Protocol for analysis of DszA GSSM library. Steps in italics describe protocol modified after transfer to and testing on robotic platform.

\section{Optimization of robotic DszA GSSM screening.}

Although the protocols described above were developed for transfer to the robotic platform (Figure 11), it was essential to further refine the robotic high throughput assay by additional experiments to insure robustness and reproducibility. Wild-type DszA clones were inoculated with colony picker and assayed on robot using the described conditions containing $\mathrm{DBTO}_{2}$ and 4,6diMeDBTO${ }_{2}$. 
As can be seen in Figure 12, a consistent level of activity on all substrates was obtained with a minimal number of culture wells exhibiting anomalous levels of activity. Average levels of activity of wild-type reaction wells across plate compared to 'vector-only' negative control wells (column 12) demonstrate a high signal-to-noise ratio.

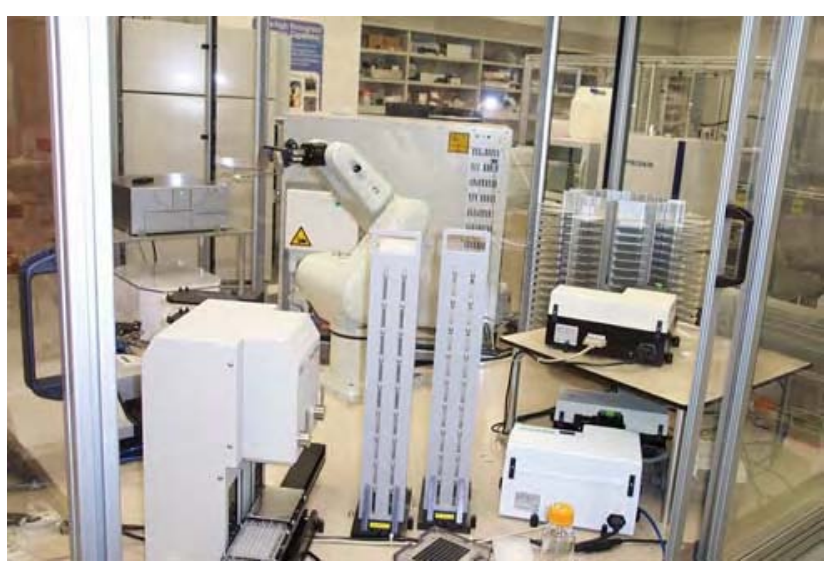

Figure 11. Photograph of robotic platform used for DszA GSSM assay.
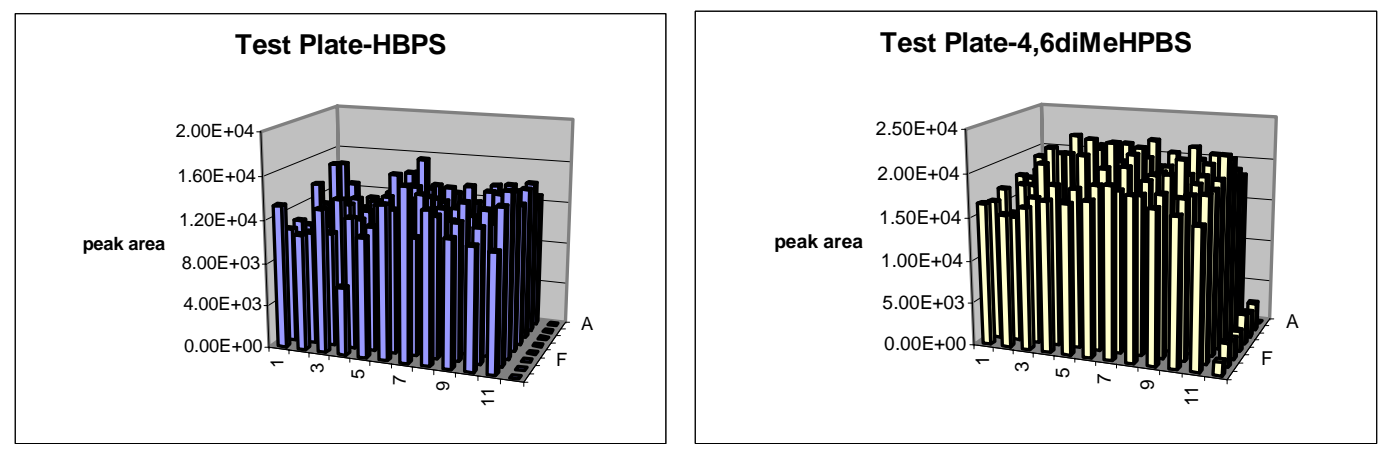

Figure 12 Example of DszA test plate data. Graph shows conversion of $\mathrm{DBTO}_{2}$ and 4, 6dimethyl $\mathrm{DBTO}_{2}$ to products HBPS and 4,6-dimethyl HBPS, respectively. Peak areas do not reflect actual product concentration.

To determine the reproducibility and robustness of the robotic assay using a "real world" example, a plate of DszA variants at residue 112 (randomly selected, leucine in wt DszA) were tested. Figure $13 A$ and $B$ shows results for the activity of the variants on $\mathrm{DBTO}_{2}$ and 4, 6-dimethyl $\mathrm{DBTO}_{2}$ for a single run. Wells were assayed to determine if consistent levels of activity were seen between the two substrates. It can be seen that levels of activity on the substrates vary substantially with a number of active and inactive variants to be seen. Comparison of the two substrates shows similar patterns and demonstrates the feasibility of assaying multiple substrates in the same reaction. 


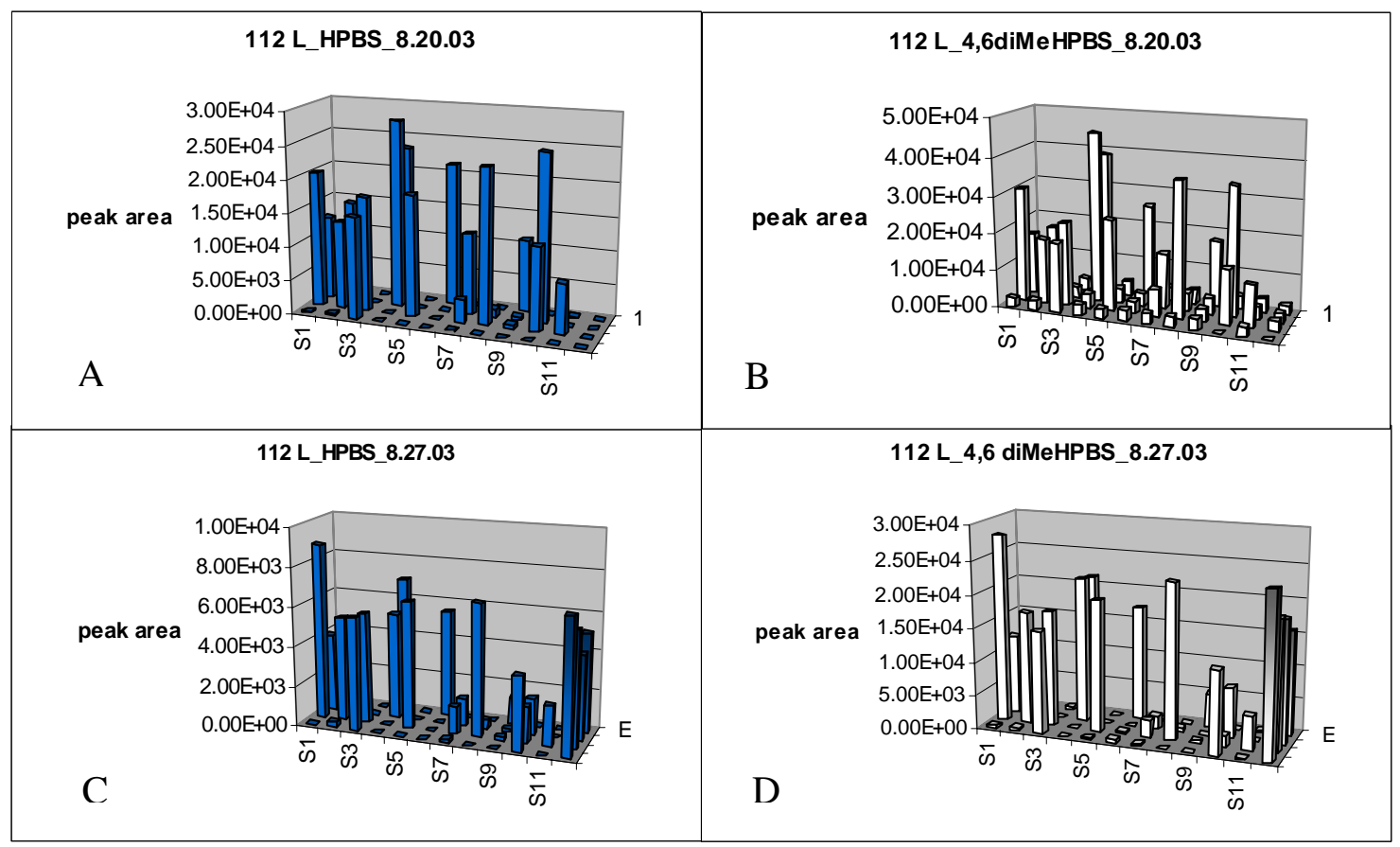

Figure 13. Example of DSZA GSSM data of mutations at residue 112 (leucine). A and B show data from first run, $C$ and $D$ show data from second run, one week later.

To determine if the pattern of activity was reproducible between runs, an experiment where the same plate of mutants, prepared by two separate inoculations and robotic manipulations, was carried out. As can be seen in Figure13B and C, the additional run exhibits a similar pattern as seen with the run one week previous and provides confidence in robustness of the GSSM screen.

To further provide confidence in the results, each of the $112 \mathrm{~L}$ variants was sequenced to determine the nature of the substitution at residue 112. In cases where there were identical residues generated by synonymous codon substitutions, the activity data was nearly identical (data not shown). In addition, substitutions that restored the wild-type leucine residue generated activities indistinguishable from the wild-type controls.

\section{GSSM data analysis}

To carry out the GSSM screening of the IGTS8 DszA variants, the following data analysis protocol was used. To determine activity of DszA on $\mathrm{DBTO}_{2}$ and 4, 6-dimethyl $\mathrm{DBTO}_{2}$, products were determined by LC/MS/MS. To identify interesting mutants, the data was analyzed by evaluating the absolute converstion of substrates (to identify overall up mutants) as well as activity on 4,6-dimethyl $\mathrm{DBTO}_{2}$ relative to activity on $\mathrm{DBTO}_{2}$ (reflecting change in substrate specificity).

\subsubsection{Primary screening of $d s z A$ GSSM library}

Primary screening consisted of transformation of the GSSM library into E. coli (one transformation per residue-reaction), robotic colony picking into 96 well format (96 
clones for each mutated residue), growth of the cultures, inducing the expression of the variant DszA protein, cell lysis, in vitro end point DszA assay with $\mathrm{DBTO}_{2}$ and 4 , 6diMeDBTO$_{2}$, in the presence of added $E$. coli Fre reductase and analysis of the reaction products and substrates. Additionally each plate of mutants was sequenced to determine the nature of the mutations. Sequencing was done in 96-well format and only one sequencing reaction across mutated residues was performed in order to determine the responsible base substitution. This approach reduced material cost three times comparing to the standard protocol, with three independent sequencing reactions, and provided sufficient quality data. From these combined data, apparent enzyme activity and nucleotide sequencing, putative hits were identified.

Although perhaps not readily apparent, there are definite advantages in sequencing of plates of mutants as a component of primary screening. The most important advantage is that it allows for a higher confidence level in confirming hits. Because of the relative complexity of the high throughput assay, a significant challenge in the DszA GSSM screen is to distinguish signal from noise. This noise can have many sources as the substrates of the reaction are only poorly soluble, the MS analysis is not very sensitive and has an error range of $10-15 \%$, and the many steps of robotic additions also contribute to experimental error. By determination of the nucleotide sequence, confidence in the apparent levels of product formation in different wells increases if clones have the same or similar substitutions. For instance, Figures $\mathbf{1 4}$ and $\mathbf{1 5}$ show examples of how determination of sequence was crucial in identifying potential hits.

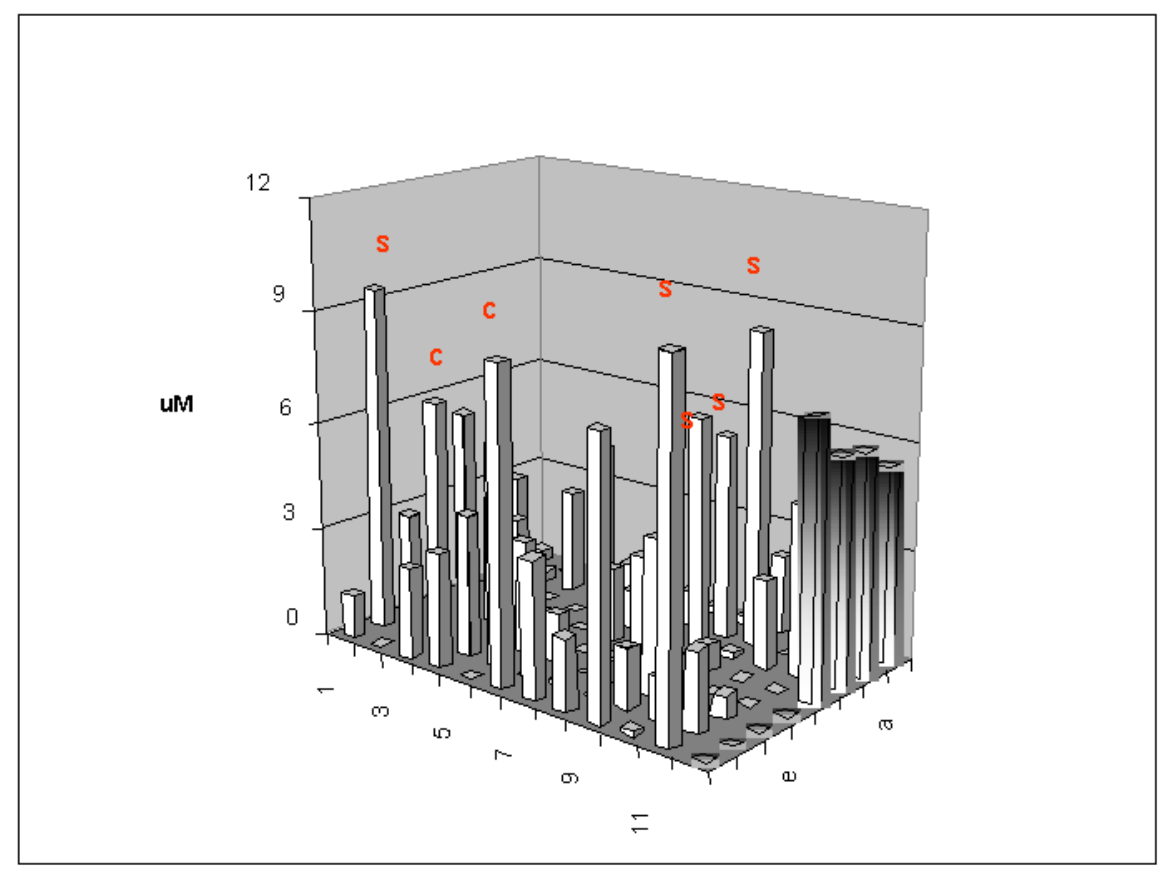

Figure 14. Results from sequencing and enzymatic assay for an alanine residue. Graph shows the amount of 4,6-diMeHPBS in 96-well plate. Red labels over the higher bars indicate amino acid substitution determined after sequencing. Shadowed bars in column 12, rows A-D are wild type (wt) controls. Negative controls (vector only) are in column 12, rows E-H. 
Figure 14 shows the primary results of screening an alanine residue, where a large number of residues showed activities equal to or greater than wild-type but the apparent variability of the overall plate was very high. After determination of the nucleotide sequence of the entire plate of mutants it became apparent that all substitutions of serine for alanine resulted in a slightly improved rate of activity on 4, 6methyldibenzothiophene. In addition, substitutions with cysteine, a residue with many similar properties to serine gave similar levels of activity.

Similarly, Figure 15 shows the results of mutagenesis of an aspartate residue, where fairly high levels of activity were seen with a number of clones and an occasional well with higher than wild type levels. By determining the nucleotide sequence of this plate it became apparent that mutations with asparagines substitutions were solely responsible for the higher level of activities seen. With these data, confidence in the validity of these putative hits was increased. In addition, it was therefore necessary to advance only a small subset of mutants to secondary characterization as they all contained the same or similar substitutions. Although not shown, the sequence data from both of these examples contained the expected distribution of overall amino acid substitutions without apparent bias for ultimately selected residues.

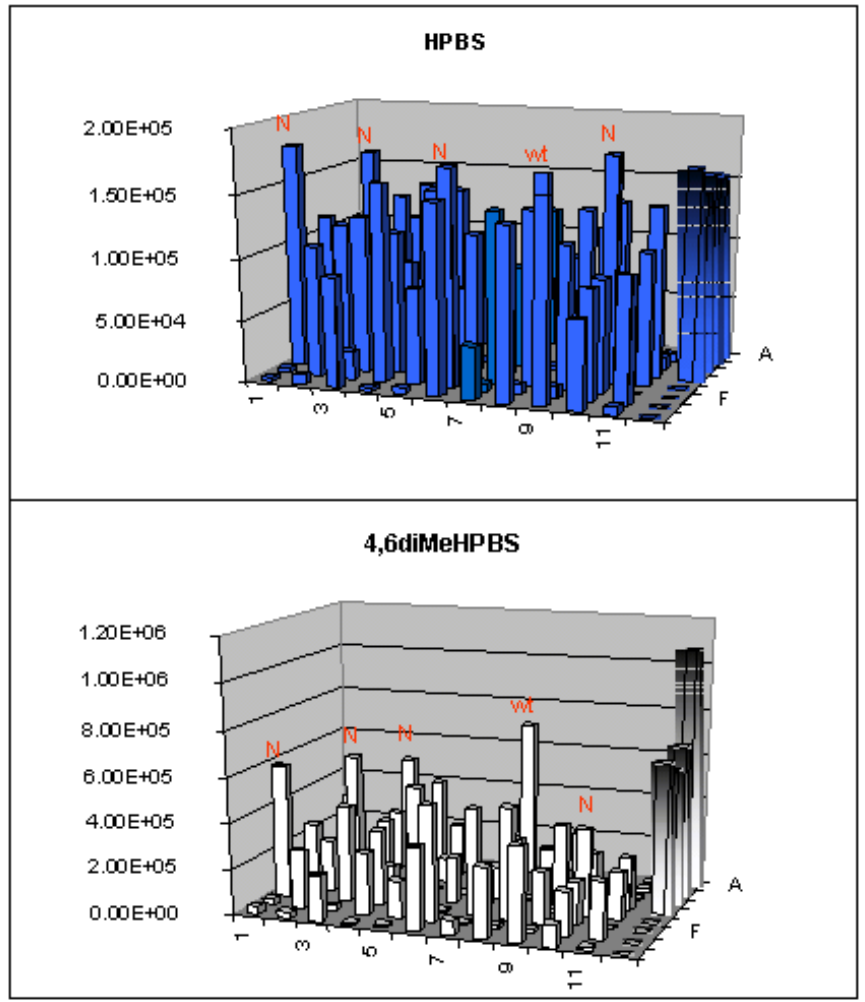

Figure 15. Results from sequencing and enzymatic assay for an aspartate residue. Top graph shows the amount of HPBS in a 96-well plate, bottom graph shows the amount of 4, 6-diMeHPBS from the same plate. Red labels over bars indicate amino acid substitution determined after sequencing. Shadowed bars in column 12, rows A-D are wild type (wt) controls. Negative controls (vector only) are in column 12, rows E-H. 
Another advantage of sequencing is that it provides insight about the residues that are crucial for DszA activity and allows us to classify amino acids into three groups:

1) Non-permissive amino acids that are crucial for the protein function and can be seen by most substitutions resulting in null phenotypes. An example of such a residue is shown in Figure 16.

2) Permissive amino acids that can be seen as residues where most substitutions result in no change in DszA activity. An example of such a residue is shown in see Figure 17.

3) Amino acids of intermediate importance where different substitutions have differing effects on enzyme activity.

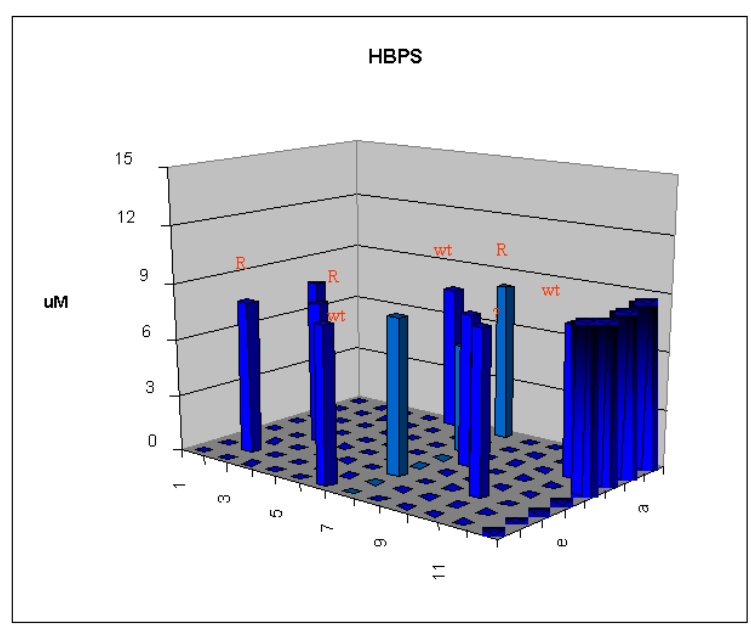

Figure 16. Results from sequencing and enzymatic assay of a non-permissive residue (Arg). Graph shows the amount of HBPS in 96-well plate. Red labels over the higher bars indicate amino acid substitution determined after sequencing. Shadowed bars in column 12, rows A-D are wild type (wt) controls. Negative controls (vector only) are in column 12, rows E-H.

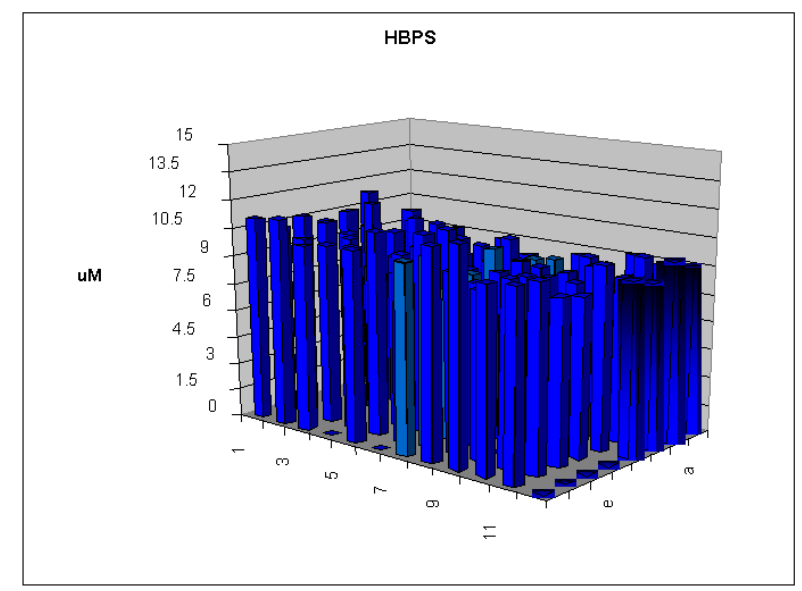

Figure 17. Results from sequencing and enzymatic assay of a permissive residue (G/u). Graph shows the amount of HBPS in 96-well plate. Shadowed bars in column 12, rows A-D are wild type (wt) controls. Negative controls (vector only) are in column 12, rows E-H. 


\subsubsection{Secondary screening of dszA GSSM library}

Approximately 150 clones from the primary screening were shown to have either higher activity towards both of the substrates or increased ratio of 4, 6-diMeDBTO $\mathrm{O}_{2}$ to $\mathrm{DBTO}_{2}$ oxidation and were validated by nucleotide sequencing (having the same or similar substitutions with altered activity) and were selected for secondary confirmation.

These assays were performed in a similar format to the primary screens and putative hits were inoculated randomly into 96 -well plate, in replicates of ten, together with wild type controls. End point assays were performed and the average product formation and standard deviation were calculated and compared to results with wild type controls. An example of secondary analysis is shown in Figure 18, where a hit from a primary screen is confirmed in secondary assay.

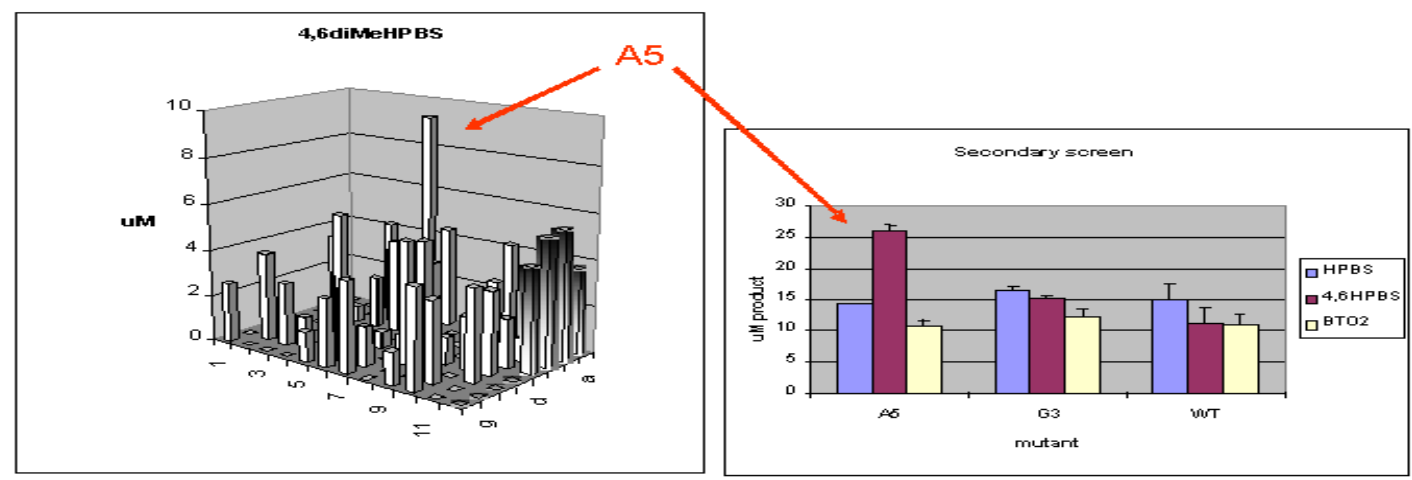

Figure 18. Results from primary and secondary screen for a selected mutant. Graph on the left shows the amount of 4, 6-diMeHBPS product in 96-well plate primary screen. Shadowed bars in column 12, rows A-D are wild type (wt) controls. Negative controls (vector only) are in column 12, rows E-H. Well A5 was selected as a putative hit with increased activity towards 4, 6diMeDBTO ${ }_{2}$. Graph on the right shows results of the secondary analysis, where the A5 clone was assayed in triplicate and compared to wild type.

From the secondary screenings 28 variants were identified. The mutants fell into three distinct classes:

1) mutants with higher level of activity on 4, 6-diMeDBTO ${ }_{2}$ but wild-type activity on $\mathrm{DBTO}_{2}$;

2) mutants with higher level of activity on 4, 6-diMeDBTO ${ }_{2}$ but lower than wild-type activity on $\mathrm{DBTO}_{2}$; and

3) higher activity on $\mathrm{DBTO}_{2}$ relative to 4, 6-diMeDBTO${ }_{2}$.

\subsubsection{Tertiary screening of $d s z A$ GSSM library}

After identification of putative hits by primary and secondary screening, a total of 28 variants were carried through to a more rigorous by a tertiary analysis. Here, DszA proteins expressed from putative were purified and the specific activity of the variants, 
with $\mathrm{DBTO}_{2}$ and 4, 6-dimethyl $\mathrm{DBTO}_{2}$, both separately added to the assay or in combination. The inclusion of both substrates in the characterization mirrors both original primary screening conditions as well as conditions that are likely to be similar to those in diesel fuel, where multiple DBT species are found and competitive inhibition may be seen.

Figures 19-27 show results for three DszA residues (with two variants each) and follows their progression through the primary, secondary and tertiary screening.

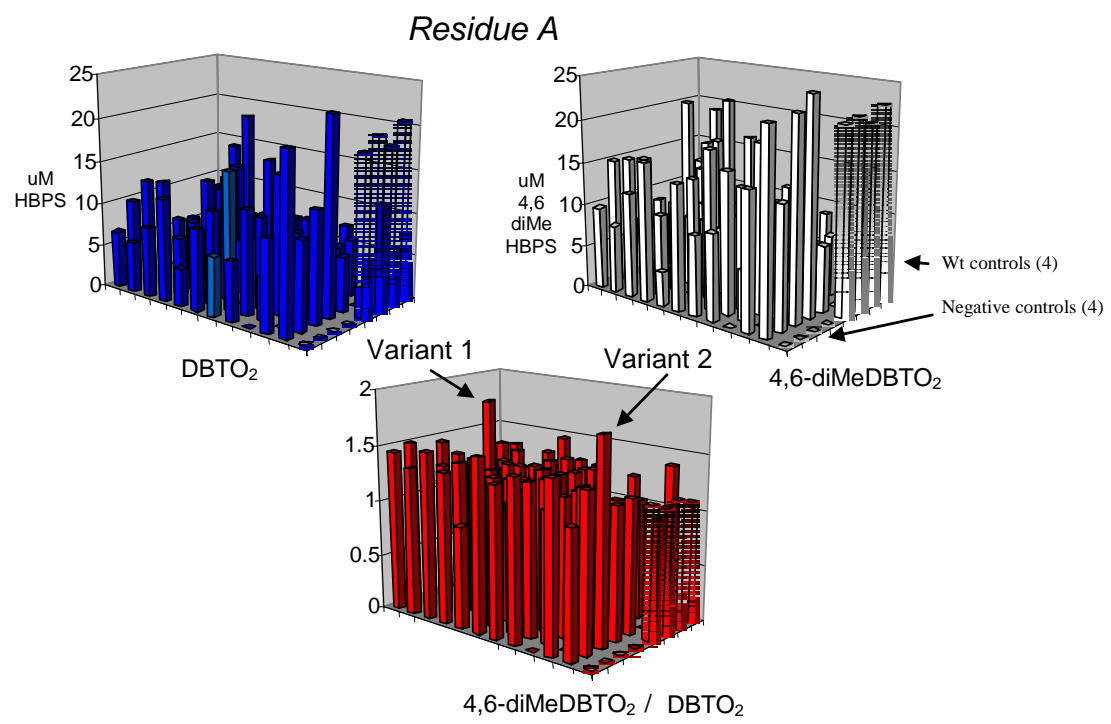

Figure 19. Primary screening data from GSSM optimization of DszA residue A. Results from

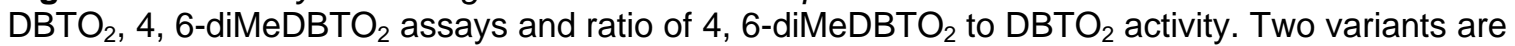
indicated.

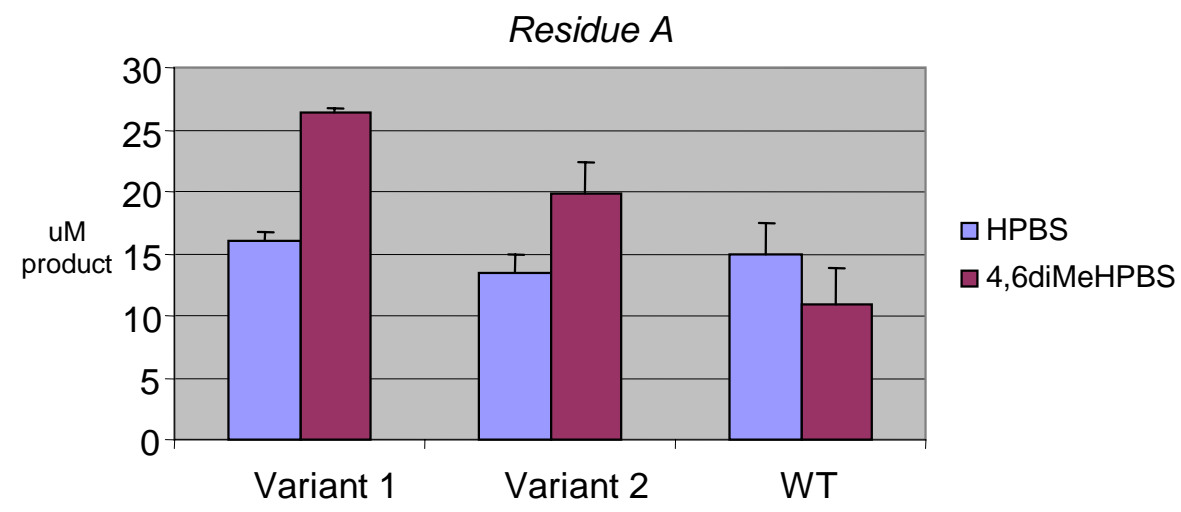

Figure 20. Secondary screening data for two DszA residue $A$ variants. Conditions were same as used for primary screening but in replicates of ten. 

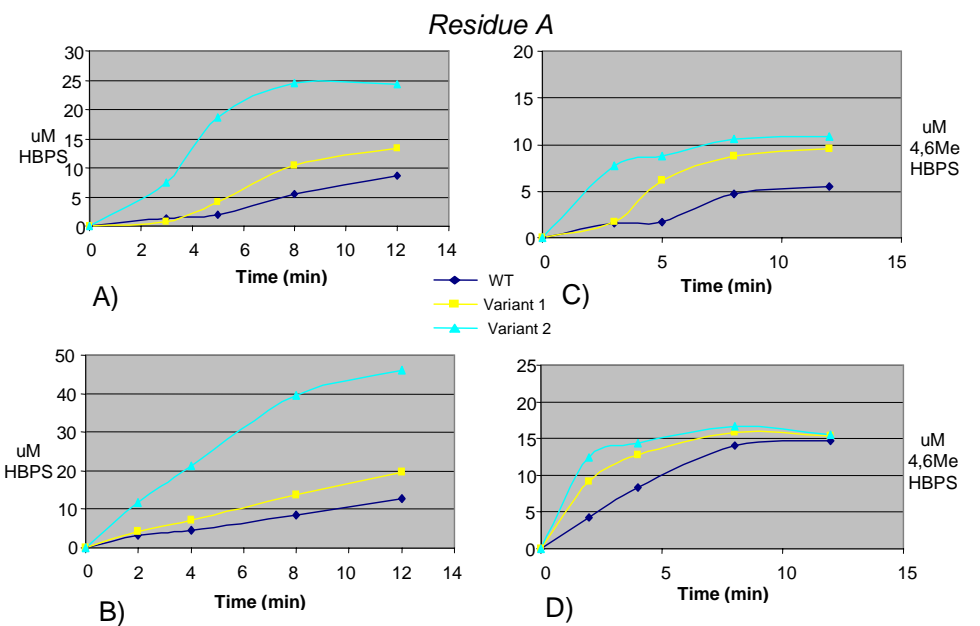

Figure 21. Tertiary screening data from two DszA residue $A$ variants. The following conditions were used- A) $\mathrm{DBTO}_{2}$ alone (100uM) and assaying HBPS product; B) $\mathrm{DBTO}_{2}(100 \mathrm{uM})$ plus 4,6diMeDBTO$_{2}$ (100uM) and assaying HBPS product; C) 4,6-diMeDBTO 2 alone (100uM) and assaying 4,6-diMeHBPS product; D) 4,6-diMeDBTO 2 (100uM) plus $\mathrm{DBTO}_{2}(100 \mathrm{uM})$ and assaying 4,6-diMeHBPS product.
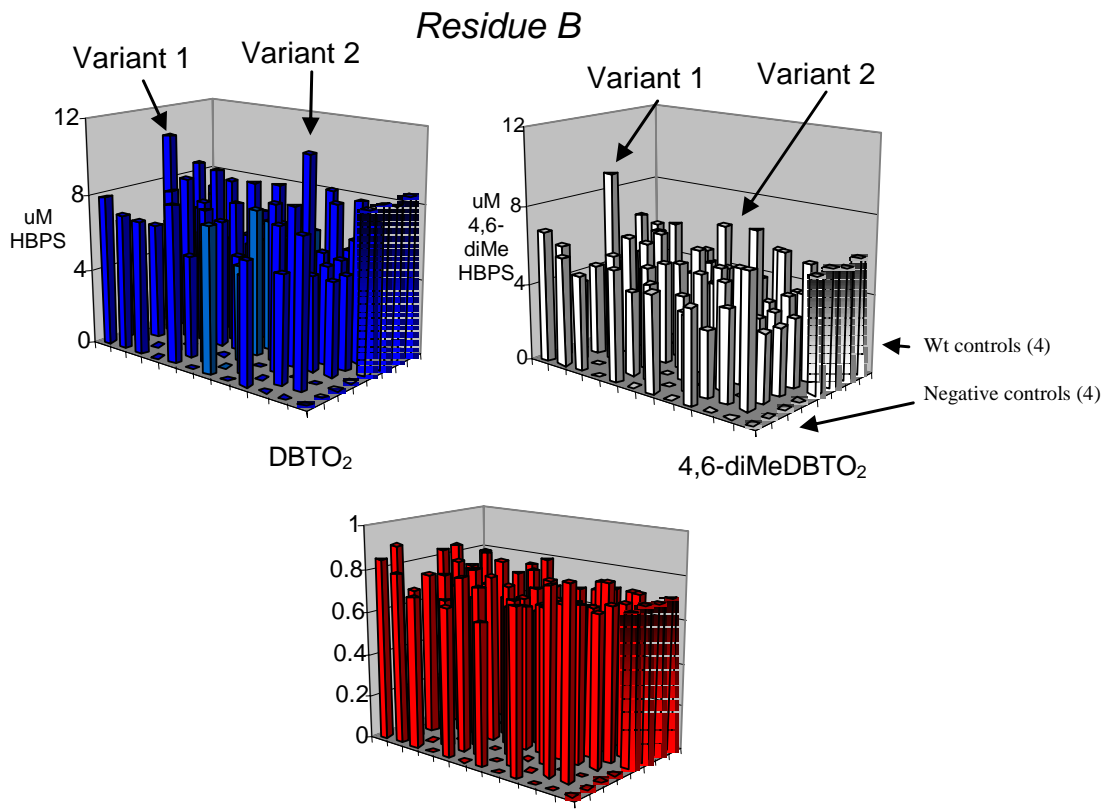

4,6-diMeDBTO $/ 2$ DBTO 2

Figure 22. Primary screening data from GSSM optimization of DszA residue $B$. Results from

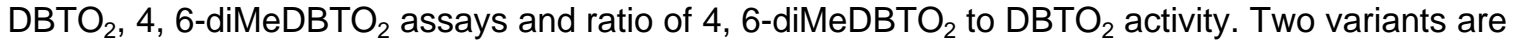
indicated. 


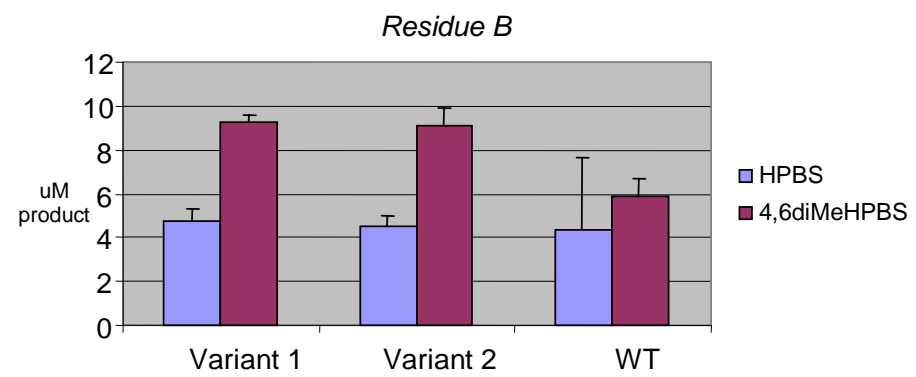

Figure 23. Secondary screening data for two DszA residue $B$ variants. Conditions were same as used for primary screening but in replicates of ten.

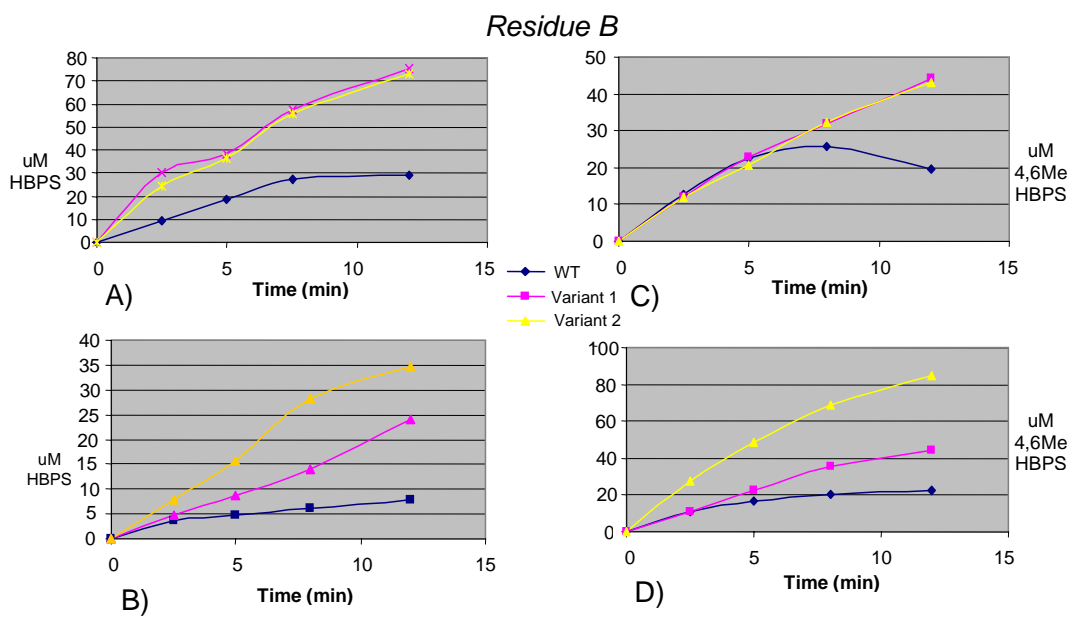

Figure 24. Tertiary screening data from two DszA residue $B$ variants. The following conditions were used- A) $\mathrm{DBTO}_{2}$ alone (100uM) and assaying HBPS product; B) $\mathrm{DBTO}_{2}(100 \mathrm{uM})$ plus 4,6diMeDBTO$_{2}$ (100uM) and assaying HBPS product; C) 4,6-diMeDBTO ${ }_{2}$ alone (100uM) and assaying 4,6-diMeHBPS product; D) 4,6-diMeDBTO 2 (100uM) plus $\mathrm{DBTO}_{2}(100 \mathrm{uM})$ and assaying 4,6-diMeHBPS product.

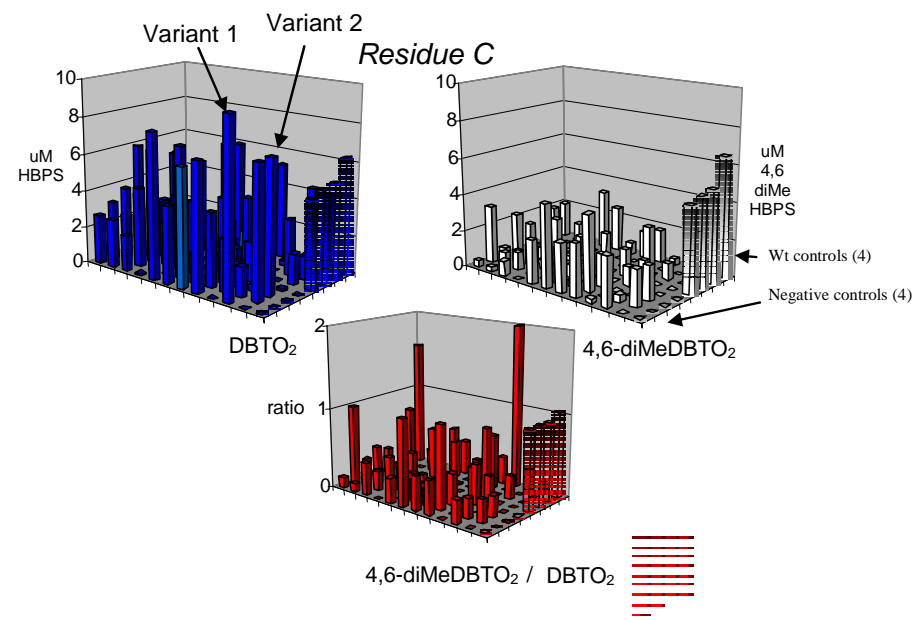

Figure 25. Primary screening data from GSSM optimization of DsZA residue C. Results from

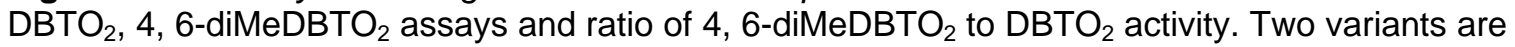
indicated. 


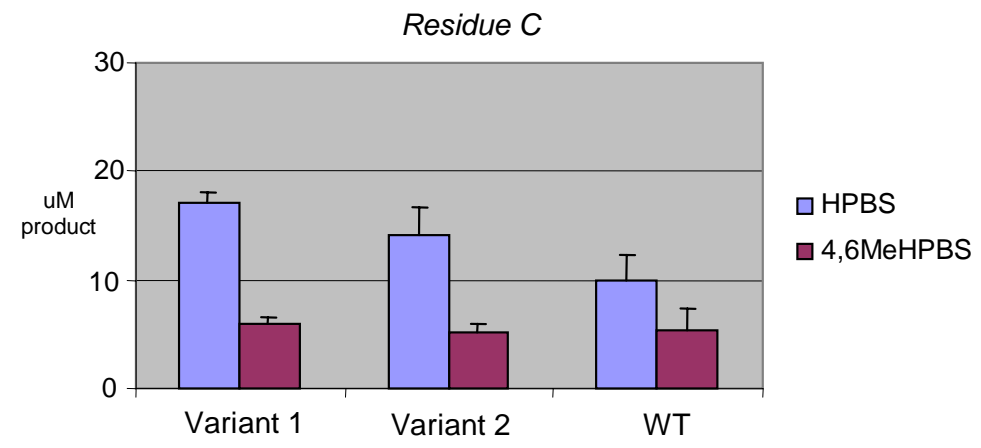

Figure 26. Secondary screening data for two DszA residue $C$ variants. Conditions were same as used for primary screening but in replicates of ten.

Residue C
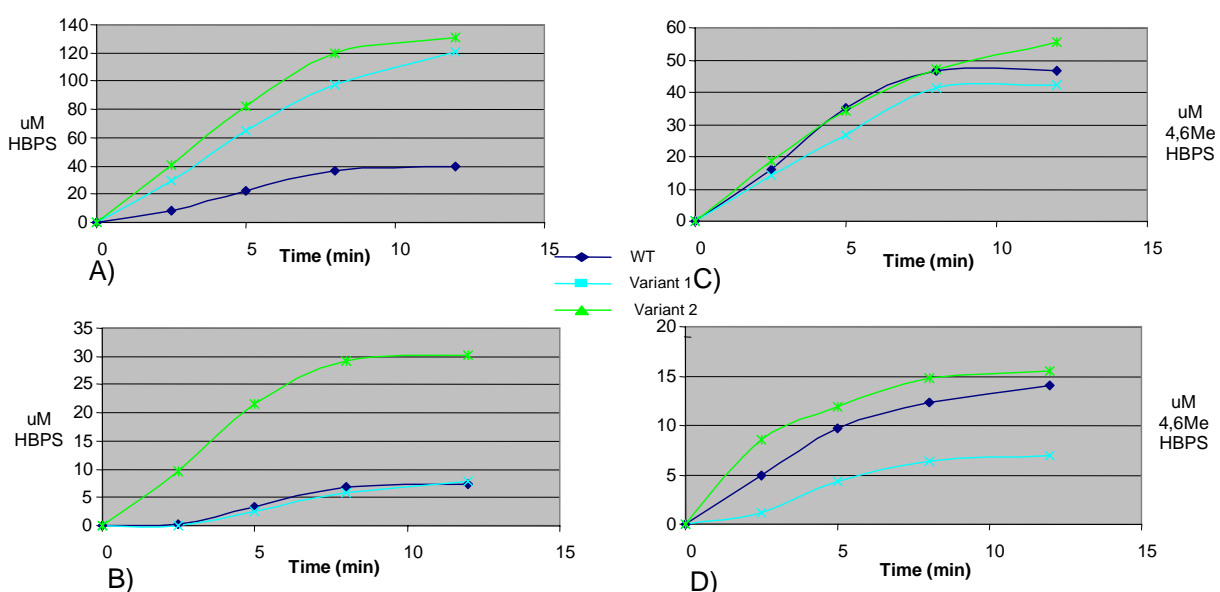

Figure 27. Tertiary screening data from two DszA residue $C$ variants. The following conditions were used- A) $\mathrm{DBTO}_{2}$ alone (100uM) and assaying HBPS product; B) $\mathrm{DBTO}_{2}(100 \mathrm{uM})$ plus 4,6diMeDBTO$_{2}$ (100uM) and assaying HBPS product; C) 4,6-diMeDBTO ${ }_{2}$ alone (100uM) and assaying 4,6-diMeHBPS product; D) 4,6-diMeDBTO 2 (100uM) plus $\mathrm{DBTO}_{2}(100 \mathrm{uM})$ and assaying 4,6-diMeHBPS product.

Three mutants with pronounced improvement of activity on 4, 6-diMetDBTO 2 vs. $\mathrm{DBTO}_{2}$ were purified at larger scale. As the DszA reaction requires the transfer of reducing equivalents from NADH to $\mathrm{FMNH}_{2}$ via a flavin reductase, activity was determined as a function of the concentration of either the flavin reductase Fre (used in GSSM screen) or DszD (the IGTS8 flavin reductase).

Figure 28 shows the results of these experiments and it can be seen that while the ratio of diMetDBTO ${ }_{2}$ to $\mathrm{DBTO}_{2}$ activity catalyzed by the wild-type enzyme is unaffected by the amount of flavin reductase added, the three mutants all showed a pronounced effect of flavin reductase levels. At the lowest concentrations of reductase tested, the ratio of diMetDBTO ${ }_{2}$ to $\mathrm{DBTO}_{2}$ activity is highest, while increasing the amount of flavin reductase results in a less pronounced ratio of diMetDBTO ${ }_{2}$ to $\mathrm{DBTO}_{2}$ activity, approaching that of the wild-type enzyme at the highest levels of reductase. Although there are differences in the activity supported by Fre vs. DszD (may be a reflection of the specific activity of each preparation), the trend is the same for both. 

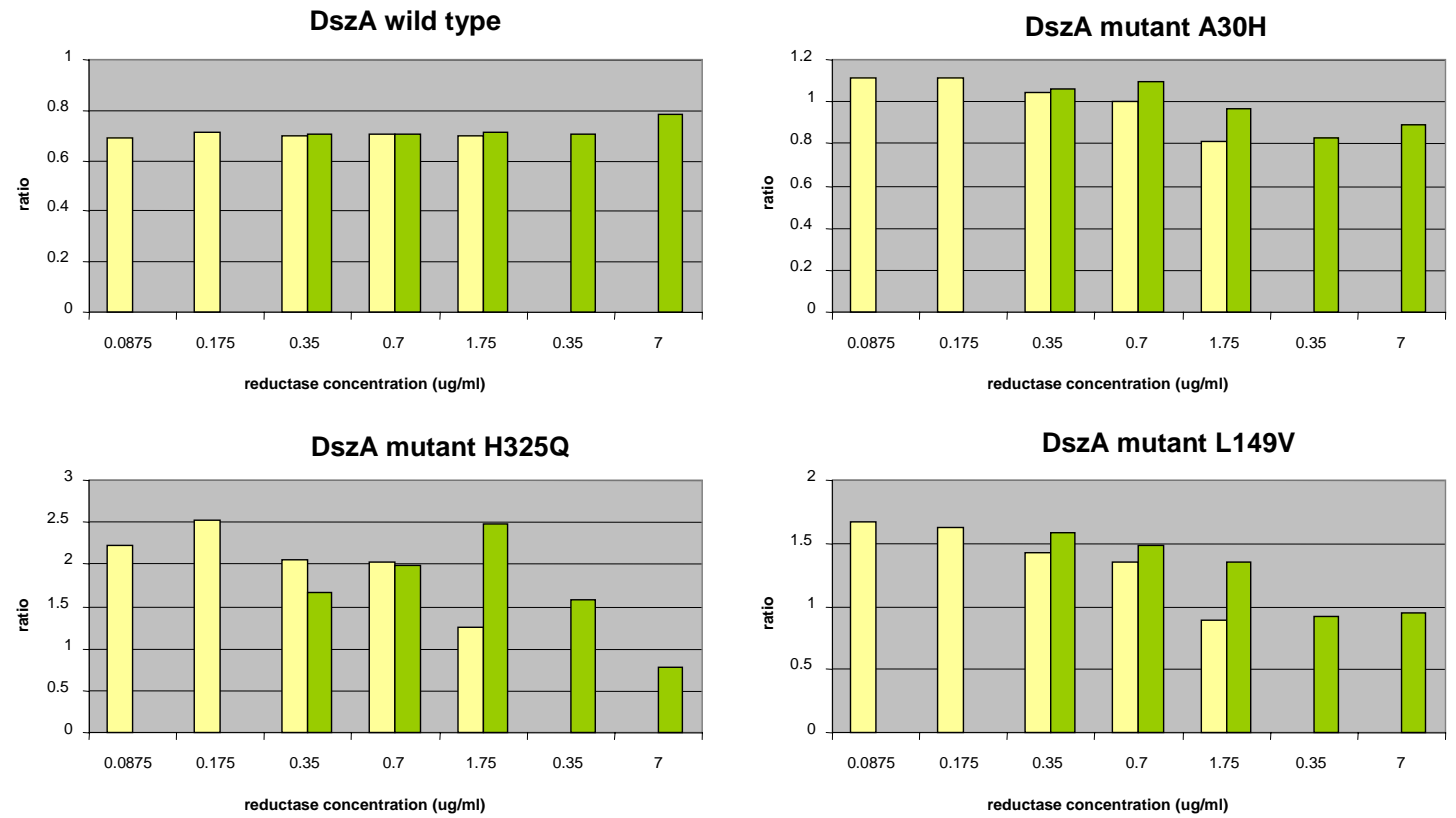

Figure 28. Effect of varying the amount of flavin reductase on ratio of diMetDBTO $\mathrm{O}_{2}$ to $D B T \mathrm{O}_{2}$ activity of DszA mutants. Yellow bars are data using Fre; green bars are data using DszD.

One possible explanation of this result is that the DszA variants have resulted from a decreased $\mathrm{Km}$ (higher affinity) for diMetDBTO 2 relative to $\mathrm{DBTO}_{2}$. The higher ratio activity that results from lower reductase levels may reflect the lower availability of reduced $\mathrm{FMNH}_{2}$. As DszA must simultaneous bind three substrates, $\mathrm{O}_{2}, \mathrm{FMNH}_{2}$ and DBT sulfones, an increase in affinity for sulfones will result in a greater likelihood of full substrate-enzyme occupancy.

\subsubsection{Bioinformatic analysis of DszA GSSM mutants}

To obtain the most information possible from the GSSM mutations to address the structure-function relationship of DszA, we developed a hypothetical model of the DszA structure derived from previously determined structures of related proteins. To construct this model, a template structure was selected. A BLAST search was performed, querying the DszA sequence against the sequences of experimentally determined structures in the Protein Data Bank. DszA's nearest structural neighbor is the $E$. coli alkanesulfonate monooxygenase SsuD (BLAST score of $5 \mathrm{e}^{-7}$, PDB ID 1m41).

DszA is predicted to have a classic $\beta 8 \alpha 8$ fold also known as a TIM barrel (see Figure 29). Typically, substrate binds to the loops that are C-terminal to each canonical strand. The DszA model was generated using the homologous structure of SsuD from $E$. coli (PDB code 1m41, SwissProt entry SSUD_ECOLI). The homology model was created by aligning the homologous sequences and replacing residues of the template structure (1m41) with those of the target sequence (DszA). Labeling corresponds to canonical secondary structural elements. 


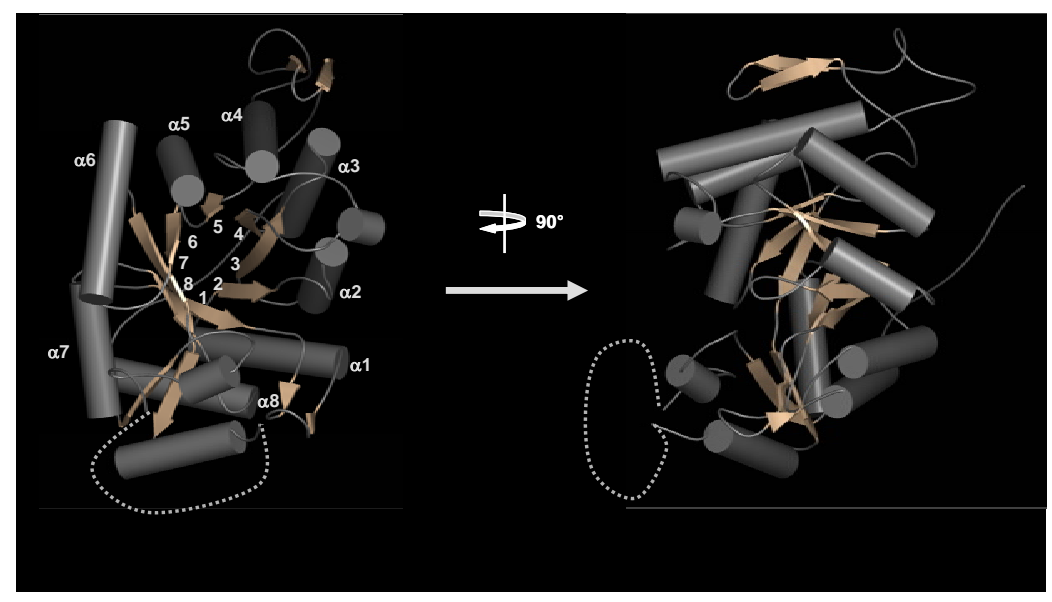

Figure 29. Ribbon model of DszA derived from threading of E. coli SsuD protein.

Using this model, we then superimposed the data from the GSSM experiments by mapping tolerant and non-permissive positions onto a space filling model derived from above. In addition, mutants confirmed to have either increased activity on both $\mathrm{DBTO}_{2}$ and 4, 6-dimethyl $\mathrm{DBTO}_{2}$, or increased activity on 4, 6-dimethyl $\mathrm{DBTO}_{2}$ relative to $\mathrm{DBTO}_{2}$ were also mapped. Finally, those mutants that were selected for GeneReassembly were also mapped to this model (Figure 30).

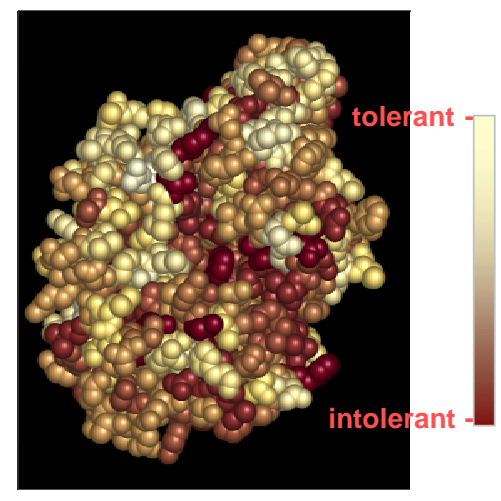

GSSM substitution tolerance

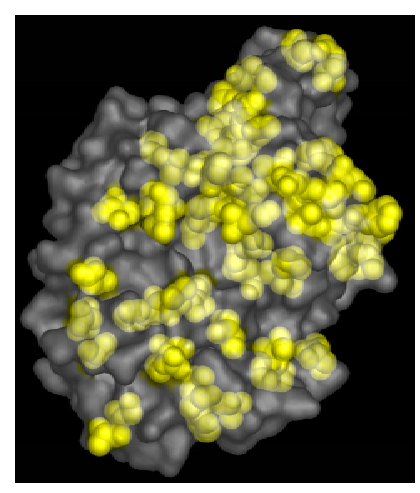

Confirmed variants (tertiary screening)

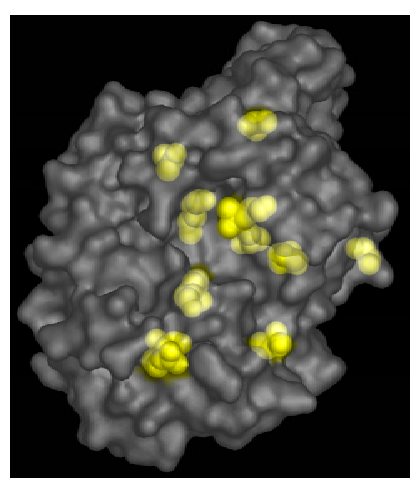

Variants selected for GeneReassembly

Figure 30. GSSM data superimposed onto DszA space-filled model.

We have also attempted to use this information, together with sequence alignments from other known DszA enzymes as well as an endosulfan monooxygenase recently described (11) and the SsuD protein to determine candidate active site residues. Of all positions conserved across these proteins, 31 residues were investigated that could act in a catalytic fashion (CDEHST). Of these only 3 did not tolerate substitution as assessed by GSSM (134D, 153E, 174E). Of these, only one (153E) is near the putative 


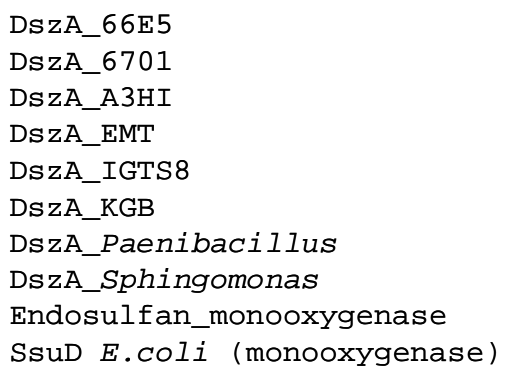

HLSRGRAGWNVVTSLNQAEAQNFGIENHL . . HLSRGRAGWNVVTSLNQAEAQNFGIENHL . . QLSGGRVSWN-WTSLNDAEARNFGIDQHL . . HLSRGRAGWNVVTSLNQAEAQNFGIENHL . . QLSGGRVSWNVVTSLNDAEARNFGINQHL . . HLSRGRIAWNIVTSLSQAEAQNFGFDDHV . . HLSKGRAAWNVVTSLNNAEARNFGYEEHL . . QLSSGRVSWNVVTSLSNAEARNFGFDEHL . . HLSRGRVAWNIVTSLTQSEAQNFGHDDHL . . RLSNGRALFNLVTGSDPQELA- - GDGVFL . .
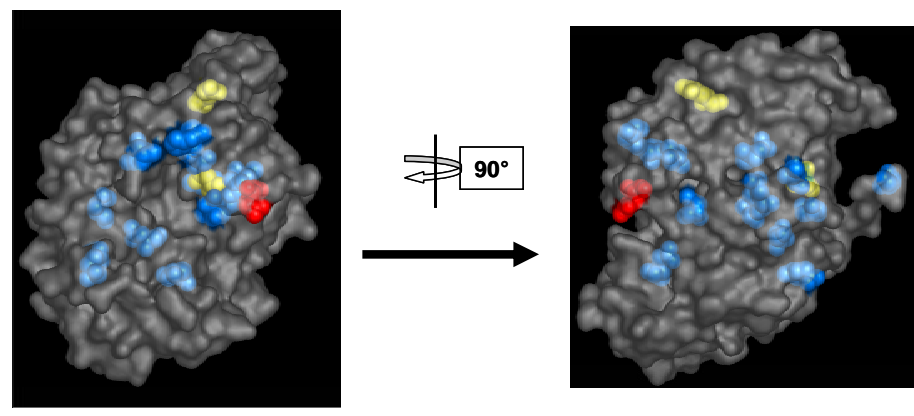

Figure 31. Hypothetical active site glutamate active residue, indicated by asterisk, as determined by homology and GSSM data.

active site in the DszA model (Figure 31) (the 31 positions investigated were: 57E, 62D, 68D, 95E, 132T, 134D, 137S, 147T, 153E, 174E, 165H, 187D, 189D, 194D, 202D, 210D, 211H, 240S, 252E, 283D, 297E, 323S, 336D, 365E, 371E, 422E, 425D, 440E, $445 \mathrm{~T}$ and $449 \mathrm{H})$.

\subsubsection{GeneReassembly mutagenesis of DszA GSSM mutants.}

Thirteen DszA GSSM variants shown to have either a higher ratio of activity with 4, 6diMetDBTO ${ }_{2}$ versus $\mathrm{DBTO}_{2}$ or higher activity on both substrates were selected as parents for GeneReassembly (Figure 32). GeneReassembly is a proprietary Diversa technology for the complete synthetic blending of parental genes in order to create a random combinatorial library of variants to be screened by high throughput methods for further improved properties relative to the input parents.

Using proprietary software and design parameters, the combinatorial introduction of these variants and their subsequent screening for activity on 4, 6-dimethyl $\mathrm{DBTO}_{2}$ and $\mathrm{DBTO}_{2}$ was carried out. With thirteen parents, the predicted complexity of the library was $2^{13}$ or 8,192 unique combinations. A total of 15,000 individual transformants was generated, which represented an approximately $2 \mathrm{X}$ coverage of the total possible combinations. 


\begin{tabular}{|c|c|c|c|c|c|}
\hline $\begin{array}{l}\text { RESIDUE } \\
30\end{array}$ & $\begin{array}{c}\text { Original } A A \\
A\end{array}$ & $\begin{array}{c}\text { Mutated AA } \\
\mathrm{H}\end{array}$ & $\begin{array}{l}\text { DBTO }_{2} \\
1.47\end{array}$ & $\begin{array}{c}\mathbf{4}_{\mathbf{6}} \text { diMeDBTO }_{2} \\
2.11\end{array}$ & $\begin{array}{c}\text { 4,6diMeDBTO } \\
{ }_{2} / \text { DBTO }_{2} \\
1.43\end{array}$ \\
\hline 69 & G & $\mathrm{N}$ & 1.59 & 1.86 & 1.17 \\
\hline 75 & $S$ & $\mathrm{~T}$ & 1.53 & 1.89 & 1.24 \\
\hline 83 & G & V & 1.33 & 1.97 & 1.48 \\
\hline 149 & L & V & 1.08 & 1.84 & 1.70 \\
\hline 167 & A & $T$ & 1.60 & 2.13 & 1.33 \\
\hline 247 & A & M & 1.08 & 1.91 & 1.77 \\
\hline 358 & $\mathrm{~F}$ & L & 1.23 & 1.78 & 1.45 \\
\hline 374 & $\mathrm{R}$ & C & 1.97 & 1.97 & 1.00 \\
\hline 114 & A & $\mathrm{F}$ & 0.76 & 1.20 & 1.57 \\
\hline 325 & $\mathrm{H}$ & $\mathrm{Q}$ & 0.36 & 1.14 & 3.16 \\
\hline 382 & $\mathrm{~F}$ & V & 0.74 & 1.11 & 1.52 \\
\hline
\end{tabular}

Figure 32. GSSM variants selected for GeneReassembly and activity on substrates relative to wild-type parent.

To assess the quality of the GeneReassembly library, 142 random clones were selected and sequenced. Using proprietary ParentFinder ${ }^{\mathrm{TM}}$ software, the parental contribution of each clone was determined. Figures 33-35 demonstrate the relatively random positional and numeric distribution of the library generated.

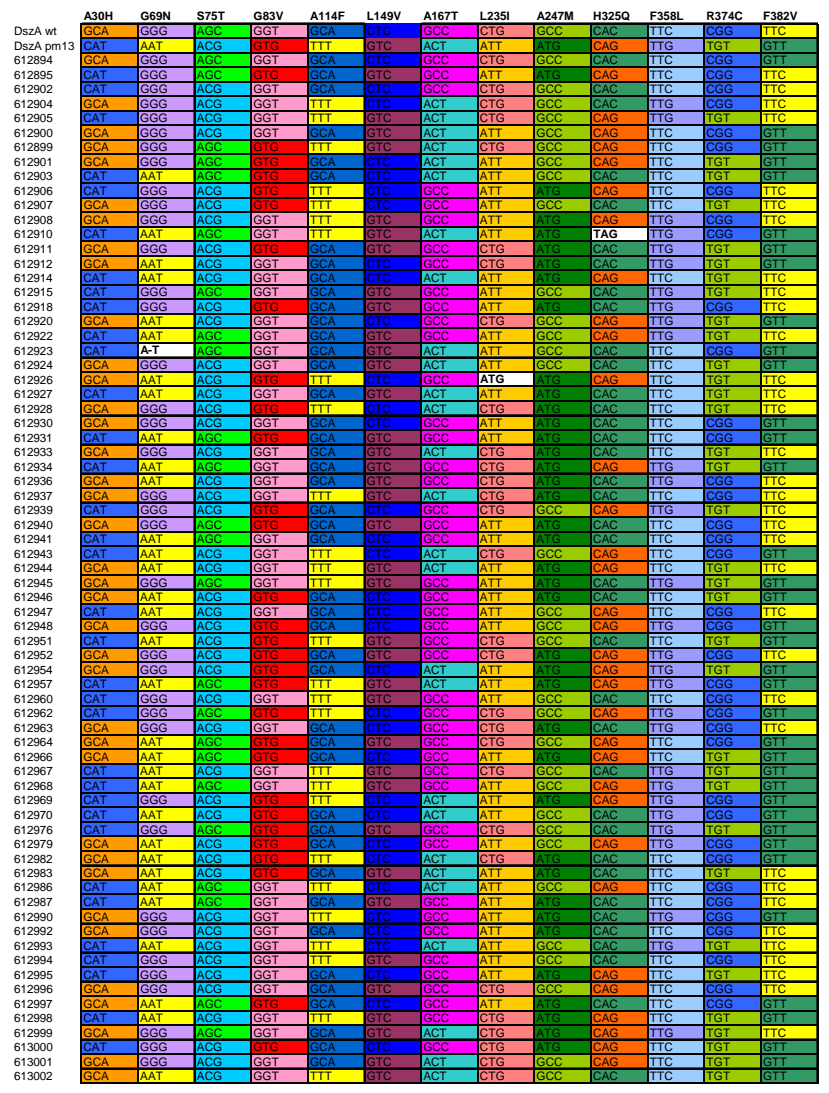

Figure 33. Parental contribution to each of the 142 randomly selected and sequenced GeneReassembly mutants. 


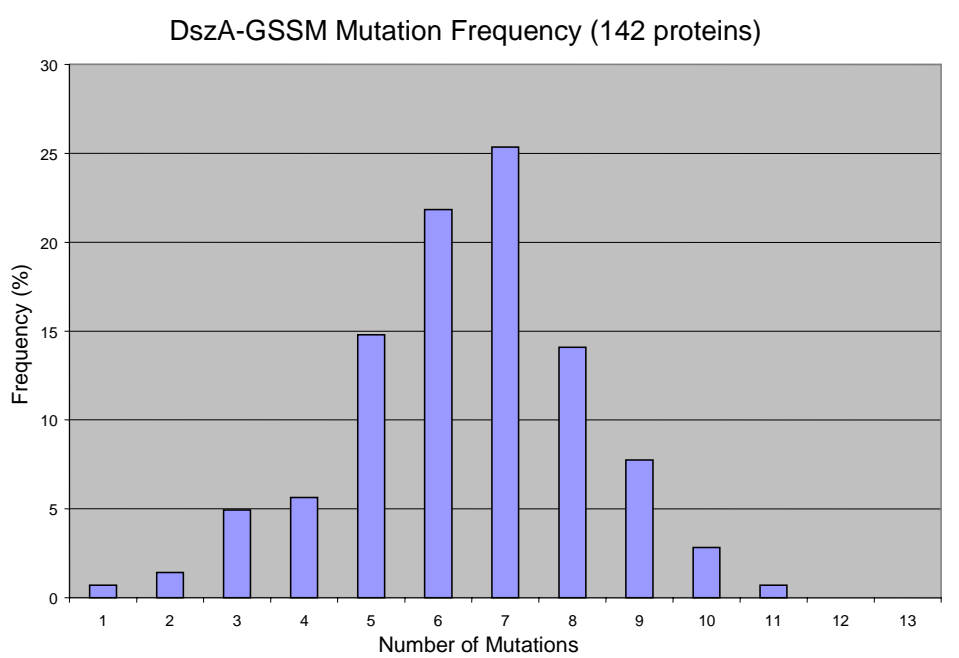

Figure 34. Distribution of the number of parental contributions to each of the 142 sequenced GeneReassembly mutants.

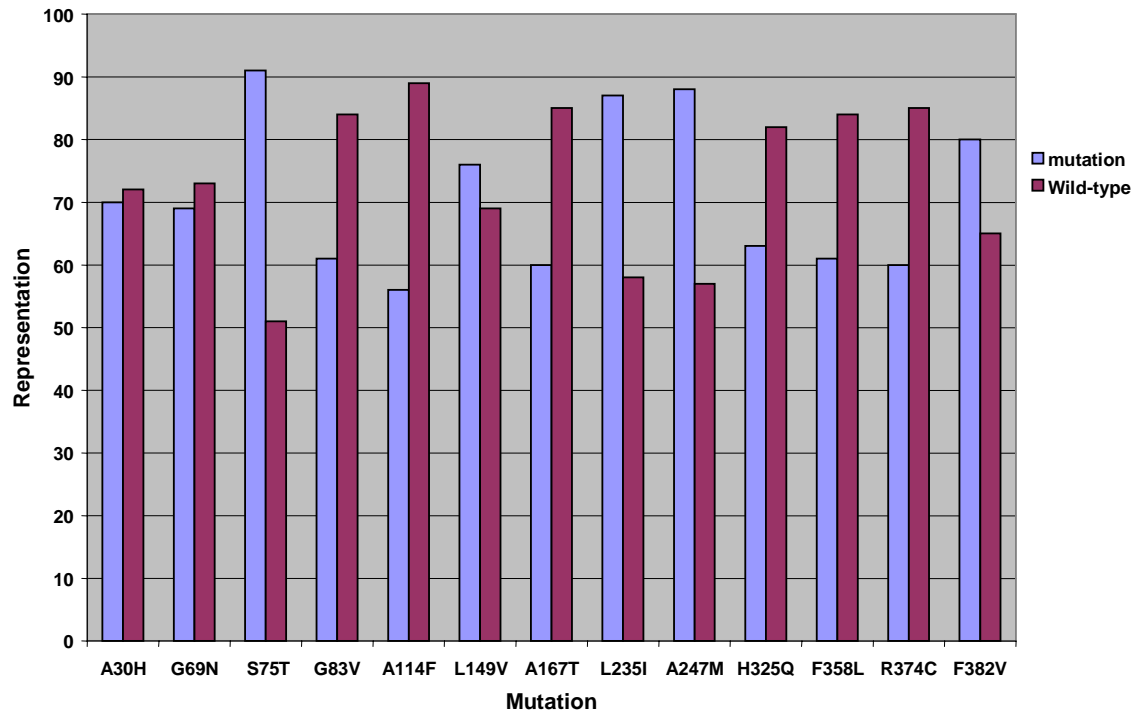

Figure 35. Relative contribution of each of the parents to the final library as seen in the 142 sequenced GeneReassembly mutants.

\subsubsection{Characterizations of DszA GSSM variants and reassembled derivatives.}

As stated above, a total of 15,000 clones were generated. $\sim 4,000$ of these reassembled clones were assayed for activity using a similar high throughput assay as described for the primary screening of the DszA GSSM library.

Although a large percentage of clones appeared to have lower levels of activity than the original parent clones (likely as a result of negative combinatorial effects of active site mutants as discussed previously) approximately 70 mutants were selected that had 
activity equal to or higher than one of the original parent clones selected as benchmark. Six mutants were selected for further study.

As can be seen in Table 6, each of the mutants shows increased activity on 4, 6dimethylDBTO ${ }_{2}$ (bold numbers) from the wild-type and the benchmark mutant. Although the average number of mutants for the library was $\sim 7$ substitutions per clone (plotted from the distribution of the 142 randomly sequenced clones), it is apparent that the clones with improved activity have an average of 3 mutations. Although there is some degree of variation between results from the two separate trials, overall it is apparent that the reassembled mutants have a 1.5-2X improvement of activity for 4, 6-dimethyl $\mathrm{DBTO}_{2}$ as compared to the benchmark single mutant and a 3-4X improvement over the wild-type enzyme. In addition, the results suggest that there is also an apparent increase in activity on $\mathrm{DBTO}_{2}$, although not as dramatic as on the larger substrate. It must be remembered, however, that these enzyme variants have not been purified, so the numbers are not true measurements of specific activity.

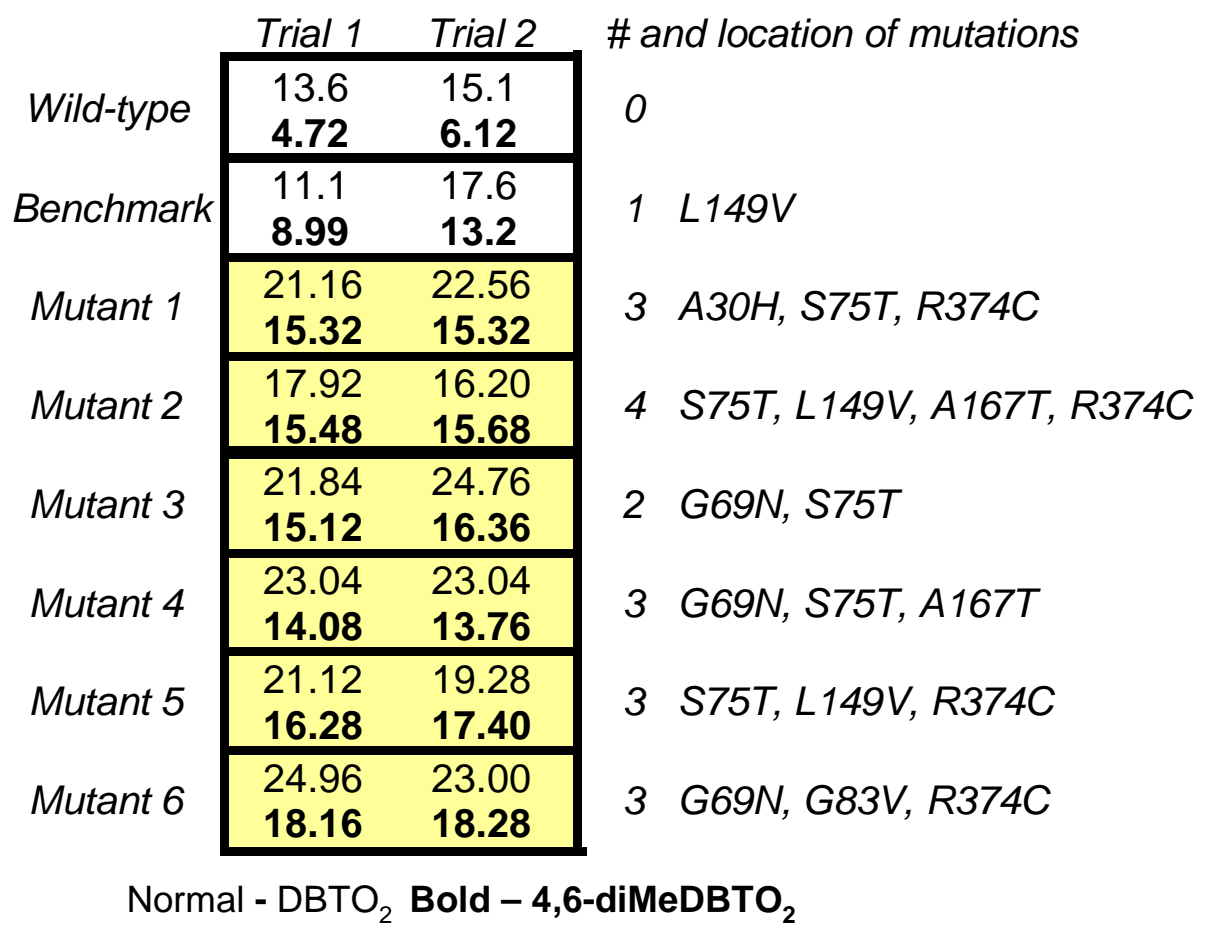

Table 6. In vitro activities of reassembled DSZA GSSM variants on $\mathrm{DBTO}_{2}$ and 4,6dimethyIDBTO 2 (in bold) (uM product/endpoint).

\subsubsection{Expression of selected variants in Rhodococcus}

To determine the behavior of the DszA variants when expressed in Rhodococcus IGTS8 two of the mutants were cloned into the expression vector pEBC1104 (Figure 36), introduced into Rhodococcus CPE648 (a derivative of IGTS8 cured of the pSOX plasmid) and purified using the following protocol: 
1) Cells grown and expression induced in BSM medium with DMSO as sole source sulfur.

2) Cells resuspended in phosphate buffer and cells disintegrated using microfluidizer.

3) Cell debris removed by centrifugation and supernatant proteins precipitated with ammonium sulfate.

4) Reverse phase chromatography (RPC) using a gradient of reducing ammonium sulfate.

5) Buffer dilution followed by ion exchange chromatography (MonoQ).

Progress of DszA purification was monitored by immunoblot analysis and in vitro activity determination. Results are shown in Figure 37.

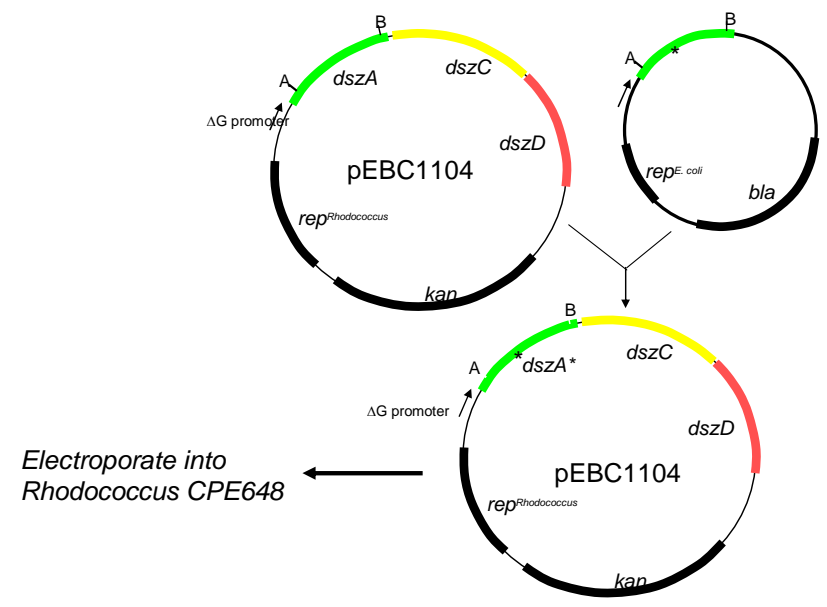

Figure 36. Plasmid pEB1104 and strategy for cloning DszA variants for expression in Rhodococcus CPE648.

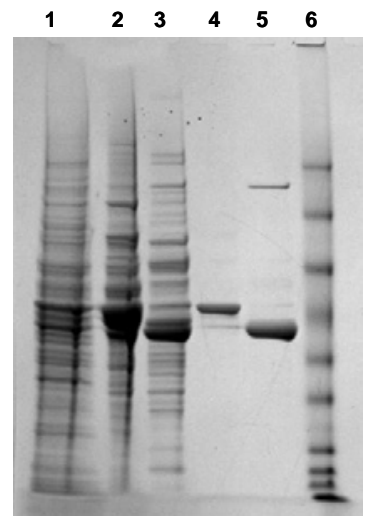

Figure 37. Purification of DszA and DszC proteins expressed in Rhodococcus IGS8 (pEBC1104). Coomassie Blue-stained SDS-PAGE gel. Lanes: 1) crude extract; 2) DszA-containing fraction from RP; 3) DszC-containing fraction from RPC; 4) DszA-containing fraction from MonoQ; 5) DszC-containing fraction from MonoQ; 6) MW markers.

From these efforts we saw a high degree of variation in the specific activity of the variant enzymes from batch to batch. Nonetheless, we have seen that the relative activity of the 
DszA variants on the two substrates $\mathrm{DBTO}_{2}$ and 4, 6-dimethyl $\mathrm{DBTO}_{2}$ is reproducibly higher with the variants than the wild-type parent. Figure $\mathbf{3 8}$ shows an example of a kinetic experiment using two variants in comparison with wild-type, where the overall wild-type specific activity is lower than normal. Table 7 shows the specific activity values of this experiment and an additional purification where wild-type activity is higher but the ratio of activity on the two substrates is similar as the first experiment. Again, the variants show a substantially higher activity on the 4, 6-dimethyl $\mathrm{DBTO}_{2}$ substrate vs. $\mathrm{DBTO}_{2}$ than the wild type parent.
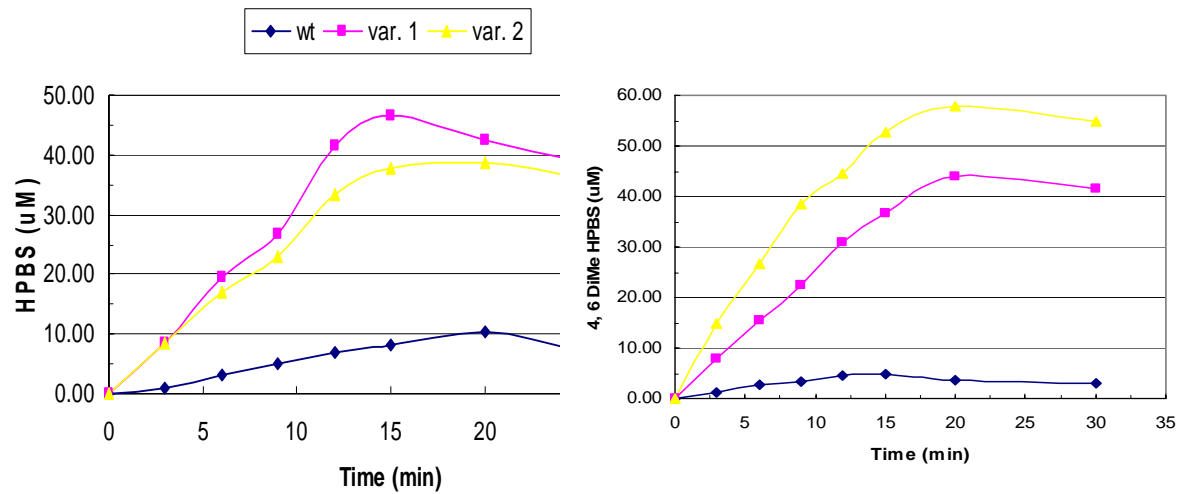

Figure 38. Kinetic analysis of wild-type DszA and variant activities on $\mathrm{DBTO}_{2}$ and 4, 6-dimethyl $\mathrm{DBTO}_{2}$.

\begin{tabular}{|c|c|c|c|c|}
\cline { 2 - 5 } \multicolumn{1}{c|}{} & \multicolumn{3}{c|}{$\begin{array}{c}\text { Specific activity } \\
\text { (UM/min/mg protein) }\end{array}$} & \multicolumn{2}{c|}{} \\
\hline Variant & w/ DBTO 2 & $\begin{array}{c}\text { w/ 4, 6- } \\
\text { dimethyl } \\
\text { DBTO }_{2}\end{array}$ & $\begin{array}{c}\text { Ratio of Sp. } \\
\text { activity }\end{array}$ & $\begin{array}{c}\text { Normalized } \\
\text { to wild-type }\end{array}$ \\
\hline Wild-type & 164 & 103 & 0.63 & 1 \\
\hline 1 & 662 & 763 & 1.15 & 1.83 \\
\hline 2 & 603 & 1096 & 1.82 & 2.88 \\
\hline Wild-type & 2468 & 951 & 0.39 & 1 \\
\hline 1 & 1073 & 917 & 0.86 & 2.22 \\
\hline 2 & 868 & 937 & 1.08 & 2.76 \\
\hline
\end{tabular}

Table 7. Specific activities of wild-type DszA and variants on $\mathrm{DBTO}_{2}$ and 4, 6-dimethylDBTO${ }_{2}$. Two separate kinetic experiments were carried out using material purified on two separate occasions. 


\subsection{Directed evolution of DszC}

Although DszC is the first enzyme in the BDS 4S pathway, it was the second enzyme for which we carried out a directed evolution effort. As in the case of DszA our target was the development of an enzyme with improved ability to oxidize the 4,6-dimethyl DBT that is recalcitrant to HDS. From the many lessons learned in the optimization of the high throughput assay for directed evolution of DszA, we designed a modified strategy to carry out the DszC effort.

\subsubsection{Revision of strategy}

As described previously, the determination of the variant substitutions by nucleotide sequencing provided a high degree of confidence in selection and rejection of potential hits. To more completely take advantage of this information, we elected to determine the nucleotide sequence of all DszC variants at each position before carrying out the high throughput activity assays. This allowed us to "cherrypick" and array microtiter plates with known substitutions and served to:

1) reduce throughput, since it was necessary to screen only preselected residues which were arrayed in duplicate, avoiding the need to oversample to insure adequate representation,

2) give a higher degree of confidence in the data since activity can be directly compared between identical or comparable residues,

3) provide information on residues whose modification gives a desirable outcome as well as information on residues critical for activity whose modification leads to a loss of activity.

There are, however, issues that this strategy creates as it is essential to have robust data-tracking procedures in place in order to carry data through from sequencing, arraying and assaying to analysis. With this in mind we developed procedures that included automated clone arraying, plate tracking, data analysis and report generation.

\subsubsection{Optimization of high throughput assay}

A variety of conditions and experiment protocols were tested in order to develop the most reliable and robust assay for evaluating DszC GSSM variants. Variables that were tested included alternate $E$. coli host strains, incubation and induction conditions, various lytic preparations for releasing enzyme from induced cells and substrate concentrations.

The induction of enzyme expression, lysis of cells and order of addition of reagents is very similar to that previously described for the DszA GSSM assays. In the assay protocol, 50uM DBT and 50uM 4, 6-dimethyIDBT were added as substrates and the reaction proceeded at room temperature for 30 minutes. Reactions were quenched by the addition of an equal volume of acetonitrile and products were assayed by LC/MS/MS as described previously. 


\subsubsection{Results}

As in the case of the DszA GSSM effort, we were able to identify sites that were both permissive and restrictive positions to substitutions. Figure 39 shows a typical permissive residue where the wild-type can be replaced by any other substitution tested. Figure 40, in contrast, shows a residue where not other residue other than wild-type can substitute. Throughout the protein were seen residues that were of varying degrees of permissiveness or restriction.

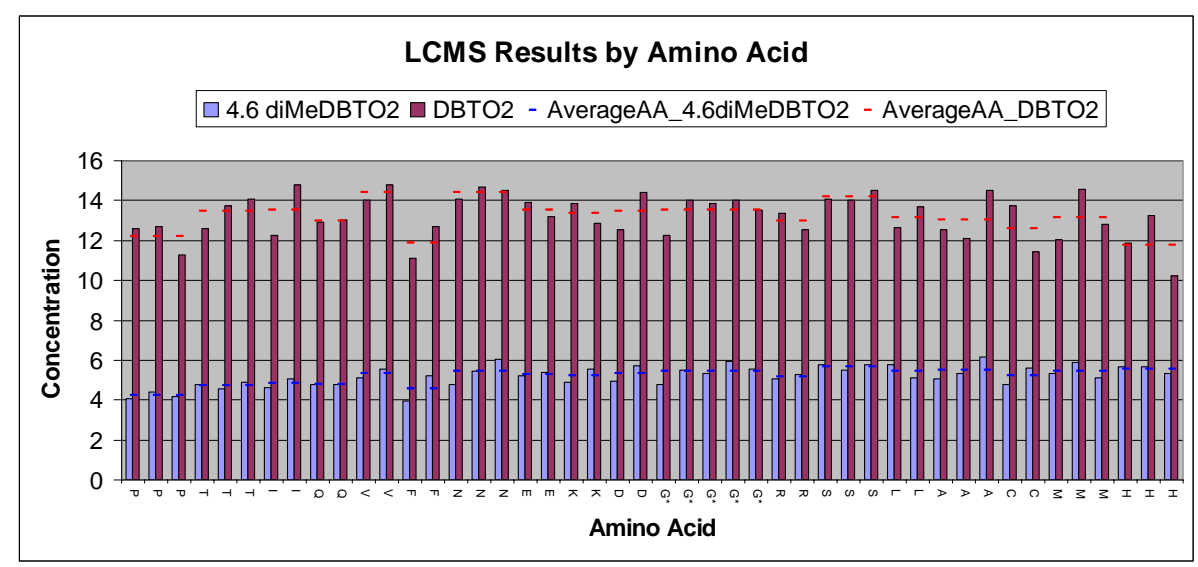

Figure 39. Result of DszC GSSM variant activity screening for a "permissive" residue on DBT and 4, 6-dimethyIDBT.

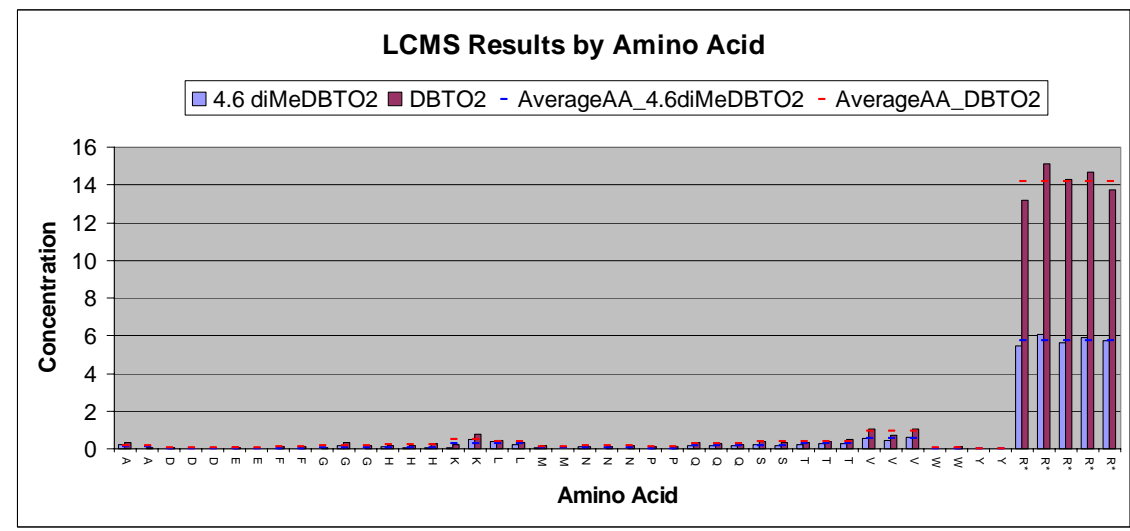

Figure 40. Result of DSZC GSSM variant activity screening for a "restrictive" residue on DBT and 4, 6-dimethyIDBT.

In the initial primary screening of the DszC GSSM library, a total of 91 putative "upmutants", with either an improved ratio of activity on 4, 6-diMeDBT vs. DBT or an increase in apparent total activity, were identified. These were then characterized by a 
rigorous secondary screening effort, with a high degree of replication for each during assaying. Of the original 91 mutants, only three were found to be significantly improved relative to the wild-type benchmark.

To further confirm the properties of these three mutants, plasmid DNA from each were purified and used to retransform a "naive" $E$. coli host. The resultant transformants were then reassayed for activity on 4, 6-diMeDBT vs. DBT.

Unfortunately, none of the three mutant variants demonstrated significantly different activity than the wild-type control, although the original parental clone still showed the improved activity. This suggested that it was a change in the E. coli host background that affected the change in activity. Upon reevaluation of the DNA sequence data from the plates of mutations that the three variants were derived, it was apparent that each was the sole representative of that particular substitution, providing further evidence that the effect was due to an infrequent change in the host itself. Although an interesting observation, we did not follow up further because of the irrelevance of the observation to the development of a final biocatalyst host.

Interestingly, we were able to identify residues whose modification resulted in a significant loss in activity on the 4, 6-dimethyl DBT substrate relative to DBT. Figure $\mathbf{4 1}$ shows an example of one such residue and it can be seen that the substitution of some residues, such as tryptophan (W), have a profound effect on the ability of the enzyme to convert the 4, 6-dimethyl DBT substrate. These results would suggest that this residue either directly or indirectly contributes to the shape of the substrate binding pocket and certain substitutions prevent interaction with the more bulky dimethylated substrate.

\section{LCMS Results by Amino Acid}

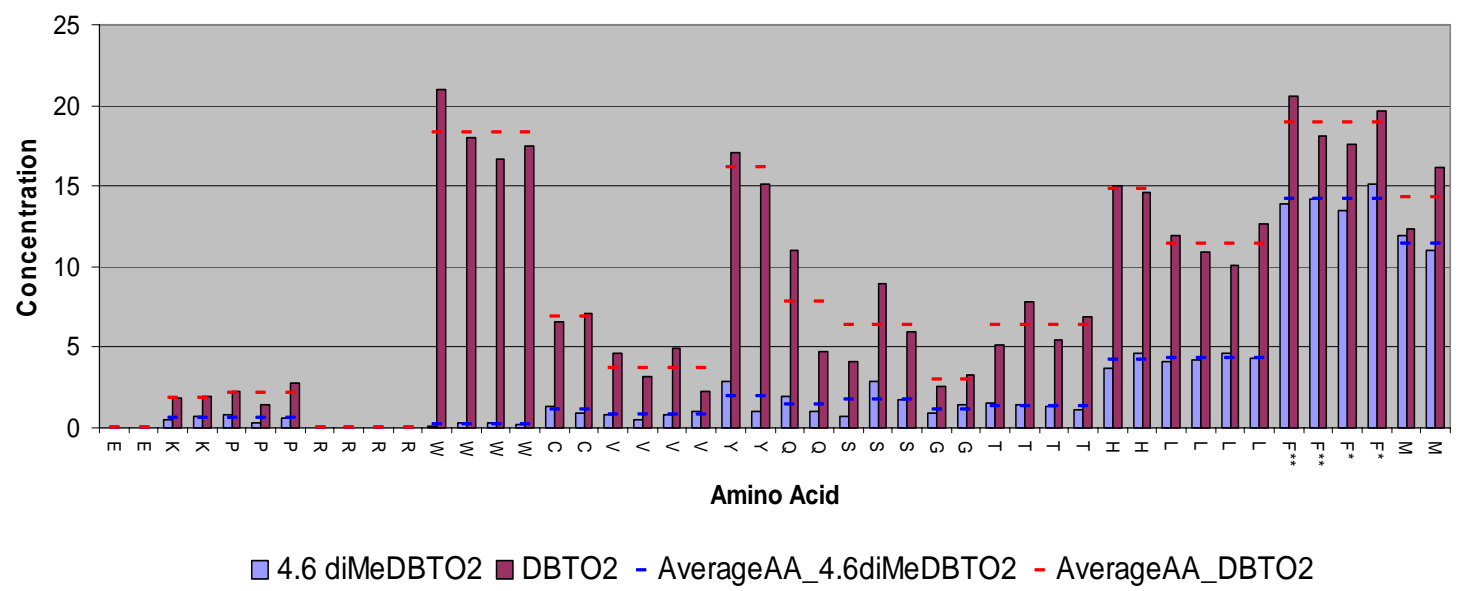

Figure 41. Example of a DszC residue showing substitutions leading to a selective reduction in activity on 4, 6-dimethyl DBT. Wild-type residue is indicated by asterisk; results reflect amount of product in a predefined time. 


\subsubsection{Bioinformatic analyses of DszC GSSM mutations}

As described for DszA, it was necessary to identify a protein homolog with a know structure in the PDB database. This model was built by threading onto the known crystal structures of an acyl-CoA dehydrogenase, to which DszC shows significant homology when comparing secondary structure and known protein folds

Figure 2 shows the results of this DszC structural prediction. From this model, a few observations can me made. First, substrate binding site appears to be at the nexus of the three major structural domains, with key residues indicated in yellow. It is this region, at the vertex of helical domains that appears to impact substrate specificity since it is this region where mutations that selectively reduce activity on 4, 6-diMeDBT, relative to DBT, are found.

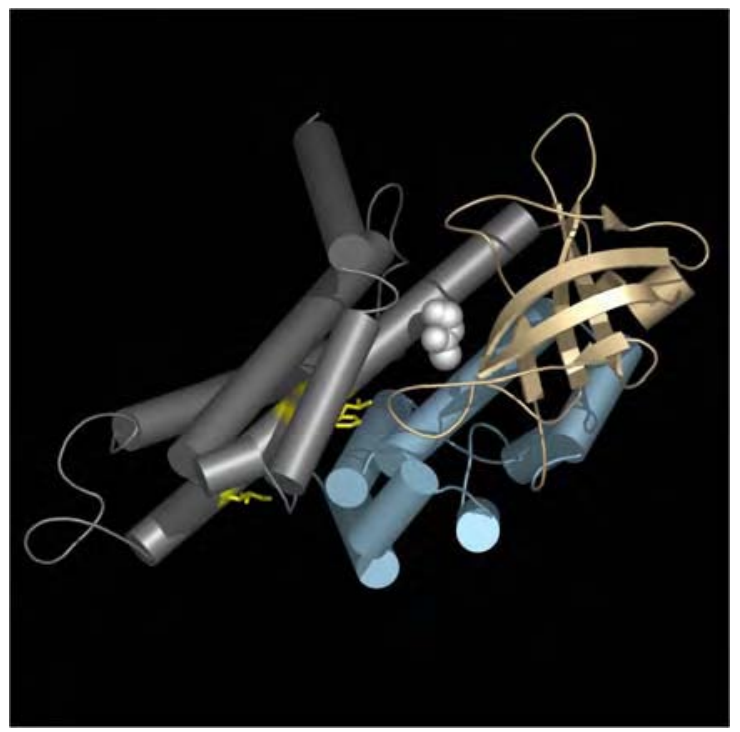

Figure 42. Structural model of DszC derived from threading to the known structure of an acyl CoA dehydrogenase.

Figure 43 summarizes the results of the DszC GSSM by mapping restrictive-permissive residue substitutions onto a space-filling model of the DszC protein. As can be seen, restrictive residues tend to cluster and may define substrate binding sites as compared to the known binding sites of the acyl-CoA dehydrogenase. In addition, as it is known that the acyl-CoA dehydrogenase is found as a homotetramer, composed of two symmetrical dimmer structures, it appears that a number of non-permissive residues may map to a hypothesized dimmer contact zone. 


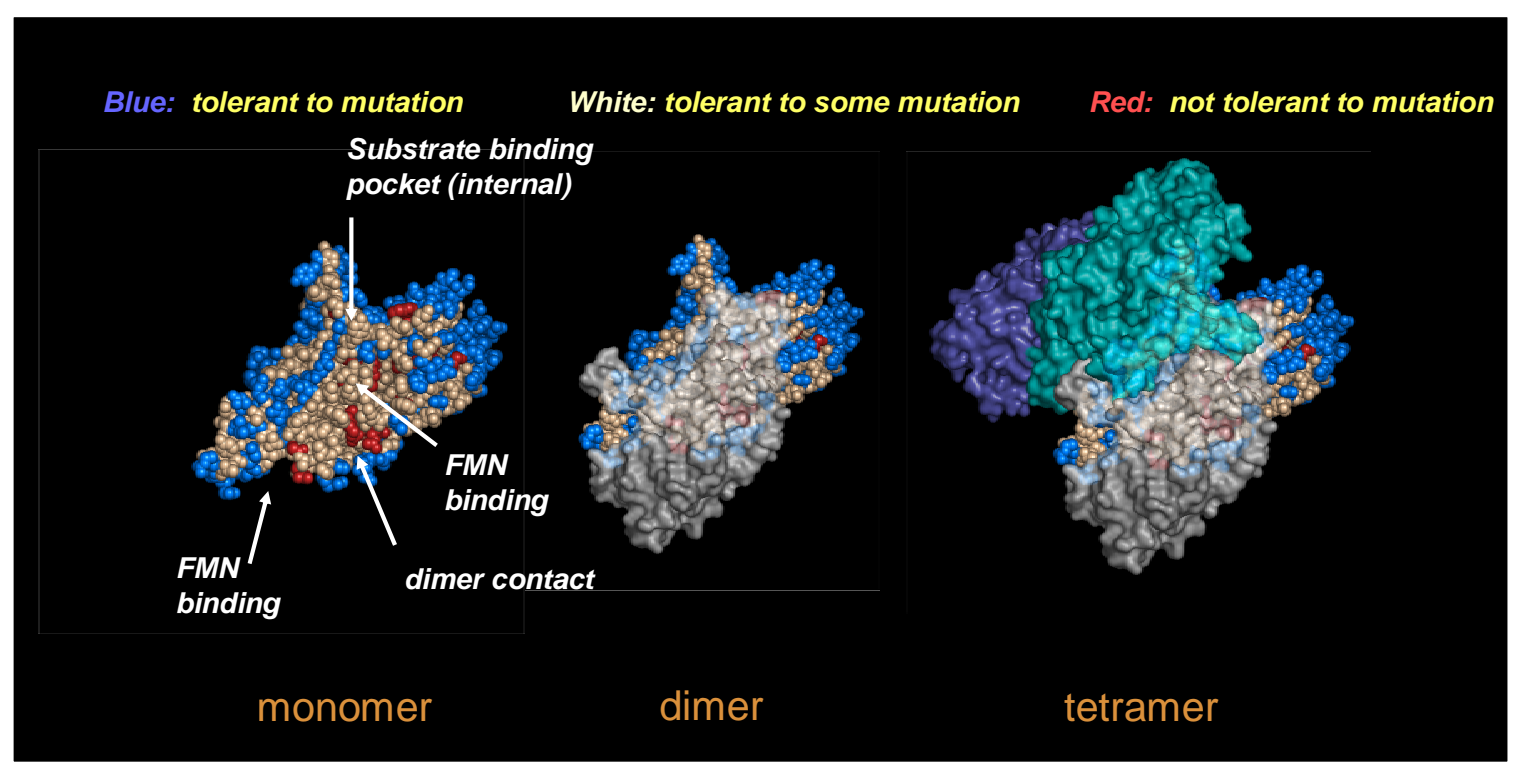

Figure 43. Mapping of DSZC GSSM residue substitutions to a predicted structure of DszC. Based on homology to the acyl CoA dehydrogenase monomer, homodimer and tetramer.

\subsection{Identification of alternate hosts}

Although Rhodococcus IGTS8 has been the prototype strain for biodesulfurization, it is possible that other organisms may represent potentially better organisms for biocatalysis. Properties such as faster growth rates and general robustness may also be important properties in the development of an economically viable biocatalyst. With this in mind, we set out to characterize $>130$ Rhodococcus and related strains to identify potential hosts for the introduction of the biodesulfurization genes and comparison of properties to Rhodococcus IGTS8. Rhodococcus erythropolis, R. globerulus, R. opacus, $R$. ruber and $R$. rhodochrous, as well as Gordonia ruberperfincta strains were obtained from both the ATCC and DSM culture collections. Phenotypic characterizations included the ability to utilize rich and minimal media under a variety of aeration conditions, and ease of plating to identify strains that could most easily be genetically manipulated.

\subsubsection{Growth characteristics}

From initial experiments it is clear that a subset of strains have significantly more robust growth properties as compared to Rhodococcus IGTS8. In particular, a number of isolates, primarily Rhodococcus opacus strains, were able to grow to higher density with significantly less aeration and may represent strains that are capable of utilizing available oxygen more effectively. These strains all demonstrated extremely robust growth characteristics in liquid and solid, as well as rich and minimal, media. Each was capable of reproducible formation of large uniform colonies on minimal agar medium and growth to maximum density on minimal liquid media within $18-24$ hours at $30^{\circ} \mathrm{C}$. 
From descriptions in the literature, $R$. opacus strains are capable of growth on a wide variety of carbon sources including acetate and ethanol and are also able to store large amounts of triacylglycerols when grown under appropriate conditions (Figure 44) (12). Stored triacylglycerols represent a ready source of cellular reductant upon mobilization and could be envisioned to minimize the need to provide exogenous carbons sources to provide reductant for biodesulfurization reaction.

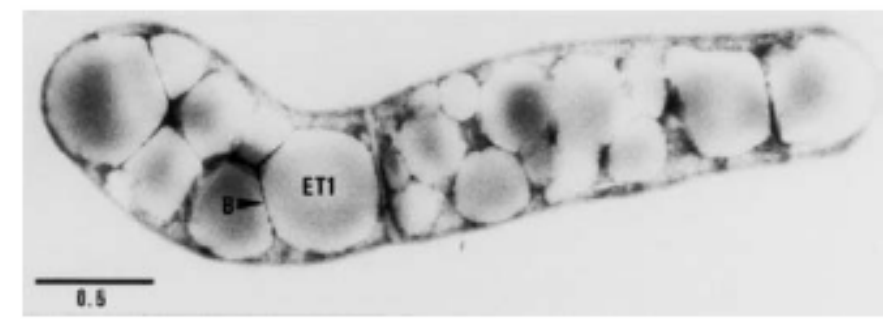

Figure 44. Electron micrograph of Rhodococcus opacus DSM 43943 and accumulated droplets of triacylglycerols.

\subsubsection{Genetic amenability}

As mentioned above, another important criterion for the potential development of new strains as biodesulfurization biocatalyst is the ability to be genetically manipulated. To assess this property of the twenty $R$. opacus strains, each was prepared for electroporation using a method developed for IGTS8. Plasmid pEBC1100 was then used to test the ability of each to receive DNA. This plasmid expresses the entire dszABC operon as well as DszD, encodes kanamycin resistance and can confer the complete 4S pathway phenotype onto a recipient.

Kanamycin-resistant clones were selected after electroporation of the strains. From these experiments, it was determined that seven of the twenty strains were not able to be efficiently electroporated and were dropped from further consideration as biocatalyst hosts due to the need for their genetic manipulation at later stages in the project. Results for ten of the strains, in terms of qualitative efficiency of electroporation are shown in Figure 45.

\begin{tabular}{c|c|c|c|} 
Strain & Efficiency & Strain & Efficiency \\
43250 & + & 44236 & +++ \\
43251 & + & 44305 & ++ \\
43252 & ++ & 44311 & +++ \\
43943 & ++++ & 44312 & ++++ \\
44193 & ++++ & 46027 & ++
\end{tabular}

Figure 45. Electroporation efficiency of representative Rhodococcus opacus strains. Measured with $\mathrm{pEBC} 1100$ as electroporation substrate. All strains are from the Deutsche Sammlung von Mikroorganismen und Zellkulturen (DSMZ). 


\subsubsection{In vivo biodesulfurization activity}

The remaining thirteen strains, each containing $\mathrm{pEBC} 1100$, were then tested for their ability to use DBT, $\mathrm{DBTO}_{2}$ or HBPS as sole source of sulfur when grown on minimal medium, Figure $\mathbf{4 6}$ shows the results of these experiments, with growth being expressed qualitatively.

\begin{tabular}{c|c|c|c} 
Strain & $D B T$ & $D^{2} \mathrm{O}_{2}$ & $\mathrm{HPBS}$ \\
44236 & ++ & ++ & $+/$ \\
43252 & + +- & ++ & ++ \\
44311 & +++ & +++ & +++ \\
43943 & +++ & +++ & +++ \\
46027 & - & +++ & - \\
44186 & + & ++ & + \\
44315 & - & +++ & ++ \\
44307 & + +- & +++ & + \\
44193 & +++ & +++ & +++ \\
44251 & - & ++ & $+/$ \\
44312 & +++ & ++ & +++ \\
44305 & - & ++ & - \\
43250 & + +- & ++ & $+/$
\end{tabular}

Figure 46. Utilization of organosulfur compounds by $R$. opacus strains as sole source of sulfur in strains containing pEBC1100. All strains are from the DSMZ.

\subsubsection{Survival on exposure to hexadecane and diesel}

Another important property of a potential biocatalyst strain is the ability to survive exposure to organic solvents and diesel fuel. Both the ability to survive a transient exposure as well as continuous exposure was tested for the subset of $R$. opacus strains that demonstrated activity on DBT, $\mathrm{DBTO}_{2}$ or HBPS.

To determine survival after transient exposure to hexadecane, various amounts of hexadecane were added to a diluted suspension of the organism $\left(10^{5} \mathrm{CFU} / \mathrm{ml}\right)$ to be tested and samples were taken after 15 minutes of exposure. A high degree of variability was seen between experiments, most likely due to difficulty in completely separating cells from the hexadecane/water interface. Overall, patterns of survivability could be seen with each strain in comparison to IGTS8 and derivatives JB55 (dszABC deletion mutant - retains the remainder of the pSOX plasmid) and CPE648 (cured of pSOX plasmid). From these patterns an overall score was generated for each strain that qualitatively describes survivability under these conditions (Figure 47).

Using this scoring scheme, strains exhibiting pattern 1 are most tolerant, and pattern 5 are least tolerant. By combining results from multiple experiments, it can be seen that overall, IGTS8 and its derivatives are more tolerant to hexadecane than most $R$. opacus strains. Some, however, such as DSM43250, DSM43236 and DSM44312, appear to be nearly as tolerant. It is interesting to note that IGTS8 is noticeably more tolerant than its 
derivatives CPE648 and JB55, which appears to correlate with its more pronounced mucoidy and presumed production of biosurfactant (see below).

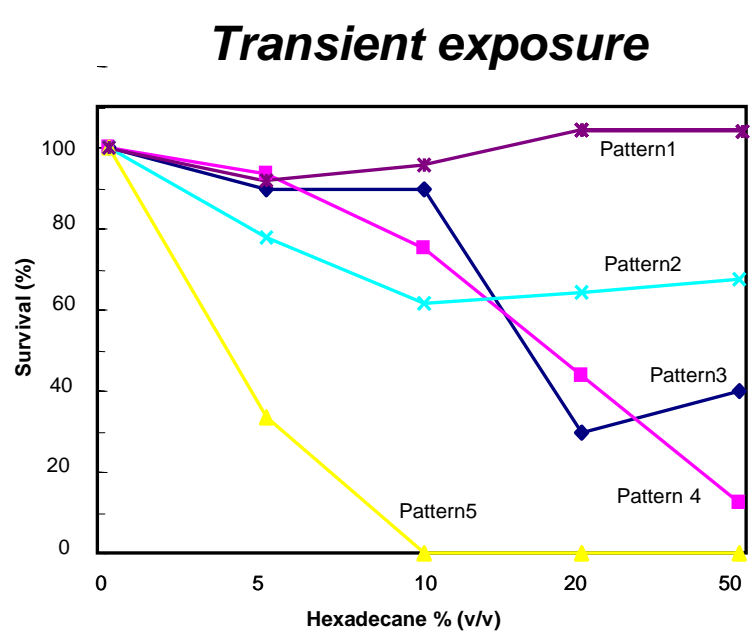

\begin{tabular}{|c|c|c|c|c|c|c|}
\hline & A & B & C & D & E & AVG \\
\hline IGTS 8 & 1 & 1 & 1 & 1 & 1 & 1 \\
\hline CPE 648 & 2 & 3 & 2 & 2 & 2 & 2.2 \\
\hline JB-55 & 3 & 2 & 2 & 3 & 2 & 2.4 \\
\hline $\mathbf{4 3 2 5 0}$ & 2 & 2 & 1 & 2 & 2 & 1.8 \\
\hline $\mathbf{4 3 2 5 1}$ & 4 & 4 & 4 & & 4 & 4 \\
\hline $\mathbf{4 3 2 5 2}$ & 2 & 4 & 2 & 2 & 4 & 2.8 \\
\hline $\mathbf{4 3 9 4 3}$ & 5 & 4 & 5 & 5 & 4 & 4.6 \\
\hline $\mathbf{4 4 1 8 6}$ & 4 & 5 & 4 & 4 & 5 & 4.4 \\
\hline $\mathbf{4 4 1 9 3}$ & 5 & 5 & 4 & 5 & 4 & 4.6 \\
\hline $\mathbf{4 4 2 3 6}$ & 3 & 2 & 2 & 2 & 2 & 2.2 \\
\hline $\mathbf{4 4 3 0 5}$ & 1 & 4 & 1 & 1 & 4 & 2.2 \\
\hline $\mathbf{4 4 3 1 1}$ & 4 & 5 & 4 & 4 & 5 & 4.4 \\
\hline $\mathbf{4 4 3 1 2}$ & 3 & 2 & 3 & 2 & 3 & 2.6 \\
\hline
\end{tabular}

Figure 47. Transient survivability of strains with hexadecane. A) Patterns of survivability seen in multiple experiments; B) Scoring of strains, 1-5, using pattern in A) with 1 being best, 5 being worst. All strains are from the DSMZ.

To determine the ability of each of the strains to survive continuous exposure to either hexadecane or diesel strains were diluted to $10^{4} \mathrm{CFU}$ and plated onto medium that had previously been covered with a layer of either hexadecane or Petro Star diesel. After 2 weeks of incubation, the number of CFU as compared to a control plate with no treatment was determined and survival rate indicated (Figure 48).

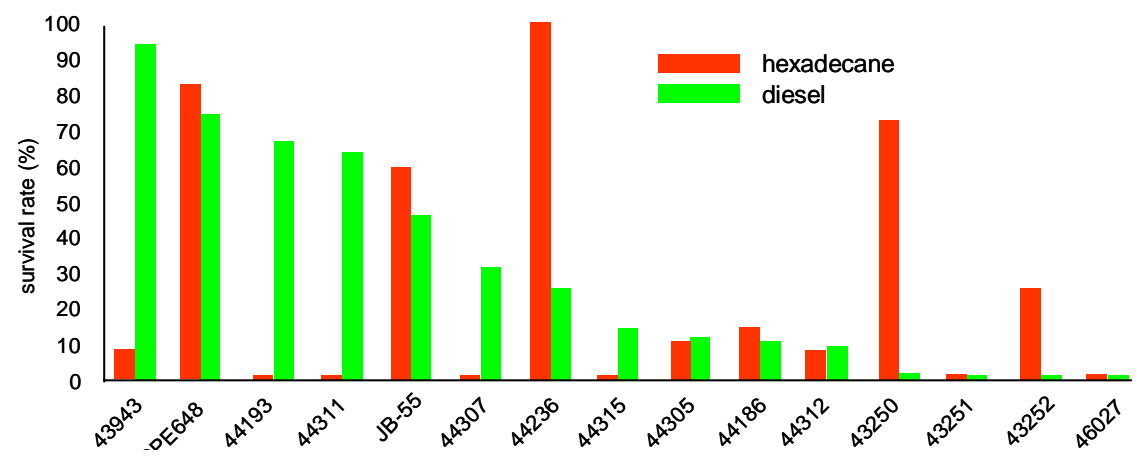

Figure 48. Continuous survivability of strains with hexadecane. All strains are from the DSMZ.

From these experiments, it was apparent that many of the strains that were most tolerant in the transient exposure, such as DSM44236, DSM43252, DSM43250, were also found to be tolerant in this experiment. Surprisingly strains such as DSM44186 and DSM43943 
did not stand out in the transient exposure experiment but did survive continuous exposure. This may be a result of inadequate partitioning in the transient exposure experiment, with cells remaining associated with the hexadecane phase and not being plated for CFU determination.

It is clear from these experiments that IGTS8 and its derivatives are comparatively resistant to hexadecane and diesel and suggests that they express specialized properties for this resistance. It has been suggested that IGTS8 produces a biosurfactant that contributes to both its survival to hydrophobic compounds and its biodesulfurization ability. Extensive characterization of surfactants from Rhodococcus, Nocardia and Mycobacterium species suggests that they are sugar ester derivatives (primarily trehalose as sugar) of the cell wall mycolic acids that are found in these organisms. Surprisingly, little is known about the genetic basis of Rhodococcus biosurfactant production. Any use of alternate Rhodococcus strains for biodesulfurization is likely to benefit from the expression of these biosurfactant compounds

\subsubsection{Whole cell two-phase BDS activity}

From the above experiments we selected four $R$. opacus strains for further evaluation by carrying out two-phase whole cell oxidation experiments, using DBT substrate in a hexadecane matrix. Into each of the strains, we introduced plasmid pEBC1104, which encodes $d s z A, d s z C$ and $d s z D$, but is missing the $d s z B$ gene. Since DszB is not expressed, the final product of oxidation from DBT is HBPS, whose accumulation was monitored to evaluate BDS activity. As can be seen in Figure 49, the R. opacus strain DSM 43943 was noticeably more active only a longer period of time, in comparison to DSM44311, which although it had a faster initial rate, appeared to reach a maximum, extent at $\sim 40$ hours.

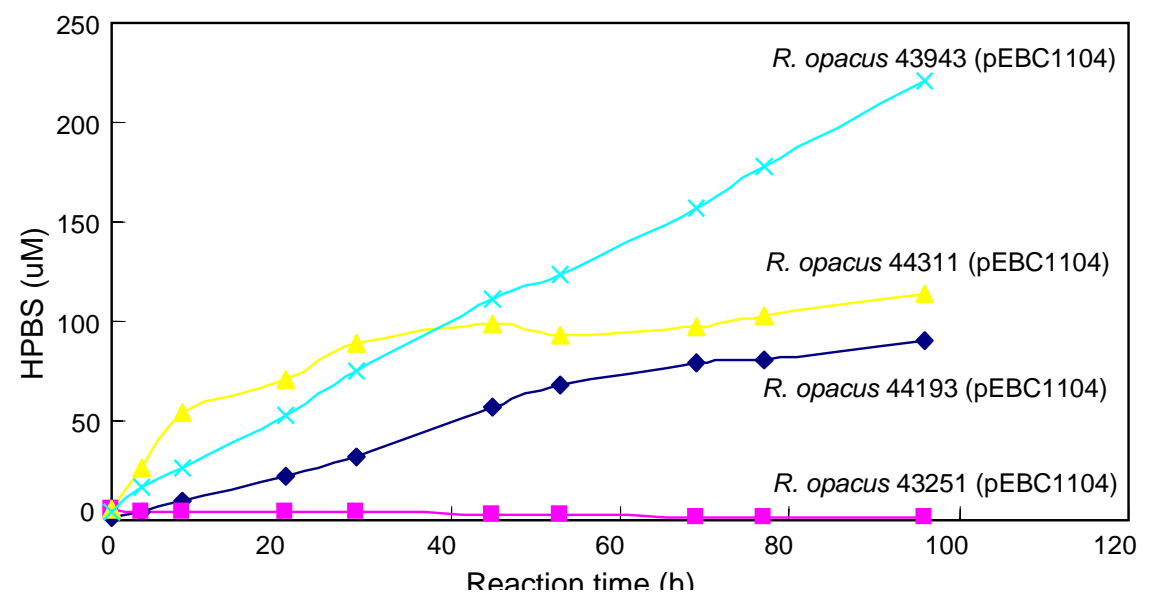

Figure 49. Whole cell BDS activity of R. opacus strains containing pEBC1104.

To further evaluate this difference between DSM43943 and DSM44311, we carried out additional whole cell reactions and evaluated both HBPS appearance and DBT depletion in order to insure mass balance of the constituents and that HBPS was not being converted to another product. As can be seen in Figure 50, the disappearance of DBT 
and appearance of HBPS was reciprocal, demonstrating again that DSM 43943 was clearly able to sustain DBT oxidation to completion in this assay.
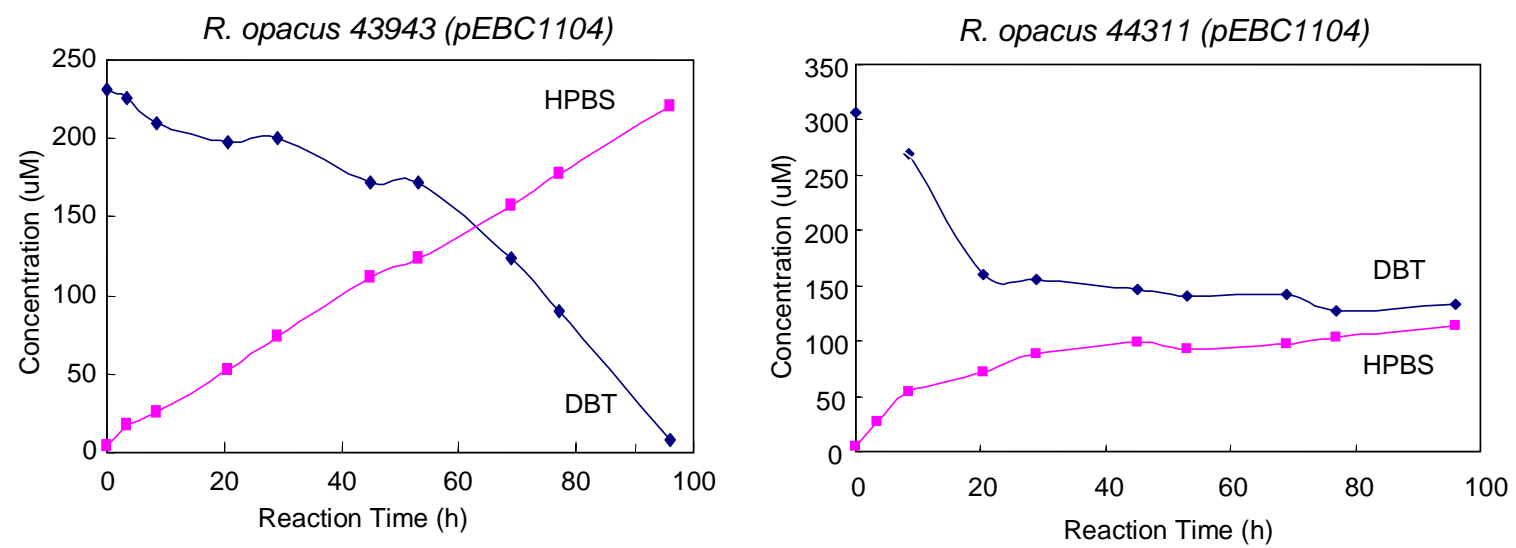

Figure 50. Whole cell BDS activity of R. opacus strains DSM 43943 and DSM 44311 containing $p E B C 1104$.

\subsubsection{Contribution of pSOX genes to BDS activity}

To determine if additional genes are present on the IGTS8 pSOX plasmid that contribute to biodesulfurization activity, plasmid pEBC1104 was introduced into CPE648 (a derivative of IGTS8 cured of the pSOX plasmid), JB55 (a derivative of IGTS8 with the dsz genes deleted from pSOX) and R. opacus strain 43943. In addition, Rhodococcus IGTS8 derivative BKO53A was included.

BKO53A is a strain previously constructed by Energy Biosystems that demonstrated the highest level BDS activity of any strain previously reported and served as their benchmark for any further development. This strain contains the pSOX plasmid modified to include cointegrated, additional copies of the $d s z A,-C$ and $D$ genes, under the control of the $\Delta G$ promoter, a variant of the normal $d s z$ promoter that is less sensitive to inorganic sulfur repression. In addition, the $d s z B$ gene has been deleted and replaced with a chloramphenicol acetyltransferase gene, conferring chloramphenicol resistance to the strain.

As can be seen in Figure 51, JB55 (pEBC1104) shows more long-lived rate of BDS activity compared to CPE648, suggesting that pSOX may express additional gene products that contribute to continuing activity of the biocatalyst over time. It can also be seen that the $R$. opacus host shows higher levels of sustained activity than either of the IGTS8 backgrounds, suggesting that longevity of the biocatalyst is more robust in this host in the hexadecane model system. BKO53A, however, still demonstrates the highest level of activity and completely removes all substrate after twenty hours. 


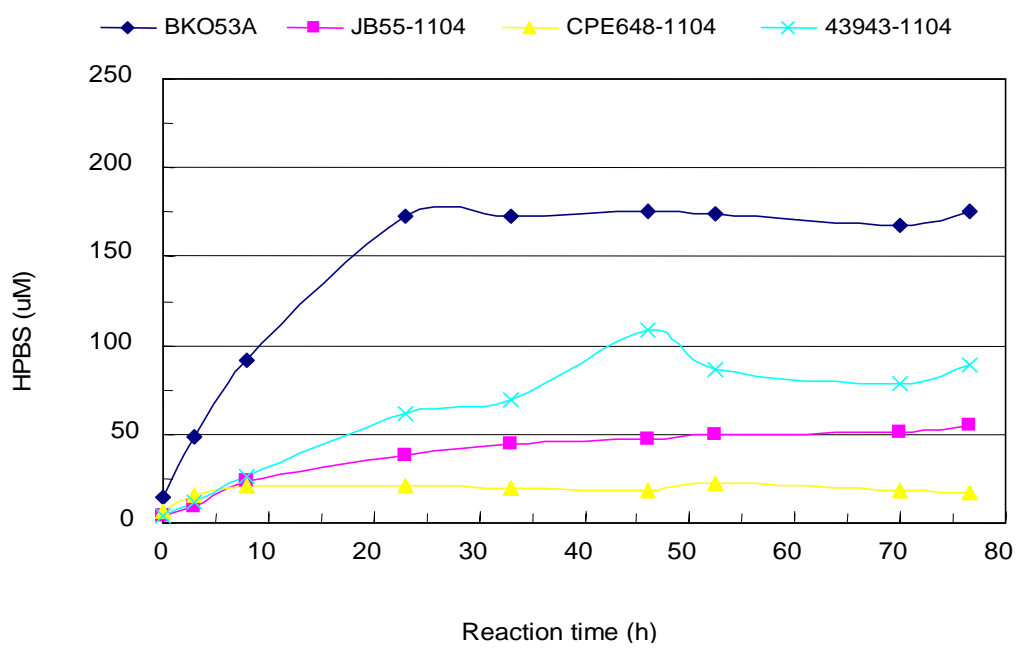

Figure 51. Activity of pEBC1104-containing strains in DBT-hexadecane shake flask assay. Reactions contained $0.6 \%$ DBT in hexadecane at a 3:1 WOR. Equivalent dry cell weights were added for each.

\subsubsection{Introduction of pBKO53A into Rhodococcus opacus}

Since we had observed that $R$. opacus DSM 43943 exhibited higher levels activity than either IGTS8 derivatives CPE648 or JB55 containing pEBC1104, we wanted to determine if the introduction of the pBKO53 plasmid (modified pSOX plasmid) from the IGTS8 derivative BKO53A would confer comparable or better BDS activity. It had been previously reported (3) that the pSOX plasmid might be conjugatable and we had also evidence that transfer genes were found in the pSOX plasmid (see below).

With this in mind we attempted to transfer the pBKO53A plasmid to DSM 43943 by conjugation. A spontaneous rifamycin-resistant derivative of DSM 43943 was generated and used as a recipient in a biparental mating with IGTS8 BKO53A as donor. Since pBK053A was generated by cointegration of a chloramphenicol-resistant $E$. coli cloning vector into the native pSOX plasmid, rifampicin- and chloramphenicol resistant exconjugants were isolated. Two independent colonies were purified and confirmed to be $R$. opacus DSM 43943 by sequence comparison of the 16S DNA to the parent strains.

The two R. opacus 43943 (pBKO53A) clones were tested for BDS activity in a two phase hexadecane-DBT assay and compared to the IGTS8 BKO53A strain as well as a DSM 43943 control strain (Figure 52). Both strains exhibited comparable activity and equal or better activity to the IGTS8 BKO53A benchmark strain.

\subsubsection{Characterization of biocatalyst activities on partially hydrotreated diesel.}

As previously discussed, we are considering the combination of the hydrotreatment and biodesulfurization processes as having the most likelihood of success. With this in mind, we set out to characterize the activities of candidate biocatalysts on the partially hydrotreated material. 
Final Technical Progress Report DOE Award No. DE-FC26-02NT15340

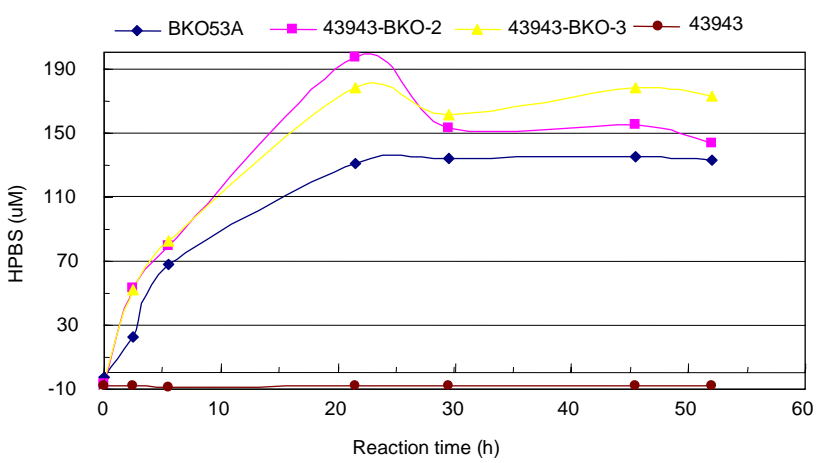

Figure 52. Whole cell BDS activity of IGTS8-derivative BKO53A compared to two independent isolates of R. opacus DSM43943 containing pBKO53A plasmid. Reactions contained 0.6\% DBT in hexadecane at a 3:1 WOR. Equivalent dry cell weights were added for each.

Both the Rhodococcus IGTS8 (pBKO53A) and the Rhodococcus opacus (pBKO53A) strains were tested for their ability to remove sulfur from partially hydrotreated diesel the 418ppm (Figure 53).

Rhodococcus IGTS8 (pBKO53A)

Rhodococcus opacus (pBKO53A)
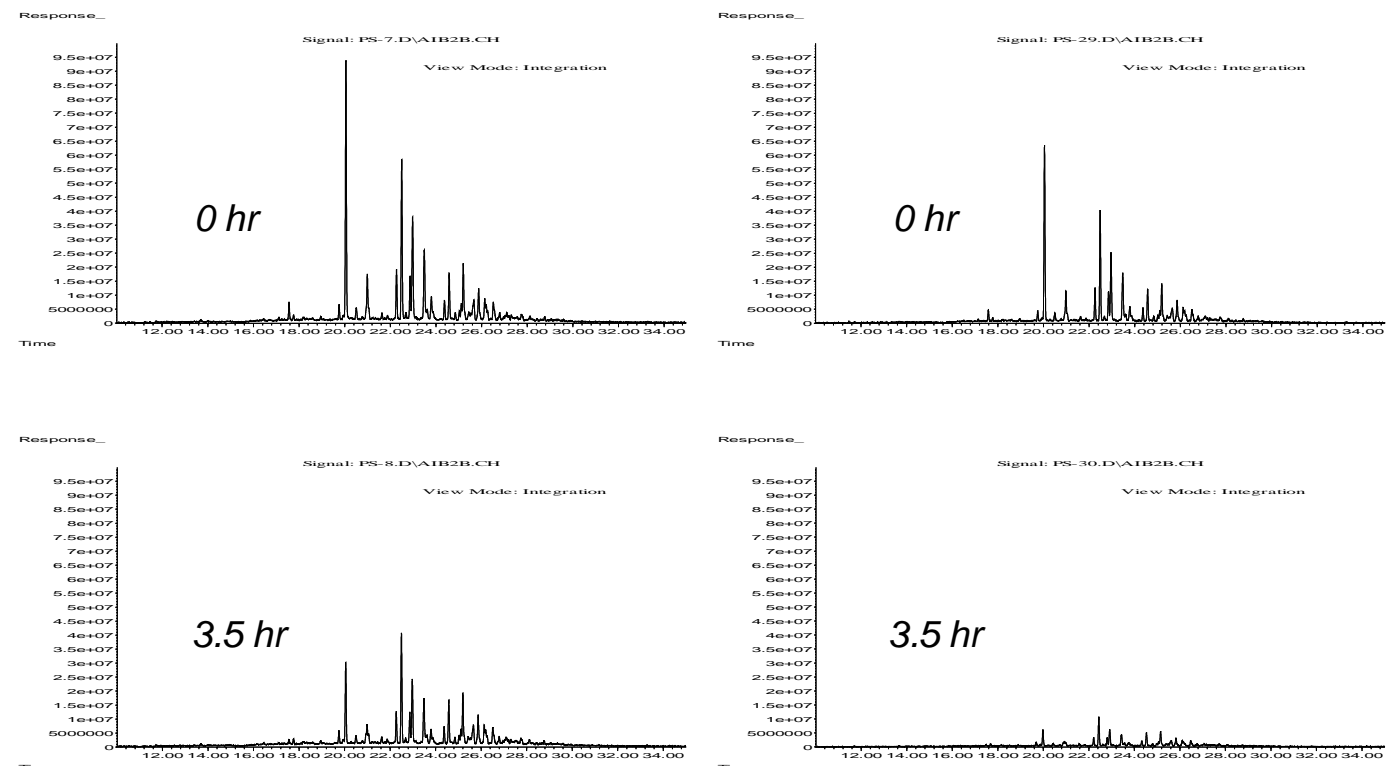
nime
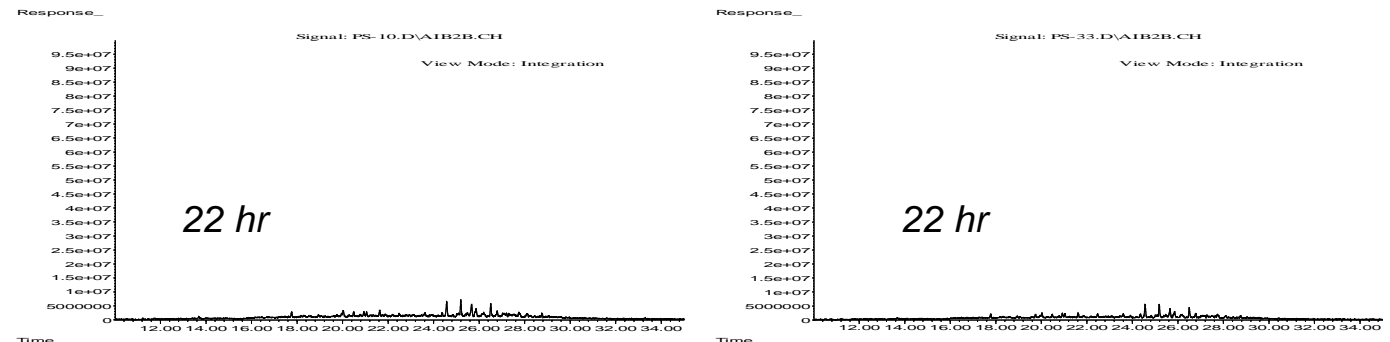

Figure 53. Time course of biocatalyst activity on partially hydrotreated diesel fuel (418ppm) showing reduction of $C X-D B T$ compounds. 
When the quantification of each of the Cx-DBTs was determined, little difference was seen between the performances of the two different biocatalysts. As can be seen in Figure 54 (showing the results of the IGTS8-BKO53A biocatalyst) the reduction of CXDBT compounds is nearly complete at fifty hours while the remaining total sulfur content remains levels off earlier and does not appear to be acted upon by the biocatalyst. This residual sulfur is likely to be represented by the "hump" that is seen to remain at the 22 hour time point (Figure 55).

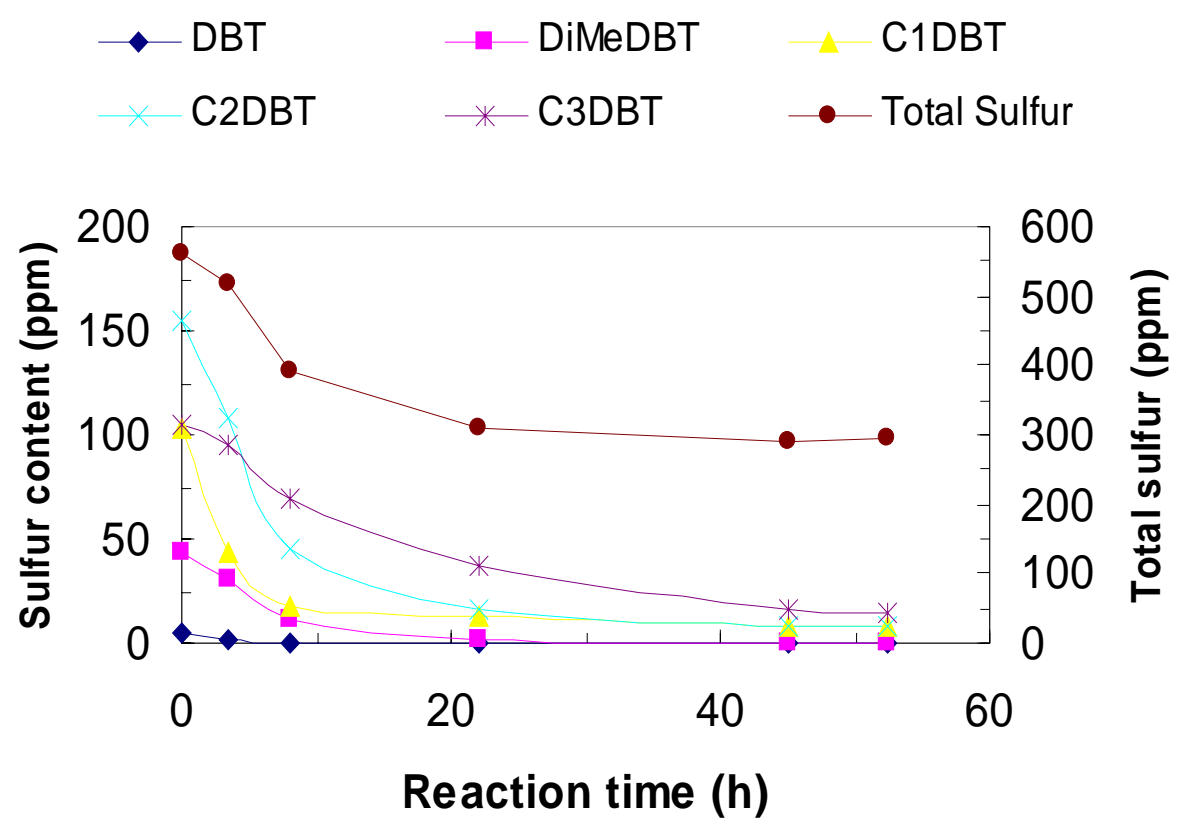

Figure 54. Time course of IGTS8-BKO53A biocatalyst activity on partially hydrotreated diesel fuel (418ppm).

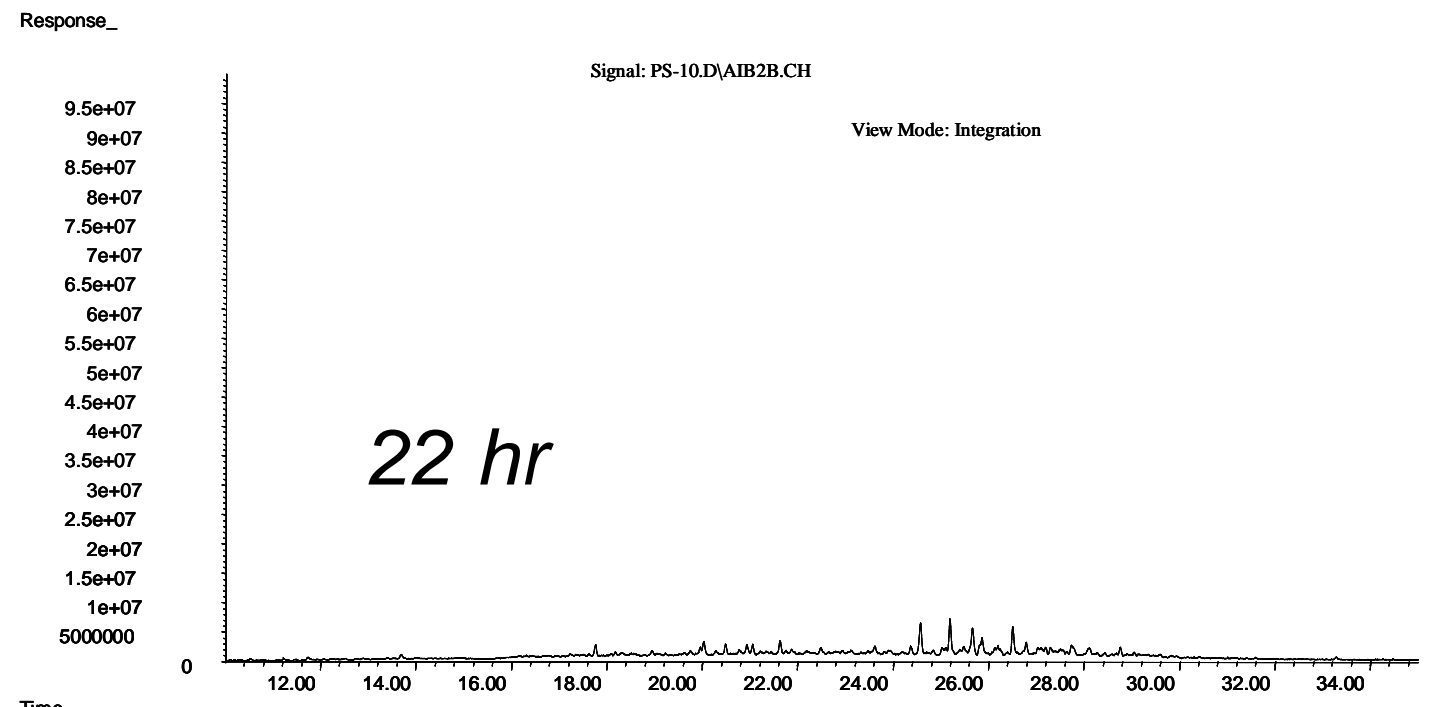

Figure 55. Expanded chromatogram of 22hour time point. From Figure 53 (IGTS8-BKO53A) showing residual "hump" of sulfur compounds not acted upon by biocatalyst. 
This suggests that there are compounds that are resistant to the effects of the biocatalyst even in this partially hydrotreated diesel. It is unlikely to be $C_{x}$-DBT compounds of $\mathrm{C} 4$ or higher because of their negligible contribution to total sulfur. Instead, this would appear to be the presence of a highly heterogeneous group of compounds that are not substrates for the biocatalyst. To further illustrate this point, we carried out the BDS treatment of diesel that has been hydrotreated further to 43ppm total sulfur. Figure 56 shows the results of this treatment and again demonstrates that, while the $\mathrm{C}_{\mathrm{x}}$-DBT content is nearly completely removed, a significant amount of sulfur remains that does not appear to be a substrate for the biocatalyst. As can be seen in previous GC/SCD traces, a small but detectable "hump" of sulfur-containing compounds is found even in the deepest hydrotreated fuels (Appendix 2). No obvious peaks however can be seen, suggesting again that this material is a highly heterogeneous population of species with apparent masses of less than $\mathrm{C}_{5}$-DBTs. This indicates that the failure of the biocatalyst to remove this material is not as a result of mass transfer or solubility problems with high molecular weight $\mathrm{C}_{\mathrm{x}}$-DBT compounds but due to the inability of the biocatalyst to act on these unknown species.

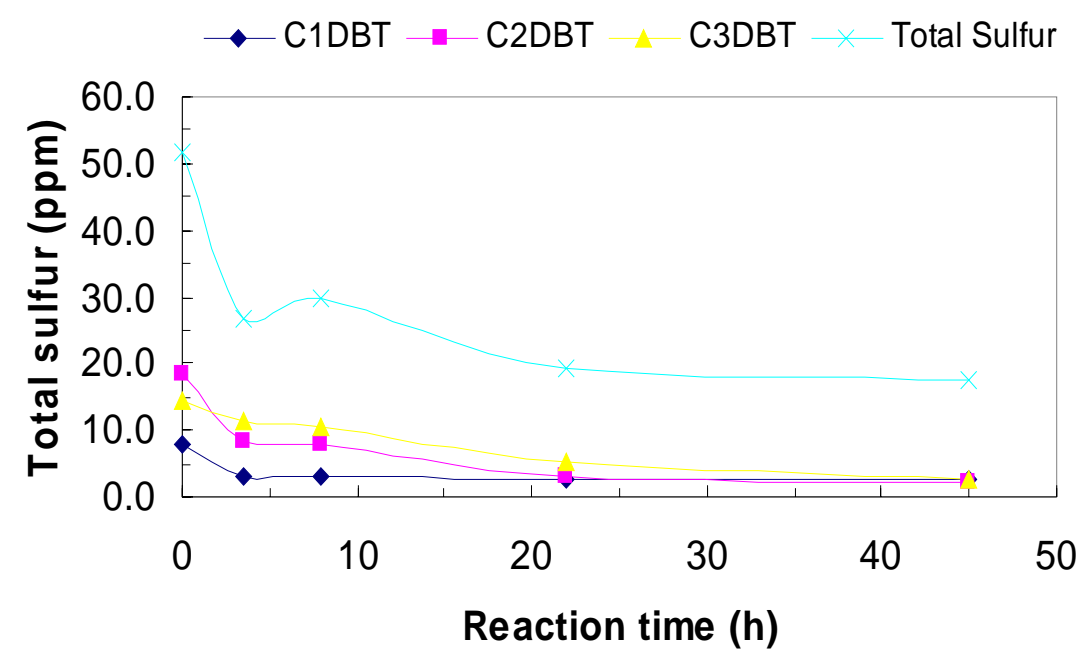

Figure 56. Time course of IGTS8-BKO53A biocatalyst activity on partially hydrotreated diesel fuel (43ppm).

To determine if there may be differences in the ability of these two hosts to use endogenous reductants as sources of redox equivalents in the oxidation of DBT, both strains were tested with and without the addition of glucose for their ability to oxidize partially hydrotreated diesel fuel. As can be seen in Figure $\mathbf{5 7}$ both strains required the addition of glucose for full activity, but it appears that Rhodococcus opacus 43943 is able to sustain a low level of oxidation over the course of the assay. In contrast, activity of IGTS8 appears to level off after 25 hours. To further define this observation, oxidation of individual DBT species was determined. Again, it can be seen that the $R$. opacus strain appears to sustain a low level of oxidation of each of the Cx-DBTs monitored over time where the IGTS8 levels off (Figure 58). 

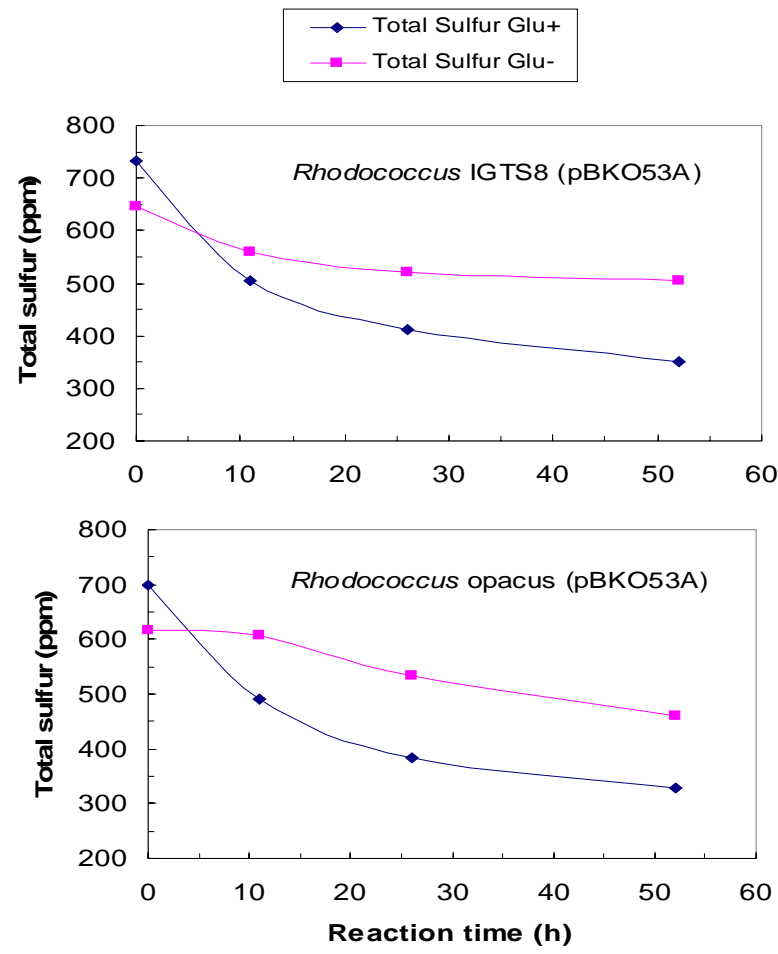

Figure 57. Activity of biocatalysts on partially hydrotreated diesel fuels with and without the addition of glucose as reductant and the effect on total sulfur.

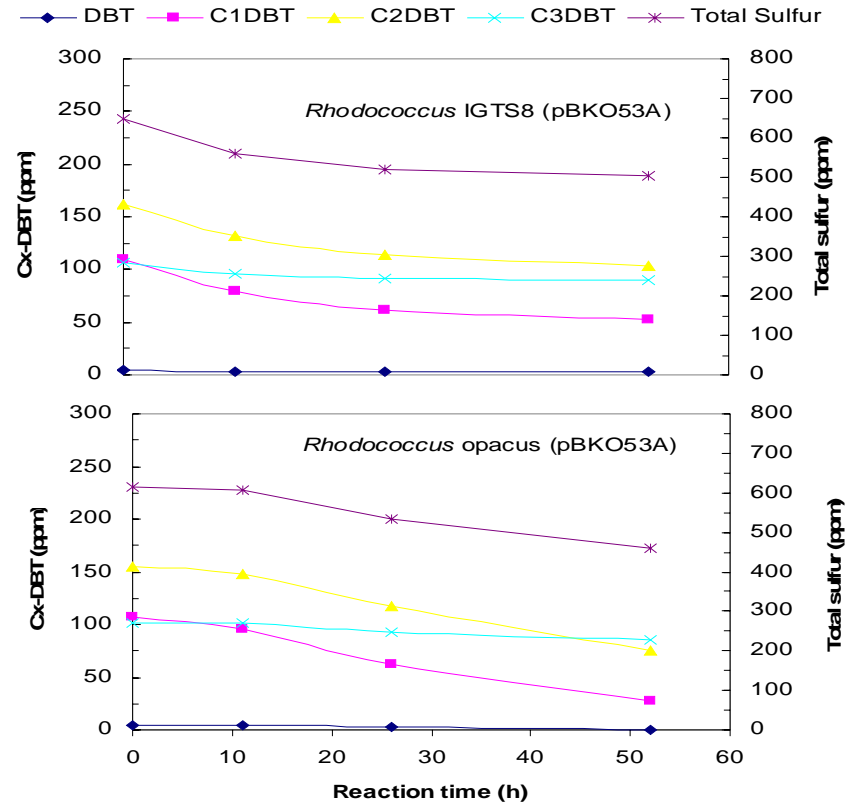

Figure 58. Activity of biocatalysts on partially hydrotreated diesel fuels with and without the addition of glucose as reductant and the effect on $C_{x}-D B T$ compounds. 


\subsubsection{Stability of pBKO53A in host strains}

As we had previously noted, there are some differences in the tolerance of the two strains to diesel fuel. In addition, we have noticed there is also a general instability in the maintenance of the pBKO53A plasmid as detected by loss of chloramphenicol resistance upon passage. To test this we carried out another plating efficiency experiment with each of the strains containing pBKO53A by plating dilutions cells onto BSM-glucose, BSM-glucose with chloramphenicol and BSM-glucose with chloramphenicol overlaid with diesel. Figure $\mathbf{5 9}$ shows the results of this experiment where the total colony forming units of the latter two conditions were compared to the cells plated onto BSM-glucose.
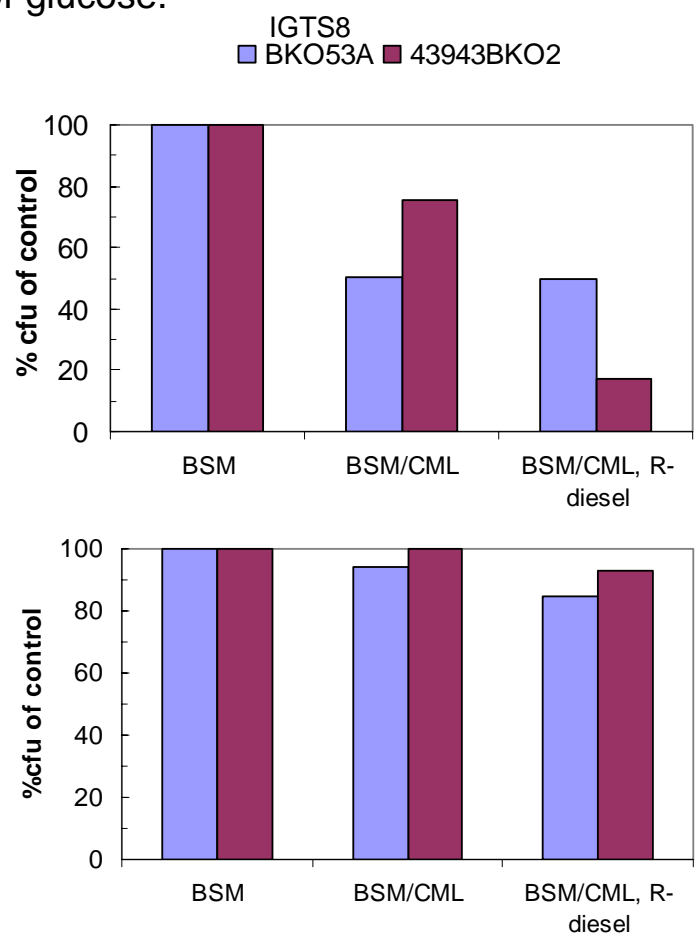

Figure 59. Relative plating efficiencies of strains. CFUs compared upon addition of chloramphenicol and diesel overlay to number of cells plated on BSM-glucose alone.

From these results it is apparent that there is instability of maintaining pBKO53A in both hosts although it appears to be less pronounced in the $R$. opacus strain. We have, however, seen that the growth of $R$. opacus 43943 carrying the pBKO53A plasmid is less vigorous than the parent strain and is of concern if robustness of growth is a distinguishing characteristic of this strain. When cells are plated on BSM-glucose with diesel overlaid, it is apparent that $R$. opacus 43943 is less resistant to the diesel fuel.

During these experiments, it was noticed that $R$. opacus 43943 colonies that survived the overlay with diesel had a different morphology than the originally plated cells. These colonies appeared "dry" and spread across the plate (Figure 60). To test whether this phenotype was associated with resistance to diesel, colonies were resuspended in BSM liquid media and replated onto BSM-glucose with chloramphenicol and a diesel overlay. 
As can be seen in Figure 60, these cells retained there resistance to diesel fuel as well as maintaining the pBK053A plasmid.

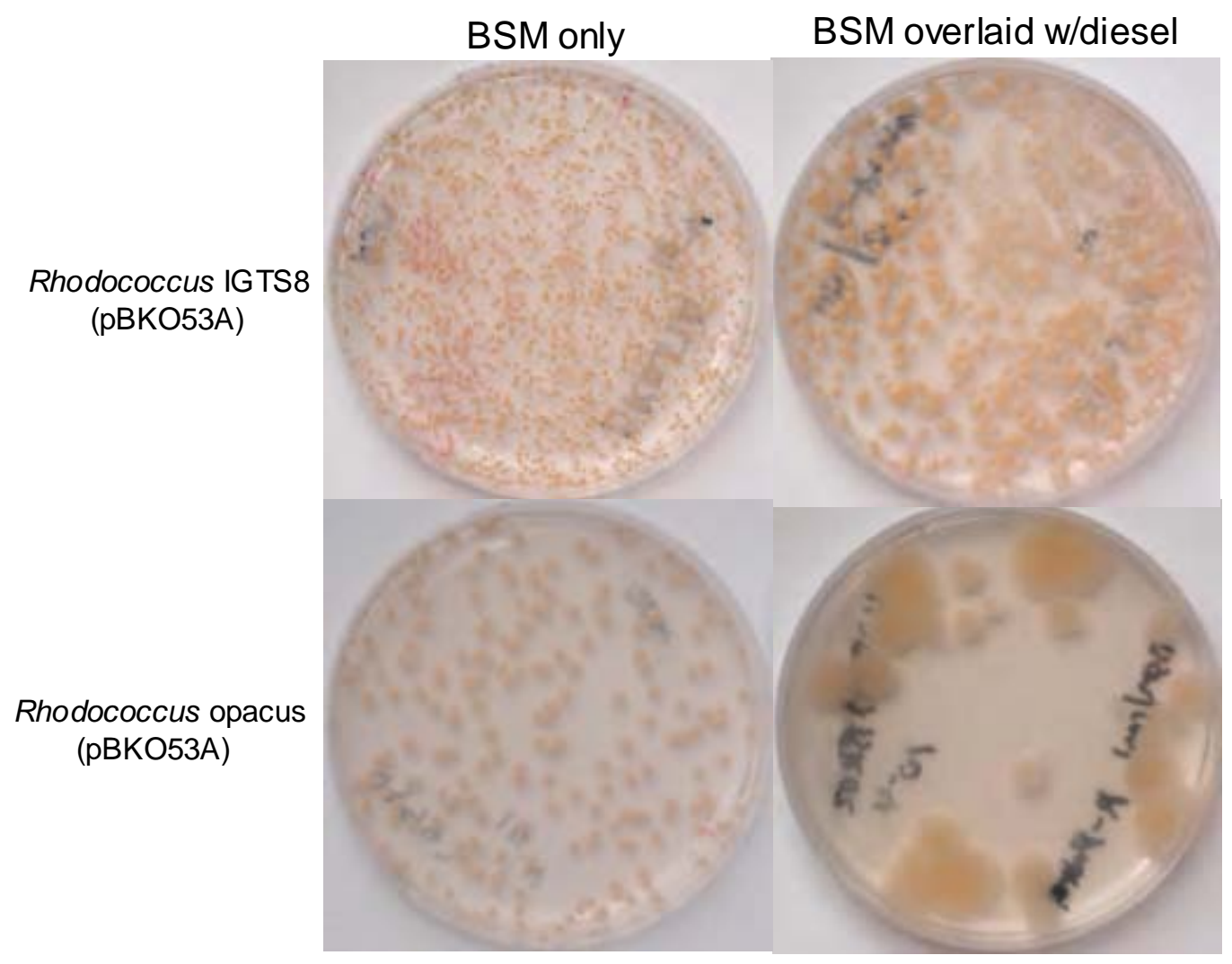

Figure 60. Phenotypes of Rhodococcus hosts on BSM-glucose and BSM-glucose overlaid with diesel.

\subsection{Generation of an antibiotic-free stable $d s z$ expression system}

\subsubsection{Background and strategy}

The development of a commercially viable BDS host requires that the catalytic activity be stable throughout the entire process. Since the results described above indicate that the modified pSOX plasmid is unstable in the absence of antibiotic selection pressure, it was necessary to generate a system for stabilizing expression without the need for addition of antibiotics. In a commercial large-scale fermentation unit of the sort required for BDS it would be very difficult to insure that antibiotic resistance genes from the host organism are not released into the environment, as mandated by the EPA, without an expensive addition to the process to sterilize the waste stream and remove all DNA.

An alternative strategy to antibiotic selection would be to use a chromosomal auxotrophic mutant (requirement for a growth factor that the wild-type prototrophic strain does not require) as host and a complementing gene as selection marker on the vector for expression of recombinant genes. 
With this in mind, we selected thymidylate synthase (ThyA), an essential gene for purine and nucleic acid metabolism, as the selectable marker. This enzyme is absolutely required by all living organisms for prototrophic growth (Figure 61). Mutations in this gene result in strains that are absolutely dependent on the presence of exogenous thymine or thymidine in the medium. By deleting the gene from the biocatalyst chromosome and then providing the complementing gene by cloning into a dsz expression vector, we could then insure maintenance and stability of dsz genes introduced into a biocatalyst host.
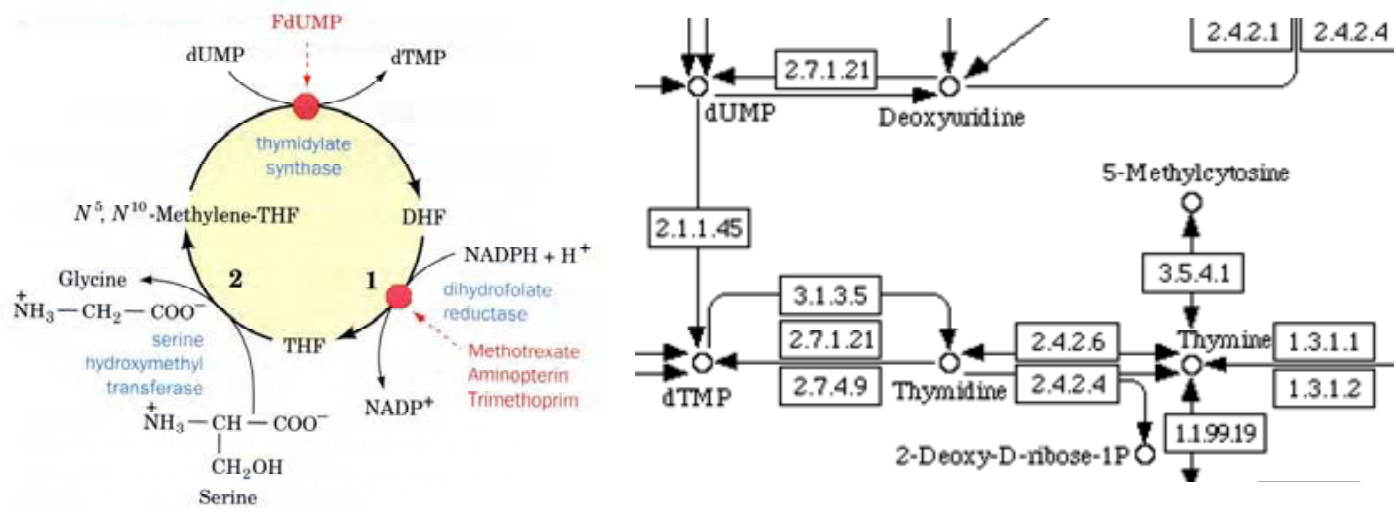

Figure 61. Role of thymidylate synthase (ThyA) in cellular metabolism and thymidine salvage pathway.

\subsubsection{Generation of thyA deletion strain}

The overall strategy for generating a host for the stable expression of evolved dsz genes is diagrammed in Figure 62. To construct a thyA deletion mutant, the Rhodococcus IGTS8 derivative JB55 was selected as host. This strain contains the entire pSOX plasmid, with the exception of the $d s z$ genes, which have been replaced with a tetracycline resistance gene. This enabled us to construct gene replacement "cassettes" consisting of 1) pSOX sequences flanking the dsz genes to provide homology for recombination, 2) a thyA gene to complement the chromosomal deletion and enable prototrophic growth once integrated, 3) a strong promoter for expression of the dsz genes, 4) the optimized $d s z$ genes including $d s z D$, in various gene orders to test optimal organization of expression and 5) a terminator to prevent read-through transcription. This cassette could then be integrated into the JB55 thyA strain, as a linear fragment, by homologous recombination, selecting for prototrophic growth and screened for the loss of tetracycline resistance. 


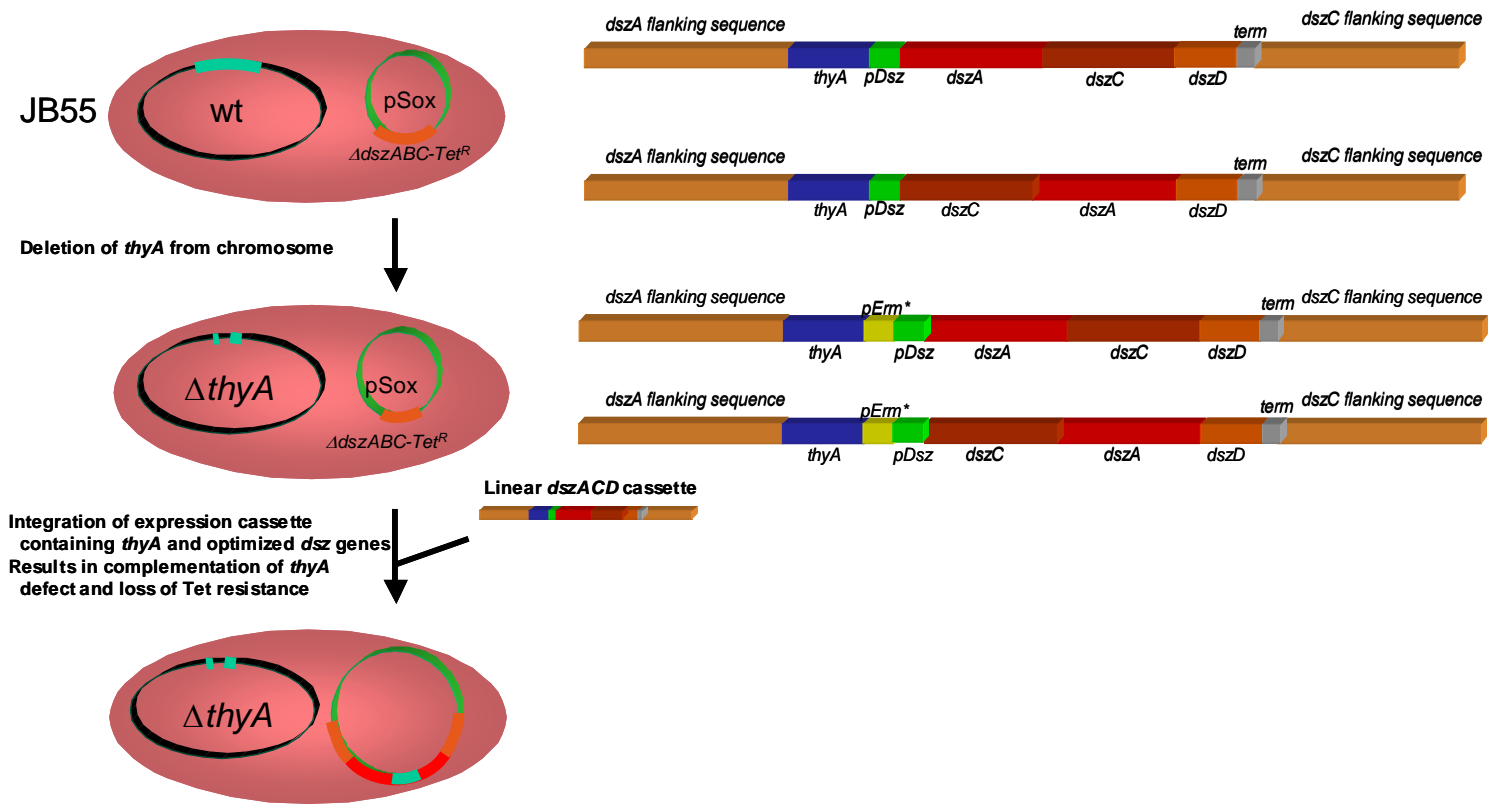

Figure 62. Diagram of the strategy for generating an antibiotic markerless selection system for the introduction and maintenance of modified dsz genes.

We had previously identified the thymidylate synthase (thyA) gene from the genome sequence of Rhodococcus strain RHA1 (http://www.rhodococcus.ca) and used it to isolate the corresponding gene and flanking sequences from Rhodococcus IGTS8. We then constructed a vector containing the thy $A$ flanking regions, a kanamycin-resistance gene and a sucrose synthase gene (sacB) (Figure 67). By electroporating this vector into JB55 and selecting for kanamycin resistance, we generated a primary cointegrate.
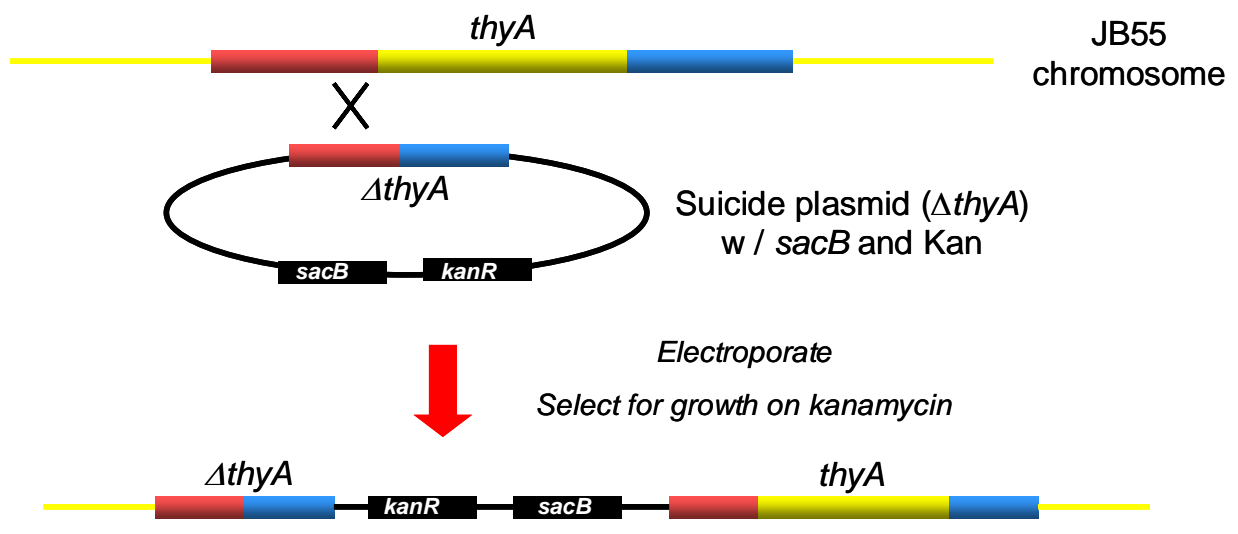

\section{$1^{\circ}$ cointegrate}

Figure 63. Generation of primary cointegrate for construction of JB55 thyA deletion mutant. 

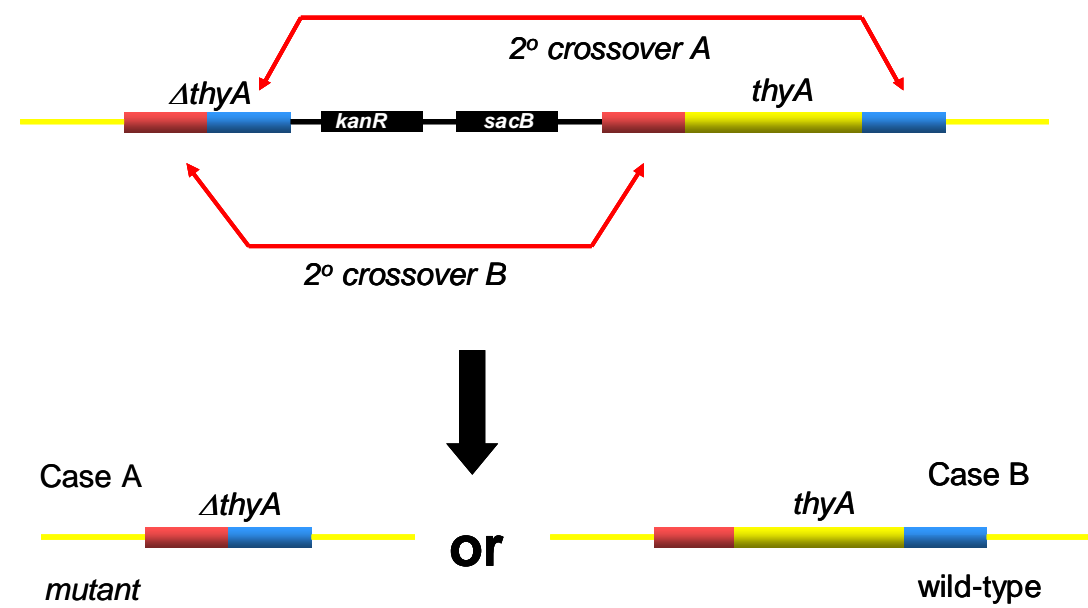

Figure 64. Generation of second crossover and creation of JB55 thyA deletion mutant.

The correct integration of the vector by homologous recombination was confirmed by Southern hybridization. To generate the thyA deletion, we then selected for the ability to grow in the presence of $5 \%$ sucrose. In this manner we could then force a second recombination between homologous duplicated sequences to remove the sacB gene, which is toxic if expressed in the presence of sucrose. As can be seen in Figure 64, two different recombination events can lead to loss of $\operatorname{sac} B, 1)$ recombination through the right-hand duplicate sequences (relative to the thy $A$ gene, $2^{\circ}$ crossover $A$ ) resulting in a thy $A$ deletion mutant or 2$)$ recombination through the left-hand duplicate sequences $\left(2^{\circ}\right.$ crossover $B$ ) resulting in restoration of the wild-type.

When sucrose-resistant clones were selected in approximately $50 \%$ were found to be unable to grow in the absence of added thymidine. Figure $\mathbf{6 5}$ shows the growth of one such mutant in liquid medium (nutrient broth) in the presence and absence of added.

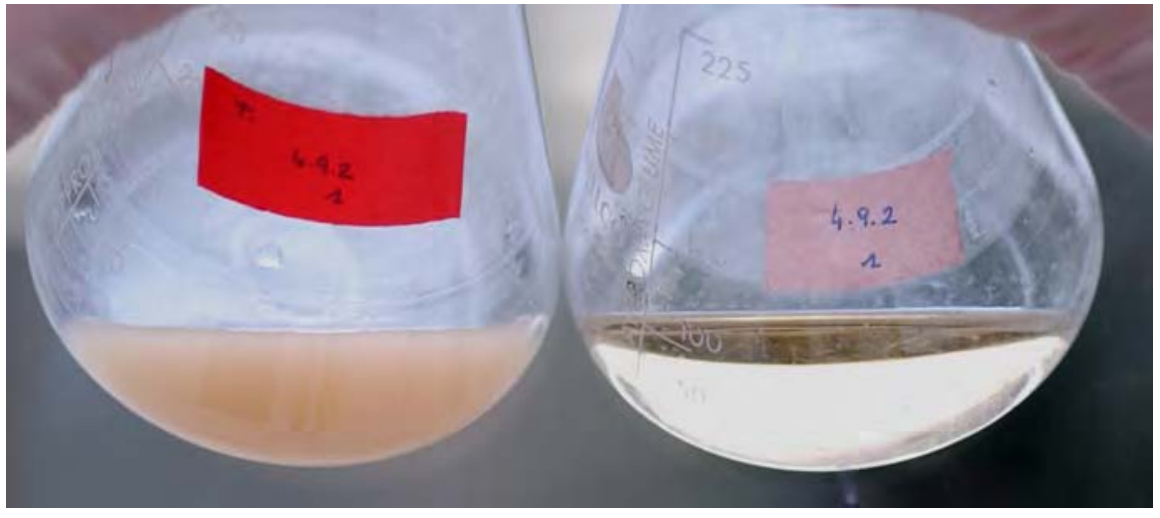

Figure 65. Growth phenotype of thyA mutant in the presence and absence of added thymidine. Thymidine added to $10 \mathrm{ug} / \mathrm{ml}$ in left hand flask. 


\subsubsection{Complementation and construction of $d s z$ expression cassette}

With the successful generation of the JB55 thyA mutant it was necessary to test for the ability to complement the mutation by the introduction of a wild-type thy $A$ gene. Our strategy for stable $d s z$ gene expression required the ability to introduce and incorporate a linear DNA fragment by recombination (Figure 62). To determine if this was feasible, we amplified a copy of the thyA gene from the IGTS8 chromosome, along with $1 \mathrm{~kb}$ of flanking sequence on either side, to produce a linear fragment of DNA. This fragment was then electroporated into the JB55 thyA mutant and cells were plated onto agar medium without added thymidine to select for prototrophs. Prototrophic colonies were obtained at high frequency $\left(-10^{-5}\right)$ after electroporation while no colonies were obtained without the added thy $A$-containing fragment.

To construct the dsz expression cassette, we combined a number of different elements using an in vitro recombinant technique. These elements are shown in Figure 66 and consisted of; 1) pSOX sequences flanking the $d s z$ genes to provide homology for recombination, 2) a thy $A$ gene to complement the chromosomal deletion and enable prototrophic growth once integrated, 3) a strong promoter for expression of the $d s z$ genes, 4) the optimized $d s z$ genes including $d s z D$ and 5) a terminator to prevent readthrough transcription. In addition, the construction of this cassette allowed for varying the dszACD gene order to enable testing the optimal combination for effective biodesulfurization activity.

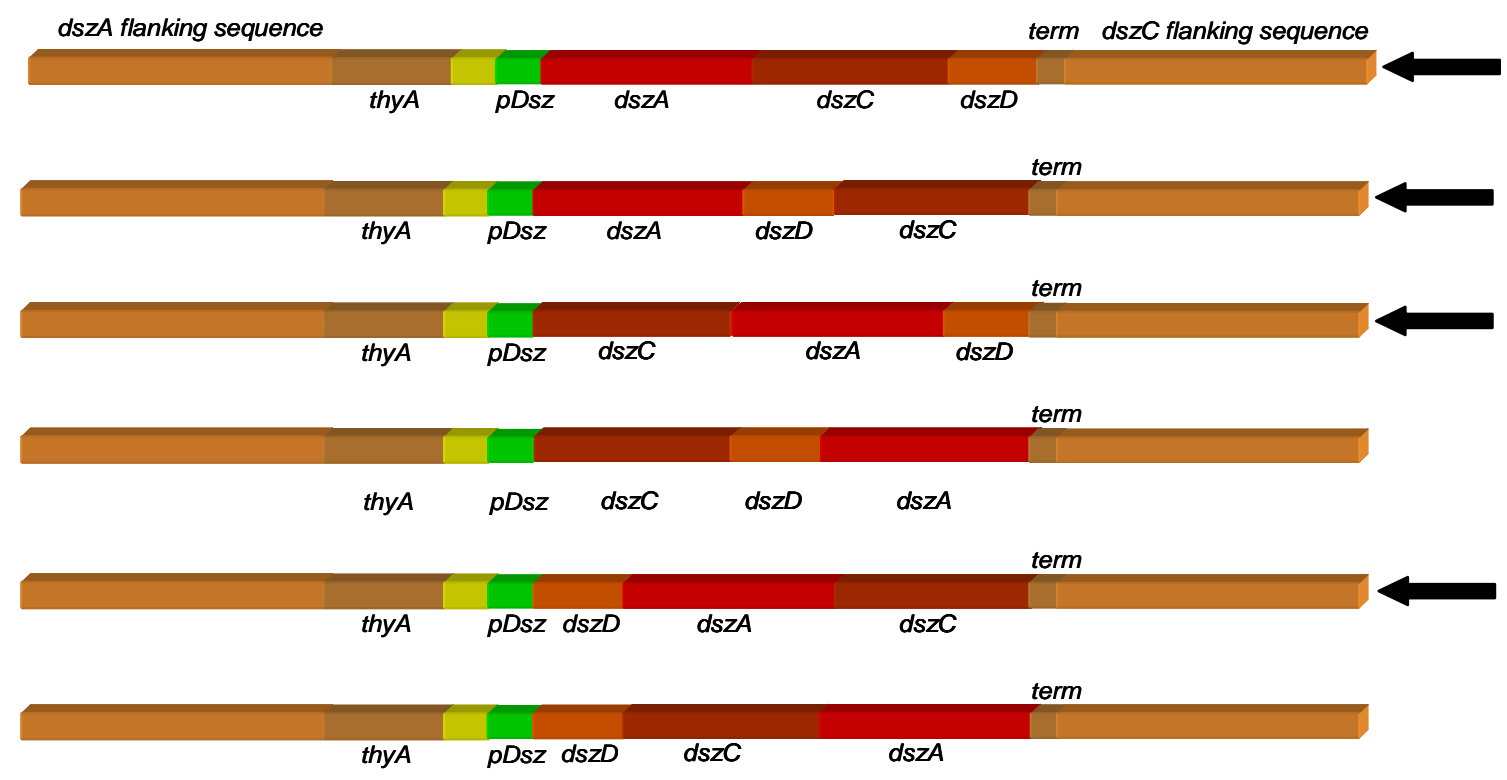

Figure 66. Diagram of dsz gene cassettes for integration into JB55 thyA deletion mutant. Only those marked with an arrow were successfully generated. 
As of the end of the project, four of the six gene cassettes were successfully constructed. Although multiple attempts were carried out to generate the remaining two constructs, instability of the clones seem to preclude their construction.

Two of the constructs, with dsz gene order ADC and ACD, were prepared as linear fragments by restriction digestion at flanking sites and used to electroporate JB55 $\Delta$ thyA. Correct integrants were verified by the restoration of prototrophy, loss of tetracycline resistance and by Southern hybridization.

Two independent clones from each of the two constructs were purified and tested for whole cell BDS activity and compared to the benchmark BKO53A strain. As seen in Figure 67, each of the constructs showed substantial BDS activity but neither approached the levels and initial rates seen with BKO53A.
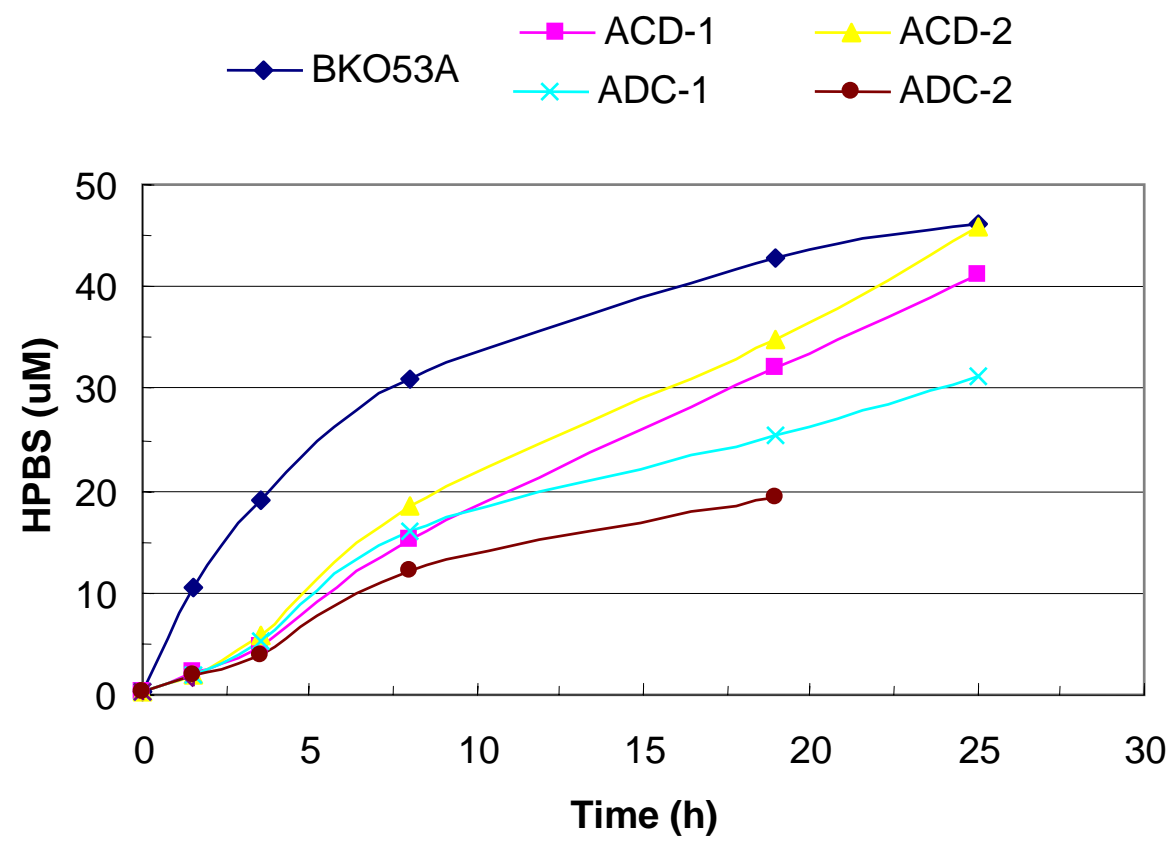

Figure 67. Whole cell BDS activity of BKO53A vs. JB55 $\triangle$ thyA - dsz integrants.

\subsection{Sequencing of IGTS8 plasmids}

As our previous data suggested, it is likely that pSOX-encoded genes in addition to dszABC play a role in the ability of IGTS8 to carry out biodesulfurization. To attempt to identify additional genes, we carried out an effort to sequence the plasmids of this organism.

Studies in the literature have suggested that there are at least two plasmids, one of $\sim 150 \mathrm{~kb}$ and one of $\sim 90 \mathrm{~kb}$ in this strain (13) but prior sequencing efforts have been limited to the $d s z$ and rep regions of the $\sim 150 \mathrm{~kb}$ pSOX plasmid. 
To prepare IGTS8 plasmid complement, total plasmids were prepared from IGTS8 using a modified alkaline lysis procedure and two libraries were generated, a small insert library of 1-3kb inserts derived from randomly shearing IGTS8 plasmid DNA and a large insert cosmid library using randomly sheared DNA with inserts of 35-40b fragments in pCC1Fos.

To analyze the library for plasmid composition, fragments containing either the known $d s z$ genes or the pSOX rep region were used as hybridization probes against 600 randomly selected cosmids. Only 15 were obtained that were derived from the pSOX plasmid, suggesting that the copy number of the $90 \mathrm{~kb}$ plasmid is much higher than the $150 \mathrm{~kb}$ pSOX plasmid. In addition, many of these 15 clones prove to be unstable upon further purification, suggesting that the pSOX plasmid contains many repeated or unstable sequences prone to recombination in the $E$. coli host.

To carry out the sequencing, $>5000$ randomly selected small insert clones were sequenced and assembled to generate a complete sequence for the $90 \mathrm{~kb}$ plasmid (Figure 68, Appendix 3). Although we could not generate enough sequence to assemble the entire $150 \mathrm{~kb}$ pSOX plasmid, we were able to assembly five contigs that in total $=\sim 120 \mathrm{~kb}$ of sequence (Figure 69-70, Appendix 3). In a number of regions there were highly repeated sequences which created ambiguous assembly into a complete sequence.

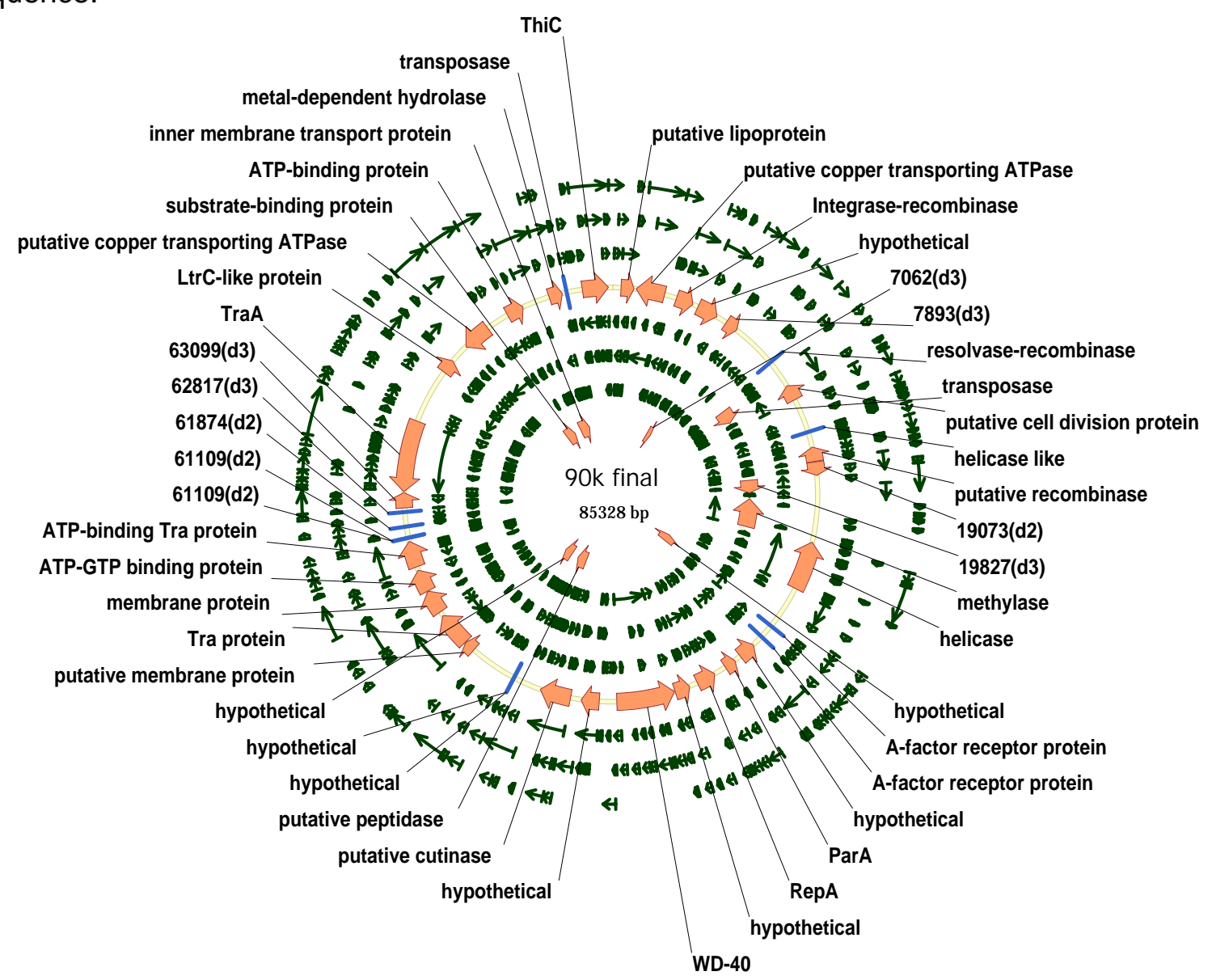

Figure 68. Map of the $90 \mathrm{~kb}(85,328 \mathrm{bp})$ IGTS8 plasmid. 
Annotations of the both the "90kb" and incomplete pSOX sequences suggest that they encode a variety of transporters for heavy metals and other cations as well as a number of transposons and insertion sequences that suggest horizontal transmission from other organisms. This implies that IGTS8 normally finds itself in an environment containing a variety of toxic compounds and employs numerous efflux mechanisms to protects itself.
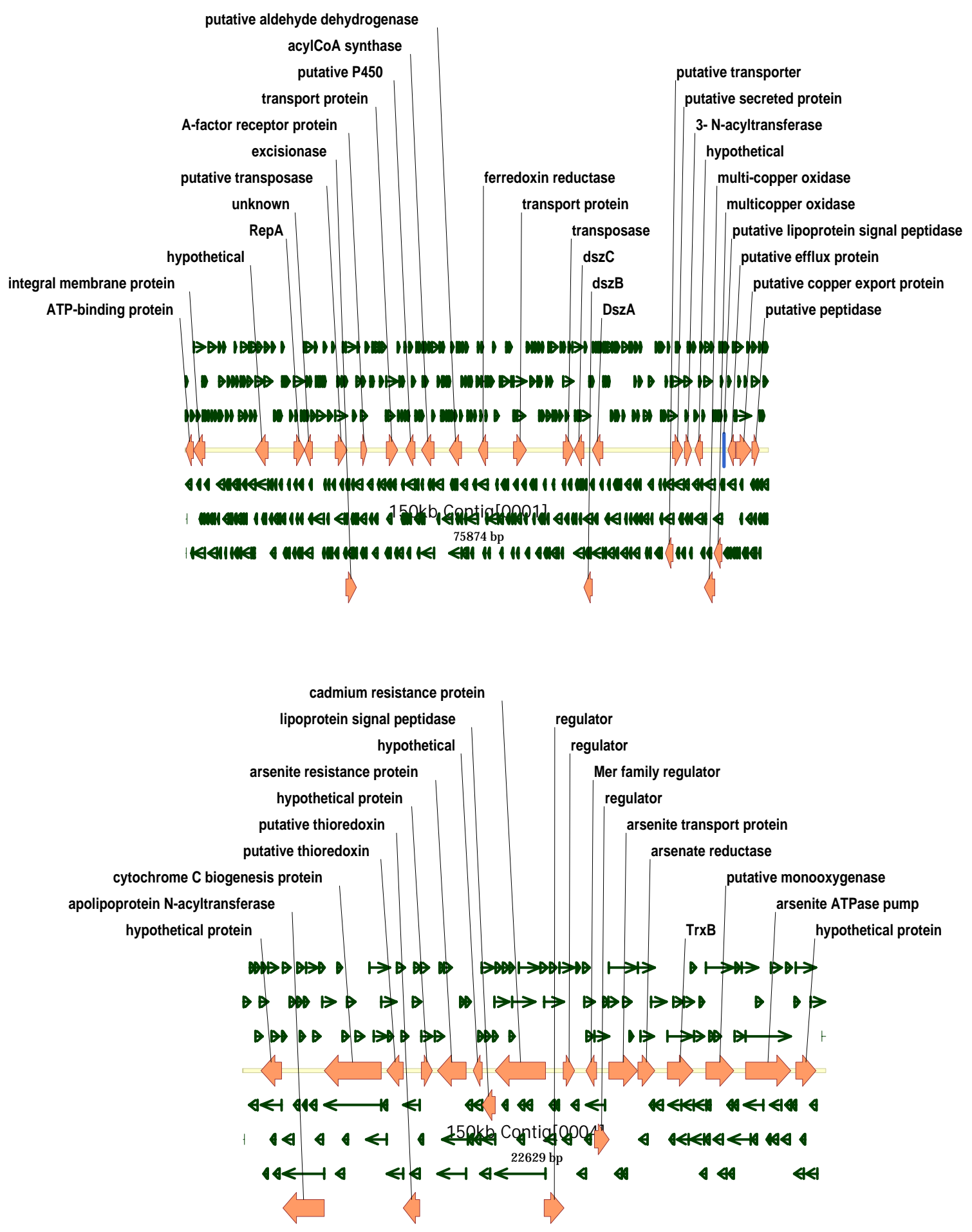

Figure 69. Maps of the two largest IGTS8 pSOX contigs. 

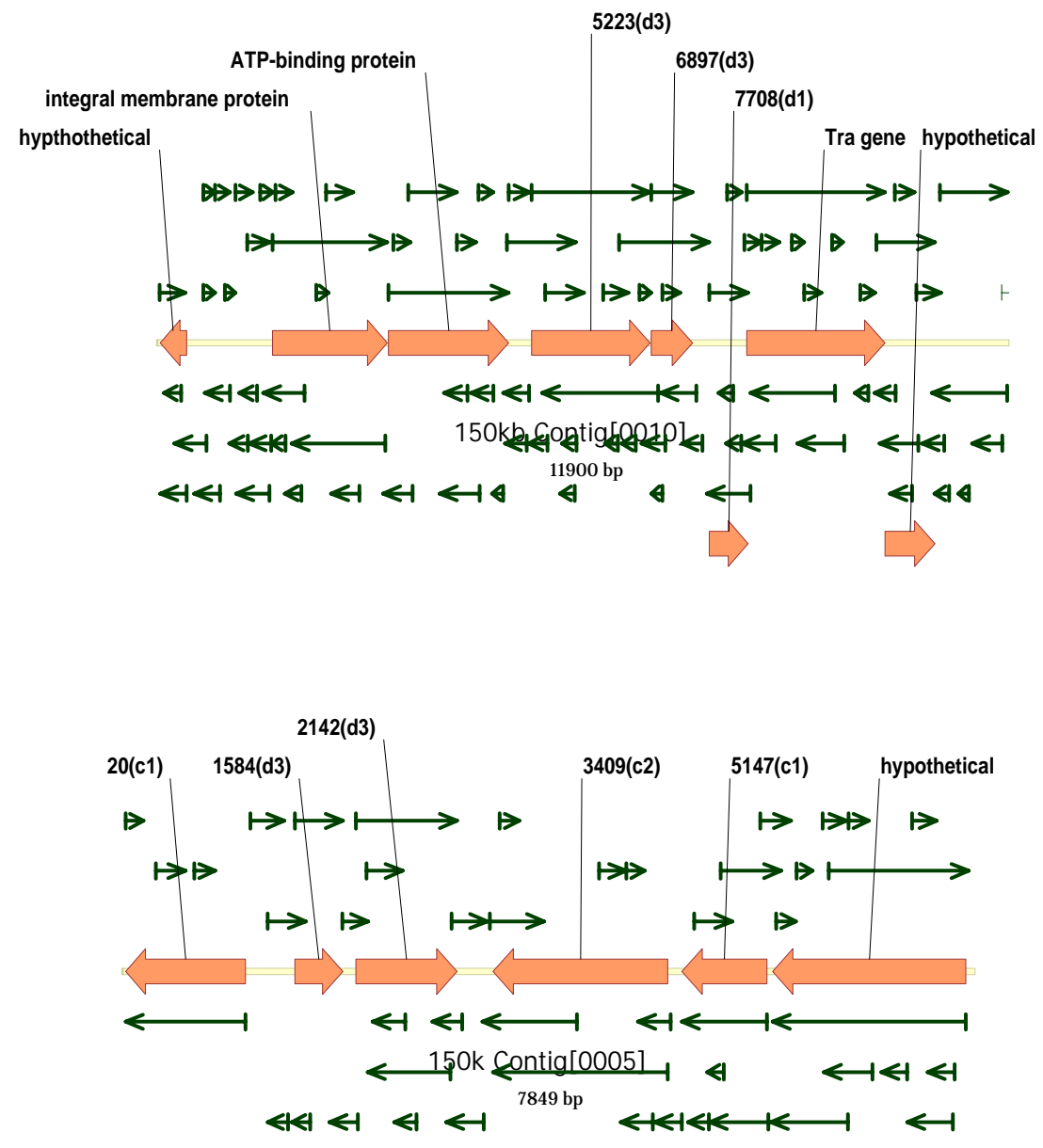

Figure 70. Maps of the two smallest IGTS8 pSOX contigs.

\subsection{Economic study of BDS standalone and combined processes}

To address the economic costs associated with biodesulfurization as both a stand-alone process as well as in combination with HDS, a study was carried out by Pelorus, Petro Star and Anvil and is reported in detail in Appendix 4.

The design throughput for the study is $6,000 \mathrm{bpd}$ of straight run diesel containing 0.5 weight percent sulfur from PetroStar's Valdez Refinery. The specification for the ULSD product sulfur content is $10 \mathrm{ppm}$.

To simplify the economic study and avoid biasing the study results, the study excluded all outside battery limits (OBL) facilities. The approach is consistent with the 1999 BDS study which excluded all utility systems improvements, offsite tankage, and waste stream disposal. 


\subsubsection{Study approach}

The HDS/BDS Study looked at five cases to determine if a BDS process is a viable means to produce USLD when compared to traditional hydro-processing methods:

- A standalone HDS process, used as a benchmark to assess the viability of a BDS process

- A standalone BDS process

- A BDS process followed in series by an HDS process (BDS/HDS Case)

- A HDS process followed in series by a BDS process (HDS/BDS Case)

- A Diesel Splitter followed by an HDS Unit operated in parallel with a BDS Unit (Pre Fractionation Case)

\subsubsection{Study details}

This Task 4 Final Report includes this Summary as well as details for each of the studied Cases that were developed as three standalone reports:

- HDS Baseline Case Report and Cost Estimate

- BDS Baseline Report and Cost Estimate

- BDS/HDS Combination Cases Report and Cost Estimates.

The HDS and BDS Baseline Reports cover their respective process operated as a standalone unit.

The Combination Cases Report covers three possible sequencing options when a BDS process is operated in conjunction with an HDS process. All three reports are included as sections of this Task 4 Final Report.

\subsubsection{Study results}

The results of the study are contained in the summary table below. From this table, the following conclusions can be made regarding the viability of a BDS process to produce ULSD at PetroStar's Valdez Refinery:

- An HDS Unit has a lower installed cost than a comparable BDS Unit

- An HDS Unit has substantially lower operating costs than a comparable BDS Unit

- The combination of a BDS Unit and an HDS Unit is not economically viable when compared to either of the standalone units. 


\subsection{CONCLUSIONS}

In this final technical report we have described our efforts to develop a biodesulfurization host optimized for its ability to meet the many challenges of implementing a commercially viable BDS technology. Many of these challenges were apparent from the outset, while a number of new challenges and levels of understanding of the existing problems became more obvious during the course of this work.

These challenges are not only technical in nature but include the economics of introducing and implementing this new technology and the competitive threats posed by competing, established technologies. In addition, new regulatory challenges have been imposed, to attain levels of sulfur in ULSD, which may prove extremely difficult in achieving using BDS.

Here we highlight some of the most important technical challenges to the success of a BDS technology, whether as standalone or in combination with HDS.

\section{Organosulfur composition}

From the two analytical efforts we have carried out to determine the organosulfur composition of both straight-run diesel and partially hydrotreated diesel, it is apparent that the overall composition of the organosulfur compounds is highly heterogeneous. While major species, alkylated benzothiophenes and dibenzothiophene, can certainly be identified that make up the bulk of the organosulfur compounds, the remaining minor species contribute to the total sulfur and must be effectively removed to attain the ULSD specifications mandated. As our data on the action of a biocatalyst on deeply hydrotreated diesel demonstrates that even the nearly complete reduction of the most HDS-recalcitrant species, 4-alkyl and 4-dialkylbenzothiophenes, to low ppm levels is not enough to reduce the "hump" of heterogeneous, undefined sulfur compounds to acceptable levels.

It is probably this challenge, more than any other, which presents the greatest obstacle to successful implementation of this technology. The enzymes at the core of the process must be able to act upon a large variety of substrates at sub-Km levels in a reasonable amount of time to be commercially viable. Optimizing the enzymes to be simultaneously high affinity and broad specificity presents a formidable challenge and is perhaps counter to our current understanding. Alternatively, one could propose to introduce a number of enzyme systems in a single biocatalyst, or use multiple biocatalysts with unique enzyme systems, but is likely to be as technically challenging and costprohibitive.

\section{Biocatalyst host properties}

Although these challenges are significant, we chose to focus on the optimization of Rhodococcus IGTS8 DszC and A to improve activity on the HDS-recalcitrant 4dialkylbenzothiophenes. If BDS were used as a front-end to a combined process, it could 
be envisioned that a reduction in this species would afford a reduction in the operating and capital costs required for hydrotreatment.

While we were successful at identifying mutants of DszA that were $\sim 2 X$ more active on 4, 6-dimethyl $\mathrm{DBTO}_{2}$ we were unable to identify variants in DszC with significant improvement on 4, 6-dimethyl DBT. Interestingly, in both cases, evidence of the effect of other factors contributing to apparent specificity was apparent.

In the case of the DszA variants, we observed that the relative ratio of DszA, flavin reductase and substrate had a pronounced effect on the activity on 4, 6-dimethyl $\mathrm{DBTO}_{2}$ vs. $\mathrm{DBTO}_{2}$. This suggests that the intracellular redox state and availability of reduced flavin will have important consequences on the ultimate activity profile of the Dsz system. As mentioned in the introduction, other observations from the literature have also shown that in spite of the identity of the expressed dsz genes, the host contributes significantly to the apparent range of substrates acted upon (2).

During our screening for DszC variants, we were able to identify three clones that reproducibly showed increased activity on 4, 6-dimethyIDBT vs. DBT. Unfortunately, retransformation of the three responsible mutated plasmids, expressing the DszC variants, into a "naïve" $E$. coli host results in the loss of this phenotype. This likely resulted from a mutation in the chromosome of the original $E$. coli host that somehow altered the cell physiology and again demonstrates the apparent contribution of the host to the activity profile of the enzyme.

These observations highlight the need to develop not only the enzymes required for BDS but to carefully consider the properties of the host organism, not only in terms of contributing the enzymatic activities, but for other important properties relevant to a commercial process including robustness, efficient generation of cellular reductant and tolerance to organic matrices.

With this mind, we set out to identify alternative hosts to Rhodococcus IGTS8. We had noticed during our initial characterizations of this strain that its properties were less than ideal as it had a slower growth rate and achieved lower total biomass concentrations than desirable.

After screening >100 different Rhodococcus, Nocardia, Mycobacterium and Gordonia strains for their growth characteristics, we focused on a number of Rhodococcus opacus strains for further work. These strains grew at a comparably fast rate to high densities, were non-pigmented (in contrast to IGTS8, which produces a red pigment that partitions to the oil phase) and had a reported property of being able to accumulate triacylglycerols when grown under nitrogen-limited conditions.

As one of the important requirements for a BDS host is an ability to efficiently produce the necessary cellular reductant from an oxidizable substrate. Previous efforts in this area have focused on the addition of glucose, acetate, ethanol or alkanes during the catalytic stage of a BDS process. The use of an organism that can accumulate triacylglycerols during the biomass production stage, when the cells are at their most robust and healthy would allow for the mobilization and utilization of these storage compounds to provide cellular reductant during the following catalytic stage. Unlike glucose, acetate, ethanol or alkanes, which require an activation cost (transport, phosphorylation, alkane oxidation) and input energy for their utilization if provided 
exogenously, intracellular accumulated triacylglycerols can be directly used, without activation, by $\beta$-oxidation and are very efficiently converted to cellular reductant.

We have demonstrated that a number of $R$. opacus strains are also able to tolerate hexadecane and diesel fuel nearly as well as IGTS8, although it appears that IGTS8 and its derivatives do possess an inherent resistance that may be due to the production of a biosurfactant. It will be an interesting to determine the mechanism of IGTS8 biosurfactant production and whether the responsible genes can be transferred to other hosts. Most descriptions of biosurfactants in the Actinomycetes, particularly Mycobacterium, describe sugar-mycolic acid conjugates as the most prevalent, suggesting that the genes necessary to glycosylate the already present cell wall mycolic acids of Rhodococcus would be minimal.

\section{Expression of dsz genes}

From these studies, it is apparent that the stability of expression of the dsz genes in IGTS8 is questionable and development of a biocatalyst strain will require a mechanism to insure robust and stable expression of the pathway. In addition, the proper stoichiometric balance of expression of each of the Dsz enzymes will be important to most efficiently oxidize the organosulfur compounds and avoid bottlenecks. It is not readily apparent from either this work or from the literature what is the proper balance of the two or three Dsz proteins (depending on whether DszB is included). This is likely to depend on the specific organosulfur compound and the relative affinity of each of the enzymes, as well as the indirect contribution of host factors, as described above.

For this reason, in the last stages of our work we set out to generate a system for the stable expression of the Dsz proteins without the need for including antibiotics in a fermentation that would create environmental issues with the potential release of resistance genes. We also designed the system to allow for the introduction of modified genes and construction of cassettes with various combinations of $d s z$ gene orders to study the effect of altering relative expression levels of each of the components. This would hopefully enable the empirical optimization of both genes and stoichiometry of expression in a BDS host.

\section{Economics}

Finally, we have presented an in-depth analysis of the operating and capital costs of BDS as both a standalone process and in combination with HDS. Current cost estimates of a HDS-treatment facility of comparable scale served as the benchmark case. Since performance specifications of the biocatalyst strain have a major effect on the economics, assumptions of significant improvement (50-100x) over current biocatalyst performance were made to derive these values.

In short, the study concludes that implementation of a BDS facility by itself or in combination with HDS would not afford a significant cost reduction and in some cases would be significantly higher. Factors that contribute most significantly to the relative costs of the processes are the reductions in operating costs and catalyst performance in hydrotreating plants that were not the case ten years ago when intense work began on the development of the biocatalyst host and BDS process by Energy Biosystems and others. 


\subsection{REFERENCES}

1. Kilbane, J. J. and B. A. Bielage (1990) Proceedings: 1990 First International Symposium of the Biological Processing of Coal, Electric Power Research Institute, Palo Alto, CA

2. Noda K, K. Watanabe and K. Maruhashi K(2003) J. Biosci. Bioeng., 95:354-360.

3. Kayser K.J., H.S. Park, J. H. Kwak, A. Kolhatkar and J. J. Kilbane II (2002) Appl. Microbiol. Biotechnol., 59:737-746.

4. Sambrook, J., E. F. Fritsch, and T. Maniatis. (1989) Molecular cloning: A laboratory manual, 2nd Ed. Cold Spring Harbor Laboratory Press.

5. Gray K.A., O.S. Pogrebinsky, G.T. Mrachko, L. Xi , D.J. Monticello and C.H. Squires. (1996) Nat Biotechnol. 14:1705-1709.

6. Jackson C.J., D.C. Lamb, T.H. Marczylo, A.G. Warrilow, N.J. Manning, D.J. Lowe, D.E. Kelly and S.L Kelly (2002) J. Biol. Chem. 277:46959-46965.

7. Munro A.W., D.G. Leys, K.J. McLean, K.R. Marshall, T.W. Ost, S. Daff, C.S. Miles, S.K. Chapman, D.A. Lysek, C.C. Moser, C.C. Page and P.L. Dutton. Trends Biochem. Sci. 27:250-257.

8. Jaspers M.C., W.A. Suske, A. Schmid, D.A. Goslings, H.P. Kohler and J.R. van der Meer. (2000) J. Bacteriol. 182:405-417

9. Kolisnychenko, V, G. Plunkett, C.D. Herring, T. Feher, J. Posfai and F. R. Blattner (2002) Genome Research. 12:640-7.

10 Sirko, A., M. Zatyka, E. Sadowy and D. Hulanicka (1995) J. Bacteriol. 177:41344146.

11. Sutherland TD, I. Horne, R.J. Russell, and J.G. Oakeshott (2002). Appl Environ Microbiol. 68:6237-45.

12. Waltermann M, H. Luftmann, D. Baumeister, R. Kalscheuer, and A. Steinbuchel. (2000) Microbiology. 146:1143-9.

13. Denis-Larose, C., D. Labbe, H. Bergeron, A.M. Jones, C.W. Greer, J. al-Hawari, M.J. Grossman, B.M. Sankey and P.C. Lau (1997) Appl. Environ. Microbiol. 63:2915-9. 


\subsection{LIST OF ACRONYMS AND ABBREVIATIONS}

\begin{tabular}{|c|c|}
\hline BP & Boiling point \\
\hline bp & Base pair \\
\hline BDS & Biodesulfurization \\
\hline BSM & Basal salts medium \\
\hline CAT & Chloramphenicol acetyltransferase \\
\hline CED & Conversion-extraction desulfurization \\
\hline DBT & Dibenzothiophene \\
\hline DBTO2 & Dibenzothiophene sulfone \\
\hline DCW & Dry cell weight \\
\hline DiMe(t) & Dimethyl- \\
\hline DMSO & Dimethylsulfoxide \\
\hline DNA & Deoxyribonucleic acid \\
\hline Dsz & Desulfurization \\
\hline EPA & Environmental Protection Agency \\
\hline $\mathrm{FMN}\left(\mathrm{H}_{2}\right)$ & Flavin mononucleotide (reduced) \\
\hline GC & Gas chromatography \\
\hline GFP & Green fluorescent protein \\
\hline GSSM & Gene Site Saturation Mutagenesis $^{\mathrm{TM}}$ \\
\hline HDS & Hydrodesulfurization \\
\hline HBP & Hydroxybiphenyl \\
\hline HBPS & Hydroxybiphenylsulfinate \\
\hline His & Hexahistidine affinity tag \\
\hline HPLC & High performance (pressure) liquid chromatography \\
\hline $\mathrm{kb}$ & Kilobase \\
\hline LC & Liquid chromatography \\
\hline MS & Mass spectrometry \\
\hline MW & Molecular weight \\
\hline $\mathrm{NAD}(\mathrm{H})$ & Nicotinamide adenine dinucleotide (reduced) \\
\hline OD & Optical density \\
\hline PCR & Polymerase chain reaction \\
\hline $\mathrm{RPC}$ & Reverse-phase chromatography \\
\hline RPM & Revolutions per minute \\
\hline ULSD & Ultra-low sulfur diesel \\
\hline
\end{tabular}




\title{
Analysis of Sulfur Compounds in Petrostar Chemical Extraction Desulfurization Materials
}

\author{
Michael D. Levin, Ph.D. \\ Integrated Chemical Processes Department \\ Diversa Corporation
}


Final Technical Progress Report DOE Award No. DE-FC26-02NT15340 


\section{TABLE OF CONTENTS}

Introduction $\quad 5$

Background 5

Experimental 6

CED material 6

Sample manipulations 6

Data analysis $\quad 7$

Results and Discussion 8

Separation 8

Assessment of accuracy of various aspects of analysis

Conclusions

12 
Final Technical Progress Report DOE Award No. DE-FC26-02NT15340 


\section{Introduction}

This report describes the analytical approach used to determine the composition of sulfur-containing compounds in Petrostar chemical extraction desulfurization (CED) material. The origin and nature of the samples, their handling, methodology of analysis and detailed procedures are explained. Qualitative and quantitative results of analysis are discussed.

\section{Background}

Petrostar marine diesel is obtained by straight run distillation from North Slope Alaskan crude oil. It is not subjected to cracking, reforming, hydrotreatment, or any other condition, which might change the chemical composition of substances present in the crude. Only the boiling point range of the diesel fraction and the crude composition limit the diversity of structures. Thus, the number of sulfur-containing compounds present in detectable concentrations is very high. Attempts to analyze the composition of these compounds by individual methods of separation yield only very broad unresolved humps with few structural features. The retention properties of sulfur-containing organic compounds are very similar to each other, and to those of hydrocarbons, which are present in a much higher concentration in diesel. This makes speciation even more problematic.

Sulfur-containing compounds found in diesel fractions may be divided into two large groups: aromatic thiophenes and aliphatic sulfides. In aromatic thiophenes, sulfur is part of an aromatic ring structure, while in aliphatic sulfides sulfur atoms do not participate in aromatic conjugation. Aromatic thiophenes may be divided further into thiophenes (Thios), benzothiophenes (BTs), and dibenzothiophenes (DBTs). In aliphatic sulfides the sulfur atom may be attached to saturated hydrocarbon substituents, cyclic or aromatic substituents, or be part of a cyclic structur/8/2006 itself. The presence of thiols and disulfides in dißalge 90 fraction distillates has been reported as well.

Petrostar marine diesel contains around 3500 ppm total sulfur. The distillation range of the diesel fraction defines the range of molecular weights for each class of compounds. For all classes but DBTs, this range corresponds to heavily alkylated core structures. The number of possible isomers grows exponentially with the degree of alkylation, and so does the complexity of mixtures.

One of the commonly used techniques to simplify the separation of sulfides and thiophene derivatives is initial oxidation to the corresponding sulfones and analysis of the resulting mixtures. With the notable exception of Thios, oxidation of sulfides to sulfones can be done selectively and quantitatively. Oxidation usually produces one well-defined product, which can be easily traced back to the original sulfide. Sulfones can be easily separated from the matrix of hydrocarbons since they are more polar than sulfides. This separation may be achieved by selective extraction or by chromatography. Sulfones also differ in retention properties among themselves much more than the original sulfides.

Oxidation of thiophenes occurs at a lower rate than oxidation of benzothiophenes, dibenzothiophenes, and aliphatic sulfides. The reaction results in a complex mixture of products of side reactions, the most prominent of which is Diels-Alder addition. Thiophene sulfoxides and thiophene sulfones are strong dieneophiles, and can react with themselves, or with starting thiophenes and benzothiophenes. Extensive alkylation of the thiophene framework reduces the Diels-Alder reactivity of the corresponding sulfoxides and sulfones, rendering them somewhat more stable. 


\section{Experimental}

\section{CED material}

The CED material was obtained by chemical oxidation of Petrostar stock diesel with peracetic acid and extraction of polar products with acetic acid. A sample of CED material was provided by Petrostar and analyzed according to a procedure outlined in a flowchart in Figure 1.

\section{Sample manipulations}

A sample of CED material was separated into fractions by preparative HPLC on a $\mathrm{C}_{18}$ reverse phase column. Fifty fractions of equal volume were collected. Solvents were evaporated from each of the fractions. An $i$-propanol solution of two internal standards, butyl sulfone and pentyl sulfone, was used to redissolve residues from fractions. The reconstituted fractions were analyzed by gas chromatography-

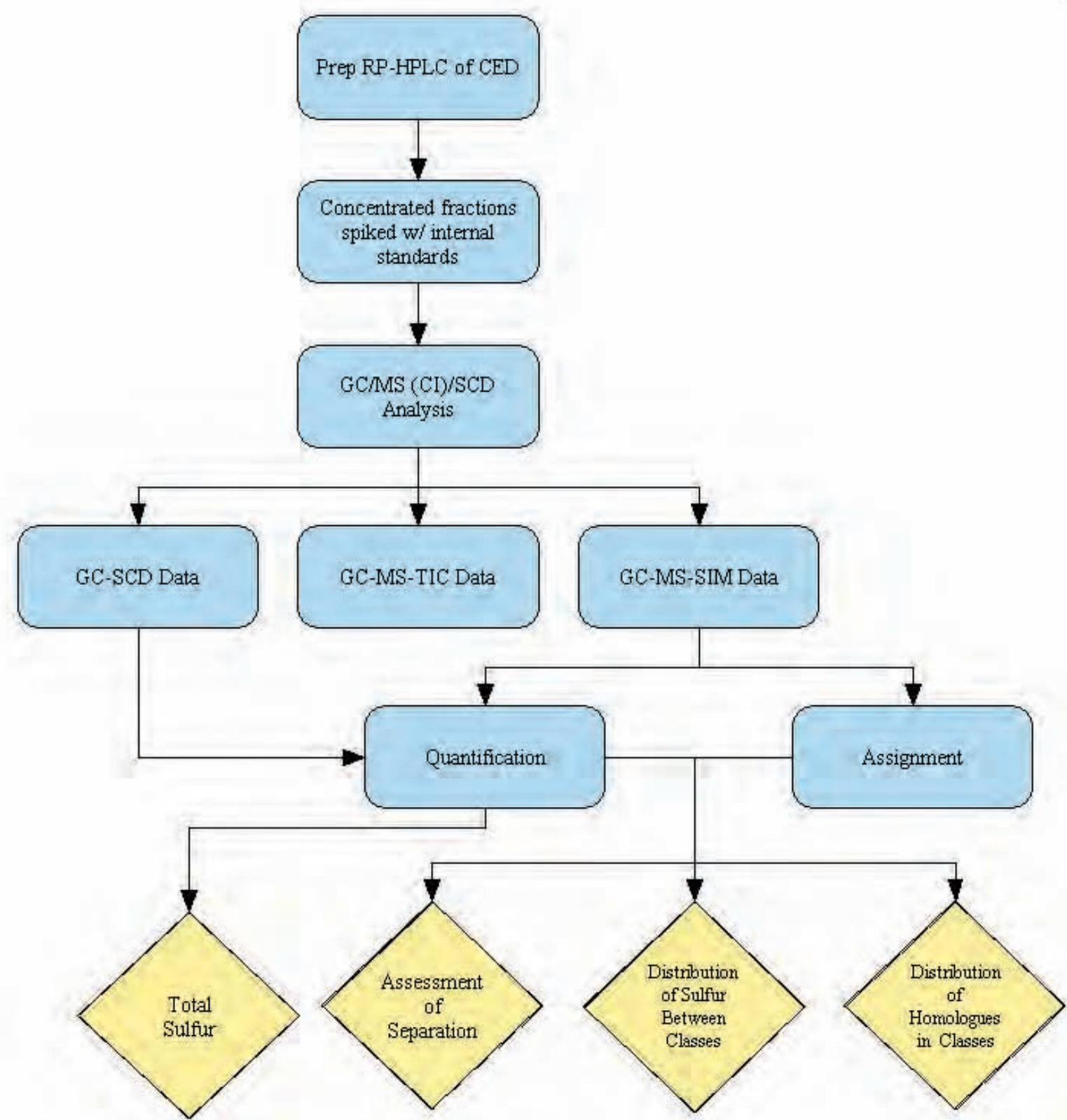

Figure 1. Flow-chart describing the analytical techniques used to analyze and characterize $\$ / 8 / 2000$ tar CED material. 
mass spectrometry (chemical ionization)-sulfur chemiluminescence detection (GC/MS(CI)/SCD). GC inlet split ratios were optimized for each fraction such that the maximum signal before saturation was obtained in the SCD detector. Two injections were done for each sample. The first injection was used to obtain an estimate for the optimal split ratio and to acquire a mass spectrometer total scan total ion chromatogram (TIC). Ions observed in the TIC mode were used to perform selected ion monitoring (SIM) during the second run with the optimal split ratio. Only the data of the second set of chromatograms were used in analysis.

\section{Data analysis}

The sulfur-containing species from the CED material eluted between 13 and 21 minutes under the GC conditions. The SCD trace was integrated between these times, and the integral was converted into molar concentration of sulfur by comparison with the integrals of two internal standards. The integrated intensities of individual ions from SIM data were used to estimate concentrations of isomeric species in each fraction. These calculations were done under assumptions of (i) equal ionization efficiency for different species and (ii) no significant ion suppression in cases when compounds were not separated before they entered an ionization chamber of the mass spectrometer.

Five classes of compounds were identified in the CED material fractions based on the mass spectrometric data: benzothiophene sulfones $\left(\mathrm{BTO}_{2} \mathrm{~s}\right)$, dibenzothiophene sulfones $\left(\mathrm{DBTO}_{2} \mathrm{~s}\right)$, thiophene sulfones $\left(\mathrm{ThioO}_{2} \mathrm{~s}\right)$, bicyclic aliphatic sulfones $\left(\mathrm{BicycO}_{2} \mathrm{~s}\right)$, and monocyclic aliphatic sulfones $\left(\mathrm{MonoO}_{2} \mathrm{~s}\right)$. Compounds within these classes were identified as members of a homologous series differing from one another by an integral number of $\mathrm{CH}_{2}$ fragments. Masses of observed [M+H] ions for different homologues are listed in Table 1. Concentrations of various homologues belonging to the same class of compounds were summed up across fractions and provided the total concentration of compounds belonging to this class. SCD data from different fractions were normalized for different split ratios.

\begin{tabular}{|c|c|c|}
\hline Classes & Base structure & {$[\mathrm{M}+\mathrm{H}]$ for $\mathrm{Cx}$ homologues } \\
\hline $\mathrm{DBTO}_{2}$ & & $217+14 x$ \\
\hline $\mathrm{BTO}_{2}$ & & $167+14 x$ \\
\hline ThioO $_{2}$ & & $117+14 x$ \\
\hline $\mathrm{MonoO}_{2}$ & & $121+14 x$ \\
\hline $\mathrm{BicycO}_{2}$ & & $189+14 x$ \\
\hline
\end{tabular}

Table 1. Masses of observea//,/2006ons for different homologues. 


\section{Results and Discussion}

\section{Separation}

HPLC-UV traces of preparative separations (not shown) are not informative due to overloading of the detector and a lack of resolution on this step. GC/ SCD/MS traces of individual fractions show various degrees of separation. The first four fractions (eluting at $11-12.5 \mathrm{~min}$. on HPLC) show good separation of peaks down to baseline. In the next seven fractions (13 - 16 min.) many peaks overlap to some degree. Starting with the fraction eluting at $16.5 \mathrm{~min}$. on HPLC, the GC chromatograms develop an unresolved hump between 14 and 20 minutes with peaks on top of it. The contribution of the hump steadily increases from fraction to fraction relative to the contribution of resolved peaks. The retention time of the signals increases as well. The last nine chromatograms (fractions 32 - 35.5) display mostly unresolved signal between 17 and 21 minutes. Every gas chromatogram contains peaks for two internal standards: butyl sulfone (retention time $8.3 \mathrm{~min}$ ) and pentyl sulfone (retention time $11.5 \mathrm{~min})$.
Integration of $\mathrm{GC} / \mathrm{SCD}$ traces normalized to internal standards revealed the total sulfur distribution between fractions (Figure 2). The graph shows four peaks and three valleys between them. The first peak (fraction 12.5) is attributed to elution of $\mathrm{C} 1 \mathrm{DBTO}_{2} \mathrm{~s} . \mathrm{C} 2$ $\mathrm{DBTO}_{2} \mathrm{~s}$ and $\mathrm{C} 5 \mathrm{BTO}_{2} \mathrm{~s}$ form the second peak (fractions 14.5 and 15). The peak at fraction 17 is due to elution of $\mathrm{C} 6 \mathrm{BTO}_{2}$ s, the most abundant homologue among alkylated $\mathrm{BTO}_{2} \mathrm{~s}$. The last broad peak around fraction 19.5 is composed of a variety of compound classes with some dominance of $\mathrm{C} 7 \mathrm{BTO}_{2}$ s. Total sulfur content in the initial CED material is $15.4 \mathrm{~g} / \mathrm{l}$.

A contour plot of SIM data (Figure 3) allows the assessment of the separation achieved by 2D chromatography. Separation must be assessed along several lines: (i) separation among classes of compounds, (ii) separation among series of homologues within classes, and (iii) separation among individual compounds within series of homologues.

(i) The contour plot of SIM data reveals that $\mathrm{DBTO}_{2} \mathrm{~s}$ were nicely separated from all other classes of compounds present in CED. $\mathrm{ThioO}_{2} \mathrm{~s}$, on the other hand, overlap extensively with $\mathrm{BTO}_{2} \mathrm{~s}$ and $\mathrm{BicycO}_{2} \mathrm{~s}$.

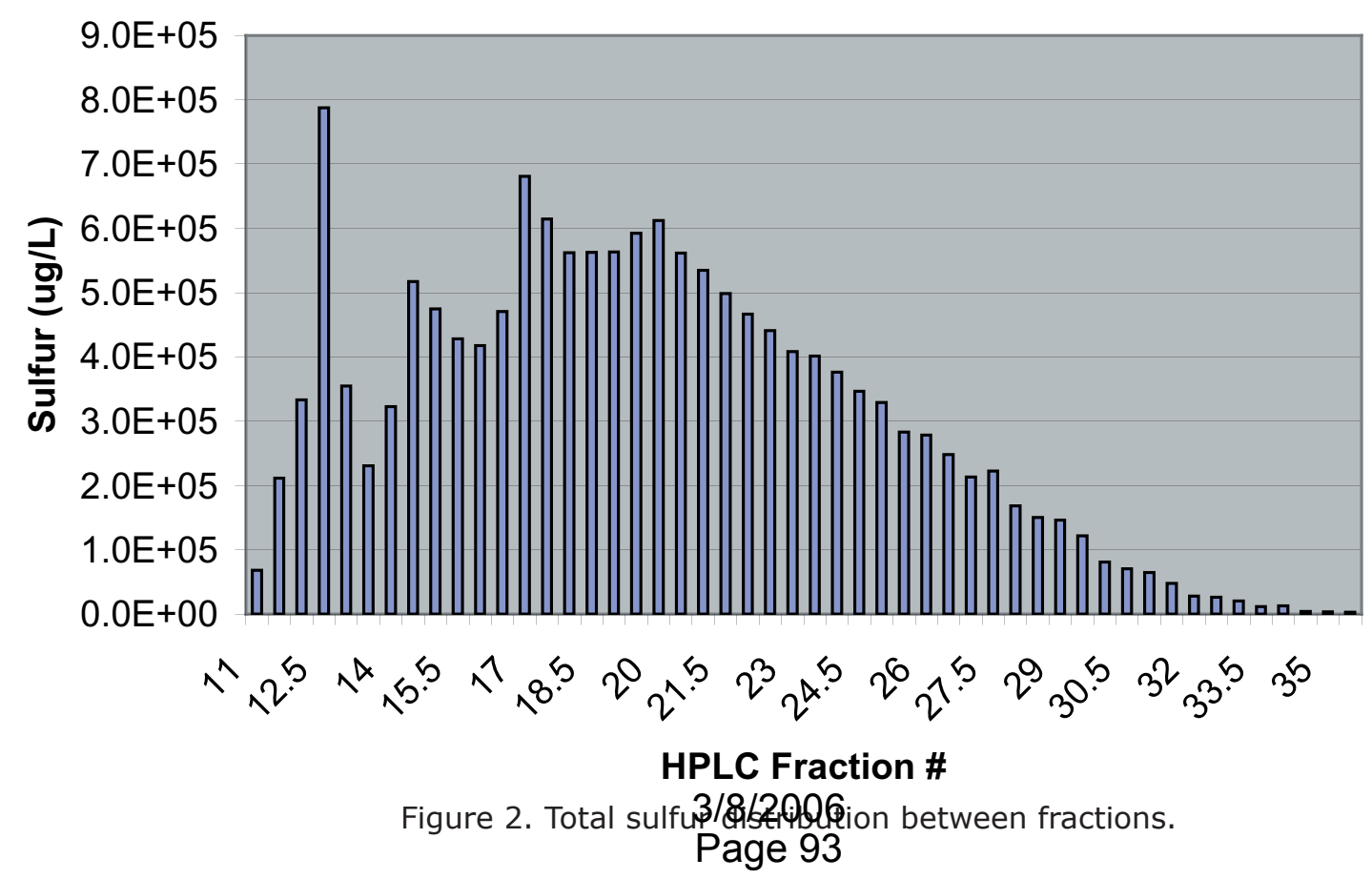




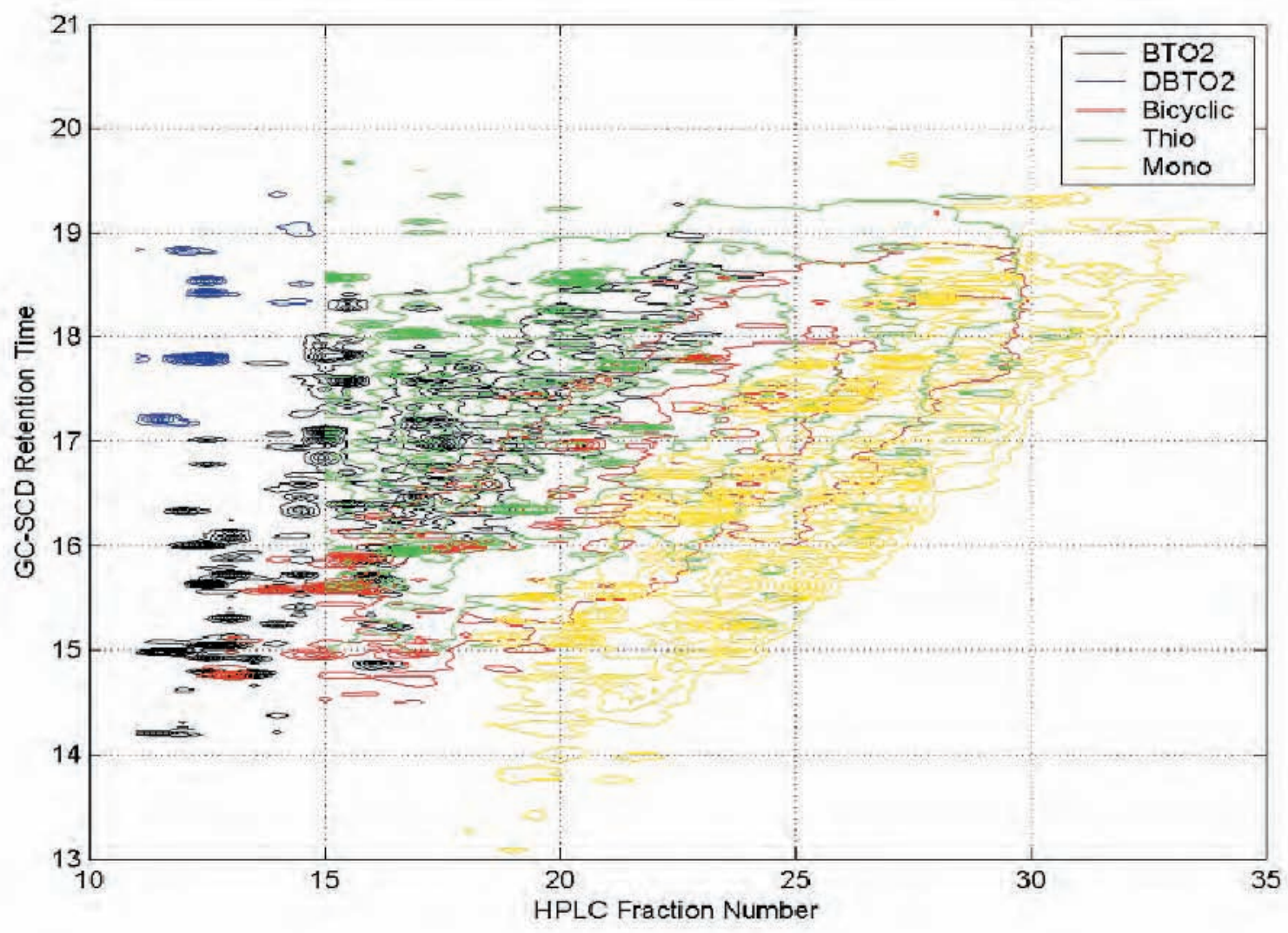

Figure 3. A contour plot of SIM data.

$\mathrm{BTO}_{2} \mathrm{~s}$ and $\mathrm{BicycO}_{2} \mathrm{~s}$ have some overlaps, especially in the area between HPLC fractions 12.5 and 16.5 and $\mathrm{GC}$ retention times between 14.7 and 16 minutes. Quite good separation was achieved for $\mathrm{MonoO}_{2} \mathrm{~s}$. Contours of $\mathrm{ThioO}_{2} \mathrm{~s}$ and $\mathrm{BicycO}_{2} \mathrm{~s}$ overlapping with the $\mathrm{MonoO}_{2}$ area represent only a low concentration of the contaminating species.

The intensities of the peaks corresponding to different classes of compounds are shown on surface plots of SCD data (Figure 4). The landscape is clearly dominated by a single peak of 4-methyldibenzothiophene sulfone (Figure 4a). All $\mathrm{DBTO}_{2} \mathrm{~s}$ combined contribute a minor fraction to the overall sulfur content of the CED material, but the relatively small number of isomers of $\mathrm{DBTO}_{2}$ in the mixture provide for high intensity of peaks corresponding to individual compounds. In order to observe peaks corresponding to other compoumo/8/2006

Page 94 the SCD count vertical scale has to be expanded. Peaks of $\mathrm{BTO}_{2} \mathrm{~s}$ (Figure 4b) cover an underlying plateau of $\mathrm{ThioO}_{2} \mathrm{~s}$. The intensity of individual peaks steadily decreases along with the HPLC fraction numbers and the number of possible isomers corresponding to the homologues. Some smaller peaks on the front edge of the $\mathrm{BTO}_{2}$ ridge can be assigned to $\mathrm{BicycO}_{2} \mathrm{~s}$. These peaks are more visible in Figure 4c, along with the $\mathrm{MonoO}_{2}$ ridge in front and to the right of them. 
Figure 4. Surface plot of SCD intensities and contour plot of SIM intensities for 2-D chromatography of CED material

a) Full vertical scale for SCD and contours for all five classes of compounds

b) Expanded 10x vertical scale for SCD and contours for $\mathrm{DBTO}_{2} \mathrm{~S}$ and $\mathrm{BTO}_{2} \mathrm{~S}$

c) Expanded 25x vertical scale for SCD and contours for $\mathrm{BicycO}_{2} \mathrm{~S}$ and $\mathrm{MonoO}_{2} \mathrm{~S}$
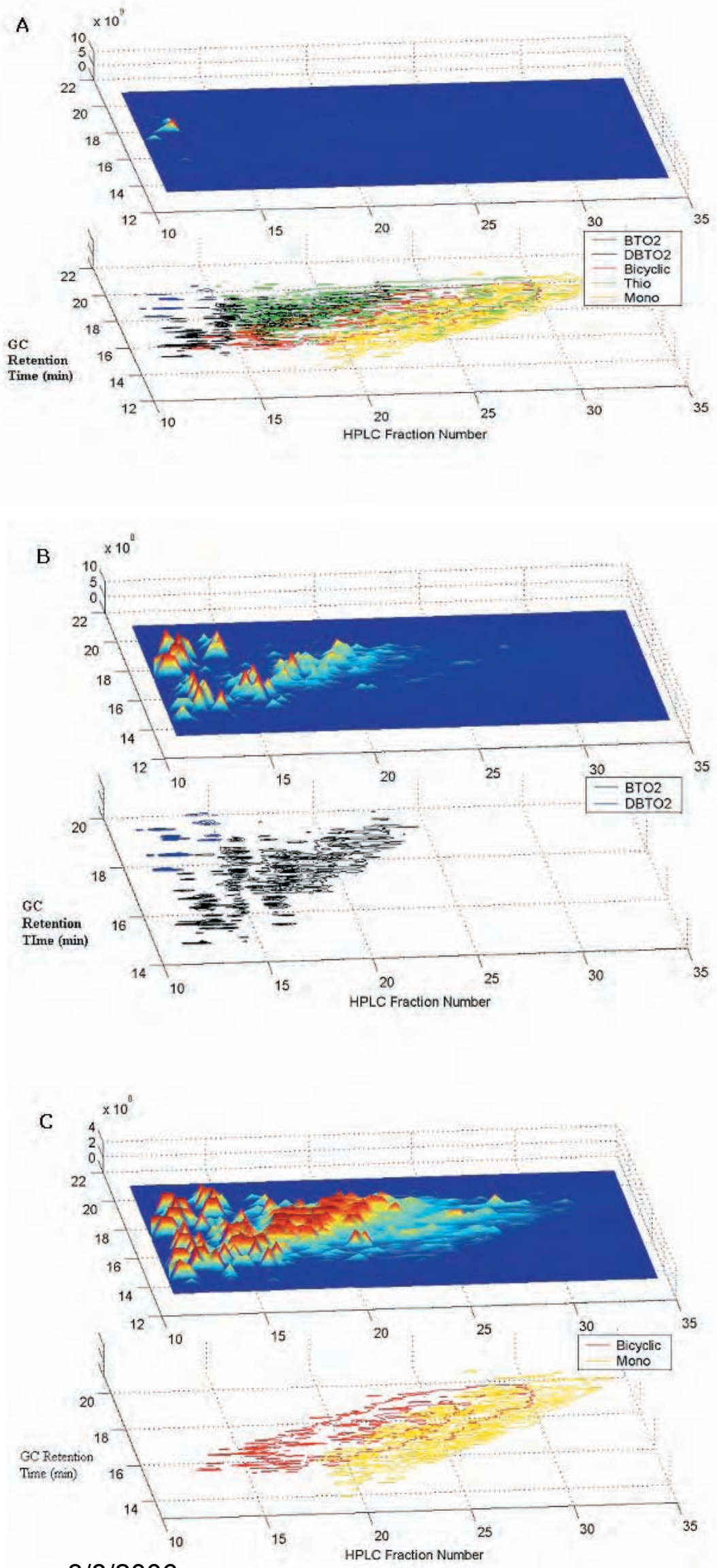

$3 / 8 / 2006$

Page 95 


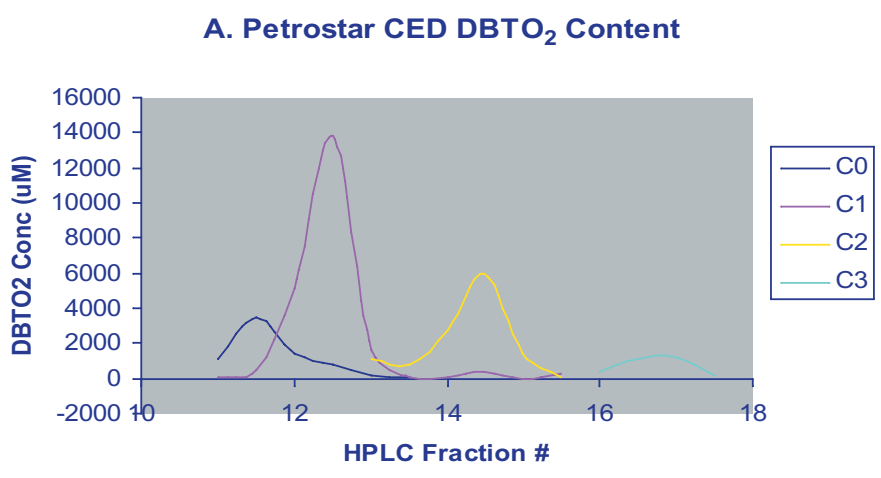

B. Petrostar CED BTO ${ }_{2}$ Content

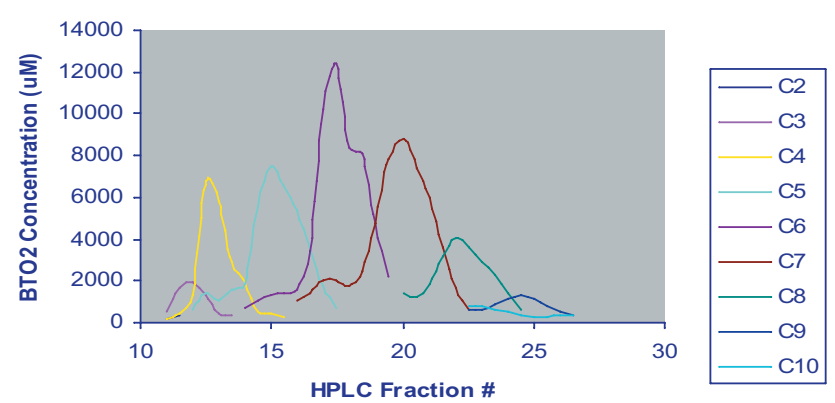

Figure 5. Elution of homologues series of sulfurcontaining organic compounds belonging to the same class a) $\mathrm{DBTO}_{2} \mathrm{~s}$, b) $\mathrm{BTO}_{2} \mathrm{~s}$, c) $\mathrm{ThioO}_{2} \mathrm{~s}$, d) $\mathrm{BicycO}_{2} \mathrm{~s}$, e) $\mathrm{MonoO}_{2} \mathrm{~s}$.

(ii) Separation within series of homologues within classes is achieved primarily by HPLC (Figure 5). GC improves separation only when the number of isomers for homologues is small and individual compounds can be separated (fractions $11-12.5$ ). In the latter fractions, signals corresponding to different homologues overlap significantly. Distributions of sulfur among homologues within classes of compounds have broad maxima characteristic for each class $\left(\mathrm{C} 1\right.$ for $\mathrm{DBTO}_{2} \mathrm{~s}$ (Figure 5a), $\mathrm{C} 6$ for $\mathrm{BTO}_{2} \mathrm{~s}$ and $\mathrm{BicycO}_{2} \mathrm{~s}$ (Figure 5b), $\mathrm{C} 11$ for $\mathrm{ThioO}_{2} \mathrm{~s}$ (Figure 5c), and $\mathrm{C} 10$ for $\mathrm{MonoO}_{2} \mathrm{~s}$ (Figure 5d). The boiling range of the diesel fraction probably defines these maxima. All distributions $3 / \mathcal{H}_{\mathrm{i}} / 2096$ ndividual compounds precludes their separation. 


\section{Assessment of accuracy of various aspects of analysis}

The experiments described here were designed to obtain the most accurate analysis possible. However, we worked under several assumptions, and we feel obliged to discuss the possible effects that these assumptions may have on the accuracy of our analysis. CED material was obtained by oxidation of a sample of Petrostar marine diesel and the subsequent extraction of polar compounds with acetic acid. We assumed that (i) oxidation quantitatively converts all sulfides and thiophenes into the corresponding sulfones and that (ii) acetic acid completely and selectively extracts all sulfones out of the matrix of diesel hydrocarbons. Assumption (i) is not true for substituted thiophenes. Thus, the concentration of their oxidation products can be significantly lower in the CED material compared to the concentration of thiophenes in diesel. In addition, acetic acid may be less efficient in extraction of saturated aliphatic sulfones due to their lower polarity. Thus, the fact that we did not find dialkyl sulfones in the CED material may be attributed to an incomplete extraction.

The SCD data is quantitative and does not require any assumptions to yield the values for total sulfur content of individual fractions and of the whole CED material. However, TIC and SIM data were processed under two major assumptions: (i) ionization efficiency of individual compounds is the same, and (ii) compounds do not compete for ionization when they enter the mass spectrometer as a mixture. In order to remove the dependence of our analysis on the first assumption we would have to synthesize all individual compounds and measure standard curves for them under the ionization conditions used. This is a formidable task. The second assumption was validated by an extensive separation achieved by the $2 \mathrm{D}$ chromatography and by the fact that the amount of overlapping samples entering $3 / 8 / 2006$ 


\section{APPENDIX 8.2}

GS/MS characterization of hydrotreated diesel.

As we had previously reported that we are exploring the combination of biodesulfurization with hydrotreatment of diesel fuel, partially hydrotreated Petro Star diesel was obtained. In order to determine the effect of the biocatalyst it was necessary to determine the composition of the sulfur-containing species in this material.

To quantify the sulfur containing species, GC/MS and GC/SCD methods were developed and used. For the initial determinations, a table of predicted masses was prepared for all potential sulfur-containing compounds and their alkylation-series derivatives expected to be found in diesel fuel (Table 1).

\begin{tabular}{|c|c|c|c|c|c|c|c|c|}
\hline & $\begin{array}{l}\mathrm{Cx}^{-} \\
\mathrm{DBT}\end{array}$ & $\begin{array}{c}\text { Cx-bicyclic } \\
\text { sulfides }\end{array}$ & $\begin{array}{l}\mathrm{Cx}- \\
\mathrm{BT}\end{array}$ & $\begin{array}{c}\text { Cx- } \\
\text { thiophenes }\end{array}$ & $\begin{array}{c}\text { Cx-monocyclic } \\
\text { sulfides }\end{array}$ & $\begin{array}{c}\text { Cx-alkyl aryl } \\
\text { sulfides }\end{array}$ & $\begin{array}{l}\text { Cx-dialkyl } \\
\text { sulfides }\end{array}$ & Cx-ThioIndans \\
\hline $\mathrm{C} 0$ & 185 & 157 & 135 & 85 & 103 & 125 & 63 & 137 \\
\hline $\mathrm{C} 1$ & 199 & 171 & 149 & 99 & 117 & 139 & 77 & 151 \\
\hline $\mathrm{C} 2$ & 213 & 185 & 163 & 113 & 131 & 153 & 91 & 165 \\
\hline C3 & 227 & 199 & 177 & 127 & 145 & 167 & 105 & 179 \\
\hline C4 & 241 & 213 & 191 & 141 & 159 & 181 & 119 & 193 \\
\hline C5 & 255 & 227 & 205 & 155 & 173 & 195 & 133 & 207 \\
\hline C6 & 269 & 241 & 219 & 169 & 187 & 209 & 147 & 221 \\
\hline C7 & 283 & 255 & 233 & 183 & 201 & 223 & 161 & 235 \\
\hline C8 & 297 & 269 & 247 & 197 & 215 & 237 & 175 & 249 \\
\hline C9 & 311 & 283 & 261 & 211 & 229 & 251 & 189 & 263 \\
\hline C10 & 325 & 297 & 275 & 225 & 243 & 265 & 203 & 277 \\
\hline C11 & 339 & 311 & 289 & 239 & 257 & 279 & 217 & 291 \\
\hline C12 & 353 & 325 & 303 & 253 & 271 & 293 & 231 & 305 \\
\hline C13 & 367 & 339 & 317 & 267 & 285 & 307 & 245 & 319 \\
\hline C14 & 381 & 353 & 331 & 281 & 299 & 321 & 259 & 333 \\
\hline C15 & 395 & 367 & 345 & 295 & 313 & 335 & 273 & 347 \\
\hline C16 & 409 & 381 & 359 & 309 & 327 & 349 & 287 & 361 \\
\hline C17 & 423 & 395 & 373 & 323 & 341 & 363 & 301 & 375 \\
\hline C18 & 437 & 409 & 387 & 337 & 355 & 377 & 315 & 389 \\
\hline C19 & 451 & 423 & 401 & 351 & 369 & 391 & 329 & 403 \\
\hline
\end{tabular}

Table 1. Structure-molecular mass $(M+1)$ correlation table for sulfur compounds.

To begin the characterization efforts, a partially hydrotreated sample (1746ppm S as per producers specification) was analyzed. From this dataset, all possible Cx-DBT compounds were identified by GC-MS and their retention times compared to known standards (Figure 1A). The same sample was also analyzed using GC/SCD (sulfur compound detector) (Figure 1B). and the two chromatograms were compared. As can be seen, the major peaks in both the GC/MS (integrating only Cx-DBT compounds) and the GC-SCD (detecting all sulfur-containing compounds) correlate strongly suggesting that Cx-DBT compounds make up a majority of the sulfur-containing compounds present in this partially hydrotreated diesel. 


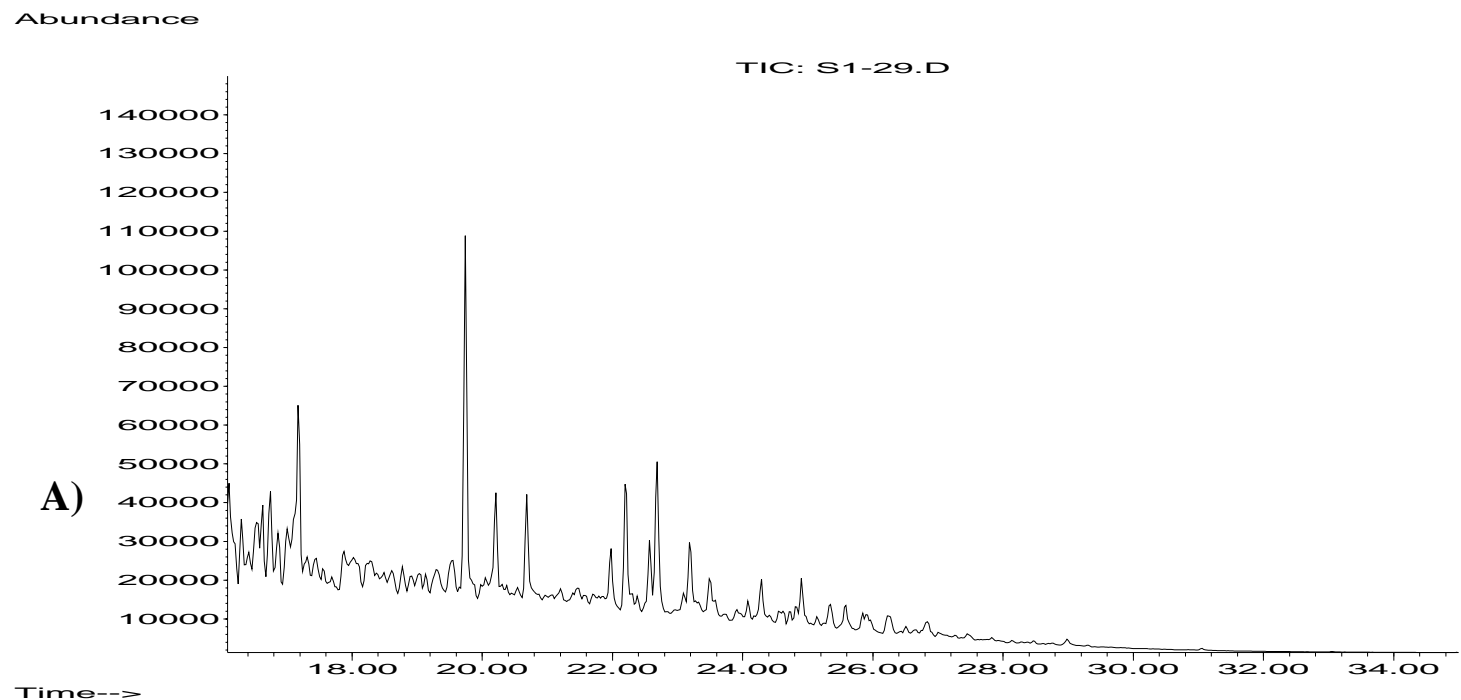
Time--> Response

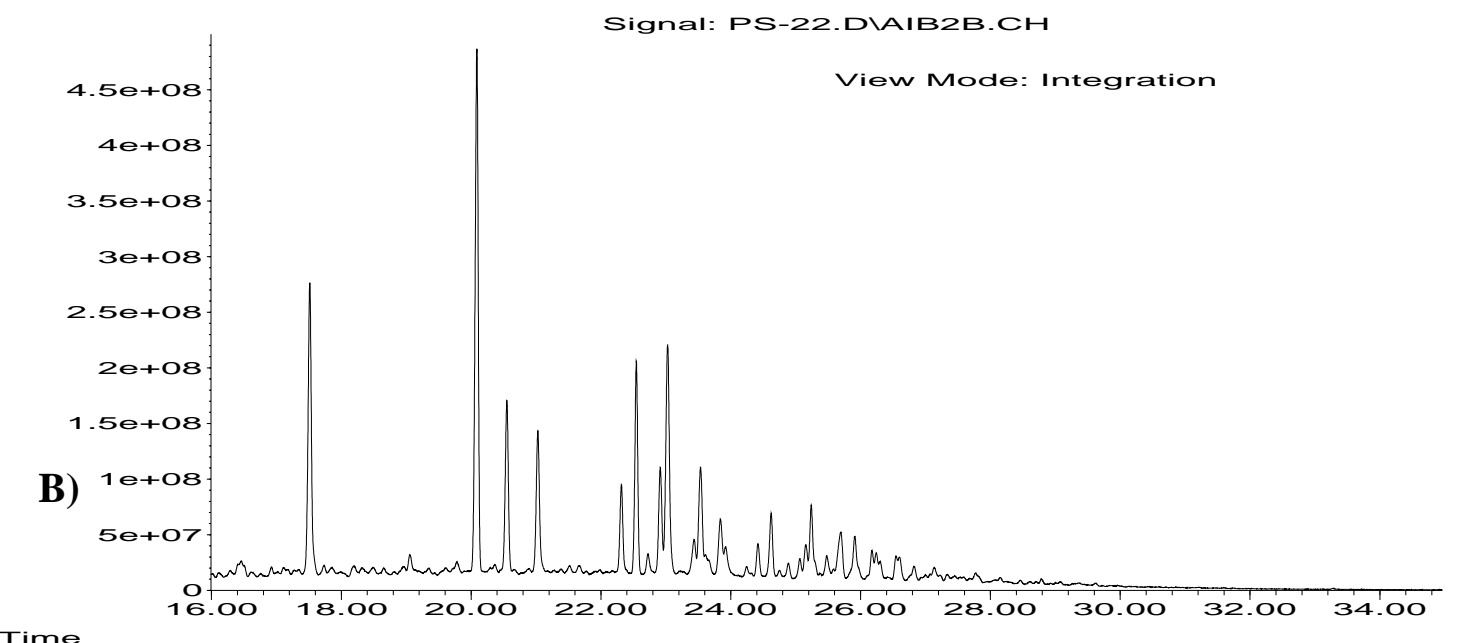

Time

Figure 1. GC chromatograms of partially hydrotreated diesel fuel (1746ppm S).

A) GC/MS trace detecting masses corresponding to Cx-DBTs (M+1); B) GC/SCD trace of total sulfur compounds.

To roughly define the composition of the partially treated diesel for its Cx-DBT composition, a similar comparative analysis was carried out, focusing on the individual members of the DBT alkylation series (Figure 2). In the case of DBT (with only one isomer), the retention time could be determined by using of a known standard. In the case of the other compounds, because of the complexity of the isomeric composition, GC/MS and GC/SCD traces were compared to identify each compound. From these data it can be seen that C1-, C2-, and C3-DBT derivatives compose the majority of the sulfur -compounds with minor amounts of C4- and C5-DBT compounds present. 
Final Technical Progress Report DOE Award No. DE-FC26-02NT15340

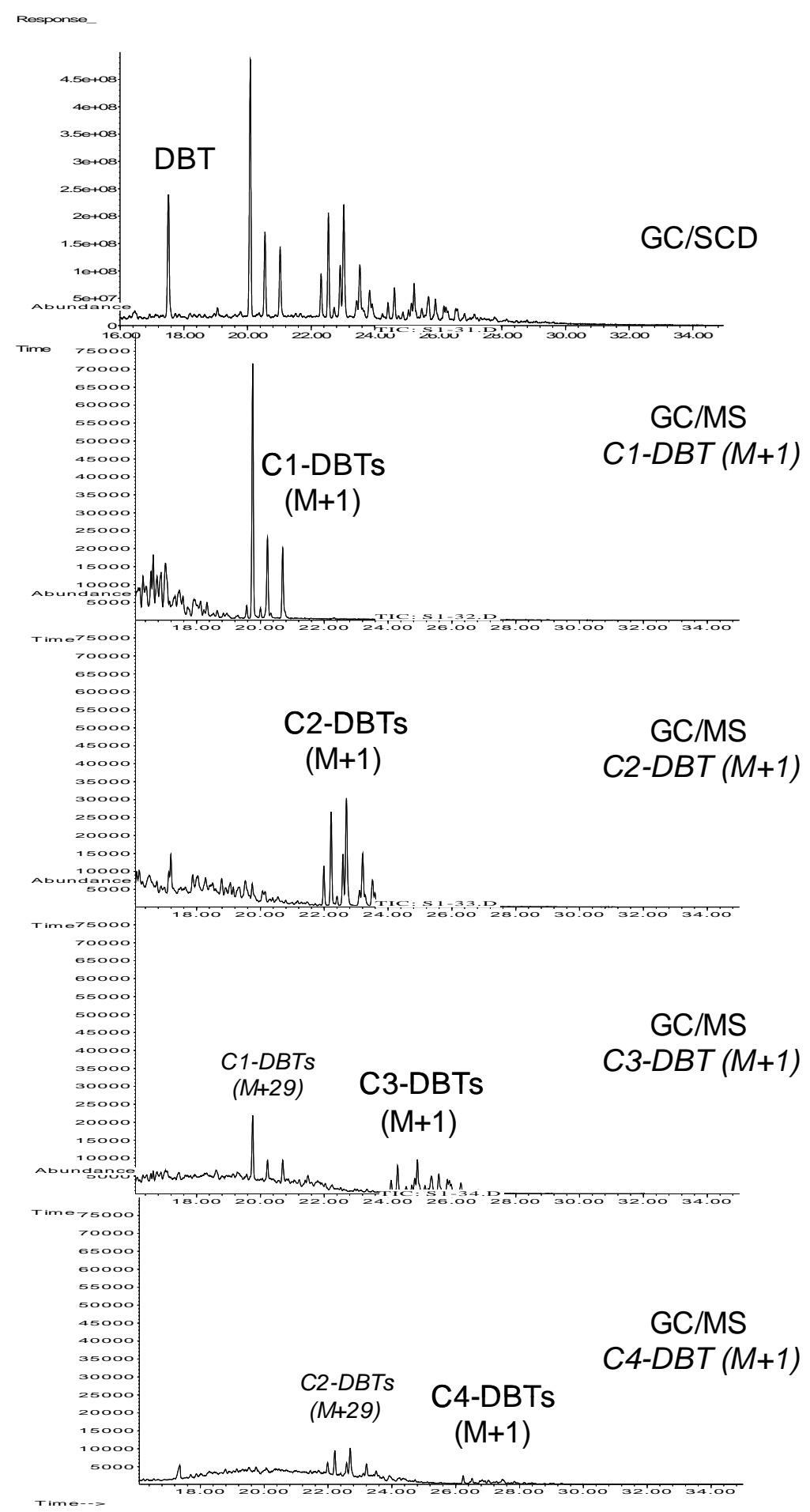

Figure 2. GC/MS traces of partially hydrotreated diesel fuel (1746ppm S). Each trace is detecting masses corresponding to the indicated $C x-D B T(M+1) . M+29$ peaks can also be seen of the C1-DBT and C2-DBT compounds in the C3- and C4-DBT traces, respectively. 
To quantify the DBT species in the partially hydrotreated diesel fuel, an internal standard, benzothiophene, of known quantity was added to each of the samples before separation. The response factor of this standard and known DBT standards was compared and seen to be similar (Table 2).

\begin{tabular}{|c|c|c|c|c|}
\hline Compound & $B T$ & $D B T$ & 4,6-dimethyl $D B T$ & 4,6-diethyl DBT \\
\hline Response Factor & 1.000 & 1.003 & 1.003 & 0.970 \\
\hline
\end{tabular}

Table 2. Detector response values for benzothiophene and DBT standards.

Since the response factors of each of the compounds was similar, 0.4mM DBT was added as internal standard, peak areas were determined and the quantity of the DBT compounds was determined (Figure 3, Table 3). This analysis shows that upon deeper hydrotreatment, DBT and certain isomers of Cx-DBTs are removed selectively. These results suggest that Cx-DBT compounds substituted at certain positions (presumably the 4- and 6-) are most resistant to hydrotreating. It can also be seen that the contribution of Cx-DBT to the total sulfur increases upon hydrotreatment but does so less between the 418 and 43ppm material.

\begin{tabular}{|c|c|c|c|c|c|c|c|}
\hline $\begin{array}{c}\text { Diesel } \\
\text { samples }\end{array}$ & $\begin{array}{c}\text { DBT } \\
(p p m)\end{array}$ & $\begin{array}{c}\text { C1-DBT } \\
(p p m)\end{array}$ & $\begin{array}{c}\text { C2-DBT } \\
(p p m)\end{array}$ & $\begin{array}{c}\text { C3-DBT } \\
(p p m)\end{array}$ & $\begin{array}{c}\text { C4-DBT } \\
(p p m)\end{array}$ & $\begin{array}{c}\text { Total sulfur* } \\
(p p m)\end{array}$ & $\begin{array}{c}\% \text { of sulfur in } \\
C x-D B T\end{array}$ \\
\hline $43 p p m$ & 0.0 & 5.0 & 12.1 & 10.1 & 0.86 & 58 & 48.4 \\
\hline $418 p p m$ & 2.87 & 61.3 & 92.7 & 59.3 & 4.63 & 487 & 45.3 \\
\hline $1746 p p m$ & 58.9 & 178.6 & 226.0 & 119.1 & 10.4 & 1780 & 33.3 \\
\hline Straight & 110.2 & 237.6 & 274.9 & 161.8 & 13.3 & 4130 & 19.3 \\
\hline
\end{tabular}

* determined with Antek SIO analyzer

Table 3. Determined values of Cx-DBT compounds contributing to sulfur in Petro Star diesel and hydrotreated samples. 
Final Technical Progress Report DOE Award No. DE-FC26-02NT15340

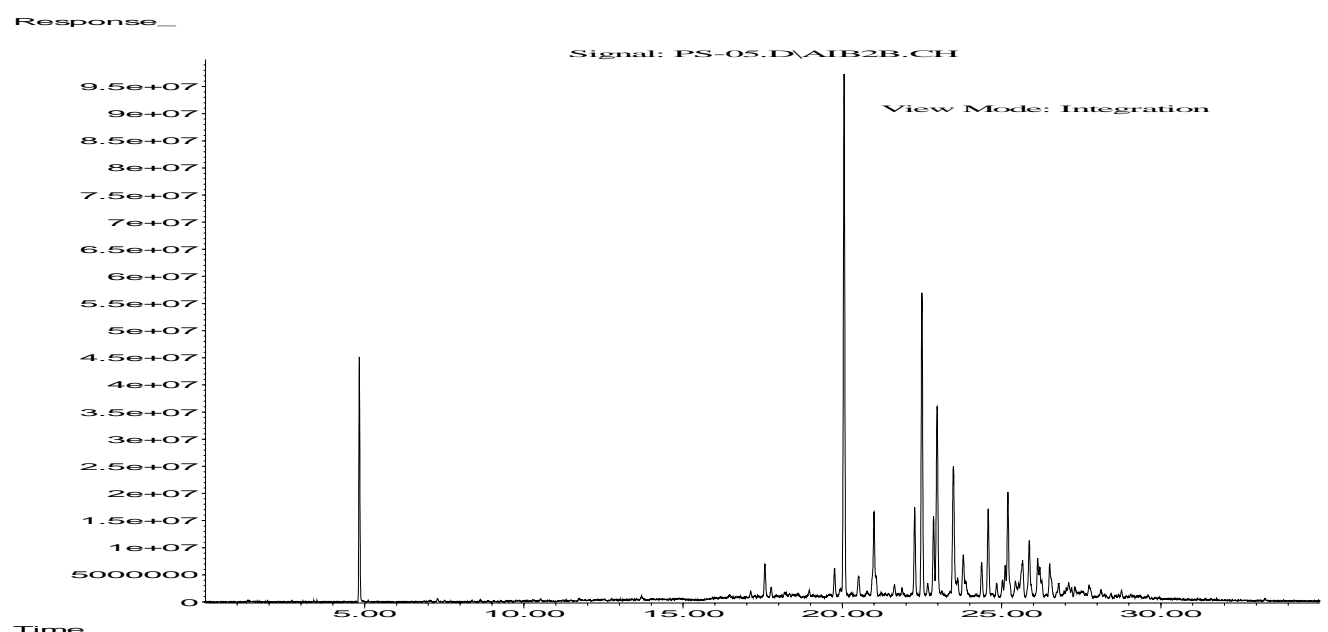

Figure 3. Example of a GC/SCD trace with BT spiked to aid quantification of CX-DBT compounds.

Since these results suggested that additional non Cx-DBT sulfur species are present in the partially hydrotreated diesel, the $1746 \mathrm{ppm}$ material was analyzed by GC/MS and compounds of other classes were specifically analyzed and compared to the chromatogram of Cx-DBT compounds (since the peaks appear essentially the same as the GC/SCD trace the GC/MS trace was used for direct comparison of retention times) (Figure 4A-F).

A)

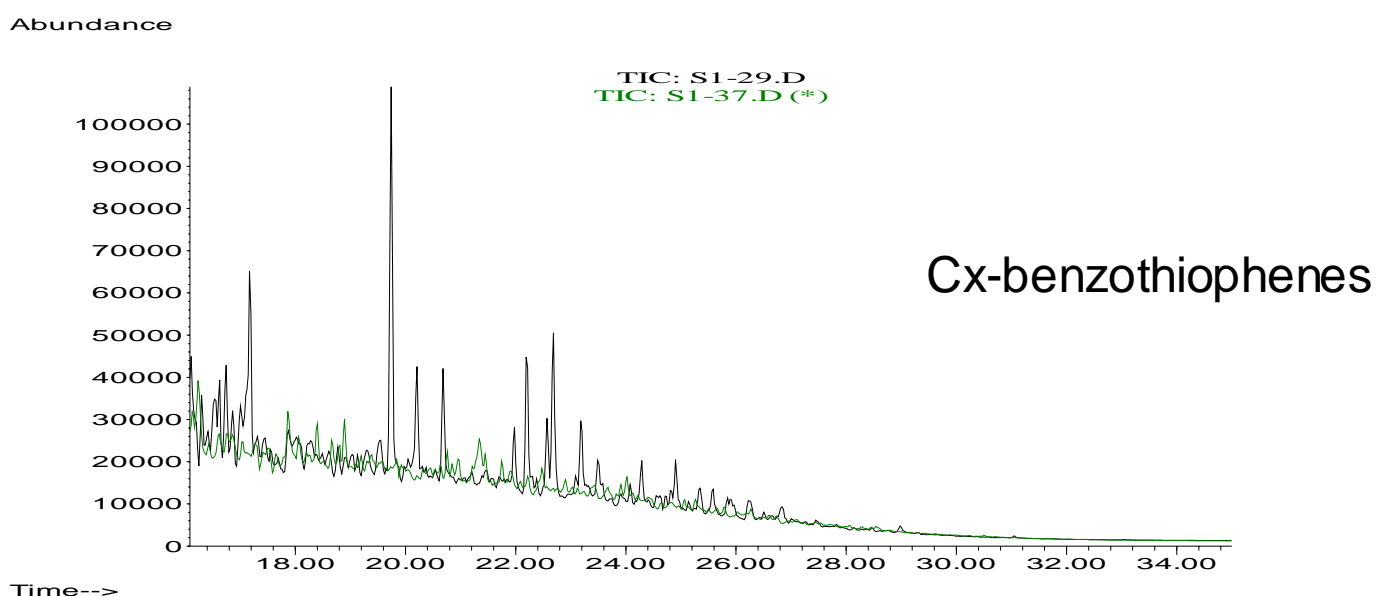


Final Technical Progress Report DOE Award No. DE-FC26-02NT15340

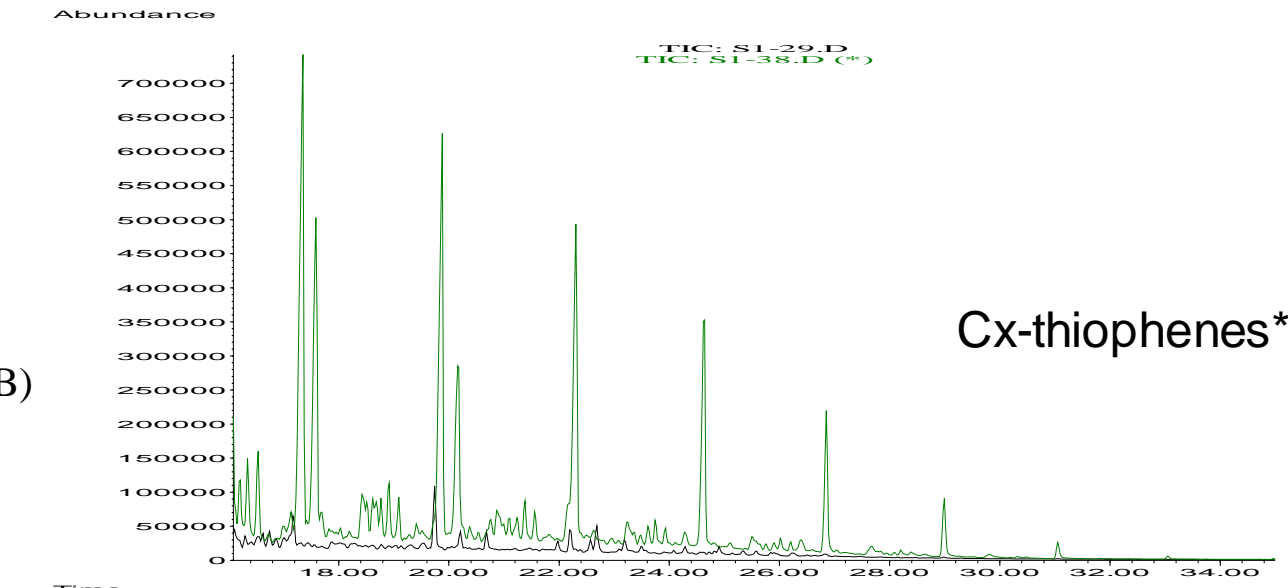

* the ionization of $C x$-thiophenes is $M+1$, the ionization of alkanes is $M-1$, giving the same relative mass of these series of compounds.

C)

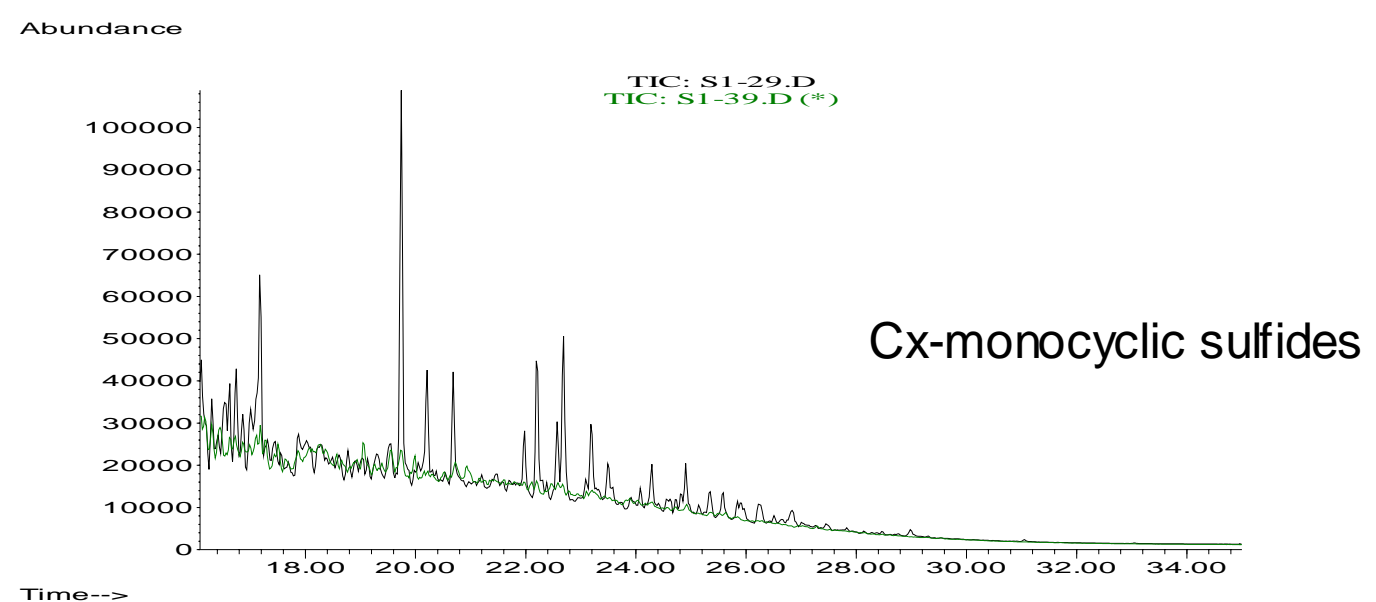

D)

Abundance

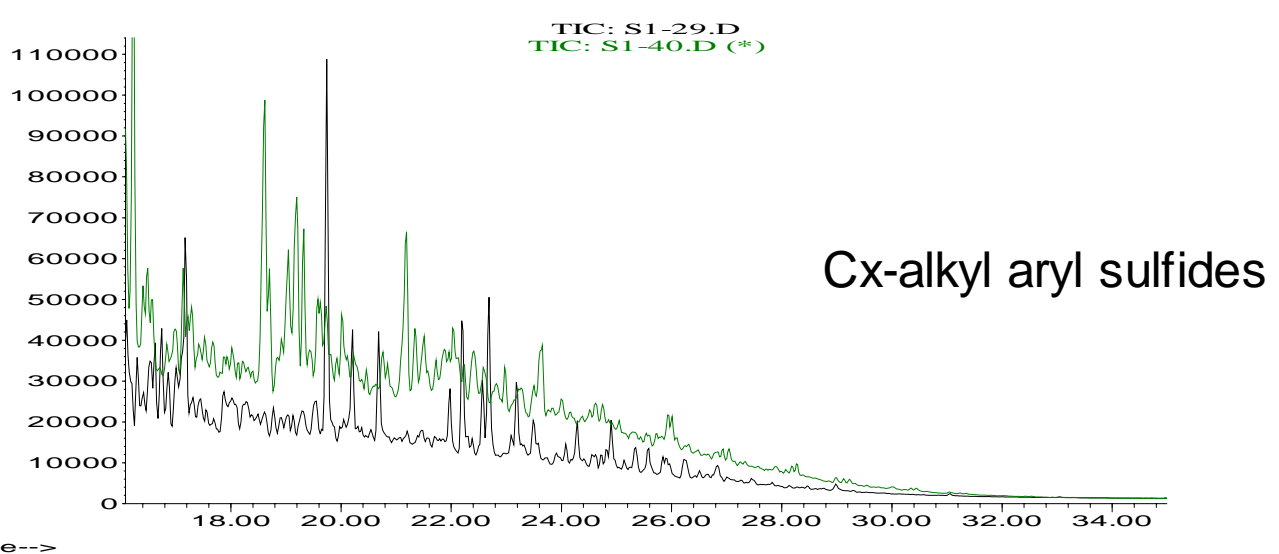


E)

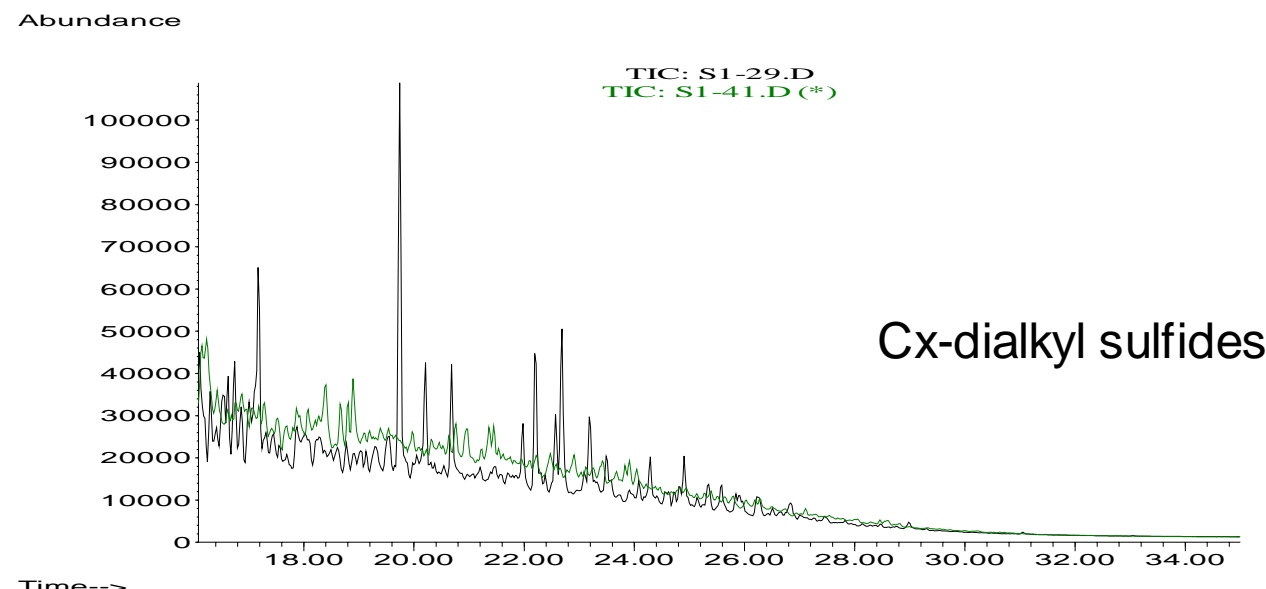

F)

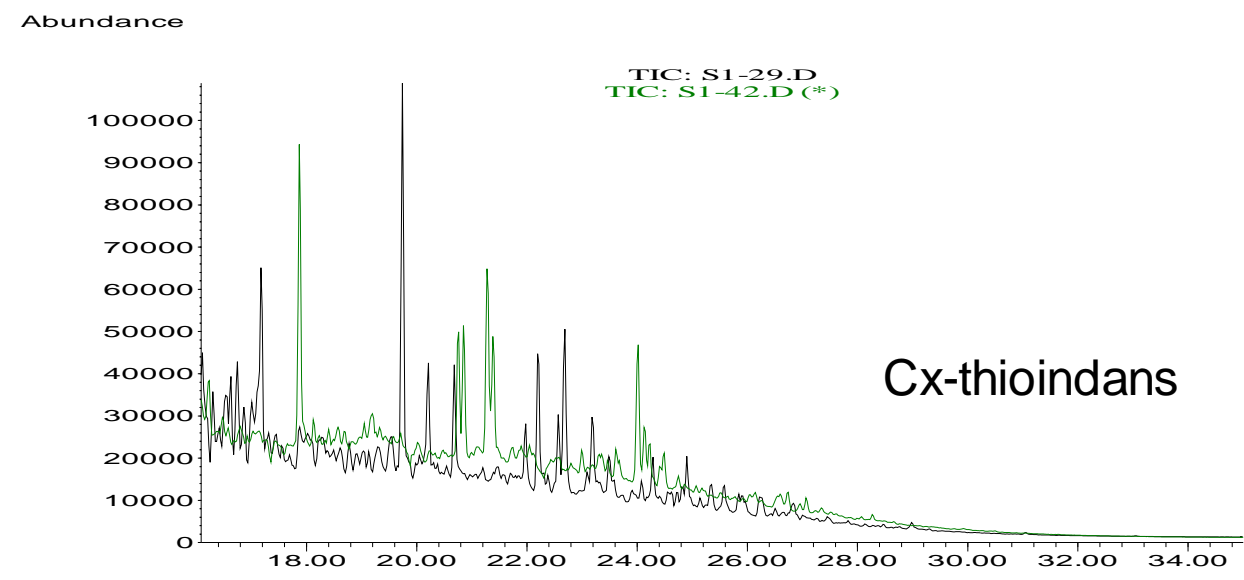

Figure 4. GC/MS traces to identify alkylation series of each of the possible classes of compounds that may contribute to total sulfur. Traces in green are experimental; trace in black is GC/MS of CX-DBT compounds.

As the retention times of none these compounds matches significant peaks in the GC/SCD traces (again as correlated to the GC/MS trace of Cx-DBT) it argues against their major contribution to sulfur as individual species in the partially hydrotreated diesel. It is, however, likely that trace amounts of many of these compounds composes the residual "hump" of sulfur that is seen on the traces and the remaining $\sim 50 \%$ of the nonCx-DBT sulfur that is seen in these samples. 
Final Technical Progress Report DOE Award No. DE-FC26-02NT15340

\section{APPENDIX 8.3}

\section{Nucleotide sequence of the IGTS8 plasmids}

>IGTS8 "90kb" plasmid - complete sequence TCCGGGCCATAGCCTCGCATCACTCGAACATTCACAAAGAAGGGTGTGAAATTGCATTCGATGCAAAACA GAGCCGCGGTTGGCCGCGTGTCGTGTTAACCCAGCATCGGTTTACCGACAGACGTTCCTCTTCAGGTGCA AACCCATCGTCCCTCCACCACCTCACATGTACCCCCGGGGGTATAGCTTGGTATGGTCGACGACATGCAT TCAGTGATCCGGCGGCTTCGACGGGGCGTAGCCTCGTCGGCCGGATTGCTGATCGTGTTGACGGTCTTCG GGGTGCTGCTGATGCACTCGGTGACACCGACCACGTCGATGGCTGACCCGATGTCCACGTCGATGAGCGG TCGCCACGCCTCGATGGCGCCGCCGTCCGCTGTCGAGAGTTCGGTTCAGGTGGCGTTCGGGAAGCATGAC TGTCCGTCGGCGCATCAGATGATGCATCCCTGCGTGGGAACCACGGTGTCGTGGCCTGCGCTGACGGTCC CGGCGTTGAGCGTCGACATCGACTTCGCGCCGACAACTGCGTCCGGAATGGGTGGTCGCACCGAGTCGAC CCGGGGGCGGGCGCCGCCGTGGACCCTGTGGGAATTGGACAGATCGGTGACCTTGCGAGTGTGACAGGGA GCCCGGTAGATCAGGCCTACCGGCGGATCGAAACATATCCACTTCCCTGTTTTCAACACTCTTGCAAGGA GTTTCACATGCGTACCAAACTAGTCGCTTCGCTCGCTGCTGCCACCGTCGGCGCATTCCTCGTCGTCGGC TGTAGCGACAACTCGACCGACTCGCCCATGGACGGGCATTCCATGGGCTCGACCTCGTCTGCGCCGATGT CCTCGGCCCAAGCGAGCACGTCGGCGCAGTTCAACGACGCCGACGTGACGTTCGCGCAAATGATGTACCC GCACCACGCGCAAGCAGTCGAGATGGCCGCTATTGCGAACGGCCGCACCGACAACCCGGAAGTGCTGTCG TTGGCGTCGGCAATCGAAGCTGCACAGCAACCAGAGATGGACCAGCTGACGGCGTGGCTCGCCCAATGGG GCCAGCCTGCACCCTCCGCCGACATGGGCGAGATGAGCGGAATGGATCACAGCGCCGACAGCGGCATGAT GACCCAGCAGGACATGGACGCGCTGATGGCGGCCACGGGGCCCGAGTTCGACCGCCAGTGGCTGACGATG ATGATCGCCCACCACACCGGTGCGATCGAAATGGCGAACACCGAAATCGTCGACGGGTCCAACCCTGACG CTCAGGAAATGGCCCGCACGATCGTTTCCACTCAGCAGCAGGAGATCGAGACCATGCAGCGACTGCTCGG CTGACCTGGTTCATTATCGACGGGAAGGCCGCACCCTCTACTTTGGAGAGGGTGCGGCCTTCGATCATTC GGCGGTCAGGTCTGTCCAGTTGCGAGGCTTAGCTTTCCGACACCGGTGAGCTCCGGGGCTACGCTCCACG TCGGTCATCGCTCACTGCGCCGGTGATGGCGTCGGGTGTCAGATCGAGTCGACGGAGCAACTGTGCGTTG GCGGCGACGATGATCGTCGATAGCGACATCAGGATCGCGCCGATCGACATCGGCAGTACGAACCCGACCG GTGCCAACACCCCGGCCGCGAGGGGAACGGAGATGAGGTTGTATCCCGCTGCCCACCAGAGGTTTTGCTT CATCTTTCGGTACGACGCGCGCGAGAGTTCGATCACCGACAACACAGATCGCGGGTCGTCGCTCGCCAGA ATGACTCCTGCCGATGCGATGGCAACGTCGGTGCCTGCACCGATCGCGATCCCGACATCGGCCTGCGCGA GAGCCGGGGCGTCGTTGACTCCGTCACCGACCATCGCCACGGTGCGTCCTTCCTGTTGCAGCTGGGCGAC GTTGTGCGACTTGTCTTCCGGTTTGACTCCGGCGAAGTACCGGTCCACGCCGAGTTCGGTGGCCACAGAT TTTGCGACGGCGTCGGCGTCGCCGGTGATCATGACGACTGTGATTCCGCGTTCGTGGAGTGCGTTGATTG CGGCGCGTGATTCGGGCCGGATCTCATCGGCGAGCGCGAGCGCACCGATCACGGCACCGCCGGCGAGGAC GTGCAGGATGATGGCCCCCTCACCGCGCCACCGATCGGCGACGGCGAGTTCGGACGCACGCTCCTGCTCA AGCAGCGCGGGCCCGCCGACCTGGATGCGGGTTCCGTCGACGTCGGCGGATACCCCGACAGCTGGGGACG ACTGGAAGTCGGTGGCCGCCGGCACGGTCAGGTTCCGGTGCCGTGCGGCGTCGACGATGGCGCGTGCAAG AGGGTGCTCGGAATCTGTTTCGGCGGCTGCGGCCAACGCAAGGACCTCATCGGCGGTGCGCCCGCCGGTC ACTTCGATCGCGGTGACCGTGGGTTCGCCCTTGGTCAGGGTGCCGGTCTTGTCGAACAGCACGGTGTCCA CTGTCCGCATGGTCTCCAATGCGAGCCGGTCTTTGACCAGGACACCGCCGCGGGCTGCTCGTTCGGTGGC GATGGAGACCACCAGCGGGATGGCCAGGCCGAGGGCATGCGGGCAGGCAATGACCAGCACGGTGATCGTG CGGATGACGGCTCCGTCGGGCATCCCAAGCAGCGTCCACACAGCGGCAGTGATGACTGCTGCTCCGAGGG CGAACCAGAACAACCAGCCCGCTGCAATGTCAGCGATGCGCTGGGCCCGCGACGTCGAGTTCTGGGCGTC GGCGACCAACCGGCCGATCCCGGCGAGAGCGGTGTCCTCCCCTATCGCGGTGACCTTCACTCGGAGACCG GAGTCGGTGGCCACCGTCCCGGCGACAACCTGGTCACCCTCGCTGCGGCGGACGGTGCGCGATTCCCCGG TGATCATCGATTCATCCATACTCGCCGATCCGGAAACGATGGTGCCGTCGGCGGGCACTCGCCCGCCGGG GCGGACGACGACGACATCACCGAGCTGCAGCTGGTCCGGGGACACCTGCACCACGTCGTCGCCGTCGACG CGTTCAGCGGTGTCGGGGAGCAGCGCCGCCAGAGAATCCAGAGCCGACGTCGTCTGCGCGAGTGACCGCA TTTCGATCCAATGCCCGAGCAGCATGATCACAATCAGCAATGCCAGCTCCCACCAGAAGTCGAGCTGATG GTCGAGAACGCCGAGGCTCGCTCCCATCGATGCGAGGAAGGCGACTGTGATCGCCAAAGCGATGAGCAGC ATCATTCCTGGTGCACGTGAACGTATCTCACTGACAGCGCCGGTCAGGAACGGCGAACCTCCCCAAACGA ACATCACCGTGCCCAGCACCGGGGAGATCCAGCCGATCCACGACGCGTCGGGTAGCGAGTATCCGACGAG CATCGCGAAACATGCCGGAGGCGAAGATCACCGGGACAGCGAGGACCAGCATGATCCAGAACAAGCGGCG GAACATGCCGACGTGATCACCGTGCCCAGCGTGACCGCTACCGCCGTGCCCTGCATGGCCACTGTGGTCC GTGTGCCCACCGTGTCCGGAAGGGGCCGTGCTGTGACCGGACATCTCGTGGCCGGCGTCATGACCGCTGT GGGACACCGGAGTGGGTTGATCCGTTGGCACGGCGTCGACGCCGGGATGTGTGTGATCGTTCATCGTGCG TGTCCTCTCGTCCACCGAGTTCGATGGCTACGTAGTGCGCGAGTCTGCGGGTTGCGGTTCGGCTGTGTAT CCGGCCTCACGGACCGCTGCGACGACCTGGGCCGGGTCGAGGTCTACGGCGCTTGTCACCGACAGTGTTC CCGCCGACGCGGACGCTTGGACTGTCGCCACCCCGGCCACTGCACTGACTTCCTCTCGTACCGAGGCCTC GCAATGGCCGCAGGTCATGCCAGTGACTAGGTAGTTCGTGGTGATCACGAGGTGCTCCTTCATTCGTTTC 
Final Technical Progress Report DOE Award No. DE-FC26-02NT15340

ATACCCCCTAGGgGTATCCGACAGAACAGTCCGGACCATACCCCCTCCCCGTATGTTTGCAAAGGGACGG CAGGTCACCGTCGGTCTGTGTGCTGCGGTTTACTTCCGGGCGGACGCGAACTCGTCGATGACGTTCGAGG GCGACGAGGCGATTCGAGCTGTCCAAGGGTTCACACGTAGCGACTGAAGTAGCGGCACGGAGACCCCTTA GATCATCGGTAAAGCGGCGGCGCGCGCGGCATCGGCGGGTACGTGATCGACAGGCCAGGTGCCGACATCG CGTGAACATGCATCTGATGACTGTTACATTCATCCGGTGCCGGTCGATCGAGCTGCCAGAAAACCCACGT CCGAAGCCAAGGGATCCGCCGACCGCCTCCTGAGCGACGGCGTCGTGCTGGTGCTTCCGGAGGAGACCGT CCTGGAAGCAATGATCGAGGGGTGGGAGATACAGCAACGCGCTCGGATGCTTGCGAACTTGACGATCGAG CGGCGGACCCAGATCGTGCGGAGGTTGATAGCGTTCACGAACGACTACCCCTGGAACTGGTCTTCGGTGG ACGTCGAGGAGTGGACCGCTCATCTTGTCGATTCGGGGGTCGCCCATTCGACCGTGCGGCTCTACCAGAT GGCCGCGTCGCTGTTCTGCGCCTACATCACTGATGTCCGATATCGGTGGGATGAGGTCTGTCAGCGGTAC TTTCGCGTTCACCCGGTGCAGGTCTTCCACGAATGGAACACCGTCGTCCACCGCGGGGAGTACGAAGGTC GACCGGGAAACCGGCCGCTGACCAGGGACGAGTTACAGGCCTTCTTCGACTATTGCGACGACCGGGTCAG CCGGGTCGGGTTGTCCGGCCGGAAGGGCTGGCTTGCCAGCTACCGAGACGCGACCTTGTTCAAGGTGATC TACGCCTGGGGGCTACGCCGCCGCGAGGCATCGATGTTGGACGTCGTGGACTGGACCGCGAACTCTGCGG CCAGCGAGTTCGGCCGCCATGGCGCATTATCGGTTCGATACGGCAAAGCCCTCAATGGCGGGCCCCCGCG GCGGCGAACGGTGCTGACCACGATGGGGTGGGCTGCCGAAGCCGTCGCCGAATGGATCACCGAGATTCGG CCCCTGTACGACACCGACCATCTCGCTATGTGGCCGACAGAACGTCGGGCGCGCGTCGGGACCGAGTCGA TTTCGCACCGTTTCGCCGAGTATCGGGACGCCATCGGATTGGAAAAGCATCTCGGTCCGCACTGCTTGCG GCACTCGTATGCGACACACCTCCTCGAAGACGGTTTCGACCACCTGTTCGTTCAGCAACAACTCGGACAC TCCTGGGGATCGACCACCGCGATCTACACCAGCGTCGGTACCGACTACAAGAACAATGCCCTTCGGCGGG CATTGGACCGTGCGTTCACCGCAGACTAGGACGGAGACAGGGATGCAACAACCACGCTTGAACTACGACT GGCACCTACGTCGACTCTTGGCCGAGAGGGGGATCTTCGCAACGACAGGTCTGGGACCGCTGCTCGCCGA ACGCGGGATCAATCTGTCCGAAGCCCAGGTCTATCGCCTGGTCACTGGGACGCCGGAACGCCTCAGCCTA CGTACCCTCGCGGCGCTCTGCGACATACTCTCGTGCACGCCGAACGACTTGATCGAACCAGTCGCCGAAC GGGCGGCTGTGAGAGCGACCGGCACAACCACCCACCGGCCCAAGACTGCGGCAACACCGCCCGGCGCGAA GGCACGCTCACCGCGGCGAGCGGAAATCAAGCGCGATAAGTGACCAATACTGTTTGTGTACAGTGCAATT CAATCGTGGACCGCGTCAGGGTATGTGACCGCTGCAGAAAGAGGCGCCGGCAGCATCGCGGCGCGTGCAC CGGCTGCGCGAAACCCGACAGGCTGTTGGACGACAACGCACTGTGCCGATGGTGTCGCGAGAAAGCGCAA CAAAGCTGCCCGCGGTGCCCTAGCGTCGGTCAACCCCTGACCACCATGGACGGCGAACGCATGTGCGCAC GGTGCGCGCTCCGCTGCCACCTCGAGCACGTGTTGCCACCCACCGCCAACGGACTCTTGGAACCACTTCG TGACGCCATTTTGCAGGCCGAACCGAAAACCACACGGCGATGGCTGAACCGCAACACCGATCTACTGCGC GGCCTCCACGACGGCACAATTCCCCTCACGCACGCAGCACTCGACCTACTGCCCCGCCCCAAGTCCGTCG ACTACCTCCGATCACTTCTCATCGCCACGGGCATCCTCGACCCCGACGCCCATCGCAGTCTCCGGCGACT CGAGACGGAGCTACCTCAACTTCTGAGTGGACTCGACTCAGGCCACCACAAGCTCGCAAGCCAGTGGACG CACTGGGCTGTGCTGCCGCGCTTGCGCGGACTCGAATCGCACCGACCTATCGGCACCGCTGTTCAGAACG CACGCTGCCAAATCCTCCAAGTCATCGAGTTCCTCAACACCCTCGAACAGCGAGAAATCGAGATGCACCG TTGTGAGCAACACGCCATAGACGCCTGGTTCGCAGAGCCCGGCACGTCTCGTTGGCACGTGCGCCCGTTT CTGACCTGGGCACGCAGAACACGGCACCTACCGAAAGAAATCGACCTCCCGAAAGGTCGTCGAGTCCACG CAGAACTACCGACCGACGCCGAGGACCGCTGGCAGATCGGTCGACGCCTGGTCAAAGACGAATCACTCGA CCCAGTGGATCGACTGGCAGGCGCACTGGTCGCCCTCTACGCGCAGTCGCTACGGCGAATCGTCAGCCTC ACTACCGCCGACGTGATCGCCACCGCGACGACCACAGGCCTACGACTCGGTGCCACCGACGTCCTCGTCC TGCCTGATCCGTTCGCGGCGATCGCCCGAACACTGCCCCTCAGTCGACGCACCGGAACTGTCGAGCAATT GCCCACGACATGGCTCTTCGCGGGAGGCCACGCCGGCAAACCGTTGACTGCGACCAGTCTGGGAAACCGA TTGCGCGCCATCGGAATCGAGCCCCGAAAGCTACGACTGTCCGCCACCGAACAGCTTGCCCGTGAGCTGC CCCCGAGTGTGCTCGCGGGAATCTTGGGCCTCAATGCTCACACCGTCGCCCAACACACCCTCAAGACGAA CGGCCAATGGGCGAACTACGCCGCCAACCGCCGATCCTGATCAGTGGGAAAGCATTCACAGGTTCGAATA CACCCACACTGCCAGCACTGTAGGAGTGGAACGCTGCTGCTGCGGTCAGGTAGGCGGCCCATGCGTCGGT GTCGGTGAGCTGGGCGACCTTCGCGGTGATGCTCTCGTGCAGTGCGGCGGCCTGCTCGCGTCGTGCCGCG CTCTTGTCTGCCGTGGTCTTCGCCTGTGCGCCCATAACTTTTCGGCCTCCGCTGATCCGGTGGCGTCCCC CTGCTCGGTGGCCGCCAATTTTTTTCCCTTCTGGCAAAGGAGAATCGCGTCGGCATGAGGGGGCCGGAG TGCCACCCCTGGGCCGGGAAAATTTCTCTGCGAGCGCGCGAGTGCGGGTTGAGAAAATTTCTCGGAGCCT CGGGCCGGCATCGCCGCCAGGGGTTGCGCGCAGGTCACCGCCGACGAGAATCGGAAGGCCAGAAGGACAA ACAAATCCACGCTTTTCGCTGGTGCGGAGCGCAGCGGAGCTCATCTCGGGGGACACCGGTAGGTGGCCGG CCGCCGGGCAGGCGCCAACACACCGCCGGCAACGGGCCGGGCGTTCGCACCTCCCGCTCGGGTGGCTGGC GGGGTGATTGTGCGGGAGCGACGGAGGCCGGCCCCGCTTGGTGTCGCGGTAGCTCTGTGCTTTGGTAGGT AATCTCGACTTCAGACGCCGGGTGGTGGTCTTAGCCAATCCTGTTGGAATTCATGGTGCATAATCACCGT ATGGAACTGGGTATTTATCTCGACTCAAAAGCAATTAACGCCCTCAATGAGGAGATTGCGCCTGCGGAAA TGAAATCTTTCATAGCCCGAATAGTTAAAAAGAATGCAGATGAGAGGGCGGGCTCGTCGGACGAGATCCA TCGAGTGATGTTCTACAACACCGTTATAAACTCAGCGAGCGATATTACACAGAGACCGGGTTACGTTCTT GAGGATGATGGTTATGGCATGTACGAGCCGGCCGTGGTGGCCTGGCATGACGGTGAGATTAGTGCGTCAA TTAGAGCAATGAGTTCGGCGGAAATTGCTGAGTTCTCGGCAGAGATGGTGGCTCATCGTTATTTGAGCTT GGAGTCGATCAATGAGGTGTTCGAAGCGTTCAACTTCGGTGCCCGAATTCGTCGGCCAGAGCGGGGAAAG ATTGAGGTCGAGATTCTTGAGGCCACCGATCTCGATCAGACGGTTGAGGAGTCAGCTCATCAGAACATTC GAGCGCTTTTTGCTCGGGCGAGTTCCAACCTTGACGCCGGCGATTACACGGGTGTGCTGGTAGCGGTAGC 
Final Technical Progress Report DOE Award No. DE-FC26-02NT15340

GAGTGCGTTGGAAACACTCGCGAAGGAAACCGTCGGCACAAGTGCAGTCGCGAATAAGTCCATGGGGCAA TTCAAGACTGAGTACAAGGCTGCGTCGAATTTGCCGGATCCGGTGGTGGACTACATGCACGCGATCTTTT CACGTCGAAATACAGAGCCAACCGCAGGTCACGGAGGTTTGGGGGAGCCAACGTTTACTCGTGCGGAGGC GGTGGTGATGTTGAATATGGCCAAAGCGTTTGTTGCTTGTGAGAATGCTCTGCGTGATTCCGAGTGATCG GCCGTACCGCTGGTTGTGGCCGCTTCCTGCTGGTCTTAGGGTTATCCGCTGGGCGACCTGAAATGGGGGA AGCAGTTGTCGGATAGTTGGAGTCCGGATTTTCGGCCGCGGAGGTGGGAGCGAGACAAGGAACAGTTCGC GGCGTGGACGGCCGGTGCGGATGTTCGTTCTCCGTGGAAGCGTCGCCGGTTCGGAATCTGGCGTGAGGAG CAGTTTTCGGGCGAGATGCGCGTTGCTCGCGAGGCTGAGCAGAAGCGTCGTGCGGAGCTGATGGCGAATC CGAGTGCTGAGCTTGTTGAGGCGTATCGGGAGCTGCGGGCTGCGGAGGCGGCATCGGGCTCCCAGATTGG TTGTGCGCGTGGCGGCGATATGAGTGACCCGAAAACGGTTCGGGCTCTTGCTCGGTTGCTGAGCGATCAC GCCGAGAAGTTTCAGTAGCGCTGACGGCCGGGCCGCATTACTCCTGGTGTCCGGCCTGATCGCGGATGGA ACTTGTCTGATGCGTCCCCGGTCGTGGTCGCGTGTCGCTGGCACGGCCGCGCGGTGAGGTCTTCGGGAGC TACTGCGCGGCTTTGATGTGTTCGCCGCTGTCTTTCTGCGTGCGGTAATCAGTGCGTGACAGAGATAGTT ACGAGATCTATCTTCAGTTATTTTTTAGACACAGATCACAGTTATTTTTTAGACATATTTTTGCCGGATT CGTTGTTATAAAAACCAGACACGCTTGAGCGAGCGCGGTGGCGTCGCGGTATGTGAATCGGTGTTTCTGA ACCAGTTTCATGCGTTGAACTGGCGATTCAATCCACTGCAAAGTGGGGTCGCGGCCGGCCGCAAAGCTGT GCATAATGACTTTCATCGGGCCTTGAGTGAATTCAAATTTTCCTTGTGCAGGGCTTTAGTCTGTCTACAA CCGGGAGTTCAAATGATTGAGTCTGTCAGTGCTTTGTCGTCAGCGCCGTCGGTGGGTTCTGGTGATTGGT GGCCGGTTTTGATGATGGCGTCAGCGGTGTCGGTCGGTGTCGTTGTGGTCGGTGCGATTGTGGCGGCGTC GGAGCGGTCGCCTTGGCCGTTTGCGGTGGTCGGTGTTGCATGCCTGAGTGCGTGGGCTTTTGCCGATGCG GCGTTTGCGAGTGCCACGGCGAGTGCGGCGCAGCTGGCGGCTTCGGGGCCGCTGCCTTTGTCGCTGCTCA ATGGGATTGGAACGGCCGTTTTCGTCGTCTATGTCGGTTGCGCGGTCGTGCTGGGAATTATCGCCGGTTC TGGATGGATCGAATCGTGACGGCGGCGCGGTACGCCGATGGTGCTGACAGTGCGGCGTTTGCCTCAGTCA TTGCTGGTCGGAGTGTCGGGCGCGCCCGGCGTCTGGTGATGTGCTGGCGCGAGTTCGTGGCTGCGATGTG GGGTTTTGGGCGGAGTCCGTTCGAGCGGCAGAAGCGCGTCCGATCGCTGCTGTTTTTACTGGTACTCGCG GGGTGGGTTCCGGGTCTCGTTGCCCTGGGCTTGACGTTGCAAGCTGCGGTGCAGCTCGGCGGGGTGGCGG CGCTGTCTTTCGCGGCGGTGTTGCTGGTGATTTGGCCGTTGGCCTCGGTGTTTTCGCCGGGGTTTTGCCT GGAAAGCTCGGATAGTTCCGCGTCGCTGCGGGTGGTGCCGAGAGATGAGGTGCCCGGGGTGTTCGAGGTT CGGAGTTTTTCGCGGTGGCCGATGACGGAGGCGACTGCCAACTCTGCGGGCGAGGTTGCGGGTGTGGTGG GTGGTCTTGCTGATGAGTTCGGTGTGACTTTGGTGGCGTTGGCCGCTTCGGATTCTCTGCGGCGTAGTTA CATTCGGAAGTTCGGGTTCGTTGGAGCGCCTGAGGACCTGTGCAGGATGCTGACGGGTAAGCGGTTGAAG CCGTTTTTGGTACGCACACCAAGGATTGCGGTTGAGCGTTCGGAAGCAACTATCTGATTTGTGCTGGTGT TGGGAGTGTGCGACACGCCCGAGCGGGTCGGTTCTTGTTAGAATGTGCGCACCCCCGTAGGGGGTGCGCA ACCTCATTGTCGCGTGAAAAGTGCACTGCTTCTTGACTTTTCTGGCTTACTGTCGCACGCATGACGACCA TATTTGCTCAGACGTACATGTCTGCTTCGGATCGTGATGGCGTGGAGTTGTTCTCACTGGAACCTCTGTG GGATGTGATTCGGGATCATGCCTTGTGGTTGTTGGTGATCGCTCTCGCAGTGGCTCTGTGGGGTCACGCG AAGGTGCAGGGGCGATTCAAATTACGCGCGTCGATGGGTGTGGGCGGCACGGCTGTTATCGCTGCCGCGG CCTTTGCGGTGGGTGTGTTGGTGGTGTGGTGGTAGCGCGCAGCCCGTCTATGTTGGGTGAATGACTGAGG CCATGCCGAGTGACGATTTTGTGGTTTCGTTTTCCACCACGGAGGATGCGCGGGCCTGGTTGATTGACCG TGTGGCGTATCTGGCTGGGGTGGCGGGACAGCAGGGTTCGCCGCGGGTTTCGTTCAGTGCGCTGCGGACG GCGAATGTGGCCGGGTCGGGTGCGTCTACTCCTGCGATGTATTACTACGAGACAGACGAGTTGGTGTTTC ATCCGCGAGAGGTGGCGACCGGGATCATCAACACTCAGATTGCGGACGCAATCGTGTTGCATGAGTTGGG CCACCGGGCCGACCCAGGCCCTATGCTGCGGAAACGGATCTCTCAGGGGTTGTGGATCGTCGCGGCCGTG GCCGGTATGTGGTTCCTGCTCTCCCATTCCGGGTGGGCTGTCGCGCTTCTCTCCGTGACTGGGCTGTTGG GTTATGTGTGGTTGTCGTGGAACGCGTCGGCTCTACGGAAGGAAGAGGACATGGCTGACGATTATGCGTT GGCCCACGGGGGTTCTGAGCCGATTCTGTCGTTCTTGAGGGCTTCTGTCGTCGATCCCACGGCCGAAGGG ACTGCGGTTCATCGGAAACCTGCTGATCGGTTAGCTCGAATGGAGCGCAAGGTCGCGGAAACAGGGGCGG TACCGCGTCGGTTTTGACGGTTCGGCTTGTGTTTGGTTCGTGTGAGGATTACTGCGTCTCGCCGGCCCTC TCGGGTGCGAGGGCGCGACTGACGGATGATCTACCGACACGGAGTGTCGAAGCTATCGTGTCGAGGCTTT TGCCCTGTTCACGCATCGTGCGGGCGAGTTCGGTGCGTTCGGGCGTCATAACGGTGGGCCGGCCGCCGTG TCGGCCTTGGCGGCGGGCGGCTTCGAGGCCGGCGTGGGTGCGTTCGACGATCATGCGGCGTTCCATTTGG GCGAGGGCGGCCATGATGTGGAATAGGAATTCGCCGCCTGCGGTGGTGGTGTCGAATCCCTCTGTGAGGG AACGGAATGCGATGCCACGTTCGCCGAGGTTGTGGACGGTGTCGATGACGTGGCGGACGGATCGTCCGAG GCGATCGAGTTTCCAGACGACGAGGACATCACCGTCGTGGTCGCGTAGGTAGTTCAGGCATTCGGTGAGT TGAGGGCGCTGTGCCGTGGAACCGGATTCGTAGTCGGTGAAGATGCGGATAGCGCCAGCTTGGCGGAGTG CATGTTCTTGCAGCTCGGGGTTTTGTTCGAGGGTGGAGACGCGGGCGTATCCGATGATGCTCACAGTCTC GGCCCTTCTGGTCTGCTGCTGTGTTGTGGGATGGTGCGGCTGTTCATGAGATGGTGGTCGACTTCTGGGG AGAAATGATCAGAACTTGTGGATCCCGGGAGGGCGTACCTTCCCGGAAGATGATCTGCAAGGTATCGAAA ATTCTGATCAAGACCAGCGCGCCAACGCTGGCGGGAAGGTACGCCCGTGCTCACGGTAGTACACGAGAAC GACCAGTCCAACGACGACAGCGGACGGTCGCTGCTCGACGAGATCGTCCGCGACGGCGCCCGTCAGATGC TCGCGGCCGCACTGCAAGCGGAGGTCGCCGCCTACATCGACCAGTTCGCTGACCAGGTCAATGAGGACGG GCACCGCCTGGTGGTCCGAAACGGATACCACACCGAACGCGACGTTCTGACGGCCGCGGGAGCGGTCACC GTGAAGGTCCCCCGGGTCAACGACAAGCGTATCGACCCGGACACCGGAGAACGGCAGCGATTTTCGTCGT

CGATCCTGCCGGCCTGGGTGCGCAAGTCCCCGCAGATGAACGAGGTCCTGCCGCTGCTGTACCTGCACGG

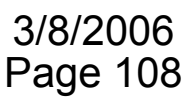


Final Technical Progress Report DOE Award No. DE-FC26-02NT15340

GCTCTCCACTAGCGACTTCGGACCGGCGCTCGAGCAGTTCCTCGGATCGGGTGCCGGGCTCTCGGCCTCC TCGATCACCCGGTTGACGACCCAGTGGCAGGACGAGGCGAAGACCTTCCAGGATCGGGACCTCTCGGGTA CCGACTTCGTCTATCTGTGGGTCGACGGCATCCACCTCAAGGTGAGGCTCGAGCAGGAAAAACTATGTCT GCTGGTGATGATCGGGGTCCGCGCGGACGGCCGAAAGGAGCTTGTCGCGCTCACCGACGGGTACCGCGAA TCGGCCGAGTCGTGGGCGGATCTACTGCGCGGCTGTCGCCGGCGCGGCATGACGGCACCTGTCCTCGCGG TCGGTGACGGCGCTCTCGGATTCTGGAGGGCGTTGCGGGAGGTGTTCCCGGAAACTCGTGAGCAGCGGTG CTGGTTCCACAAACAATCTAATGTTCTTGCTGCGCTACCGAAGTCGGCTCATCCCGGAGCGCTCGCGGCG ATGAAGGAAATCTATATCGCGGAGGACATCGACAAAGCGCAAGTGGCGATCAAGGCCTTCGAACTCGACT ACGGAGCGAAGTACCCGAAGGCCGTCGCGAAGATCGTCGACGACATCGATGTGCTGCTCGAGTTTTACAA GTATCCGGCTGAGCATTGGGTGCATCTACGCACGACAAATCCCATCGAATCTACTTTCGCCACAGTGCGA TTGAGAACCAAGGTAACCAAGGGGCCCGGATCCCGTGCGGCGGGACTTGCCATGGCCTACAAGCTGATCG ACGCCGCCCAGGCTCGTTGGCGGGCGGTCAATGCACCACATCTGGTTGCGCTCGTGCGGGCCGGAGCGGT GTTCCACAAGGGGAAACTGCTCGAACGACCCACCGACATCACCACATTCGACGCCGGGGCAGTCGAGCCC GCAAGCACCGGATCGGAGGTCGCCTGAAACAATCTCATCCACAGGATTTGACAATATCTCACTTCTGGGC TCCAGTGGGTGTGGAACTTGGTGGTGTCGAGGTCGGCTAGTTCTCGCTCCGGAGCGACTGGTGCGGGAGT TGGCCGAATCTCGGTCGACCGTGGTTCATGTTGTGGTGCTTCTGTTTTGATCTGGCTTGCCCACGTGTAG GTCTGTTCGGGCATGGTGTCGTAATGCTCGATCATGGCCTGCCAGTGATCGGGGAAGGTCATGACGTTCT CGCGGGGTGCGGTGGCGATGGAGTCCGCGGGTACTTCGGTGACGGGTTTGATCGCGGGCCGTAGGACGTC GGCGAGAATGGTTCCGCCATCGATGTATGCGAAGTTTCGGGCTGCTTGGACGGCGTCGACGCTGAGTTCG CGCCACTCGATTGCCCATCCGACATCGCGTGCGAGTTGGTCGAAGAAAACCCTTTGGGTGCGGGAGTTTC CGTCGACGAACGGATGGACGCTGGTGAGATCTCCCCAGTGTCCGGCGAGATCCTGGACGAACGCTTCGTG GCTGTGGTGGTTGACGTAGACGCCGCTGTTGATGTCGGTGAACACTCGGTCGAGTTCGCCTGGGATGGCG GCGGGTGGGTCGTGGAGGATGCCTAGATCGCCTGGCGCGGTGTCGATGGTGCGGATTTCACCGGCCCAGT CGTAGAGGTCACCGAGTAGGTGTCGGTGGATTGCTTGCAGGTGCGCGAAGTCGAAGTGTCCGTCGATCGG GTTCGCTGCCAGTTCTGCCATTCGGGCAGCGGCGATCGCGCCTTCGACCTCGGCGAGGATCTGAGGGTCT TTGTAGCCGAACTTGTTTCGGAGTACCTGTGTACCCGGGTAATGGTAGGAATCGGTCACAGTCCGTGCCG CCGGCGGATCGCTGCATGCGCGAGCTCGATCGCCTCGTCTCCCGTCAGGTCCCCGGCGAGTACCGATCGA GCAGCTGTTTCGGCTTCGGCGGTGAAGGGGACGCCACCCATTTCGAGGCCGGCGCGGATGCCTGCTATTT CCCGTTCGATCTCGCCCGCGGTCAGGATGCGGGTGGGTTTACGCGGAATCTCGTCTGGATTCGTCATGGT CTGCTCCCCCGGTTCTCGTTTTGCGGCTGTACGCGTTTCTGCGGTTTCGATGCTGCCGACCCTCGGTCGG TGGTCGCCGGCGGCGTGTTCGCCGTTCTGCGTTGAGAGTGATGCTATCGAACCGACCGAAAACGATCTAC TCGGCAATTTCGGTCGAACCAGTTTCGGTCATGAGTTTCGGTCGACGGTTTCGCGTGGTCGCTCGCTTGC GCCGATTCGGGTCGATTCGTGGGCCGAATACGATCGTTTCCGGCCTATCGGCCTTATCGTTAGAGTGCTA AACATAAGGCATGAATGATGAAGATCGGCAGAACGCTCTCGCGGATGTCGTGGAGCTTGCACGCCTCCTC CACGCTCTCCGGGGGTTGTCCGACGAAGTCGAAGCGCTTCTTGCGGATGCGATGAAGATCGCTCGCAGCT CGGGCCTCAGTCAGGCGCTCATTGCGGAGGCTGCGGCGCTGTCACCTGGCCGAGTGAGCCAGATCGTCAA GTCCGATGAACCTGAGGTGTCGCGATCACAGGTACACGACCGGGTATACAAGATCACCGAATGGCCCAGC GACGCATTGCGGACTTATTGGGCCTCGTTTCCCGGCCGTATGACTATGCCGCCTTACCCCCGTCGCCGCC ATGCCTCTGATCGATAACAACATCTACGGCCGGGGGCTTCTGTCGGTGGACGATTCGACTGCGGTACGCA GGATTTCGTTTTCCTCATCAGTGAGTGCGCGGCCCATCGCACCTTCGACGAGGCCAGCGACCATGAGTGT CGTTCGGGTTTGCTCGCTCATCGGTTCATCTCCGTGTGATTGCTCGGCCAAACAGCGGTGCGCTGCGCCT GTTTGGTGGTGTGGTTGGTAGGGATGGGCGCGAGATGGTGGAGTCCGGGGAGGCTGTAGGCGGTCAGGGC GAGTCGGGTGCGCCAGGCTTTGTGTTTGAACGCCCAGATCCAGTGTCCGGTCATCCAGATTGCAATGCCG GCGGCGAGGATGCTGTAGGCGGTGGGGGTGCTTCCGGTGAAGCCGATGCGGAGCAGTCCTTCGAAGACTG CGATCGCTCCTGCGAGGCGGGCGATGATCGATCCGAATCCCCAGATCAAGAGTGCTGCTACGGCGATGAT CCCGGCGATAGCCATGCGGTTGTCCTCCTTCTCGTGGGTGATTCGGGGTTCGGGTTCGTTGTGGTCGAGC GTCGAAGAGTGCGCTGCTAGCTTTGTCCGCTGTACCGGCGCCATACGAATGGGGCGGCGCTGAGGAGAAC CAGCAACAGCGTGACGAGGGCGAAGATCATGCCGAATTGCCACGGCTTCATTGCGTCGGGAAAGAACAGC CAGGCGATCAGTGTTCCGAGTACGAATCCGGCTACTGCTGACCCTGCTCCTGCTTTTGCGACTGGGGCAA CGATGGTGGTGATCTCGTTGTTCATGGCGGTCCTTTCTGTCGTTTCTGGTGAGTGTGCCGGTTAGGTCCG ACAGGGATTGCGGTGCCGAGGGTCAGAACAGGGTGTTTTGCGCGGTGTGGGCTGCTGCGTCGGTGCGCCT CTCGTAGGCGGCTGCGAGGGTTTGCGCAGTGGTGGGGCTGCATCCCATTTCGGTGCCGATCTCGTCCCAG CTGGCTCCGTGGTGGTGCAGTTTCCAGGCGTCGTGTTCGTCGTCGCTCATGGCCGCTCTTTCTCTTCGGT GGGTGTGTTGAGTTCTCGGCGGAGGCGTAGGGCTACGACGAGGTGGTAGATCGCGAGTCCGAGGAGGCTT GTTCCGGCGATGAGGGAAATGGTTGTTCCGCCGGTGAGGAAGATGATGCCTACGGCGAGGCTGATCAGCG CGACGACGGTGTGCGCTTTCCAGCCGGGTTTGCTGGTGCCCTTTTCGGTCGTCAAGGTCGATTCTCTCTG TCTTTTGGAGCTTTTCGGAGGCTGGCGGCTTTGGCGAAGCTGTAGACGGAGGCGGTGAGGGCGATGATTC CGGCGGTGATCACATGTTTGGTGCCGATCGTGAAGTCCAGGGCTGCGACGGCGAGCGCGAGGACTCCGAA GATGCTGTGTTTCACCACACCTGGCTTGTTGGTCATGCTCGTCCTTCGCGGATGGCTGCGAGGCGTTGGG AAGCTGCGCGGCGTTCGCGGCCGATGCTGAGCAGGTGCTCGAACAGTTGGTCTTCGGGGAGCTCTTCGGC GGCTTGGGCGAGGGATCGTGCTGCGTCGAAGAATCCGCGCCGGTAGGCCTGGGTGAGGACTGCTGCAATG TTGTCCTCGAGTTCGGCGGACGGGACGCCGATGGTGGTGATGTCGGTGAGTGCTTTGGTGAGTTGTTTGC CGAGGTGTCCGCTGGTGCGGCCTTCTTGCTGCAAGGTGGTGAAGACGTGGGCACTGTTGTGGGGTTTGCC TGCGCTCACAAGGTCCGCGATGACGGCAAAGAGTTCGGCGTGGACGGGTCGGTAGAAGTCTGCGCTGGTC 
Final Technical Progress Report DOE Award No. DE-FC26-02NT15340

AGGGTCGCGACGGTTCGTTTCGCGCTCTGGGCCGGTGCCCAGAGCAGTGAGCACAGGAGTTGTGCTTCGA CGTCGAGGGCGATGCTGTATTCGTCGATGTCGTCTTCGTTGTCGGTGAGGTCGTCGAAGTCGGGGACGGT GGCCAGTGAGAGTGTCACGTCGGGTTCCTTGCTGTCGCCGGTGGGCATGGGAGGTCAGGGGCGCGGTGAG GTGAGTTGCCGGTGGCTGCGCAGGATCTCGGCGGCTGCGCGGAGGTTGCGGGCTGCGTCCTCGAGGTGGG TTGCGATGTCCTGGTCGTGGCCGGCAACAACTTCGGCGGTGTCCGTGTCTGGTGCGGTGTAGAGGGTGTG CAGTTCTTCGCGTGCGTCGCTGATTCCGGCGAGGGAGTCGTCGAGGGCGGCGAGCATGGTGCGGAGCTTG CGGTCGACGGATTCGCTGTCGCGGCGGAGCTCGGCGTCGAGTGCGCGGTCGACGAAGTCTGCGTCGGGGG TGAAGGTGAATGCTGCTGCGGTCATGGTGTCCTCGTTTCGCTGGTGAAAGTGTGTGGTCAGGCGGCTGGG ACTGGTGGAGTGTCGGTATTCCAACCGTGCACTATTCGTTTACGCAGGTCCGCTTGGGTTTTCACTGATG AATCCATATCGCCGCCTCTCATCGGTGGTCCGGGCACAGGAGGGCGGGCGTCAAATTCCACCCACTGGCA GTCAGCCACTGTTGAACAGGAACCGGGAGACCATCGACTGCCGCGGCGTGCGCGAAGGTGTCGGCAAGAA TTGGACCGTCACCGCATACGGCGCACGCGACGTCGAAAGCGGGTGGCCCGGGTTCGCAATGGCGACAGTG CGAACGGATGACGACCGCAGTCGGATCGTCGACATCCGGATGGAGCCATGCAACGGCCAGGTCGCCGCGT TGATGAGCGGCTTCCGCTCGCGTACTCGGCTCGTAACCACACGTCGGGCAGGTGCGTGGCACTCGCATGG GCACAACAGAATTCACAGGAACGTCCCCAGGAGGATGCGCTGTGGAGGTCGAAGCTGACTTGTTCTTGTT GAGATGGCCGTACACAGTCGTGCGGGGCACGTTCAACATCGCCGCAATCTGCGCGACCGTATTCTCCCCA GCGTCGTACAGCCGCTGAGCGAGCGCAATTTGATCGTCGGTGAGCTTCGATGGGCGCCCGCCCTTTCGGC CACGCGCCCGCGCAGCGGCCAGGCCGTCCCGGGTGTTCGCCACAATCAGCTCACGCTGAAGTTCCGCGAG TACCGACAGCATCCCGAACATCGCCCGACCTTCCGCGGTGGCGGTGTCGATTCCCTGGTCGAGGACCTTC AACTCGATCCCGCGCTCGCGCAGCTGCGCACCGAGGGTGATCAGATGAAGTACCGAGCGCCCCAATCTGT CGAGGCGAGTGATCACCAACGTGTCCCCACCCCGCAACCGCGCCAGAACGACATCGAGTTGCGGGCGTGA GGCTTTCGCACCGCTGGCATGATCGAGATGAATGTTCTCGGCCCCGACGCCCGCCCGCGCCAGCGCATCG ACCTGGTGAGCGGGATTCTGATCTGCACTGGAGACTCGCGCATAACCGAACAACATCTGTCGAAAATATC TTACTCAACCGTTTTTCGACAGACTTTCCATCGTGGTAATTCAGCACACTCGATCAGCTGGACCGCTCGT CTACACTCCGTCTGTGCTCAGACGCCTCGCATTGCCAACGCGTTCCGCCTTCCGTGATCGCAGCGGACGC TCCATCGGTGCCGCTGCGACTGCGCTCGTGGTGATGTCGCTGCTCGCGGCCTGCTCCTCGGAGAGCGGCA ATACCGAGAACCCTACGGTGGAGCCCTCGCCCACGACGGTCTCGTCGACGATTCCCTCGCCAACGACAAC CTCTGCGTCGGCCTCGCACACTGTCACGCCGTCCAACGCCGTCGCGACGCCGCCCTCTGCGCAGAATCTG CTCCTTTCCGCAGCGCAATTACCGGGTGCGGGCTGGACACCACAATCGGACACCGACCCGGAAGCCACGG AGCTCCAAGAAACGACGCAGCCAGAGTGCAGTAATCAATCGTGGATCCTGCGCAGTGCGGACATGACCGA CATGGCCGGCGCGTCATGGACGTTCGATGCTGCCGCCGAGGTGGAAACGATCAGCAGTCAAGCCTTCGTC TACCCCGATGCGGCGGCAGCGACCGCAGCAATGGCGTCGTATCGCGATCTCGTGGGCCGCTGCACGAGTT GGCAGAGCGTCCTCACCGCCAAGGGATACACCTACGCCGAATCACAGGAAATGTTCGATGCATCGGTCGG CGAGGAATCGGTTGCCCGGCAAGCCGGCACCACGCACGTGCGCTTGACCGATCTCCCCACCTTCCGGATG TATTGGGTGACATCGCGTGTGGGGCAGACGATCGTGCAAGTCACCTACCGGCCCGGCTCCCTGCTCGGCA CTGATCAGGGAAGAGACCAAGCCCTCGAACTCGCGGCGACCGCAGTGTCCACTGCCGAAAACCGTACGAA ATAGAACCAGCCCGATAGTCGGTCACCTCGGTGGAGGCTGGGCGAAGAAAGATCAGCGCGGTAACCAATC TGCCACTAAGGCCGATCCACAGTTCGGCGGGACATCGGCCATACGAGCAAGCGCCTCCCGCAATCCGTTT CGGGCGATGAAGGGCACAGTAGTGAAGAAACTGGACTACGACCTGGTCGGTGAACAACTGTTCTCGAACC CGAAGGTCAGAGCACGTATCGAACGGCACTACCGCCTCCCCACCGATGCAGCCACCGCGTCGCAGTTCCT CGACCACGCGGTACGCCACCTCGATCAGGAAGCGATGGACAACCCCCTCACTCAGACCTACAGCAACCAA CAGGTCTTCAAAGCTGCAGTCAACGCCCTCGGCAGTAACTCCCGAAACTGGGCGAGTTTCGTCAAGGTCC GTGACCAGCACCTCGAAGGACTTCTCTACGGCTTCGACCCCGAAAAGACGCACACCGCCATCCTCGACGG GACGTTGACCCTCACCGATATCCGTGAGCACCTCAGCGGGCAAAGCGGAGCCGGCGATGCGAGGGCAATC ATCCGCTGGGCCGCCCTGCTGACCGCTCAACCGAACTACTACCAACAGATCCTCGTAATCGCCCGCGCCT TCGTCGACCTCGCAGCCGATGCCGACCACCCGCTCGACAACGAGCATCTGATGATGTGCCTCGCCGGCTA CCTGATCGCACCTCCACGCCGCTGGCCAGGCGACAAATACATCGACACCGACACCCTGTTAATGACGCCC CGCGAACGAGACCTCCCCGGGATGGGCTACCCCCTCGCCAGCGAATTCCTCCGCAACCTGCATTGGAACG GCTTCAAACCCGACCGCCACATCATCAGGCTCCTGAGGCTCTGGGTTCCCGAACTCCGAGACACCGTCGA CCCCGAGGTCGATCGTCTCTGCACGTTGATCGGAAGCCGAAACCAGTCCCTGCGCTACTTCCTCAAATAC TCACTGATCGGCGTTGCCATCACCCCGGACGGCAACTACTCCCGCGCCGACAACCTCATCTGGATGCTCG GCGCGTACCTGGAAAAGAAGAACAAGGAATCGGACGTGCAATACATCCACGACTAGAAGTCTGTCGCGAT GGATCGAGTTCGGACACCACTGACTACTCGTCCGCTGCCAACACCCATGCGGTTGGAGTAGAGCACACGG CACACCTCGAGGTGACCCACCCGGCTGGTCGTCAGTACCAGCCAGGTGGGTCGAGTGCGCCCCAACTCTG GAGGGCCGGGGCACGTATTTTGGGAGCGGCTCGATGGACGGTCTGTGACGGCATCTCGACGAACCCCGTG CATCACCATCTCGAACAGAGGGCGCGGAAGGGGAATGTAGCGCACACGGTTCCACGGATCTGCAACCGCC CGGCGGGTTTAGCGGCCATCCTGTTTGTCGAAAAGATGGAGACAGCAGATTATTCGACATAGATTATCGA CACGAGTTCTCGACATCTTCAACTCGGCACGAGCTGGGGATACGCGTTCTTCACAGAAGCGTCGAATACC GATCTAАTTTCGACAACCTGTTGGCGTGCATCGAAAATTCGAACACGTTCACTCGTGAATGCCTCTTGAC TTCATGATTTGCATTCTCCACAAAGCTGCGTGGCATAAAAATTGAAGAGTTAGATCAGCTACGAATCCAC CGCGGATATAAGATCGGCATATCGGTCAAATAATGCCGAAGATATTTCAAGATCGGTCGAATTACATGCT AGAGAGTAGGCCTCAAGCACAGCTGAATCTAGATTCATGTGCGCCTCACGAACCTCAGATGGCATCGTAT TTCTATCATACATATCAGAAAGAGAAAGGTGAGAATTTTTATCACGCTCTTCAAGTAAAACCTGGGCAAA GGATGCTATCTCGACCCTAGATTTATCACTTAAATCAGGCCATGGGAAACAATTATAAACGACTCCGGCT 
Final Technical Progress Report DOE Award No. DE-FC26-02NT15340

GATAGCTGAAАATCAGATTTCATTCGACTTGATACAАTTTTCAАCCAАTTCGTAAATACTCGACTTTGAA GAATTCCGAAGGTAGACATATCGGCAGACTCAATTGCGAATACAGAATTATTAATAATGTATTCGCTCCC ATAGAATGACATGGGAACGTAATCCCTATTTTTCGAAGAGACGCTCGGCACGAGGAGGTATTCACGATTT GGCTGAAAAATTGCACCGAAAGACCAAGGCCTATCTACCGCGCGCCCCTTTGCGCCTTTTGCGCCTCTCC TCTCCACTTCTACTGCCTCCACCCTTTGCCGAAGAACCGGAGAGGACCGTATCTCAGACTTAGTTGCATC TTGCAGCCAAAGGCAGAATCTGTGCCGATCATTCAAATGCTCGTCAGCGCCGACCAATGGACGAAGATAT TTGGCTGCGATTGGATCTTCCGAACGAATCATCTCAACATCCTGGGCTGTCAGCTTTGAGAGATACCCCC CGTCGCGAGGCTCATTACCTACCGATATCAGAGGAATACCCGAGACAAGCGGACTCGTTCGCCCCCAGAC ATAAACATCTGCCGCCGACGAAAGAAATGGATTAATATTCTTTACATACTGGAGACCGCCACCTAGTTCC TCTTCCAGCCATAATTTCTTGGGACCCACACGCCCCGAATCGAACCCAATAATAACGCAATGAACAGCCG СTTGATTACTTCCCGAATTTTCCCAATTAAAAGTTCTATAAGCAAAATTGATTCGCATACCTAGTCTATG CATCTGATTCCACAAAATTGCAGGTTGCCCACCCTGGCTTATAGAGTTAGTAGAGACGAATGCCGCACGA ATTCTTGTCCCTTCCATATACTGAGCCGCCTTCAGGAACCAATTGGTCACGTAGTCAAGAGTCCCACCAC CACGAACATCATCCCAAATCAACTTTTGATCCACCTTTTGTGACACCGACTGAAGTCGGGCTCCAAGATA AGGAGGATTTCCCATCAGTATCACATCATCGGATCTAGAACAAACGTCTTCCCATCGGATTCTCAGCGCA TTATCATGACGGATTATCGGCGCCAGTTCAATCGGAAGCCTATCCGGAGCTTGGCCGAATTCTAGCACCA TCCGCTGATTTGATAGATGATCAACCAGCAGCATTGCAGTTTCCGCAATGCGAGCAGGCCATTCTTCAAT TTCTATACCAAAAAAATGGTCCAGGGTCACTTTAATGTCACTCGTGACGTCAAAACTAAGTTGCGATCTA TCAACCCCTGCAACTTTTATATGAGTTTTATCGAGCAGATCAAGATCGCGGCGGCGTTTTAGCAACTCCA GTTCGAGCGCCCGCAATTCACGATAGGCGACGATCAAGAAATTACCGCATCCGCACGCAGGGTCAAAGAA TCTAAGTCGCCCTAGATCATTATGTAGTTTCGTGAGCTGAGATTTATCGTTCCACGACTTCTCAAGCCGT TCAGTAAATTCGTCAAGAAATAAGGGACGAATTGTTTTGAGAATATTTTCTTCGGTGGTGTAGTGTTCAC CCCCATCTCGGCGGGCTTCCTTACTTTTGACGGTCTGAAACATCGAACCAAACACAGCCGGAGAAATTAT CGACCAGTCAAATTCGCAAGCCTCAATTAATGCATCACGGAACCGTGGAGTGAACTTATTAAGCTTGAGA GGATCGGCATAAATTCCTCCATTCACATAACGAAATATCAAATAGGGGCTACCATCGGGCAAAGCCCTCT TACCCTCTGGCGTATTCATCACTTCAAAGAGGGTAAGTAAATCGGCGTGGAGACTTTCAGCTGTCGTATG CTCGAGTAAATAGTTTTCGAAAACATTCTCCGGCTTCCACATGTCTGCGTCATCACCGAAGAGCAGAAAC AACAACCGCGCCAGAAGGAGAGTCGCGTCGTGATCCTCGATACCAGCTGCTTCAAGCTCACCGTAGAGAG CGCCCATCAAGCGAGCGGCTTGGACCGACGCAGTTTCTGCCTCTCTACCGAACGCGTCACGCATCCGCTC AAGCAATGTGACAAGAGAGATGTCCTCATCAAATCCAAGCTCGATGGCCTCAAGACGGTGCGTCAGGTGT GTACTACGGTCACCCACGACATGTTCATGGGCAAGGAGTTTGGCGTTGACACTGCCTAACTGAGCACGAC GCGGCCAACGCCATTCTTGGCGTTTGTCACCAGTGAAGATGATCAACCGTTCGTGACTGTCCTTACCGAC AAGATCATCAAGGATGCGCTGGACCTTACGGGGCGGCAGGACAGGGCAGTGCCAGATGCGCAAACCTTTG TAACCAGCAACCTGTATAAGGTTGTAGACCTGATCGTTGACATCGAACTTCGTCTCGTTCTGGTCGGGGT TTCCCCAGCCGAGTTCGTCGATGAACAAGGTCCGGAAGTCTCGGGAGTCTACTAGGTTGAGTAGCCGATT TTCGGTCATGCTGTGACTCCCATGCTGCTGACGACCCGGATCGGGTCCTTACCTGTTCGTGTGGCGATGA CCAGCCGCTGATCGCGGTGCAGTGTCCTGATCATGGTGGTGAGGTCGTCGTCGCTGGCGCCGTTGCGGAC GGAACGTTTGAGTCGGTTTTCCGCGTCGCGCTGCAGCGGGTGTTGGAACAGCTCGTCGAGGGCTTTCTCA GTCTCATTGTCGTGCGCATTCAGTGATTCGCCGAGTTTGCGCCACAAAGTGCGTCGGACTCCGCGCAGAC GGCCGGCAGCGACCGTTGGTGTGGCTAGCGGGCCGCGGACCAGTTTTTCGATCAGTTCGTCGTGATCTTC TCGGTGCTCGAGGCCGAGTTCTTCGGGTGTTGCTTCGAAGGCACGCAGGACCTCATGCCCGGTGAGCAGT CGGGTTTCACCGTCGGAGGTGGCGATCCCGAAGCCGTCCATTCCGGACTCGGTACGCACGTAACAGATCA CCCCTTCGTCCTTGTCGCTCAACCGTTTTGCGCGGGTGGCGTCGATCATGTCCGGCAGTGCCGCGATACG CGCGGCGAGATCCGGATCCTTTTCCTGAGCTTGGTGCCAAAGCTGATAGGCCAAACTCGAAGCGTCGACA TCCTCTGCGGAGTCTTGGTCGTCGAGGGTGCCGTTGTACAAGTCCGTGATGGCTTGCACTTCGCCTTCGC TACCGAAGAATTGTTCGTCGGATCCGAATGCTTCGGCGTTGGCGCTGAGTCGATCACTGATGCGTTGGCG TAGTGAGATGACGTTGTCGACGGATTCGTGGAAGAACGAGTACAGCAGGACCTCGTCGGATTGCTGGCCG ACGCGGTCTACACGGCCGGCTCGCTGGATCAGGCGGATGATTGCCCAGGGCAGGTCGTAGTTGACGACGA TGTGCGCGTCCTGCAGGTTCTGGCCTTCGCTGAGGACGTCAGTGGCGATCAGAACGCGTAGTTCGTTGTC GACACCGAAGCTGTCGTGCCCAGGAAGTGCGTTCGAGCGTGGAGAGAAAAGGTGTGCGAGGGCGGTCGGG TCATCGCTGTCTCCAGTCGCCAATCCAACACCGCTGATCCCAGCTTCTCGCAAAGCTTCGGCGATGTAGT TGGCGGTGTCCTTGTATTCGGTGAACACGAGCACTTTGTCGGCGGGGTGATCGGCGGTCAGCAGTTTGGT CAGGGCTGCGAGTTTGGAGTCGGTCGCTACTTCCCAGCCGCCGTACGACTGCAGCATGCCGTGCAATGCG GTGGTGTCGCAGTCGAGGTCCTTCCGCAACTCAGTGGTGAACAGCTCCGGCCGAATCCACGTCACGGCAG CAGAATTGGAGGATACGAGCGCTTCGTAGCGGCGGCCGGGATCGTCGGTAAGCCGTTCGGCAGTGTCGAT GTCGGAGGCAGTGGGGTCGTCGTCGTCAGTGAGGCTGGTATCGACGATCGTGCCCGTCGGGATACGGAGT CCGTTGTCGATCGCGTAGCCGAACAATTCGTTGCGGGCGATATGCCGTTGCAGGGACAGTGTGAACGAGT GCCCGCAGGAGGACAGTCTCTTGTAGAACGTGGTGCGGACGAAGCCGGCGACATTGCCGCGGCCACGGTT GAGGTTGTCGACGAACTCCTTCTCCTCATCCGTCAGCTTCGCCGTCTTCTTCAGGTATTTTCCGAGCTCG TACCGGGGCAGGACCAGGTTGGTAAGGGTGTCGAGGGTGGTATCGCTCGACATGATCGCCGCCGGGTCGT CCGGTCCGAAGGAGTGATCGACGGGCTTGGGGATGCGCTCCGGAAATGTGAACCGTGTCCCGTTGGAAAA TTCGAGGTATTGGCGACCGTCCTCATTGGTTTCGGAGTAGTTGTTCTTGATGAAGGTTCGGGTGCGGCGC ACCAGATGCTCACTCATCAAGCGTTTCCAGTCGTCCGGGTCCTCCGATTTACGGAAGGCCGCCATTGTGG TGATCTTGCCGTCGACTTTGTCGATGAGTCTGGGGTCGGCGGCCATGGCGTTGGTCGGGGAGATACCGAG 
Final Technical Progress Report DOE Award No. DE-FC26-02NT15340

GTCATCGTCATCGTCGATGTAGAGCGAGAGCTGGTTGGCGACGTCCCGGAATCGGATGTTGTACGGGGTC GCGGTCAGCAGAAGCGTTTTGCTGTCATTGGCGTGAATGTATTCCTGGATCGCCTTGTAGTCGCGGCGTT CGTCATTGCGCAGAGTGTGAGACTCGTCGACGATCACGAACGCGTACCTACGCAGATCCGGAAGAATCGT ATGGGCCATGCTGTACGGCACGACGCGCCCGTACAGGTTGTATGCCTCGAAATGCTCCTCCCACATCTTG GCCAGGTTCTTCGGACACACCACCAACGGCATGTAACCGTGTTCGTCCCTCAGCATCAGGGCCACAGCGA TGGCGGTCAGGGTTTTCCCCAAACCGACGACGTCGCCGAGCATGGTGCCCTGTTTGGTCATGATCCGCCG CGCCAGCGTCCGGACGGCAGTGGATTGGTATTCGAGGAGTTGAGAGCTGACCTCGGAGGGGACGGAGTAC TCGGCCAGTCCTTCGCGTACGTCGCGGGAGAGGTCGTAGCAGACCTTGAGGAACACCTCGTACGGGCGGC GGGGTTCTTTGCGGGCCCATGATTCGTCCAACATCGTCAATAGATCCGCGGTGACTTCGCGACTGAACTT GTCGGTCCATCGGTCCTCGAACCACTCCGCCAACGCCTCCGCGGCATTCGTCTCGAGTACATCGACGTTG AGTTCATAGTTATGAGTCAAACCGGGCGCGGTGAGGTTCGAGGATCCGACGAATCCGGTGATCGGATTGT TCAGGTCTTCCCGGTGGAAAATGTATGCCTTGCCGTGCAGTGGCCGGCGGGTGAAGACTCTGATCTCGAC AGCTTTGGATTCGAGGAGATCCCGAAGTGACTGCAGCGCCGCACGATCCGCAGTGGTGGGCAGTCCTCGC ATGAGTTGCGTACGAAGTTGTTCGAGGAGTTGAGCTTTCCGCTCACGACCGACATTGAAGTCTGCATCGA GCACTTCGGTGCCTTCGACCGCAGCTTGCAGTTCCTCGAGGGCTTCTTCCTGCGGTCCGGTCATGACCAT GCCGATCAGGATTCGCACGACCGGAACGGGCCTTCCCTGCTCCGCTTTCTCCTTGACCAGAGAGTCGAAC ACGGACCACCCTCGCAGGTTGAAATACCCGACGGCGACATCCATCCGCTCCGATACCTGCAATGTCTGCT GCAGGTGCGAGCCCAGTTTCTGATGGATGTTGTCGAAAATCCGCGTCACGGGAAATGTCGCTCCGTTCGA GTGCCAGCCTAGGAAAATCCTAGGAGATACACCCGAACTCGGTGTCCGGGTGGGATGAGTCTTTCAAATG ATCACAGGAGGGCATCGAGACACCCGGCGATTCCATCAAACCCGCCTTGTCGGACCTGACTGGTTAAGTG AGGCTCCGGTATCTCGATTCGCGAAAACCGGCAAATCGCCACAGTTTTACCTCGCCAACCGAATTCAACG AGAAGAGAACATCGTGGGCAAGACGAATCGGCGCCAAAAGCAAAAGCGGGACAGTCAATCACGACCACAG AATGCATCGCACAACTTAGAGACACCTGAAGCTGTGATGATGCGCAGTATCTTGTCCCGCACCCTGATCC CCTCTCGTAACCGGGTCGAATCGCGGGCGAACGTGGCCCGCCGCTTCGAGGGTTGGCGAGTCAATCCCAG CCACCCCGAGCCAGGTAGCCCGATCGACTTGGACGACGCAGGGTTCTCCGCGACCATCACCAAGCACGTC ACCGATCATCCAGTTCTGCACCATGCTCTATTTCCGGCCATGAGTGCCGCTGAGAATCTGACCACTGTCG TAAACCTGGTCGATCAATGGGACTCAACGGGCAAATTGCGCACAGTTTCGATCGTCACCCTCTGTCGATC AGCATTGGAGTCGGCATCACGGGCAGTGTGGATCTTGTCCGATCCCAGCCGCGACGAACGCCGTAGTCGG GCACTACGAGTCACCAAAGACGAAGTGCTCGCTCAAAAAAAGTATCTTGATGAACAGATCAAGCGAATCG ACGAGGGCTCGATTTCTGCCACGGATAGCAAACGTGCTGAGCTGGCAGAGAGCCTGCAGGCGGCTAAGGA CGTTCTCGACGAACTCAGCTCGGTGAAGGGCGCACTCAAATTCGACCAGACCATCGAGAAAGCTGCGGAG TGGATCGACGAAAATATCCCCAATGGCTCGGTCAAGCCGATGGCCGACTTATCGAAGTCGATGTACGCAA TCGCGTCCGGGATCGCACACGGCTACACGTGGACAACCAGCTATCTGCACGGGGCTACTGACCTGTCCAA TATCGTGGCCGATTTCTTGTATGCGGCTACTACAACTACGGAAGCAGCTGTCGCATTGTACGAGGCACAA GCTTCAGCAACAGGGGCAATCGCAGACAACTGTCCATCTCATCTTCGCGACGTAGCTGAACGATTCCATC CTTGCTACACGCCCACTGCATCGACCGATTCGGGCGAACGGGCGCCGACCGCGGACTGCTCGTCAACACA ATGATCGCCCGTCAGCACTGGCGATGAAGAGCGATTGCTCAGAGATCCCGATGGCGGCGTTCTCCACGAA GTTCATGACGCGGTCGTAGAAATCTATCCGCCCCATCGGGTCCTGAAAAGCGTTTCCGCTGGGCCACGAC CATAGCGGTCGCCAGAGCCGCTCTGCGAGACAATTCGGGTGTCCGTCCTGGCGAGGAGTTTGACGAAGAG TCCGTCGTGGGCGGCGGCCGAGAAGACTTGCCCTGACCGTTCTGTGAGCGGTCCTTGGACGACGCCTCTG TTGTGCTTCGACCTGGAGAAAGAGAGTCTTTCACTCGTCGGGGTAGTCCTTCGGTTCGTCGGACGTGCTG CAGGAGCCCAAGTCCCTGTAGTAGACAAACGTGAAGCTCGATCACCGTCGCGGGTACGTGAGCAAGGCTA TTCCGTTGACTGCTTCCGGAGATGGATAGATCTTGAGACTGCGCTTCTGCGGGCGACACCTAGAGCACGT CGTCAGTGATCATCTAGTTGCCTTTGATTCGTGGGTTGCTTCGGGCCCCGAGGTAGTGCGCCCAGTCGCG GCGAGCGTAGTCGGCCCACCGACTGACGGAATGGATATGCAACCCGAGCAGGTCGGCGACGACCGGTGCT GGTAGATCTTCGGTGAGAATACGGAGAGCGGAGTTGCGACTGATCCTGACCGGGATATCGAGTGCGGTCA TCGCGACCCCCACCGGGCGGGCATGACGAGCCGTCCCGGGCACGGTGCGGCTAGGAAACAAGAACGGCGA ACCTGTCTTTGTAGCCAGAGGATGGGCGATGGCGGTTTCTACCAATTCATTGACCAATGTTGCGACCGTC GGTGGCAGCCTGACCGGGTTGCGAGCAAAGACGATGCTGGTTTCACCATCTTGTTGCTCGACGGCACTGA TGCTGAGTGCGAGGATTCGGGTGAGAGGGATCCCGTACAGCAGCACCAGAAGTCCGGCGACACGCAGCGG CAGTTCGATGGTGGCGTCGGTGAGAAGTCGTCGGATTCTTCGTGCGTGTTCGTGTGCTTCCGCGACGTCG GTGGGGAATGCCGATTGCCTCCTCGACACGGCGGCGTCACCGATGATGTCTCGTTGTCGTAGCCAGAGGA CGAAACCGGTGATGCTGTAGGTTCGGTGCCCGTTGATGGAGGTCCAGCGGTCGAGTTGGTGCTGGTTCAG ATCGGTTATCGCGAGGTCGTTCTCATCGAGCCATTCCAGTAAGTGAACTGCTCCCCAGATGATGGCTCGG TCACTTTGCGCGCTGGAGTCGGTGTAGCTGCGGCGGACGGCGCGGCGGCGAGCACGGCGCAGCACACTCC ATTGTGCGTACGGCCGGATCAGCGCCGCGTGTTCTGCGGTCAGCGTTCCCGTCAGATGGTGGAGCCATTG TTCGAGTCGGCCCAATGGCTCGATTCGAGGCGGGAGGACATCAGCGTGGACGAGGACCGCACGAAGGTAG TGCAACGCAGTGGACGGGGGGAGGGCGTCGAGGTCGGCATGCACGATCGGGTGCTCTACTCGTGCGATGT GTTGCAGCAGTGCAATGGTTTTCGGGTGGTCGAGCCACCGCAGAGTCGAGTCGGGACGTCGCTCCTCGGC GGCGAGGTACACGATGAACGGCAGTATCGCACGGTCGGTTCGGTGTTTACTGGGTCGGCCTCGCGTTGCG CAGTTGTCCCGCAACTGCATCCTCGATGCGGGTTTCGAAGTCGACGTCTTCTCGAAGGATCGCTGAGGCG TGGGTGGCTCCGATGGTGCCGGCGCAGAGGTTCCATGCGAGTCGGTGCGCGGTGGGGGTGTCGGGGATTT CGCCTGTGGTGATGGCGGTGTCGACGATGTCGCTAACGGAATCGACCCAGCGTGCGAAGGCTGCATCGTT GTCGACGGCTGGTTCGAGGATCAGTTTCATCCCGGCGCGCAGATTGGTGTCATCAGCGATCTTGTGTGCC 
Final Technical Progress Report DOE Award No. DE-FC26-02NT15340

AGGGAAGTGAAGATCGCAGTCAACCGATCGAACGTGGTCTCTCCAGCGGTGCGTTCCGATGCGTCGCGGA TGGACTCGTCGACCGCGAGATTCCAGTCTGCGATGAGCTGTTGCGCAATCGCCTCCTTGGATGGGAAGTG GTAGTAGACCGCACCTCTCGCGAAGTGGCCAGCGTCGAGGATTGTGTTGATGGTGGCGTTGCCGTACCCG TCGATGTCGAACTGTTGGGCAGCGACGGCGATGATCGCTTCTCGCGTTCGGATCGCGCGATCTTGCATTG CGGCGTTCTTGCTGCCGTGGTTTCGGCCGATGTGGGGGCGAATAGGATTGCTCATGGCTACAGGTCCTGT CTGTGGCTCGGTCCTGGCGGTGTTGCGAGCACTGCCAGGACCTCTGTTTGTACTGTTGGTTCGGCTGAGG CGCGAGGCCTCTCCATATGCACTGTCGAACGATCATGGCGGTCATCGGTTTTCAGCGTGGCCGGCATGAC TGTGCAAGATCGCAAGGGTTCAGCGTCGAGCCTGTGACTCGGTCAGGGCTGTCGGTGGTTCTTGAAAGTC GGAGAACAACTTTCGGTGGATCTTTTCGGTGTACGAGTCGGGCCGGGGCGTGTGATCCGGTGCGGAAGTG TTCACAGCGTCGCTCCTTCGATCATGTGGACGGACGCTGGGTCGGTTCCGCGCCACATCATCAGCAGATC ATCGACACGCCTGGCGATGGTGTCGTTCTCGCTTCGACTCGCCGAAACTTTCACCGCGCCGACGAATCCG GCGCAGAGAGTGTCTGCGAGCCTTGACATGCTTTCGGCGCAGTCGAGCGCGCCGGAGCGGATCGCGTCTA TGACGATCGGCGTGACGGCTTCGGTCCATGTCTCGTAGGTGGCACGGGCATCACTGAGCGATTTGTCGAC GGACAGGATGAGTCCGGCTCGGGTGATCGCTTCTGTTTGGGTGCGGCGGGCGAGTTCGCGGTAGATCATC AGCACTTGCCGGTCGGCGGGTTGACCTGTCGCTGCGGCTTTCTCGACTGTTTCGGTGACGGCGTCGGACC AGCGGCGGAGCATTTCGCTCGCGATGGATTCTTTCTTCGAGAAGTGGAAGTACAGCGCGCCTTTGGTGGT CTCGGAGATCGCCAGGATGTCGTTGATGCTGGTGCCTGCGTATCCAGTTTGGCTGAACAGGGTGGCGGAC ACCTCGAGGATCGCTTCTCGGGTGATTTTCGCTCGTGGCTGCATAGGTGGTCGGCCGGTTTTGCCGTGGT CGATGCCTTCGGATCGGTCGGTATGATTGTTCATGGTCACAGGTGCTTTCTGTGGTCATCGGCCCTGACG GTGTTCGAGCACTGTCGGGGCCACCCTTTTGTTGGTGGGTGTGGGTCAGACGGTGGTGAGTTCGTGGTCG GCGGGGCTCTCGGCGGACCGGAGGTCGGGCAGTGGCGGGCTCGTGAATCCCGCTTCTCGTGCTGCGTTAT CGAACTTCACGTACCGGCGCCATGCGGTCGATATTGCGACGGGGGGCTTCGACTCCAAAGCGATGGTTTC CCATGTGAGGTTCTGGTTCGTCAGCTTGCGGACCGCTGTGTCTTTCACGTTCTTCGCCAGATCCGAGCTG GCAATGAACGCCATGAGGTCGTCTATGCCGGGGTCGATATCGGCATGGGCTTCTTCGGCGTCTTGGCGGT GAGGAAACTCCGCAATGGTGTCTTGGGTGCCAATGTCGTTGAGGCCCTCATCCATCATTTCGTCGACGAA AAACGGCTCCGCAAAGTCCGCAATAAGGTCGCCCTGGTCGGTCTGCGTGTCCGCCGTTGTGGCGTCCTCG TTTCGTTGCGCTTCCGCCGGCTCCGCAATATTGTCGGAGATCTTTTCGCCGGAGCTAGGGTCGCGGGTCG GTTCGTGACCGGCGGCGAGATCAGACGCAATACTCGTTGCAACCTCACTGGCGGTGGCATTTTCGACGAT CGCACGGTAGGCGGCGTAGGCCATTCCGACTGCCTTCACCAGGATTGTGATTCCGTGGGTGAATGCCAGG ACCAGGGCCGGTGGAATGATCGCTATGGCGGCTGCAATGGCGGGCGCGAGTGGAGTGAACCCGAGGTCTC CCCCACCGTCGATCGACTCCTGCGCGGCGATTGCTGCGTGATAGGCGTGGTAGGCGTTTCCGAGGATGCT GATCACGACGACGCTGACGGCGAGTGCGATGTAGAAGCCTCTGTCGCGTTTGTTCATGTTGAGCTTGCTG ATGATGACGATGGCGATGGTGGCGCCGAGGATGGCGCTGTCGACGATTGCGGGGAAGATCCAGGTCAGGT GTTCGGGGATCAGTGCTTGCCGTGCGAGGTCGCGTTGTACGGCGAAGGAAAGTACGAATGCACCGGTGGT GATACCGAGGGCAATCACGATCGCGAGTGCGAGTGCGGCGAGCAATGCGCCTAGCTGAATACGGGCAGCT CTGAGGCTAAAGTCGGTCATGACTGGGTTCTTTCCTTCAAGTGGTTTCCCGGTCTGGAGCCTCGCCGGGT GCATCCGGCGGGGCTCCTTTTGCGTTTTCAGATCAATCAGCGCGCTCGCTATCGGGTACTGCGGCCGTGC TTTTTGTAGTGCTCGGGGTAGGTCGACTGAATTGCCTGCTCGATGATCGAACGGATGCTGTGTCCGGTTT CGTCTTCGACGGCGTCGATAGTTTCCCGGAAGCTCAAGCCGAGTCGGGTTGAGAACTGGATGACGGGTTC GGCTTTTTCCTTCTTCGGCCGCCCTACCGGCCTGCGTGCGGCTGGAACCTCTGCCGAATCGACAGCCTCG TCGACGGCCGGACGCGGCCCTCTCCGTGGGGCGCTGAGGTCAGGTTCTTTGCTCATTAGTCCATTCCTTC GTTCGGTAACTCATGAGTTCATGCATTGCTGCATTCGTTACGTAACTCACGCATGAGTTAGTGCATTCAC TCATTATTGCACGCGTGCATGAATTAATTAAGTCGACTCACGCACTCATGCGTTAATGAATGCGGAAAAT ATTTGCTGGTAAGCGGCAAAAACGGGCTCACCCTTGTCGAATTTTCCGTACCACTCGCCGGTCATGCGCG ACTGGTCGACGATGGTGCGATTCGGAATGATTGGCGTCAGAACGAGGCCCGTTTCCCTTAGTTGCTCCAG CGCTTTCACTGAAACTTTCGTCATCACTGTCTCCTTGACGGCGCCGACGACGATGCCTGCCTCACTGAGA CGGCAGGGGGCACCGATCGCTTCGCGACTTTTGCGGAACTTTGCGACGGACTCTTGCGCACCTTCGACGC CGTCGATCCCGTCTTGATTCGCGGTTGCGGCGTAGATGACCTTGTCGCTGGCGGCGAGCGCATTTTGCGT GAGCGTTCCACCTTGGCGGTTAGGGCAGTCGATGATGCGAATTTCCGCAGTGTGCCCTTCCAGCGCCGTT GCGAGCCTCAGTTCGGCGTGATCCTCGTAGTCCCTCTCACGGTTCGAGAGCGACCTCGAGGACGGAATGA TGCGCAGATTGTCGTCCCACCCGCTGTCAATGGCGAGATCTTCGGCCCATCCTTCCGGGTTTTCGTCGGC CAAGATCGCGCCCACGTGCTGCCACGATTCCTCCGGGTAGCTGCCGATCCATTTGGTGGCTGCACCTCGG GGATCTAGGTCGATGAGGGTGGTCTTGAACCCCGACAGGGCAGCCACCATTGCAAGGGAAACTGCGGTCG TCGTCTTGGTGACTCCACCAGCCTCGCTGTACACCATGATTGTCGTCATGCATGAATGCTAGCACTCATG CGTTAACTCTGTCTGTAGTTAATGCGTTCATGCATGCATTAATTAACGCCGCCTTGTCGATTGCTTTTAG TCACGGCATCACCGCTGGTGAGGAAGTTTTGCGGTCCGCTGCACTTCCGCTCCCACACCACTGTCAATTC GAGGCAAAGACGCAAAATGCGCAAAATGTGGAGCGTTCCTGGCGTTGCCTGACTCTTCCCACTGTCGCAA AGGTCTTATATCCGGGGGCGAATATTTCGCCGTGCTGGGCCGAAGACTGTCACTCCGCGTTCGACGTCGG CCGGCGTGGAGCTGTGGTGAACGAGAGAAACCCGGTCTGTTGCGTGCACTGCGGCTTGGTCGTAGGAGTG CACGGTCGCGTTGTCGTAGGCATAGGTGGTGGTGTCTTCTCCGACGTCGCCGAAGGAGTCGTCGACGAGG TTGCTTGTGGCTCGGTTGGTGGCGCGGACGCGTGTGGTGTCGAATGCGTCGGTATGGGCGTTCCCGTAGG CGAAGAGAGTTGTGTGCCCGAAGATTTGGGATCGTGCGGCGTCTGTGGCCACGATCTGGGCACTGCGCTT AGTTTCGGGTGTCAGTCGGGTGATGATGACCCGTGAGACGCCGACGATGCGGATCGTGATGCCGTCGGGC AGCTCGGCGTCGGTGAGTTCTAGAGGTTCGTCGTTCCGGTGGTCGATGACAATCGTCTTTGCGCCGGTGC 
Final Technical Progress Report DOE Award No. DE-FC26-02NT15340

GCAACCGTTCGTGGATCTGGGTGACGGTCTGGCATGGGGAGAGCGGCCGGCGCTTGACGGGCGGCCGCTT CGGGGACTGGCGTTTTCCGTTCATCGCTTACCCCGATCTGAGAGATGGGTGATCCACACGGGCGAGGTTT CATTCTCGCGCGCGGATGCCTGGGTCTTTCGGATGCGGGCGCTGTCGGCGGCTCGGCGGGCGACGAAACG GGCAGCTTGGCGGCCGGGGGAGTCCTCGGATGCGGCCGATGCGTAGCGGTTGCGTTCGGCCTCGAATGCT GCTGCCTGTCGTTCGCGCTCGGCTTTTTCCTGGTCTGCGGCTATCTGAGCGAGAACGTGTGGTGCGAAGG CCAAGTCCTGCTGCTTCATGAGCCAACGGATGAACGCGATGGGGTGCTTCGGGGTCGGTGCCATCCGCGT GGCCTTGCTCCAGGCGTCGATCGCGTCGTTCAGATCGGCGGCCGTCCAACCGTGTGCGGCTGGGTCGGTC AGTGCGGGTGCCCACCCTCGGGGGGTGTGCCGCCGCGCCCAAACGGGAGTTCGTGGATTGCTGAGCCATT TGGATGCGAGCAACAAGCCTTTCCGGACGACTCCGGCCTTCTCGACATCGGCCTTTGACTCCTTGCGCCG CGAAGCGGCTCGTTTATCCACAGATCGTTTTGTATTAAGTACCTCTCTACGAGAGGTAAAGCTCTTAAAG TGACCGCTATGCGGGTGGGGTGCCATCTGTATGGAACCGGCGACGACGATGCGGGTTTTATCCACAGGCT TGCTCGGGTGCAGTGCCCAGACAGACGCCCAGCCGCGGGCCTTGTCGTTGAACCTCCATGAGGCATACCG CTCGTCCTTGGTGCGGAGTCGCCCGCGGAGAACCTCGGTGGCCACGCGCAGGAGCTTGAGGGCTTGTCGT GCTCGCTGAACAGTCCGCTCTTTCTTGCCGGTGCGTTCGACGATGGTTTCCACGGTCAAGCGTGAGTTGC GGCCGGTGTCGAAGTCCGCGGCTTCGGCCATGACGGTAGCGACTGCCAGCAAGGACTTGAGGCTGATGCC GCCGCCAGACGACCGAACCAGCGCCGGTCGAATCCGTGTGTATTCGGCGGTGAACGCACACCGAACGACG ATCTCGACCCAGTGCTGGGCGCCTCGCCATCGAGCGATGCCGCCGTATGCGCCGTCTTCGAGGGCGAGTG CGACCTTGCAATAGCGCCGGCACGCGGCTTCCTTGCCGAGCGTTTTGAGCAGCATCGGGGTCAGGGCATG GCACTGGTGCCCGTACAGGTGCCCCTGCATCCTGCGGGGTGTACGAGCGGTTCCGTAGCGGCGCCGCGTA GGGCTGCTACCGCAGTACGGGCGCTGTATGGGTGCGGGCGTGTGCTCCATCGCTGAGACACGCGAAAAAT CGGTTATGGATTTTGCATCAGGACGCGATGTACCCTGAGACCTGTCGCGCAAGACAGTCCCTTCGGGGAA AGCAGCAAGACCCTAAGATCCGAGCGTCAACTCGTTTCTTACGACATCCGAATCCCCCGGCCCGTCAACC GGGGGATATCGTCGTTTTAAGGGCGATCAACTGCGCATATGAGACCGAAGCGGGGTTTCCGCATCTTCGG CGTTGTGGTCACCATTAGGCGGTCATCGTCAGCCTCTCTGTGTGGACTGCGGCCGGGTCGAATCGGGTTA CCGCAGCGCTGAGGATGTCGGCGACGAGTTCGCTCTCAGTCAGTCCCCGAGCTTCGCGCATCAGGGTGAG GCGGTCTTTGACATCCACGGGCACTCGCGTCTCGACACGACGCCGCTCGCCTTTACTCGGACGACCTCTC GCCATGACAAGCCCCTTCCAGTTCGTCCGCGCGGTGGCGCGGGCATTGTGTTGCCTGCAAATACTCCCGC AGAAATGTCGAGATACGCAGCCGACACGCCGGTTCAATTCGTTCGCTCTATATGGGTCAGTGTAAATCGG CTAGAAAAAGCCTGACTGCGACGGACTCCATCGGTTCCGTGAGCGGATTGCTGTAGGTCGTTGCTCCTGG AAGCTGCGCGGCACGGGCACGCGCTGCATCAGCGAGCGCATCGAAAAGGCCACTGGACCGTTGAATAGGG GATTTACGTGTCCAGGCCAGGTGCTGCGCAGTTTCCGGAAGCAGGCGCTCACATGCCAGCCGGACTGCGA GTTTGTCGTGCGCTGCGCCAGTGACGTAGCCGAAGAGAGCGGGTGGCGCTAGTTGTTCAGCGAGTTGCCA GAACCGTTCTGTCTGGAATACATGGATGAGTGCTTGGGCGTCGTCGCCTAGCAGTCGTTCGTAGAAATCC GGGACCAATCGATCGCGAACGAAGTTCGTTCCCGTCTCGGTGCGAACTACTTCGTCTAAAGCCTGGTTCG CTGCGGCGGATTCGATCGGGTGCTCGATCACCCGGCCTCCTGCAAAAATTGCATCTGCACCGCTGCCGGT GAGAACAGCTGCCGGCGCCGGGGACGCTGCGCTGAATCGGCGGATCAATTCTGAGGCAGCGAGGATCGGG ATAGCGGCTGCGACCTCCCACAGTTCGTTGACTCCCAGAGCGACGACTGCCGATTTAGCGAGGCTGACGA CGTCTGATGAGCTCAGGACAACAACTTCATGCCGCAGTCCGAGTGCTTCGGCAGCGGCTATCGCGGGTGC TCGATCGACGGTTTCCCCGTCGACGACGATCGTGATCGCTCGCGGTGTGGTGCCGAGGGCGACTGCGGTC GCTGCGACGAGGATCGAATCAACGCCACCGGAAAGCAACACGATCGGATCTGCGGGGTAGCTGTCGAGCA CCACTCGTATTCGATCGAGGGTTTCGGCGGCTATTGCCTTCGCCGCGATGTCAGGGTCTGTGGTGGTGCG GAGGCTGGAACCATCGACGTCGAGTGCGTGATCGTCTGCGACGAACTGCATTGGCTTTGCGGTCTGGAGT GCAGTCGGTGAGCGCACGGTGCCCTTTCCAAGGATGAGTTAGCAGGTGGTTCGGTAGGGGAGTTCAGGGA ATAGTTGGTCCCACGTCGCGTCGGTAAGAACATTGTTCGTGTTCTTGCACACTCGGGTTTCCGCCGCGCG CTGGGTGAGATTCCATAGCCGGACAGCGCCGCCCTCTCCACCTGCCGCGAGGTGATTGGAAGTGGGGCTG AATGCGATGTACCAGCGGGTGGCTCCGATGGATGCGATGGAACCGCCGAGTTTGATGGGAGCTGCGATGT TTGTGGTGTCCCAGAGCTGGACGGTTCCCTCGTCGCTGCCCGAAGCCAGAGTCCGCCCGTCGGTTGCGAA AGCGACGGACCGGATCGTGCTTGATTGGCCGATGAGTGGGTCACCGATCCCTTGCGCCTCGCTTGGCTTC GTTACGTCCCAGAACCGAATCGTCTGATCGTCACCAGCGCTGGCCAGGGTTTTCCCGTCCGGCGAGAAGA CAACGGATTCGGCCTGCTTCGTGTGCCCGCTCAGTGGTTGAAGCATCTTGGGACGGCTCGGATCTGAGCT GTCGAGGAGCGTGATGTCGTTGGTACCTGACGCCGCAATCGCGAGCGTTCGGCCGTCCGGTGAGAAGGCC GCGTTGGTGAGCAGGTCGCCGGGGACCGGCAGCGGCTGCCCCAATGGGGCGGGCGCAGAGATGTCGGTGA TGTCCCAGAACTGGAGCGAGTTGTCGCTGCTACCGGTGACTAGGGTGCGGCCGTCTGGAGTGATCGACAT CAGATACGCGTATCTGGTATCGAGTTCGAGGCGGCCGAGAGGACGGATGGTGTCGGGGTCTGAGGCATCC CAGAGCCGTACTGCCCGATCGTTTCCGCCCGTTGTCAGCACGGTTCGGCCATCCGGTGACATTTGCAGGT TGTCGATTTGGCCGTTGGGCACTACGACTCGTCCCCGTGCGATCGGGTGATCGGGATCAGAGGTGTCCCA CACTTGAAGGGTGCCGTCGACAGAGCCCGTGGCCATGTGAGATCCGTCCGAGCTGAATCTGGGGGTCATG ACACGGTCGGTGTGGGCGTCGATGACGGATGTAGGGCGGTTCCACATCCTGAGGACGCCGTCCTGGCTTC CTGTTGCCATAGTTGCGCCGTCGGGAGCGAAGGCGACTGTGATCATGCCCCCGTTACTGCCGGCAAGGGG CTGTCCTAGGGAGCGCGGGCGGGAAGGGTCTCGGAGGTTCCACAGTCGGGCGGTGCCGTCCCAGCTTGCG GTTGCGAGCGTTTGACTGTCCGGTCCGAACGCGATGGACCACAGCGCACCGGTGTGACCAGTGAGAGGCG GCCCTTCCAAGGTCGGCTGCGCGGGGTTTCCGATGTTCCAGACTCGGGCGGTTCGATCGTCGCTGCCGGC GGCGAGAAGTTGGCCGTTTGGACTGAACGCCACCGAGTGCGCCGCTCTGGCAAACCCGGTGAGCGGTGCT GTCGCGATTACGGGGTTTGACGGATCGCTGACATTCCACAGCCACACCGTTTTGTCGTCACTCCCGACCG 
Final Technical Progress Report DOE Award No. DE-FC26-02NT15340

CGAGTAGTTTCCCGTCCGGACTGAACGCGCTTGTTCTGACGGGACCTGCTGGCCCGGAAAGTGGGGGAGT GAGGAGAAACGGGGCTTCCGGCTTGCTTATGTCCCACAGCCCTGTGGTTCCGTCGTCGTTCGGTGCTGCG AGGGTGCGTCCGTCTGGGCTGAACGACACCACGTACACGGTTCCGCCGGCGGATTGCAGGGGGCTGCCGA TTGCCTCGGGCGAGAGCGGATCGCGTACGTCCCAGAGTCGCACTGTGCCATCACCGCTCGCAGTGGCAAG CATCGCACCGTCAGGGGACCAGGCGGCCGCAGTGACGAAGCTCGTGTGACCTTCCAGCGGCGATCCGATC GGTGTCGGGACTTGTGGATCGCTGACATCCCATAGTCGCGTTGTGTGGTCTCCACTCGCCGTCGCGAGAA CTTTGCCGTCAGGTCGGTATGCCGCGGTGTAGATCGCATTTTCATGCCCGGTGGCCGGCGTCGCCATCGG TGCATCTTGAGTAGCGAGAACTCGGGACGCGGTGGCGGTCTCGGCAGGCTGCATGTCGCGGGCTGCAAGA ACTAGCTGCGCCGACAGAGACGGATTGGACTGTTGTAGTCGGTCCGCTGTCGATAACACTTCTCGGAATT GTGCACTCGATGCAAGGACCTCTGAGCGCGCACTCGCTCGGAACGCAAAGACGGACACGACCATCAAGAC CGCGATAAAGGCTGCGATCGCGGACACGGCGCCCCGTTTTTGGAGCTTCCGTCGCTCTTGCTTCCTTATT GCATCCCGGCGAAAGCTGTACGCCGTCGGTGTCAGTGGGTGATCGTCGAGTTGCGCGATGACATCGTGCA ACCTGTCGCCTGCCCACAGGTCGGCGGACTGTCGACCTCGCCGGTCCCATTCGGCCGCGTCGCGTTCAAA CGTCTGCCGAAGCATGTTGCTCGCCCTGTTGGCGCTGATCATGTTGTGCAACCGGTTCCAGCTGTCGATG ACCACTTCATGGGCAAAGGCCACGAGTCCGTCGTCGTCGACGGTCAGTAGTCGAGCGGTGGTGAGTGCTT CGATGACCCGGCCGTTCCGGGCATCCGCGCCGCCCACGCGTAGCAGCTCGGCGCGGGGTACAGCCCTCCT GGTGTCGCGGGCACCGTCTCCGATCTGAACCAACCTCAACAGCAGGGCCGGCGCCACTGCACGATCGTCG GCGTCGAGCACCTTCCAGGCGTTTTCGGCGGCGCGGTTGACGGAACCTTCGATTCCTCCGGCAGCTTCAT ACGCTCGAAGTGTGAGGTTTCCGGCTGGGTCACGGTGCATCCACATGCTTTCAAGCACGTGGGACATCAG GGGGAGAACGCTCTGATTGTGCCCTACTCGTGTGGCGTCGAGATCACTGATGATTCGTTCGGACAAGCCT TGTTCGAGGTCAGCGTTCGCGGCGGCTGCCGGCAGTTTGATGGCTTCGGCCAGCTCTTCGGAGCTCATAG GCGCGAGCACGATCTGCCTGTGCTGTAGAGCGTCGGCAAGGGGAGCGTGTTGCAAGCATTCGGGCACGAA GTCCGCTCGTACGCCGATTACAACTCGTATGGTGCTCGTTGAGCTGACCACGCGAGAGAGACGGTCGAGT CGATCTATGAACTCGTCGCGGGTCGCGCCATTCGCACCCGCTGTGAGTACCTGTTCAAACTGATCGACGA TGAGTAGTAGGGCATCGTTCTCGGAATCAGCGAAGTAGTCATCGAGCAACTCCCGGTAGTCGTCGCTCGA CTCGGCTTCTCGTATGCGCGGGAAAATGCGGGTAAGCGCGCCGAAGGGGTCCGGGCCGGGGTCTACGGTC TCAATGCTCCACGGTTCCGTAGATCCCCACGTCAACCCGTGAGTAGCTGCACGCCCCGCGATACCGGCGT GAAGAAGCGAAGATTTACCAGTCCCGGAGGCTGCATATAGGACGAACAACCCGGGGGTAGTGGCGCCTTG GAGGGTGGAGACAGCTTCGTCGATGCTGCGTTCGCGTCCGAAGAATCGGTCACGATCTGTGACTGTGTAC GAGGCTAGGCCGAGATATGGGCAGGCAATTGGCGCTGTTTTCGACGCGTCCGGGCCTTGCCTGCTACCGG TCTGCGCCTCTGCCCACCATTCCCTCCACACCGCCATGTCATACAGACCATGCTCGGAAGGCTGTATCGA TTTCGCCCTCGCCGCCGATATAAGTACCCAGAGGACAGGCTCGAGCGATTCGAACTTTTCAGGGACGCGG GCTCCGCGCCGCCAATCGCTGATGCGCTGGTAGCTAGGACCCTCGGAGCTACGTTCTTTAGCCCTCGCTG CTACTGCCTTGAGCGTCGGATTATTCGCGGCCGCCCAGAGTGCACTCAGTCTTCCTGCGAACTGGTTCCG GTCCGTCACTTGAATCCTCGCGCCACTGTTTTCCTGCCGTTTCCCCAATTCCGCACCCAACAAAGTTCCG CGTGCGGAATATTTGTCCGAAGTGCCCGGACCTGCAAACTTAGCGTGTTGCTTGGTGCGGAATCGAACCG TGGTGTGGAAAATCGGGAAAAGTTCGCCGCATGACGGTAGAAGACGCGCCTATCGCGGCGTGCGATTCGT GCGGTGCATCGAGAATGCGATCCGCGCACTCTTCACACAGGGGTGTGCGGAGGTCTGCTTCTCGCGCGGT TGAATGCGGAACGAATATCGCTCAAGGAGTAGTCGAGCTCAGCGGCGAGCATCTCCGCGACATCCTCGCT GAGGTTGATCTCACCGCGCTCGAGGGCACCCATCATGGTTTGGGAAAGACCGATCCGCTCGGCAAGTTGA GACTGTGTGATCAGCCGCAGGGTGCGAAGGTCGCCAAGGAAGGTCTCTCCTTTGGGTACGGAAACCAAAG CGGAAATATCGATATCGAGCACGCTAGGGTCGACCAATTTAACGAGTGTCTCGATCTTGGGCGTGCCTCT ACCCGCTTCCCATGCTTTGACTGCGCCGGGCGTCAAACCAAGAATGCGCGCAAGTTCGGCTTGAGTGAAT CCTCGCTCCAACCGTCGATCACGGAGCACGTTGGGTTGAAAGCCGCGAACGGATCTCCTGCCCACTCATC CCCCGTCCACTCGTGTTCGGTGCGATTCACCTAGGCGTGAAGATTAGCCATCGTGGGATTACCCCTACTG TCTAGCCGTTTTAAACTGACTGTTATAGACTGACCCTCATAGATTGATCTTGTGTCCGGCTCGGGCCGTC ACCTGGGCCGGACACATCCAACGAATCGGGGGAGCTGTGAGAACGAAAGAGTGGGGTGAACTCCAAGTTC GAGGCCGGGCGGGCTCGCTGTGTGCCGGCAGGAGCCTGGGCCACACATGCATTTCGGTGAGCATTCCCGG CACGCGAGTCTGCCCCGTTGGCCATTCACTTGTTCCTCACCATCGGTCCTCGCTGCCTTCGGCGCAGGAC CCCTCCCCGCTTTCTGATGCCAACGCATCGGAAACAGTGGCCGCGTAAGCGCCACCTCAGTCCGCATCAA TTCCCCGTATTTACAACCCAATTGGAAGGTTCCACACAATGAAGCACCACAAGTCTCGACCTACCGGTCG CCACCGCGCACCGTCCGCGCCTGTCATCGCCGCCGGCACCCTCACCGCTCTGGTTGTGGCCACTTTCGCA ACGGCAGGGATAGCGGTGGCAGCCCCGCAGCCAGGGGTAACAGGGCCTGCACCGGGACAGCCCGGAGTGA CTGCCCCGTCACCTTCACCTTCACCTGCACCTGCACCTGCACCCTCGACCGAGCCTGTACCCGAGCCGGT CTACTGGGTGCCGCCGCCCGCAGAGTACGCGAACGTTCCGTCGCAGCCGCTGCCGAACTGGGACTATGAT TCCGGCACCTACACGGCCCCGGCCGACTACAGCGTCGCACCGATCGACTACGGCAACATTCACTTGCCCG GACCCGTCGAGATCGTCGCACCGATCATCGCACCGAAAGAGAAGGGTCGCATTGGTGATTGGGTCTTCGA GCAGAAGACCTGGATGAGTGACGACGATCTCGCCCGCACCAACAACACCTCGGCCGTCATCGAAAGCCAG GTCTCAACCTTCTATGCCTCGATCGGTGTCCCCGTCGACCGCGCGAGCCGCATCGCCGCCGCACAACTGG CCGGTGGTGCCGGTGGCGCTTTGGCCGGAGCCGCCGCCGTTGGTGTACCCGCAGCAACAGTGGGCGCGTT GATCGGCGGAACCATCGGCGGAACGTCGGCCCTCGGCTTGTTCAGCCCCGTTCTCACGCCGATCGGTGCC GTGCCTGCTGGTGTGGTGGGCACAGCCACCGGAGCAGGTATCGGTGCAGCGGCTCTGGGCGTTCCCGCAG CGGCGATCGGCGCAGTCGGCGGTGGCATCGCAGGCGTTGCGGCCGCAACCGCCTACGGCGCAGGCGAATT GGGAGAACCTGTCGATGTCAGCGACCAGATCCCCGACATCGACCAGCCTGCCGTGACCACTCAGACGCAG 
Final Technical Progress Report DOE Award No. DE-FC26-02NT15340

AACGTTCTTACCGACTGGGAGAACTCCGGCCCCGTCGGACAGGCAGTGGCCACCACCGTCCGCGACACCG TCGCCGTCGCACCCGCGATCGACCAGCAGGCCCGAGACTTCGTCGCAGCCCAGCCCGGAGGCGAGCAGAT CATCGAACAGGTCGACACAGCCCTCACGGACTTCTTCACCAACGCCAGCCCTGGCCTGGCATCGCAGCTG ATCAGCGATGCAATCGGAGCAGGCACACAGGCATAGCCGGATTGGCCGGGACGGGCACGCCCGTCCCGGC CATCTGACATGGATTGCCTGGTCGAAGAAGGAGTTTGAAACGATGTTTGGCTCGAAGGGATCTACCCCTA AACCGGCGGTACGTAGCGCGACCGGCGGAACTGGTACCCGCCCAGTCCGCCTCCCAAGGCGTCCTCGTAT GCCGCATGCGCGGACACCTCGCCGTCAGGTCGCGGCCGTGACGGCACTGGCGATCGCGGTGCTGGTGTTG TTGGTCGGGGTCGTCGTGGGCGCTGCGTCGGTCATCGGCCGGGTCACCGACGACGAGCCGTCGAACATCA GCGAGATCGTCAATTCCGCTGCGACACCGTCGACTACACCGTCTCCGGAACCTGCCGGATGCCCGGTGTC TGCTGGTGATCAGAACAGCGGCGAGGGTGTGATCGCAGCGTTCGAACACGCGTATTACACGCTGCGCTCT GCCGATGCAGCGATCGCGCTACTCACCCCTGGTGCAGCTCCCTTCGCAGCGGACATGCAGTCGGCGATCA ATGCGACGCCGGAAGGAACGACCTACTGCATGACGGTGACCGGCGTTTCGGCCGGAACATACGCAATGGA GCTGGACGAGACCCGCCCCTCCGGCAAAGGCGCGTCGCTGTATCAGCGCATCACCACCGCCGTGATCGAC GGTCGGCATTTCATCACCTCCATCACTGATGCAGAAGGAATCCGATGAATATCTCTTCCACCGCCCGCTT CGCGTCGGCGTTCGCCATCATTTCGACGCTGGTCGCAGGATTCGCGGCCGCACCGCTCCTGGCCAACGCC GCACCGTCCACTGCGGCGGGCTGCACCAAGTACATCGGTGTCTTCGCTCCCGGAACGACCGAGACCCGCG CCGACGCCGATCCGTCGAAGCCTGCCGGAATGTTGGGCCAGGTGGGGGAGAAGCTCGAAACGATGTACGG GCCCAACACCTTCCGTGCCATCTATCCGTCGTACCCGGCCGAAGCGTTTTTGCAGTCCGGCATGACCTAC TCGGCCTCGAAGACCGCCGGTGATCAGGCAATGGCGACCGCGATGGAGCAGTGCCCGAACTCGAAGTTCG TCATCGGCGGCTATTCACAAGGCGCACAGGTCGCCAACGACGTCGCCGTCAACATCGGGGCAGGCAGCGG CCCCGTTCCCGCCGCGTCTGTTCTCGCTGCGGTCATGCTCGCCAATCCGAAGCTCGGCACCGCCGGTTCG CAGGTCGTCGGCCCCAAGCTCGACGGACAGGGCATCGCCGGTCCGGCCGCCGAGGGCTACGGCGCTCTGT CGGGTCGGGTCTTCGACATCTGCCACCCGGAAGACAAGTACTGCAACACCAACACCGGCAAGGATTCATT CTTGGCGTCGTTGGGACGAGTGCTTGCCAACCCACCCGGAACAGTGGCACCGACCACCGCGACTGCTGTC TCGGCGACAACGACTCCGTCCGTCGCAGGTCAGACCTCGACCGACACGGCTGCGAAGCCGGCGTCGACTC CGGCGGCCGCCGAAACTGCGAATACCTCGGCAGGCGCCGGAACCGCGAACGCGTCGGAGTTCTTGGGCGC GGTGACCTCGAACTACGGTTACGCGGATCTCGCGGCTGCTCCAGGGGCAGCGTCCGCGTTGGCGACCTCG GTGGAGAAGCTGCAAAGCGCAGGCGCTCCCACCTCGACGTCGGCGGCGGTCGATGTCGCCGCGGTTCGTC AGCAAGCGCAACTGTTGGCCGGAACCTTCAAGCCGGTCCAACAGACACAGGCATGGATGGCAGCGAACCC GGCCGCGCGCGACGGGTTGCGCAGCGCTCCGGCTGATTCTCCTGAGGCCGCCACGAACGCGGTCGTCGAT GCGCTCGACAAGGTCGACGTTCCGCAGGTTCTGGGTGCTGCTGACCAGGTGGTGACGTTGGCGAACAGCA TCCTCTCGGGTGGTGGCACCGGTACCGGCGGCACGTACGCGGGTCTGACTGGTCCCGCACAGAGCTTGTC GAACGGTGTGGCTCCGCTGGCGGATACACCGACCGATCAACTCACCACCGCCACGTCGATCCTCTCCGCG ATCCAACCGGTGACGATCATCAACCAGATCCTCGGCGTCGTCTCGGGTGTGACCTCGGTGGACTACAACG CGGTTGCGGCCAACCTGCAAATACTTCCCGGCCATCTGTTGGCCGGCAACGTCAAAGAAGCGCACCGGGT CGCGGGGGAGCTGAACAACCAACTCTCGCCTTTGGTGAAGATGGCGGCCGGGGTCGACCTGAAATCGGTG TCACGGATCTTGGCACTGATCCCCGATCCGTCCGGCACAGCCGCCTCGGCGGCGCTGGTGGCGAACCTGC TGGGCAACGTCGACATCATCCGCCTCGCCCGCGACGTCGGGCAGCTCCAGGAAGTCGCGTGGCAGGTCGC CGAAACCGGGAACGTCCTGGCCCTCGGGCAGCTCCTCCCGATCTGTGTGGATCTTGCGAACGTTGCGCTC GGTGTCCTGACACCGGGGCAGAAGATGACCGCCGAGCAGCTCCACGAACCGGCCGATTCGATGACCACGT TGATGGGTGCGCAGGCGCAGAACTCCGACTTCGTCGGTCTCGGTAACTCGGTCCTGGCGACGGTGACCTC CCAGGATGCGCAAGCACTGACCTCGGTCGTCGACGCCGGATTCACCGCAGCGAGTTTCCTCGGTTCCCGC GTTCACGAGTCCTACGGGCAGTGGTTGATCGACGGAAAGCGAACCGCTCTGGACGCGATGGTCGACATCT ACCGACAAGCGATCGGCGGCTAGCGCGCGGCGGCGGGTGAACCGAAAACGCTCTTGGGCGAGAGCTTTTG ACGTTCACCTCCCCACTCCTCATACTCACGGCCCTCTCGGGCCGCGATAGGTCCCACACGTTGCCCAGGC GGGCACGGCACGACACAACGACAAGGACATGACGATGGGTGATTCACCCAGCTCGACGAAGACCGGCCTC AAGGCCACTGGCGGCGTCATCGTCGCAGTGGTCCTGATGCTCGGTTTCGTCATGTTCGGTACGGACAACA ATCCCAAACCTTCCAACGATTGCCTGCCCACAACCGCAACTACCGGATCCGTGGCACCAGCTCCGGGAAC CAAAACGCAGCCGCTCAAGGCCGGTACCTACCAACTGACGTCGGGTTTCGGTCCTCGCGGTGGTTCCATG CACCGCGGTACCGACTTCGCCGGCCCGCTCGGTACTCCGATCTATGCCGCTACCTCCGGTGTCGTCGCAG AGGCCGGCCCCGCGTCGGGGTTCGGGAACTGGGTCATCATCGACACCGACGTCGACGGGAAGCGGATTTC CACCGTCTACGGCCACATGGCCGCTTCGACGATCAAGGTCAAGACCGGTGATCAGGTAACCGCCGGTCAG GAAATCGCCGCTGTCGGAAACGAAGCCGATTCCTCAGGCCCGCACCTGCATTTCGAGGTCTGGGAGGGTG GCCGACTTCCCGACGGCGCCGGAACGCCAACCGATCCCGCACCGTGGCTCGCCGGTGCTGCCGAGCCGTC CGGACCCGCACCGGCACCGGCTCCCGCGCCCGGCACGGGTGTTCCGTCTCCCGCGATCGTCAACGCTGCC GCTATCACTCCACTTCCGGCAGGTGCCGGGTGCGGTGGACCGAGTCTGGGCGGCGGGGGACTCAAGCCGG GCAGCGTACCTGCGGAGTTCGAACCGTGGATCCTCAAAGCCGCCGGGACCTGCCCCGAGATCACCGCTCC GCTGCTGGCAGGGCAGTTCAAGCAGGAAAGCGGCTTCAACGTCAACGCGCACAACAACGACTCCGGCGCG GACGGCCCGGCACAGTTCCTTCCCTCAACTGCTGCACAGGAAGGCGTGGACGGGGACGGGGACGGAAAGC TCGACATGCGCTCACCGGCCGACGCTGCGATGTCGATGGCGTCCTACGACTGCAAGATGCTCGAGCTGAC GAAAAAGGCGATGGCTGACGGCCGCCTGCAAGGCGAGCTGATTCCGCTGACGTTGTCCATGTACAACTGC GGCCCCGGAAACACCTTGTCGCAGGGCCAGGTCTGCCAGAACGAGGAAACACGCGGCTACGTCACCAACA TTCAGAAGTTCGCGATCGAGGAATTCACCGATACGTCCGCCCCGGCGGGCCCGATTCCAGGCGGCCCGTC CACCGGCTCGGCCGTCGTCGATACCGCACTGCGGTGGCTCGGCACCCCGTATGCCTGGGGCGGCGGCGAC 
Final Technical Progress Report DOE Award No. DE-FC26-02NT15340

GAGAACGGACCGACGCGCGGCGTCCGCGACGGGGGAGTGGCAGACAGTTTCGGAGACTTCAACAAGATCG GGTTCGACTGCTCCGGACTGGTGAAATACGCAGTGGCGCAAGCCACGGGCGGCCGAACGGTCCTCCCTCA CCAGGATCAGATGCAGATCGACGACTCCCGCGGAACCTCGATCACCAACCCCGCCGACCTGCGACCCGGC GACATCATCCAACCCCACAGCGGCCACATCTTCATCTGGATGGGCGGCGGCAAGGTCGTCGAAGCTCCGC AATCGGGCGATGTCGTCAAAATCTCCGACTGGACCCCACCATCCTCCGGGTTGGCAGCGAAACGCTTTGC CTGACAGAACAACCCAGACCAGAACATCGCAACCCGATGACCACCCCGGAATGAAGTTTCACCCGAAGAA AGGTAGATCCCAGAAATGACGGTATCCGCAGCCACCGAATGGGAACAGGCCGCCGACGCAGTACGCGCCG CCGCTGACGAGTTGCGCACCAGCGATTCCTCAGAGATCCGCGCGTGGGCGAAGAAGAACAAACTGCTCTC GCGCAGCATGTGGCCGAAGGTCAAGCGCGAGTTGGTCAAACAACTCGATCTCGACTACGACGTACTCCGC GACGCGGAAGCGACCAAACGCAAGAAGGAGATCGCCGAAGCCGCCGCCACCGCACCGTTGGTGGAACTGT TCGCCGCCGGTGACGAGCGGGGCAGCTTCGCTGTACTCGGACCGGTCGACGACGCGGCCTGGTACGGGAC GTTCCACAAGAACGACACGGTCTTCAAGGAAGGCAACCAGCGCTCGGCAGACAACTCCGCAGCCGGTAAG GCGGTATTCCTCGCCGGCAAAGCGCGTGAGGACGCGAACGTTCCCGCGGTGCGGTTGCTCCTGCACATCT CCAATCCGGAGATCGACGGGAACTCCCTCGCCGGTATGGCAGCCAAGCACGGTGTTGCACTCGACCTCGA CATCACCGACAACAACCGGGCCGTGGACTGGTGCGAAGAGCCTGGTTACCAGGCGTGGCAGGCGATTCGG TTGTCGGATCTGTTCATCGAGGACGAGTCGTGAAACGCCACCTCGCGGCCGCCGCCGCTGCATCTGCCGT CGCTATTGCCCTGGCCGGGTGCTCCTCGGGCACGGAAGGCACCGCAACCGGCCCGTCCACCACGACGGGC GCACGGGCGCAGCAATGGTCCCCGAACGAAACCTCGGCGGCCGAGCCCACACGGCCTGCGGCCACCGTTC CGGAACACGCGCCCGAACAGGTCGCCATCGTGGACCGCAGCGATCCGGCTGCCGTTGCCGACGCCGTGGT CACCACCTGGTTCACCTGGAATACCGCTGACGATCGCGGACCCAATGACGCGGCCGCCCGCACCGGCGAC CTGCTCACCGAACGCTTCCTCGGCGATGTCACCGGAACCACCCCGACCGGAAGCCCCGGCGGTCAGTGGC TCGAATGGGCCGCGAAGGACGCGCAGGTCTGGCCGACGGTCAGCTCGGTACCGAACCAGGGCGCCGTGGA CACCGCGACTGCGGTGCACCGGATGTATCAGGTCGAACAGACCGCGATTGCGTCCGATGGCTCCATCGTC GGCGTCGACACCGCCATCGTCGCCGTCCTGGTCGTCAGTCGCGGCGGTCTGTGGGCAGTCGACGACCTTA AAAAGCTATGACCAGCGAGAAGAACGTCCCCGGAACCACCCCCTCCCCGAAAGGAACTCCCGTGAGCGCT CCTGACCCCTACGACGAGCACCAGGACACCTACACCACCGAAAAGGCCGTTGACCTGCGCGGCCAGCGTC TACGTCGGCAATCTCGGCCCGAAGCCGAGACCGCCCCGATCGAGACCCCTGAACCAGCCCCGCAGATCGA GTCAGCCGCCGAATCCGGCGATACCGAGTTGGTCGACGAGTCGGCAGGATACGACGACCCGTATGCGGTC GGGCAGGACGCGCTGGCAGAGAACGACGAACCCGAATTCGACTACCGGTTCGAGCGGGAGACAAGCGAAG TCGACGACAGCCCCGCAACATGGGGCTGGCGCGGACGTGTCAACGCGGCGGTCGGCATGAAGATGGCGCC GAAGCCTGACTCGGCGGAGGTGCGGTTCCGCCAATCCGTCACCCGCGTTCAGCAGCCGCTACCCGGTTGC ATGGTCGTCTCGGTGATCAGCCTCAAAGGTGATGCCGGAAAGACTTCGACGACCCTGACCTTGGCCAACA CCTTCGGTTCGCACCGCCACGGCGTTGTCGCGTGGGATGCCAACCAGTCCACCGGGACTCTCGGAGACCG CGCGGCCAATCCCACCGATCCCGACGTCGGCCCCTGGGATGTTCTCGAGAACGCCCGCGATCTCACCTCT GCGGGAGCCGTGTCGGGTGCGCTCGGCAGGTTCCTGCGGCTTCAACCCACGCACGACGAAGTCCTCGGAG CCGACACTTCGACCTCACGCGATCATGGCATCGGCTGGGACGAGTGCGCGGCGATCATGGCTGTACTCCG CCGTCATCGGGACCTGATCTTCATCGACACCGGCAACGAACCTGTGGCCGCGAATTGGCAATGGGCCGTG CAGCACTCGAACCTGTTGGTCGTTCCCCTGCCGCTGCGCCTCGACATGACAAAGATGGCGTTGCGGATGT TCAAAGGGATCATCGCCCGCGGCTTCGGCCATCTGGTGTCCTCCGCGATCGTGCTGACTGTCGTGACACC CGGCTCCGATCCCACCCTCGAGCCTGAACTCGTCGAGGGACTGGCTCGACTGGGCCTTCCGGAAGAGAAC GTCATCCGGGTTCCCTACGAGCCGTCGTTCGCGACTGGGGAGCGCATCGTCTACGACCGTCTCCTTCCGT CCACGATCGAGGCTTACACCAATGTCGCCGCCGAAATCGCGGATGCTCTCGCTGCCAGCGTGTACGGCCG CGCTGCCGAGTACGCCGACCCGTACACCCCCGAGCAGATCCAACGTCCTCCGGAAGTTATTCGACGTACA GCCCGACCCGAACCGGTCGCGCAGTACGAGGACCGATACGCGGAGTACGAGCCGGAACCTCGGCCGAACC CCCGCCCCCTCCGTCCTCGCCCGGTAGCTCCACCCCACCCGCGAAGTCGCGGTCGCCTGGATTGAAAACC ACTGCACCACAGCCAAACACCACATCGAAAGGGAACACGATGAACGAACAGAACACACCGGTGAGCCCGT CCACCCGCGGCGGCCGGCTGCGTCGGGCGGCGGCCATCACCTCCGGCACCATCGCCCTGGCAATCATGAC CGCCGCTCCTGCCGCAGCTCAGGACGCGCCGAAGTCGAAGAACCCGTTCGAAGGGGTCACACCCACCCTC GAGTTCCTCGGACCTGCGTTCCAGAACGTCTGGGTCCGCGTGCTCGGCACCATCTGGGCACTCGCAATGG GCGGTGTCGGCGTGAAACTGATCTTCGCGCTGTACAAGATGCGAGCAGCGAAGAACGGCGGCTACGCCTC GGAGATGACCGACAACGCCGCTGAGGTCAAGACCGCAGCCACCGCCTTCGGCGGCCTGACACTTCTGACC GTCATCATCGGCGCCATCATGTTCGTCACTCAAGGCTGAGACGAATTCCGCTCCAGCTCAGCATTGTTCA CGAGAAAGAAGAGAACTCATGGTCAAAGATTTTTCTCAGGTAGGTACGGGCAACCGTCCGGTCGCGGCGA CTGGATCCACAGGTGGCGGATCAGGGTTCTTCTCTGACCGCAAGCGCGTTATCGCCACTGGTGTTGTCGC AGTGGTGTTCCTCGCGTTGGTGATCGCGCTCCTCACCTCCGGTGGTGGTGATGACAACAAGGGGAGCGGA GCCGATGCAATTCGTGCAGCCCACGGTCCGACGACGACCATCGACGGAGTTCCGGCCGGATACACCCGCG ATCAGGCCGGCGCACAGACCGCGGCCGTGAACTTCATCCAGGCCAACGGTCAGACGTACCAGGGCCGGAT CGACGCCGAAAAGCTGCGAGAAGTTGCTGTCTCGGCGAATGCGACCGATGCGCTCAATACCGTCATCGAC ATCTCCAGCGGCCGCACCGATCTGGGGAAAACCGTCAACAACGTGCCACTGATCGTCACCGTCCCCGACT TCACCCAGGACTCAGCCACCGTGTCCGTGTGGGGCATGGGCGTCGGTCAAAGCCCGATCAGTGAAGGAAG CGACAAGGTCGGCATCCTCACCGTCTACTCGACGAGCACCGTCACGGTGAAGTGGGAGAACGGGGACTGG AAAGCCCAGGACTGGAAGTTCGTCAGCGGCCCGTCCGCAGATGATGCCCAGTTCCCGGCCGCTGATTCGG CGCTTTCGAAGCCGGGCGCGAACGGCTTCTACACGTTCTACGTCAACTAGAAAGGGGGCCTGACTGATGT GGCGCAAAATCATTCTGACCGCGGTCGTGGCATTGTTCGCGATGCTCGGACTGGCGTTGCCGGCGGCAGC 
Final Technical Progress Report DOE Award No. DE-FC26-02NT15340

GGAACCAGTTGCTGTCCCCAACACCGACCCGGCTCCTGGCGTGCAGAATGCCACTCAGTCGGGCAAGGAA CTCCCCAAAGGATTTCCCGATGATCTGAAGAAGTTCGTCGCCGGCACAGACGAATTCAAATCCGCCTCGT GGTTCACCGGAGTCTGCAAGGACAAAGGCGGCGACATGGGCCAGTACTCGACACAGGTCATGCACAACGA AAACCGGTTGATGTACTGGACGGCAAAAGAATCCGAGCGGATCGCCATGTGGGCACCGCAGGTGATGATG TCGAAGAACCCGGCTCTGATCGACGCCCTGGTCGCCGCCGGCGGCGCCGGTACACAGATGTCCGGCATCG ACATGGGTGTGGAGGCGAACCAGAAGGCCGCCTTGAGCATCACGGACGAGTCGATCCTCAAGGGCGTCTA TCCGAACACGTTCCCCCTCGATGAGGCTGCGGACATGTACCCGCCGTCTCCGACCTGTGCGCAGGATCTG GCGCGGTGGACAACGAAGACGACCAGCACATGGGGTTTCGAGTGGAGCCCGACACCGGACGCGGCCTCAC TCGCGGCGATGAAGAAGCAGCCGAACGCCGACAAGGTGCCTGACGCTGCCTGGACTGCTCCCTGCGCCGG CAATGGTGTTCCCGGCGTCCTCTGCATCCACGCGATGTTCGTCAACTGCGAGCGCGCAACACCTGGGGAA GACATGTTCGCGTGCACGGAATGGAACACCAGGGTCGGGAAACTGTTCGGCGGCACCATGAACTGGATCG ATCAGAACACCAGTTTCAGTGACCGCCTCGGCGAGACCCTCGGCGGGATGATGAGGGCAACGCCGGCCTA TATGGGCGGCAAGATCTTCGTCGACAGTTTCACCTTCGCGTGGGATACCGTTGCCGGCCCGCTCGGTGAT CTGGTCGCATTCGTCAAAGACCCGTCGGACGTGATCGATTCCTGGGCGAACAAATTCAAGAGTTCGGCGA TGTCGATGACCGATTCGGTCCTGCCCGGTTTGGCAGGCATCGGCGAATTCGATCCGTTGGAGCCGCAGTT CTTGACTGTGTACGCCATGTCGACGGGGTTGGGTCTCCTGGTGCTGGCTATCGCCACGATCTTCGCGCTG TACAAGTCAGCCGACGGAAGCCGCCCAGGCGAAGAACTCGCCAAGGACTTCTTCGGATACTTGCCCGCCG GAATCATGCTGATGCTCTTCGCTCCCTCTATCGCCCAATTCGTCATCAGTCTCGCCCACGAGATCTCGGT CGCGTTGGTAGGCGCGATCGGCACCACCACCGACGAAGTGATCAACAACGTATCGACCTCCCTGGGCGCT CTCAACGACGAAACCCTGGTCGGTGGAGCCGTCGCCGGCATCATCGGATTCGGGTTGATGGTCATCGGTG TGCTCTCGGTCTTCTTCGGCTTCTTGATGCACGCGGCCGCGCTTCCCGTCCTCGCAGTGGTCAGCGGTAT CGCATTCGGCATGTGGGTGCACCCCACCTGGCGAAAGAAAGCGCTTCGGCCGGTGATGATGTTTCTCGGT GTCGTGCTGTCCAAGCCCATGCTGTTCGTACTGCTGGCCGCGGTATTCGTCGTCATCAACGTCTCAGCGC AAGGATCAGTCGCCGACGACGGCAAATTGGAATCCCTGGGCCAACTCGCCCTGATCGCCGTGTGCTTCGT GATGATCGGTCTGGCACCGTTCGCGTTGCTCAAGTGGTCACCGATCCTGCCGACCGCTGCGGATTCCGCC GACTTCGGTAGCTCGGGCTCAGCCTCGGGCGAGATGATCGGCTCGTCGGCGAGCATGTACCAGCAGCAAG CCGGAGGCGGATCGGGCGGCGGCGGCGACGGGGGAGCTGCGTCCCGCAATGCCGACGTCGCGACCTCACC CGGACGCGGCACCGGACCCTCCGATCACCTCGGCGGCCACAATTCCTCCCCCGACGCATCGTCAGCATCG TCCGATCACGCGTCCAGTCGATCGGGCCACGGAGCGCAAACCTCGTCCGGTTCCGTCGGGATCGGTGGCA AAGACTCCGGCAAGGGCGGAGCCAAAGCGGGAAGCGCCGAATCGAAATTGATGAGCGCGGCGAAGACAAC CGCCGGCGCAGCGGCAGGTGCAGCGACAGTGGCGGCGCCCTTCGCGGTGGCCGCTGCCGGCGCGGCGATG AACAAGGCCGGTGCCTCGGCGCAAAGCGCTCCCGAACACGCGGATGGAGAGAACTGATGAGAAAGCACCG CACCACCTACCTCGGCCGGGACGCCGCACTGCACGGTCTCGCTCAGTTCCGAGATCCAGTGGTACTCACC AGGATCGGAACAGTTGCGATCCCGCTGTACCTGACGGTGATGATCGGGCTGTCCGTAGGGACTCTCGCGG CCGCGTGCCTGGTGATGTTGGCCGGGTTCGTCGCGAGCACCGAGTTTTCTCGACCGGACACCTACGTTGA ACGGCTGGTCCGCGCACGCGCCGCCCGCCGCCGAGACGCCACCGAACCAGTGACCGGAGAACAGTGATGA GTGAACAACGCACGTCGATCCTGGGGCGTCAGATCGCCCGCAAAGGAAACTTGCCTTTCGCCGAACCGGT CTTGATCGCCTGGAGCGCGACACTCGTCCTAGCGCTGGCCCTGATCTTCGTCATCGGGCAGAACATCTGG GTGCTCGGGTTCGACTTGGCGTTGATCCTGGCCGTCATCGGTGCGACGTTCGAATTCGGGCAGCGTGAGT CCTATGCGGCAAGCAAAGCCAAGCAGATTCGAAACCGTCAGCGCCGCAAGCGCGGTGAGCATGTCTACGT CAGCGCCAGGGACGAAAACTTCGGCGACCCCGACCACGATCCGGGGTGGGAGTTCCCCGTCCCGCTCTAC CAGACGAAGCCGGTGAATCTGGCCGGTACCGGCCTCGACGACATGTTCATCCTCGAACACACCACACCGG GCGACAACAACTACTACTCGGTGATCGTGGCGATGCAAGGTTTGGCCGAAGGTCTACGCGGTGATGCGGA GTGGGCGGTGACCTCAGCTGCGTTCGGTCGTATCCTCGCCGGGTTCGCGAAGCGGTCCTCGTTCGTTCGG GGTGTGGCGCTGCTGCACCGCAGCGTCCCGGCCGACATGGCGCCTCACGAGAACTGGATGATGCGCATGA TCTCGGCCGCCGGTGAATCCGTGCGGCGCATCGACGAAGCGATCCGTTCCTACGGTCAGCTCCTCGACCG CAACGCCCCGTATGTCGAGGAGCACCGCTCGTACTGCGTACTGATCATTCCGAAGTCACCGACGTTCCTC GCCGAAGCTGCCCGCATCGCGCGCCGCAAGGATGCCGCCCTGGTGGGCGGTATCGCTCAAGTCATTCGCG ACGAAACGGAGAAGGCGCAGCATTCCCTCGAACGCGCCGGTATGGGCCGGGTGGAGGTTTACGGCGAACA GCGAGCCTGCGCGGTGATTCGCGCATTCCAAGACCCCACCTTCCCGCTCGACGCGCACAAAGGTGTGCGG TGGAACAACTGCTGGCCCTCTTACATCGGCCGCGACGAGTCGGTCGAGATCGCGGGGGCGTGGCACACAC GGGCCGGGGTGATCCCCCCGCGGGCGATCGAACCGCGTGAACTCGGTCCGTTGTGGCTCGGTCCGTTCCT GACCGGAGTCGAGCCCGACGTCGGAGACGACGAGATCGCCGCCGCCCCGACCATCCGGACGGTGATGGTG CGCATGGATTTCGTGGAAGCCTCCCAATCCCGCGAAGCCGCGAAGAAGGACGTCACCCGCGACGAGGCCA AGCGCATCGAGGAGAGCCGCAAGGGAAAGACAACCGACGGCGCCTCCGACGTGGCGATCACCGAATCAGC TCGCCGCCGACAGGATCTTCTTCCTGGTAGCGGCCACCACGGCTTGATCTATTCGATGGCCGTCTCGGTC ACCGGCCGAAACGAAGACGACGCATTACGCGCCGGTACACGTGTGGAGCAGGCCGCCGACAACTGCGGTA TCGGAGAAATCGTGTGGCAGGACAACCGCCACGACGTGGCCGCGTTCTTGACGTTGCCTCTCGGCCGCGG ACTGGCGGCCACCAAATGGACTCGAATCAAGACAAGGGGCTGAACCGACGATGACGAAGACAGAACAGAG CACCGCGCACCGTCGCGGCCACAAGAAGTCCAACCGATGGACCCTCACACCAAGCCTCGGGAAGAAGAAA GTCCAGGTCGAGAGCGCGGAGGAAGTCGTAGTCGCGCAAGCGGTCCACGCCTCCGACAGGATCACCACCC GTCGGCCTGCCCCCAAACGCAACCGATGGTTGGACCGGTGGGGCTGGTACGAGGTGCGGGCGGAAGGCGC GTGGACCACCACCCGGCAAGGTGAAGCGCTGAACCTGGCCACGATGCGCCGCTCGGTGCGTCACGAAGGA

CTCATGACCGGGCTGAACAAGATCAACCAGTCGATGGTGATCACCGATCCGTTCGCGCTCTACGGCTCGG

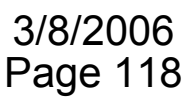


Final Technical Progress Report DOE Award No. DE-FC26-02NT15340

AAATCGAGAACATCAACGTCGCCATCGTCGGCGACATCGGCAAGGCGAAGTCGTCGCTGATCAAAACCGC GTTCTGCATCCGGCAACTCGCCTTGGCGAACAACCGGCAGTGCGTCGTCATCGACAAAAAGCGGCAGGGC AACAAGGGCGGCGAGTATTCGGGTATCGCAGAGGCGATGGGCGCCCGATCGATCCGATTCCTCGCCGGCG GTGACGGTGTCCGGATCAATCTGCTCGATCCGGCTATCGCCAGCGGAACCCAACGTACGGACGGTGTTTC CCCGGCCGGTCAGGAAGCGCTGGTCGGTGCCGTCATCGAAGACACGATGGGTCGTTCCCTCGTCGAGACG GAAAAAGCAGCTGTGCGCCAAGCACTCAAGTCCGTCAACGAACGCGCCGATGCGGAAAACCGCGAGCCGA CGTTGCGCGAACTCGCAGCGGAACTGCTCGAAGCGAGCCCCGGCAGCGGCAACATCTTCGGCTCACTGCA CGCCGAGGATTCGCGCCGCTGGGGCCTCAATCCTGGTCTCGCGCTGCAAGAACTGTGCGAAGGAGACCTC AAAGGCCTCGTCGACGGCGCTACTTCCCCGGAGGTCCGCGAGGCACTCGATCATTCGTTCGTGCACTTCG ACGTCTCGGCCCTCCCGAACAAGGGGCCGGCGTTGCGGGTCATTATGACCGTGATCAACACCTGGCTCGC GAACGTTCTCGCAACCCGCTCCGCCAAGCACATGCAAACGCTGCTGCTGATCGAGGAAGGCTGGCACGTC GCCGACGGCTCGACCGGAAAGGTGTTCCGCGAGAACATGAAACTCTCTCGCGGCCTCGGTCTTTCGACGG TTTCGGCGTTCCATCACATCTCGGACCTCCCGGTCGACTCTCCCGCGCGTGCGCTGATGAAAGAGGCCGG AATCGTCTTCTTGTACGGGCAGGACCGTGTCGAGGATGCAGAGGAAACAGCTCGCATGTACCACCTGCCG GCCGGGTCGACGGAAATCATCATGTCCCTGGCGAAAGGACATTGCCTGGTCAAGTACGGCAGCGCCGACC CGATTCTCATGCAGCACGTCCGTTCCGATCTTGAAATCGAACTCACCAACACCGACGAAGTGATCAAGGG AGAGGCAGCATGATCGCCACAGACGCTCCGGATCGCCTGACGACGCTTCCTGTTCCGCCGCTCGTTGCCT TCACCATTCTGGTCGGCGGGTCGGTAGCTGCTTGCGCGCTCATGATGTGGGTTCTGGTAGCGCGGCGCAG GTCCAGCGGCCGTAGGCCGCCCCTGTCACCGCGGGGCCGGACATCCGATACGGCATCCACCCGCACCACA CTCTCGCGGTCCGAGCTTGTGATGTCCGACCCGATCGAAGGAGACAAGTCGTGAGTACAACTCAGCGTCC TACGCAACCGATGGTGCCGCCTGGCGTGCTTCCCCTTGTGAGTCTCGTTGCTTCGGCCGTCGTCAGCGTC GTTGCCGGCGGATACATCTCAGGTGTCGTGTCCGGTCATGGCGGCGCTCTCCCCACCGGTGTGTACGGAG TTCTCGACGTGTTCGAAGGGCTGTTCCGGAATCCGGGCGACCCCGCTCTCGCGTGGCCGGAAGGTTCTCG GCCGGGCGGACCGGTACTGACGTGGGTTTCCATCGCGATCGTTGCGGCGTTGTGCCTGTGGATGGGGCTG TTCTTTTCCGGGCAGCAGATGAAACGCGCCGCCGCTCGGCGGGTGCGAGAGTCCGGATTCGCTGACCGCA CCGAACTCGCCCGAACCGGTATCAGCGAAAAGGCGGCCGTCCGGAAGGCGAAAGCGACCCGCCTCTCACT CAAAGACGTTCCGGTGCGGCGCATCGATGCCGCTGTCGAGACCACCCGAGTCGCGGAGCTGTACGACCAC AAAGGCGAGGGCGTATTCCTCCAGTACCGCGACGGACTGACCTGCACCGGACCGACTGGTGCTGGTAAGA CGTGGCGTGTCTGCTGGAAAGGTGTCACCGACGCCGTCGGCCCGGTCGTCGCTACCTCGACCCGCGGCGA TCTGTTGCGCTCCACGTGGGCGCAGCGCCGGGAAGTCGGGCACGTCGAGGTCTTCGACCCGGAGGGGCTC ACCGCGATCCCCAATCCGATGCGGTGGTCGATCCTCGACGGGTGCGAGGATCCGGAAGTCGCCAAGCGGC GTGCAGAAGCACTGGTCCAAGCCATGCCGATGGACGACACCAGCAATTCCGGCTATTGGAACGGCAAGGC CGCGATGCTCATGCGCGGCTACCTCTACTCCGCCGCCGTCAAGAACAAGAACATGATCGACCTGCGGATC TGGGCGTCCTCCCGCAACGTCCAACTGGTCCGCGATGTTCTCGAGCAGGACCTTCCGGACTGGCACGCCG AACTCGAGCAAGCCTTGGACTCACAGTCCGACTCCTCCGATGACGTGGTCAGCGCGTGCGCGCGATTGCT CGAACCGTTGGCGTCCCCGAAGTTGATGAGCGCGATCAACGTCCCCCGCAGCGAGTCCGCGGATCTGCAT GCGCTGATGACCGAGGGCAAGAACACCGTGTATCTGGTCTCGGAAGGACACAGCGCTTCGGCGGCCGCGT TCACCACCGTGCTCTCGGCAGAGCTCTATCACATCGCGAAGACACACGGGCTGGGCAACGAGGACGACAA GATCGATCCGCCGTTGCGGATGGTCCTCGACGAAATGAACAATGTCGCGGCGATTCCGCACATGCCGGAT CTGATCACGGACTCGGGCGGCCGCGGAATCCAGATCTGGGCGTTCGTGCACAGCGCCTTGCAGAACGAGA AGCGTTGGGGCCGCATCGACGGGCAACGGTTGTCGAGCGATTCCCCGGCACGGATCTACTTGCCAGGCCT AGGTGACGAAGGCGAATTGTCGGCGCTCTCACGGTTGATGGGTAGCCGCGACGAATACATCAGCCTGGAC CCGCGTTCGGCACCGCGCAGCGTTCCGGTGATGGCACTCAACGAAATTCGTGAAATGCCGGAAGATCAAG CGCTGATGATCTACCGCAACGCCAGGCCGATGAAGGTGCGGCTGCCATCGGTGTGGGATGTTCCGGATCT TTCGAAGCGGGTGCGGGCGAATCAGTCGGCGTTCGACGCGTTCTGCGCGAAAGCCGAACTATGAGCTCAG AATCTGCGACCTCGCCGTGGGAGTGCGCGGACCACTGGGCGCCCGGGGATTCCGCGTTGTGGATTGGCGT CGGCTCCAGCGGGGTGTACGAGCGCCCGATCATCGTCGTCTCCCCGGCCGGCGCCCTCGTTCGCGATCCG GAAGTAACGTACAACGACGGTCTCTTCACTCTGTCCGACGGATTACGGGATGTGCGGCACCGTGATTCGT GCGAACCGTGCGCAGCTTCGGTGCGCCTGCTTCATCAAGGAGGTGGTGTGTGATGAGCATTCCGGACTAC CATCCCGGTCCGGCTGATTGGCGCCACCTGGATCGCGAGGCTGCTGCGGAGCTGTGGCTCGAGCTCGGAC AGTGGGTCGAGTGGCTGCGCCGGCGCTACAACGTCGGTGGGCAGCAGATCCGATCGTGTTGGTACAAACA TGATCCGATGGTCGAAGAGCTGACGGCGGCGATGTGGGCGCACCGAGAGGTGTACCAGCAGCTGGAGAAG AACCCGTATCACGGCGGTATGGCAGCCTGGCACAACCACGTGCTGTGGCCGATGATCTCTCGGCTGCCCA AGATGGGCTTCAACGAGGAATGCCGTGGCGGAGAGTGCGGCTACCAGCCGAACGACCCGAAGATCGGCAC GGATTTCGCGGAATTCGTCGCCGCCGACACCGACCCTCGACCCGAGACCGCGCCCGCACCGCTGATCGAT CAGGTCCTCGGCTTGGTCGATAGCGCAGCCGGGAAGGTGACTGCGCTGACGATGGATCAGGTTCTCGAGA TGATCGACGGCGGCCGCGCGGTCGCCGAAGATCCTTCCGACGATTTCACGGCGGTCGAGATCGACGGCGA CCGGTGGGAATTCGACGATGACACCGAAACATATCGGCCCGTCGAATAACCCTCTCAAGCCGAAGCATCA ACAGCCCCACGCCCTTCGGGCGGTCTCGCCCACCGTTCGAGAGTCCGGGCCTCGGGGTAGTTGATGTTTC TCGTGTCCGCCACGGTTGCGGCGATCGTGGGTTGTCCCGCCTGGGGCCTGCGCGCCGTGCTGTGGCGGAC ACACCСTCTTTTTCATAGGATGCTTTGCATCACAACTCGATTCGGATTGGTGGACTACCATGCAGGAGCA CGACCCCCTCGGACGGATGGATTTTCCTTTGATCCGGTCACTCTCACGCATGAGTGGAATGTTCATCCGG TCAGGATTGATTTTTCTCGCCCTGTTCGTCGCAGCATCGCTGATTGCGCAGGAGACACCAGGCCAGTGGG CGGTCGGCGCGTTCGTCGTGCTCTGGCTGGGTTTCGGCCTCACGCTCCGGTGGCTCTCGGTATCGCGATT 
Final Technical Progress Report DOE Award No. DE-FC26-02NT15340

TACCGACCTGTGACAGTTCCCTCCGCTTGCATGACTGTGGCCCTCGACCGGAAAGGTCGAGGGCCACAGT CGTACAGCGAGTTCGTTAGGGCAGTCCGAGTGCGATCGCGAGTGCTTTGTTCAGTTTGACCACCGTTGCG GGGGAGAGCGCTCCTGCCGGCCTGCTCGCGGCGATCTCGTCGTCGAAGAGCTGGATGAGGTCGTCCGCCA GGATCGAACCGACAAGTGGATCTGAGGCTCCGAGGGGGACGGCCGTCGGAACGCCGGGCTTCGGTGTGGT GGTCACGCGTGCGGCGAGGACCGTGTTCAGGTTGCGGTTACGGACGTTGTTGGAGACCACCACCCACGGC TTCTCGCCGTGTCCCATGTCGAACCAGTAGATCTGCCCGCGGAGCGCGGCCGTCATTCGCCGTAAGCCTG GTCGACGCGCTCGGCGTAGCTGCGCCGGCGCGCTCGTTGTTCCGTTAGGCCTTCTTCTTGATCCTTGGCG AGTTCGGCATAGCCGGCTTCGAGAGCCTTCTCGCGGAGTGCTTCTGCACCCACACGGAGAAGTGCGCGGG CGATCCCGGACTCGGAGTTGTCGCGGATGGTGATGTGCTGCTGCTGCGCCCATTCCCGCAGGATTCCTGC TTCGAGGCGGTCGGGGTCGGCGAACACGGCGATTTCCGCTTGGTCGTCGCTGTCTAGTGTTACAGGTAGC CGTTTCACATCCATGTTTCACAGTATGACTCACTTTGTGGATCACGGCAATGAGTTTTCGGACACGCCGC AGATCCTGTCGGCGGGATTGGCGATACTCGCCAAATGAAACTGTTTGCCCAGAAGCGGGATGAATCGGAA TTCGTGGAGTACGGCTTCGGCCTCGTGAATGAAGTCGGCATCGATCGCGTCAACACTGAGGACTCGACAC TGGTCGATCTTTACTACTGGAGCGAAGGAGTACGCGAAGCGCTCCGGGAAGTCCGCGACTTCAAGGTCAC GGACTGAGCTCGGCGTTTCACACTCACGTGCACGCCTCGTCGAGGCGACAGGCAATGTGCGATCGGTAGG GTCTAGCTCGTCGAGCAGGCTGAGGGGAGCAGCGACGGTGGTGGGTCGAATATGGCTGGATGTTCCGTAT GTAGAGAAGGATCTGGCGAAGGCCGGAGGCGCGAAGTGGGATCCATCAGCCAAGCGATGGTATGCGCCCC GAGTCGAGATGGCAGGTCTGTCGCGATGGGTCGCGCTCCCTGACATTCCGGAGATCCTGCCGGGTGAAGA CCGGTCTCTCGGTCATGGTCTGTTCGTCGACCTCGTCCCTGATACGTGTTGGTTCACCAATGTCCGGTAC TGCGTCGAACCGAAGGATTGGGAAAGACTGCGCCGCATGGTCACGCGGCGCGCCGGGATGCAGTGCGAGA TCTGCGGTTTCCCGGAAGACCGAGCGTCGCAGCGGTGGCTCGAAGTGCACGAGAGATGGACTTACGACTG GCCGACCAAAGTGCAACGTCTGGGCCGGCTCATCTGTCTCTGTACGCCCTGTCACCAGGTGACGCACTTC GGATTCGCGCAGATGAAAGGCAATGAAGAGCAAGCTTTGCAGCATCTGATTGCGGTGACCGGAATGACCC GTCCGCAGGCGCGGAGCCATGTTGCTGCCGCGTTCGATGAGTGGCGAGCCCGCTCCATTGGGGAATGGGC TCTCGATCTGAGCATCCTCACCGACGTCGGGGTTCAGGTTCGTCGACCTCCGGCGCCCGCCGACCGCCGG ATCATCGCAAGCGGCCGGCCCACCGCGACACAAATCCGGCAACCACAACCGGTGGTTCCCCCGCTCTGTC CTACCCCTGCGATCACTGCACCTAGATTGCGCGATCGGATCGCGGCATGGTGGCGATCACGCTGAGACCA CCCGCCCCCTCTGTCGTCTGACGAAACGCCAGCCTGCCGGCTGTCACAGACGCGGTCCGCGGCGTTTTTG TTCGTGTTCCACGTGTTGGGCGATGGTGTGGTGATGCTCGAGTGTCGCCTGCTGCCGCTCGGCGCGCCGC TGTTCTTCGCGGTGCTCTTGCATGCGGTCGTGCAGGGTGTCGATGTCGCGGTGGACAGTGTTGAAGGGTG CGATGTCGGCTTTGTACTCGGGCCGGTCGCCGGCCACTGAGGTGGTGTCGGTGATGTTGTGGCGGTCGCG GTATGCCTGGATCTCGGCGGCGGTGCGATCCCATTGCGCTGCCAGCTCGGGGTCGTCGGGCCGCGCTTCG AGGTGCTCGGTGAAGCGCAGTTCGGGGTCTTTGCCGCGAGCATCGTCGGCGCTGATGCGTCGGGTGTTCG CGGTGTGTCCGACGGCGATGTCGGTGTAGGTGGTCTCCAGCTGCCCAGCGAGGGTGGGGTCGACGAGGTG GGCGTCGAGGTCGGGGGAGGGCACCCAGTGCGGCGCTGCGGGGTCGACGGGCTCTGATGCGGCCGGCGCG ATGGTGTCGAGTGCGTCGGTGATCCCGGTGACGTGTGCGGTGGAGTGCGCAGCGGTCTCGATCGTGCGGC ACAGTTCGTCGACGTCGAAACCTGCGTGGGTGACGAGTTCGCGGATACGGCCTACTGCTGCGTTCCACCG CGGGTCTTCGGTGCGTTCGGCGGCGGCCACCGCGAGAGGGAAGTGCTCGCGCAGGATGTCGTCGTGTTCG GCTGCCCAGCGGGTGTTCGTCTTCTCGGTCGCAGTCGTGATGGCTGGTGCCATGTCTTTGATCAGGTTCG CGGCGGTGACGTCTTCACCCCGTGCTGCGAGTTGCTGCGCGTGCCAAGACAGAACTGCCTCGGGCGCGAT CCCAGCGAGTGCTGCTTTACGTAGGTGGCGGCCCAGTCGCTGCACCCCGGCTTCGGCGAGGATGCGTTCG GCGATCTCGGGTTCGAATGCTTCCCGCACGGGTCGGTGGATGCGGGTGGTGTCGAGCTGCGCCCGCGTCT CGTCGTACCGGCGGATGGTCGCGCCGATGCCGTCGGTGCGCTCGTGTTCCGGCCGGGCGGTGGCGTAGCG ATCGGCGACGATGCGGGCGTAGTCGTCGAGTTCTTTGTCGGTGTTGGTGGTGCGCGGGGGCAGCGGGGGG AGCGCTGTGTCGGAGCGCTCGGCTGCCGCTTCTGCTGCGGTCTGAGTGGCTTCGACGATGCGGGAGTTCA GGACACGCGCAACGTCGCGGGCGGTGTGGAGTTCACCGGATGCGAGTGCGTCGTTGAGGATGCGCCGGGC ACTGTCACCGCGGTCGTGTGCCTGTCCGAGAGTGGTGCGGAGCGCGGCGAGGCTGCCGGGGTTTTCGGCG GCGATCGTCGCGAGCAGTGCTGCGGGCAGCGCCCGGTCGAGGACGTCGTCGAGCCAGCGATCGCGCAACC TGTTGTGTGCGGCGTGGTACGCCTCGCGGAGTCGTTCGGGGCTGCGTGCGTGCTCGAGTGCGTCGCGGAT CTCGGTGATGGCGGCTTTGTGGCCGTTGTCGTTGGCGAGGATCTTTTCGAGGACTTCACGTGAGGCCGGG TTGTTGATGGACCCCAGGTGCATGCCCTCGGTGTCTTGGGTGACGCCGGGTTCGATGGGGACGTACAGGT GGTTTTCGGTGCGGCCGCGGGTGGTGGCGACGTAGAGGCCGGGCCGGTTGGTGGTGTGTCCGACGGTGGT GTGGGAGGTGTCGACAGTGATGCCTTGGCTGCGGTGCACGGTGGCGGCGTAGCCGAGTTCGGTGTTCCGT TTCACGTACCGGGCGGGCAGGGTGATCGTGCCCTCGTGGGTTTTGCTGCGCAGGTTCAGTGAGCCGTTGT CGTTGACGGCGGTGACGGTCCACAGGTCGCCGTTGCGGACGAACATGTTGCGCCGTTTGCCGCCCTTGGT GCGCAGACGGCGCTTGTTCTTGCGGGTCAGGACGATGTCACCGACTCCGGCGTCGAGTTCGTCGGACAGG GTCACGGTGTGTCCGGCTTTGGCGGTGCCGGTGGCGCGGTAGTGCGCTTGGATCGCGAGGTTCACTTCGC GGACGTCGTCTCGGGTGGAGAGCATGACGATCGAGGTTTTCCCGGCGCCGATGTCGGCGAGGTAGGCGGT TGCGGCGGCGTCTTTCATCTCGGCGGCGGTGCCCTCGTGGACCCATCCGCGGGAGTAGAACAGCTCCAAG CTGCTCTCGTCACCGTGCCGCAGTGCGAGGGAGTTCTCGGCTTGTTCGGTGTCTTTGCCGAAGCGCACGA CGACTGTGAGTTCGGGTGCGTTGGTTTCTTCGGCGAGCAGTCGCAGCGCTCCGCCGGTTTCGACGGCGTC GAGCTGGTTCGGGTCACCGAGTAGCCGGACCACTGCGCCCTTGGCTGCGGCGATTTCGGTGATGGCGGCG AGGTCTTTGGTCGATGCCAGGGACGCTTCGTCGACGAGGAGCATCGCCCCGGCGGGGATCTCGATGTGAT CGGGGAGGCTGCGTGGTTCGTGGCCTTCGAGGCCGCGCCACGGGTACGTCAGGGTTGCGAGGGTGTGGGC 
Final Technical Progress Report DOE Award No. DE-FC26-02NT15340

GGTGACGTCGATTTCTTCGCCCAGGGTTTCGGCCGCAACTGCGGATGGTGCGAGCGCGATGACTTCGTGT CCGGTCGCGCGCCAGGTGTCGGTGACCGCTTTCATCGACGTCGTTTTGCCGGTGCCGGCGGGCCCGACAC CGACGGCGAGGAGCGTTCCGGCGCCGACGAAATGCTCGGCAAGTTCGCGCTGTCCGGCGTTGAGCGGGTG ATCGCTGGTACGGGCGATTTTTTCAACGACCTTGTCGAGTCCGGCTTGTGCCGCGATGTAGGCGGTCGGT GTCCGAGTGGCGTCGAGCATGAACTGTTCGGCGGTGATGATGCTCGCGGAGGTATGCAGTGCGGTGCCAT GGTTGGTGAGGATGAACTCACCATCACGTCGTCTGAGAGCCTCCGGTGCGGGGGGAAGTTCGGCCGCCGG CAGGTGCACGGACGCGCCCAGTACGTGTTCGGTGACGGCTTTCACTGCGGCCGTGCGTGCGGCTTCGTCG GGGAAGGAGACGGCTGCAAAGTTCGCTTCGACGGCGCTGTGAATGTGGGTGATCGCCCACGTTGCGCGGC CGTTCTCGAGGTGTTCGACGACTCGTTTCGCTTCCTCGGCCGGTGTTCCGGCCTTGCGCATCGCCTGGTG CTCACGCACTCCAGCGGCGTGTTCGCGCTGCACGCCGCGCCATTGTTCGGCGAGGGCTCCCACAGCTGTG TCGTCGCCGAGGATCTGCGCAGATCGGGTGATCCAGTTGTTCTGCATTTCGCGCAGGGTTTGCGGTTCCT GTTTCCCGGCGCGGGTGTCGAGGTTCGCTTGCTGGTAGAGCGCGTACATCGCCTTTGCGCTGGGCATCCG GTCGTGCTTTTGGCGGTATTCGGCGACCAGCTGATCGCGCCGGGTCTCGATCTGAGTGCGCCGCGAGGAG AАCATTTCGCACAGCTGCTGCGGCACTTCGGCGATCTCGACGACGTTCTGCCGACCACGTCCGGTGCTGC GCTCTGTCAGTCCGAACCCAAGCTCGGTGTGCATTTTCGACATGATCGCTGCGTTGTAGCGCTGCGAAGC CGCGACGGCGTAGCGGTGCAGGGTGGCACCGTCGAGAGCTCGCCACTTACCGTCCTTTTCGGTGCATACC TTGATGGACACAACGGCGTGGGTGTGCAGGTTCGGATCCCCCGCGCGGGAGTCGTAGTGGTCGTAGAGGG TGGCGACCATGCCGGTGGTGTCCTGGTGCTCTTGGCTCGTCGCGCCGGTGCGGGTGAAGCATGCTTCCTT CTCGATCCAGCCAAGCGTGCCCTTGACGGTCTCGTGGTGGATTCGTTCGATCTGGCGGCGCAGATCATCG TCGGCGAGCGCCCACATCGTCGACACGCTCTTGGGCGGTGTGAACACCAGATCTTGTCCTGCGACGGGTT GCCGGACACGGTTCTTCTGCGCCGAAATCCAGGACCACAGCTCTTGCGGGTCCTGTGCGGAACGCTGGTG TGTCTCCTCGAACAGTGCGGTTCCGACCTTCTCCATCAACGCCCCGGACTCCGCTTTGGAGGGCCGGCGG GCGTTGTCGACGGCGAACTGCTCCAGGGCGGCGCGGTGGGCCTTCAGGAGGGGGATGTCGTTGGCGAAGT CCGGGAACTTGCGGCCCAGTCGAGCCTCTTTGACGGCCTTCTTCGGGTCCATCCCACCCTTGATCGATTC CTCAACCATGGGGTCTGCGTTGGGGTGCATGCCCTCCCCGAAGAGTGCCTTCATCTGAGGTTCGGACACT GTGGCGCCGTCGGTGATCCGATGCTGGTCGGCGATCTCGGTGTCGGCATCCATTCGTGCGAGATCTTCGA GTCCGCCGCCGAACCACGTACCGGGCGGGGTGCCGTGGGCGGTGTAGTAGTCGGTGAGTTCTTGCCCGTG CTCCAGCTCGCGGTCTGCGGTGGCGACCTGCTTTGTCAGGTACTCGTAGCCATCGCCCGCGTGCAGTACG TGGAGCGTCATCACAAGGCCACCATAGTCGCCGCAGGCGGCCTGGTGGAGGGCAGACACCCCCAAAGTCT GAAATTCAGCAAGAGGTGTGTCTGAATTTGGGGTTTTGGCTGAGTGAAACGAAGTGCATTTGGTGAGGGA CGAGCTGTCTGTGCTGAGGGAAGCGAAGTGTTTGAAAATATGGATGAGCTGGATGGATAGATGGTTTGAC ATGGTGATGTGTGAAAAGGTTCAGTGGAGTTGTGATGGTTGGCCCGAGTTTTTGAGCCGGGAACCGTTAG GTGGGCGGGGAACAATTGATGGCCGATCGCCGCTGCCGGCGGTAAGGTTTGCGGCATGGCGTCCTCAAAC ACATCCACGATCAGCGCGAGGGATCGGGCACGCGCAGCGAATGCCGCTCGTCTCGCGGAAGAGCAAGAGC GATTGAAGAAGGTCGAGGACGATCTCGTCCGGTTCTTCAAGGCGCAGGAGTCGCGGGAGAAGATCGCGGA AGATGCGCGAGTGCGTATCGAGCGCATTCGCGAAGAAACCGATACCAAGATCGCGGACGCGGATCGTTCC GGTGCGGCTGCGATTGCGGATCTCAAGTCTCGCGGTGAAACGGTGGCGAGCATCGCGCAGCTGACCGGTC TGACGACGAGTGAGGTCAACAAGTTGCTGCGGTCGACCAAGCCGAAACAGAAATCGGAGAAAGTACCCGC CGACAAGGCCGCGTCGAAGGAAGATTCTTCGCCCGCGCCTGCTTCGACTTCGTCGACTGATTCTGCCGCG CTGGCGTCGTAGAAGCGACCAGCAAACCAATGTCGGCCTTCGCGGTTTTCCCCGGTTGGGTGTCAGCGAC CGATTGTGTGTTGCCTGAGATCGAGCGGAGCGGGAACAGAAATCTCGGGCATCCACATGAGGGATATCGC TGTCCCCGGTTGGGGGAAACCGCGGCCGATTCGTCGGCAACTCTCACAAGCGTTAGGAGTTCGTTCTGAT GGCGGACGTCCTAACGCTTCCGGCGCTCGGCGATCCGGAGCGGGAACGTTTCGCGCGGTTCATCGTGCGC GGACCTGGCGAACGTGATTGCCATTTTTGGACCGGCAGTATTTCTGATGACGGGTACGGAAGATTTTGGT TGATGCGCGATGGCCGGCAGCGGGTCGTGCGCCCGCATCGGTACGCGCTGATGCTCGAACTCGGTCGGCC GTTGACCGATTCGGAGTTCGCGATCCACGAGGTGTGTGACAACCCGATCTGTGTCCGGGCGACGACGAGC ATCGCCGGTCGTCCACATGTGGTTGCGGGTACGCAATCGCAGAATCTTGCAGGGATGAGCGCGAAGGGGC GCGGCGGCGGCCGGGGGCAGAGCTGGCATTGGTACGGCCCGGATCGCGCCGCCCGCGCAGCGCGATCGAA AGCTCTACGGGATGCATTGCGCAATGGATGGGATGCGGACGCGGTGGAGTCCGCTTTGCTGCGCACGGAT ACTCCAACACTTTTTTGACGACCGAGAGGCATGTAGATGACTGAGAACGGCGTTCCGCGTGAGCGGTCTG AGGTGGAGGCTTCTCTGTATCAGGCAACGAAGCATGTGAATGTCATTACCGATGCGGATCTCGCGGCCGT TGTCGAGGGATTGACTGGCGAATCCGTTGCGCGCCCGCGGATCGATTTCGCTTTTCCCGAGCCGGGGACC ACGGATCATTTGTTCAATTGATCTTTGGCGCGCCGCATTGCACGGGTGGCGCGCTGAGGAGCTGACGCAG CGGTTGTTCGGGCTCGATGCCCGAACAACCGCCACTGCATACGGGGGCGGCCCCGACTTCTTGGCGAAGT CGGGGCCGTTGTTGTTTTCAGCTGTCGAGGTTGTCGACGTCGACGCCGAAGGATCGCGCTGCCTCGGCGC TGATCGCGTGCAGCTGCGTGGTGACTGCGGCGGGCGAGACTGCGTGCTCGAGTGCGAAGTCGAGCAGGTT GCGCAGTGAGGTGTCGTGTTCGCCGTTGCCGTCGATCACTGCGGCTTGGGCGGCCTCCAATGCGATGCCG GTACTGGGTCCGTCGCCGCTGATGTACGAGCAGTACGCCGCGACGGTGAGAATCTGAATGCGAGCGTTGC CGCGTACCTGGCGGGCGATAGTCGCGAACGCGTGGGCGGCGGCGTGCTCATTTCCGTTGGCGGTGAACAT CAGAGCATCGCGTGCGGTCGGGCTGATGGTGGCGCACAGTCCGACACGTGCAGCCAACTCGGCGTCCGGG TTGCCGCGTGTGCGGATGAGTGTCACGAGTTCGGTGACGGTGTGGCGAACGAAGTCCGGTCCCTGCGCGT GCAGCGCTGCGTGTGCCGGTGCGGTGTCCGCTTCGACGGTCACGGCGTAGCGGGCGGCGATGTCCTCGCG TGAGGCTGCGGTGCTGCGGCCCTCTTCAACGGCGTTGTGAATGCTCAACGTGGAGGCGTTCGGGTCTTCG GTGAGTCCGGTCGCGTACGTGTCGAGGTCCGTCCACAACGCTCCGGCTTCGAGCGCGGTGACGTGCAGGG 
Final Technical Progress Report DOE Award No. DE-FC26-02NT15340

CGAGCATGTCGTGGATTCCGGCGTCACGGAATCCCTGGCGCAGGATCGCGATCTGGTCAAGCGCGATGAC TGCGGCGGCGTCTTCGGCGACGGCAACGCAGATCGTGGCGGTGGCCTCGGCGCGTGAGGCAGCTTCGAGG TACGGGGCCGGATCGAATTCGGCGCCCTCGCCTGCGGCGGCGTCGGTCCGCGCGACAAGGGCAATCTGCT TGGTGGTGCGTCCGTCGACGATTTCGTCTTTGAGCATGATCACGATGACCGATGCGGTCGGGATGAATCC GAGCATGGTGGGGATGGAGGCAAGTACCTCGGTGGCGGTGTTCAAACGGATGCGCTGCATGTCGTTCCCC TCGCTGATAAAAGTGACCGGGCCAGGTGCCCCGCCAATTTTTTTCGCCTTCTGGCAAAGGAGAATCGCGT CGGCATGAGGGGGCCGGAGTGCCACCCCTGGGCCGAGAAAATTTCGGCGGGAGCTTGCGAGTGACGAAAA TTTCTCGGAGCCAACGGCCGGCGCTTGCGCCGCCAGGGGTTGCGCGCAGGTCACCGCCGACGAGAATCGG AAGGCCAGAAGTGAAAACAAATCAATGCTTTTCACAGAACGGAGCGCAGCGGAGTTCATTTCGGGGGACA CCGAAGGTGGCCGGCCGCCGGGCAGGCGTCAGCGTTCCGCCGGCAACGTGCAGTCGCGGCACTCCCACTG CCCACCAACGCGGGTACAGGTGCGGCGCTGCTCGCACAGCCAGCACCGTTGATTGCGTCGGGTCAACAAC TGCGCTGCGGTCGCGGCGGGGCGCGCGGGTTCGGTGGTCTCGGCGCGCTCTCGCTCGGATACGGGCCTGA TCATGTCGCCGAAGATGCGGCGGCACTGCGAACAGATTTCTCCGTCCTCGGCCACGATGTTCGAGCATCC CGCGAGAACGCATCCGGTGAACATCTGCCGATCCACCATCGTCTCTGCCCTCGGCGCGCTCACCATCGGA ATCGGATTCCGTGCGGTGCGGCGGCGCGCTCGACTCGGCCGTAAACCCGATTCACCCGAGAGGGTGCGAT CTGTCCGGCGTCGTAGGCGCGGCGGGTCGCGACGGCGAGCAGGGCGACAGCTTCCCGGGCGCTCACTTCG TGCGCGAGGGTGAGCCACCCGATTTCCGCTTGCATCCCTGCCGCGCTCGTGGCGGCGGTGATCTTTGCGG CCAGCATCTCGAGGATCTCGGCGGCCGCCATTCCTGACGGTGTGATCGCGGGATACGCGGCGTCGATGGC GTTGCTGTACATCGTGTCGGCCCGCAGCTCGTCGGTCGTGATGCTCATGTCGTAGCCCTTTCGTAGACTT CCAAGCGCGACACCGGGCGGGCTGCCTCCACCCGGTATCGCACCTGTGTTTGCGGGGTGCTGCCGTCTAT GCGGCTGCGGTGTCCTCGGCTTCGTCGGCGTCGGGTTCGAGTGCCTCGGCGAGGGTGTGGACCGTGGCCA GGACTGCGCGGGCGGTGTCGGTGATGACCTCGAGGTCTCCATCTCCCCATCCGGCGATGTAACCGATGCT GTATTCGCTGGTGTCGAGTCCGAGCAGTCCAGCCAGGACGTAGGCGACGGATTCGGCTTCCACCTCGGCG CGGCCTCGGTGCAGCTCGGCGGGGTCGATATCTCCGCTGGCGTGCATGAGCGCATGGGCGGCTTCGTGAA TCATCGTTTTCGCGGCCTGGGCGTCGCTGATCGTGTCGTCGACGATGATGCGGCGCGATCCGTCGAGGCT GGTGCGGCCGTTCGATTCTCCGGCGATGGGCTCGCGGGTGACGGTCCATCCCTGCTCGGTCATCACCTGC GCGACGCGGGTATAGATCGCGGCGGGGTCTTCGCCTTCAAGTCGGGTGGTGATGGTTTCGGCGGCTGCTG CGGGGTGTCCTTCGATCGGGTCGGTCTGGGCGATGTCGAACACCGACAGAATCGGGAACCGCGGCGCGCG GCGGGTGGTTTCCTCGCCGGTCTCGGTGTCGGTTTCGGTGATCTTCTTGGTCGAGTAGCCGAAAATCTTG ATCGACTTTTCGCCCTTGCGGACCTGCCGTCCGAGTTCCTGCCACTTGCGGAATCCGGCGACCTGGCTGG CGTCCGCGGCCTGCGAGAAAATCAGCATGACGTTGTTGAAGAAGGGTGTGAAATTGCATTCGATGCAAAA CAGAGCCGCGGTTGGCCGCGTGTCGTGTTAACCCAGCATCGGTTTACCGACAGACGTTCCTCTTCAGGTG CAAACCCATCGTCCCTCCACCACCTCACATGTACCCCCGGGGGGTATAGCTTGGTATGGTCGACGACATG CATTCAGTGATCCGGCGGCTTCGACGGGGCGTAGCCTCGTCGGCCGGATTGCTGATCGTGTTGACGGTCT TCGGGGTGCTGCTGATGCACTCGGTGACACCGACCACGTCGATGGCTGACCCGATGTCCACGTCGATGAG CGGTCGCCACGCCTCGATGGCGGCCGCCGTCCGCTGTCGAGAGTTCGGTTCAGGTGGCGTTCGGGAAGCA TGACTGTCCGTCGGCGCATCAGATGATGCATCCCTGCGTGGGAACCACGGTGTCGTGGCCTGCGCTGACG GTCCCGGCGTTGAGCGTCGACATCGACTTCGCGCCGACAACTGCGTCCGGAATGGGTGGTCGCACCGAGT CGACCCGGGGGCGGGCGCCGCCGTGGACCCTGTGGGAATTGGACAGATCGGTGACCTTGCGAGTGTGACA GGGAGCCCGGTAGATCAGGCCTACCGGCGGATCGAAACATATCCACTTCCCTGTTTTCAACACTCTTGCA AGGAGTTTCACATGCGTACCAAACTAGTCGCTTCGCTCGCTGCTGCCACCGTCGGCGCATTCCTCGTCGT CGGCTGTAGCGACAACTCGACCGACTCGCCCATGGACGGGCATTCCATGGGCTCGACCTCGTCTGCGCCG ATGTCCTCGGCCCAAGCGAGCACGTCGGCGCAGTTCAACGACGCCGACGTGACGTTCGCGCAAATGATGT ACCCGCACCACGCGCAAGCAGTCGAGATGGCCGCTATTGCGAACGGCCGCACCGACAACCCGGAAGTGCT GTCGTTGGCGTCGGCAATCGAAGCTGCACAGCAACCAGAGATGGACCAGCTGACGGCGTGGCTCGCCCAA TGGGGCCAGCCTGCACCCTCCGCCGACATGGGCGAGATGAGCGGAATGGATCACAGCGCCGACAGCGGCA TGATGACCCAGCAGGACATGGACGCGCTGATGGCGGCCACGGGGCCCGAGTTCGACCGCCAGTGGCTGAC GATGATGATCGCCCACCACACCGGTGCGATCGAAATGGCGAACACCGAAATCGTCGACGGGTCCAACCCT GACGCTCAGGAAATGGCCCGCACGATCGTTTCCACTCAGCAGCAGGAGATCGAGACCATGCAGCGACTGC TCGGCTGACCTGGTTCATTATCGACGGGAAGGCCGCACCCTCTACTTTGGAGAGGGTGCGGCCTTCGATC ATTCGGCGGTCAGGTCTGTCCAGTTGCGAGGCTTAGCTTTCCGACACCGGTGAGCTCCGGGGCTACGCTC CACGTCGGTCATCGCTCACTGCGCCGGTGATGGCGTCGGGTGTCAGATCGAGTCGACGGAGCAACTGTGC GTTGGCGGCGACGATGATCGTCGATAGCGACATCAGGATCGCGCCGATCGACATCGGCAGTACGAACCCG ACCGGTGCCAACACCCCGGCCGCGAGGGGAACGGAGATGAGGTTGTATCCCGCTGCCCACCAGAGGTTTT GCTTCATCTTTCGGTACGACGCGCGCGAGAGTTCGATCACCGACAACACAGATCGCGGGTCGTCGCTCGC CAGAATGACTCCTGCCGATGCGATGGCAACGTCGGTGCCTGCACCGATCGCGATCCCGACATCGGCCTGC GCGAGAGCCGGGGCGTCGTTGACTCCGTCACCGACCATCGCCACGGTGCGTCCTTCCTGTTGCAGCTGGG CGACGTTGTGCGACTTGTCTTCCGGTTTGACTCCGGCGAAGTACCGGTCCACGCCGAGTTCGGTGGCCAC AGATTTTGCGACGGCGTCGGCGTCGCCGGTGATCATGACGACTGTGATTCCGCGTTCGTGGAGTGCGTTG ATTGCGGCGCGTGATTCGGGCCGGATCTCATCGGCGAGCGCGAGCGCACCGATCACGGCACCGCCGGCGA GGACGTGCAGGATGATGGCCCCCTCACCGCGCCACCGATCGGCGACGGCGAGTTCGGACGCACGCTCCTG CTCAAGCAGCGCGGGCCCGCCGACCTGGATGCGGGTTCCGTCGACGTCGGCGGATACCCCGACAGCTGGG GACGACTGGAAGTCGGTGGCCGCCGGCACGGTCAGGTTCCGGTGCCGTGCGGCGTCGACGATGGCGCGTG CAAGAGGGTGCTCGGAATCTGTTTCGGCGGCTGCGGCCAACGCAAGGACCTCATCGGCGGTGCGCCCGCC 
Final Technical Progress Report DOE Award No. DE-FC26-02NT15340

GGTCACTTCGATCGCGGTGACCGTGGGTTCGCCCTTGGTCAGGGTGCCGGTCTTGTCGAACAGCACGGTG TCCACTGTCCGCATGGTCTCCAATGCGAGCCGGTCTTTGACCAGGACACCGCCGCGGGCTGCTCGTTCGG TGGCGATGGAGACCACCAGCGGGATGGCCAGGCCGAGGGCATGCGGGCAGGCAATGACCAGCACGGTGAT CGTGCGGATGACGGCTCCGTCGGGCATCCCAAGCAGCGTCCACACAGCGGCAGTGATGACTGCTGCTCCG AGGGCGAACCAGAACAACCAGCCCGCTGCAATGTCAGCGATGCGCTGGGCCCGCGACGTCGAGTTCTGGG CGTCGGCGACCAACCGGCCGATCCCGGCGAGAGCGGTGTCCTCCCCTATCGCGGTGACCTTCACTCGGAG ACCGGAGTCGGTGGCCACCGTCCCGGCGACAACCTGGTCACCCTCGCTGCGGCGGACGGTGCGCGATTCC CCGGTGATCATCGATTCATCCATACTCGCCGATCCGGAAACGATGGTGCCGTCGGCGGGCACTCGCCCGC CGGGGCGGACGACGACGACATCACCGAGCTGCAGCTGGTCCGGGGACACCTGCACCACGTCGTCGCCGTC GACGCGTTCAGCGGTGTCGGGGAGCAGCGCCGCCAGAGAATCCAGAGCCGACGTCGTCTGCGCGAGTGAC CGCATTTCGATCCAATGCCCGAGCAGCATGATCACAATCAGCAATGCCAGCTCCCACCAGAAGTCGAGCT GATGGTCGAGAACGCCGAGGCTCGCTCCCATCGATGCGAGGAAGGCGACTGTGATCGCCAAAGCGATGAG CAGCATCATTCCTGGTGCACGTGAACGTATCTCACTGACAGCGCCGGTCAGGAACGGCGAACCTCCCCAA ACGAACATCACCGTGCCCAGCACCGGGGAGATCCAGCCGATCCACGACGCGTCGGGTAGCGAGTATCCGA CGAGCATCGCGAAACATGCCGGAGGCGAAGATCACCGGGACAGCGAGGACCAGCATGATCCAGAACAAGC GGCGGAACATGCCGACGTGATCACCGTGCCCAGCGTGACCGCTACCGCCGTGCCCTGCATGGCCACTGTG GTCCGTGTGCCCACCGTGTCCGGAAGGGGCCGTGCTGTGACCGGACATCTCGTGGCCGGCGTCATGACCG CTGTGGGACACCGGAGTGGGTTGATCCGTTGGCACGGCGTCGACGCCGGGATGTGTGTGATCGTTCATCG TGCGTGTCCTCTCGTCCACCGAGTTCGATGGCTACGTAGTGCGCGAGTCTGCGGGTTGCGGTTCGGCTGT GTATCCGGCCTCACGGACCGCTGCGACGACCTGGGCCGGGTCGAGGTCTACGGCGCTTGTCACCGACAGT GTTCCCGCCGACGCGGACGCTTGGACTGTCGCCACCCCGGCCACTGCACTGACTTCCTCTCGTACCGAGG CCTCGCAATGGCCGCAGGTCATGCCAGTGACTTCAGCCGTGGGACGCGAGTTCTGGTCGCCGGTCTGATG GCGACGGCAGCGGTTGCCTCAGGATGCGCGACGGGGCAAGTCGCCAGCGAAGCCGATCAAAACAACCCGA CGTACCAGGCCGCCCAGAAAGAGGGCCAGGTAACCTGGTACACCGCGCTTGATCCCACGACCTCGAAGTC CTTCATCGCTGCCTTCAACGCGCGATACCCTGACATACAAGTGAACCTGCTCCGCCTGACCTCGGGACAA CTGACTTCCCGATACTCCCAGGAGCGCTCCGCCGGTGCCGTCCCCGCCGATGTTGTTACCACGGGCGACG ACGTCTTCGAGGCCGACGCCCGCCAGCAGGGTTGGTTCGAGTCCTCGCTCGAACTTCCTAACCTCGCGTC GTGGCCGGATGCTTACTACACCGAAGGTAGCGCGAAGGTCAGCATCATCCCGCTCGGCATCGCGTACAAC ACCGACATCGTCGACACCCCTCCCAGCGACTGGGAAGACCTTCTCACCGCGGACTACGTCAACCAGCTGC AACTCGGTGATCCGCGCAACGTGCCCGCGTATATGCAGCTTGCCTACCTCCTGCGGCAGAAGTACGGCGA CGATCTCCTCTCGCGGGTACGCGCCCAGAACCCGACGGTCGCCCCGAGCATCGTGAACGCGAACCAGACT ATCGCCGCCGGCGGCACCCGCGCAGTCTTGCCTGGAACCTATTCGCTCGTCGAGGAACTCGCGACCAAGG GCGCGCCGATCGGCTTCGCTCAGCCTGACACCACCGTCGGCGTCGAGTTCCGCACGATGATCTCGGCGGG CTCCGCGAACCCGAACGCTGCACGCTTGTTCATGGATTTCATGCTCGGCCCGGACGGCCAGCGAGCGATG AACGTCCGCGCCTACTCGCCGCTGGGTGAGGAGTACGGAGCGGTTCCCCTGCCCGCCGACTTCCACACCA TTCCGACCTCGGAAATGTCGGGCAGCGAGAAGGAGCTCATAGCACTGCTCGGTCTGCAGTAGGCATCAGT CCGCAAGAAGGAACGGAATACCACAATGACCACACCAACGAAGCGCCGTGCGCTTCACAGCGGCGATCAG GCGAAACGAACCGGCGATGCTGTACCCGGCGTTCAGATCCGTCACCTGACAAAGTCGTTCACCCGCGCCG ACGGAACCGAAGTCCATGCCGTAGACGACATCTCGCTCGACGTGGAGCCAGCAGAATTTCTCATTCTGCT GGGCCCCAGTGGCTGTGGGAAAACGACTCTGCTTCGCGCAATTTCGGGCCTCGACCAGCCGGACTCCGGC ACAATCAGCGTTAATGGGACCGAGGTGTTCAACTCGGCCACGAACTGCGCCGTCCCGCCGGAAAGCCGAC CGATCGGTATGGTCTTTCAGTCCTACGGCCTGTGGCCGCACATGACGGTTTTCAACAACATTGCCTACCC GCTGCGCAACCACAGGGTGCCCAAGAGCGAGATCCCAGCGCGCGTCGAGCAAGTCGCCGACATGGTTGGT ATCACCGCCGTGTTGAAGCAGCACCCGTCCCAGCTGTCGGGCGGTCAGCAGCAACGCGTCGCGGTAGCAC GCGCCATCGTGTCCGCCCGGTCGTTGCTGCTCTTAGACGAGCCTCTGTCGAATGTCGACGCAAAGGTACG CGACCAGCTACGCATCGAACTCCTGCGCATGCAACGCGAACTCGGCTTCACTGCGGTCTATGTGACCCAC GACCAGGAGGAGGCGATGGCACTCGGCACCCGCATCGCAGTGCTGCAGGAAGGCCGTATCGCACAACTCG CGCCGCCCCGGGAGATCTACGAGAACCCCGCCTCGCCCTACGTGGCACGCTTCGTGGGCACGGTCGACGA GTTCGATGGCACGATCGTGGCGCGCAAGCGAGGAGAGGTCGCCGTCGAAACGGCCTCGGGTCTGCGTCTG CCCGTCGCCACCTCGCGCACCGACCTGCCCGACGATGTCGTGGTCATGGCTCGTCCCGAGCGGTGGACGC TTGAGCGGACCGAAACCGTCGATCCCCAGCACCTGTCGGGCGTGATCGAGGCGTCTTTGTTCCTTGTCGG ATCACGCACGGAGTACGTCGTCTCGACGGGCGAGGGCGACGTGCGCGTACTGGCCTCGCACGGCAAGCAG ATCCCACAGGGGAGCACGGTTGCACTGCGCTGCCAGCCCGAAGACCTGCTTATCTTCGGGAGGGACGAAT GAGCGCACTCGACACCCGCACACCGGCCACCGAGACACCGGAAGCCACCTCTTCCCGCGGCGGGGGCATC AGCGCCTTCCTATCGTCCAGCCGGATCGTGTCGGTGCTGGCAGTGCTGGCGGTGGTGGGGCCACTCGCGG TCATGGTGATCAAGCTCTTCTTCCCAGATGGCAAACTCGATCTGTCCGCGTTCTCGAAGGTCTGGGCTGA GCCGTGGTTATGGTCGATGTTGGCCGACACACTTATTGCGGTCCTGCTCGGCGCAGGTATAGCTCTGTTT CTCGGCATGTTGTTCGCATGGATCAACGAACGCACCAACGCAAGCCTGGGCTGGCTCGGCAAGACGTTGC CGGTCGTGCCGCTGTTCATGCCCCCGCTCGCGAGCGCGATCGGATGGGTCCTGCTCGGTACCCCTGGCCC CGGCGTCCTCAACACCACCGCCACCGCGGTGCTCGGATGGGTCGGCATCGATGCGGGACAAGAGGGGCCG ATCAACATCTTCAGCTGGCCCGGCATCGTGTTCCTGTTCTCGGTAAACCTGCTACCTCATGTTTATCTGG TGGTCTCCGCGGCGCTGGGAAACCTCGATCCCGCACTGGAAGAGGCGGCGCGCATGAACGGCTCAGGACC GCTGCGGACCCTGATGACGGTCACGATGCCCGCAATCAAACCCGCCGTGATCAGCGGGGCCATGCTCGCC CTCGCCGCAGGATTCGCATTGTTCTCCGTACCGTTTCTCATCGGCACACCGGCCGGCATCGACAACTTGA 
Final Technical Progress Report DOE Award No. DE-FC26-02NT15340

CCGCCCGCATCGTGCACATGACAGCCAACGTCTACCCGCCCGCACTCGACGAAGCAATGGTGCTCGGTCT CATCGTGGTCTTTTTCATCGGCTCGAGCTGGCTGATCCAGCGGCGAGCCAACAAGCTCTCACGTCACTCG ACCATCACCGGAAAGACAAGCAGCCCTTCCCGAATAGACCTCGGCCTGATAAAATGGCCCGCGCGACTGG TGATGATCGGCTACATCTGCTCGGCCATCGTGCTACCGGCGATCGCTCTCGTGTTCGTCTCCCTGCAGAG CTACTGGTCGGGCAAGTTCGATCTCGACACGCTTTCCCTCGATCATTACCGACAATTGTTCGCCCCGTCA TCGAACACCCTGGCGGCGTTACAGAACAGCCTGGTGCTCGGTGTGGTCACTGCTTCGGTGGCACTGATCG TCACCGCACTCATCGCCTACTACGGTGACCGCCGTCCCGCGAGCTACCTCGGTCGTTTCGCGGATGGAGT GACGAAACTCCCAGCCGCGCTGTCGCACGTTGTGATCGCGGTAGCCCTGATCGTCACGCTCGCCGGCTCA CCATTCGGGTTGCAGGGCACCGCCGTACTTCTGCTTCTAGGTTACCTAGTCATCTATCTCCCCCAAGCGG GTGTCGCCGCGAGTTCCGCTCTTGCACAGATCGGCCCGACGATGTCGGAGGCATCCTTGATGTCCTCGGC CTCCCAAGGACGGACCTTCCGCAAGGTCGTCCTGCCGCTCATGTCGCCCGGCCTGATCTCGGGATGGGTA CTCGTTTTCGTGCTAGCCGCGGGCGATCTCACCGCGTCCGCGATGCTTTCCAGTCCCCGCACTCCGGTGG TCGGGTTCGTGATGCTCGACCTGTCCACGAGCGGAACCTACGGGGTGGTGGCGGCGCTCGGCGTCGTGAT CACGCTCATTTCGGCAACCGTCGGTTCGCTCGCGCTGGCATACGGGCGCCGGGTAAGCGGACGCCGCACT CGCCAGTCGATCTGAATCTGATCACTCTGCACCGAAAGCACCGAAAGCACCGAAAGCACCGAAAGGATGT TCATGACCACCCGTTATGAATACGCCGTCGACTTCGACGCGATCACCGCGCTGGACTTCCATACCCACGT CGAGATCGACGGATGCGGCCACAAGGCCCTCGATGACGAGCTCATCGCCGCCTCCGAGAAGTACTTCAAG TCCGGGTCCGATCGCACTCCGACGATCCAGACCATCGCACAGATTTACCGCGAACGGAACATGGCTGCCG TCGTCTTCACCGTCGACGCGAATTCCGCGACGGGGCATCCAGTCAATTCCGTCGAGGAGATCGCCGAGGT CGCCGCCGCGAACAACGACGTACTCATCCCGTTCGGATCCGTTGACCCTCTGCAGGGTCGCGCGGCCGTT CGCCGCGCACGCACCCTCATCGAGGAATACGGTGTCAAGGGGTTCAAGTTCCATCCCTCCCTGCAAGGCT TCGAGCCCAATGACCGTGACATGTACCCCTTGTACGAGGTACTCGCCCAGCACGGAGTGCCCGCCCTGTT CCACACCGGACAGACCGGCATAGGTGCGGGCATGCCCGGCGGCCACGGAATCAAGCTGCGTTACTCGAAT CCGATGCTGCTCGACGACGTCGCCGCCGATCACCCGCACCTCACGATCATCATGGCTCATCCTTCCGTTC CGTGGGTCGACGAACAGATTTCCATCGCCACTCACAAGGAGAACGTGTACATCGATTTGTCGGGCTGGTC GCCGAAGTACTTCCCGCCGCAACTCGTCCGTGCCGCCAACACGATGCTGGGAACGAAGATGCTGTTCGGT TCGGATTTCCCGGTGATCCAGGTGGATCGGTGGATGTCCGATTTCGAGTCGCTGGATATCAAGCCTGAGG TGGTGCCACTCATTTTGAAGAAGAACGCGCTTCGGGTGTTACGGATCGCGACGTAGGTCTGCTCCGCTGG ACGCCGATTCCAACGAATACCTTGGCCGAGCCACCGACCGCACGTTGCGTTGGAGGAACAGCCGTGCGTC CAGGCGCATCAAGGCCGAGTGACCGTGATGAACGCGACATCTCGGGGTATCCCGAAGGGGTGCCGTTCGG CTCGACGGTGGATCCGGGCCGGACGAACGACGGTAATAGTTGAGCTTGGAAGCGCGCTCCTTACCTGTGG ATCCCGGGTGGGCGTAACTTCCCGGGATGTGACTCGTGGTCCGCAACGGCTTTTACTAGGGAACGCAACG TGCTGACCTCTTCGGGTGTGATCTCGGTGACCGCACCACGAGTCAACGACAAACGCATCGGCCTGGAACT GTTGAACGGCAACGATACTCGTCGACGATCCCTCCCGCCTTGGTGCGCAAGTCGCCGCAGACGGCCGAAG TGTTGCCGCTGCTGTATCTGCATGGTTTGTCCACCAACGATTTCGAGCCGGCGTTGGAGCAGTTCCTCGG TACCGGCGCGCCGGGTTATCGGCGTCGGCGATCACCTGGCTGACCACTCAGTGGCAGGACGAGGCGAAGG GTTTCAGCCCGAGGGTGATCTCTCGAACTTGGATTGTGTGTACCTGCGGGTCGACGGAATCCACCTCAAG TTACGTCTCGATCGACGATGTAGACAAACCCGTCGTCGTTCAGATATCGGATGTCTCCCATGTGGTGCCA ACCGGATTGACGCAAAACTACGTTCTCGTCGGCGCGGTTGAGATAACTAGGTGCGACGAGGGGACTTCGC AACTACCAGCTCGCCAGGCTCTCCGGCAGGCAGGATCGCGATCCGCGGTGCTCCGGGAGCTGATCAATCG AGGCTCGACCGGCGCCCGCTGCAGTCGCGGATGCGGAGCAGCAGCTGGGGATGTCGCCGGCGGTAGGTGT AAATAATGCTCGGCAATCTTCGCAACGGCGAAGGACAGTCACCGACCTTTGATCGCCGCTCCATCGGGCG GCCCGGGTCGTTTCCCGCGTGCGGCCCGAGGGAAGAAGTGATCACCGAGTACATCAACCGCCAATTTCTG GATCGTCAAAACTCAGTGTCACTGCGGTGCACGTTTCGAGACGATGTGCACTCCCGCACGTGAGGAAGAG TGGATCGAATACCTGGCCGACCGCGGAAAATGCGATGTCGAGGCCAAAACCGTGGCCACACGGAACTTTC GAGTAGCGAACTCCGCGGTCACAGGATGTTAGGGTCGGTGCTATCCACACGGGTGCCCCATTGCAGGGGC TGAGATCGGGCTGATGCAGCCTGCGACCGTTGAACCTGTCCGGGTAATGCCGGCGAAGGAAGAGGAGATC CATCATGAGCGCATCTGCCCAGCATGTCTTGGCTTATAACACTGACCAGGAGCAGGTCATTCGTGTTCCA GTCACGGAAATCTCCCTGGACGACAGCCCCGATGGCGTCTCGAATCCGCCGATTCGGATCTATCGCACAG CTGGGCCCGGCAGCGACCCGGTGGTCGGGTTGCCGCCTCTGCGGGCGTCCTGGATCGCAGGCCGCGGCGA TGCCGAGACTTACGCGGGCCGGGAGCGAAAACTGCTCGACGATGGCCGCTCCGCGATCCGGCGTGGCGCA GCGTCCCAGGAGTGGAAGGGCGAACGGCCGGTGCCGCTGCGATCGATGAACGGTCGTACGGTCACCCAGA TTCACTACGCCCGCGCCGGAGTCATCACCGAGGAGATGCGATTCGTTGCGTTGCGAGAAAACTGCGACGT CGAGCTCGTCCGTTCCGAGTTGGCAGCAGGACGGGCGATTATCCCAGTCAACATCAACCACCCCGAGTCG GAGCCGATGATCATCGGCAAAGCATTCATGGTCAAGATCAATGCCAACATCGGTAACTCGGCAGTCACCA GCTCGATCGCCGAGGAGGTCGACAAGATGCAGTGGGCCACCCGGTGGGGTGCGGACACCGTGATGGACCT GTCCACCGGTGACGACATCCACACCACCCGTGAATGGATCATCCGGAACTCCCCGGTGCCCATCGGCACC GTGCCCATCTATCAGGCGCTGGAGAAGGTCAACGGAGAGGCCAACGAGCTGACCTGGGAGATCTTCCGCG ACACTGTCATCGAGCAGTGCGAGCAGGGCGTCGACTATATGACGATCCACGCCGGGGTGCTACTTCGCTA TGTACCCCTGACCGCCGAGCGGGTCACCGGCATTGTCTCCCGCGGCGGGTCCATCATGGCCGGATGGTGC CTGGCCCACCACCAAGAGAACTTCCTCTACACCCACTTCGACGAGCTCTGCGAGATCTTCGCCCGCTACG ACGTCGCCTTCTCCCTCGGCGACGGGCTGCGTCCCGGCTCGATCGCCGACGCCAACGACGCCGCCCAGTT CGCCGAGCTGAACACTCTGGCCGAGCTCACCACACGAGCCTGGGCGTACGACGTGCAGGTCATGGTGGAG GGCCCCGGCCACATCCCCCTACACCTGGTGCGGGAGAACGTCGAGCGACAACAGCGCCTGTGCCACGGAG 
Final Technical Progress Report DOE Award No. DE-FC26-02NT15340

CCCCGTTCTACACACTGGGGCCCCTGGTGACCGACATCGCCCCCGGTTACGACCACATCACCTCCGCGAT CGGGGCCACCGAGATCGCCCGGCATGGCACGGCCATGCTCTGCTATGTAACCCCGAAGGAGCACCTGGGA CTGCCGAACAAGGACGACGTGAAGACCGGGGTCATCACGTATAAAATCGCCGCCCACGCCGCTGACCTCG CCAAGGGCCACCACGGTGCCGCGGAGCGCGACGACGCGCTGTCGAAGGCCCGCTTCGAGTTCCGCTGGCG CGACCAGTTCGCCCTCTCCCTGGACCCGGATACCGCCGAGGCCTTCCACGACGAGACGTTGCCGGCCGAA CCGGCCAAGACCGCGCATTTCTGCTCGATGTGCGGGCCCAAGTTCTGTTCCATGCGCATCTCCCAGGACA TCCGCGACCAGTTCGGCAGCGCTGCCCAGCAAGAGGCCATCGCCGGGATGGAGGAGAAATCCGCCCAATT CCTCGGATACGGCGGCACTGTCTACCTTCCCGCCCCCACAGTCTCGCCATCCTGAGCCCATTGGGCAGTA CAGATGCACTGACCCCGCAACAGCGATGTGAAGTCGGAGCTGTCATCCCGGCGAGTTGAGGGGAACGGTG GCACACGGTACTGCAGTTCTGCAGTCCCTACCCGACTACACTGCTGCCGCAACACCTTCTCGAACCCA

\section{>IGTS8 pSOX plasmid - contig001}

GATCTCGGTGGTGGTGTGGCCGTTGAAGATTTCACCGAACGAGCCCTTGACCAGGCCGCGCAGAGAACGT TGCAGATCGCGGGTCGCTTCGCGGTACTCGTGCTCGCCGACGGCGGCCGCGTTCTCCATCAGCTCCGGAT GGCCTTCTTTGACGAGCTTGTACATGTCCTCGATCAACGGCGGGTTCTCCGCGGTGAAGCCCTTTTCGTT GTAGAGGATCTCGATGCAGGACGAGATGATCGTTTCCTCGTAGTCGCGCACCGCACTGCCACGGGATAGT TCGATCAGTCCGGACATGGTCTGCAACTGGCGTGCCCGTAGTTCGGTGAGCATCTTGCGGGCCAGCTCCG GGAACACCGCCAGTCGAGGCAGGATCGAGCCGAGGACACCACCGGCGAGAGGGTTGACCCGGCCGTACCC GTATCCGAGGTCGATGACCTGACCGTCGACGGCCTCGATCAGATTGCGGTAGTCCGGCTTCACGTCGGCG AGAACGAGGGTGGTCACTTTCTGCGCGACGCCGCCGAGAACCATCCGGCGCACGAACGAGGACTTGCCGT AACCGTTGAGTCCGAGAACGAACCCGACCGGGGCGGTGATCAGGCCTTCGACGAACCAGGACAACAGGTC GAACAGGACCGGGGCGCCGGTGAGCAGATGACGCCCGACCGGTGTCCCGATCAGCGGAGCACCAGCTCCG ACGGCCCACGGCCACATGCCGGCCGCCTGCGCCGAGGAGGCACGGTATTCGACCTGACGGCCGACCGAGG CCATCCGGCCACCGCCCGCACCGCGATAGCCGCGGTCGGAGAGCTTGAGGGCAGGCAGGAGGAACCCTTC TTGTTCTTCGCTTGCCTTCTGCGCCTCCAGGTTCCGGCGACGTTTGTCGTCCATCTTGAGGTGATGGCGA GCCAGTACACCGGCCAAGGTGGGGCCGTGGGAGCGTGCCAGCACTTGCCGGCGCTGTTCTTCGGTGAGGA GCTTGACCATGACCGAGCCCCCTATCCGGCCATCGCCGACGGCATGGAGGCGTGCTCGGGCAAGATGACG CCGATGCCGAGGGAGCCGTAGAACCCGGCCGCCTGGTAGCGGTACGCGCGGCGCACCTTCAACCGCGCCT GCACGGACAGGTCCTTGATGATCGCGTCGATCGCCGGAATGTCCCCGTCGAGAGGTTCGGTGATGGTGAT CAAGATGCCGAACCGCACCAGTCCGTGACCACGCGCCTGTTCCTCACGGGCACGGGCGGTCGCTTCCACC TTCAACGTTGCGGTGACCGAGCCGATGCCCTTGCCGCCCTGTTCGGCGATCAGAGCGTTCTTGTAGTCGT TGTCGACGATCTTGACTGCATCCGCGGCCGTGTGCGGGCGGTAGACGATCGCGACCCGCTTACGCGGAAT GTCGGGGTTCGGAGCGAGGATGCGGCGCAACACGCCTTCGTCGACCGCGCCGGTCGGCGCCATCTCCATT TCCCAGGTCACCGAGCGGGCACCGTCGTGGATGTACTGGTCGAAGCGTTCTTCGTGCGAGATCGGGCCGG CGTCGGACCAGTCCAACCCGTGTCCTTCCACCGTCGCGTCGGCGCGTTCGATGTCTTCCTGCGAGGCCGG ATCAGTCGAGCGGCGAACGAAGGAAATGACGTCCGAGGCTGCCATCGGGCGGACACCGACTCCGGCGGAG CGCATCGCGGCGCACAGACCGGGAAGCCTGCGACCGATTTCGACGGCCTGCTCCGAAGGGTCCTTCTTAC GAGCATCGGTGGTCGCTCGGAACGTGACCGCCAAGCGCGGCAGAAGTTGAGCCTGCTCGGTGGCGAACAG TTCAGCCGCCTCGTAATTCGAGTCCTGCGCCAACTGCGGTGCATCCGGTTCGGTCTGGCGAATGACCTCC GCCAGCAACCGGTTTCCGGTCTCGGGCACGCAGTCGATCACCGGGACGACCGAGACCACGTCCGAGGTCT GACCCATCGAGGCGAGGACGACGCCCCACTCCCCCACCCATTCGTTGATCATCGACTGATCGACCGCCTC ATCGCCCTGCGGCCACGCCTTGAGGACCACCGTGTACAGGTGAAGTTTGGGCATGTGGATCATCCCGAAC CGGTACCCGGCGGCGTCCTCACCCTCGTAGAGTTTCGTGTCCGCGGCCACGCCGGGCAGAAGTGCCTTCC CGCCGGGGATTTCAGAGAACAGTCCACCGCGGTAAATGTTTTCGCGCCGACGGCGCGTCCGGAGCCATTG САTTCTCAATACCGTCAGTTCGTATCCACTCCGTCCGTCCCTGCCCCACACCAGCGGGGCAGCAACGACG ACGGAAACCGCCAACACCGTCAGGCCGACCTTGAAACCACCGACCAACATCGATCCCATAACCGCGATCA CCAGCACGAAGCAGACGATCGTCGTGCCCCACGTCAGACCGAACAACCCGGAATTACGTGGCTTGGTCCA GCCCGTATAGAGCAAACCGTTCTCGTTTGTGGTGCTCATCGCCGAATCGTGTTCCTTCCCAGATCGGTCT GACTACTCGAATACCGGTCCCCGGCAAGGTCTCCCGCGCCGCGATCGGAGGACTTCGTGCCGGAGCCGAC TGCGCCGATCGCCTTACTCGCCTTCGATCCGCCGCCGGAACCCTTGCCGCCACTGCCCGCGCTGCGATAT CCGCTGGCACCTTGGGACGTGCGGCCCGATGCCGGAGAGGTCCGCGTGTTCGGCGGCTGACCGAACGCTC CTCCGCGGCCGCCGCCGCGTGAACCTCCGCCGCCACCGCCACCGCCTCCGCCGCCACCGCCCGGACGACC GCCGCCTGCGAAGGACCCGCCGCTGTTTCCGCCGCCGGGTCCGCCGCTGAATGCGCCCGCAGACTTGCTG CCTGCGCCGGCCGACTTACCGGCCGCCATCGACTTGCCGCCGGTAAATGCCGCAGCACCGGCCATCAATG CCATGCCCGGTCGCCGCCGCGCCCGAGCCGCCGGACCCCACGGCTGCGACCGCCGGAGCGATCAACCGCT GCAACGCCGGCAGTACCGCAGCGGCGAGCGCGAGCAAGATGATGCCGATCAAGGACTGCTGGAACTGATC CGCCGACGGCGTCTCGCCTTCGGTGATCCGCGCCGAGTCCTGAGCGGAAAGGAATGCCATCGCATACACC AGCGCGCCGATGGGCTTGAACAGCAGAAACGCGAGAATCCAGGACACCAACTTCTGGTACGACTGCGATC CGGTACTCGTTCCGGACGCAGCGGCCGCAACCGGCACAGCCGCGACAGCCACGATCAACAACCCTTGACG AACCACGAGCAACGCAGCCTGAGCCAGCGCGCCCAAGATCCCCACCAGGCCGATCACGAACAGCAGCCCC GGAGAAGCACCGGTGAAGGCACTGACCGCGAGCATCGAGTTCACCATCCCGATCGGGTCTTTGTTCGAGG TGTCGTCGACGATCCACTCGGCGAACCGGTCACCGGCAAGAGTGGCCAACGCGATGATCGAGGCGAACAT 
Final Technical Progress Report DOE Award No. DE-FC26-02NT15340

CGTCGTCGCGAACACCGTGCGCGCCAACGCCTTGTACGTCTCCTCCGCTTCGTTGAGCAGGGCGTGACGT TTCGCGGCCGCCAATTTGATGGCCATGAAAATCACCGACGCGGTCAGCAGTGCCAACTGAATCCAGCCGG TGTACTCCGTGATCGCCTGGAGCGGAGTCGAATTCGCCAGGTCCGGATTTGGCAGCTTGATCCACCACGT CATCGCCATCGTCAGCACCTGAGCCATAGCCTCGAGGAACGACTTGACGATGTCGCCGAACTGCGAATCC CACATCTCGTTGACTTTGTCGCCGGCGAAGGACGTCACCGCAGTGACCGGATTACCTCCGGCATCACAGA TTCCTCCGGCAGTTGGGCTGATCAGGGCGCCGCTGCCAGGAATGATGACGTTAGCTATTCCTCCAGCGAT GCCCCCGACGGTGTTGCACAGACCGCTCGAATCCTCTTCCTCGGTCTGTGAATCCCCGGGAGTTTGTGGG ACATCCTGCGCGGAGGCGACAGACGCACTCCCGAACATGACCAACAGCGCGAGGACGAGCGCGAGGACTA CTTTCCTGGCCACCGCGTCCACCCCGCTAACGTCGGAGTCTGGCCGACCTCGTCGGCGTTGCGTTGTGTG AGCTTTAGAGACCAATCCCCGTCCCGCCAGAGCAACTCCAACGGAACGGTGGTCCAGCCGTTGCCGGAAA CGTTGTTCCCGGCGTATTCGACAACGGCGTAGTCCGGCGACCAATACTTGAGGCGGTACGCCTCCTGCCG GATGAGTGGAACGGATACGCGGCCGGTTTCACCGTTGGACAGGTGTTGTTTCGCGTTGGAAATGTTGTTC GCCCGTTCAGCTTCAAGTTCAGGTGTGGCGGCGATCGTGCGCTTTTCCATCACGTCGAGGGCACCTTCGA CGCTCGACGCTGCTCGATTCCACAGACTGATCCCAGCAAGCGCTGCACCTTGGGGTGTCTGGGCGAAACC GGTGGCCAGGGCGTCGTCGACCTTGGACGGTCCGTCCGATGTGGAGAACGGCATCGGGAAGTTCTGCACC TTCTGCCACATCACCCCGGCAGGCGGAAGTGCTGCGTAATCAGCCGCGCCCCGGTCCGGTTGGCCGGAAC GATCCTGATCGAGAGCGACACCGTTCGGATTCGGTGGTACTTCGACCCGGAAGCCGAGTTCGTCGATGTA CGGCAATCCCCAGTCCGAGCGGTCTTGCTGAGGAGCGATCGTCGTTCCCGGTTGAGGCTGCGGGGTCGGT TCGTCGGTCTTACTGCGATTGATCACCACGGCGATCACCCCGCCCACTACAACGACGACCACGATGATCA GCGCAAGGTAGAACGTCCATTTGCGACCGATGAAGGACTCTTCCTCGCTGCTCTGCGGCTGCTGCTTCGT GGCCCTCATCGGTTGGCCGCCAATGCAATCGCCGACGACGGAAGCCCGTCGACCGAACTCACCCGGCCGC TGGTCTCGTCGTCGCCTGGGAGCAGTAGCTTCCAGTCGTCGCCCGACCACACCACCGTTACCTGTGATGC TGCGAATGACTGATCGGGGAACGCGGAGTACACTGTCACCGCTGCGTTGTCGACGTTGTAGTCGCTGACC TCGTAGCCCACGATCTTCGGTGCTTCTGTTGGAGCCGGCGGGGCAGTGAACGAGAGCAGCTGTCGGCTGA TCGCGAAGTCGTCACGAGCAGGACCAGGCGCAACACCTGCGGCCACGACACCGGGCCACTGGTGATCCGG AGCGACCGAAAGCCGCACCGTCTGCTGAATCGCAGCGAGCGCCGCGCCCTGCGGATCGTGGGTGAAGCCG CTGACCGGGCCGGAACCGGAGTTGGTTTTCGGTCCCTGATCTGCGACAGGCAACGCGATTGTTTGGTAGT CCTGCCACCGGACGTTGCTCGGCGGTGCCGACACGTCCGGAGCGTCGGTCGTCGCTACGTCGGTGGGAGT ATCGGAGTCGTCGGACCCGCATCCTGCCCCGACCGCCACGACAGCGGCAGCCATGACGGCGAACCCTATG CGTTGGGTGAGTTTCATTGGAAGGAAATGCCTTTCGTCAGATGAGGGTCAGAACGACAACAGGGCGGCAG CGATACCGGACGCACCGGATGCCATGACCGAAACGATCAGGATCACGACAATCGTTTGGGCGGAATCTGT TTCGTGTGGTGACTGTGAGCGGGTGTATGCCAGCCGTCCTGCCGCCCAGATCATCGAGATGGTGAAGATC GCGAACGCGAACCACAGCAGGTAGCGCAGCAGGGTCATGATCGGGTCGGTGACTTCCGGCGGGGGCGTTT TGAATGCCAGAGCTGTCTGTGCGGCCGCCAGCGTGAGGTTCACCATGGGGAAACCCCGTTAGCTGCGGAG ACTCCCGCGGTGACGCTCAGGACACCAGCGCCGAGCAGGGCAGACCACTGCGCCCGATCCGACATAGAGC ACGGGGTGTACCCCCGAAACCTCTCCCATCCCGTGACCGCAGCCCCGCCGACCGTCGCGACAACCGCGAT GACGGTCACCGACCACAATGCCCACTGCGCCGCGCTCACTGGATCGGACCCACGAACAGGAACGTGAGCA GCGCGATCACGACAACGACGATCCCGGCGACTGCCAGCAGAAAGCCCCTGTCCAGCACGCGCATACCCGT GGCCGTCGGCCGACCGTTCGCGTAGTCGTCGGCCGCCTTCGCATGGCCCTGGGTGCAGAGACGATTGCCG TACAAGGCGAGCGTGCCGCCTCCGAGTCCGACCAGGAGCATGATCACGCGGGCAATCACGACAACACGAC CGTGTTCATGATCGTTCCTGCGCTGGTGGCGATCACACCGCCGATGAGTGCGCCGACGACGATCTTCGGG GATTCGAGCTGGCCGCCGGAGTACTTTTCCCAGCCGAACTTTCCGCCGGCGTAGACGAGTGCGCACACCC CGGCGAGGACGACGCCCCACAGCAGCCACCGACCGAGTCGGAGGAATCCTTCGGACTTCGGAGGCGCTTC GGGTGTGAGGTCGATCTGAGCGAGAATGTCGATGCCGGTCTGGAGCGTGTGAATAACCATGTGGTGGGCG ACCTTTCACTTTTGTTGGGTTTTGATGCGGGCGATGATGGATTCGCGTAAGGCCTTGCCGAGTTCGGCGA TGTCCGCCGGAACGTCGTCGGGGCCGCGTTTGCGTTCCGGTTCCGGATCGAACGGTGACCACACCTGCAA CGAGGTCGGATCACGTACCAGCGGATACTGTTCGATCCACCCGACCCGCCACTGCGCTGCCGTGAGCGAG GTGACGAGGTCGAGAGTCTGTTGAATCGGCTTGGGCACCTTGCGCCCTGGCCGATCGGCGACGGTGATCA AACCGACGACCTGGCTGGGTCCGCCCTTGCCCGCCGCGTGCTGACGCAGCAGCTCGTGAGCGCGCACGAG ACCGGACACCGTCTCGCGGGCGACGACGGCAACGAACGGACTCTGCCCGCCGTGACCGCCGGGCCACCGG CGACCACATTCAGCAGCGGGCGCGAGAACCTGCGCCAGAGTCGACGTTCCGGCCCCGCCGTGAGCGCCGA GCAGCCACACCATCGGCTTCGGAGCACCAGGCGGCAGCGGGATCGGTTCATCCCAGATCGGCGCCACCGC ATCAGGAAGACGAACCGACGCATCTGTGAGGTCGACCCGGTGTCCCGGTTCGGCGGACAAGCGTTGTGCA CCCATCAGCGATCACTCCGGCTGTCCGCCGCGCGCACACTCACACTGCGGATCTGCTCGTACGCGGCAGC GGTGTCGGGTGCGAGGTCGGTCGCCGAGATCGTCAACCCCGACGCAAGGTGCTGGTCGTACGGGATCTCC TCGACCGCGAAGACCTTGCCGGTCAGATGGTCGCGGAGCTGCGAGACATCGGCAGCAGAAGGGCCTGCGG TCTGGTGCGAGATGACCACGATCGTGCGATCGAGCGCCTTCTCTCCGGCGGTGAGCCGGATCGTCTCGAG GACGGCCCGCATCCGGTTGAATGCATCGGCGCGGCACGGTACCGCGACAACGATCGTCGACCACGGTTCT CCCCGGAGCGACTGCGCACTCTCGCGGCCGAACGCGCGGTGTCCCAGGTCGTAGACCGTGGTGTCGACCT GAGCGCGGACGTACCGATCGACCTGACGGAAGTCGGGTTCGATGGTTTCGAGCCAGTGGCGGCCGAGGAC GTGGGCGCCGGCCGAGGTTGCCGAGGTGGACGCGCTGAACGACTCGTCAGCGTGCAGCGTCTCCGAGGAC CCGAACATCGTCTCGATCGAGGTTTCCGAGACGGTCGAATCCGCTCCGCGCTCGACCAGGTCGCCGCCGC CGACCGTGGCATCTACGACGGCACTCGTCGCACCGATCGTCGACCACGCGTTCGCGAGACCGATCGCCGT GGTGGTGCTCCCCGCTCCCCCGCACGCACCGACGACCAACGTCATCGGCGGGACGGGATCGAGAACCTGG 
Final Technical Progress Report DOE Award No. DE-FC26-02NT15340

GCAGCGGCCGTGGATGTTTTGGCCGCAGAACTCTTCGGTTCGGGAGCGACGAGCGGAAACACCTGAGCTA CCGGCGCTTCCACGACCTCCGGTTCCTCACCGAGCCAGCCGAGCCAATCCTGAGAGTTGTCGTTGTCGGT CACGATGCTTTTCCAATCGAGGTGATGAGCGTGCGGCCACCGACATCGGTGACCTTGACGATCTGACGGC CACTGACGCGGGAGCCGTCCAGATAGCGCTGCTCGAGGGTGACGGCGAAGGTGTTCGGTTCGGTTGCGCG GATCTCGACGCAGTGCTCGGTACCAGCGGGAACCGTGTCGATACCGGCTTGCAGTTCTTCGGCGGTTGCC GACTTCACCTGCGCATCGGAGACAAGCAGTGCGCGGGCCTTGGCCCCGTTGCGTTCGACGTAGTAGGCGT GATCGAACGCGAGAATGGCATCGGGACCCGACCCGGTTCCGCCGGGGCCGGACCCGCGAACGACTCCGGC CTCCGTCGACGGCGAGCACCACGACTGCTGAGCCACCGAGGTCGGAGTCTGCGAGGTGGTTGCCGTGCTG GTTGTTGCGGGAGCGATCTCCGCGACGTTCATCGGTGCGTCGTCGTCACCGCCGCCGAGCGCAGAAGCGG CGATCGCACCCCCGCCGATCACGAGGACCGCGACCGCTGCGAGCGCGCCGATGACCGCAGCGCGGCCACG CAGGCGCTGCGACGGTGAAGCCGAAGTAGCAGCCAGGGGTAGCGGATCGGCCGCTGCCTGGTCCTGCGTG TACTGCATCGCTTCCCGCAGCCACGCCGCGCCGCTGTCGTCGACAGAGACGTCGTTGGAGTCAGCAGCAG CAGCAACAGACGGCGCGGGAACGGAATCGGGTTTCGGAGCGGCCGGAGAGGTGTCGTCGTCGGACGCGAG GTCCACGACCGGAAGCGGCTCTACCGGCGCAGAGGTCTGCGCTCTCCCGTCCGCCGCAGGCGTGAACGGT GGGAACCACTGGTCGAGAACGGGATTGGAAGACTTGGGATCGTCATTGTTGTTATTTGTCACCATCGAGT CCTTTCTCCGGAGGTCCTGGTGCGGGGCACGAACACCCCGCACCAGAACGCCCGATCAGTTCGCCGCAGC AGTCGGTGTAGCGACCGCCGTGGTCGAGGTGATCGAGGTGGAGGTGCTGTTCGGACGGGGACTCGTCGTC GTTGTCGCCGAAGAAGACGAGGACGGGCGAGTCGCGACCGCCGTGGACGTGGCCTTCGTAGTGGAAGAAG GCGATCCACCTGCACGATCTGCCAGGTCCTTCGCCAACGCGACGAACCAATCGGTGACGAACGCCGTCGC CGATGTCCCGGTCGCCGTGGCCGGCACCGAGCCGTAACCGCCGTGGGCGGCCACCGTGGACGAATACTTC GTCAGCGTCGCAAGGTTGAGCAGATCCTTGTTGTCACTGACGTTGTCCTTGACCGCCGCGAACGCCTGCG TCGTCAACCGAGACGTAGTGGCCGGCGGAATCAGCTTCGTTGCCGCTCCGACCAACTTCGTGCCCAGAAG AGCAACCGCGCCAGCGGGATTGGCCAGCCCGACCGTCGCCAACTCCACGATGTTCGCCGGGGTCAGAACT TCCTTCGCGACAGTCTTGACCGCGTTGAACCCGATCGTGCCGACCTTCATGATCGCCTTGATCGCATCAT CGGTGGACGGCAACGGAGTCCGAGGATCGGAGGCCACCGCAAGACGCTCGGACAGCGACTTCTTCGGCGC GATCGACAGATTCGCCAACGTCGTACCCGAGATGTCCTCGTTGACCACGGTCACCGCAGTCTTGATCGAC GTCATCGCCAGAGCGTCACCGACCGAGGTCAACGACCGAATCGGATCACCCGAGGACAGCTCCGACTGAC CGGCAATGTTGGTCACCACCCGCAGGATCGGAGAATCCGACGGCGCATCGCACGCGAGGTCACCGGACTG GCAGAAGCTCGCCACCCGCCCAGTCAGCGAGCCGTAGTTCTCCGCCACGTCCGCGACCGGCCCGATACCG CCACCAGCAGGAGGGACCTGATCGAGAGCGGAAACCTTGGACACCTCCGCGCCGTCCGTTCCCGGAGCAG GCTTCGGGGACGTCTGACCGGGCGAGCCCGGAAACAGCGGTGCACCGGCAGCGCGGGTCGGGTCACCGAA CGTCGCGACACCGACAACCTTGTCCGCAGCGATCACGCCCGAGCCCTTGCCGACCTCTTCGGCGAACAGT GAAACCACATGTGCGCCTTGGCTGTAACCGGCGAGGGTGAACTTCGTGTCCGGGCACGCAGCCGCGACCT CCGAAGCCATATCCCGCAAATTCTGCAAGCCGCCCTCGACGGACTGCGAATACGGCACGTTGCCGCCGAG AACAGCGCCGCCGAACCCGGCCTCGTACGGCACGTACGCGCGGCCGGCCAATTCCTTGTCACCGTCGACC GCCTTCGCGATCATCGGCATGAACACCTGCGACAACATGCCGGTGTCCGTCGTCGGCGCTGCATCCGGAG CAGACTGCCCTGTCCCCTGCACTCCAAGGCCGAACAGCGCGGGGCACTCCCCCTTCACCAGATCGGTACC GGACGCGGGAGCTGCATTCGCAAGCGGAGCAAACGAAAGGACCACGGCGGTAGCACTGACCAGCGACAGG CCCACTACCGCCGACCTGGCACCGAGACCAGCAGTAGAGCTGATCCCGGTGCCCGTCGAACGAGAACGAG AACGAGTGAACATCACATCAGATCCCTTGATCGAAGTTCAGAGCAGACAACGCGCCCACCCCGGATGGAC GGCTCACGCCGGGATACCGGCACCGATGGCGTCGGCGATCATCCCCAGCGGAAGACCGGGCACAGCGGTG TTGGCCTGAAAATCCGTCACAGCCTGGTCGAATGCCGCGACAGCACCCTCACCGCCGGGCAGCGAGGTCA CAGCATCGCGGATCTGCTGATCGACAACTGGTGCAGCAGCAGCGGTGTTGCGGACCGCGTCAGAGGCCGC AGTACCAACCGGCGGGCTAGCGGACCACTGATCGAGCGTGGTTTCGGTCTGCTCAGTGATGGCGGGCTGA TCAATGTCCGGGATCTCGAAATCGATCGGCTGCCCCAAATCGCCTGCACCATAAGCAGATGCGGCAAGAG CGCCACCTGCGCCGCCGACGACCGCGCCACCGATAGCGGCAGGTACGCCGAGCACAGCTGCACCGATGCC CGCACCCGTCGCCGTTCCCACAACCCCAGCCGGRAACCGCACCGATGGGAGTCACGATCGGAGCGAACAA CGTCAAGGCGCTAGTCCCGCCGATGGTTCCCCCGATCAACGCGCCAGCGGTTGCAGCCGGAACAGCCGCC GCCGTAGCGCCAGCGAACGCACCAGCGGCAGCGCCGCCGACCTGAGCTGCTGCGAGACGTTCCGCCTCAT CGGCCTCGAGGCCGGTCGAGCGCCAGAAGTCCGTCACCTGAGCTTCGATGACAGCGGTCTGACCGTTGGT CTTGTCCGCGGTGTCCTTGGCGATCCAATTCGGGCCGATCAGCGAGGTAGCGGCCGAAACGGATCTTGTC TTCCGGAGCCTGAATCGGTGCCGTGAAGGTCTCGAGCTGCGTCGGGAGGTGAATCGACGTGTAGTCGACA GGAGCGACATAGTTGTCGTTGCTGTTGGTCGGCGGGGTGTACTGGTTGGTGTCGTAGTCCCAGTTCGGGA GCGGCTGCGCAGGCTTCTGGTACTCGACGGGAGCCGGAACCCACGTCTGCACAGGAGCGGGTTCGGGCGC CGAAGGAGCAGGTGTGGTCACTCCCGGCTGGCTCGGCACCGGAACCTCACTGGTGACGCCCGGCTGATCG CCCGGAGCAGCCACAGCAAAACCAGCCCCTCCGAACGTTGCCACCACCACAGCGGCAAACGAAACCGTAG CGACCGCAGTCGTAGACGGGGCGCGATGACGACCGCCCGAACGTGGCTTCTGGAAATGCTTCATGAGTGC ССTTTCAAACTTGTTGTGGTTTAACAATTAACCGGAATGGTGGATGTGTACGGCCGCACAAAAATTGAGA GCTACGCGGCCAGCGATCCCGACGCGAGCGCATCGGGAAGTCGAGTTGCTACGGGCGGAAATGCCCTTGT TGAAAGGCGCCTGATGTACCAGGAGTGGCGTGTGTCCGACCGCACGCTGCTGGAAGAACCCAGGAAACTG GCACGTTGACGTAGATATGTCAGGTCTTGTGACGTGAACAGTTAATGACATTGGCGGACAATGGAATACA TCGTTTGGGGGTGGGGCACCCACCTCGCTCTCCGGGCAGGGAGAGCGAGGTGGGTGCTTCGCGGGCCGCA TCATCAATTCGCGCAGGGAGCGCTGGGTGATAGGCCGAGTTCAAGGTGGCACAGCGGCTTTCAGGTACGA CGAACCGCCCGACCTCCGGGTAAATGGAATCGGGTAATTCTGTCGCCTGGGCTATCCAGGTCGAACGGGT 
Final Technical Progress Report DOE Award No. DE-FC26-02NT15340

GTTTTGAGCAGAGCTGTTCCCCATGACTGCCTCCGGCTTGTTGCGATTTCAACCAGATCGCTTATGCTCG GAAGCCTGCGGTCTGGGTGCCTTAGTGCCTCTTGCACTGCAGGCTGGAAAGAACCCCAGGACCGGAACGC GACGCCAATCACAAGTCCGCTTCTGGGGTTTTCTGCACGACAAGGACCGGCCCGATTCGCACCTGGCCGG TATTTGTCGTCGAGTCGAATGTCACGAGCTGGCCGAATGCCGCTCTGTGCATGAAAAATAGTCACGATCC СTGCCCCTGCCCGGGAGAAAGTGGGCACCCCTGAATAGGGCTGCGCCACTTGCGTGGTGCTGCATCTGGA ACCGACCTCGACAAGATCCAGGAAACCCCCGGGTGGGAGCGCTCTCGAAGCGTATTGCAAACCGGCTGGT TTGTGCAACTTATACACGCGCCTTCACCTCGATCCTCACTACGCTCACCGACGTTCCGGGCTAGCATTGT TGTCAACAATAACACGCAACTATGACGCGTGCTAGTGGCGGGTACCACTCCAGGGAGGCGCAAGAATGAG CAGGCAAGCAGGCGATCTGACCCCAGGAGTTGATGAAGTGCCACGGAGTGGAGTGCGCGGCTTTCGGGCC GACAGGCTCCGTGAGTTGCGTGTCAAAGCGGGGCTGACGCCAGACGATTTGTCTGTACGACTAGGGTCCA GCCGTCAATCCGTTTCCCATTGGGAGACGGGGCGTTCCACGCCAGCTCCCCCGGTGCTCAAGCAGATTGC ACAGGAACTTGACGTGTCGATCAGTGTGTTGGTCCCGATTCCGGACAACAGATTGCGTATGGGCGACCTT CGGGTGCGTGCCGGCCTAACTCAAATCCAAGCTGCCGAACGTCTCGGAATCTCGCCCACCTCCCTTGCCG AGATCGAAAAGGGCATGAAACCGGTGAATGACCAGCGTGTCTCCGCGATTGCGGAGCTTTACAACGTCGA GACGGCGCAGGTTGTCGAAGTTTGGGAACGTGGCCGTGAGACACGCGAGACACGCGCAAGATCCAAGTAA TAAATCACGCAACAAAATCACGTGCCCGCAGCATTAATTCGGTGATCTAACATTCAGTGCGGCGTGTCAC GCGAAGGAAACCGCCAACTTGGCGCAAGATGGCGGTGAACCTGGCGCACGTTCAGGCGCAGGATCGAAAG GAGAGGCATATGCCTAACCCAAGTAAAGGCAATCGCACTCGCGTGTCGACACGCCTTCCTGTGGACGTGG CCCAGGCGCTCGAAGAGCTTCGGGCTAAGACGGGTGTCAGTTCGGAGAGCCAACTCGTAGCCGATGTCTT GGCACTGATAACTGGTCACTCTGAACTCGTGGTCGAGCTTGACCAGGGAATCCTGTTCGATCTTGGACTC GATCAGAAACTGCACCGTGAGGAGGTTCTGCCGCTGACTGCGTAGCGAAACCCCTTCTCCGGCAGGAGAA TACGTGACAATTTCACACGCTTTGCAGTAGCACGAACCCCCTAGAAATCGAAAATCCCCCGGCGGGCAGG CCGAGGGTTTCGAAGTGGGTGAGAGGTTGAGCTGGTAACTCGCGCTCTCGGTGGTACTGCGCTTCCCCGA AGGGACAGTCTTGGAGGACTGGTCTCAGGGTACATCGCGCCCTGTTGCAGATTCAATAACTGATTTTTCG CGTGTCTTGGTGATGGAACACGCGCCCACATCCGTACAACGCCCGTACTGCGGGAGCAGCCCCACGCGTC GCCGGTACGGCACAGCCCGCGCACCCCGCACAGTCCGCGGGCACGTCCGCGGGCATCAGTGCCAATCGCT CACGCCGATGTTCATCAAGGCACTTGGCGGGCCGAAAGAAGCGTGCCGGCGGTACTGCCAGATGCGGCTC GACCTCGGCGACGGTGCATACAACGGCATCCCCATCTGGCAGGGCGCTCAGCACTGGGTGGAAATCGCTG TCCGCGAGGCATATACGGCCGAATACAAGAACATTCGCCCCGCCCTGGTTGAGACGACAGGCGGCGGAAT AAGCCTCAAGACTCTCCTAGCTGTCGCCACAGTCATGGCGTCAGTCGCTGAGTTCGACACTGGCCGTGAA TCACGTTTGTCTCTCGATAAGACCATCGAACGTACGGGCAAGGGTGAGAGAACCGTGCAGCGTGCCCGTC AGGCCCTGAAACTGCTTCGAGTGGCCACCGAGGTCTTCCGTGGACGGCTTCGTAGGAAGAAGGGCGAACG ACAGGGTTCGTACCGGGTGGGCGACAAGGGCCGGGGATGGGCGTCAGTCTGGGCACTCCACCTGCGTAAG CCTGTGGATAAAACCCGTGTCTACCTGGACGGATCCATAAAGATGGCACCCCATCCCCGTAGGGGTCATC TTTTGTCTCTTCCCTCTCGTAGAGAGGTTATTAATACAAAACGATCTGTGAATAAACGAGCCGCTCCGCG GCGCAAGGAATCCAAGGCCGAGGTCGAGAAGGTTGAAGCCATCCGAAAAGGCCTGTTGCTTGCATCCAAG TGGCTCAGCAATCCGCGAACGCCGGTCTGGGCGCGTCGGCACACCCCCCGAGGGTGGGCATCGGCACTGA CCGAACCAGCCGCGCACGGTTGGACCGCAGCGGATCTGAACGACACGATCGACGACTGGGCGAACGCCCA GAACATGGTTCCGACCCCGAAGCACCCCATCGCATTCATTCGTTGGCTCATGAAGCAGCAGGATCTGGCC TTCGCACCGCATGTTCTCGCTCAGATTGCCGCTGACCAGGAGAAAGCTGAACGTGAACGCCAGTCCGCCG CGTTAGAGATGGAGCGAGAACGCTACGCATCGGCCGCGCCCGAGGACTCCCCTGGCCGCCAAGCAGCGCG TTTGGTTGCTCGTCGAGCAGCCGACACGGCTCGCTGCCGCAAGGTGGATACCTCAGCTCGTGAGAACGCC GCGCAACCTGTGTGGATCACGCATTTACGCGACCTCGGACCGCAGTGAGCAGCCGCGATCAGCTTTCGGT GAGCGGAGAAATCTGTACGATCGCCAAACGCTCGGTCAAAGGTCCTGGGTACGGGCTGATCCGCAGGGTC GAAGGACGCCAATCATCATTTTCAGTCCGAAGACGGGCCACGACGTGGACCGGATCGGTCGAACCTAGAT TGAACTTGTCCTGGGCCGAAGACAACAGATGTCGATCGTCAGCGTGGATGACCTGGTTCGCGCCAGGAAT GTCGCGCCACTTCACCCACGGCGCTGGTTCAGGCAACCACAACGCCAACACAGCGGTCTGTGTGCAGAAC ACGGCCGTGTAGATGCCCGAATCCTTCACACTCTCCTGCAACCCCAGCTCAGCGAGTGTCGGCCGGCGCG GCGGCGTCGTATCGGTCACGTCGTGCCAGAGCAACCGCAACCCGCGCTCGCCTTCCTCCACACACCCCCG CGCCCACATATGCCAACGCATCACGCGGCCGTCCGCGTGCAGGACACTCATATCGGCGTCGAAGGTCTGA TCGTCCACCGGATTGAGGCCGGCCGCGAGCATGGCCTCCGAATCGTCGAATTTGATCGCCTTCGCGTAAT ACTCCGCAGGCGTGCGCTCCGGAACGTGATCCTCGGGCTTAACTCCCGACATCAGCGCAGCTTCGAGGGT TTGCGCGATGACGAAACGATCGAGCAGCCAGGAGAACCCGGACACGATTCGCGGCGGTGTCGGTGGGGCT TCGGGATCGCCGATCCACAGCTGAACAGCGTGAACCTGGCCTGTCGGACCGACAACAGGAAGGCATTCGA TGCGGTAGCTCCGCTTGTCGCGGGCGCTGTCGAGGGCCGGCAGGACGATCTTGGTTCCACTGCTCCGAAC CTGCTCGATGATCGGGTCGATTCGCACGTCCGCGCTCGGCGAGAGCCGTTTGGTAGTTGCCCAGTTCTTG ACGTTGCCACCGACGCTGACGACGGTCGGACTCTTCCCCAGCGTTTCGATCAAATGCCACTCGTTCACAG ACACCTCGCTTACCGCTGCCACCCGCCCCCGCGGGAGCTGCCCCACCCGGGAAGACCCGGACAATTACGG CAACTCGGCAGACTACCGGCCAGTAGCGTACTCGGCGTTACAGACGTTGCAAGGAGAGCTCGAGCCCGAC TGGCTTGAGCGTCAGCGCTTCTCGGACGTCCAACTTATATTCCGGATCCGATTGAAAGCTGTAGCGCTGC AATATGGTTGCCAACGACAGGACCGCTTCGTGCAAAGCGAACTGCCGACCGATGCAGGACCTTGGTCCTG TTCCGAACGGACGGTAGGCGTGAGCTGGGCGTTTCTTCACATTCTCCGGCTTGAAGCGGTCCGGATCGAA GGAATCCGGATTCTCACCCCAGACCGGGTCGCGGTGCAGCTGTGGGAGGAGGACGAATACCCATGATCCC TTCGGCATCGCGTAGCGACCACCCAGGGTCGTGTCGGTACGGACTTTGCGGAAGTATCCCGGAGCGCTCG 
Final Technical Progress Report DOE Award No. DE-FC26-02NT15340

GCCACAGGCGCAGCGTCTCGTCGACGACTCGGCGTAGGTACCGCATTCGGGCTACGTCTTCAAAGGCCGG TGTTTCACCACCAGTGATGTCCGCGACCTCCGCACGAGCAGCGTCAGCGATCTCAGGATGCCGAGACAAG AAGTACAGCGCGAAGGCGATAGCGCCGGCGGTTGTCTCGTTGCCAGCGACGAGAAACGTCAGGACCTGGT TCCGAATGTTCACCGGGTCCAGCTTCTCGCCCGTCTCGTCGTCGATGTTGTTGAGCATCAGGTCGAGCAG ATCGTCCTGATGCTCCCCCGGCTTTGCCTGACGCTCAGTGATTACGTCGTCGACGACCGTCCGAACAAAC GCGATGTCCTTCTCGTATTGCAGGTCAGCGCCGTTTCCGCGCAACTTCCGAAGGAATGGAATGTCATTAG AGGTGCGGTTTACGTGCTCGAGAACCCTCGACATCGCACCGACGAACGGGTGCTCATCGCCATCGAACGA CGCGAAGTCATAGCCGAAGCCAGCCAGGCCGATCACGTCCAAAGCGAGCTTGTTCGCATCAGCGGTGGCA TCGATTTTTCCGCCGGCGGCCGCCGCCGCATCCCACGCTGCCGTGAGTCCGTCCAAAGCTCGCAGCATCG ACGGGTGGTACTTACGCAACGCTGCCTGGCTGAACCCGGCGTTGAGGATGTTGTGCGCCTTCTGCCAGTT GGGTTCGGAGTTGAAAGCGGTGAAAAGGCCGTCCTCCGCGACTGCCCGCAGCTTTCGGATCGGCAGACCC ACCGATTTCGCCCACAATGCCTCGTTGTTGACCTCGGCGACCAGCTCCACCCCGGAGACCACGGTCAGTC GGTGATTGATGATCTTGCGCTCGAAGATCGGCCCCAGCCTCGAAGCCATGACCATTTCCTTCTGGACAGG CTTCACCGAGTCGACGCTGAGCAAGTCCCCCAACACAGGCAGCCGCCAGGACGGCGACGGAATCGACTCG AACTCAAGGACTGGTTTGACCGCGACCTCGCTGGTGGGTGTCTCACTGCTCGGATACGGGCACTTGTCGG AAGACATGGTGGACAGTCTTGCATCAGTTCCGGACTAGATGCCGACTCGACGCAAAATGGGCGATGGAAC GCAAAGCCGCCATCCAGGATGGAGCAGCGCGCCAAGAACCATGCAAACCAGCGCTACTTGCGCAGACAAG CGGAAGTCGAACGTGTCGTTGTTCCCCCTCATCTTCACTTCCCCACTGCTCTACGGAACCTTCTGAGCTG GCATTTTGCATGATTTCCGAGCCCCGGAACCAGCTATACAGTTTTACGGCAAACCGGGAAACTGGAAAAC CGTATAGACGGCTACCCGGCAAAACGATATGCTGTCTATACGGATAGACGGGAAACCGGATGCCTGGGAA ACTGGCTAAGGTGTGTATACGGCTAACTGGCAAGCCGGGAAGCTGTTTTACAGGTAACTGTTTTTACAGC TTTCCGGCAAACCGGGAACTGTAAAACCGGCAAACTGGGAACTGTAAAACCGGCAAACTGTAAACAAGCG AAAGGGCCCTTCTCGATGATCATCAGTCTCGTCAACACCAAAGGCGGAACGGCCAAGACGACGAGCGCGA TCTACCTTGCACTCGCGTTTCATAATCGGGGGAGGAAGGTTGTCGTCCTCGACTTGGATAAACAGGGTTC AGCAACTGACTGGGCTGACCGCGCCACAGAGGCCGGAGATCCACTCCCGTTCCCAGTGCATGTCGTGAAC ATGAAACGGCTGGTGAAGTACGCCACCGATGGCGATGACCAGGTAGTAATCATCGACACCCCGCCCGGTG ACGGGCAAGTTATCGACGCTGCAATCGGGGCGTCCAACTTCGTCATAATGCCCACGGCGGCTACAGGACT CGACACCGCCAGGGTCTGGGAGACGCTGCCCTCGGTGCAGGGCCGCCTTCCCTACGGAATCCTCATTACC TCCGCACGTCTCGGAACGAACCTGCTTGAAGATGCCAAGGCAGCGTTCGACAGCAACGACGCAGCCCGAT TCGACACCGTCATCCCCATGCGCGAGCGCATCCGGTCAACATTCGGTACTACCCCCAAGCACGACGAAGG GTATTCCGACGTCGTCGACGAGATCACAGAGGCGCTGACAGCATGAGCATCCCCAAGGCAAAGACGACAC CGACGCTCGGGCCCCGCAAGTCCGCGCCACCGATCGCGACGCCCTCAGTGACGTCCAGCTTCGTCGAACC GGACGCAGACCGCACGAAACTCACCGTCCAGATCGACGCTGAACTGCACCGACGCTTCAAAGCTGCTGTT GCAGGAAGCGGCAAGAAGATGCGCGACGTGGTCGAGGAAATGATCGAGCAGTGGACCGACGCGAACAGTG GTCGCTCATAACTCGGTGACGCTGTTACGCAAAACCAAAGGGAGCCTCGCCGGTTGCACCCGACGAGGCT CCAGACCGGGAAACCCCTGGAAGACATCTCACGGGGTGTAGGAGTCAAGCCGCGATCGGGAGGGGAGGTG GAAATGCGTGCAATTCGTCGAAGAGTTCGCGGGTGATGAGGCAGTGGTGGAGTTGGCCGAGGAATCGGTT GAACAGGTGTCGTTGAGCTTGGTGGTTCCAGTCCCCGCCTGCGCGTCGGGCATCGAAATGTCGCCTGGCT CCGGGGGACGCTTGGAGGGCGCTGAACGCCCAGATCGTTCCTGCTGCAGCCAGTCTTCGGTTCTTGACGT GCCGGTGCAGGACGAAGGTCTTTTTCCCGCTGGCACGGGTGATCGGGGCGGCGCCGGCGTAGGCCTTGAG GCCACGCGCGGTGGCGAACCTGTTCCGGTCGTCACCGATCTCGGCGAGAACTCGGGCCCCGGCCAGTGGC CCCAGCCCGGGGAAGCTGGTGATGATCTTCGCATCGGGGTGTTCGGAGAAGAGCACGCTGACGTGTGCGG CGAGGGTCTCGGCGGCTGCGCAGGCAGCGTCGAACTGCCGCAACAGGGCCATCAGACTGATTCCCATCGC TGCTTCGATCTGCGGTGGCTGGCGAAGCGCCGGGGTGCGCAGGATGCCCCGCAGTCGTTCGACGTCTCGG TCGAGGAATCGGGAGCGTCCCGCCCTCTTGAGCGCCGCCCGAAGTTGGGGCCGGGTCAGGGCGGCAGCTG CGGTCGGGGTCGGAGCCGCAGCGAGCACGCTTCGGGCATCAGGGCGGGTCAGTCCGTCTTCGAGGGATTG AAACGCGGTCAACGCTGCCGGGAAATATTCTTTGAGGAGCGAGCGGACCTCGTTGCCGAAGCGTTGCCTG GACCAGACCGCGTCTTGCTGGGCGCGGGCGAGAACCCGGATGGCCAGAGCGCTGTCGGTGTCGGCCGGCA ACGGACGGTGCGCGGTGGCGTCGGTGCGCACGATATTCGCGAGCATGACCGCGTCGGCGGAATCGGATTT CGATCCGGAGACCGAATGCCGGGACCGGTACCGGGAGGCGGCGAGTGGGTTGATCGGGTAGATCACCCGG CCACTTGCTCGGAGTGCGGCGACCCACAGGCCGTGGTCGGTTTCGATCCCGACCGGGATCAGGTTTTCGG CGGTGTCGCCGGCTTCGGCGAATGCCTCGAGCAGTCGGGCGAACCCGGCGGCGTCGTTGTCGACTCGTCC CCGGCTCACGACTTCGCCCAGGTCGTTGAGGATTGCGAAGTCGTGATGGTTTTCGGCCCAATCGATTCCG CAGTACAGTCCCACCGCGCCTCCCAGTGTCGATGTGCAGGTTGCGAACCCGTGTGGTGCACGCGGCGACC TAATTGCAGGACTCGACGGTCCGTCATCTCACCAGCCGTTCGTGTCACCAGCGCACCGCAGGGCACTCGA TCTGCCGAAGAGCTCACGGCTCGGGAATGCAGAAGAAGAGATCGACCCTGTGGCGGGCTCGTACCACGAC АTTTCACCACGTCGAATGGTGCACTGCCTGGCGCTGTCTCTCTTCCAGAAGGCGTCGTGTGCGCCGGGAA AAAACCGGGAGTCGGCCGGGCAGTGCACCGGCAGAAACATCAGACCAGCGTGAGCGTTGCAGCCCGTCGG TCCGTGCTTCTACACACCAATTAGGAAGAAACCCAGTCATGACCGACTTTAGCCTTCGGGCAGCTCGTAT TCAGCTAGGCGCATTGCTCGCCGCACTGGCACTCGCGATCGTCATTGCGCTCGGTATCACCACCGGCGCA TTCGTACTTTCCTTCGCCGTGCAACGCGACCTTGCACGGCAAGCACTGATCCCCGAACACCTGACCTGGA TCTTCCCGGCGATTGTCGACAGCGCTATCCTCGGCGCCACGATCGCCATCGTCATCATCAGCAAGCTCAA CATGAACAAGCGCGACAGAGGCTTCTACATCGCACTCGCCGTCAGCGTTGTCGTGATCAGCATCCTCGGA AACGCGTACCACGCCTATCACGCAGCAATCGCCGCGCAGGAGTCGATCGATGGTGGGGGAGACCTTGGGT 
Final Technical Progress Report DOE Award No. DE-FC26-02NT15340

TCATTCCACTCGCGCCCGCTATTGCAGCGGCCATTGCGATCATTCCGCCGGCCCTGGTCTTGGCGTTCAC GCACGGAATCACGATCTTGGTGAAGGCAGTCGGAATGGCGTACGCCGCTTACCGTTCGATCGTCGAAAGT GCCACCGACAGTGAGGATGCAACGAGTATTGCATCTGACCTCACCGTAGGCCGGGGACCGATCCGCGTCC CTCAGCCCCGCGAGAAGATCGCCCACAATATTGCGGAGCCGGAGGAAGAGGATCTAGACGTAGACGCCAC AGCGGTGGATGCGAAGGCCGGCAACGAGGACGCTTTTGCGGACTTTGCGGAGCCATTTTTCGTGGACGAA ATGACGAATGAAGACGTCGACGACATCCTCGATCCCCAAGACACAGCTACGGAGTTCCCAGCCCGTCGAG ACATCGAAGGAACACARGGGCGACACGGAGGCGGGCATAGACGATCTCATGGCCTTCATTGCCGGGTCGG ATCTCGCGAAGAACGTGAAGGGACACAGCGGTCCGCAAGCTCACGAACCCGAACCTCACGTGGGAAACCA TCGCTCTGGAATCGAAGCCGCCTGTCGCGACATCGACCGCATGGCGCCGGTACGAGAAGTTCGATGCCGC AGCGCGAGAAGCAGGCTTCGACAGTCCACCATTGCCCGACCTGCGAGCCGCCGAGAACCCGATGCGCAGC GAACTCGCCACCGTTTGACCCACACCCACCAATAAAGGGGTGGCCCTGGCGGTGCTGTAACACCGTCAGG GCCGATGACCACAGAAAGCACCTGTGACCATGAACAATCATACCGACCGCTCCGAAGGCATTGACCACGG CAAAACCGGCCGACCACCTATGCAGCCACGAGCGAAAATCACCCGAGAAGCGATCCTCGAGGTGTCCGCC ACCCTCTTCAGTCGAACCGGGTACGCGGGCACCAGCATCAATGACATCTTGGCGATCTCCGAGACCACCA AAGGTGCGATGTACTTCCACTTCTCGAAGAAGGAATCCATCGCGAGCGAGATGCTCTGCCGCTGGTCCGC CGCAGTCTCCGAGACAGTGGGGAAGGCCGCAGCGACAGGTCAACCCGCCGATCGGCAAGTGATCATGATC TACCGCGAACTCGCCCGCCGCACCCAAACAGAAGCGATCACCCGAGCTGGACTCATCCTGTCCGTCGACA AGTCGCTCAGTGATGCGTGTGCCACCTACGAATCGTGGACCGACGCGATCACACCGATCGTCATAGACGC GATCCGTTCCGGCGCCCTCGACTGCGCGGAAAGCATGTCCAGGCTCGCAGACACTCTCTGCGCCGGATTC GTCGGCGCAGTGCAGGTTGCAGCTAGCCTCGACGAGAACCACACCATCACCAAACGAGTCGACGATCTGC TGCTGATGTGGCGCGGAACCGACCCGGCCGCCGTCCGTATGGTCGAAGGAGCATCCCTGTGAACAGCCCC AACGAACCGGCACACACCACGGAAAGCACTTCCGCCACGCCAACCCGCCCCCGGATCGGGCGCATCCCCG GCAGCAAGAACTCGGCAATGCAGGACCGTGCAGTGCGGACACGAGAAGCGATCATCGCCGTCGCGGCACG GCATTTCGACACCGACGGATACGGCCACACCAGCATCAACACCATCAGCGGTACCGGAAAATTCGCCAAA GGCGCTATGTACTACCACTTCCCATCCAAAGAAGCGATGGCCGAACACCTCATCTCGGACTGGAACCACA CCCTCGACGAAACCATCAGCGAAGCACTGGCGGCCGGTTCGAGCAGCACAGCAGGGGAGAAGCTGGCAGC GATCTTTATCTCGCTCGCACAGCACATCGGCGAAGACACCAACCTCCGCGCCGGCATGAAACTCACCCTC GAACCCACCATCAACAACGGCGCAGCATTCGCACACTGGGTCGACGCCATCAGCGACATCATCGAACCCG CCATCACCGCAGGCGAACTCACCGACACCCCCACCACACCCCGACTCGCATGGAACCTCTGCGCCGGCAC CCTCGGAGCCGCCCACGCATCCGCAACCTTGCGAGAGGACATCGACCTCGCAACCCGCATCGCAGACGCG GTAACAGCGCAGCTGCGCAACGCACTCATGCCTGCATGACAAAATCTCGTTCGACAGAATCCGATGCGGG CCAATCGAGAGCAACTCAGGCAACGATCGGATCAACCCTTCATCCGTCCGGAGCTATGGGACTGAAGCGA GATGCCCCGGCCAGTGGAGGCCGGGGCATCTTCGCTACCGAACCGGGCTACTTTCCCGGCCCTTCCCTGA GCCCCTGGCCAATGAGGGCGAAGAAGGCGCACTCAACCAACCAAAGCCCCGTCTGCCCCTGATAGGGCAG ATCTGCTACCCCATAACTCTCGGCAACAGATCGATTGGGGTCGAAGGCATCTCGCATGATCTGACGAGCG AGTAGCAACTCTGCCTCCGCGATCTGGCCGTCTGGCAGCCCCGGATCCAGGTTGGTCAAAAACGCCCAGT CCACCTGCCCATACAGATTGGGAAAAGACTGACGTATCAACTTGGAAGCCGTCTTGGCGATGTGCTGCGA CAGATCCTCGGCGAGGCCGAGTTGTGCGAACACAAAAGCAGTTTCGGCGTCACCAAAGAAGTCCTGCAAA CCGAAAACGACTGTCGGATCGAGCAAATGCCGCTCAAGTATCTCGTCAACATTGGCGTGAATTTGCAACG GGTTGAGTACCAAGGACTGTCCGTCGGTGACCGAGGCCTCCCGGTAGTCCGGATCAATAAAACGCGGTAA ACCCTTATCTCCGAATGACATAGCTGAACCCCCACCTTGCGGGAAAAATAGAACCGTCACCCAATCTACC TAATTGTGAACGTATTTGGGTGGAGTGCGGACAAGGCCGATGTCAAGCAGTTTCCGAAGCCCTACGATCG TGGTCATTGCGCTCAATTCGGGCAAGCTGAATATTCGAATACAGTGCCGAACGCTTCAAACCCGTGTTCT TGCAAAGAGTTTCGATTTTGAACTGTCCGGTTTCGTGCACCTGCAACAGGTACGCACGCTCTTCCGGCGT GAGCTTATGTTGACGGCCTTTCATCTTGCCGGCCGCGGCAGCCGCTGCCACTGCGTCCCTGGTCCGACTT CGGATCAGGTCTGACTCGAATTCGGCCATCAGCCCGAGCACTCCGATGAACATTTTGCCCATCGGATCCG ACGGGTCGTAGATCTTCCCGTCGATCGACAACGCAACACCCTTGTCCGCCAGTCGCCCCACCGTTTCGTG GAGATCCTTCGCGGACCGGGAGAGCCGGTCGAGTTTGGTCACGCAAAAGACGTCGCCTTCGCGGGCCGCG TTGATCGCATTGTCGAGCCCGGGCCGTCGAGTCTGCCGGCCACTGATTCCGTGATCGACGTAGACCCGGT CTCGGTCGACTCCCTCCTTGGCGAGAAGCTCGACCTGAATCGACGAATCTTGAGCCGCCGTACTGACCCG TGCGTAGCCGATCTTCATTCCACTCCCGATCGTCACCGTTCACGATGTCCCGCACACAAACTGTCCGTTA GAAACTATGCCATACCGGATTTTTTCAACGGACAACAAAATGGACGGAAGGCGCTGAAATTTCCCCAGGT GAATGTCAGTTTCAGAAGACGGCTGCACGATGTCAGAAGTGCACTGCAGTGCAGCTCCGAGTTCTTGAGC ACACGTCCGGCCTTATCCAGTGAGCGCTGCGATCCCTGACTGGATAGCGCATTGATGGTCAAATTTCGCA TCAAGTTTTACTGGCCGTCGGTGAGCTGCGGGTCTAGCGTCGCGGCCGGCCGTGGACGGCTGTTGTCGCG CGAAGTGCTCGATCGGACACTTGCGGCATGGGCGGCGAGAAAGTTGTCGACGATTTGGATGCAGTCGTCG GCGTGGATGTGGCGCAGTCCTGTGAGGCGAGCGAACACTATCGGTCCGAGGAGCTGAATGAGGGCGAAGG TGGTGTCGATCTCGCCGAGCACAGTGCGGGCGTCAGGGGCCGTCAGGATCTGATCCAGGGACCTGCGGTA TTGCTCTATGACACGGGCCCGAAGCGAGACCACCGCGCCAGGATCGAGATCGCGCCGGCTGGCGTCGCCG CTGTTGACGGGTCCCATTGCCAGCCAGGCCAGCGTGGTCATCTGCACCGGAGCTTGGTCGATGAGGTCGG CCTGGGTGGTGAGCACCGAAATCAGACGTTCGCGTAGCGGACCGGTATCGATATCGGTGTCGACTGGGGG TAGTAGTCGCTCGAAGGTTGCAGCGAGCAGGTCAGTGGTACTTCCGAAGTGTCGGTACAGAGTGGCACGT GCAACCTTCGACATGCGGGTGACCGCCTCCACGGTGACCGCCTCGACGCCGCCGGTGGCTAGCAGCTGGC CCGCCGCTTCGAGTAACCGGTTACGTGAGCGCGCCAGCCGGGGGTCGGCCGGGCCCTCGGCCGCAACGAG 
Final Technical Progress Report DOE Award No. DE-FC26-02NT15340

AGGGTCGACCATGAACGGGCTCCGCTCCATTTTGACTGACAAAACATGTCCTAGCATGACCATTCTGTCA CACCCGCAAAGTTATGATACTAATAGTCTCGCACCGAGACGATCAGTCTCAAACTGGGAGGCTGGCCGTT GAACGAACGTAGATCCGACTCTGCCAGCGTCGCTCGATGGAGCGGTGCCCAGTGGTGGATGTTGATTGTG TCGTGCATGGCAGTCGCGTTGGTGGTGGCCGCAATGGCGGCATTGTATTCAGCGTTGCCACAGATCGCGG TCGCGACAAGGGCGACGCAAGCTCAACTGACCTGGATAGTCGACGGTTATACGCTGGTATTGGCGTGCTT CGTTCTGCCTGCCGGTGCGATCGGTGACCGGTACGGTCGCCGGGGGGTGCTCGTGGCGGGGTTGGCGTTG TTCGCTTTCGCGTCGGCATTGCCCTTGTTTCTCGCCGACCCGGCGTGGCTGATCGCTGCTCGCGCGTTGG CTGGGGCGGGTGCGGCGCTGGTCATGCCCTCGACGCTGTCGATACTGACCGCGGGATTTCCACCGGCGCA TCGCGGTCGCGCTGTAGGTGTGTGGGCCGGGGTTGCTGGATCCGGGGCGGTCCTGGGCATCCTCGGGGCT GGAGTGCTGCTCGAGCAGTGGTCGTGGACCTCGGTGTTCGTCGGGTTGACCGTCGCCGGAGCGGTTCTGG CCGGGTTGGCATGCAGTATCGCGGAGTCCCGCCAGCAGGAGCATCCACCGGTCGATTGGGTGGGCGCGGT GACCGTAGTTGTCGCGGTCGGGGCGATCGTCTTCGCTGTGATCGAGGTTCCGGCCCGTGGCTGGTCGGAT TCGCTCGTCGCCATCAGCACTGCGGTGGGCTTGTTTGCGGTTGTGGTGTTCGTCGTCGTAGAACTACGTT CCTCGGCACCCCTACTCGATGTCCGGTTGTTCGCCCGCCGTGGTTTCGGTGCCGGCGCGCTGTCTGTCTG TATCCAATTTCTGGTGACCTTCGGGGTGTTCTTGTTGTTGGTGCAGTATCTGCAGTTGATTCTCGGTTAT GGACCGCTCACCGCCGCGTTGGCGTTGACCCCTATGGTCGTGCCGCTCATCGTGATCTCGGTGATTGCAC CCTGGCTGTCGAACATTGTTGGGCTGCGGGTGATGACCACTGTAGGTCTGCTCACCATCGCGGCGGGACT GGTGATGGTGAGCAGACTGACCGTCGCGGCGACTTACCCTGACCTGCTGTGGCCCTTGCTCATCATGAGC GCGGGTTTGGGGTTGTGTACCGCCCCTGCGACTTTCGCCATCGTCTCGGACACTCCTGAGGCCAAGCACG GGGTGGCTGCCGCGGTCAACGACGCAGCTCGCGAAATCGGTGCCGCGATTGGGATTGCGGTAGCCGGTAG CGTGCTCGCGGCCGGTTACGTTCAGCACATTCAGCCGGCTCTGCCCCAGCTACCCGAGCCTGCCCGTGGT CCGGTGGCCGATTCTCTGGCCGCCGCGTTGCAGGTCGCCGACCGCGCAGGACCAGCTGGTCAGCCGCTGG CCGAGTTCGCCAGAGCCGCATTCGTACACGGAAGTGGTCAGGCGACGCTGTCGTTGGCGGCGCTGACCGC CGCTGGGGCACTCGTCCTCGCCGTCTTTGCGCCAGGTAGACGCAGCCGTACGACAGCCACCGCGGGTGAT GGACGTCGGTGAAGGTCGGCCAGGGTGTCTATTCTTTGGTGTGATTCGTGTCTGTGGCCCGGAGTTGGGC TCGGGCTTGGGACTGCATGAAGTGTTCGACCTCGACCACCACGAACAGGGCAGTAGCGGTGGCTACGGAG CTGCCCTGCGCATCGTCCATCGTGGCCGTGAGATCCAGTTTGCGCCCATCACGAGACCGAACGGCAGCAC GCAACACGTATGGAGTGCCGAGCAGAACCGGCGCTAGGTAGTCGAGCTCGAGACGCCGCGTCACGGCGAG CTCACCCACTGTGTAAAGCAGGAAGCCGAACAGATCGTCGAGAACGGTCGCCACCGCGCCGCCGTGTGCT ATCCCGGGTGCCCCGACATGACGTTGATCGAACACATGGTGAGCTACCACGCCGTTCTCGTCACGCTGGA CCGACAGGGCGTGGCCGTGCGGGTTCTCCGGCCCACAACCAAGACAGTCGGGGTGGTGGGGCGGTAGATC AGGCGACTCGGAAGTCGTGCTCCAGAACGAGCGCGCCCAGTTCTCGACAGCGGGGCCGGCCGACCCGCTG ACCTCGTCCGGAATTCTTTTCATGGTCGGACCTCTCTGTCGTGAAACCGTGCCGAGTCTGTCGCGTCTTC GCGCGCTCGGCCTGCACCGCAGCATCCGGTCGGTCGTTCGTCCGACCGGTCGGCGCACTCGCCCCCGCCA TGATTACCACGAGCGGGAGTCTTCTCACTCAACGCACCTCCCGGCCCGATAGCGCACACGACGCGGCCGT CGAGCCGAAAATGGTGACCGCGAAACATCGAGGGCTTCCGTCGGCGGGCTGAATTGTTGTCGGCGTGGGG CAGGGATCAGGGTCTGTTGCCGAGTAACGACACGGCATCGATGCAGCGTCCACCGAGAGGCGTCCGACTG TTCCGTCCGGCAACTCTTCGGTCGTTCGGTGCCTCCGCACGCCTGGGGCGATCCGGCGCGGGCGGCTGCA GCACAGTGCGATGCGCCGTTTACCGCGAGGTGGTTCGGTCGGGTTCAACTCCCGAGACTTCGAGCCCTTC TGGTGGGGGGTAGGGGAGGGCTCATCTGGTTGCCACCTTTAAACTCGATAGCCCGCGGAAGGACAGCCCG GTACGCCAGCGAGGCTGTTCGACGAGCTGGAGACCGGGAAAACGAGCCACCAGGATCGGGAACAGTAGGG ATCCTTCGAGCCGGGCCAGCGGCGCGCCGAGACAGAAGTGAATGCCGGCGCCGAACGACAACGGTGGGAC TCCGGTTCGGGTGATGTCGAGCTTATCCGGGTCACAAAATCGGTCCGGATCTCTGTTGGCCGCCCCGAGC AAGGTCAATACGATCTGACCGGGATGTAGATCGACTCCTGCCAGCCGTGTCGGCTCGAGCACCGTGCGCC CGTTGGTCTGGACGGGGGAGTCGTAACGCAACAATTCTTCGACGGTATTGCTCGCCAAGTCGGGCCTGGC ACCCAGTTCGCGCATTTGGTCGGGGTGAGCGAGCAGCGCGGCGACACCGTTGCCGATGAGGTTTGTGGTG GTTTCGAACCCTGCGGCGAACAACAGAATCGCGGTGCCGACGCATTCGTCGTCATCCAGGATGTCGTCGC CGTGCGCGACGGCGAGTCGGCTGAGTAGGTCATCGGCGGGGCGGGCGCGTTTGACCGCCAACAGGTCAGC GAAATACATGGCAAGTTGGTTCTCGGCTTCACAGGCGGCGGTCAGAGCCTCAACATTGGCTCCGGGTTCC AGTGAGGCGAGCAGGGCACGCACCAATGGCGCGGCAACTGCCCGATCGGCCGCGGGAACTCCCAGAAGTT CGCCTATCACATTCACAGGCAGCGGGAGCGCCAGATCGGTGATGATGTCGACGAAGACCTGTACCTCCAT GACGTTAAGTAGGTGATGGATCTGTGTGATGATGCGGCTGCGCAACTCGTCAATGTGTCGAGGGGTGAAC ACGTCGGCCACCAGTCGACGCAGCCGGTGATGGTCAGGGGGGTTACGGAACAGCATGGTGCGCCGGAATC GATGCATGGCCCGACGTTGCAGCTCGTCAGGGATCTCGGACAAGCCGAAGCCCAGCGACTCGTCGGCCTT GCCGAGTCTGCGGTCGCGGAGCGCCGCCGCGCAATCGTCGTAGCGACTCAATACCAGCACCCCCGATCGC GTAACCAAGACGGGGCGCGTCTCTCGCAGCCGTCGGAACGCTTCGTAAGGTCCGGCTGCGCCGTGATCGG TGAGTAAGGCGTCCAGGAGCAGTTCCCTCTCGGTCGGTACATTCATGCTTGCCTCTTAGTATTCGCCTGT GACTGCAGGAAATGTTCGACGTCGACCAGGATGAACAGGGCGGTCGCCGTGGTGACCGATCGACCCTCTA TATCCTCGATCCTGGCCGCGAGATTCAATTTCCGGCCATCGCGAGACCGTGTATCGGCGTGCAGGGTGTA AGGCGTGGCAAGCAGAACCGGCGCGAGGTAGTCGAGTTCGAGACGGCGCGTCACGCCGAGTTCTCCGACC GTGTAGAGCAGGAAACCGAAAAGATCGTCGATTACGGTCGCCACCGCGCCGCCGTGTGCTATCCTGGGCG CCCCTACGTGACGTTGGTCGAAGAGGTGGTGAGCAACGACGCCGTCTCGGCAACGCCGTACCGACAGGTA ATGCCCGTGCGGGTTCGTCGGCCCGCACCCAAGACAGTTGGGGTGGTGGGGTGGCAACTCAGGCGAGCTA GATTCTTCCAGCGGCCGGAATGAGCGCACCCAGTCCTCCATGTCTGGGTCGACCGACCCCCCGACCGCAT 
Final Technical Progress Report DOE Award No. DE-FC26-02NT15340

CCGAAGCTTTGCCCATGGTCCGACCTTCCTGTCGATGAAACCGCGCTTAATGCCGGTCTCGAGTGTGACC GTTATGCAAATACACCTGAGCGCACTCCGGAGAGTGCGGTGGCAGCCCTTCGCTCAACTCCACGTTGGTC AGGCGAGGTGTCCCGCCCAACGGGAGATCACGACCCCATCTCCTTCACCAGTGCCGTAGTCTGCGATGGA CGCCGGTGTCGTGAAAAACTTCCCGCCCCGCGTTCCATCTGCCTCGTCCTATTCAGAACTCCGGTTGGAA AAAAAAATTTCCTAGATTTCGATGACAGCTAGCTCTCCTTCGGCGTGCAGGGAGCGTAGGCGCTTCTTGT CGAACTTGCCGACTGAGGTCTTGGGCACCTCGGAGATGAAGCACCAACGCTCGGGCAGCCACCACTTGGC GACCTTGCCGTCGAGGAAGTCGCGCAGATTAGCAGGTGTGGGCTCGTGGCCCTCACGTGGAACGATCACC GCGAGGGGGCGTTCTTGCCACTTCTCGTCCGGCACTCCGATCACAGATGCCTCGAGCACGTCTGGATGAC CGGCAAGCAGCAGTTCGAGCTCGACCGAGGAGATCCACTCCCCGCCGGACTTGATCACGTCCTTGGCGCG GTCGGTCAGCGTCACGAAATTGCGTGCATCGATCCGCCCGACGTCGCCGGTGCGTAACCACCCTTCCTGA AACTTCTCACTGTCATGCTCCTCGCCGACGTACGAGCCGGTAATCCAGGGCCCGCGTGCTTGCAACTCAC CGACCGCTTCGCCGTCGTGCGGGAGCTTATTGCCCTCGTCGTCGACAATCCTCAGCTCCACCCCTGCAAC CGGGCGGCCTTGGGTGGCGCGCAGGCGCCAGCGCTCCTGCACATCGAGCGATACCGGTGGGCGTGCGATG GCGGCCAGCGGCGAGGTTTCGGTCATTCCCCATGCCTGGACGACCTGCACGCCGTACCTCTCCTCGAAGG CCTCCATCAGGTGAAGGGGTACTGCGGAACCACCGCAGGCGACCAGACCGAGAGAGGAGATGTCATAGCT GGAGTTTGCCTCCAAGAAGTTTAAGACGTCATTCCAGATCGTCGGAACTGCGCCCGCGACGGTCGGCCTC TGCGCGTCGATGAGCCGCACCAGCGGCTCAGCCTGCAGGAACCTGTCAGGCATGAGTAGGTCGGCGCCCG ACATAAGGGCGGCATAGACTAGTCCCCAAGCGTTGGCGTGGAACATCGGCACGATGGCAAGGATCCGATC ATCGCAGCTGACTCGCAGTCCGTCGGCGGCGCAGGCCGCCATCGAATGCAAGTACGTCGACCGGTGGCTG TAGGCGACCCCTTTGGGGTTGCCGGTGGTACCCGAGGTATAACACATAGCGGCTGCGGACTGCTCATCGA GATCAGGCCAGTCGAACGTATCGGACTGAGAGGCGAGGAACTCCTCGTACCCCACGACCCTGACCCCGTC ACGGTGCAGCGGGGTAAGGTCCACTTCGCCGGTCACGACGACGGTGTGCACGCTGGTGAGCTGCAGCAGT ACAGGGGCCAGCAAGGGCAGCAGGCTTCCATCGAGAATTACGACCTGATCCTCGGCGTGGTTGCCGATGT AGACAAGTTGATCAGGGGTGAGACGTAGGTTCAGGGTGTGCAGCACTGCGCCCATCGAGGGCACCGCACA GTAGGCCTCAACATGCTCCTGGTTGTTCCACATAAAGGTCGCAACACGCTCATCGCCTGTGATCCCGCAA CCGCGCAATGCGTTGGCCAACTGTGCGGCTCTCCGTCCGACTTCGGCGAAAGTGCCGACCTTAACGCTCC CGTCGGACTGCAAGGTCCGTACGGTTGCCGAGCTATGCACACTCGAACCGTGGCGCAGAATCGCCGCAGT TGTCAAGGGAAAGTTCATCATCGTGCTCTGCATACCATTCCTTCATTCGATAGTGCCTGCGAACGAACAG CGATCCGCAGATCAATTCACCGGGCGTCCTGGTTAACAGCGGGCTGTCAGAGACCTGCAGCCGTAAGTGA GGTTGAAGCCAATTCCAGGGACTCGACCAGGTTCGACTCGAGTTCTGGTAGTCGGCTAGCCGCCGTGACC ACCGCCGCTAAGCGAGCGCGCGCATCAATGTCGCGTTGGGTCCCGGCACGTGCAGTTTCGGTCCATGCCT GCGCACAACGGCTGGCGGCGACGGCGAGGTCGGCGGTGGCTAGATCACCGGTCAGGCGTGCGACATCCGC GCAGCCATCGGCGAGCAACCGGCGGAATAGGCCGCCGCCGGTGCCCGCCTTCTCGATGAAAGCGCCCAGA CTGAACAGGAAGATCTCAAGATCTCTTGTCGGAAGGTCTGCCCAGGCTCGAAGGTCGGCCGCCAACTGCT CGACTGCAGCTAGCCCCTCGGCCCCGGCCTCCATGGTCGTGAGATCAACGATCCCCGGCTGTGACGGGTG GCGCATGTTGGCCGCGGATTGGCGAAATGCCTCTGCCGCCACTGTTGCCATGTCTGGCAGCGCGCTCGGC CAGGTGATGCGGTATGTACAGTGGCGCGTCGGTTGAGGGAAGGACGTCGAGGAACGCGCCCGAGCCAGCG AATCGAGCGGGACTTCCTGCACCTCGGCGCGATCATTGTCTACGACGAAGGCAATCCCCTTAGCTTCGTC GTAACCGATCACCACGATGTCGTGGCGACTCATCTGCAACTGGACCTGCAGGTACGGTAGCTCGGCTATG TCGCCCCACACCAGGCTGGGTCTGCCTGCGTCGATCTCGTCGAGAACCCAAGACCATCCCTCACTGGGAT CGTCGGTGGTGAGAACGTGGACCTGTCCGCCTAGCCGGCGGGGTAGATCGATTTCGAAGTCGGCCCCTCG TCCCACCAAATACAATGGTGGAACCAGGTCATTCGATCGGAGGTACGCTAGCCCCAGCGAGCCGCCGAGT GCGAACACCAGACCTTCATCGGGTGGCCCTTCCCACCCCAGTCCATGCCAGTGCAGAAGATCCCGCATTG CGCCAGAACCACAGTGCCCCCCGATCTGATGGGGGTAGTCCTTGATCAGGATCTTGCGGGGCATGTATCT TCCTTTTTTGGGTGGTGGGTTCGAAGACCAGGTCAGTTGTTCCGAATTCTGTCCAGGTAGGCCTGACGTG CCCCCATGTCCACGTCGTCGAGGAATACAGAGTCTGTGTCCAGGGCCGATCCGAGCCTGGTGGCGAGCCG TTCGGGGACGAACTTCATCGCTGCGACCATGCTCCCTGCGAGGCGAGTGACGCGCACGAAGGGCCGCGGT TTCTCGATCAGTTCGGCTGTCACTCGAGCGATTTCCTCGGGCTCTGCATTGCGCAGTCCCTTGGCTCCTG CTGTCCCAGCAACGAGTTCGGTGTTGGTGAACGTGGGCCGCACTGTGGACAGCCGCACGCCTGACTTGCG GTATTCCAGTCGCGCCGCCTCCGTGAATCCGATGACCGCGAATTTGGTGCCGCAGTAGGTCGCCAGACCG GGGACGGCGAGCTCGCCGGCCAGTGATGCGGTGTTGATGATGTGTCCAGTACGACGAGGGAGCATCCGTT GAAGGGCGAGTTTGGTCCCAAAGATGACTCCCAGGACGTTGATCTCGACCTGCCGCCGGGTCACCGCGTC GTCTTCCTCGTGGGTGTGCCCGGTGGGCATGATGCCCGCGTTGTTGATCAGCACGTCGAGGGGGCCGAGG TCTCCCTCGACTAGGTCGAGAAAGTCTTTGAACGAGTCAGGGTCGGTGACATCGAGGCGGGTGACAACCT TAACCCCCAGTTCGGCGGCGGTCTCCTTAGCACGTGCCTCGTCGATGTCGCCGATGGCCACGCGGTGACC TCGTCGGATCAGCTCCTTCGCGGTCTGGTAGCCGATGCCGCGGGCTCCCCCGGTGATCGCCACTGTTTTC GCGGCGGTATGGGGATTGTGTGTCGTCACTTGCTGTGCTCCTTGTTCAGGCCGAGGCGTTGTCGTAGACC GCGACCGGCTGCTGCACGCAATACTTTTTCGGCGATGGCCGTACGTCGGGAGGTGTACGGGTACCACGTG AGTTCTCGTGTCAGGACAGGGATCCGCGGCTYGGTCACGGACTGGACCCGGCAGTATTTGTGAAGTCCGT AGGCGCCGCCTAGTCGCGCACCGATACCTGAGGCCTTCCATCCACTGTGCGGCAGTGTGGTGGCGAAGAG GTTGCTGAAGACGTCGTTGATGTTGACTGCACCCGCGTCCAGACGTTGTGCGATACGCCGCGCACGAGCA TGGTCGCGGGTCCACACTGTGGCAGACAAGCCGAACGCCGAGTCGTTAGCCAGGTGGATGGCCTCGTCCT CGTCCGCGACACGCATCACAGGGATCGTGGGGCCGAAGGTCTCCTCGGTCATGCAGGCCATTGTGTGGTC GACGCCGACCACAACGGTTGGAGCGTAGTAGTTCCCGACCGCGCTTAGGGTTCCGCCGGCCAGTACCTGC 
Final Technical Progress Report DOE Award No. DE-FC26-02NT15340

GCACCTGCCGCCACTGCCTGGCTGACGTGGCGATCGGCTATCTCGACCTGTCTCGCCGTGACCATCGCAC CTACGTCGTCACCTGCCGCGGCACCTTGGGTAAGTGACCGTACTCGTTGAGCCAGCCGGGCCACGAACTC GTCGTAGATCTCAGAGACCACATACACCCGCTCGACTGAGATGCAGACCTGCCCCGCGTTGAACAGACCG CCCCACGCGATCCCCTCCACAGCACGAGCAAGGTCGGCGTCCTCCAGCACGATCGCCGGATCTTTACCGC CTAACTCCAAGCTCACAGGCTTCAATTGCTCGGCACAGCGGATCGCGATTGTCCTCCCGGTGGCTGTCGA GCCCGTGAACTGAACGAAGTCAGCGGCGTCCACGACCGCAGCACCTGCTTCGCTAGCACCCACAACATGC GCCAAAACTGGTGGGGCGCCGATCTCGTGCCAGCCCTCGACGACTCGTGCACAGGTCAGTGGCGTCTCTT CCGAGGACTTCGCCAGGACTGCGCATCCGGCAGCCAGCGCAGGAGCTGCATCCATGAGGAAGAGGGTGAT CGGGAAGTTCCACGGTGTGATGAGCCCGACAACCGGGTACGGGTGGTAGGTCTTGGACAACCGTTTCGGC AGACTTAGCAGACCTGCGGATCGGACTTGCTCGTCAGCGAGAAACTTCTCGGCATGGTTTGCGTAGTAGG TGATGAACTCGCACGATGCTGTCGTCTCAACCAGGGCGTCGGGGCGCACCTTGCCTGTTTCGGCTTGCAG CAGATCGGTGAGTTCGTCGGTGTGAGCCAGGATCCAGTCTCGCCAGCGATGCAACCATCGGGAGCGTTGC CGCGGGCTTGCCTGAGCCCACCCGAGCTGAGCGCGGCGGAGATCGGCCGCGATGCTGTTCACGGTCTCGC GGGTATCGATGGGGACGGTTGCGACCAGCCGGCCGTCGGCGGGGTTGCGTACGTCCAGGGCATCCGCTGT GGTCGCCGTGCTCATCGGGCCTCCGGGAGTGAGTGGAGCCCGCGGGCGATCCCGTCGGGCTGGGTGCATC TGTCGGGTGTGTCGGTGAAAGCGTCAGCGAGCACGGGGGTGCGCTCAAAGATTCGCTGTCCATAGGTATG CCGAAGCTCGGTCAAGATCGTGTTCAAAGGCAGGTCGTCGAAGACGCCGCGCTCGCGGACATACTCCATG TGCAGGGCTCCGTCGGCGGCGAAGGCGTGCAGGAGGGCGTCGTGCTCGCCGTCGAAATCCACCGGCGGCA CCCCGAAACGGGCACACAACTCGGTGTTGAACGCCGGGGTCGCCTTCATCCACCGGCCGTTGACGTAGAG ATGGCTGTAGCCGTGATAGACGAACAGGTCTGTGCCGCCCATGAGCGCTCGCAACGCTTCGGTCTGCAAA TGATTTCGGACGTCGGCAAACCCCAGTAGCGCCGGAATCCCTGCCGCACGGCACACCGCGGTCAAGAGCA CCGCCTTCGGGACGCAATAGGCGCGCCCGGTCTCGAGGACGAAGCTTGCTCGATAATGGGCGGGATCGTC CGACACCGTGTAGGGGTCGTACCGGATCTGGTCGCGGACGGCGGCGAAGAGGCGCTTCGCCTTGTCCCGG TCGGTGCCCGCATCCCCAACCGCGGCGGCGGTGAACGCCCGGACGGACTCGTGCTCGATGTCGAGGAAGT CGGTCGCCCACGAGTGCGCCAGCGGTGCCTTATTCATCTGCGCTCCAGAGTTATCGGCAACCCGTCGACC GGAATGGGGAGCGAGGTGTTGTCCCACCTCGCTTCATACTGATCGGGGACCGTCCAGGTGTAGGTGCGTA GCATGTGGTGCAGAATCGCCTTGACTTCGAGGGTGCCGAAGTGCAGACCGATGCACTTGTGTACCCCTCC TCCGAAGGGGATCCACCCGAGGCGGTGATTCTGGTCCTCACGCCGCGGTTCGCTGAAGCGGTCAGGGTCA AAGGTGTCGGGGTCGGTCCAGCACGACTCGTCGAAGTGGTTCACCGACGGAGCCACGGCCACCAGGGTGT CCGCCGGGATGTGGTGGCCGGCGATATCCACGTCTTCGACCGTCTTGCGCATCACGATGGGCACGGGCGC CACCAGACGGAGTGTCTCCTTGATCACCAGGTCGAGAGTCTCCAGTGAGTCCAGGTCGTCGATGTCCGGG CGGCGTCCACCGAGTCGGTCGGACTCGGCCCGCGCCCGTTCCTGCCAGGCCGGGTGCTTGGCGAGGAAGT AGGCGGCAGCGGTGGTGGTGATGGTCGATGTGTCGTGGGCCGCCATCATCAAGAAGATCATGTGGCTAAC GACGTCCTCATCGGTGAACGCCTCACCGTCGGCCGTGCGCGCATGGCACAGCCCCGCAAAGAGGTCGCCT CCTTGGCCACTTCGAGCCGCCGGCAGGTGCCGGGTGAAGTAGTCCTCCAAGAGCGCGCGCCCACGGACTC CCGCTCGCCAACGAGTCCCCGGTAACGGGAAACGGATGATCGAGCTGGCCGCACGCACGGTGGCCACGAA GGCGTCGTTGATGGCATCGGTATCCTCGCTACCCCCCCGACCTGCCATGAAGACCTTCGTGGCGACGTCG AGAGTTAGTGCCTTCAGCAGCGGGTAGATGCGCGTGGTGGATTGTCCCGCTGTCCACCCTGGGATGCTGG CCTCCAGCGTCGGGCCGAGCTGGTCGACGTAGCCGGCGAGTCGGTCGCGGGTGAACGCCTCCTGCATGAT CCGCCGATGCATCCTGTGTTCGTCGAAGCTCATCAACATCAGGCCCCGATGAAAAAAGGCATCGATGAGA TAGGTCCACCCGTCCTGGGAGAACGACTTCGCCTTGGTGGTCAGCACCTCCTGTATGGCATCCGGTCCGG CAACCGCCACTATTTTCGTGCCGAAGGCGCCCATCCACGACACAGGTCCTAGCCGCTGGTACCTGGCCCG GGAGAAGTTAGATCCGAACCGGATGTAGTCCAGGGTGTGACCGAGCAGAGGCATGCCAGCGGTCCCTAAG ACCGGTTTGAGGCCTGACCCCGCCGGGGGCGGGGCGAGCTCTCGGACAGGCCACCGGTCACTGGCCCCGA GCAGGGCCGCGTCGACCCGACTCGGCAGTGGCACCATGAGGACCGACGAGAGTCGCTCACGACCCTTCGC CAGCGGGAGATGGATAATGTTCGTCACTTGTGTTCCTTTCCGAAACGCAGCCCGCACTCACCATTTCGTA CCCATCGCCCGAAGACCCGTCATCTCAGGCGGAGCTGGCAAGCGCCAGTTCAGTTCGGTCGATGGGGAGT TGCTGCGTGATTACCCGCTTGCTGAACATGAAGTCACGGGGGCGGTTGATGCAGTCGGCGCCAATAAGCT CTCCCTCACGGAGGTAGAAGCAGGTGAAGTCGCGGTCTCGGGTCGGGTCTCCGCTGAGGATGACTTCGTC GTAACCGGTGTTCAGACCGGCGATCTGGAGCTTGAGATCATATTGATCCGACCAGAACCATGGAAGCGCC GCTATCTTCTTGGACTTCCCACACACCGTCGCGGCAGCGACCTTGGCTTGCTCGTGCGCGCTCGGCACAG ACTCCAGGCGTAGAGGGCGGCTGTAGCGAGCCATGCTGTGGCTTGCGCAATCCCCGGCGGCCACGATGTC GGGGTCGCTGGTCCGGGCCTGGTCGTCGATCACGACGCCGTTATCAATGACCAGACCTGCGGCGGCGGCA AGATCGGTGTTCGGCTCAACGCCGATGCCGACAATGACGAGGTCAGCGGGAATTGACTCGCCACCGGACA GAACTACTTCACGGACCCTGTCCTCGCCGGACAGAACCTCGACCAGCACACCCGTCCGGATTTTCACGCC CTCCTGCCGGTGAATACGGTCGAAGAACGCTGATACTTCGGGGGCAGTGACCCGCTCAAGGACGCGCCCA GTCGCCTCGAGCACGGTGACCTCCAGCCCCAGTGCACGCAACGAGGCAGCCGTCTCCAGTCCGATGTAAC CGCCGCCGATGATCACTGCCCGACGTCCTGCGTGGGCGGCCACTCGGATCATCTCGACGTCTGCGACTGT GCGTAAGTAGTAGACTCCGGCCAAGTTGGCTCCCGGGGTGGGGAGTCGCCGAGGGCGGGCGCCAGTGCAC AGCGCAAGCTGGTCGTAAGCTAGTGCGCCGCCGGTACTCAGTGAGAGATGACCACCCGAGCGGTCGATCG CCTCCACCGTCGCATTCAGGACTTGGATTCCCTGCTTGCTATAGAAATCGGCATTGCGGATCGCAAGCTC GTCGAGTGTGCTCTTCCCGGCCAGGTACGCCTTCGACAAAGGAGGGCGTTGGTAGGGCAACGTCGACTCA TCGCCGATGAGAACGATCTCGCCGGTCCACCCTTCCTGTCGAAGACTGGCCGCGAGTTGGGCTCCGGCAT GGCTGGCCCCGACGACGAGGGCCCGATGTAAGGTCATACGTCGGATTTCGGGGTGAGACGGACCATCATC 
Final Technical Progress Report DOE Award No. DE-FC26-02NT15340

TTGCTGATGCCCCTGACAAAGTTGGACTGCACATACTCGGGCTCGCCGACGACCTCGATCTTTTCGAAGC GAGAAAGCAACTCTTCCCAGAGAATCCGCAGCTGTAATTCCGCCAGTCGGTTGCCCATGCAGCGGTGGAC ACCGAAGCCGAAGGAGATGTGGTTGCGAGCGTTGGCCCGGTCGATGATGAAGTCGTCGGGCCGATCGAAC ATCCGCTCATCACGGTTGCCTGAGGCGTACCACATCACTACCTTGTCGCCCTTGCGGATGAACTGCCCGT TCAGCACGGTGTCGGCCTTGGCGATCCGGCGCATGTAGGCCAACGGTGTTTGCCACCGAATGATCTCCGA GACCATATTGGGGATCAGGTCAGGGTTCGCCTTCAGCTTCTCGAACTGGTCAGGGAACCGGTTCAACGCG AGAACACCGCCGCTCATCGAGTTCCGCGTCGTGTCGTTGCCTCCGACGATCAGCAACACAAGGTTGCCCA GGAACTCCATCGGTCGGTCGATCAGATCCTTGGTGTCTTCGTTGCTCTGCAACAGGGTGATCAAGTCGAA TCCGGGTTCCTCCCCGGCAGCTGTCCGGGCAGCCTTGTCGCGCCAGAGAGTGCTGAGACCTCGCGCCATG TCGCGCATGCCGCGGAACACCTCGTCATTGTCCGAGGGGCCGCCGTTGGCTTGCTCCATCGAGGATGCGA GATCAGACCAGTAAGCGAGCTTGCGGCGCTGCTCGTAGGGAAAGTCCAGGAGGGTCGCAAGCATCCGAGC GGTCAACTCGACCGAGACGTTCTGGACCCAGTCGAACGGCTGATCCACGGGCAGGTTGTCCAGCACCTCC TGGACGCGCGATCGAATCAGATCCTCCATCTCACGCAGGTTCTTCGGTGCGACCACGCCTTGGACAGCGG CCCGCTGCGCGTCGTGTCGTGGCGGATCCATCGCTATGAACATCTCGATATCAAGAAAACGAGGTGGAAC CCCGATGACGATCAACGGCTCGGCGGAGAAGACCTCGTGGTTCTTGTCGACGGCCATGATGTCGGCATGC CTTGTGACGGACCAGAACGGCCCGAACGGACTGTTAGGCTGGTAGTGGACCGGAGCCTCGTTGCGTACGC GCTCGAAGTACGACTGCCAGCGCCCCTGGCGATAGAGGAAAGGGTTGCTGAGGTCGATGTCGGCGAGTTC GACGTCCTCCACAGGAGGGATGGGGCTCTCGATGAAAAGCCTCTCTCCGTTCGCTCCGGTCACCCATCGC CGCGTCTTGTCGTACAGTTGTGCGCCCTGGATCTGCCGCTCCATCGGGATAGTCGACTGAACCTTAGCGG CGATTGCCTCAGAAAACTTCATTGCGTCTTATCGTCTCTCTCGTCGTCACATCTGAAACTCAGGCATTAG GACGGTCAGGCCGTCCCACGCCTCGGAGACCGGAATCTGGCAGGACAGTCGAGAGGTCGGTTGACGCTCA GGGTTCATCGCGAGCATCTCCTCCTCGTCGGAGCCGGAGAGGCCCACCTCCTCGGACCACTGCGGATCGA CGATCACATGGCAGGTGCCGCACGCGGCCTCGCCTCCGCAGTCGCCGTCGATGCCGGGCACTGCATTGTT GGTCGCGACCTGCATCAGCGACTGTCCTTCTTGGAAAGGGACCTCGTGCTTCTTGCCGTCGTGTGAGACA AAGGTGACAACTGCCATAACCAACTCCTGATACGTTGTCCTCGCCTTGGGAACTTGATCAAAGTGTCGTC GGGGATGACCGCTACGGCTATGTTCTGCGGAGTCAAAAACTTGTGAATTCGGCTCAAAGGGATCTGGTGA ACCAATGATCCTCGATGACGAGGGTGTGCCCCCGCTCCTGTTCGTGCAATTGCTCGAGCGCCAAGCATTC GACCCAGACGCCGTCGCGCGGCTTCGCAACATCATGTCTCGCGAAGGAACCGACGAGGCGACGCTGATTC AGCGCGACGTTCAGGCCCCCATACGGTGGTTTCGGGAGGTCTACCCCGAACTCGACGTCGACCAGGCAAC ACTGCTCGGCTTTGCGTTCGCTGAACAGGCCCAGTTGACGTCCTTCGGCCCGTTGAGTTCTCCGCTGGTC AGCGCAGGCTCGGTAGCCGAGATCGTGGAGTTGCTCACCTATCTGCCGTTGATCTCGACAGCTCTCAGTC CACAGTTCCATCCAAGTGACCACGGCCTCACCATCGGGTTCACCGCACACACAAGCGATCCGGCCCTGGA CTGCCTGGTCGTCGCCTACTGCGGGTCGGCGCTACTGCGACTGCTTGACATGCTCGCCGGCGACATGCCT ACCGTCACACTCCACTTGAGTTGGCCGGTGCCGCCCCTCCTGTCCAATCATGAGGACTTAATAGCCGGGC GCCTGTTTTTCGGCGCTCCGATGTCCTTCCTTCATGTTCCCGCGGACACGCTTGACGAGGTTTGCCGGTT CTCCGATCCCCTCACATACCGACTCGCCATCGGCGATCTGCAGCGAACTCTCGACCAGCGGGGCAGGACC ACGTCGTTTTCGGAGAAGACGAGACGGCTGCTGGAGAAGGAGCCCGCACAGGGGAGTATCCACTGGGTCG CACGCGAGTTGTCGATATCCGTCAGCACACTCAAGCGGCGCCTCTCCGAAGAGGGGACCACTTTCCGCGA GTTGCGCCAGTCATTCCTGCGCGAGCGTGCGATGCTGCGGCTACTCGACCGATCAATGTCGGTGACTGAG GTCGCCACGGAGCTCGGATACAGTGACCTCACCAACTTCTCACACGCCTTCAAGCGATGGACCGGCCGTT CTCCGAACGAGTTCCGGCATCCATGACGCTGAGCCGCCCGGTCTCCGGCGGGTTGAGGGGGCTGGCGGAT GCGGTAAATCAGTGTGGGGCCGTTCCCAGTGCATGAGATCAGTGGTACTCCAGGGGCCGACCAAGATTTC GGTCCGACAGGATCTTCCTGAGTCTCGGGGTCACCGCAACGGCGTGATCAGTGGTGGAATCCGCGTCGAC GTGGGCACTTTGCAGCCAAAGCGAGGGGTTATGCCTGCTGTGTGGGCATGGGCATGTGCTCGGGCATGAT CATCTTTCTCGGAAGTGACAGCTCCGCATGGGAACCTAGATTTTCCGGATTGAAGGCAGGTGCTGCGGCA GTTGTGGCCGCGGGCCGGAGCGCCAGCCGACCACCTTTGTTCGAGAACGCCTGCTTGGGCACGTCAAGAC ACCCCCGAAGTTGGGCGCGGCCGCGTCGAGTAACCGGTTGCGTGAGCGTGCTAGCCGCGGATCGGCCTGC TCGTCGACTGCGGTGAGTGAGTCGGTCGTGGCGTGGTCCGGGGCGGTCATGGGAGCGCGCTCCTCCGATT CGTCTCGTTCAGCCCTGGGCGTATGTGTCGACTCTATCCCGAGCCCCCACCACTATGATACGAATGGTCT CGTACCGAGACTGTTGGTCTTTGAGTTGGGAGGCGCCGGTGGACACACCTGGGACGGGCGCTGTTGAGAT CGACGGGTGGACCAGGGCCGAGTGGTGGATGCTGACGGTGTCGTGCCTGGCGGTGGCGCTGGTGGTCGCG GCCATGGCGGCGTTGTACTCGGCGTTGCCGCAGATCGCGGTCGAGACCGGGGCGACGCAAGCCCAGTTGA CCTGGATCGTCGACGGTTACACGCTGGTGCTGGCGTGTCTTGTGCTGCCTGCCGGAGCAATCGGTGACCG TTACGGGCGTCGCGGTGTGCTGGTCACGGGGCTGGCGGTGTTCTCGGTCGCGTCGGCGCTGCCGCTGCTG CTGGCCGACCCGGTGTGGCTGATCGCAGCGCGCGCGCTGGCCGGGGTGGGCGCGGCGCTGGTGATGCCTT CGACGCTGTCGATCCTGACCGCGGGGTTCGCCGCTGCCCATCGTGGCCGGGCGGTGGGGGTGTGGGCCGG AGTTGCGGGGTCAGGGGCAGTGCTGGGCATCCTCGGCGCCGGGGTACTGCTCGAGCGGTGGTCATGGCCT TCGGTGTTCGTCGGGTTGACCGTGGCGGGGGTGGTGCTGGCGGGGTTGGCGTGCACTATCGCCGAGTCCC GAGAGAGCGAGCATCCCCCGGTGGACTGGGTGGGTGCGGCGGTGGTGGCCGTCGCGATCGCGGCGATCGT GTTCGCCGCGATCGAGGTTCCCGCGCGGGGCTGGGCCGACCAGCTCGTCGCCGCTATCGCCGGGGTCGGT CTCCTGGCAGCGGTGGTGTTTGTGATCGTAGAGCTGCGGTCTTCTGCGCCACTACTGGACGTGCGGTTGT TCGCCCGCCGCGGTTTCGGGGCCGGCTCGCTGTCGGTGACCATCCAGTTCCTGGTTACCTTCGGAGTGTT CCTGCTGTTGGTGCAGTACCTGCAGCTGATCCTCGGCTATGGGCCGCTGGCCTCGGCACTGGCGTTGGCG CCGATGGTGGTGCCGCTGGTCGTGATCTCGGTAATCGCACCGTGGTTGTCGAGCCTCGTCGGGTTGAAAG 
Final Technical Progress Report DOE Award No. DE-FC26-02NT15340

TGATGACCGTCTCAGGACTGCTTGCCATCGCTGCCGGACTGGTGCTGGTAAGCCGGTTGACCGTGGCCGC GGACTATCCCGGCCTGCTGTGGCCGCTGCTGATCATGAGCGCGGGTCTGGGATTGTGCACCGCCCCGGCG ACCTACGCCATCGTCGCCGACACCCCTGCGGCCAAACAGGGGGTCGCCGCCGCGGTCAACGACGCTGCCC GCGAGATCGGCGCCGCGATCGGGATCGCCGTGGCTGGCAGCGTGCTCGCCGCAGGCTATACCCACCACAT CCAGCCCGCTCTGCCCCAGCTTCCCGAGCCCGCCCGCGGCCCGGTCGCCGATTCCCTGGCCGCTGCTCTG CAGGTCGCTGACCAGGCCGGACCGGCGGCCCAACCGCTGGCCGAGTTCGCCAAGGCAGCGTTCGTGCACG GCAGCAGCCAGGCCGCGCTCACGCTGGCCGCCCTCACCGCCGCCGGGGCGATCGTGCTGGCAGTCCTCTC TCCCGGCCCCACCACCCGCCGCTCCCGCGCGTCACGCACTCACACGAACACCCCTCCCCGCTCGAACACC CCCTTCTCGTGAAAGAGGCCCCATCATGACGGCCTATCGCACGATCCTCGTCGACACCGACGGATCCGAC TACTCCTACCGCGTGGTCGACCACGCCGCCGAGCTGGCTCACGTCACCCACGCCCGACTCCTGATCATCT GCGCCCGTCACGACATCGACGAACGCGCACTCGGTACTGACGTCGACCGGCTGCGAGCTACCACACCCAC CGACGCCATCCTGCGCACCGCGCACCAACATGCGATCGCCCACGGCGCCGTCGACATCGAAACTCGCATC CTGCCCGAGCCCACCGTCCGCGCACTGTTTCACCTAGCCGGCCCGACCGCCGACGACCTCATCGTCACCG CGAACCCCCACCGGTCACCACTGGCCCGGCTGCTATTCACCTCGCCGGCCGAGCTTGCCCACAAAGCCCA CTGCGACATCCTCATTGCTAATGGTGAGGACACCTACGGAAGGCGTCCATGACCGAGCACACACCCAACA CCAACACACCCGAGACCGCCGGCCCCGAACCTTTCGAGGCCGAGGACTGGATCCGTACCTTCGCTGTGGC GCCGCCTGAACACGTCGACCTGCCGCCTCACCACCCCCACTGCCTTGGTTGCGGACCGGCTAACCCTCAC GGCCACCAGTTGCGCGTCCGACGCGACGCACACGGCGTCTACGCCCACCACAGCTTCGATTCCCGCCATG TCGGCGCCCCAGCGATTGCCCACGGGGGCGCGGTCGCCACCGTCCTCGACGACTTGTTCGGGTTTCTGCT CTACACCGTGGGAGAGCTCGCCGTCACCCGCCACCTCGCTATCGACTACCTCGCCCCGGTCCTGCTCGAC ACCCCCTACACCCTCCGCTCTCACATCCACTCCCGCGCAGGCCGCAAACTACACCTGCGAGCCACCATCG AAAACAACCACGCCCACGTGGTCACTACCGCCACCGCACTGTTCCTCATCGTCGACATCGACCACTTCCT GACCCCCCGACACAACGCCTCGGCGGCGACCGCGCCCACTACACACGAAGACCAACAAGGCCCTCAGATG GACAGTCCCTGACGTGTCCCTCTAGCCGATCCGCGATCGGCAGAGCGCTGTGCCGTCCGTGGCGGCAGTC GGAGACGTGATCGAACCGGGTAAATATCTCCGGCTGTACACCCCCAACCGGAGGCCGGTGCGCGCCATCA GGTCGCAGAACGCGGCGTTGCGGCCGTGTTCGGTCACGACGACGGGACGGTGCTCATGGTTTCAGGGTGA GAGATTGCGTGAGGATCGCATCCCGGTTCCGCGCACCCGTCGCCGGTGTGGGGCGACCTCGGCGGTCGGT TTCGGCTCGTGGCAGCGCAGCGTGACTAGGTGCTGATCGTTGCGCGTAGTCGATAGTCGTCTCCTGTTGA ACTATGGGCAGGTGGGGCGCAGTATTTACCGATGAAAACCGAAGAGGAAGAACGACTGATCGCTCATGCG GTGACGCGGTTGTCGGTGAAGTATCCGTCGATATCGGCTGATGTCGTTGCGGACGCTGTTGCGTCTGCTC ATGCTCATTTCGACGGTCACAAGGTGCGTGATTTTGTTCCTCTGCTCGTCGAGCGCATGGCGGGTGAGAA ACTTTCCTCGCTGGTCGCTTCATAATGGCTGTGTCCGCGGCAGAAGAGTCCGATGTTGCGCAGGCGAGAG TGTTTCTGGCTCTGTTGAATTCGGAGATCGACGAGTTGTCGGAGCGCATCGAATCCGCGCTGCGGCTGGT GCGGGGTGCGCGTCCGGTCGCACCGGTGTTGGCGTCGGCTGCGCGCACCGATGCGGCGGCTCTGCGCAAG GACCTTCATCGGGTGCACGCTCTGGTTGAGCAACTGGTACGCCGCTTTCCTGCCGTCAACAGTCCTACCG ATGTGCCTGCCGCACGTTCTCTCCGCAGCTGATCGGTTTCCCTCGCAGCATTGATCGCGCTGAGTACGCG GTCCGGAGTCTCCCTCTATCCACAGGCAGCAATGTACGGGGACGGTGAGCTGCGGGGATGGATCGACAAC CAGCATCGAGTGCTGCCCCTGCTGTGGTCGTCTGGGCGGCCTATATCGGCGGGGTGTGCCCTAGCGCACA TACTCCGGAGAGTGCATTGGATGAGGTTCGGCGCCGTGGCTTGGGGTTCGTGGTCCTGGGTAACAGTCCG GGATCGAGCGTCAGGAGCAGGTGACGGTCAGGGAATAGATCATTTCCAGATTTGTGGGCTCGCCGTTGGC TTGTTCAATTCGCTGCCTGGTGGGGGCGCGAATTGCGCCTACCTGGACGCGTCGTTTCCGGGTTTTCTTG TCGGCACTGCGTAAAGGTCGGTCGTACGTTGTATTGCTCTTCTGCATCTGTCGGCGGTGAGGAGGTATTT TTCGGCGGCGGTGTAGTCGGTGACGGCGGTGCCGTCCGGGCCAAGGGTGTGGGTGATGAGGTCGAGGTAG CGACGCCGGTAGAGGTAGGCATCGGGCTCTGCGAGTAGAAATTCTACGGCGGCCATGACGTCCGGGGGCG GGGCCGTTCGGGTGCGCCGGGCGCGTGCGTTCAGTTGGTGTGCCGGCGGCACCTCGTGACTGTTCGCGGC AGAGTAGAGCCGCGCGATGCGGCGAGGACTGCTCATCGGGGCTCCGCCGCTGCAGGCAGAATGGTCATCG CGTATCCGAGCCGTATGTCGTGGTGGTGCGAGTGGTGGTAGCGGGGCTTTTCCTGCCGGATGTGGTGGGA AGACCTCGGTGTGTAGGCGCGGCGACGCCGGAGGCAGTCATCGAGGCATCACGAACGAGAAAAGGTCCAG CATGTTCATCGGTCTCCTTGTCGTTAGCCGGGCACATGAGATATGTATACCTTTCTGTCCCGCCGGGTCA CAGATATCGAAAGTTACAGATTGGAAAACTCGCGCCACGAGCGAACGTGCCGATGTTCTAACGGATCGGG TTCCGGGGCAGCGACCGAATGCCGTCGCCGGCGCTTCGCGTTCGATCGCCAAAGTTGAATGTGCCGGATG TGTTGCGGTCAATAGATTGCCGCCCATACAGCTGGTAACGGTGCGTGCTGGAGAGGTGCGAGTTGCCTTA CGTAGGCGTCATCAGCTGCGGCGCTTTATTCTCGCGCAAATGTCTCAGGAGGATGTCCCGAGTTCCGTGG AACTTCGGTGGCGGTCCGGTGAGCATCATGCTGCGCTCACCTGATCATTGTGCACGACGGGTACGACATG TTCGGCAACCTCGCGTTCGAGGGCTGCTCGCAGTGCCGGCAGGTGCAGGTGGCCGTTCACCCGGCGGAAT TGCTTGCCGGCCTCGACCATCCCGGCGGCGCACCAGCGCAGCGCCATCTGCCCGTCGCGCCAGCGCTTGA CGTTGCCGGCATGTTCGCGGCAGACCGAGATCATCGACTCGATGCAGTTGGTCGAGCGCAGCGTGCGGGC CAGCGTGGGCGGTACCCCCAAACGCAAGACCGTGAGCGTCTCATCGAGGCCCTCGCGCAGACTCGCCGCC GCGCTGGGGTGGGTGCGGTCGAGTTCCTTGGCCAGGGCCAGCAGCGCGGCTTCGGCCTCAAGCGCCGATC CGGCATGGTAGGCATCAGTCATCCTGCGGCCTACAGTGGTTCGAAGGCGTGGGGGCAGATGATCTTTCAC GTTCCGAACCTTGTGCAGCTGACAGCGCTGAATGACCGGGTGATCGAGCACGTCGAGCACSSCTTTGCGC AGCGCCTTGGACCCGTCCAGGCCCACCAGCATCGGACGGGTGACGTCCAGACCCCGTTCCCGCAAATCCA CCAGCAGCTCGGTGACCAGGGTGGCGTTCTCCGTCGAGCCCTCCACCAGCGCCAGCGGGTGCTTGGTGCC CTCGATGTCGATCCCCAGCGCCACGACGCAACACGATTCGGCGAAGTGCACCCCCGTCGATCATCAACGC 
Final Technical Progress Report DOE Award No. DE-FC26-02NT15340

CACCAGATCCAGCCCGGACAGATCACGCGACAGCAGCTCAGCCAGCGCGGTCTCGGTCATCGCCACGAAC CGGCGGGATACCGCTGATTTGCTTGTCGCCGTGGCCTTTTCCGTGATCTGGACGCCGACCGGCTCCAGCC CGACCGGGTAACGACGAGTGGACAGCCCGGCCAGCATCTTCTCCATCGCCAGCCGGCCCAGGATCTCCGT GGAACTGAACAGCTCATAGGACGCGACCGCCAACTCGCCCGTCTCGTCGGCGGCGCGCACCCGGGGCCGG GTCACCGGCACCCGTCGCCCACCCAAGGTCACCGAGCCGCGTTCACGACCGTGCCGCACCGCGGTCCTGG CCTGATCGTGGCGACCCTTCGGGCCAGCCAGCGCGGTGACGTCAGCCTCCATCAACGCCGCCATCACCTG CAGGCCCGCGCCGACAGCCAACGCCAGCAGGCCCTCGCTCATGTTTTCGGCGATCTCGCTCATCGCCACA CTGACTCGTTCGGGAACCGCGGATGCACTCACATCGACGGACTGAATCTGGTTACTCTTCTTCATGGTGG TCCCCTTGCTGTAGGAGTTCTTGGCGGAACGCCCGATACCTACCACACGGCAGGTTTCGAGCAGGGGACC GCCACCTCAACTTCTACGAGACTCGGGACAACCTCTGTCTCAGGCAGCTCCGCTAGAGGCAGGCCAAGTT GTGTGCAACAAGAGCAATGATCAGAACCTGTGGATGGTTCTCGGCGAGGCGGCGTGAGTGGGCGCACCTT CCCGGGAATGATCTGAAATTCCATCAGCTCTGATCAGGACCGGCGTTGGCGCGCTGGTTCGGGAAGGTAC GCCCATGCTCACGGTAGTTCAGGATCAGCAATCCTCCAACGACGACAGCGGTGTTGGTCGGTCGTTGCTT GACGAGATCGTTCGCGACGGTGCCCGGCAGATGCTCGCGGCGGCGTTGGCCGCCGAAGTCGCCGCATACA TCGAGCAGTTCGCCGAGCATCTCGATGAGCACGGCCGGCGGCTGGTGGTCCGCAACGGCTATCACCACGA GCGTGACGTGCTCACCGCGGCGGGGGCGGTGACGGTGACCGCACCGCGGGTCAACGACCGCCGTGTCGAC GCCGAGACCGGTGAACGGCAACGGTTTTCCTCGGCGATCCTGCCGGCGTGGTCACGCAAGTCCCCGCAGA TGACCGAGGTGTTGCCGCTGCTGTACCTGCACGGGCTGTCCAGCGGCGACTTCGGTGCGGCGTTGGAGCA GTTCCTCGGCACTGGTGCCGGGCTGTCGGCCTCGACGATCACCCGGCTGACCGCGCAATGGCAGGACGAG GCGAAGGCCTTCGCCGACCGGGATCTCTCGGGCACCGATTTCGTGTACCTATGGGTCGACGGCATCCATC TCAATGTCCGCCTGGAGCAGGAGAAGCTGTGCTTGCTCGTGATGATCGGCGTCCGCGCCGACGGCCGCAA GGAGCTCGTCGCGCTCACCGATGGGTTCCGGGAGTCCACAGAGTCGTGGGCTGATCTGCTGCGCTCATGC CGACGCCGCGGGATGACCGCCCCGGTGCTGGCCGTCGGTGATGGGGCGCTGGGGTTCTGGAAGGCACTGC GGGAGGTGTTTCCGGATACCCGTGAACAGCGCTGCTGGTTTCACAAGCAGGCCAATGTGCTTGCTGCGCT GCCCAAGTCGGCTCATCCAGGTGCGACCGCGGCGATGCGGGAAATCTACAACGCCGAGGACATCGACAAG GCCCAGGTCGCGATCAAGGCCTTCGAGGTAGATTACGGCGCGAAGTACCCCAAGGCGGTCGCCAAGATCG TCGACGACGCCGACGTGCTCCTGGAGTTCTACCGGTACCCGGCCGAGCACTGGATCCACTTGCGCACTAC GAATCCGATCGAATCGACGTTTGCGACCGTGCGGTTGCGAACCAAGGTCACCAAGGGCCCTGGGTCACGC GCAGCGGGGATTGCCATGGCCTACAAGCTGATCGACGCCGCACAAGCCCGCTGGCGGGCGGTCAACGCCC CCCATCTGGTCGCGCTGGTCCGTGCCGGCGCGGTGTTCCACAAAGGCAAGCTGCTCGAACGACCCATCGA CATCTCACCCGCTGAGCCCGACGAATCAAGCGAAACGGAGGTCGCCTGAAACACCCTCATCCACAGGTCT TGACAATATCTCCTTGTGTGCACCCGTACGTATGGAGAAGCTGAGTATCGGCGGGCGATTCGCGACTCGG CCGCGCGGTGGCCTCGGCCTCGATCAGCGCCTCAGATCCTCAGGAGGTGAAGCCGGGAATCGGGTATTGA CCGTTCAAGGTGTGCTTGCCGACGTCGGCGATCTTGTAGGACACCGGGTCGTGCAGGGAGTGGGTGCGCA CGTTGCGCCAGAAGCGGTCGAAACCGTACCTGGGATGTGTTCCGCGCGCGCCGATCACCTCGAAGACGCC GCTGCTGATGTTGAGGGCGGCGTTGGTGGCCAACGCTTTGACTCCCGAGACCTTCACCATCAGTTCGCCG CGGTCCTCGGGGGTGAGCGCGTCGCCCTTGTCCCACACCGTCTGCAGCAGGTGGGCCGCTTCACGGGCGG CGGCGTCAGCTCCCTGCAATGCGATGGTGAACTCACCGTAGGAGCGGATGGTGTAGGGATCCTCGGTTGC CTGTTGAATACCGGCCGGTGTCCAGGGCCTCGCCTGGGTACGGGTGTACTCCCTGGCGGCATCGAGTGCG CCGTGCGCGATCCCCAGATAGACGTTGGCGAAGATCAATTGCGCTATGGGCGCGAAGAGGCTGCCGCGCT CGGATTGTATGAAGGCGAGAACGAAGGCGTTGGGCGCGCCCAGCACTTCGTCAGGCTCGACCTTGACGTT GTGGAAGTCCGTGGAACCGCTGTCGGTCTGCCGCATGCCGATGGCGGCCCAGTCGTCGTTGGGCGTAACG CCAGCCCGCGATGTCGGGATAGCGGCAGCAATGATCGCACCCTGCTGCGGAGAATCATCCTGGACGACGC CGAACACGAACAGCAGGTCCGACCCCTTGGCGCCGCTGCAGAAGTGCTTCGTGCCATTGAGCACGTAGCC GCCGTCTTCGGTCGGGGTGGCGCTGACCTTCCAGTCCAGCACGTGGCTGTTGTTCTCGCTGGAGGCATTT CCGGTCCACCAGTTGTTCTGCGCGATCTGGGTGTACAGGTGTTCTTCTTGTTCCTGCGAGCCGATCAGTT CGATCATCGGGGCGTTGGTGAGGTGGTATCCGAACAGGTGTCCCAAAGATCCATCGGCTGCCGCGATTTC GCGGACGACCTCGATGGCGGTGGGCCAGTCTGCGCCCCAGCCGCCGTATTCGCGCGGGACGAGGAGCGAC AGCAGGCCGCTCGCGCGCAGGTCTTCGCGCTCGGCTGTTGCCGAACCCCCGGCGCGATCACGCTCGACGG CGGTGGCTCGCCACTTTTCGGCTAGCCCACGGGCAACCGCGACGGGATCGTTGTCGGCGGCGTCGCGTGG TCGAACGTGCTGCTTTTCAGGTGACAGTGTCATGCGGATGTTCCTATCGGTGGCGATTGAGGCTGTTGTT CAGAAATTCCGGAGCCGCCCACTGATCGAGGGCGACGGGTTCCTGCAGCAAGTTGTTGGTGAGCAGGAAT TGCTGTGTGCGCTCCAGGAGGGCGAGGGCGTCGTGATCCAGGCGTGGAACCAGACGCTGCTGGAAGTCGG CGCCGAAGCCCTGGCCTACTGCTCCGGTCGATACGCCCAGGTTCGCGGCGTGCAGGCTGGTCACCGCGTC GGAATGATCGCGTGCCCACAGCCCGGCGTCGACGGCCGCGTCGACCAGTCGTTGAACAAGGCCAGGTCGC TGGCGAACCAGCCCGCTGCTGACCGTCCACACACTGGCGTAGGCATTGCGCTCATCGAGGCCGAGATCCA CCACTGGGCGGGCCCCGGTGGCTTGCAACTCCCCGGCCCAGGGCAGCCAACTGTACAGGGCGTCAACGTC TCCGCTGGCCAACACCGCGGCCTGACCGCGGGCGACATCGGGAAAGAGGTCCGCACCCTTGACGGTCGCC GATTCTTCGAGCTGCTCAGCGGGAACATCGACACCAGGACTGCTGATCGGCACCAGCTCGACGTCGTCCA CACCCAGTTCACCGTGCTCAAGGGTGTGCAACAAGGCGCGCGCCTCCCACGAGCCCAGCGCTACCAGCGT TTGCCGCCAGGGATCCAACTCGAGGTAGTCGCCCAGCTGGCCGCGCAGGATGCGAATTGCCGAGGCCGAG ACGCCGATTCGACGTCCGGCAAGGTCGGCGGCCGCTGTGATCGGGCTGTCGTCGCGGACAAAGAAGCCCT GGCGCCCCAAGAGCGGGGTGATGCCGAGTAGACGCGTGCGCCCAGGTGCCCGCAACCCCTCGCTGAGCAG TGGCGGGATCTCACCCCCAAAACGGGTGTAGGCAGGCTGGTCGTAGGTGAAATGAACCGTGCCCTGCTGG 
Final Technical Progress Report DOE Award No. DE-FC26-02NT15340

CCGCTGAGGACGTCGAGTTCGATGCCGGCGGCGTCGAGGAAGCCCGATTCCGATGCCGTGAGCAGAGCGT TGGGTACCGGGCAGTTGCTGTAGGTCAGTGTGTCGCGGATGGCGGAATCGAGTTCTGAACCGGGGTTTGC GGGGTCGACGCGGCTTGTCATGAAGGTTGTCCTTGCAGTTGTGGTACGCGCAGACCCAAGTGGTCGCGCA GAGTGTTGCCCTGGTACTCGGTGCGGAAGTAGCCGCGATCCTGCAGAACCGGAACCACCTGGTCGACGAA CTCGTCGTAGGAGCCCGGCAGGAAGGCCGGAGAGATGATGAAACCATCCGCGGCGCCGCCCTCGAAGTGG CGGATCAGCTCGTCAGCGATCTGCTCCCCGGTACCGGCCCACTGAGGAACGAACCCCACGTTGGTTCCAT AGCGCCGACCCATTTCCGCCAGCGTGAGCTCTTCGCTGTGCGTTGCGGCGGCGAACATGTGCAGTTGCGT CGGGACATTCCGATCCTGCAGATCCCGCAGGATGTCCTTGATCGGAGTGTCGAGAGGGTACGCCGCCAGG TTGATGCCGGTGTGACTGGATAGCGTCGACAGTCCCACTTCCGGATGGACCAGACTGTTGAGATATTCCA GTCGTTCCTGTGCCACCGCCTGGCTTTCGCCGAGTACCGGCATCACGGCGGTGAAGATTTTCGTCTGATC GGGATCGCGCCCCGCAGCGTCGACCTCGGCTTTGATGCCCTGGTAGGTGGCCTGCATCACCTCGAGGTTG GGTGCAAGACTGAAGACGGCCTCGGCCCACTTCCCGGCGAAGCGCCGACCCCGGGGCGACAGGCCGGCCT GCAGGATCACCGGCTCACCCTGAGGTGAACGCGGTACCTGCAGAGGTCCGCGCACATTCAGCCACTCCCC GTGGTGATCGACGTAGTGCACCTTCGCGGGATCGGCGAACACGCCGGCCGCCTTGTCCAGCACGAGGGCG TCCTCGTCCCAGCTGTTCCAGAGTTTCTTGACCGCTTCCAAGAACTCATCGGCGCGGTCATAGCGGGCGT CGTGTTCCAGATGCTGATTAATGCCGAAGTTGCGCGCTTCAGCGTCGTTGAGCGAGGTGACGACGTTCCA GGACACCCGACCCCCTGACAACTGATCGAGCGTCGCGAACACCCGAGCAACGTGATACGGGGGATAGTAG GTCGCCGAAATGGTTGCCCCAAGACCCAGGTGCTCGGTCACCGCGGCCATGGTTGCGACCACACTGGCCG GCTCCAAGGCGACTGCACCCTGCCCGCCCAGGCCGACACCGGTGTCCAGGTTGTCCCCGTAGCTGTCCTC GACGGCCAACCCGTCAGGCAGAAACAACAGATCGAACTTGCCGCGCTCCAGAGTACGGGCGATGTGTTGG TAGTACTTCCCCGACAGAAAGTCATTCGACGCGTCCGTGTGCCGCCACGCCCCATGTGCATGAGTCACAT TGCCGGCCGAGAAGAAACCGGCCAGATGCATTTGTCGTTGTTGAGTCATCGCGTATGCGTCCTTTAAATG TCAATCATGGCTAGATTCCTGTCGCTAAGCAGTCTCACCCCCACCCCCGGGGTGTGCCCACCGTTAAACT TCACCTGATTTCAATGCTCCACCAGCCAAATTCAGGCCTTGAACCACGGGGCACGTCGGGTCAAGGTGAC GGTGACGGTGCCGATGACTTGGCCGCCTTCGCCGCTGCCGGCCGTCCGCATTTGGGTCGATCCGAGGTCC TTCGAATCTCTATCCTTGTCGAGGTCAATAGCCAAGCACCACAAAGGATCTCACTCGCACGGCGCATCGA GTCGACGTTGACCGACGCTCAAAACCGTCGATCTGGTTAGATCGTCGTGTATGCACCGGACGCCATGCCA ATCGCGAGCGTGTCCCGTCGACCACGTCGATCGGCAACATCAGCCGATCTGCGAGCAAATGACCCTAACG GTCGATCACCTCCGACCGCTGCGGGAAGGGGTGGTCGACTACCTGCGGAGGAGGGGCAAGCCAGCTGGCC GATGTCCCGCCGTGCGCACCCGTTAAGCGGCGCTACACCACACACGTCGGGTCGGACCTCCAGCGTCAGT CCAGCCGCGTCGCCACGTAGCGTGCAAAGGTCGATCCGGATGCTCGCGCATGAGCTTCCAATGTCTGAGG GCTGTAGCCGAGGGTTTCGGCGAGGATCGCTATCGGCGTGGTTTGGGTGAGTTCGTTGAGGGTGCCCATC CGTGCCTCCAGCGCCGCGAGAACTGCCCGGAGCCGTGCGCGCAAATGCGTCGGCGCGACGTGCATGCCGG GACGGTAGCCGCGAAACACCCACGGCGAGTTGGGATGTGCTGCGGTTTGGTTGTTGTAGTTGCTGGCTGC CAGCGCGCGAACGGGTTCATCGAGCGGAGGCGGGAGGTCGATCGGCATCGCCGCGAGGCTGATGGTCACG GTATCGGCGCTAATGGCGATTTGTTTCCACGTGAGGGCAGCGACGTCTTCTGCGCGTTGAGCGAACACGA TGATCAGAATGGCGGCCAATCGGTCGCGGGGGTGAAGAGTCTGCTGGTGGGCGACCTTCTCTATGACGGC GTTCTGTTGGTCGATCGGCATCCGTGGGGCGGTTCCGCGGCGGTGCGGTGTCATTTCCACGTCGGACGGG ATCAGCTTGGTCTTGATGGCCCATCTGATGAAACGCAGGAGGTGCTCTCGGGTGCTCGGTCCGGTCGCTT GCCATAGATCGATGTGGGCCTGGGTGAGCTGCTCGACGGTGGTGCCATGTTCGGTGGCCAGCCAGTTGCA GAAGTCGATGGTCACGGTGAGGGTCTGTTTGGCGCGCAGGAACGCCGAGTCGGTCACCACGTTCATCGAG CGGAGCCGTTTCTCGAGACTCCAGCGGATGAACCGGGCGAGGAGCTTTCGGTGCTCGTCGGTGGTGATGC GCGTGCYKSGCGGTGACCGCCCAGGACTGAAACCGGGCCAACCGCTCGTCACGTGACGCCAGGGCGCCGT GCTCGACGAGCAGSSTGCGCACGTAGTCGGTGGTGCGGCCCGGCGGCAGGTGATCGAGCACCTCGTGAGT GAGAGTTGGGTGAGCCGCTAGCTCCTTCAGGACCCGCTGGACGTGTGCTTGGCGGATCCAGGTCAGACCG CTGTTGGGGCGGTCCATCTCGGCGAGTGCGTCGACGATCGCTTGCAGTTGTGGTGCGATCACGCCGGTGT CGGGATGGGCGAGCAACCGTTGGGCGGTTTCGCGCAGCCCGCACCGTTGGCATCGGCCGCCGCTATAAAG TTCGGCCTCATCGCCGCAGCCGGTGCAGTCAACGTTGAGCCTGACGCCGCTACACCGCCGGCATGAGGGC CGTCCCTCGGCCAGGCCGGGCAGCACGCCCTGGTGGCCGCACGCGCAGATCCCAACGGTGCGTTTGGCGG CCTGGTAGCAGTATCCGCAGATCGATCCGTCGGGCCACCTGGCCACCAGCTGGTAGTGCCCGCGGCATCG GGCACAAGCCGTTGGCCAGCGCCCGGGGTCCTCAGGTTTGCGGGGCCGGGCCATCGTCGTCCTGCACGAT CCGTATCCGTTTGGCGACGGGGTTGCCCGGTGCGATCCCCAAGTCCCGCGGGCGCGGCGCGTCCACGGTG ACCGCGGCTCGCATCTCCACGTAAGGCTCGAACAGATCGTTGGGCGAGCAATCGAAGATGTCACACAACG CAGCGAAGGTGCGGGCCGGAATGCGTTGAGGTTCACCGGTCACGAGGCGATACACCTGAGCATCGGAGAG ATTGATTCCCCGCGAGCGCAGCAGCGGGCGCAGCTCGGTGGACTTCCACAGGTTGTGGGCCGCCATCACT CGCCGCAGATGCCAGTGAAAGCCGATGCGGCGCTGCTCAGCCATCAGCTTCCCCCATTCGGAGGCGCCGA GCGATCATCTGCTGCACTACGCGTTGCTTGAAGTCCGAACCCACCGAGGTATACAGCGCCGTCGTCGAGG CGTAGGAATGCCCTAATTGCTGCTGCACGAACAACGGATCGTAACCGGCCTCGAGAAGATGCGTGGTATA GGAGTGACGCAGACAGTGCAGCTTCAAGTCCGGCGGCAGACCCGCCCGCGCACGCAGCGCAGTGAACGAG CGTCCCAGGGCATTGATCGTCAACCTGCTGCCGCGTTCACTGGGCCACAACGCGGGGGAGCGGTCCGCGG TCCTAAACAGCTGCCGGCCCTCGGTGCACCAGTATTGCAAAAGCTCTACGGCCCAGGGGAACTCCGGCAC GGTCAGCACCGTCCGCCGCCGCGGCCCCGACCCCGTCGTGCCCTTGGCCCACCGCACGGTCATGGCGCCG TAGTCGCCGAAGCGCGGCACGTGCGGGTTGGGCCCGAAGTCCGTCAGGTCCAGCATGACCAGTTCGCGGC GCCGCAGTCCGTAGGCATACCCGACCTTGAACGCGATCGAATCACGCAACGCGGTCAGCCAGCGCTTGGA 
Final Technical Progress Report DOE Award No. DE-FC26-02NT15340

GCCGACCCGGTGCTGCTCGTCGACGAAGTCGTCGACCACGTCGAAGAAGTGTTGCAACTCGGTCTTGGTG AACGCTCGCCGCCTGGTCGGCACGGCATCGTCGGTGGTGTGTCGAGGCGTGTTCCACTCGAAACAGATTT GAGACGGCACGTCGCCGAACTGCTTCTCGCAGAACGGAACCCAACCGTATCTAGAGTCAGTGACATAGGA ACAGAACATCGAGATCGCGTTGCTGTAGGACCGCAACGTCGACCACTCGATCGGTGTTTCTCGCGATCTG AGATCGGCAAGGAACTGATCAAGATCAAGCGGGCGCCAGCGCCACGGATACTATACTCGTTGGCGAACTC GACGAACCGGCCGATGAGGCCGCATCTGGCCGTGATGGTGCTCGTGGCGAGACCTCGGGCCAGCATCTGA GCACGCCACCCGTCCACCATCGCCTTCAAGACCGCCTCATCGGCCCGCAGCAGTGAGACATCGCCACGTT GAAGGACCCGAGGAATGAACCCCGACAAATCCTCAGCCATGTGTACGCCCGAACTCGGCTTGCCCCAATG GCGCAGCGCCATAACGCAATGCGTCACTGTGACGAATTGCTGGCGATCCAGGCGGAGGGGTACAGGGGCA GATAAGGCCAGTCGCATCGTCGACATAGCGCAGCCCGCGGCCGGCGACGGGATCGGCAGCCTCGGGTTCG CGGCTTGCCGTCAACTGACGCCTGCGGAACATCCTCATGGATCACCTAACAACCGAATCAATCACTAAGT GATTGAACCATACGACGACCACGGCGTACTGACAACAAATGCATTACTCATCCAGGCCGTCTGTACCGTC ACGCGACGCGCTGAGCAGGGTCGATCGACCAGTCAGCTTGTGGTGCGACCACTTTCATTAGATGCAACTC GCAATGGTTGTTCGCCGATAACCTATATTATGTCATTTTGTGGATGAAAGTCGCTGCACCGCAACGAAAT ACACCCACATTGAACGTGCATCGTCAGTATCGAATGGCAATATTCGCTACCAGAGCGAGAGCGGACGTCC TGCAGCCTCGGCCGCAGCGATGCGCTCTTCGATGACGTCGACTCCTACGTTTTGTTCGATCCCGGTACGG ATCGCGGTGGCAATGGCGCGAGTGCGACCCGCGGGTCCTCGAACGTCGGCGAGTGGGCTGGCGATGTTGC GTCGCCGGAGCTCGTATTCGCTGCGGTTGCTGCGATTGGTTCCCAACTGCATGTGTGCCGGGTTGGTGCA GCCGGCGAAGTCGCATTGGTGGCACAGCACCGCGTCGTCGGTGTTGGACCAGCCCAACCTCGAGATGACA CCGTCTTCGAGTTGGTAGGCGAACAAATGCGCCGGCACGGTCCCGCGGCGGGACGGCCCAGGCAGACTCG CCGCCCGGAAGGAACCATGCCCGGTCGAAGACACACCGCCGATCCACGGCCAGCACTGCTCCCGGCGGCG TCGGAATCGCTTGGACTCGAAACGAGAAACGACGGCAGGATTGTCGAGCCAAGAACGCCACAGACGCTCC GGAATGCGTACAGGCTGGGGCTGCCCGATCAACGTCATCTGGAACGCCCGCAGCGCGGGAGCGCTGTCGG GCATGCCGGGCAGCGGGGATGGAGCATCGTTGACGAACGTCATCGGCCCTGCTCCTGACACTCGATCCGG ACTGTTACCCGAGTTGACTGTAGAGACACGTGCCGACAACCAGTTGAAGATCCGGGGTATGACCTGTGGT GGGCTTCGGTAACAGTCCACTCGTACCTGCCATTGCGGCTGCCCTTGCGATCATCCCAATGGCCCTGGTT GCCGAGAACGCCAACCTTTGCGCAGGAATGAAACTCACCCTCGAACCCACCGTCGTCAACGGCGCAGCCT TCGCGCACTGGGTTGACGCGATCAGTGACATCGTCGAAAACTCCATCACCGACGGAGAACTCACCGACAC CCCCACCGCGCACCGACTCGCATGGAACCTGTGCGCTGGCACCGTCGGAGCAGCCAACGCATCAGCAACC CTGCGAGAAGACATCGACCTCGCAACCTGTCTCGAAGGCGTGGTAACAGCGCAGTTGCGCAATGCCTTCA CGCCCGCCTGATCTAAAATACGGGTGTCGACCTCAATATCAACTGGTCGAAATTGCTTGAGGGACAACTA GACCGACAACATCTGCACCATTGACTTAGCGGCCGGATCCCACTTTCGAGCTCAGTTCTCGACCCGAGGG GTCCGGCCGCTAGCTTCCCGCTCGATCCGCGCAAGCTGGATATTGGTATACAACGACGAACGCTTCAAAC CCGTGTTCTTACATAGTGTTTCGATCTTGAACTGGCCCGTCTCATAAATTTGCAGTAGGTACGCACGCTC CTCGGGCGAGAGCTTGTGCGGCCTACCTTTCATCTTCCCAGCAGCTGTCGCGGCGGCAACAGCATCACGA GTGCGGGAACGAATCAGATCCGACTCGAACTCCGCCATCAACGCAAGAATGCCAATGAACATCTTCCCCA TCGGATCAGCCGGGTCGTACACCTGACCATCAATCGACAGCGACACACCCTTCTCCGCGATCCGCTCCAC CGTCTCATGCAAATCCTTCGCCGAGCGAGACAGCCGATCGAGCTTCGTCACACAAAACACGTCGCCGGCC CGAGCCGCGTTGATCGCATTATCCAAACCCGGCCGGCGAGCCTGCCGGCCACTGACACCATGATCGACAT AGACCATGTCACGATCCACCCCGGCCTTCGCCAGAAGATCAACCTGAACCGCCGTGTCTTGCCGGACCGT ACTGCACCGCGCATACCCGATTTTCACGCCACCCCCACTGCCGAACACGAATTGTCCACTAGAGCCTATG CCATAACGGACAAATCCAACGGACACCAAAATTGGACGTTTCGGTACGAAATTTCGCCACGTCAGTCGCA CCCAGACGCCCGATCTTCACCGTCCGGTATGACGAGGGTCAAAACGCACACCCAAAGCCGCAGGAAGCCG ATCAGCGCCCAAATCGAAATTTTCGGCCCCGTCGCGGTCTGTGGCAGCAGCTCGCACACAGCTCGACAGA ACAGCTCACGATCGAGGCCGCCACACTCACCAGGCGAAACCCGCTAAATGGCGTCGGATCTTGGCGCCGG CCGAAGCACGACGCCACCGAATGCAGCTTCGAGATACTCACCGAACCCCACAAGATTCGGACAGCTGGGG TTCGGATCCGCGTTTCCTCCCGCAGCAACACCTGACAAGATGCGAATCGGACACATCTGCGCTGACTCAA TTGCACAGCATTCGTAGGGTTCGACGGACTGCGGAACGATCCGCGTTTTGCTACCGCGACTCTTCGTGTG GATGCGAACGGACCGCGCAACGACTCATGCGACTGTTTACAGACCGTAGGACTAGTCGCAAACAGTCTCC ACAACGATCTTTCGCCGCCAAACAGACTGCGCAACGCCTTGCGCAGCTGAATACAGACACGAGTACGGTT CGCACAGGGGCTGCACAGCGAACTTTCAGTGCTCAGACGGACTGCGGAACGACTCATGCAGCTGTTTTCC AGTTGCGAAAGGTTTCCATAGACTTGCAATCGCGATCGTCTGGGCAGTCAAATAACAGCACAATGAGATC GAATAAGTTTAAATAACATCAGCAGTCTGCTACTGTATAAGCAATGACGTAAGAAACCCGCCAGTCCCGT AGGACTGACGGGCCGCGCGCCGACAATTAAATCGTGACGAGCTGGTCGAACAGTTGATCAAGCGTGAATC GTGCGAAGACGCCGCCAAGCCACTTGCCGATTCCACGCCAGATGCTCTCAGTTGAGCGTTTGGCGATCAT GTTTCACCTCCTTCGTATTTAGTTGTGTCACATAGGACAGGCCGTCACCCGAGGGTGAACGGCCTAGTCG TATGCCTGGAAACAGCATAACAGACGGAACGGTCTTCCGTTACATAACACGAGAATCGGCGCGTCAACTC GATCCCGCGGCGCAGAGCACGGTCACTTTTGCCTGTAAAAGCGCTGGTCAGGTCTTTTTTAAGATTCTGG GATCTTCGCCTTCCTTCGTCGCCGCACACTGCAACTCCCTTCCGCGCATCCACTCCCGGCCTCATCGTCG ATTGTCGGTTTGCCGGGTGGCAGTTCGGATCCGCGACTGCCGACACCCAAACCATGTCCGACCTCTTGAG TACGATCAGACCGCGACGAGCATCAGGTCGCCACTCTGACCAGACCGAAGCGGAAGCCGAAGTCCTGACC TGAACGCAAACCCGTCCAGATCGGTCACACGTGGCGACCTGATACTCATTCACCGACCGAAAATCAGAGC TACCGCGACACCGCCCGTGGCCGGCCTGCGCAGCTCCCGCACAATCACCACGCCAGCGCACTGCGCGGGA GGTGCTCACCACCGCACTTACCCACCGCCGGACACACTCCAAAAACTGAACGCCACCTCCGGTGCCGTTG 
Final Technical Progress Report DOE Award No. DE-FC26-02NT15340

CCGCTACGCGGCCCCGAGTGGTCAAGAAGTCGTCGTACAAGTCGCCCCAGAACACCATGCCGGGGACCCG ACCCGTCAACCCACGCTTCGCTCTACGGGGTTGACGGCCCACCCTCGACATGGTGACAGGGGAGCGACCC TACCGACTCGCAGGAAGGCATCACCATGACCGAAACAGTCACTCCCACACACTTTGTCGTGATACCCCGC ACCGAAGAACACGGCGGATACGAGAACGAGTGCTTCACCCATTACGTCCAAGACAACCGCGGCCGCTACT GCCTGCGAGGACCGATGGTCCAGTCGGACGCGGAATGGCTTGCAAGCAAGATGAATACACAGATCAGCTG ACCAACCAAGAAGGCCCGCACCGGATTCGGTGCGGGCCTCACTGCTGTACTCCTACGCCGGGGCAGGACG CTCCGGCATCTCCATCAGAGTTGGCTCAGCACCCTCGGTGTACCGGTAAGCCCACTGACTGCCCCGACGA CGATCGCGTCGTAGTGCGTCATGAGAGTTCTCTTTCGGTCAGGAAGTGATCGGAATCGTTGTGGTGGCGT GGAGGGCGGGGATGGTTGCTGCGCTGATCACGGCGCCGACGAGGGCGATCGCTCCGAGGACAAGGTAGGC GTGGGCGTAGCTTCCGGTCCAGGTGGAGAGCACGGTGCCGGCCCACGGCGCCAGTGCCATGACAATGACG ATCGGTGCGGACATCAGCCCGTTGAGGCGCCCGTAATGTTCACCGCCCCAGCGGTCGGTGATAGCGGTGG CCTGGATCAACGTGAAGATACCGCGGACCAGTCCGGCGCCGATGGCGATGCCGATCAACGCCGCCGCCGA GCTCGCCAGACCTAACCACGCGGTGCTCAGACCGGCAACGACGATGACCGATACTGTGCGCACGCGGACA CTGGTCTTCGCTGCGAGAGGTAGGTAGAAGATGCGGCCGATGACCTGACCGGCGCCGCCCAACCCGAGCG TGAGTGCGGCGAGGCTGGGACTGAAACCGCGTTCGATCAGCAGCGGTACGAGGTTGAAGACGCCGGCGGA TGCGGCGAAAGCGGCTGCAGCGAGGGCGACGATCAAAGCAAGAAAGGCGAGGCTGCGTGAGGTGCGGTGA TAGTCGCCGGGCTGGGCATGGGGCAGCCGGATGGGGTCGGGCCACCCGCCCCGCAACCCCCAGATATGTC CGGGGATGGTGATCACAGCCAGTATGGCGGCGAGCACCAGGTAGGTTGTGCGCCAATCGAAACGATCGGC CAGAGCGACAGACAGTGGCGCGAACACCGTGCTGGCCAGACCCGCAGCGAGGGTGAGAATCATCAGCGCT TTGACGTAGTTGTCGCCGTACCAGCGGGTCAGGGCGGCGAATGCCGGTGGATACAGCACCGCGCCCATCG CAATTCCGGCGAACACCCACCCCGCGAAAAACAGAGGGAGGGTGTGAGCTGTCGCGATGACCACCAGTGC GGGTACAGCGAGTACAGATCCGGCGGTCATGACCCAGCGGGGCCCGCGGCGATCGAGGATCCGTCCGACA GGTATGCCGACGACGGCGGCGACGAGTTGGCTGACCGAGAACGCCGCCACAATCACCGAGGTCGACCACC CGGTATCGCGGGAAATGGCCACCGACAGAACAGGAAACGCGTAGTACAGGATTCCCCAGCTGGTGATTTC GGTCAGACACAGCGCCACCAGGACCCGCCGCAGCCCCGACGGGTCGAGGCTGACGGGGCGCTGCGGCGGA TCCTCGATGGTCGTCATCGGCTGCGCCTTATCCGCAGGAGTTGGAACTACCGGAGGGGCTGGACAGCGAG ATCAGCTCCGGAGCCGGCGTCGCGCAGCAGCCACCCTCGGATTCCGCGGTGTCGGGTTCATCGAAAACAC CTGCGCCGCCGCAGACTCCGGTTTCCGGGAGGATCAACTCCACCCGAGCAGCAGCCTCGTGGTCACCGGC AAGTTCTGCGGCGATGCTGCGAACCTGCTCGTAGCCGGTCATCGCCAGGAACGTCGGCGCGCGGCCGTAG CTCTTCATCCCCGCCAGGTACACACCCGGTTCGGGATGGCGAAGTTCTTTCGCGCCGTGCGGATACACGG TGCCGCAGGAGTGGACGTTCGGGTCGATCAGAGGCGCCAACTGTGTCGGTGCCTGCAGGACGGGGTCGAG GCCCAGTCGGATTTCCGACAGCCAGGACAGGTCAGGGCGGAATCCGGTCAAGGTGACGACGTTGTCGACG TTCTCGATCCGGGTGCCGGCATCGGACACCAGTGCGAGCTGACCCTTGTCGGCGGATTCGACGGCGGCGG TGCGGAAACCGGTGACCACGGTGAGAAGTCCGTCGTCGACGGCCTTCTTCGCACGCAGTCCGAGGGCGCC GCGGGCGGGCAGCTCGTCCGATTCGCCGCCGCCGAACGCGGAACCGATAGCGCCGCGGCGAACCGCCCAC GTGATCCGCGTACCGGGGGCGTCCACCTCGAGGCCGGCCAACGCGACGATCGCGGTCAATGCGGAATGGC CGCTACCTGCGATGACAGTGTGCTTGCCAGCGAACTGGGCACGCACTGTCTCGTCGTCGAGATCGGGCAC ACGGTAGGTGATCGCGGATCCGGCAGCCCTCTCGCCGAGGGCGGGCAGTCCTTCCCCGCCGAGCGGGTTC GGGGTGCCCCAGGTGCCGGACGCATCGACGACGGCCTGAGCGAAGACGCGGTCCTCACTGCCGTCGGCAC GGCGCAGGTGAATGGAGAGAGGTTCGGTCTCGCGGCCGGCATCGACGACCAGGTCGCGGCCACGTCGGGC GACACCGATCACCTCGGTACCGAACTGCACCGCATCACCGAGGGCGGCGGCGAGGGGTTCGAGGTAGAGC TGTCGCCACTGCTGTCCGGTCGGGTACGTCTCGCCGTCGGGGGCGCTCCACCCGGTCTGCTCGAGGAGGA GGCGGGCAGCCGGGTCGACCAGCTCTGACCAGCGGGAGAAGAGACGGACATGATTCCATTCGGCGATCGC CGTACCGGCGACGGCGCCTCGCTCGAACACCAGCGGCGTGAGTCCTCGTTCGCGGAGTTGCGCGGCGGCG GCGAGGCCGATCGGTCCGGCTCCGATCACGACAACGGGTAGTTCGGTCATCACAAATTCCTTTCATCGAC AATGATCGATCTTTAATTCGAACTGTATCGACATCAGTCGATGAATGCAACCATCGACGAAGGTCGATAT AGTGTGTGTATGACATCGCTGCAGACCCCGACGGTGCGCGTCGGCGATTCGGACCCAGGTGCGGTCGACT CGAATGTGCTCGAGCAAGCTGACGCCGTCACCTACGCGCAGTGGTTCGCGTGCCTGGCCGAACCGATGCG GGTGCGGCTGCTGCATCGCGTAGCGAGTGCTGCCGGCGGGATCACGATCGGGGCTCTCGCCGAGGCGTTG GGGATCGGGCAGCCCACGGTCTCCCACCATGTGCGCAAGCTCGCCGACGTGGGATTCGTCAGCCTGCGCA AAGAGGGGACCAGCACCGTTGTCGCGATGAATCCGACGTGCTGCGTCGCGTTGCCGCAAGCCGCCGACAT CGTGATGGGAGTGCTCGCCCCGCGGCCGTGCTGCCCGAGCGACCTTCCCGATGACGTCGCCGTCCGGGTC ATGTCCGATGACGACTGGGACGCTGTTCTGCGGATTTACGGCGAAGGAATTGCCACGCGCAACGCCACTT TCACCACCGAAGTCCCCAGCCGGGAGTACCTCGATGCGCAGTGGCTGCCGAATCATCGCTGGGTCGCCAC CATCGACGATGTCGTCGTCGGCTGGGCGGCTCTGAGTCGGGTGTCCGGGCGGGACTGCTACCGCGGAGTC GCCGAGAATTCCGTCTATGTCGCTGACGGGATGCGCGGACGGGGTGTCGGAAAAGCGTTGCTACGCAAGC AAGTCATGGCAGCCGACGCGGACGGACTCTGGACGCTGCAGACGTCGATCTTCCCCGAAAATCGCCCCTC CGTCTCGCTGCACCATTCGGCAGGTTTCCGCACCATCGGGATCCGGGAAAAGATCGCCCAACTCGACGGC ATTTGGCGCGACACGGTCCTGCTCGAACGCCGAACGGCAGCGGATTCCTGATTTTCTGAACCGCTAATCG AAGCATTTTTCGGCAATCGATCCGCCATATGTGATCAGCAACTCGAAGCAACCCCTTAGCTCGAGTGCAG AGTGAGTCTGCTGAGACGTGATCCCCGCTGTAACTGGCAAATTATTCGTGGGCTGGTGTCACGTCCTCCT GCAGTGAGTCCTTGAGGCTCGGGATCAGCGGCTTCACAGCAGAGCGGTCACCGTCGGGGATGTACGTGCA GACGAACTGGAGCGGGTGAAACTGAAAGTCTTCGCGAGTCGCCGATCGGAGTGTGCAACGCCGCCTGCAT CTACATGCCGGTTGAGTATCACATCTGCTGCTCTCGCTTGCCTGTCACTCGGCTGCCCGGTAGCTCGGTT 
Final Technical Progress Report DOE Award No. DE-FC26-02NT15340

GGGCAGGACTGTGCAGATTGTTGCTGAAAAGCGGTCGGTCCATGGCCAGGGGCGCGGAGGTCCAGCGAGG ACGGCTAGACCACGGGTTGCGCGGCGTCCTTGTCGAGAATTCCGTCTCTCTCCCACGGCCACGTCATCAG AGCCCAGATGATGAATACGATTGTCATCCCAGAAAACAGATACACCGGCCACGGACCCATCAGGTCCAGC AGCGATGCGGTGGAAGGTTTGGCGTTGAGAAAGCCGTAATTGGTATCGGCGATGCGATTGAAGATGAAAG TGACTATCGCCCATGTCAGCGTGACCAGTACCGCGAGGCGGTAGCTGCGCCATGTAGGTCGCATTCTCCG GCCCCACGTGAGATAGATCGCGGCCCAGACGACAAACAGGTGAATCGCCCAGAAGGCAAGAAACTCGTAA CCGGGGAAGTCTGGCCCGGCCAGAGCCGGTGAAATCAACGCCTGCGTACTCAATACAAGCCCCCAGTAGT AGGTGAGGGCGAACGGCCAATGTCGGTGCGACCACAATGCGTAGATCGCGGACACTGTCGCGAGATCGGT GAGTTGTAAGGGTACGGATCCCCCTAGCGTGGGTGAGGACAACGAGTAGAGCACCGCCGAGCCGTATATC ACCGCCAGTATTCCGCCGAGGGTGCGGCTGAGGTGGCGAGCTTGCGCTTCGGTCTGTCTACGTCCCGCCC AGATCAGAAACACCGAACCCGCGACAAACAGAGCGATGGCTGCCCAATGCGATAGGCCGTACGCCGAGAA CTCGGGTTGTGCAGAATACACATCTCTCACTTCCTCAGCGCCGCCGAACGCACTGTTCCATAACAACCTT CGCAGATAGCTGTTCCAGTTCGGAAGTGCCGTTCTCCGGCTTAGTTGACGATGATCGTGTCCGCGGTGCG AGAAAGGGCGTAGCTGTGCAGTGGCTTCTGAGCAGGTCGTTGCACGACCGAGCCATCGACGATGGAGTAT CGGCTGCCATGACATGGGCAGTTGATCGTTCCATCGCTGACCTCGGAGACTACGCATCCCTGGTGGGTAC ATACCGCAGAGAAGGCTTGGAACACGCCTTCCTGCGGCTGGGTGATCACGACTTGCTCGTCCGCCAGAAT GACTCCGCCGCCGACGGGAACCGCTGATATCGGAACCTCGGCACTGCCCAGGCTTACGCCGGGTGCCTTG CTGTTGTTCTGGGTGCTACATCCGGCGACGGCGAGACAAGTTGTTGCGCAGGCTGTGAGGAGGACCGTTC GGCGCCCGACCTTGATGTCGGATGGATCGATCGTGCTCATGCGGGGACCACCAAACCAGTGAGAAACATC AGGAGTATTCCGACGCCGACGCCGACGAGGACCATCGGATCCAGGTCCGAGCGGCCTTGTGTGCGCAGGG ACGGTGCAATTTGGATGACGACTTGAATGATTGCCCCGACGCCGACTCCGAGCAGCAACGCAGATACTTC ACGGTTGTCGACGCCCGCCCCGATAACAGCACCGAGGATTGCCGGCGCACCCGCGATCAATCCCAACCCC AACAAGGTCAGCAGGGGGGTGCGTCGCCGAGCCAACGGTGCGATGATCGCGAGCCCCTCTGTTGTGTTGT GCAGGGTGAATCCGATAACGAGGAACGCGCCGAGCGCAAGCTCACCTACCGCATACGCGGATCCGATCGC GAGGCCTTCTCCGAGATTGTGCAATCCGATGCCGATGGAAATCATCAGTGCCAGTCTGAGTTCGGATGCG CCTGTCGTACCGGCTGGGCGTCTTCCGCGCAGGTATCGGTCGATGCACGTGAGCGTCAGGAAGGCGATTG CCGCGCCGAGAACGACGAGAGTGCCGCCGCCGAAGGCTCCGCTCGAGGCTGCTGCCAGTTTGAACCCCTC GCTCGTGCCGTCGAAGATCAAAAAGGCGAGCAGACCGACCGTGACGGCCAGCACGAATCTGACTGCCCTG ACGCCGGCGCCCCGCATCACAGGCAGCAAGAGCATCCCGAGCAGGACCGGGATGACTCCGACGTATGTGC CCAAGAGAGCCATCAAGCCGAAGAACGCCACTCCCGGGGCGGGAGTTGCTACGGCAGCGGGAATTTCGTG TTCGATGACCAACCCTGTGGAGGTGAGCATCGAGACGAGGTACGGCTGTCCGGTGATCCACGGGTAGTCG AGGGTGATCGTCTGGGACTCGAGTCTGCCGATGGGATCTGCAGCTCCTACCAAATCGACGAATGTGTCGT TCACGGCAACTTGCGCGACGGTCACCGCGTCGGGGCCGGTGTTGCGTAGCGTCAACGTGATTCGGCCTGG TTCGAGGGCCGTTCGTTCGACGGAGATGTTCTCGATCGGTGGGCCGGTGCGTTCGGGGAGGCTTTGTCCT GCGAGCATTGCCAGCGCAGTCAGGGCGATGACGATGATCGCGAGTACTGCGGCGCCTCGGAGCCAAAGGC CGGTGCGCGATGCGTCGCTTTGATCGGACTCTGTCTGCGCGGAACCCGCAGCGCCAAGGGAATTCTCATG AGTACTCGTCGGAGAGGGAGGATCAGGGGTGCCGGTCATTCGGTCACCTCGAAGAATCCCATCCAGCCCA GATCTGCGAATTCGGTTTTGTGGGCGTGGAACATGTACTTGCCCAGGTAGGGAAAGCGCAGTTCGCATAT TCCGCGCTGACCTTGGGCCTGGATGATCGTGTCCGTGAATTCGCTGGGTTCGAGGCGTGTTCCGGTCGGG TAGTAGTCGAAGAAGTTTCCGTGTAGGTGGAAGCTGTTGATCGGGTCGTATTCGAGGATGTTGACCAGGT AGATGCGAACCAGTTCGTCTCGTTTCACCTGTACAGGTTCGTGCATGTAATGGAACGGGATGCCGTTGAC GGCGTAGAGCTGGTTTCCTTGCCCGTCGAATGTGGTGTTGTAGCCGTGCATCACCATCACCATTTCGTCG GCCGCAGGGCGCCCCTGAGCTGGATCGACGATGAATGTGCCGTACATGCCGCGGGCGATGTGCTCTGCCA GTGGATTGACGTGGCAGTGATAGAGATGCAATCCGAAAGGGATGGCATCGAATTCGTAAGTGAATGATTC GCCCGGCTCGATGATTCCCCGCCCCACCCCGGGAATTCCGTCCATGTCAGCGGGATGGATTCCGTGAAAG TGCATCGTGTGTGGGTGCGCGGAGCCGTTGACGAACTTCACTCGGAGCCGGTCTCCCTCACTGCATCTCA ACGCGGGACCTGGAATGCGGGAGTTGAAGGTCCACGCCGGAAAATAGACTCCTGGTGCTACCTCGATCTC TTCGTCGGCTGCGAAGATCTCCCACTCGCGTAGCGTTCGGCCGTCAGGCAATTCGGAGGTCGTCCCGTAG TCGAAGTCCCGCAGCAGTGCCGTGGGATGAAACCCGTTGGCAGTGTGGTTCACCTGCTGACCGGCCCGAA AGGTCGCGCCGCTCGTGCCGCCGTGACGGACTCCGCCGTCTCCGTCAGGTGTGGCGGTACCCGGGTTGGT GTTTCCGGGATGCTCGGTAGGCGATGCGCCCGCAACTCTTCCTGCGTTCATCATGAGTCCGGCTGATCCG GCGAGCGCCCCACCGATCAACAATTGTCGCCGCGACGGTGCGCTCATGGCCACCCGACCGAAGCATCGAC ATTCCTGACAGTCAAAAGAGTCACACCTCAAAGTTTGTCATGCCAAACTTTATTTTCATAGACACCAAAC TATCCGAACGCATTCGACCGAAAACAGGCGGGCCGCGGAAATTTTCGACGAGTGGGGGCGTCGTGGTTTC GCCACCTCGAACCGCAGAAGTTCGCAGAGATGGACTGCTTCCCTGCCGACAGCGCTCCGGCTCCCTCCTC GCTCGAATCAGGTCACACGGATTGCGGACGCTGAATCTCACGTTCGGACCCGCGCGAGGATTCGACCAGC AGCGACACGACGACTAGGATGAATCCACAGGTGAGGTAGGTATCGGCAAGATTGAAGGTGGGCCACCAGC CGGTGTGGAAGTAGTCGGTGACCTTCCCGTCGAGGGCTCGGTCGACCACGTTGGAGGCAGCTCCGGCGAC AATCAGAGCGAAACCGATCACGGCCACCCCGCGACTTTGCGGGACACTCCGCCAGGCGTATACGGCGATT CCCATGGTGGCGAGGCCGGTAAAGGCGATGATGACGGCACTCGGCAGCTGATTACCCAGGCTGAAGGCGA CACCACTGTTGTATGCCAGCCTCAAACTGACGACGCCCAGATCCATCTCGTTGACCCCGGACAGGTGCTT CCGCGCAACGGGCTCAATCGTCAGGGCGACGACGACCGAAACGAGTACCGTCGTAGCGAAGAGAATTCGT TTCTTGTGATGGGCGGATTTGTCGTTCGTGGTCACCTCGTTGGTACTCACCACGGAAGCTCCTGGCAACG CCCGTCACCCGCGGCGGGTTCTACCTGCAGGGTCGCGTGAACGAGATTGAACTGGGTGGCCAACAGATCC 
Final Technical Progress Report DOE Award No. DE-FC26-02NT15340

TGGGCAGCAGCGAGAACCTTGGTCGGATCGGACTCCTCGTCGATCGTCAGATGCGCCGACGCAACTTCCA TGCCCGATGTGAGCGTCCAGATGTGAAGGTCGTGGGCGTCCTTGACTCCGGGGATCCCTTGCAAGGCTTG CGTCACCTCGGTGATGCTCACGCCGGCGGGGGCGTGCTGGAGCAGGATCCGCAGGGCATGGCGGCCGAGA TTGTAGGCACGCGGTAGGACGAACAAGCCGATCGCAACACCGATCACCGGATCCGCGTACCGCCATCCGA AGATCAGTGTGACCAATCCGCTGATCAGGACTCCAACGGACCCGAGCATGTCAGCCATGACCTCGAGATA GGCGCCCCGGACGTTGATGCTTTCCTTCGCCCCGCTGCGAAGCAGCAAGAATGCCGCGATGTTCATCACG AGGCCGACGACGGCGACGATGCTCACCGGGAGTCCAGGGACCTCAGGCGGATCGGAAAGTCGACCGAGAG CTTCGTAGAGAACCCATCCGGCGACACCGAACAGGAGTAGGGCATTGAACAAGGCAGCGAACACCTCCGC CCGGTACATACCGAACGTGCGATCCGGGCGCGATCGCGCTCTTTGCGCAAGCAAGATAGCGGTCAATGCC ATGAGGATGCCGAACACATCGGTGAAGACGTGTGCGGAGTCGGAGAGCAGGGCGAGCGATGACGTGGACA ACCCCACGATGACTTGCGTGACGAACGTGACCAGACCGAGCCCGACGGCTATCCATAATTTGCGGACATG TTTACCCGATGCACTCGCAGCTTGTTCCGCAACGCCGTGCCCATGGCCGTGACCCATCTCGATATGCCTT TCTCGCCGATGACTGGAGCGTCGTATTCTCATATGCGCATATTTGCCAACGAGAGAGTTTCAGTAAACCC TGGCTGAAGCACCCATGCAACTATCCGTCATAGTATTTTGGTCCGGAGCACCGATCGCGCGGGCGAGATC CACAATGCTGAGAGGTCCACCCGCAAATGATCGAAACCGAACCGTCCGGCGCCGATACCCTCGTCGTCGG ACGACCCATACGGAAAGTATTGGGATGGGGTGTCATAGCTGCAATCACTGCGGCGGCATTGGCGCGCGCA TCAACTTCCGAGGCGCTGCGATTGCTCGGACTTCCAGATCCCGGGCCCTTGACGATTTACGGACTCCCGG CGGTGATGGCGCTCGGCGAGGTTGCCGCCGTGATGACCGTAGGCAGTCTACTTTTGGCTGCCGTTCTCGT TCCGCCTCAAGCCTCAGGTGTACTCGATGTCGACGGATATCTGGCGCTACGAACCGCAAGCGTCGCCGCC GCTATCTGGGCGGTATGCGCGGCGCTGCTCGTTCCACTCACCCTCTCGGACAGTTCAGGCCAACCTCTCT CCGTCGTGTTCGCCGATCCAGGGCTGTTCGCTCGCGCGATTTCGGACATCGATGTCACAACTGCATGGGC ATGGACCGCAGGACTCGCACTCGTGCTCGCGATTGCGTGTCGTCTGACCCTGCGATATTCGTGGACACCG TTACTTCTCGCATTCGCTTTGTTCAGCCTGCTCCCCAGGGCATTGTCGGGTCACTCCTCCTCGGGCGGAT CCCACGACATCGCCACGAACAGTTTGGTTCTGCATATCCTTGCGGCGTCGATCTGGATGGGAGGAATAGC GGCTCTCGTGCTGCATGCCCGCAGAAAGGGGAGCTATCTCGACTTCGCCGCGCGCCGCTTCTCGGCCGTG GCACTCATGGCGTTCGTCGTCATAGCGATCAGCGGTGTGGTCAACGCTGCCGTGCGGGTGCCGCTCACCG CGGTGTGGAGTAGCACCTACGGTGTCTTGATTCTTGCCAAAGTCGCCGCCTTGGTCCTCGCCGGACTGGC AGGTTGGCGCCAACGGAAAATCGCGATCGCCGAACTGACGAAGGACCCTAGCGACGGTCGCACCTTCATT CGTCTGGCAGTGGTGGAGTCGGTGGTTCTCGCAGTAACCGTCGGTATCGCCGTCGCCCTGGGCCGCACGC CGCCCCCGGCGCCCACGGAACGACAGGATCCGAGCAGAGTCGAGGAGGTACTCGGGTATGCGCTCGATGG CGTACCGACTGCGGGCCGCTTGCTCGTCGACTGGCGATTCGATCTCATTTTCGGAACTCTCGCCGTGGTG TTGGCTGCGTGCTACGTCGTCGGCGTTTTTCGCCTCCGACAGCAGGGCGTGCAGTGGTCGACGTTCCGAT CGGTCAGCTGGGCTCTGGGGTGCCTGGTGTTGCTTGTCGCAACATCATCGGGAGTGGGTAGATATGCACC CGCAGTGATGAGTATGCATATGGCCGCAACGTCCGCCGTGGCGGTCCTTGCTCCACTCTGTCTGGTCCTG GGCGCCCCGTTCACGCTGTTTAGGCTCTCCGTTCGACGATCGTCCGGAGATGCGCCCGGGCTCAGGGAAT GGGCGCAAAGCGTGTACAGAGGCTCCTGGTTCCGATTCATGTCCCAGCCCTATGTGATTGCTGTGATTTT CGCCGGAGCCCTGCCTCTCCTTTACCTGGGTGGGATGTACAGCGCTGTCGCCGGCTCGCACGTCCCCCAT CTGGTGATCAACGGAACCCTGCTGATCTGGGGTTTCATGTTCTTCTGGGTCATGGTCGGCGTCGATCCGT TGCCGAGGCGGGCGAGCCTCGCGGTCAGGATGGCCCTGCTCCTGGCGGCGTTCTTGGCCTACGGGGGCCT CGCAGCAGCACTGATCACCACTGACACGGTGATTGCCGATCAGTACTTCGCGTCGTTGCAGCGCGGTTGG CTCGACGATCTCTCGCGAGATCAACGGTTCGGTGCCGGACTGATAATCGCGATCGGAGCAATGCCCCTGG CAATCGCGACAGGGTTTCTCCTCACCGCGTGCTGCTCGCCGAGATGCCACCGCGCGGCGCATGAGTAGGA GAAGGTGCATTCGCAGCGGGACGTTTCGTCGAGATTCTGAACTTTGTCAACGCTCGCCCAAAAACTATGC GAAATAGTAGTACGCTGTCTGGGACCGTCACCATTCTGTGACCTTCCGCGACGCAAGGAATGCGATCTTG GCCCGACACCGCAAGCCCGACACCGACGATCTCGACTGCCCACTTCGGGAAAGTTATCTTTTCGGCACGC CACACAGCGATCGAGACCTGCCCGTCGACACGATGTATATCTCCACCGCATCTGCAGTCGACCGTGAATC CGTCGCTGGAAGCGTTCAGGCGCGACGCGGCGGTGCTCATCGTCGACAGTCCCGCAGTCGTCCTGGTGGA CGGGCTTTGGTTCTCTCTGTCGCCACCGGCGCTTTGCTTGCCGCCGGCACATCCGCCTCACACGCCTCGG CAGGGAGCGTCAGTGCTCCCGTCGAGCTCGACCAACAACCGCTGGCACCTCTCGACCAGGCACTGGCATC GGCTCCGGAAATTCTCCAGGTTCCCAAACTGGCCGACCTGGGGAGGCTGGCCGAACAGTTGGCTCGAGGC ACCGAACGCGACACCGATCGCGCGTCGAGAGAGGCAACCGATCGCGCGTCGAGAGAGGCAGCTGCACGCC AACCGCTGACGATGGCTCCTGTCAGCGGAACATTGACGTCGAACTACGGGCCGCGTTGGGGGTCGACTCA TTACGGGTTGGACATTGCCAATGAGATTGGTACACCTATCGTGTCCGTGACCGACGGTACGGTACTTGAG TCCGGACCTGCGTCGGGTTTCGGACTGTGGGTCCGTATCCTGCAGGATGACGGGACAATCGGGGTCTTCG GACACATCAACGAGACTCTGGTAACCGCGGGTCAAAAGGTGCGTGCCGGAGAATTGATTGCGACGGTAGG AAACAGAGGACAATCAACAGGGCCACATCTGCATTACGAGGTGTGGCAGGCAGACGGCCAGAAGGTCGAT CCCATGGCATGGTTGGACGCGCGCGGGGTACAGATCCAGCCTCCGGCCATCAGTGACTGACGCAATCGAC GATTTTCGAAGGGGGAAGGAAGATGAAGAGGCTCGGCGGGCTCGAATCCGAAGTGATGGATCTCCTGTGG GATGCAGAAGAGCCGCAGTCTGTGCGGGATCTGGTCGACCGCTTCGAGGGCGGCGCCCCGAAGGCATACA CCACGATCTTGACCGTCGTGACGCACCTTCACGAAAAAGGGTGGGTACAGAGGGAAAAACGAAGCCGCGC GTACATCTATTCGCCATCGCGTAGCCGCGATGAAGCGACCTCACTGGCCATGCGTGAATTACTCGACAAC AGCAATGACTCTGTTTCAGCGCTTCTGCACTTCGCGCAAAACGTCTCGGATGACGAATACGAGGCACTGC GGCGAGCACTGGATCACGGCAAGGTTCGGTAGATGACTCTCGCCGCAGTCCTCTTTCTCGGGGCCGTGCT CATCGGAGCGTTCATGCCCAAGGTTTTGCGGCGTTTCGAATCGACCACTCTGGCTCCTGCAGCGATTCTC 
Final Technical Progress Report DOE Award No. DE-FC26-02NT15340

GCGGCATGGATCGGCAGTATCGTGGGTCTGGGATTCCTTGCCGTATCGGCAGTAGTGATCCTTCTGTGGC CGAGCCATGCACCAGCGGAAGGACTCTCGTACGCCCGGCGCGCACCCCGTTGGCGATGACGACGATCTCG GCGACCTCGTGGACGAGCACGACCGCAGCCAATCCGAGTACACCGAACAGAGCCAGAGGCATCAGAACGG TGATGATCGCCAAAGACAGGCCGACGTTCTGCAACATGATCGACCGCGATCGTCGCGCGTGCTGCAGAGC CTGAGGAAGATGCCGCAGGTCCTCGCCCATCAAAGCGACATCGGCGGTCTCGATGGCGACGTCGGTTCCC ATCGCACCCATCGCGATACCCAGATCGGCGGTGGCCAGGGCGGGGGCATCGTTGACACCGTCCCCGACCA TCGCCGTCGGTCGGCTCGCACGCAAGGTCTCCACAATGCGCGCCTTGTCCTCCGGGCGCAGATCGGCGTG CACGTCGTCGATGCCGACATCGGCGGCCAGAGCGCGGGCAGTGCGCTCGTTGTCGCCGGTGAGCATCGCG ACGGTGTAGCCGCCGCGGCGTAGTTCGGTGACCACTTCGCGTGCTTTCCGGGCGGAGTTTCGTCGCGGAC GGCGACCGCCCCCGATCACGGTTCCGGCGCGTTTCGATCAGGACCGCAGTGGCGCCGGCGTGCT

\section{>IGTS8 pSOX plasmid - contig002}

GTCGATTCGGCTTGCTGTGTGGCGGCGATCTGTCGAGCAAGATTTGTCACGACGGGGAGATCGGCATTGT CGGCGGCGTATTGGGCCATCGGAATTCCCCCTTGATGGTGGCGCAACAGCAATTGCAGATAGCGAATTTC GAATGCCGGCCCTGTCAGCACGCGTAGTTCCGCGAGTTCGGCAGGAGAAGCCATGCCGGGCATCACAGCT GACGAATTTGTGCCGGAATCATTTTGTCCGGTGCTCATGCCGGGCATCGAGTGATCCATCGCCGAGGTAG TCGAGGGCATCCACTCCATGTATTCGCCCGTGCCCCGGGCCGGTCGATCCCAGAGGGACAGCCACCCCTG CATCATTCCTATCTGACTTTGCTGAGTAGTGACGACGTCGTAGGCGAGCGTACGGATAGCAGGGTCGGCT GTGTTCGTCAGTGCGAGGGATGACATCTCGACCGCTTGCCCGTGGTGAACGGACATGTCCTGCGCGAAGC CGACCTCTACTGCGCCGACGGCTGGGGCGCTCGGCGAGGTGTTTTCGATTCGTCCCTTTGCCCACAGTCC GATGACGATTCCGACCAACAGGGCGATGACAACACCGAGAACCAACGCTGTGGGCCTCGTGTCACGGCGT TTGCCCGATGAATCGGACATCGACTCGGACTCAATGGGTACCGGAGCGTGTTCAACCATTGCTGCGTTCT CCCGTGATCGCGGGCATCGGTGTGGCCGTTGCCCCGGGGAGACCAGTTGCTGACTGCTCCGATGCTGCGG GAATCGCATCAGGGCCTGGCGGAGATGCGTCGAAGGGGGAAGGAGCCTTCGGGTCGAATCCGCCGGGGAC GGTTGCACAGCTGGCCCCGACCTCCGGATGGGTGTTCGGGTTCTGTTTGAGGGAAGTGACGAACTTTGCG ATACGGGGGTCGTCGACGCTGTCGACTTTGAGTTGGTGCCCCCACGATTGAATAGAAACAGGTGAATCGA TGCCGGGGAACGGTGACATCAGAGAATACGGTTCACCATCTACTTGGGAGGCAAGGGCATCGACGTCGGA CGAGTTCAAGGAGTCAGGATCGTAGGACACCCAAACCGCGCCGTGCTCGAGTGAATGCACCGCATTCTCT GTGCGAATCGGCTCCGGGTAGACGATGCCTGTGCACGTTGCCCAGTACTGATCATGCGCTCCGCCCAAAG GCGGAGTTTGATCGTATGCCACACGCTGAGGCGCTTGGATATGGTTGGCGGCAGCATATTCGATTTCGGT CACCCCCTCGATCGACTTCGACGGGTCCGGGTTGTCCGCGCTCGGAGCGTATTTTTGCGCTTCGGTTCTC TCGATGGCCTTGGGCACCATGTTGTAGGCGATGACCGAGACCAGGCCGGCGATAATTAGGACTGCTATCG ATATCGACCACGGAAAACTTCTGCCACCAGGCACCCCACCGCCGCGGGGGCGAGGTGTGTTCTTCTTGCG GTTCTTCGACATTGGCGGACTCGTTCCCTAGTGCGTTGGCACCGACACGGAATTACTATCAATCATAGTA ATTCATGGTGTTGTTCCTTCTGCCGAGACAGAAGCGGTACGCGCCCCCGAAGAGAGGTGCGGTGGCGAGA GAAAGGACGATCACTGCCAGGGCGATCTCGGGAACCGCGCCCAGACGTGTTGCCGGTGTGAGATCGGAAC GCAGGGCGATCTCGTCGGTCAGTGCCTGTGCTGTGAAGATCTCGCTCTGTTGGCGTACTTCCCCCGTCGG CGTGATGATCGCGCTGACACCGCTGGTTGCTGCGATCAGCACACTGCGGCCGTGTTCGACCGCCCGCACC CGCGACATGGCCAACTGCTGAAAGGTCATCTCGGTCCGGCCGAAGGTCGCGTTGTTGGTGGGAACCGCGA TGACGGTGGCTCCTGCCTTGACGGACTCGGTCACCAGATCGTCGAAGGCCACCTCGTAACACGTGGCAAC AGCTACCTGAACTCCGTTGAGGCCGACGACGCCGTTCCCGTTCCCGGCAACGAAATTGCCCGCAGAATCG GCATATGACGACACCGCACTAACGATCGATCGGAGCGGCAGGTACTCCCCGAAGGGCACAAGCTGCCGCT TGATGTGTTGCTGCCCAGGGCCATCCGTGGGGTCCCACACCATGACACTGTTGACCGAAGTACCCGCCGC ACCTACGAGAACCGTACCGACCAGGATCGGTGTCCCGATCGAACGGGCTGCTCGGTCCAGTCGCTCTGCC GCATCCCGATTCACCAAAGGATCTATATCCGAAGCATTTTCGGGCCAGATCACTAGTCGTGGCCGGGCTA CGCGACCGGCCGCGACATCGGCTGCGAGCTGCTCGGTGCGGGCAACATGATTGTCGAGCACCGCTCGGCG TTGGGCGTTGAAATCGAGGCCGAGTCGCGGCACGTTGCCCTGAACGACGGTCGCAGTGACGACCTCGTCA GGGGCGGCACCTGGAGAACCGGTCACCAATGCACTCACAAAGATGAGGACGATGCAGGCGCCACCTTTTG CCAATGCTTGCCATCGAACAGTTAAGGGCCCGGCCAGTGACCAGGCGGTGAGGGTTCCGATCAGTGCAGT CGCGAAGGAAAGACCTGGCGCCCCCGCGATCGAAGCCAACGGCAGCAGCGGACTGTCAGCCTGCCCGAAG GCCAAACGCCCCCATGGGAAACCGCCGAACGGAACTCGGCTACGAAGTGCTTCTGCGGTGACCCATGCAC CCGCAATCCACACCGGGGAAAGGGGAGCGCGACCGACGGCCACCGCCAGTACGCCGAACACGCCGAACGC GAGCGCTTCAATCGCCGCCAACGCCAACCAAGGCGTTGCACCTACGTACGCTCCGACCCACGGCAGCAAG GGAACGAAGAATCCCAGTCCCGCGGCATAGCCGAATCCGAAACCCGCACGGAGACTGCGACCCTGGAGAA CGGCGGTCAGCAGGGCGATGGCTACAGGGGCGAGAAACCACAGCGTGCGCGGCGGAAAGCTGAGAAACAG CAGCCACCCAGCGCCCACCGAAAGCGCGAGAGGCAAAGCGATCCCACCGTGGCGCCGTTTGGGGCGACCA CTCTTCAGGCATGCATCGAGTTCACCGTCGGCAGTGATTGCACGGTCGACCGAAAGGACCCTCACAGGCC CGCTCTCGTAGAAGGAGAACTGTGCTGGTCCGCCAACAATTTCTCACAGAGATCGTCGAATTCGCCGCCC CATCCGGCGTGGTCGGTCCGCGCCAGTCCACCGAGTTCGAGATCAACCTGCTCCGGTCCCGTCCCCGGGC GTAGGCGGATCCACACCCGCCGCCTCCTGATCACGAGCGAGATCAACAGTCCGAGCATCATCGTGATCGC AAAGACGAGAACCCACTGTTGCACAGGGTCGTACGACACCTGCAGATTGGCGAATTCCTTGGCCCCGTCG

\section{$3 / 8 / 2006$ \\ Page 142}


Final Technical Progress Report DOE Award No. DE-FC26-02NT15340

AACCGAACCTGTGTTCCATCGGTGAGAGTCCGCGTCTCTCCCGGGCGAAGGTTCACACGATCTTGTTTGA CCAGCCTTCCTTGGTTGATCATTTCCTTGTTCAACGTGAAGATCGATTGCGGGATGCCGGTATCCAGACC CGTATCGCCCTTGTAGATGTCGATCGCGACCGCAGGGTCACGCGCGTCGGGAAACGACGAAGTCAGGAGC GTCCCGCTGAAGGCCGCGGTCGGTGCGAAGAGTCCCTCGATGGCGATCTGATTCTTCCGGCGCTCGTTCG GATCGGGGAACGAACCGCCGGGTGGATCGAACCGCAACGCCCCACTGCTCAGGAACGTCACCGCGTCGTC CGGTTGAAACTGCATTGTCTCGGTACGGGATTGTCCATCGGGGAATGTGACCGTGAACTGCGGCGCGTAC CCGTGGCCGGTCAGATAGACGCGGGCGCCATCGGTACGCAACGGTTCGTTGACCCGAAGGCGATAGTCCT TCCATGTATTGGTCGCGATGTCCTGCCCGCTCTGGTACGAGATGTTCGAGGTGAACATTTCGGCCTGACC GGTATCGAGATAGTCCGCGGAGAAGTCGTTGACCTTGATGCAGAACGGTGCGAGACCCGTGCCGTCAGTT GCACTTCCAGCCCGAAACGAGTCGTACACGGCGGGTGACGTGTTGCAGAATCCAGGGCCGCCGTCGGCAA TGACGATCACCGAACCCTCATATCCCACCAACTTGCCGATGGCGACGGTGACGAGCAATCCGATCAGCGA GAAGTGGAACACCAGATTCCCTGCCTCTCGCAGATACCCCTTCTCCGCGGAAACCGTTACCTCGGAATCT TTTTCGGACCTGATCGCTACCCGCCAGCCCTTGAGCGACTGCCGCACTCTGGTGAGGGCCTCGTCATGCG AGATATCTAGAACGCCGCGAAAATGGTGAGGCAACCTGGACAGATTCCGAGGCGCTCGCACAGGTTGGCT CCGAAGCGCGCCGAGATGCGCCTGCAAGCGTGGAACCAGACAGCCGATGAGAGAGAGAAACAGGAGGACA TAGATCGCAGTGAACCAGAAGCTGGAAAAGACGTCGAACAACTCCAGCTTGTCGAACAACGGGCCAAGGG TTGGTTGGGCCGCGATGTACTGGGACACTTTTTGTACGTTGAGATTTCGTTGAGGCAACATAGCGCCTGG AAGTGCTGCGAGTGCCAAGAGGAACAGGAGCACGAGCGCGGTACTCATCGATGTCAACCTGCGCCAATTC TGGCGCAGTAGCCGAATGCTTTCTCGGATCATCAGTCTTGGGAGGCGACGGATGAGAGCTCCTGCGGTGG CGTTATCGGTCATATCGGCAACACGACCGAACTGACGAATTCGTTGCGCAACCAGCCGATGAACATGCTC CAGACGCCGGTGACCAGGGCCAGGCCGACAACGACGAGCATTATTCCGCCGATGATCTGAATCGTCCGTG AATGGCGTTTGAGCCATCCGACACCGCGGAGCGCTTTTGCGGACCCGAATGCCAGCACGATAAACGGCAG TCCCAATCCCAGACAGTAGGCGACGATCAGTATCACCCCACGCGCTGCTGTCGTGCCTTGAGTTCCGGCC GCAACCGAAATAACCCCGGCGAGAGTGGGCCCGAGACACGGTGTCCAACCCAACGCGAACACACCCCCCA ACAAGGGTGCGCCGGCAAGGGAGGAGATGCGCCGTGGGGCGAACCGTGCGTCTTTCTGCAGGGCAGGAAT GAAGCCGATGAACGTCAACCCCATCACGATGGTGATCACACCACCCACGCGTTGGAGCAGCTCTTCGTTG ATGCGCAGGGTGCCGATCACCCCGAATACCGACGCCGTGGCGAGAACGAACACCACCGTGAATCCCGCAA CGAACATCGTGGCGGCCCCTGTCACCCGCCATCTGCCCGCGGGTTGTGCATTCTCGTCGCCGGGGGTCAC CGACGGAGAATCAGCTCCGGAAATGCCGGCCAGATACGACAGGTATCCGGGAACCAGTGGCACCACGCAC GGCGATGCGAAAGACACCAGACCGGCCAGAATGCACGCTGCCACGGCGAGCAGCAACGGACCGCTCGAGG CGGCGGTCTGGAAGCTGGACCCGACGCCTTGGGCGAGTTCGTTCACTGTTCGGCCGCCACACGCTCGATG ACAGGTTGCAGATCCTCGGTGAGAAGTGCGCGAAGGAATACGGCGGCGACGCGCTGCTGTCGATCCAACA CCAGCGTGGAGGGAATCACGCTTGTCGGGTAGTTTCCGCCGAGTGCTGTCAATGTTCTCATCGACGGGTC GTAGATCGAGGGGTAGCTCACGTTGTTGTCGACGACGAAGTCCTGGGCCTTGTCACGTTGAGGATCTCGA ACATTGATCCCGAGAAACTGAACACCGAGACCTTTGGTCGCTTCGTACGCGCGCTCGAGATCATCGGCCT CGGACCGGCACGGGGCGCACCATTGCCCCCACAGATTGATCACGACGACCTGACCTGCGTAATCAGCCAG TGAAATATCGCTACCGTCGGTCATGAGGTCAGGTCCGGCCAATACGCCGATCGTGTTTCTCGACTCGGGT GGGTCGTACAAAATATCGGTTTGCCCTCCCGGTGAGACGAAATCGAAATTCGCACCCGAAGCGACTGCGT CGGTGCCGGTCGCGCACCCGCTCGCGGCGACGACGATCGCGGTGAGAATAGCGACGAGTGAGAGGGCGCG GGTGGGTGTTCCGAGTGCGCGTTGTCTCGGTTTTCGAGGGAGCGTGGGGGCGGTCATTTCATCAACTCAC TTACAGCGGAGCGTATTTGGTCAGTGTTCTCGAAAACCATGGGGGTTGTAACCTGGTCGACGGACCCGTC ACGTTGGACGAGGAAGCTCAGCGGCAGAACTGGCGGCGCCGACAGCGCAACTCCGATGTCGTCGACGGCG GCGAACGAGGGATAGCGCACGCCTGTGTCCGTGAGAAGTGCTAGTGCTGTTGACATGTCGTCCTGGACGT TGATTCCGACTACGCGGATGGCGCCCGGAGTACCTGCATAGTCATTCAGTACCGGGATTTCCGATCGGCA CGGCCCACACCACGAGGCCCAGAGGTTGATCAAGGTAGGTTCCCCCGACAACGCTGCACCGAGGTCGACT TCGGTGCTCTCGCCGAGGCACTTCGCTTTGATTCCCAGGAGTTCTCCGTTTACGGGCGGTGGTGCCGGGG CCACTGGACAGGAAACGTCCGCTGCCGCGGGCGACGAGGAAAGCACCGAGCCGGGAGCTTCTTCTTCGCC TTGTGATGCGGCCGGGGGCACTTGCGGCCGGGTCGAGGTGTGGGCCTCGGTCGAGGTGGATCGAGGCCAG ATCGCCACGACAAGGAGGGCGATCACGACCAGGAAGAACAGGAGCCATCGCGAACGTGCCTGCATGCACT TCCTCCAAGCGTGGTGCGATGAACCGGAAACAACACTGAACTACTATTGTGCATAGTAGTTATGTGGTTC GTCTGTCGCCCCCCGCCAAACAGAGGCGGGATCCGAACCCGAGAATCGAGCATCGTGAAACGCCAGTCCG CTCCGAAGGGCCGCCCCAACTCACCAGTCAGGCCAGAAATTGCGGAAGTGGATGCCCATCAAACGCAGCA GACACGCCTGGCGCGAATCAACCGTGTGGCACGTTCAAAGCTCGTGCGCCTGATGCTGTACTACACCGCG GCTCGGCTCGCCCTGATCGCCGGTCTCGCAGTGATCATCATGGGAATCAACGCCATATTCGATCTTGAAA TCCCCGGCGTCTTGGCACTGTTCGGGGCCCTGCTGCTCGGATTCGCGATCTCCGCGATCATATTCCGGTC GTTACGGCGCCGGATCAATGAAGAAGTGATGGTCGTAGATGAGAAACGTCGCGCAATGCGTACTCAGTGA GGAGTCGCCCTGCGGCCCGACACGCATAGAAGCGCCCAGTCGCCGAACGTACCTCGCAATTCACAAAATC GTGCCCGCACGGTCCCTTTTCGGTACGTGACCGCTCAAAGATCTATTTCTGGATGGCGCCGGTGGGAGGG TCGAGCGTTTTCATGGCATCGAGTGATGAAGATGTCAGGTGGCGGGCTCAGATTCGCAAGTCAGTGGAAT TCGTTGTGGCATCAAATTTCGACATCGGTACGAGCCGAGGGATCAGGCGGACGTAGATCACCATGCCGAC GAGCTCGACGAGAGTCTGGGTCACCACGACCACGGCAGCCAGTGACAACGCGGGCGGAAGCGCCAGCGCG AGCGGCAGAACGACGAGGGAATTACGGGTGGCACCGGAAAATATCAACGCCCTCGTCGCGGCCGGTTTCA CGCGTGCTGCCTTGCCGACACCTTGACCGACGAATGCCATGACCACCAGGAACACGGCGAAAATCGGAAC CACTACGATCAGCGAACTCAGTTGTGTGCGGACAGATTCGATCTGGGAGGCAACAACCACTGCCAGGGTC 
Final Technical Progress Report DOE Award No. DE-FC26-02NT15340

GCCATCATCACCGGAACCATGATCGAGGCCATCACCTCCATCACGATGCGACCAGCCCCATGCCGTCGAG CAACGCCCTGCGTGAGGGCCGCGAGAACAAGCGGCACCACGATCAGAAGTAGGAAAGCCTCCACGAACGG AGCGACGTCGATGACATCGACAAGTTCGGATCCGACGAACAACCACAAGTAGACCGGAAGAAGCGCCATC TGCAGCAACATGAGCAGCGGCGCGGCAGCGAGGAGACGTTCGGACGCTGCGCCGGCCAACCCGCTGAAGA CGATGACGTAGTCGATGCACGGCGCCAACAGCACCAACAGCACGCCGAGTAATACCGCTTGATCGTCGGC GACGAAACGCGAGAGAGCGAACACGACGATCGGAACCAGAACGAAGTTCACACCGAGCAGTGCAGTGAGG AATCGGCCGTCCTTCAACGACGTCCGGATCGAGGAGAACGGTACCGCGAGGAAGGTGGCGTAGAGCAGGA CGATCAGAACGGGGTTGATCGCGTGTTCGAAACCCGGAGCGGTGTCGGGCGCAAGGATCCCCACAAGGCC ACCGATCAGCAGGGCCAAGAGATAGAGCGGGATTTGATGACGTTCGAGGGCCTCGACCAGGCGAGTAACA CGTGGAGCAGTCATGAGTGGCAGCGGTGTCATTTCTGGTCGGATTCGGCAGGAACAACGAGATGAGTACG TCATCGGCCCGACGTAGGGCTCGACGGTAGAAGCGTGCGAGGGAGTCATCTCCTGGTGATGATCGTTCGG AGCACGAACCGGAAAACGGCGGCGCTGCCAATTTCGACCACCTCACCGGCAACGATATCGGTCAAGAGAC TTCTGACCGTGACACCGAGGACTGTTGCGGCAGCGATTGCCGGTGCGACATCAGGGGGTGCCGGACGAGA ACTGGTGATCGCATAACCCTTCCGCGGCGAGTCGACGACGTACTGGGGAGAGCATGACACGGTGCTAGTG CACGTTGGACCGCCTCCGACAACAACACCCGTGGCGGTTCAGGCGCTGCGAGAGATGGTTTCGGCGAGAT CGTCAGGTATCGGCATCGGTGTCATCAGACCGACACGAGTGCGTCCGGTGTTGCCCTCCGGATAGCGTCC CAGGGCTGACCCCGGTGCGGCGACGATGATGCGAAGAATCTGTCCGAGTGCCTCGCGGCGATCACGCTGA CGAAGGGCGAGGAGGAGCATGGCGAGGTGGTTGTGGGTGTGAGGCCACGGATAGGGCTGGGAGATGACAT GTGCGCGTTCGAGGTGATGCCAACGCCTGATGTCATCGCCGGTGCGGCCAGCTGCGGCCATCTCCGCGGT GTAGGTACGGCGCAACTGTGCGGGCATTCGTTTCATCAGATGTGTTCCCTGCTAGTGGATTCGAGGAGCA GCCCGCCGATGACGAGGACAACGCCGCCGGTGATGTAGGTGTCAGCGAGGTTGAACGTGGGCCACCATCC GGTATGGAAGTAGTCGGTGACTTTGCCGTCGAGAAAGCGGTCGACGACGTTGGCGGCGGCACCGGCCGTG ATCGCGGCCAGCCCGATGGTCTGGAGTACGGGACGCTCAGATGCTGTGCGCCAGGCGAACACACCGATCG CGGCGGTGAGGGCAGCGGTGCCGGCGAGAACGACGACTGTGGGGAGCTGGTCACCGAGACTGAACGCGAC ACCGCTGTTGTAGGCCAATCGCAGTTGCAAGACCCCGAAATCGACTACCTGCCCACCGGACAGCGCGCCA CGTGCCACCGGGTCGAGGAGCAGCGCGACCGCAGCCAAGGCACCTGCGAGTATGACGAGAACACCCCGGC CGCGACGCATTCCGACCACGGACACCGATGATGACCCGGAGCTGGCGGCAGATGTCATCGCCGTACTACC GTGGCCGGCTCGGACGCCCTGGTCGACTGCGGTACAGCAGCCAGACTTCGGGTACGCCCGGCGCGCACCC CGTTGGCGATGACGACGATCTCGGCGACCTCGTGGACGAGCACGACCGCAGCCAATCCGAGTACACCGAA CAGAGCCAGAGGCATCAGAACGGTGATGATCGCCAAAGACAGGCCGACGTTCTGCAACATGATCGACCGC GATCGTCGCGCGTGCTGCAGAGCCTGAGGAAGATGCCGCAGGTCCTCGCCCATCAAAGCGACATCGGCGG TCTCGATGGCGACGTCGGTTCCCATCGCACCCATCGCGATACCCAGATCGGCGGTGGCCAGGGCGGGGGC ATCGTTGACACCGTCCCCGACCATCGCCGTCGGTCGGCTCGCACGCAAGGTCTCCACAATGCGCGCCTTG TCCTCCGGGCGCAGATCGGCGTGCACGTCGTCGATGCCGACATCGGCGGCCAGAGCGCGGGCAGTGCGCT CGTTGTCGCCGGTGAGCATCGCGACGGTGTAGCCGCCGCGGCGTAGTTCGGTGACCACTTCGCGTGCTTC CGGGCGGAGTTCGTCGCGGACGGCGACCGCCCCGATCACGGTTCCGGCGCGTTCGATCAGGACCGCAGTG GCGCCGGCGTGCTGCATTCGTTCGATATCCCCGGTCAGTGGCCCCGGGTCGATCCAGCCTGGTCGACCGA GCCGGACGGGGATGCCGTCGATGTAGCCGGTCAGGCCTGCACCGATGACTGCGTCCGCGTCATCAGCGGG TGTGTAGTCCTCGACGGCGGCGAGAATCGCGCGAGCGAGCGGGTGCTCGCTGCGTGCCTCCAACGCTGCG GCCACCGCGAGAACGTCACCGCGGTTGGCGCCGTTCGCGGTCGCGACGTCGATCACGGCGGGTTGGTTGC GGGTGAGGGTGCCGGTCTTGTCCAATGCGATGGTGCGGATCCTGCCGAGCGCTTCGAGGGCTGCGCCGCC CTTGACCAGGACACCGAGCTTGGATGCTGCGCCGATTGCGGCGACGACCGTGACGGGTATCGCGATGGCA AGCGCGCACGGGGACGCGGCGACCAGTACCACCAGGGCTCGTTCGATCCACACCAGCGGATCACCGAGCA CGCTGCCGACCGCGGCGATAACCGCGGCGACGATCATTACGCCCGGAACCAGCGGTTTGGCGATCTTGTC GGCGAGGCGTTGCGCCTCGCCCTTGCGGGACTGCTCAGCTTCGACGATCCGCACGATCTTCGCCAGCGAA TTGTCCTGCGCCTCTGCGGTGACCTCGACTTCGAGGACGCCGGTGCCGTTGATCGACCCGGCGAATACCT CGTCGCCGGGACCGGCTTCGACGGGCACCGATTCTCCGGTGATCGCGGAGGTGTCCAGGGCGGTGCGGCC GGCGCGAATGACACCGTCGGTGGCGATCCGCTCCCCGGGCTTGACGATCATGGCCTCACCGAGCTCGAGG TCCGAGGGTGGGACGGTTTCCTCCCGGCCGTCGCGGAGCACTGTCGCGGTGTCCGGGACCAGGGACAGTA ACGCGCGCAGGCCACGGCGGGTGCGGGTGACCGCGTACTCCTCGAGTCCCTCGCTGATCGCAAACAAGAA TGCGAGCATGGCTGCTTCGCCGACCTCGCCGAGCAGTACCGCGCCGACGGCCGCGATCGTCATCAGGGTG CCGACCCCGATTTTGCCCTTGGCCAGGCGTGTGAGGGTGGACGGTACGAAGGTGTAGCCGGCAATGATCA GCGCGCCCGCTTCGAGGCCGATCCCGAGCGCATCAGGTCCGCCGGCCAGCCCGACTGTCAGGGCCGCGAT CAGCAGCACGCCCGCAGCTGCAGCCGCCCGGATCTCGGTAACCTGCCACCACTTTTCGGGCCCGTGTTCT TCCTCGCCCTCGGCACGGGGTTCGTCGTGGCCGCAGCCGCACGCGTCACTCACGGCTATCTCCGATGTCG GTGCCGTAATTCGGGCACAGGGCAACCGCGTTCCCTGTCGCGGCAAGCAGCGTCTCCGCCGAGGCGAGTA GGTCCATCAATTCCGGCCGGGCCAGCGAGTAGAACACCTGCCGACCTTCCGGTCTGCCGTCGACCAAACC GCAATCACGTAGGCATGCGACATGTGCGGACACCGTCGACTGGGCGAGGCCGAGTTCGCCGATCAAATCC GCAACCCGTGCCTCTCCTTCGGCGAGACGGCGCACGATCGACAATCGGGTGCCGTCCGAGAGACTGTGGA ACAGGGCCACTGCCGCATCCAAGCTGGACCCGCTGCCGGTTGCGGCGAGGCACCCCGACTCTTTATTTAT CGTCATATAACGATGATAGCGATAGTTGGCGATTTAAAAAAGGTGCGGATGTGCAGGACTTGAAAGTGGA TGTGGTACGCCAGCCGAACGGTGGTCGGCCGACGTACCTCACCAGGAATGGCGCAGTGGCATCTGTAGTT TGCGAGTTGCTCGCCACGGCATCACATGGGGGGACAACAAGGACCCATCGCTCGACACAGCCAGTCGCAC AACATCTCCAGTGGATTCATCTTCATTCACCTCCTTCTGAGTTCGTCGCCAGCGCGGCGACGAACGGTCC 
Final Technical Progress Report DOE Award No. DE-FC26-02NT15340

GAGTCACCTGCGGTTCCCGAACGTCATCCATAATGGACATTCCAGTGCGATGGAAGGTCAAGGATCTCGT GAAAATTGGTGAACTGGCCGCTCGAAGTGGCGTTGCCCCTAAGACACTGCGTTTCTACGAGGACAGCGGG GTGATTCCCGTCCCGGTCCGAAAGTCCAACGGCTACCGGGACTACGGTCCGGAATCTCTTGCGCGTTTGC GGTTCGTCCGCAGCGCCCAGTCTGCAGGTCTCACCCTGCGAGAGATCCGCGACATCCTCACCACCCATGA CCGCGGGGCGGCTCCTTGTGAACAGGTCGTGGAGATGCTCTCGGAGCATCTTTCCCAGGTGCGTGGCAAG ATCCGCGAACTTACCGCCCTCGAAACAAACCTTGTTGCTCTGCTGAGTAGAGCGGAAGGTGAGATTCCGT CGGAAGCCGACGATGTCGGTGTGTGCTGGATCCTGGAATCCGAGTCTCAGGAAGGTGTTGCCGAATCGTG AAATTGATTGCGACCCCTTCCCGTTTCAGCAGGTTCGGCGCTCTGCAACGTGATGAGGTGCGCGAAGCGG TTGTCGTGGTGTTGTCGGTGTATTGATCAGTCCGGTACGGAAGTAGCGGCAGTGGGTGTATCCCGTTCGA ATGCATTCCTCAGCGCAGTCACGGTAGGAGCGCCACTGACCGCACCCACGGCGTCGCGGTAGAGCCTGCA GGACACGCTTGGCATCTGATCCGGTTCGGCAAAGGGGTCGCGGCCGTCAACGAGCAACGTCGGAGATCCG GCCATACCGGCAAGTGCTGCTGATCGAGGATCTTCGATGATCACGTGGCGCAGTTCCCATTCGATCGGCA CGCCTGAAAGAGCGTGCTCGATTCGTTGTTCGAGGAGCGGCACGTTAGGGCAGTCCGGGACTTGCAGAAT TTCGAGATTCATCATGGTCGTCCTTTGTCGGTGTGCAGTGTCTGGAGCATGGGGCAGTGCCGGTCTGCTC GCGGTCGCGGTCGCGCGCAGGTGGACGCGAACTCGGCCAGCGAGGCCCGCATGCGTTGCAGATCTCGGAT СTTTTCGTCGAACTCGGTGATCTTCGTCTGCGCCAGTCGCCGAGCTGTGTCGCAATCATCCGGTCCGCCG CCAGCGAGATGGAGAAGTTCGTTGATTTCGTCGAGGCTGAAGCCGTGTTCCTGGGCGCGTTTGACGAACC GGACAATTTCGACAGTTTCGTCCGGATATGAGCGGTATCCCGAGGCGGATCGCGGCGGGTGCTGCAGGAG TCCGCGCCGTTCGTAGTACCGCAATGTCTGTACGTTCACCCCGGCGCGGGTGGCCAGTTCACTGCTACGC ATGTCGCCATTGTTAACCCTGTACCCGGCTACGGAGTCAAGTCGTCTCTCTCCTCGTCGGGAATTCATCT GAGGTTGGTTGCGTGTTATTTCGAAAACTGTCAATATCGAAGTATGTCGAATCAAGTTGTGGTGGAGACC GGCGCCTGCTGCTCCCCGCTCGTACGTGAACCGTTGACCGAAGACTGGGCCGGCGGCCTGGCCCGCATGT TCAAAGCCCTCGGCGATCCGGTTCGCCTCCGCTTGCTCAGCTTGGTGGCAAGTCACGAGGGTGGTGAAGC CTGCGTCTGCGACATCTCCGACACCTTCGACCTGTCCCAGCCGACGATCTCGCATCACCTCAAGGTGCTG CGACAGGCCGGTTTACTCGACTGTGAACGACGCGGGACCTGGGTGTACTACTGGGTTATTCCGGCTGCGC TGCAACAGCTTTCCGCCGTTCTACTGGTTGAAGGTACGAGCGTGAGTGTCGCGGACGCGTGCGCAGAGGT CAGCGCATGAGCAACACCGCTGCCACCACCGAACACCCCGCAGTCGTCGGAAAACTCTCCACCCTCGACC GCTATCTGGCGGTCTGGATCGGCGTCGCCATGGTCGTCGGCCTGCTGGTCGGGCGCTGGATCCCCGGTGT GAACACTGCCCTGGACAAGGTGCAGATCGACGGCATCTCCCTGCCGATCGCCCTCGGACTGCTGATCATG ATGTACCCGGTGTTGGCGAAAGTTCGCTACGACCGCCTCGACACAGTCACCGGGGACAAGAAACTCCTCG CCGGCTCCCTGTTCCTGAACTGGGTTCTCGGCCCGGCGCTGATGTTCGCCCTCGCCTGGCTGTTTCTGCC TGACCTGCCGGAATACCGAACCGGTTTGATCATCGTCGGTCTCGCTCGCTGCATCGCGATGGTGATCATC TGGAACGACCTGGCCTGCGGAGACCGCGAAGCCGCCGCGGTGCTGGTCGCCATCAACTCGGTGTTCCAGG TGATCATGTTCGCCGTGCTCGGTTGGTTCTACCTCTCGGTCCTGCCCGGGTGGCTCGGCCTCGAACAGAC CACCATCGACACCTCGCCCTGGCAGATCGCGAAATCGGTCCTGATCTTCCTCGGTATCCCGCTGCTGGCA GGTTTTCTGTCCCGCTTCTTCGGCGAACGCGCCAAAGGCCGCGACTGGTACGACACCAAGTTCATCCCCA AGATCAGCCCCTGGGCGCTCTACGGTCTGCTGTTCACCATCGTCGTGCTCTTCTCGATGCAGGGCGAACA GATCACCTCCCAGCCTTGGGATGTCGTCCGGATCGCGTTGCCGCTGCTGGCGTACTTCGCGCTCATGTGG GGCGGAGGCTACATCCTCGGCGCCGCAATCGGATTGGGCTATGAACGCACCACGACCCTGGCGTTCACCG CCGCGGGCAACAACTTCGAACTCGCGATCGCCGTAGCCATCGCCACCTACGGCGCCACCTCGGGGCAAGC TCTTGCCGGTGTTGTCGGCCCGCTGATCGAGGTACCGGTGCTCGTCGGGCTGGTCTATGTCTCCCTTGCT CTGCGTAAACGCTTTGCCGTCAAAGAGGTTTCACCCGCACCTACCGGCACGTAAGTCACCTACAACTCTT CGGGAGTCATGATGAACGAGCACCGCGCCCAGCCCGATCTCCTCTCACCCCAAGCTGTTCTCTCGCGCAC CGCGCACGACCTGGCAGCGAAGTATGTCGGAGTCTTCTCCCCGCAAACCGTCGAACGCTACGTCTTCGAG TCCTATACGGCGCTGCGCCGCACCGCGAAGATCCACACCCATCTCACCTCGCTCGCGGCGCGGTTCGCTG CCGACCGCCTCACCGCTTTGGCTCAGTCCAGCGGAGCACTCGCCAAGGACGTGCCCGAAGTTCTGTTCGT GTGCGTCCATAACGCCGGCCGGTCGCAGATGGCGGCGGCACTGCTCGATCACCACGCCCGCGGCCGCGTT CATGTGCGCTCGGCTGGATCCGCGCCGGTCCACGAGATCAACCCCGCCGTCATCACGGCGATGGCCGAGA TCGGGATCGACCTGGCCGACGAGTTCCCGAAACCGCTCACCGATGACGTGGTCGCCGCAGCGGACTGCGT CGTCTCGATGGGCTGCGGCGACGCGTGCGTCGTCTACCCCGGGAAGCGGTACCTCGACTGGGCTCTCGAC GACCCGGACGGGCAGCCCCTCGAGGTGGTGCGGGCAATCCGCGACGACCTCGATGCCCGGGTGATCGCAC TGCTCACCGAGCTGACCTCACCCGTCGCCGTCTGACCGACCTCTTACTTCCGTCTTCGAAGGGAACCTCC ATCCTCATGTCCGACAAGCCCAGCGTCCTGTTCGTCTGTGTCCACAACGCCGGCCGATCGCAGATGGCCG CCGGATTCCTGAGCCACCTCGCCGGCGATGCCATCGAGGTCCGCTCCGCCGGATCCGCCCCCGCGGAGAC CATCAACTCCGCCGCGGTTGAGGCGATGGCAGAGCTCGGAATCGACATCTCCGCCCAGACCCCGAAGATC CTCACCACCGACGCCGTGCAGGATTCGGATGTCGTGATCACGATGGGCTGCGGTGACACCTGCCCGATCT TCCCCGGCAAGAGTTACCGCGACTGGGTCCTCGAGGATCCGGCCGGTCAGGGCATCGATGCGGTTCGCCC GATCCGCGACGAGATCAAAACCCGCGTACAGGACCTGATCACCGAACTCACCGCCCCGTCGGTATCGCGC TGACCGCCCACGCTTTCCACATTTCCCCTGTGAAAGAAGCTTCCATGACCACTCCGTCAAGGGTCCATGA CGTCATCATTGTCGGTTCCGGACCGGCCGGCTACACCGCTGGTGTCTACACCGCGCGCGCTGAGCTCGCG CCCCTGCTCTTCGAGGGAACCCAGTTCGGTGGCGCCCTCATGACCACCACCGAGGTCGAGAACTTCCCCG GATTCCGCGAGGGAATCATGGGCCCCGATCTGATGGAACAGATGCGTGAGCAGGCACTGCGCTTCGACAC CGACATCCGCACCGAGGACGTCGAAGAGATCGACCTCAGCGGCCCGATCAAGACCGTCGTCGCGAACGGC GAAACCTACGCCGCGCACGCGATCATCCTCGCCATGGGCGCAGCAGCCCGCTACCTGGGCATTCCCGGTG 
Final Technical Progress Report DOE Award No. DE-FC26-02NT15340

AAGAACGCCTCCTCGGCCGCGGCGTCAGCGCCTGCGCCACCTGCGACGGCTTCTTCTTCCGTGACCAGGA CATCGTCGTGGTCGGCGGCGGCGACTCCGCGATGGAAGAAGCAACCTTCCTCACCCGCTTCGCTCGCTCG GTCACCCTGGTGCACCGCCGCGAGGAGTTCCGCGCCTCACGGATCATGCTCGAGCGCGCCAAGGCCAACG AGAAGATCCGCTTCCTGACCAACGCAGAGCCCGTCGAGGTGCTCGGTGAGAACAGCGTCACCGGCCTCGT CGTGCGCGACACCGTCACCGGCGAGACCTCCACACTCGAGATCACCGGCATGTTCGTCGCCATCGGCCAC GACCCGCGCAGTGAACTCGTCAAGGGCCAGGTCGATCTCGACGACGCCGGATACGTGCGCGTCGCTCCCG GTTCCACCGCGACCTCCGTCGACGGCGTCTTCGCCGCCGGTGACCTGGTCGATCACACGTACCGTCAGGC GATCACCGCTGCCGGCACCGGATGCTCCGCAGCAATCGACGCCGAACGCTGGCTGGCCGACCGCGGCGAC ATCACCGCCAACACGCTCGACGCAGCCGGCCACACGGTCGACGCCGTCTGACTCCCACACCCAGCGAAAG GAACTTCGATGAGCAAGCCTTCAGTGCTGTTCGTGTGCGTCAAGAACGGCGGCAAATCCCAGATGGCCGC CGGCCTCATGCGCAAAGCCGCCGGTGACCAGGTGGACGTGTACTCGGCCGGCACGAAACCAGGCTCGGCG GTCAACGCCCTGTCTGCCGAAACCCTCCTCGAGGTCGGGGTGGACATCTCCGGACAGACCCCGACACTGA TCGATCCGCAACTGGTGCGCGATGTCGACCTCGTCGTCACCCTGGGCAGCGAAGCGAAGGTCGATCCCGT CGCAGGTACCGATTTCGAGAACTGGGACACCGACGAGCCGTCCGAACGCGGCATCGACGGAATCGAACGA ATGCGGCTGGTCCGCGACGACATCGCCGCCTGCGTCGACGCCCTGGCCGGGCGTCTGACCACGTAATCGA CGTATCACCCCGGTTGTCAGCAGTAGATCTGGATGAGGAAAGGACACTCGTGCACTCGCAAGGGACACCG ATCACGGTCGACGTTGTCGTGATCGGTGGCGGTCAAGCCGGAATGGCCGCCGGGTACTACCTGCGCCGCT CCGGCCGCGACTTCGTGATCCTCGACGCCGAAACAGCGCCCGGTGGATCCTGGCAGCACTACTGGGACTC ACTGCACCTGTTCTCACCAGCCGAATACTCCTCGCTGCCCGGCTGGCCGATGCCGGGCTGGAGTCCCGGT TTCCCGCCCGCTTCGCATGTCGTGGATTACCTCACCGCCTACGAGACCCGATACGACCTACCGGTCCGCC GCCCGGTTCGTGTCACGTCCGTGACCCGTTCCGGTGACGGGTATCTCGTCGAATCCGATCACAGCAGCTG GTTCGCGCGGGCTGTCGTCAACACCACCGGGACTTGGCGGCAACCGTTCTGGCCGATCTACCCCGGTATC CGCGAGTTCACCGGCACCCAAATCCATACCACCAGCTACCGTTCGCCGACCCCGTTCGCCGGTAGCAAGG TCGCCGTGGTCGGCGGCGGAAACTCTGCTGCGCAGATCGTCGCCGAAGTCTCCACCGTCGCCGAGACACT CTGGCTGACGAGCCGGGAACCGCGGTTCCTGCCCGACGACGTCGACGGCCGGATCCTGTTCGACGTCGCC CGTGAACATGCCCGTGAAATTGCTTCAGGTGAAACCGATTCCGGGGGCATCGCCGGACTCGGCGACATCG TGATGGTGCCCCCTGTCAAAGCCGCCCGCGAGCGCGGCGTGATGGTGGCGCACCCCATGTTCGACCGCCT CACCGCCACCGGACTGGTCTTCGGCGATCACGTCGAACCGGTGGACGCCATTATCTGGTGCACCGGATTC CGCCCCGCACTACGGCACCTTCAGCCCCTTGGACTCCGAGACGGTGACACCGGACGCATCGAACTCGACG GAGAGCACGTCGCTGATGAACCTGGACTGATTTTCCTCGGATACGGCGACTGGACCGGTCCCGCATCGGC CACCCTGATCGGAGTCGGCCGCACCGCACGCGACGCCATCACCGCACTCGACGACGACCTGTTCACAGTC CCGGCCATCTGAGCAACATCCGAAACAAAACAGAGGACACATCATGAGCACCATCGAAGTATTCGAACCG GCCCTGTGCTGCAACACCGGCGTCTGCGGCGACGACGTCGACCAAGCGCTGATCACCTTCACCGCCGACC TCGACTGGGTCGCGAGCAACGGCGGCACCATCACCCGCCACAACCTCGCCAACGACCCCCTTGCCTTCGC CGGAAACGACACGGTGGCAGCGTTTCTCAAAATCTCAGGCTCTGAAGGACTCCCGCTGGCGATCGTCGAC GGAGTCACCGCCCTGACCGGCACATATCCCACCCGCAACCAGCTCGCGAAATGGGCCGGCATCACCGAAC CGGGAGCCGCGGACGCGCCGGCCGGGATCACGATGCTCGGACTCGCCGACGGCTCAGCATGCTGCTCCAC CGACGCCGCCGACTCCACCACCTGCTGCTGAAAGCGCCGACACCACAAAATGAAGTTCCTGAACAACGCG CCCCGATTCCTGTTCTTCACCGGCAAAGGCGGTGTCGGCAAAACCTCCATCGCCTGCGCGAGCGCGATCA CCCTCGCCCGCGCCGGGAAGAAAGTGCTGCTCGTGAGCACCGATCCAGCCTCCAACGTCGGACAGGTCTT CGGCGTGAGCATCGGCAACACCATCACCGACATTCCCGCCGCTCCCGGATTGTCTGCGCTCGAGATCGAC CCCGAACAAGCCGCCGCCGCCTATCGCGAGCGGATCATCGGCCCCGTCCGCGGATTGCTCCCCGAGAAGG AGATCGCGGCGATCGCCGAACAGCTCTCCGGGTCCTGCACCACCGAAATAGCGTCGTTCAACGAATTCAC CGGACTGCTCTCCGGCCAAGGCGACATCACCGCGGACTTCGACCATGTCCTCTTCGACACCGCTCCGACG GGGCACACCATCCGGCTGCTGCAGCTTCCCGGATCGTGGACCGAGTTCCTCGACGACGGTAAAGGCGACG CATCGTGCCTGGGGCCGCTGTCCGGGCTCGAGAAGCAGCGAGCCGTCTACGCCGACGCCGTTGCCGCGCT CGCAGATCCGCAGCGGACCCGCCTCGTCCTGGTCTCGCGCGCCCAGCGTTCGACGCTCGCCGAAATCACC CGCACCCACCGTGAACTCGCCGACATCGGACTGACCCATCAGCATGTCGTGATCAACGGTGTCCTCCCGG GCCCGGCCGACGACACCGATCCGCTCGCGACCGCGATCTACCGGCGTGAACAAGCCGCGATAGCCGGCCT GCCCGACGAATTGGCGCAATTGCCCACCGATCAGGTCCCGCTCAAAGCGACCAATATCGTCGGTATCGAC GCACTCGAGAGCCTCTTTGCTGCCGACCTCGGCCCGGTATCCGCCGCCTCAGCGGACCCGTCCCTGCAGC TACCCAGTTCTCCACTCTCATCCCTGATCGACGAACTCGACACCGACGATCACGGCCTGATCATGTGCAT GGGCAAAGGCGGAGTCGGCAAGACCACCATCGCCGCCGCCATCGCTGTCGCCCTCGCCGAACGCGGCCAC CAGGTGCACCTGACCACCACCGACCCGGCCGCACATCTGACAGAAACCTTGAACGGGGAACTCGACAACC TCCAGATCTCCCGAATCGACCCCGCCGAAGCCACCGAGCAGTACCGCACCCGCGTCTTGACCACCAAAGG CAAGAACCTCGACGACCGCGGACGCGCGAACCTCGCCGAAGACCTCCGATCGCCGTGCACGGAGGAAGTG GCCGTCTTTCAGGCGTTTTCGCGGGTGATTCACGAATCGAGTCGCAAATTCGTCGTCGTGGACACGGCCC CGACCGGGCACACCTTGTTGTTGCTCGACGCCACCGGCTCCTACCACCGGGAAATCGCCCGACAAATGGG TGAGAACAGCAACTTCACCACCCCACTCATGCGACTACAGGACCCGAACGCCACGAAAGTCCTACTCGTG ACCCTCGCTGAAACCACCCCCGTCCTCGAGGCCGCCGGTCTCCAAGCCGACCTACAACGCGCAGGCATCG AGCCCTGGGCATGGGTGGTCAACAACTCCCTCGCCGCAGCCGAACCAACCTCGACGCTCCTGCAGCAACG CGCAGCCGGCGAGATCACCGAAATCGACACCATCACAGACCAATACAGCCAACGAACCGCCGTCGTTCCG ATGTTCGCCGAAGAACCCATCGGCGAAGCCGCCCTGGCAGCTCTGATCAAGGCATAGCCCCTCCCCCCAA AAGTCGAGCACTACGAAGCCCGACTGCGGTCAACAGCTAAAGAAAACGACCCGAGCTTGCTCGAGCCGGT 
Final Technical Progress Report DOE Award No. DE-FC26-02NT15340

GGCCGGCAGTAGCGATCCGGACCTTTCCGCCACCGTTCTTATCGAACTTGTGTTCGAGTACTCTGGACGT GCGGGAAGTGTGCGCGAATCCAGGAAAGCGGGGCGACGATGAGCCAACAGCCCCAGTCATCAGCAACAAC GGTGCTGACCAAGGCGGACAAGCTCGAACAGTTACGCCGCAAGATGGCCTCGATCCCCGCTCGCTCGGAC GGCACCGAACCAGCCACGCCGTCGGTGATGCTCCGCCCGGTCGACGAGCGTGAGCCGGCCCCGATCGTGG CCACCGCGCAATCAGCCCTCCGGATGCTTCCCGTTCCGGCGCCGATCGGTGATCTGCTCCCGCGCGGCGG GTTGGCCCGAGGAACCGTCGTCAGCGTCGACGGCGCAGCATCGGTCCTGATCGGACTGCTCGCCACCATT ACCGCAGCCGGCGGGCATGTCGCCGTAATCGGAATGCCCGGCCTCGGACTTCTCGCTTTCCATGAGCAAG GGGGAGATTTAGCGAAGCTCGCCCTCGTCCCTCAGCCGAAGGATGCCGGGATCGACTGTGCCGCAATTCT GCTGGAGGGTTTCGATGCCGTTGTGCTGGGCCTCGCCGGAGGCGCTGTGACGCCCTCTCGCGGGCGGGCA GTGACAGCAAGGGCGCGAAGTAAGGGATCGCTGCTCATCGTGACAGAAGGCCAGTGGGACGGTCCGGATC TGCGGATCTCGTCCCGTGTTTTCGGATACTCGGGTCTATCCGCGGGGAGAGGGCGGGTGACAGGCGTTCG GCTCGACGTCGCGGCCGTCGGGAAAGGATTCCAGCAACGCGCCGGACGCGTGGAGATCCGCGACGACGTC GGACAGCTCCGCTGGGCGACCGTCGACGACACCACCCTCGCATCGACACCGCCATTGAGGGCAGCACAGT GATCAACACCGACCACTGGTCGGAACGCTGGCCCGATCACCCCGTGATCAGGACATTCGACTCACCGAGG GCAGTGAAAGTCGACATCGGCCGCGCGCTCCCGCTCGGCCGCGGGGGAGCCTCACGCGCCGATCATGTGA GCATGCGGGTGAAAACATCCGCGCTCTACATGTCCGGATACCTCGACGGCATTCTTAAATTCTGGGCAAG GATGCACGATGGTCAATGGTTGGCCGGGGTAACCGTGCGAGTCACCGATGCCGGCGGAACCAACCTCGTA GAGATCGACATGCTCGTGACCGCCGACGCCATCGCGGAAGTGCCGGCCACCAATTGAGCTGGGCAATTAC GGACACGCGGAAAGGGCCG

\section{>IGTS8 pSOX plasmid - contig003}

CCGGCCCTACGCGTACAAACTCGACCTATGTCACCACTCCTGGACGATATCGACGGTGCTGCGCCAGATC GCCGGGTTGTTCACACTGAAAGAGACCTGGCCTTGGTGATTAGCAGTCTCGTCAACCAGCTGGCCGATAG TGGCGCGGACGATAGTCCCGTTGTTGTAGACCGATCCTTCGCCGGAATACACGCCCCAGTATTCGCCGGG GATGGCGTTGAGGAAATCAGCGGTGTTGGTGTCGGCGAGGAAGCGCGTCTCGTTCTGATCGAACCGAACA CCGACATATCCGTCTCCGGAATACGGCGTAACGGCAGATGCGGCACCCGCGCCGAGGAGGGCGACAGCGC CGGCTGCGACCGGGACCAGCAGCGCAGCTACGGCCATACGTGAACGGGACATGGAAACCTCCTGTGACGT GAAGGCTGCATCGACCGCAACCAACTTGATGTAACTGACGGTATCGCAAATGCTGGTAACCGAACATTTG CGACAACATGACCTCGGTCACGTGGTCGAGGAACTGGAAGTCTGACATTCGTGCCGCTCGCACTTTATGA CTAGGCGAAACAGTAGATAAGGGCCGGGAGTTTGTACACGGAAACGGGCCGCTTGTCGCTTTTCGTTCCC TCTGAGTGAATGCCTGGCACTCAACCCGGTCGGTCGGACCACCCACTGCATCCGCCTGCCCGGCGGCCCG GCCACCTGCGGTGTCCCCCGAAGAGAACTCCGCTTCGCTCCGTTCTGGAAAAGCGTTTTATTTGTTTCTC СTTCTGGCCTTCCGATTTTGACCGGCGGTGACCTGCGCGCAACCGCTGACTCCGCTTCGCTCCGGCCTTC GGCTCGAAAGACTTTTTCAACCCGCACTCGCAAGCTCGCAGAAAAACTCTTCCGCTCACCAGTTGCACTC CGCCCCCСTCATGCCGGTCCAACTCTCCTATGCCAGAAGGCGAAAAAAATTGGCGGGGCACAGGATTGGT CCCCAGCCGCGAACGAAAGGTCACCGATGAGCGACGACTACGACTACGCCGAGGGCGACGACTACAGCGC GGCAACCATCCACTACGCCGGACTCAGTTGCGGGTGCGGTGACTACTCCTGCCCTCGCATGCACGACGAC GCCGTGCAGTGCGTCAACGCCGAGTAACGACAACCACCCACCAATCTCGAGCAAAGGAAAAACGATGAGC AACGAGACGAACTGCGACTGCGGCCAAACCTCCTGCCCTGCAATGACAGACGACACGCAGATGTGCATCT ACCCGGACCCCGGCGACTACGCCTGCACCGCGCAACGATCCGGCGACGAGGACTGCATCTGCGGTTGAGC GACCCACCACCCACCACAAAGGAGCCCCACATGAGCACCGAAACCGACACCCTCGCACCCGCGCTCGTCG CTGTTGTCGAGGCGACCGCAGCGCTCGATGCAGCGATGTTCGACCGCAGTCGCGAAGGGTTCATCGACGT CACCGCCCTGGTCGACGCATTCGCAGCCGCCCGCGCCGCAGCCCGCGACGCCGGACACACCGAGGAGGAC ATCGACGCCGCCGCCACCCCCACCAACTAACCACCCACCCATTCGTCACCAGCAAGGACGCGCAGCATGA CCGACCGCAACCGCCCGACCGAAGGAGAAAACGATGTACGGATACGACTTGCACAACATCTGCGAGAAAG CGGATGCGTGGGCGAAGTTGTTCGAGACAGCCGAGTATCTCGCCAGCGTCGTCGAGCACAACTGCTGCTC GGAAATCGACGTGTTCGTGGAGTTGCTCATCGCGCTCGACTACCACGGCCACGCCTCCGACCTGATGGAC GCACACATCGAGGCCGATGGCTGCCGAGAGCGCCACCCCACGGCCGATCAAGTGAGGTCGGCCTGACGGT GTTGGCGGTTTCCGTCGTCGTTGCTGCCCCGCTGGTGTGGGGCAGGGACGGACGGAGTGGATACGAACTG ACGGTATTGAGAATGCAATGGCTCCGGACGCGCCGTCGGCGCGAAAACATTTACCGCGGTGGACTGTTCT CTGAAATCCCCGGCGGGAAGGCACTTCTGCCCGGCGTGGCCGCGGACACGAAACTCTACGAGGGTGAGGA CGCCGCCGGGTACCGGTTCGGGATGATCCACATGCCCAAACTTCACCTGTACACGGTGGTCCTCAAGGCG TGGCCGCAGGGCGATGAGGCGGTCGATCAGTCGATGATCAACGAATGGGTGGGGGAGTGGGGCGTCGTCC TCGCCTCGATGGGTCAGACCTCGGACGTGGTCTCGGTCGTCCCGGTGATCGACTGCGTGCCCGAGACCGG AAACCGGTTGCTGGCGGAGGTCATTCGCCAGACCGAACCGGATGCACCGCAGTTGGCGCAGGACTCGAAT TACGAGGCGGCTGAACTGTTCGCCACCGAGCAGGCTCAACTTCTGCCGCGCTTGGCGGTCACGTTCCGAG CGACCACCGATGCTCGTAAGAAGGACCCTTCGGAGCAGGCCGTCGAAATCGGTCGCAGGCTTCCCGGTCT GTGCGCCGCGATGCGCTCCGCCGGAGTCGGTGTCCGCCCGATGGCAGCCTCGGACGTCATTTCCTTCGTT CGCCGCTCGACTGATCCGGCCTCGCAGGAAGACATCGAACGCGCCGACGCGACGGTGGAAGGACACGGGT TGGACTGGTCCGACGCCGGCCCGATCTCGCACGAAGAACGCTTCGACCAGTACATCCACGACGGTGCCCG CTCGGTGACCTGGGAAATGGAGATGGCGCCGACCGGCGCGGTCGACGAAGGCGTGTTGCGCCGCATCCTC GCTCCGAACCCCGACATTCCGCGTAAGCGGGTCGCGATCGTCTACCGCCCGCACACGGCCGCGGATGCAG TCAAGATCGTCGACAACGACTACAAGAACGCTCTGATCGCCGAACAGGGCGGCAAGGGCATCGGCTCGGT 
Final Technical Progress Report DOE Award No. DE-FC26-02NT15340

CACCGCAACGTTGAAGGTGGAAGCGACCGCCCGTGCCCGTGAGGAACAGGCGCGTGGTCACGGACTGGTG CGGTTCGGCATCTTGATCACCATCACCGAACCTCTCGACGGGGACATTCCGGCGATCGACGCGATCATCA AGGACCTGTCCGTGCAGGCGCGGTTGAAGGTGCGCCGCGCGTACCGCTACCAGGCGGCCGGGTTCTACGG CTCCCTCGGCATCGGCGTCATCTTGCCCGAGCACGCCTCCATGCCGTCGGCGATGGCCGGATAGGGGGCT CGGTCATGGTCAAGCTCCTCACCGAAGAACAGCGCCGGCAAGTGCTGGCACGCTCCCACGGCCCCACCTT GGCCGGTGTACTGGCTCGCCATCACCTCAAGATGGACGACAAACGTCGCCGGAACCTGGAGGCGCAGAAG GCAAGCGAAGAACAAGAAGGGTTCCTCCTGCCTGCCCTCAAGCTCTCCGACCGCGGCTATCGCGGTGCGG GCGGTGGCCGGATGGCCTCGGTCGGCCGTCAGGTCGAATACCGTGCCTCCTCGGCGCAGGCGGCCGGCAT GTGGCCGTGGGCCGTCGGAGCTGGTGCTCCGCTGATCGGGACACCGGTCGGGCGTCATCTGCTCACCGGC GCCCCGGTCCTGTTCGACCTGTTGTCCTGGTTCGTCGAAGGCCTGATCACCGCCCCGGTCGGGTTCGTTC TCGGACTCAACGGTTACGGCAAGTCCTCGTTCGTGCGCCGGATGGTTCTCGGCGGCGTCGCGCAGAAAGT GACCACCCTCGTTCTCGCCGACGTGAAGCCGGACTACCGCAATCTGATCGAGGCCGTCGACGGTCAGGTC ATCGACCTCGGATACGGGTACGGCCGGGTCAACCCTCTCGCCGGTGGTGTCCTCGGCTCGATCCTGCCTC GACTGGCGGTGTTCCCGGAGCTGGCCCGCAAGATGCTCACCGAACTACGGGCACGCCAGTTGCAGACCAT GTCCGGACTGATCGAACTATCCCGTGGCAGTGCGGTGCGCGACTACGAGGAAACGATCATCTCGTCCTGC ATCGAGATCCTCTACAACGAAAAGGGCTTCACCGCGGAGAACCCGCCGTTGATCGAGGACATGTACAAGC TCGTCAAAGAAGGCCATCCGGAGCTGATGGAGAACGCGGCCGCCGTCGGCGAGCACGAGTACCGCGAAGC GACCCGCGATCTGCAACGTTCTCTGCGCGGCCTGGTCAAGGGCTCGTTCGGTGAAATCTTCAACGGCCAC ACCACCACCGAGATCGACCTCGACGCCCCGGCGATCTGCATCGACGTCTCGCACATCCCCGAAGGCCACA CCCGTCTCAAGGCCGCGGTCATGTTGGTGTGCTGGTCCGACGGCTTCGCCGCCATCGATGCCGCCCACGC CCTGGCCGATGCCGGCCTGGGACCGCAGCGCCGCTTTCAAGCTGTCATGGACGAGCTCTGGCAGGTGCTC GGGCTGGGTGAGTTCATGGTCGATCGCGTCAACTCGCTGACCCGATTGAACCGCACGATTGCGACCGCGT TGTGGATGATCACGCACACGATCACCGACCTCGAGGCCATGGGCTCGGTGTCGGCGGCGAACAAAGCGAA GACGTTCATCGAACGTGCCCGCGTGAAAGTCATCGGCGCTGTTCCGGAAGCGGAAATCGACCGCCTCGAA CGGATCGTGAAGTTCACCGGCACCGAAGCCGACATGGTCACCGGTTGGTCTTCACCGCCTGCTCGCACCG GTGAGCGGCGTAAGGAAGGTGAGCCGAAAGAGCCGCCGCCCGGTCAGGGCAAGTTCCTGATCAAGGTCGG TGAGACCCGCACTCCCGGAATTCCCGTGCAGCTGATCTTCACCCCGTCGGAAATCGCGTCCGGCATTCAC GACACCAATTCCCGGTACGGCGACCTCACCTCGGACGCCGTGAGCGATGACGACAGTTTCGAAGGAGAGG GCGCGGCATGATCGCCGAACGCAAGTACAAGTCGCTCACCCCGCAATTGCGGGCAGCCGCGGTCAAGAAG GTCGCCGAACTGAGCACCGAAGTGTCCTCGCGGTGGAAAGCGATCGTGCTGATCGCCGAAGCGTTGGAAG TGCACCCGAACACCCTGCGGAACTGGGTCAACGCAGATCCGGAGATGGCCGCGACCAGGCGCGTCGACAC CGCTCGTCTCGAACAAGAGCACCGAGTCCAGCTCGCGGCCGCCACGGAACTGATCAACGAACTCTCCGAT CACCTCCACGGCCGCAGGCAGTAGGGGCAGGTCACTTCGGTCATGGACGTGAAAAAGATCGGCGCATTCT GCGGCGCCGCGCTGCTGGGTTTGGTGATGATCGTGGTGGTCATCATCGGCCCGGCCACGGAGAATCCCGA TTGCGTACCCGGCGGAGGCCGGGTCGGATCGGTGGCTCCCGCCGGCTCACTGGTCAAGCCGACCAAGAGC AGCGAGACCACCGATACTTCGGGATTCGGTGCGCGCTGGGGAACCCAACACCAGGGCATCGACCGCGCCG GTCCCGTCGGAACACCGATCTACGCGTTCACCGACGGCCGCGTTGTCGCTGCCGGCCCCGCCGCCGGATT CGGCAACTGGATCATTCTCGATCACCAGATCGACGGGAAGGTCTACTCGACCGTCTACGGGCACATGTTC GACGACGGCGTTCTGGTCAAGGTCGGCGACCAGGTTTCCGCTGGTCAGGAAATCGGCAAGATCGGCAACG CCGGCGAGTCCACCGGCGCGCATCTGCATTTCGAGGTCTGGGACGGTGGCCGCTTGCCCGACGGTGCCGG AACCGCAGTCGATCCGGCGCCCTGGGTCGACAAGGCCGCCGAACCCGGTGCCGCCGCCCCGACTCCCGCA CCAGGACAGGAACCTGCACCGAGCAACCCGGCTGAACCGGGCACCGGCGGGCTCAATTCCAGCCAGCTCG CTCTCGCGAAGCAAGCGGTGGCCGTCGGGGAGTCGATCGGAGTTCCCGATCAGGGAATCGTCGTCGCCCT GGCCACGATGAGCCAGGAGAGCACGTACCGGATGCTTGCCAGCTCCAACGTCCCGGAATCGCTGCAATAC CCGCACGACGGCGTCGGCTCCGATCACCTCTCGGTCAACCAGTACCAGCAGCAGGTCACCATCTGGGGAA CCGCGGAAGAGCTGATGAACCCGGTGACCGCGAACGTGAAGTTCTACGACGCCCTGCTCAAAGTGTCGGG CTGGCAGTCCATGCCGGTCACCGTGGCTGCTCAGACGGTGCAGGGCTCGGCGCACCCGGAGGCGTATGCC GACGACGAGCCGCTCGCCCGTCAGCTCGCCGCCCAGTTTAAGGGCGCCGGCAAGGACCTCACTCCGCAAG AGTTGGCCGACATCGCCAAGGTCGGTGTTGCGACCGCCGTCGTCGACGGCGGTGTCGGTGTCTGCACTCC GGGAGTCTCGAACTCCGGCGGACCGCAGTTCAAGCCGGGTGGACCGTTCGGTGCGAATGTCATCGCGGCC GCGTCCCGGTGGATCGGTACGACGTACGCGTGGGGCGGCGGCGATCAGAACGGACCGACCAAGGGCATCA GCGACGGCGGCGGCGGCGGAGATGCGAACGGCGATCCGAACAAAGTCGGCTTCGACTGCTCCGGTCTGAC GCTGTACGCGGTGTTCCAGGCCTCCGGCGGACAAATCCTGCTTCCACACTTCACCGGAAGCCACGGCAAC CCAGGGCAGCTGTACGACGCGCGGGGACAGGACATTTCGTTCGATCAGAAGCAACCCGGCGACCTGATCT ACTTCGGCGCCGGTGGGGACACCCACCACGTGGGCATCTACTACGGCAACGAAAACGGCCAGGACATGCT GCTCAACGCCCCGCAGTCCGGGCAAACCGTCTCCATCATGCCGCTCTCGGGTTGGGCCGGCGAAGACATG TACGTCAAGCGTTTCGGATAAAGGGAGAACACGATCATGACTCGCACACGCAACACTGCGGCCGCTGCCC TGCTGGCCGGGGCGCTTCTGCTCGCCGGATGTGGTTCCGGAGGCGACGGCCACGACAACCATCCCCTCGA CGAGGGAGGCCCCGCCGACAGCTCACTGACGACCCCGCCCCCGCCGCCACTGCCGGGTGCGTTCGCCGGA GTCGACCAGAACGATCCGGAATCGGTCATGGTCGGCGCAGCGCAAGCCCTGTTCTCCTACGCCCCCGCCG AGGATGCGAACCAGCTGGCCGCAGCGCAGCGTGCCGCGCCGCTCTTCGACGAGCGGTACTACTCGGAGAA CTCCGGTTCCTTCATCGCCCTCGCCCCGATCACCGGCAAACAGTGGCAGAGCTGGGCGGACGAGGGCGCG ATCGTCACAGCATCGGCGAGTGTGACCAGCGACGAACACCCGGCCGATCAGCCGGCGAAAGTTTCCCGCG TTCTCGCGGTGACACAAACAGCAACCACACCGGAGGGGAAACCATTGAGCAGCAACACCTTCGCGGCATA 
Final Technical Progress Report DOE Award No. DE-FC26-02NT15340

CATGAGCGCGACCAAACTCGGCGTCTGGCGCGTGAGTGCGGTGGCCGTGCGATGAGCCACGAACTTCTCT ACGCGACCGGAACCGCTGCCGGTGAGCCGGCGGCGGCACTGGGCATCGCCCTCGAAACCTTGCAGGAGCT GCTCGGCGAATTGGAGTCGAACAACACTTTCGTTGTCGTGCAGGGACATTCGCACACCATCACGCCCACA TCCGTGCAGCAGCCAGGGATGATGCGCAGCGAACGCCCCGGCTACATTGCCACCGTCACCACCGTCGTGG AGATCTCATGAGCGGGGGAGCGCACCGGCTGCTGAACTGCGCTGTTGCGCTCGCGGTCGTCGGGGCCGGT GTCTGGGGAACGGTCACTGTTCGCGAGTTGATCGCACCGGTCACCGATTCCGCGGCGTCGACGTCGGGTC AGGTGGTCCGGGTCGTCGACGGCGACACTGCCGATGTCCGTTTGGATTCCGGCGAGGATCTGCGGGTCCG GATCTTGGGGATCGACACTCCGGAGACGGTCAAATCCGATGCTGCGGTGCAGTGTTGGGGACCGGAAGCA AGCGCCTGGGCGCACACGATGCTCGACGATGCAGCGGTGACGTTGACGGGCGACCCTCGCACCGGCGATG TCGACCGATACGGCCGATCGCTGCGTTACCTGACCTTGCCGGACGGCACCGATTACGGCGTACTTGCGGC GCGGTCGGGCATGGCGCGGGCGTACGTCTACGGCAACACACCTATTGAGAAGACAGCGGAAATAGCTGGG GCGCAAGAGGATGCGCGTTCGGCAGGAATCGGACTCTGGGGTCCGCCGTGCAACGGAGAGGCAAACTGAT GAGTACCAGGGCAGTGAAAGATCCATCAGCTTCGGACGGGCTGTTGCCGGCAGTTCTCATCGCCGGTCTG GTGGCGGTGGTCGGCGGCAGCGTGTGGGCGGGCATCCACCTCGGGACCGACCAGACCGCGCCGGGAAACC CGATCGCGATCGTCAAGGGCTTGATTCGAGGTGAACTCGTGTGGGAGACCGGGGCGACCCTGGTGGTTGC TCTGGTCCTGGCAGCAGTGATCGCTCTCGTGGTGGCCACGGCGGTGCTGGTGCGGCGGGTGCAGGGGGAG AAGACCCGCATCGACGCGAAAGCGAAGGTCATGGGCACCGGACGCGATATCAAGGGCATCACCGAGAAGG AATGCCGCTCGATCGCGTACAAGAGCTTGGGCATGAAGGAAGAGCTCAAGGACCGAACGAAAGCGGTCGG GGTGCAGATCGGTAAGCACCTGCTGACCGGGCGGATGCTGCGCGGCAGCTACCAGGATCTTCACTGCGAC ATCTGGGGACCGCGTACCGGTAAGTCGTCCTCGCGGGTCATTCCGGCGATCCTGTCGGCGCTGGGAGCGG TGTTGACCACGTCGAACAAGCGTGACGTCGTCGACGCGACTCGGGCATGGCGGTCGGTACGGCTGATCGA CCGGGAAACAGGAGACAAGGTCAAGGCGCGGTCTGTGGACTGGGGAACCTACAGCGACCCGGCCAAGCCC GTCGCGAAGGGCAAAGTGCATGTGAACACCCTCGTTCCGCGGAAGGTGTGGGTGTTCGATCCGCAGGGCG TCGCCGGTGAGGAGCCGACGTGGTGGTGGAATCCGTTGTCCTGGGTCACCGACGAGGTCAAGGCCGCTGA CCTGGCAGAACACTTCGCTGCCGGTGACGACGGAGTCGAGGCGAAAAAGGACCCGTTCTTCGATCCGGAA GGGCAAGACCTGGTCGCCGGGCTGCTCCTCGCGGCCGCTGTCGACAACCGTCCGATCACCGATGTGTACC TCTGGGCGACACAGGAGACCAACGACGAACCTGTCGCAATCTTGAAGGAGGCGGCCGGCGGGAAGTACGC GATGCAGGCGAGTGGCCTGGCGGCGCAGTACAACCAGACCGAAAAGCAGCGTTCGGGTGTTTTCACCACG GCGAAGAAGATGATTCGCTCGCTCAAGCTCTCCGCGGTCCATCCGTGGGTGCGACCGATGGGCCCGGGGG ACAACCGCCCGCATTTCGATCCTCGGGCGTTCGTGGCGAACGACGAGACGATGTACAGCCTCTCGAGAGA AGGCAACGGTTCGGCCGGTCCGCTGGTCACAGCCCTGACGGTCGCGATCATCGACGCCGCTGAGCAGCTG GCCACCAGCTCACCGAAGGGCCGTCTACCCAAACCGCTGATTGCAGCTCTCGACGAGGTCGCCAACGTGG TGCGCTGGACGAAGCTCCCCGGCCTCTACAGCCACTACGGCTCACGCGGCATCCTGATCATGGCGATCTT GCAGTCCTGGTCACAGGGTGAGGACGTGTGGGGCGCCAAGGGCATGAGCAAACTGTGGTCCGCCTCGAAC GTGCGCGTCTACGGCGGCGGTGTCGCAGTCGACGACGGCAACTTCCTCAAGGACATGTCCACCGCGATCG GTGAGCACTGGGAAATCACCGGCTCCGTCTCCAACAGCGGCAACGGTCGCAGCGTCTCCAAACATCGCAG CAAGGAACGCACCCTCACCGAAGCCGAACTCGAAGCGCTACCCCGCGGCCGCGCGGTCGTACGTTCCTCC GGTAACTCACCAACCCTGGTGAAGACGGTGCCCTGGTGGGAGGGACCGAACAAGGACGCTGTCGTGGCCT CGATCAAGGTTCACGATCCCGAACCGGAGAAGACCCTGGCGAAGGCCGTCACCAAGGTCGGCGCCGTCGA CGCAGTGGAGTTGGTCGCATGAGCACCGATTTCGATTTCCCCGGATTCGCCGACGACTTCGAGCCGCCAC CGGAAGGCGCCTACGACGAGGTACCGATCGACGACCCCGCGCCGGCTGTTGATCCTGGCATCGCCGCGCC CGCCGAACCTGCCGGTGCACTCAGTGCGCAGATGGAAGCGTTCGTGGCACGGGCGATGGACAAACGGATC AGCCAAGAAGCGGATGCGATCGCGGCCGCGAAGTTCGAGGAGATGCTCACTCCCGAACTGCACACCCAAC TCCAAGAAGCCGCACAAGCACGCCTCGCCGCGCAGCTCGCGGCGGCGCTCAAGATCCCCGACGATCCCGA ACCCGAGCCGGAACCACAGATGGTGTTCGGCTCGGCAGAAGAATTCATGCGCGACTGGCTCGCCCCGACC TACCGCCGCCAGGTGACGGACACCCCTTCGCGGGTGTGGTGCCCGCAGTGGTGGAAGCACGAAGAAGCGG TCAACCGCGTCGAAGCTCTCTGGCGGGCGTGGGAGTACCTACGCCTGGATGCGAAGACCGGCATGAGTGT GTGGTGGCGCGATCACGCCGATCACCACATGGAAAAGCTGTTCGCGGGCGATGGCCCCTTCAAAGCGTGC TCGGCCGAGCGGGGCCACAAAGGTTCCGTGAAGCCGCTGCCTACCGACAAGGCTCCGGACGCACTGTTCC CCGATCAGCGCGAGACGTGAACCCCCGCGAAACCGCACTTTCGGCCCGCACCCGGCGGACCGGTCCGACG AAAGGTGAGAACAGATGTCCGATGACGAAGTAGGCACGATCGGCCGCGAAATGGGCATGGCGATGCGCGT GGCCATGCAGGTGATGAGCCGACTCGCCGAGCAGATGGCACGCTCCCGCGAACAACGAATGCGGGACGCA CAGCATCGCAGCGAACAGGCAACTCGCGAGTACGCCGAACGTCTCAAGGTCGAACGGACGGCCGCCGAGA CGCAGCTGCGAGGAGTCTCGGACAAGCAGTGGTGGGATCACGCCAAGCCGGAGGCGATCACCGACGCCTA CTTGCAGGCAAAGGCGTGGAGCCCTGAATCGGAGATCGCCGCCCAGGCCGCACACAAGATCCGCTCCGAA GTCCACCAGCGATACGGCTTCGACGTCGACAACGATCAGGCAGCACGGGACTTCCTCGCCTACGGCCGTG CATACAAGGACGTCGCGGGTACCGAGCAGAAGCGGGCCGCTCAGGATCGCGTCGAAGCTGGTGCCGCGAT GACCGCGGCCGAGAGCGCCGACCGTAAAGCCGAACGCGCGGCGGTCGAGCTCGAAGAAGCGAAGACGTGG GCGCGGGAGAACACTCCGGAATGGTTCGCCGTCTGGGAGCAGACGTACGGACCTGAGGCGGGGAAGGAAG AACGGAGCGAAGCAGAGAAAGAACTTGTCGGGGACTGGCAGCGGTCGCAGCCCGAAACGTCGACGACAGT CGAGGAAGGCAACCGCGTCCAAGGCGGCGATGAGCGCGGTGAAGCCGTCGAGAAGTACGACTCCGCGGAC CGGCGCACCGCGTTCGCGAACAGTATGCACGCTGCCGGCGTCGACGCCGAAGCGATCGCGGCGCGGATGT CCGCCGACACTGCGCAGGGGCGTCCCGCTCGGGATGCTGTCCGCACCGGACCGGGCAAGCCGCCAGTCGC GCGGAAGGGGAAGCCGAACAAGTCCCAAGGCCGCGACCGGCAACACGCCGGCCGGTAGGGACTCGACTGG 
Final Technical Progress Report DOE Award No. DE-FC26-02NT15340

>IGTS8 pSOX plasmid - contig004

CCTTCCAGTCGACGTTCTAACCGGTGATACCTCCGATTGCGAGGGCTCGATCAGCGAATTCCTGTCCGGC TACTGTGGCGATCTGTGTTGAAGCCACCGCGAGCGTGGACCGAAGCCAGTCCATGCCTCGTTTAGCTCCT TTGCGGAGTATCCCGTCGATATCGTCGAGAACATCGTCGAGATCCTCTTGATCAGCATCGACGACTGCTC CCGCCATGTCTTCCCTGAGCACCCGCAGTGCCTCGAGTGCCTCGTTAAGGGTCATGCCGGCAGTGATGTT CACAGTCGCAATGTTGGTGTGCCCCTGTCCTCCGAGCACCTGGTTACCGACGGTCATAGTGCCGTAGTTA TCGGACGAAATCTTCGAAATATTGGTCACCGAGGTCCGATCGATAGGAGAAGCGAGGATCAGATAAGGGT CTCGCAATGCGCGTCGGCCGACCGAGGTGGTTTCCACATAGAAAAACTCCCCGTTCGCCTGTTTACTGCC TGTGAGCATCCCGAGTTCTTCCAGTTCCTCCGCAGTATGTCGAATCTCCAGTGGTGTCAACGCACCGGTG AAGTCTTCACCCAGTGAGCTTTCCACGAGCCCTTGAGTGCTGTTATAGGACGAAATCCACTGAAGGACCC GACGGGCAAGCATCTCCGCGCGGTGGCTCTCACGCGCATTGCGCGCGGGCACAGAGCCCGCAGAGGTCAG TATGAAATCGAAGCTTCCATCCATGGTGGCAGTGCGATCGATGTAACCCAACCCGCTCAGTTCCTCGTAC ACGGTGAAAGCATCAGAGGTGGGACAGACGTCGTCGGGCAAGGAATCCGACTGTGGATTGTCGGACAACC AACGTAGGGCGCAAAGGGCACGAAACGTCAGACTCAAAATTTCCTCGATTCGAAGTGCGCCCACGCGGAA CGCATCCTCATAACTTCTCGGTCTAGCCGAACGTCCCGAGACAGGAACTCGACAGTGCCGAAACTGCGGC GATGGGTAAGAATGCTGTAGTCCTTTGAACAAAAACGTCTGTGGCGTCCGGATCGGGACCATAAGCGTCA TGAGCTGGGTACCCGGACTACATTTTCGAACTTGAGGAAGGCCGGGGAGCGATAGATGTCTCCCCGACCT TGCCACTGTTGTCGAGCTACTTCTTCTTGGATTCGACGACCGTGGTCTTCGGATGACTCTTGGCGTAGCC CTTGGTCACGTATTTACCGGTAATCGCACTCCGTGCACTTCCTTTGGAGCTGCGTCCGCTGTTCTTGGCC АTTTCACCTCCTCTCCTGGATCCCAAGCAGATCGGGGATGCGACAGGGCATCCCCCAGATCTGTTTTACG GAGTGATCCGGGTAGTTCGGGTGACGGTGTTGACCTTGACGGGAACGGTCTTCGTGGTGGTGGATCCGCC GCGACGGATCTTGACCGGCACGCGGACAGTCTGGGTCCGTCGAGTTGTATTCGTACTTCGGGTCGGGCTC ACGAGGAGCCTCCTTAGATTTTCAGCGCCGGCACAAAAACCTGTCCTCACCCACGGGCCCGACTCGGCGG GCCCGACTTGTCAGGTGTACCGTTGCCAACGCATCCTCAGAAAATGCAACAACAAGCACCCCGACTGGCG GACAGCCCCTGGTCGGGGCATTTGTGTTTCTCCAGCGCTCAAGACACTACACCTAGTGGCCGACAGGAAC ACATAACGCGAGATGTTGTGACTCACATCGTTGAAATTCACACGCCGCCCACTGTCCTCGAGCTCTTTTT ACAGGGGATCCGACATTGCGACATTGGACAGCAGTCACCACTTACTGTCACTGCGCTTAAAGAAACGATG GCCTCCGAACGCAAACGCCTGCGGCCTTCCTTCACTGACCACAGGCGCCCTGAACTGCTCAACTCTCGAT TGAGTGGACTACATCATCACGAGTTCGCGAACCTTACCCGTCTTCGGATCCTCGATTTGAACGGTCTTAC CTGCCCGGGTCTGCTGTTCAACAAAATGCGCAACAGAGACTGCGCGGCGGACAGCTTCAGTGACTGAGAC GCCGTACGCCTCTGCGATTTCCCGAAGGGCGTCAGCGGTCTCGGCGTTCATGTTGACACTGAGCCGAATC GGCTTGGCCTGCTCAGCGGCAGAGGTGGTGGTGGGGACTTCATGCTTCTGTTCATTCATTTTTTCTCATC TGACCTCTCTGGTCACCGAGCGTTGGAGATCTTTCGATCTCACGTCGGTCTCACGCGGTCCGCACTGGTC ATATGCGGAATCATTGGCCTAGGCAGGGCCTGCAAAGTACTTGTGGAACCGACATCTCACAGGTTCGCCG TTCGTTTGGGCGAACCGTACCCAACAATAGCCGCAAGTGCGCCCTCAATGCAACAAATATACGTACGCAA ACCATACGCAGACCACCCGCAACCCACCCGCAAGTCATACGCAAAACGCAGTATGTACGCATTGAGTACA CACCACATGTGTTCGACATGTGCAGCGGGCCACTGCTGGCTGCTACACTTGCCTGTACAGAGCCTCGTAC GGAAGAAGCGGGCCCGACCACGAAGGGCACCGCCGATGAGCAACGAAGACCGTGCTGAGGACGAAACGGG TTCCACGAGTCCCGGTGAAGCCGAGCGGGCGCCGGAATGTCAGAACAAGTTTTGGATTTTCGGGAACTAT CCCGAGAGCCACGCTGGGCTGAATATAGAAGGGTACGAACACATTTTCCCGGTGGTCGTGACAAATCTGG CGATGTTGCCCTCGCTGAGCCCGGAGGACCTAGCTGATTTGGTGGGCATGGACCGCCTCCTCGGCCCCGG GGAGAGAAATCCAGCAATTTCTCATCTCCCCGAGCATGGTCCCAGCTTTCACAGCCCTCCCTTCGGAAAG CCGGAGGAAGAATCACTAAACTCCACATCATTCGTTGAGGACGAGCGCCCCGCCTCCGCAAACTTGAGCC ACTTTTGGACACGCGAGGTTGGAGAAGACCAGTCAGAAGATCGGTACGAAGAAGACGAAGCTCCTTCGCC ATACGGCAGGTTGAATGTTTTCGTTCTTTTCGCCGAGGAAGAGCCTGAAGATGACGCAGCCTCCAGTTGA AGGCACTTCACCCGGTTCCTTCGAGGAAGACCTTCCGCCATCGACGGCGGGCCTGGTTCCTATGGCGCCC GCTCGCCCAACACACACAGACCAAACCCGTAGATATATCGCATACGGATTGCTCTGCTTACTTGGCTTAT TAATTGGGGGGACGCTGCTTTCCTTCATCGCCGGCTGGCTCACAGTCGACGAGATGAAAGAGAGCGGCGT АСTTATTACCCCAGTTTTCACCCTCGCCGGCACCGTCCTCGGCTTCTACTTCGGAGCAAACAAGAACTGA GTTCAAGCCTCATCCCGCGTGCGCGCGTATGAAAGTGCGCTCTGGTCAGAACGGGTTGTTCATCAGGTGA CGTTCGATCAACGCTTTGACCGCCTGTACGGCCGCGGATGCGCGCGCCGTCGAACCACCGAACAAGCCGG CTTGAAGCGGCGGCCAGGCATCGCCTTCCGCCTGGCAGTTGTTCAGGTACTGCGCCCACACGGTGTTCGA CTCGTCGAGCGACCAGGTTCGTTTCGCAAATGTGCCCAGCCACGGCTCGGCATGAACCTGACCGCCGTCC ACGGCGTCGGCGGTAATCAGCGAGAAGAGCACCTCCGCTTGATCGAACAGCTTCTCGTACTCCGTCGAGA AGACCCCCACCTTGTCGAAGTTGGGTCGCAGCGTCTCTCGGATGTAGTGAGAAGCGGGAAAACTGGTTCT CCGGCGCAATTGGCCGGCCACGATCGCTCTGAGCATTTCCTCGGTCAGAGTTCCCTGCTGATTCGAGAGC AGTGCTTGTGTGATTGCCTCGCTGTGGAGTGAGTAGTAGGGGGAGGCGCCGACGATCATGGGGCGATAGA AAGTAACTCCGCCGAACTGCTCGGATACATCGAGTTTGGCGTCGCAAGTCAACGCCTGAATCAGGCGATA GTTGCCCTCTCGGAGGGCTGCAACCGTGGCGGCGTACATCAAGAGCGTCGTCGGAAACAGTGCTAGCCCC GTCAAGTATTTGGAGCGTGCTGGCTGGATGAGTGAGCCAGGCTCCGACGCGTCGACTCGCGTCGCTGCGA 
Final Technical Progress Report DOE Award No. DE-FC26-02NT15340

TGACATCGAGCGTTCGTTGAAAGATCGGAACGTAGTCCGCTGCTCCCCACATGCCCGCGCAAATAAAGAG ATCGATCGCCGGATCCGCGACGTCGCGGTAAGTGCCGGCGCGATCGACCATCTGCCGTACCTGACTTGCG GAGTCGCTTCCGGGGACGAATGCCGGAGGGAACTTGTCGATGTCCTGGAGTTCGCTGTATACCGGGCGCA GGATTCCCTGAACGCCGCGATCGACGCTGGGTTTCTGCCCCGGCGTGCTGATGATGCGCTCCAGCTCTTC GAGTCGAGATCCGACGGGAATAGCTGCGGTGGGGATGGCTGTCGATGCCAAAGTGGTCGGGGCTGAACCC GGTACCGGCGCAGTGGTCGGGGTTGATCCCGGCACTACGGGTGCAGCGGTGGGCGCTGAGCCAGCTGGAG GCTCTTCATCGAGCAGCGTTTTGATCTGGCTCACGAGTTCCTGGCCGTCGGCCGCCTTGCTTTCGCCGAA GAAATCGAGGAACTGAATGTCGCTGAGGAAATCGCCGTTCCCGATGTTGTATTCAGGGATCTCCGAGTCG TCGAAGCGAACATTGAACAACCACATAGTTTCGGGACGCCGCTTGCGTAGTTCACCGGCTGCCAGGTCGA GTTCCTCGTTCTGGCCGTTCCGTTTCTTCTTCTCCGCAGTCTTGGAGTAGCACGCCACGAACGCGAGGCT GTTTTGCGTGATGGCTTTCCGGATCTCGGCCTTCCAACGTGTGCCACCAAGTATCGACTTCCGGTCTATC CAGACTGAAAAGCCTTCCGCCTCCAACAACTCTTTGAGTTCGTCGGCGACTTGACTGTCCTCGCGAACAT AAGACAGGAACACATGGCCCGGACGCGGGGGAGCAGGATTCGACACCCCGACATCTTACTGGTCGTCCGG TTTTCACTTGCGGCTTCGCTCTGGGCCGGCCGGTAGTCAGCTCTACCAAGAAGACCCCTCACATGACGTG CGGGGTCTTCTTCACTGGTGCTGTGGACTTGGTTCACGTTCCGCTCGGCGGCGTGGTTGGCGCGATTCGC AAGAGGTTCGCGATGTCGTCATTGAACTTTCCGCGATGGGTTTGAGCCCATTGGGCGGTCTTCTCCGCTG ATTCGGTTAATGCTTCTGGTGTGGCCGCACGCGGGCCCTCCGGCAACAACTCCAGATCGGAGAGTGATCC GATCAACCGTGGCAAAGGCTCGGCGCCATCCCAGGTGTCGAGGATCGCCGCCAGAGTCATCAAGTGCGAT AGCCACTGCTCAAGCAGGGTCTGACGAACAATCGCCTCCAGCCCCACGAACGAGAACGGTATTGAGTTTC GCTGTAGCTCGGGCGCTTCGGCGAAGGTTGAGATACGGAGCAGATGGTAGGCAGCAACAGTCTTCGCGGC ACGCGTCAGGACGGAAGGTCCGAGCGCAATTGTCAGTGTGCTCGCCGAGTCCGACAGTCTCACGAACACT TCTCTGGAACGGGTGAAGATCGCCCGGAAGTCGGCTTCGTCTGTACCGTGTCGGCCGTACGAGCCATGAA TCTCTGCGAGCTCTACAAGAGCCAGTCGGGCGTCCTCGACGTAGCGCTGTGCGTAGGCGCGTCGACGTTC TTCCGTAACCAACCGGCGGTCGTGCGCGAACTGTTCCGCGATGAGTCCGGCGTCGTGCTTGAACTGTGCC TTGAGAAATCGGTAAGCCACGTACAGAGCGACGAGGGAGACGATCAACGGAAGTCCGGTGTCTGCGAGCC ACTCGGCGTTCCATATCTGCGACCAGAAGTTCTTCACGGCACATTTGAGCACAACAGCCAGACACAGGTC CGCACCCTGCGCGTTACCGGAAAGGTCTGGTCACACGTCTCGTCCGGTGTCGCGGCCGCGGTCGGGTTGT GCGGCGGTGCGCGGTTTGGGTTCGAGCTTCGGTGGGATCGGTGACGGGCGCACTGCTTCGGTGGGGCTGC TCGGGTACGACAGGCGCAGGGACTTCTGTGCGGCGTCGATCTCTGCTTGCTCTTCGGCGGTGTAGGTCCG TTCGACCGATTCGGGTGCCGTCTCCGGTGTCGTGGTGCTGACCTCGTGGGCGGGTTCGTCGCGTTCGGCG ACCGCGACGGAACCAGTCTCGGCGGATCGTTGTGCGGCGGCGTTGAGTCGTCGGTGTACCGATTCGAGTT GCACTCGGCTGACGGTCTCGAGTTGGGCTGCGTAGGCAGCGGCTCCTGCCGCGCGTGATGATGCTGCGAT CGCTTCGGTTGCCTTCATCAGTTGCCGCAGTAACAGGGCGTAGCCGGCGGCTGCGTTCAGTCCCGGCTTG CATTGCATGACGATCATCGCTGCGCCGCCCGCGGAGACGATGCCGGCCTTCTTCGGGGCGTGCTGGTGTG CGGGGATCTGCGCGGACCGTGCGAGTTGGACGGACGCTCTCGCGAGCGGGCCGGGTGTGGCTTCGACTTG GGAGGACCACGCGGCGAAGACACCGGAGGTTCGTCCGGCGGCGCGTGCCCACTGCGCTCGGTCGGTGACC GGAATGCTGGTGAGGTAATTGTTCCACTGTTCGATGTCATGCGCGGCGCGTGCCAACAGTTCGGGATCGG CGATGACGCTCTCGCGTCCTTTACGCACTGCGGGCTTCTCGCGTCGTGCTGCGCGCCATTCTGCGACGGC TTCACCGGAGCTGGTGGGGGTGTCTTCCCATTCCTTGCGGAGGCGGGGGAGTGTGAGGTCCTTACCGAGG TGTCCGCCGCCGTAGAAGATGGGCTTGGCATCGGTTCCGGTGGCATCGCGGTCGGGTCTGACGGCGACCT TGTAGCCGACTACGACGTCATCACGTCCGGAGGCGAAGCGGGGCCGGACGATCAATCCTTGCCCGCGAAG TCGGCGTACGAATTCCGCTTCGGACTTCGACGATGTTGCACAGGCGCGCACGCGCCGTGCAAGGAGATCG CGGTCGAGTTCGGTCTGCCCGTTGCGTACTGCGCGCGCATTTTCGGCGCGGTGGTATCCGCGTTCGCCGG TCTTGCTCTGGCGGGACTCGATGACAGCGAGGCCGTGTTCACGTTCGATGCGTGCGGCCGCCACGCTTGC TCGTTTCCAGTCACGGTAGAGGTTGACCTTGGTTCCGTCTTCGCGCACGGCGGATGCGGCGATGTGGATG TGGTCGTTGCCTTTGGCGGACACTCCGTGTCGGACGGCGACCCACCTTGCGGGACTGCGACCGGAGGTTT CGGTGAAACCCATTTCGTCCATGAACTCGGTGGCGATCTTGTTCCACTGCTCGTCCGTCAGCTCGCCTTC GTCGGCCCTGAGCGAGAGCGAGCAATGCCAGACATTTCCGTCGCGATACGGGTCGATCGGATCGCGAACC TTCTTGCCGTGACTGTCGCGAACGTCTTGGCCGGCATCGTCGCGCAGGTAGCTCGGGATCTTGATTTCGG TGCCGAACACTTTGCGGGGCTGGTCGATCTGCTTTCCGATCGCGAGAGCGTCGACACCGCTGAGTTCGTT GTCGTCGTGCCACGCCATGATCGCGTAGTCACCGGCAACAAGATGCGGGTCGGTGTGCTCGTTGCTCGTG CCCGGACCAGCGAGGTACGACATCAGTCCGGTGACTTTGGCACCGCGGGAAATCTTGACGATCACGGCGC GGACATCTCGTCGAGGGTGCCGTGAATCCGGTCGGCCAAACGCCGCACGCTCGCCAATGCGCCGTGCAGT TCGGCGGGG 
Appendix 8.4

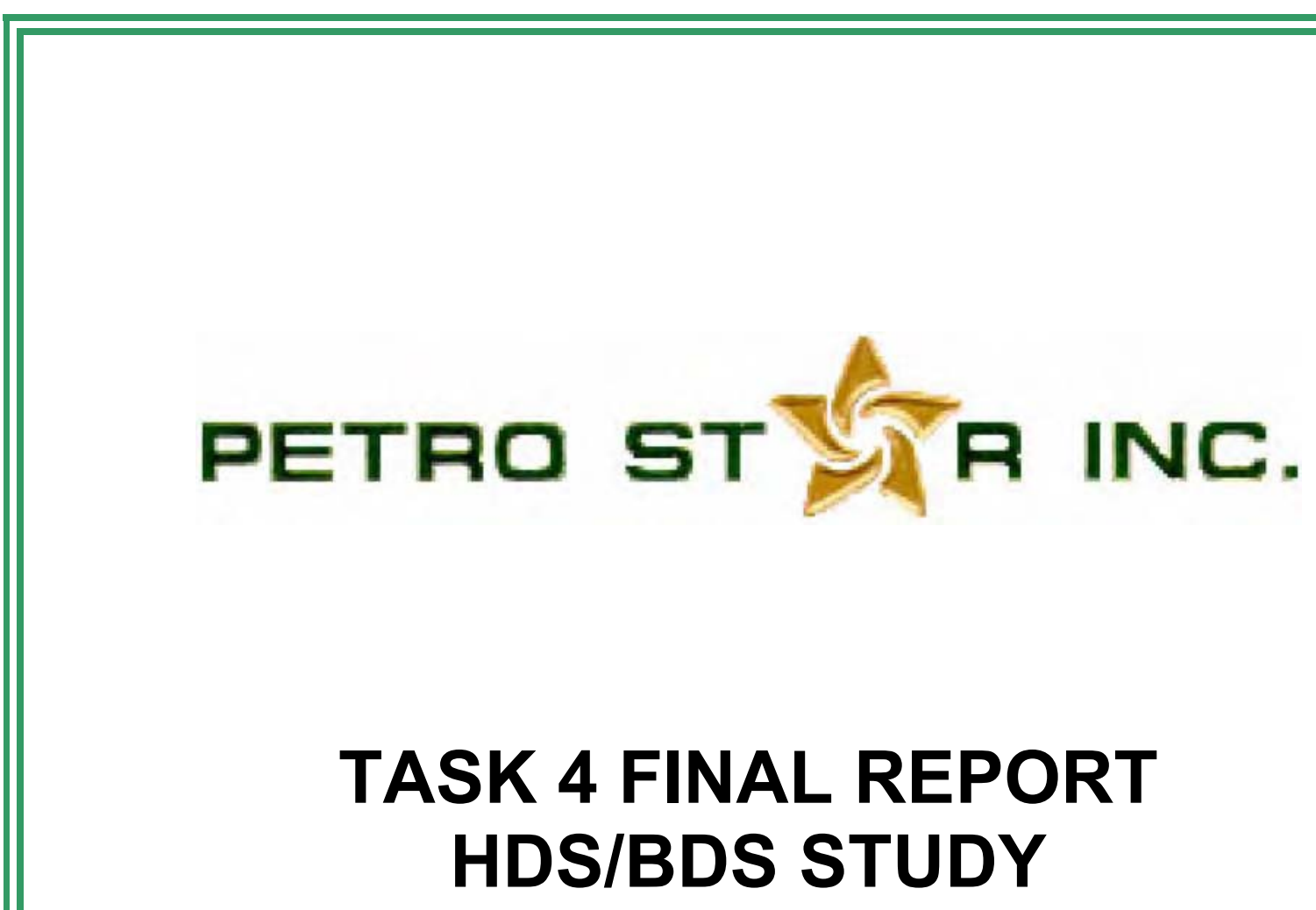

ALASKA ANVIL NO. AE1416

REVISION 0

AUGUST 2005 


\section{TABLE OF CONTENTS}

\section{SUMMARY}

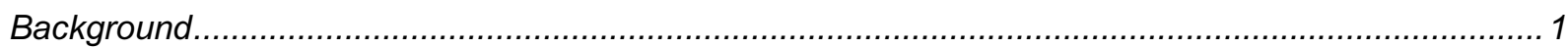

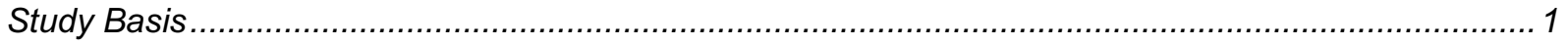

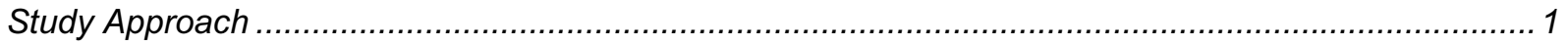

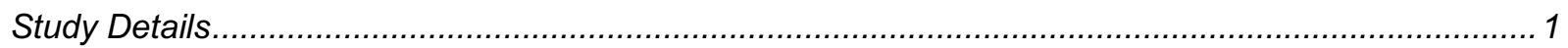

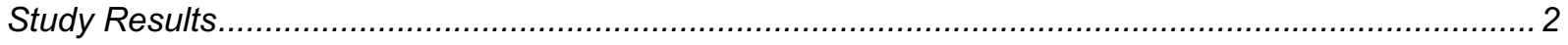

HDS BASELINE REPORT AND COST ESTIMATE

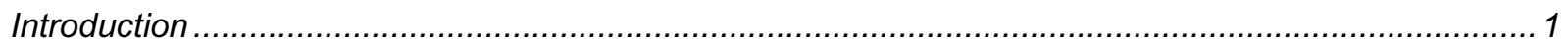

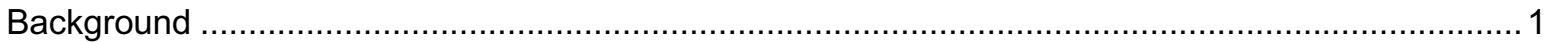

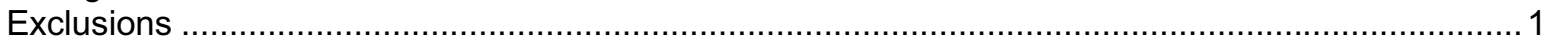

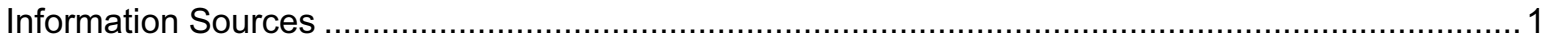

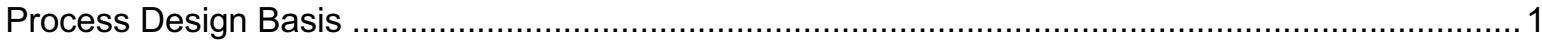

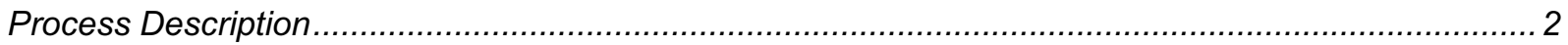

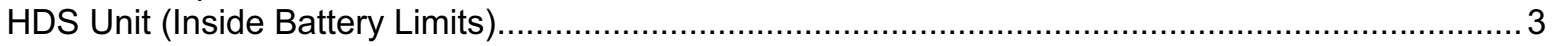

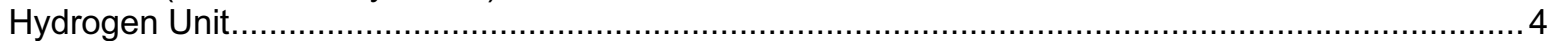

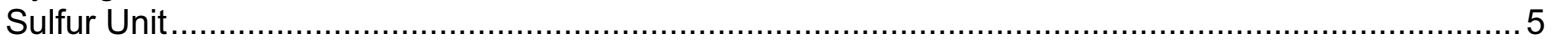

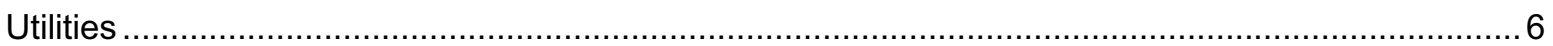

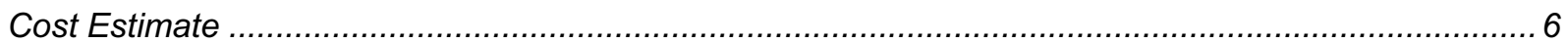

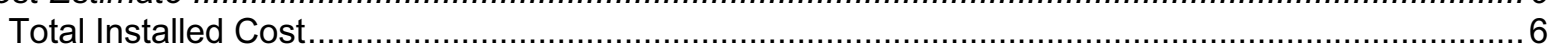

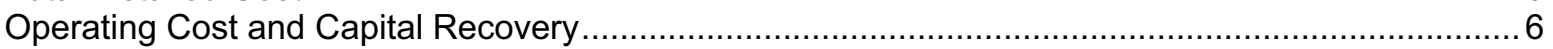

Appendices
A. Diesel Feed Properties and Sulfur Speciation
B. HDS Unit Process Flow Diagrams and Material Balance
C. HDS Unit Equipment List and Budgetary Equipment Data Sheets
D. Hydrogen Unit Flow Diagram
E. Sulfur Unit Block Flow Diagram
F. Utility Requirements
G. Cost Estimate Basis and Cost Estimate
H. HDS Baseline Case Operating Costs for 6,000 BPD Diesel Feed

\section{BDS BASELINE REPORT AND COST ESTIMATE}

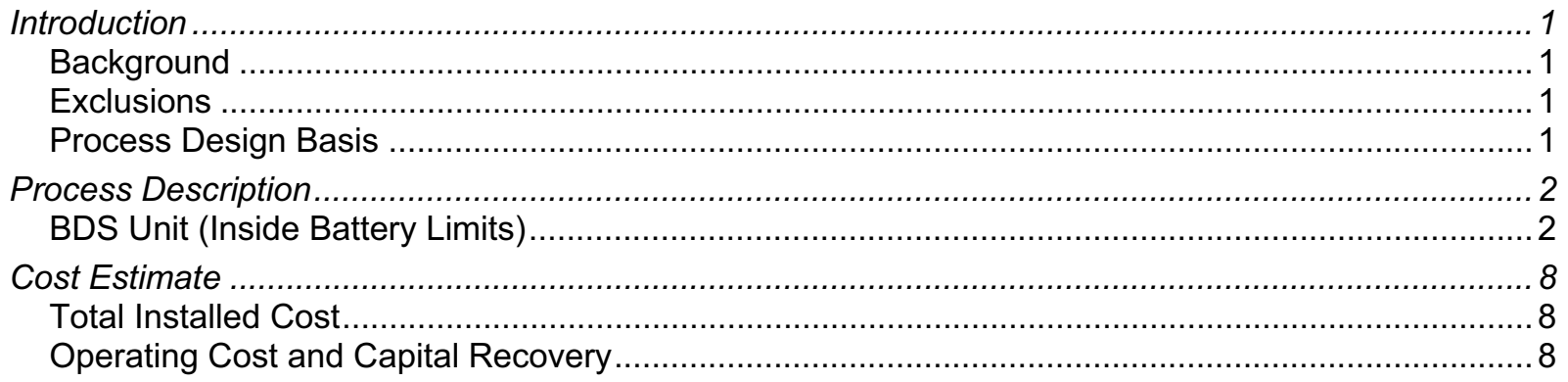

Appendices
A. Diesel Feed Properties and Sulfur Speciation
B. BDS Unit Process Flow Diagrams and Material Balance
C. BDS Unit Equipment and Budgetary Equipment Data Sheets
D. Cost Estimate Basis and Cost Estimate
E. BDS Baseline Case Operating Costs for 6,000 BPD Diesel Feed including Utilities 


\section{BDS/HDS COMBINATION CASES REPORT AND COST ESTIMATES}

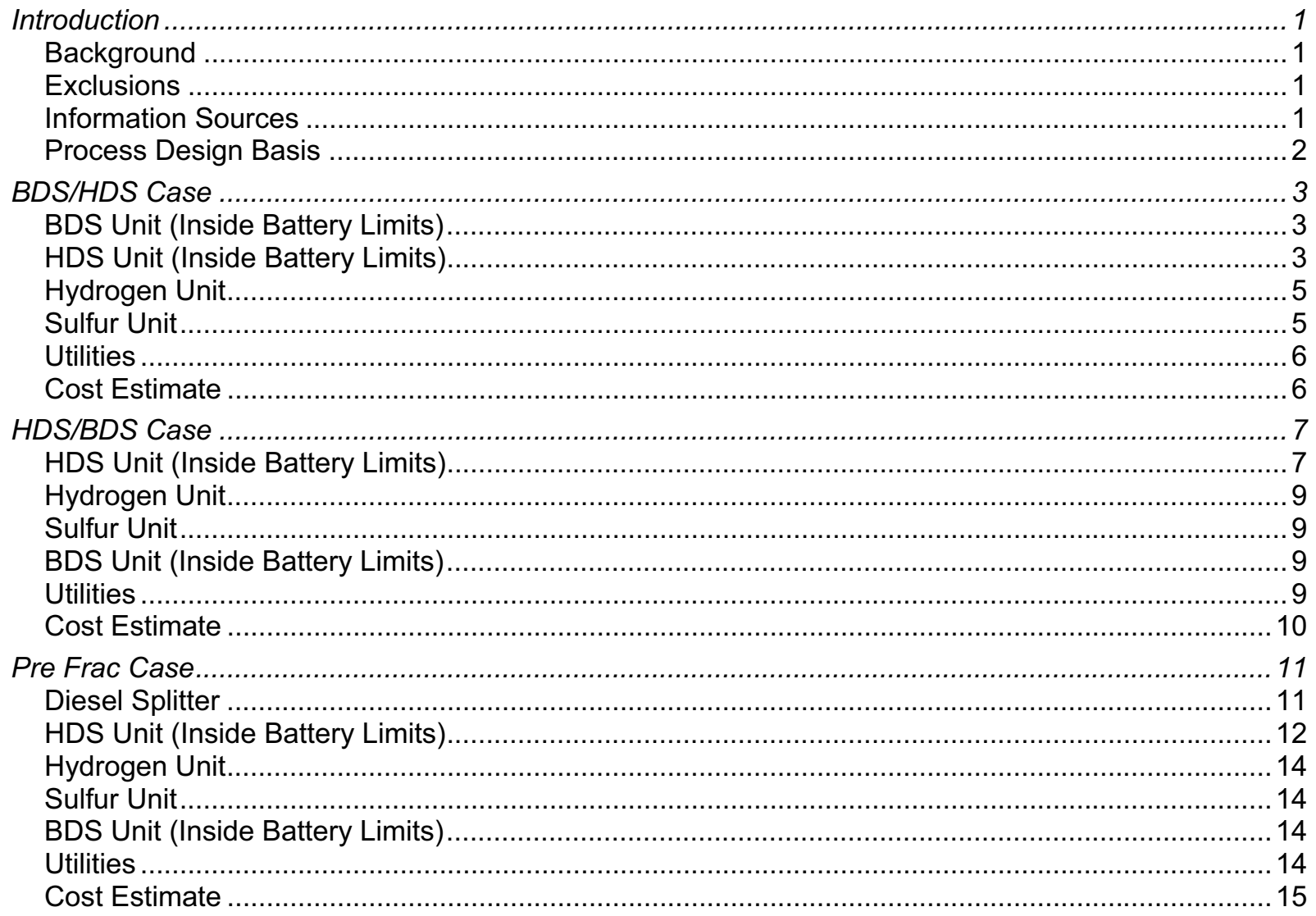

Appendices

A. Raw Diesel Feed Properties and Sulfur Speciation

B. BDS/HDS Case

C. HDS/BDS Case

D. Pre Frac Case 


\section{SUMMARY}

\section{Background}

To meet the EPA's 2006 sulfur content requirements, Petro Star is leading a Department of Energy study to determine the viability of ULSD (i.e. $<10 \mathrm{ppmw}$ sulfur) production from a biodesulfurization (BDS) process. This study is a continuation of a 1999 study that investigated the feasibility of ULSD production using a BDS Unit.

\section{Study Basis}

The design throughput for the study is $6,000 \mathrm{bpd}$ of straight run diesel containing 0.5 weight percent sulfur from PetroStar's Valdez Refinery. The specification for the ULSD product sulfur content is 10 ppmw.

To simplify the economic study and avoid biasing the study results, the study excluded all outside battery limits (OBL) facilities. The approach is consistent with the 1999 BDS study which excluded all utility systems improvements, offsite tankage, and waste stream disposal.

\section{Study Approach}

The HDS/BDS Study looked at five cases to determine if a BDS process is a viable means to produce USLD when compared to traditional hydro-processing methods:

- A standalone HDS process, used as a benchmark to assess the viability of a BDS process

- A standalone BDS process

- A BDS process followed in series by an HDS process (BDS/HDS Case)

- A HDS process followed in series by a BDS process (HDS/BDS Case)

- A Diesel Splitter followed by an HDS Unit operated in parallel with a BDS Unit (Pre Frac Case).

Axens North America, Inc. provided the HDS Unit reactor design and unit yields for this study under a proprietary agreement. Pelorus provided the BDS Unit design as well as the operating cost estimates and equipment costs for the specialized equipment.

\section{Study Details}

This Task 4 Final Report includes this Summary as well as details for each of the studied cases that were developed as three standalone reports:

- HDS Baseline Case Report and Cost Estimate

- BDS Baseline Report and Cost Estimate

- BDS/HDS Combination Cases Report and Cost Estimates.

The HDS and BDS Baseline Reports cover their respective process operated as a standalone unit. The Combination Cases Report covers three possible sequencing options when a BDS process is operated in conjunction with an HDS process. All three reports are included as sections of this Task 4 Final Report. 


\section{Study Results}

The results of the study are contained in the summary table below. From this table, the following conclusions can be made regarding the viability of a BDS process to produce ULSD at PetroStar's Valdez Refinery:

- An HDS Unit has a lower installed cost than a comparable BDS Unit

- An HDS Unit has substantially lower operating costs than a comparable BDS Unit

- The combination of a BDS Unit and an HDS Unit is not economically viable when compared to either of the standalone units.

\begin{tabular}{|c|c|c|c|c|c|}
\hline & HDS Baseline & BDS Baseline & BDS/HDS & HDS/BDS & Pre Frac \\
\hline Diesel Yield -- vol \% & 96.6 & 98.6 & 95.9 & 96.9 & 96.5 \\
\hline HDS Unit & 96.6 & "-- & 96.3 & 97.2 & 95.6 \\
\hline BDS Unit & -- & 98.6 & 99.6 & 99.7 & 98.3 \\
\hline Capital Cost -- \$MM (Note 1) & 27.8 & 33.9 & 44.5 & 43.3 & 46.5 \\
\hline HDS Unit & 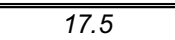 & "-- & 14.3 & 14.8 & 11.6 \\
\hline H2 Unit & 6.7 & -- & 5.8 & 5.5 & 4.1 \\
\hline Sulfur Unit & 3.6 & -- & 2.6 & 3.4 & 2.3 \\
\hline BDS Unit & -- & 33.9 & 21.8 & 19.6 & 22.0 \\
\hline Diesel Splitter & -- & -- & -- & -- & 6.5 \\
\hline Operating Cost -- \$MM/year (Note 2) & 5.8 & 9.2 & 9.4 & 8.0 & 10.2 \\
\hline HDS, $H 2$, and Sulfur Units & 5.8 & "-- & 5.4 & 5.2 & 4.2 \\
\hline BDS Unit & -- & 9.2 & 4.0 & 2.8 & 4.7 \\
\hline Diesel Splitter & -- & -- & -- & -- & 1.3 \\
\hline Total Cost - cpg of ULSD (Note 3) & 13.4 & 18.6 & 21.4 & 19.4 & 23.1 \\
\hline HDS, H2, and Sulfur Units - Capital & 6.6 & --- & 5.4 & 5.6 & 6.7 \\
\hline BDS Unit - Capital & -- & 7.9 & 5.0 & 4.5 & 14.4 \\
\hline Diesel Splitter - Capital & -- & -- & -- & -- & 1.5 \\
\hline "HDS, $\mathrm{H} 2$, and Sulfur Units - Operating & 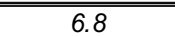 & $\overline{-1-}$ & 6.5 & 6.2 & 7.8 \\
\hline BDS Unit - Operating & - & 10.7 & 4.6 & 3.2 & 15.2 \\
\hline Diesel Splitter - Operating & -- & -- & -- & -- & 1.5 \\
\hline
\end{tabular}

Notes

1. The capital cost is the expected IBL installed cost based on 2005 US Gulf Coast (USGC) pricing. OBL costs, owner costs, licensing/royalty fees, and BDS R\&D costs are excluded from the estimate. The estimate accuracy for each case is $+50 \%$ to $-15 \%$.

2. Includes variable and fixed costs, excludes raw material costs.

3. The total cost of ULSD production includes both capital recovery and recurring operating costs. The capital recovery component was calculated using the expected TIC and a 5 year recovery period. Interest expense was not included in this analysis. 


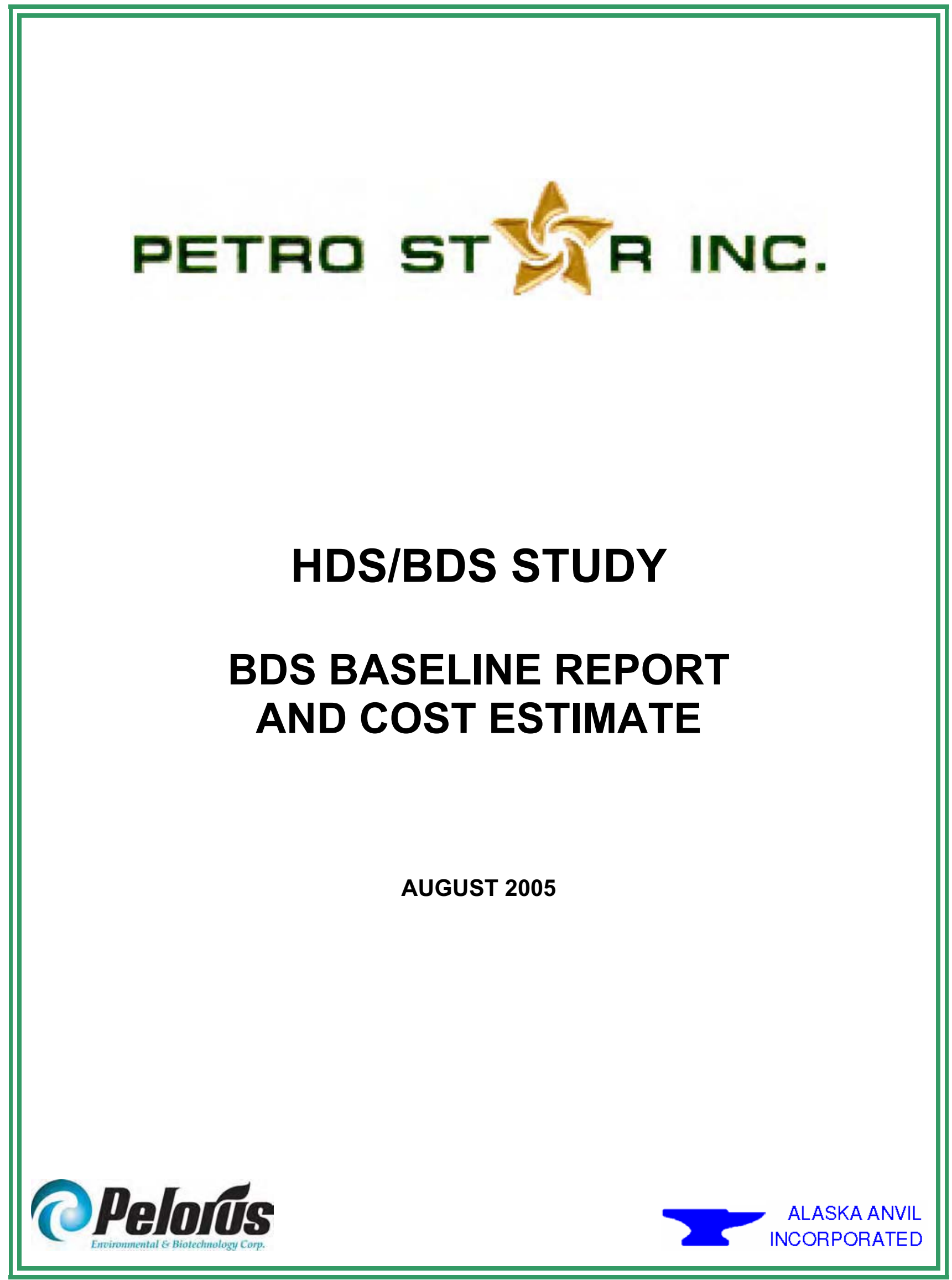




\section{EXECUTIVE SUMMARY}

To meet the EPA's 2006 sulfur content requirements, Petro Star is leading a Department of Energy study to determine the viability of ULSD production from a biodesulfurization process. This BDS Baseline Report, a part of the larger investigation, presents the BDS process and the total installed costs that will serve as the baseline economics that the BDS process must surpass.

The effort is based on an installation at Valdez, Alaska that produces 6,000 bpd of ultra low sulfur diesel meeting a 10-ppmw sulfur requirement.

The design includes the following proposed IBL facilities:

- A new BDS Unit

- A new Fermentor to produce biocatalyst on site

- New facilities to treat biocatalyst waste

The cost estimate for the BDS Unit includes only the inside battery limits (IBL) portion of the BDS Unit. Based on equipment-based costs for the BDS Unit, the total expected installed cost for the BDS complex is $\$ 33.9$ million in 2005 dollars based on USGC equipment pricing. The expected accuracy of the estimate is $+50 /-15 \%$.

The annual BDS Unit operating cost is $\$ 9,232,900$ which translates to 10.7 cents per gallon of ULSD. The total incremental selling price for ULSD would be 18.6 cents per gallon to recover the capital in 5 years (excluding interest expense) and pay the yearly operating cost. 


\section{TABLE OF CONTENTS}

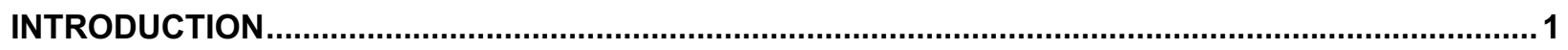

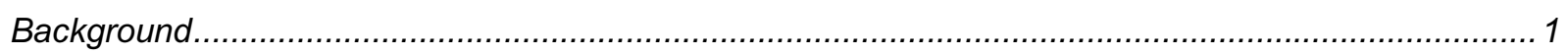

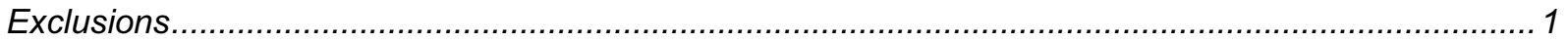

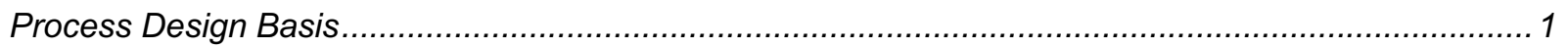

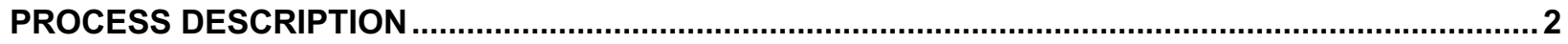

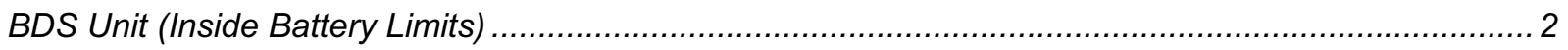

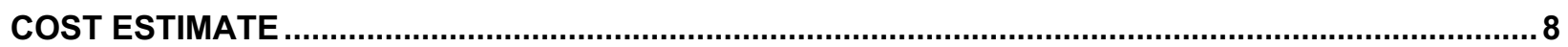

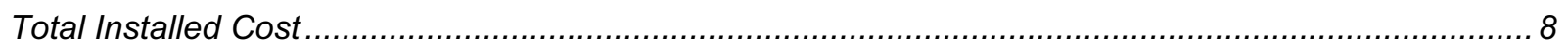

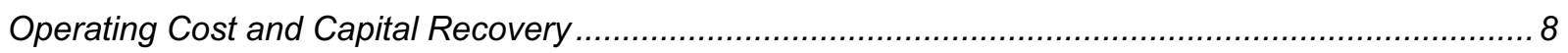

\section{APPENDICES}

A. Diesel Feed Properties and Sulfur Speciation

B. BDS Unit Process Flow Diagrams and Material Balance

C. BDS Unit Equipment and Budgetary Equipment Data Sheets

D. Cost Estimate Basis and Cost Estimate

E. BDS Baseline Case Operating Costs for 6,000 BPD Diesel Feed including Utilities 


\section{INTRODUCTION}

\section{Background}

All U.S. refineries will be required to meet the EPA's third quarter 2006 sulfur content requirements for highway diesel. The EPA requirement for maximum sulfur content of ultra low sulfur diesel (ULSD) is $15 \mathrm{ppmw}$. The refinery product should have about $10 \mathrm{ppmw}$ sulfur or less to ensure that the product specification can be met on a reliable basis after product shipping and distribution logistics.

To compare different strategies to accomplish this requirement, Petro Star is leading a Department of Energy HDS/BDS study that evaluates the following processing routes:

- A standalone hydrodesulfurization (HDS) process

- A standalone biodesulfurization (BDS) process

- A combination of the two.

To be economically viable, a BDS process must be competitive with other commercially proven desulfurization routes. The route most chosen and best known to refiners for diesel is HDS. A new BDS facility must be less costly than a comparable new HDS facility. Or, in the various combination scenarios, using the BDS facility as a pre- or post-treatment facility combined with an existing HDS unit must be less costly than modifications to an existing HDS Unit that would be required to achieve the lower sulfur requirement.

As part of the study of potential BDS economics, this baseline report provides the process description and total installed cost of a new standalone BDS Unit producing 6,000 bpd of highway ULSD at 10 ppmw sulfur.

\section{Exclusions}

To simplify the economic baseline study and avoid biasing the study results, we have excluded all outside battery limits (OBL) facilities. A prior 1999 Kellogg report exploring a BDS baseline also excluded all utility systems improvements, offsite tankage, and waste stream disposal.

\section{Process Design Basis}

\section{Unit Throughput}

The design throughput for the BDS Unit is $6,000 \mathrm{bpd}$ of straight run diesel containing 0.5 weight percent sulfur.

\section{Unit Feed Characteristics}

The design basis composition and characteristics of the diesel feed, including a sulfur speciation, are included in Appendix A.

\section{Product Specification}

The specification for diesel product sulfur content is $10 \mathrm{ppmw}$. 


\section{Utility and Infrastructure Availability}

The study assumes the refinery has the following utility systems and infrastructure available to support the BDS Unit:

- $\quad$ Refinery fuel gas

- Water (available as feed to a new demineralization unit)

- Instrument air

- Electricity for drivers

- Nitrogen

- Process vent system

- Product tanks

- Cooling fluid system

- Steam/boiler feed water

- Naphtha fuel

- Flare.

\section{Refinery Ambient Conditions}

The new BDS facility design is based on a Valdez, Alaska location.

\begin{tabular}{|l|c|}
\hline Ambient Temperature Condition & Degrees F \\
\hline Highest monthly average & 62 \\
\hline Lowest monthly average & 17 \\
\hline Record high & 86 \\
\hline Record low & -23 \\
\hline
\end{tabular}

\section{PROCESS DESCRIPTION}

The design includes the following proposed IBL facilities:

- A new BDS Unit

- A new Fermentor to produce biocatalyst on site

- New facilities to treat biocatalyst waste.

\section{BDS Unit (Inside Battery Limits)}

\section{Overview}

The BDS process employs a selected strain of microorganisms (biocatalyst) which, through the action of produced enzymes, convert a portion of the sulfur contained in a liquid petroleum fuel into an oil-soluble compound, hydroxy biphenyl (HBP), which partitions to the diesel fuel, resulting in higher diesel yields. For the Petro Star BDS application, the petroleum fuel is diesel with a total sulfur content of 5,000 ppmw. Through processing in the BDS Unit, the sulfur content of the fuel is reduced to $10 \mathrm{ppmw}$.

The BDS Unit is operated as a continuous flow system at low pressures and moderate temperatures in an aerobic environment. Water is used both as a medium in which to sustain the microorganisms as well as the carrier for the microorganisms within the unit. 
The BDS Reactors are operated at a 50:50 oil-to-water volumetric ratio, a biocatalyst cell density of $20 \mathrm{~g} / \mathrm{L}$ (combined phase basis) and a biocatalyst residence time of 200 hours. The majority of the water and biocatalyst supplied to the reaction section is provided by internal recycle. A portion of the biocatalyst inventory is, however, continuously purged from the system, with an equivalent amount of fresh biocatalyst added, to maintain biocatalyst activity. The biocatalyst makeup is continuously grown in an on-site Fermentor. See Appendix B for process flow diagrams and the material balance. See Appendix $\mathrm{C}$ for the equipment list and budgetary equipment data sheets.

The main processing sections of the BDS Unit include a feed section, reaction section, and a separation section. The feeds to the reactors include the raw diesel feed, biocatalyst-containing water recycle and biocatalyst makeup streams, oxygen (supplied air), and the various substrates and nutrients necessary to sustain the organisms. The reaction section consists of three identical, series-flow, airlift reactors in which the biodesulfurization reactions occur. The emulsified water-oil-biocatalyst effluent from the last reactor is forwarded to the separation section. The separation section consists of two stages of gravity separation; the first to recover the desulfurized diesel product and the second to recover a biocatalyst-free aqueous stream that provides a water effluent. The remainder of the reactor effluent, consisting of the bulk of the biocatalyst and water plus some entrained oil, is recycled back to the reactors.

The biocatalyst-containing sections of the process are operated under aseptic conditions. Aseptic operation requires that all inputs to the system are treated to remove or destroy any foreign organisms. Foreign organisms, if introduced into the system, could inhibit the performance of the biodesulfurization organisms, even to the point of complete failure. Sterilization of the inputs to the system is accomplished either through removal of the foreign organisms in cartridge filters or by deactivating (killing) the organisms with heat in continuous heat sterilizers. Where sterilization is by means of filtration, a pre-filter is provided to remove relatively large suspended matter that could plug, and thus shorten the life of, the more expensive bio-filters. Where heat sterilization is employed, the sterilizers would be provided as packaged systems complete with heater, holding loop and cooler; heating being provided by a separate circulating loop of steam heated hot water.

Ancillary facilities are included for feed and chemical handling, treatment of the reactor offgases, drying of the treated diesel product, and disposal of the waste biocatalyst purge stream. A support utility system includes a chilled water system. The chilled water system removes the external heat of reactions in the BDS Reactors and regulates the biodesulfurization reaction operating temperature. The design biodesulfurization temperature is $86^{\circ} \mathrm{F}$. Temperatures much higher than $86^{\circ} \mathrm{F}$ result in microorganism deactivation, while temperatures below the optimum result in a decrease in the desulfurization reaction rates (and a corresponding decrease in the overall level of desulfurization).

\section{Feed}

Feeds to the BDS Unit include the raw diesel feed, compressed air, process water, and various additives and chemicals. 
The diesel charge to the BDS Unit will normally come directly from the Crude Unit. For proper operation of the downstream BDS Reactors, the diesel charge must have a relatively uniform rate, have its temperature regulated to the operating temperature of the downstream BDS Reactors, and be sterilized.

Diesel feed to the unit is received into the Diesel Feed Drum (D-101). D-101 serves as a surge vessel to dampen fluctuations in the diesel feed flows, thus ensuring a more uniform rate to the downstream reactors. The drum provides approximately 30 minutes of residence time at the design diesel feed rate when operating at a maximum level of $80 \%$.

From the Diesel Feed Drum, the diesel feed is transferred to the No. 1 BDS Reactor by the Diesel Charge Pumps (J-101A/B). Prior to entering the reactor, the temperature of the diesel feed is regulated to that of the downstream reactors in the Diesel Feed Cooler (C-101), and the diesel feed is sterilized by the Diesel Prefilters (L-101A/B) and Diesel Bio-Filters (L-102A/B). Chilled water from the chilled water system provides cooling within the Diesel Feed Cooler.

Operation of the Fermentor, BDS Reactors, and Seed Tanks requires oxygen for the desulfurization oxidation reactions and to maintain the aerobic environment necessary to sustain the biocatalyst. Oxygen is supplied to the various vessels in the form of air by the Air Compressors $(\mathrm{J}-109 \mathrm{~A} / \mathrm{B})$. The air supply is cooled to the BDS operating temperature in the Process Air Cooler (C-103), and then routed through the Air Pre-Filters (L-103A/B) before distribution to the various users. Chilled water from the chilled water system provides cooling within $\mathrm{C}-103$. Air feed sterilization is accomplished downstream of the pre-filters in individual bio-filters located at the various points of use.

The majority of the water feed to the BDS Reactors consists of recycled water. However, process water is required to supply the Seed Tanks and Fermentor. Process water makeup may also be required to maintain the water balance in the reaction section should water losses become excessive. For aseptic reasons, the process water is routed through the Makeup Water Pre-Filter (L-113A/B) and the sterilizing Makeup Water Bio-Filters (L-114A/B) before distribution to the end-users.

Substrates and nutrients are required to sustain the microorganisms, and chemicals are required for $\mathrm{pH}$ control. Glucose serves as the substrate source for the Seed Tanks and Fermentor, while ethanol is used in the BDS Reactors. A common nutrient cocktail is fed to both the Fermentor and the BDS Reactors. For pH control, potassium hydroxide is used in the BDS Reactors while ammonia is used in the Fermentor. Ammonia is a preferred alkali in the Fermentor since it also serves as an additional source of nitrogen, a necessary nutrient.

Glucose is stored in Glucose Storage Tank (F-101), and fed to the Fermentor by the Glucose Pumps (J-104A/B). F-101 provides 14 days storage of the $50 \%$ glucose solution at the design usage rate. The glucose feed to the Fermentor is sterilized in the Glucose Sterilizer (L-109) before entering the Fermentor. 
The premixed nutrient cocktail is stored in the Nutrient Storage Tanks (F-102A/B), and fed to the Fermentor and BDS Reactors by the Nutrient Pumps (J-105A/B). Two storage tanks allow for the batch preparation of the nutrient cocktail. Each tank provides 2 days storage of the nutrient solution at the design usage rate. The nutrient feed stream is sterilized prior to entering the Fermentor and BDS Reactors in the Nutrient Sterilizer (L-110).

Ethanol is stored in the Ethanol Storage Tank (F-103) and fed to the BDS Reactors by the Ethanol Pumps (J-106A/B). F-103 provides 7 days storage of the $70 \%$ ethanol solution at the design usage rate. The ethanol feed is sterilized in the Ethanol Bio-Filter (L-111) before entering the reactors.

Potassium hydroxide $(\mathrm{KOH})$ is stored in the KOH Storage Tank (F-104), and fed to the BDS Reactors by the $\mathrm{KOH}$ Pumps $(\mathrm{J}-107 \mathrm{~A} / \mathrm{B} / \mathrm{C} / \mathrm{D})$. F-104 provides 7 days storage of the $4 \mathrm{~N} \mathrm{KOH}$ solution at the design usage rate. The $\mathrm{KOH}$ feed is sterilized in the $\mathrm{KOH}$ Bio-Filter (L-112) before entering the reactors.

Anhydrous ammonia is stored in the Anhydrous Ammonia Storage Drum (D-102). D-102 provides 7 days storage of the $100 \%$ ammonia solution at the design usage rate. From the storage drum, ammonia is fed to the Fermentor as a vapor stream. Vaporization of the liquid ammonia stored in the tank is accomplished by means of external heating coils. The ammonia is injected into the air stream which feeds the Fermentor, and is routed, along with the air, through the Fermentor Air Bio-Filter (L-104) for sterilization.

\section{Reaction}

The reaction section consists of the Fermentor, main BDS Reactors, and Seed Tanks.

Biocatalyst makeup to the BDS Reactors is grown in, and provided from, the Fermentor (D-201). The Fermentor operates in a continuous mode, with the rate of cell growth adjusted to meet the makeup requirements of the BDS system. The Fermentor is initially inoculated with biocatalyst from Seed Tank B.

Water, air, glucose, and nutrients are added to the Fermentor at controlled rates to provide the necessary environment for cell growth, and anhydrous ammonia is injected to control the $\mathrm{pH}$ and to provide additional nitrogen nutrient. The water can be supplied from fresh makeup or recycled from the effluent of the separation section. The remaining additives, chemicals and air are supplied from the feed section. All inputs to the Fermentor are sterilized.

The Fermentor is operated as an airlift reactor vessel. Chilled water is circulated through the vessel jacket to remove the heat generated from the glucose (substrate) oxidation and to control the Fermentor temperature at approximately $86^{\circ} \mathrm{F}$. Biocatalyst makeup to the BDS Reactors, in the form of an aqueous slurry of $20 \mathrm{~g} / \mathrm{L}$, is transferred at a controlled rate by the Fermentor Transfer Pump (J-201A/B).

Diesel desulfurization occurs in three identical BDS Reactors (D-202, D-203, and D-204), each of which provides a hydraulic residence time of approximately 1.0 hour at the design flows. The 
reactors are operated in series flow, with the raw diesel and recycle plus makeup biocatalyst fed to BDS Reactor No. 1. The biocatalyst recycle is predominately a water stream with as much as $25 \mathrm{wt} \%$ entrained oil. Fresh makeup water can also be supplied if necessary to maintain the desired volumetric water-to-oil ratio of 50:50. The fresh biocatalyst makeup is set to maintain an operating cell density of $20 \mathrm{~g} / \mathrm{L}$, total fluid basis, in the reactors. The oil-water-biocatalyst emulsion is transferred from the first reactor to the second reactor by the BDS Reactor No. 1 Transfer Pumps (J-202A/B), and from the second reactor to the third by the BDS Reactor No. 2 Transfer Pumps (J-203A/B). The BDS Reactor No. 3 Transfer Pumps (J-204A/B) transfer the effluent from the third reactor to the downstream separation section.

Ethanol, nutrients, and air are supplied to each of the reactors to sustain the microorganisms and potassium hydroxide is added for $\mathrm{pH}$ control (the desired operating $\mathrm{pH}$ being 7). The air supply to each reactor is sterilized in individual Bio-Filters (L-105, L-106, and L-107, respectively) before entering the reactors. The air rate to each reactor is regulated to limit the carbon dioxide content of the off-gas to a maximum concentration of approximately 7 vol\%. Carbon dioxide is formed from the oxidation of the ethanol substrate fed to the reactors.

Chilled water from the Chilled Water System is circulated through the vessel jackets as well as internal coils to remove the reaction heat and to control the reactor temperatures at the desired operating temperature of $86^{\circ} \mathrm{F}$.

When the plant is first started up or when it is restarted after a shutdown, the required biocatalyst inventory must be re-established. The required microorganisms are grown in the two seed tanks, Seed Tank A (D-205) and Seed Tank B (D-206). D-205 is a packaged 40 L seed system and D206 a packaged $2000 \mathrm{~L}$ system. The seed tanks are operated in a batch mode, with the tanks and associated piping sterilized between batch runs.

\section{Separation}

The separation section serves two functions; to recover the desulfurized diesel product and to separate a biocatalyst and oil-free water stream. The separation section consists of the 1st Stage Separator, the Coalescer Separators, and the Electrostatic Dehydrator.

The pumped effluent from BDS Reactor No. 3 is discharged into the 1st Stage Separator. A surfactant is injected into the reactor effluent stream upstream of the Separator to enhance phase separation. Phase separation within the 1 st Stage Separator occurs exclusively by gravity. The Separator is constructed with an internal overflow weir and effluent section for recovery of the separated light liquid (diesel) phase. The Separator is sized to provide 30 minutes of residence time at an operating level of $80 \%$.

The recovered diesel product from the Separator will contain a small residual amount of water and biocatalyst that must be removed prior to transfer to storage. The 1st Stage Separator Overflow Pumps (J-301 A/B) transfer the diesel from the effluent compartment of the 1st Stage Separator to the downstream Electrostatic Dehydrator. 
The underflow from the Separator, consisting of the majority of the water and biocatalyst plus approximately $25 \mathrm{wt} . \%$ retained oil, is split into two streams downstream of the 1st Stage Separator Underflow Pumps (J-302 A/B). Approximately 25\% of the stream is directly recycled back to the reaction section, while the remaining $75 \%$ is forwarded to the Coalescer Separators. The amount of direct recycle is limited by a maximum sulfate concentration $5 \%$ in the effluent from BDS Reactor No. 3. Sulfate concentrations in excess of $5 \%$ can inhibit the activity of the biocatalyst.

A small quantity of air is continuously injected into the 1st Stage Separator. The air serves as a purge to control buildup of any gases that may be released from the BDS Reactor effluent stream. The vent stream from the separator is sent to the plant off-gas handling system.

The majority of the water and biocatalyst retained with the separated diesel product is removed in the Electrostatic Dehydrator (L-302). Recovery is accomplished by imposing an electrical voltage to the fluid that serves to coalesce the entrained water droplets. A wash water stream is injected into the dehydrator feed stream to enhance water removal. The effluent from the precipitator is forwarded to the Salt Dryers for removal of the last traces of moisture. The recovered biocatalyst-containing water stream from the dehydrator is recycled to BDS Reactor No. 1.

The Coalescer Separators (L-301 A/B) receive a portion (approximately 75\%) of the underflow from the upstream 1st Stage Separator. The purpose of these separators is to recover an essentially oil and biocatalyst-free water stream. Each of the separators is equipped with an internal coalescer element to promote phase separation. Two separators, one in-service and a spare, allow for cleaning the coalescer elements during a run.

The light phase from the Coalescer Separators consists of a water-oil-biocatalyst mixture of approximately $65 \mathrm{wt} \%$ water and $35 \mathrm{wt} \%$ oil plus biocatalyst. This light phase is recycled back to the reaction section by means of the Coalescer Separator Overflow Pumps (J-303 A/B). A strip stream, representing the biocatalyst purge, is taken from the light phase recycle and forwarded to the Oxidizer. The absolute biocatalyst purge quantity is equivalent to the fresh makeup supplied to maintain catalyst activity.

The last traces of residual water are removed from the diesel product in the Salt Dryers (D-401 A/B) before transfer to storage. A final removal step is required since even trace amounts of water will cause the diesel product to have a hazy appearance. Flows through the paralleloperated dryers are upwards through the salt beds. While salt replacement in the dryers would normally occur during a scheduled plant shutdown, two dryers are provided to allow for salt replacement during a run if required.

\section{Biocatalyst Waste Oxidation}

Waste biocatalyst purge stream disposal is through thermal oxidation. The biocatalyst purge stream from the separation section is forwarded to the Thermal Oxidizer (L-801), which is a horizontally-fired, liquid injection-type incinerator. 


\section{Refrigeration System}

Cooling is required to regulate the temperatures of the feed streams to the BDS Unit and to remove the heat generated in the Fermentor and BDS Reactors. A closed loop circulating chilled water (water/glycol mixture) system is used for this purpose, with water chilling provided by the Chilled Water Refrigeration Package (L-601).

The chilled water is pumped from the Chilled Water Expansion Drum (F-601) to the Chilled Water Cooler (C-601) by the Chilled Water Circulation Pumps (J-601 A/B). In C-601, the water-glycol solution temperature is reduced to the desired operating temperature by exchange with refrigerant. The supply temperature of the solution will be low enough to achieve the necessary thermal driving force so that process heat transfer areas are reasonable. From the chiller, the water is distributed to the various users and then returned to the expansion drum.

The largest heat loads on the system are the exothermic heat removal requirements of the Fermentor and BDS Reactors. Additional heat loads are associated with cooling the diesel feed, the process air from the air compressors, and the reactor system vent gases, with smaller loads associated with the package sterilization systems. With the exception of the Vent Gas Chiller, the cooling medium for all applications is circulating chilled water. Refrigerant will be directly used as the cooling medium for the Vent Gas Chiller due to the lower required operating temperature.

\section{COST ESTIMATE}

\section{Total Installed Cost}

The conceptual cost estimate is based on installing an BDS Unit processing 6,000 bpd raw diesel feed and producing ULSD meeting the 10-ppmw sulfur requirement. The cost estimate is for the IBL portion of the BDS complex only.

Based on equipment-based costs for the BDS Unit, the total installed cost estimate for the IBL portion of the BDS complex is $\$ 33.9$ million in 2005 dollars based on USGC pricing. The accuracy of the estimate is $+50 /-15 \%$.

The cost estimate excludes other owner costs such as startup and commissioning costs, initial operator training, startup procedures, and pre-startup operator staffing. A contingency of $25 \%$ is in the total installed cost.

See Appendix D for the Cost Estimate Basis and Cost Estimate.

\section{Operating Cost and Capital Recovery}

The total annual operating cost of the BDS Unit is $\$ 9,232,900$. This cost is based on a $95 \%$ onstream availability for the plant. The operating cost can also be expressed in terms of cents per gallon of ULSD, based on a $98.6 \%$ yield on the ULSD. The total operating cost of the BDS Unit is 10.7 cents per gallon of ULSD. The basis for developing the operating cost including utilities can be seen in Appendix E. 
Based on the expected TIC of the BDS Unit, the incremental selling price for ULSD would be 7.9 cents per gallon to recover the capital in 5 years (excluding interest expense), and the incremental selling price to recover operating costs would be 10.7 cents per gallon. The total incremental selling price for ULSD would be 18.6 cents per gallon to recover the capital in 5 years and pay the yearly operating cost.

The operating cost includes lost diesel sales due to loss of diesel with the biocatalyst purge stream. 


\section{APPENDICES}

A. Diesel Feed Properties and Sulfur Speciation

B. BDS Unit Process Flow Diagrams and Material Balance

Process Flow Diagrams

Material Balance

C. BDS Unit Equipment and Budgetary Equipment Data Sheets

Equipment List

Budgetary Equipment Data Sheets

D. Cost Estimate Basis and Cost Estimate

E. BDS Baseline Case Operating Costs for 6,000 BPD Diesel Feed including Utilities 


\section{Appendix A - Diesel Feed Properties and Sulfur Speciation}

\begin{tabular}{|l|l|c|c|}
\hline \multicolumn{4}{|c|}{ Diesel Feed Properties } \\
\hline Test & Units & Results & Specification \\
\hline Gravity @ 60 ${ }^{\circ}$ F, Min/Max & API & 33.3 & $32-36$ \\
\hline Flash Pt, Min. & Celsius & $63.5\left(146^{\circ} \mathrm{F}\right)$ & $60\left(140^{\circ} \mathrm{F}\right)$ \\
\hline Cloud Pt, Max. & Celsius & $-12.3\left(10^{\circ} \mathrm{F}\right)$ & $-9.5\left(15^{\circ} \mathrm{F}\right)$ \\
\hline Pour Pt, Max. & Celsius & $-15\left(5^{\circ} \mathrm{F}\right)$ & $-12\left(10^{\circ} \mathrm{F}\right)$ \\
\hline Distillation & & & \\
\hline IBP & Celsius & $171\left(340^{\circ} \mathrm{F}\right)$ & Report \\
\hline $10 \%$ Recovery & Celsius & $249\left(480^{\circ} \mathrm{F}\right)$ & Report \\
\hline 20\% Recovery & Celsius & $265\left(509^{\circ} \mathrm{F}\right)$ & Report \\
\hline 50\% Recovery & Celsius & $287\left(549^{\circ} \mathrm{F}\right)$ & Report \\
\hline 90\% Recovery & Celsius & $317\left(603^{\circ} \mathrm{F}\right)$ & $282-338$ \\
\hline Final Boiling Pt. & Celsius & $336\left(637^{\circ} \mathrm{F}\right)$ & Report \\
\hline Recovery & Vol \% & 99.9 & Report \\
\hline Residual & Vol \% & 0.1 & Report \\
\hline Viscosity @ 40 C, Min/Max & Vol \% & 0 & Report \\
\hline Ash, Max & CSt & 3.57 & $2.0-4.3$ \\
\hline Carbon Residue on 10\% Bottoms, Max & Wt $\%$ & $<0.001$ & $0.01 \%$ \\
\hline Btu Gross, Min & Btu/Gallon & 139,190 & $0.35 \%$ \\
\hline Calculated Cetane, Min & Index & 48 & 136,000 \\
\hline Copper Strip Corrosion, Max & Code & $1 \mathrm{a}$ & 45 \\
\hline Total Sulfur & Wt. \% & 0.500 & 3 \\
\hline
\end{tabular}




\begin{tabular}{|l|c|l|c|}
\hline \multicolumn{5}{|c|}{$\begin{array}{c}\text { Sulfur Speciation } \\
\text { (Ratioed to 5,000 ppm*) }\end{array}$} \\
\hline Component & ppm wt sulfur & Component & ppm wt sulfur \\
\hline Hydrogen sulfide & $<1$ & 2-Ethyl thiophene & $<1$ \\
\hline Carbonyl sulfide & $<1$ & 2,5-Dimethyl thiophene & $<1$ \\
\hline Methyl mercaptan & $<1$ & 3-Ethyl thiophene & $<1$ \\
\hline Ethyl mercaptan & $<1$ & 2,4\&2,-Dimethyl thiophene & $<1$ \\
\hline Dimethyl sulfide & $<1$ & 3,4-Dimethyl thiophene & $<1$ \\
\hline Carbon disulfide & $<1$ & Methyl Ethyl thiophenes & $<1$ \\
\hline Isopropyl mercaptan & $<1$ & Trimethyl thiophenes & $<1$ \\
\hline Ethyl sulfide & $<1$ & Tetramethyl thiophenes & $<1$ \\
\hline tert-Butyl mercaptan & $<1$ & Benzothiophene & $<1$ \\
\hline N-Propyl mercaptan & $<1$ & Methyl benzothiophene & 2 \\
\hline Ethyl Methyl Sulfide & $<1$ & Dimethyl benzothiophene & 29 \\
\hline Thiophene & $<1$ & Trimethyl benzothiophene & 119 \\
\hline sec-Butyl Mercaptan & $<1$ & Tetramethyl Benzothiophene & 354 \\
\hline Isobutyl mercaptan & $<1$ & Dibenzothiophene & 229 \\
\hline Ethyl sulfide & $<1$ & 4-Methyl benzothiophene & 213 \\
\hline MN-butyl mercaptan & $<1$ & 3-Methyl DBZT+2-methyl DBZT & 147 \\
\hline Dimethyl disulfide & $<1$ & 1-Methyl dibenzothiophene & 94 \\
\hline 2-Methyl thiophene & $<1$ & 4,6 Dimethyl dibenzothiophene & 97 \\
\hline 3-Methyl thiophene & $<1$ & Dimethyl dibenzothiophene & 354 \\
\hline Tetra-hydro thiophene & $<1$ & Trimethyl dibenzothiophene & 75 \\
\hline Ethyl methyl disulfide & $<1$ & Tetramethyl dibenzothiophene & 4 \\
\hline 2-Methyl-tetra-hydro-thiophene & $<1$ & Unidentified volatile sulfur & 3,283 \\
\hline
\end{tabular}

* Raw data from the speciation analysis ratioed to $5,000 \mathrm{ppm}$ to reflect the design basis sulfur content. 
Final Technical Progress Report DOE Award No. DE-FC26-02NT15340

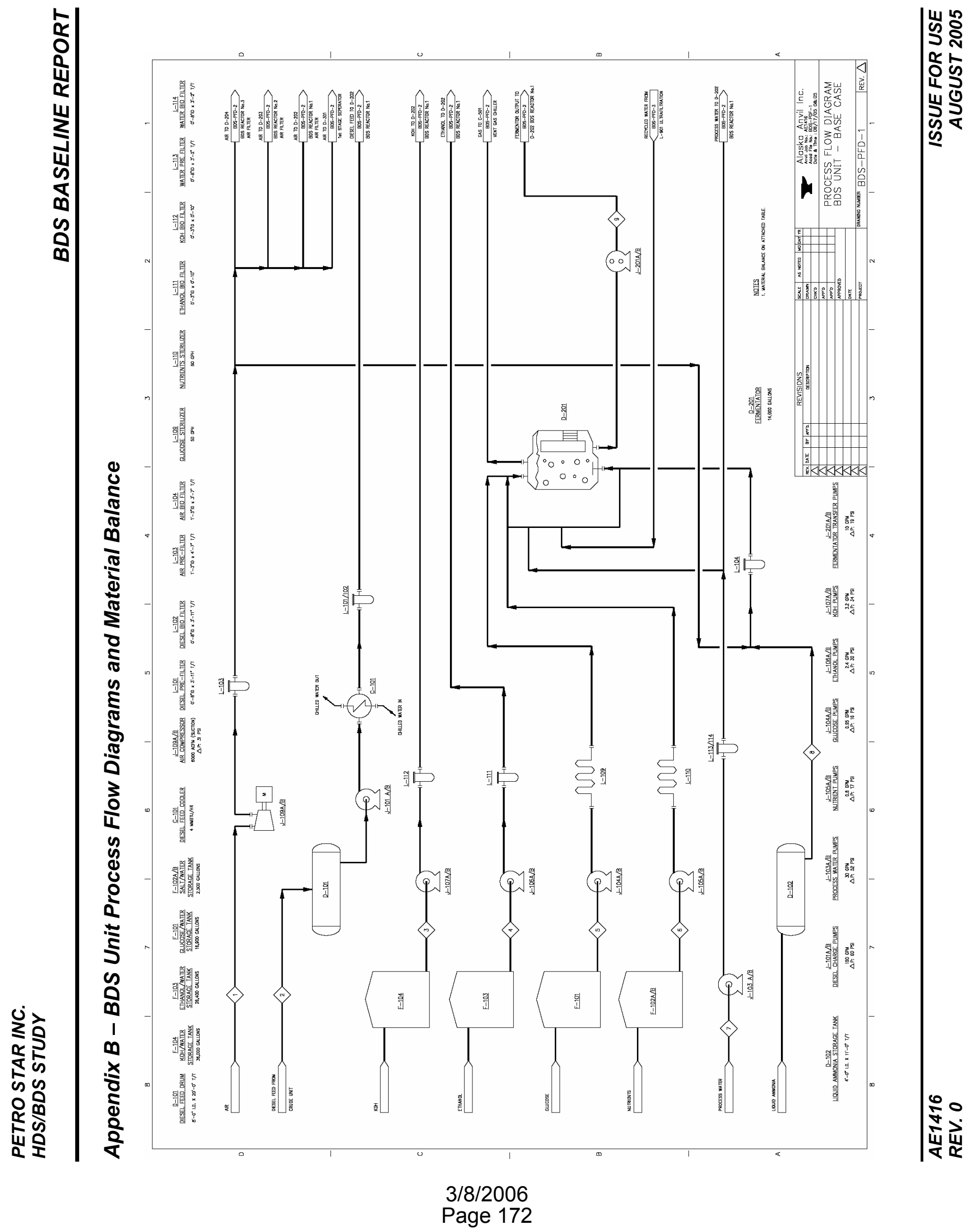


Final Technical Progress Report DOE Award No. DE-FC26-02NT15340

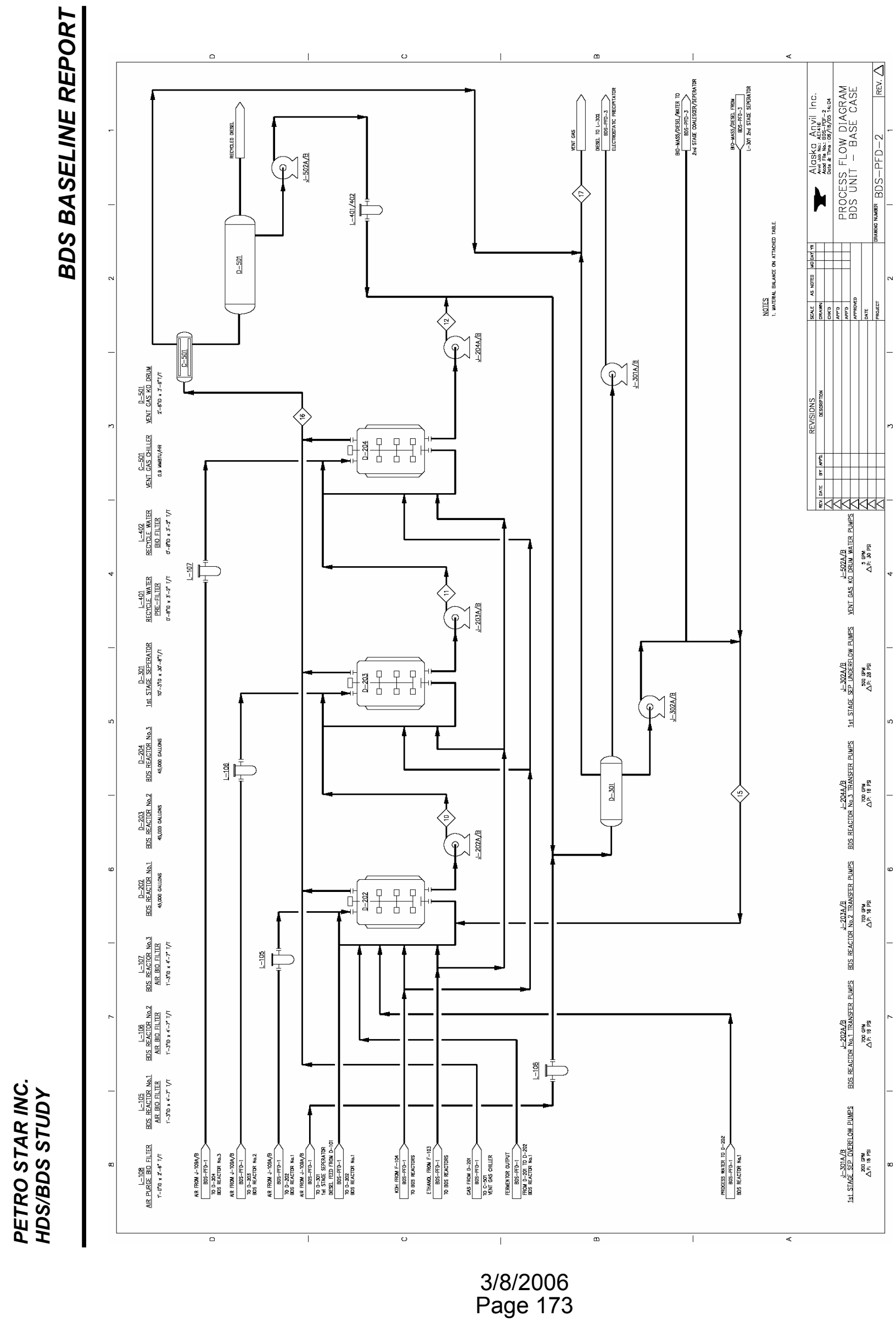

崩另

ว

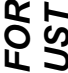

너

乌

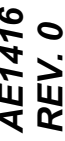

Page 173 
Final Technical Progress Report DOE Award No. DE-FC26-02NT15340

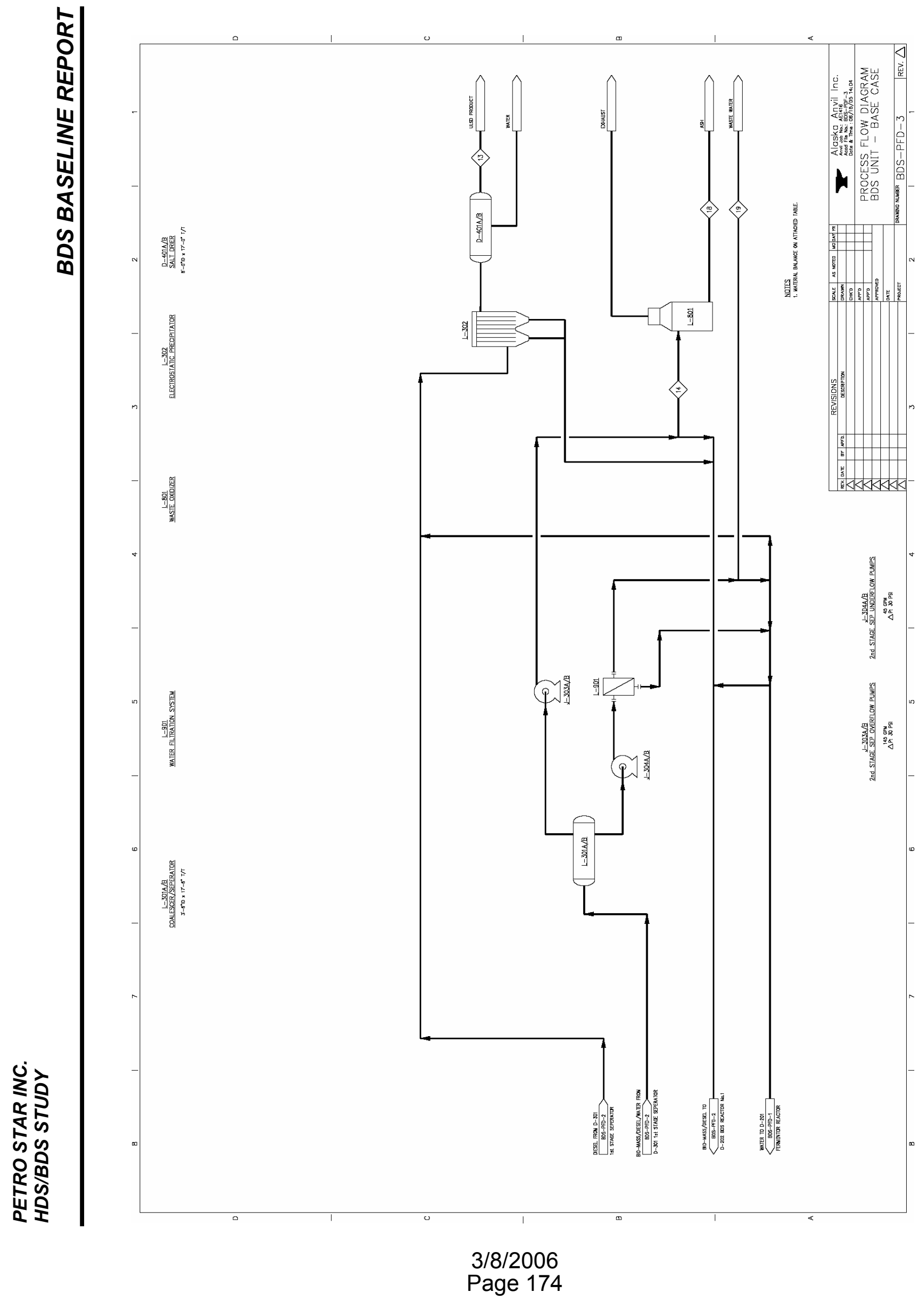


Material Balance

\section{BDS Unit Material Balance -- BDS Baseline Case}

\begin{tabular}{|c|c|c|c|c|c|c|c|c|}
\hline PFD Stream Number & & T & 2 & 3 & 4 & 5 & 6 & 7 \\
\hline Simulation Stream Number & & Air & Diesel & $\mathrm{NaOH}$ & Ethanol & Glucose & Nutrients & Process Water \\
\hline Stream Description & & Air Feed & Diesel Feed & $\mathrm{NaOH}$ Feed & Ethanol Feed & Glucose Feed & Nutrient Feed & $\begin{array}{c}\text { Process Water } \\
\text { Feed }\end{array}$ \\
\hline Phase & & VAPOR & LIQUID & LIQUID & LIQUID & LIOUID & LIQUID & LIQUID \\
\hline Hydrocarbon Mass Flow & $\mathrm{LB} / \mathrm{HR}$ & & 74483 & & & & & \\
\hline Aqueous Mass Flow & $\mathrm{LB} / \mathrm{HA}$ & & & 1900 & 926 & 456 & 370 & 13270 \\
\hline Biomass Mass Flow & $\mathrm{LB} / \mathrm{HR}$ & & & & & & & \\
\hline Sulfur in $\mathrm{HC}$ & PPM & & 5000 & & & & & \\
\hline Total Mass Flow Rate & $\mathrm{LB} / \mathrm{HR}$ & 26663 & 74483 & 1900 & 926 & 456 & 370 & 13270 \\
\hline Temperature & $\mathrm{F}$ & 68 & 176 & 68 & 68 & 68 & 68 & 68 \\
\hline Pressure & $\mathrm{PS|G}$ & 0 & 0 & 0 & 0 & 0 & 0 & 0 \\
\hline Standard Liq Flow & $\mathrm{BPD}$ & & 6000 & 85 & 69 & 26 & 25 & 912 \\
\hline Vapor Flow & MSCFH & 359 & & & & & & \\
\hline Wt \% Vapor & & 100 & 0 & 0 & 0 & 0 & 0 & 0 \\
\hline MW & & & & & & & & \\
\hline
\end{tabular}

\begin{tabular}{|l|l|c|c|c|c|c|c|c|}
\hline PFD Stream Number & & 8 & 9 & 10 & 11 & 12 & 13 & 14 \\
\hline Simulation Stream Number & & Liquid Ammonia & S-16 & S-17 & S- 18 & S-19 & S-27 & S-31 \\
\hline Stream Description & & $\begin{array}{c}\text { Liquid } \\
\text { Ammonia Feed }\end{array}$ & $\begin{array}{c}\text { Fermentor } \\
\text { Output }\end{array}$ & R\#1 Output & R\#2 Output & R\#3 Output & Diesel Product & Biomass Purge \\
\hline Phase & & LIOUID & LIOUID & LIOUID & LIOUID & LIOUID & LIOUID & LIOUID \\
\hline Hydrocarbon Mass Flow & LB/HR & & & 124764 & 124517 & 124322 & 73413 & 907 \\
\hline Aqueous Mass Flow & LB/HR & 33 & 3367 & 146244 & 147805 & 148092 & 0 & 1660 \\
\hline Biomass Mass Flow & LB/HR & & 456 & 32181 & 32181 & 32181 & 0 & 456 \\
\hline Sulfur in HC & PPM & & & 1269 & 177 & 10 & 10 & 10 \\
\hline Mass Flow Rate & LB/HR & 33 & 3823 & 303189 & 304530 & 304594 & 73413 & 3024 \\
\hline Temperature & F & -40 & 86 & 86 & 86 & 86 & 86 & 86 \\
\hline Pressure & PSIG & 210 & 33.1 & 36.4 & 36.4 & 26.8 & 5 & 5 \\
\hline Standard Liq Flow & BPD & 3.2 & 262 & 21751 & 21813 & 21813 & 5914 & 212 \\
\hline Vapor Flow & MSCFH & & & & & & & \\
\hline Wi \% Vapor & & 0 & 0 & 0 & 0 & 0 & & 0 \\
\hline MW & & & & & & & & 0 \\
\hline
\end{tabular}

\begin{tabular}{|c|c|c|c|c|c|c|c|c|}
\hline PFD Stream Number & & 15 & 16 & 17 & 18 & 19 & & \\
\hline Stream Number & & $5-32$ & $5-38$ & $5-39$ & Ash & Waste Water & & \\
\hline Stream Description & & $\begin{array}{l}\text { Reactor } \\
\text { Recycle }\end{array}$ & $\begin{array}{c}\text { Reactor Air } \\
\text { Effluent }\end{array}$ & Gas Purge & $\begin{array}{c}\text { Incinerator } \\
\text { Waste }\end{array}$ & Waste Water & & \\
\hline Phase & & LIGUID & GAS & GAS & SOLID & LIQUID & & \\
\hline Hydrocarbon Mass Flow & LB/HR & 50562 & 561 & & & & & \\
\hline Aqueous Mass Flow & $\mathrm{LB} / \mathrm{HR}$ & 128530 & 405 & & & 16398 & & \\
\hline Biomass Mass Flow & $\mathrm{LB} / \mathrm{HR}$ & 31725 & & & & & & \\
\hline Sulfur in $\mathrm{HC}$ & PPM & 10 & 487 & & & & & \\
\hline Total Mass Flow Rate & $\mathrm{LB} / \mathrm{HR}$ & 210816 & 25936 & 25100 & 61 & 16398 & & \\
\hline Temperature & $\mathrm{F}$ & 86 & 86 & 60 & 68 & 86 & & \\
\hline Pressure & $\mathrm{PS} \mid \mathrm{G}$ & 37.4 & 10 & 1 & 0 & 10 & & \\
\hline Standard Liq Flow & $\mathrm{BPD}$ & 14561 & & & & 1074 & & \\
\hline Vapor Flow & MSCFH & & 342 & 331 & & & & \\
\hline Wt \% Vapor & & 0 & 100 & 100 & 0 & 0 & & \\
\hline$\overline{M W}$ & & & & & & & & \\
\hline
\end{tabular}



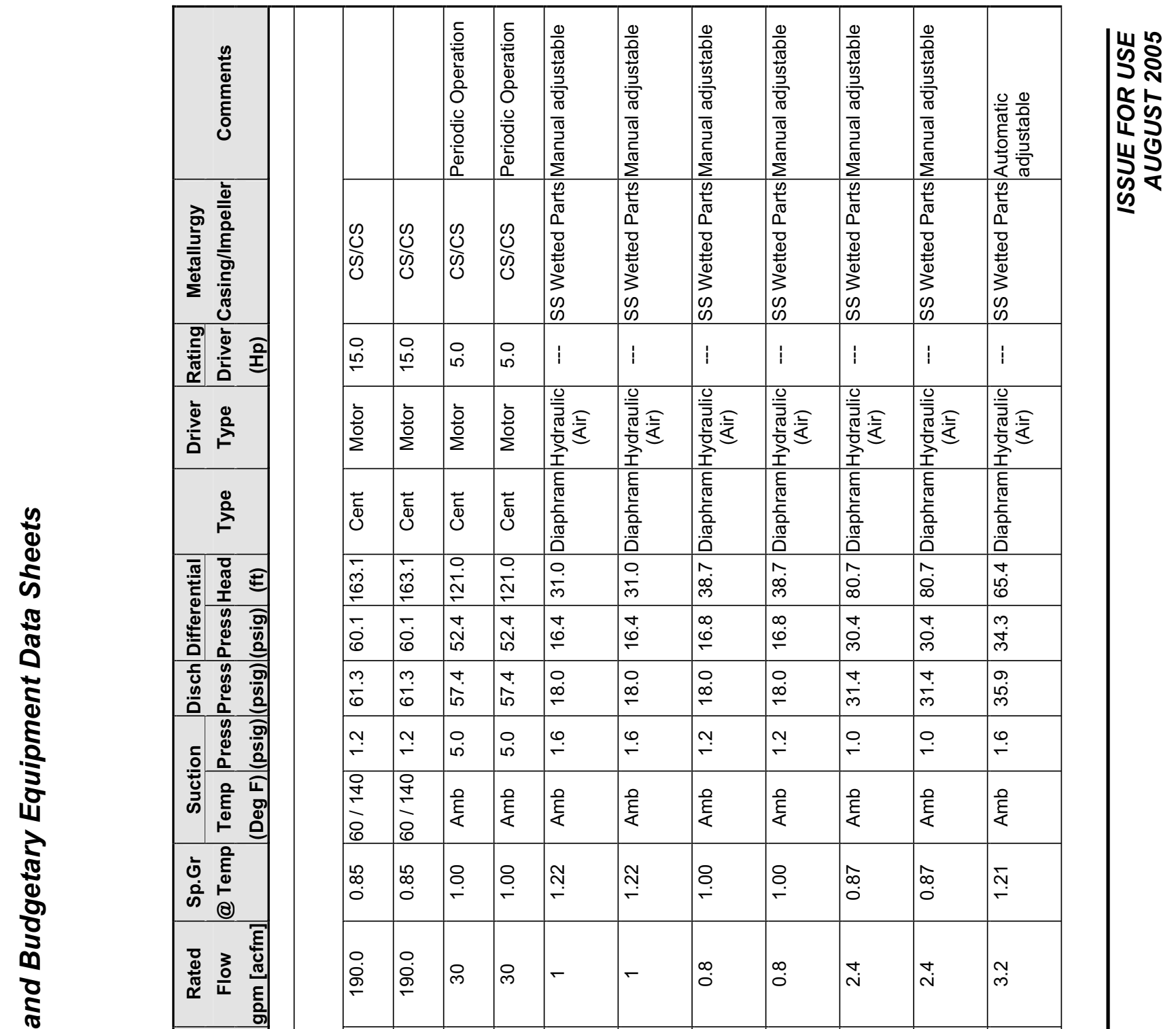

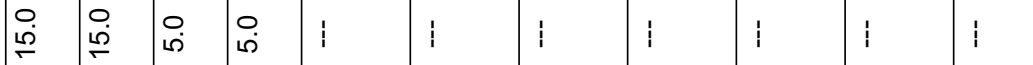

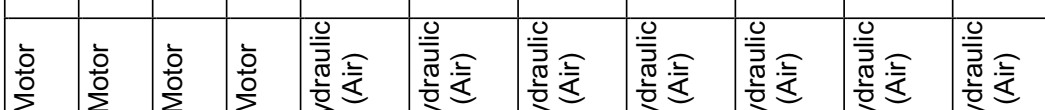

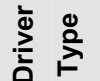

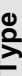

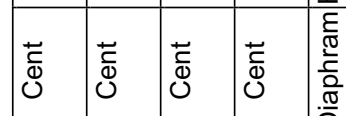

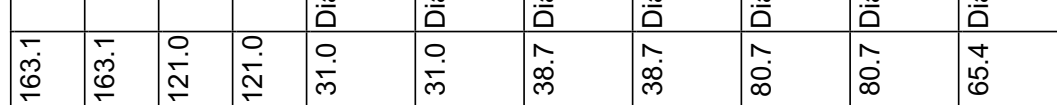

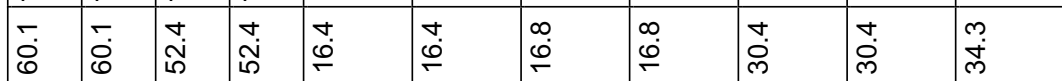

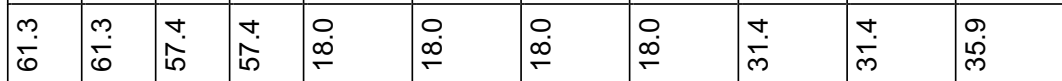

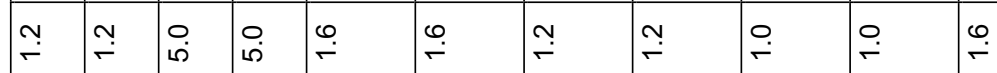

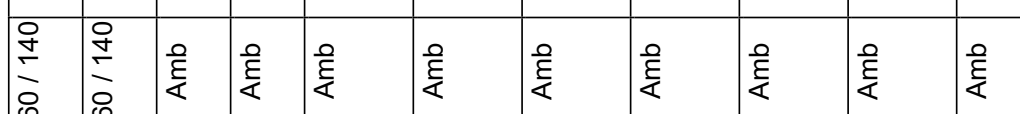

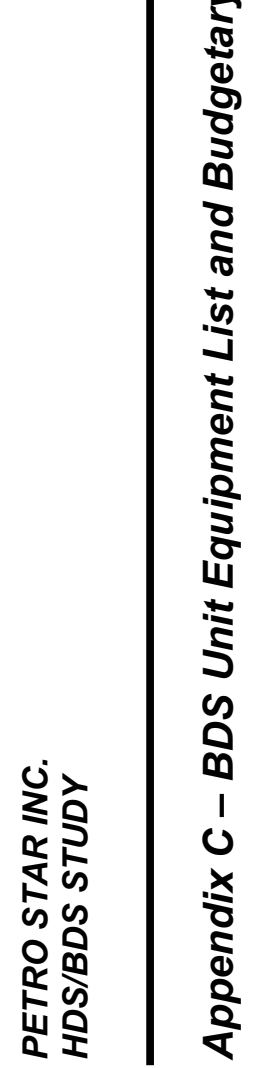

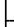

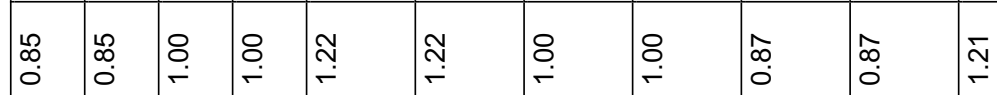

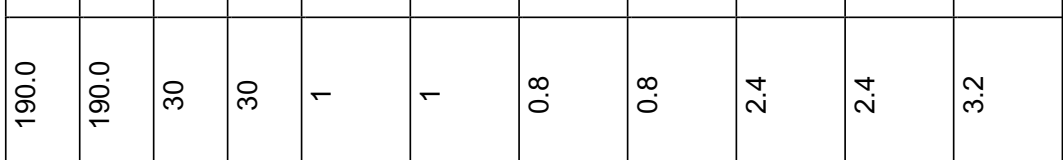

$\sum_{\substack{\mathbb{U} \\ 心}}^{\text {U }}$

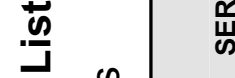

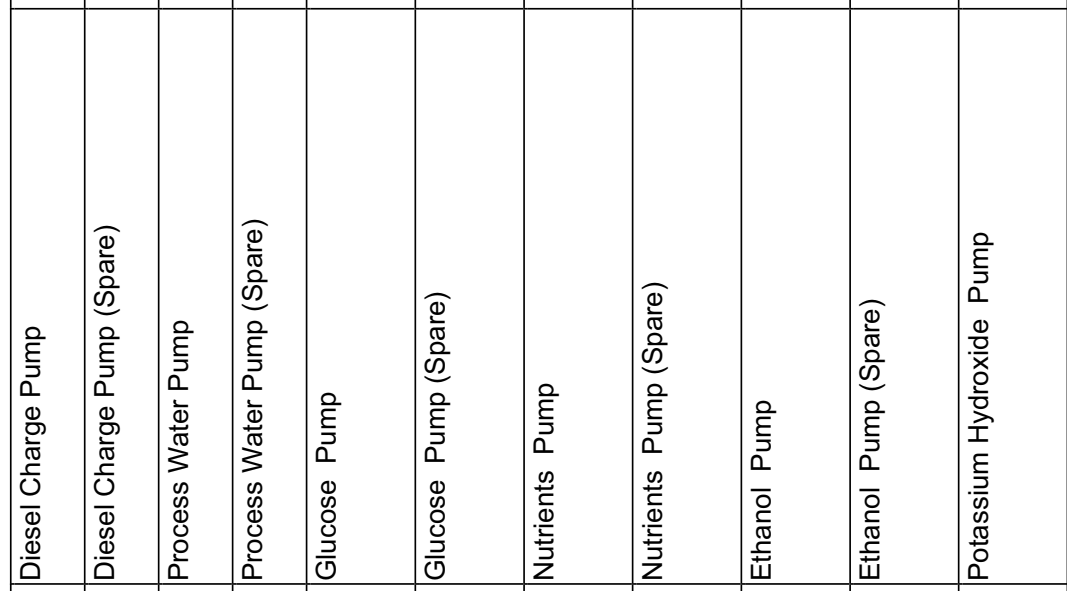

$\pm$

œ

$\sum_{\substack{2 \\ 0}}^{\infty}$

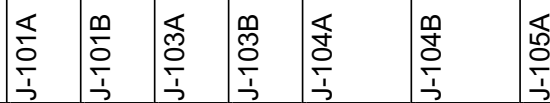

$\frac{1}{1}$

$\stackrel{\mathscr{\bigotimes}}{\frac{\infty}{1}}$

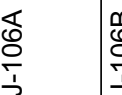

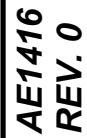


Final Technical Progress Report DOE Award No. DE-FC26-02NT15340

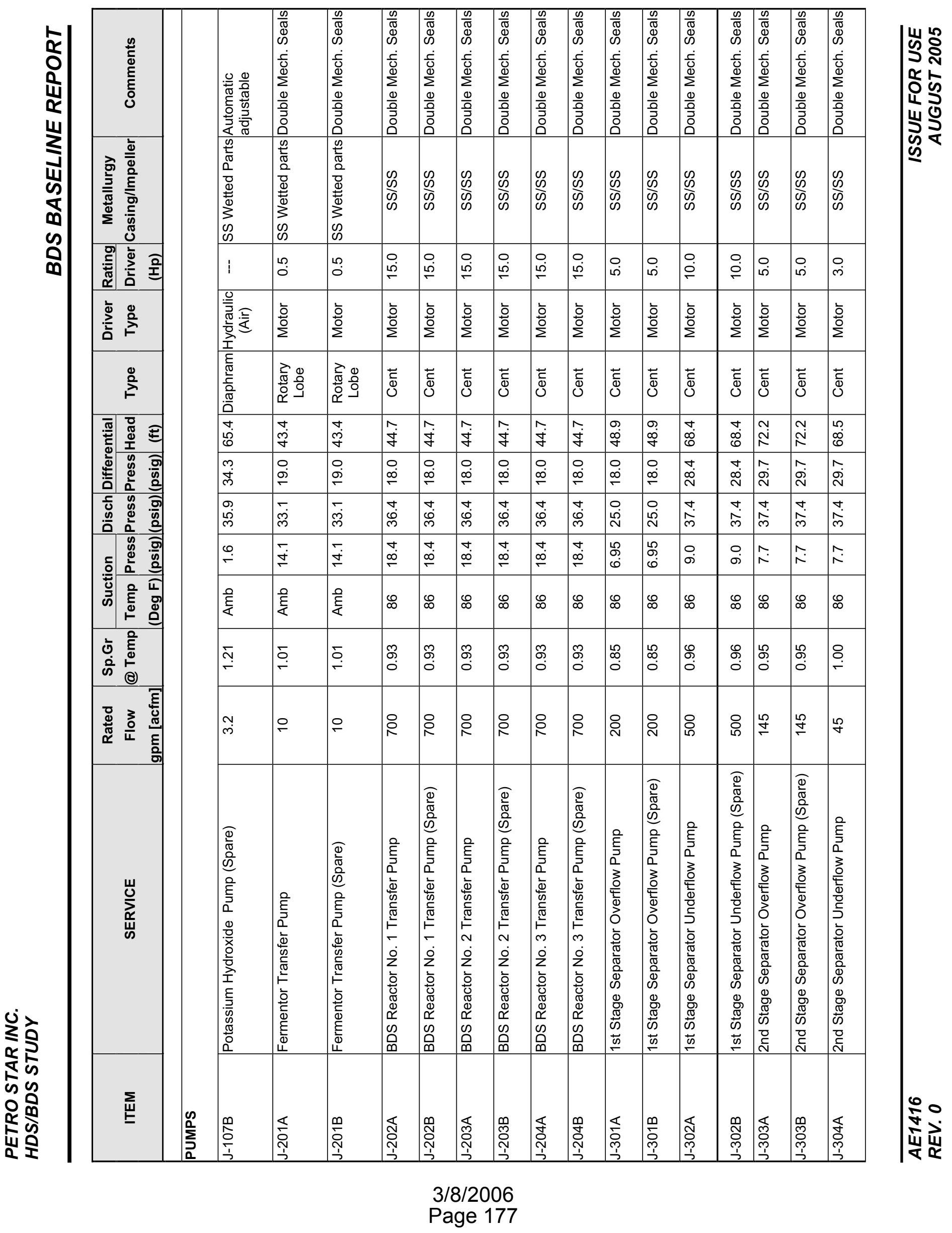


Final Technical Progress Report DOE Award No. DE-FC26-02NT15340

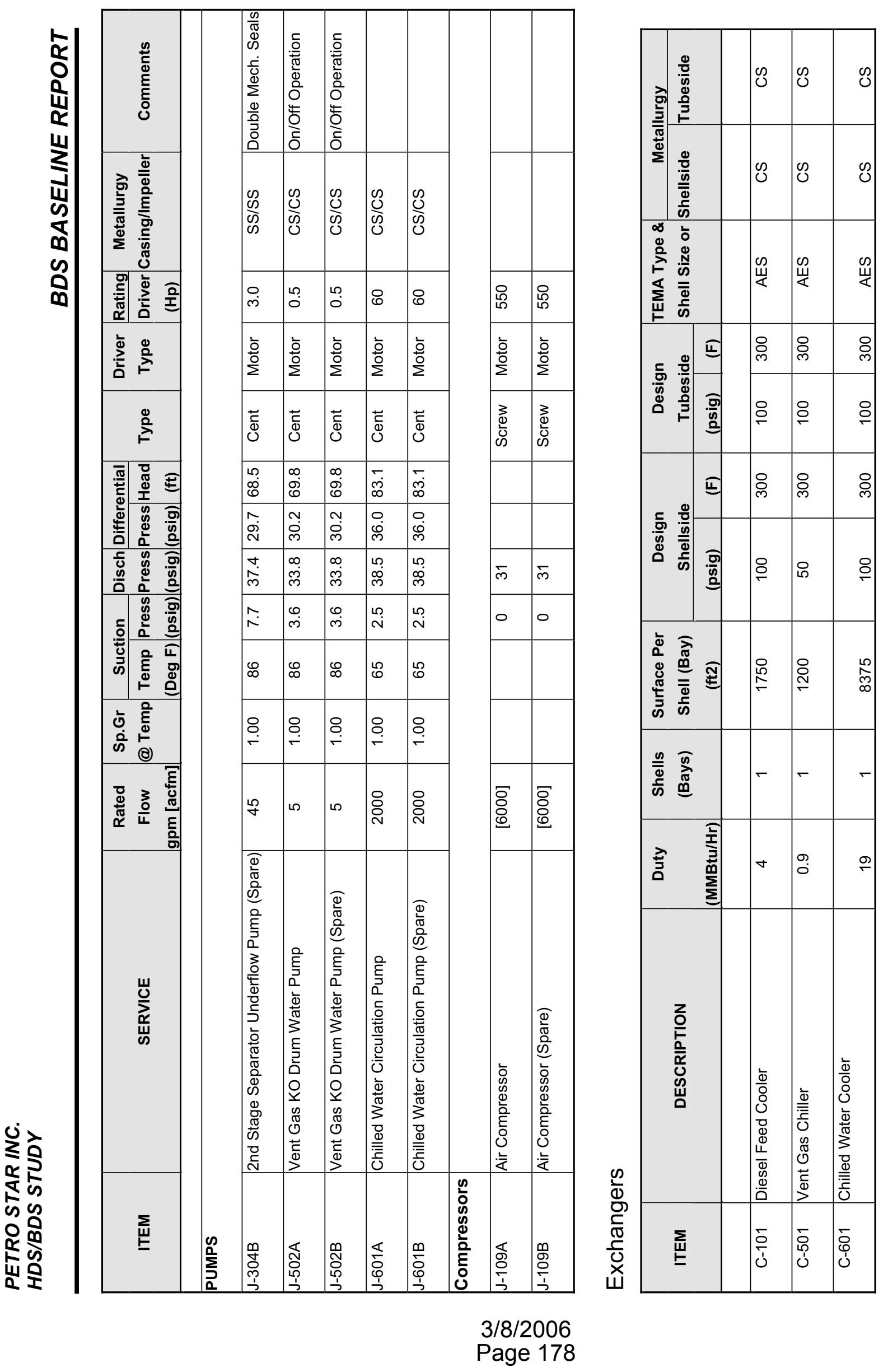

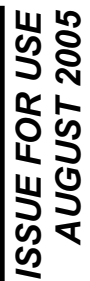




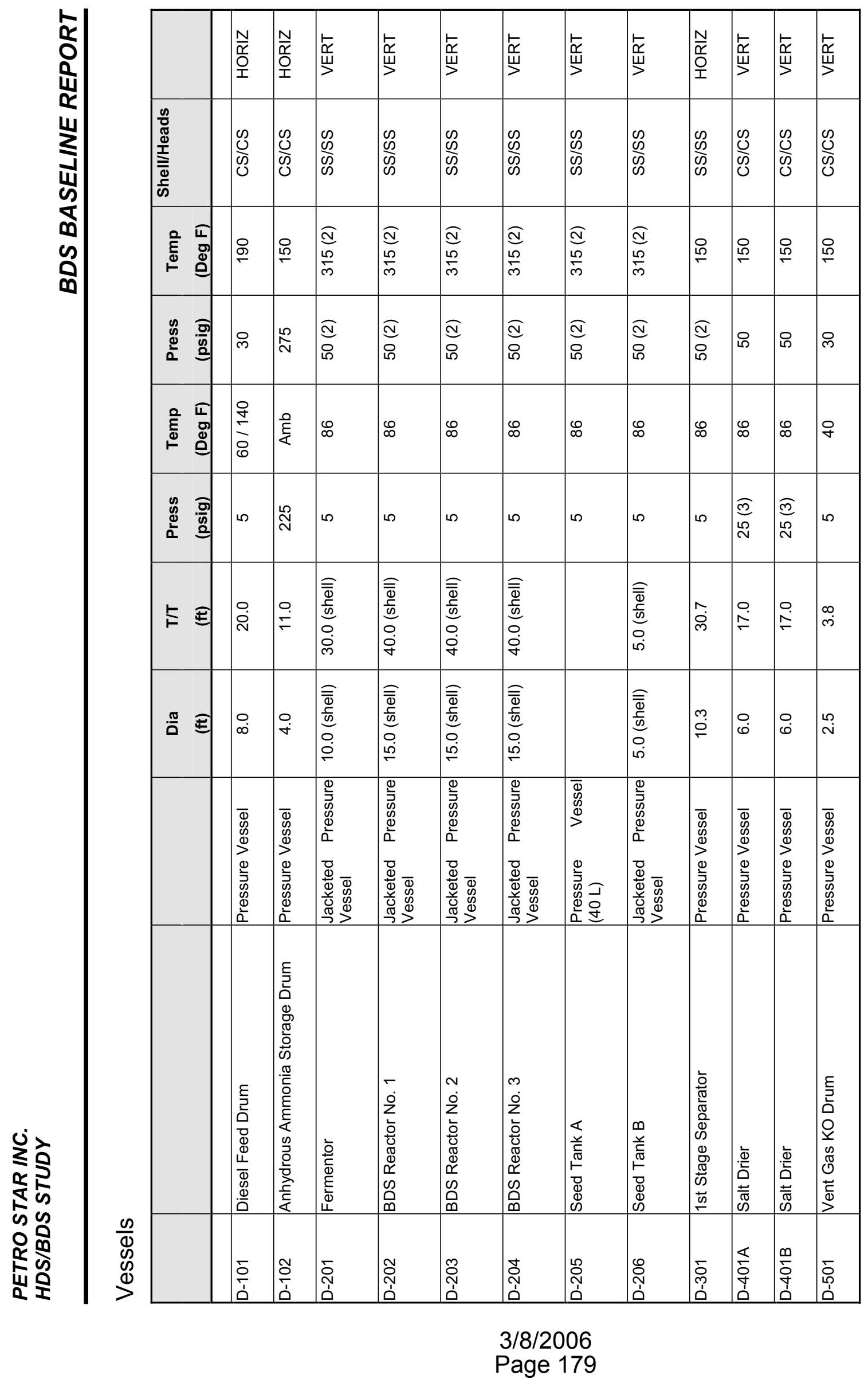


Final Technical Progress Report DOE Award No. DE-FC26-02NT15340
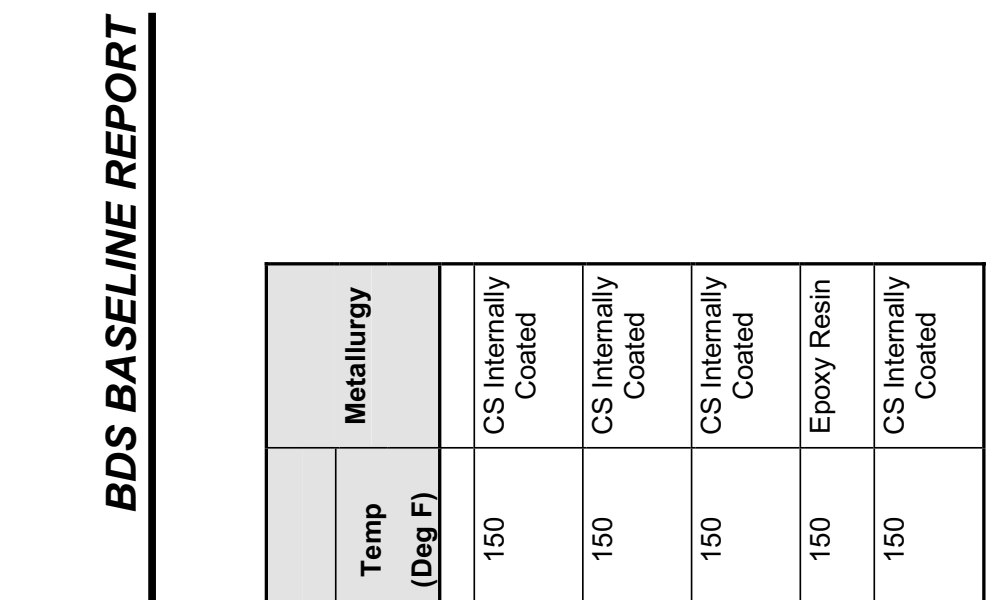

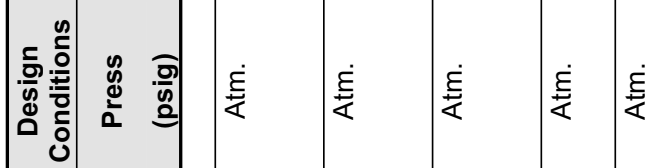

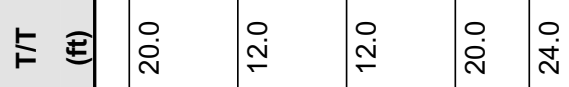

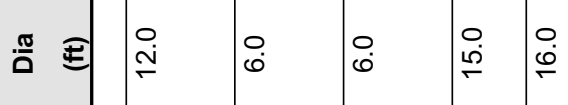

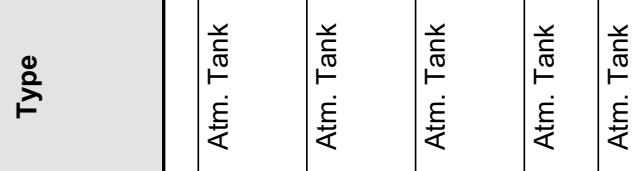

约

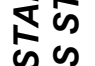

ำ

点

岂全

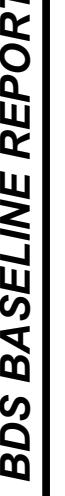

\begin{tabular}{|c|c|c|c|c|c|c|}
\hline 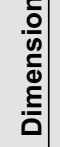 & $\frac{\pi}{\Delta} \Xi$ & $\stackrel{\stackrel{O}{\stackrel{\mathrm{V}}{ }}}{ }$ & $\begin{array}{l}0 \\
0\end{array}$ & $\stackrel{0}{0}$ & 吊 & $\begin{array}{l}\stackrel{\circ}{\dot{\theta}} \\
\stackrel{0}{*}\end{array}$ \\
\hline
\end{tabular}

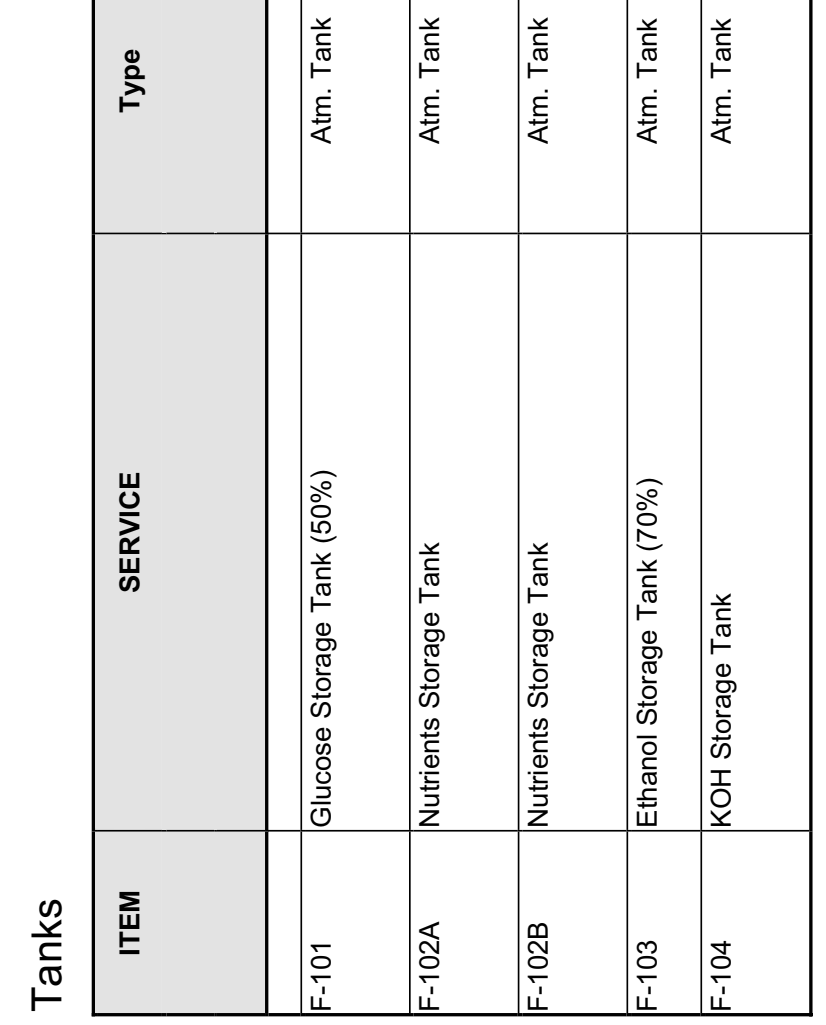

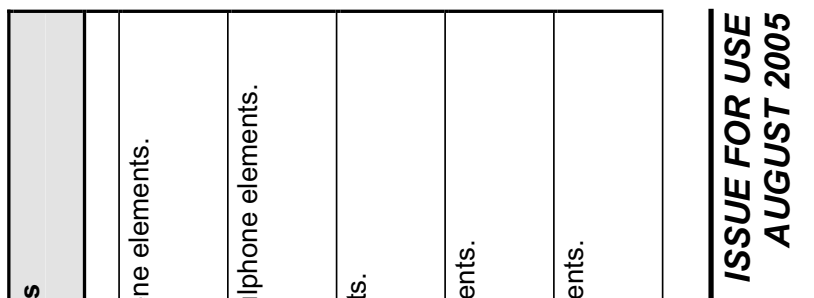

$\frac{\frac{n}{\pi}}{\frac{\pi}{20}}$
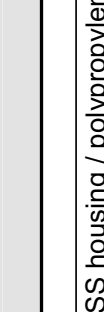

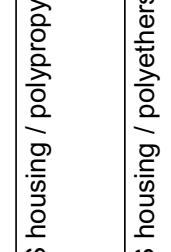

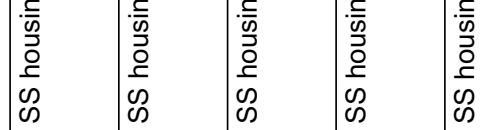

\begin{tabular}{l|l|l|l|l}
$\frac{\omega}{\omega}$ & $\frac{\omega}{m}$ & $\frac{\omega}{m}$ & $\frac{\omega}{m}$ & $\frac{\omega}{m}$
\end{tabular}

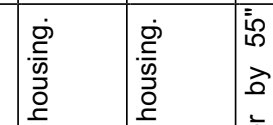

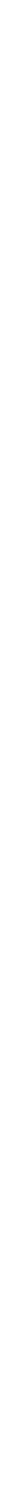

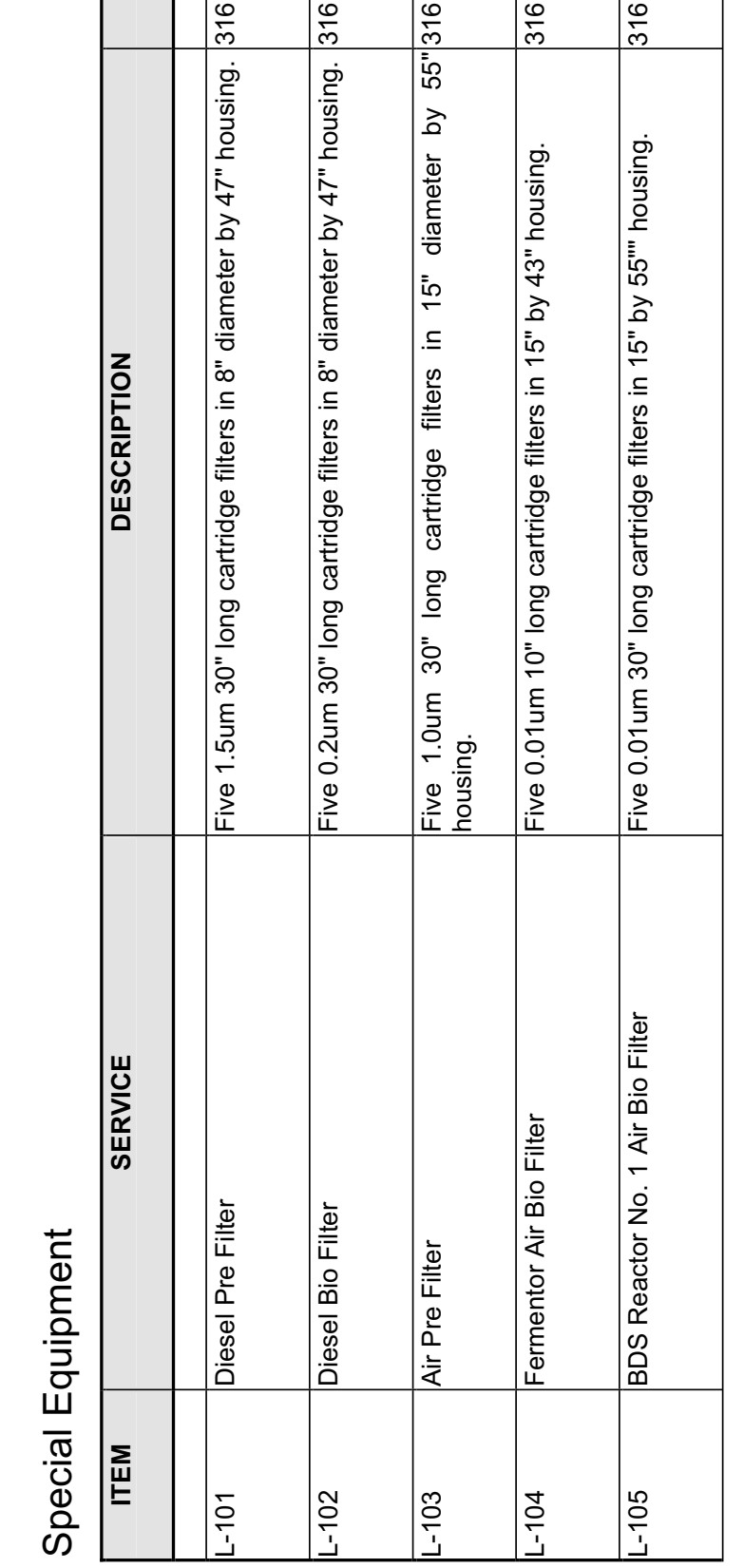

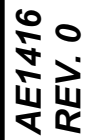




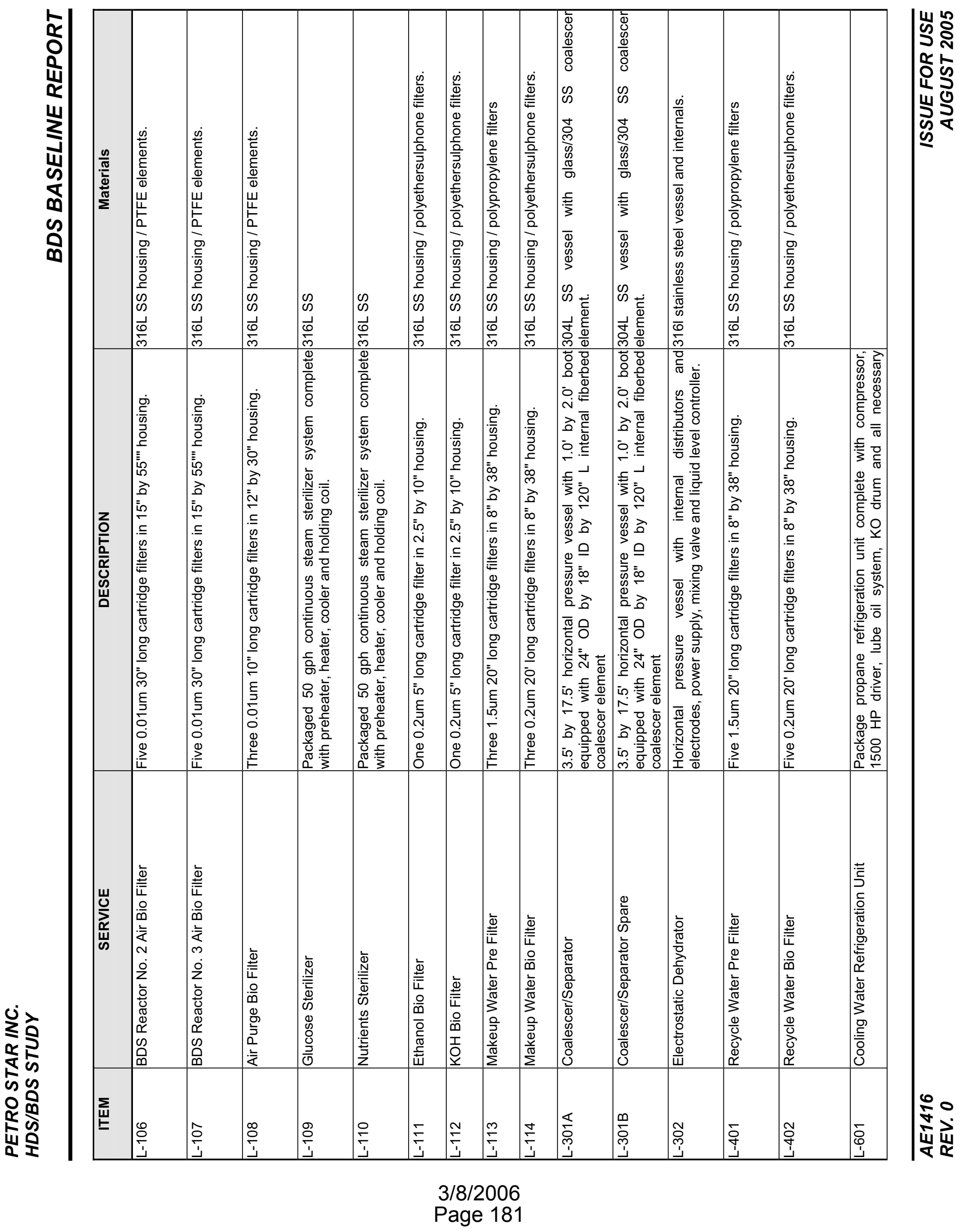




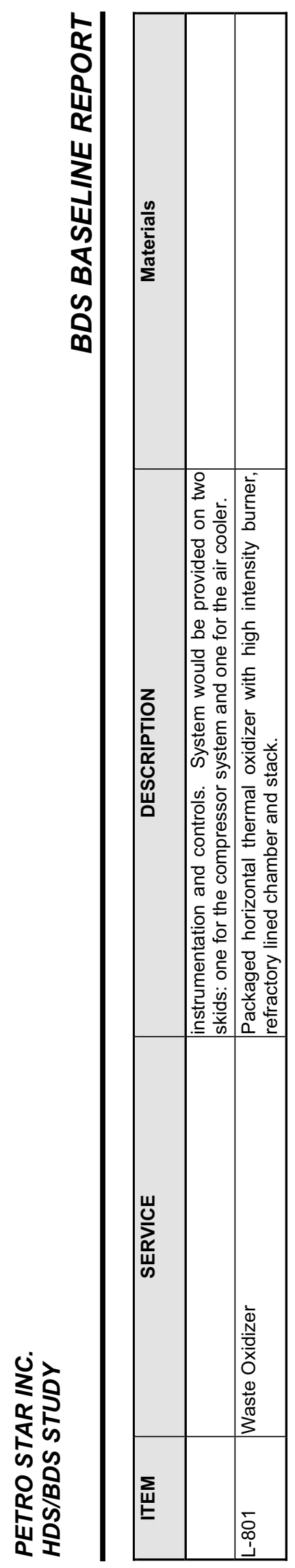




\begin{tabular}{|c|c|c|c|c|}
\hline ANVIL & & $\begin{array}{l}\text { BUDGETARY DATA S } \\
\text { RESSURE VESSELS-ASME }\end{array}$ & & \\
\hline $\begin{array}{l}\text { CUSTOMER } \\
\text { LOCATION } \\
\text { PROCESS UNIT } \\
\end{array}$ & & $\begin{array}{l}\text { HDS/BDS Study } \\
\text { Alaska } \\
\text { BDS ULSD Baseline }\end{array}$ & $\begin{array}{l}\text { PROJECT ENGINEER } \\
\text { PROJECT NO. } \\
\text { DATE } \\
\end{array}$ & $\begin{array}{l}\text { PMD } \\
\text { AE1416 } \\
3 / 17 / 05 \\
\end{array}$ \\
\hline ITEM NUMBER & $|x|$ & 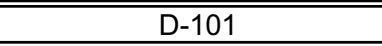 & & \\
\hline SERVICE & $x$ & Diesel Feed Drum & & \\
\hline FLUID & $x$ & Diesel & & \\
\hline ASME SECT VIII DIV 1 OR 2 & & & & \\
\hline POSITION ; HORIZONTAL, VERTICAL & $x$ & Horizontal & & \\
\hline DIAMETER, FT-IN & $x$ & 81 & & \\
\hline TANGENT TO TANGENT LENGTH, FT. & $x$ & $20^{\prime}$ & & \\
\hline SKIRT HEIGHT (FT-IN) & $x$ & & & \\
\hline DESIGN TEMPERATURE $\left({ }_{\mathrm{i}} \mathrm{F}\right)$ & $x$ & 190 & & \\
\hline DESIGN PRESSURE (PSIG) & $x$ & 30 & & \\
\hline MATERIAL OF CONSTRUCTION & $x$ & CS & & \\
\hline INSULATION (YES/NO) & & & & \\
\hline TRAY OR PACKING TYPE & $x$ & & & \\
\hline NUMBER OF TRAYS & $x$ & & & \\
\hline TRAY MATERIAL & $x$ & & & \\
\hline PACKING VOLUME, FT ${ }^{3}$ & $x$ & - & & \\
\hline PACKING MATERIAL & $x$ & - & & \\
\hline INTERNALS & $x$ & & & \\
\hline LINING & $x$ & - & & \\
\hline PLATFORMS AND LADDERS & $M$ & & & \\
\hline BOOT (YES / NO) & $x$ & $\mathrm{NO}$ & & \\
\hline & & & & \\
\hline & & & & \\
\hline & & & & \\
\hline & & & & \\
\hline & & & & \\
\hline & & & & \\
\hline REMARKS & & & & \\
\hline LEGEND: & & $\begin{array}{l}X=\text { PROCESS INFO REQUIRED } \\
O=\text { PROCESS INFO OPTIONAL } \\
M=\text { MECHANICAL INFO OPTIONAL }\end{array}$ & & \\
\hline
\end{tabular}




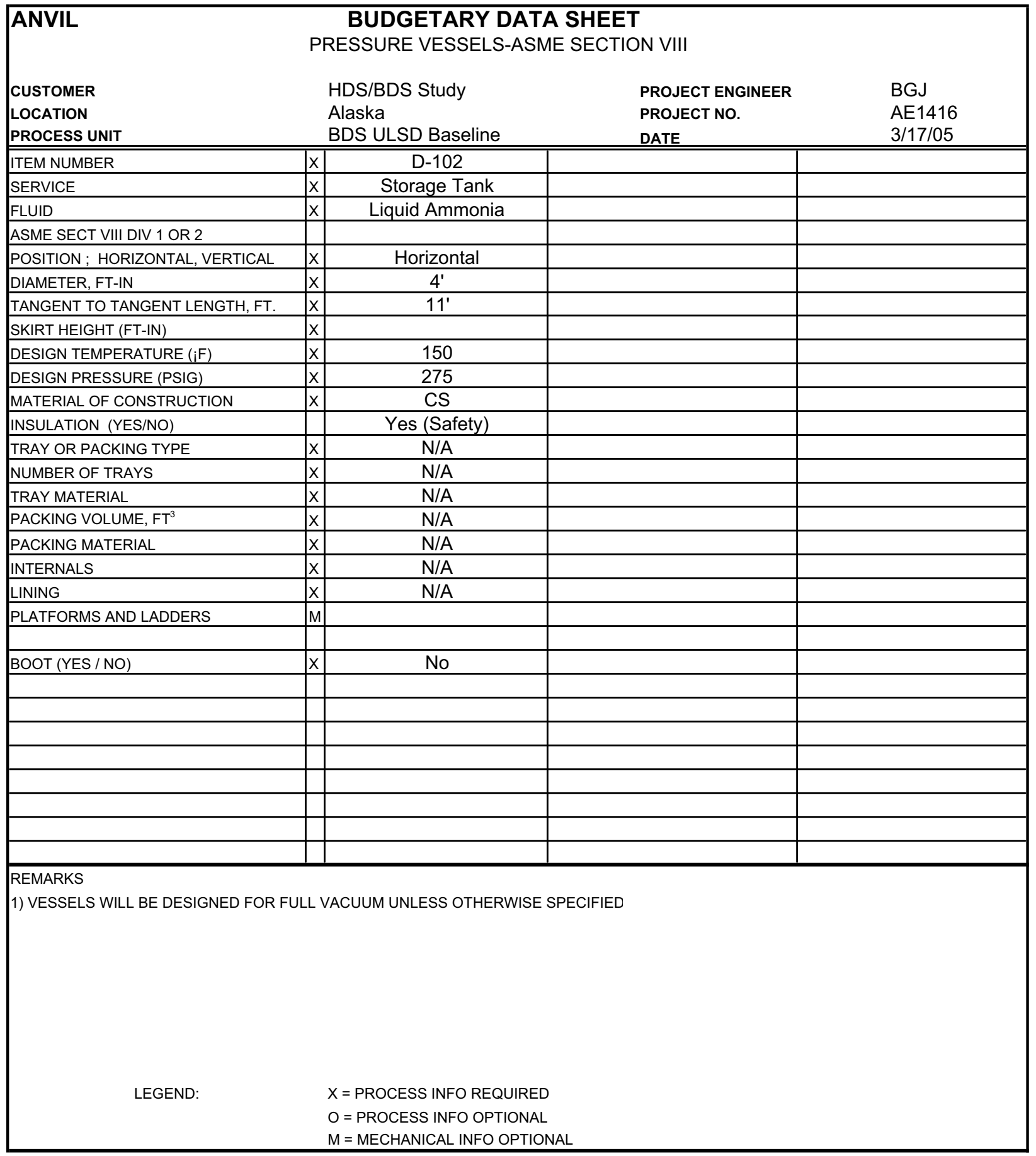

Macintosh HD:Users:phil:Documents:Pelorus:Petro Star:Economic Analysis:BDS Baseline:[Budgetary Data Sheets.xIs]D-101 


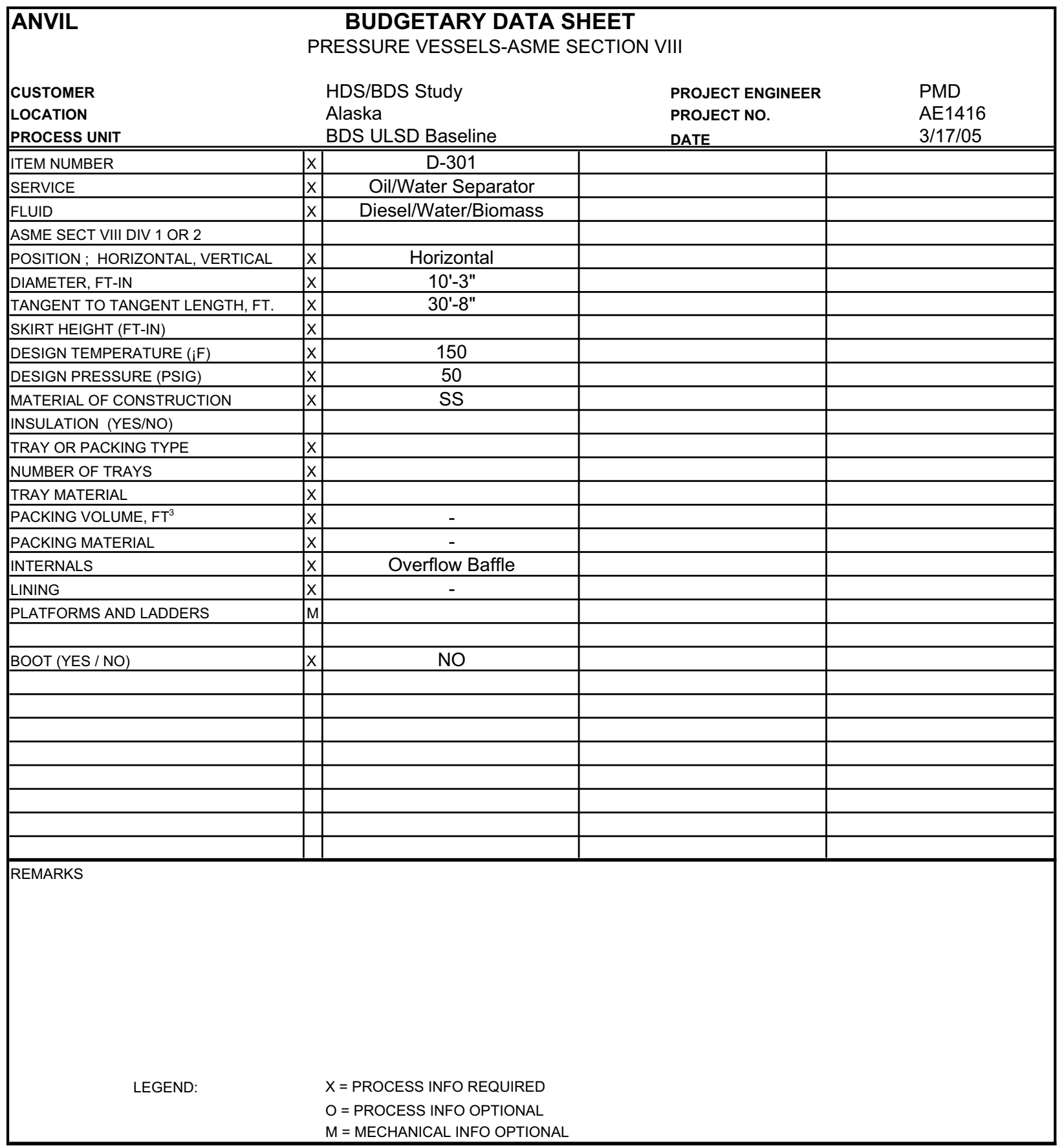




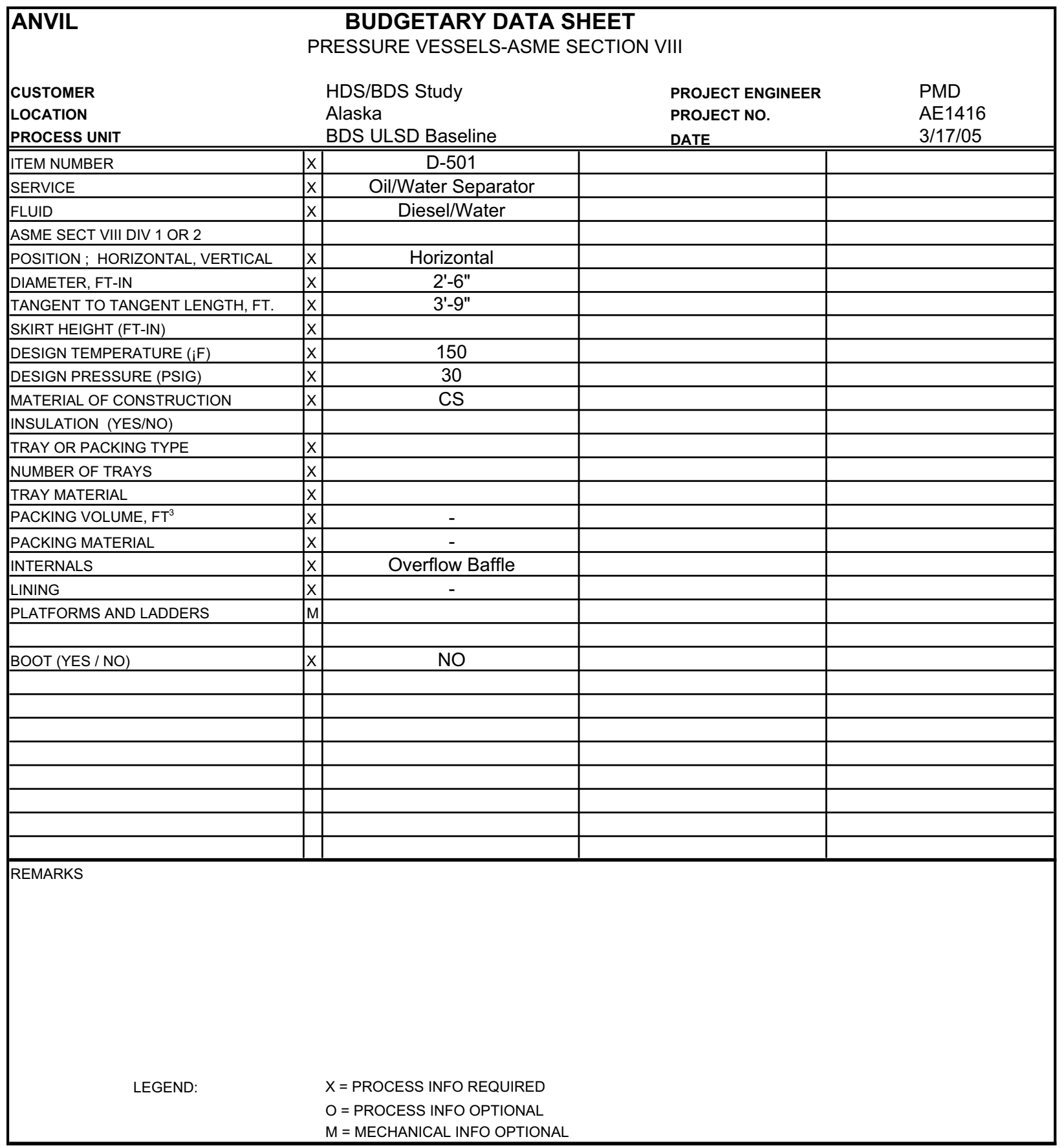




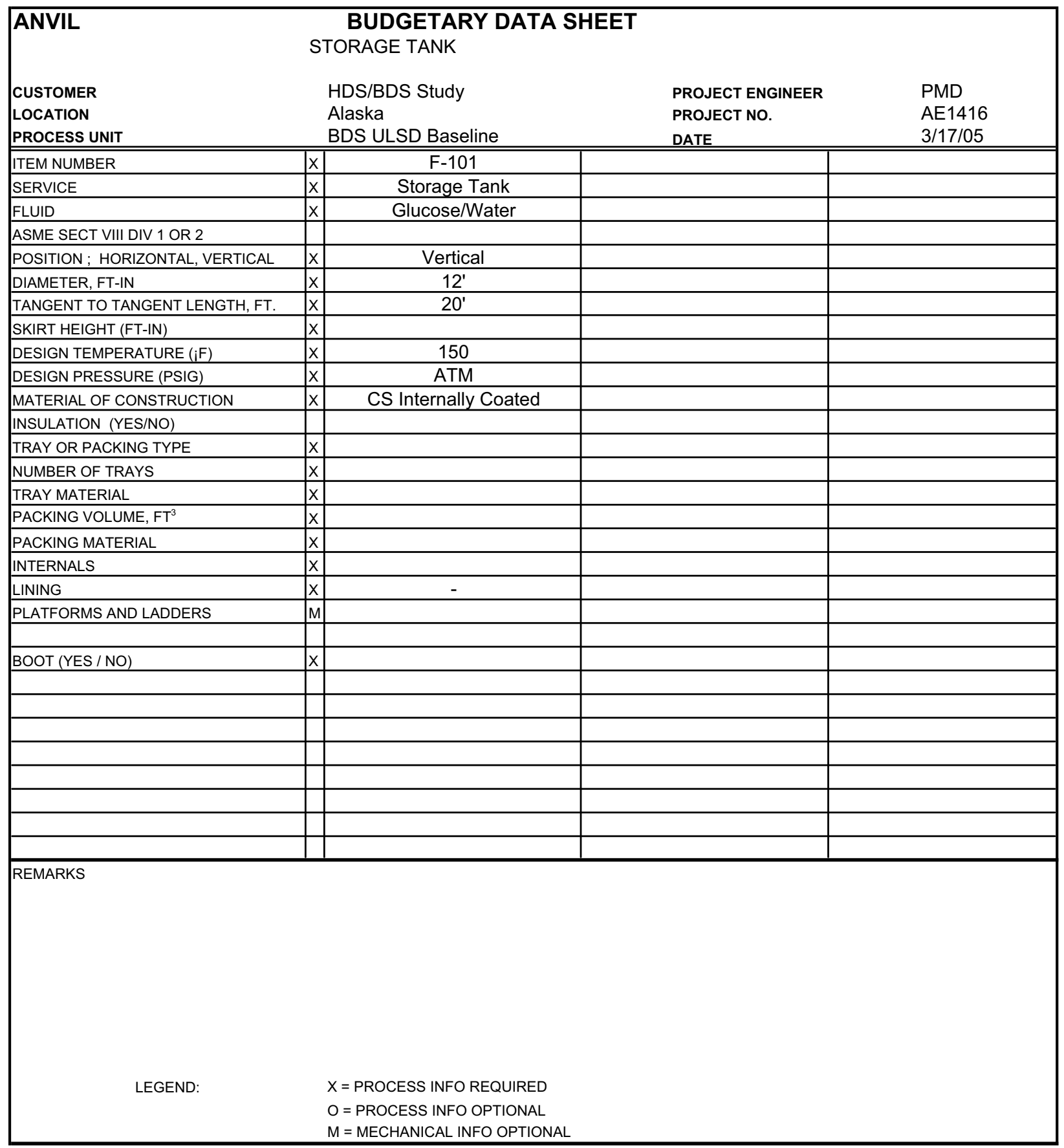




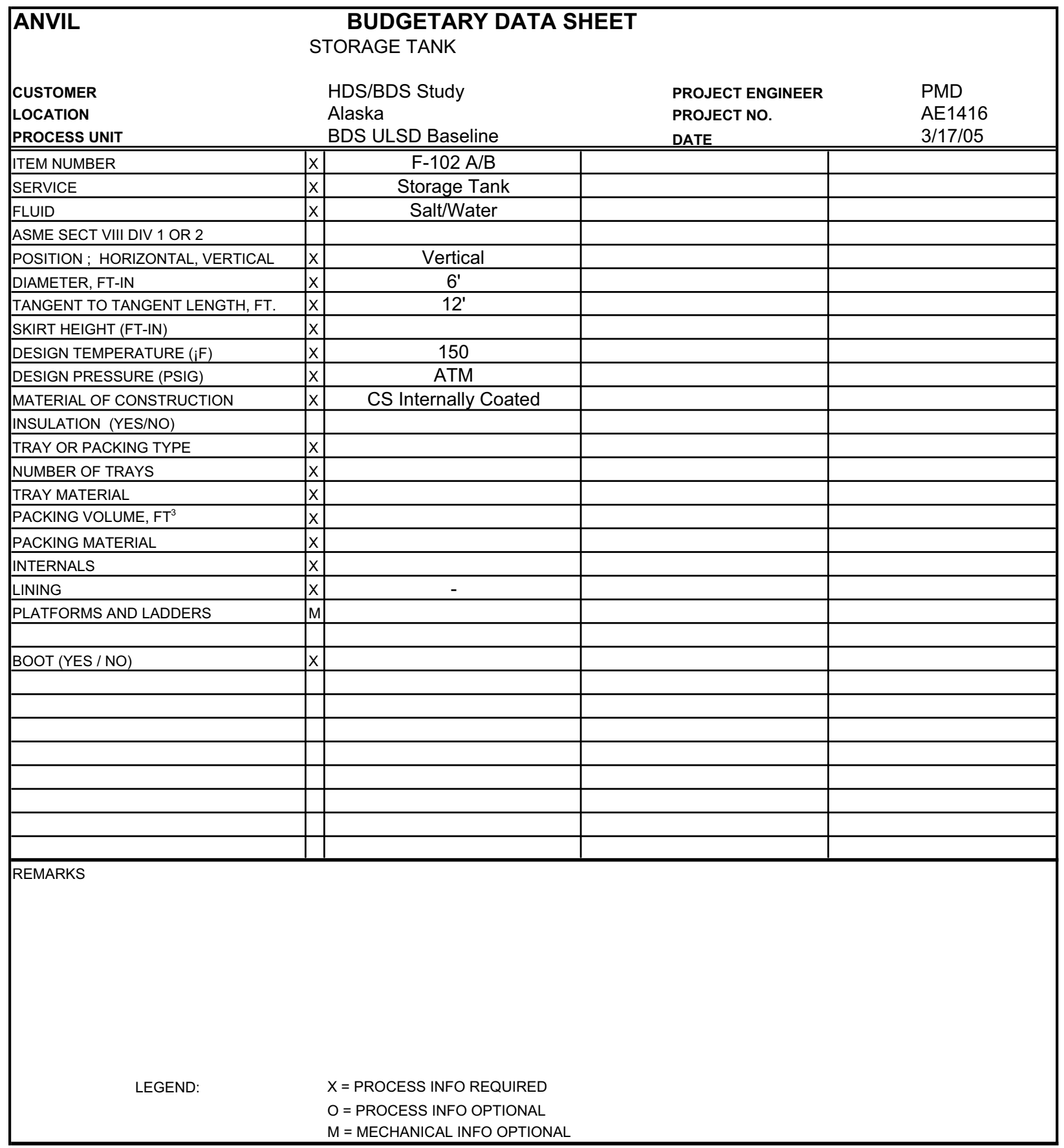




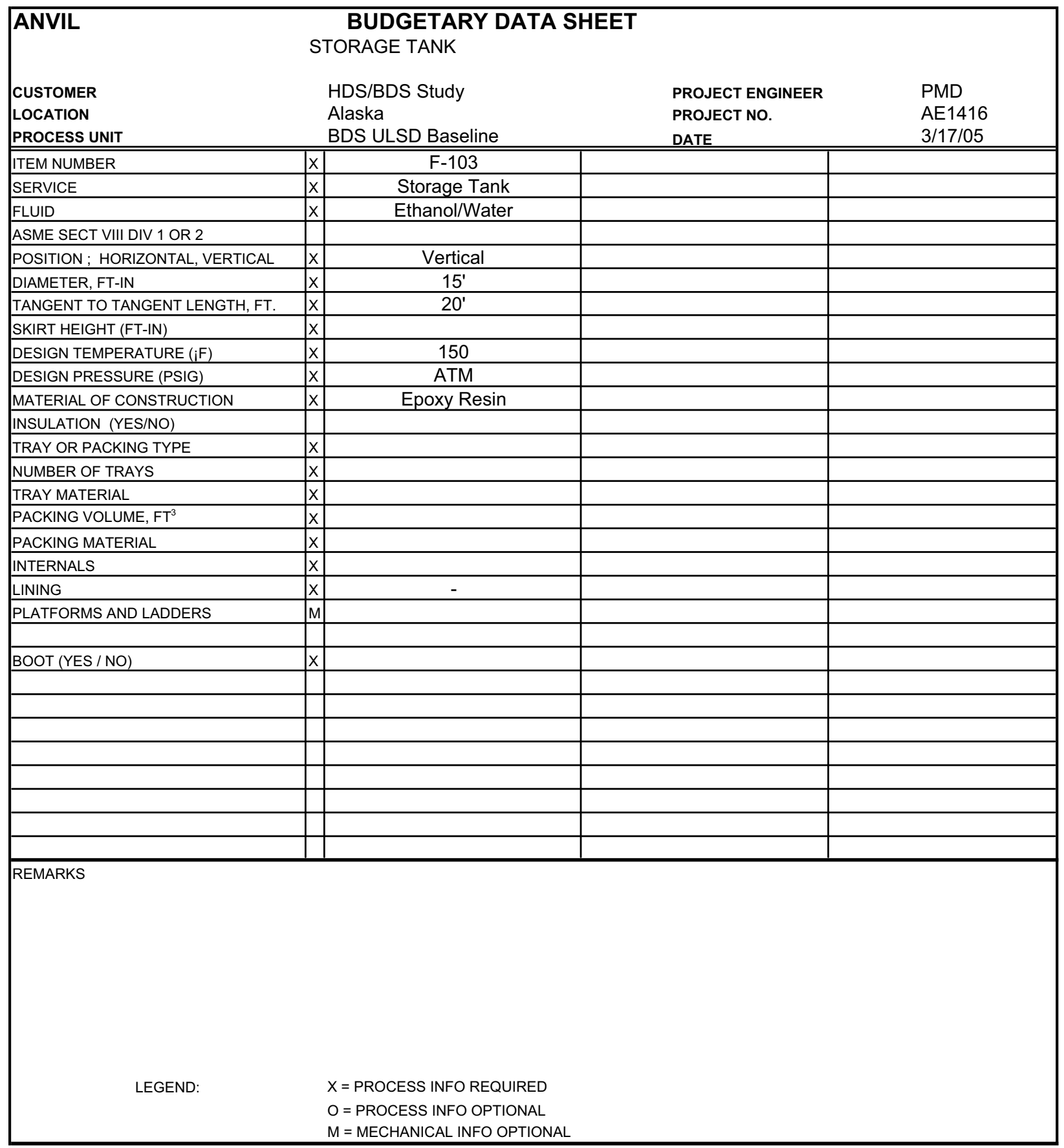




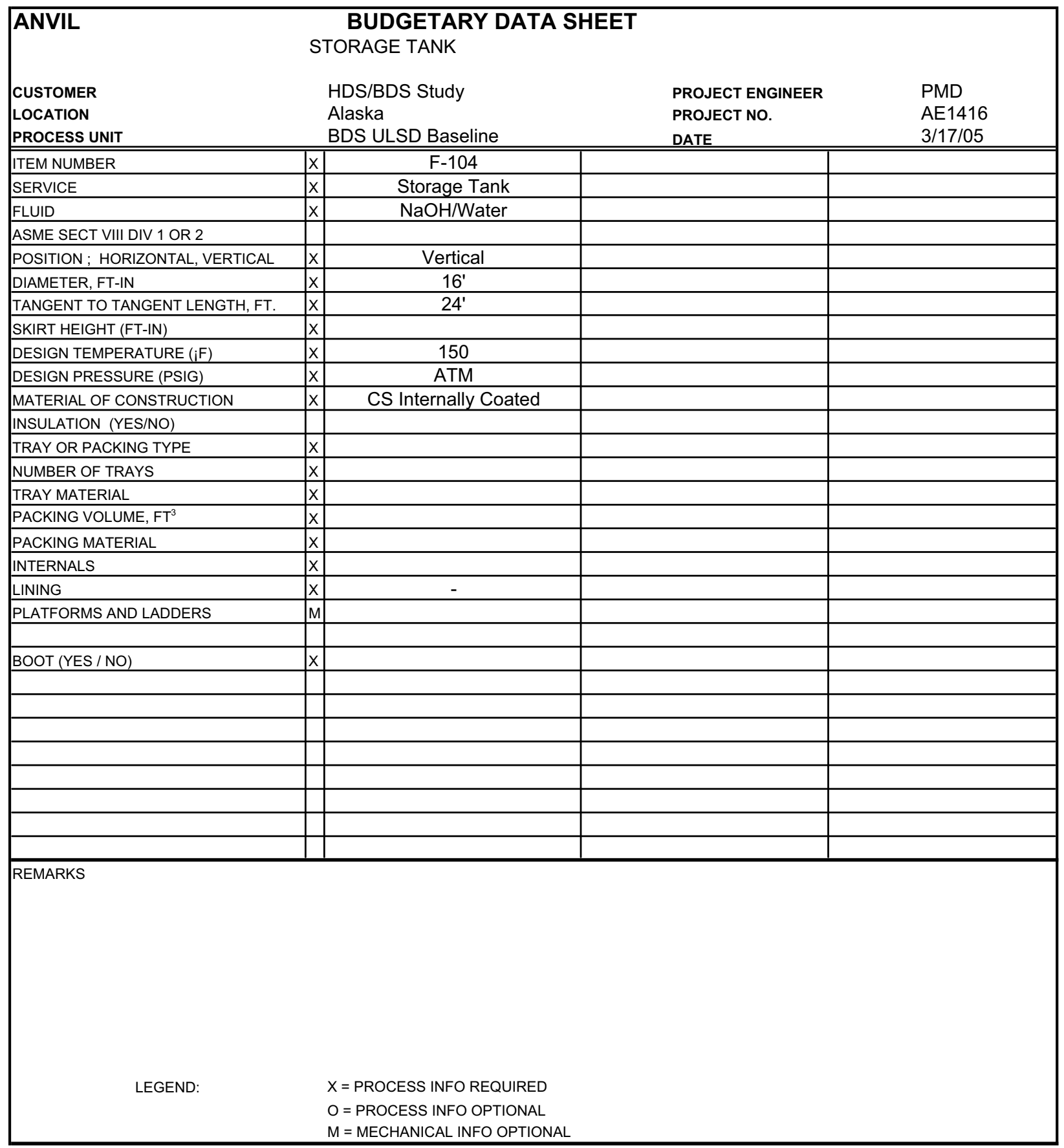




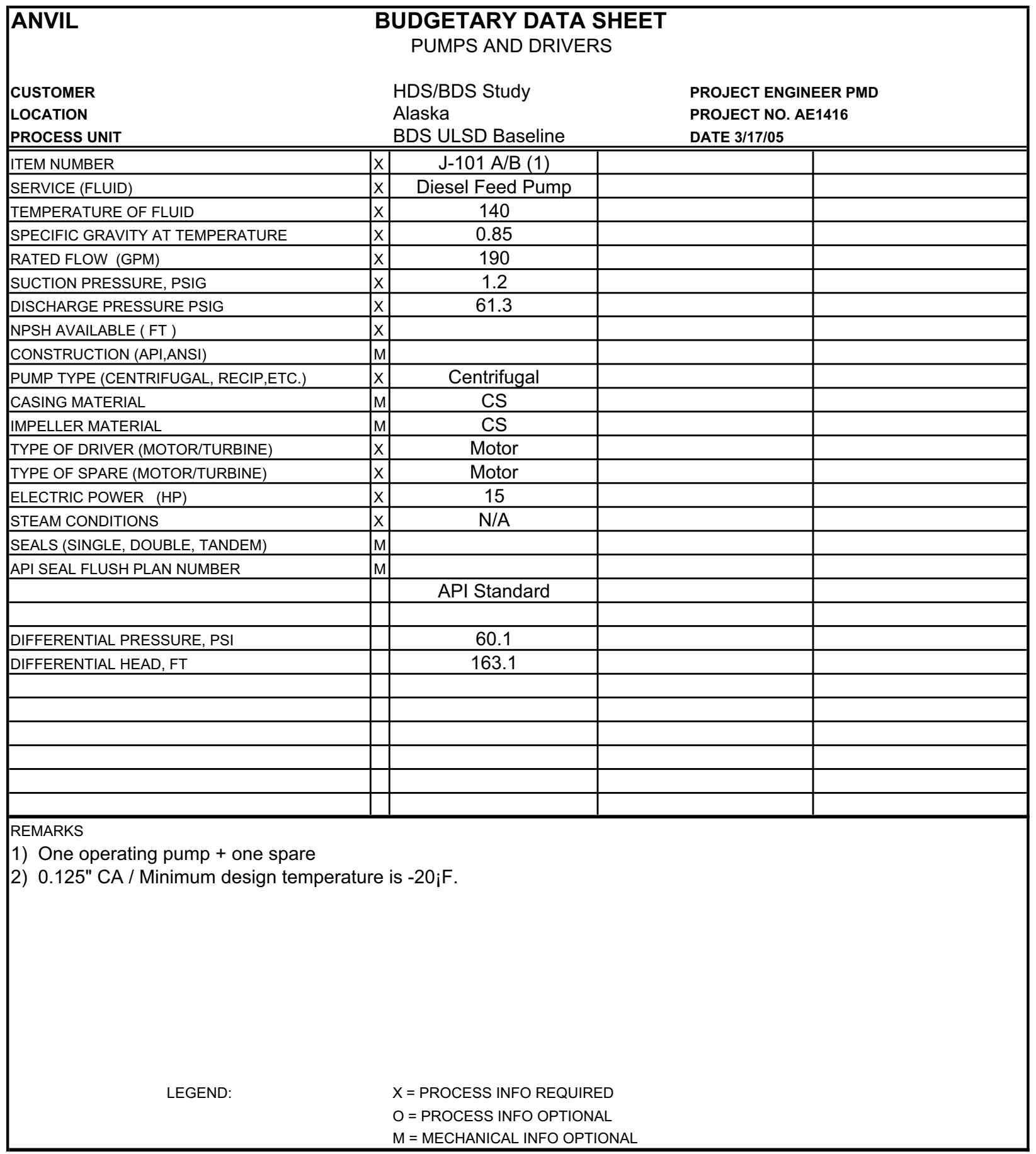

Macintosh HD:Users:phil:Documents:Pelorus:Petro Star:Economic Analysis:BDS Baseline:[Budgetary Data Sheets.xls]D-1 


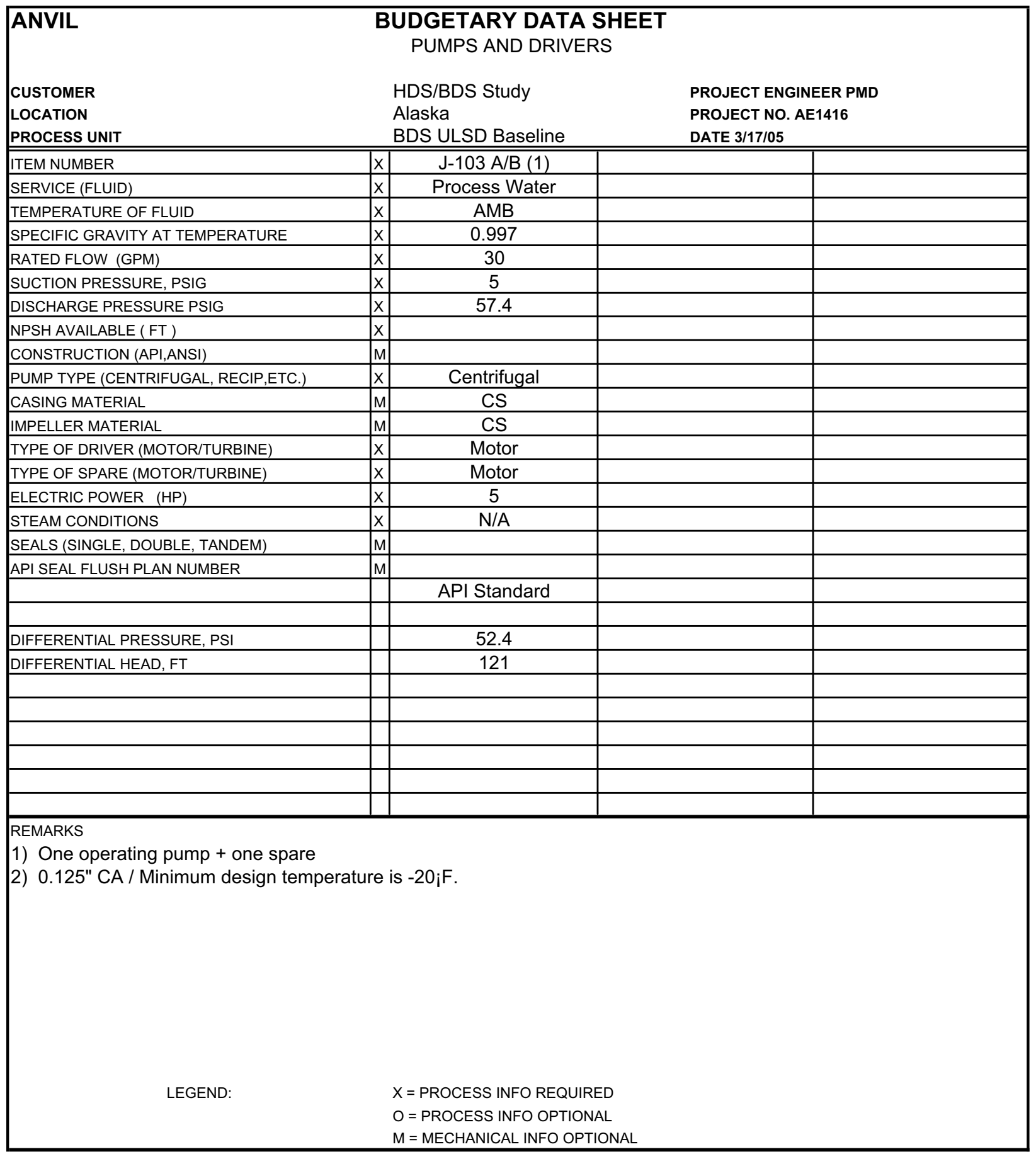

Macintosh HD:Users:phil:Documents:Pelorus:Petro Star:Economic Analysis:BDS Baseline:[Budgetary Data Sheets.xls]D-1 


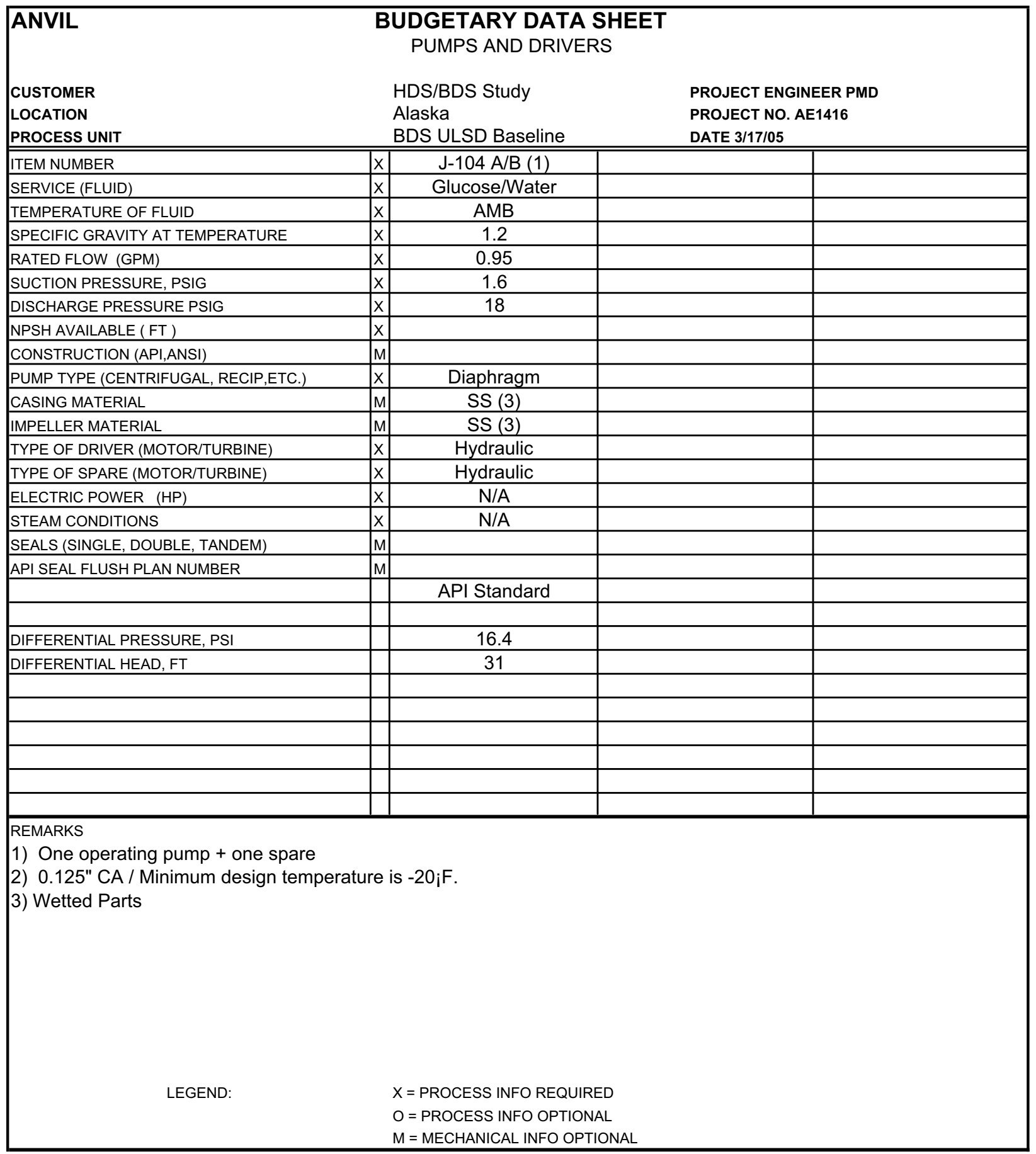

Macintosh HD:Users:phil:Documents:Pelorus:Petro Star:Economic Analysis:BDS Baseline:[Economic Spreadsheets.xls]BI 


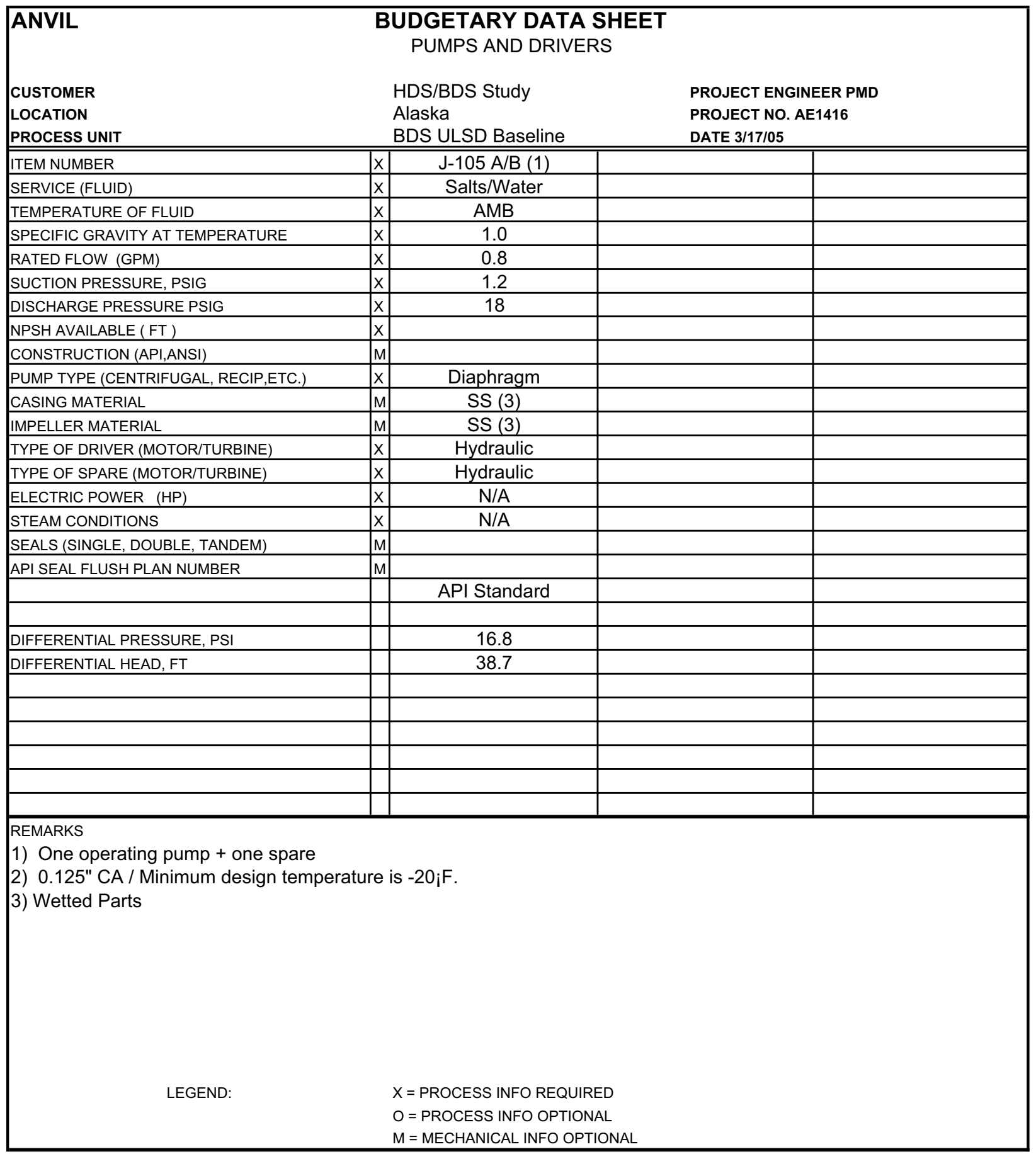

Macintosh HD:Users:phil:Documents:Pelorus:Petro Star:Economic Analysis:BDS Baseline:[Economic Spreadsheets.xls]BI 


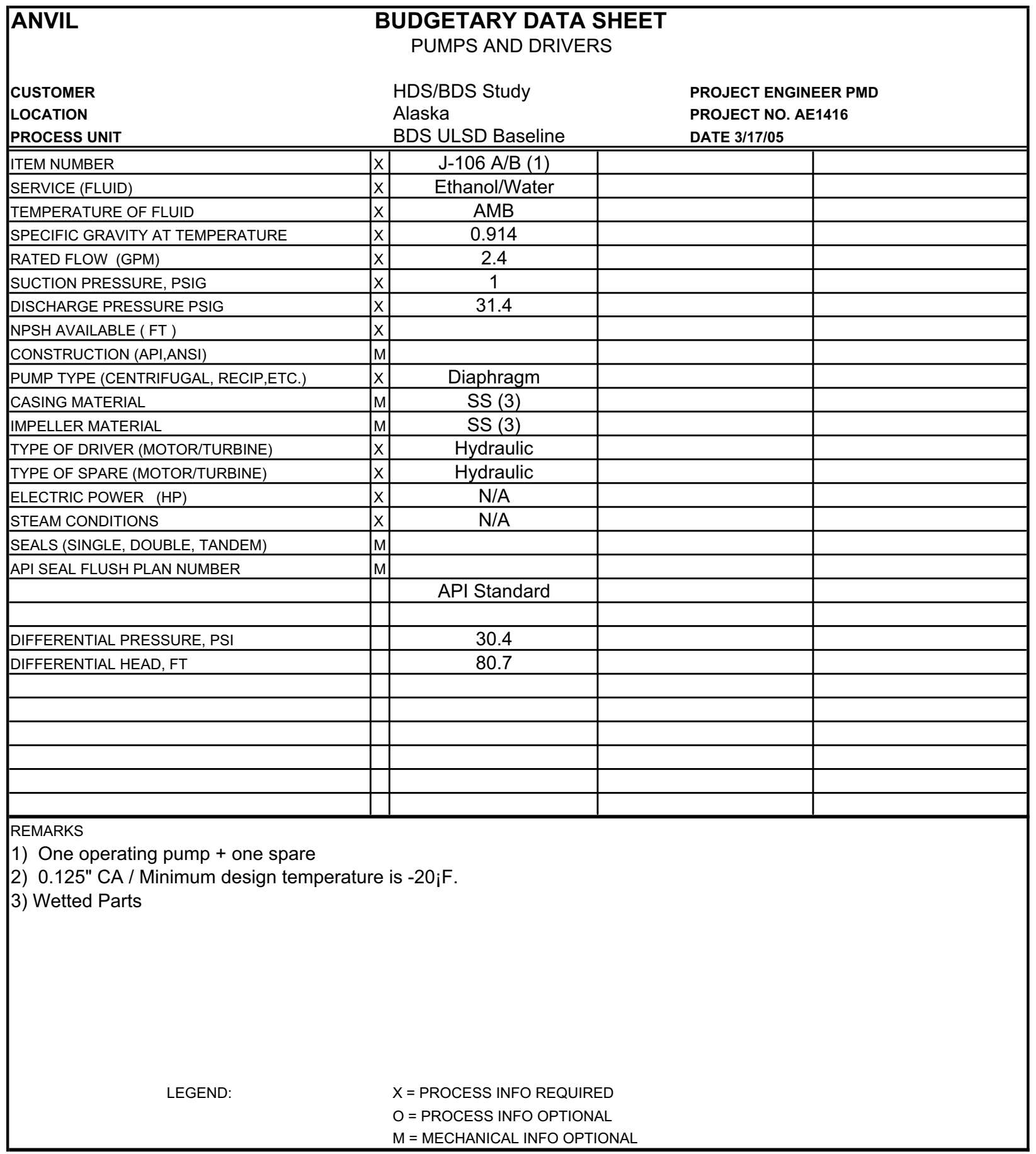

Macintosh HD:Users:phil:Documents:Pelorus:Petro Star:Economic Analysis:BDS Baseline:[Economic Spreadsheets.xls]BI 


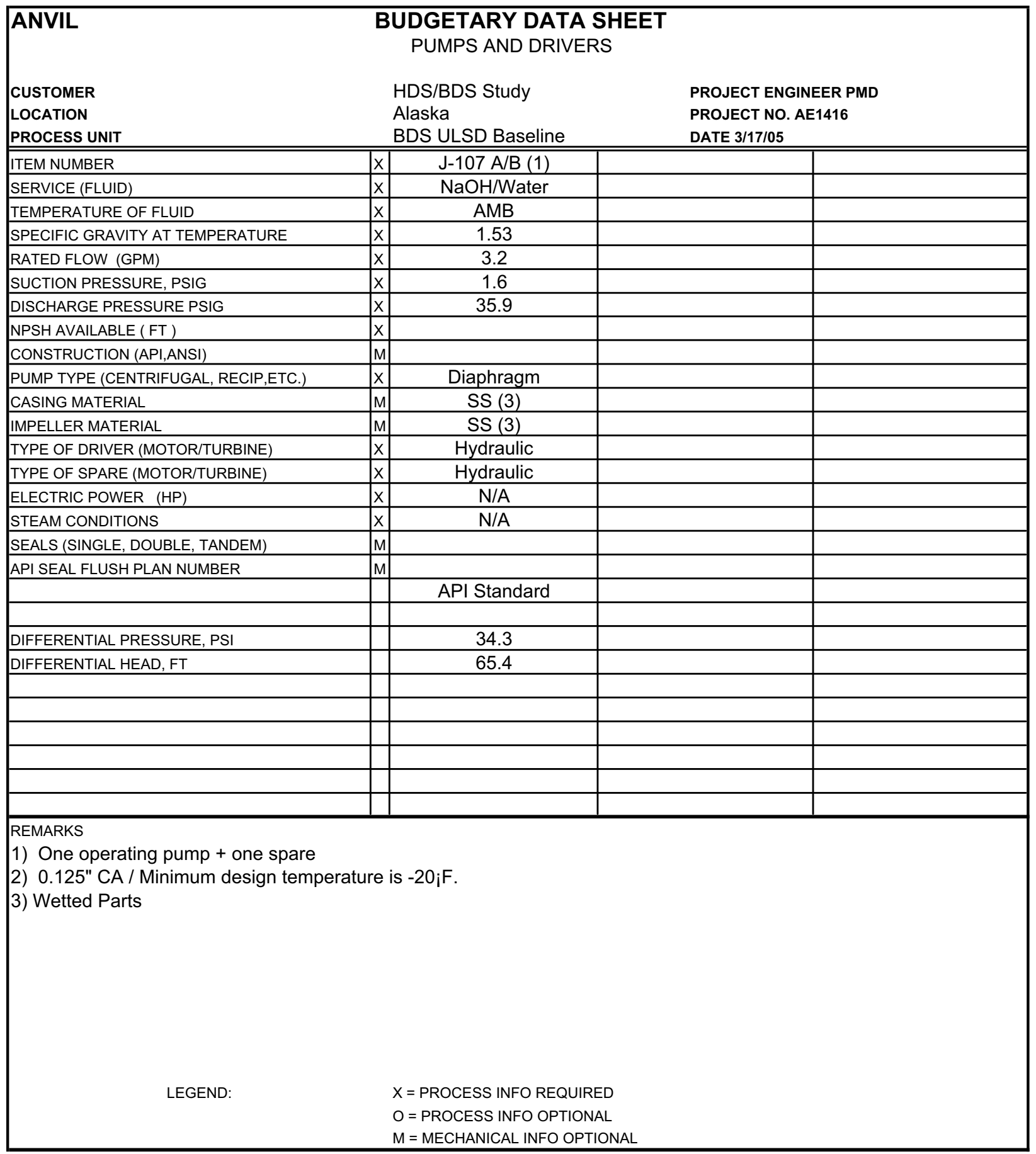

Macintosh HD:Users:phil:Documents:Pelorus:Petro Star:Economic Analysis:BDS Baseline:[Economic Spreadsheets.xls]BI 


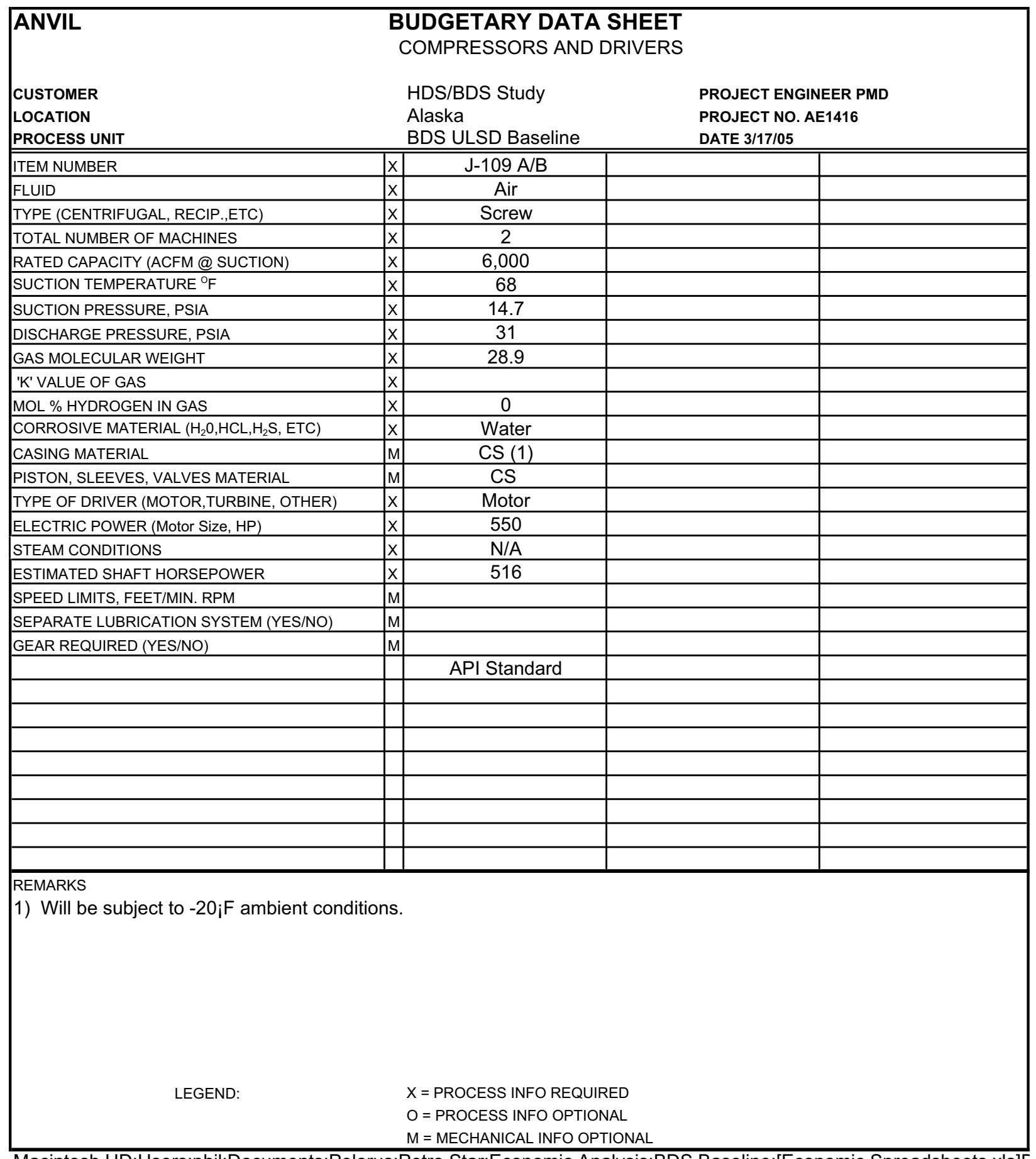

Macintosh HD:Users:phil:Documents:Pelorus:Petro Star:Economic Analysis:BDS Baseline:[Economic Spreadsheets.xls]E 


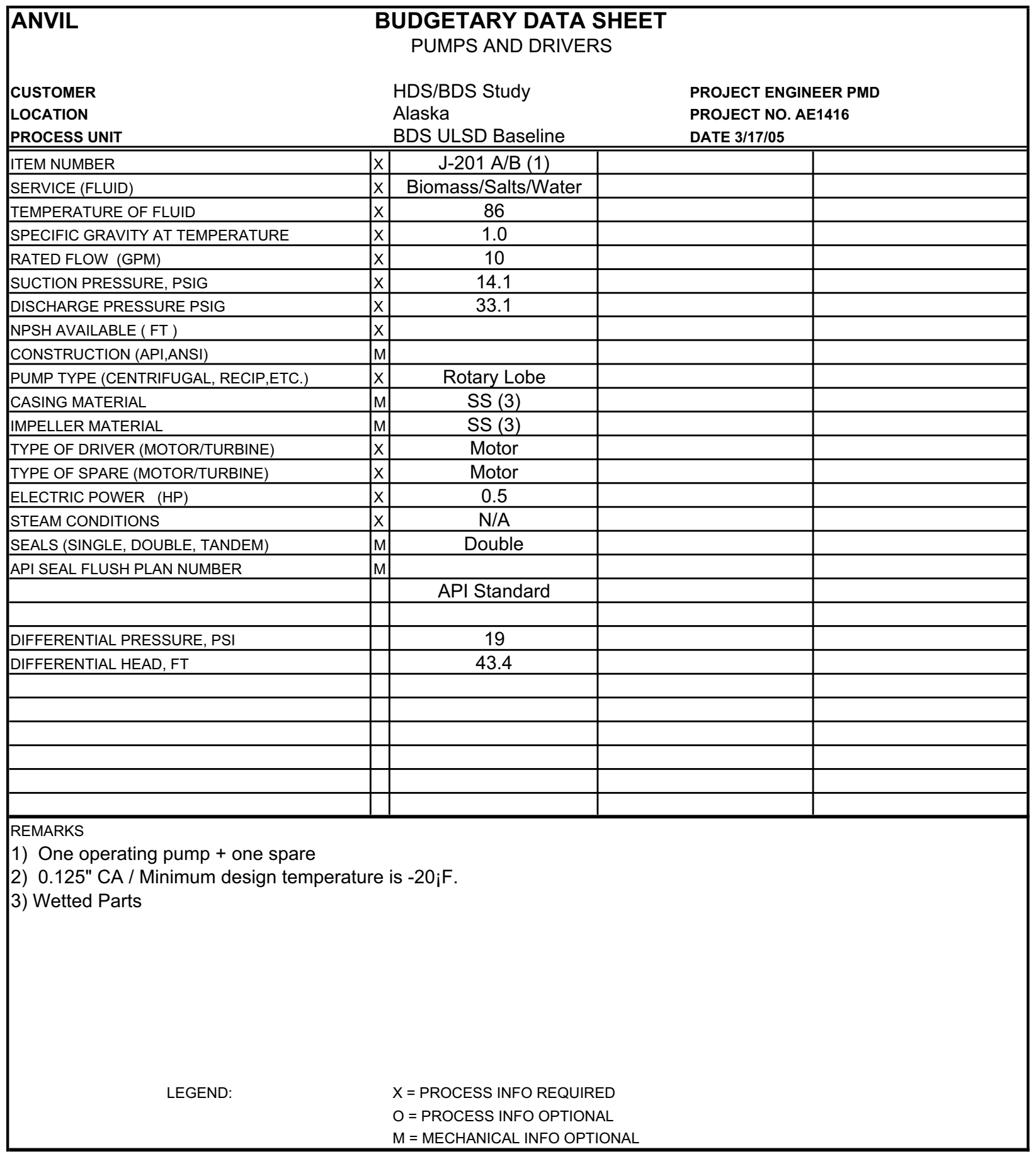

Macintosh HD:Users:phil:Documents:Pelorus:Petro Star:Economic Analysis:BDS Baseline:[Economic Spreadsheets.xls]BI 


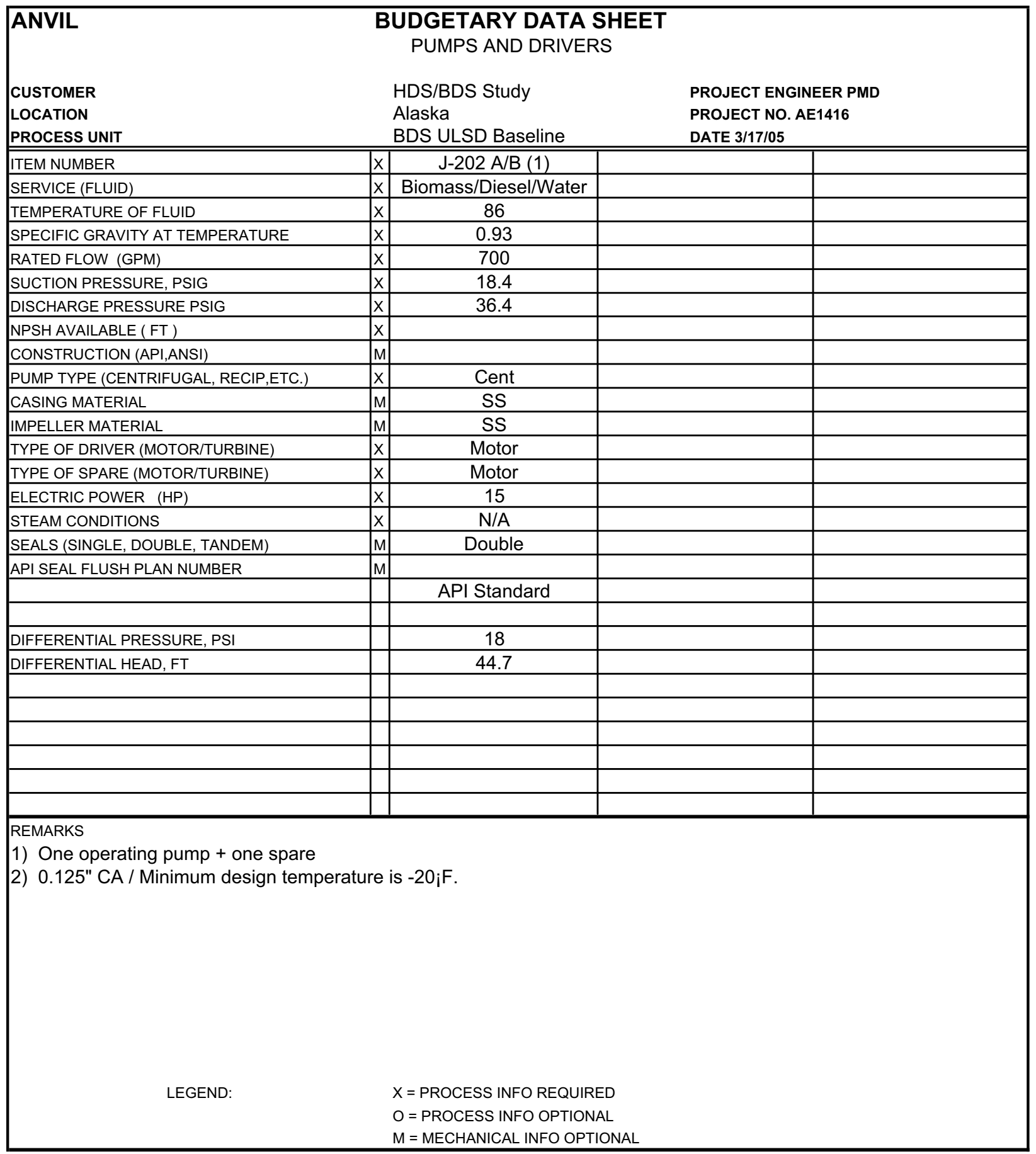

Macintosh HD:Users:phil:Documents:Pelorus:Petro Star:Economic Analysis:BDS Baseline:[Economic Spreadsheets.xls]BI 


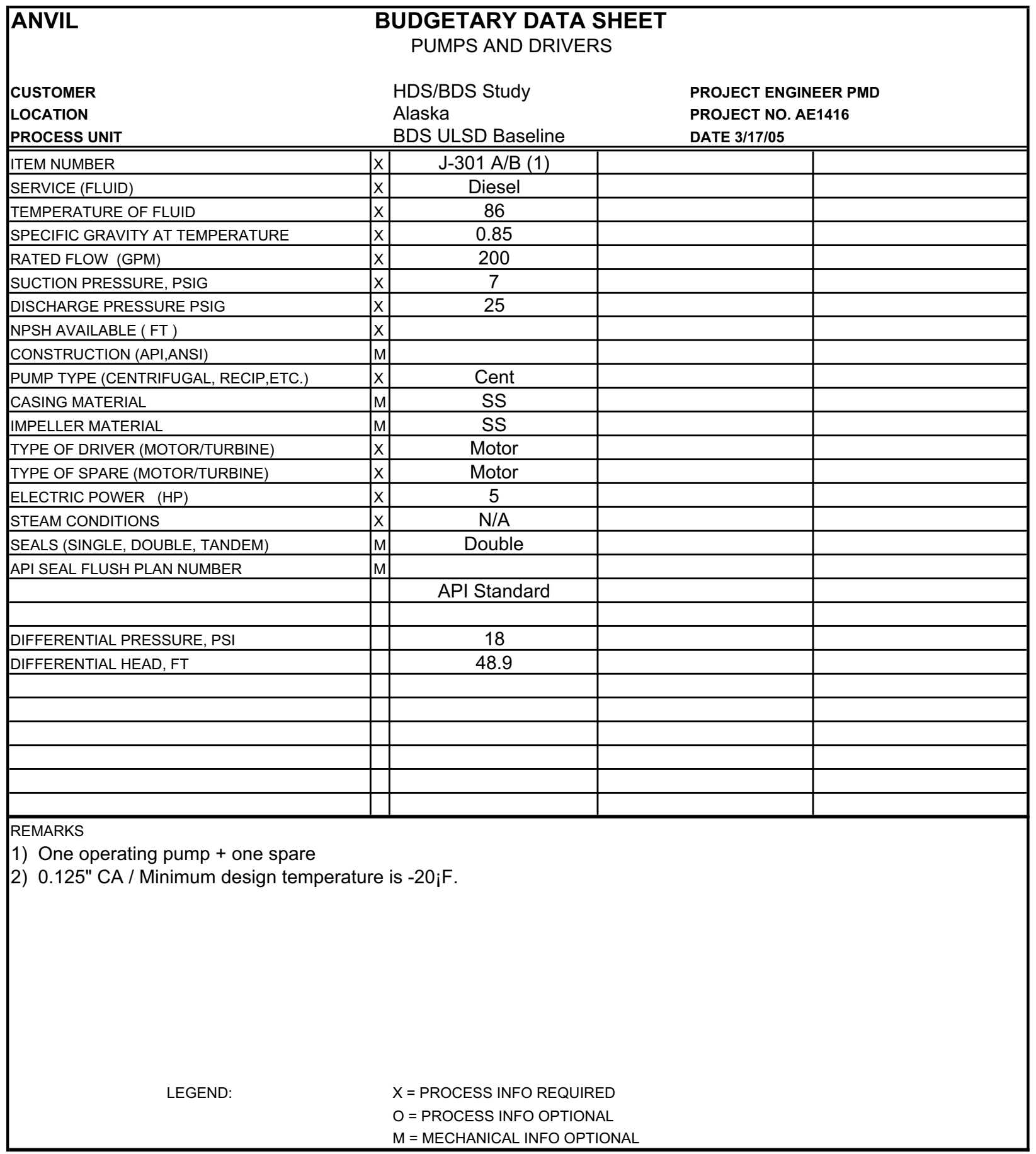

Macintosh HD:Users:phil:Documents:Pelorus:Petro Star:Economic Analysis:BDS Baseline:[Economic Spreadsheets.xls]BI 


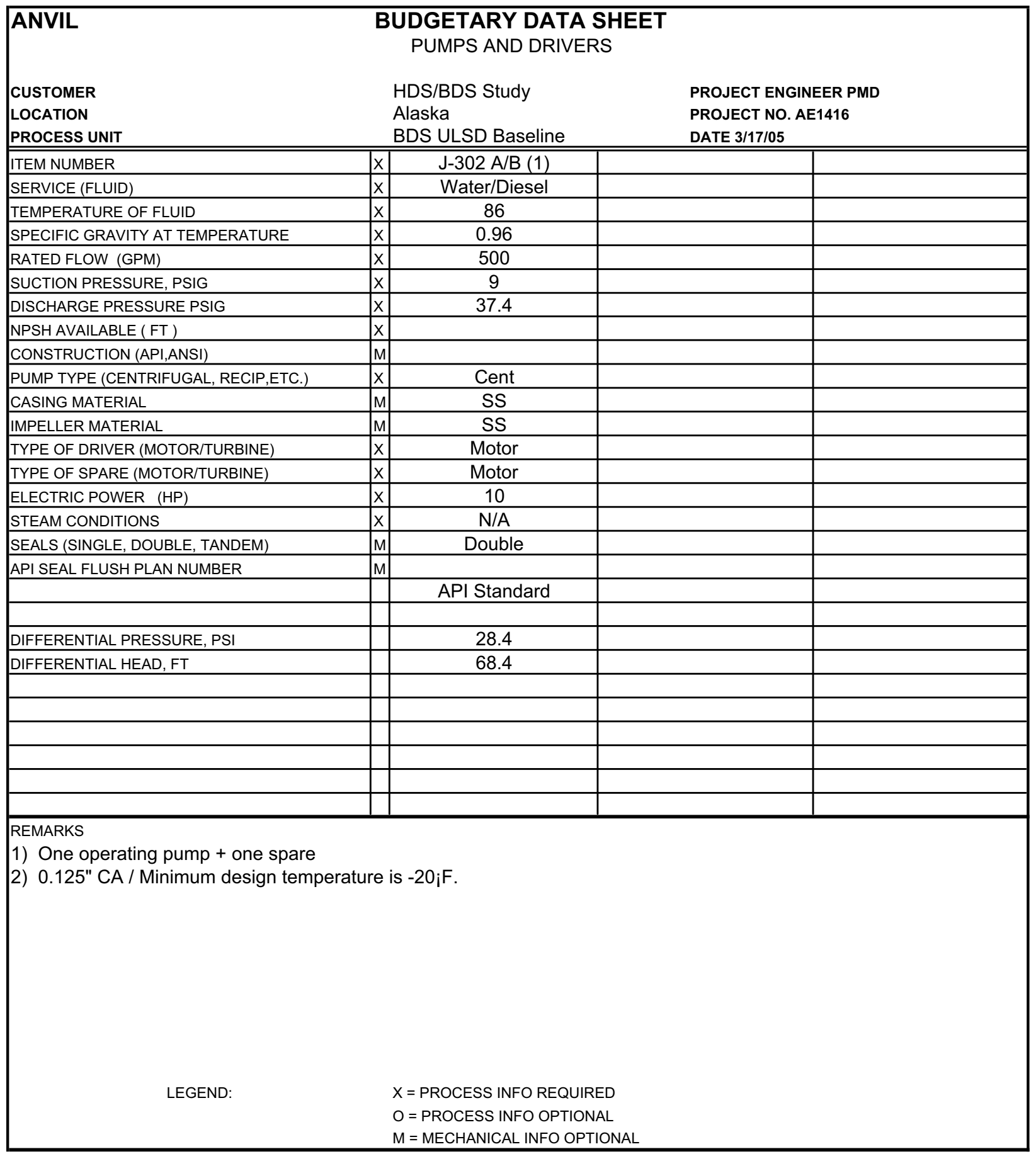

Macintosh HD:Users:phil:Documents:Pelorus:Petro Star:Economic Analysis:BDS Baseline:[Economic Spreadsheets.xls]BI 


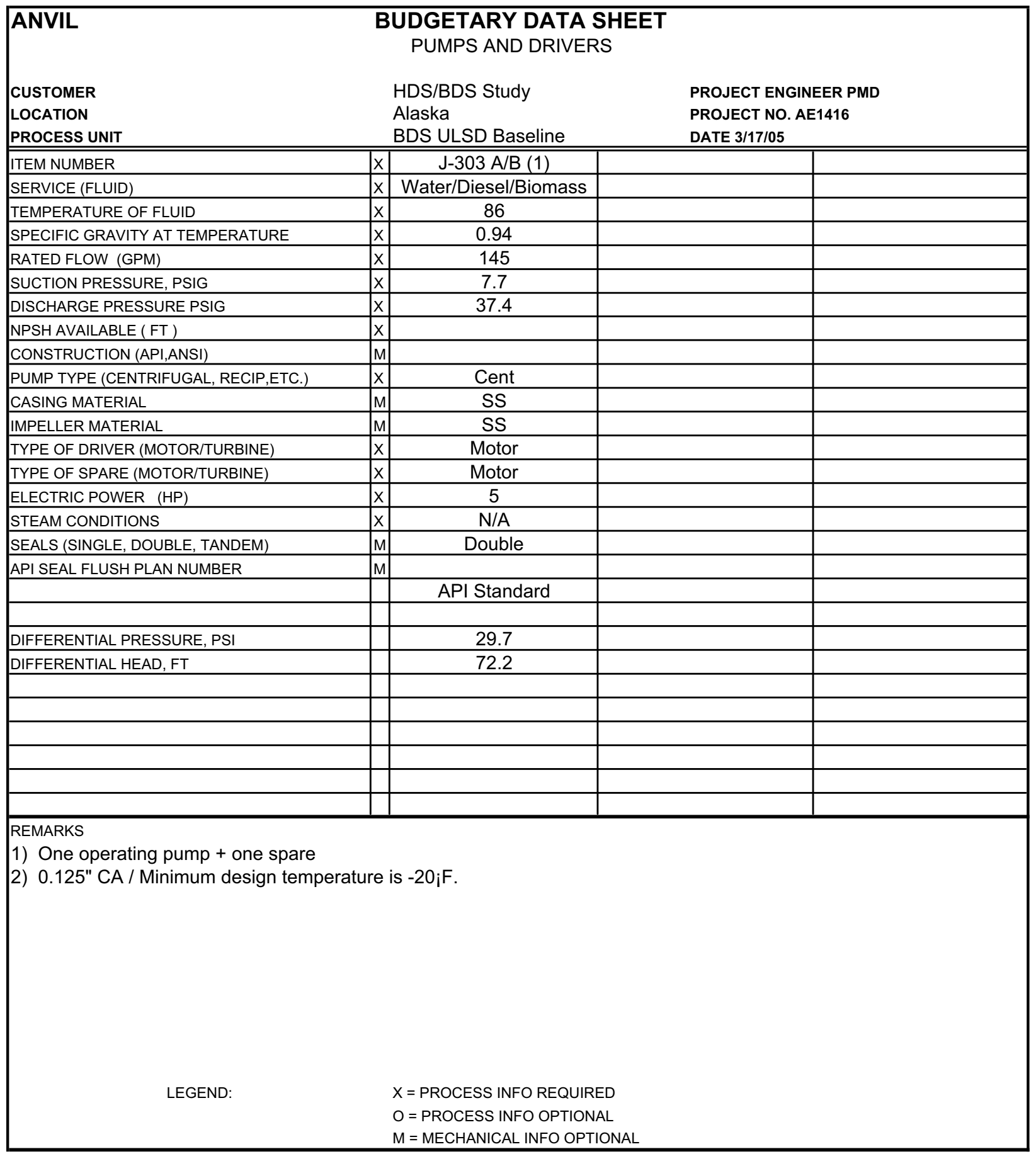

Macintosh HD:Users:phil:Documents:Pelorus:Petro Star:Economic Analysis:BDS Baseline:[Economic Spreadsheets.xls]BI 


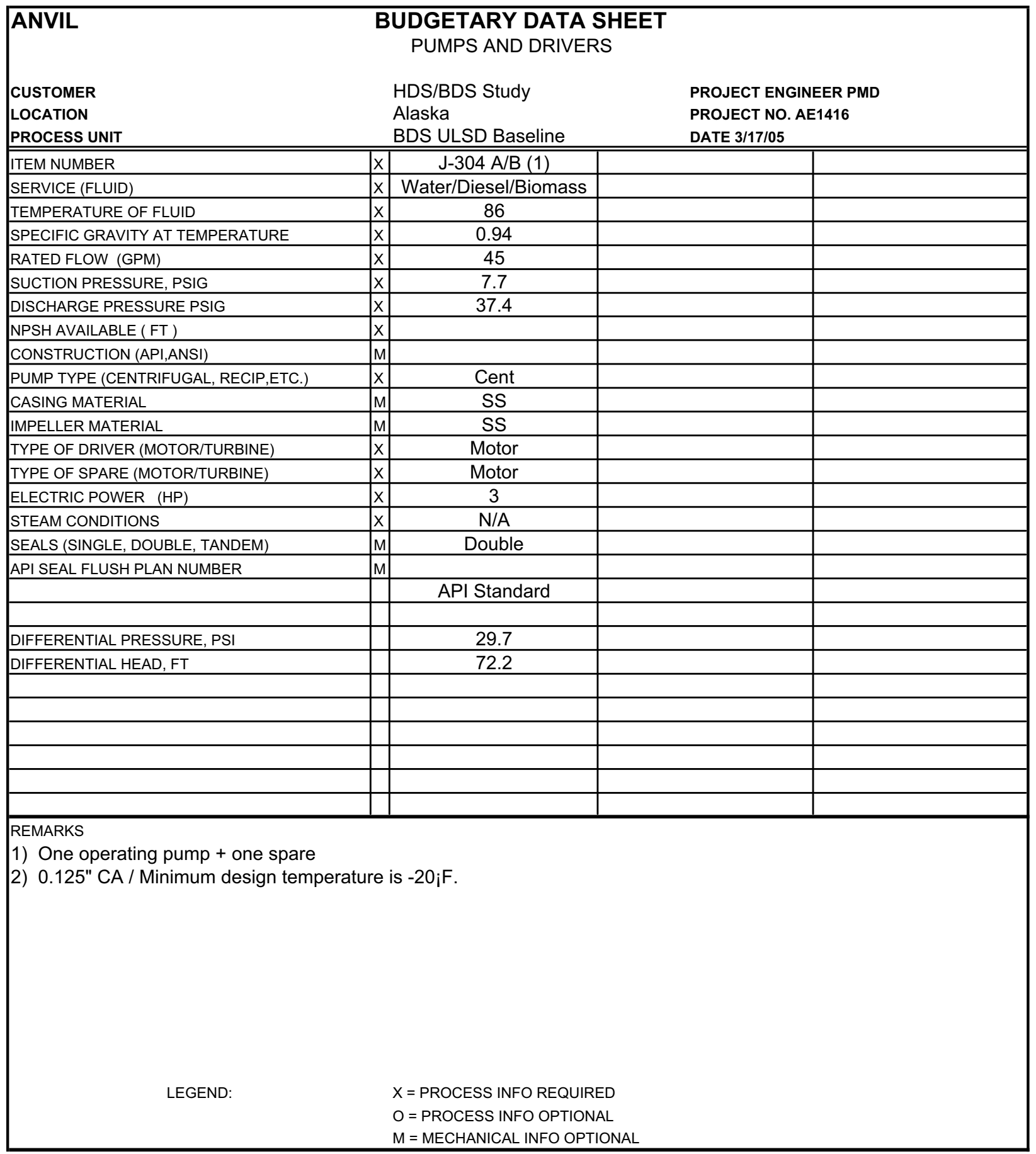

Macintosh HD:Users:phil:Documents:Pelorus:Petro Star:Economic Analysis:BDS Baseline:[Economic Spreadsheets.xls]BI 


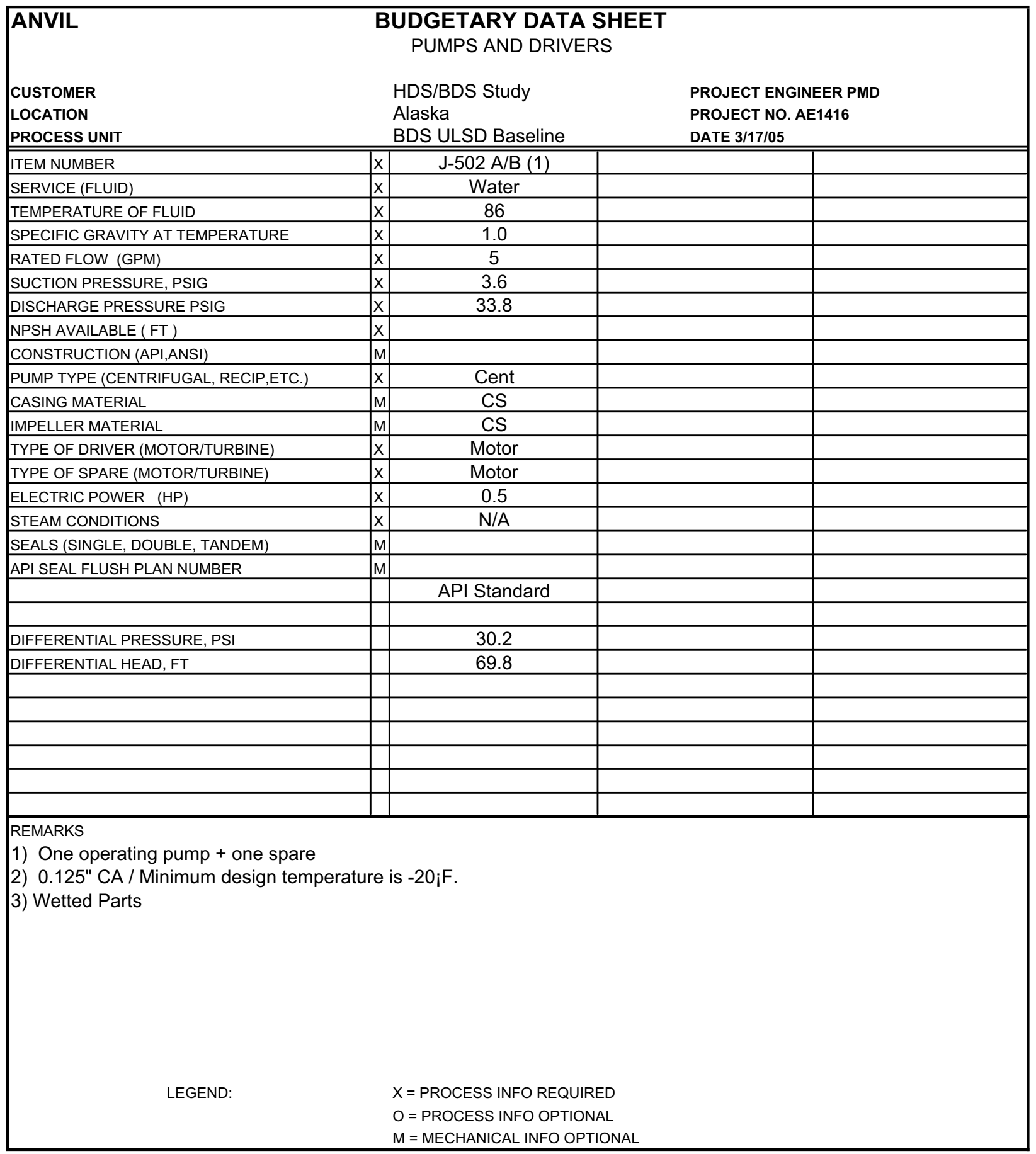

Macintosh HD:Users:phil:Documents:Pelorus:Petro Star:Economic Analysis:BDS Baseline:[Economic Spreadsheets.xls]BI 


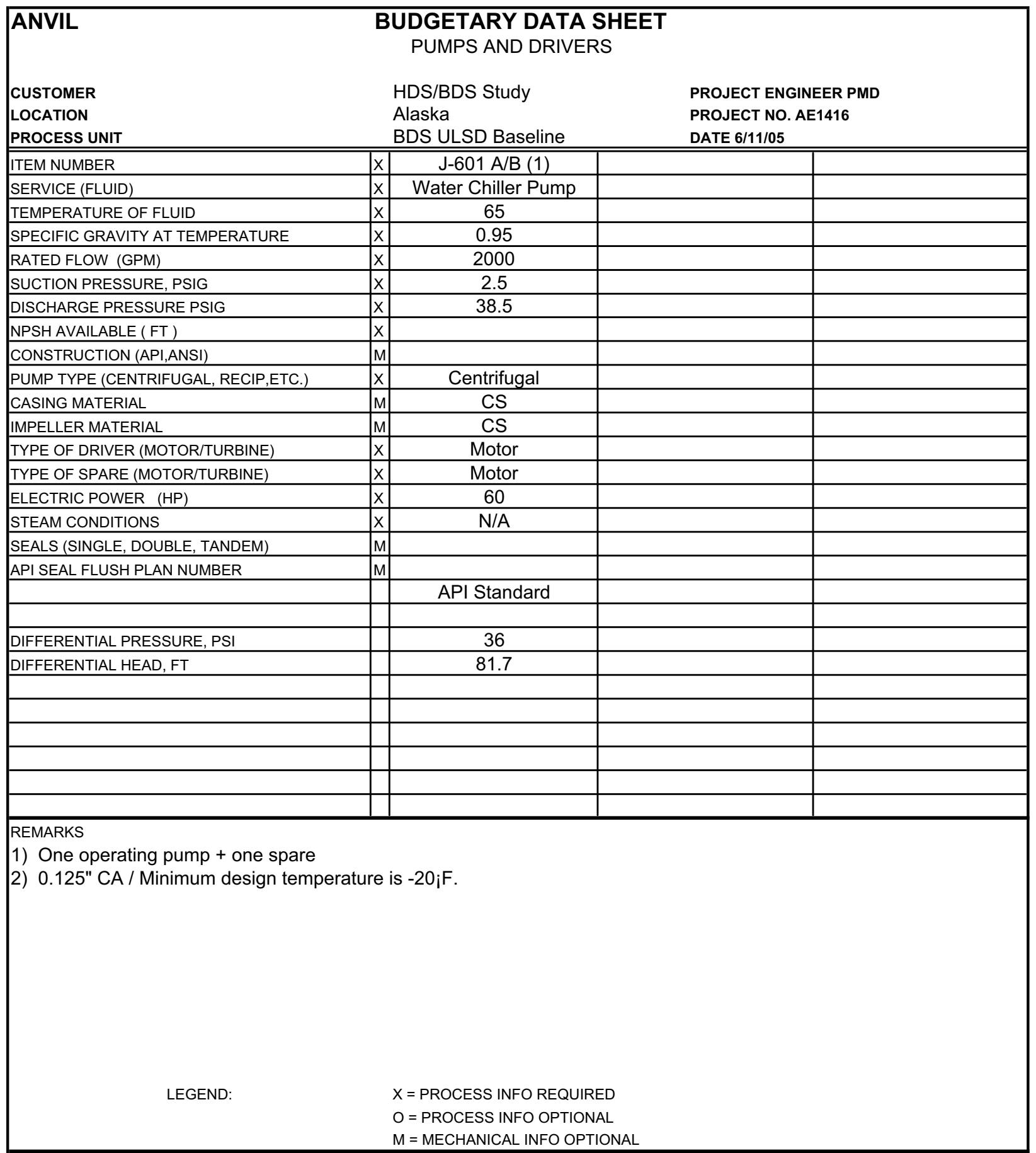

Macintosh HD:Users:phil:Documents:Pelorus:Petro Star:Economic Analysis:[Budgetary Sheets Add.xls]J-601(D) 


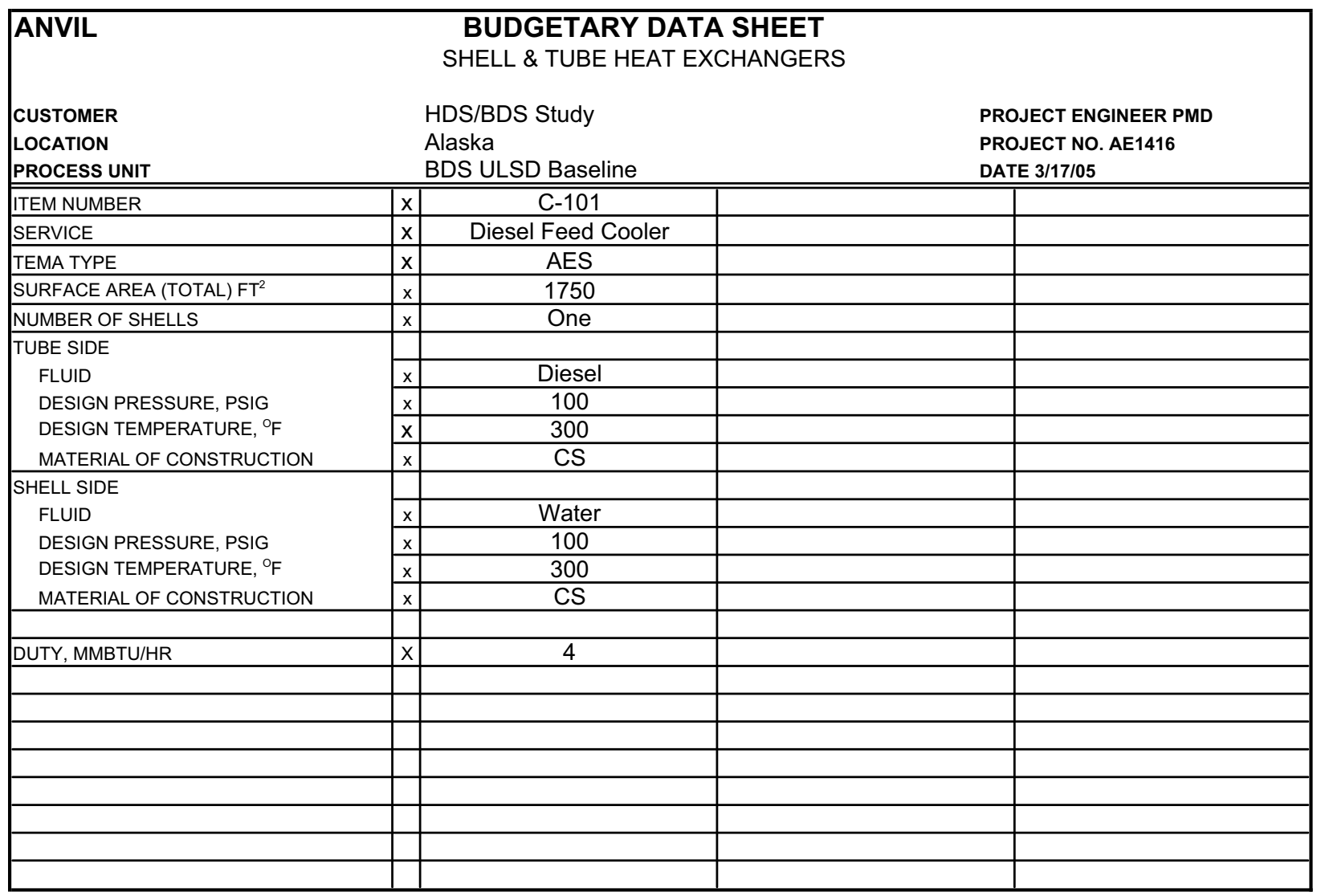




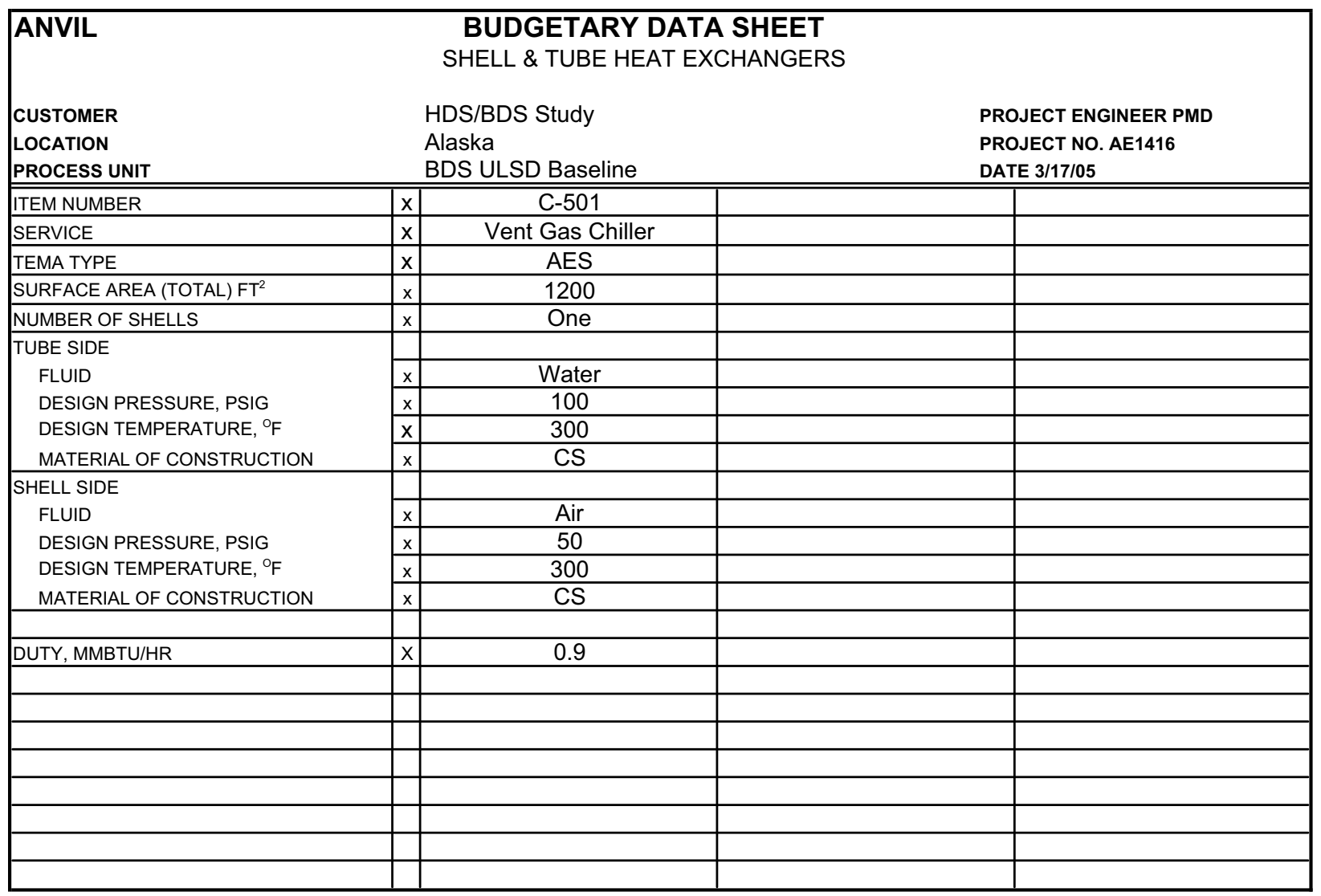




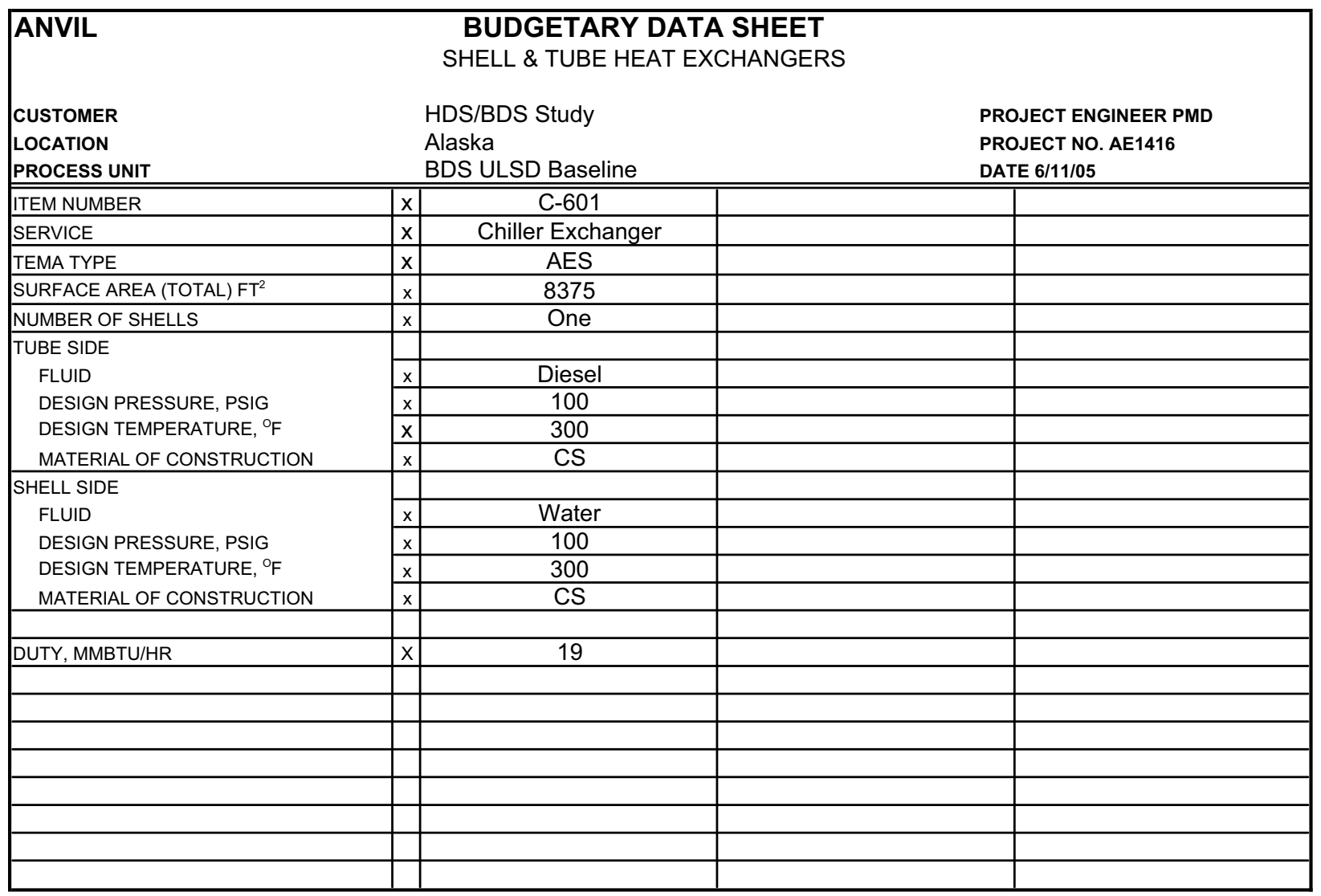




\begin{tabular}{|c|c|c|c|}
\hline ANVIL & $\begin{array}{l}\text { BUDGETARY DA } \\
\text { FILTER }\end{array}$ & & \\
\hline $\begin{array}{l}\text { CUSTOMER } \\
\text { LOCATION } \\
\text { PROCESS UNIT } \\
\end{array}$ & $\begin{array}{l}\text { HDS/BDS Study } \\
\text { Alaska } \\
\text { BDS ULSD Baseline }\end{array}$ & $\begin{array}{l}\text { PROJECT ENGINEER } \\
\text { PROJECT NO. } \\
\text { DATE }\end{array}$ & $\begin{array}{l}\text { PMD } \\
\text { AE1416 } \\
2 / 22 / 05 \\
\end{array}$ \\
\hline ITEM NUMBER & L-101 & & \\
\hline SERVICE & Diesel Pre-Filter & & \\
\hline FLUID & Diesel & & \\
\hline & & & \\
\hline HOUSING LENGTH, IN & 47" & & \\
\hline HOUSING DIAMETER, IN & 8" & & \\
\hline FILTER CARTRIDGE LENGTH & $30 "$ & & \\
\hline NUMBER OF UNITS & 5 & & \\
\hline DESIGN TEMPERATURE $(\mathrm{i} F)$ & 30 & & \\
\hline DESIGN PRESSURE (PSIG) & 50 & & \\
\hline MATERIAL OF CONSTRUCTION, HOUSINCX & $316 \mathrm{~L} \mathrm{SS}$ & & \\
\hline MATERIAL OF CONSTRUCTION, ELEMENTS & Polypropylene & & \\
\hline PORE SIZE, MICRON & $1.5 \mathrm{um}$ & & \\
\hline & $X$ & & \\
\hline & $X$ & & \\
\hline & $x$ & & \\
\hline & $x$ & & \\
\hline & $\mathrm{X}$ & & \\
\hline & $\mathrm{x}$ & & \\
\hline & $\mathrm{M}$ & & \\
\hline & & & \\
\hline & $x$ & & \\
\hline & & & \\
\hline & & & \\
\hline & & & \\
\hline & & & \\
\hline & & & \\
\hline & & & \\
\hline & & & \\
\hline & & & \\
\hline
\end{tabular}




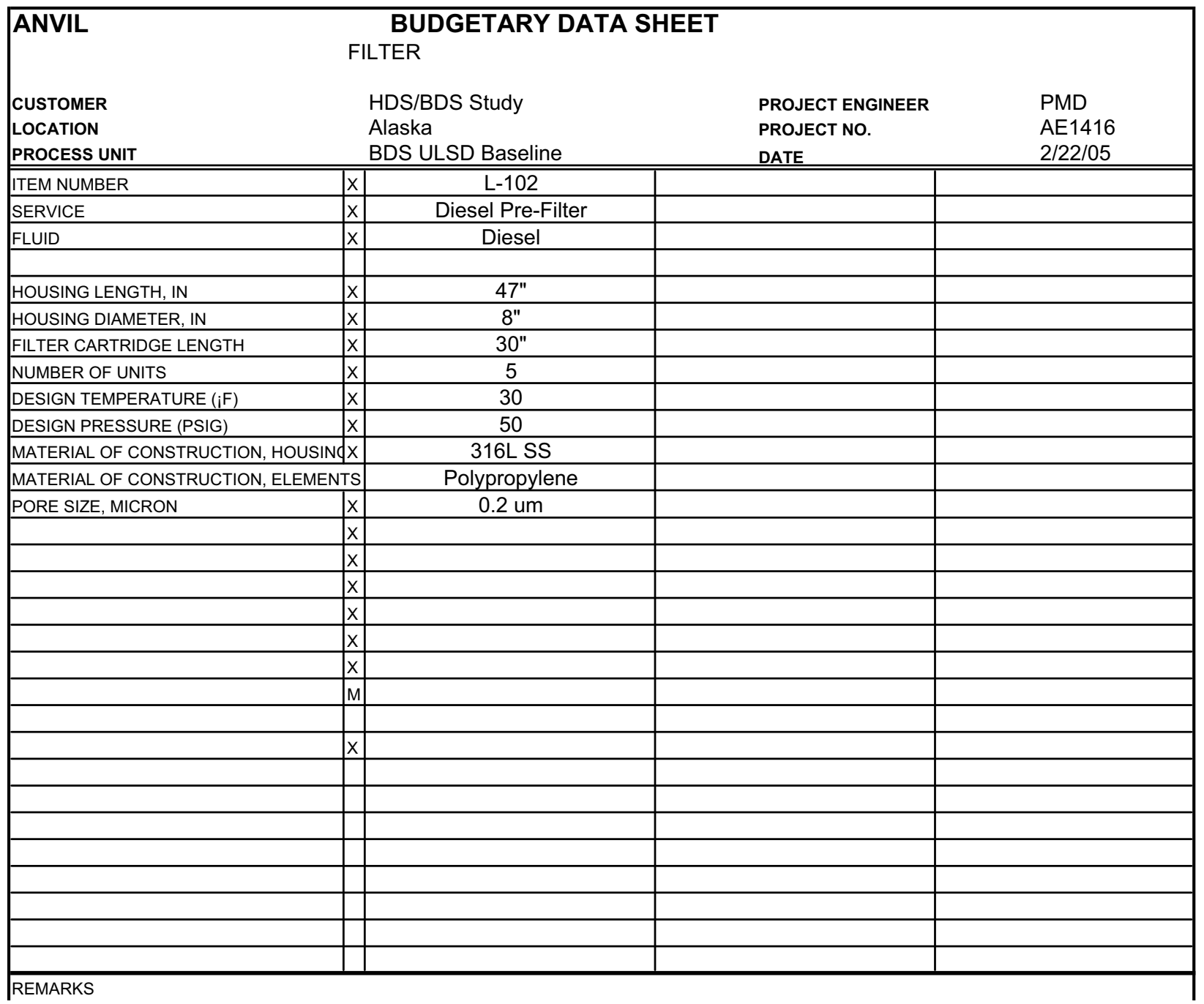




\begin{tabular}{|c|c|c|c|}
\hline ANVIL & $\begin{array}{l}\text { BUDGETARY D } \\
\text { FILTER }\end{array}$ & & \\
\hline $\begin{array}{l}\text { CUSTOMER } \\
\text { LOCATION } \\
\text { PROCESS UNIT } \\
\end{array}$ & $\begin{array}{l}\text { HDS/BDS Study } \\
\text { Alaska } \\
\text { BDS ULSD Baseline }\end{array}$ & $\begin{array}{l}\text { PROJECT ENGINEER } \\
\text { PROJECT NO. } \\
\text { DATE }\end{array}$ & $\begin{array}{l}\text { PMD } \\
\text { AE1416 } \\
2 / 22 / 05 \\
\end{array}$ \\
\hline ITEM NUMBER & L-103 & & \\
\hline SERVICE & Air Pre-Filter & & \\
\hline FLUID & Air & & \\
\hline & & & \\
\hline HOUSING LENGTH, IN & $55 "$ & & \\
\hline HOUSING DIAMETER, IN & $15^{\prime \prime}$ & & \\
\hline FILTER CARTRIDGE LENGTH & $30 "$ & & \\
\hline NUMBER OF UNITS & 5 & & \\
\hline DESIGN TEMPERATURE $(\mathrm{i} F)$ & 30 & & \\
\hline DESIGN PRESSURE (PSIG) & 50 & & \\
\hline MATERIAL OF CONSTRUCTION, HOUSINCX & $316 \mathrm{~L}$ SS & & \\
\hline MATERIAL OF CONSTRUCTION, ELEMENTS & GF & & \\
\hline PORE SIZE, MICRON & $1.0 \mathrm{um}$ & & \\
\hline & $X$ & & \\
\hline & $X$ & & \\
\hline & $x$ & & \\
\hline & $x$ & & \\
\hline & $\mathrm{X}$ & & \\
\hline & $\mathrm{x}$ & & \\
\hline & $\mathrm{M}$ & & \\
\hline & & & \\
\hline & $x$ & & \\
\hline & & & \\
\hline & & & \\
\hline & & & \\
\hline & & & \\
\hline & & & \\
\hline & & & \\
\hline & & & \\
\hline 1 & & & \\
\hline
\end{tabular}




\begin{tabular}{|c|c|c|c|}
\hline ANVIL & $\begin{array}{l}\text { BUDGETARY D } \\
\text { FILTER }\end{array}$ & & \\
\hline $\begin{array}{l}\text { CUSTOMER } \\
\text { LOCATION } \\
\text { PROCESS UNIT } \\
\end{array}$ & $\begin{array}{l}\text { HDS/BDS Study } \\
\text { Alaska } \\
\text { BDS ULSD Baseline }\end{array}$ & $\begin{array}{l}\text { PROJECT ENGINEER } \\
\text { PROJECT NO. } \\
\text { DATE }\end{array}$ & $\begin{array}{l}\text { PMD } \\
\text { AE1416 } \\
2 / 22 / 05 \\
\end{array}$ \\
\hline ITEM NUMBER & L-104 & & \\
\hline SERVICE & Air Bio-Filter & & \\
\hline FLUID & Air & & \\
\hline & & & \\
\hline HOUSING LENGTH, IN & $43 "$ & & \\
\hline HOUSING DIAMETER, IN & $15^{\prime \prime}$ & & \\
\hline FILTER CARTRIDGE LENGTH & $10 "$ & & \\
\hline NUMBER OF UNITS & 5 & & \\
\hline DESIGN TEMPERATURE $(\mathrm{i} F)$ & 30 & & \\
\hline DESIGN PRESSURE (PSIG) & 50 & & \\
\hline MATERIAL OF CONSTRUCTION, HOUSINCX & $316 \mathrm{~L}$ SS & & \\
\hline MATERIAL OF CONSTRUCTION, ELEMENTS & PTFE & & \\
\hline PORE SIZE, MICRON & $0.01 \mathrm{um}$ & & \\
\hline & $X$ & & \\
\hline & $X$ & & \\
\hline & $x$ & & \\
\hline & $x$ & & \\
\hline & $\mathrm{X}$ & & \\
\hline & $\mathrm{x}$ & & \\
\hline & $\mathrm{M}$ & & \\
\hline & & & \\
\hline & $x$ & & \\
\hline & & & \\
\hline & & & \\
\hline & & & \\
\hline & & & \\
\hline & & & \\
\hline & & & \\
\hline & & & \\
\hline & & & \\
\hline
\end{tabular}




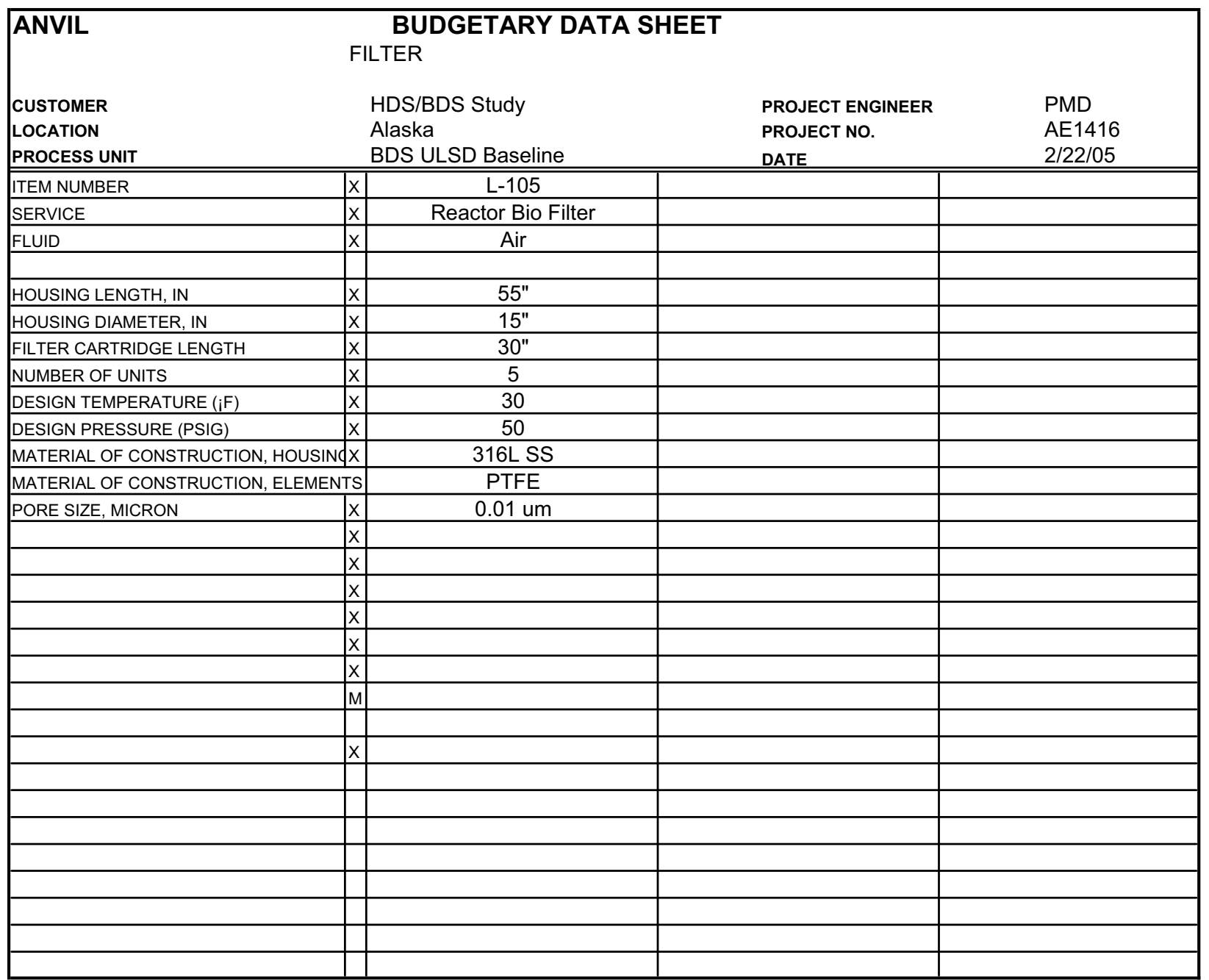




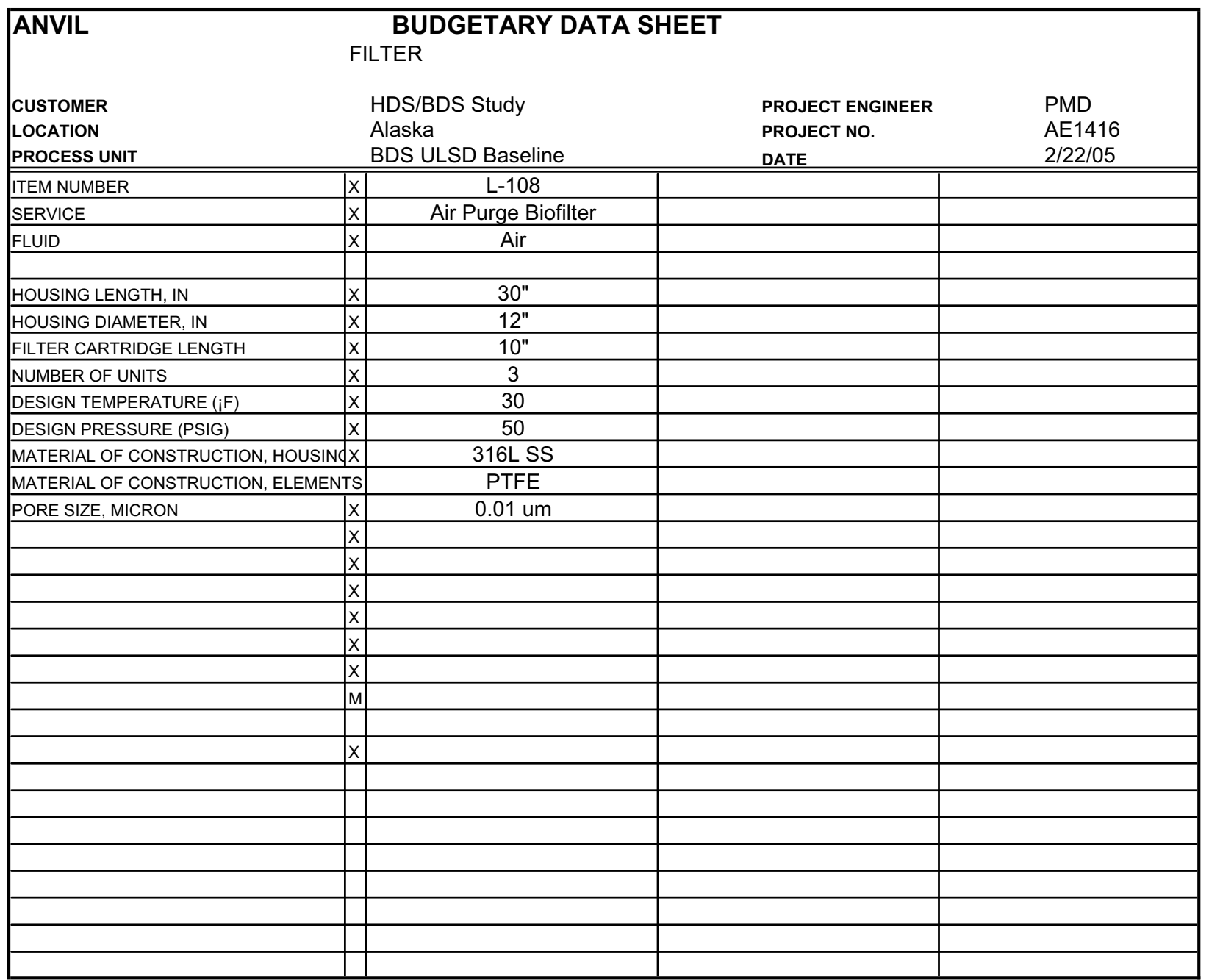




\begin{tabular}{|c|c|c|c|}
\hline ANVIL & $\begin{array}{l}\text { BUDGETARY DA } \\
\text { FILTER }\end{array}$ & & \\
\hline $\begin{array}{l}\text { CUSTOMER } \\
\text { LOCATION } \\
\text { PROCESS UNIT } \\
\end{array}$ & $\begin{array}{l}\text { HDS/BDS Study } \\
\text { Alaska } \\
\text { BDS ULSD Baseline }\end{array}$ & $\begin{array}{l}\text { PROJECT ENGINEER } \\
\text { PROJECT NO. } \\
\text { DATE }\end{array}$ & $\begin{array}{l}\text { PMD } \\
\text { AE1416 } \\
2 / 22 / 05 \\
\end{array}$ \\
\hline ITEM NUMBER & $\mathrm{L}-111$ & & \\
\hline SERVICE & Ethanol Bio Filter & & \\
\hline FLUID & Ethanol/Water & & \\
\hline & 10" & & \\
\hline \begin{tabular}{|l} 
HOUSING LENGTH, IN \\
HOUSING DIAMETER, IN
\end{tabular} & $2.5^{\prime \prime}$ & & \\
\hline FILTER CARTRIDGE LENGTH & $5 "$ & & \\
\hline NUMBER OF UNITS & 1 & & \\
\hline DESIGN TEMPERATURE $(\mathrm{i} F)$ & 30 & & \\
\hline DESIGN PRESSURE (PSIG) & 50 & & \\
\hline MATERIAL OF CONSTRUCTION, HOUSINCX & $316 \mathrm{~L} \mathrm{SS}$ & & \\
\hline MATERIAL OF CONSTRUCTION, ELEMENTS & Polyethersulfone & & \\
\hline PORE SIZE, MICRON & $0.2 \mathrm{um}$ & & \\
\hline & $x$ & & \\
\hline & $x$ & & \\
\hline & $x$ & & \\
\hline & $\mathrm{x}$ & & \\
\hline & $x$ & & \\
\hline & $x$ & & \\
\hline & $\mathrm{M}$ & & \\
\hline & & & \\
\hline & $\mathrm{X}$ & & \\
\hline & & & \\
\hline & & & \\
\hline & & & \\
\hline & & & \\
\hline & & & \\
\hline & & & \\
\hline & & & \\
\hline & & & \\
\hline
\end{tabular}




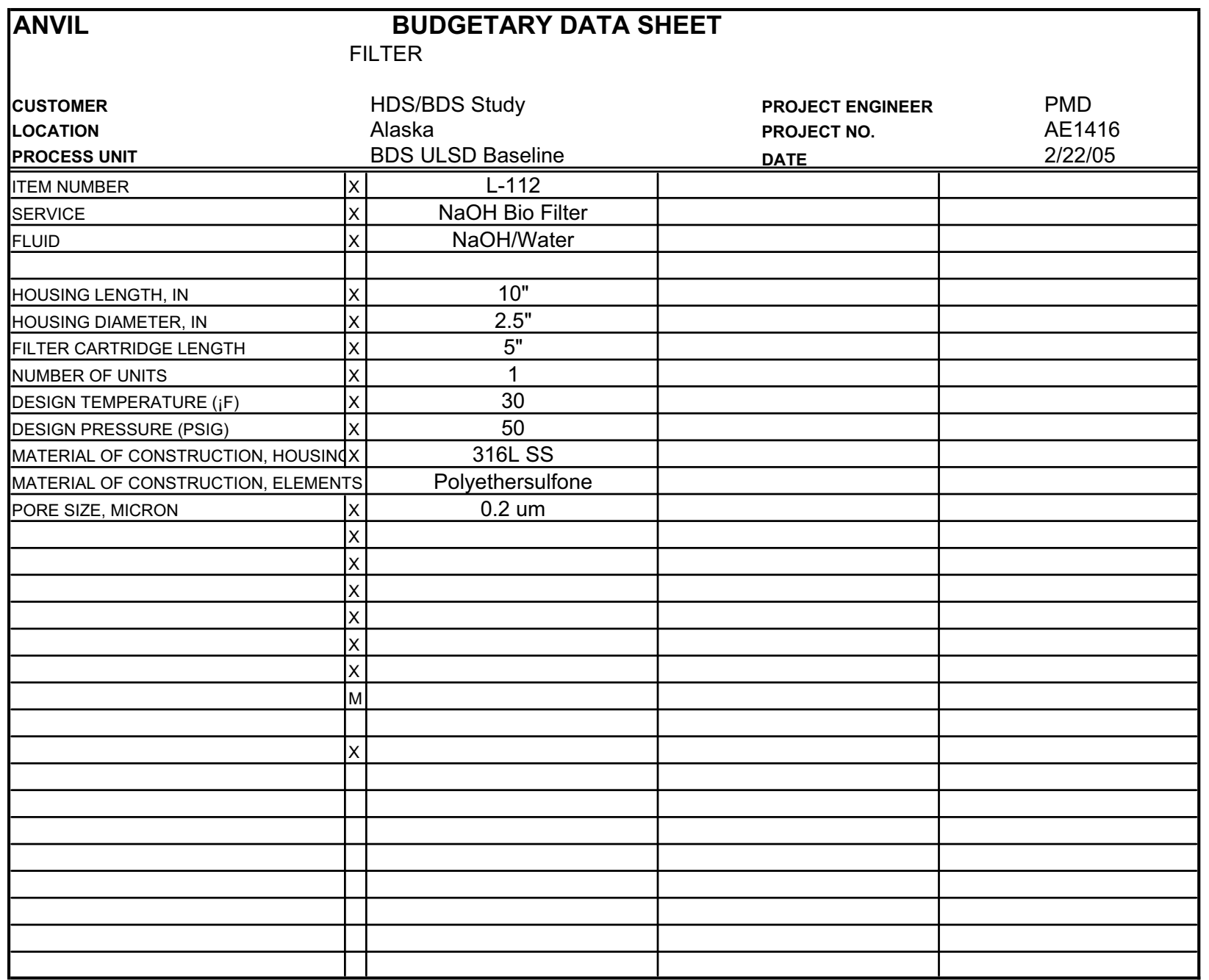




\begin{tabular}{|c|c|c|c|}
\hline ANVIL & $\begin{array}{l}\text { BUDGETARY DA } \\
\text { FILTER }\end{array}$ & & \\
\hline $\begin{array}{l}\text { CUSTOMER } \\
\text { LOCATION } \\
\text { PROCESS UNIT } \\
\end{array}$ & $\begin{array}{l}\text { HDS/BDS Study } \\
\text { Alaska } \\
\text { BDS ULSD Baseline }\end{array}$ & $\begin{array}{l}\text { PROJECT ENGINEER } \\
\text { PROJECT NO. } \\
\text { DATE }\end{array}$ & $\begin{array}{l}\text { PMD } \\
\text { AE1416 } \\
2 / 22 / 05 \\
\end{array}$ \\
\hline ITEM NUMBER & L-113 & & \\
\hline SERVICE & Water Pre Filter & & \\
\hline FLUID & Water & & \\
\hline & & & \\
\hline HOUSING LENGTH, IN & $38 "$ & & \\
\hline HOUSING DIAMETER, IN & $8 "$ & & \\
\hline FILTER CARTRIDGE LENGTH & $20 "$ & & \\
\hline NUMBER OF UNITS & 3 & & \\
\hline DESIGN TEMPERATURE $(\mathrm{i} F)$ & 30 & & \\
\hline DESIGN PRESSURE (PSIG) & 50 & & \\
\hline MATERIAL OF CONSTRUCTION, HOUSINCX & $316 \mathrm{~L} \mathrm{SS}$ & & \\
\hline MATERIAL OF CONSTRUCTION, ELEMENTS & Polypropylene & & \\
\hline PORE SIZE, MICRON & $1.5 \mathrm{um}$ & & \\
\hline & $X$ & & \\
\hline & $X$ & & \\
\hline & $x$ & & \\
\hline & $x$ & & \\
\hline & $\mathrm{X}$ & & \\
\hline & $\mathrm{x}$ & & \\
\hline & $\mathrm{M}$ & & \\
\hline & & & \\
\hline & $x$ & & \\
\hline & & & \\
\hline & & & \\
\hline & & & \\
\hline & & & \\
\hline & & & \\
\hline & & & \\
\hline & & & \\
\hline & & & \\
\hline
\end{tabular}




\begin{tabular}{|c|c|c|c|}
\hline ANVIL & $\begin{array}{l}\text { BUDGETARY DA } \\
\text { FILTER }\end{array}$ & & \\
\hline $\begin{array}{l}\text { CUSTOMER } \\
\text { LOCATION } \\
\text { PROCESS UNIT } \\
\end{array}$ & $\begin{array}{l}\text { HDS/BDS Study } \\
\text { Alaska } \\
\text { BDS ULSD Baseline }\end{array}$ & $\begin{array}{l}\text { PROJECT ENGINEER } \\
\text { PROJECT NO. } \\
\text { DATE }\end{array}$ & $\begin{array}{l}\text { PMD } \\
\text { AE1416 } \\
3 / 17 / 05 \\
\end{array}$ \\
\hline ITEM NUMBER & $\overline{L L-113}$ & & \\
\hline SERVICE & Water Bio Filter & & \\
\hline FLUID & Water & & \\
\hline & & & \\
\hline HOUSING LENGTH, IN & $38 "$ & & \\
\hline HOUSING DIAMETER, IN & 8" & & \\
\hline FILTER CARTRIDGE LENGTH & $20 "$ & & \\
\hline NUMBER OF UNITS & 3 & & \\
\hline DESIGN TEMPERATURE $(\mathrm{i} F)$ & 30 & & \\
\hline DESIGN PRESSURE (PSIG) & 50 & & \\
\hline MATERIAL OF CONSTRUCTION, HOUSINCX & $316 \mathrm{~L} \mathrm{SS}$ & & \\
\hline MATERIAL OF CONSTRUCTION, ELEMENTS & Polypropylene & & \\
\hline PORE SIZE, MICRON & $0.2 \mathrm{um}$ & & \\
\hline & $X$ & & \\
\hline & $X$ & & \\
\hline & $x$ & & \\
\hline & $x$ & & \\
\hline & $\mathrm{X}$ & & \\
\hline & $\mathrm{x}$ & & \\
\hline & $\mathrm{M}$ & & \\
\hline & & & \\
\hline & $x$ & & \\
\hline & & & \\
\hline & & & \\
\hline & & & \\
\hline & & & \\
\hline & & & \\
\hline & & & \\
\hline & & & \\
\hline & & & \\
\hline
\end{tabular}




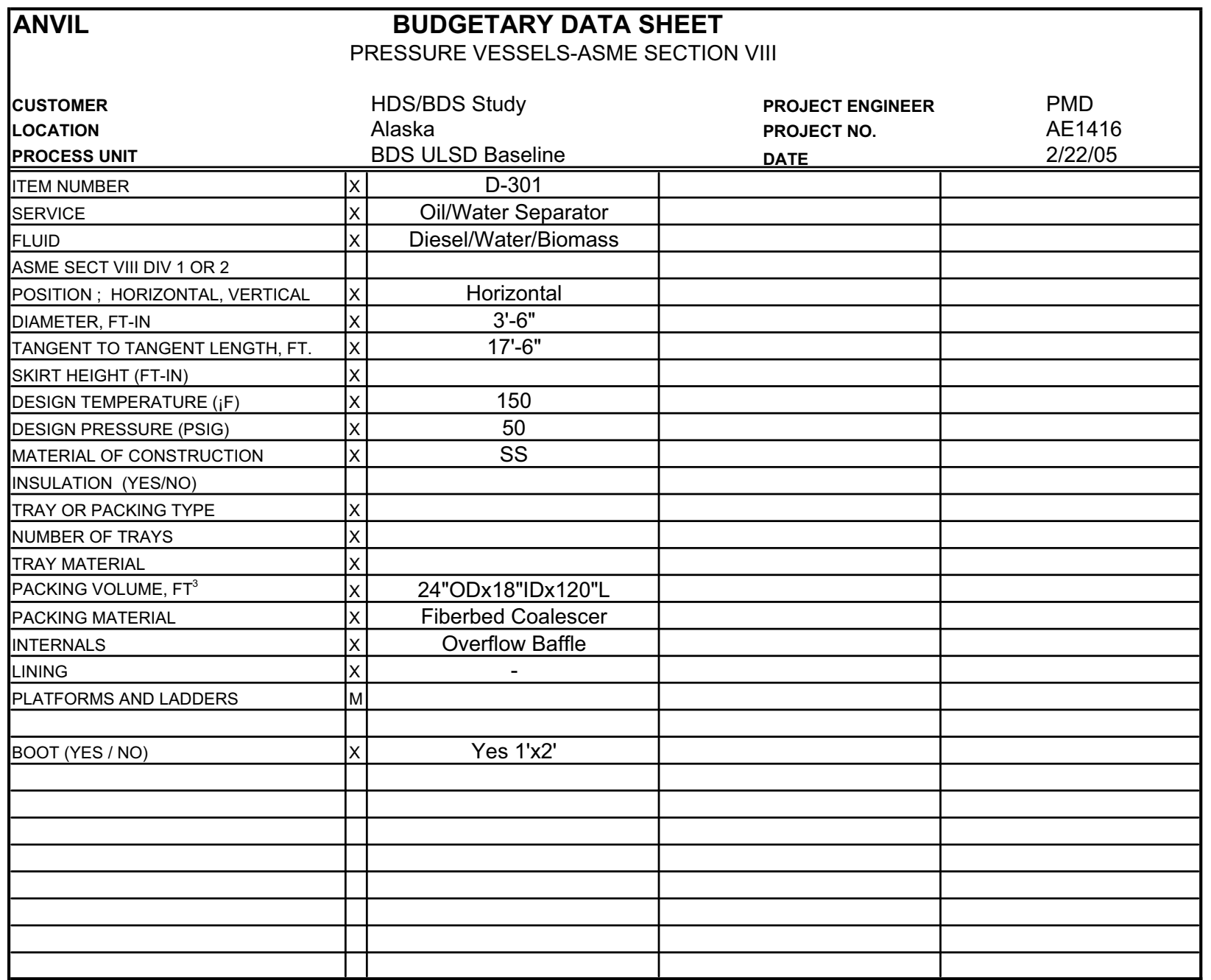




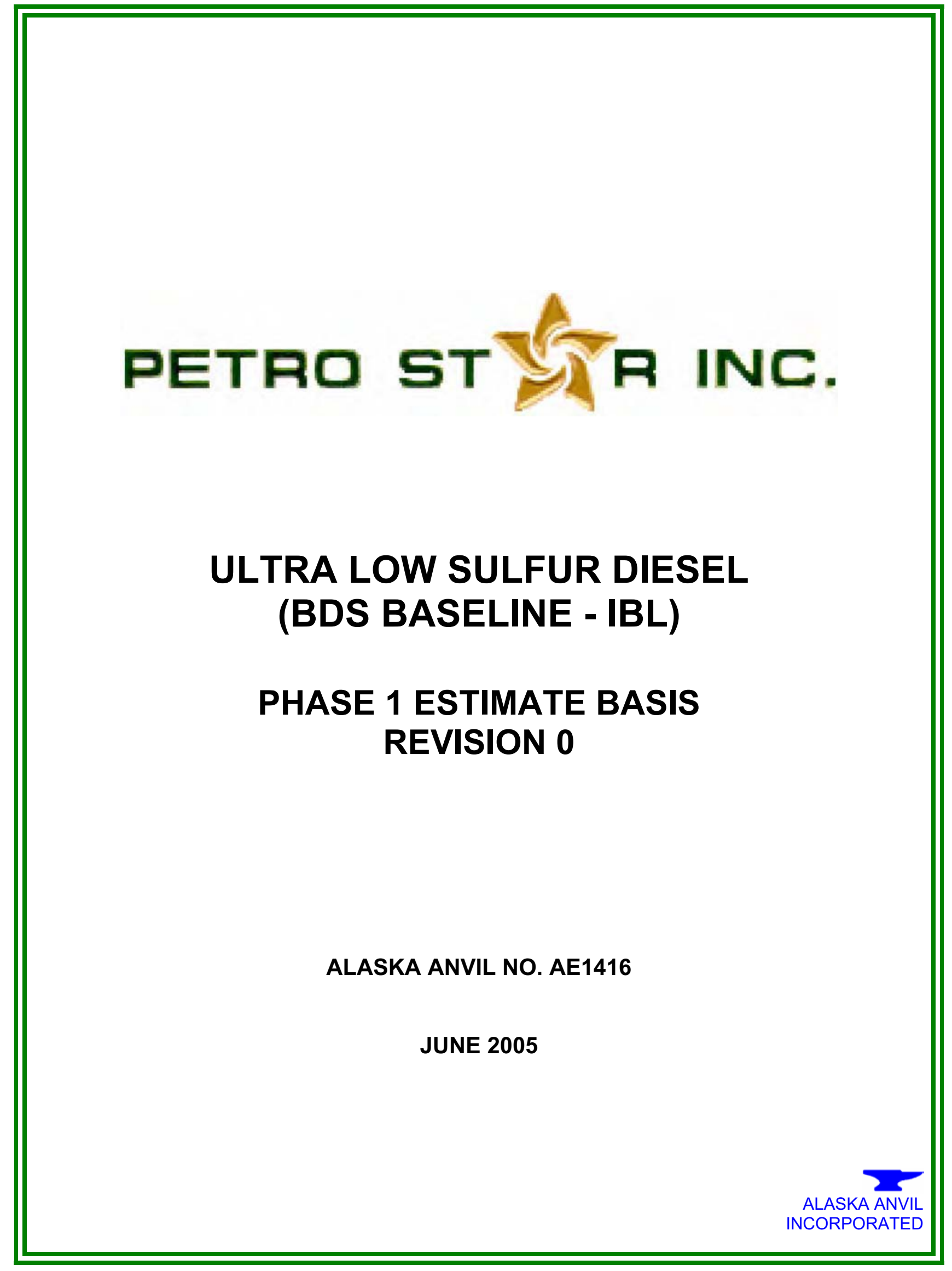




\section{ESTIMATE BASIS GOAL}

This Estimate Basis identifies information, qualifications, exceptions, and assumptions used in developing the cost estimate.

\section{ESTIMATE BASIS PURPOSE}

During the estimate review process, the project team uses the Estimate Basis for the following purposes:

- As a checklist of items to consider during estimate preparation.

- To document what is included and not included in the cost estimate.

- To assess cost risks of estimate components.

- As part of the decision support package for assessing the BDS process feasibility.

\section{GENERAL INFORMATION}

- The purpose of the project estimate is to determine if the ULSD BDS process is economically viable as a standalone process or in combination with an HDS Unit. This estimate addresses the BDS standalone process.

- $\quad$ Estimate type:

- The estimate was developed using equipment based factored estimates for Inside Battery Limits (IBL) costs.

- Most of the equipment pricing in the Diesel Biodesulfurization Unit was derived from the ICARUS estimating program.

- Pricing for equipment marked with an asterisk* in the estimate was provided by Pelorus.

- Outside Battery Limits (OBL) costs have been excluded from this estimate.

- $\quad$ The project will be installed in a brownfield location within a Valdez Alaska Refinery.

\section{PROCESS BASIS}

\section{Facility Data}

- $\quad$ Facility type - Ultra Low Sulfur Diesel Treating Complex, which includes:

- Diesel Biodesulfurization Unit

\section{Design Basis}

Product specification - Feed 6,000 bpd of untreated diesel to produce 10-ppmw sulfur maximum ultra low sulfur diesel.

\section{COST BASIS}

\section{Labor, Indirects, Equipment, and Bulk Materials}

- Included in the equipment factor. 


\section{Project Services}

- BDS - estimated based on 15 percent of TIC.

\section{Owner Services}

Not included in the TIC cost. Historically, owner services will cost from 5 to 7 percent of TIC, not including licensing, royalties, or catalyst.

\section{Escalation}

Project is based on 2005 costs. No escalation is included.

\section{Location Factor}

All costs for this estimate have been developed from a U.S. Gulf Coast (USGC) basis. No location factor is included.

\section{Other Costs}

- Catalyst and chemical initial charge has been added as an additional line item.

\section{ASSUMPTIONS}

- Process licensing and royalty costs are not included.

- Assumes fully installed pump spares, but no warehouse spares. 
Final Technical Progress Report DOE Award No. DE-FC26-02NT15340

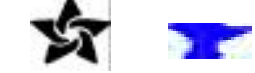

CLIENT: Petrostar

PROJECT: BDS Baseline

STAGE: Phase 1

PROJECT DESCRIPTION:

PROJECT COST \& SCHEDULE ESTIMATE SUMMARY

CLIENT PROJECT NO.:

ANVIL PROJECT NO.: AE1416

REV NO.: 1

PROJECT RISKS:
CLIENT PE:

ANVIL PE: B. Johnson

Date: $6 / 17 / 05$

\begin{tabular}{|l|l|}
\hline PROJECT DESCRIPTION: & PROJECT RISKS: \\
& \\
\hline
\end{tabular}

\section{PROJECT COST ESTIMATE SUMMARY}

\begin{tabular}{|l|c|}
\hline COST ESTIMATE STRUCTURE & $\begin{array}{c}\text { TOTAL PROJECT } \\
\text { COST - USGC }\end{array}$ \\
$\begin{array}{l}\text { COST ESTIMATE PARAMETERS } \\
\text { Estimate Classification } \\
\text { Estimating Method }\end{array}$ & Phase 1 \\
$\begin{array}{l}\text { COST ESTIMATE SUMMARY } \\
\text { Expected Cost }(\$ M M)\end{array}$ & Factored \\
$\begin{array}{l}\text { High Range }(\$ M M) \\
\text { Low Range }(\$ M M)\end{array}$ & \\
\hline
\end{tabular}

\section{PROJECT COST ESTIMATE ANALYSIS}

Total Project Expected Cost Component Analysis
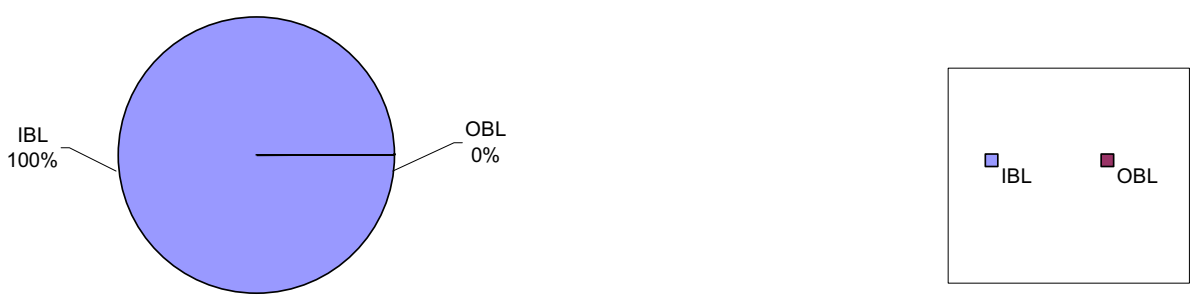

Total Project Cost Profile (\$MM)

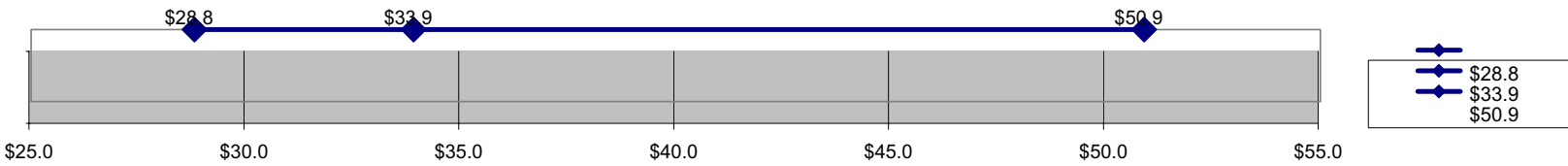

PROJECT SCHEDULE ESTIMATE ANALYSIS

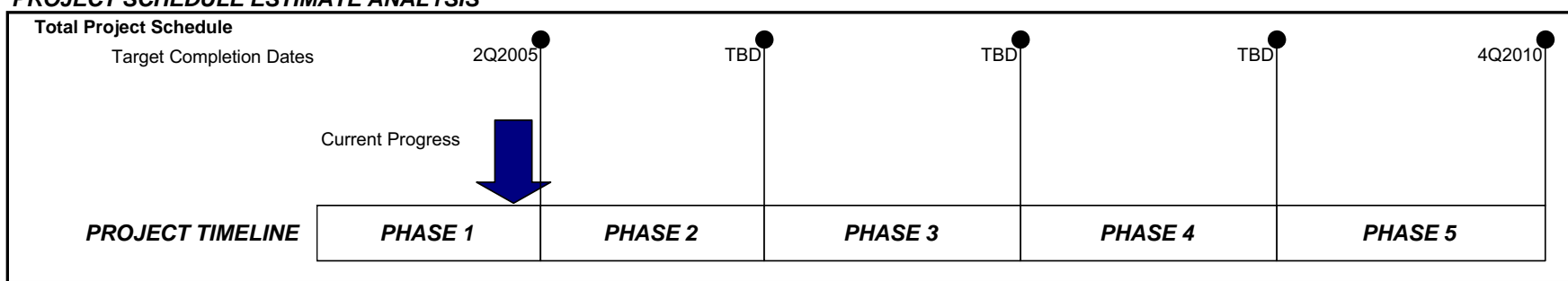


Date: $6 / 17 / 05$

REV: 0

PHASE 1 ESTIMATE - FACTORING SUMMARY

\begin{tabular}{|c|c|c|c|c|c|c|}
\hline $\begin{array}{l}\text { EQUIP. } \\
\text { ITEM NO. }\end{array}$ & $\begin{array}{l}\text { QUAN- } \\
\text { TITY }\end{array}$ & DESCRIPTION & $\begin{array}{l}\text { TOTAL } \\
\text { EQUIP. } \\
\text { COST, \$ }\end{array}$ & $\begin{array}{c}\text { FIELD } \\
\text { COST } \\
\text { MULTI- } \\
\text { PLIER }\end{array}$ & $\begin{array}{l}\text { TOTAL } \\
\text { FIELD } \\
\text { COST, \$ }\end{array}$ & NOTES \\
\hline \multicolumn{7}{|c|}{ SHELL \& TUBE HEAT EXCHANGERS } \\
\hline C-101 & 1 & Diesel Feed Cooler & $\$ 31,500$ & 4.0 & $\$ 126,000$ & $\begin{array}{l}1 \text { (ea) - Shell \& Tube TEMA type Heat Exchanger, } \\
\text { AES, 1750SF, CS }\end{array}$ \\
\hline C-501 & 1 & Vent Gas Chiller & $\$ 25,600$ & 4.0 & $\$ 102,400$ & $\begin{array}{l}1 \text { (ea) - Shell \& Tube TEMA type Heat Exchanger, } \\
\text { AES, } 1200 S F, \text { CS }\end{array}$ \\
\hline \multirow[t]{2}{*}{ C-601 } & \multirow{2}{*}{\multicolumn{2}{|c|}{ SUB-TOTAL }} & $\$ 83,000$ & 4.0 & $\$ 332,000$ & \multirow[t]{2}{*}{$\begin{array}{l}19 \text { MM BTU/Hr, } 8375 \text { SF w/ } 1 \text { shell, TEMA type AES, } \\
\text { Tubes: CS , Shell: CS }\end{array}$} \\
\hline & & & $\$ 140,000$ & & $\$ 560,000$ & \\
\hline \multicolumn{3}{|c|}{ PRESSURE VESSELS } & & & & \\
\hline D-101 & 1 & Diesel Feed Drum & $\$ 39,500$ & 4.2 & $\$ 165,900$ & 1(ea) - Horizontal Vessel, 8'0" DIA x 20'-0" T-T, CS \\
\hline D-102 & 1 & Liquid Ammonia Storage Tank & $\$ 16,400$ & 4.2 & $\$ 68,880$ & $\begin{array}{l}\text { 1(ea) - Horizontal Vessel, 4'0" DIA x 11'-0" T-T, CS, } \\
\text { Insulated(safety) }\end{array}$ \\
\hline D-201* & 1 & Fermentor Reactor & $\$ 185,000$ & 4.2 & $\$ 777,000$ & 1 (ea) - 14,000 gal airlift fermenter \\
\hline D-202* & 1 & BDS Reactor \#1 & $\$ 595,000$ & 4.2 & $\$ 2,499,000$ & 1(ea) - 45,000 gal airlift reactor, S/C Field Erected. \\
\hline$D-203^{*}$ & 1 & BDS Reactor \#2 & $\$ 595,000$ & 4.2 & $\$ 2,499,000$ & 1(ea) - 45,000 gal airlift reactor, S/C Field Erected. \\
\hline$D-204^{*}$ & 1 & BDS Reactor \#3 & $\$ 595,000$ & 4.2 & $\$ 2,499,000$ & 1(ea) - 45,000 gal airlift reactor, S/C Field Erected. \\
\hline D-301 & 1 & Diesel / Water / Biomass Separator & $\$ 112,300$ & 4.2 & $\$ 471,660$ & $\begin{array}{l}\text { 1(ea) - Horizontal Vessel, 10'3" DIA x 30'-8" T-T, } \\
\text { 304SS, w/ overflow baffle }\end{array}$ \\
\hline $\mathrm{D}-401 \mathrm{~A} / \mathrm{B}$ & 2 & Salt Drier & $\$ 62,600$ & 4.2 & $\$ 262,920$ & 2(ea) - Horizontal Vessel, 6' DIA x 17' T-T, CS \\
\hline D-501 & 1 & Diesel / Water Separator & $\$ 9,600$ & 4.2 & $\$ 40,320$ & $\begin{array}{l}\text { 1(ea) - Horizontal Vessel, 2'6" DIA x 3'-9" T-T, CS, w/ } \\
\text { overflow baffle }\end{array}$ \\
\hline \multirow[t]{2}{*}{ L-301 } & 2 & Diesel / Water / Biomass Separator & $\$ 61,600$ & 4.2 & $\$ 258,720$ & $\begin{array}{l}\text { 2(ea) - Horizontal Vessel, 316L SS, 3' 6" Dia. x 17' 6" T- } \\
\text { T, } 24 \text { "ODx18"IDx120"L Fiberbed Coalescer, Overflow } \\
\text { Baffle and 1'x2' Boot }\end{array}$ \\
\hline & & SUB-TOTAL & $\$ 2,272,000$ & & $\$ 9,542,000$ & \\
\hline
\end{tabular}


Date: $6 / 17 / 05$

REV: 0

PHASE 1 ESTIMATE - FACTORING SUMMARY

\begin{tabular}{|c|c|c|c|c|c|c|}
\hline $\begin{array}{l}\text { EQUIP. } \\
\text { ITEM NO. }\end{array}$ & $\begin{array}{l}\text { QUAN- } \\
\text { TITY } \\
\end{array}$ & DESCRIPTION & $\begin{array}{l}\text { TOTAL } \\
\text { EQUIP. } \\
\text { COST, \$ } \\
\end{array}$ & \begin{tabular}{|c|} 
FIELD \\
COST \\
MULTI- \\
PLIER \\
\end{tabular} & $\begin{array}{l}\text { TOTAL } \\
\text { FIELD } \\
\text { COST, \$ } \\
\end{array}$ & NOTES \\
\hline \multicolumn{7}{|c|}{ PACKAGED SKIDS/SYSTEMS } \\
\hline $\mathrm{D}-205^{*}$ & 1 & Seed Tank A & $\$ 94,000$ & 2.5 & $\$ 235,000$ & Packaged Seed Fermenter System \\
\hline$D-206^{*}$ & 1 & Seed Tank B & $\$ 328,000$ & 2.5 & $\$ 820,000$ & Packaged Seed Fermenter System \\
\hline $\mathrm{J}-109 \mathrm{~A} / \mathrm{B}$ & 2 & Air Compressor & $\$ 714,600$ & 2.5 & $\$ 1,786,500$ & $\begin{array}{l}2 \text { (ea) - Air Compressor, Reciprocating, 6000ACFM, CS } \\
\text { body/internals, 600HP Motor Driven }\end{array}$ \\
\hline L-109* & 1 & Glucose Sterilizer & $\$ 98,000$ & 2.5 & $\$ 245,000$ & $\begin{array}{l}\text { 1(ea) - Packaged } 50 \text { gph continuous steam sterilizer } \\
\text { system complete w/ preheater, heater, cooler, \& } \\
\text { holding coil }\end{array}$ \\
\hline L-110* & 1 & Nutrients Sterilizer & $\$ 98,000$ & 2.5 & $\$ 245,000$ & $\begin{array}{l}1 \text { (ea) - Packaged } 50 \text { gph continuous steam sterilizer } \\
\text { system complete w/ preheater, heater, cooler, \& } \\
\text { holding coil }\end{array}$ \\
\hline L-302* & 1 & Electrostatic Precipitator & $\$ 457,000$ & 2.5 & $\$ 1,142,500$ & Packaged ESP Unit \\
\hline L- $601^{*}$ & 1 & Water Chiller & $\$ 860,000$ & 2.5 & $\$ 2,150,000$ & $\begin{array}{l}\text { 1(ea) - Packaged propane refirigeration unit complete } \\
\text { w/ compressor, driver, lube oil system, KO drum \& all } \\
\text { necessary instr. \& controls. }\end{array}$ \\
\hline L-801* & 1 & Waste Oxidizer & $\$ 723,000$ & 2.5 & $\$ 1,807,500$ & $\begin{array}{l}1 \text { (ea) - Packaged horizontal thermal oxidizer w/ high } \\
\text { intensity burner, refractory lined chamber \& stack. }\end{array}$ \\
\hline \multirow[t]{2}{*}{ L-901* } & 1 & Water Filtration System & $\$ 240,000$ & 2.5 & $\$ 600,000$ & 1 (ea) - Cross flow filtration system for water purification \\
\hline & & SUB-TOTAL & $\$ 3,613,000$ & & $\$ 9,032,000$ & \\
\hline \multicolumn{7}{|c|}{ ATMOSPHERE STORAGE TANKS } \\
\hline $\mathrm{F}-101$ & 1 & Glucose / Water Storage Tank & $\$ 34,500$ & 2.9 & $\$ 100,050$ & $\begin{array}{l}\text { 1(ea) - Atm. Storage Tank, Flat Roof, Flat Bottom, 12' } \\
\text { DIA x 20' T-T, Epoxy Resin coated CS }\end{array}$ \\
\hline F-102 & 1 & Salt / Water Storage Tank & $\$ 14,900$ & 2.9 & $\$ 43,210$ & $\begin{array}{l}1 \text { (ea) - Atm. Storage Tank, Flat Roof, Flat Bottom, 6' } \\
\text { DIA x } 12 \text { ' T-T, Epoxy Resin coated CS }\end{array}$ \\
\hline $\mathrm{F}-103$ & 1 & Ethanol / Water Storage Tank & $\$ 53,100$ & 2.9 & $\$ 153,990$ & $\begin{array}{l}1 \text { (ea) - Atm. Storage Tank, Flat Roof, Flat Bottom, 15' } \\
\text { DIA x 20' T-T, Epoxy Resin coated CS }\end{array}$ \\
\hline \multirow[t]{2}{*}{$\mathrm{F}-104$} & 1 & $\mathrm{NaOH} /$ Water Storage Tank & $\$ 60,500$ & 2.9 & $\$ 175,450$ & $\begin{array}{l}\text { 1(ea) - Atm. Storage Tank, Flat Roof, Flat Bottom, 16' } \\
\text { DIA x 24' T-T, Epoxy Resin coated CS }\end{array}$ \\
\hline & & SUB-TOTAL & $\$ 163,000$ & & $\$ 473,000$ & \\
\hline
\end{tabular}


REV: 0

PHASE 1 ESTIMATE - FACTORING SUMMARY

\begin{tabular}{|c|c|c|c|c|c|c|}
\hline $\begin{array}{l}\text { EQUIP. } \\
\text { ITEM NO. }\end{array}$ & $\begin{array}{l}\text { QUAN- } \\
\text { TITY }\end{array}$ & DESCRIPTION & $\begin{array}{l}\text { TOTAL } \\
\text { EQUIP. } \\
\text { COST, \$ }\end{array}$ & $\begin{array}{c}\text { FIELD } \\
\text { COST } \\
\text { MULTI- } \\
\text { PLIER }\end{array}$ & $\begin{array}{l}\text { TOTAL } \\
\text { FIELD } \\
\text { COST, \$ } \\
\end{array}$ & NOTES \\
\hline \multicolumn{7}{|l|}{ PUMPS } \\
\hline$\overline{\mathrm{J}-101 \mathrm{~A} / \mathrm{B}}$ & 2 & Diesel Charge Pump & $\$ 55,000$ & 4.5 & $\$ 247,500$ & $\begin{array}{l}\text { 2(ea) - API Centrifugal Pump, } 190 \mathrm{gpm}, 163 \mathrm{ft} \text {. Head, } \\
15 \mathrm{HP} \text { Motor driven, CS, } 1 \text { operating, } 1 \text { installed spare }\end{array}$ \\
\hline $\mathrm{J}-103 \mathrm{~A} / \mathrm{B}$ & 2 & Process Water Pump & $\$ 7,600$ & 4.5 & $\$ 34,200$ & $\begin{array}{l}\text { 2(ea) - Centrifugal Pump, } 30 \mathrm{gpm}, 121 \mathrm{ft} . \text { Head, } 5 \mathrm{HP} \\
\text { Motor driven, CS, } 1 \text { operating, } 1 \text { installed spare }\end{array}$ \\
\hline $\mathrm{J}-104 \mathrm{~A} / \mathrm{B}$ & 2 & Glucose Pump & $\$ 14,200$ & 4.5 & $\$ 63,900$ & $\begin{array}{l}\text { 2(ea) - Diaphragm Pump, } 0.95 \mathrm{gpm}, 31 \mathrm{ft} . \text { Head, } \\
0.125 \mathrm{HP} \text { Motor driven, 304SS, } 1 \text { operating, } 1 \text { installed } \\
\text { spare }\end{array}$ \\
\hline $\mathrm{J}-105 \mathrm{~A} / \mathrm{B}$ & 2 & Nutrients Pump & $\$ 13,800$ & 4.5 & $\$ 62,100$ & $\begin{array}{l}\text { 2(ea) - Diaphragm Pump, } 0.8 \mathrm{gpm}, 39 \mathrm{ft} . \text { Head, } \\
0.125 \mathrm{HP} \text { Motor driven, 304SS, } 1 \text { operating, } 1 \text { installed } \\
\text { spare }\end{array}$ \\
\hline $\mathrm{J}-106 \mathrm{~A} / \mathrm{B}$ & 2 & Ethanol Pump & $\$ 15,800$ & 4.5 & $\$ 71,100$ & $\begin{array}{l}\text { 2(ea) - Diaphragm Pump, } 2.4 \mathrm{gpm}, 81 \mathrm{ft} . \text { Head, } \\
0.125 \mathrm{HP} \text { Motor driven, 304SS, } 1 \text { operating, } 1 \text { installed } \\
\text { spare }\end{array}$ \\
\hline $\mathrm{J}-107 \mathrm{~A} / \mathrm{B}$ & 2 & Potassium Hydroxide Pump & $\$ 16,400$ & 4.5 & $\$ 73,800$ & $\begin{array}{l}\text { 2(ea) - Diaphragm Pump, } 3.2 \mathrm{gpm}, 65 \mathrm{ft} . \text { Head, } \\
0.125 \mathrm{HP} \text { Motor driven, } 304 \mathrm{SS}, 1 \text { operating, } 1 \text { installed } \\
\text { spare }\end{array}$ \\
\hline $\mathrm{J}-201 \mathrm{~A} / \mathrm{B}$ & 2 & Fermentor Transfer Pump & $\$ 12,800$ & 4.5 & $\$ 57,600$ & $\begin{array}{l}\text { 2(ea) - Rotary Lobe Pump, } 10 \mathrm{gpm}, 43 \mathrm{ft} \mathrm{Head,} 0.5 \mathrm{HP} \\
\text { Motor, 304SS, } 1 \text { operating, } 1 \text { installed spare }\end{array}$ \\
\hline $\mathrm{J}-202 \mathrm{~A} / \mathrm{B}$ & 2 & BDS Reactor No1 Transfer Pump & $\$ 21,400$ & 4.5 & $\$ 96,300$ & $\begin{array}{l}\text { 2(ea) - Centrifugal Pump, } 700 \mathrm{gpm}, 45 \mathrm{ft} \text {. Head, } 15 \mathrm{HP} \\
\text { Motor driven, 304SS, } 1 \text { operating, } 1 \text { installed spare }\end{array}$ \\
\hline $\mathrm{J}-203 \mathrm{~A} / \mathrm{B}$ & 2 & BDS Reactor No2 Transfer Pump & $\$ 21,400$ & 4.5 & $\$ 96,300$ & $\begin{array}{l}\text { 2(ea) - Centrifugal Pump, } 700 \mathrm{gpm}, 45 \mathrm{ft} \text {. Head, } 15 \mathrm{HP} \\
\text { Motor driven, 304SS, } 1 \text { operating, } 1 \text { installed spare }\end{array}$ \\
\hline $\mathrm{J}-204 \mathrm{~A} / \mathrm{B}$ & 2 & BDS Reactor No3 Transfer Pump & $\$ 21,400$ & 4.5 & $\$ 96,300$ & $\begin{array}{l}\text { 2(ea) - Centrifugal Pump, } 700 \mathrm{gpm}, 45 \mathrm{ft} \text {. Head, } 15 \mathrm{HP} \\
\text { Motor driven, 304SS, } 1 \text { operating, } 1 \text { installed spare }\end{array}$ \\
\hline $\mathrm{J}-301 \mathrm{~A} / \mathrm{B}$ & 2 & 1st Stg Sep Overflow Pump & $\$ 47,600$ & 4.5 & $\$ 214,200$ & $\begin{array}{l}\text { 2(ea) - API Centrifugal Pump, } 200 \mathrm{gpm}, 49 \mathrm{ft} \text {. Head, } \\
5 \mathrm{HP} \text { Motor driven, 304SS, } 1 \text { operating, } 1 \text { installed } \\
\text { spare }\end{array}$ \\
\hline $\mathrm{J}-302 \mathrm{~A} / \mathrm{B}$ & 2 & 1st Stg Sep Underflow Pump & $\$ 18,400$ & 4.5 & $\$ 82,800$ & $\begin{array}{l}\text { 2(ea) - Centrifugal Pump, } 500 \mathrm{gpm}, 68 \mathrm{ft} \text {. Head, } 10 \mathrm{HP} \\
\text { Motor driven, } 304 \mathrm{SS}, 1 \text { operating, } 1 \text { installed spare }\end{array}$ \\
\hline $\mathrm{J}-303 \mathrm{~A} / \mathrm{B}$ & 2 & 2nd Stg Sep Overflow Pump & $\$ 11,800$ & 4.5 & $\$ 53,100$ & $\begin{array}{l}\text { 2(ea) - Centrifugal Pump, } 145 \mathrm{gpm}, 72 \mathrm{ft} \text {. Head, } 5 \mathrm{HP} \\
\text { Motor driven, 304SS, } 1 \text { operating, } 1 \text { installed spare }\end{array}$ \\
\hline $\mathrm{J}-304 \mathrm{~A} / \mathrm{B}$ & 2 & 2nd Stg Sep Underflow Pump & $\$ 10,200$ & 4.5 & $\$ 45,900$ & $\begin{array}{l}\text { 2(ea) - Centrifugal Pump, } 45 \mathrm{gpm}, 72 \mathrm{ft} \text {. Head, 3HP } \\
\text { Motor driven, 304SS, } 1 \text { operating, } 1 \text { installed spare }\end{array}$ \\
\hline $\mathrm{J}-502 \mathrm{~A} / \mathrm{B}$ & 2 & Vent Gas KO Drum Wtr Pump & $\$ 6,200$ & 4.5 & $\$ 27,900$ & $\begin{array}{l}\text { 2(ea) - Centrifugal Pump, } 5 \mathrm{gpm}, 70 \mathrm{ft} \text {. Head, } 0.5 \mathrm{HP} \\
\text { Motor driven, CS, } 1 \text { operating, } 1 \text { installed spare }\end{array}$ \\
\hline \multirow[t]{2}{*}{$\mathrm{J}-601$} & 2 & Water Chiller Pump & $\$ 25,000$ & 4.5 & $\$ 112,500$ & $\begin{array}{l}\text { API Centrifugal Pump, } 2000 \text { GPM, } 0.95 \text { SG, } 81.7 \text { Ft. } \\
\text { Head, CS Case, 12\%CR Impeller, 60HP Motor }\end{array}$ \\
\hline & & SUB-TOTAL & $\$ 319,000$ & & $\$ 1,436,000$ & \\
\hline
\end{tabular}


Date: $6 / 17 / 05$

REV: 0

PHASE 1 ESTIMATE - FACTORING SUMMARY

\begin{tabular}{|c|c|c|c|c|c|c|}
\hline $\begin{array}{l}\text { EQUIP. } \\
\text { ITEM NO. }\end{array}$ & $\begin{array}{l}\text { QUAN- } \\
\text { TITY }\end{array}$ & DESCRIPTION & $\begin{array}{l}\text { TOTAL } \\
\text { EQUIP. } \\
\text { COST, \$ } \\
\end{array}$ & $\begin{array}{l}\text { FIELD } \\
\text { COST } \\
\text { MULTI- } \\
\text { PLIER } \\
\end{array}$ & $\begin{array}{l}\text { TOTAL } \\
\text { FIELD } \\
\text { COST, \$ } \\
\end{array}$ & NOTES \\
\hline \multicolumn{7}{|l|}{ FILTERS } \\
\hline $\mathrm{L}-101$ & 1 & Diesel Pre-Filter Vessel & $\$ 5,210$ & 4.2 & $\$ 21,882$ & $\begin{array}{l}\text { 1(ea) - Filter housing, } 316 \mathrm{LSS}, 47 " \text { T-T x 8" Dia. w/ } \\
5(\text { ea) - Polypropylene element, } 30 " \text { long, } 1.5 \text { um pore } \\
\text { size }\end{array}$ \\
\hline L-102 & 1 & Diesel Pre-Filter Vessel & $\$ 5,280$ & 4.2 & $\$ 22,176$ & $\begin{array}{l}\text { 1(ea) - Filter housing, 316L SS, } 47 " \text { T-T x 8" Dia. w/ } \\
5(\text { ea) - Polypropylene element, } 30 " \text { long, } 0.2 \text { um pore } \\
\text { size }\end{array}$ \\
\hline L-103 & 1 & Air Pre-Filter Vessel & $\$ 11,400$ & 4.2 & $\$ 47,880$ & $\begin{array}{l}\text { 1(ea) - Filter housing, } 316 \mathrm{~L} \text { SS, } 55^{\prime \prime} \text { T-T x } 15 " \text { Dia. w/ } \\
5(\text { ea) - GF element, 30" long, } 1.0 u m \text { pore size }\end{array}$ \\
\hline L-104 & 1 & Air Bio-Filter Vessel & $\$ 11,125$ & 4.2 & $\$ 46,725$ & $\begin{array}{l}\text { 1(ea) - Filter housing, } 316 \mathrm{~L} \text { SS, } 43 " \text { T-T x } 15 \text { " Dia. w/ } 5 \\
\text { (ea) - PTFE element, 10" long, } 0.01 \text { um pore size }\end{array}$ \\
\hline L-105 & 1 & BDS Reactor \#1 Air Bio Filter Vessel & $\$ 12,420$ & 4.2 & $\$ 52,164$ & $\begin{array}{l}\text { 1(ea) - Filter housing, } 316 \mathrm{~L} \text { SS, } 55 " \text { T-T x 15" Dia. w/ } \\
5(\text { ea) - PTFE element, } 30 " \text { long, } 0.01 \text { um pore size }\end{array}$ \\
\hline L-106 & 1 & BDS Reactor \#2 Air Bio Filter Vessel & $\$ 12,420$ & 4.2 & $\$ 52,164$ & $\begin{array}{l}\text { 1(ea) - Filter housing, 316L SS, 55" T-T x 15" Dia. w/ } \\
5(\text { ea) - PTFE element, } 30 " \text { long, 0.01um pore size }\end{array}$ \\
\hline L-107 & 1 & BDS Reactor \#3 Air Bio Filter Vessel & $\$ 12,420$ & 4.2 & $\$ 52,164$ & $\begin{array}{l}\text { 1(ea) - Filter housing, 316L SS, 55" T-T x 15" Dia. w/ } \\
5(\text { ea) - PTFE element, } 30 " \text { long, 0.01um pore size }\end{array}$ \\
\hline L-108 & 1 & Air Purge Bio-Filter Vessel & $\$ 7,011$ & 4.2 & $\$ 29,446$ & $\begin{array}{l}\text { 1(ea) - Filter housing, 316L SS, 30" T-T x 12" Dia. w/ } \\
\text { 3(ea) - PTFE element, 10" long, 0.01um pore size }\end{array}$ \\
\hline L-111 & 1 & Ethanol Bio-Filter Vessel & $\$ 1,308$ & 4.2 & $\$ 5,494$ & $\begin{array}{l}\text { 1(ea) - Filter housing, } 316 \mathrm{LSS}, 10 " \mathrm{~T}-\mathrm{T} \times 2.5 \text { " Dia. w/ } \\
1 \text { (ea) - Polyethersulfone element, } 5 \text { "long, } 0.2 \text { um pore } \\
\text { size }\end{array}$ \\
\hline L-112 & 1 & $\mathrm{NAaOH}$ Bio-Filter Vessel & $\$ 1,308$ & 4.2 & $\$ 5,494$ & $\begin{array}{l}\text { 1(ea) - Filter housing, } 316 \mathrm{LSS}, 10 " \text { T-T x } 2.5 \text { " Dia. w/ } \\
1 \text { (ea) - Polyethersulfone element, } 5 \text { "long, } 0.2 \text { um pore } \\
\text { size }\end{array}$ \\
\hline L-113 & 1 & Water Pre-Filter Vessel & $\$ 2,850$ & 4.2 & $\$ 11,970$ & $\begin{array}{l}\text { 1(ea) - Filter housing, 316L SS, 38" T-T x 8" Dia. w/ } \\
3(\text { ea) - Polypropylene element, } 20 " \text { long, } 1.5 \text { um pore } \\
\text { size }\end{array}$ \\
\hline L-114 & 1 & Water Bio-Filter Vessel & $\$ 2,892$ & 4.2 & $\$ 12,146$ & $\begin{array}{l}\text { 1(ea) - Filter housing, } 316 \mathrm{~L} \mathrm{SS}, 38 \text { " T-T x 8" Dia. w/ } \\
3(\text { ea) - Polypropylene element, } 20 \text { "long, } 0.2 \text { um pore } \\
\text { size }\end{array}$ \\
\hline L-401 & 1 & Recycle Water Pre-Filter Vessel & $\$ 2,850$ & 4.2 & $\$ 11,970$ & $\begin{array}{l}\text { 1(ea) - Filter housing, } 316 \mathrm{LSS}, 38 \text { " T-T x 8" Dia. w/ } \\
3(\text { ea) - Polypropylene element, } 20 \text { "long, } 1.5 \mathrm{um} \text { pore } \\
\text { size }\end{array}$ \\
\hline \multirow[t]{2}{*}{ L-402 } & 1 & Recycle Water Bio-Filter Vessel & $\$ 2,892$ & 4.2 & $\$ 12,146$ & \multirow[t]{2}{*}{$\begin{array}{l}\text { (ea) - Filter housing, } 316 \mathrm{~L} \mathrm{SS,} 38 \text { " T-T x 8" Dia. w/ } \\
3(\text { ea) - Polypropylene element, } 20 \text { " long, } 0.2 \text { um pore } \\
\text { size }\end{array}$} \\
\hline & & SUB-TOTAL & $\$ 91,000$ & & $\$ 384,000$ & \\
\hline \multirow[t]{3}{*}{ FREIGHT } & & Freight Allowance (7\% Equip Cost) & $\$ 462,000$ & & $\$ 462,000$ & \\
\hline & & SUB-TOTAL & $\$ 462,000$ & & $\$ 462,000$ & \\
\hline & & TOTAL & $\$ 7,060,043$ & & $\$ 21,900,000$ & \\
\hline
\end{tabular}


A N V I L C ORPORATION Final Technical Progress Report DOELANPrFetl'stafE-FC26-02NT15340

PROJECT: BDS Baseline

Date: $6 / 17 / 05$

ANVIL NO: AE1416

REV: 0

PHASE 1 ESTIMATE - FACTORING SUMMARY

\begin{tabular}{|c|c|c|c|c|c|c|c|}
\hline $\begin{array}{l}\text { EQUIP. } \\
\text { ITEM NO. }\end{array}$ & $\begin{array}{l}\text { QUAN- } \\
\text { TITY }\end{array}$ & DESCRIPTION & $\begin{array}{l}\text { TOTAL } \\
\text { EQUIP. } \\
\text { COST, \$ }\end{array}$ & \begin{tabular}{|c} 
FIELD \\
COST \\
MULTI- \\
PLIER \\
\end{tabular} & & $\begin{array}{l}\text { TOTAL } \\
\text { FIELD } \\
\text { COST, \$ }\end{array}$ & NOTES \\
\hline & \multicolumn{4}{|c|}{ DESIGN SERVICES } & $\$$ & $5,000,000$ & \\
\hline & \multicolumn{4}{|c|}{ OWNER'S SERVICES \& COSTS (provided by owner) } & $\$$ & - & \\
\hline & \multicolumn{4}{|c|}{ INITIAL CHEMICAL CHARGE } & $\$$ & 229,000 & \\
\hline & \multicolumn{4}{|c|}{ ESCALATION (provided by owner) } & $\$$ & - & \\
\hline & \multicolumn{4}{|c|}{ UNADJUSTED COST ESTIMATE (UCE) } & & $27,129,000$ & \\
\hline & \multicolumn{4}{|c|}{ CONTINGENCY } & $\$$ & $6,771,000$ & \\
\hline & \multicolumn{4}{|c|}{ EXPECTED COST (P50 VALUE) TOTAL INSTALLED COST (TIC) } & $\$$ & $33,900,000$ & \\
\hline
\end{tabular}

\footnotetext{
${ }^{*}$ Note: Equipment pricing by Pelorus.
} 
Final Technical Progress Report DOE Award No. DE-FC26-02NT15340

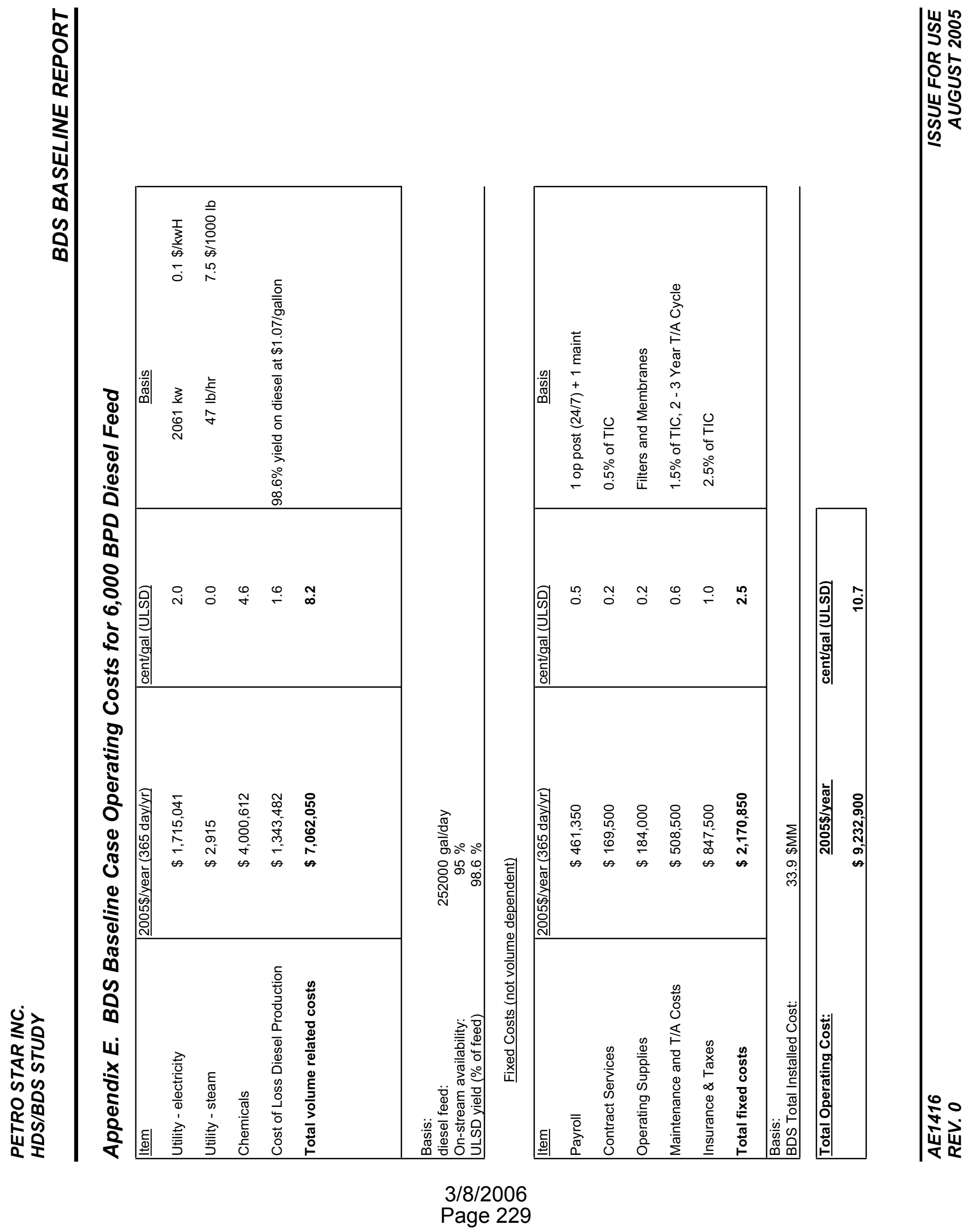




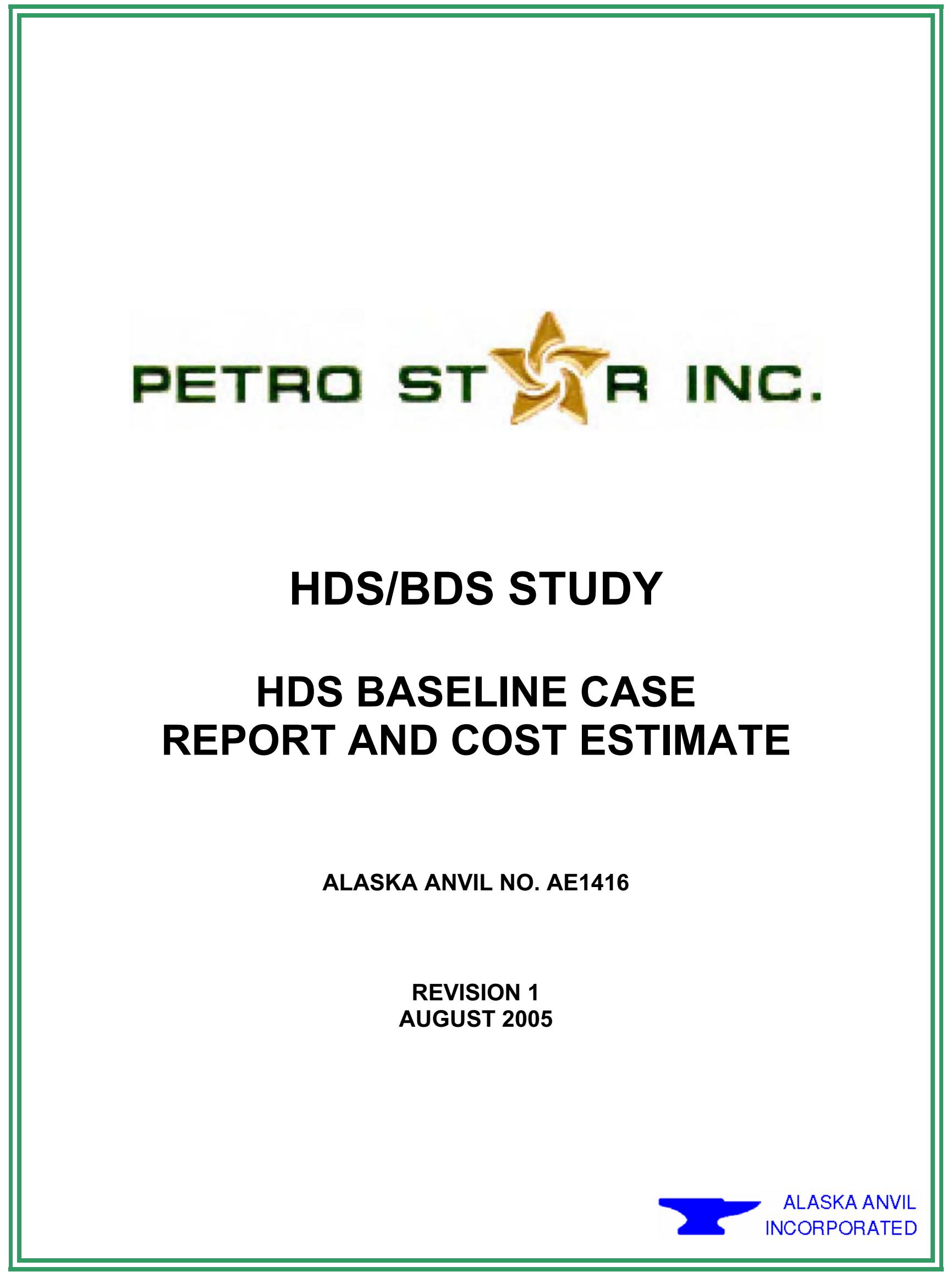




\section{EXECUTIVE SUMMARY}

To meet the EPA's 2006 sulfur content requirements, Petro Star is leading a Department of Energy study to determine the viability of ULSD production from a biodesulfurization process. This HDS Baseline Report, a part of the larger investigation, presents the HDS process and the total installed costs that will serve as the baseline economics that the BDS process must surpass.

The effort is based on an installation at Valdez, Alaska producing ultra low sulfur diesel meeting a 10-ppmw sulfur requirement.

The design includes the following proposed IBL facilities:

- A new 6,000 bpd HDS Unit

- A new 2 MMSCFD Hydrogen Unit

- A new 3.7 LTD Sulfur Unit to treat the off-gas from the HDS Unit.

The cost estimate for the HDS Unit includes only the inside battery limits (IBL) portion of the HDS Unit. Based on equipment-based costs for the HDS Unit and licensor quotes for new Hydrogen and Sulfur Units, the total expected installed cost for the HDS complex is \$27.8 million in 2005 dollars based on USGC equipment pricing. The expected accuracy of the estimate is $+50 /-15 \%$.

The annual HDS Unit operating cost is $\$ 5,800,000$, which translates to 6.8 cents per gallon of ULSD. The total incremental selling price for ULSD would be 13.4 cents per gallon to recover the capital in 5 years (excluding interest expense) and pay the yearly operating cost. 


\section{TABLE OF CONTENTS}

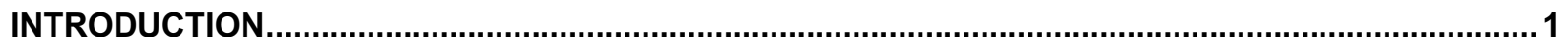

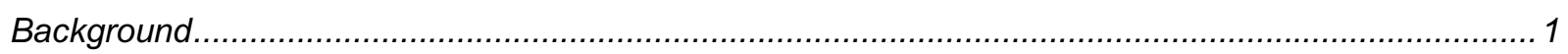

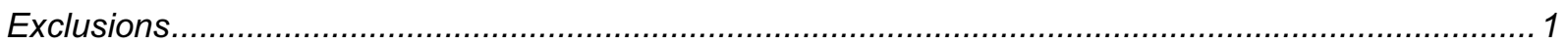

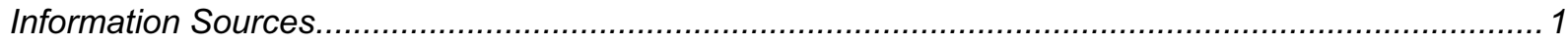

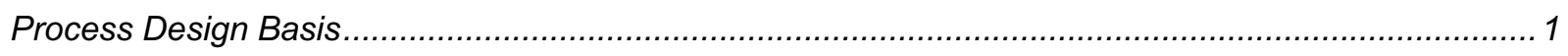

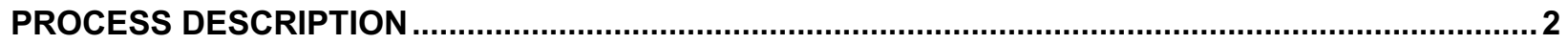

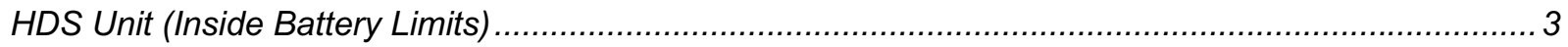

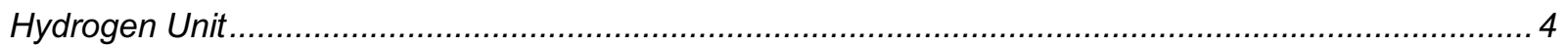

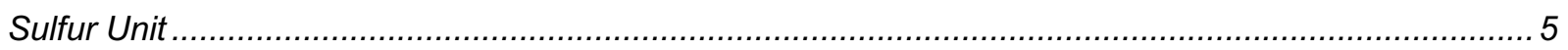

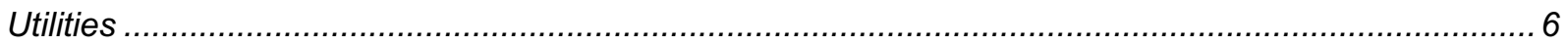

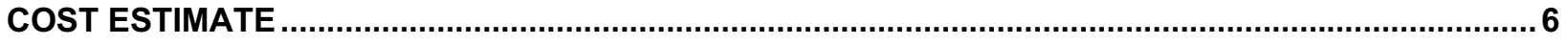

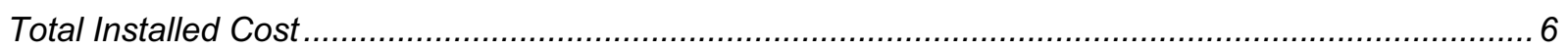

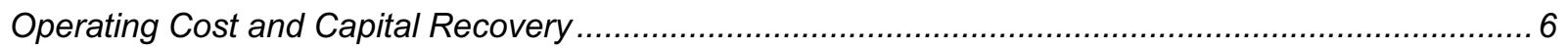

\section{APPENDICES}

A. Diesel Feed Properties and Sulfur Speciation

B. HDS Unit Process Flow Diagrams and Material Balance

C. HDS Unit Equipment List and Budgetary Equipment Data Sheets

D. Hydrogen Unit Flow Diagram

E. Sulfur Unit Block Flow Diagram

F. Utility Requirements

G. Cost Estimate Basis and Cost Estimate

H. HDS Baseline Case Operating Costs for 6,000 BPD Diesel Feed 


\section{INTRODUCTION}

\section{Background}

All U.S. refineries will be required to meet the EPA's third quarter 2006 sulfur content requirements for highway diesel. The EPA requirement for maximum sulfur content of ultra low sulfur diesel (ULSD) is $15 \mathrm{ppmw}$. The refinery product should have about $10 \mathrm{ppmw}$ sulfur or less to ensure that the product specification can be met on a reliable basis after product shipping and distribution logistics.

To compare different strategies to accomplish this requirement, Petro Star is leading a Department of Energy HDS/BDS study that evaluates the following processing routes:

- A standalone hydrodesulfurization (HDS) process

- A standalone biodesulfurization (BDS) process

- A combination of the two.

To be economically viable, a BDS process must be competitive with other commercially proven desulfurization routes. The route most chosen and best known to refiners for diesel is HDS. A new BDS facility must be less costly than a comparable new HDS facility. Or, in the various combination scenarios, using the BDS facility as a pre- or post-treatment facility combined with an existing HDS unit must be less costly than modifications to an existing HDS Unit that would be required to achieve the lower sulfur requirement.

As part of the study of potential BDS economics, this baseline report provides the process description and total installed cost of a new standalone 6,000 bpd HDS Unit producing highway ULSD at 10 ppmw sulfur.

\section{Exclusions}

To simplify the economic baseline study and avoid biasing the study results, we have excluded all outside battery limits (OBL) facilities. A prior 1999 Kellogg report exploring a BDS baseline also excluded all utility systems improvements, offsite tankage, and waste stream disposal.

\section{Information Sources}

Axens North America, Inc. provided the HDS Unit reactor design and unit yields for this study under a proprietary agreement. A cursory inspection of other licensor data revealed little difference in yields, process conditions, and reactor size. Therefore, only the data received from Axens was used for this study.

\section{Process Design Basis}

\section{Unit Throughput}

The design throughput for the HDS Unit is $6,000 \mathrm{bpd}$ of straight run diesel containing 0.5 weight percent sulfur. 


\section{Unit Feed Characteristics}

The design basis composition and characteristics of the diesel feed, including a sulfur speciation, are included in Appendix A. The hydrogen from the Hydrogen Unit is $99.7 \%$ pure.

\section{Product Specification}

The specification for diesel product sulfur content is 10 ppmw.

\section{Utility and Infrastructure Availability}

The study assumes the refinery has the following utility systems and infrastructure available to support the HDS Unit:

- $\quad$ Refinery fuel gas (for fired heaters and Hydrogen Unit feed)

- Water (available as feed to a new demineralization unit)

- Instrument air

- Electricity for drivers

- Nitrogen

- Process vent system

- Product tanks

- Cooling fluid system

- Steam/boiler feed water

- Refinery fuel gas

- Naphtha fuel

- Wastewater treatment

- Flare.

\section{Refinery Ambient Conditions}

The new HDS, hydrogen, and sulfur recovery facilities design is based on a Valdez, Alaska location.

\begin{tabular}{|l|c|}
\hline Ambient Temperature Condition & Degrees F \\
\hline Highest monthly average & 62 \\
\hline Lowest monthly average & 17 \\
\hline Record high & 86 \\
\hline Record low & -23 \\
\hline
\end{tabular}

\section{PROCESS DESCRIPTION}

The design includes the following proposed IBL facilities:

- A new 6,000 bpd HDS Unit

- A new 2 MMSCFD Hydrogen Unit

- A new 3.7 LDT Sulfur Unit to treat the off-gas from the HDS Unit. 


\section{HDS Unit (Inside Battery Limits)}

\section{Feed Section}

In an integrated refinery, the diesel feed to the HDS Unit comes directly from a refinery feed/product exchanger without being cooled or routed through an intermediate storage tank. The diesel feed enters the Diesel Feed Drum, V-500, at $216^{\circ} \mathrm{F}$. The feed drum provides 15 minutes of surge capacity and a stable level for the Diesel Feed Pump, P-500 A/B. See Appendix B for the IBL HDS Unit's process flow diagrams and material balance. See Appendix $\mathrm{C}$ for the HDS Unit equipment list and budgetary equipment data sheets.

The feed is pumped through two Reactor Feed/Bottoms Exchangers, Nos. 1 and 2, E-500 and E501 , where it is preheated to $650^{\circ} \mathrm{F}$. Makeup hydrogen from the Hydrogen Unit, described below, is compressed by the Makeup Hydrogen Compressor, C-500, and enters the diesel feed stream upstream of the first Reactor Feed/Bottoms Exchanger. Hydrogen-rich recycle gas enters the diesel feed stream upstream of the second Reactor Feed/Bottoms Exchanger.

The combined diesel and hydrogen feed stream to the HDS reactor is heated to reaction temperature in the Diesel Charge Heater, H-500. The Diesel Charge Heater has an absorbed duty of 3.5 MM Btu/hr and uses refinery fuel gas or naphtha as its fuel source.

\section{Reaction Section}

The diesel and hydrogen feed stream enters the HDS Reactor, R-500, at $678^{\circ} \mathrm{F}$ and 660 psig at end-of-run (EOR) conditions. The catalyst in the reactor reduces the sulfur content of the diesel to $10 \mathrm{ppmw}$, and will effectively hydrogenate the most resistant sulfur species, 4,6 dibenzothiophenes. The hydrogenation reaction converts sulfur compounds to $\mathrm{H}_{2} \mathrm{~S}$ and also converts a small portion of the diesel to naphtha.

\section{Post-Reaction Cooling}

The hydrotreated diesel leaves the reactor at $721^{\circ} \mathrm{F}$ and $600 \mathrm{psig}$, and is cooled in the reactor feed/bottoms exchangers to $330^{\circ} \mathrm{F}$. The diesel is further cooled in the Separator Feed/Bottoms Exchanger, E-502, to $326^{\circ} \mathrm{F}$ by preheating the bottoms stream from the Low Temperature Separator, V-502.

The diesel stream then enters the High Temperature Separator, V-501, to separate the diesel and naphtha from lighter hydrocarbons, hydrogen, and $\mathrm{H}_{2} \mathrm{~S}$.

The bottoms stream from the High Temperature Separator is routed to the Naphtha Stripper, V503, to separate the diesel from naphtha and sour hydrocarbon gas.

The overhead vapor from the separator is mixed with wash water to mitigate potential plugging and corrosion from ammonium sulfide salts which could otherwise precipitate in the air cooler. The overhead stream is then cooled to $120^{\circ} \mathrm{F}$ in the High Temperature Separator Overhead Cooler, E-503, and is then routed to the Low Temperature Separator, V-502. Like E-503, all coolers in the HDS Unit are air coolers to minimize the size of the cooling fluid system. 


\section{Low Temperature Separator}

The Low Temperature Separator (V-502) separates the naphtha from lighter hydrocarbons, $\mathrm{H}_{2} \mathrm{~S}$, and hydrogen. A water draw is included to remove sour wash water.

The hydrogen-rich overhead stream is recycled to the reactor feed stream via the Recycle Gas Compressor (C-501). An amine absorber is not required to treat the recycle gas because of the diesel feed's low sulfur content, the high purity of the makeup hydrogen gas, and consequently low $\mathrm{H}_{2} \mathrm{~S}$ content of the recycle gas.

Excess gas from the separator overhead is bled via pressure control to the Sulfur Unit's $\mathrm{H}_{2} \mathrm{~S}$ scrubbing section and is ultimately used as refinery fuel. The Low Temperature Separator pressure setting dictates the pressure level in the reactor system.

The bottoms stream is routed through the Separator Feed/Bottoms Exchanger (E-502) for preheating before entering the Naphtha Stripper (V-503) at the upper feed point. Because of the low flow rate of Low Temperature Separator bottoms, the duty of the E-502 service is small; in the design phase, the size or need for an exchanger in this service could be optimized.

\section{Naphtha Stripper}

The Naphtha Stripper (V-503) separates naphtha and sour hydrocarbon gas from the diesel product. The bottoms stream from the Low Temperature Separator enters the stripper at $325^{\circ} \mathrm{F}$ via the Separator Feed/Bottoms Exchanger. The bottoms stream from the High Temperature Separator enters the stripper at $580^{\circ} \mathrm{F}$ via the Stripper Feed/Bottoms Exchanger (E-505).

The Naphtha Stripper employs 22 distillation trays to the separate naphtha and sour hydrocarbon gas from the diesel product. The diesel yield from the stripper is approximately 97.0 volume percent of the diesel fed to the HDS Unit.

The overhead from the stripper is condensed in the Stripper Overhead Condenser (E-504) and is collected in the Stripper Overhead Accumulator (V-504). The non-condensed sour gas from the accumulator is routed to the sulfur recovery unit and the sweetened gas is ultimately used as refinery fuel. The Stripper Overhead Pump (P-502A/B) delivers the naphtha from the accumulator to the plant liquid fuel system. A part of the condensed naphtha is returned to the top tray of the stripper as a reflux stream.

The bottoms stream from the stripper is routed through the Stripper Feed/Bottoms Exchanger (E505) where it is cooled to $417^{\circ} \mathrm{F}$. The product diesel is further cooled to $120^{\circ} \mathrm{F}$ in the Diesel Product Cooler (E-506) before being routed to a product storage tank. The Stripper Bottoms Pump (P-501A/B) sends a portion of the stripper bottoms stream through the Stripper Reboiler (H-501). The Stripper Reboiler has an absorbed duty of 5.6 MM Btu/hr and uses refinery fuel gas or naphtha for firing.

\section{Hydrogen Unit}

A new steam reforming-type Hydrogen Unit will supply hydrogen for the HDS Unit. This process is generally used in refineries to generate hydrogen needed for hydroprocessing. The 
unit size for this project is $2 \mathrm{MMSCF} / \mathrm{D}$ of hydrogen. The hydrogen produced in the unit has a purity of $99.7+\%$. See Appendix D for a typical steam reformer's general flow scheme.

Feed to the Hydrogen Unit is off-gas from the Crude Unit. At the Crude Unit, this gas is treated for $\mathrm{H}_{2} \mathrm{~S}$ removal. This feed gas is first compressed up to a pressure of about $350 \mathrm{psig}$. The feed is then heated and passed through a guard bed to remove any remaining $\mathrm{H}_{2} \mathrm{~S}$. The guard bed vessels are arranged so that the adsorbent can be changed while the plant operates. The feed gas is then mixed with steam and superheated in a feed preheat coil of the Reformer furnace. The feed mixture goes through catalyst filled tubes in the Reformer where the feed reacts with steam to produce hydrogen and carbon oxides. The reforming reactions are endothermic and heat is supplied by controlling the firing of the Reformer furnace.

The steam used by the process is produced by steam generation in the Reformer. This Reformer Unit would be designed to be self-contained from the viewpoint that no net steam would be produced in the process.

The reformed gas is cooled by the process steam generator and then sent to the shift converter in which $\mathrm{CO}$ and water vapor are converted to additional $\mathrm{H}_{2}$ and $\mathrm{CO}_{2}$. The gas leaving the shift converter is cooled with feed preheat and a trim cooler and then condensate is removed and the gas then goes to a pressure swing adsorption (PSA) hydrogen purification system.

The PSA is an automated system and runs on repeated cycles, first in adsorption mode and then in regeneration mode. An off-gas stream from the PSA is routed to the Reformer furnace and provides a large portion of the fuel required. The hydrogen stream from the PSA unit is very high purity and available at a pressure of about 300 psig to the HDS makeup hydrogen compressor.

\section{Sulfur Unit}

The purge gas from the HDS Low Temperature Separator (V-502) and the off-gas stream from the HDS Stripper Overhead Accumulator (V-504) are combined and sent to the Sulfur Recovery Unit in which the $\mathrm{H}_{2} \mathrm{~S}$ is removed from the hydrocarbon gas before use as fuel gas. The Sulfur Unit converts the $\mathrm{H}_{2} \mathrm{~S}$ into elemental sulfur.

The process that converts the $\mathrm{H}_{2} \mathrm{~S}$ is the Thiopaq Biological $\mathrm{H}_{2} \mathrm{~S}$ Removal Process. This process provides an economic means to recover small tonnages of sulfur from commercial plants. The process has been successfully applied in over 40 locations. See Appendix E for a Thiopaq process simplified block diagram.

The Thiopaq process operates at very mild conditions and requires little attention from an operational point of view, making it an attractive process compared to conventional amine/ Claus/ TGU schemes. The Thiopaq process is fairly simple and uses microbial oxidation. The process uses a soda solution and air for regeneration and operates at atmospheric pressure and a temperature of about $110^{\circ} \mathrm{F}$. 
In the Thiopaq process, the sour gas is fed to a scrubber and contacted with Thiopaq solution that removes the $\mathrm{H}_{2} \mathrm{~S}$ from the off-gas. The sweetened gas goes overhead in the scrubber and on to the plant fuel gas system. The rich Thiopaq solution goes to a low-pressure flash vessel in which a flash gas is taken overhead which can be used as fuel gas in a low-pressure burner. The solution then goes to the bioreactor where the $\mathrm{H}_{2} \mathrm{~S}$ is oxidized under controlled conditions to elemental sulfur in the presence of microorganisms. The elemental sulfur produced is hydrophilic and is separated from the aqueous effluent in a separator. This sulfur is in cake form that can be filtered to reach $90+\%$ solids and used for agricultural purposes or land filled.

The project does not include a storage area for the sulfur product to hold the sulfur between outhaul shipments. The sulfur product would be about 3.7 tons per day. This translates to about a truckload of sulfur per week.

A water bleed stream is taken from the bioreactor, which along with caustic injection, is used to control the $\mathrm{pH}$ inside the reactor.

\section{Utilities}

Anvil has identified the preliminary utility requirements for the proposed facilities to serve an HDS Unit. See Appendix F for an itemized list.

\section{COST ESTIMATE}

\section{Total Installed Cost}

The conceptual cost estimate is based on installing an HDS Unit processing 6,000 bpd raw diesel feed and producing ULSD meeting the 10-ppmw sulfur requirement. The cost estimate is for the IBL portion of the HDS complex only.

Based on equipment-based costs for the HDS Unit and vendor quotes for the Hydrogen Unit and Sulfur Unit, the total installed cost estimate for the IBL portion of the HDS complex is \$27.8 million in 2005 dollars based on USGC pricing. The accuracy of the estimate is $+50 /-15 \%$.

The cost estimate excludes other owner costs such as startup and commissioning costs, initial operator training, startup procedures, and pre-startup operator staffing. It also excludes licensing and royalty fees. A contingency of $25 \%$ is in the total installed cost. See Appendix G for the Cost Estimate Basis and Cost Estimate.

\section{Operating Cost and Capital Recovery}

The total annual operating cost of the HDS Unit is $\$ 5,800,000$. This cost is based on a $95 \%$ onstream availability for the plant. The operating cost includes fuel, power, chemicals, utilities, sulfur disposal, other consumables, operating and maintenance labor, maintenance expense, and taxes and insurance. The operating cost also includes lost diesel sales due to conversion of diesel to naphtha and fuel gas in the HDS Unit. However, a credit is taken for the value of naphtha and fuel gas as refinery fuel. The basis and methodology for developing the operating cost estimates is presented in Appendix $\mathrm{H}$. 
The operating cost can be expressed in terms of cents per gallon of ULSD, based on a 97\% yield on the ULSD. The total operating cost of the HDS Unit is 6.8 cents per gallon of ULSD.

Based on the expected TIC of the HDS Unit, the incremental selling price for ULSD would be 6.6 cents per gallon to recover the capital in 5 years (excluding interest expense). Therefore, the total incremental selling price for ULSD would be 13.4 cents per gallon to recover the capital in 5 years and pay the yearly operating cost. 


\section{APPENDICES}

A. Diesel Feed Properties and Sulfur Speciation

B. HDS Unit Process Flow Diagrams and Material Balance

Process Flow Diagrams

HDS Unit Reactor Section

HDS Unit Naphtha Stripper Section

Material Balance

C. HDS Unit Equipment List and Budgetary Equipment Data Sheets

Equipment List

Budgetary Equipment Data Sheets

D. Hydrogen Unit Flow Diagram

E. Sulfur Unit Block Flow Diagram

F. Utility Requirements

G. Cost Estimate Basis and Cost Estimate

H. HDS Baseline Case Operating Costs for 6,000 BPD Diesel Feed 


\section{Appendix A - Diesel Feed Properties and Sulfur Speciation}

\begin{tabular}{|l|l|c|c|}
\hline \multicolumn{4}{|c|}{ Diesel Feed Properties } \\
\hline Test & Units & Results & Specification \\
\hline Gravity @ $60^{\circ}$ F, Min/Max & API & 33.3 & $32-36$ \\
\hline Flash Pt, Min. & Celsius & $63.5\left(146^{\circ} \mathrm{F}\right)$ & $60\left(140^{\circ} \mathrm{F}\right)$ \\
\hline Cloud Pt, Max. & Celsius & $-12.3\left(10^{\circ} \mathrm{F}\right)$ & $-9.5\left(15^{\circ} \mathrm{F}\right)$ \\
\hline Pour Pt, Max. & Celsius & $-15\left(5^{\circ} \mathrm{F}\right)$ & $-12\left(10^{\circ} \mathrm{F}\right)$ \\
\hline Distillation & & & \\
\hline IBP & Celsius & $171\left(340^{\circ} \mathrm{F}\right)$ & Report \\
\hline $10 \%$ Recovery & Celsius & $249\left(480^{\circ} \mathrm{F}\right)$ & Report \\
\hline $20 \%$ Recovery & Celsius & $265\left(509^{\circ} \mathrm{F}\right)$ & Report \\
\hline $50 \%$ Recovery & Celsius & $287\left(549^{\circ} \mathrm{F}\right)$ & Report \\
\hline 90\% Recovery & Celsius & $317\left(603^{\circ} \mathrm{F}\right)$ & $282-338$ \\
\hline Final Boiling Pt. & Celsius & $336\left(637^{\circ} \mathrm{F}\right)$ & Report \\
\hline Recovery & Vol \% & 99.9 & Report \\
\hline Residual & Vol \% & 0.1 & Report \\
\hline Loss & Vol \% & 0 & Report \\
\hline Viscosity @ 40 C, Min/Max & CSt & 3.57 & $2.0-4.3$ \\
\hline Ash, Max & Wt $\%$ & $<0.001$ & $0.01 \%$ \\
\hline Carbon Residue on 10\% Bottoms, Max & Wt\% & 0.08 & $0.35 \%$ \\
\hline Btu Gross, Min & Btu/Gallon & 139,190 & 136,000 \\
\hline Calculated Cetane, Min & Index & 48 & 45 \\
\hline Copper Strip Corrosion, Max & Code & $1 \mathrm{a}$ & 3 \\
\hline Total Sulfur & Wt. \% & 0.500 & 0.500 \\
\hline
\end{tabular}




\begin{tabular}{|l|c|l|c|}
\hline \multicolumn{5}{|c|}{$\begin{array}{c}\text { Sulfur Speciation } \\
\text { (Ratioed to 5,000 ppm*) }\end{array}$} \\
\hline Component & ppm wt sulfur & Component & ppm wt sulfur \\
\hline Hydrogen sulfide & $<1$ & 2-Ethyl thiophene & $<1$ \\
\hline Carbonyl sulfide & $<1$ & 2,5-Dimethyl thiophene & $<1$ \\
\hline Methyl mercaptan & $<1$ & 3-Ethyl thiophene & $<1$ \\
\hline Ethyl mercaptan & $<1$ & 2,4\&2,-Dimethyl thiophene & $<1$ \\
\hline Dimethyl sulfide & $<1$ & 3,4-Dimethyl thiophene & $<1$ \\
\hline Carbon disulfide & $<1$ & Methyl Ethyl thiophenes & $<1$ \\
\hline Isopropyl mercaptan & $<1$ & Trimethyl thiophenes & $<1$ \\
\hline Ethyl sulfide & $<1$ & Tetramethyl thiophenes & $<1$ \\
\hline tert-Butyl mercaptan & $<1$ & Benzothiophene & 2 \\
\hline N-Propyl mercaptan & $<1$ & Methyl benzothiophene & 29 \\
\hline Ethyl Methyl Sulfide & $<1$ & Dimethyl benzothiophene & 119 \\
\hline Thiophene & $<1$ & Trimethyl benzothiophene & 354 \\
\hline sec-Butyl Mercaptan & $<1$ & Tetramethyl Benzothiophene & 229 \\
\hline Isobutyl mercaptan & $<1$ & Dibenzothiophene & 213 \\
\hline Ethyl sulfide & $<1$ & 4-Methyl benzothiophene & 147 \\
\hline MN-butyl mercaptan & $<1$ & 3-Methyl DBZT+2-methyl DBZT & 94 \\
\hline Dimethyl disulfide & $<1$ & 1-Methyl dibenzothiophene & 97 \\
\hline 2-Methyl thiophene & $<1$ & 4,6 Dimethyl dibenzothiophene & 354 \\
\hline 3-Methyl thiophene & $<1$ & Dimethyl dibenzothiophene & 75 \\
\hline Tetra-hydro thiophene & $<1$ & Trimethyl dibenzothiophene & 4 \\
\hline Ethyl methyl disulfide & $<1$ & Tetramethyl dibenzothiophene & 3,283 \\
\hline 2-Methyl-tetra-hydro-thiophene & $<1$ & Unidentified volatile sulfur & \\
\hline
\end{tabular}

* Raw data from the speciation analysis ratioed to $5,000 \mathrm{ppm}$ to reflect the design basis sulfur content. 


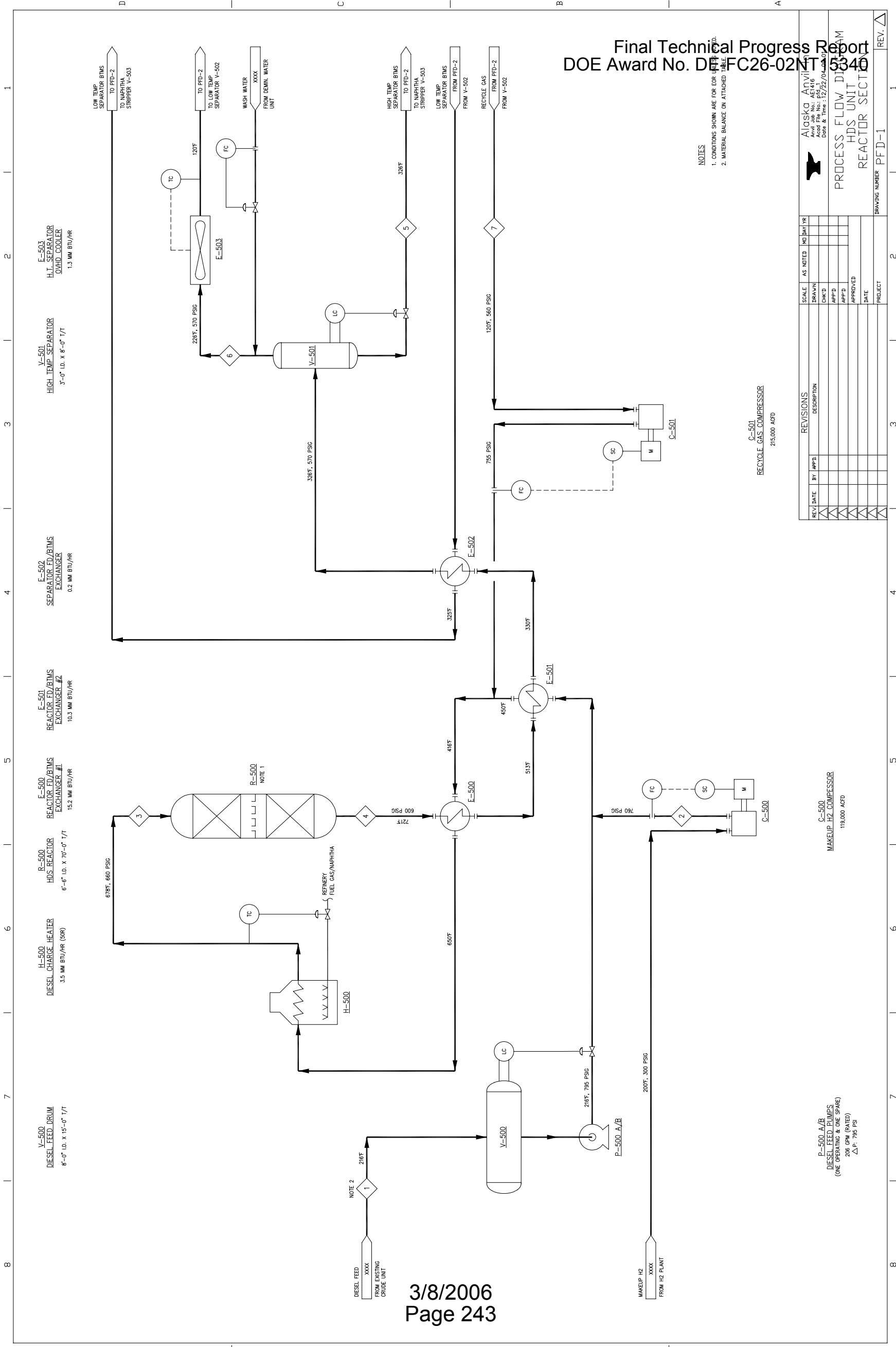


Final Technical Progress Report

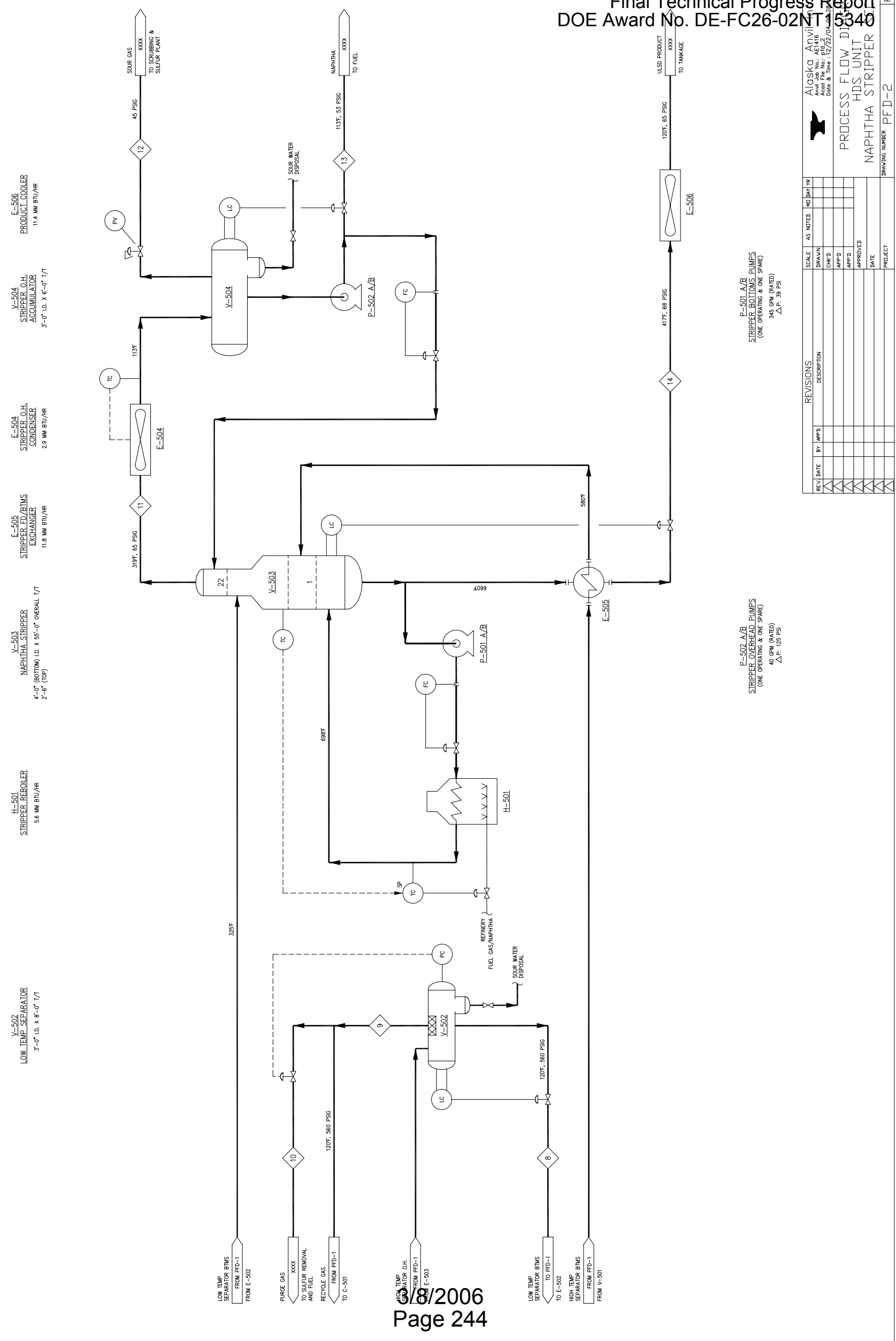




\section{Material Balance - EOR Conditions}

\begin{tabular}{|c|c|c|c|c|c|c|c|c|}
\hline Stream Number & & 1 & 2 & 3 & 4 & 5 & 6 & 7 \\
\hline Stream Description & & ANS FEED & $\begin{array}{l}\text { Compressed } \\
\text { Make-up Gas }\end{array}$ & Reactor Feed & Reactor Outlet & V-501 Liquid & $\begin{array}{l}\text { V-501 Vapor + } \\
\text { Wash Water }\end{array}$ & Recycle Gas \\
\hline Phase & & Liquid & Vapor & Mixed & Mixed & Liquid & Mixed & Vapor \\
\hline Mass Flow Rate & LB/HR & 75,128 & 463 & 79,713 & 79,714 & 74,910 & 5,504 & 4,123 \\
\hline Temperature & $\mathrm{F}$ & 216 & 227 & 678 & 721 & 300 & 226 & 120 \\
\hline Pressure & PSIG & 20.0 & 730.0 & 660.0 & 600.0 & 570.0 & 570.0 & 560.0 \\
\hline Standard Liq Flow & BPD & 6,000 & & & & 6,055 & & \\
\hline Volumetric Flow & MSCFH & & 85.3 & & & & & 306.9 \\
\hline Wt \% Vapor & & 0.0 & 100.0 & 43.0 & 66.6 & 0.0 & 93.1 & 100.0 \\
\hline MW & & 218.8 & 2.1 & 57.8 & 64.9 & 194.5 & 6.2 & 5.1 \\
\hline
\end{tabular}

\begin{tabular}{|l|l|c|c|c|c|c|c|c|}
\hline Stream Number & & $\mathbf{8}$ & $\mathbf{9}$ & $\mathbf{1 0}$ & $\mathbf{1 1}$ & $\mathbf{1 2}$ & $\mathbf{1 3}$ & $\mathbf{1 4}$ \\
\hline Stream Description & & $\begin{array}{c}\text { V-502 HC } \\
\text { Liquid }\end{array}$ & V-502 Vapor & Purge Gas & $\begin{array}{c}\text { Column Vapor } \\
\text { to Condenser }\end{array}$ & $\begin{array}{c}\text { Sour Gas } \\
\text { Naphtha }\end{array}$ & $\begin{array}{c}\text { Diesel from E- } \\
505\end{array}$ \\
\hline Phase & & Liquid & Vapor & Vapor & Vapor & Vapor & Liquid & Liquid \\
\hline Mass Flow Rate & LB/HR & 547 & 4,267 & 144 & 12,614 & 574 & 2,292 & 72,591 \\
\hline Temperature & F & 120 & 120 & 120 & 319 & 113 & 113 & 352 \\
\hline Pressure & PSIG & 560.0 & 560.0 & 560.0 & 65.0 & 63.0 & 63.0 & 68.0 \\
\hline Standard Liq Flow & BPD & 48 & & & & 10 & 208 & 5,817 \\
\hline Volumetric Flow & MSCFH & & 317.6 & 10.7 & 61.8 & 10.0 & & \\
\hline Wt \% Vapor & & 0.0 & 100.0 & 100.0 & 100.0 & 100.0 & 0.0 & 0.0 \\
\hline MW & & 110.0 & 5.1 & 5.1 & 77.4 & 21.7 & 88.2 & 214.9 \\
\hline
\end{tabular}




\section{Appendix C - HDS Unit Equipment List and Budgetary Equipment Data Sheets}

\section{HDS Unit Equipment List}

\begin{tabular}{|c|c|c|c|c|c|}
\hline \multirow{2}{*}{ Item } & \multirow{2}{*}{ Service } & \multirow{2}{*}{ Description } & \multicolumn{2}{|l|}{ Design Conditions } & \multirow{2}{*}{ Metallurgy } \\
\hline & & & Pressure & Temp. & \\
\hline $\mathrm{R}-500$ & HDS Reactor & $\begin{array}{l}\text { 6'-6" ID X 70'-0" T/T w/ } 2 \\
\text { sections of HR-526 Co } \\
\text { Mo catalyst. }\end{array}$ & 710 PSIG/FV & $750^{\circ} \mathrm{F}$ & $\begin{array}{l}\text { SA387 Gr. } 11 \mathrm{w} / 321 \mathrm{SS} \text { or } 347 \mathrm{SS} \\
\text { weld overlay. Internal trays are } 410 \\
\text { SS or } 321 \mathrm{SS}\end{array}$ \\
\hline V-500 & Diesel Feed Drum & $\begin{array}{l}\text { 8'-0" ID X 15'-0" T/T } \\
\text { (Horizontal) }\end{array}$ & $50 \mathrm{PSIG} / \mathrm{FV}$ & $275 /-20^{\circ} \mathrm{F}$ & Killed Carbon Steel \\
\hline V-501 & $\begin{array}{l}\text { High Temperature } \\
\text { Separator }\end{array}$ & $\begin{array}{l}\text { 3'-0" ID X 8'-0" T/T } \\
\text { (Vertical) }\end{array}$ & $620 \mathrm{PSIG} / \mathrm{FV}$ & $380 /-20^{\circ} \mathrm{F}$ & Killed Carbon Steel w/0.15" CA \\
\hline V-502 & $\begin{array}{l}\text { Low Temperature } \\
\text { Separator }\end{array}$ & $\begin{array}{l}\text { 3'-0" ID X 8'-0" T/T' } \\
\text { (Horizontal w/ Boot) }\end{array}$ & $610 \mathrm{PSIG} / \mathrm{FV}$ & $350 /-20^{\circ} \mathrm{F}$ & $\begin{array}{l}\text { Killed Carbon Steel w/ 0.1" CA } \\
\text { (PWHT) and Monel Demister }\end{array}$ \\
\hline V-503 & Naphtha Stripper & $\begin{array}{l}\text { 2'-6” ID (Top), 4'-0" ID } \\
\text { (bottom) } \\
\text { X 55'-0"T/T (Overall) } \\
\text { w/ } 22 \text { valve trays }\end{array}$ & $125 \mathrm{PSIG} / \mathrm{FV}$ & $750 /-20^{\circ} \mathrm{F}$ & $\begin{array}{l}\text { Killed Carbon Steel w/ 0.2" CA and } \\
\text { Type } 410 \text { SS trays, supports, and } \\
\text { downcomers }\end{array}$ \\
\hline V-504 & $\begin{array}{l}\text { Stripper O. H. } \\
\text { Accumulator }\end{array}$ & $\begin{array}{l}\text { 3'-0" ID X 6'-0" T/T } \\
\text { (Horizontal w/Boot) }\end{array}$ & $125 \mathrm{PSIG} / \mathrm{FV}$ & $250 /-20^{\circ} \mathrm{F}$ & $\begin{array}{l}\text { Killed Carbon Steel w/0.125" CA } \\
\text { (PWHT) }\end{array}$ \\
\hline E-500 & $\begin{array}{l}\text { Reactor Fd/Btms } \\
\text { Exchanger \#1 }\end{array}$ & $\begin{array}{l}15.2 \mathrm{MM} \text { BTU/Hr, } \\
4770 \mathrm{FT} 2 \mathrm{w} / 2 \mathrm{shells}, \\
\text { TEMA type CEU }\end{array}$ & $\begin{array}{l}\text { Tubes: } 650 \text { PSIG } \\
\text { Shell: } 800 \text { PSIG }\end{array}$ & $\begin{array}{l}775^{\circ} \mathrm{F} \\
700^{\circ} \mathrm{F}\end{array}$ & 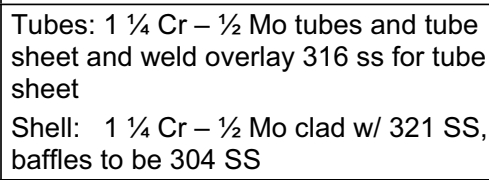 \\
\hline E-501 & $\begin{array}{l}\text { Reactor Fd/Btms } \\
\text { Exchanger \#2 }\end{array}$ & $\begin{array}{l}\text { 10.3 MM BTU/Hr, } \\
2904 \text { FT2 w/2 shells, } \\
\text { TEMA type CEU }\end{array}$ & $\begin{array}{l}\text { Tubes: } 650 \text { PSIG } \\
\text { Shell: } 810 \text { PSIG }\end{array}$ & $\begin{array}{l}540^{\circ} \mathrm{F} \\
475^{\circ} \mathrm{F}\end{array}$ & $\begin{array}{l}\text { Tubes: } 1 \frac{1 / 4}{4} \mathrm{Cr}-1 / 2 \text { Mo for tubes and } \\
\text { tube sheet, SA } 387 \mathrm{Gr} 11 \text { channel, } \\
\text { weld overlay } 321 \mathrm{SS} \text { tube sheet, } \\
\text { channel, and channel cover } \\
\text { Shell: Killed C. S. w/ } 0.125 \text { " CA }\end{array}$ \\
\hline E-502 & $\begin{array}{l}\text { Separator } \\
\text { Fd/Btms } \\
\text { Exchanger }\end{array}$ & $\begin{array}{l}0.2 \mathrm{MM} \text { BTU/Hr, } \\
70 \text { FT2, double pipe. }\end{array}$ & $\begin{array}{l}\text { Tubes: } 625 \text { PSIG } \\
\text { Shell: } 610 \text { PSIG }\end{array}$ & $\begin{array}{l}355^{\circ} \mathrm{F} \\
350^{\circ} \mathrm{F}\end{array}$ & $\begin{array}{l}\text { Tubes: 516-70 Carbon Steel } \\
\text { Shell: Killed Carbon Steel }\end{array}$ \\
\hline E-503 & $\begin{array}{l}\text { H. T. Separator } \\
\text { Overhead Cooler }\end{array}$ & $\begin{array}{l}\text { 1.3 MM BTU/Hr } \\
\text { 838 FT2 (Bare Tube) } \\
\text { 17,800 FT2 Extended } \\
\text { Surface, } 10 \text { HP Fan }\end{array}$ & Tubes: 620 PSIG & $350^{\circ} \mathrm{F}$ & $\begin{array}{l}\text { Seamless Carbon Steel tubes with } \\
\text { aluminum fins }\end{array}$ \\
\hline E-504 & $\begin{array}{l}\text { Stripper O. H. } \\
\text { Condenser }\end{array}$ & $\begin{array}{l}\text { 2.9 MM BTU/Hr } \\
\text { 838 FT2 (Bare Tube) } \\
\text { 17,800 FT2 Extended } \\
\text { Surface, } 10 \text { HP Fan }\end{array}$ & Tubes: 125 PSIG & $370^{\circ} \mathrm{F}$ & $\begin{array}{l}\text { Seamless Carbon Steel tubes with } \\
\text { aluminum fins }\end{array}$ \\
\hline E-505 & $\begin{array}{l}\text { Stripper Fd/Btms } \\
\text { Exchanger }\end{array}$ & $\begin{array}{l}\text { 11.8 MM BTU/Hr } \\
\text { 3,371 FT2 w/3 shells, } \\
\text { TEMA type CEU }\end{array}$ & $\begin{array}{l}\text { Tubes: } 620 \text { PSIG } \\
\text { Shell: } 100 \text { PSIG }\end{array}$ & $\begin{array}{l}630^{\circ} \mathrm{F} \\
710^{\circ} \mathrm{F}\end{array}$ & $\begin{array}{l}\text { Tubes: } 18 \mathrm{Cr}-8 \mathrm{Ni} \text {, Channel is CS w/ } \\
0.25 \text { " CA, tube sheets are } 410 \mathrm{SS} \\
\text { Shell: CS w/ } 0.25 \text { " CA, CS baffles }\end{array}$ \\
\hline E-506 & Product Cooler & $\begin{array}{l}\text { 11.4 MM BTU/Hr } \\
\text { 2,203 FT2 (Bare Tube) } \\
\text { 46,700 FT2 (Extended } \\
\text { Surface, } 17 \text { HP Fan }\end{array}$ & Tubes: 100 PSIG & $450^{\circ} \mathrm{F}$ & $\begin{array}{l}\text { Seamless Carbon Steel tubes with } \\
\text { aluminum fins }\end{array}$ \\
\hline $\mathrm{H}-500$ & $\begin{array}{l}\text { Diesel Charge } \\
\text { Heater }\end{array}$ & $\begin{array}{l}\text { 3.5 MM BTU/Hr } \\
\text { Fired Heater, Convection } \\
\text { Section shared w/ H-501 }\end{array}$ & Tubes: 800 PSIG & $750^{\circ} \mathrm{F}$ & Tubes: $9 \mathrm{Cr}-1 \mathrm{Mo} \mathrm{w} / 0.1$ " CA \\
\hline $\mathrm{H}-501$ & Stripper Reboiler & $\begin{array}{l}\text { 5.6 MM BTU/Hr } \\
\text { Fired Heater, Convection } \\
\text { Section shared w/ H-500 }\end{array}$ & Tubes: 230 PSIG & $770^{\circ} \mathrm{F}$ & Tubes: $5 \mathrm{Cr}-1 / 2 \mathrm{Mo} \mathrm{w} / 0.1$ " CA \\
\hline
\end{tabular}


HDS BASELINE CASE

\begin{tabular}{|c|c|c|c|c|c|}
\hline \multirow{2}{*}{ Item } & \multirow{2}{*}{ Service } & \multirow{2}{*}{ Description } & \multicolumn{2}{|l|}{ Design Conditions } & \multirow{2}{*}{ Metallurgy } \\
\hline & & & Pressure & Temp. & \\
\hline C-500 & $\begin{array}{l}\text { Makeup Hydrogen } \\
\text { Compressor }\end{array}$ & $\begin{array}{l}\text { Capacity: 119,000 ACFD } \\
\text { Motor Driven } \\
\text { Shaft HP: } 136 \text { BHP }\end{array}$ & $\begin{array}{l}\text { Suction: } 300 \text { PSIG } \\
\text { Disch: } 760 \text { PSIG }\end{array}$ & $\begin{array}{l}\text { Suction: } \\
200^{\circ} \mathrm{F}\end{array}$ & $\begin{array}{l}\text { Casing: killed C. S. } \\
\text { Internals: CS }\end{array}$ \\
\hline C-501 & $\begin{array}{l}\text { Recycle Gas } \\
\text { Compressor }\end{array}$ & $\begin{array}{l}\text { Capacity: } 215,000 \text { ACFD } \\
\text { Motor Driven } \\
\text { Shaft HP: } 126 \text { BHP } \\
\end{array}$ & $\begin{array}{l}\text { Suction: } 560 \text { PSIG } \\
\text { Disch: } 755 \text { PSIG }\end{array}$ & $\begin{array}{l}\text { Suction: } \\
120^{\circ} \mathrm{F}\end{array}$ & $\begin{array}{l}\text { Casing: killed C. S. } \\
\text { Internals: Stainless Steel }\end{array}$ \\
\hline $\begin{array}{l}P-500 \\
A / B\end{array}$ & $\begin{array}{l}\text { Diesel Feed Pump } \\
\text { (One Operating + } \\
\text { One Spare }\end{array}$ & $\begin{array}{l}\text { Rated Capacity: } 206 \text { GPM } \\
\text { Diff. Press: } 794 \text { PSI } \\
\text { Head: } 2282 \text { feet } \\
\text { Motor: } 150 \mathrm{HP}\end{array}$ & 955 PSIG & $250^{\circ} \mathrm{F}$ & $\begin{array}{l}\text { Casing: killed C. S. } \\
\text { Impeller: } 12 \% \text { chrome }\end{array}$ \\
\hline $\begin{array}{l}\text { P-501 } \\
\text { A/B }\end{array}$ & $\begin{array}{l}\text { Stripper Bottoms } \\
\text { Pump } \\
\text { (One Operating + } \\
\text { One Spare }\end{array}$ & $\begin{array}{l}\text { Rated Capacity: } 345 \text { GPM } \\
\text { Diff. Press: } 125 \text { PSI } \\
\text { Head: } 489 \text { feet } \\
\text { Motor: } 50 \text { HP }\end{array}$ & 225 PSIG & $710^{\circ} \mathrm{F}$ & $\begin{array}{l}\text { Casing: killed C. S. w/ } 0.2 " \text { CA } \\
\text { Impeller: } 12 \% \text { chrome }\end{array}$ \\
\hline $\begin{array}{l}\text { P-502 } \\
\text { A/B }\end{array}$ & $\begin{array}{l}\text { Stripper Overhead } \\
\text { Pump } \\
\text { (One Operating + } \\
\text { One Spare }\end{array}$ & $\begin{array}{l}\text { Rated Capacity: } 40 \text { GPM } \\
\text { Diff. Press: } 39 \text { PSI } \\
\text { Head: } 124 \text { feet } \\
\text { Motor: } 5 \text { HP }\end{array}$ & 115 PSIG & $125^{\circ} \mathrm{F}$ & $\begin{array}{l}\text { Casing: killed C. S. w/ } 0.125 " \text { CA } \\
\text { Impeller: } 12 \% \text { chrome }\end{array}$ \\
\hline
\end{tabular}




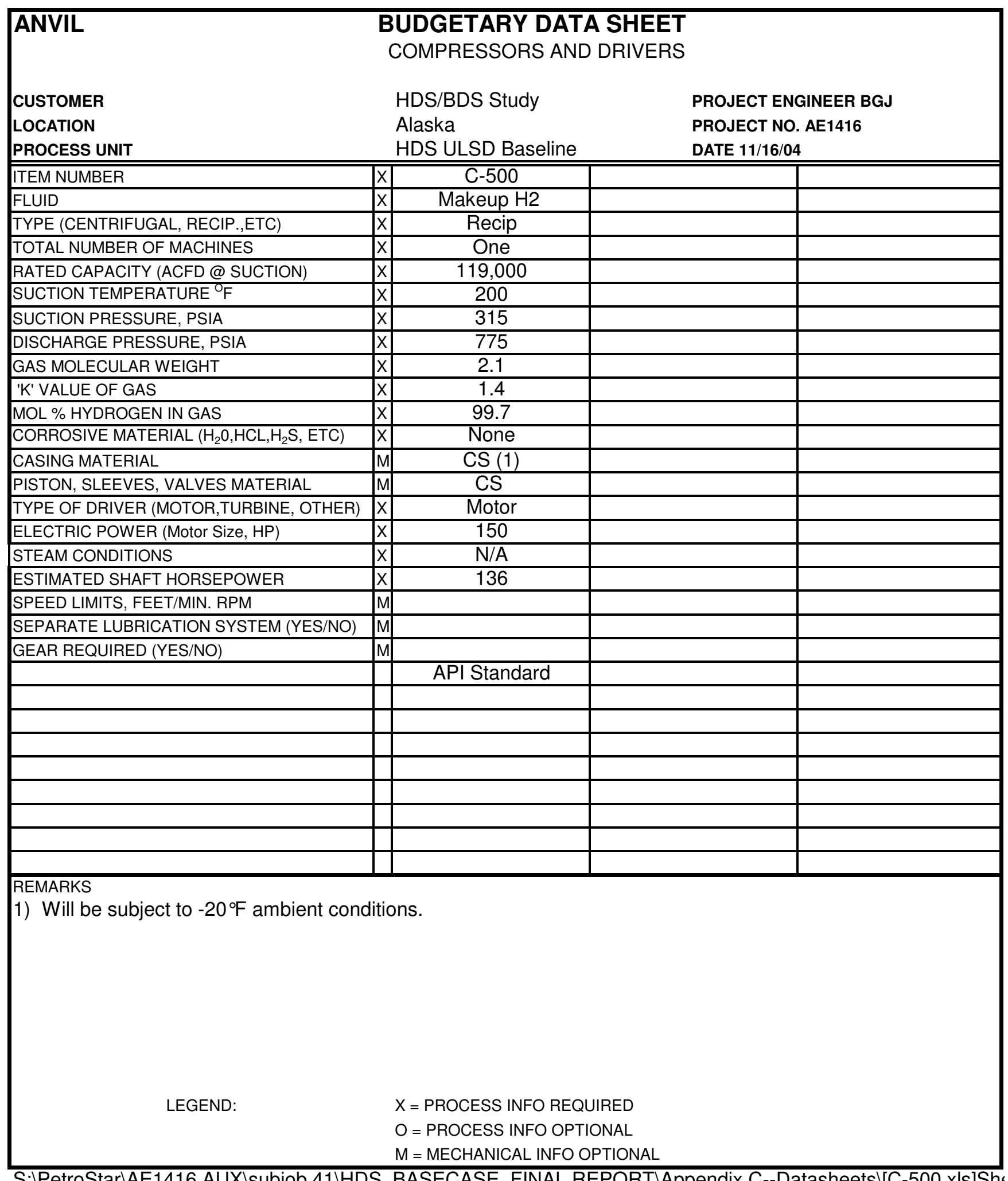




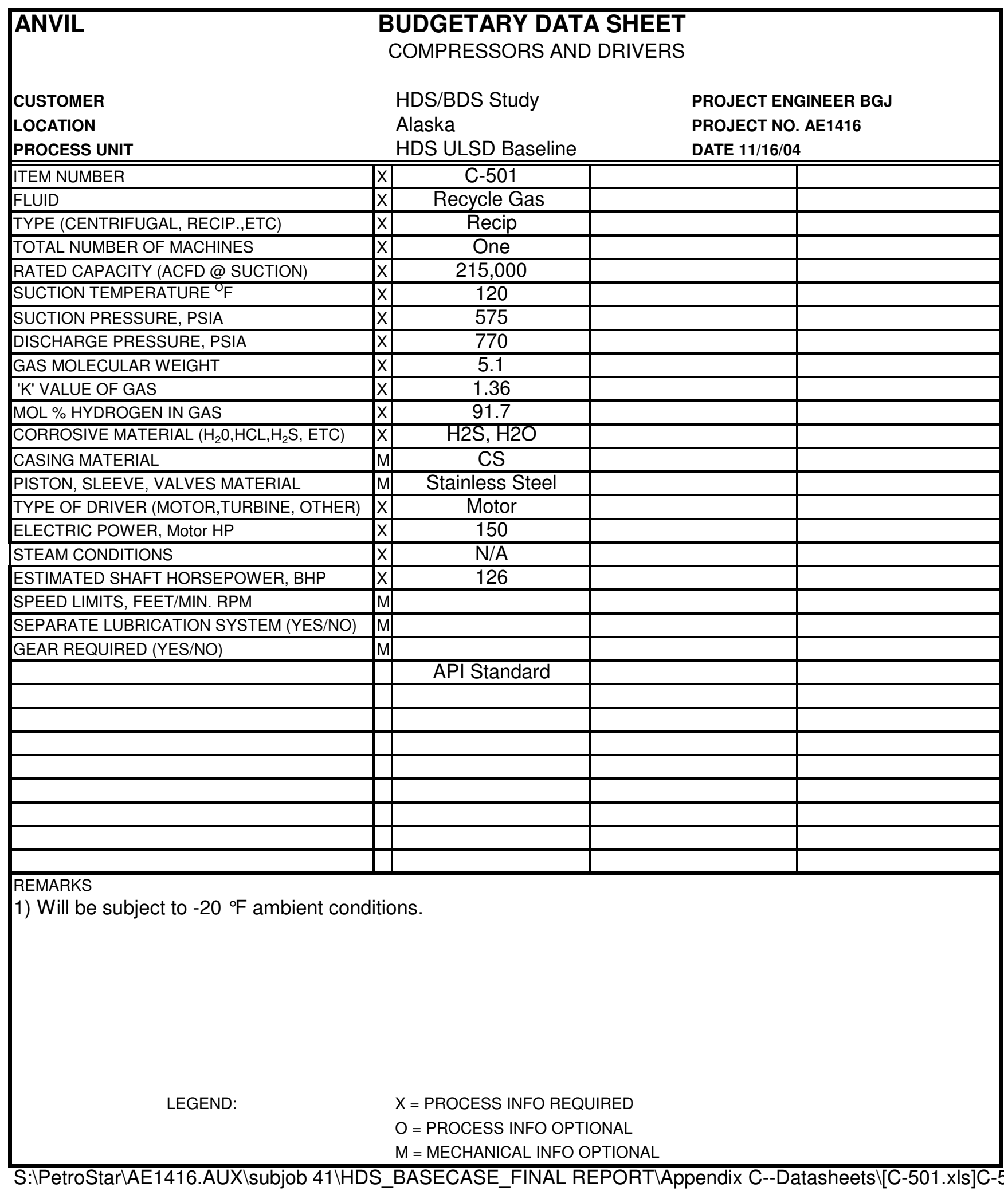




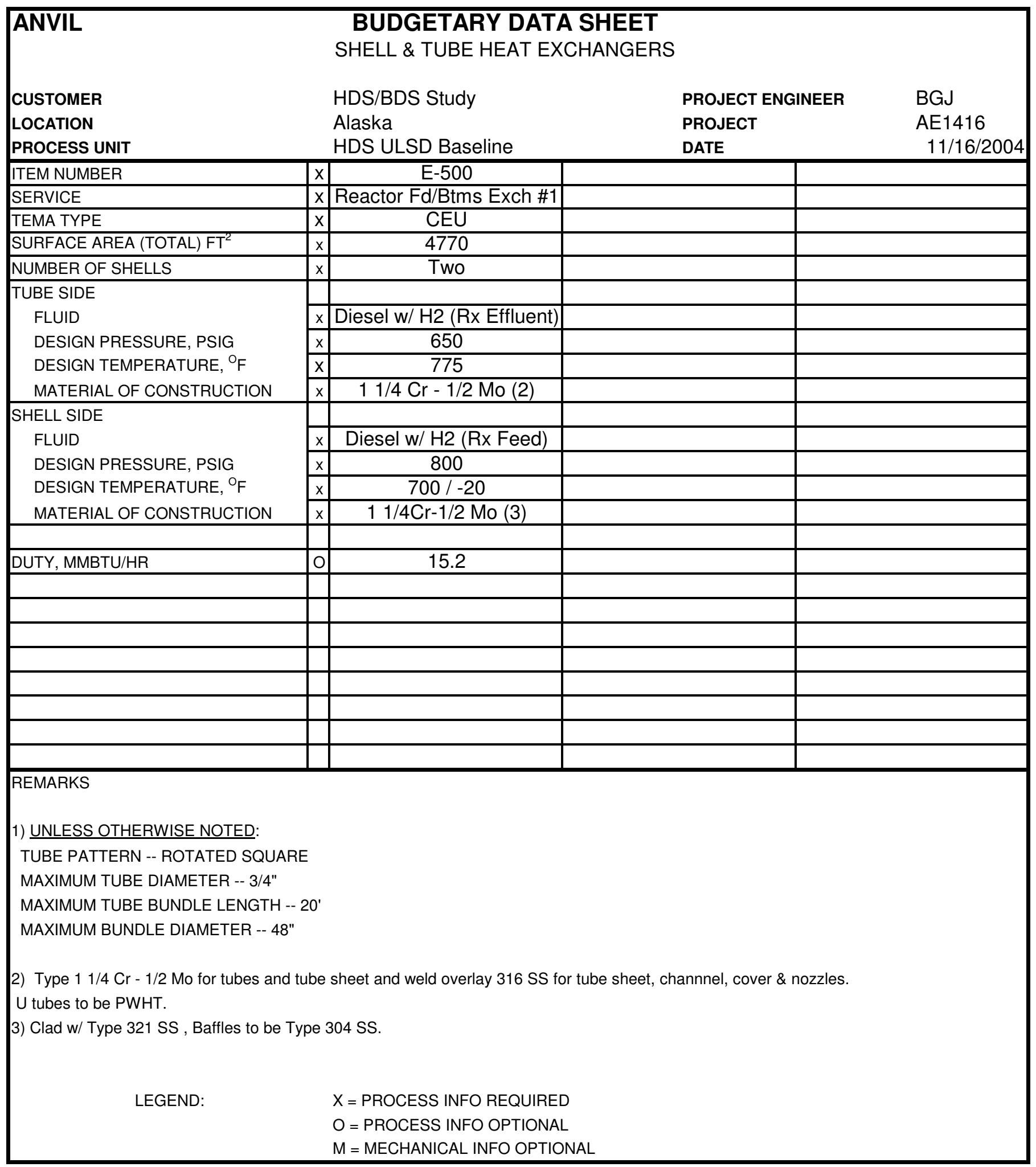

S:IPetroStar|AE1416.AUXIsubjob 41|HDS_BASECASE_FINAL REPORT\Appendix C--Datasheets|[E-500.xIs]Sheet1 


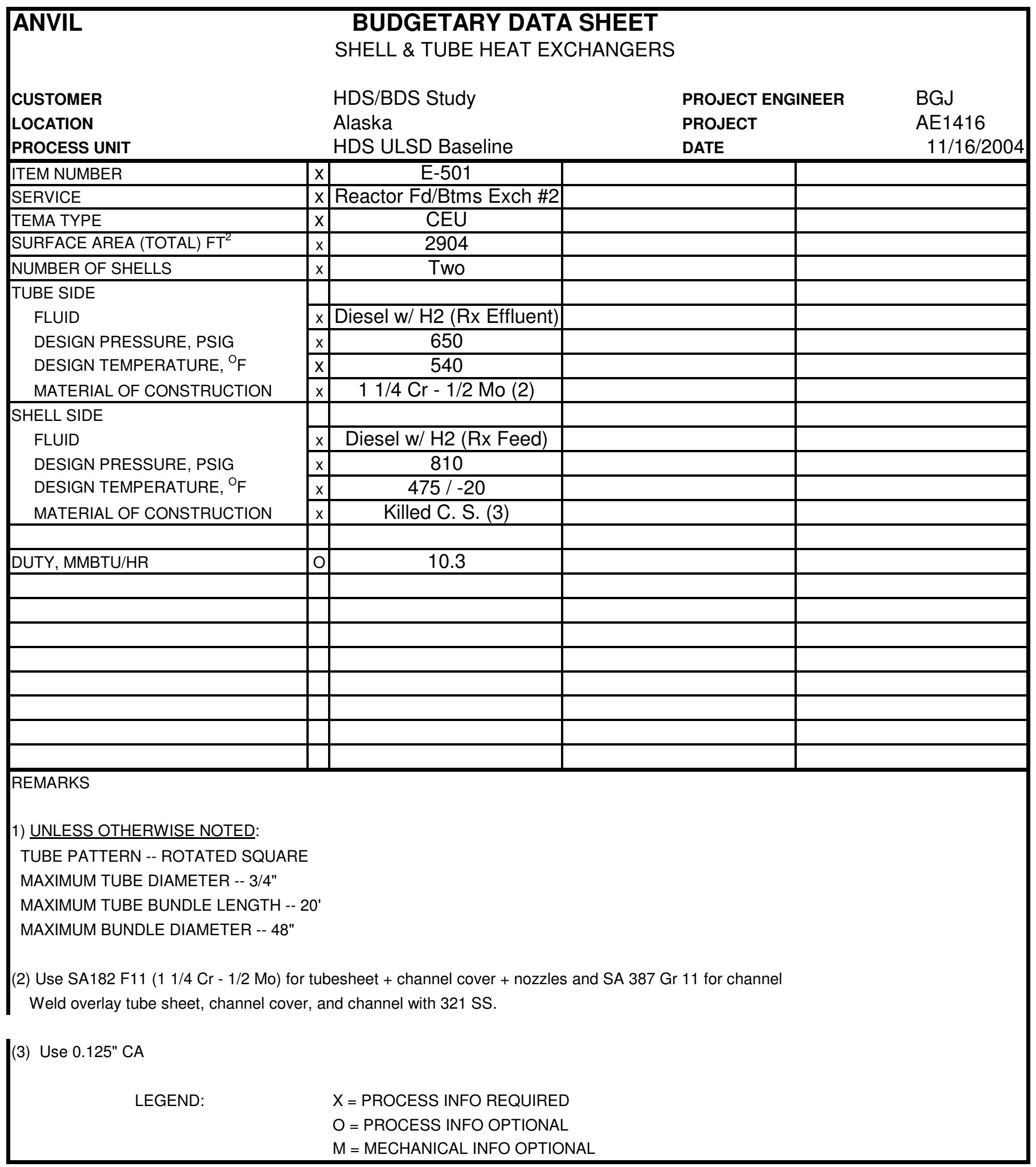

S:IPetroStar|AE1416.AUXIsubjob 41|HDS_BASECASE_FINAL REPORT/Appendix C--Datasheets|[E-501.xIs]Sheet1 


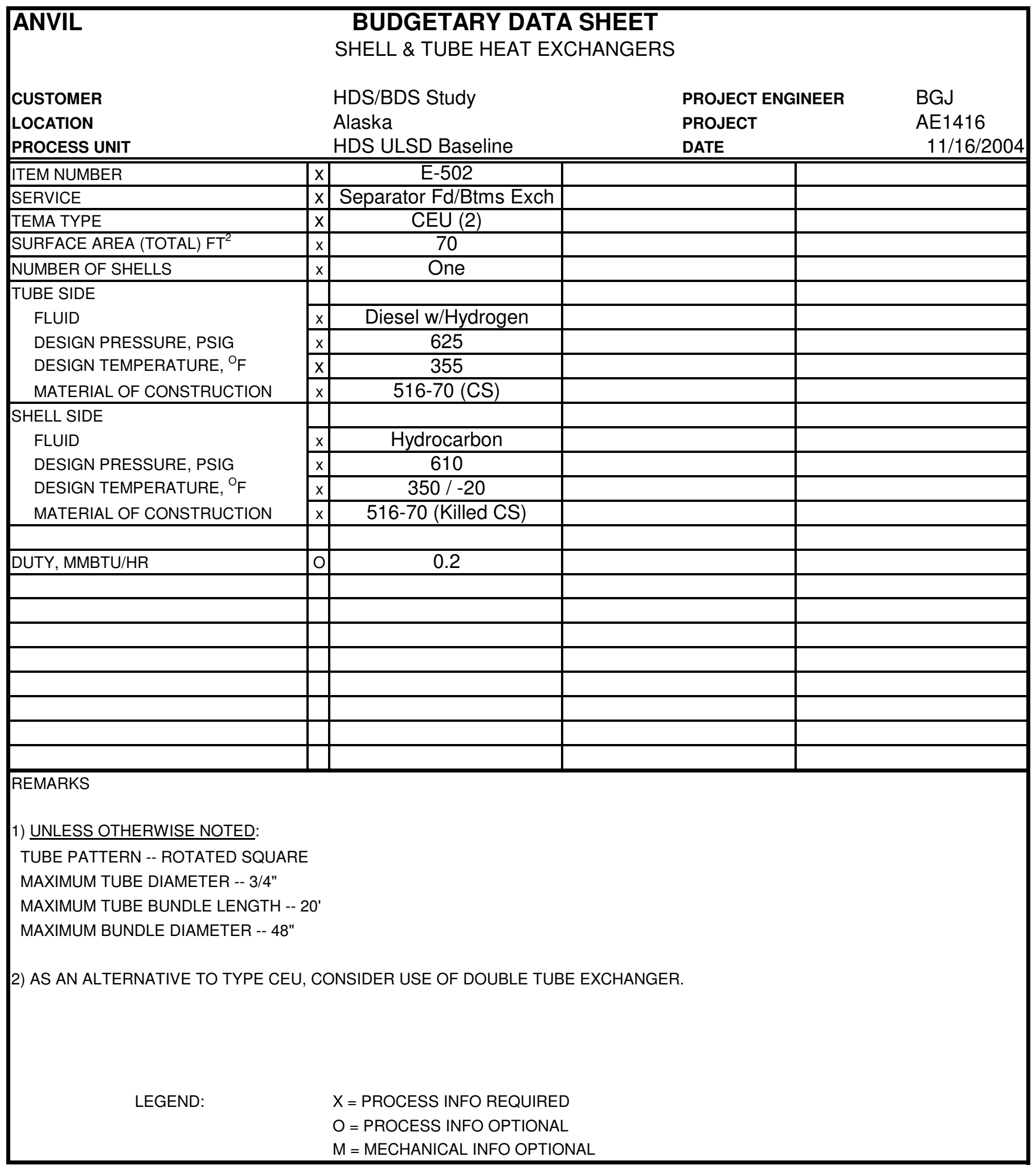

S:IPetroStarlAE1416.AUXIsubjob 411HDS_BASECASE_FINAL REPORT\Appendix C--Datasheetsl[E-502.xls]E-502 Rev B 


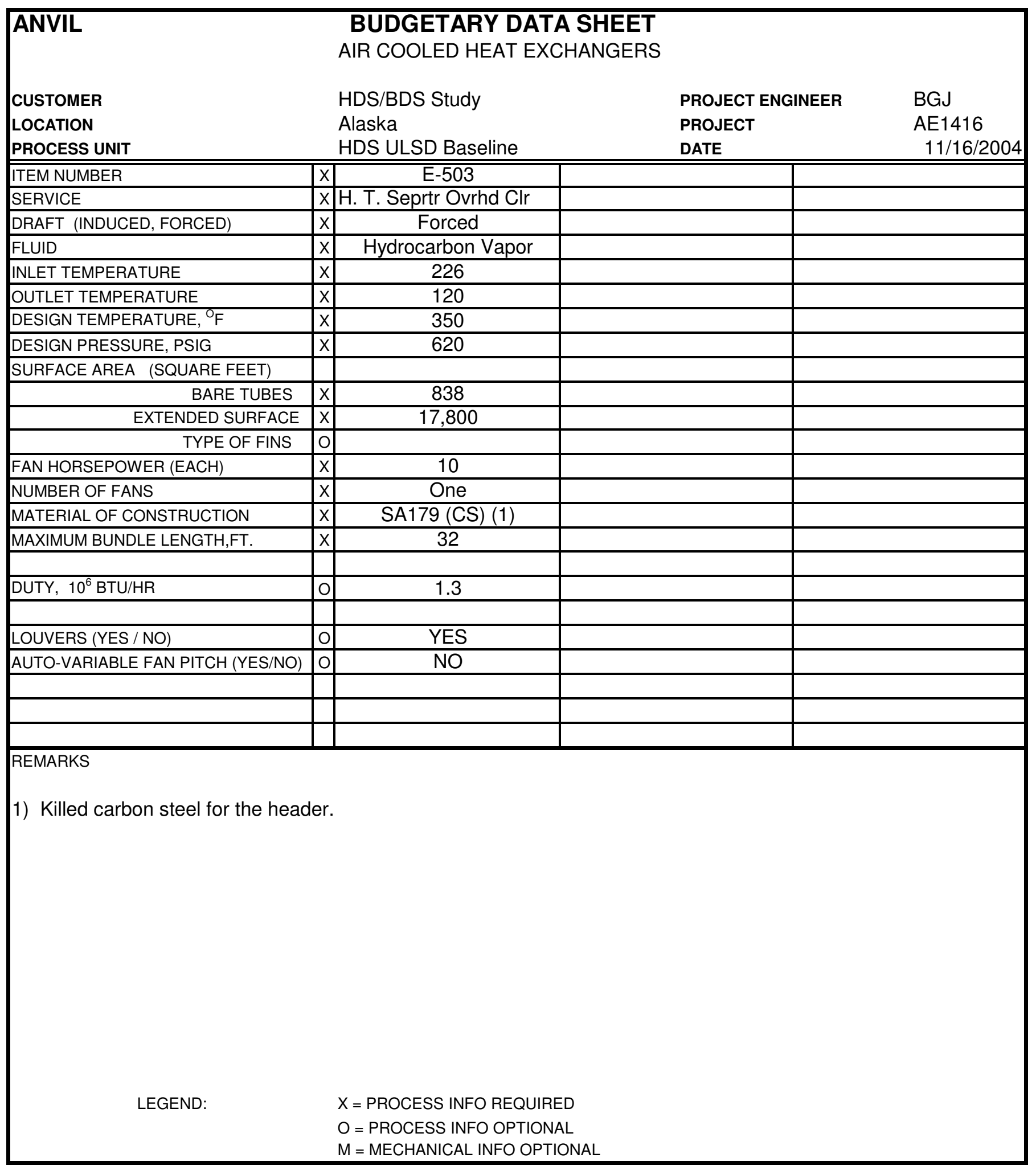

S:IPetroStar|AE1416.AUXIsubjob 411HDS_BASECASE_FINAL REPORT\Appendix C--Datasheets|[E-503.xls]E-503 Rev B 


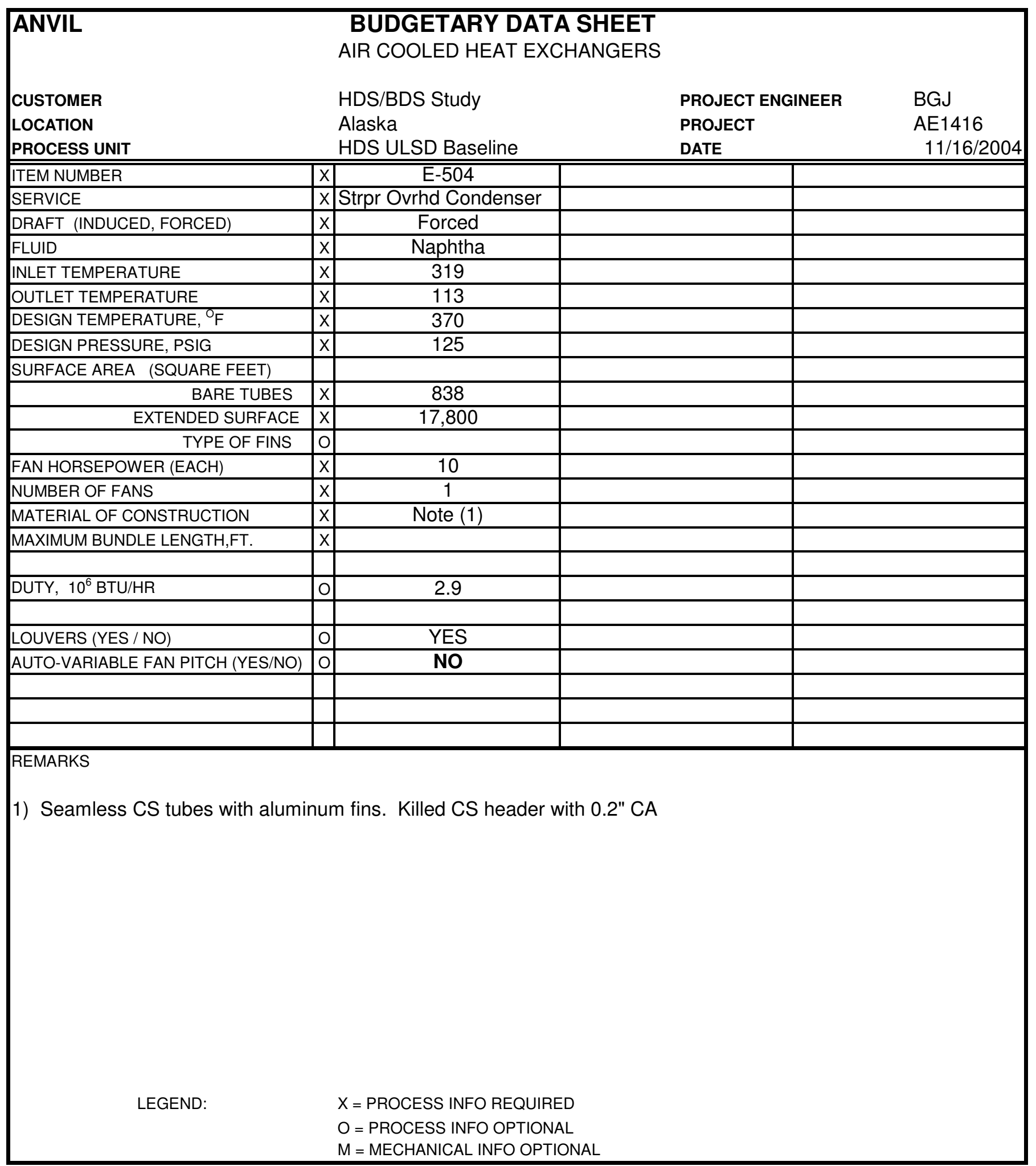

S:IPetroStar|AE1416.AUXIsubjob 411HDS_BASECASE_FINAL REPORT\Appendix C--Datasheets|[E-504.xIS]E-504 Rev B 


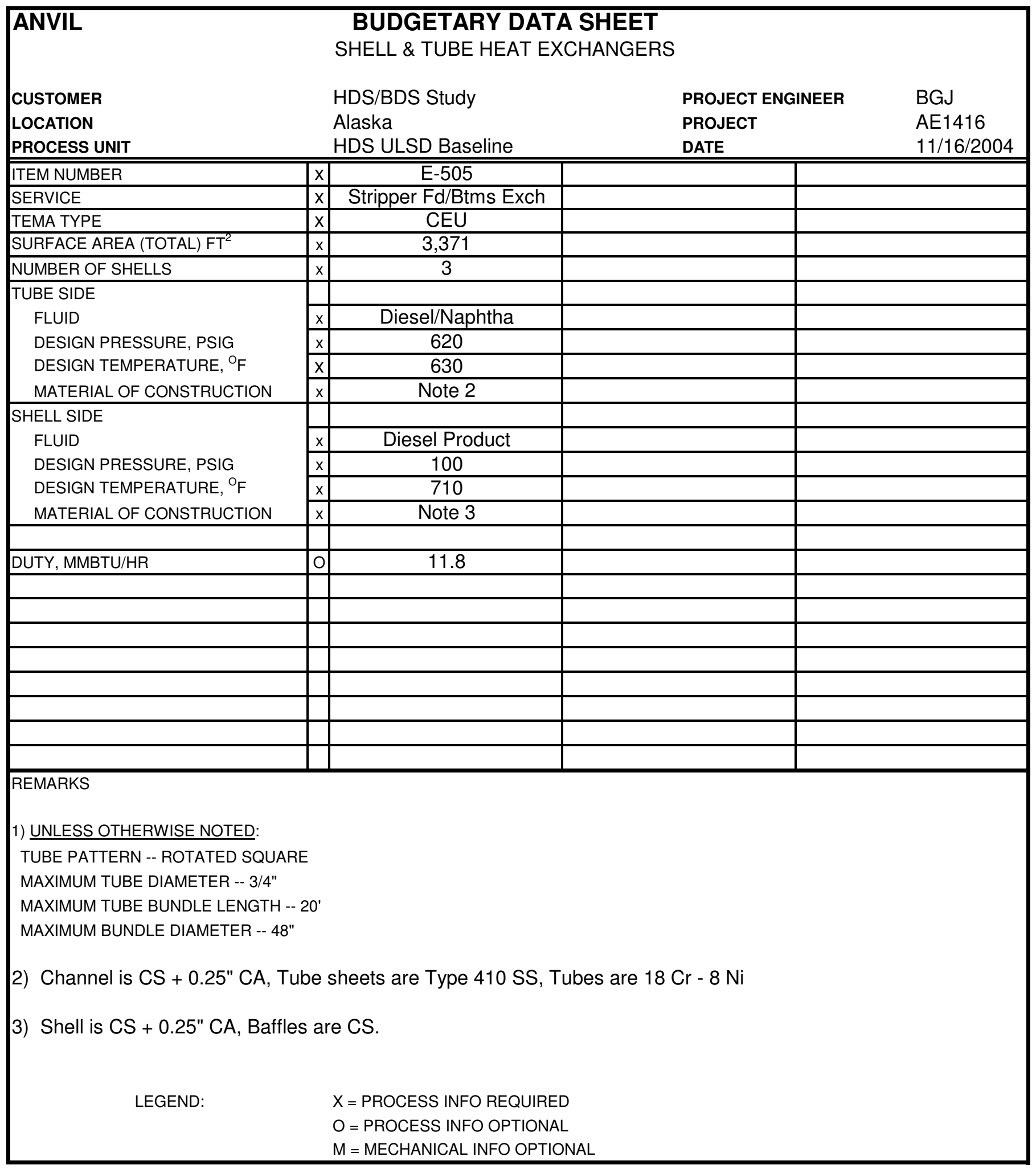




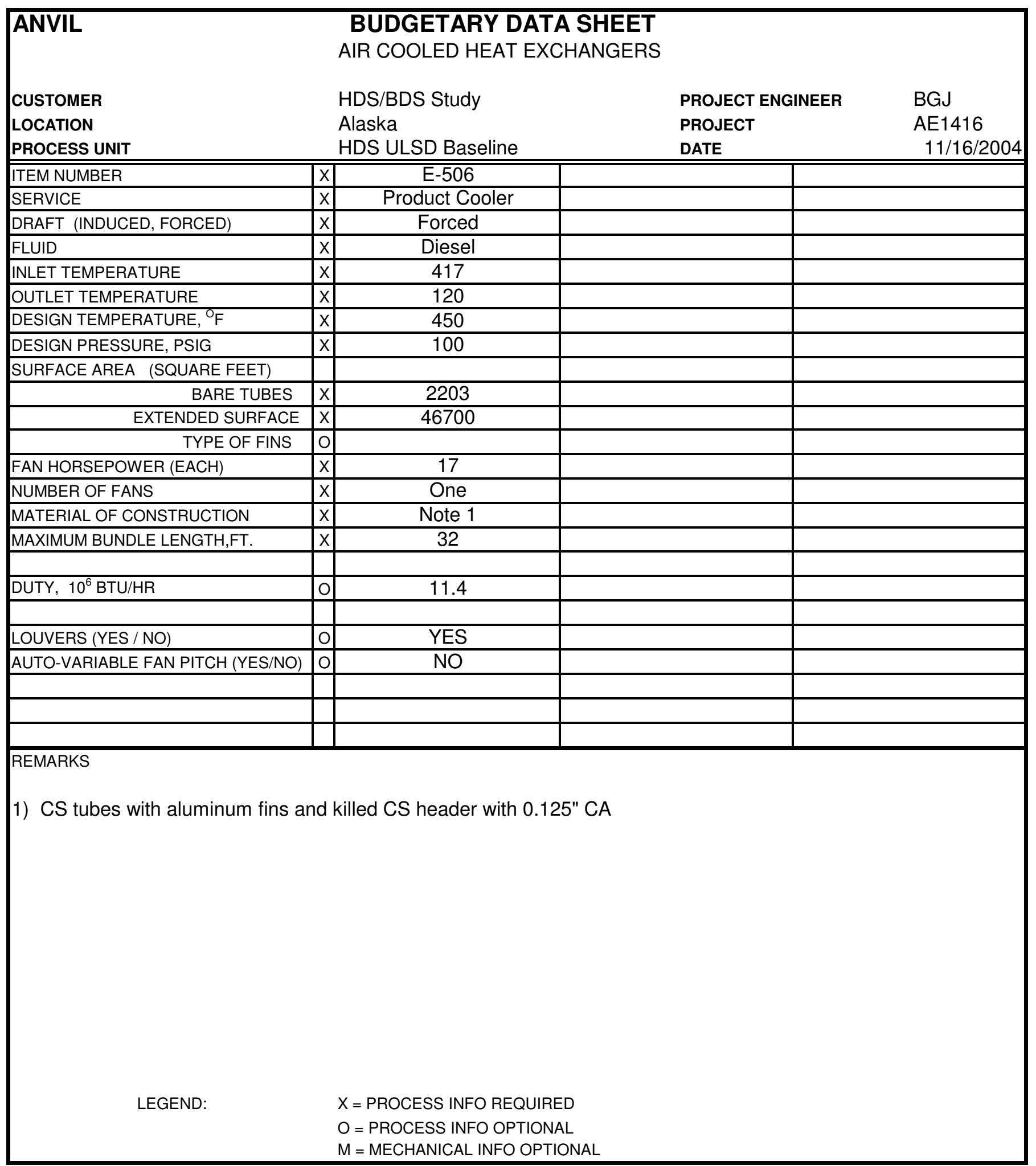

S:IPetroStar|AE1416.AUXIsubjob 411HDS_BASECASE_FINAL REPORT\Appendix C--Datasheets|[E-506.xIS]E-506 Rev B 


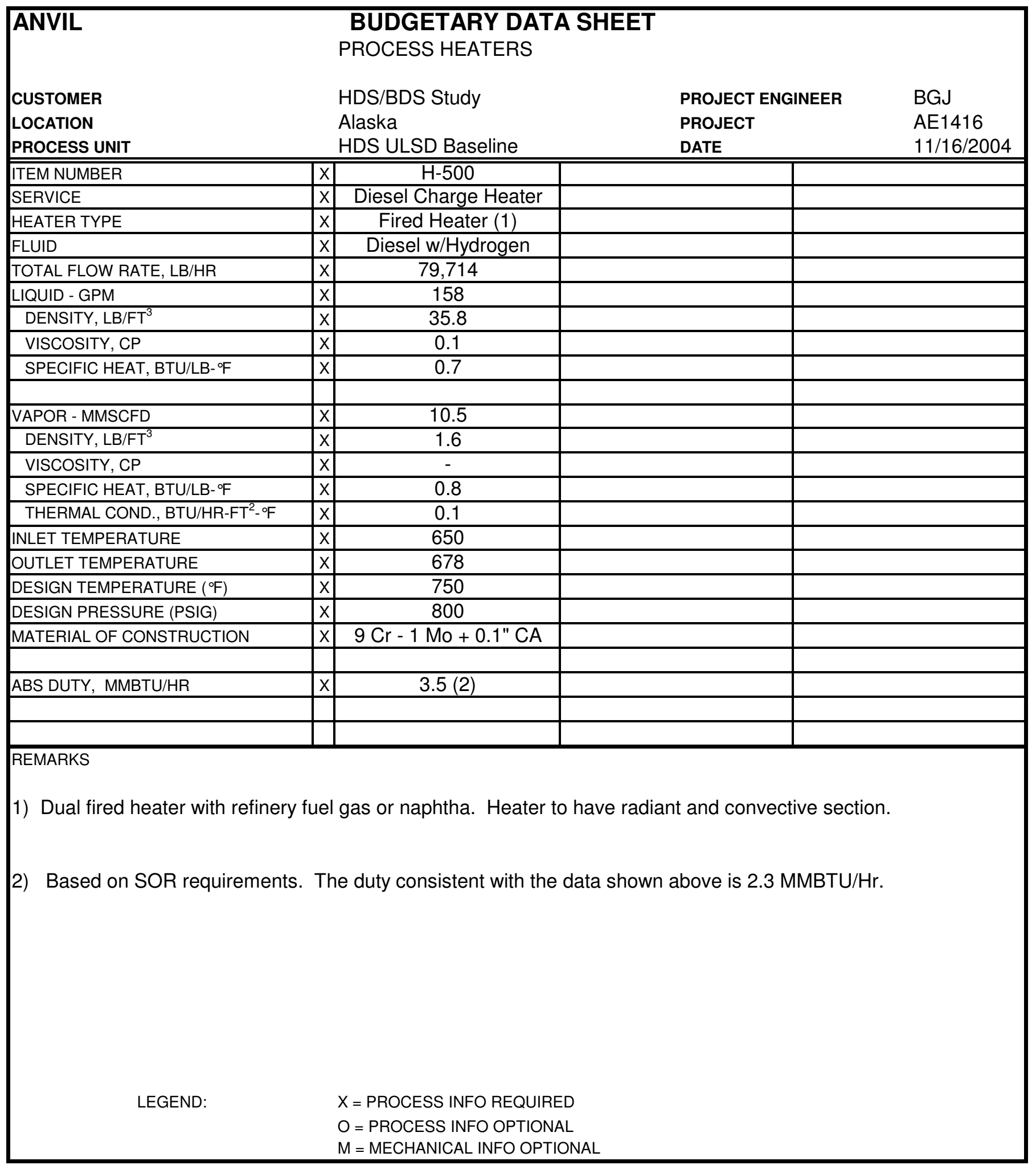




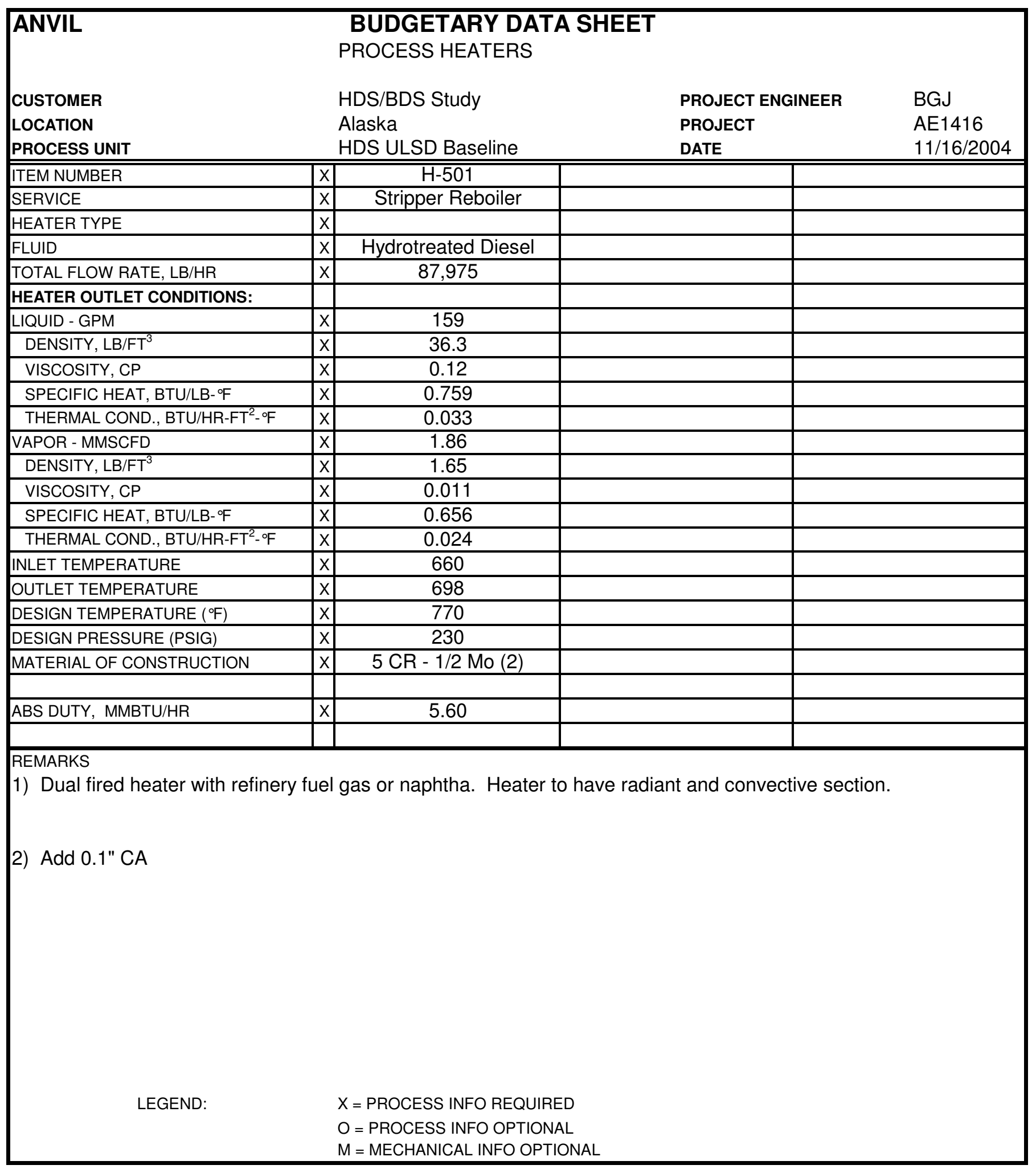




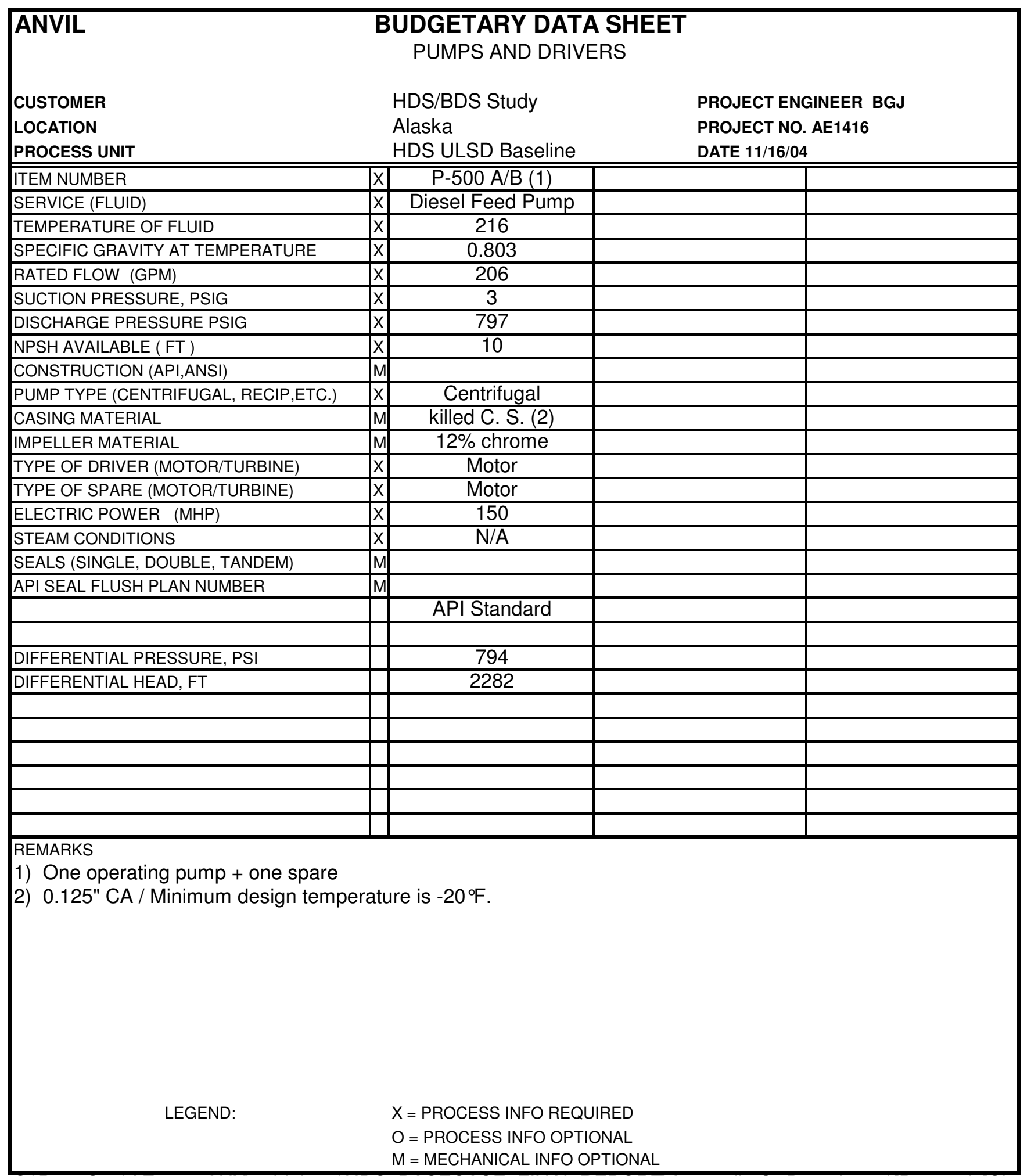

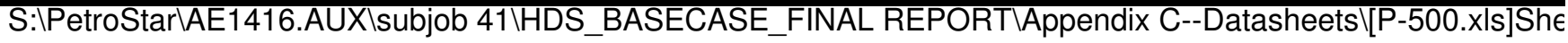




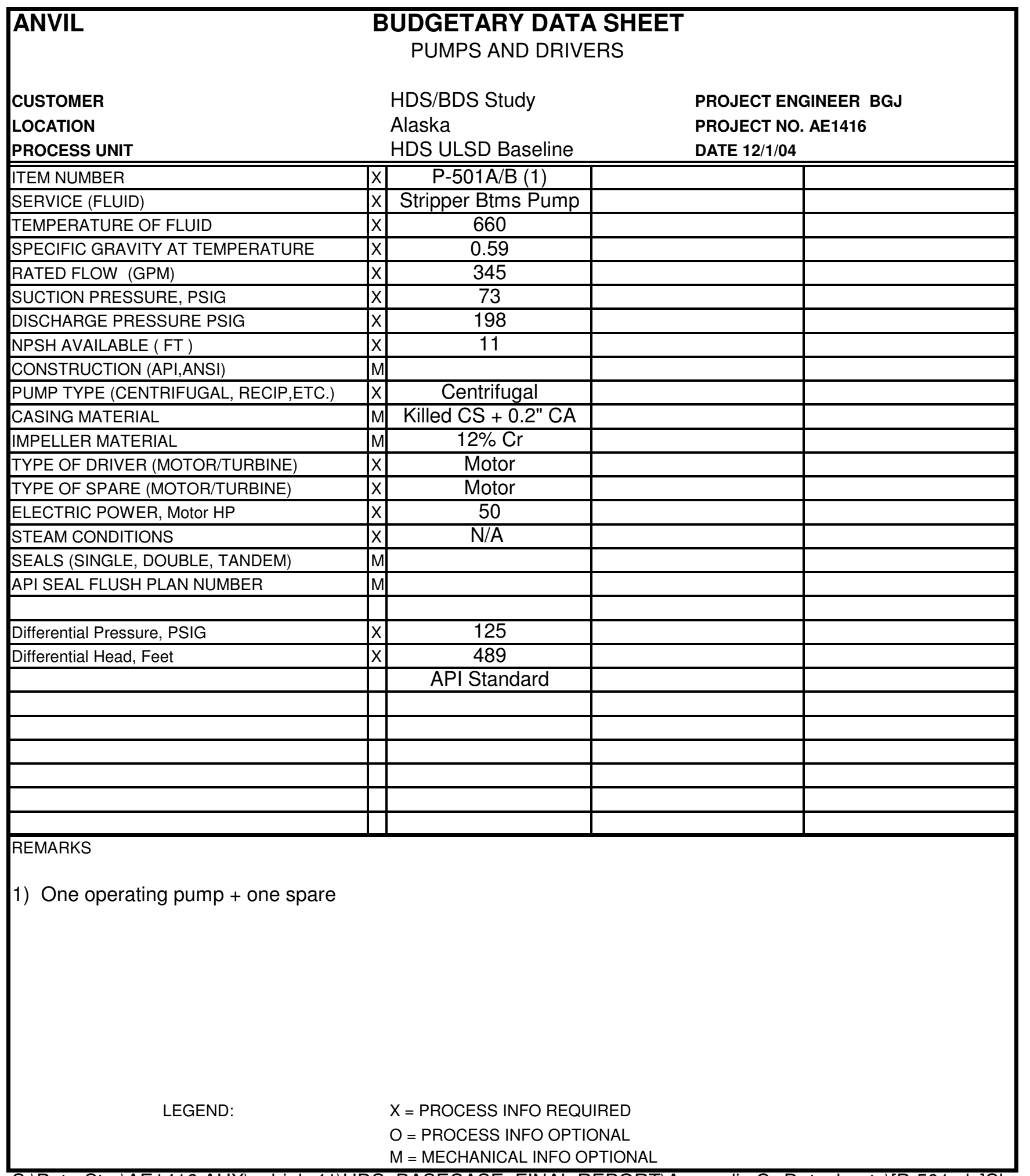




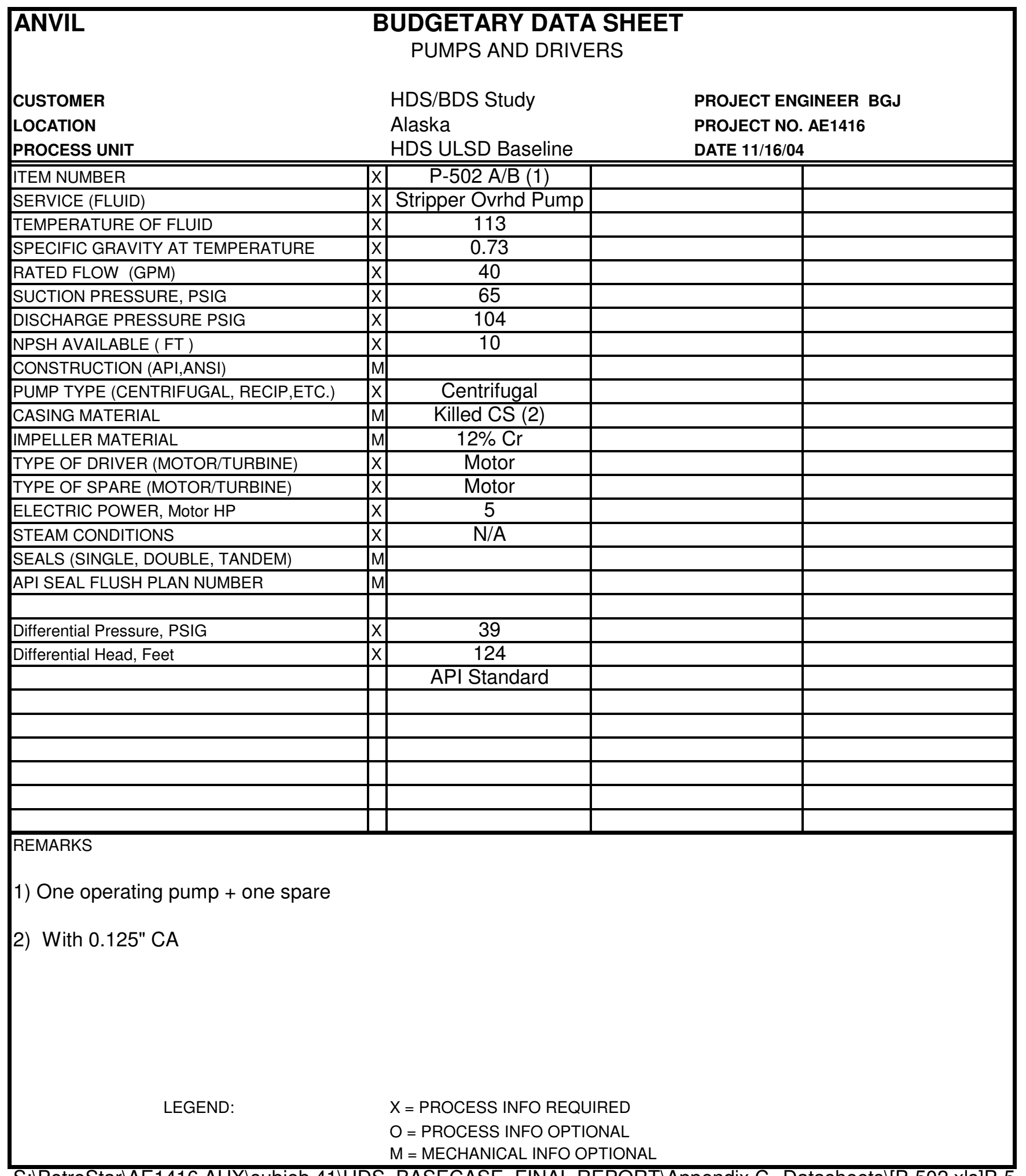




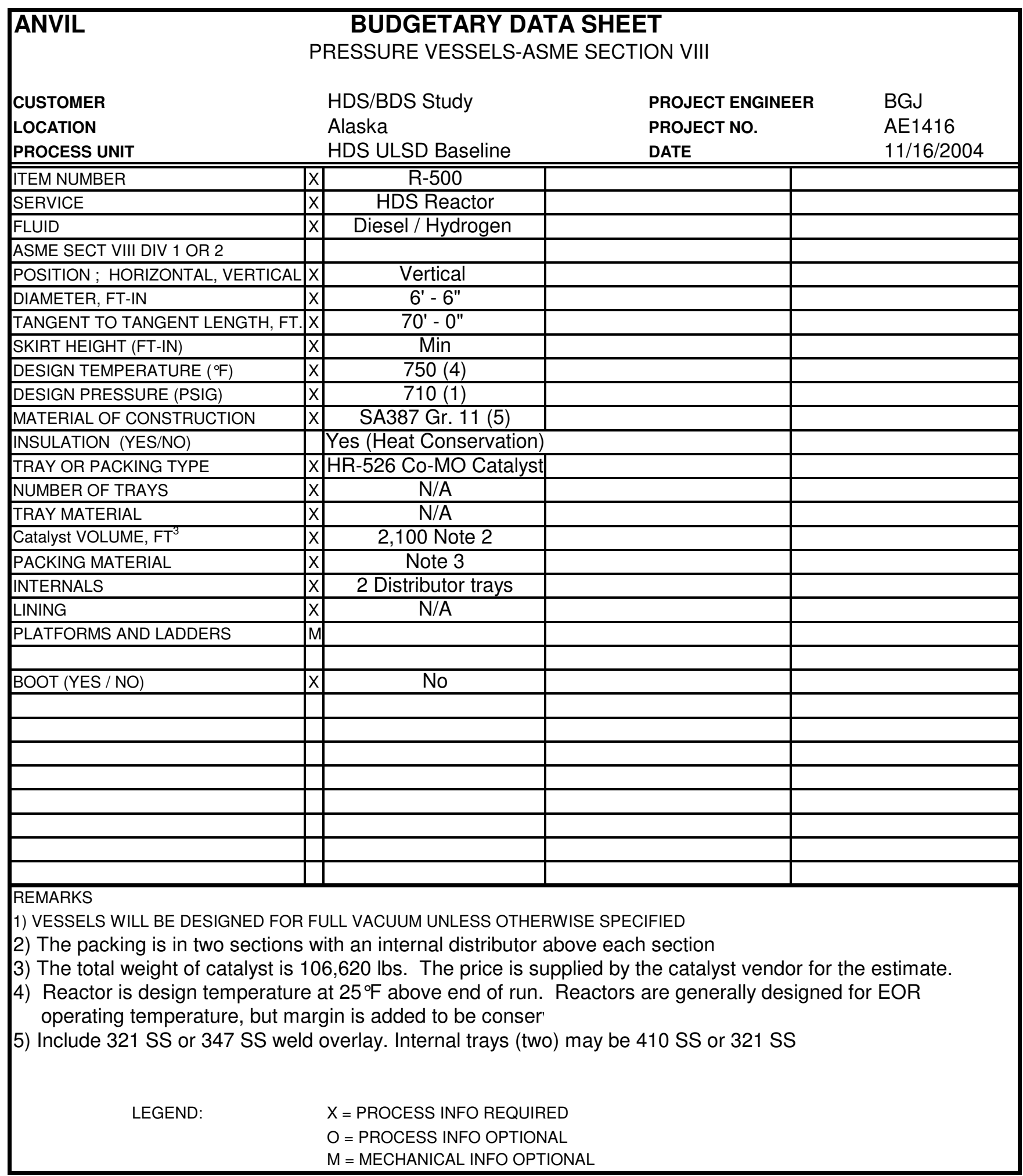

S:IPetroStarlAE1416.AUX|subjob 41|HDS_BASECASE_FINAL REPORT/Appendix C--Datasheets|[R-500.xls]Sheet1 


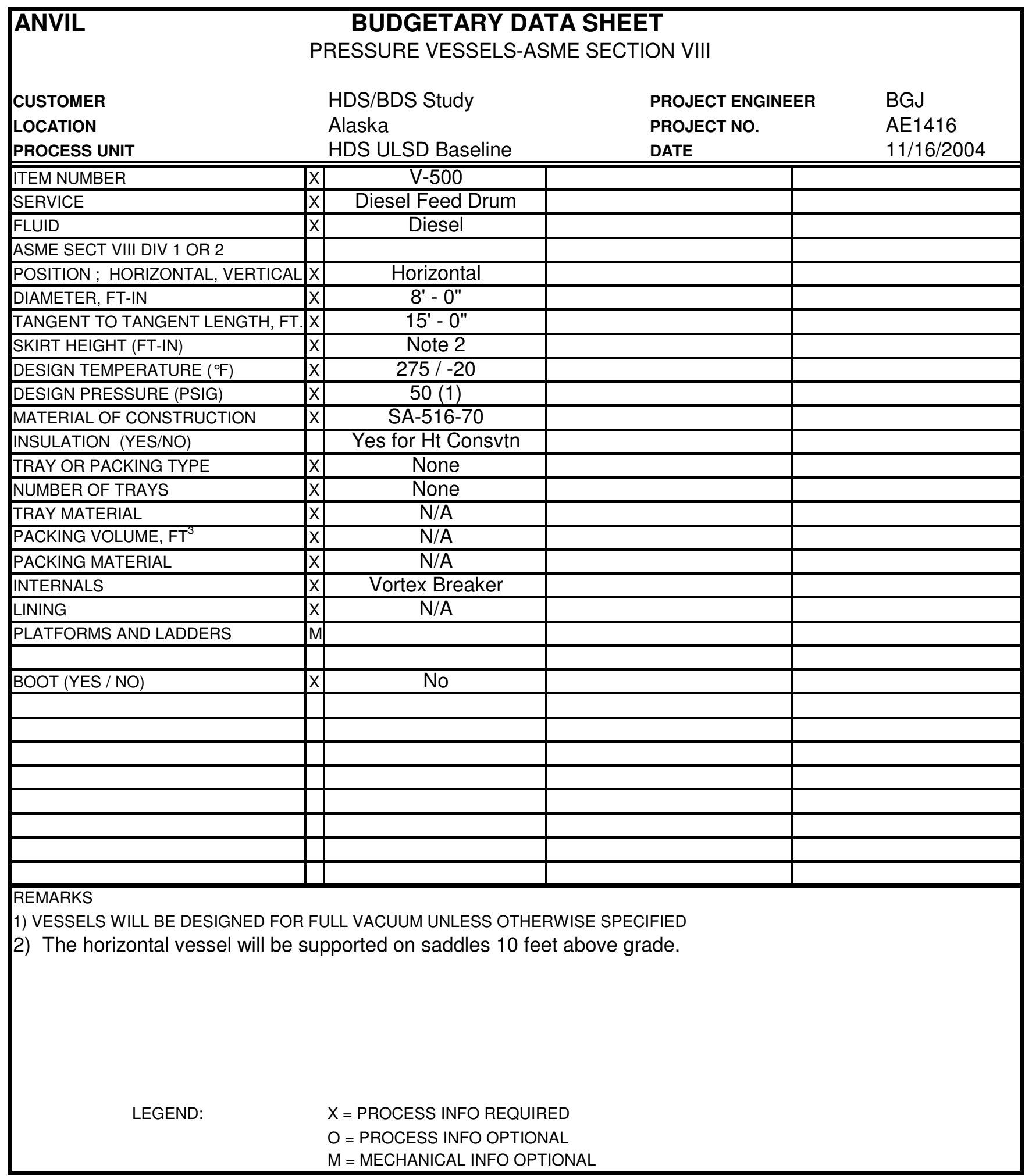

S:IPetroStar|AE1416.AUXIsubjob 411HDS_BASECASE_FINAL REPORT\Appendix C--DatasheetsI[V-500.xls]V-500 Rev B 


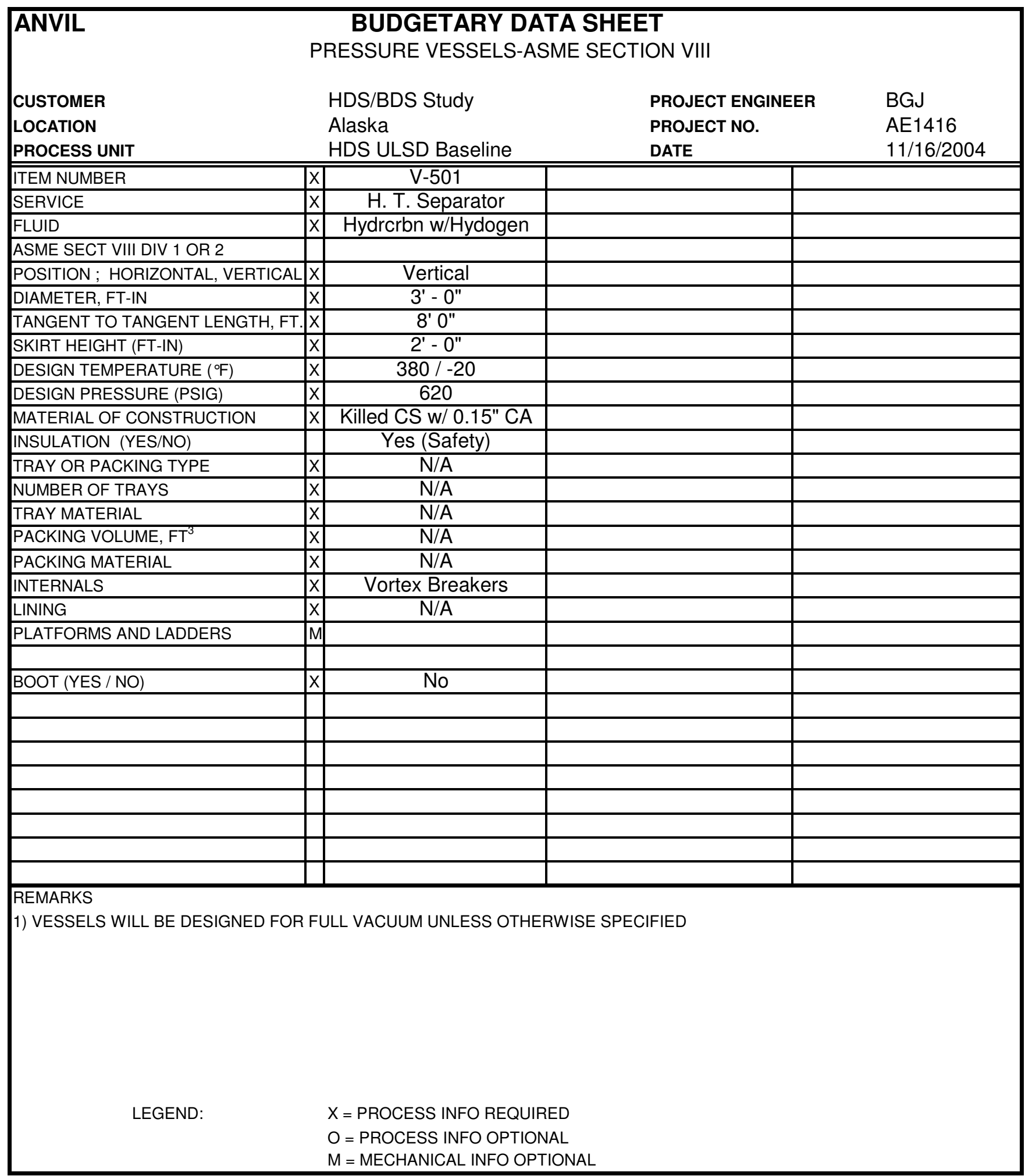

S:IPetroStar|AE1416.AUXIsubjob 411HDS_BASECASE_FINAL REPORT\Appendix C--DatasheetsI[V-501.xls]V-501 Rev B 


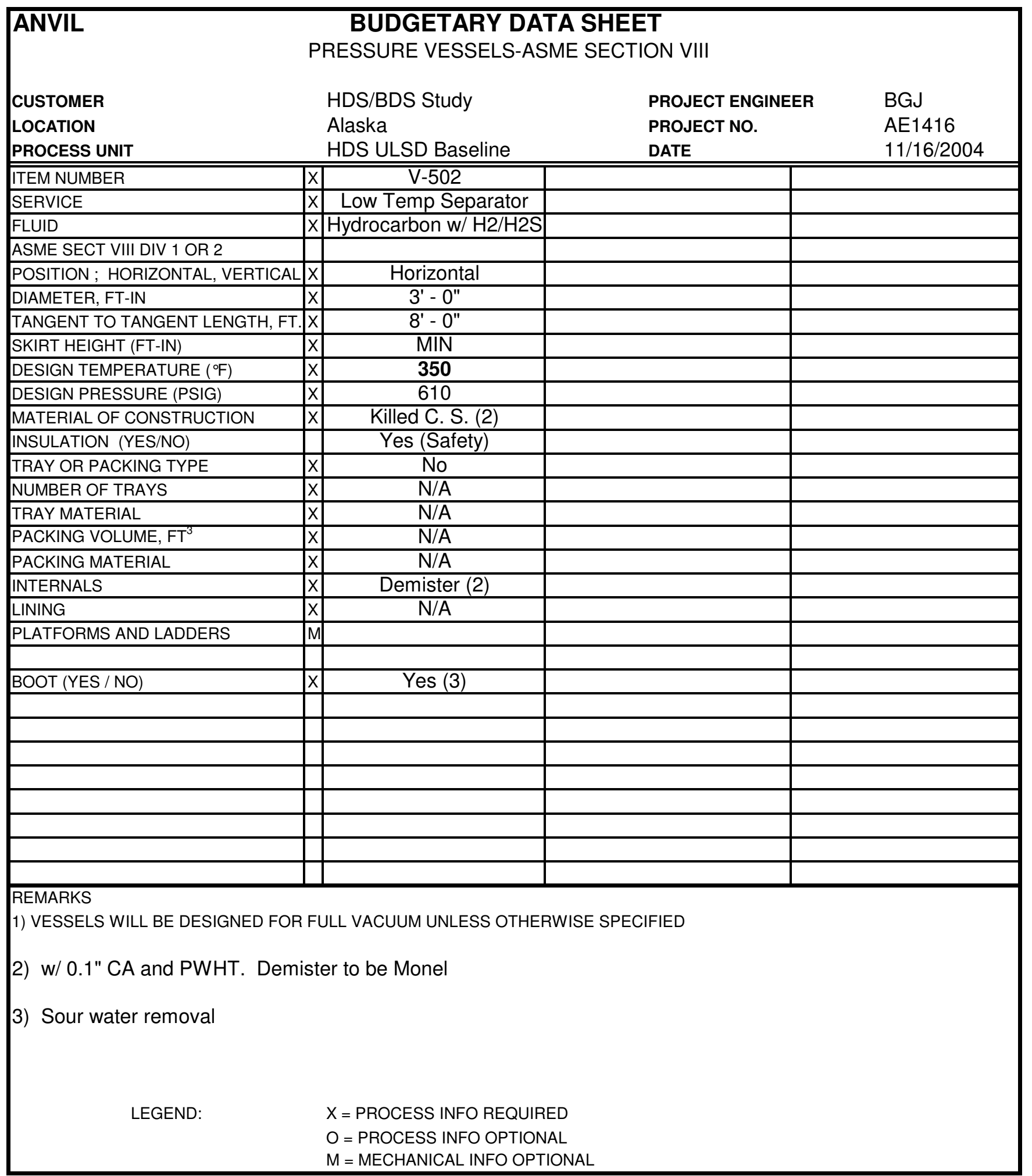

S:IPetroStar|AE1416.AUXIsubjob 411HDS_BASECASE_FINAL REPORT\Appendix C--DatasheetsI[V-502.xls]V-502 Rev B 


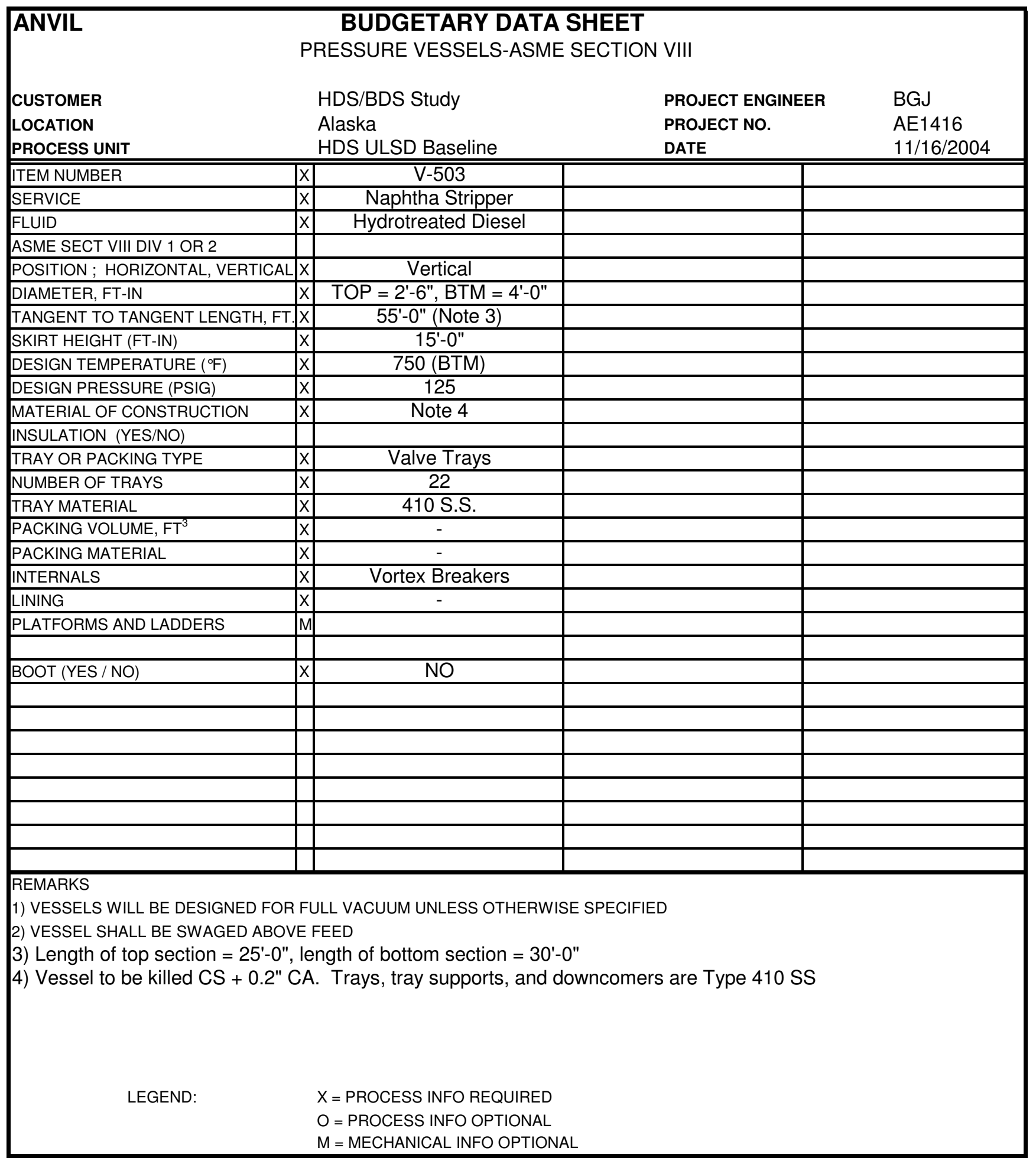




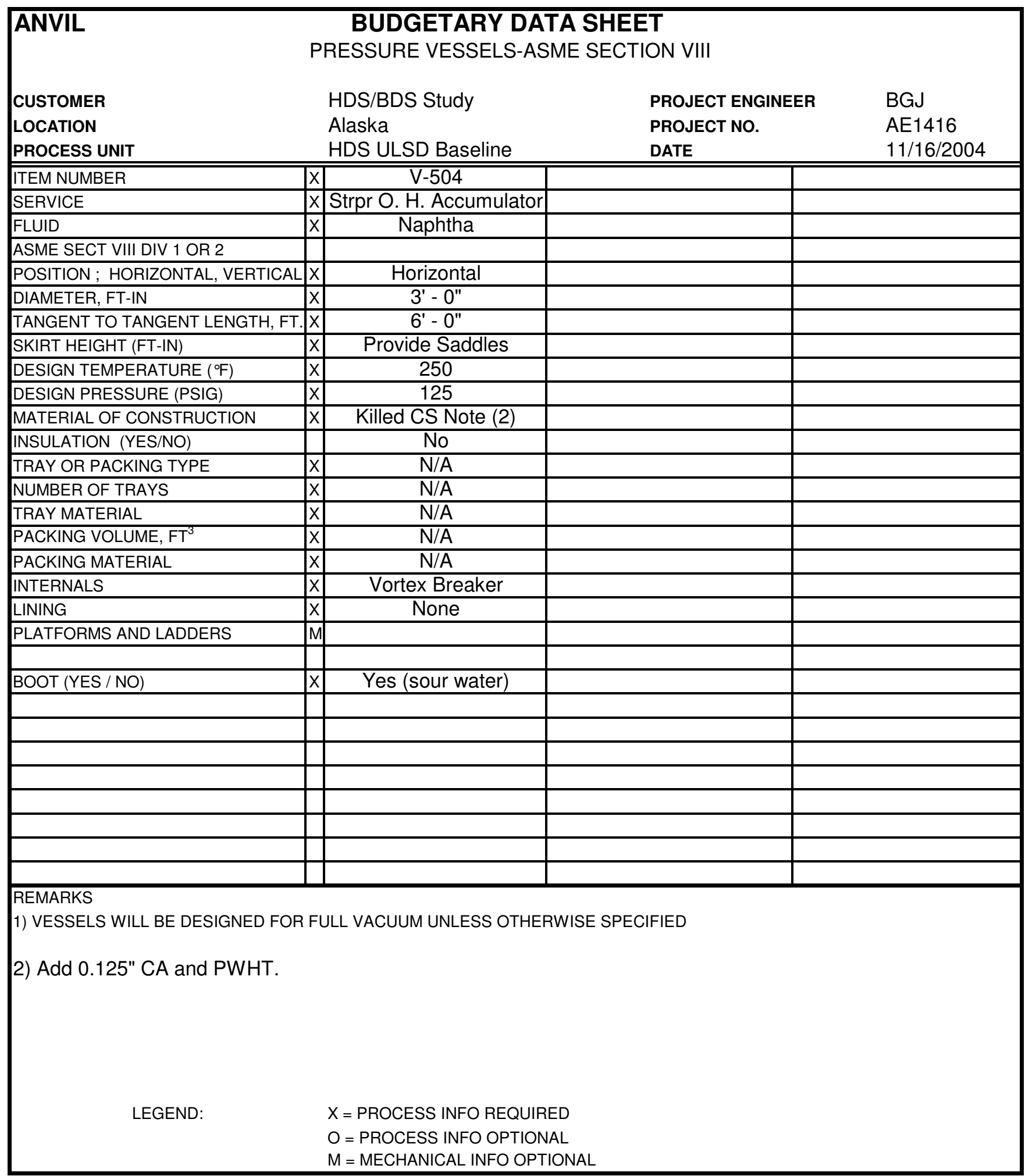

S:IPetroStar|AE1416.AUXIsubjob 411HDS_BASECASE_FINAL REPORT\Appendix C--DatasheetsI[V-504.xls]V-504 Rev B 


\section{Appendix D - Hydrogen Unit Flow Diagram}

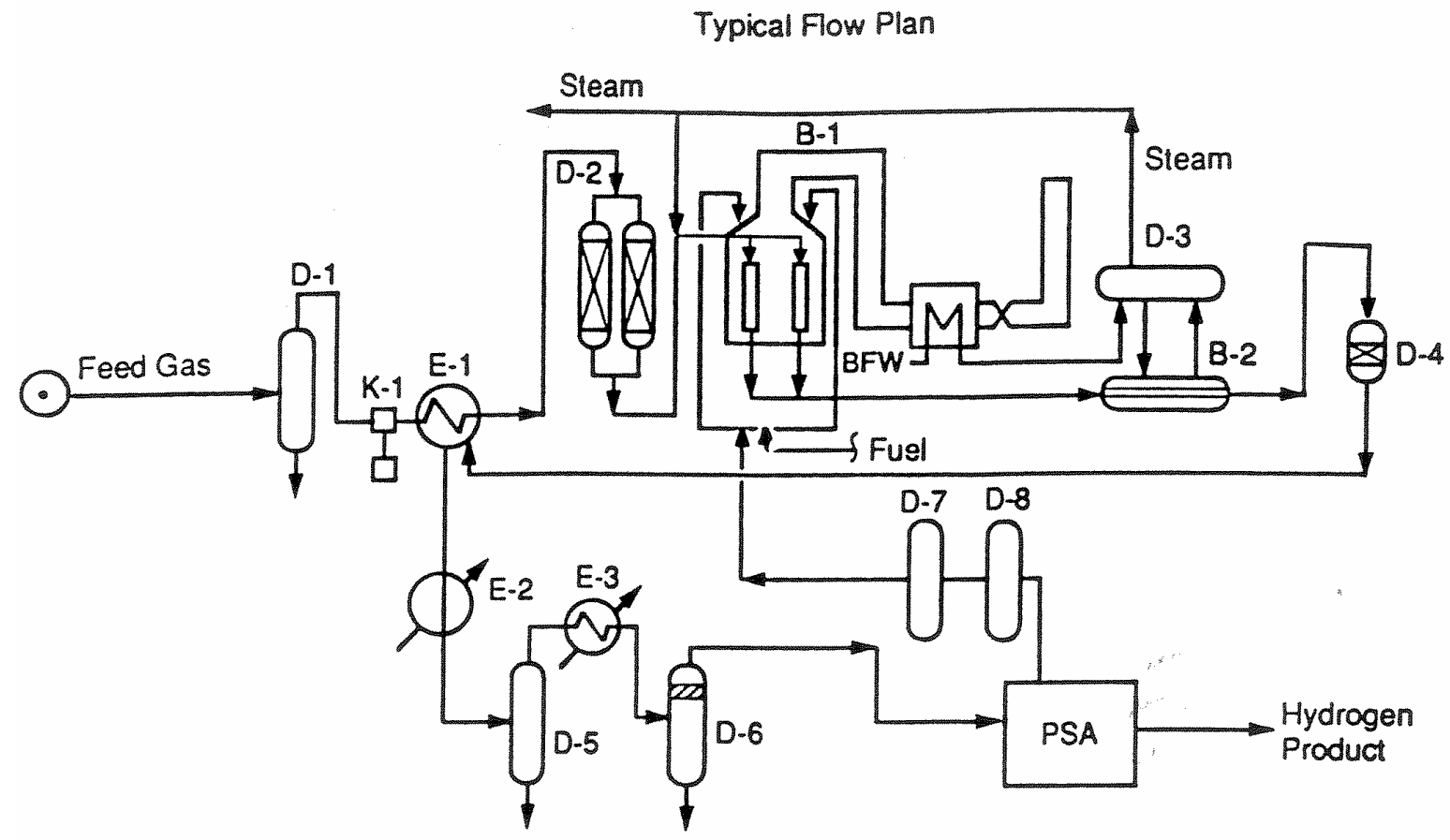

Equipment List

\begin{tabular}{|c|c|}
\hline ltem & Description \\
\hline$K-1$ & Feed Compressor \\
\hline$D-1$ & Knockout drum \\
\hline $0-2$ & Desulfurizer Drums \\
\hline 0.3 & High Pressure Steam Drum \\
\hline$D-4$ & High Temperature Shift Reactor \\
\hline D-5 & Knockout Drum \\
\hline D-6 & Knockout Drum \\
\hline D-7 & PSA Surge Drum \\
\hline 0.8 & PSA Mixing Drum \\
\hline$E-1$ & Feed Preheater \\
\hline$E-2$ & Cooler \\
\hline$E-3$ & Cooler \\
\hline B-1 & Reformer Furnace/Flue Gas/Heat Recovery \\
\hline B-2 & Waste Heat Boiler \\
\hline PSA & Pressure Swing Adsorbers \\
\hline
\end{tabular}




\section{Appendix E - Sulfur Unit Block Flow Diagram}

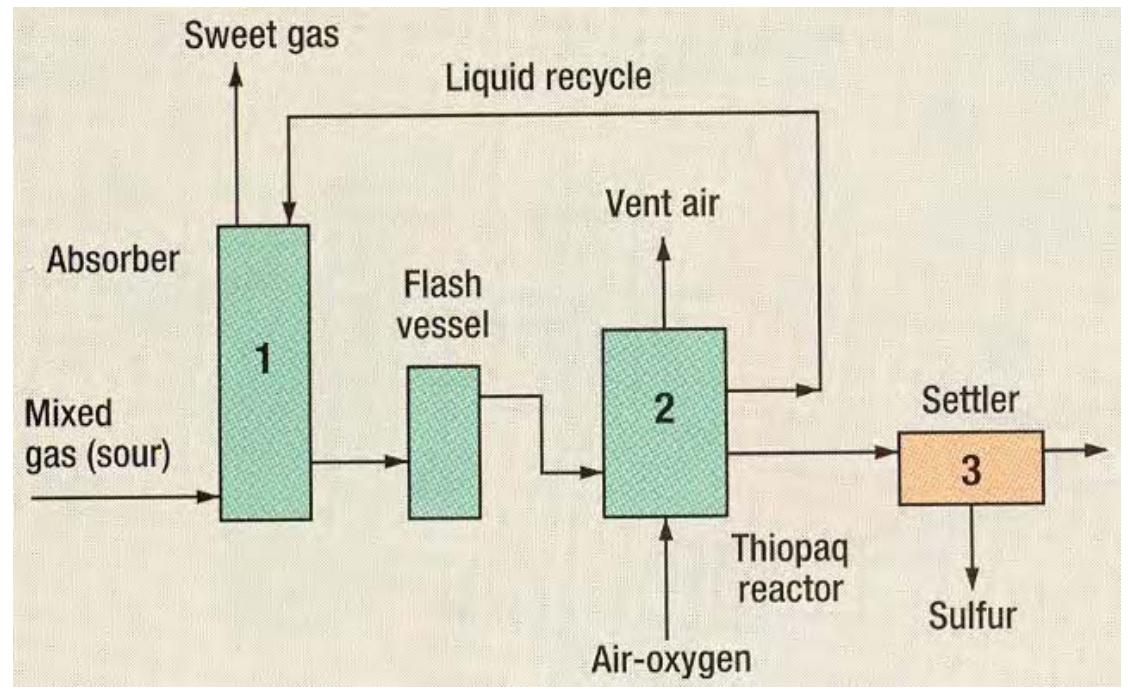




\section{Appendix F - Utility Requirements}

HDS Project Utility Requirements

6,000 BPD Feed Rate, 10 ppmw Sulfur ULSD Product

$\begin{array}{cccc}\begin{array}{c}\text { Power } \\ \text { kW }\end{array} & \begin{array}{c}\text { Fuel Gas } \\ \text { MMBTU/Hr }\end{array} & \begin{array}{c}\text { Process Water } \\ \text { lb/hr }\end{array} & \text { BFW } \\ & \underline{\mathrm{lb} / \mathrm{hr}}\end{array}$

IBL

Feed gas compressor

75

H2 Makeup Compressor

105

Recycle gas compressor $\quad 100$

Charge pump

120

Stripper reflux pump

1

Stripper Btms Circ

40

Effluent air cooler

10

Prod stripper condensor

5

Prod rundown cooler

12

Wash water injection pump

2

Charge Heater

4.4

Product Stripper Reboiler

6.8

Wash water

OBL

H2 Plant

62

32.9

5554

Sulfur recovery

105

118

Total

755

44.1

700

5554 


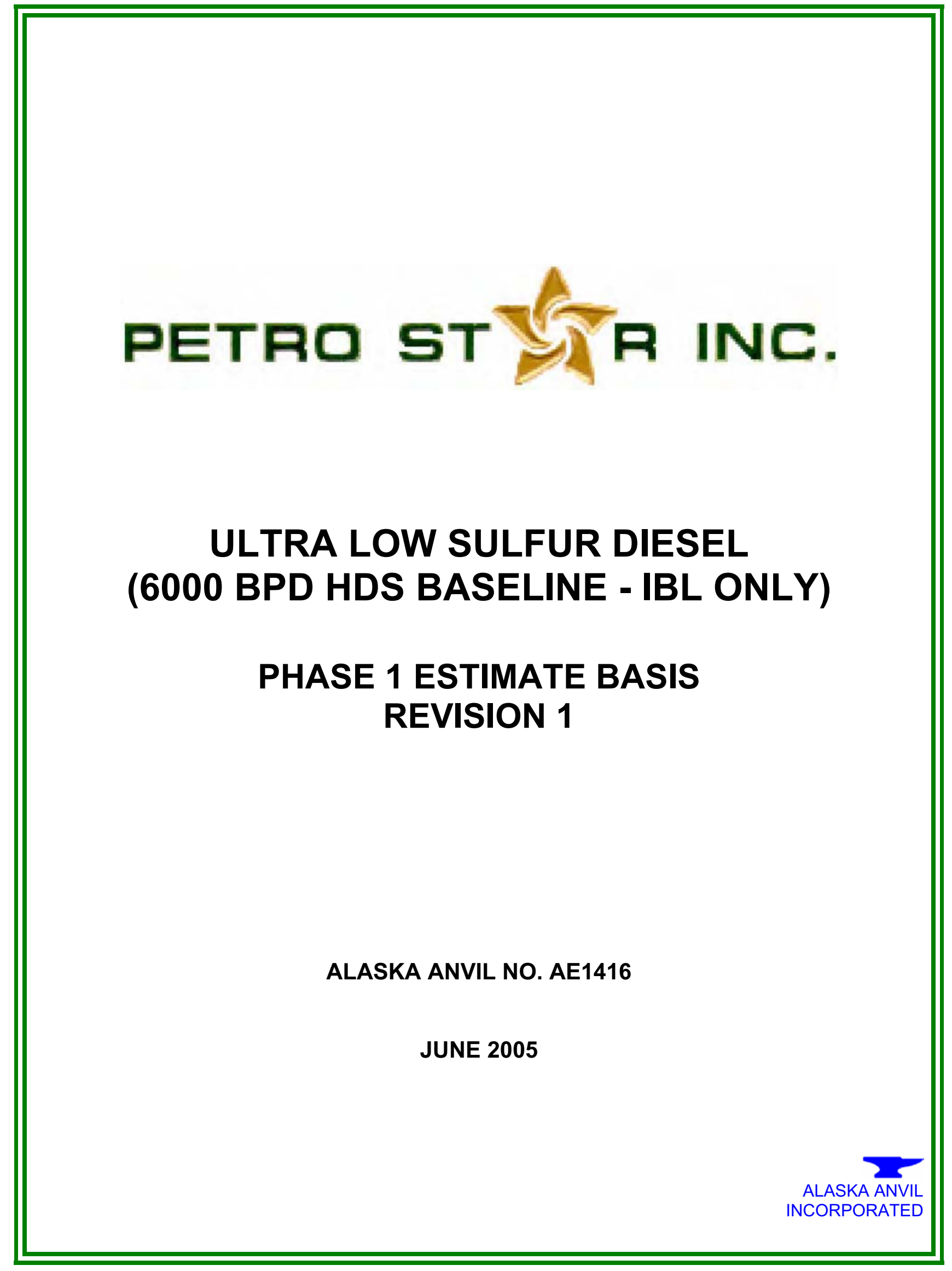




\section{ESTIMATE BASIS GOAL}

This Estimate Basis identifies information, qualifications, exceptions, and assumptions used in developing the cost estimate.

\section{ESTIMATE BASIS PURPOSE}

During the estimate review process, the project team uses the Estimate Basis for the following purposes:

- As a checklist of items to consider during estimate preparation.

- To document what is included and not included in the cost estimate.

- To assess cost risks of estimate components.

- As part of the decision support package for assessing the BDS process feasibility.

\section{GENERAL INFORMATION}

- The purpose of the project estimate is to determine if the ULSD BDS process is economically viable as a standalone process or in combination with an HDS Unit.

- $\quad$ Estimate type:

- The estimate was developed using a combination of vendor quotes \& equipment based factored estimates for Inside Battery Limits (IBL) costs. Licensor quotes were obtained for the Sulfur and Hydrogen Unit costs. Equipment costs were derived from the ICARUS estimating program for equipment in the Diesel Hydrotreating Unit.

- Outside Battery Limits (OBL) costs have been excluded from this estimate.

- $\quad$ The project will be installed in a brownfield location within a Valdez Alaska Refinery.

\section{PROCESS BASIS}

\section{Facility Data}

- $\quad$ Facility type - Ultra Low Sulfur Diesel Treating Complex, which includes:

- Diesel Hydrotreating Unit

- Hydrogen Production Unit

- Sulfur Recovery Unit

\section{Design Basis}

Product specification - Feed 6,000 bpd of untreated diesel to produce 10 ppmw sulfur maximum ultra low sulfur diesel.

\section{COST BASIS}

\section{Labor, Indirects, Equipment, and Bulk Materials}

- Included in the equipment factor. 


\section{Project Services}

- $\quad$ HDS - estimated based on a typical percentage for brownfield type projects.

- $\quad$ Sulfur and Hydrogen Units - included in the package price.

\section{Owner Services}

Not included in the TIC cost. Historically, owner services will cost from 5 to 7 percent of TIC, not including licensing, royalties, or catalyst.

\section{Escalation}

Project is based on 2005 costs. No escalation is included.

\section{Location Factor}

All costs for this estimate have been developed from a U.S. Gulf Coast (USGC) basis. No location factor is included.

\section{Other Costs}

- CEMS, air preheating, and burner management allowances have been added to the fired heater costs.

- Catalyst and chemical initial charge has been added as an additional line item.

\section{ASSUMPTIONS}

- $\quad$ Process licensing and royalty costs are not included.

- Assumes fully installed pump spares, but no warehouse spares. 
Final Technical Progress Report DOE Award No. DE-FC26-02NT15340

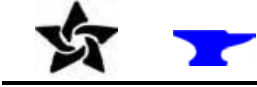

CLIENT: PetroStar

PROJECT: PetroStar Valdez ULSD - 6000 BPD HDS Baseline STAGE: Phase 1

\section{PROJECT DESCRIPTION:}

Install Ultra Low Sulfur Diesel Complex.

PROJECT COST ESTIMATE SUMMARY

COST ESTIMATE STRUCTURE
COST ESTIMATE PARAMETERS
Estimate Classification
Estimating Method
COST ESTIMATE SUMMARY
Expected Cost (\$MM)
High Range (\$MM)
Low Range (\$MM)

PROJECT COST ESTIMATE ANALYSIS

Total Project Expected Cost Component Analysis

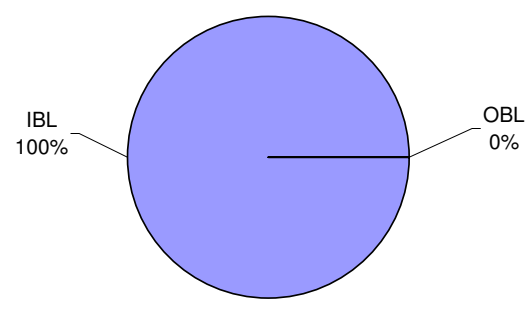

CLIENT PROJECT NO.:

ANVIL PROJECT NO.: AE1416

REV NO.: 1

ANVIL PE: L. Nace

Date: 6/15/05

PROJECT RISKS:

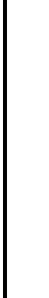

$x^{20}$

\begin{tabular}{|c|c|}
\hline \multicolumn{2}{|c|}{$\begin{array}{c}\text { TOTAL PROJECT } \\
\text { COST - Valdez }\end{array}$} \\
\hline & Phase 1 \\
\hline & Factored/ROM \\
\hline$\$$ & 27.8 \\
\hline$\$$ & 41.7 \\
\hline$\$$ & 23.6 \\
\hline
\end{tabular}

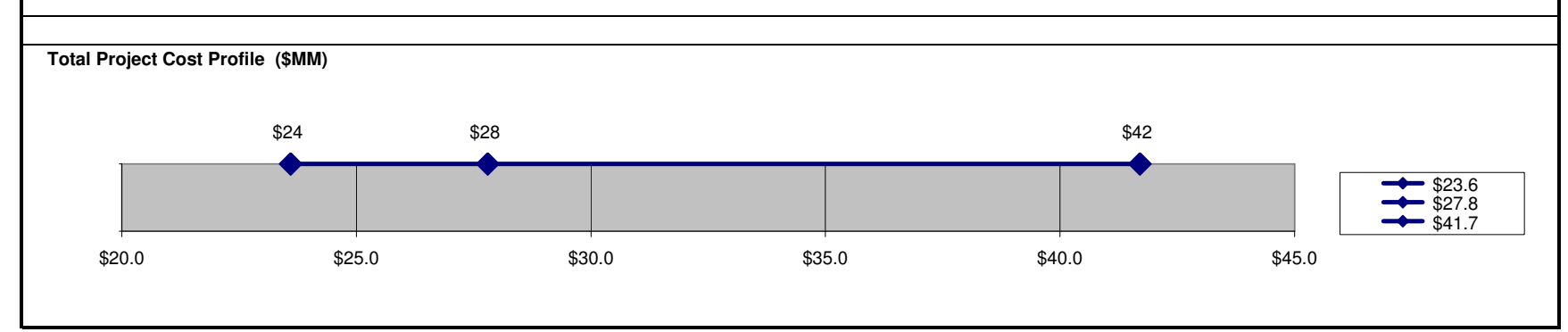

\section{PROJECT SCHEDULE ESTIMATE ANAL YSIS}

\begin{tabular}{|c|c|c|c|c|}
\hline $\begin{array}{l}\text { Total Project Schedule } \\
\text { Target Completion Dates }\end{array}$ & & & & \\
\\
PROJECT TIMELINE
\end{tabular}


Petro Star Ultra Low Sulfur Diesel (6000 BPD) Project - Valdez Refinery

IBL Impact Matrix - 2005\$ - Total Installed Costs USGC

Scenario: HDS Baseline

\begin{tabular}{|c|c|c|c|c|}
\hline $\begin{array}{c}\text { IBL } \\
\text { Component }\end{array}$ & $\begin{array}{l}\text { HDS } \\
\text { Unit }\end{array}$ & $\begin{array}{c}\text { Hydrogen } \\
\text { Unit }\end{array}$ & $\begin{array}{c}\text { Sulfur } \\
\text { Unit }\end{array}$ & $\begin{array}{c}\text { Total Cost } \\
\text { \$MM }\end{array}$ \\
\hline $\begin{array}{l}\text { Option } 1 \text { - } \\
\text { LSG }\end{array}$ & $\begin{array}{l}\text { Diesel } \\
\text { hydrotreater, } \\
\text { feed rate of } \\
6000 B / D, \\
\text { producing } \\
\text { ULSD (10 } \\
\text { ppmw sulfur) }\end{array}$ & $\begin{array}{l}2 \text { MMSCF/D H2 } \\
\text { Production } \\
\text { Plant. }\end{array}$ & $\begin{array}{l}\text { Thiopaq } \\
\text { process to be } \\
\text { used for } 3.7 \\
\text { T/D. Will also } \\
\text { need sulfur } \\
\text { storage / } \\
\text { handling }\end{array}$ & \\
\hline Cost Basis & $\begin{array}{l}\text { Factored } \\
\text { equipment } \\
\text { based estimate }\end{array}$ & $\begin{array}{l}\text { Verbal vendor } \\
\text { quote from } \\
\text { Howe Baker } \\
\text { for module = } \\
\$ 6.5 \text { MM plus } \\
3 \% \text { for } \\
\text { engineering }\end{array}$ & $\begin{array}{l}\text { Vendor quote } \\
\text { for } 3.5 \$ M M \\
\text { plus } 3 \% \text { for } \\
\text { engineering }\end{array}$ & \\
\hline $\begin{array}{l}\text { High Range } \\
\text { Cost, \$ MM }\end{array}$ & $\$ 26.3$ & $\$ 10.0$ & $\$ 5.4$ & $\$ 41.7$ \\
\hline $\begin{array}{l}\text { Expected } \\
\text { Cost, \$ MM }\end{array}$ & $\$ 17.5$ & $\$ 6.7$ & $\$ 3.6$ & $\$ 27.8$ \\
\hline $\begin{array}{l}\text { Low Range } \\
\text { Cost, \$ MM }\end{array}$ & $\$ 14.9$ & $\$ 5.7$ & $\$ 3.1$ & $\$ 23.6$ \\
\hline
\end{tabular}


Final Technical Progress Report

ANVIL CORPORATION

PROJECT: PetroStar Valdez ULSD - 6000 BPD HDS Baseline

ANVIL NO: AE1416

CLIENT: PetroStar

DATE: $6 / 15 / 05$

REV: 1

PHASE 1 ESTIMATE - FACTORING SUMMARY

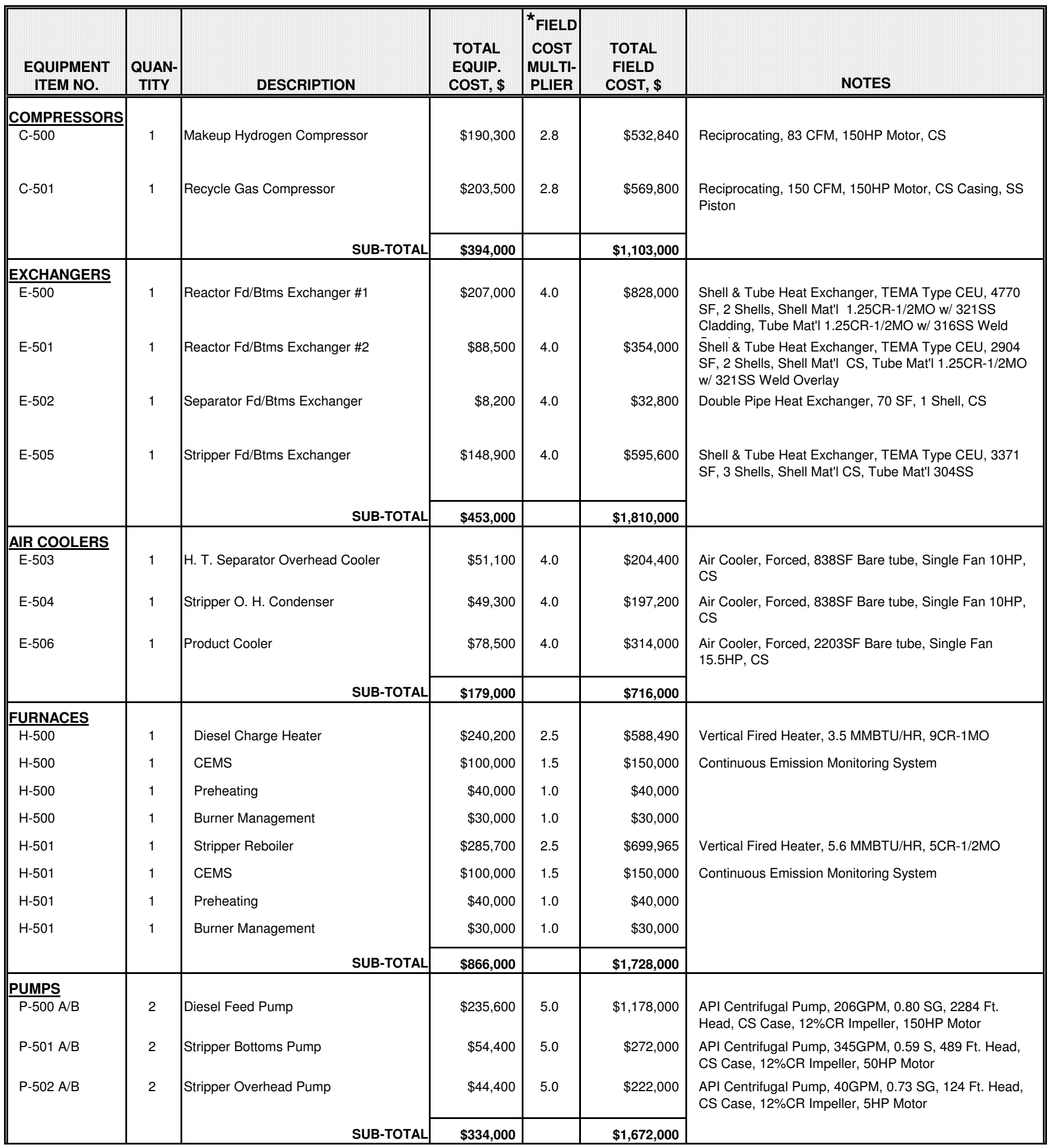


Final Technical Progress Report

ANVIL CORPORATION

PROJECT: PetroStar Valdez ULSD - 6000 BPD HDS Baseline ANVIL NO: AE1416

CLIENT: PetroStar

DATE: $6 / 15 / 05$

REV: 1

PHASE 1 ESTIMATE - FACTORING SUMMARY

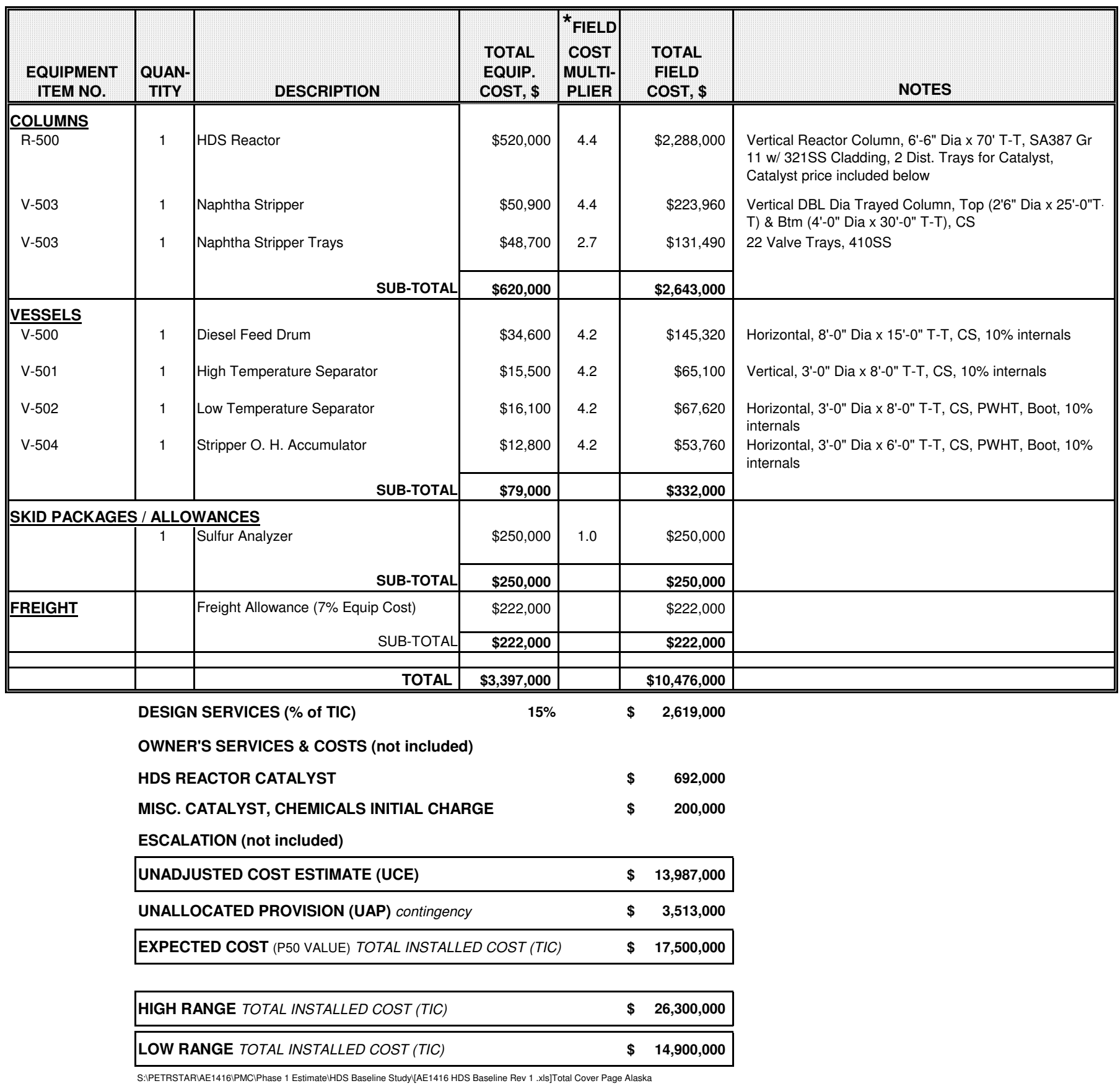

*Note: Field Cost Multiplier includes the following bulk material and installation labor: Civil, Concrete, Structural, Piping, Electrical, Instrumentation, Insulation, Fireproofing, Painting, and Testing. 
Final Technical Progress Report

DOE Award No. DE-FC26-02NT15340
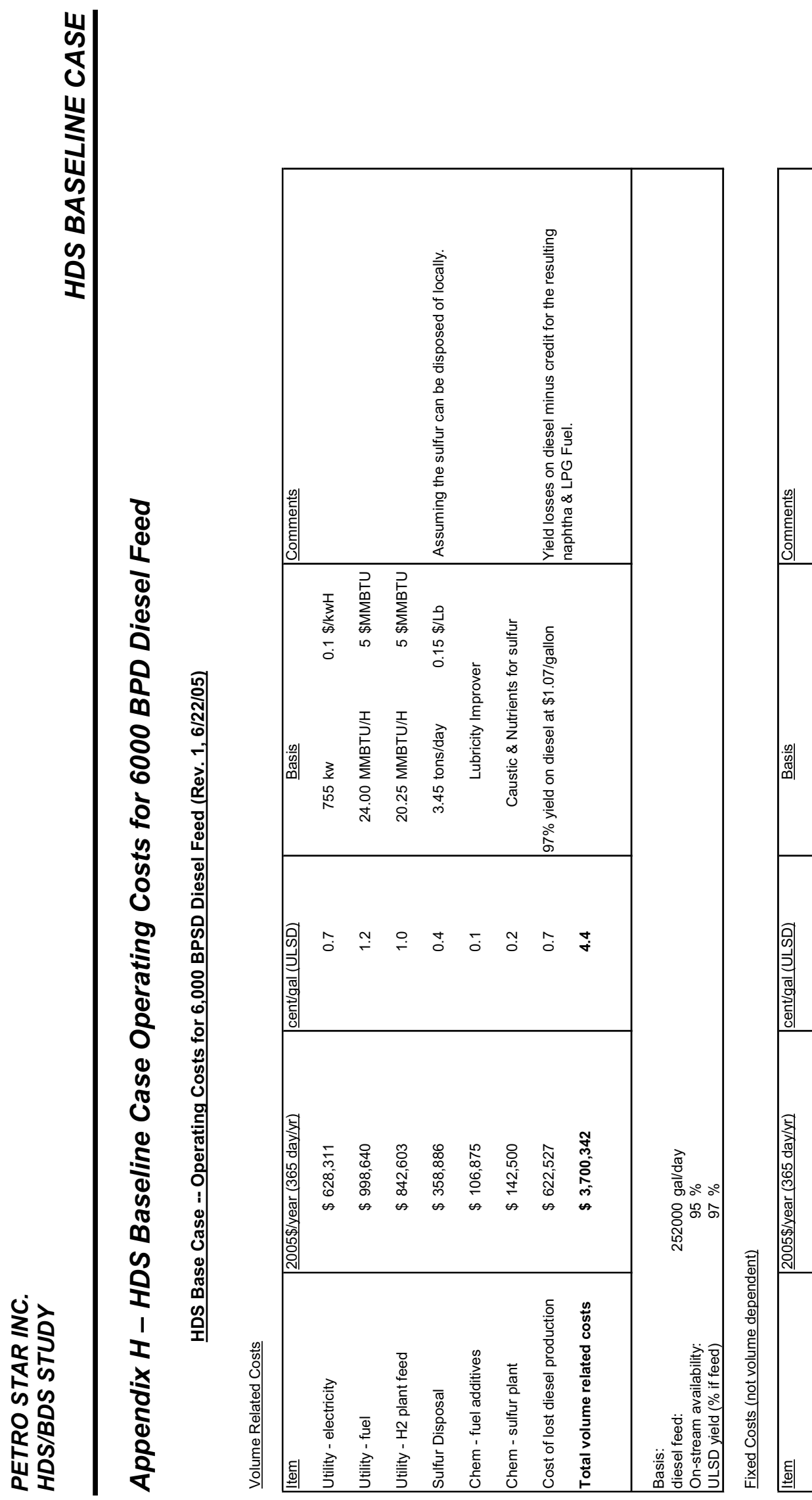

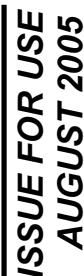

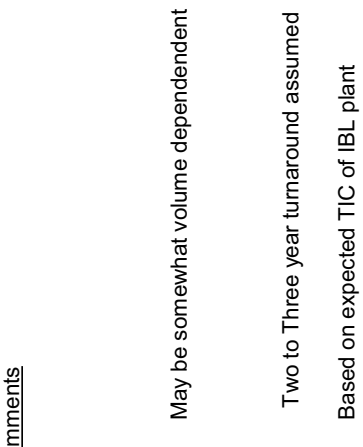

仓े।
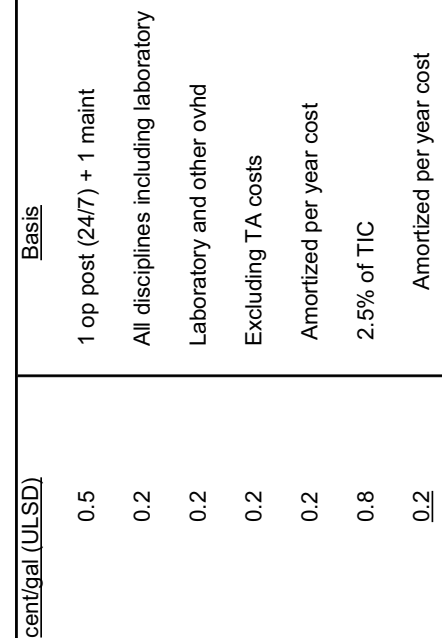

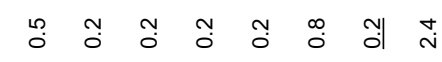

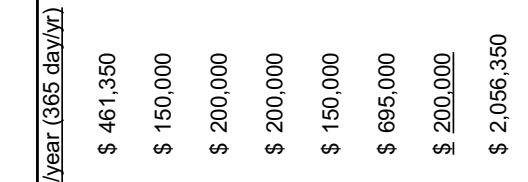

政

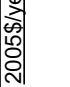

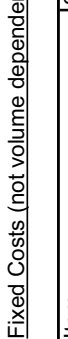

$\frac{1}{\sqrt{1}}$
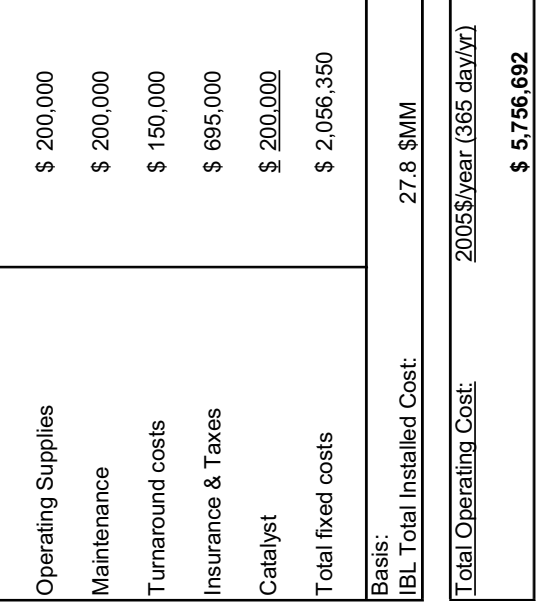

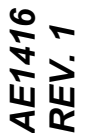




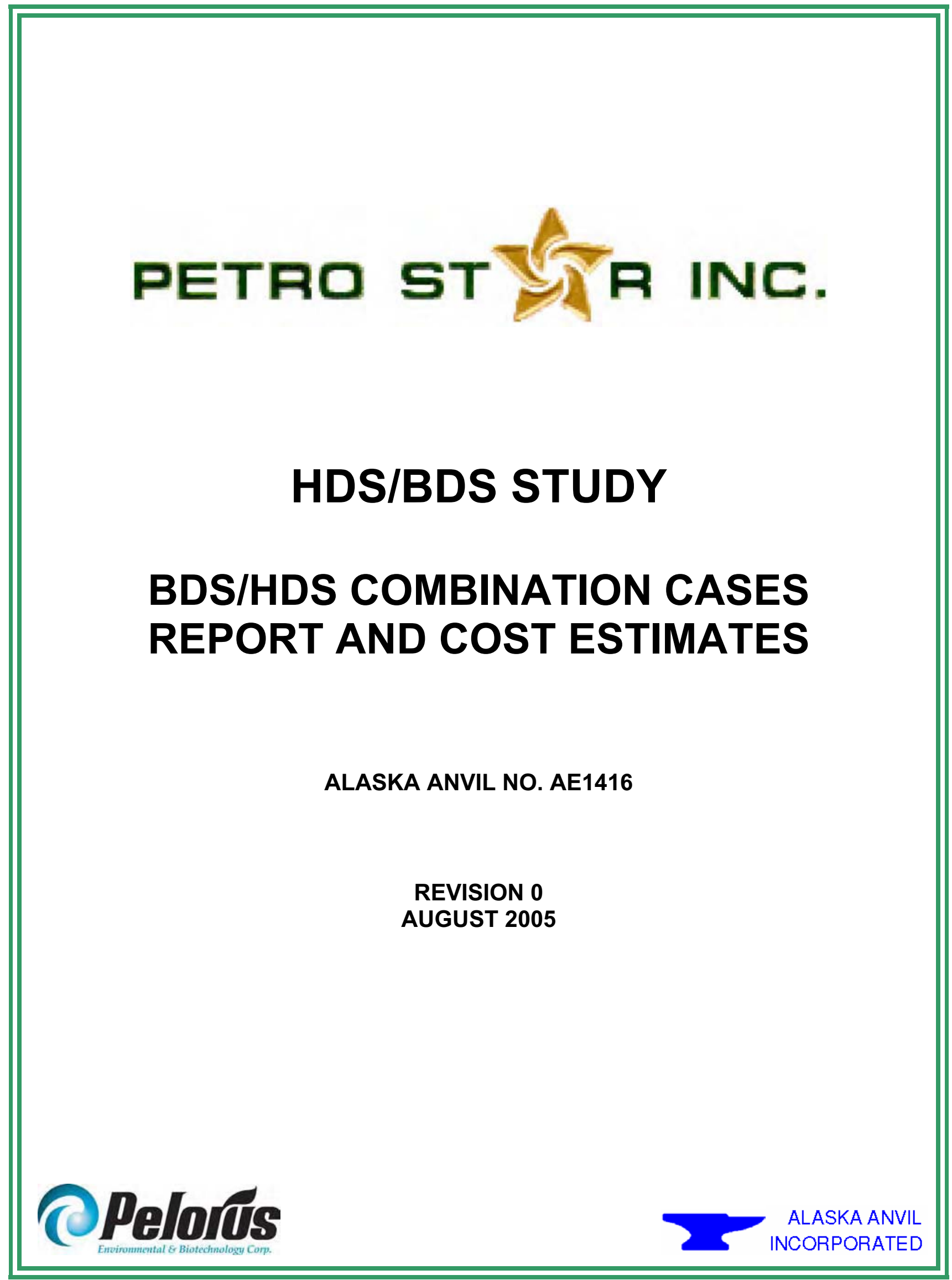




\section{EXECUTIVE SUMMARY}

To meet the EPA's 2006 sulfur content requirements, Petro Star is leading a Department of Energy study to determine the viability of ULSD production from a biodesulfurization process. This BDS/HDS Combination Cases Report, a part of the larger investigation, presents several BDS/HDS combinations to determine if a combined process has improved economics (i.e. capital and/or operating cost) over a standalone BDS or HDS process.

The effort is based on an installation at Valdez, Alaska producing ultra low sulfur diesel meeting a 10-ppmw sulfur requirement. The design feed rate to the desulfurization complex is $6,000 \mathrm{bpd}$ of straight run diesel.

The design includes the following proposed IBL facilities:

- $\quad$ A new HDS Unit

- A new BDS Unit

- A new Diesel Splitter (Pre Frac Case only)

- A new Hydrogen Unit

- A new Sulfur Unit to treat the off-gas from the HDS Unit.

The cost estimate for the each case includes only the inside battery limits (IBL) portion of the desulfurization complex. The estimates are based on 2005 USGC pricing and have an accuracy range of $+50 \%$ to $-15 \%$. The annual operating cost for each case was calculated as was the total incremental selling price for ULSD. This figure is based on a 5-year capital recovery period (excluding interest expense) plus the yearly operating cost. The cost summary for each case is presented in the table below.

\begin{tabular}{|l|c|c|c|}
\hline \multicolumn{1}{|c|}{ Case } & $\begin{array}{c}\text { Expected TIC } \\
(\$ M M)\end{array}$ & $\begin{array}{c}\text { Annual Operating Cost } \\
(\$ M M)\end{array}$ & $\begin{array}{c}\text { Total Annual Cost } \\
\text { (cents per gallon of ULSD) }\end{array}$ \\
\hline BDS/HDS & 44.5 & 9.4 & 21.4 \\
\hline HDS/BDS & 43.3 & 8.0 & 19.4 \\
\hline Pre Frac & 46.5 & 10.2 & 23.1 \\
\hline
\end{tabular}




\section{TABLE OF CONTENTS}

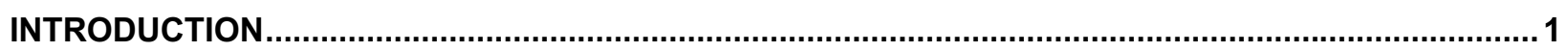

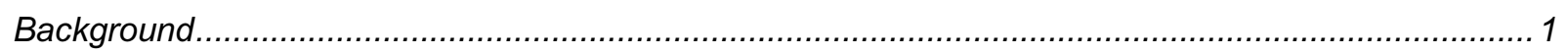

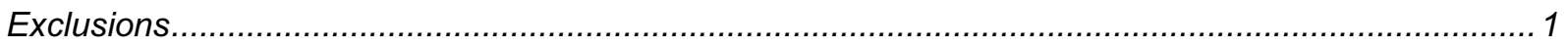

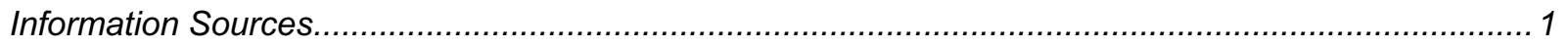

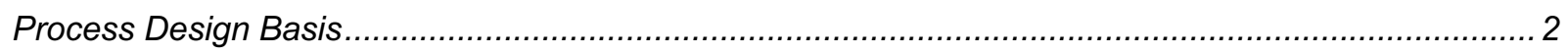

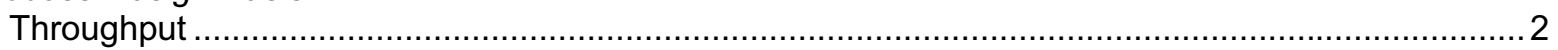

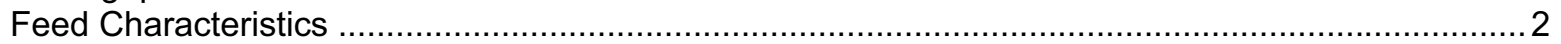

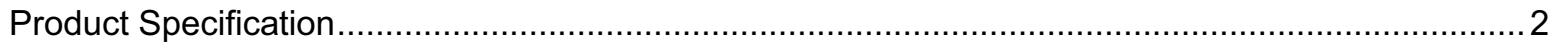

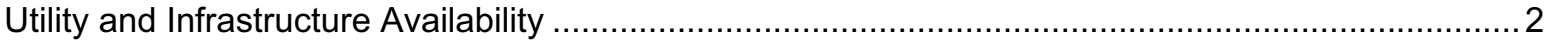

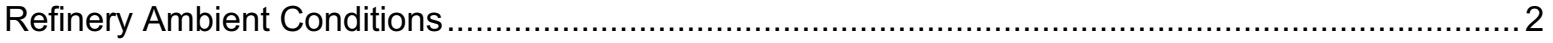

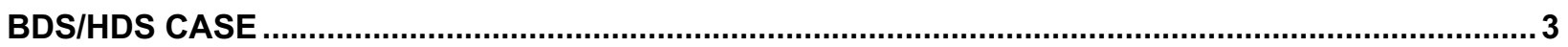

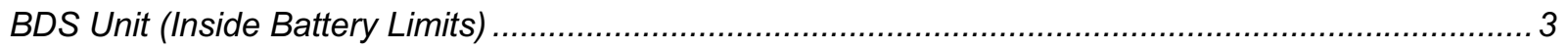

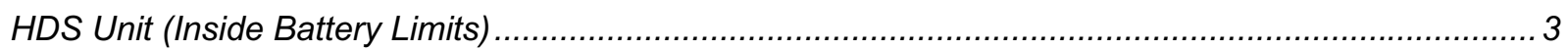

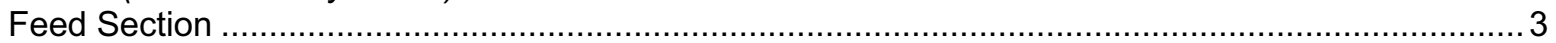

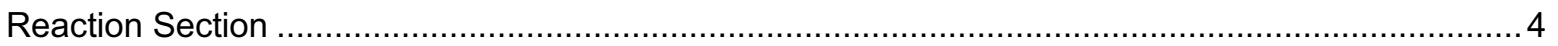

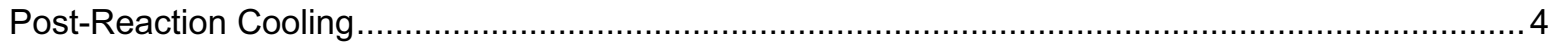

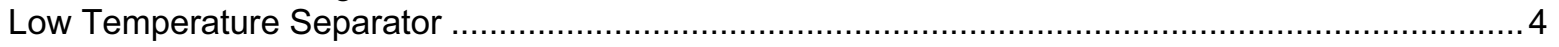

Naphtha Stripper

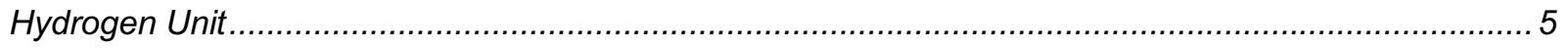

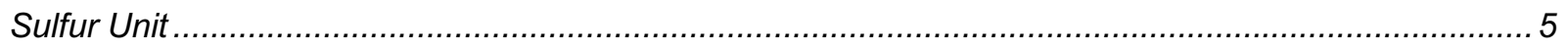

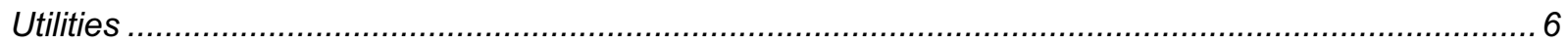

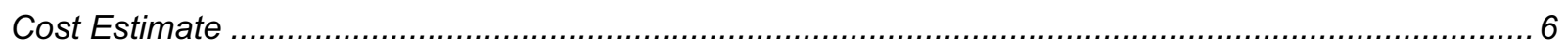

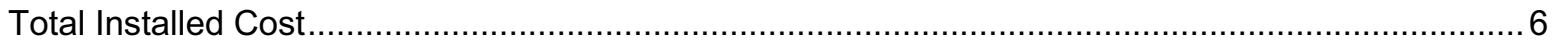

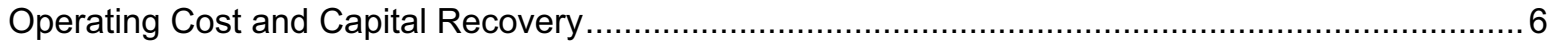

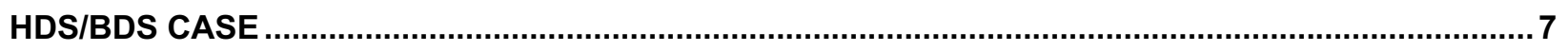

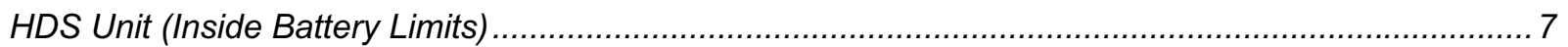

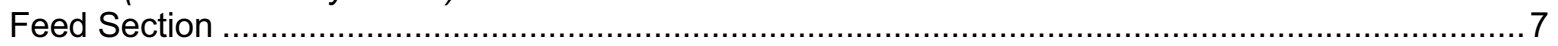

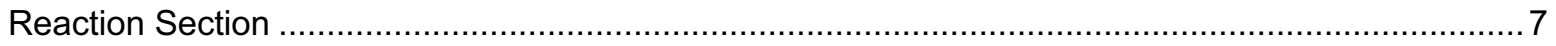

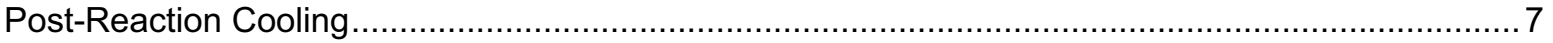

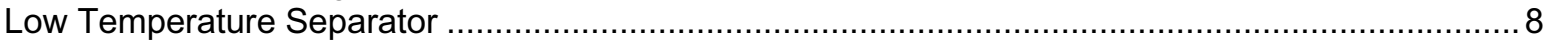

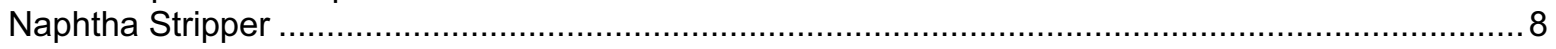

Hydrogen Unit

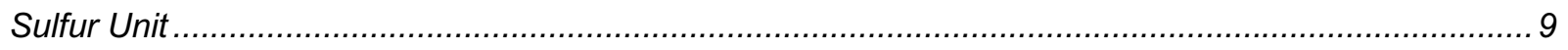

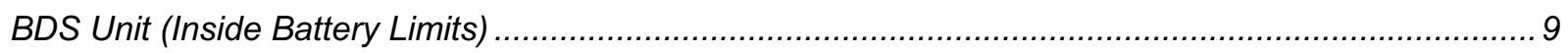

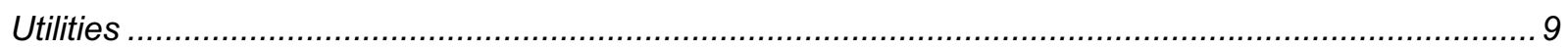

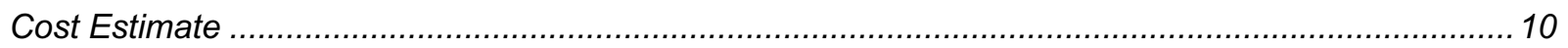

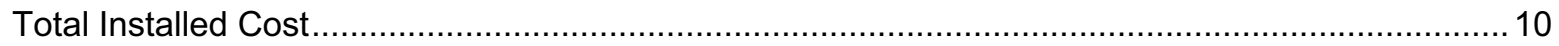

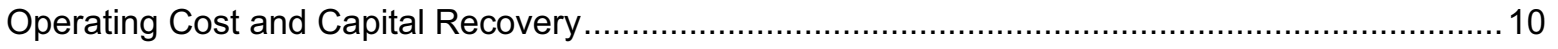

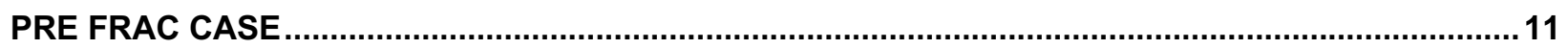

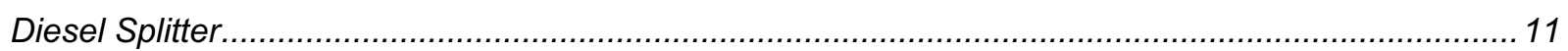

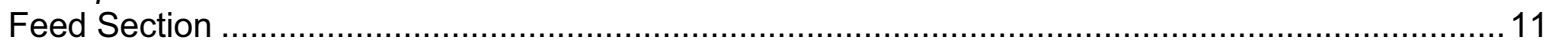

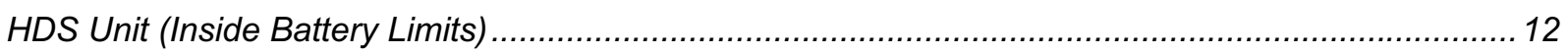

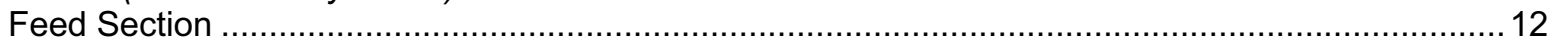


Reaction 12

Post-Reaction Cooling....

Low Temperature Separator

Naphtha Stripper

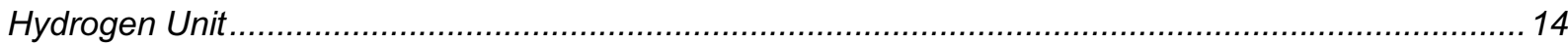

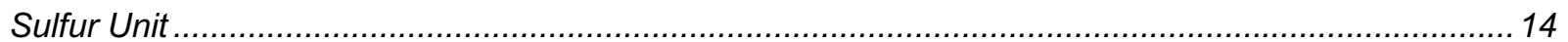

BDS Unit (Inside Battery Limits) ........................................................................................ 14

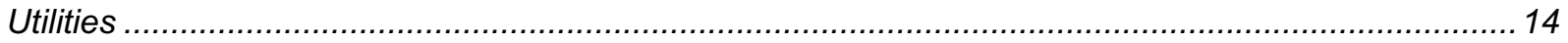

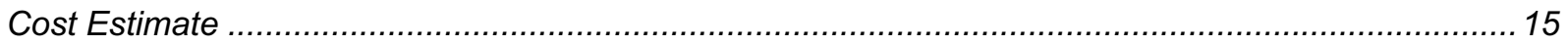

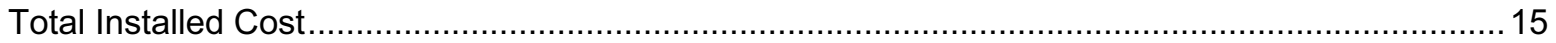

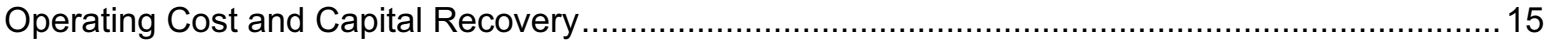




\section{APPENDICES}

A. Raw Diesel Feed Properties and Sulfur Speciation

B. BDS/HDS Case

B.1. BDS Unit Process Flow Diagrams and Material Balance

B.2. BDS Unit Equipment List and Budgetary Equipment Datasheets

B.3. Sulfur Speciation in BDS Unit Diesel Product

B.4. HDS Unit Process Flow Diagrams and Material Balance

B.5. HDS Unit Equipment List and Budgetary Equipment Datasheets

B.6. Utility Requirements

B.7. Cost Estimate Basis and Cost Estimate

B.8. Operating Cost Estimates

C. HDS/BDS Case

C.1. HDS Unit Process Flow Diagrams and Material Balance

C.2. HDS Unit Equipment List and Budgetary Equipment Datasheets

C.3. BDS Unit Process Flow Diagrams and Material Balance

C.4. BDS Unit Equipment List and Budgetary Equipment Datasheets

C.5. Utility Requirements

C.6. Cost Estimate Basis and Cost Estimate

C.7. Operating Cost Estimates

D. Pre Frac Case

D.1. Diesel Splitter Process Flow Diagram and Material Balance

D.2. Diesel Splitter Equipment List and Budgetary Equipment Datasheets

D.3. Sulfur Speciation in Diesel Splitter Products

D.4. HDS Unit Process Flow Diagrams and Material Balance

D.5. HDS Unit Equipment List and Budgetary Equipment Datasheets

D.6. BDS Unit Process Flow Diagrams and Material Balance

D.7. BDS Unit Equipment List and Bugetary Datasheets

D.8. Utility Requirements

D.9. Cost Estimate Basis and Cost Estimate

D.10. Operating Cost Estimates 


\section{INTRODUCTION}

\section{Background}

All U.S. refineries will be required to meet the EPA's third quarter 2006 sulfur content requirements for highway diesel. The EPA requirement for maximum sulfur content of ultra low sulfur diesel (ULSD) is 15 ppmw. The refinery product should have about 10 ppmw sulfur or less to ensure that the product specification can be met on a reliable basis after product shipping and distribution logistics.

To compare different strategies to accomplish this requirement, Petro Star is leading a Department of Energy HDS/BDS study that evaluates the following processing routes:

- A standalone hydrodesulfurization (HDS) process

- A standalone biodesulfurization (BDS) process

- A combination of the two.

To be economically viable, a BDS process must be competitive with other commercially proven desulfurization routes. The route most chosen and best known to refiners for diesel is HDS. A new BDS facility must be less costly than a comparable new HDS facility. Or, in the various combination scenarios, using a BDS facility for pre- or post-treatment combined with an existing HDS unit must be less costly than the modifications to an existing HDS Unit that would achieve the lower sulfur requirement.

As part of the study of potential BDS economics, this report provides the process description and total installed cost for three BDS/HDS unit combinations:

- $\quad$ BDS/HDS Case

A new 6,000 bpd BDS Unit producing low sulfur diesel (LSD) followed in series by a new 6,000 bpd HDS Unit producing ULSD

- $\quad$ HDS/BDS Case

A new 6,000 bpd HDS Unit producing LSD followed in series by a new 6,000 bpd Unit producing ULSD

- Pre Frac Case

A new 6,000 bpd Diesel Splitter followed in parallel by a new 3,860 bpd HDS Unit producing ULSD and a new 2,140 bpd BDS Unit producing USLD.

\section{Exclusions}

To simplify the economic baseline study and avoid biasing the study results, we have excluded all outside battery limits (OBL) facilities. A prior 1999 Kellogg report exploring a BDS baseline also excluded all utility systems improvements, offsite tankage, and waste stream disposal.

\section{Information Sources}

Axens North America, Inc. provided the HDS Unit reactor design and unit yields for this study under a proprietary agreement. A cursory inspection of other licensor data revealed little 
difference in yields, process conditions, and reactor size. Therefore, only the HDS data received from Axens was used for this study.

Pelorus provided the BDS Unit design as well as the operating costs estimate and equipment costs for the specialized equipment.

\section{Process Design Basis}

\section{Throughput}

The design throughput for the BDS/HDS combination cases is 6,000 bpd of straight run diesel containing 0.5 weight percent sulfur.

\section{Feed Characteristics}

The design basis composition and characteristics of the diesel feed, including a sulfur speciation, are included in Appendix A. The hydrogen from the Hydrogen Unit is $99.7 \%$ pure.

\section{Product Specification}

The specification for diesel product sulfur content is $10 \mathrm{ppmw}$.

\section{Utility and Infrastructure Availability}

The study assumes the refinery has the following utility systems and infrastructure available to support the BDS/HDS Combination Cases:

- $\quad$ Refinery fuel gas (for fired heaters and Hydrogen Unit feed)

- Water (available as feed to a new demineralization unit)

- Instrument air

- $\quad$ Electricity for drivers

- Nitrogen

- Process vent system

- $\quad$ Product tanks

- Cooling fluid system

- Steam/boiler feed water

- Refinery fuel gas

- Naphtha fuel

- Wastewater Treatment

- Flare.

\section{Refinery Ambient Conditions}

The new desulfurization facilities design is based on a Valdez, Alaska location.

\begin{tabular}{|l|c|}
\hline Ambient Temperature Condition & Degrees F \\
\hline Highest monthly average & 62 \\
\hline Lowest monthly average & 17 \\
\hline Record high & 86 \\
\hline Record low & -23 \\
\hline
\end{tabular}




\section{BDS/HDS CASE}

The BDS/HDS case includes the following proposed IBL facilities:

- A new 6,000 bpd BDS Unit

- A new 6,000 bpd HDS Unit

- A new Hydrogen Unit

- A new Sulfur Unit to treat the off-gas from the HDS Unit.

\section{BDS Unit (Inside Battery Limits)}

The BDS/HDS combination case is designed to take advantage of the substrate specificity of the existing prototype biocatalyst. For this cost estimate, it is assumed that the BDS section of the process will remove approximately $1,500 \mathrm{ppm}$ of sulfur in the feed. This assumption is based upon the removal of all dibenzothiophenes and the majority of the benzothiophenes species from the feed.

The BDS process employs a selected strain of microorganisms (biocatalyst) which, through the action of produced enzymes, convert a portion of the sulfur contained in a liquid petroleum fuel into an oil-soluble compound, hydroxy biphenyl (HBP), which partitions to the diesel fuel, resulting in higher diesel yields. For the Petro Star BDS application, the petroleum fuel is diesel with a total sulfur content of 5,000 ppmw. Through processing in the BDS Unit, the fuel's sulfur content is reduced to 3,500 ppmw.

For a complete description of the BDS Unit process, please refer to the BDS Baseline Report and Cost Estimate. The BDS/HDS combination case differs from the BDS Baseline description in the following ways:

- The petroleum fuel is diesel with a total sulfur content of 5,000 ppmw. Through processing in the BDS Unit, the fuel's sulfur content is reduced to 3,500 ppmw. (Overview section)

- The BDS Reactors provide a hydraulic residence time of approximately 15 minutes at the design flows. (Reaction section.)

\section{HDS Unit (Inside Battery Limits)}

\section{Feed Section}

The feed to the HDS Unit is taken directly from the BDS Unit without routed through an intermediate storage tank. The diesel from the BDS Unit contains approximately 3,500 ppmw total sulfur and has low concentrations of benzothiophenes and dibenzothiophenes which are the most difficult sulfur species to hydrogenate in the HDS process. See Appendix B.3 for the sulfur speciation in the HDS feed. The diesel feed enters the Feed Preheater, E-507, where it is heated against the desulfurized diesel product to $335^{\circ} \mathrm{F}$. The feed then enters the Diesel Feed Drum, V500, which provides 15 minutes of surge capacity and a stable level for the Diesel Feed Pump, P500 A/B. See Appendix B.4 for the IBL HDS Unit's process flow diagrams and material balance. See Appendix B.5 for the HDS Unit equipment list and budgetary equipment data sheets.

The feed is pumped through two Reactor Feed/Bottoms Exchangers, Nos. 1 and 2, (E-500 and E501) where it is preheated to $650^{\circ} \mathrm{F}$. Makeup hydrogen from the Hydrogen Unit, described 
below, is compressed by the Makeup Hydrogen Compressor (C-500) and enters the diesel feed stream upstream of the first Reactor Feed/Bottoms Exchanger. Hydrogen-rich recycle gas enters the diesel feed stream upstream of the second Reactor Feed/Bottoms Exchanger.

The combined diesel and hydrogen feed stream to the HDS reactor is heated to reaction temperature in the Diesel Charge Heater (H-500). The Diesel Charge Heater has an absorbed duty of 3.8 MM Btu/hr and uses refinery fuel gas or naphtha as its fuel source.

\section{Reaction Section}

The diesel and hydrogen feed stream enters the HDS Reactor (R-500) at $681^{\circ} \mathrm{F}$ and 630 psig at end-of-run (EOR) conditions. The catalyst in the reactor reduces the sulfur content of the diesel to $10 \mathrm{ppmw}$, and will effectively hydrogenate the most resistant sulfur species, 4,6 dibenzothiophenes. The hydrogenation reaction converts sulfur compounds to $\mathrm{H}_{2} \mathrm{~S}$ and also converts a small portion of the diesel to naphtha.

\section{Post-Reaction Cooling}

The hydrotreated diesel leaves the reactor at $719^{\circ} \mathrm{F}$ and $600 \mathrm{psig}$, and is cooled in the reactor feed/bottoms exchangers to $426^{\circ} \mathrm{F}$. The diesel is further cooled in the Separator Feed/Bottoms Exchanger $(\mathrm{E}-502)$ to $419^{\circ} \mathrm{F}$ by preheating the bottoms stream from the Low Temperature Separator (V-502).

The diesel stream then enters the High Temperature Separator (V-501) to separate the diesel and naphtha from lighter hydrocarbons, hydrogen, and $\mathrm{H}_{2} \mathrm{~S}$.

The bottoms stream from the High Temperature Separator is routed to the Naphtha Stripper (V503) to separate the diesel from naphtha and sour hydrocarbon gas.

The overhead vapor from the separator is mixed with wash water to mitigate potential plugging and corrosion from ammonium sulfide salts which could otherwise precipitate in the air cooler. The overhead stream is then cooled to $120^{\circ} \mathrm{F}$ in the High Temperature Separator Overhead Cooler (E-503) and is then routed to the Low Temperature Separator (V-502). Like E-503, all coolers in the HDS Unit are air coolers to minimize the size of the cooling fluid system.

\section{Low Temperature Separator}

The Low Temperature Separator (V-502) separates the naphtha from lighter hydrocarbons, $\mathrm{H}_{2} \mathrm{~S}$, and hydrogen. A water draw is included to remove sour wash water.

The hydrogen-rich overhead stream is recycled to the reactor feed stream via the Recycle Gas Compressor (C-501). An amine absorber is not required to treat the recycle gas because of the diesel feed's low sulfur content, the high purity of the makeup hydrogen gas, and consequently low $\mathrm{H}_{2} \mathrm{~S}$ content of the recycle gas.

Excess gas from the separator overhead is bled via pressure control to the Sulfur Unit's $\mathrm{H}_{2} \mathrm{~S}$ scrubbing section and is ultimately used as refinery fuel. The Low Temperature Separator pressure setting dictates the pressure level in the reactor system. 
The bottoms stream is routed through the Separator Feed/Bottoms Exchanger (E-502) for preheating before entering the Naphtha Stripper (V-503) at the upper feed point. Because of the low flow rate of Low Temperature Separator bottoms, the duty of the E-502 service is small; in the design phase, the size or need for an exchanger in this service could be optimized.

\section{Naphtha Stripper}

The Naphtha Stripper (V-503) separates naphtha and sour hydrocarbon gas from the diesel product. The bottoms stream from the Low Temperature Separator enters the stripper at $415^{\circ} \mathrm{F}$ via the Separator Feed/Bottoms Exchanger. The bottoms stream from the High Temperature Separator enters the stripper at $590^{\circ} \mathrm{F}$ via the Stripper Feed/Bottoms Exchanger (E-505).

The Naphtha Stripper employs 22 distillation trays to the separate naphtha and sour hydrocarbon gas from the diesel product. The diesel yield from the stripper is approximately 96.3 volume percent of the diesel feed to the HDS unit.

The overhead from the stripper is condensed in the Stripper Overhead Condenser (E-504) and is collected in the Stripper Overhead Accumulator (V-504). The non-condensed sour gas from the accumulator is routed to the sulfur recovery unit and the sweetened gas is ultimately used as refinery fuel. The Stripper Overhead Pump (P-502A/B) delivers the naphtha from the accumulator to the plant liquid fuel system. A part of the condensed naphtha is returned to the top tray of the stripper as a reflux stream.

The bottoms stream from the stripper is routed through the Stripper Feed/Bottoms Exchanger $(\mathrm{E}-505)$ where it is cooled to $505^{\circ} \mathrm{F}$. The diesel product than passes through the Feed Preheater, (E-507) where it is cooled against the diesel feed to $298^{\circ} \mathrm{F}$. The product diesel is further cooled to $120^{\circ} \mathrm{F}$ in the Diesel Product Cooler (E-506) before being routed to a product storage tank. The Stripper Bottoms Pump (P-501A/B) sends a portion of the stripper bottoms stream through the Stripper Reboiler (H-501). The Stripper Reboiler has an absorbed duty of 5.7 MM Btu/hr and uses refinery fuel gas or naphtha for firing.

\section{Hydrogen Unit}

A new steam reforming-type Hydrogen Unit will supply hydrogen for the HDS Unit. The unit size for this case is $1.7 \mathrm{MMSCF} / \mathrm{D}$ hydrogen. The hydrogen produced in the unit has a purity of 99.7+\%. See the HDS Baseline Case Report and Cost Estimate for a process description and PFD for this unit.

\section{Sulfur Unit}

The purge gas from the HDS Low Temperature Separator (V-502) and the off-gas stream from the HDS Stripper Overhead Accumulator (V-504) are combined and sent to the Thiopaq Sulfur Recovery Unit in which the $\mathrm{H}_{2} \mathrm{~S}$ is removed from the hydrocarbon gas before use as fuel gas. The Sulfur Unit converts the $\mathrm{H}_{2} \mathrm{~S}$ into elemental sulfur. The unit size for this case is 2.4 LTD. See the HDS Baseline Case Report and Cost Estimate for a process description and PFD for this unit. 


\section{Utilities}

Preliminary utility loads for the BDS Unit, HDS Unit, Hydrogen Unit, and the Sulfur Unit are contained in Appendix B.6.

\section{Cost Estimate}

\section{Total Installed Cost}

The conceptual cost estimate is based on installing a 6,000 bpd BDS Unit and a 6,000 bpd HDS Unit producing ULSD meeting the 10-ppmw sulfur requirement. The cost estimate is for the IBL portion of the desulfurization complex only.

Based on equipment costs for the BDS and HDS Units and vendor quotes for the Hydrogen Unit and Sulfur Unit, the total installed cost estimate for the IBL portion of the desulfurization complex for the BDS/HDS Case is $\$ 44.5$ million based on 2005 USGC pricing. The accuracy of the estimate is $+50 /-15 \%$.

The cost estimate excludes owner costs such as startup and commissioning costs, initial operator training, startup procedures, and pre-startup operator staffing. It also excludes licensing and royalty fees and BDS R\&D costs. A contingency of $25 \%$ is in the total installed cost. See Appendix B.7 for the Cost Estimate Basis and Cost Estimate.

\section{Operating Cost and Capital Recovery}

The total annual operating cost of the desulfurization complex for the BDS/HDS Case is $\$ 9,400,000$. This cost is based on a $95 \%$ on-stream availability for both plants. The operating cost includes fuel, power, chemicals, utilities, sulfur disposal, other consumables, operating and maintenance labor, maintenance expense, and taxes and insurance. The operating cost includes lost diesel sales due to the loss of diesel to naphtha/fuel gas (HDS Unit) and to biomass (BDS Unit). However, a credit is taken for the value of naphtha and fuel gas as refinery fuel in the HDS Unit.

The operating cost can be expressed in terms of cents per gallon of ULSD, based on a 95.9\% overall yield on the ULSD. The total operating cost of the desulfurization complex for the BDS/HDS Case is 11.1 cents per gallon of ULSD. The basis for developing the operating cost can be seen in Appendix B.8.

Based on the expected TIC of the desulfurization complex, the incremental selling price for ULSD would need to be 10.4 cents per gallon higher to recover the capital in 5 years (excluding interest expense) and 11.1 cents per gallon higher to recover operating costs. Therefore, the total incremental selling price for ULSD would need to be 21.4 cents per gallon above normal diesel sale prices in order to pass the cost of the investment on to the consumer. 


\section{HDS/BDS CASE}

The HDS/BDS case includes the following proposed IBL facilities:

- A new 6,000 bpd HDS Unit

- A new 6,000 bpd BDS Unit

- A new Hydrogen Unit

- A new Sulfur Unit to treat the off-gas from the HDS Unit.

\section{HDS Unit (Inside Battery Limits)}

\section{Feed Section}

The feed to the HDS Unit is taken directly from the Crude Unit without cooling or routing through an intermediate storage tank. The diesel feed enters the Diesel Feed Drum (V-500) at $216^{\circ} \mathrm{F}$. The feed drum provides 15 minutes of surge capacity and a stable level for the Diesel Feed Pump (P-500 A/B). See Appendix C.1 for the inside battery limits HDS Unit's process flow diagrams and material balance. See Appendix C.2 for the HDS Unit equipment list and budgetary equipment data sheets.

The feed is pumped through two Reactor Feed/Bottoms Exchangers, Nos. 1 and 2 (E-500 and E501), where it is preheated to $645^{\circ} \mathrm{F}$. Makeup hydrogen, from the Hydrogen Unit, described below, is compressed by the Makeup Hydrogen Compressor (C-500) and enters the diesel feed stream upstream of the first Reactor Feed/Bottoms Exchanger. Hydrogen-rich recycle gas enters the diesel feed stream upstream of the second Reactor Feed/Bottoms Exchanger.

The combined diesel and hydrogen feed stream to the HDS reactor is heated to reaction temperature in the Diesel Charge Heater (H-500). The Diesel Charge Heater has an absorbed duty of 4.7 MM Btu/hr and uses refinery fuel gas or naphtha as its fuel source.

\section{Reaction Section}

The diesel and hydrogen feed stream enters the HDS Reactor (R-500) at $684^{\circ} \mathrm{F}$ and 620 psig at end-of-run (EOR) conditions. The catalyst in the reactor reduces the sulfur content of the diesel to $500 \mathrm{ppmw}$. The hydrogenation reaction converts sulfur compounds to $\mathrm{H}_{2} \mathrm{~S}$ and also converts a small portion of the diesel to naphtha.

\section{Post-Reaction Cooling}

The hydrotreated diesel leaves the reactor at $717^{\circ} \mathrm{F}$ and $600 \mathrm{psig}$, and is cooled in the reactor feed/bottoms exchangers to $335^{\circ} \mathrm{F}$. The diesel is further cooled in the Separator Feed/Bottoms Exchanger (E-502) to $333^{\circ} \mathrm{F}$ by preheating the bottoms stream from the Low Temperature Separator (V-502).

The diesel stream then enters the High Temperature Separator (V-501) to separate the diesel and naphtha from lighter hydrocarbons, hydrogen, and $\mathrm{H}_{2} \mathrm{~S}$.

The bottoms stream from the High Temperature Separator is routed to the Naphtha Stripper (V-503) to separate the diesel from naphtha and sour hydrocarbon gas. 
The overhead vapor from the Separator is mixed with wash water to mitigate potential plugging and corrosion from ammonium sulfide salts which could otherwise precipitate in the air cooler. The overhead stream is then cooled to $120^{\circ} \mathrm{F}$ in the High Temperature Separator Overhead Cooler (E-503) and is then routed to the Low Temperature Separator (V-502). Like E-503, all coolers in the HDS Unit are air coolers to minimize the size of the cooling fluid system.

\section{Low Temperature Separator}

The Low Temperature Separator (V-502) separates the naphtha from lighter hydrocarbons, $\mathrm{H}_{2} \mathrm{~S}$, and hydrogen. A water draw is included to remove sour wash water.

The hydrogen-rich overhead stream is recycled to the reactor feed stream via the Recycle Gas Compressor (C-501). An amine absorber is not required to treat the recycle gas because of the diesel feed's low sulfur content, the high purity of the makeup hydrogen gas, and consequently low $\mathrm{H}_{2} \mathrm{~S}$ content of the recycle gas.

Excess gas from the Separator overhead is bled via pressure control to the Sulfur Unit's $\mathrm{H}_{2} \mathrm{~S}$ scrubbing section and is ultimately used as refinery fuel. The Low Temperature Separator pressure setting dictates the pressure level in the reactor system.

The bottoms stream is routed through the Separator Feed/Bottoms Exchanger (E-502) for preheating before entering the Naphtha Stripper (V-503) at the upper feed point. Because of the low flow rate of Low Temperature Separator bottoms, the duty of the E-502 service is small; in the design phase, the size or need for an exchanger in this service could be optimized.

\section{Naphtha Stripper}

The Naphtha Stripper (V-503) separates naphtha and sour hydrocarbon gas from the diesel product. The bottoms stream from the Low Temperature Separator enters the stripper at $325^{\circ} \mathrm{F}$ via the Separator Feed/Bottoms Exchanger. The bottoms stream from the High Temperature Separator enters the stripper at $580^{\circ} \mathrm{F}$ via the Stripper Feed/Bottoms Exchanger (E-505).

The Naphtha Stripper employs 22 distillation trays to the separate naphtha and sour hydrocarbon gas from the diesel product. The diesel yield from the stripper is approximately 97.2 volume percent of the diesel entering the HDS Unit.

The overhead from the stripper is condensed in the Stripper Overhead Condenser (E-504) and is collected in the Stripper Overhead Accumulator (V-504). The non-condensed sour gas from the accumulator is routed to the Sulfur Recovery Unit and the sweetened gas is ultimately used as refinery fuel. The Stripper Overhead Pump (P-502A/B) delivers the naphtha from the accumulator to the plant liquid fuel system. A part of the condensed naphtha is returned to the top tray of the stripper as a reflux stream.

The bottoms stream from the stripper is routed through the Stripper Feed/Bottoms Exchanger (E-505) where it is cooled to $426^{\circ} \mathrm{F}$. The product diesel is further cooled to $120^{\circ} \mathrm{F}$ in the Diesel Product Cooler (E-506) before being routed to the BDS Unit. The Stripper Bottoms Pump (P$501 \mathrm{~A} / \mathrm{B})$ sends a portion of the stripper bottoms stream through the Stripper Reboiler (H-501). 
The Stripper Reboiler has an absorbed duty of 5.6 MM Btu/hr and uses refinery fuel gas or naphtha for firing.

\section{Hydrogen Unit}

A new steam reforming-type Hydrogen Unit will supply hydrogen for the HDS Unit. The unit size for this case is $1.5 \mathrm{MMSCF} / \mathrm{D}$ hydrogen. The hydrogen produced in the unit has a purity of 99.7+\%. See the HDS Baseline Case Report and Cost Estimate for a process description and PFD for this unit.

\section{Sulfur Unit}

The purge gas from the HDS Low Temperature Separator (V-502) and the off-gas stream from the HDS Stripper Overhead Accumulator (V-504) are combined and sent to the Thiopaq Sulfur Recovery Unit in which the $\mathrm{H}_{2} \mathrm{~S}$ is removed from the hydrocarbon gas before use as fuel gas. The Sulfur Unit converts the $\mathrm{H}_{2} \mathrm{~S}$ into elemental sulfur. The unit size for this case is 3.4 LTD. See the HDS Baseline Case Report and Cost Estimate for a process description and PFD for this unit. The project does not include a storage area for the sulfur product to hold the sulfur between outhaul shipments.

\section{BDS Unit (Inside Battery Limits)}

The BDS process employs a selected strain of microorganisms (biocatalyst) which, through the action of produced enzymes, converts a portion of the sulfur contained in a liquid petroleum fuel into an oil-soluble compound, hydroxy biphenyl (HBP), which partitions to the diesel fuel, resulting in higher diesel yields. For the Petro Star BDS application, the petroleum fuel is diesel with a total sulfur content of $500 \mathrm{ppmw}$. Through processing in the BDS Unit, the sulfur content of the fuel is reduced to $10 \mathrm{ppmw}$.

For a complete description of the BDS Unit process, please refer to the BDS Baseline Report and Cost Estimate. The HDS/BDS combination case differs from the BDS Baseline description in the following ways:

- The petroleum fuel is diesel with a total sulfur content of $500 \mathrm{ppmw}$. Through processing in the BDS Unit, the fuel's sulfur content is reduced to 10 ppmw. (Overview section)

- The BDS Reactors provide a hydraulic residence time of approximately 12 minutes at the design flows. (Reaction section.)

- The diesel charge to the BDS Unit will normally come directly from the HDS Unit. (Feed section)

\section{Utilities}

Preliminary utility loads for the HDS Unit, Hydrogen Unit, Sulfur Unit, and the BDS Unit are contained in Appendix C.5. 


\section{Cost Estimate}

\section{Total Installed Cost}

The conceptual cost estimate is based on installing a 6,000 bpd HDS Unit and a 6,000 bpd BDS Unit producing ULSD meeting the 10-ppmw sulfur requirement. The cost estimate is for the IBL portion of the desulfurization complex only. Based on equipment costs for the HDS and BDS Units and vendor quotes for the Hydrogen Unit and Sulfur Unit, the total installed cost estimate for the IBL portion of the desulfurization complex for the HDS/BDS Case is \$43.3 million based on 2005 USGC pricing. The accuracy of the estimate is $+50 /-15 \%$.

The cost estimate excludes other owner costs such as startup and commissioning costs, initial operator training, startup procedures, and pre-startup operator staffing. It also excludes licensing and royalty fees, and BDS R\&D costs. The total installed cost includes a contingency of $25 \%$. See Appendix C.6 for the Cost Estimate Basis and Cost Estimate.

\section{Operating Cost and Capital Recovery}

The total annual operating cost of the HDS and BDS Units for the HDS/BDS Case is $\$ 8,000,000$. This cost is based on a 95\% on-stream availability for each unit and includes the cost of chemicals, utilities, fuel, operating consumables, operating labor, maintenance expense, sulfur disposal, and taxes and insurance. The operating cost includes lost diesel sales due to the loss of diesel to naphtha/fuel gas (HDS Unit) and to biomass (BDS Unit). However, a credit is taken for the value of naphtha and fuel gas as refinery fuel in the HDS Unit.

The operating cost can also be expressed in terms of cents per gallon of ULSD, based on a 96.9\% overall diesel yield. The total operating cost of the desulfurization complex for the HDS/BDS Case is 9.4 cents per gallon of ULSD. The basis and methodology for developing the operating cost and the operating cost in terms of cents per gallon can be seen in Appendix C.7.

Based on the expected TIC of the desulfurization complex, the incremental selling price for ULSD would need to be 10.1 cents per gallon higher to recover the capital in 5 years (excluding interest expense) and 9.4 cents per gallon higher to recover operating costs. Therefore, the total incremental selling price for ULSD would need to be 19.4 cents per gallon above normal diesel sale prices in order to pass the cost of the investment on to the consumer. 


\section{PRE FRAC CASE}

The Pre Frac case includes the following proposed IBL facilities:

- A new 6,000 bpd Diesel Splitter

- A new 3,860 bpd HDS Unit

- A new 2,140 bpd BDS Unit

- A new Hydrogen Unit

- A new Sulfur Unit to treat the off-gas from the HDS Unit.

\section{Diesel Splitter}

\section{Feed Section}

Diesel feed comes directly from a refinery feed/product exchanger without being cooled or routed through an intermediate storage tank. The feed enters the Diesel Feed Drum, V-1, at $216^{\circ} \mathrm{F}$. The feed drum provides 15 minutes of surge capacity and a stable level for the Diesel Feed Pump (P-1 A/B). See Appendix D.1 for the IBL Diesel Splitter process flow diagram and material balance. See Appendix D.2 for the Diesel Splitter equipment list and budgetary equipment data sheets.

The feed is pumped through Exchanger E-1 for preheating with tower bottoms. The feed is further preheated in E-2 with the tower overhead vapor stream. The feed is finally preheated to $540^{\circ} \mathrm{F}$ in E-3 by exchanging heat with the tower bottoms stream. The preheated feed from E-3 is sent to the Diesel Splitter Tower.

The Diesel Splitter (V-2) separates the diesel feed into a light diesel overhead stream and a heavy diesel bottoms stream. About two thirds of the diesel stream is recovered in the overhead product. The cut point for this light diesel overhead product is limited to a $\mathrm{D}-86$ of $560^{\circ} \mathrm{F}$ in order to minimize the quantity of substituted dibenzothiophenes in this stream. Ensuring the HDS feed has less than $10 \mathrm{ppm}$ substituted dibenzothiophenes allows a conventional HDS unit to be utilized to produce ULSD product. Cutting as deep as possible is desired to minimize the volume of feed to the BDS Unit while at same time concentrating the substituted dibenzothiophenes into an enriched BDS feed.

To achieve the desired separation, the Diesel Splitter employs 30 distillation trays. The tower is designed to operate with a tower top pressure of $10 \mathrm{psig}$ to provide the necessary lift while limiting the temperature required at the bottom of the tower. Limiting the temperature at the bottom of the tower (to below $700^{\circ} \mathrm{F}$ ) is important to avoid potential product degradation. The number of trays and reflux ratio for the Diesel Splitter were selected to provide a reasonable balance between tower diameter and energy consumption.

The Diesel Splitter bottoms are pumped via P-2 A/B and used to preheat the feed. The bottoms are cooled to $120^{\circ} \mathrm{F}$ in the Airfin Cooler (E-5) and on the BDS Unit for processing. A portion of the bottoms stream from P-2 A/B is sent to the Diesel Splitter Reboiler $(\mathrm{H}-1)$ which is a fired reboiler. The Diesel Splitter Reboiler has an absorbed duty of 17.8 MM Btu/hr and uses refinery fuel gas or naphtha for firing. The Diesel Splitter overhead vapor stream first goes to E-2 to 
preheat tower feed and then goes to the Airfin Condenser (E-4). The condensed overhead liquid goes to the Accumulator (V-3) and is pumped via P-3 A/B as reflux back to the tower top tray and also as feed to the HDS Unit feed drum.

An examination was made into the potential benefits of modifying the Diesel Splitting configuration by tailoring the streams coming from the upstream Crude Unit. An option was considered in which two diesel cuts were taken from the Crude Unit. Although the fractionation of these cuts would not be exact enough to directly be used at the HDS and BDS Units, the cuts could be fed to separate points in the Diesel Splitter, assisting separation in that tower. The results from this cursory examination showed only a modest reduction in tower diameter, however, and this option was not selected as a final configuration.

\section{HDS Unit (Inside Battery Limits)}

\section{Feed Section}

The 3,860 bpd of light diesel (4,240 ppmw total sulfur with low dibenzothiophenes) produced by the Diesel Splitter is taken directly into the HDS Unit without being cooled or routed through an intermediate storage tank. The diesel feed enters the Diesel Feed Drum (V-500) at $350^{\circ} \mathrm{F}$. The feed drum provides 15 minutes of surge capacity and a stable level for the Diesel Feed Pump (P-500 A/B). See Appendix D.4 for the IBL HDS Unit's process flow diagrams and material balance. See Appendix D.5 for the HDS Unit equipment list and budgetary equipment data sheets.

The feed is pumped through two Reactor Feed/Bottoms Exchangers, Nos. 1 and 2 (E-500 and E501), where it is preheated to $655^{\circ} \mathrm{F}$. Makeup hydrogen from the Hydrogen Unit, described below, is compressed by the Makeup Hydrogen Compressor (C-500) and enters the diesel feed stream upstream of the first Reactor Feed/Bottoms Exchanger. Hydrogen-rich recycle gas enters the diesel feed stream upstream of the second Reactor Feed/Bottoms Exchanger.

The combined diesel and hydrogen feed stream to the HDS Reactor is heated to reaction temperature in the Diesel Charge Heater (H-500). The Diesel Charge Heater has an absorbed duty of 2.7 MM Btu/hr and uses refinery fuel gas or naphtha as its fuel source.

\section{Reaction}

The diesel and hydrogen feed stream enters the HDS Reactor (R-500) at $688^{\circ} \mathrm{F}$ and 620 psig at end-of-run (EOR) conditions. The catalyst in the reactor reduces the diesel sulfur content to 10 ppmw, and will effectively hydrogenate the most resistant sulfur species, 4,6 dibenzothiophenes. The hydrogenation reaction converts sulfur compounds to $\mathrm{H}_{2} \mathrm{~S}$ and also converts a small portion of the diesel to naphtha.

\section{Post-Reaction Cooling}

The hydrotreated diesel leaves the reactor at $716^{\circ} \mathrm{F}$ and $600 \mathrm{psig}$, and is cooled in the reactor feed/bottoms exchangers to $440^{\circ} \mathrm{F}$. The diesel is further cooled in the Separator Feed/Bottoms Exchanger (E-502) to $428^{\circ} \mathrm{F}$ by preheating the bottoms stream from the Low Temperature Separator (V-502). 
The diesel stream then enters the High Temperature Separator (V-501) to separate the diesel and naphtha from lighter hydrocarbons, hydrogen, and $\mathrm{H}_{2} \mathrm{~S}$.

The bottoms stream from the High Temperature Separator is routed to the Naphtha Stripper (V503) to separate the diesel from naphtha and sour hydrocarbon gas.

The overhead vapor from the separator is mixed with wash water to mitigate potential plugging and corrosion from ammonium sulfide salts which could otherwise precipitate in the air cooler. The overhead stream is then cooled to $120^{\circ} \mathrm{F}$ in the High Temperature Separator Overhead Cooler (E-503) and is then routed to the Low Temperature Separator (V-502). Like E-503, all coolers in the HDS Unit are air coolers to minimize the size of the cooling fluid system.

\section{Low Temperature Separator}

The Low Temperature Separator (V-502) separates the naphtha from lighter hydrocarbons, $\mathrm{H}_{2} \mathrm{~S}$, and hydrogen. A water draw is included to remove sour wash water.

The hydrogen-rich overhead stream is recycled to the reactor feed stream via the Recycle Gas Compressor (C-501). An amine absorber is not required to treat the recycle gas because of the diesel feed's low sulfur content, the high purity of the makeup hydrogen gas, and consequently low $\mathrm{H}_{2} \mathrm{~S}$ content of the recycle gas.

Excess gas from the separator overhead is bled via pressure control to the Sulfur Unit's $\mathrm{H}_{2} \mathrm{~S}$ scrubbing section and is ultimately used as refinery fuel. The Low Temperature Separator pressure setting dictates the pressure level in the reactor system.

The bottoms stream is routed through the Separator Feed/Bottoms Exchanger (E-502) for preheating before entering the Naphtha Stripper (V-503) at the upper feed point. Because of the low flow rate of the Low Temperature Separator bottoms, the duty of the E-502 service is small; in the design phase, the size or need for an exchanger in this service could be optimized.

\section{Naphtha Stripper}

The Naphtha Stripper (V-503) separates naphtha and sour hydrocarbon gas from the diesel product. The bottoms stream from the Low Temperature Separator enters the stripper at $430^{\circ} \mathrm{F}$ via the Separator Feed/Bottoms Exchanger. The bottoms stream from the High Temperature Separator enters the stripper at $525^{\circ} \mathrm{F}$ via the Stripper Feed/Bottoms Exchanger, E-505.

The Naphtha Stripper employs 22 distillation trays to separate naphtha and sour hydrocarbon gas from the diesel product. The diesel yield from the stripper is approximately 95.6 volume percent of the diesel entering the HDS Unit.

The overhead from the stripper is condensed in the Stripper Overhead Condenser (E-504) and is collected in the Stripper Overhead Accumulator (V-504). The non-condensed sour gas from the accumulator is routed to the Sulfur Recovery Unit and the sweetened gas is ultimately used as refinery fuel. The Stripper Overhead Pump (P-502A/B) delivers the naphtha from the accumulator to the plant liquid fuel system. A part of the condensed naphtha is returned to the top tray of the stripper as a reflux stream. 
The bottoms stream from the stripper is routed through the Stripper Feed/Bottoms Exchanger (E505) where it is cooled to $523^{\circ} \mathrm{F}$. The product diesel is further cooled to $120^{\circ} \mathrm{F}$ in the Diesel Product Cooler (E-506) before being routed to a product storage tank. The Stripper Bottoms Pump (P-501A/B) sends a portion of the stripper bottoms stream through the Stripper Reboiler (H-501). The Stripper Reboiler has an absorbed duty of 4.5 MM Btu/hr and uses refinery fuel gas or naphtha for firing.

\section{Hydrogen Unit}

A new steam reforming-type Hydrogen Unit will supply hydrogen for the HDS Unit. The unit size for this case is $1 \mathrm{MMSCF} / \mathrm{D}$ hydrogen. The hydrogen produced in the unit has a purity of 99.7+\%. See the HDS Baseline Case Report and Cost Estimate for a process description and PFD for this unit.

\section{Sulfur Unit}

The purge gas from the HDS Low Temperature Separator (V-502) and the off-gas stream from the HDS Stripper Overhead Accumulator (V-504) are combined and sent to the Thiopaq Sulfur Recovery Unit in which the $\mathrm{H}_{2} \mathrm{~S}$ is removed from the hydrocarbon gas before use as fuel gas. The Sulfur Unit converts the $\mathrm{H}_{2} \mathrm{~S}$ into elemental sulfur. The unit size for this case is 1.9 LTD. See the HDS Baseline Case Report and Cost Estimate for a process description and PFD for this unit. The project does not include a storage area for the sulfur product to hold the sulfur between outhaul shipments.

\section{BDS Unit (Inside Battery Limits)}

The BDS process employs a selected strain of microorganisms (biocatalyst) which, through the action of produced enzymes, convert a portion of the sulfur contained in a liquid petroleum fuel into a oil-soluble compound, hydroxy biphenyl (HBP), which partitions to the diesel fuel, resulting in higher diesel yields. For the Petro Star BDS application, the petroleum fuel is diesel with a total sulfur content of 6,311 ppmw. Through processing in the BDS Unit, the sulfur content of the fuel is reduced to 10 ppmw.

For a complete description of the BDS Unit process, please refer to the BDS Baseline Report and Cost Estimate. The Pre Frac case differs from the BDS Baseline description in the following ways:

- The petroleum fuel is diesel with a total sulfur content of 6,311 ppmw. Through processing in the BDS Unit, the fuel's sulfur content is reduced to 10 ppmw (Overview section)

- The diesel charge to the BDS Unit will normally come directly from the Diesel Splitter. (Feed section.)

\section{Utilities}

Preliminary utility loads for the BDS Unit, HDS Unit, Hydrogen Unit, and the Sulfur Unit are contained in Appendix D.8. 


\section{Cost Estimate}

\section{Total Installed Cost}

The conceptual cost estimate is based on installing a 6,000 bpd Diesel Splitter, a 3,860 bpd HDS Unit and a 2,140 bpd BDS Unit, each producing ULSD meeting the 10-ppmw sulfur requirement. The cost estimate is for the IBL portion of the desulfurization complex only. Based on equipment costs for the Diesel Splitter, HDS Unit, and BDS Units and vendor quotes for the Hydrogen Unit and Sulfur Unit, the total installed cost estimate for the IBL portion of the desulfurization complex for the Pre Frac Case is \$46.5 million based on 2005 USGC pricing. The accuracy of the estimate is $+50 /-15 \%$.

The cost estimate excludes other owner costs such as startup and commissioning costs, initial operator training, startup procedures, and pre-startup operator staffing. It also excludes licensing and royalty fees, and BDS R\&D costs. The total installed cost includes a contingency of $25 \%$. See Appendix D.9 for the Cost Estimate Basis and Cost Estimate.

\section{Operating Cost and Capital Recovery}

The total annual operating cost of the BDS and HDS Units for the Pre Frac Case is $\$ 10,200,000$. This cost is based on a 95\% on-stream availability for each unit and includes the cost of chemicals, utilities, fuel, operating consumables, operating labor, maintenance expense, sulfur disposal, and taxes and insurance. The operating cost includes lost diesel sales due to the loss of diesel to naphtha/fuel gas (HDS Unit) and to biomass (BDS Unit). However, a credit is taken for the value of naphtha and fuel gas as refinery fuel in the HDS Unit.

The operating cost can also be expressed in terms of cents per gallon of ULSD, based on a 96.5\% overall diesel yield. The total operating cost of the desulfurization complex for the Pre Frac Case is 12.1 cents per gallon of ULSD. The basis for developing the operating cost can be seen in Appendix D.10.

Based on the expected TIC of the desulfurization complex, the incremental selling price for ULSD would need to be 11 cents per gallon higher to recover the capital in 5 years (excluding interest expense) and 12.1 cents per gallon higher to recover operating costs. Therefore, the total incremental selling price for ULSD would need to be 23.1 cents per gallon above normal diesel sale prices in order to pass the cost of the investment on to the consumer.

S:IPETRSTARIAE1416IWORDIFINALSI4 COMBOICOMBO_CASE_REPORT.DOC 


\section{APPENDICES}

\section{A. Raw Diesel Feed Properties and Sulfur Speciation}

\section{B. BDS/HDS Case}

B.1. BDS Unit Process Flow Diagrams and Material Balance

B.2. BDS Unit Equipment List and Budgetary Equipment Datasheets

B.3. Sulfur Speciation in BDS Unit Diesel Product

B.4. HDS Unit Process Flow Diagrams and Material Balance

B.5. HDS Unit Equipment List and Budgetary Equipment Datasheets

B.6. Utility Requirements

B.7. Cost Estimate Basis and Cost Estimate

B.8. Operating Cost Estimates

\section{HDS/BDS Case}

C.1. HDS Unit Process Flow Diagrams and Material Balance

C.2. HDS Unit Equipment List and Budgetary Equipment Datasheets

C.3. BDS Unit Process Flow Diagrams and Material Balance

C.4. BDS Unit Equipment List and Budgetary Equipment Datasheets

C.5. Utility Requirements

C.6. Cost Estimate Basis and Cost Estimate

C.7. Operating Cost Estimates

\section{Pre Frac Case}

D.1. Diesel Splitter Process Flow Diagram and Material Balance

D.2. Diesel Splitter Equipment List and Budgetary Equipment Datasheets

D.3. Sulfur Speciation in Diesel Splitter Products

D.4. HDS Unit Process Flow Diagrams and Material Balance

D.5. HDS Unit Equipment List and Budgetary Equipment Datasheets

D.6. BDS Unit Process Flow Diagrams and Material Balance

D.7. BDS Unit Equipment List and Budgetary Datasheets

D.8. Utility Requirements

D.9. Cost Estimate Basis and Cost Estimate

D.10 Operating Cost Estimates 


\section{Appendix A - Raw Diesel Feed Properties and Sulfur Speciation}

\begin{tabular}{|l|l|c|c|}
\hline \multicolumn{4}{|c|}{ Diesel Feed Properties } \\
\hline Test & Units & Results & Specification \\
\hline Gravity @ 60 ${ }^{\circ}$ F, Min/Max & API & 33.3 & $32-36$ \\
\hline Flash Pt, Min. & Celsius & $63.5\left(146^{\circ} \mathrm{F}\right)$ & $60\left(140^{\circ} \mathrm{F}\right)$ \\
\hline Cloud Pt, Max. & Celsius & $-12.3\left(10^{\circ} \mathrm{F}\right)$ & $-9.5\left(15^{\circ} \mathrm{F}\right)$ \\
\hline Pour Pt, Max. & Celsius & $-15\left(5^{\circ} \mathrm{F}\right)$ & $-12\left(10^{\circ} \mathrm{F}\right)$ \\
\hline Distillation & & & \\
\hline IBP & Celsius & $171\left(340^{\circ} \mathrm{F}\right)$ & Report \\
\hline $10 \%$ Recovery & Celsius & $249\left(480^{\circ} \mathrm{F}\right)$ & Report \\
\hline $20 \%$ Recovery & Celsius & $265\left(509^{\circ} \mathrm{F}\right)$ & Report \\
\hline $50 \%$ Recovery & Celsius & $287\left(549^{\circ} \mathrm{F}\right)$ & Report \\
\hline 90\% Recovery & Celsius & $317\left(603^{\circ} \mathrm{F}\right)$ & $282-338$ \\
\hline Final Boiling Pt. & Celsius & $336\left(637^{\circ} \mathrm{F}\right)$ & Report \\
\hline Recovery & Vol \% & 99.9 & Report \\
\hline Residual & Vol \% & 0.1 & Report \\
\hline Loss & Vol \% & 0 & Report \\
\hline Viscosity @ 40 C, Min/Max & CSt & 3.57 & $2.0-4.3$ \\
\hline Ash, Max & Wt $\%$ & $<0.001$ & $0.01 \%$ \\
\hline Carbon Residue on 10\% Bottoms, Max & Wt\% & 0.08 & $0.35 \%$ \\
\hline Btu Gross, Min & Btu/Gallon & 139,190 & 136,000 \\
\hline Calculated Cetane, Min & Index & 48 & 45 \\
\hline Copper Strip Corrosion, Max & Code & $1 \mathrm{a}$ & 3 \\
\hline Total Sulfur & Wt. \% & 0.500 & 0.500 \\
\hline
\end{tabular}




\begin{tabular}{|l|c|l|c|}
\hline \multicolumn{5}{|c|}{$\begin{array}{c}\text { Raw Diesel Sulfur Speciation } \\
\text { (Ratioed to 5,000 ppm*) }\end{array}$} \\
\hline Component & ppm wt sulfur & Component & ppm wt sulfur \\
\hline Hydrogen sulfide & $<1$ & 2-Ethyl thiophene & $<1$ \\
\hline Carbonyl sulfide & $<1$ & 2,5-Dimethyl thiophene & $<1$ \\
\hline Methyl mercaptan & $<1$ & 3-Ethyl thiophene & $<1$ \\
\hline Ethyl mercaptan & $<1$ & 2,4\&2,-Dimethyl thiophene & $<1$ \\
\hline Dimethyl sulfide & $<1$ & 3,4-Dimethyl thiophene & $<1$ \\
\hline Carbon disulfide & $<1$ & Methyl Ethyl thiophenes & $<1$ \\
\hline Isopropyl mercaptan & $<1$ & Trimethyl thiophenes & $<1$ \\
\hline Ethyl sulfide & $<1$ & Tetramethyl thiophenes & $<1$ \\
\hline tert-Butyl mercaptan & $<1$ & Benzothiophene & $<1$ \\
\hline N-Propyl mercaptan & $<1$ & Methyl benzothiophene & 2 \\
\hline Ethyl Methyl Sulfide & $<1$ & Dimethyl benzothiophene & 29 \\
\hline Thiophene & $<1$ & Trimethyl benzothiophene & 119 \\
\hline sec-Butyl Mercaptan & $<1$ & Tetramethyl Benzothiophene & 354 \\
\hline Isobutyl mercaptan & $<1$ & Dibenzothiophene & 229 \\
\hline Ethyl sulfide & $<1$ & 4-Methyl benzothiophene & 213 \\
\hline MN-butyl mercaptan & $<1$ & 3-Methyl DBZT+2-methyl DBZT & 147 \\
\hline Dimethyl disulfide & $<1$ & 1-Methyl dibenzothiophene & 94 \\
\hline 2-Methyl thiophene & $<1$ & 4,6 Dimethyl dibenzothiophene & 97 \\
\hline 3-Methyl thiophene & $<1$ & Dimethyl dibenzothiophene & 354 \\
\hline Tetra-hydro thiophene & $<1$ & Trimethyl dibenzothiophene & 75 \\
\hline Ethyl methyl disulfide & $<1$ & Tetramethyl dibenzothiophene & 4 \\
\hline 2-Methyl-tetra-hydro-thiophene & $<1$ & Unidentified volatile sulfur & 3,283 \\
\hline
\end{tabular}

* Raw data from the speciation analysis ratioed to $5,000 \mathrm{ppm}$ to reflect the design basis sulfur content. 
Appendix B.1 - BDS Unit Process Flow Diagrams and Material Balance 


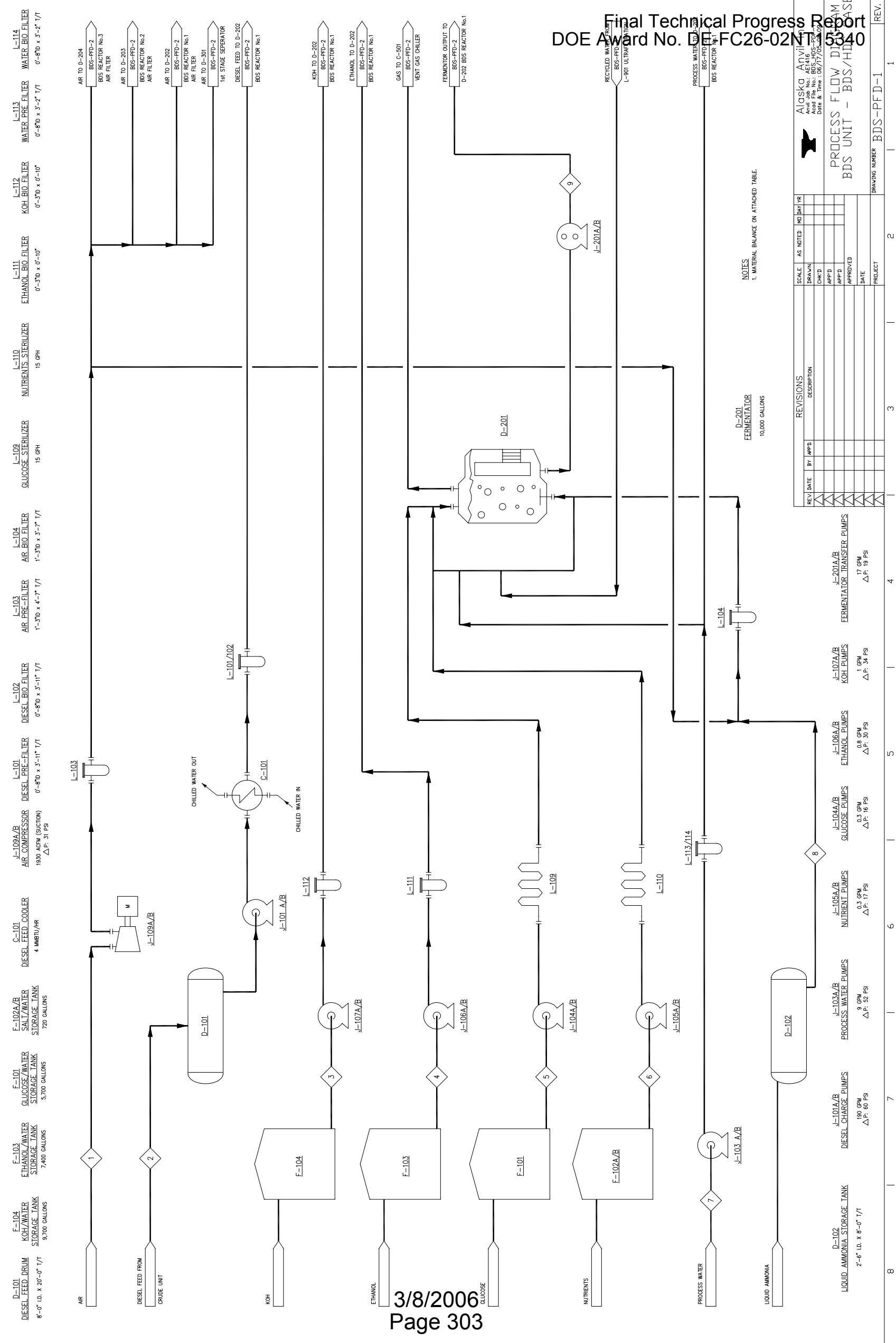




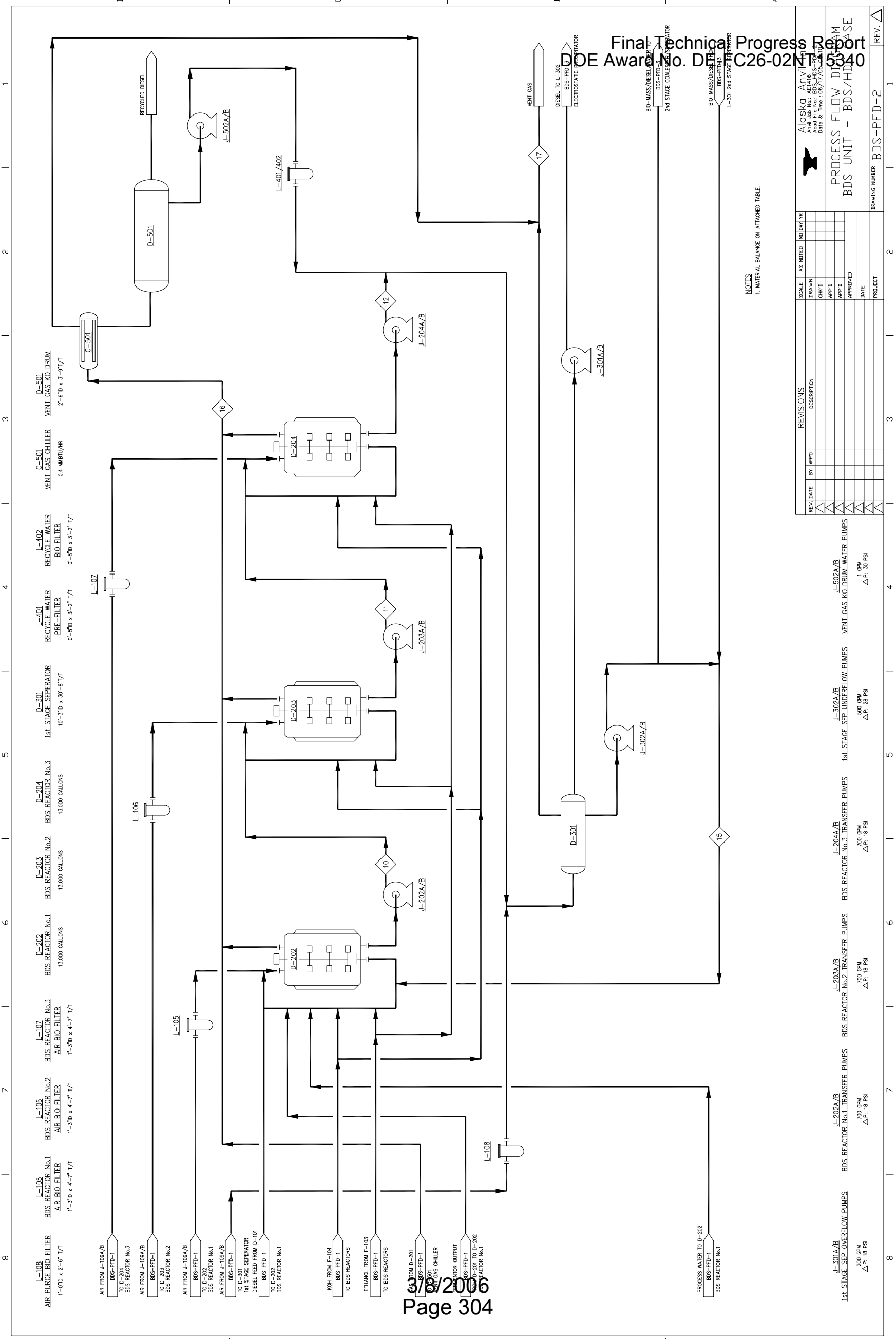




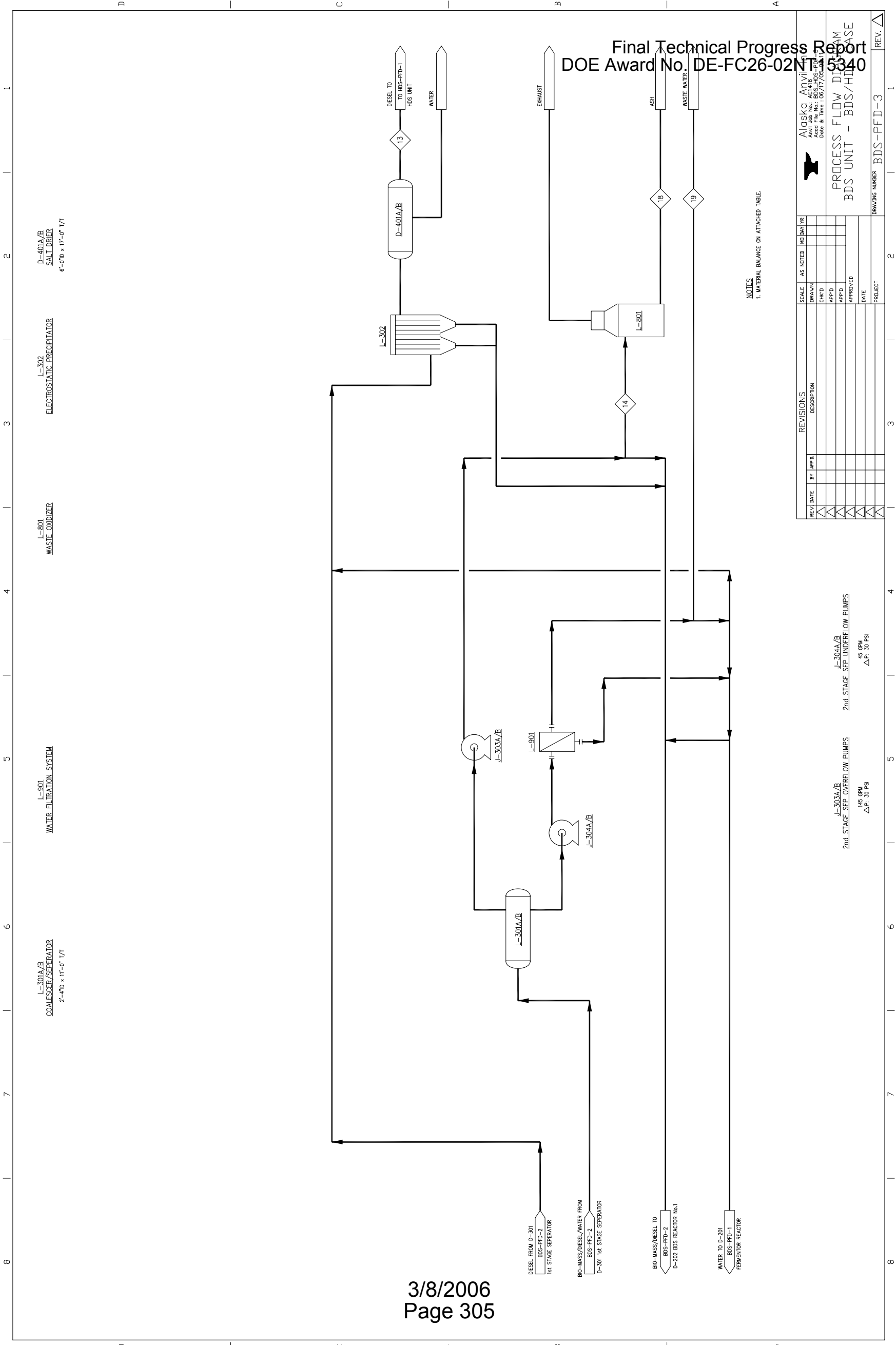




\section{Material Balance}

\section{BDS Unit Material Balance -- BDS/HDS Case}

\begin{tabular}{|c|c|c|c|c|c|c|c|c|}
\hline PFD Stream Number & & 1 & 2 & 3 & 4 & 5 & 6 & 7 \\
\hline Stream Number & & Air & Diesel & $\mathrm{NaOH}$ & Ethanol & Glucose & Nutrients & Process Water \\
\hline Stream Description & & Air Feed & Diesel Feed & $\mathrm{NaOH}$ Feed & Ethanol Feed & Glucose Feed & Nutrient Feed & $\begin{array}{c}\text { Process Water } \\
\text { Feed }\end{array}$ \\
\hline Phase & & VAPOR & LIQUID & LIQUID & LIQUID & LIQUID & LIQUID & LIQUID \\
\hline Hydrocarbon Mass Flo & LB/HR & & 74483 & & & & & \\
\hline Aqueous Mass Flow & LB/HR & & & 570 & 278 & 137 & 111 & 3981 \\
\hline Biomass Mass Flow & LB/HR & & & & & & & \\
\hline Sulfur in $\mathrm{HC}$ & PPM & & 5000 & & & & & \\
\hline Total Mass Flow Rate & LB/HR & 8000 & 74483 & 570 & 278 & 137 & 111 & 3981 \\
\hline Temperature & $F$ & 68 & 176 & 68 & 68 & 68 & 68 & 68 \\
\hline Pressure & PSIG & 0 & 0 & 0 & 0 & 0 & 0 & 0 \\
\hline Standard Liq Flow & BPD & & 6000 & 26 & 21 & 8 & 8 & 274 \\
\hline Vapor Flow & MSCFH & 108 & & & & & & \\
\hline Wt \% Vapor & & 100 & 0 & 0 & 0 & 0 & 0 & 0 \\
\hline MW & & & & & & & & \\
\hline
\end{tabular}

\begin{tabular}{|c|c|c|c|c|c|c|c|c|}
\hline PFD Stream Number & & 8 & 9 & 10 & 11 & 12 & 13 & 14 \\
\hline Stream Number & & Liquid Ammoniz & $5-16$ & $S-17$ & $S-18$ & $5-19$ & $5-27$ & $5-31$ \\
\hline Stream Description & & $\begin{array}{c}\text { Liquid } \\
\text { Ammonia Feed }\end{array}$ & $\begin{array}{c}\text { Fermentor } \\
\text { Output }\end{array}$ & R\#1 Output & R\#2 Output & R\#3 Output & Diesel Product & Biomass Purge \\
\hline Phase & & LIQUID & LIQUID & LIOUID & LIQUID & LIQUID & LIQUID & LIQUID \\
\hline Hydrocarbon Mass Flo & LB/HR & & & 126038 & 125829 & 125636 & 74229 & 244 \\
\hline Aqueous Mass Flow & LB/HR & 9.8 & 7280 & 139821 & 140303 & 140407 & 0 & 418 \\
\hline Biomass Mass Flow & LB/HR & & 137 & 34543 & 34543 & 34543 & 0 & 130 \\
\hline Sulfur in $\mathrm{HC}$ & PPM & & & 3910 & 3559 & 3470 & 3470 & 3470 \\
\hline Mass Flow Rate & LB/HR & 9.8 & 7417 & 300402 & 300675 & 300585 & 74229 & 792 \\
\hline Temperature & $\mathrm{F}$ & -40 & 86 & 86 & 86 & 86 & 86 & 86 \\
\hline Pressure & $\mathrm{PSIG}$ & 210 & 33.1 & 36.4 & 36.4 & 26.8 & 5 & 5 \\
\hline Standard Liq Flow & BPD & 1 & 511 & 21817 & 21825 & 21815 & 5977 & 56 \\
\hline Vapor Flow & MSCFH & & & & & & & \\
\hline Wt \% Vapor & & 0 & 0 & 0 & 0 & 0 & 0 & 0 \\
\hline MW & & & & & & & & \\
\hline
\end{tabular}

\begin{tabular}{|l|l|c|c|c|c|c|c|c|}
\hline PFD Stream Number & & 15 & 16 & 17 & 18 & 19 & & \\
\hline Stream Number & & S-32 & S-38 & S-39 & Ash & Waste Water & & \\
\hline Stream Description & & $\begin{array}{c}\text { Reactor } \\
\text { Recvcle }\end{array}$ & $\begin{array}{c}\text { Reactor Air } \\
\text { Effluent }\end{array}$ & Gas Purge & $\begin{array}{c}\text { Incinerator } \\
\text { Waste }\end{array}$ & Waste Water & & \\
\hline Phase & & LIOUID & GAS & GAS & SOLID & LIOUID & & \\
\hline Hydrocarbon Mass Flo LB/HR & 51769 & 567 & & & & & \\
\hline Aqueous Mass Flow & LB/HR & 128259 & 123 & & & 4746 & & \\
\hline Biomass Mass Flow & LB/HR & 34406 & & & & & & \\
\hline Sulfur in HC & PPM & 3470 & 3650 & & & & & \\
\hline Total Mass Flow Rate & LB/HR & 214434 & 8173 & 7523 & 10 & 4746 & & \\
\hline Temperature & F & 86 & 86 & 60 & 68 & 86 & & \\
\hline Pressure & PSIG & 37.4 & 10 & 1 & 0 & 10 & & \\
\hline Standard Liq Flow & BPD & 15039 & & & & 319 & & \\
\hline Vapor Flow & MSCFH & & 102 & 99 & & & & \\
\hline Wt $\%$ Vapor & & 0 & 100 & 100 & 0 & 0 & & \\
\hline MW & & & & & & & & \\
\hline
\end{tabular}


Appendix B.2 - BDS Unit Equipment List and Budgetary Equipment Datasheets 


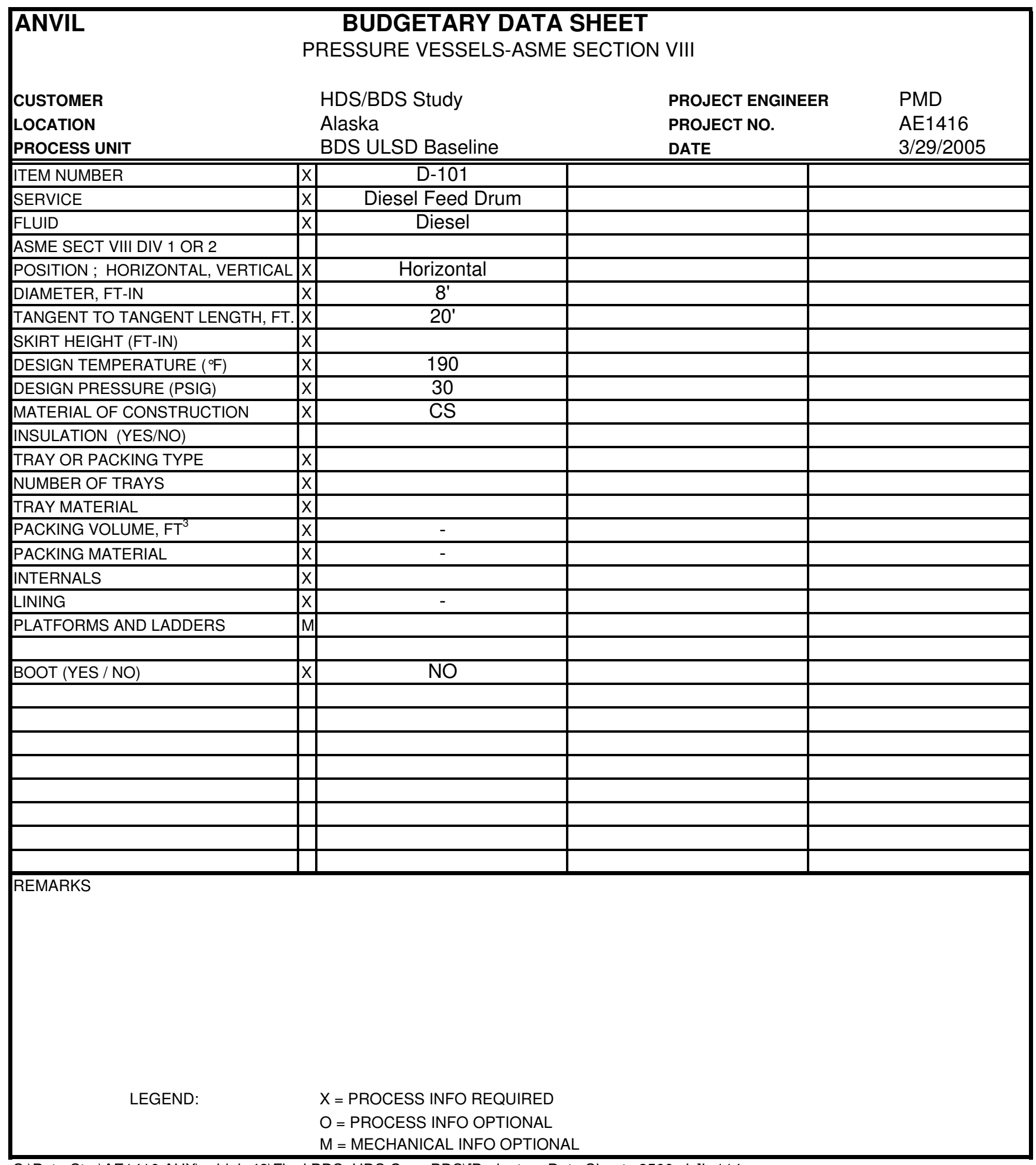




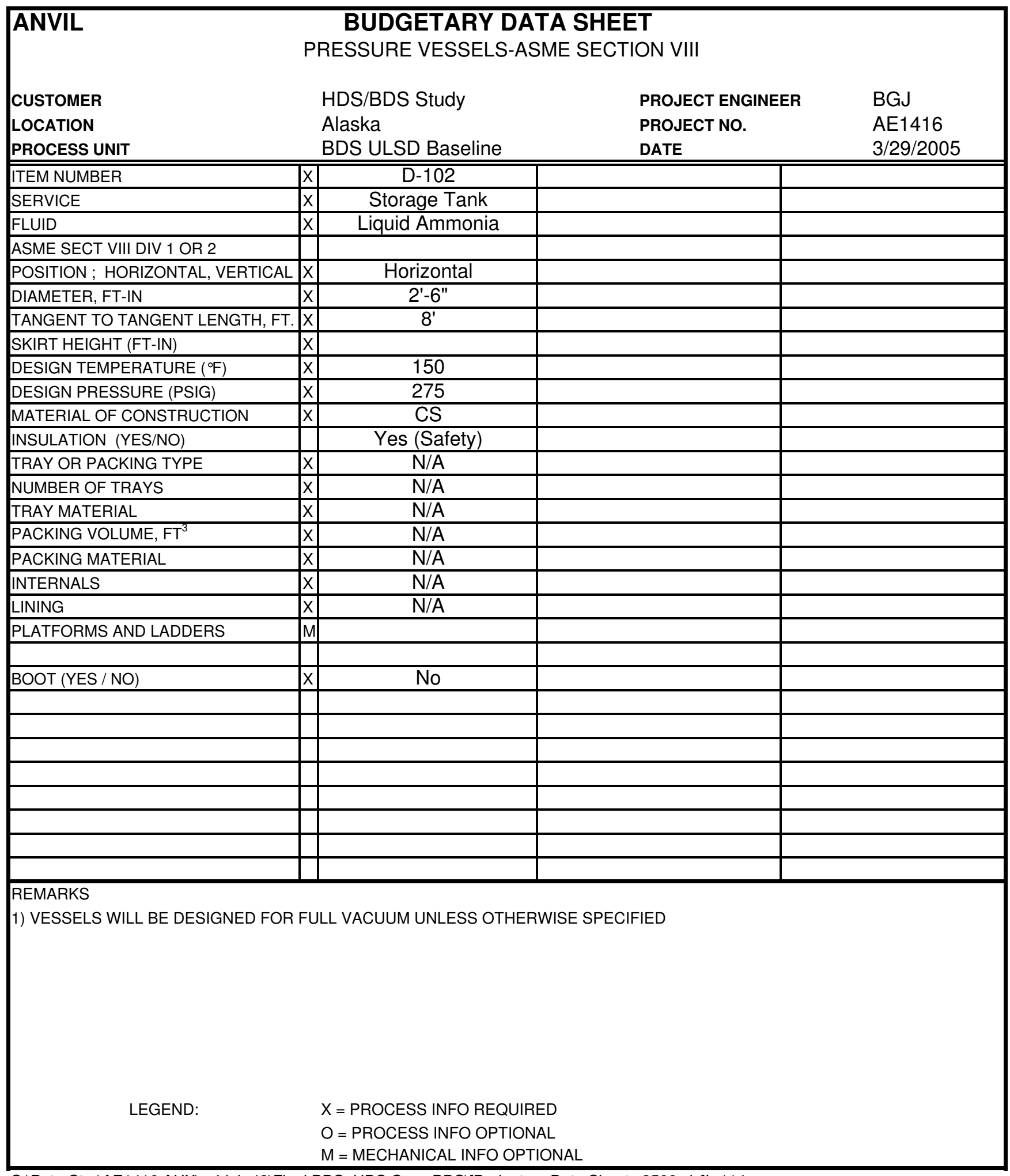




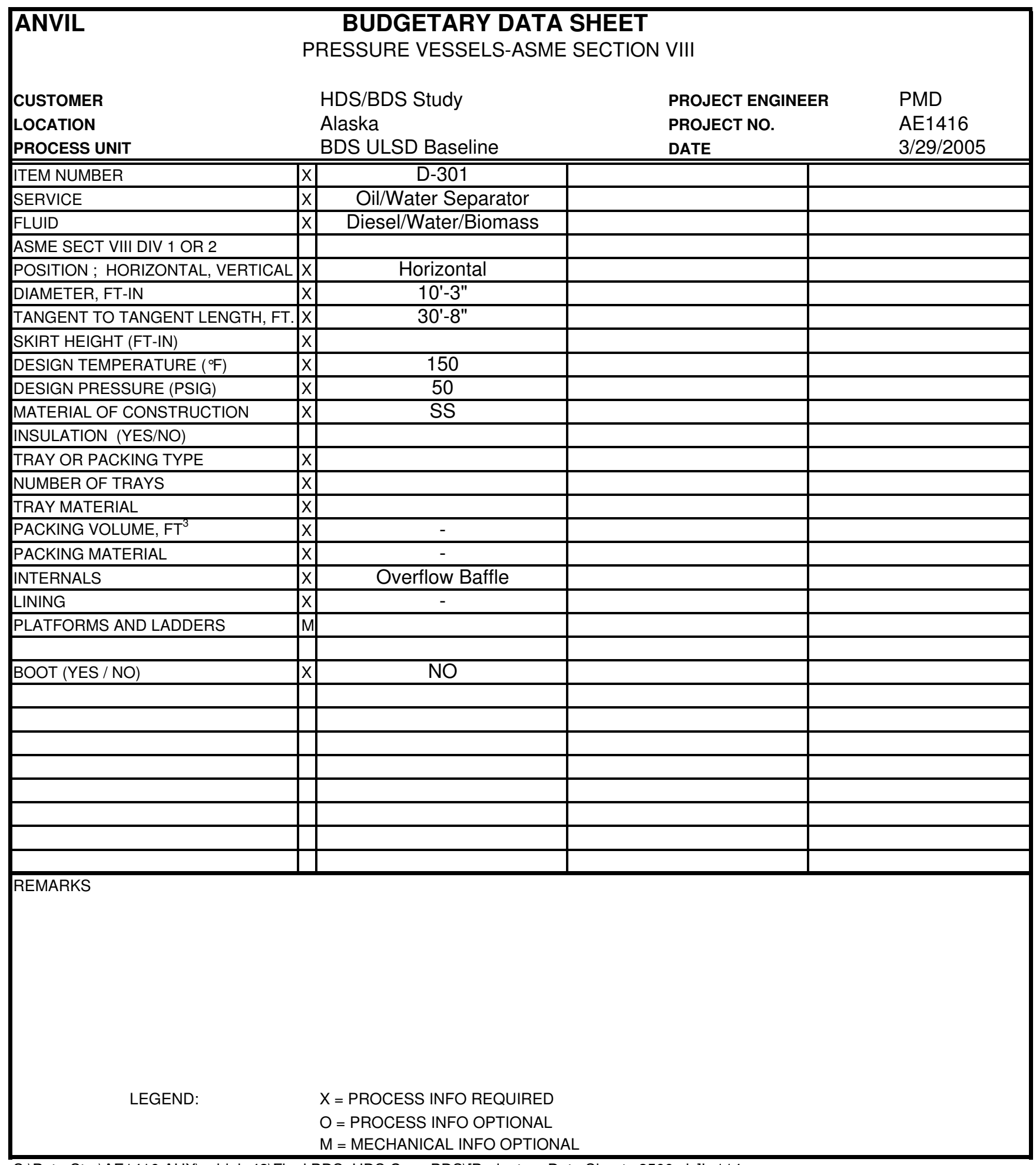




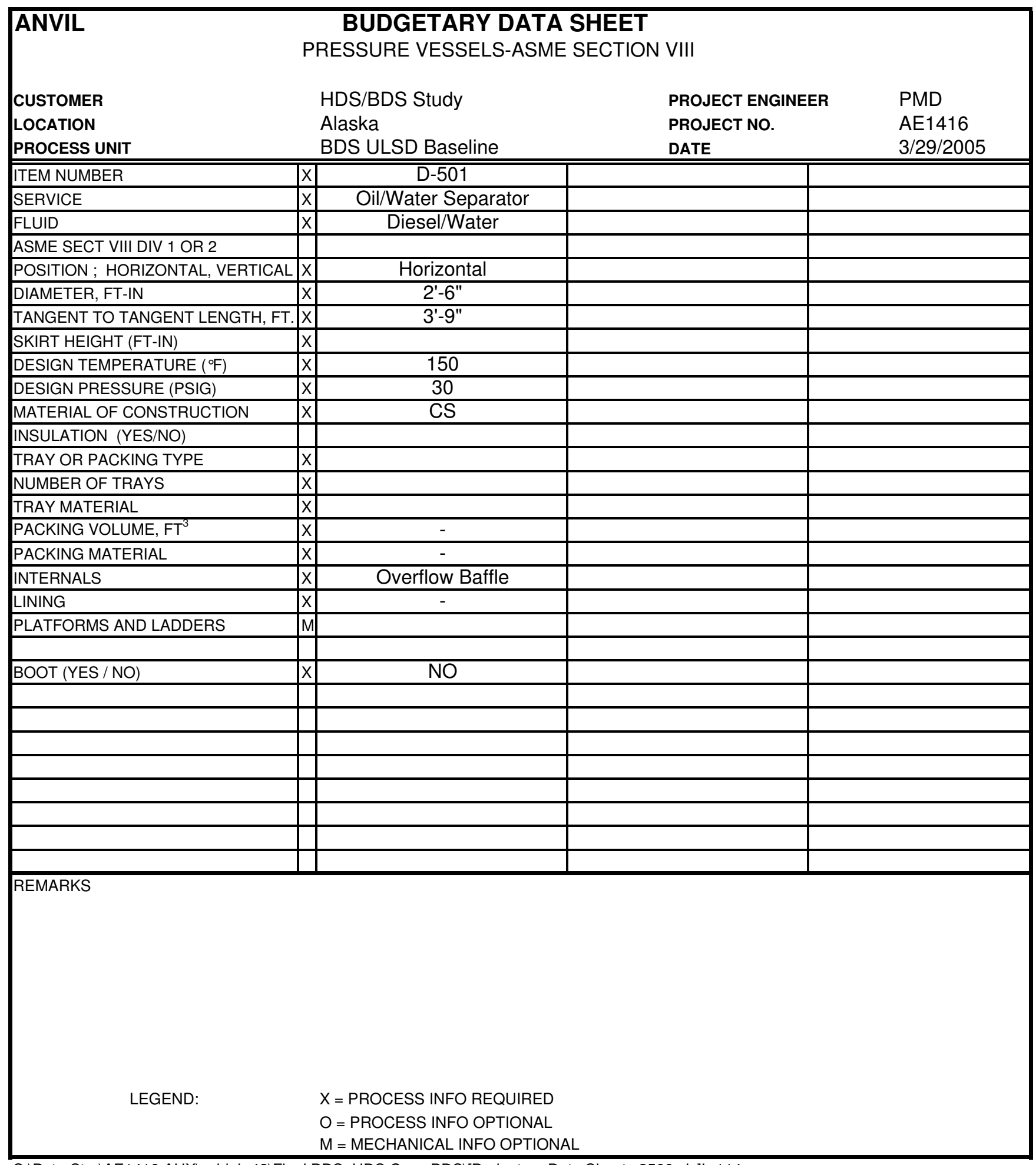




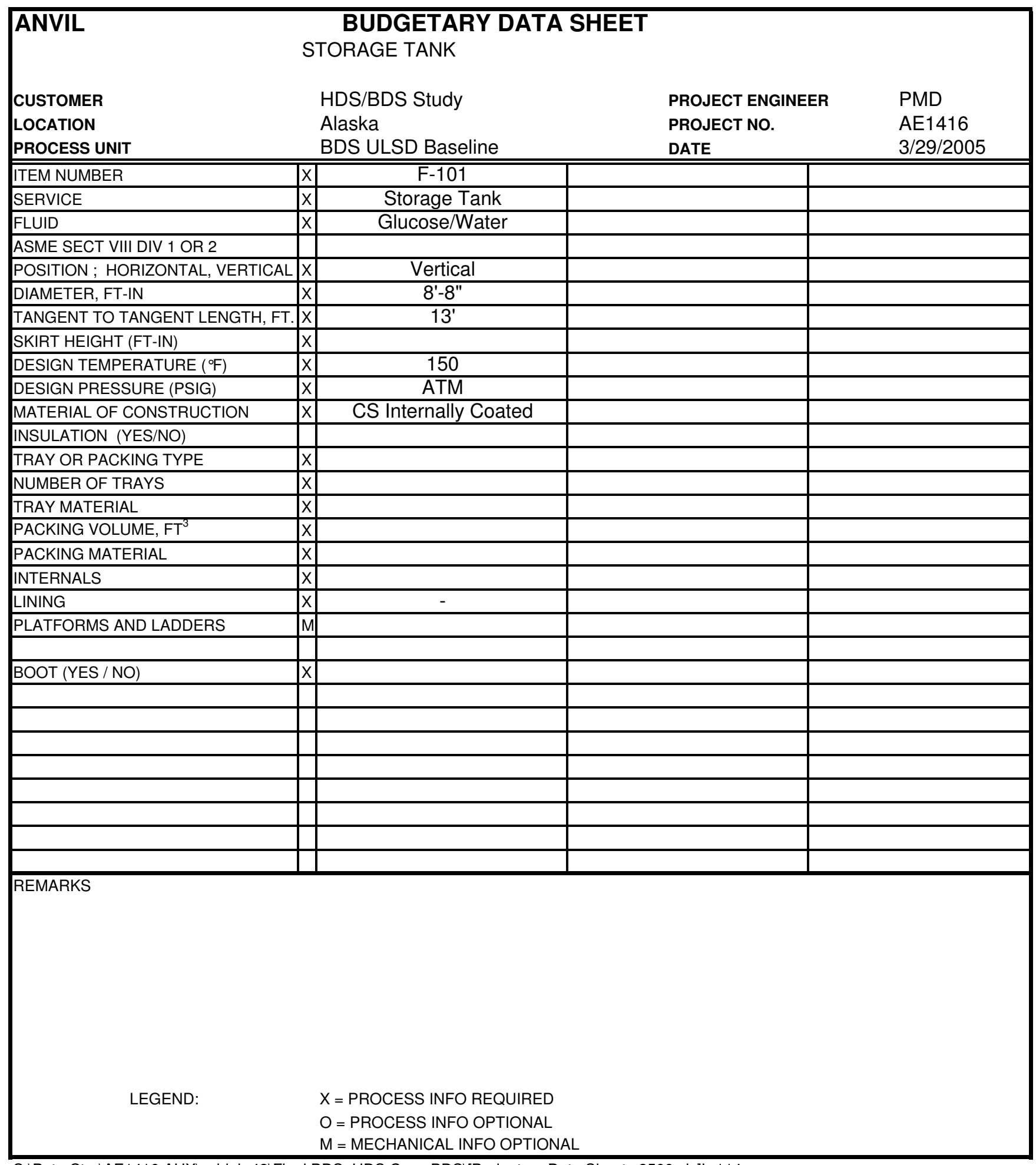




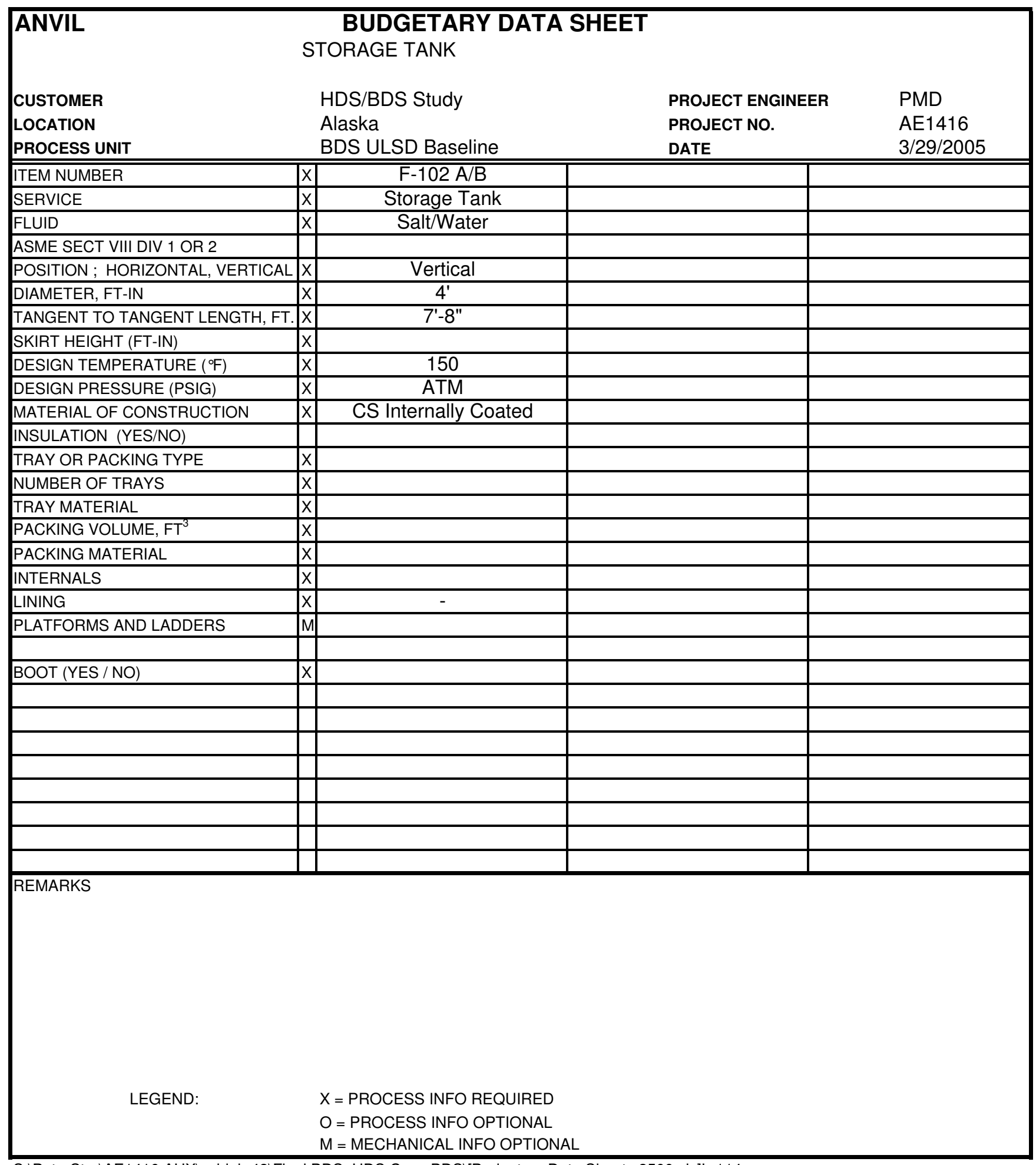




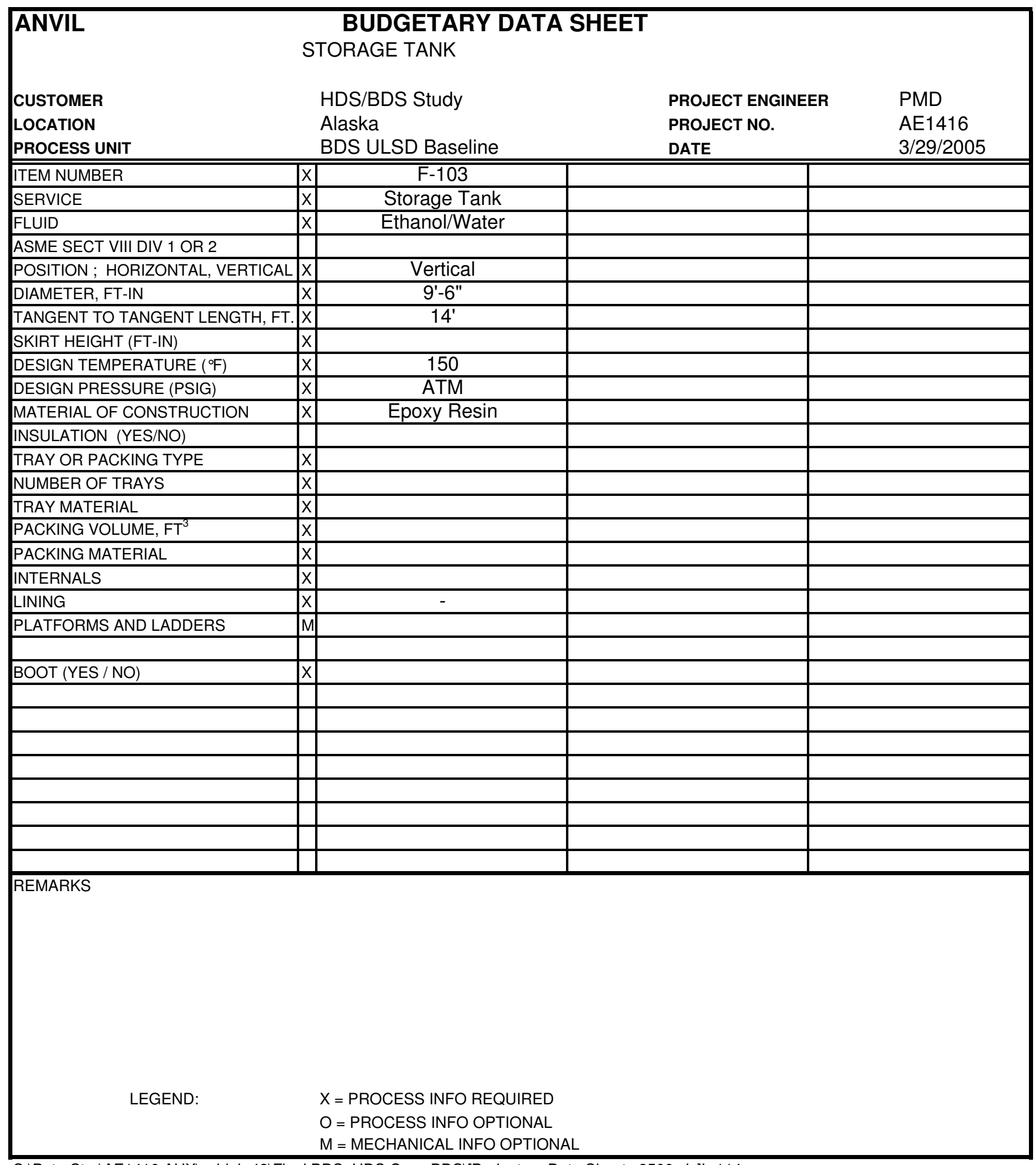




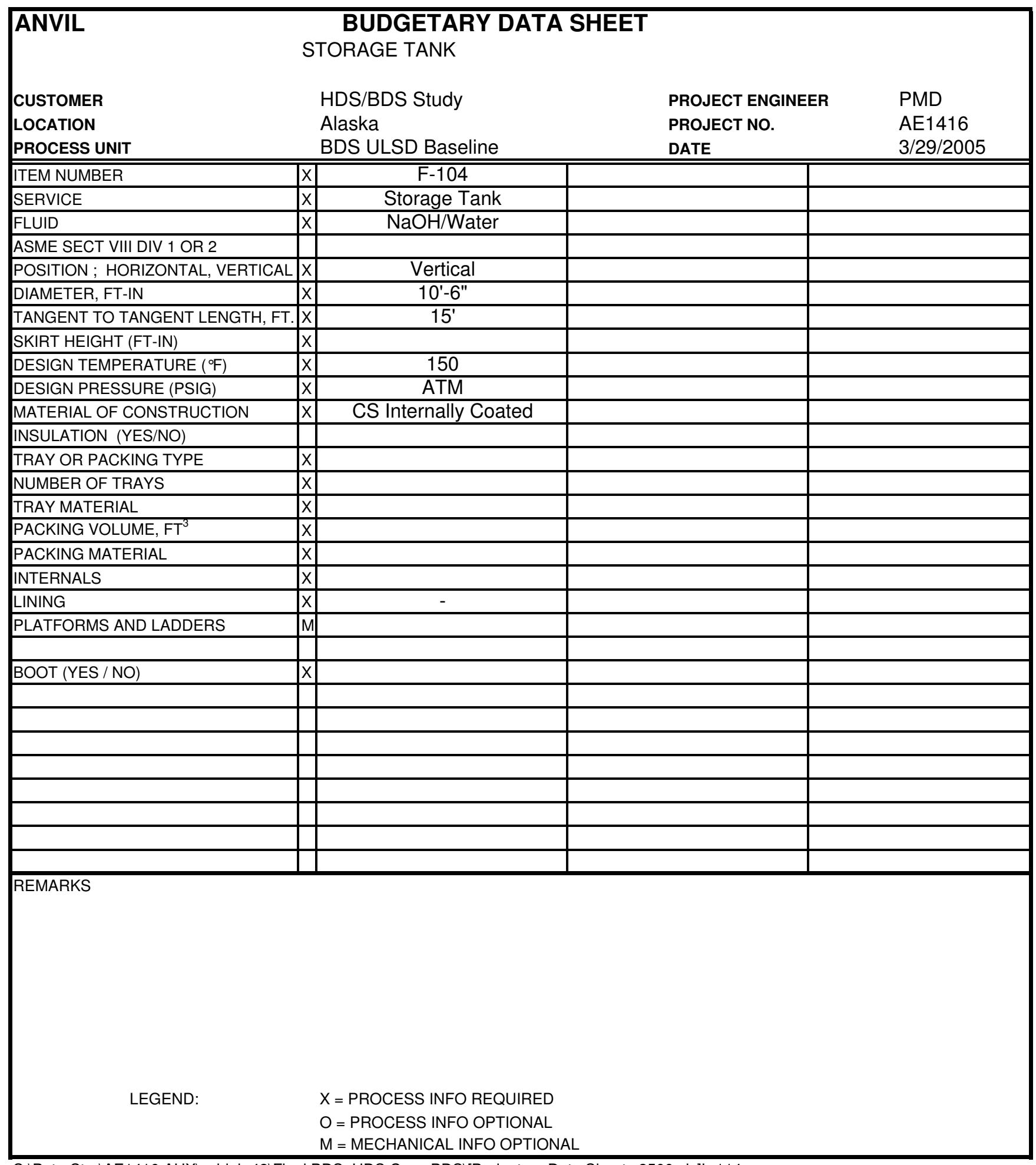




\begin{tabular}{|c|c|c|c|c|}
\hline ANVIL & & $\begin{array}{l}\text { UDGETARY DAT } \\
\text { PUMPS AND DRIV }\end{array}$ & $\begin{array}{l}\text { A SHEET } \\
\text { ERS }\end{array}$ & \\
\hline CUSTOMER & & HDS/BDS Study & & PROJECT ENGINEER PMD \\
\hline LOCATION & & Alaska & & PROJECT NO. AE1416 \\
\hline PROCESS UNIT & & BDS ULSD Baseline & & DATE 3/17/05 \\
\hline ITEM NUMBER & $\mathrm{x}$ & J-101 A/B (1) & & \\
\hline SERVICE (FLUID) & $\mathrm{x}$ & Diesel Feed Pump & & \\
\hline TEMPERATURE OF FLUID & $\mathrm{x}$ & 140 & & \\
\hline SPECIFIC GRAVITY AT TEMPERATURE & $\mathrm{x}$ & 0.85 & & \\
\hline RATED FLOW (GPM) & $\mathrm{x}$ & 190 & & \\
\hline SUCTION PRESSURE, PSIG & $\mathrm{x}$ & 1.2 & & \\
\hline DISCHARGE PRESSURE PSIG & $\mathrm{x}$ & 61.3 & & \\
\hline NPSH AVAILABLE ( FT ) & $\mathrm{x}$ & & & \\
\hline CONSTRUCTION (API,ANSI) & $\mathrm{M}$ & & & \\
\hline PUMP TYPE (CENTRIFUGAL, RECIP,ETC.) & $\mathrm{x}$ & Centrifugal & & \\
\hline CASING MATERIAL & $\mathrm{M}$ & CS & & \\
\hline IMPELLER MATERIAL & $\mathrm{M}$ & $\mathrm{CS}$ & & \\
\hline TYPE OF DRIVER (MOTOR/TURBINE) & $\mathrm{x}$ & Motor & & \\
\hline TYPE OF SPARE (MOTOR/TURBINE) & $\mathrm{x}$ & Motor & & \\
\hline ELECTRIC POWER (HP) & $\mathrm{x}$ & 15 & & \\
\hline STEAM CONDITIONS & $\mathrm{x}$ & $\mathrm{N} / \mathrm{A}$ & & \\
\hline SEALS (SINGLE, DOUBLE, TANDEM) & $\mathrm{M}$ & & & \\
\hline API SEAL FLUSH PLAN NUMBER & $\mathrm{M}$ & & & \\
\hline & & API Standard & & \\
\hline DIFFERENTIAL PRESSURE, PSI & & 60.1 & & \\
\hline DIFFERENTIAL HEAD, FT & & 163.1 & & \\
\hline & & & & \\
\hline & & & & \\
\hline & & & & \\
\hline & & & & \\
\hline $\begin{array}{l}\text { REMARKS } \\
\text { 1) One operating pump + one spare } \\
\text { 2) } 0.125 " \mathrm{CA} / \text { Minimum design temp }\end{array}$ & atur & re is $-20^{\circ} \mathrm{F}$. & & \\
\hline LEGEND: & & $\begin{array}{l}X=P R O C E S S \text { INFO REQ } \\
O=\text { PROCESS INFO OPT } \\
M=\text { MECHANICAL INFO }\end{array}$ & $\begin{array}{l}\text { IRED } \\
\text { ONAL } \\
\text { DTIONAL }\end{array}$ & \\
\hline
\end{tabular}

S:IPetroStar\AE1416.AUXIsubjob 43\Final BDS_HDS Case BDS\[Budgetary Data Sheets 3500.xls]L-114 


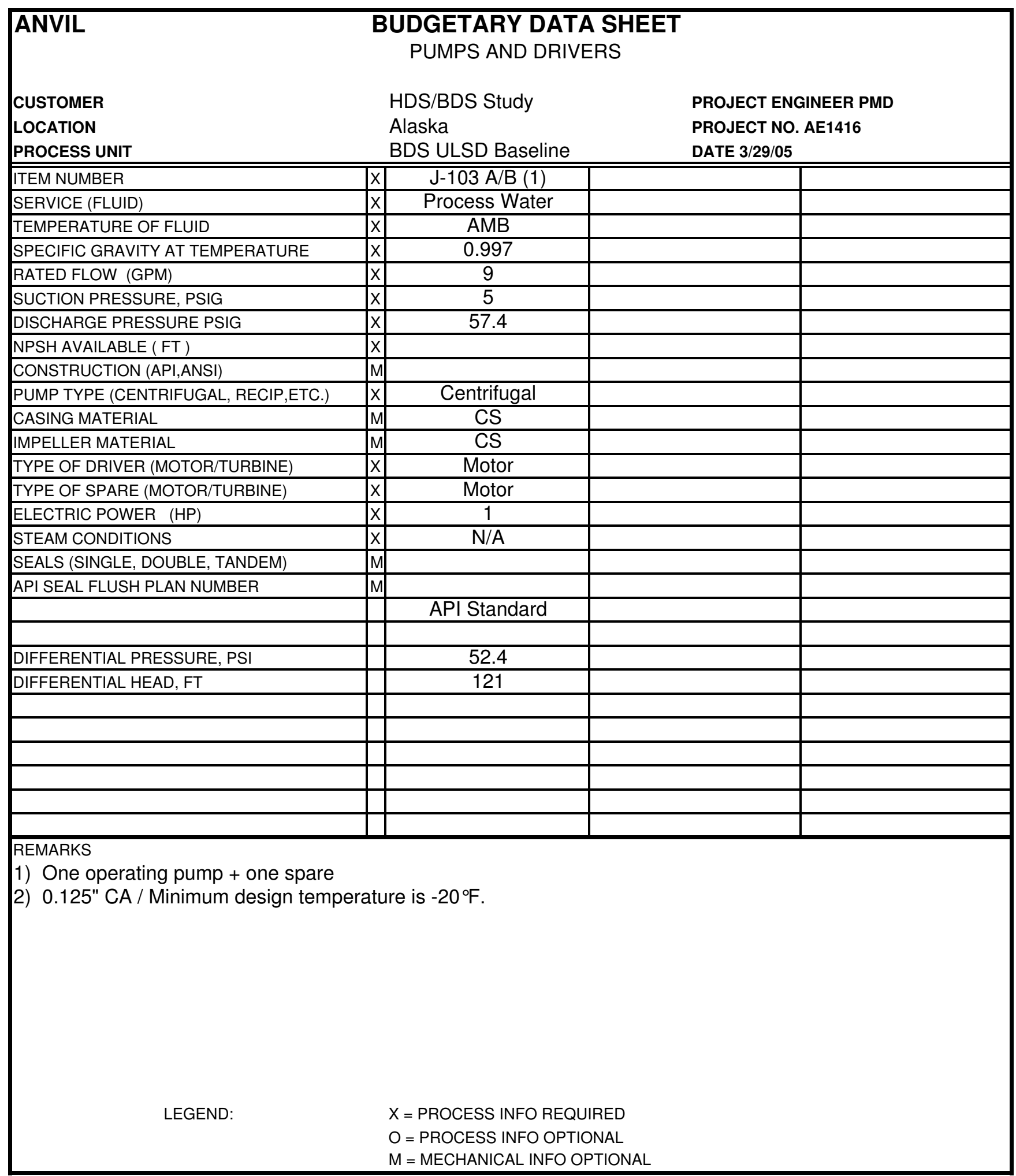

S:IPetroStar\AE1416.AUXIsubjob 43\Final BDS_HDS Case BDS\[Budgetary Data Sheets 3500.xls]L-114 


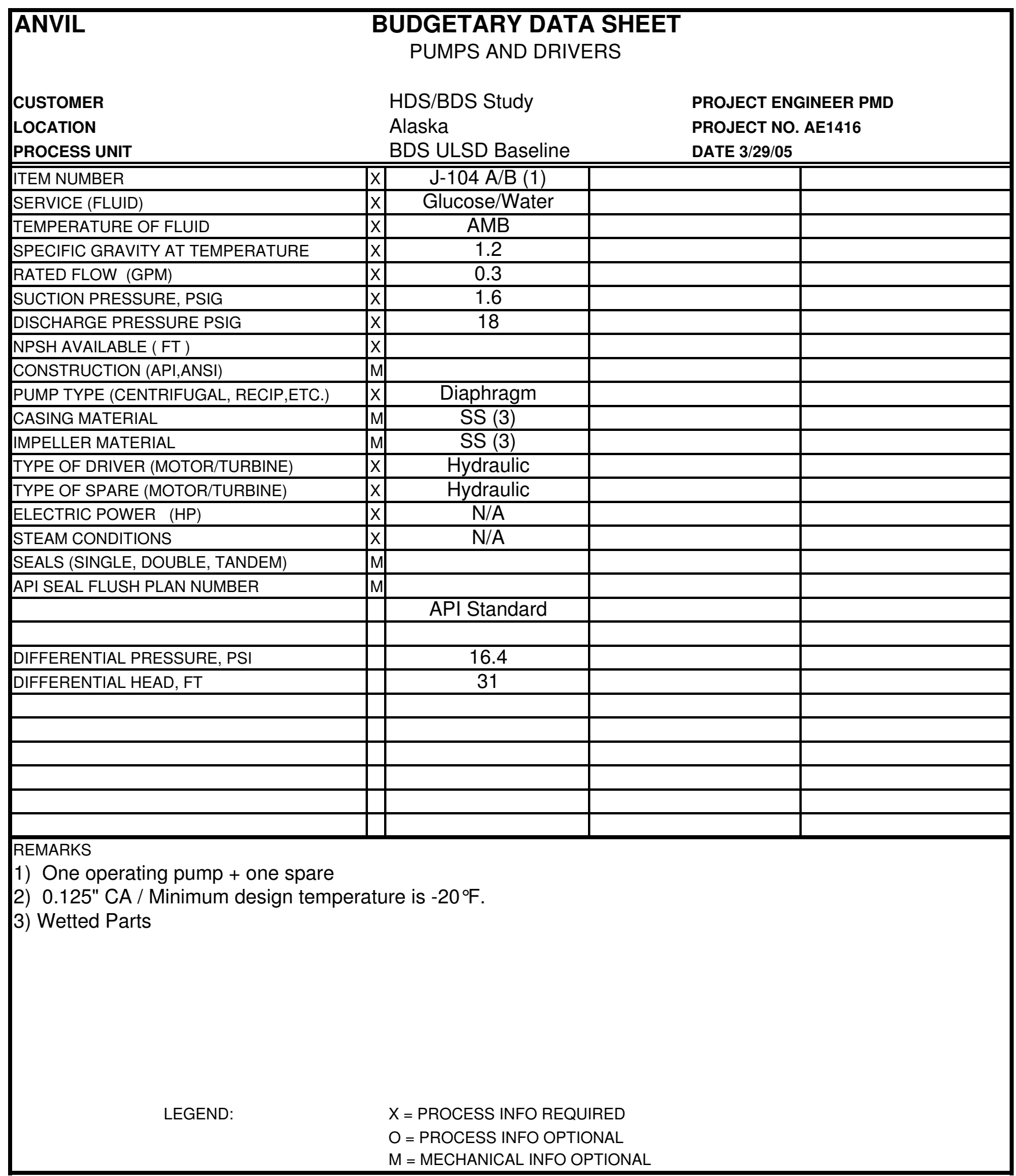

S:IPetroStar\AE1416.AUXIsubjob 43\Final BDS_HDS Case BDS\[Budgetary Data Sheets 3500.xls]L-114 


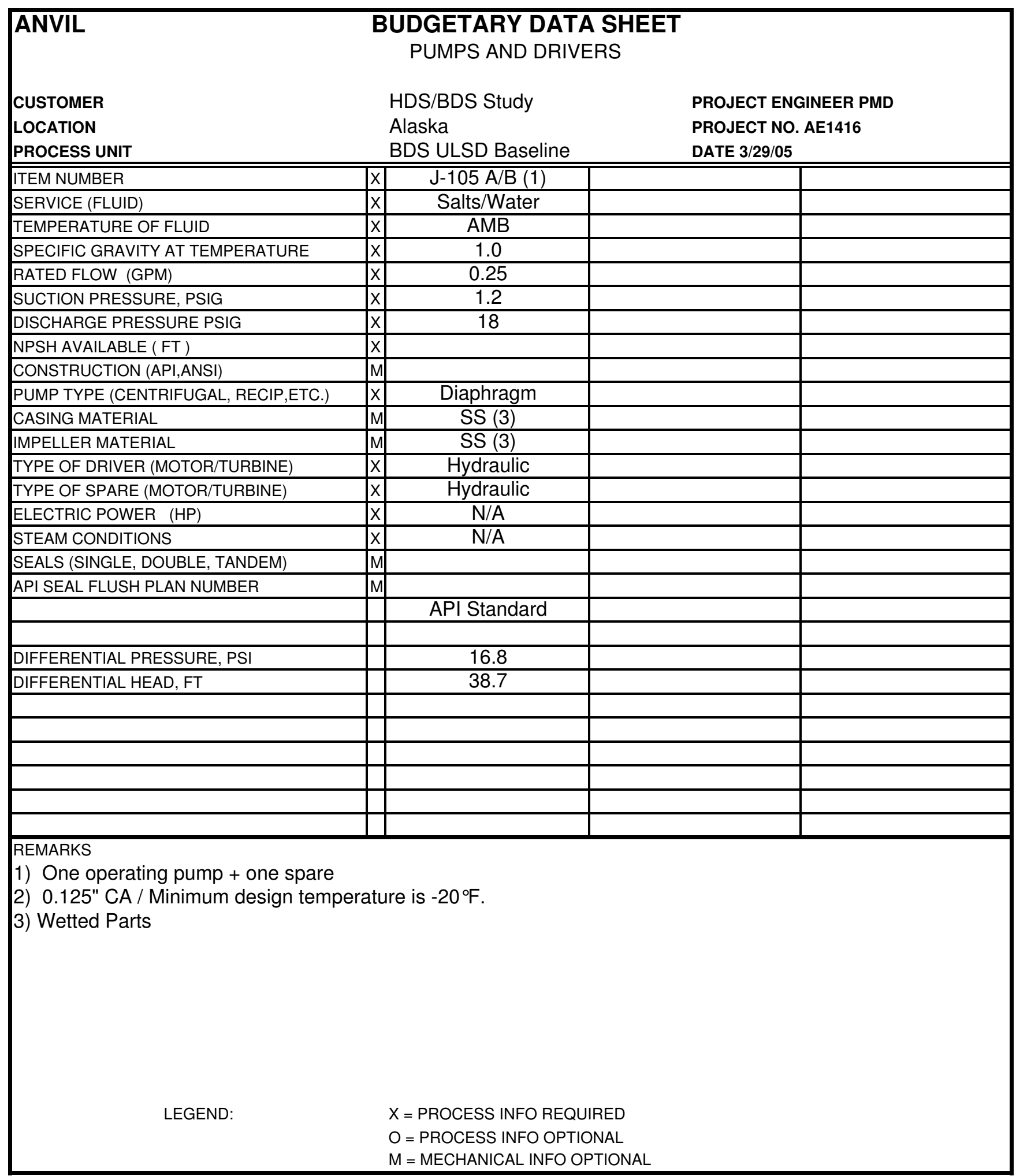

S:IPetroStar\AE1416.AUXIsubjob 43\Final BDS_HDS Case BDS\[Budgetary Data Sheets 3500.xls]L-114 


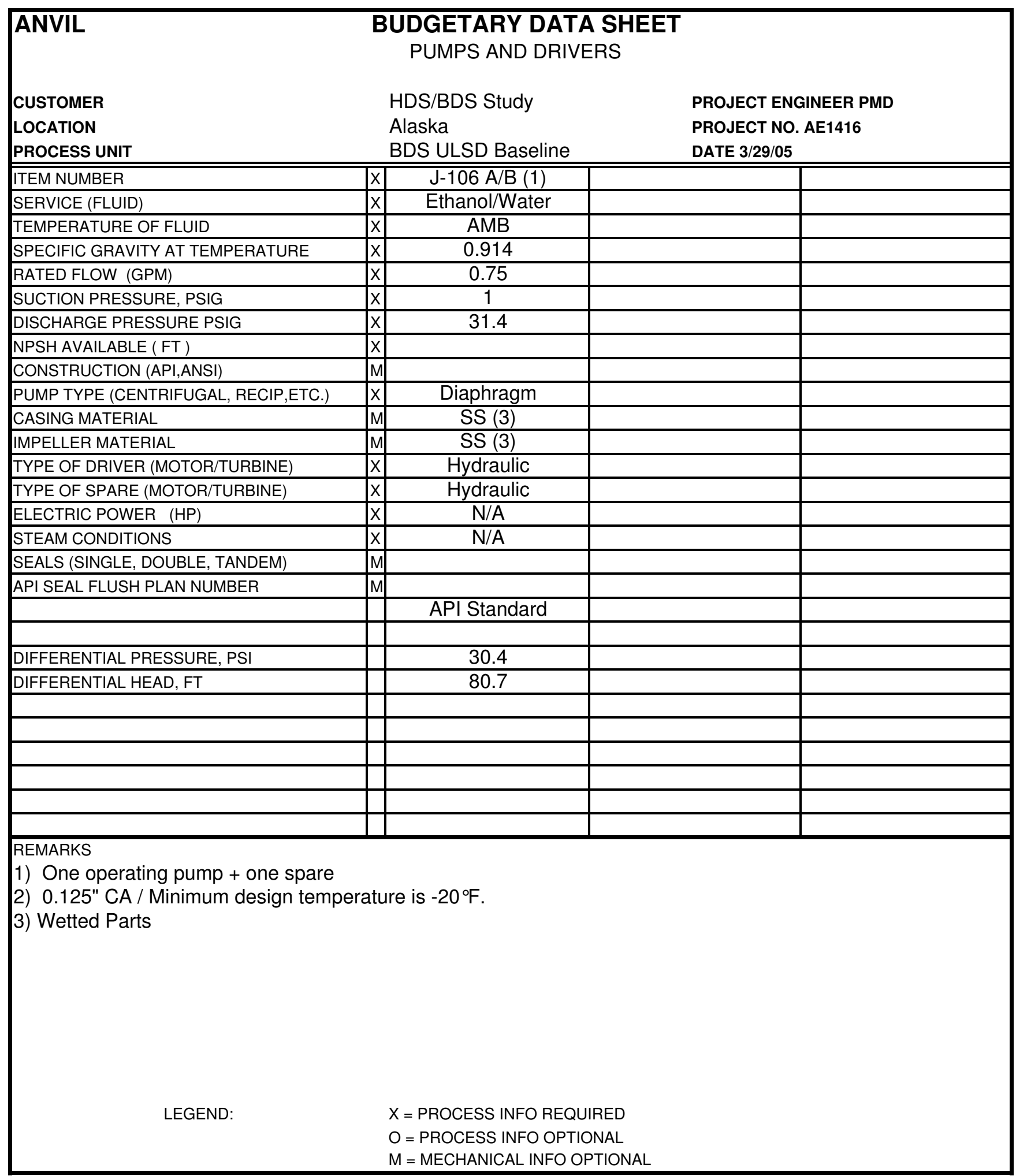

S:IPetroStar\AE1416.AUXIsubjob 43\Final BDS_HDS Case BDS\[Budgetary Data Sheets 3500.xls]L-114 


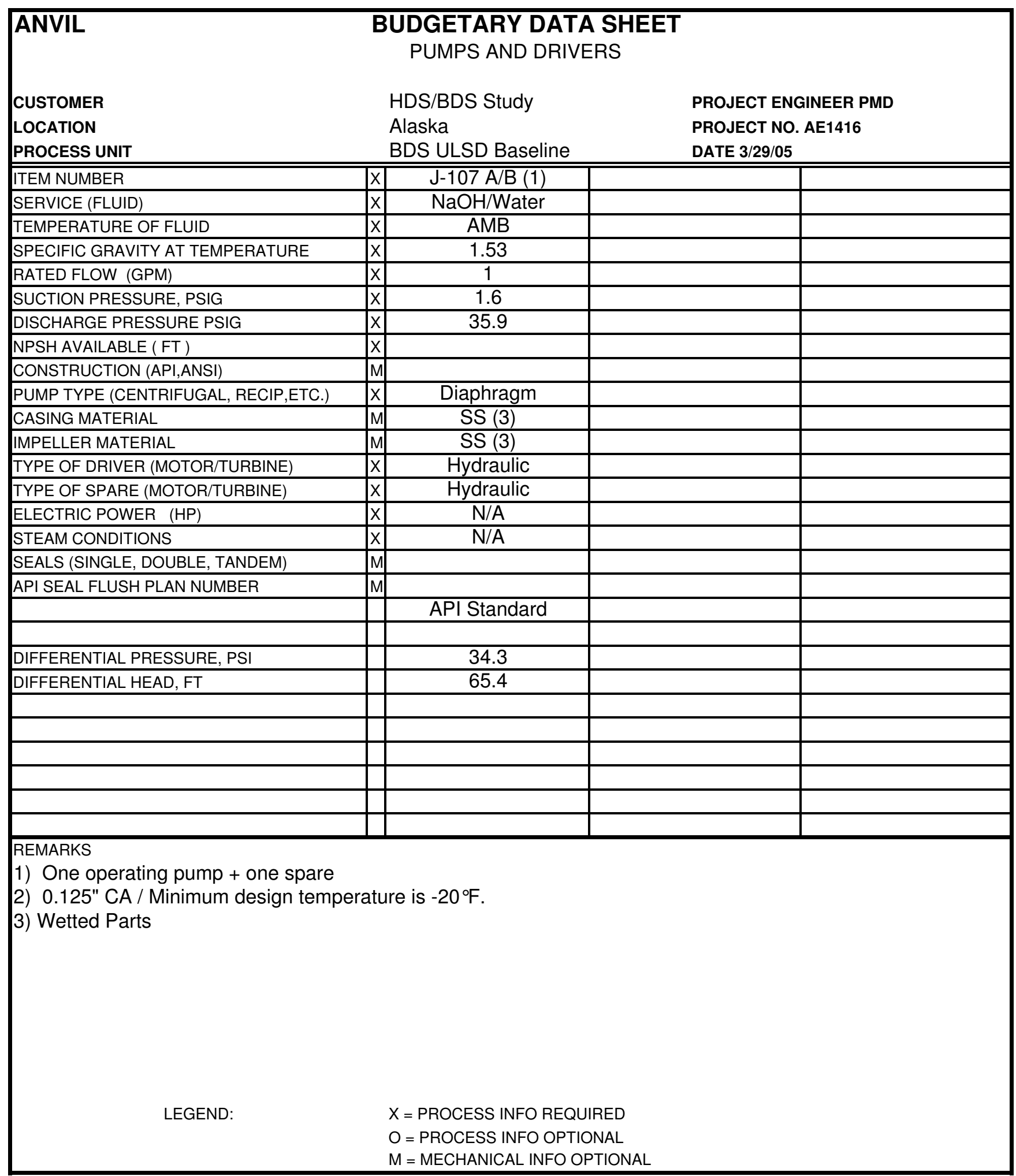

S:IPetroStar\AE1416.AUXIsubjob 43\Final BDS_HDS Case BDS\[Budgetary Data Sheets 3500.xls]L-114 


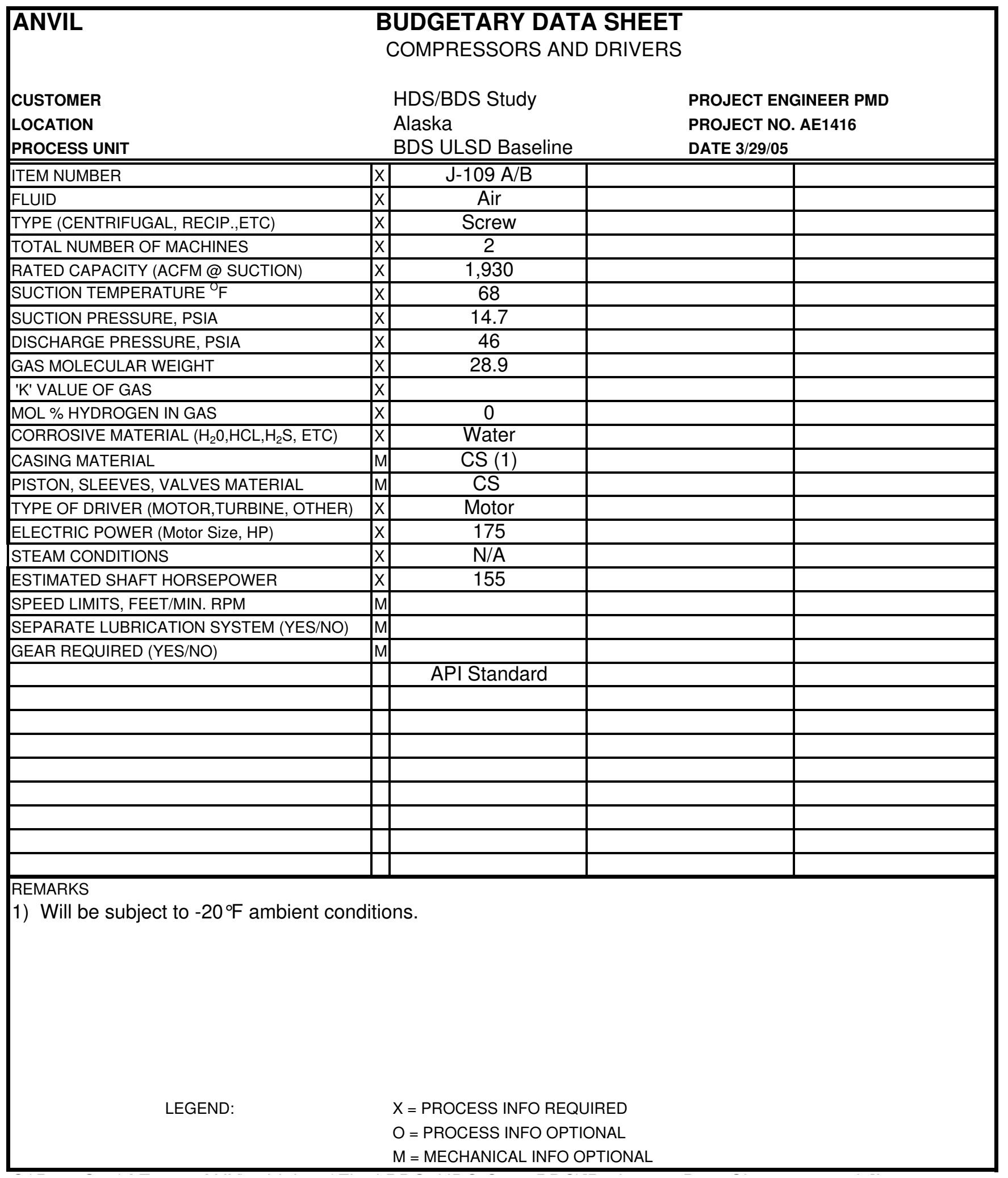




\begin{tabular}{|c|c|c|c|c|}
\hline ANVIL & & $\begin{array}{l}\text { UDGETARY DATA } \\
\text { PUMPS AND DRIVE }\end{array}$ & $\begin{array}{l}\text { SHEET } \\
\text { RS }\end{array}$ & \\
\hline CUSTOMER & & HDS/BDS Study & & PROJECT ENGINEER PMD \\
\hline LOCATION & & Alaska & & PROJECT NO. AE1416 \\
\hline PROCESS UNIT & & BDS ULSD Baseline & & DATE 3/29/05 \\
\hline ITEM NUMBER & $\mathrm{x}$ & $\mathrm{J}-201 \mathrm{~A} / \mathrm{B}(1)$ & & \\
\hline SERVICE (FLUID) & $x$ & Biomass/Salts/Water & & \\
\hline TEMPERATURE OF FLUID & $x$ & 86 & & \\
\hline SPECIFIC GRAVITY AT TEMPERATURE & $x$ & 1.0 & & \\
\hline RATED FLOW (GPM) & $x$ & 16.5 & & \\
\hline SUCTION PRESSURE, PSIG & $x$ & 14.1 & & \\
\hline DISCHARGE PRESSURE PSIG & $\mathrm{x}$ & 33.1 & & \\
\hline NPSH AVAILABLE ( FT ) & $\mathrm{x}$ & & & \\
\hline CONSTRUCTION (API,ANSI) & $\mathrm{M}$ & & & \\
\hline PUMP TYPE (CENTRIFUGAL, RECIP,ETC.) & $\mathrm{x}$ & Rotary Lobe & & \\
\hline CASING MATERIAL & $\mathrm{M}$ & SS (3) & & \\
\hline IMPELLER MATERIAL & $\mathrm{M}$ & SS (3) & & \\
\hline TYPE OF DRIVER (MOTOR/TURBINE) & $x$ & Motor & & \\
\hline TYPE OF SPARE (MOTOR/TURBINE) & $x$ & Motor & & \\
\hline ELECTRIC POWER (HP) & $x$ & 0.5 & & \\
\hline STEAM CONDITIONS & $x$ & $\mathrm{~N} / \mathrm{A}$ & & \\
\hline SEALS (SINGLE, DOUBLE, TANDEM) & $\mathrm{M}$ & Double & & \\
\hline API SEAL FLUSH PLAN NUMBER & $\mathrm{M}$ & & & \\
\hline & & API Standard & & \\
\hline DIFFERENTIAL PRESSURE, PSI & & 19 & & \\
\hline DIFFERENTIAL HEAD, FT & & 43.4 & & \\
\hline & & & & \\
\hline & & & & \\
\hline & & & & \\
\hline & & & & \\
\hline $\begin{array}{l}\text { REMARKS } \\
\text { 1) One operating pump + one spare } \\
\text { 2) } 0.125^{\prime \prime} \text { CA / Minimum design temp } \\
\text { 3) Wetted Parts }\end{array}$ & & is $-20^{\circ} \mathrm{F}$. & & \\
\hline LEGEND: & & $\begin{array}{l}X=\text { PROCESS INFO REQU } \\
O=\text { PROCESS INFO OPTIC } \\
M=\text { MECHANICAL INFO OF }\end{array}$ & $\begin{array}{l}\text { RED } \\
\text { NAL } \\
\text { TIONAL }\end{array}$ & \\
\hline
\end{tabular}

S:IPetroStar\AE1416.AUXIsubjob 43\Final BDS_HDS Case BDS\[Budgetary Data Sheets 3500.xls]L-114 


\begin{tabular}{|c|c|c|c|c|}
\hline ANVIL & & $\begin{array}{l}\text { UDGETARY DATA } \\
\text { PUMPS AND DRIVE }\end{array}$ & $\begin{array}{l}\text { SHEET } \\
\text { RS }\end{array}$ & \\
\hline CUSTOMER & & HDS/BDS Study & & PROJECT ENGINEER PMD \\
\hline LOCATION & & Alaska & & PROJECT NO. AE1416 \\
\hline PROCESS UNIT & & BDS ULSD Baseline & & DATE 3/29/05 \\
\hline ITEM NUMBER & $\mathrm{x}$ & J-202 A/B (1) & & \\
\hline SERVICE (FLUID) & $x$ & Biomass/Diesel/Water & & \\
\hline TEMPERATURE OF FLUID & $\mathrm{x}$ & 86 & & \\
\hline SPECIFIC GRAVITY AT TEMPERATURE & $\mathrm{x}$ & 0.93 & & \\
\hline RATED FLOW (GPM) & $x$ & 700 & & \\
\hline SUCTION PRESSURE, PSIG & $x$ & 18.4 & & \\
\hline DISCHARGE PRESSURE PSIG & $\mathrm{x}$ & 36.4 & & \\
\hline NPSH AVAILABLE ( FT ) & $\mathrm{x}$ & & & \\
\hline CONSTRUCTION (API,ANSI) & $\mathrm{M}$ & & & \\
\hline PUMP TYPE (CENTRIFUGAL, RECIP,ETC.) & $\mathrm{x}$ & Cent & & \\
\hline CASING MATERIAL & $\mathrm{M}$ & SS & & \\
\hline IMPELLER MATERIAL & $\mathrm{M}$ & SS & & \\
\hline TYPE OF DRIVER (MOTOR/TURBINE) & $\mathrm{x}$ & Motor & & \\
\hline TYPE OF SPARE (MOTOR/TURBINE) & $\mathrm{x}$ & Motor & & \\
\hline ELECTRIC POWER (HP) & $\mathrm{x}$ & 15 & & \\
\hline STEAM CONDITIONS & $\mathrm{x}$ & $\mathrm{N} / \mathrm{A}$ & & \\
\hline SEALS (SINGLE, DOUBLE, TANDEM) & $\mathrm{M}$ & Double & & \\
\hline API SEAL FLUSH PLAN NUMBER & $\mathrm{M}$ & & & \\
\hline & & API Standard & & \\
\hline DIFFERENTIAL PRESSURE, PSI & & 18 & & \\
\hline DIFFERENTIAL HEAD, FT & & 44.7 & & \\
\hline & & & & \\
\hline & & & & \\
\hline & & & & \\
\hline & & & & \\
\hline $\begin{array}{l}\text { REMARKS } \\
\text { 1) One operating pump + one spare } \\
\text { 2) } 0.125 " \mathrm{CA} / \text { Minimum design temp }\end{array}$ & tu & re is $-20^{\circ} \mathrm{F}$. & & \\
\hline LEGEND: & & $\begin{array}{l}X=\text { PROCESS INFO REQUIF } \\
O=\text { PROCESS INFO OPTIOI } \\
M=\text { MECHANICAL INFO OP }\end{array}$ & $\begin{array}{l}\text { RED } \\
\text { NAL } \\
\text { TIONAL }\end{array}$ & \\
\hline
\end{tabular}

S:IPetroStar\AE1416.AUXIsubjob 43\Final BDS_HDS Case BDS\[Budgetary Data Sheets 3500.xls]L-114 


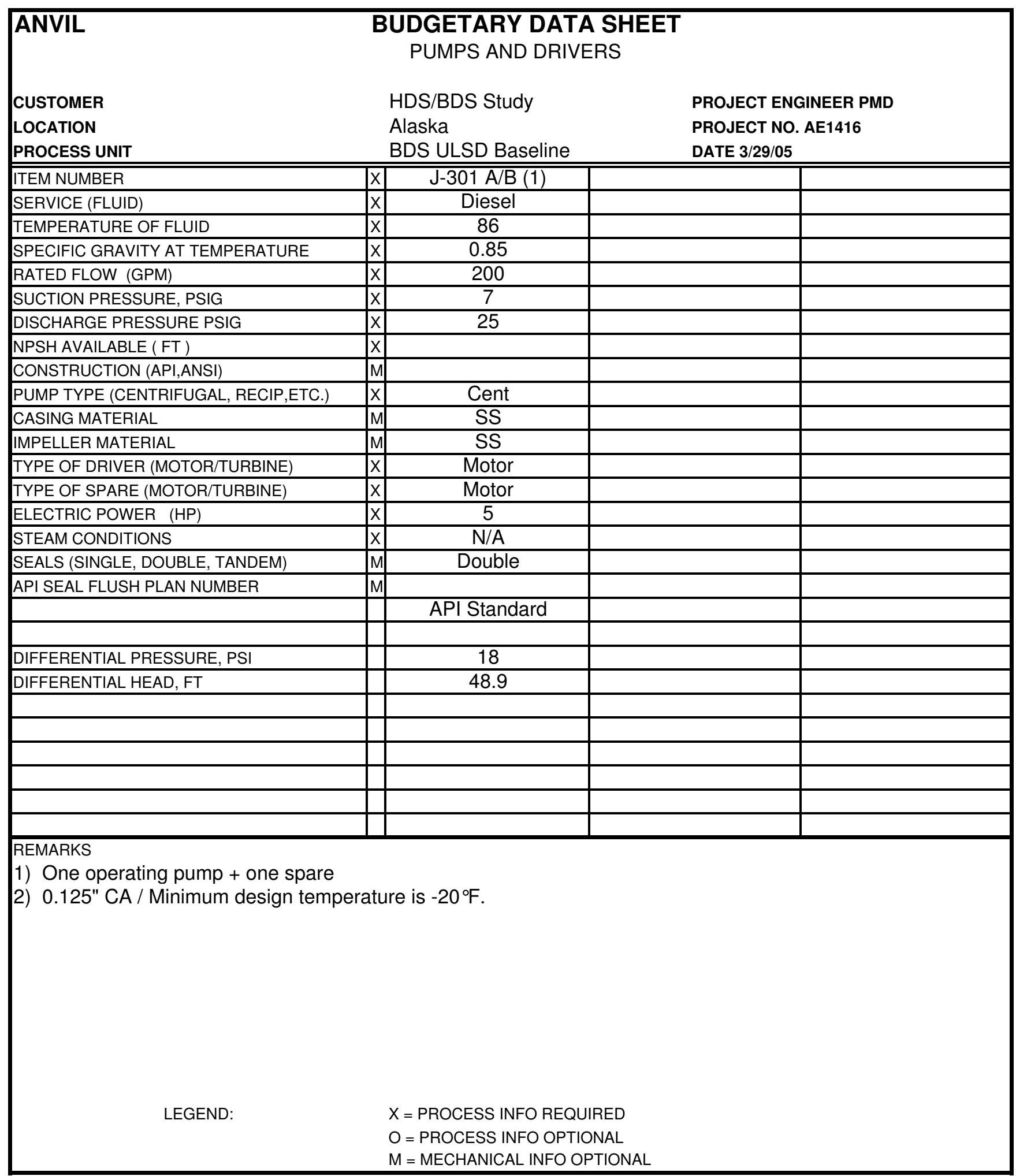

S:IPetroStar\AE1416.AUXIsubjob 43\Final BDS_HDS Case BDS\[Budgetary Data Sheets 3500.xls]L-114 


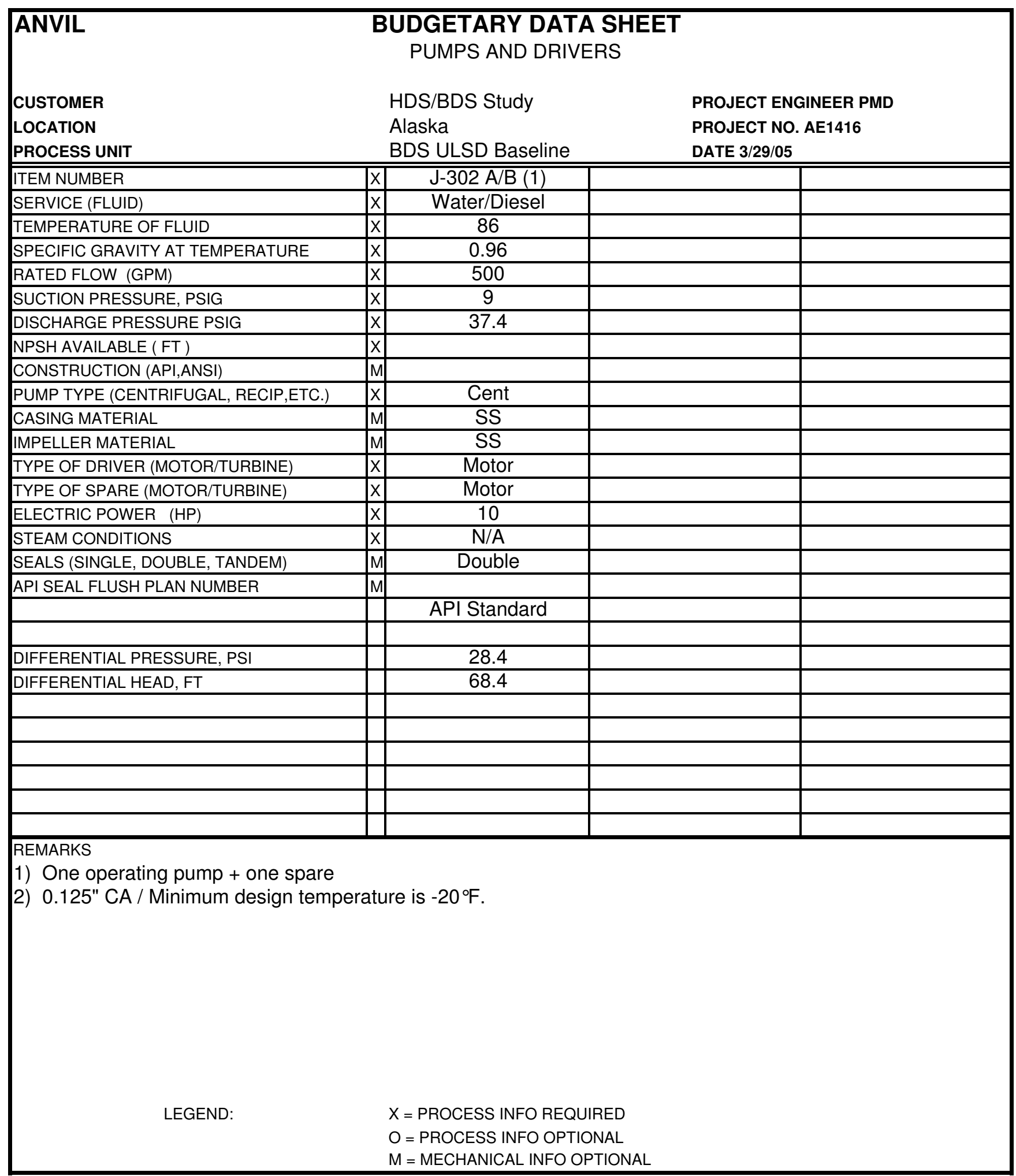

S:IPetroStar\AE1416.AUXIsubjob 43\Final BDS_HDS Case BDS\[Budgetary Data Sheets 3500.xls]L-114 


\begin{tabular}{|c|c|c|c|c|}
\hline ANVIL & & $\begin{array}{l}\text { UDGETARY DATA } \\
\text { PUMPS AND DRIVE }\end{array}$ & $\begin{array}{l}\text { SHEET } \\
\text { RS }\end{array}$ & \\
\hline CUSTOMER & & HDS/BDS Study & & PROJECT ENGINEER PMD \\
\hline LOCATION & & Alaska & & PROJECT NO. AE1416 \\
\hline PROCESS UNIT & & BDS ULSD Baseline & & DATE 3/29/05 \\
\hline ITEM NUMBER & $\mathrm{x}$ & J-303 A/B (1) & & \\
\hline SERVICE (FLUID) & $x$ & Water/Diesel/Biomass & & \\
\hline TEMPERATURE OF FLUID & $x$ & 86 & & \\
\hline SPECIFIC GRAVITY AT TEMPERATURE & $x$ & 0.94 & & \\
\hline RATED FLOW (GPM) & $x$ & 145 & & \\
\hline SUCTION PRESSURE, PSIG & $x$ & 7.7 & & \\
\hline DISCHARGE PRESSURE PSIG & $\mathrm{x}$ & 37.4 & & \\
\hline NPSH AVAILABLE ( FT ) & $\mathrm{x}$ & & & \\
\hline CONSTRUCTION (API,ANSI) & $\mathrm{M}$ & & & \\
\hline PUMP TYPE (CENTRIFUGAL, RECIP,ETC.) & $\mathrm{x}$ & Cent & & \\
\hline CASING MATERIAL & $\mathrm{M}$ & SS & & \\
\hline IMPELLER MATERIAL & $\mathrm{M}$ & SS & & \\
\hline TYPE OF DRIVER (MOTOR/TURBINE) & $x$ & Motor & & \\
\hline TYPE OF SPARE (MOTOR/TURBINE) & $x$ & Motor & & \\
\hline ELECTRIC POWER (HP) & $x$ & 5 & & \\
\hline STEAM CONDITIONS & $x$ & $\mathrm{~N} / \mathrm{A}$ & & \\
\hline SEALS (SINGLE, DOUBLE, TANDEM) & $M$ & Double & & \\
\hline API SEAL FLUSH PLAN NUMBER & $\mathrm{M}$ & & & \\
\hline & & API Standard & & \\
\hline DIFFERENTIAL PRESSURE, PSI & & 29.7 & & \\
\hline DIFFERENTIAL HEAD, FT & & 72.2 & & \\
\hline & & & & \\
\hline & & & & \\
\hline & & & & \\
\hline & & & & \\
\hline $\begin{array}{l}\text { REMARKS } \\
\text { 1) One operating pump + one spare } \\
\text { 2) } 0.125 " \mathrm{CA} / \text { Minimum design temp }\end{array}$ & atur & re is $-20^{\circ} \mathrm{F}$. & & \\
\hline LEGEND: & & $\begin{array}{l}X=\text { PROCESS INFO REQUI } \\
O=\text { PROCESS INFO OPTIO } \\
M=\text { MECHANICAL INFO OP }\end{array}$ & $\begin{array}{l}\text { RED } \\
\text { NAL } \\
\text { TIONAL }\end{array}$ & \\
\hline
\end{tabular}

S:IPetroStar\AE1416.AUXIsubjob 43\Final BDS_HDS Case BDS\[Budgetary Data Sheets 3500.xls]L-114 


\begin{tabular}{|c|c|c|c|c|}
\hline ANVIL & & $\begin{array}{l}\text { UDGETARY DATA } \\
\text { PUMPS AND DRIVE }\end{array}$ & $\begin{array}{l}\text { SHEET } \\
\text { RS }\end{array}$ & \\
\hline CUSTOMER & & HDS/BDS Study & & PROJECT ENGINEER PMD \\
\hline LOCATION & & Alaska & & PROJECT NO. AE1416 \\
\hline PROCESS UNIT & & BDS ULSD Baseline & & DATE 3/29/05 \\
\hline ITEM NUMBER & $\mathrm{x}$ & J-304 A/B (1) & & \\
\hline SERVICE (FLUID) & $x$ & Water/Diesel/Biomass & & \\
\hline TEMPERATURE OF FLUID & $x$ & 86 & & \\
\hline SPECIFIC GRAVITY AT TEMPERATURE & $x$ & 0.94 & & \\
\hline RATED FLOW (GPM) & $x$ & 45 & & \\
\hline SUCTION PRESSURE, PSIG & $x$ & 7.7 & & \\
\hline DISCHARGE PRESSURE PSIG & $\mathrm{x}$ & 37.4 & & \\
\hline NPSH AVAILABLE ( FT ) & $\mathrm{x}$ & & & \\
\hline CONSTRUCTION (API,ANSI) & $\mathrm{M}$ & & & \\
\hline PUMP TYPE (CENTRIFUGAL, RECIP,ETC.) & $\mathrm{x}$ & Cent & & \\
\hline CASING MATERIAL & $\mathrm{M}$ & SS & & \\
\hline IMPELLER MATERIAL & $\mathrm{M}$ & SS & & \\
\hline TYPE OF DRIVER (MOTOR/TURBINE) & $x$ & Motor & & \\
\hline TYPE OF SPARE (MOTOR/TURBINE) & $x$ & Motor & & \\
\hline ELECTRIC POWER (HP) & $x$ & 3 & & \\
\hline STEAM CONDITIONS & $x$ & $\mathrm{~N} / \mathrm{A}$ & & \\
\hline SEALS (SINGLE, DOUBLE, TANDEM) & $M$ & Double & & \\
\hline API SEAL FLUSH PLAN NUMBER & $\mathrm{M}$ & & & \\
\hline & & API Standard & & \\
\hline DIFFERENTIAL PRESSURE, PSI & & 29.7 & & \\
\hline DIFFERENTIAL HEAD, FT & & 72.2 & & \\
\hline & & & & \\
\hline & & & & \\
\hline & & & & \\
\hline & & & & \\
\hline $\begin{array}{l}\text { REMARKS } \\
\text { 1) One operating pump + one spare } \\
\text { 2) } 0.125 " \mathrm{CA} / \text { Minimum design temp }\end{array}$ & atur & re is $-20^{\circ} \mathrm{F}$. & & \\
\hline LEGEND: & & $\begin{array}{l}X=\text { PROCESS INFO REQUI } \\
O=\text { PROCESS INFO OPTIO } \\
M=\text { MECHANICAL INFO OP }\end{array}$ & $\begin{array}{l}\text { RED } \\
\text { NAL } \\
\text { TIONAL }\end{array}$ & \\
\hline
\end{tabular}

S:IPetroStar\AE1416.AUXIsubjob 43\Final BDS_HDS Case BDS\[Budgetary Data Sheets 3500.xls]L-114 


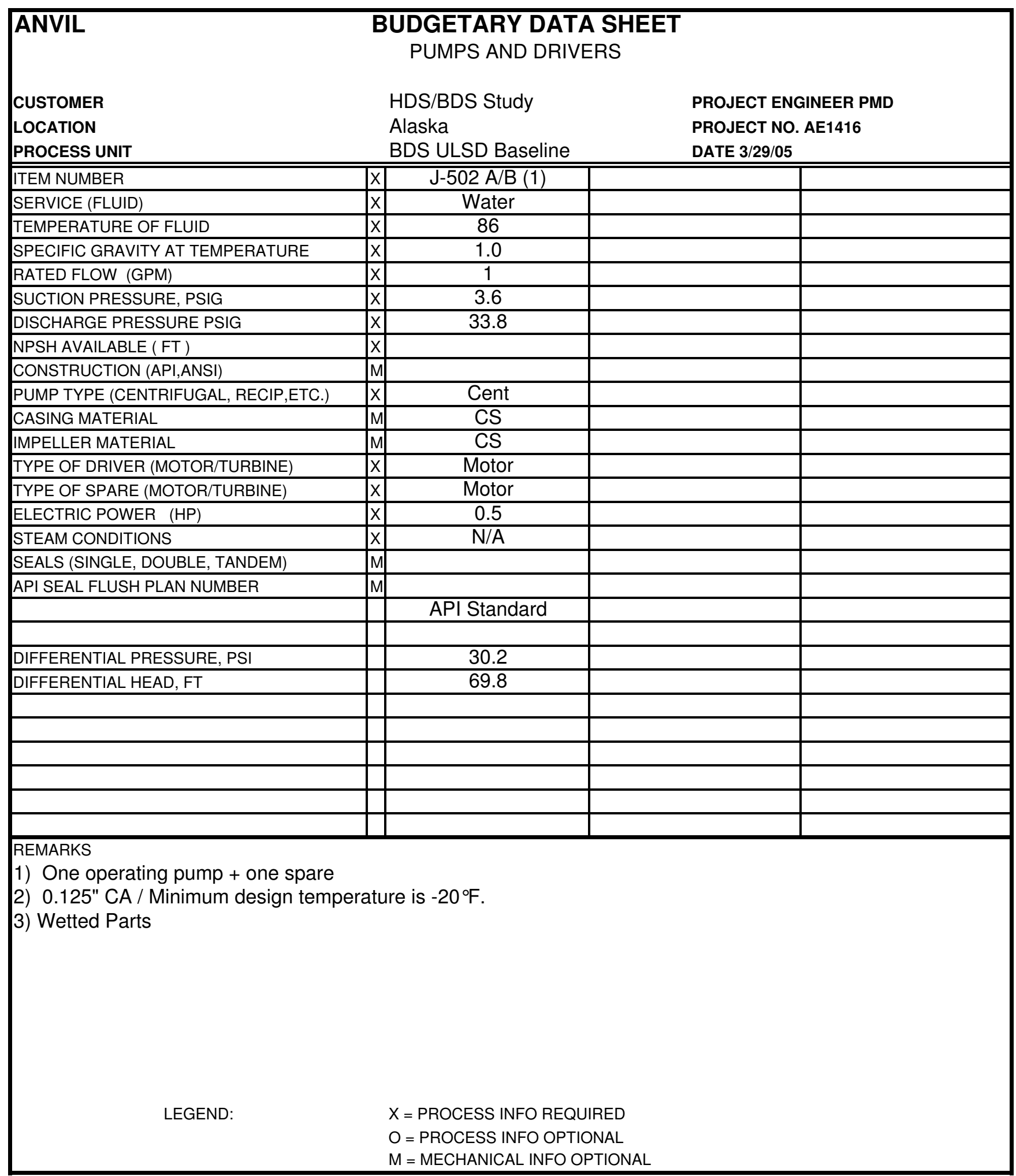

S:IPetroStar\AE1416.AUXIsubjob 43\Final BDS_HDS Case BDS\[Budgetary Data Sheets 3500.xls]L-114 


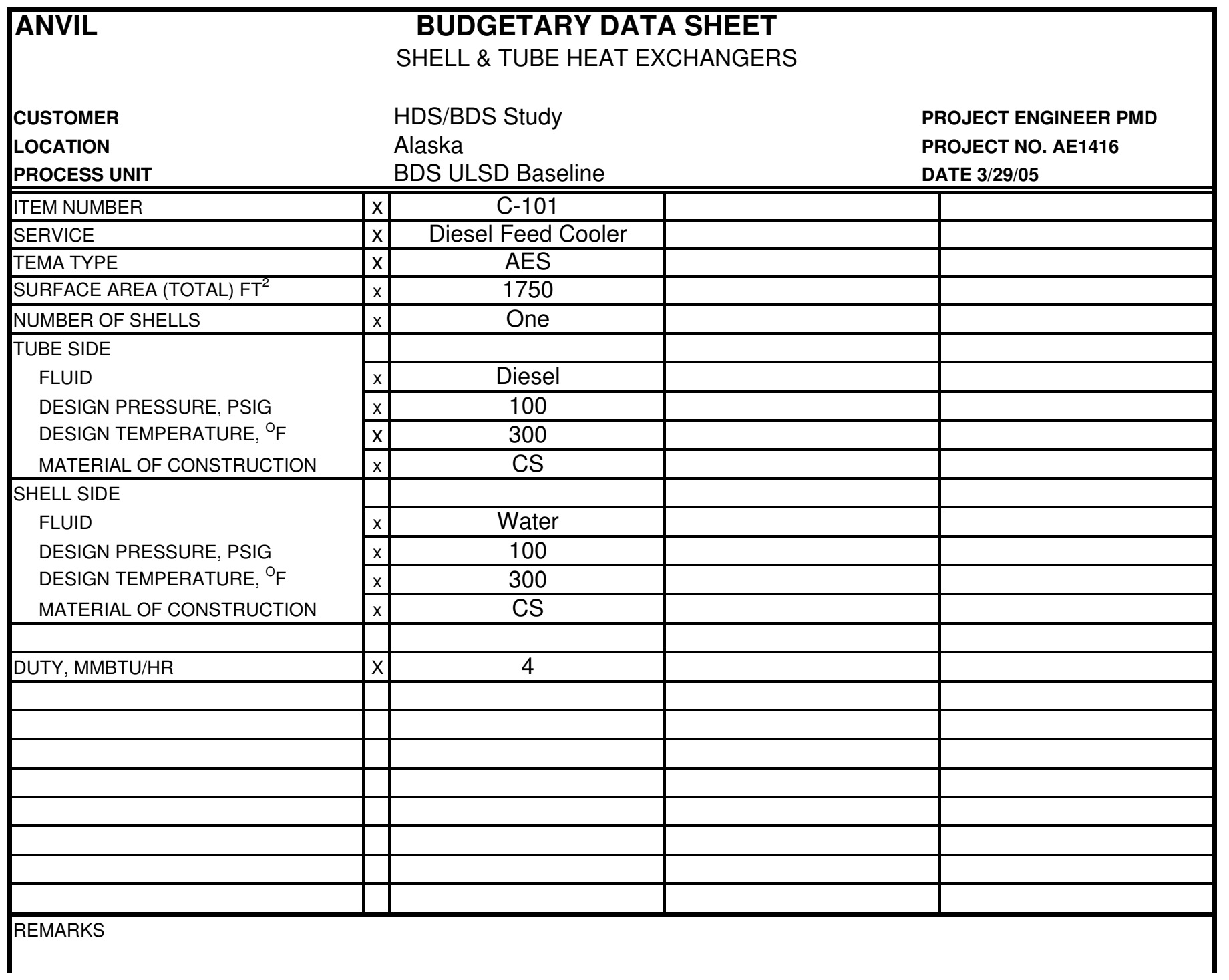




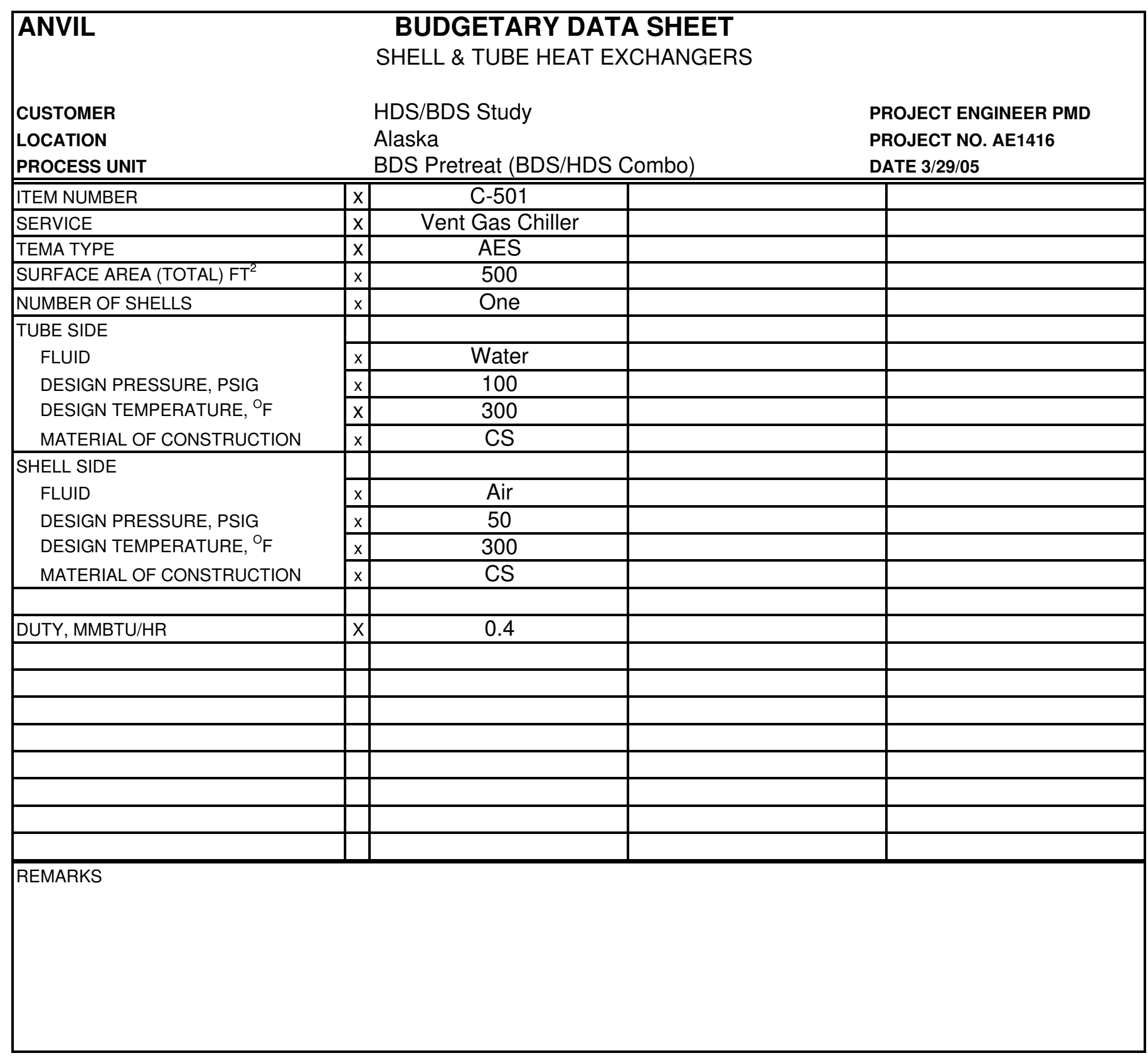




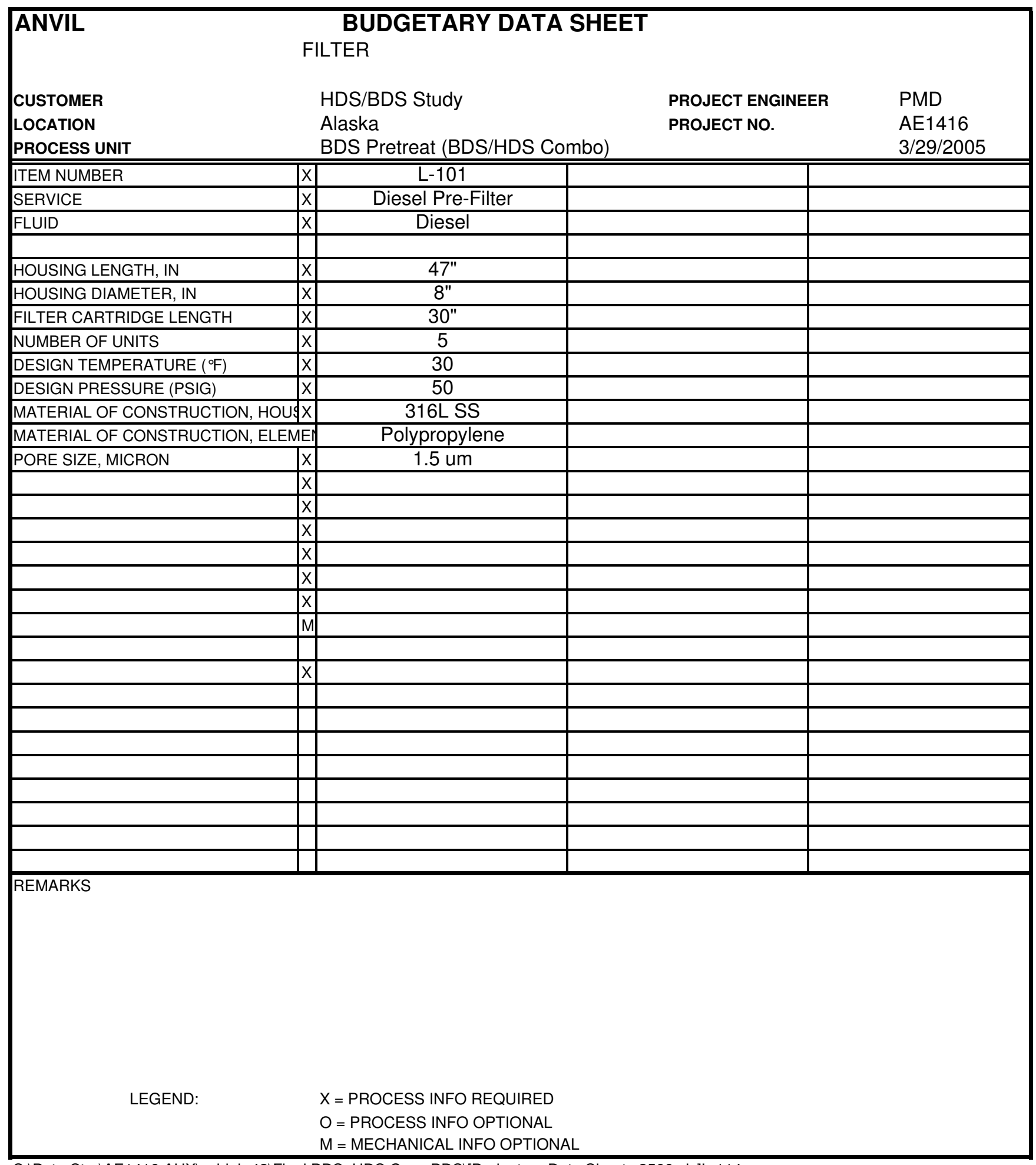




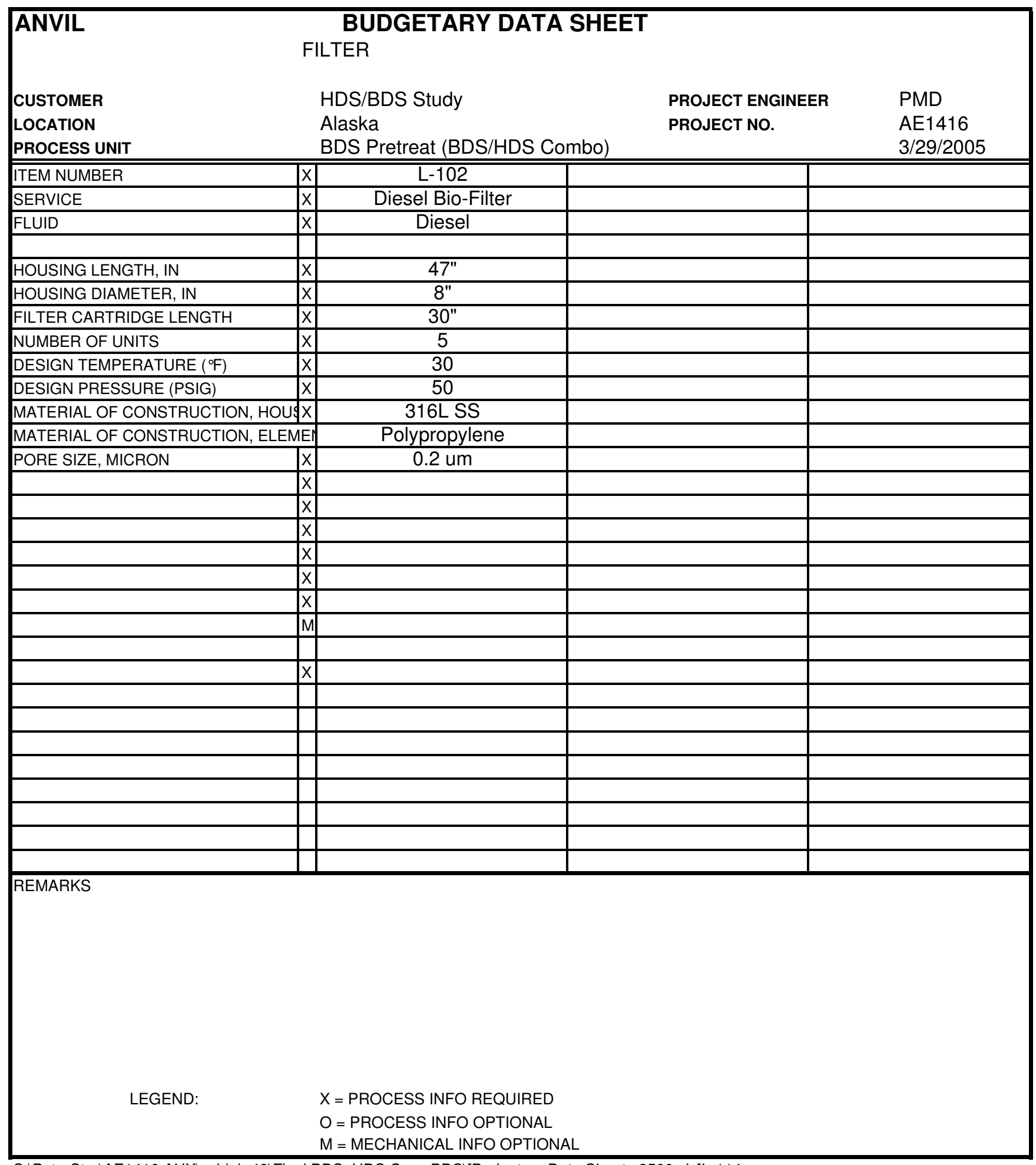




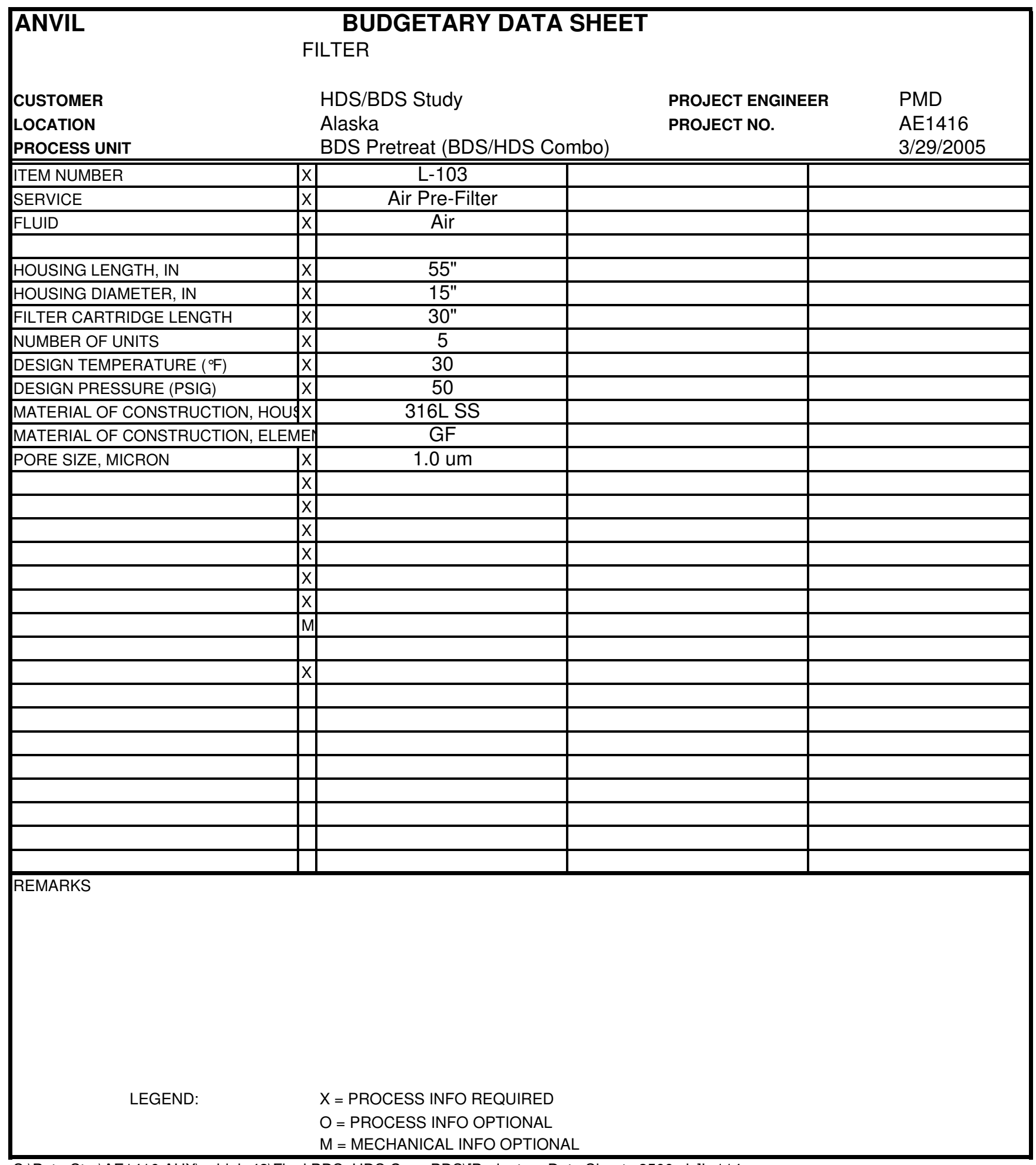




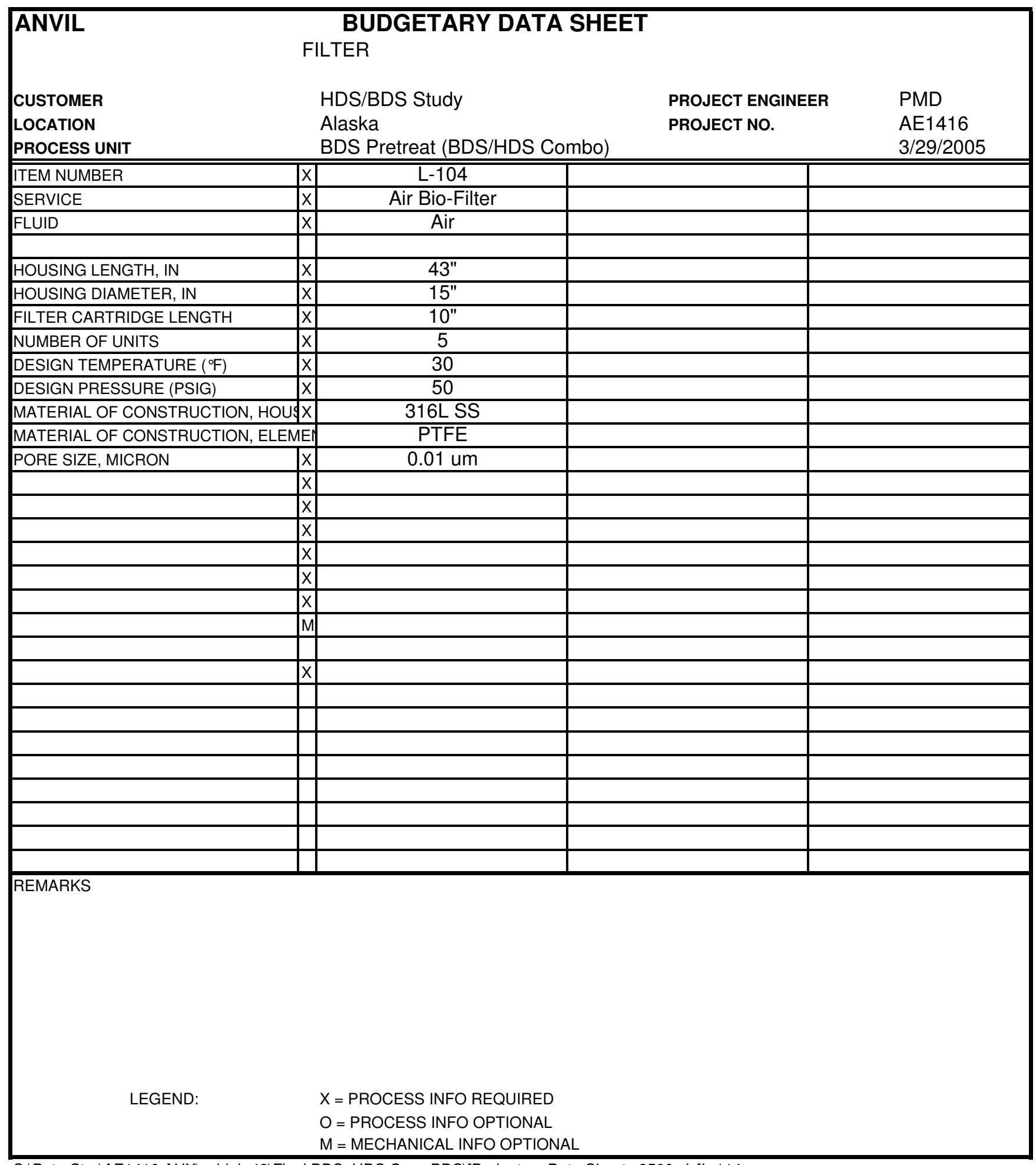




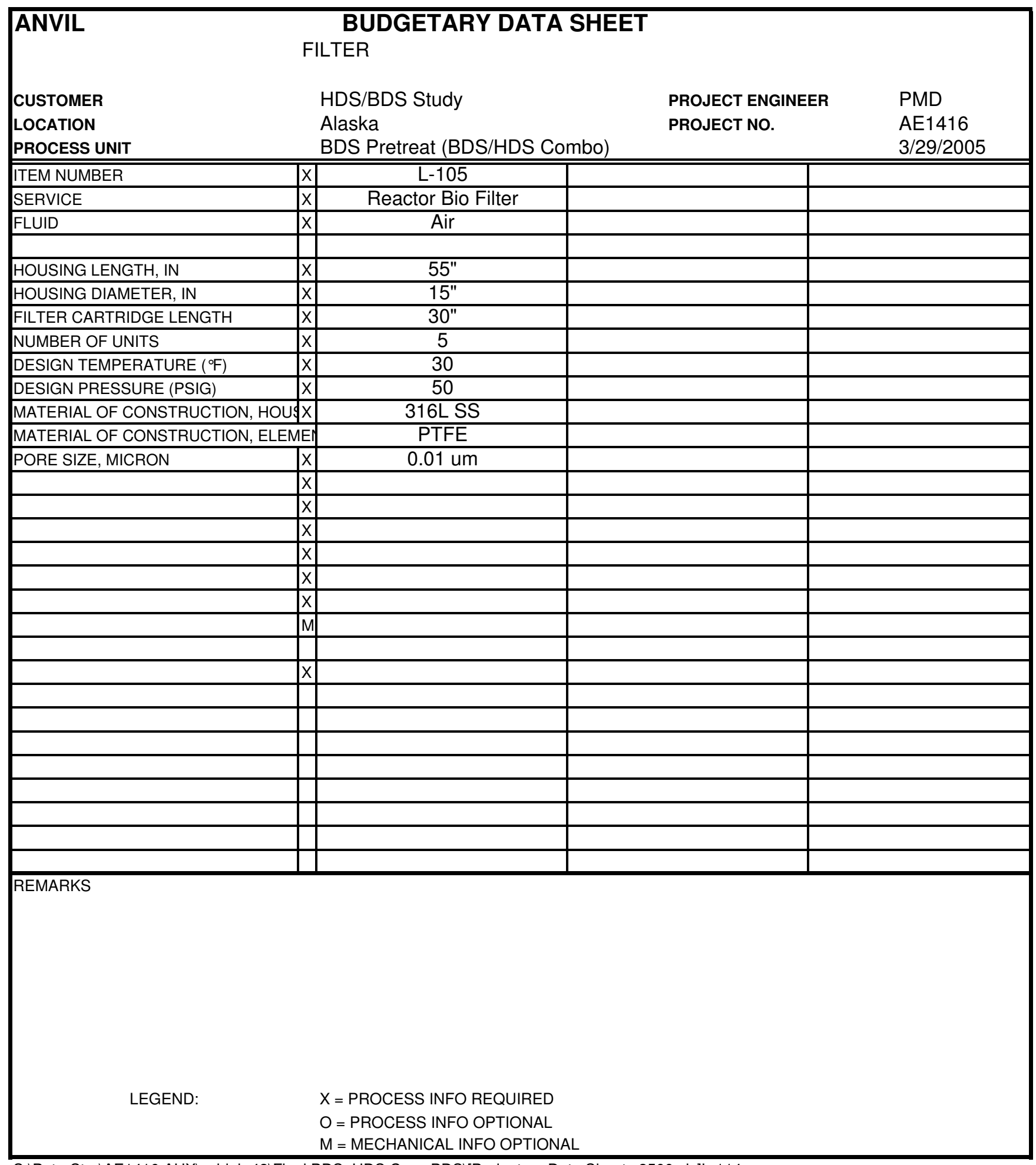




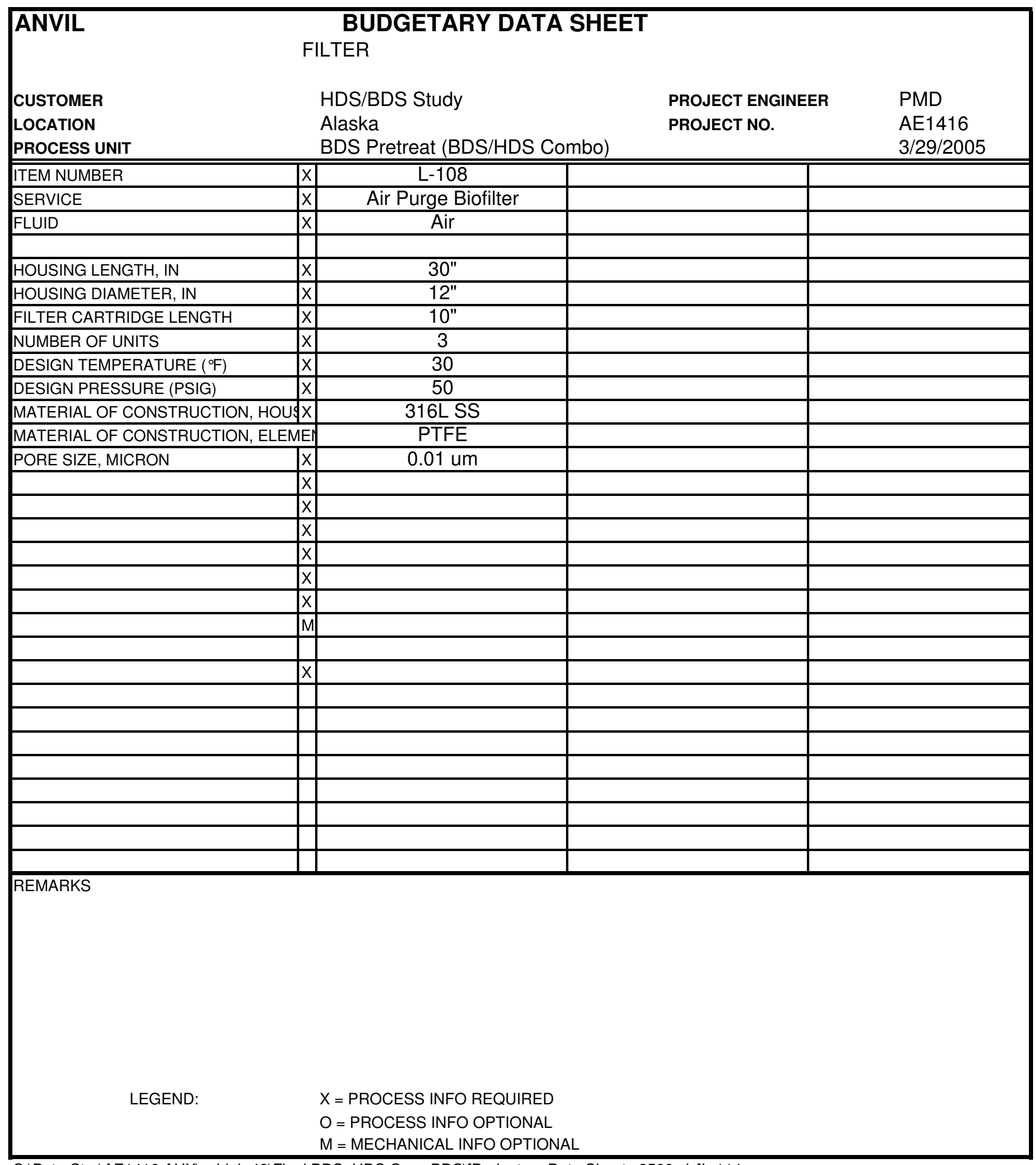




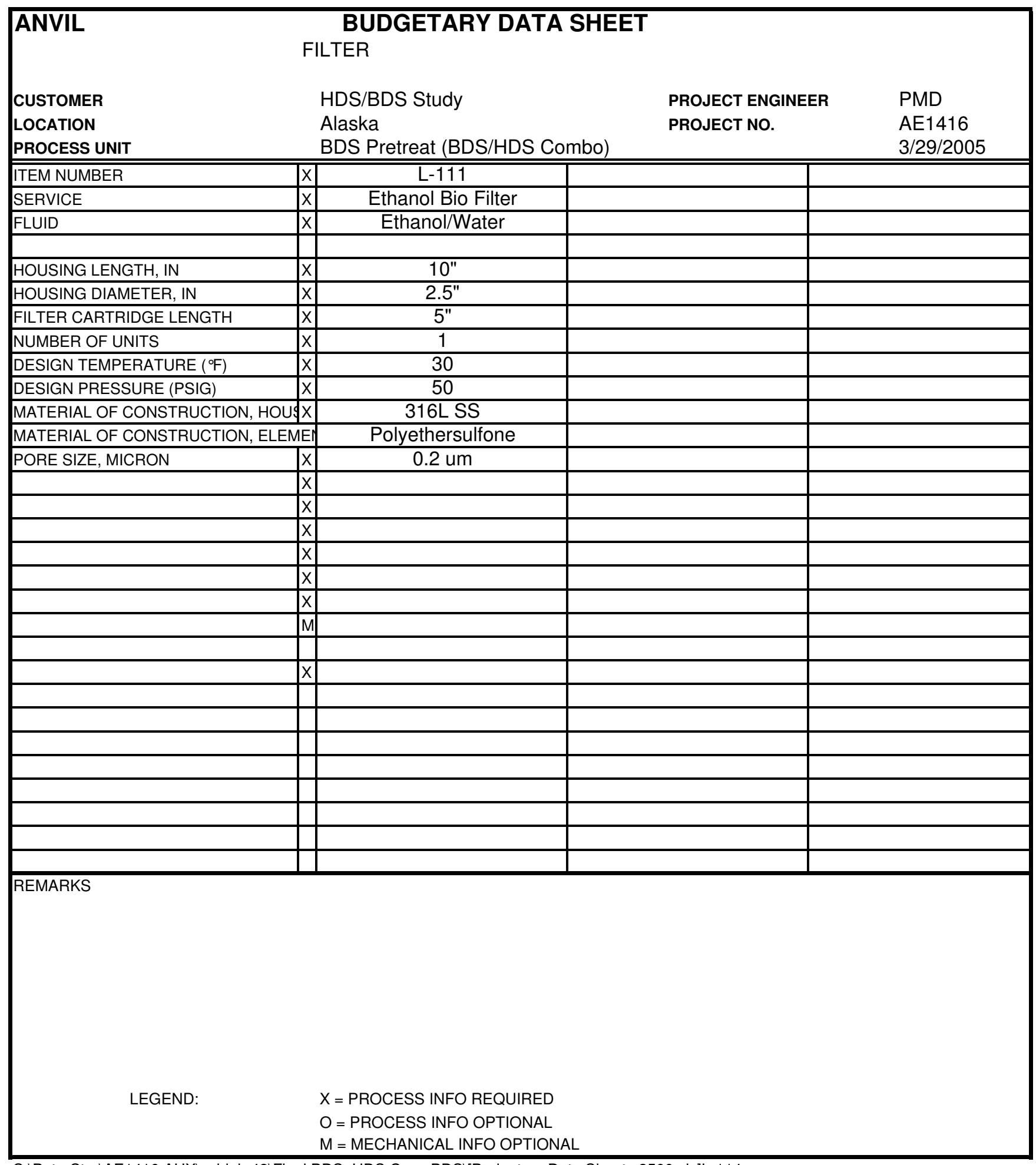




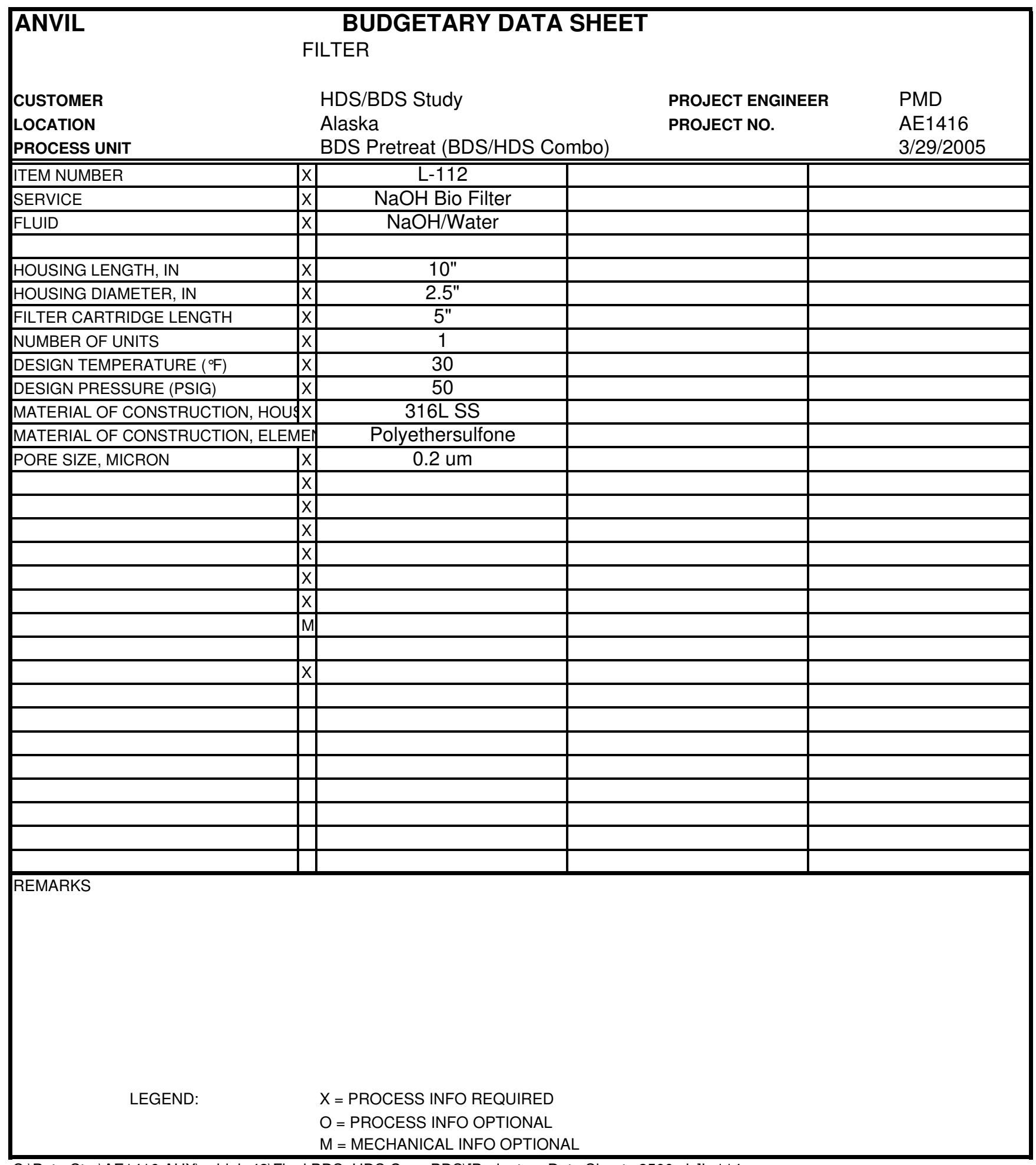




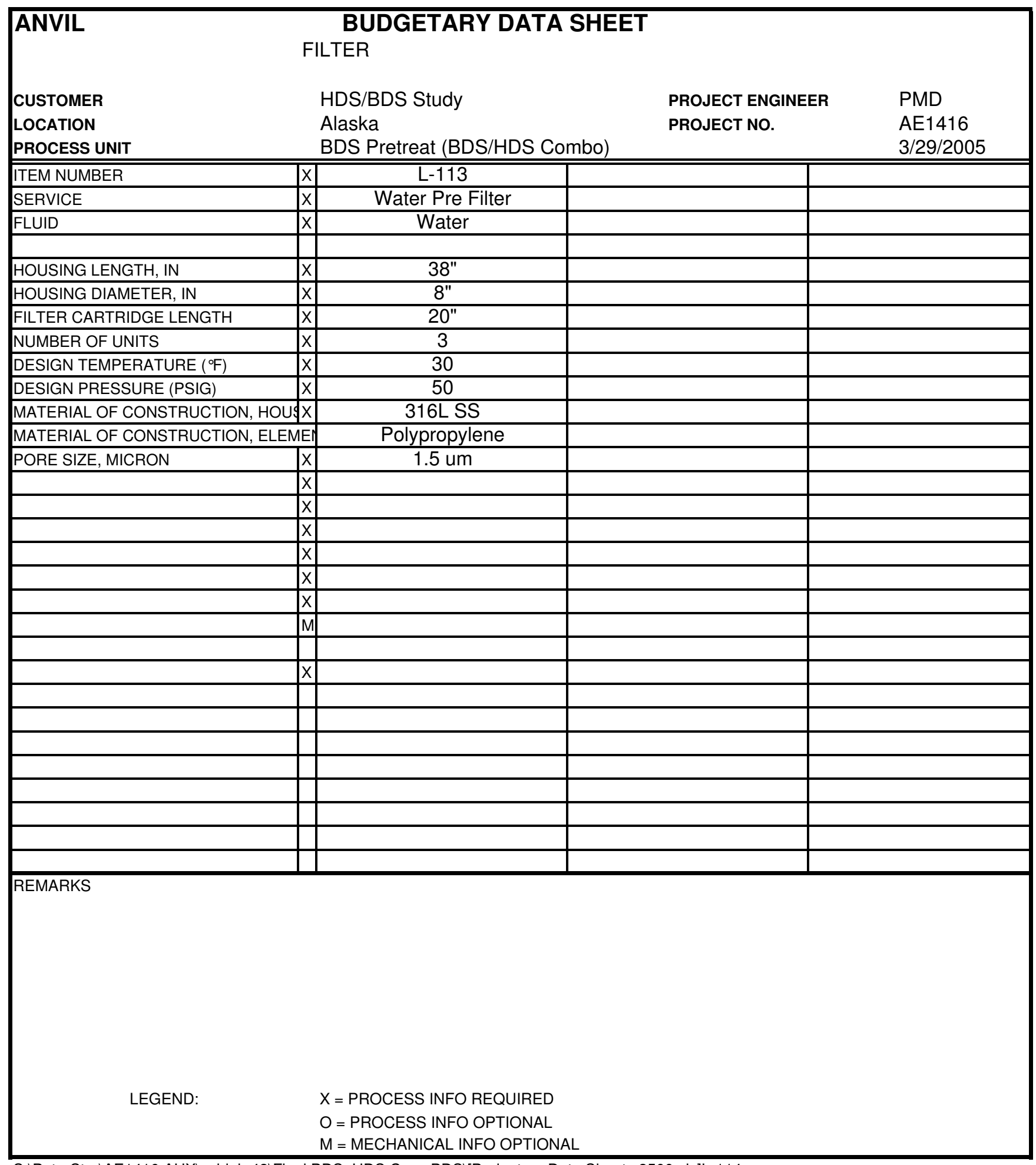




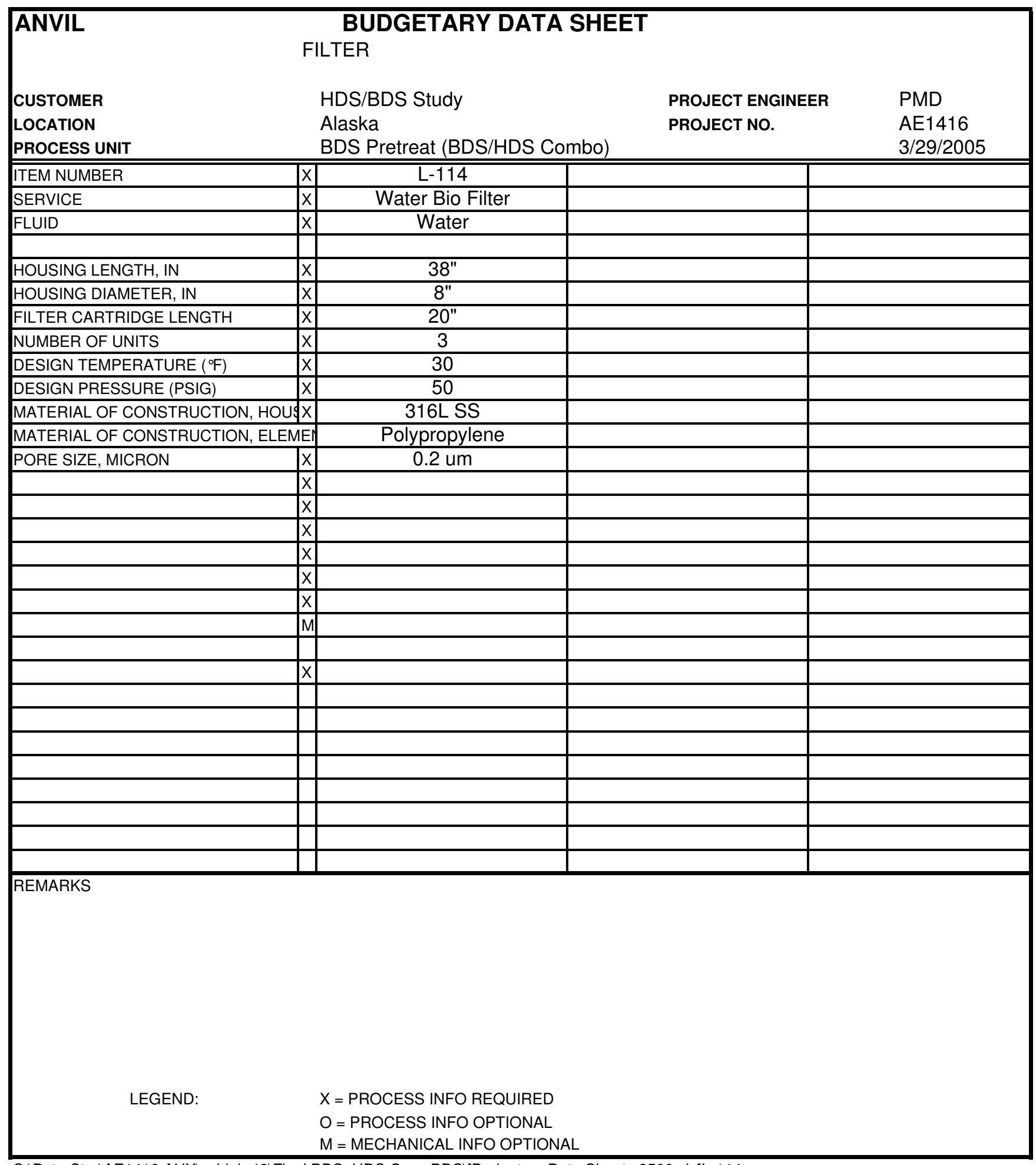




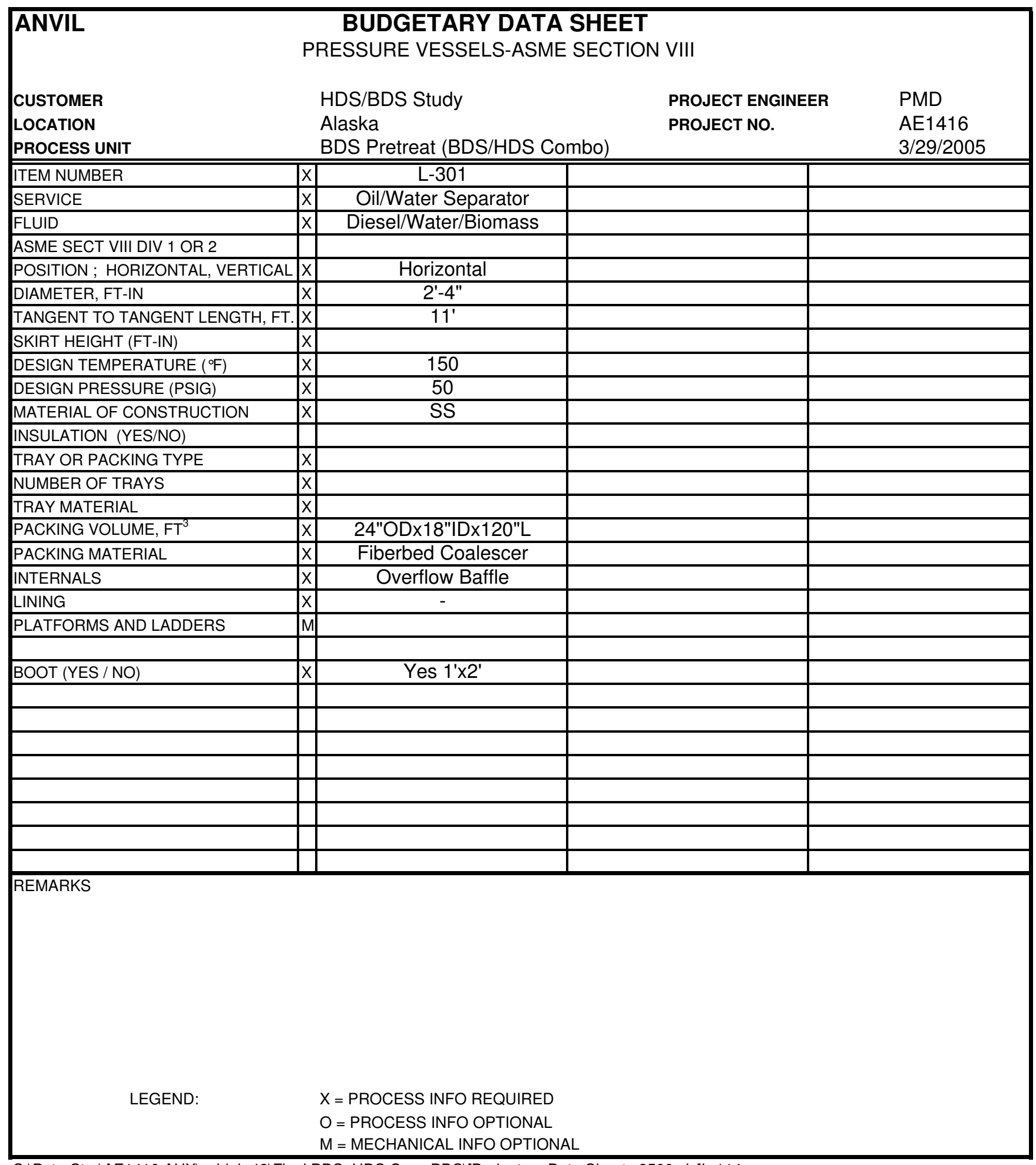




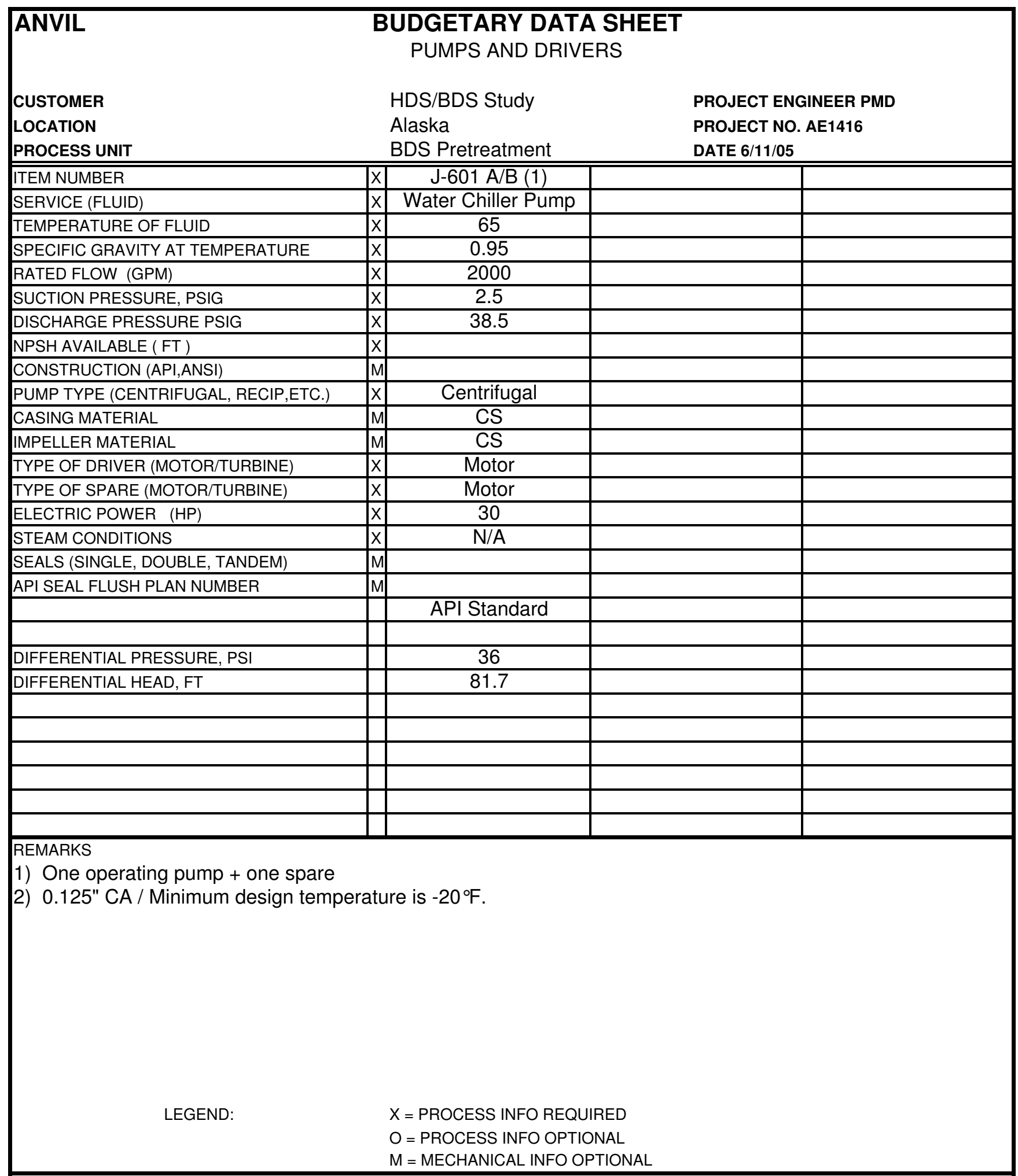

S:IPetroStar|AE1416.AUXIsubjob 43\Final BDS_HDS Case BDS\[bds_hds_Budgetary Sheets Add.xls]C-601(B) 


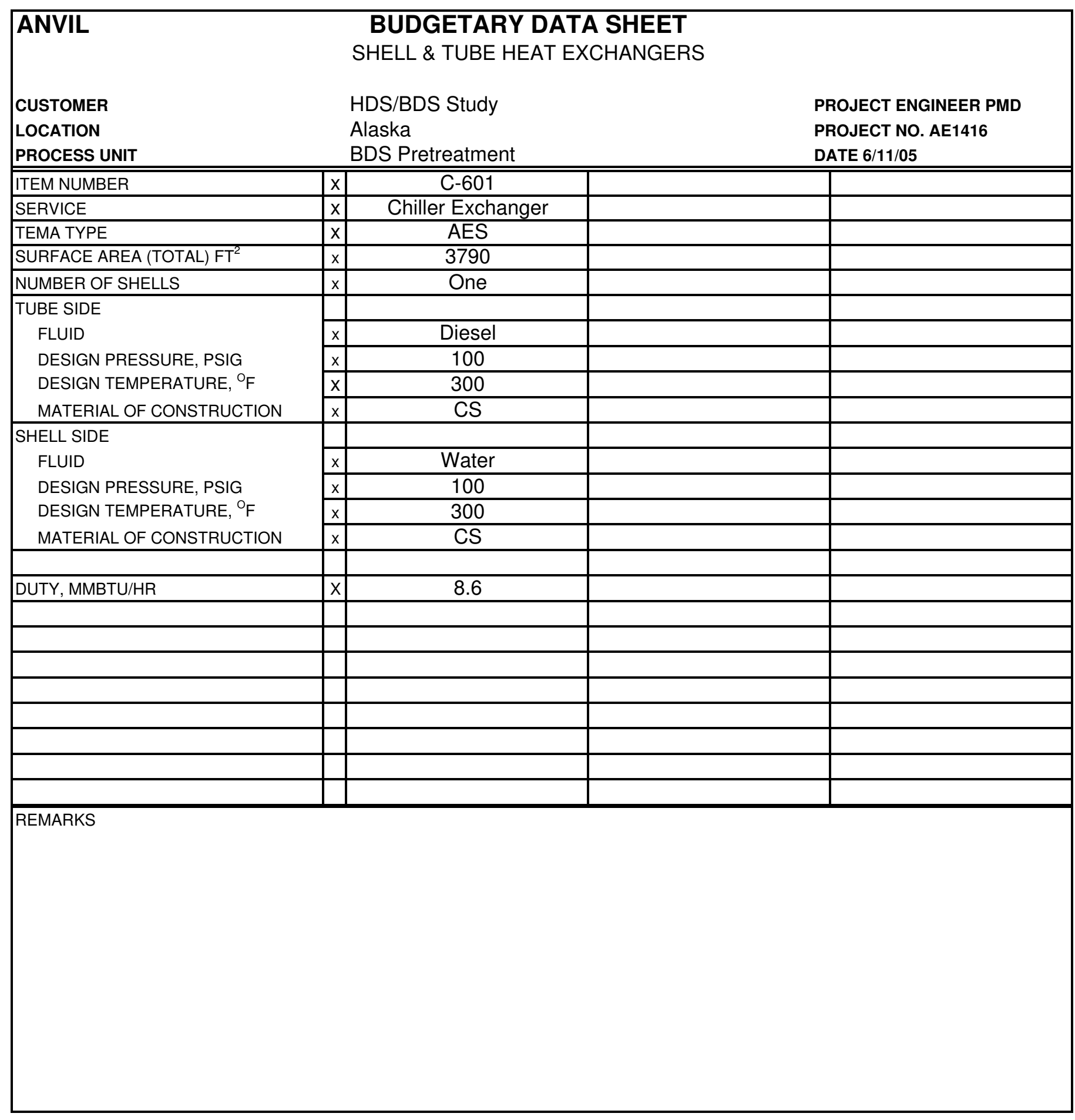




\section{Appendix B.3 - Sulfur Speciation in BDS Unit Diesel Product}

\begin{tabular}{|l|l|l|c|}
\hline \multicolumn{5}{|l|}{ Sulfur Speciation in BDS Diesel Product } \\
\hline Component & ppm wt sulfur & Component & ppm wt sulfur \\
\hline Hydrogen sulfide & $<1$ & 2-Ethyl thiophene & $<1$ \\
\hline Carbonyl sulfide & $<1$ & 2,5-Dimethyl thiophene & $<1$ \\
\hline Methyl mercaptan & $<1$ & 3-Ethyl thiophene & $<1$ \\
\hline Ethyl mercaptan & $<1$ & 2,4\&2,-Dimethyl thiophene & $<1$ \\
\hline Dimethyl sulfide & $<1$ & 3,4-Dimethyl thiophene & $<1$ \\
\hline Carbon disulfide & $<1$ & Methyl Ethyl thiophenes & $<1$ \\
\hline Isopropyl mercaptan & $<1$ & Trimethyl thiophenes & $<1$ \\
\hline Ethyl sulfide & $<1$ & Tetramethyl thiophenes & $<1$ \\
\hline tert-Butyl mercaptan & $<1$ & Benzothiophene & $<1$ \\
\hline N-Propyl mercaptan & $<1$ & Methyl benzothiophene & 2 \\
\hline Ethyl Methyl Sulfide & $<1$ & Dimethyl benzothiophene & 29 \\
\hline Thiophene & $<1$ & Trimethyl benzothiophene & 43 \\
\hline sec-Butyl Mercaptan & $<1$ & Tetramethyl Benzothiophene & $<1$ \\
\hline Isobutyl mercaptan & $<1$ & Dibenzothiophene & $<1$ \\
\hline Ethyl sulfide & $<1$ & 4-Methyl benzothiophene & $<1$ \\
\hline MN-butyl mercaptan & $<1$ & 3-Methyl DBZT+2-methyl DBZT & $<1$ \\
\hline Dimethyl disulfide & $<1$ & 1-Methyl dibenzothiophene & $<1$ \\
\hline 2-Methyl thiophene & $<1$ & 4,6 Dimethyl dibenzothiophene & $<1$ \\
\hline 3-Methyl thiophene & $<1$ & Dimethyl dibenzothiophene & $<1$ \\
\hline Tetra-hydro thiophene & $<1$ & Trimethyl dibenzothiophene & $<1$ \\
\hline Ethyl methyl disulfide & $<1$ & Tetramethyl dibenzothiophene & $<1$ \\
\hline 2-Methyl-tetra-hydro-thiophene & $<1$ & Unidentified volatile sulfur & 3,283 \\
\hline
\end{tabular}


Appendix B.4 - HDS Process Flow Diagrams and Material Balance 


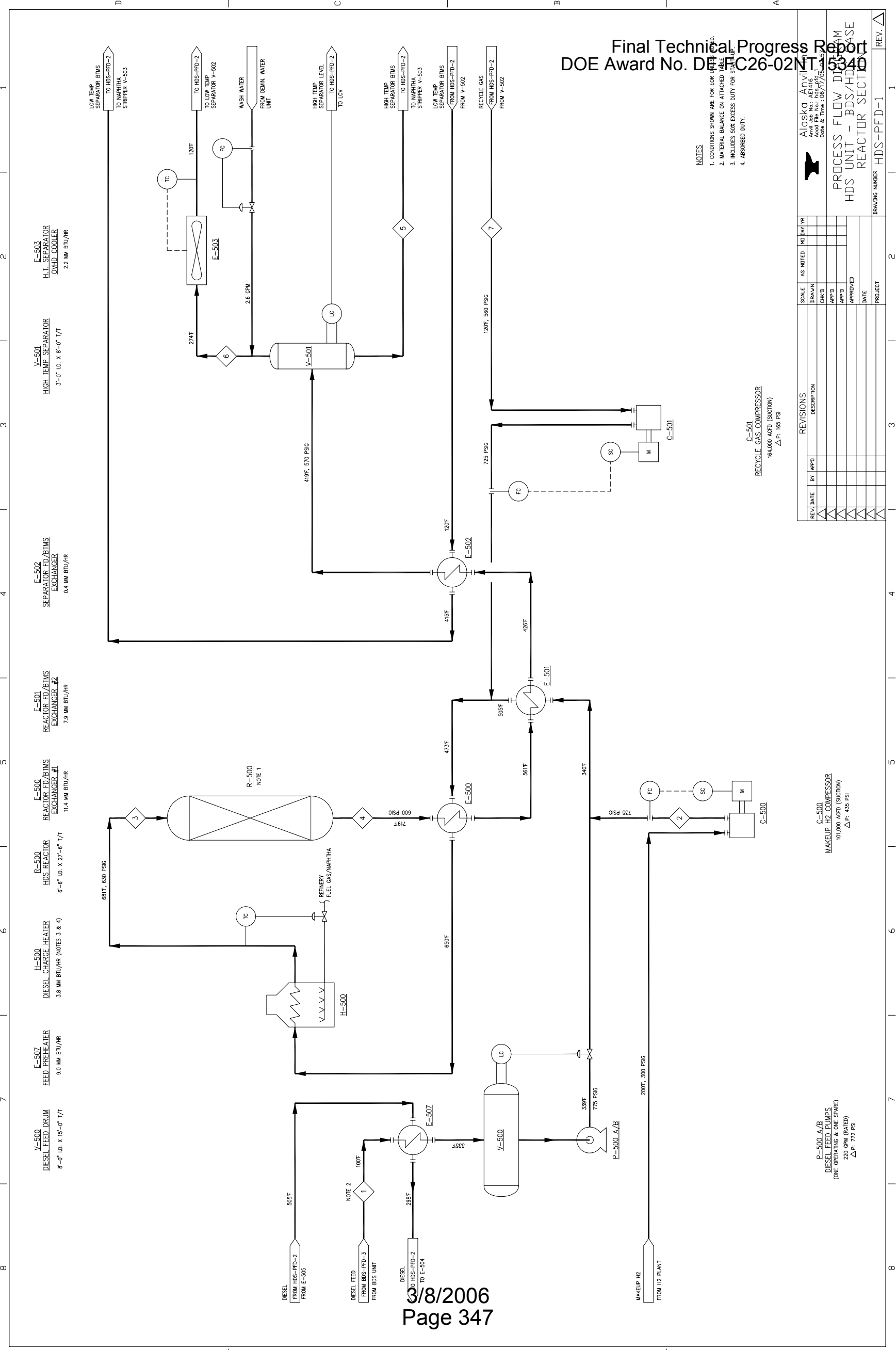


Final Technical Progress Report

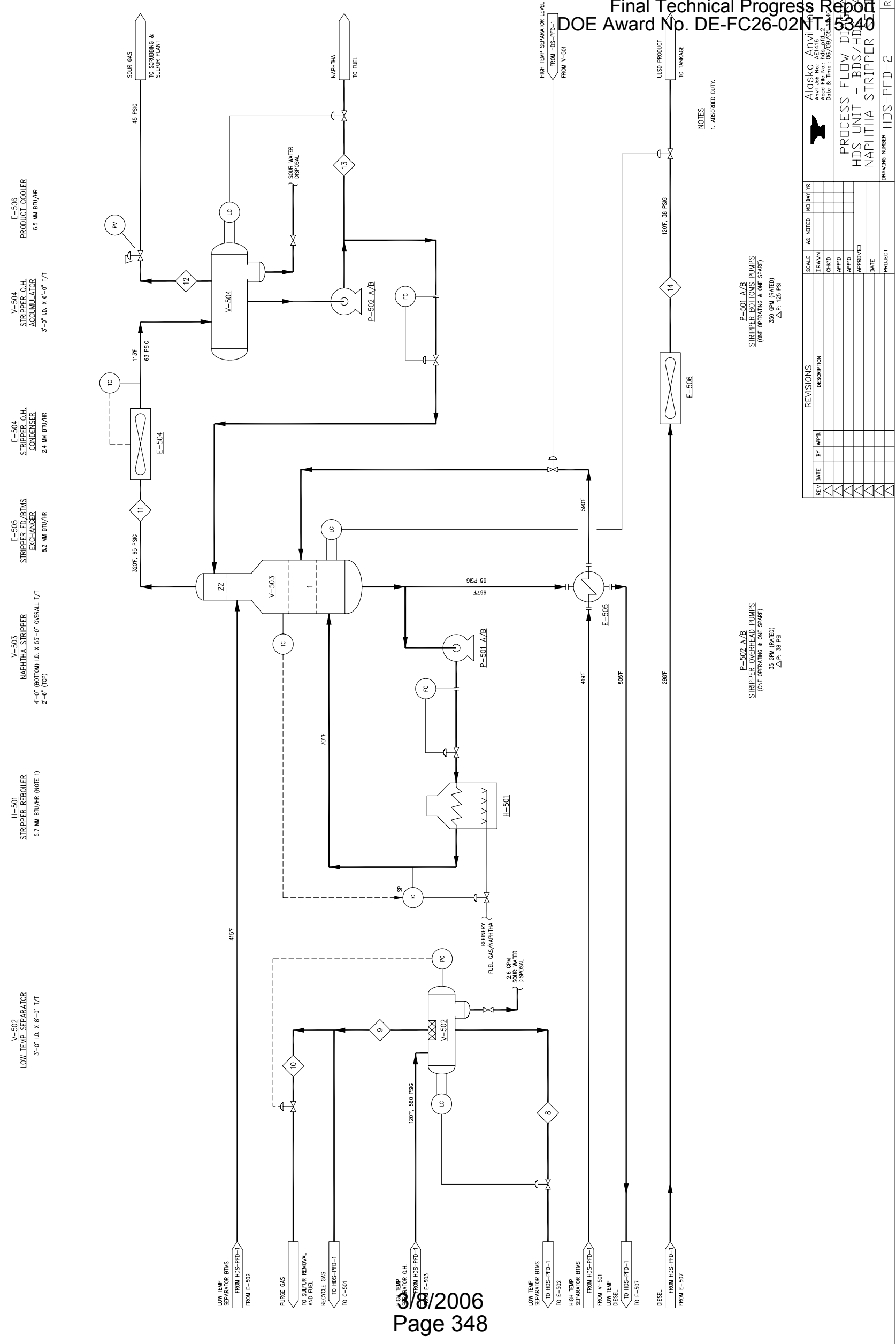




\section{Material Balance}

\section{HDS Unit EOR Material Balance -- BDS/HDS Case}

\begin{tabular}{|c|c|c|c|c|c|c|c|c|}
\hline Stream Number & & 1 & 2 & 3 & 4 & 5 & 6 & 7 \\
\hline Stream Description & & ANS Feed & Make-ıp H2 & Reactor Feed & Reactar Outlet & V-501 Liquid & $\begin{array}{c}\text { V-50t Vapor + } \\
\text { Wash Water }\end{array}$ & Recycle Gas \\
\hline Phase & & L[QUID & VAPOR & MIXED & MLXED & LIQUID & MIXED & VAPOF \\
\hline Mass Flaw Rate & LB/HA & 75976 & 381 & 79681 & 79682 & 73812 & 7170 & 3324 \\
\hline Temperature & $F$ & 100 & 417 & 681 & 719 & 419 & 274 & 120 \\
\hline Pressure & $\mathrm{PS}[\mathrm{G}$ & 13 & 735 & 630 & 600 & 570 & 570 & 560 \\
\hline Standard Lig Flaw & $\mathrm{BPO}$ & 6029 & $17 / \mathrm{a}$ & 4050 & 2994 & 5924 & 128 & $\mathrm{n} / \mathrm{a}$ \\
\hline Vapar Flaw & MSCFH & $\mathrm{nda}$ & 70.2 & 343.8 & 326.9 & $\mathrm{n} / \mathrm{a}$ & 271.9 & 295.7 \\
\hline Wt \% Vapor & & 0 & 100 & 36 & 53 & 0 & 78 & 100 \\
\hline MW & & 217.4 & 2.1 & 60.0 & 760 & 195.6 & 9.6 & 5.4 \\
\hline
\end{tabular}

\begin{tabular}{|c|c|c|c|c|c|c|c|c|}
\hline Stream Number & & 8 & 9 & 10 & $i 1$ & 12 & 13 & 14 \\
\hline Stream Description & & $\begin{array}{l}\text { V-502 HC } \\
\text { Liquid } \\
\end{array}$ & V-502 Vapor & Purge Gas & $\begin{array}{l}\text { Column Vepor } \\
\text { to Condenser }\end{array}$ & Sour Gas & Naphtha & $\begin{array}{c}\text { Diesel from } \\
\text { E-505 }\end{array}$ \\
\hline Phase & & L[GUID & VAPOR & VAPGA & VA.POR & VAPOR & LIOUID & L[OUID \\
\hline Mass Flow Rate & LB/HA & 2392 & 3486 & 162 & 10562 & 513 & 2641 & 73039 \\
\hline Temperature & $F$ & 120 & 120 & 120 & 320 & 113 & 113 & 120 \\
\hline Pressure & $\mathrm{PSIG}$ & 560 & 560 & 560 & 65 & 63 & 104 & 38 \\
\hline Standard Liq Flow & BPD & 206 & $n / \mathrm{a}$ & $n$ & $n d a$ & no & 239 & 5808 \\
\hline Vapar Flaw & MSCFH & nia & 247.2 & 11.5 & 536 & 10.8 & $n / a$ & nia \\
\hline Wt \% Vapor & & 0 & 100 & 100 & 100 & 100 & 0 & 0 \\
\hline MW & & 1160 & 5.4 & 5.4 & 748 & 181 & 89.1 & 214.8 \\
\hline
\end{tabular}




\section{Appendix B.5 - HDS Unit Equipment List and Budgetary Equipment Datasheets}

\section{HDS Unit Equipment List}

\begin{tabular}{|c|c|c|c|c|c|}
\hline \multirow{2}{*}{ Item } & \multirow{2}{*}{ Service } & \multirow{2}{*}{ Description } & \multicolumn{2}{|c|}{ Design Conditions } & \multirow{2}{*}{ Metallurgy } \\
\hline & & & Pressure & Temp. & \\
\hline R-500 & HDS Reactor & $\begin{array}{l}\text { 6'-6" ID X } 27 '-6 " \text { T/T w/ } \\
\text { one bed of HR-526 Co } \\
\text { Mo catalyst. }\end{array}$ & $680 \mathrm{PSIG} / \mathrm{FV}$ & $750^{\circ} \mathrm{F} /-20^{\circ} \mathrm{F}$ & $\begin{array}{l}\text { SA387 Gr. } 11 \mathrm{w} / 321 \mathrm{SS} \text { or } 347 \mathrm{SS} \\
\text { weld overlay. Internal trays are } 410 \\
\text { SS or } 321 \mathrm{SS}\end{array}$ \\
\hline V-500 & Diesel Feed Drum & $\begin{array}{l}\text { 8'-0" ID X 15'-0" T/T } \\
\text { (Horizontal) }\end{array}$ & 50 PSIG/FV & $450 /-20^{\circ} \mathrm{F}$ & Killed Carbon Steel \\
\hline \begin{tabular}{|l|}
$V-501$ \\
\end{tabular} & $\begin{array}{l}\text { High Temperature } \\
\text { Separator }\end{array}$ & $\begin{array}{l}\text { 3'-0" ID X 8'-0" T/T } \\
\text { (Vertical) }\end{array}$ & 620 PSIG/FV & $450 /-20^{\circ} \mathrm{F}$ & Killed Carbon Steel w/0.15" CA \\
\hline V-502 & $\begin{array}{l}\text { Low Temperature } \\
\text { Separator }\end{array}$ & $\begin{array}{l}\text { 3'-0" ID X 8'-0" T/T' } \\
\text { (Horizontal w/ Boot) }\end{array}$ & 610 PSIG/FV & $450 /-20^{\circ} \mathrm{F}$ & $\begin{array}{l}\text { Killed Carbon Steel w/ 0.1" CA } \\
\text { (PWHT) and Monel Demister }\end{array}$ \\
\hline V-503 & Naphtha Stripper & $\begin{array}{l}\text { 2'-6" ID (Top), 4'-0" ID } \\
\text { (bottom) } \\
\text { X 55'-0"T/T (Overall) } \\
\text { w/ 22 valve trays }\end{array}$ & 125 PSIG/FV & $750 /-20^{\circ} \mathrm{F}$ & $\begin{array}{l}\text { Killed Carbon Steel w/ 0.2" CA and } \\
\text { Type } 410 \text { SS trays, supports, and } \\
\text { downcomers }\end{array}$ \\
\hline V-504 & $\begin{array}{l}\text { Stripper O. H. } \\
\text { Accumulator }\end{array}$ & $\begin{array}{l}\text { 3'-0" ID X 6'-0" T/T } \\
\text { (Horizontal w/Boot) }\end{array}$ & 125 PSIG/FV & $370 /-20^{\circ} \mathrm{F}$ & $\begin{array}{l}\text { Killed Carbon Steel w/0.125" CA } \\
\text { (PWHT) }\end{array}$ \\
\hline E-500 & $\begin{array}{l}\text { Reactor Fd/Btms } \\
\text { Exchanger \#1 }\end{array}$ & $\begin{array}{l}\text { 11.4 MM BTU/Hr, } \\
2780 \mathrm{FT}^{2} \text { w/ } 2 \text { shells, } \\
\text { TEMA type CEU }\end{array}$ & $\begin{array}{l}\text { Tubes: } 650 \text { PSIG } \\
\text { Shell: } 775 \text { PSIG }\end{array}$ & $\begin{array}{l}770^{\circ} \mathrm{F} \\
700^{\circ} \mathrm{F} /-20^{\circ} \mathrm{F}\end{array}$ & $\begin{array}{l}\text { Tubes: } 1 \frac{1 / 4}{\mathrm{Cr}}-1 / 2 \text { Mo tubes and } \\
\text { tube sheet and weld overlay } 316 \text { ss } \\
\text { for tube sheet } \\
\text { Shell: } 1 \frac{1 / 4}{\mathrm{Cr}}-1 / 2 \mathrm{Mo} \text { clad } \mathrm{w} / 321 \\
\text { SS, baffles to be } 304 \mathrm{SS}\end{array}$ \\
\hline E-501 & $\begin{array}{l}\text { Reactor Fd/Btms } \\
\text { Exchanger \#2 }\end{array}$ & $\begin{array}{l}\text { 7.9 } \mathrm{MM} \mathrm{BTU} / \mathrm{Hr} \\
2740 \mathrm{FT}^{2} \text { w/2 shells, } \\
\text { TEMA type CEU }\end{array}$ & $\begin{array}{l}\text { Tubes: } 640 \text { PSIG } \\
\text { Shell: } 785 \text { PSIG }\end{array}$ & \begin{tabular}{|l|}
$600^{\circ} \mathrm{F}$ \\
$530^{\circ} \mathrm{F} /-20^{\circ} \mathrm{F}$
\end{tabular} & 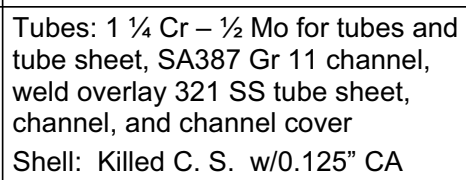 \\
\hline E-502 & $\begin{array}{l}\text { Separator } \\
\text { Fd/Btms } \\
\text { Exchanger }\end{array}$ & $\begin{array}{l}0.4 \mathrm{MM} \mathrm{BTU} / \mathrm{Hr}, \\
85 \mathrm{FT}^{2} \text {, double pipe. }\end{array}$ & $\begin{array}{l}\text { Tubes: } 630 \text { PSIG } \\
\text { Shell: } 610 \text { PSIG }\end{array}$ & $\begin{array}{l}450^{\circ} \mathrm{F} \\
450 /-20^{\circ} \mathrm{F}\end{array}$ & $\begin{array}{l}\text { Tubes: Carbon Steel } \\
\text { Shell: Carbon Steel }\end{array}$ \\
\hline E-503 & $\begin{array}{l}\text { H. T. Separator } \\
\text { Overhead Cooler }\end{array}$ & $\begin{array}{l}\text { 2.2 MM BTU/Hr } \\
409 \mathrm{FT}^{2} \text { (Bare Tube) } \\
8,790 \mathrm{FT}^{2} \text { Extended } \\
\text { Surface, } 5 \text { HP Fan }\end{array}$ & Tubes: 620 PSIG & $450^{\circ} \mathrm{F}$ & $\begin{array}{l}\text { Seamless Carbon Steel tubes with } \\
\text { aluminum fins }\end{array}$ \\
\hline E-504 & $\begin{array}{l}\text { Stripper O. H. } \\
\text { Condenser }\end{array}$ & $\begin{array}{l}\text { 2.4 MM BTU/Hr } \\
405 \mathrm{FT}^{2} \text { (Bare Tube) } \\
8,740 \mathrm{FT}^{2} \text { Extended } \\
\text { Surface, } 5 \text { HP Fan }\end{array}$ & Tubes: 125 PSIG & $370^{\circ} \mathrm{F}$ & $\begin{array}{l}\text { Seamless Carbon Steel tubes with } \\
\text { aluminum fins }\end{array}$ \\
\hline \begin{tabular}{|l|}
$E-505$ \\
\end{tabular} & $\begin{array}{l}\text { Stripper Fd/Btms } \\
\text { Exchanger }\end{array}$ & $\begin{array}{l}\text { 8.2 MM BTU/Hr } \\
2,520 \mathrm{FT}^{2} \text { w/2 shells, } \\
\text { TEMA type CEU }\end{array}$ & $\begin{array}{l}\text { Tubes: } 620 \text { PSIG } \\
\text { Shell: } 100 \text { PSIG }\end{array}$ & \begin{tabular}{|l|}
$640^{\circ} \mathrm{F}$ \\
$720^{\circ} \mathrm{F} /-20^{\circ} \mathrm{F}$
\end{tabular} & $\begin{array}{l}\text { Tubes: } 18 \mathrm{Cr}-8 \mathrm{Ni} \text {, Channel is CS } \\
\text { w/ } 0.25 " \mathrm{CA} \text {, tube sheets are } 410 \mathrm{SS} \\
\text { Shell: CS w/0.25" CA, CS baffles }\end{array}$ \\
\hline E-506 & Product Cooler & $\begin{array}{l}\text { 6.5 MM BTU/Hr } \\
\text { 1,700 } \mathrm{FT}^{2} \text { (Bare Tube) } \\
\text { 36,520 } \mathrm{FT}^{2} \text { Extended } \\
\text { Surface, } 15 \mathrm{HP} \text { Fan }\end{array}$ & Tubes: 100 PSIG & $450^{\circ} \mathrm{F}$ & $\begin{array}{l}\text { Seamless Carbon Steel tubes with } \\
\text { aluminum fins }\end{array}$ \\
\hline \begin{tabular}{|l|} 
E-507 \\
\end{tabular} & Feed Preheater & $\begin{array}{l}\text { 9.0 MM BTU/Hr } \\
\text { 1,030 } \mathrm{FT}^{2} \text { w/2 shells, } \\
\text { TEMA type CEU }\end{array}$ & $\begin{array}{l}\text { Tubes: } 100 \text { PSIG } \\
\text { Shell: } 100 \text { PSIG }\end{array}$ & \begin{tabular}{|l|}
$510^{\circ} \mathrm{F}$ \\
$720^{\circ} \mathrm{F} /-20^{\circ} \mathrm{F}$
\end{tabular} & $\begin{array}{l}\text { Tubes: CS } \\
\text { Shell: CS w/0.25" CA, CS baffles }\end{array}$ \\
\hline $\mathrm{H}-500$ & $\begin{array}{l}\text { Diesel Charge } \\
\text { Heater }\end{array}$ & $\begin{array}{l}\text { 3.8 MM BTU/Hr } \\
\text { Fired Heater, Convection } \\
\text { Section shared w/ H-501 }\end{array}$ & Tubes: 765 PSIG & $750^{\circ} \mathrm{F}$ & Tubes: $9 \mathrm{Cr}-1 \mathrm{Mo}$ w/ 0.1" CA \\
\hline
\end{tabular}




\begin{tabular}{|c|c|c|c|c|c|}
\hline \multirow{2}{*}{ Item } & \multirow{2}{*}{ Service } & \multirow{2}{*}{ Description } & \multicolumn{2}{|l|}{ Design Conditions } & \multirow{2}{*}{ Metallurgy } \\
\hline & & & Pressure & Temp. & \\
\hline $\mathrm{H}-501$ & Stripper Reboiler & $\begin{array}{l}5.7 \mathrm{MM} \mathrm{BTU} / \mathrm{Hr} \\
\text { Fired Heater, Convection } \\
\text { Section shared w/ H-500 }\end{array}$ & Tubes: 230 PSIG & $770^{\circ} \mathrm{F}$ & Tubes: $5 \mathrm{Cr}-1 / 2 \mathrm{Mo} \mathrm{w} / 0.1$ " CA \\
\hline C-500 & $\begin{array}{l}\text { Makeup Hydrogen } \\
\text { Compressor }\end{array}$ & $\begin{array}{l}\text { Capacity: 100,944 ACFD } \\
\text { Motor Driven } \\
\text { Shaft HP: } 112 \text { BHP }\end{array}$ & $\begin{array}{l}\text { Suction: } 300 \text { PSIG } \\
\text { Disch: } 735 \text { PSIG }\end{array}$ & $\begin{array}{l}\text { Suction: } \\
200^{\circ} \mathrm{F}\end{array}$ & $\begin{array}{l}\text { Casing: killed C. S. } \\
\text { Internals: CS }\end{array}$ \\
\hline C-501 & $\begin{array}{l}\text { Recycle Gas } \\
\text { Compressor }\end{array}$ & $\begin{array}{l}\text { Capacity: 163,584 ACFD } \\
\text { Motor Driven } \\
\text { Shaft HP: } 87 \text { BHP }\end{array}$ & $\begin{array}{l}\text { Suction: } 560 \text { PSIG } \\
\text { Disch: } 725 \text { PSIG }\end{array}$ & $\begin{array}{l}\text { Suction: } \\
120^{\circ} \mathrm{F}\end{array}$ & $\begin{array}{l}\text { Casing: killed C. S. } \\
\text { Internals: Stainless Steel }\end{array}$ \\
\hline $\begin{array}{l}P-500 \\
A / B\end{array}$ & $\begin{array}{l}\text { Diesel Feed Pump } \\
\text { (One Operating + } \\
\text { One Spare }\end{array}$ & $\begin{array}{l}\text { Rated Capacity: } 220 \text { GPM } \\
\text { Diff. Press: } 772 \text { PSI } \\
\text { Head: } 2333 \text { feet } \\
\text { Motor: } 150 \mathrm{HP}\end{array}$ & & & $\begin{array}{l}\text { Casing: killed C. S. w/ } 0.125 " \text { CA } \\
\text { Impeller: } 12 \% \text { chrome }\end{array}$ \\
\hline $\begin{array}{l}P-501 \\
A / B\end{array}$ & $\begin{array}{l}\text { Stripper Bottoms } \\
\text { Pump } \\
\text { (One Operating + } \\
\text { One Spare }\end{array}$ & $\begin{array}{l}\text { Rated Capacity: } 350 \text { GPM } \\
\text { Diff. Press: } 125 \text { PSI } \\
\text { Head: } 481 \text { feet } \\
\text { Motor: } 50 \text { HP }\end{array}$ & & & $\begin{array}{l}\text { Casing: killed C. S. w/ } 0.2 " \text { CA } \\
\text { Impeller: } 12 \% \text { chrome }\end{array}$ \\
\hline $\begin{array}{l}\mathrm{P}-502 \\
\mathrm{~A} / \mathrm{B}\end{array}$ & $\begin{array}{l}\text { Stripper Overhead } \\
\text { Pump } \\
\text { (One Operating + } \\
\text { One Spare }\end{array}$ & $\begin{array}{l}\text { Rated Capacity: } 35 \text { GPM } \\
\text { Diff. Press: } 38 \text { PSI } \\
\text { Head: } 120 \text { feet } \\
\text { Motor: } 5 \text { HP }\end{array}$ & & & $\begin{array}{l}\text { Casing: killed C. S. w/ } 0.125 \text { " CA } \\
\text { Impeller: } 12 \% \text { chrome }\end{array}$ \\
\hline
\end{tabular}




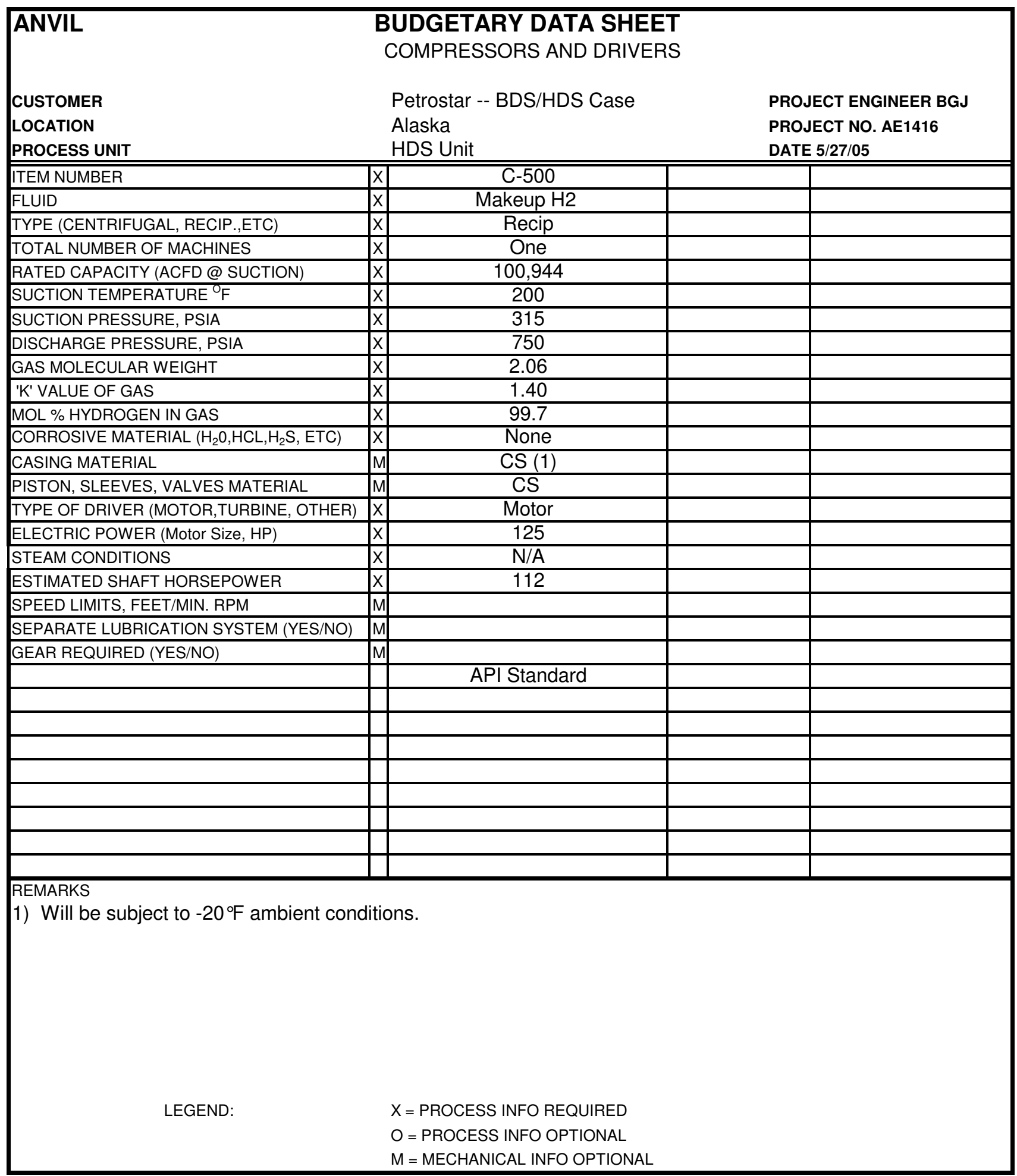

S:IPetroStar\AE1416.AUX|subjob 43|Final BDS_HDS Case HDS|[C-500.xls]Sheet1 


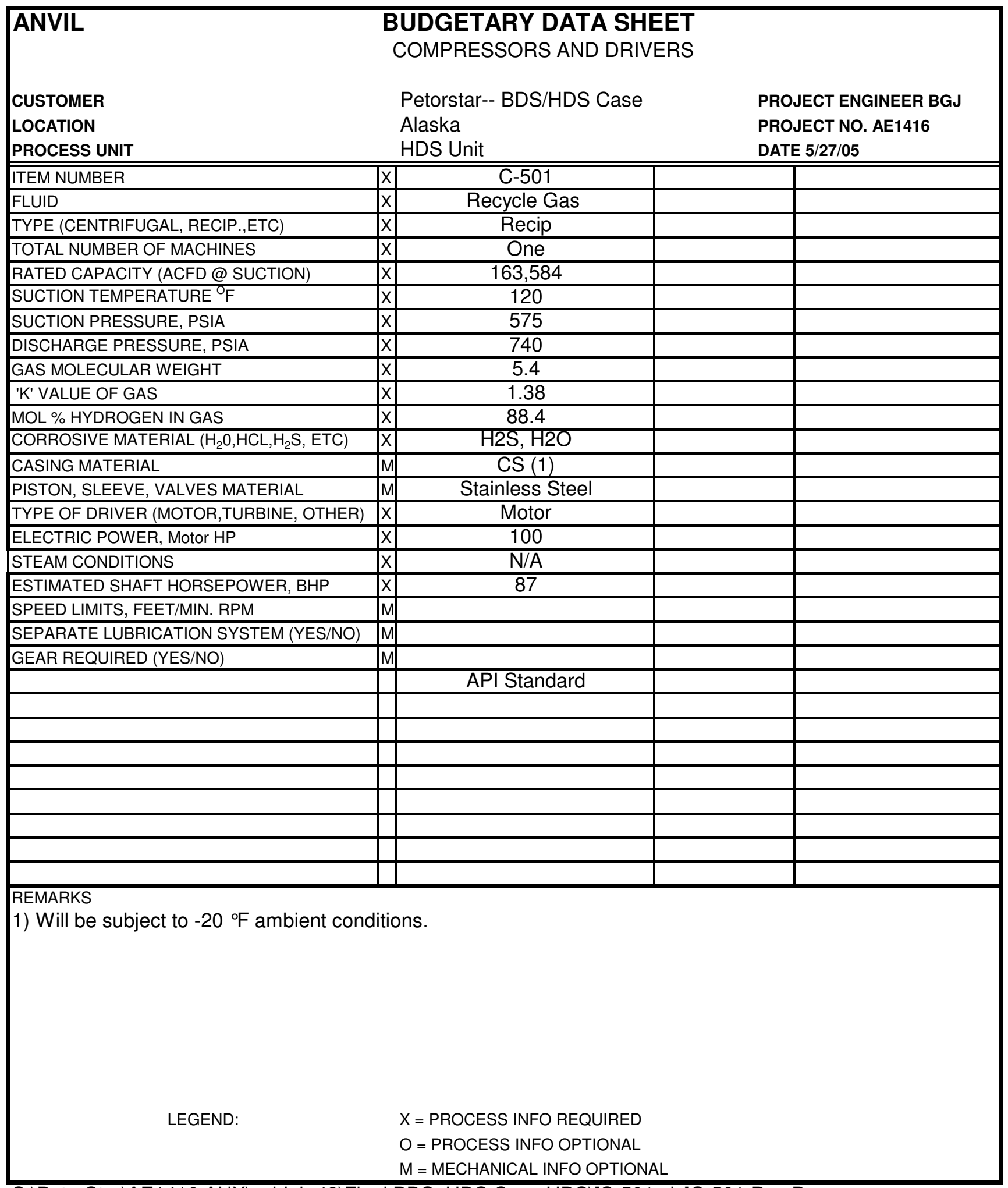

S:IPetroStar|AE1416.AUXIsubjob 43|Final BDS_HDS Case HDS\[C-501.xls]C-501 Rev B 


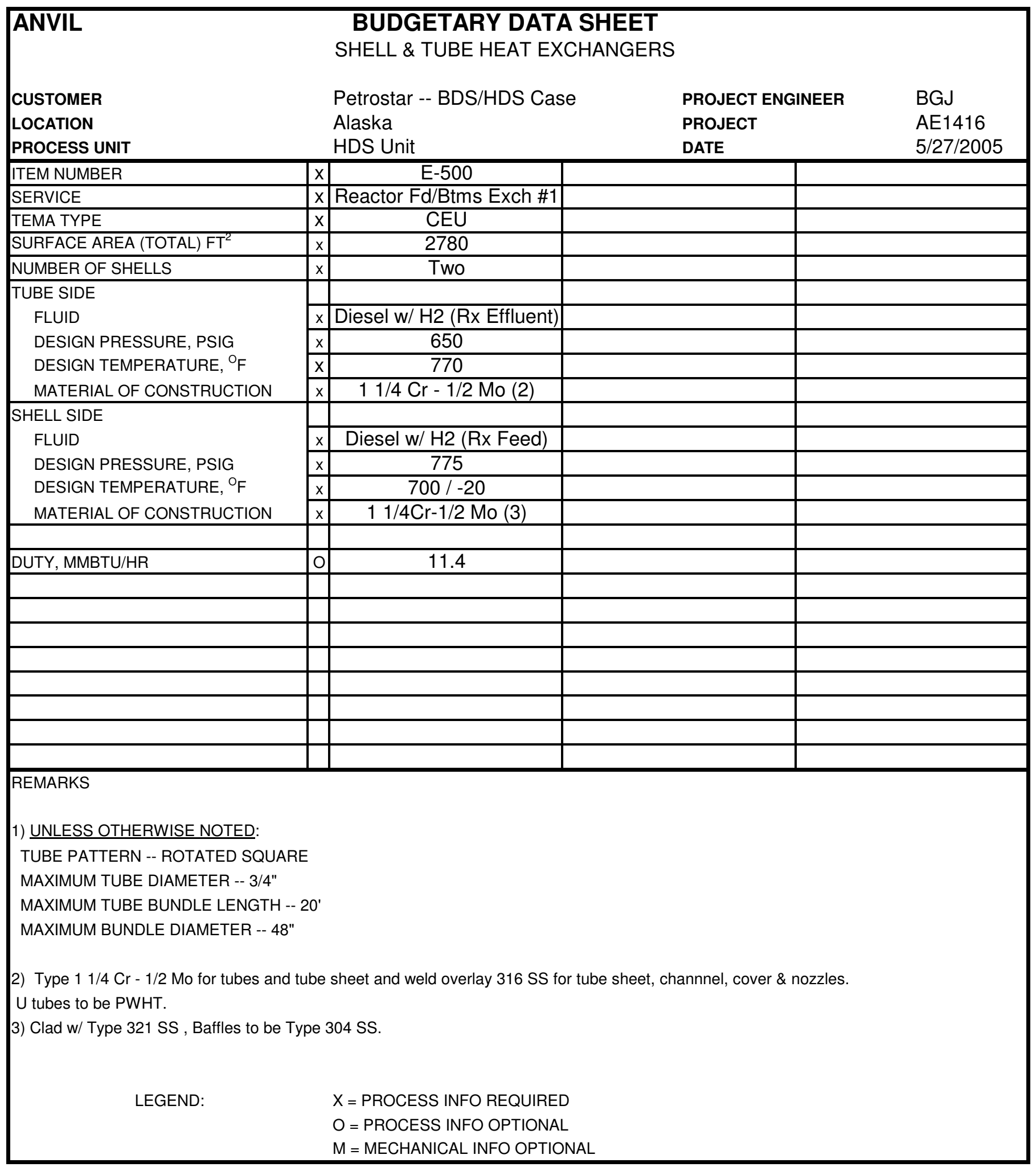




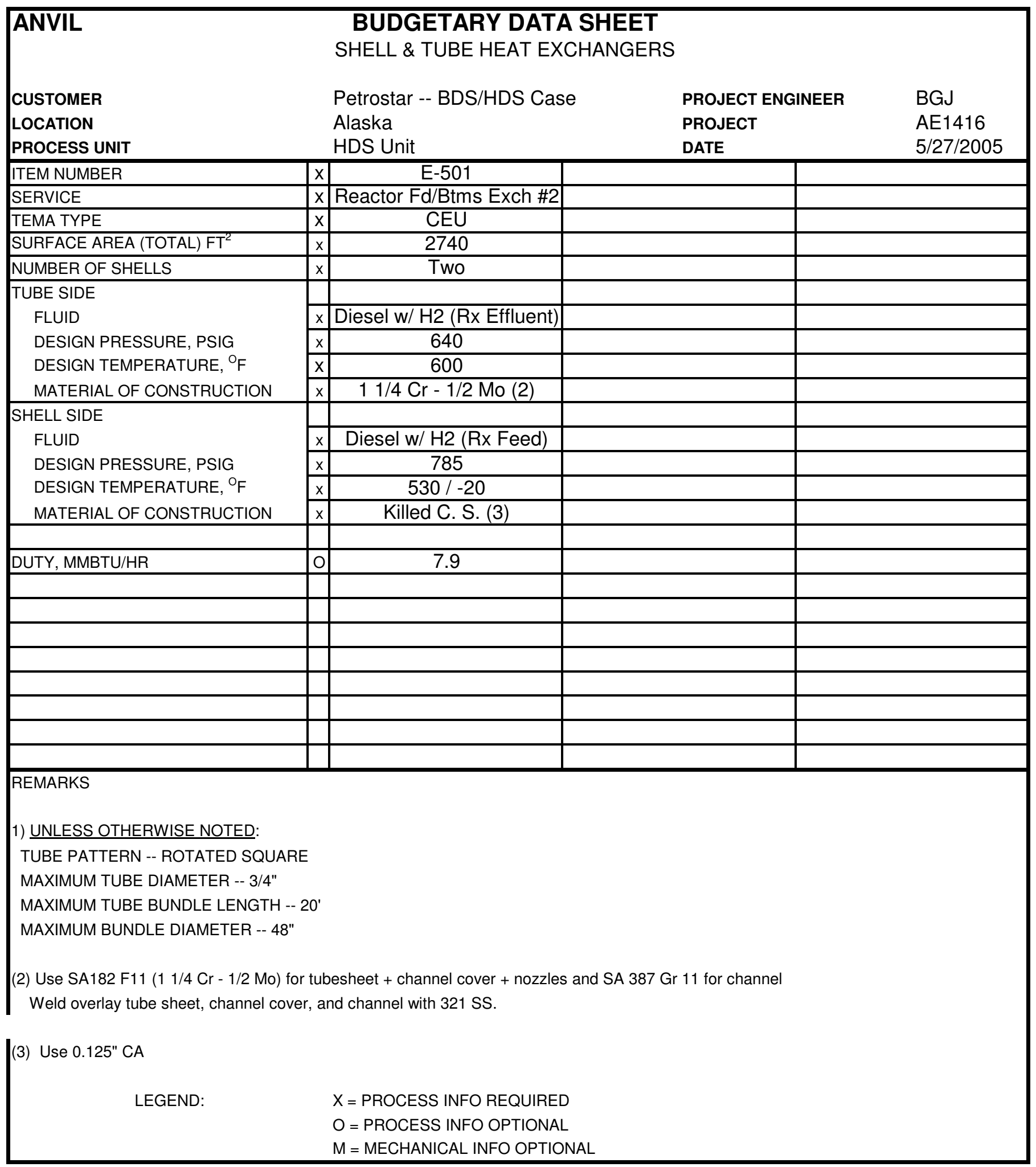




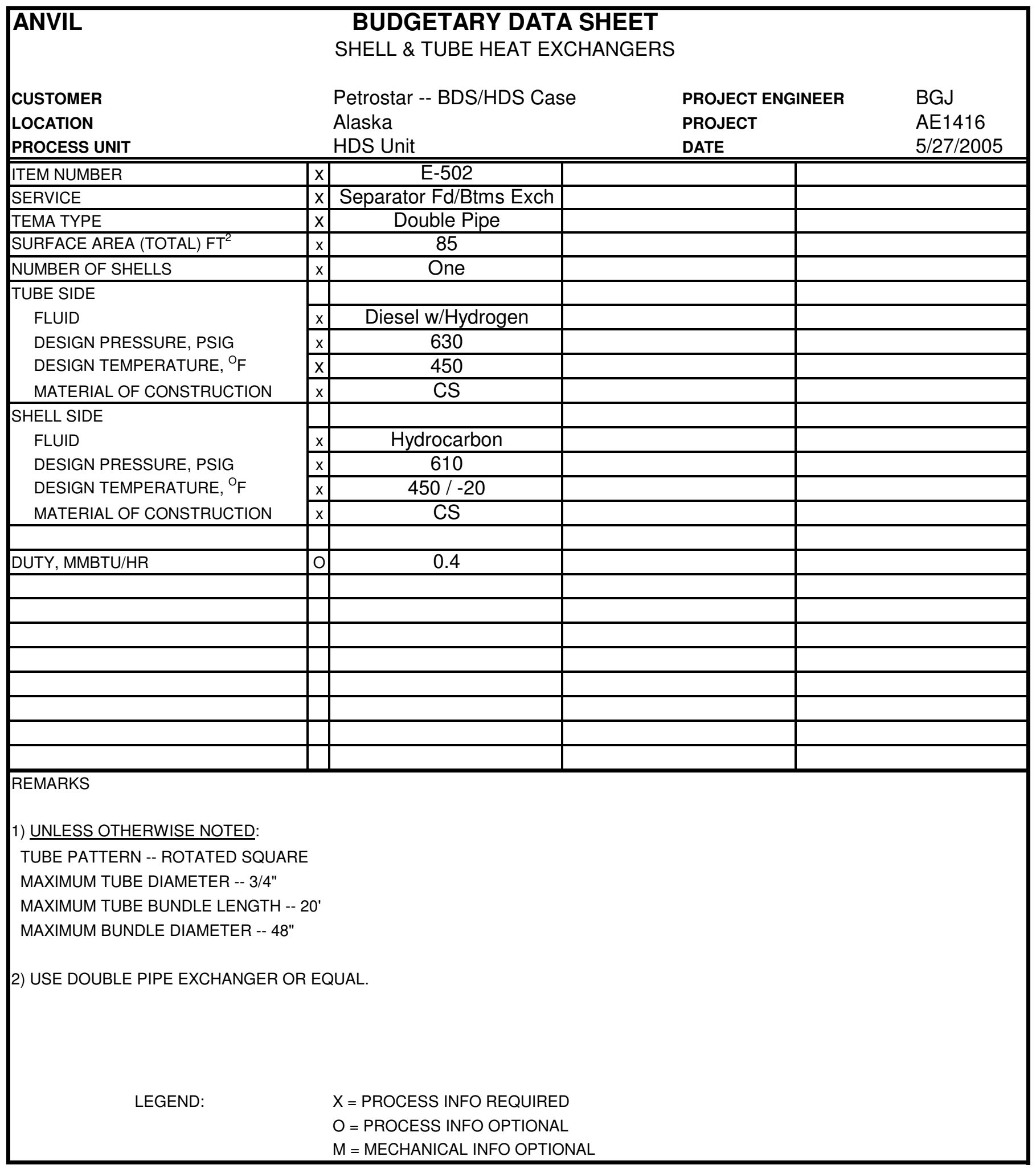




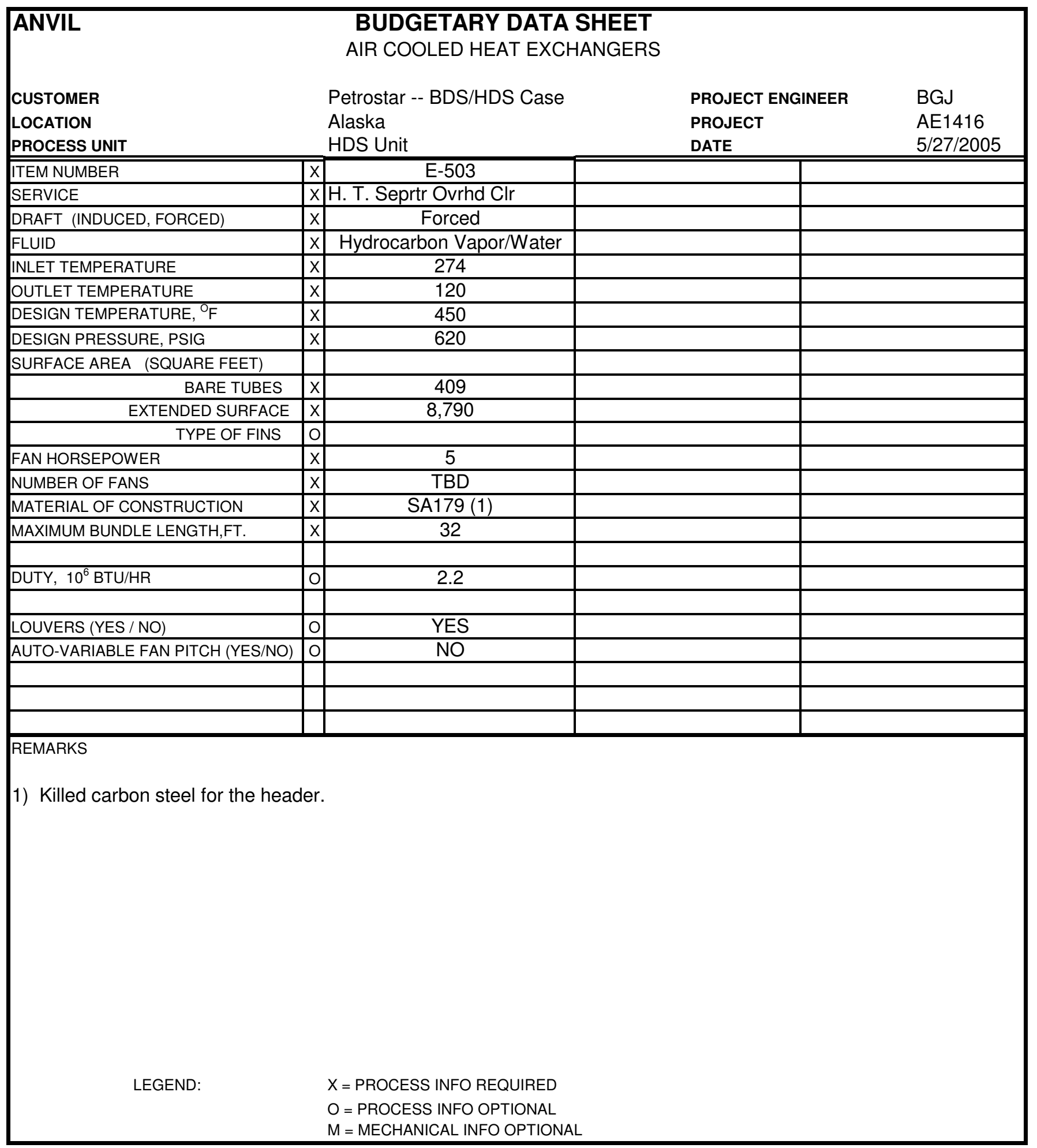




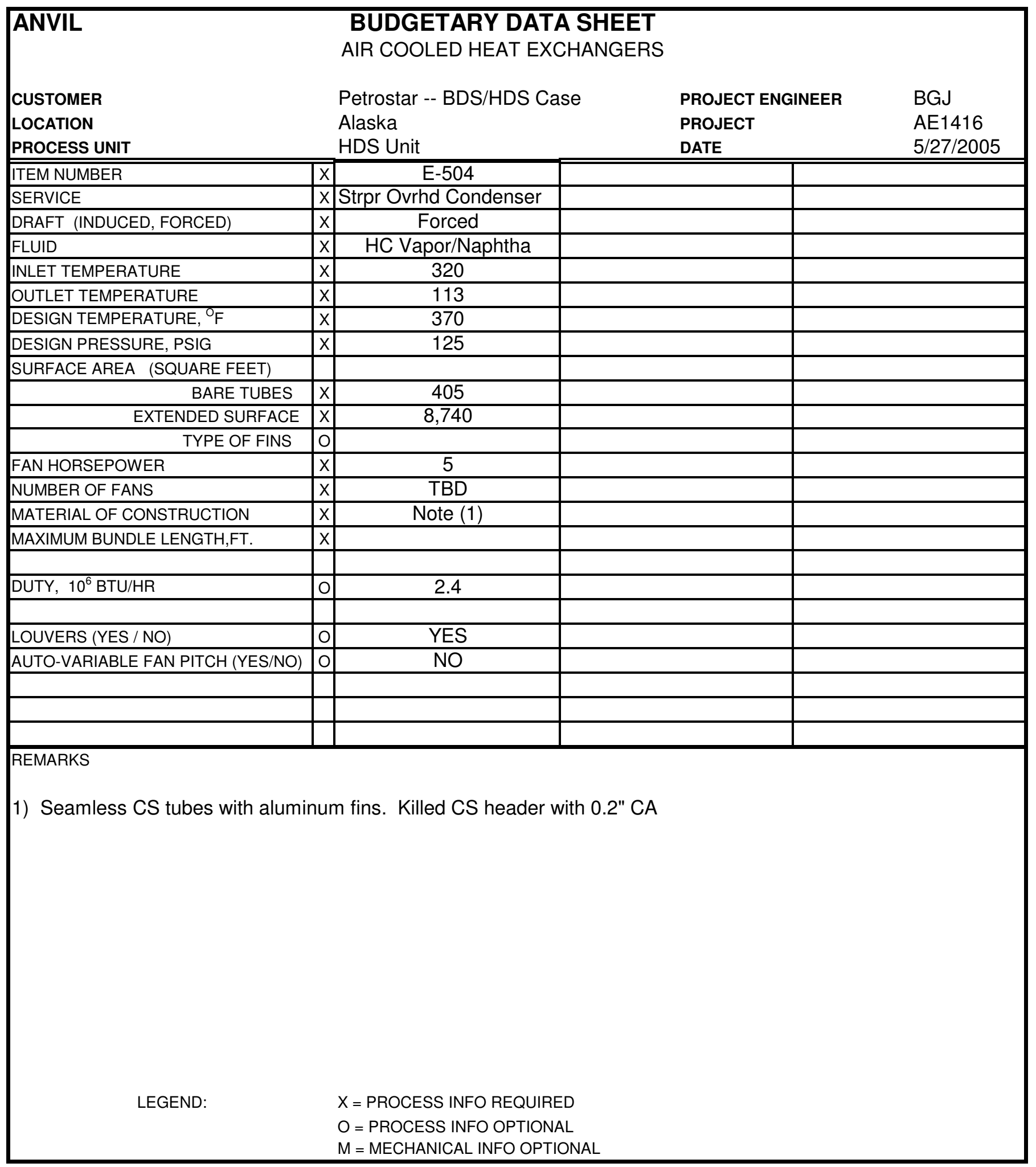

S:IPetroStar\AE1416.AUXIsubjob 43\Final BDS_HDS Case HDS\[E-504.xIs]E-504 Rev B 


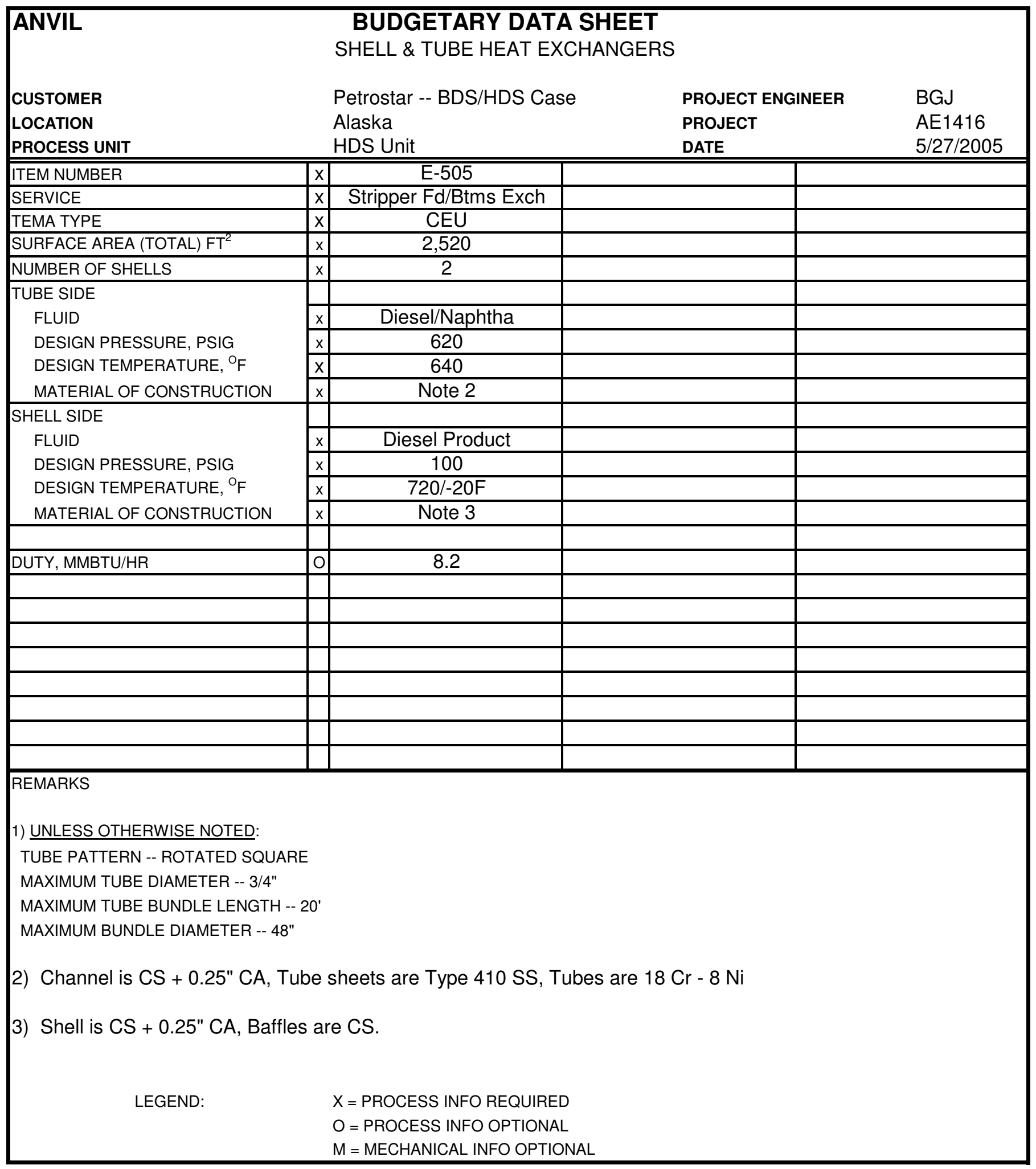




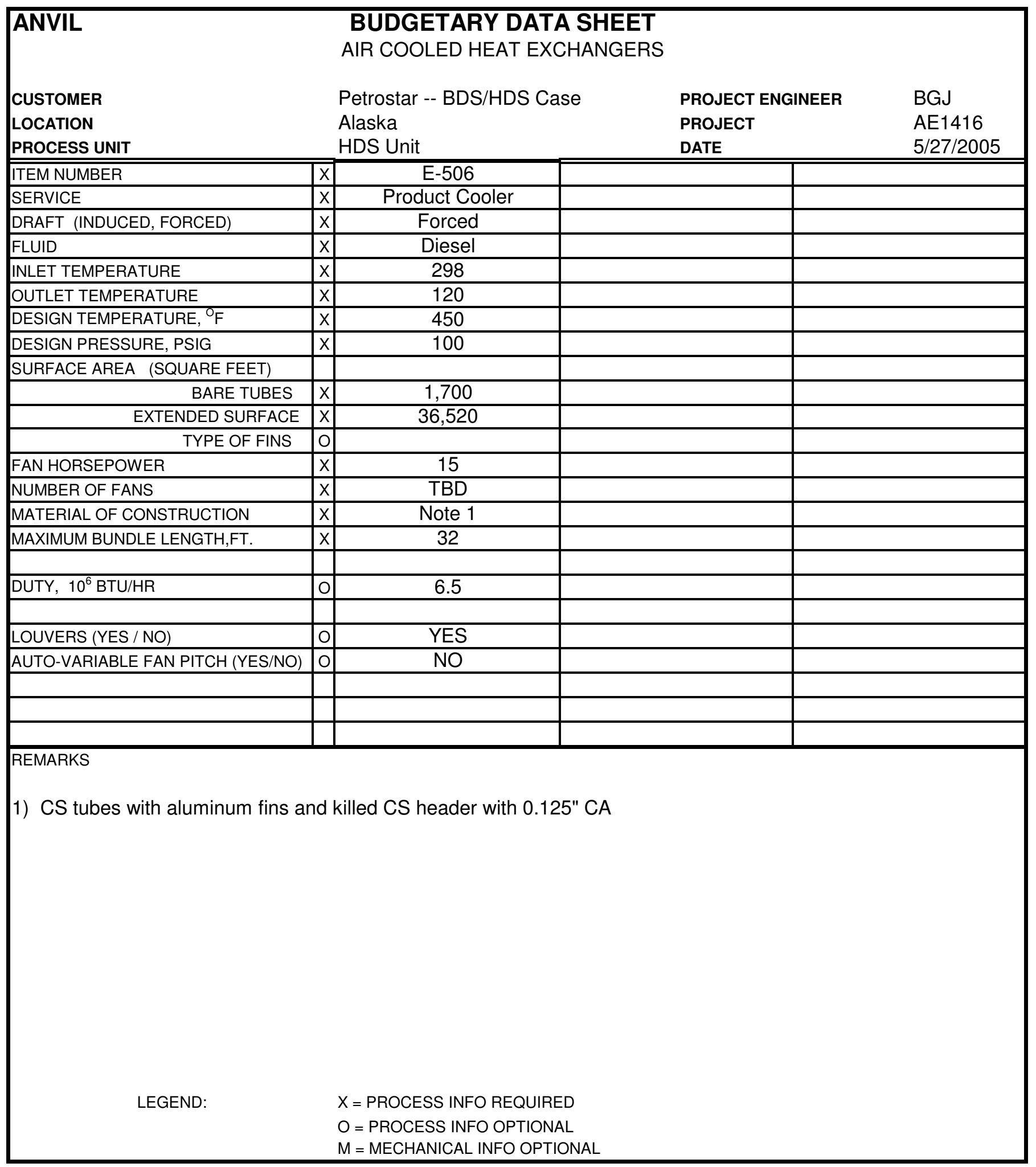

S:IPetroStar\AE1416.AUXIsubjob 43\Final BDS_HDS Case HDS\[E-506.xIs]E-506 Rev B 


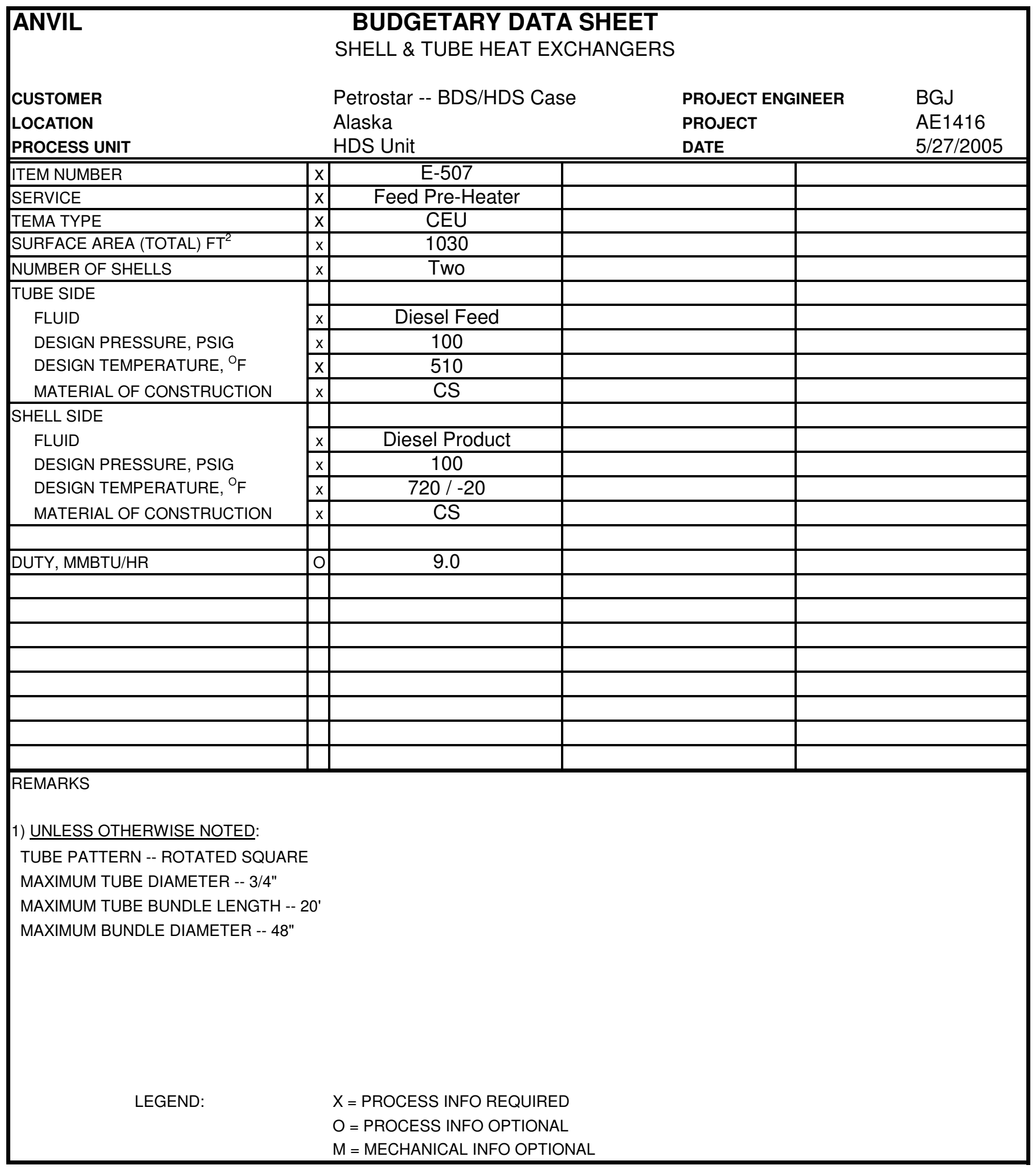




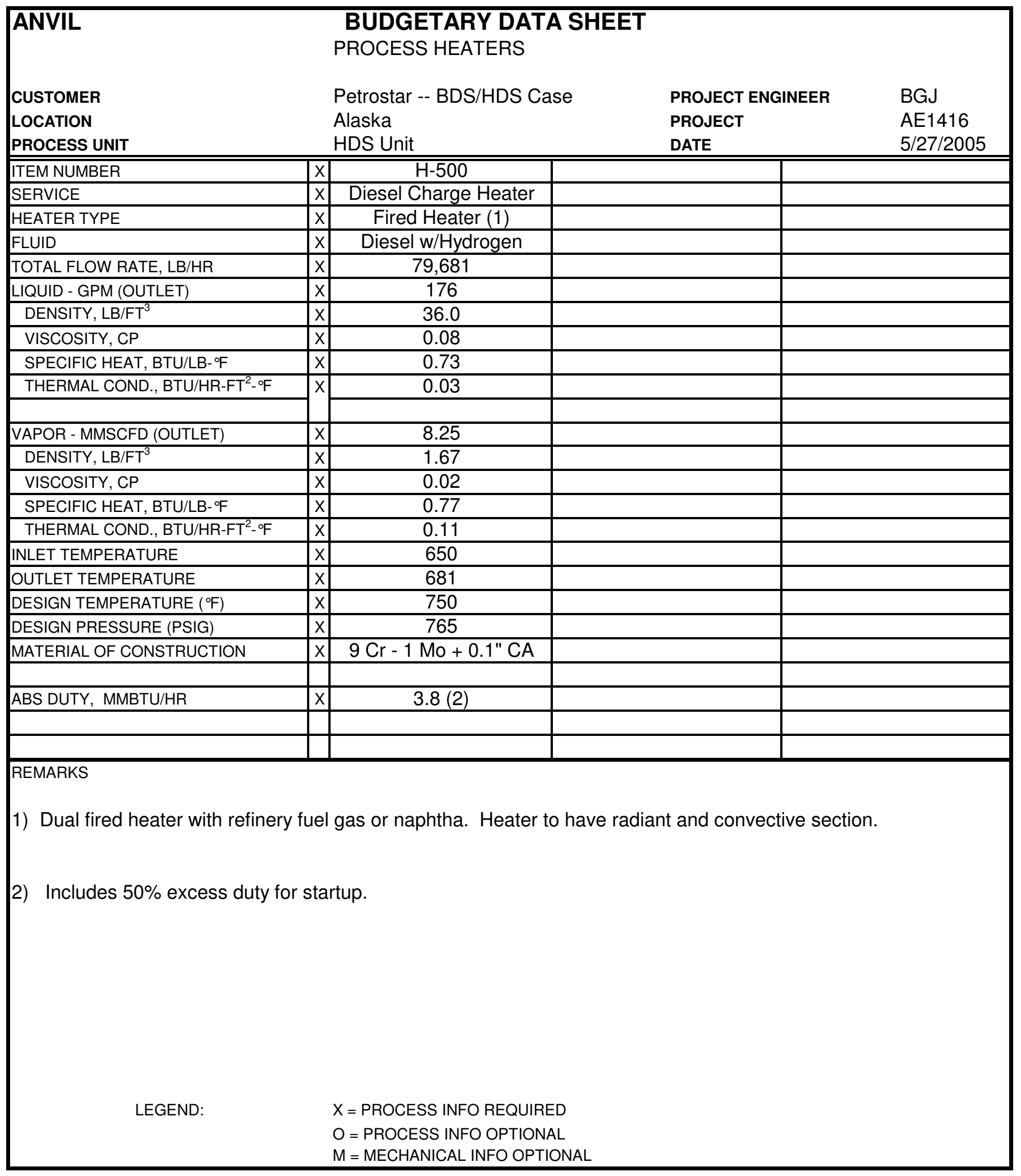




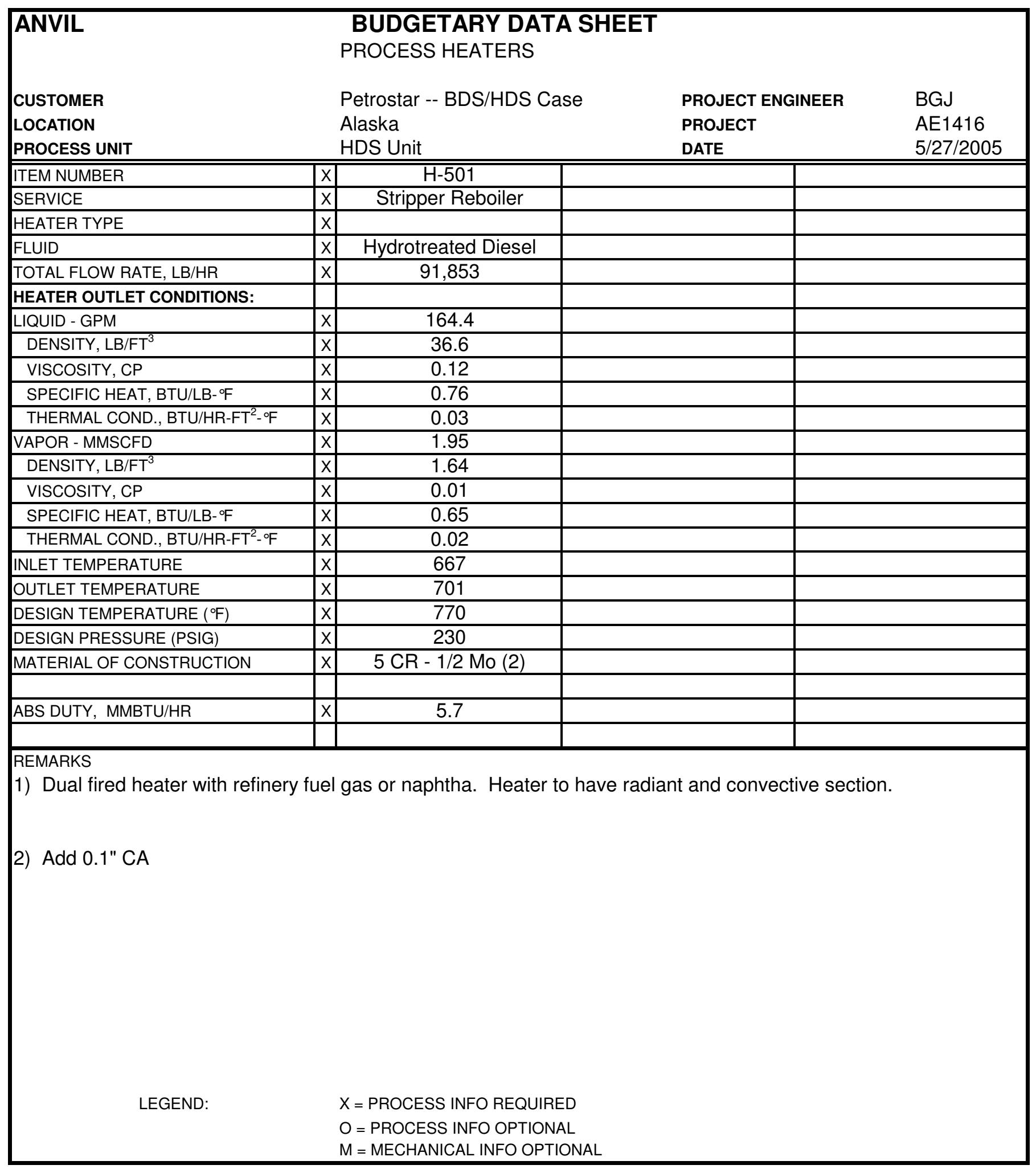




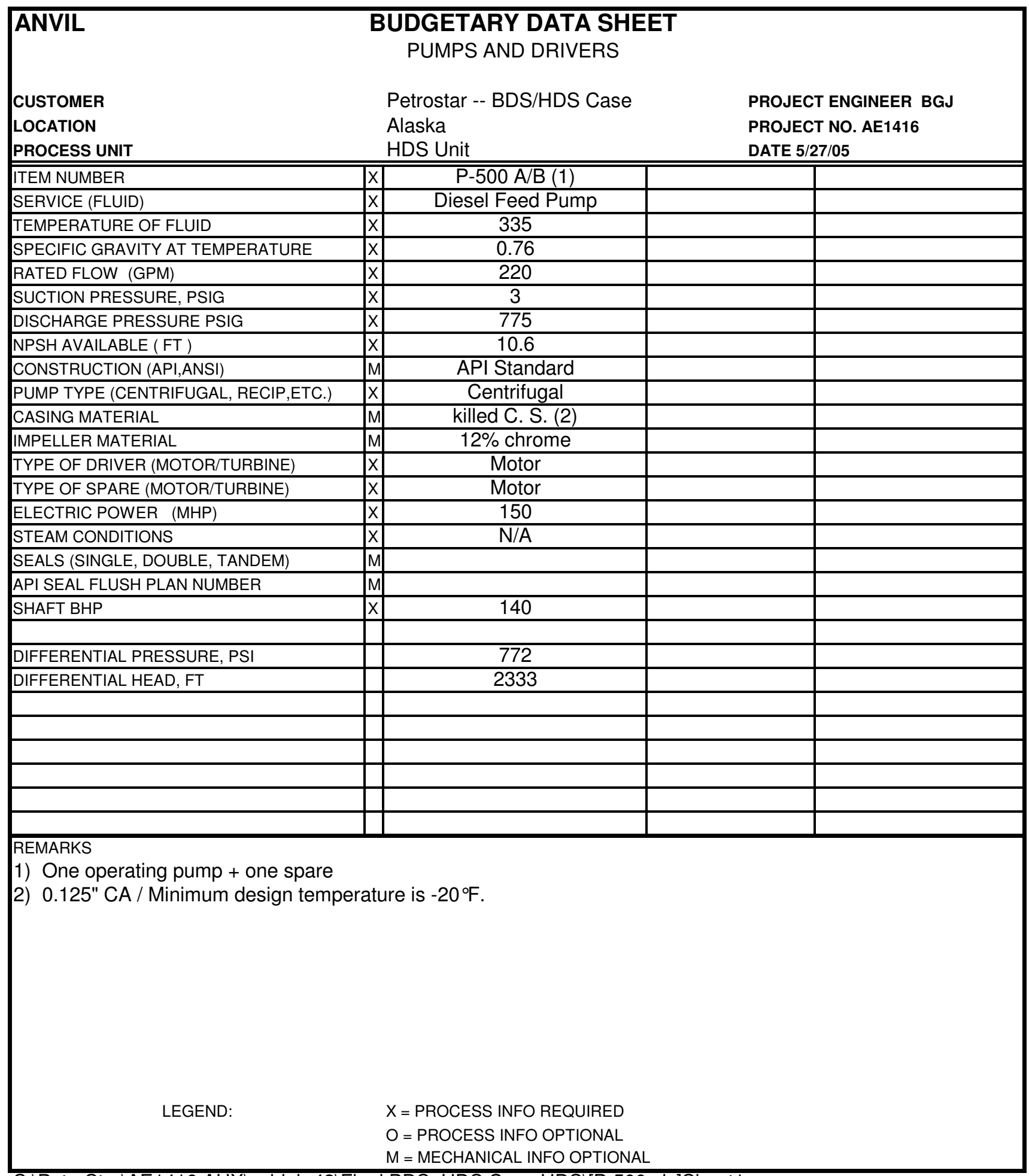




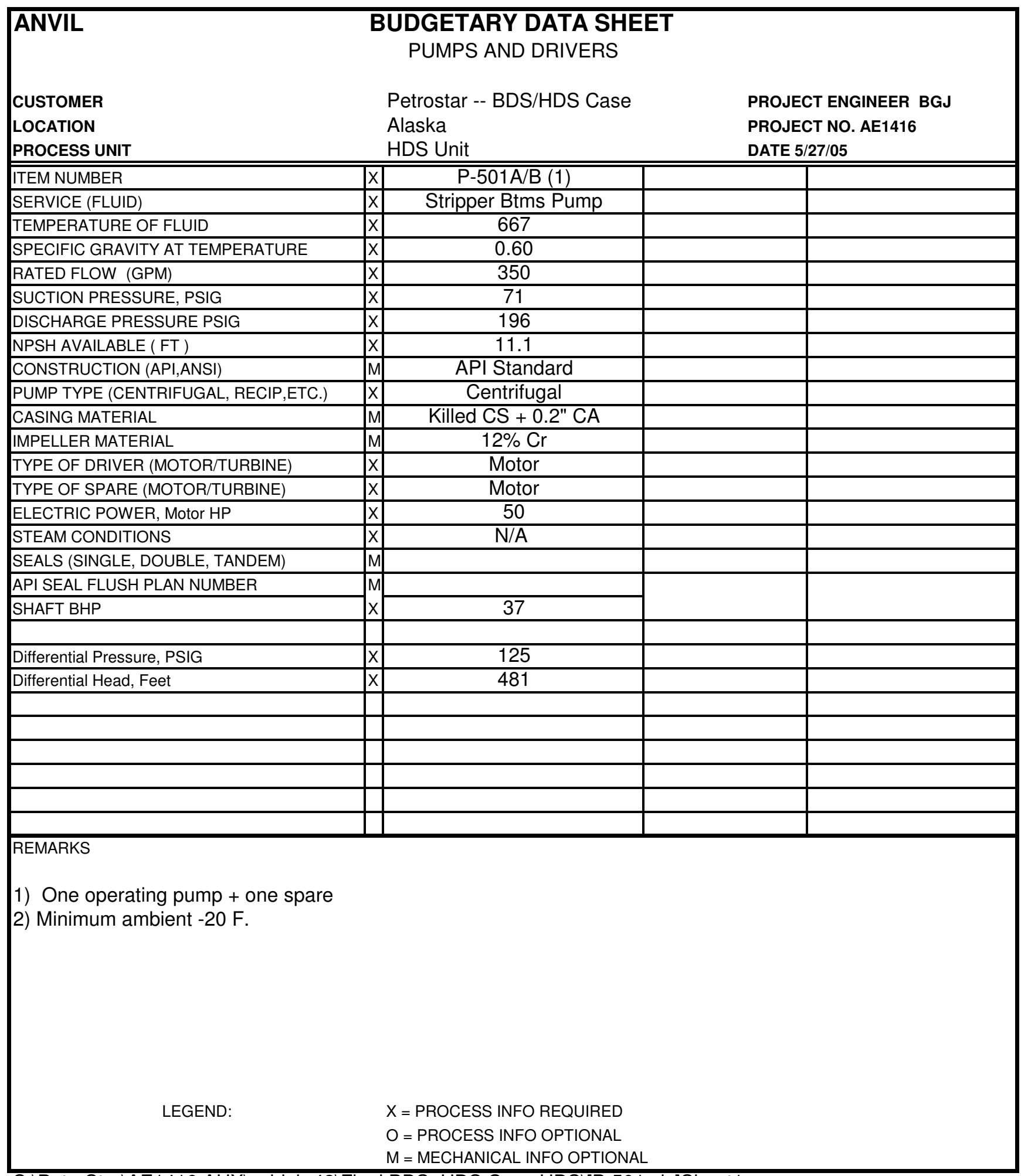

S:IPetroStar\AE1416.AUX|subjob 43|Final BDS_HDS Case HDS|[P-501.xls]Sheet1 


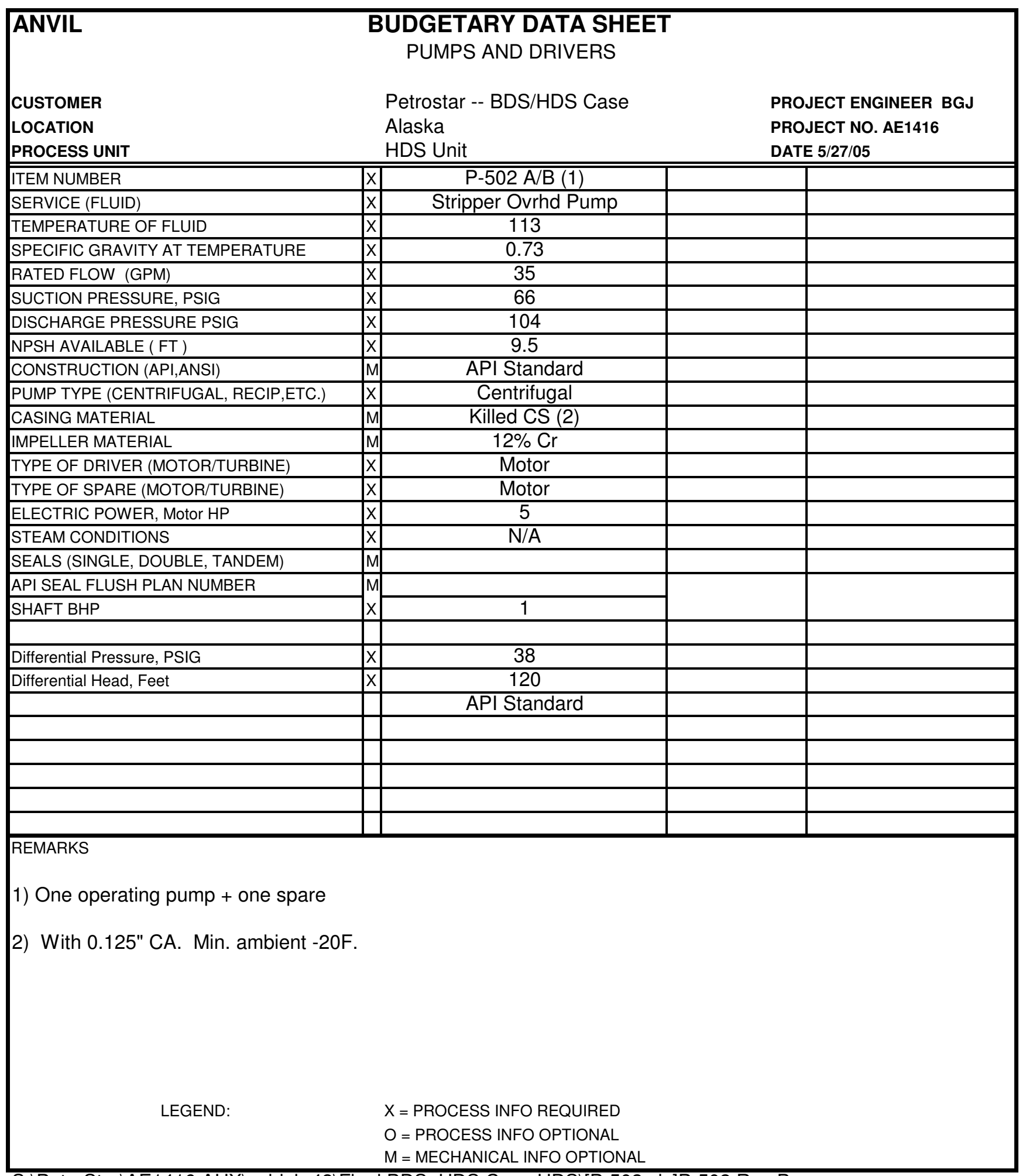

S:IPetroStar|AE1416.AUX|subjob 43|Final BDS_HDS Case HDS \[P-502.xls]P-502 Rev B 


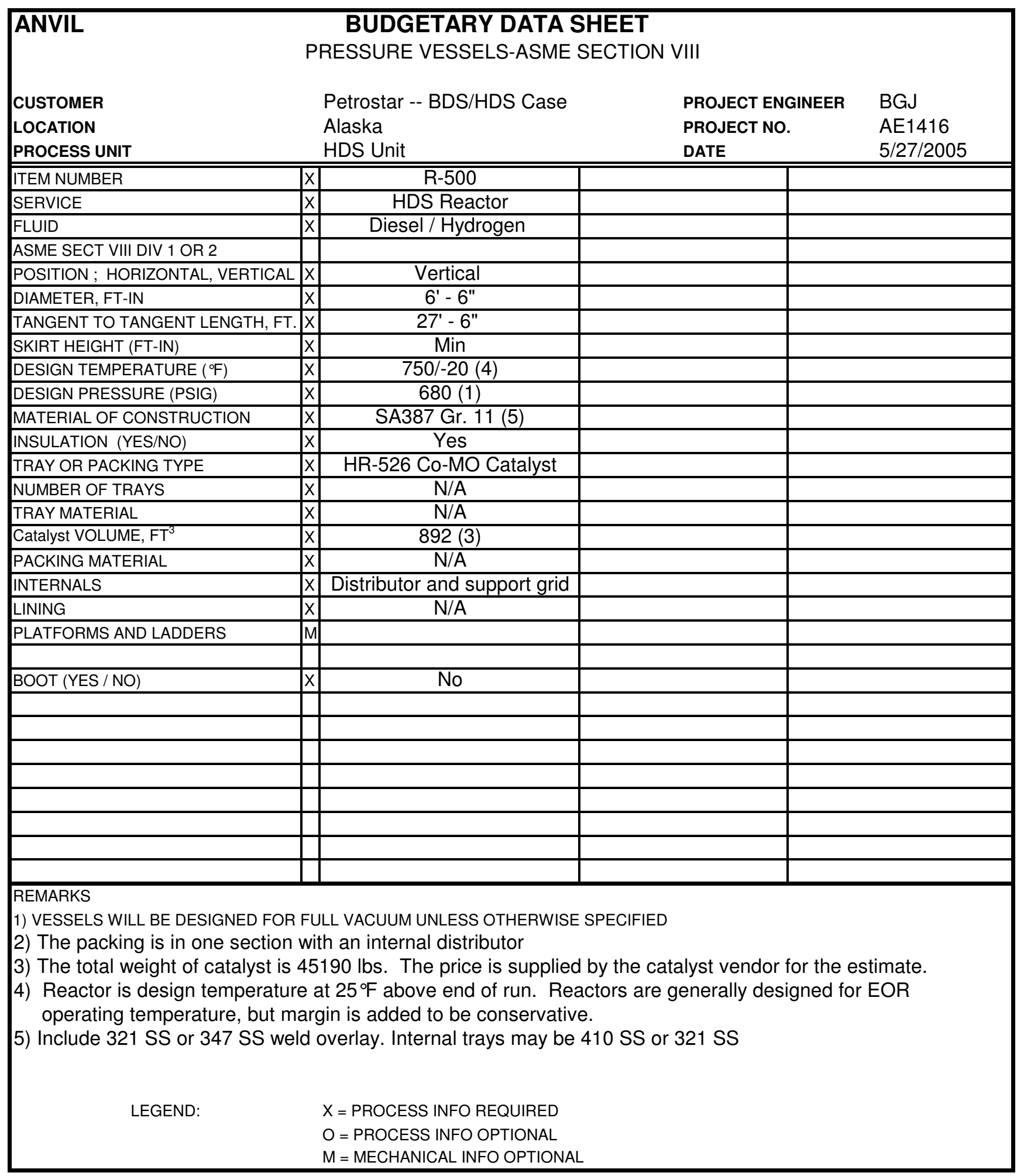

S:IPetroStarlAE1416.AUX|subjob 43|Final BDS_HDS Case HDS|[R-500.xls]Sheet1 


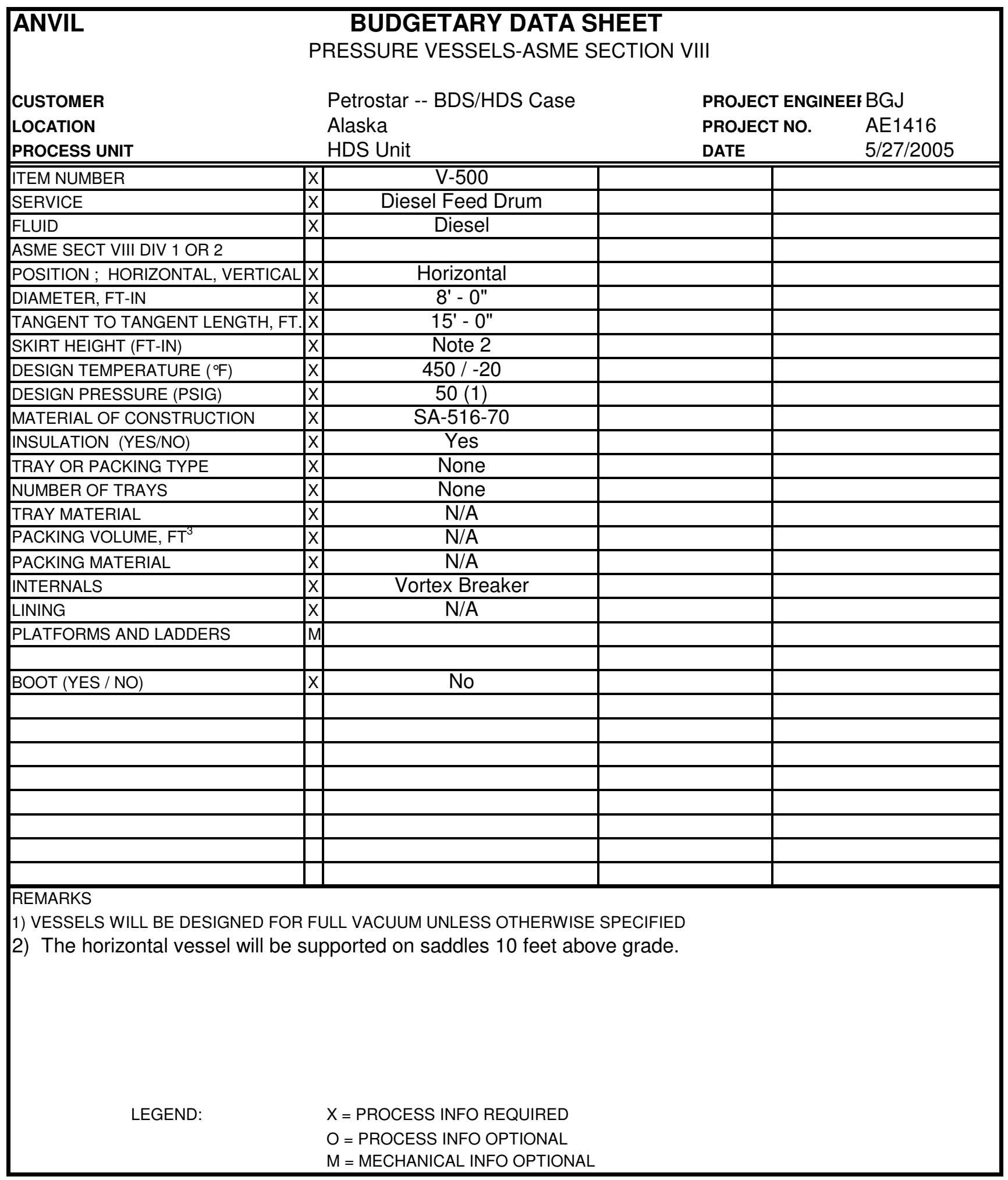

S:IPetroStarlAE1416.AUXIsubjob 43|Final BDS_HDS Case HDSI[V-500.xls]V-500 Rev B 


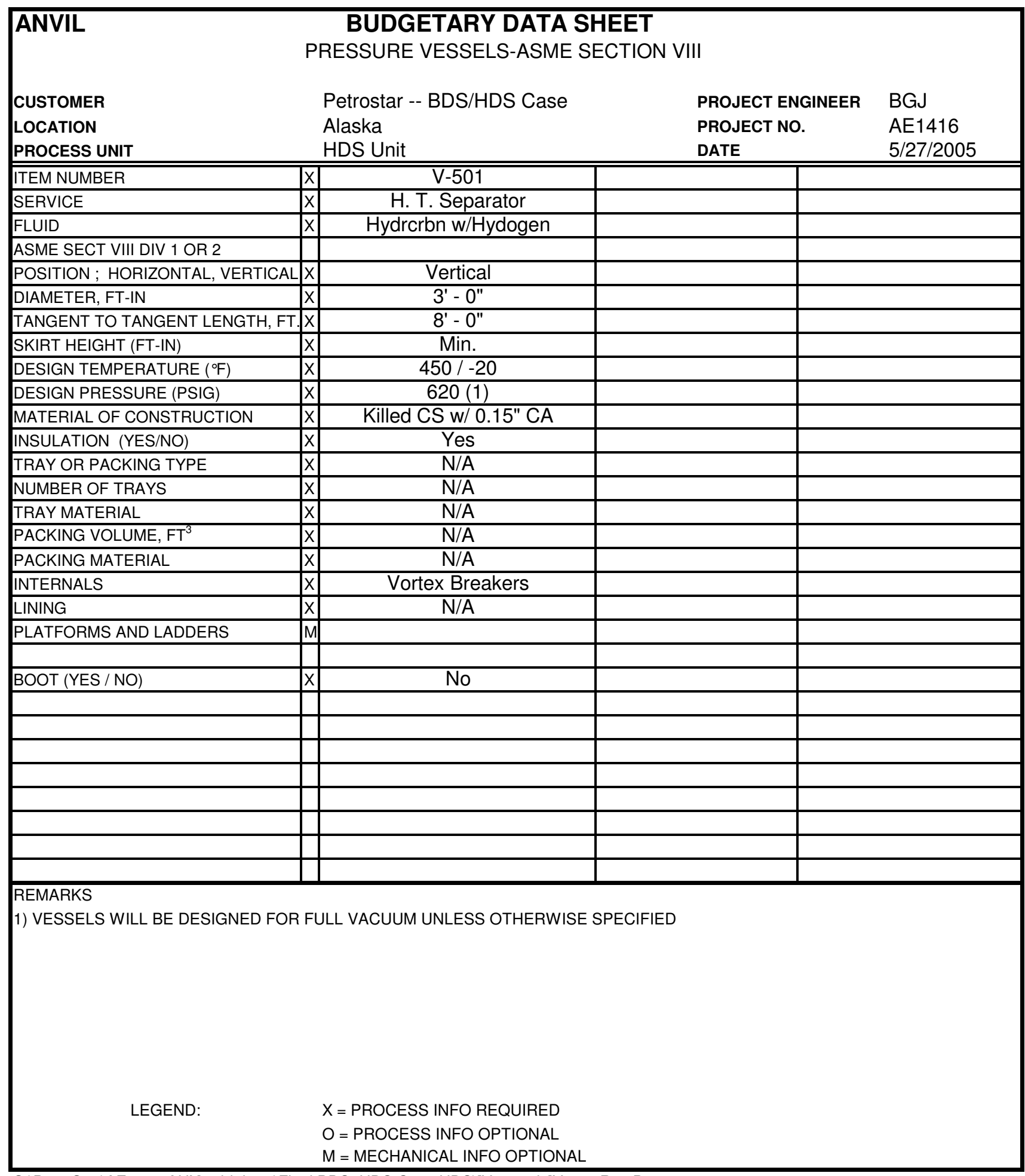

S:IPetroStar\AE1416.AUXIsubjob 43\Final BDS_HDS Case HDS\[V-501.xls]V-501 Rev B 


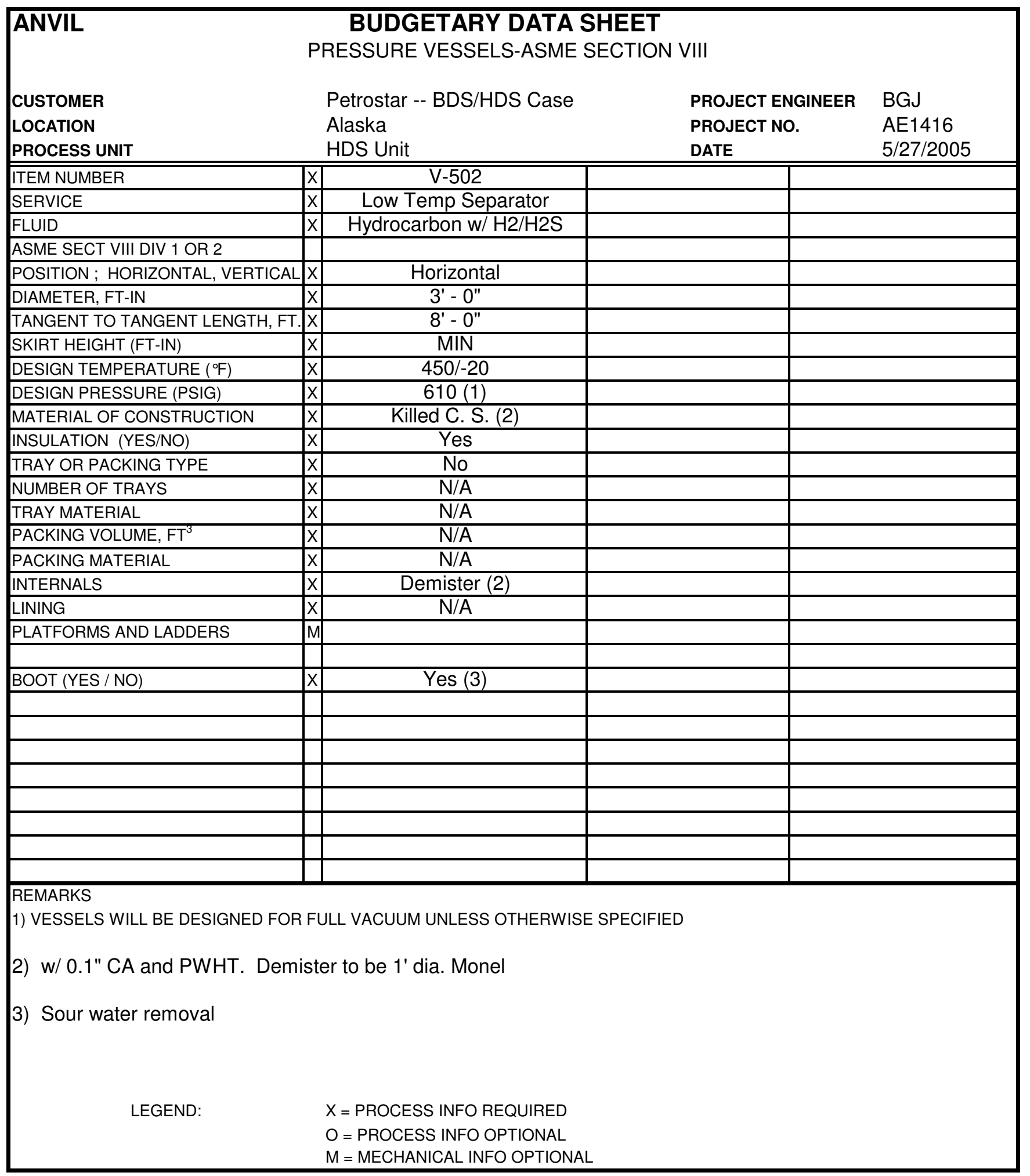

S:IPetroStarlAE1416.AUXIsubjob 43|Final BDS_HDS Case HDSI[V-502.xls]V-502 Rev B 


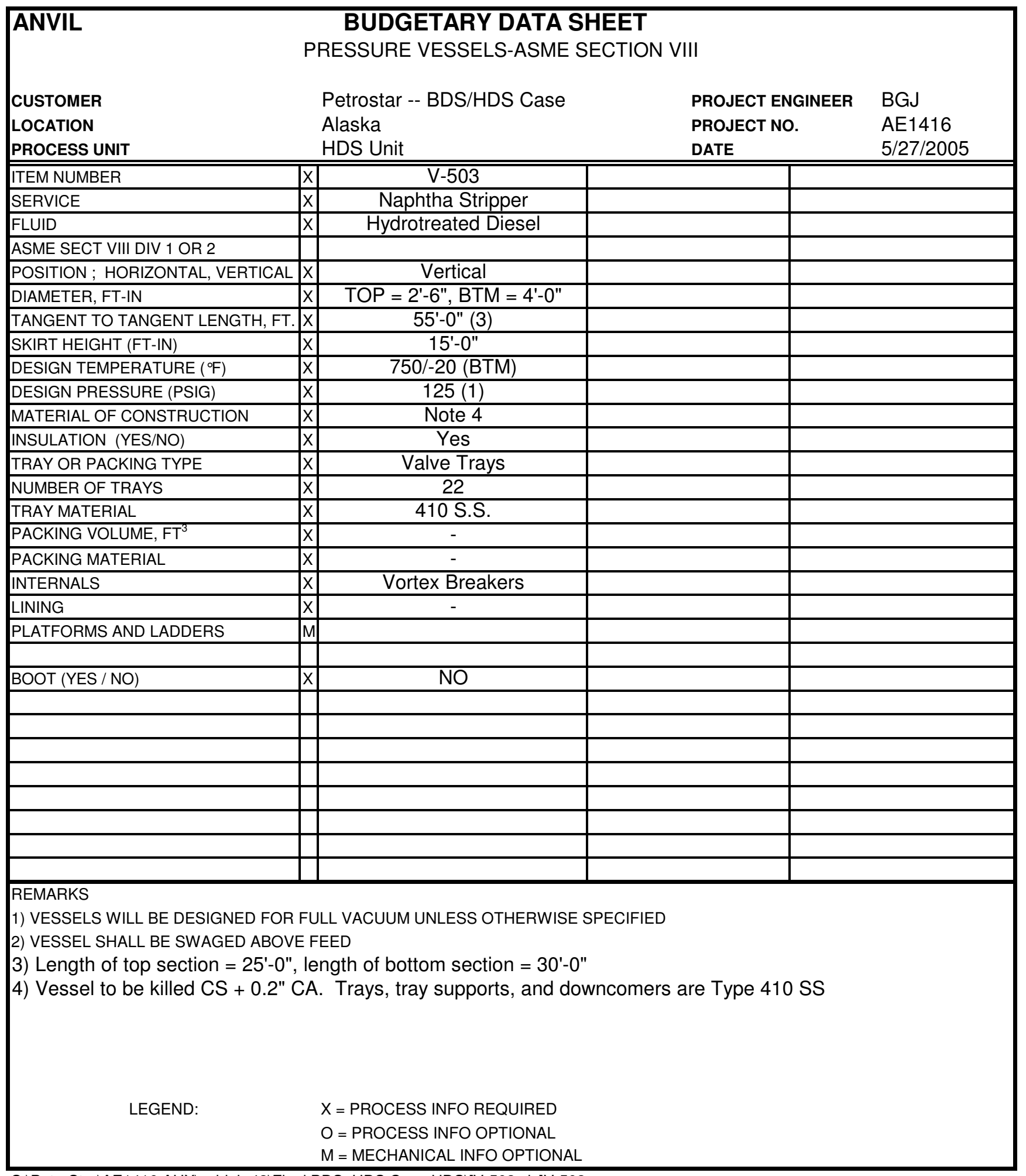




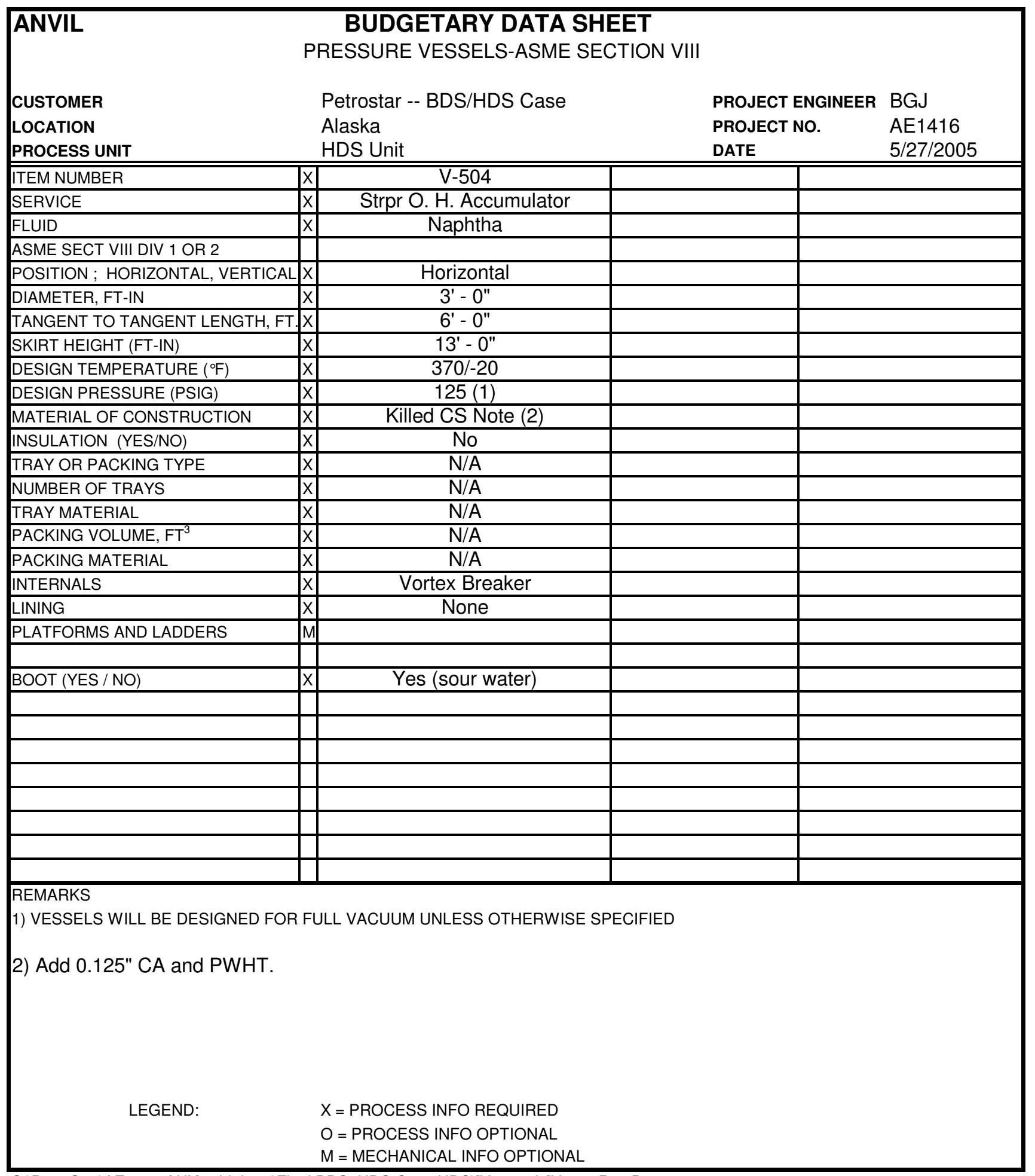




\section{Appendix B.6-Utility Requirements}

Utility Requirements

BDS/HDS Case

6000 BPD Feed Rate, 10 ppmw Sulfur ULSD Product

\begin{tabular}{|c|c|c|c|c|c|}
\hline & $\begin{array}{c}\text { Power } \\
\text { kW }\end{array}$ & $\begin{array}{l}\text { Fuel Gas } \\
\text { MMBTU/hr }\end{array}$ & $\begin{array}{c}\text { Process Water } \\
\underline{\text { lb/hr }}\end{array}$ & $\begin{array}{l}\text { BFW } \\
\text { lb/hr }\end{array}$ & $\begin{array}{l}\text { Steam } \\
\text { lb/hr }\end{array}$ \\
\hline \multicolumn{6}{|l|}{ IBL HDS } \\
\hline Feed Gas Compressor & 65 & & & & \\
\hline H2 Makeup Compressor & 85 & & & & \\
\hline Recycle Gas Compressor & 65 & & & & \\
\hline Charge Pump & 105 & & & & \\
\hline Stripper Reflux Pump & 1 & & & & \\
\hline Stripper Btms Circ & 28 & & & & \\
\hline Effluent Air Cooler & 4 & & & & \\
\hline Prod Stripper Condenser & 4 & & & & \\
\hline Prod Rundown Cooler & 11 & & & & \\
\hline Wash Water Injection Pump & 1 & & & & \\
\hline Charge Heater & & 4.8 & & & \\
\hline Prod Stripper Reboiler & & 7.1 & & & \\
\hline Wash Water Injection Pump & & & 1300 & & \\
\hline \multicolumn{6}{|l|}{$\underline{O B L}$} \\
\hline $\mathrm{H} 2$ Plant & 53 & 28.0 & & 4721 & \\
\hline Sulfur Recovery & 68 & & & & \\
\hline Other OBL Allowance & 118 & & & & \\
\hline \multirow[t]{2}{*}{ TOTAL HDS and OBL } & 608 & 39.9 & 1300 & 4721 & \\
\hline & $\begin{array}{l}\text { Power } \\
\text { kW }\end{array}$ & $\begin{array}{l}\text { Fuel Gas } \\
\text { MMBTU/hr }\end{array}$ & $\begin{array}{c}\text { Process Water } \\
\qquad \underline{\mathrm{lb} / \mathrm{hr}}\end{array}$ & $\begin{array}{l}\mathrm{BFW} \\
\mathrm{lb} / \mathrm{hr}\end{array}$ & $\begin{array}{l}\text { Steam } \\
\text { lb/hr }\end{array}$ \\
\hline BDS & & & & & \\
\hline$\overline{\text { Total Plant }}$ & 933 & & 3981 & & 14 \\
\hline TOTAL BDS & 933 & & 3981 & & 14 \\
\hline
\end{tabular}




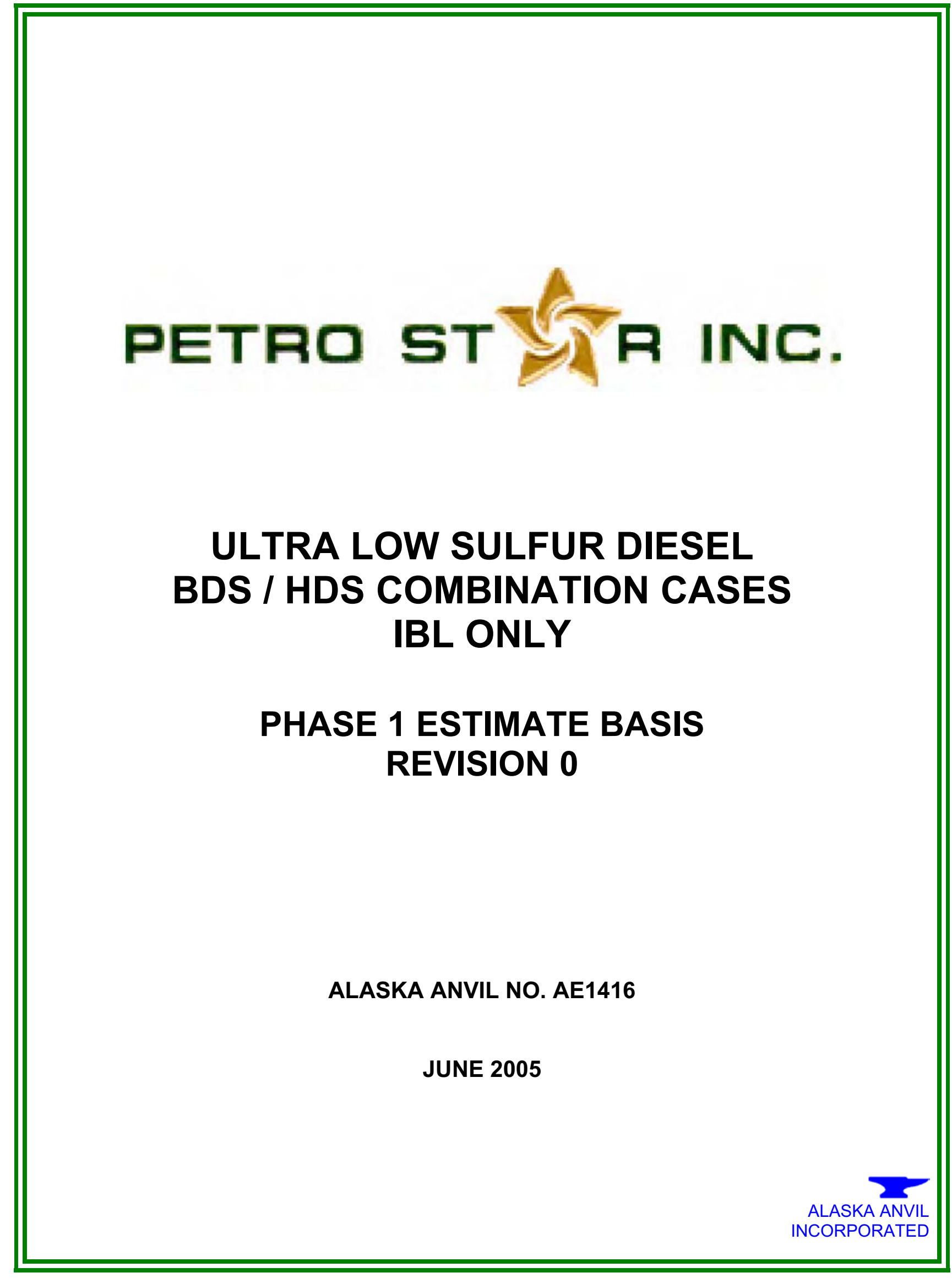




\section{ESTIMATE BASIS GOAL}

This Estimate Basis identifies information, qualifications, exceptions, and assumptions used in developing the cost estimate.

\section{ESTIMATE BASIS PURPOSE}

During the estimate review process, the project team uses the Estimate Basis for the following purposes:

- As a checklist of items to consider during estimate preparation.

- To document what is included and not included in the cost estimate.

- To assess cost risks of estimate components.

- As part of the decision support package for assessing the BDS process feasibility.

\section{GENERAL INFORMATION}

- The purpose of the project estimate is to determine if the ULSD BDS process is economically viable as a standalone process or in combination with an HDS Unit. These three (3) estimate scenarios address the BDS / HDS Combination cases.

- $\quad$ Estimate type:

- The estimate was developed using equipment based factored estimates for Inside Battery Limits (IBL) costs.

- There is a separate equipment-factoring summary for the BDS, HDS, and Pre Frac Equipment (Pre Frac Case Only)

- Most of the equipment pricing was derived from the ICARUS estimating program. Pricing for BDS equipment marked with an asterisk $\left(^{*}\right)$ was provided by Pelorus.

- The cost of the Hydrogen and Sulfur Units were factored off licensor quotes obtained for the standalone HDS case.

- Outside Battery Limits (OBL) costs have been excluded from this estimate.

- $\quad$ The project will be installed in a brownfield location within the Valdez Alaska Refinery.

\section{PROCESS BASIS}

\section{Facility Data}

- $\quad$ Facility type - Ultra Low Sulfur Diesel Treating Complex, which includes:

- Diesel Biodesulfurization Unit

- Diesel Hydrotreating Unit

- Hydrogen Production Unit

- Sulfur Unit

- Diesel Splitter (Pre Frac Case Only) 


\section{Design Basis}

Product specification - Feed 6,000 bpd of untreated diesel to produce 10-ppmw sulfur maximum ultra low sulfur diesel.

\section{COST BASIS}

\section{Labor, Indirects, Equipment, and Bulk Materials}

- Included in the equipment factor.

\section{Project Services}

- $\quad$ Estimated based on 15 percent of TIC for the BDS, HDS, and Pre Frac units; engineering costs for the Hydrogen and Sulfur Units was included in the licensor pricing.

\section{Owner Services}

Not included in the TIC cost. Historically, owner services will cost from 5 to 7 percent of TIC, not including licensing, royalties, or catalyst.

\section{Escalation}

Project is based on 2005 costs. No escalation is included.

\section{Location Factor}

All costs for this estimate have been developed from a U.S. Gulf Coast (USGC) basis. No location factor is included.

\section{Other Costs}

- Catalyst and chemical initial charge has been added as an additional line item.

- CEMS, air preheating, and burner management allowances have been added to the fired heater costs.

\section{ASSUMPTIONS}

- Process licensing and royalty costs are not included.

- Assumes fully installed pump spares, but no warehouse spares. 
Final Technical Progress Report DOE Award No. DE-FC26-02NT15340

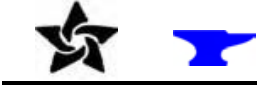

CLIENT: PetroStar

PROJECT: PetroStar Valdez ULSD - BDS/HDS Combo STAGE: Phase 1

\section{PROJECT DESCRIPTION:}

Install Ultra Low Sulfur Diesel Complex.

PROJECT COST ESTIMATE SUMMARY

COST ESTIMATE STRUCTURE
COST ESTIMATE PARAMETERS
Estimate Classification
Estimating Method
COST ESTIMATE SUMMARY
Expected Cost (\$MM)
High Range (\$MM)
Low Range (\$MM)

PROJECT COST ESTIMATE ANALYSIS

Total Project Expected Cost Component Analysis

CLIENT PROJECT NO.:

ANVIL PROJECT NO.: AE1416

REV NO.: 0

ANVIL PE: L. Nace

Date: 6/17/05

PROJECT RISKS:

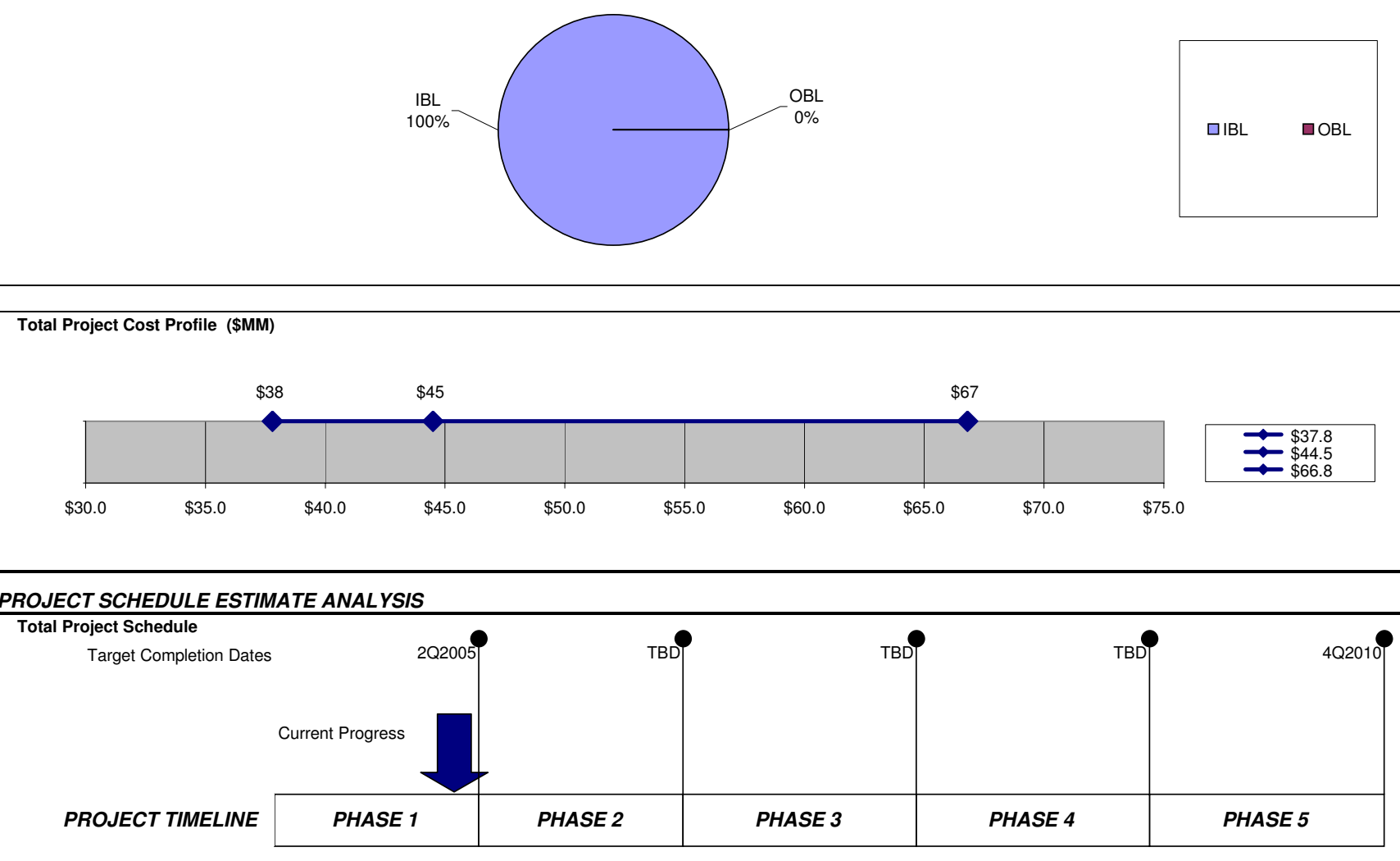

\begin{tabular}{|c|c|}
\hline & $\begin{array}{c}\text { TOTAL PROJECT } \\
\text { COST - Valdez }\end{array}$ \\
\hline & Phase 1 \\
\hline & Factored/ROM \\
\hline$\$$ & 44.5 \\
\hline$\$$ & 66.8 \\
\hline$\$$ & 37.8 \\
\hline
\end{tabular}


Petro Star Ultra Low Sulfur Diesel (6000 BPD) Project - Valdez Refinery

IBL Impact Matrix - 2005\$ - Total Installed Costs USGC

Scenario: BDS/HDS Combo

\begin{tabular}{|l|l|l|l|l||c|}
\hline $\begin{array}{c}\text { IBL } \\
\text { Component }\end{array}$ & \multicolumn{1}{c|}{ HDS } & \multicolumn{1}{c|}{ BDS } & Hydrogen & \multicolumn{1}{c|}{ Sulfur } & Total Cost \\
\hline & Unit & Unit & Unit & Unit & \$MM \\
\hline & $\begin{array}{l}\text { Diesel } \\
\text { hydrotreater, } \\
\text { feed rate of } \\
\text { 6000 B/D, } \\
\text { producing } \\
\text { ULSD (10 } \\
\text { ppmw sulfur) }\end{array}$ & $\begin{array}{l}\text { Biodesulfurizat } \\
\text { ion unit, feed } \\
\text { rate of 6000 } \\
\text { BPD, } \\
\text { producing LSD }\end{array}$ & $\begin{array}{l}\text { H.7 MMSCF/D } \\
\text { Plant. }\end{array}$ & $\begin{array}{l}\text { Thiopaq } \\
\text { process to be } \\
\text { used for 2.4 } \\
\text { T/D. Will also } \\
\text { need sulfur } \\
\text { storage / } \\
\text { handling }\end{array}$ & \\
\hline
\end{tabular}


Final Technical Progress Report

ANVIL CORPORATION

OE Award No. DE-FC26-02NT15340

PROJECT: PetroStar Valdez ULSD - BDS/HDS Combo ANVIL NO: AE1416

CLIENT: PetroStar
DATE: 6/17/05

REV NO.: 0

HDS EQUIPMENT - FACTORING SUMMARY

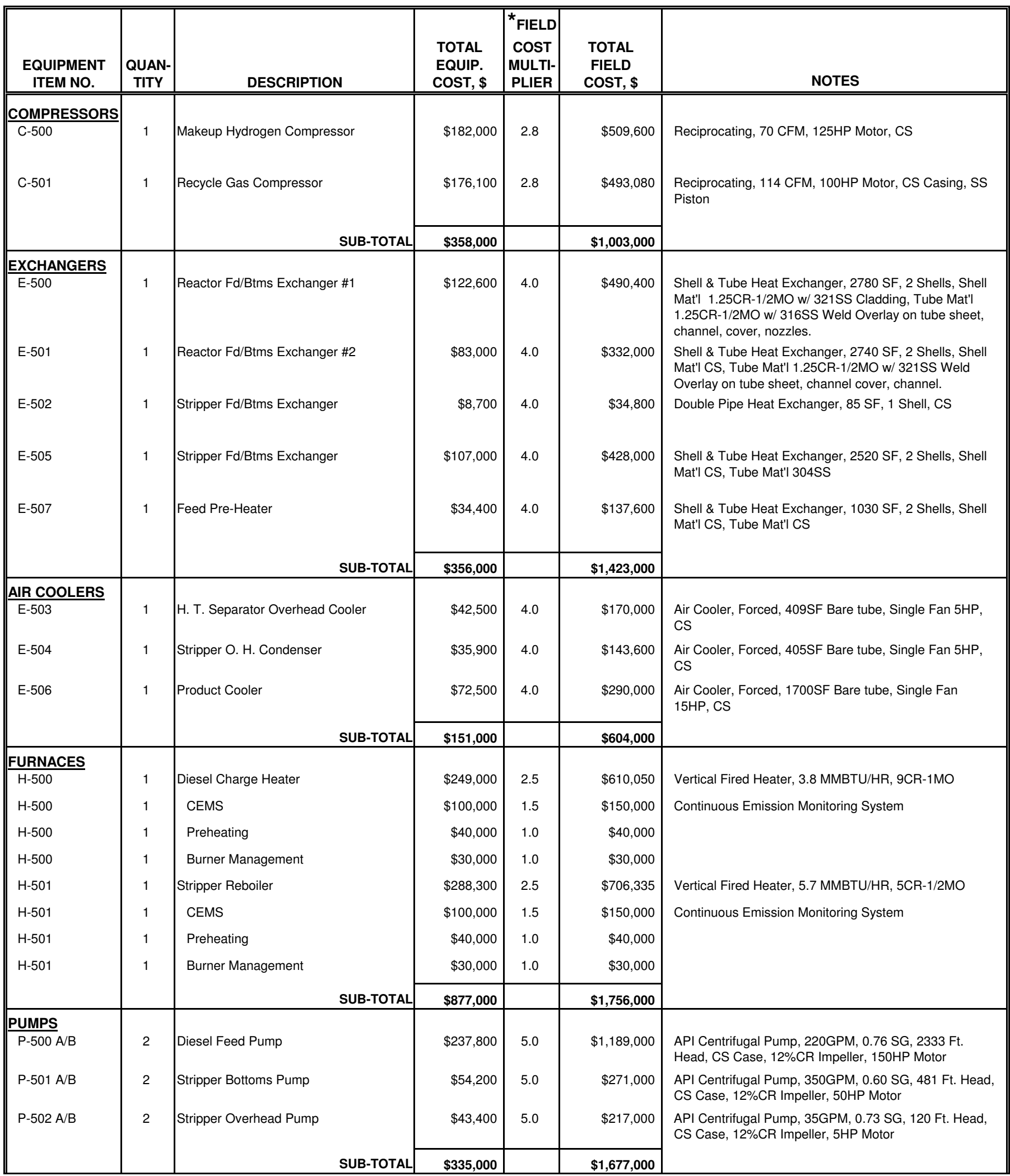


Final Technical Progress Report

ANVIL CORPORATION

OE Award No. DE-FC26-02NT15340

PROJECT: PetroStar Valdez ULSD - BDS/HDS Combo ANVIL NO: AE1416

CLIENT: PetroStar

DATE: 6/17/05

REV NO.: 0

HDS EQUIPMENT - FACTORING SUMMARY

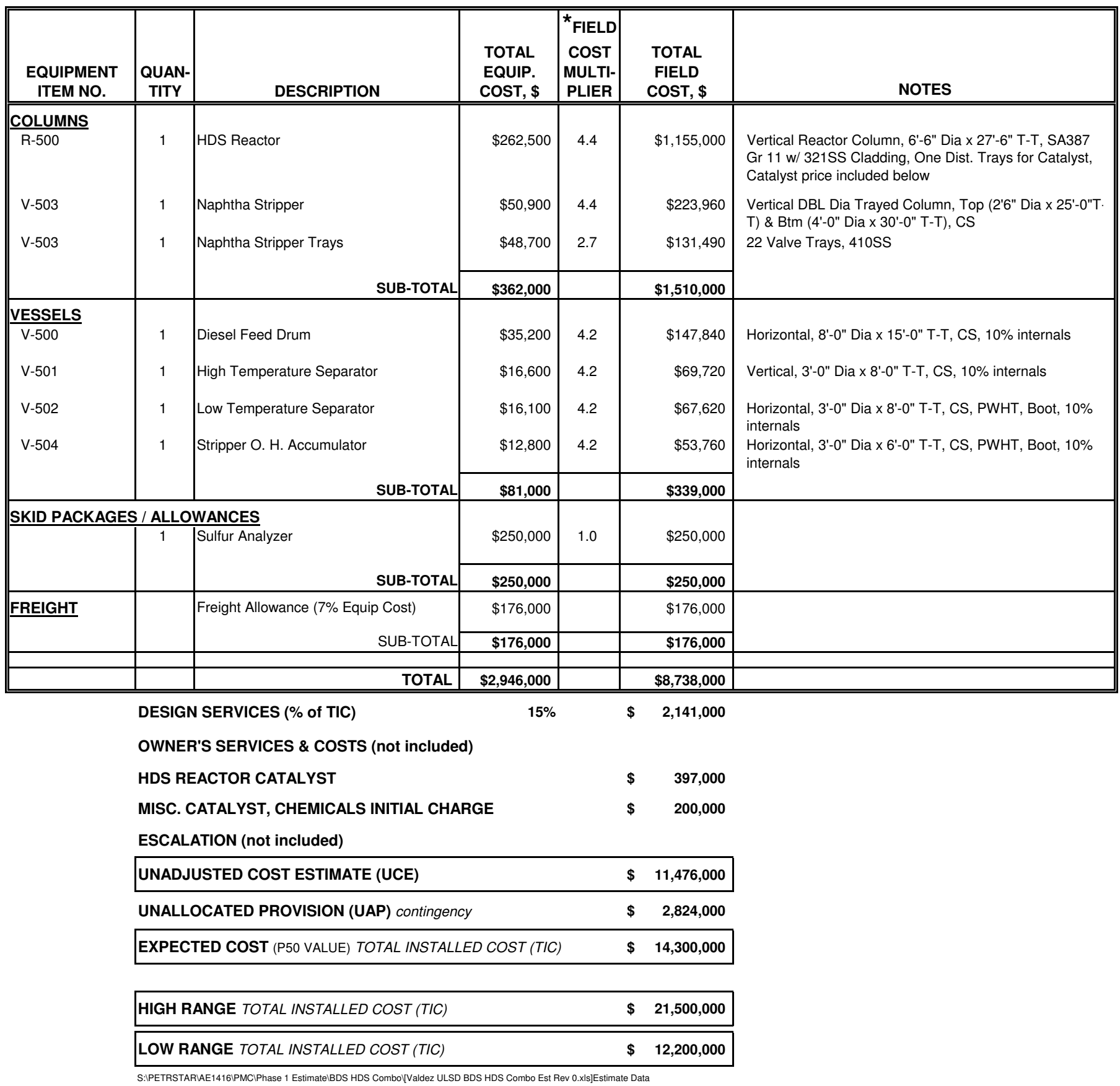

*Note: Field Cost Multiplier includes the following bulk material and installation labor: Civil, Concrete, Structural, Piping, Electrical, Instrumentation, Insulation, Fireproofing, Painting, and Testing. 
A N V I L C O R P O R A T I O N Final Technical Progress Report

PROJECT: PetroStar Valdez ULSD - BDS/HDS Combo ANVIL NO: AE1416

DOECAlvafdpetrosier-FC26-02NT15340

DATE: $6 / 17 / 05$

REV NO.: 0

BDS EQUIPMENT - FACTORING SUMMARY

\begin{tabular}{|c|c|c|c|c|c|c|}
\hline $\begin{array}{l}\text { EQUIP. } \\
\text { ITEM NO. }\end{array}$ & $\begin{array}{l}\text { QUAN- } \\
\text { TITY }\end{array}$ & DESCRIPTION & $\begin{array}{l}\text { TOTAL } \\
\text { EQUIP. } \\
\text { COST, \$ }\end{array}$ & $\begin{array}{c}\text { FIELD } \\
\text { COST } \\
\text { MULTI- } \\
\text { PLIER }\end{array}$ & $\begin{array}{l}\text { TOTAL } \\
\text { FIELD } \\
\text { COST, \$ }\end{array}$ & NOTES \\
\hline C-101 & 1 & Diesel Feed Cooler & $\$ 31,500$ & 4.0 & $\$ 126,000$ & $\begin{array}{l}1 \text { (ea) - Shell \& Tube TEMA type Heat Exchanger, } \\
\text { AES, } 1750 S F, \text { CS }\end{array}$ \\
\hline C-501 & 1 & Vent Gas Chiller & $\$ 18,100$ & 4.0 & $\$ 72,400$ & $\begin{array}{l}1 \text { (ea) - Shell \& Tube TEMA type Heat Exchanger, } \\
\text { AES, 500SF, CS }\end{array}$ \\
\hline C-601 & 1 & Water Chiller & $\$ 49,000$ & 4.0 & $\$ 196,000$ & $\begin{array}{l}\text { 8.6 MM BTU/Hr, } 3790 \text { SF w/ } 1 \text { shell, TEMA type AES, } \\
\text { Tubes: CS , Shell: CS }\end{array}$ \\
\hline \multicolumn{3}{|r|}{ SUB-TOTAL } & $\$ 99,000$ & & $\$ 394,000$ & \\
\hline \multicolumn{3}{|c|}{ PRESSURE VESSELS } & & & & \\
\hline D-101 & 1 & Diesel Feed Drum & $\$ 39,300$ & 4.2 & $\$ 165,060$ & 1(ea) - Horizontal Vessel, 8'0" DIA x 20'-0" T-T, CS \\
\hline $\mathrm{D}-201^{*}$ & 1 & Fermentor Reactor & $\$ 173,000$ & 4.2 & $\$ 726,600$ & 1 (ea) - 10,000 gal airlift fermenter \\
\hline $\mathrm{D}-202^{*}$ & 1 & BDS Reactor \#1 & $\$ 279,000$ & 4.2 & $\$ 1,171,800$ & 1 (ea) $-13,000$ gal airlift reactor \\
\hline $\mathrm{D}-203^{*}$ & 1 & BDS Reactor \#2 & $\$ 279,000$ & 4.2 & $\$ 1,171,800$ & 1 (ea) $-13,000$ gal airlift reactor \\
\hline $\mathrm{D}-204^{*}$ & 1 & BDS Reactor \#3 & $\$ 279,000$ & 4.2 & $\$ 1,171,800$ & 1 (ea) $-13,000$ gal airlift reactor \\
\hline$D-301$ & 1 & Diesel / Water / Biomass Separator & $\$ 112,400$ & 4.2 & $\$ 472,080$ & $\begin{array}{l}\text { 1(ea) - Horizontal Vessel, 10'3" DIA x 30'-8" T-T, } \\
\text { 304SS, w/ overflow baffle }\end{array}$ \\
\hline L-301 & 2 & Oil/Water Separator & $\$ 41,800$ & 4.2 & $\$ 175,560$ & $\begin{array}{l}\text { 2(ea) - Horizontal Vessel, 2'4" DIA x 11'-0" T-T, SS, w/ } \\
\text { Fiberbed Coalescer } 24 " \text { OD x } 18 \text { " ID x } 120 \text { "long. }\end{array}$ \\
\hline \multicolumn{3}{|r|}{ SUB-TOTAL } & $\$ 1,287,000$ & & $\$ 5,404,000$ & \\
\hline \multicolumn{3}{|c|}{ PACKAGED SKIDS/SYSTEMS } & & & & \\
\hline $\mathrm{D}-205^{*}$ & 1 & Seed Tank A & $\$ 94,000$ & 2.5 & $\$ 235,000$ & Packaged Seed Fermenter System \\
\hline$D-206^{*}$ & 1 & Seed Tank B & $\$ 328,000$ & 2.5 & $\$ 820,000$ & Packaged Seed Fermenter System \\
\hline $\mathrm{J}-109 \mathrm{~A} / \mathrm{B}$ & 2 & Air Compressor & $\$ 354,600$ & 2.5 & $\$ 886,500$ & $\begin{array}{l}\text { 2(ea) - Air Compressor, Screw, 1930ACFM, CS } \\
\text { body/internals, } 175 \mathrm{HP} \text { Motor Driven }\end{array}$ \\
\hline $\mathrm{L}-109^{*}$ & 1 & Glucose Sterilizer & $\$ 47,000$ & 2.5 & $\$ 117,500$ & $\begin{array}{l}1(\text { ea) - Packaged } 15 \mathrm{gph} \text { continuous steam sterilizer } \\
\text { system complete w/ preheater, heater, cooler, \& } \\
\text { holding coil }\end{array}$ \\
\hline $\mathrm{L}-110^{*}$ & 1 & Nutrients Sterilizer & $\$ 47,000$ & 2.5 & $\$ 117,500$ & $\begin{array}{l}1(\text { ea) - Packaged } 15 \text { gph continuous steam sterilizer } \\
\text { system complete w/ preheater, heater, cooler, \& } \\
\text { holding coil }\end{array}$ \\
\hline L-302* & 1 & Electrostatic Precipitator & $\$ 442,000$ & 2.5 & $\$ 1,105,000$ & 1(ea) - Packaged ESP Unit \\
\hline
\end{tabular}


A N V I L C O R P O R A T I O N Final Technical Progress Report

PROJECT: PetroStar Valdez ULSD - BDS/HDS Combo ANVIL NO: AE1416

DOECAlvard_Netrosper-FC26-02NT15340

DATE: $6 / 17 / 05$

REV NO.: 0

BDS EQUIPMENT - FACTORING SUMMARY

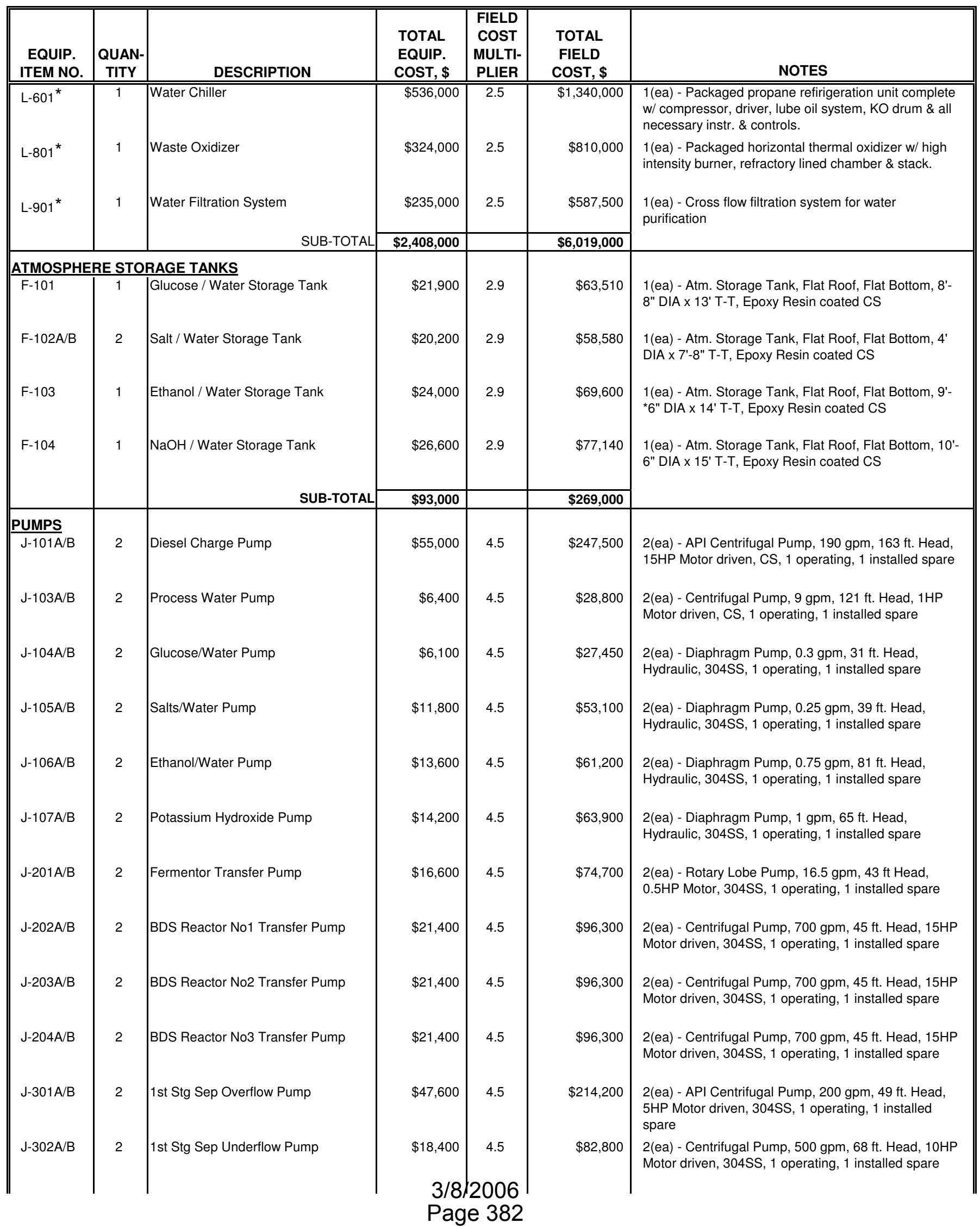


A N V I L C O R P O R A T I O N Final Technical Progress Report

PROJECT: PetroStar Valdez ULSD - BDS/HDS Combo ANVIL NO: AE1416

DOE CAleafdpNotrostar-FC26-02NT15340

DATE: $6 / 17 / 05$

REV NO.: 0

BDS EQUIPMENT - FACTORING SUMMARY

\begin{tabular}{|c|c|c|c|c|c|c|}
\hline $\begin{array}{l}\text { EQUIP. } \\
\text { ITEM NO. }\end{array}$ & $\begin{array}{l}\text { QUAN- } \\
\text { TITY }\end{array}$ & DESCRIPTION & $\begin{array}{l}\text { TOTAL } \\
\text { EQUIP. } \\
\text { COST, \$ }\end{array}$ & $\begin{array}{l}\text { FIELD } \\
\text { COST } \\
\text { MULTI- } \\
\text { PLIER } \\
\end{array}$ & $\begin{array}{l}\text { TOTAL } \\
\text { FIELD } \\
\text { COST, } \$\end{array}$ & NOTES \\
\hline $\mathrm{J}-304 \mathrm{~A} / \mathrm{B}$ & 2 & 2nd Stg Sep Underflow Pump & $\$ 10,200$ & 4.5 & $\$ 45,900$ & $\begin{array}{l}\text { 2(ea) - Centrifugal Pump, } 45 \mathrm{gpm}, 72 \mathrm{ft} \text {. Head, 3HP } \\
\text { Motor driven, 304SS, } 1 \text { operating, } 1 \text { installed spare }\end{array}$ \\
\hline \multirow[t]{2}{*}{$J-601 \mathrm{~A} / \mathrm{B}$} & 2 & Chilled Water Pump & $\$ 19,800$ & 4.5 & $\$ 89,100$ & $\begin{array}{l}\text { API Centrifugal Pump, 2000GPM, 0.95 SG, } 81.7 \mathrm{Ft} . \\
\text { Head, CS Case, 12\%CR Impeller, 30HP Motor }\end{array}$ \\
\hline & & SUB-TOTAL & $\$ 304,000$ & & $\$ 1,369,000$ & \\
\hline L-102 & 1 & Diesel Pre-Filter Vessel & $\$ 5,280$ & 4.2 & $\$ 22,176$ & $\begin{array}{l}\text { 1(ea) - Filter housing, } 316 \mathrm{LSS}, 47 " \mathrm{~T}-\mathrm{T} \text { x } 8 \text { " Dia. w/ } \\
5(\text { ea) - Polypropylene element, 30" long, } 0.2 \mathrm{um} \text { pore } \\
\text { size }\end{array}$ \\
\hline L-103 & 1 & Air Pre-Filter Vessel & $\$ 11,400$ & 4.2 & $\$ 47,880$ & $\begin{array}{l}\text { 1(ea) - Filter housing, 316L SS, 55" T-T x 15" Dia. w/ } \\
5(\text { ea) - GF element, 30" long, 1.0um pore size }\end{array}$ \\
\hline L-104 & 1 & Air Bio-Filter Vessel & $\$ 11,125$ & 4.2 & $\$ 46,725$ & $\begin{array}{l}\text { 1(ea) - Filter housing, } 316 \mathrm{~L} \mathrm{SS}, 43 \text { " T-T x 15" Dia. w/ } 5 \\
\text { (ea) - PTFE element, } 10 \text { "long, } 0.01 \text { um pore size }\end{array}$ \\
\hline L-105 & 1 & BDS Reactor \#1 Air Bio Filter Vessel & $\$ 12,420$ & 4.2 & $\$ 52,164$ & $\begin{array}{l}\text { 1(ea) - Filter housing, 316L SS, 55" T-T x 15" Dia. w/ } \\
5(\text { ea) - PTFE element, 30" long, 0.01um pore size }\end{array}$ \\
\hline L-106 & 1 & BDS Reactor \#2 Air Bio Filter Vessel & $\$ 12,420$ & 4.2 & $\$ 52,164$ & $\begin{array}{l}\text { 1(ea) - Filter housing, 316L SS, 55" T-T x 15" Dia. w/ } \\
\text { 5(ea) - PTFE element, 30" long, 0.01um pore size }\end{array}$ \\
\hline $\mathrm{L}-111$ & 1 & Ethanol Bio-Filter Vessel & $\$ 1,308$ & 4.2 & $\$ 5,494$ & $\begin{array}{l}\text { 1(ea) - Filter housing, 316L SS, } 10 " \text { T-T x } 2.5 \text { " Dia. w/ } \\
1 \text { (ea) - Polyethersulfone element, } 5 \text { " long, } 0.2 \text { um pore } \\
\text { size }\end{array}$ \\
\hline L-112 & 1 & $\mathrm{NAaOH}$ Bio-Filter Vessel & $\$ 1,308$ & 4.2 & $\$ 5,494$ & $\begin{array}{l}\text { 1(ea) - Filter housing, } 316 \mathrm{~L} \mathrm{SS}, 10 " \text { T-T x } 2.5 \text { " Dia. w/ } \\
1 \text { (ea) - Polyethersulfone element, } 5 \text { " long, } 0.2 \mathrm{um} \text { pore } \\
\text { size }\end{array}$ \\
\hline L-113 & 1 & Water Pre-Filter Vessel & $\$ 2,850$ & 4.2 & $\$ 11,970$ & $\begin{array}{l}\text { 1(ea) - Filter housing, 316L SS, 38" T-T x 8" Dia. w/ } \\
3(\text { ea) - Polypropylene element, 20" long, 1.5um pore } \\
\text { size }\end{array}$ \\
\hline L-114 & 1 & Water Bio-Filter Vessel & $\$ 2,892$ & 4.2 & $\$ 12,146$ & $\begin{array}{l}\text { 1(ea) - Filter housing, 316L SS, 38" T-T x 8" Dia. w/ } \\
3(\text { ea) - Polypropylene element, 20" long, } 0.2 \text { um pore } \\
\text { size }\end{array}$ \\
\hline L-401 & 1 & Recycle Water Pre-Filter Vessel & $\$ 2,850$ & 4.2 & $\$ 11,970$ & $\begin{array}{l}\text { 1(ea) - Filter housing, } 316 \mathrm{~L} \mathrm{SS}, 38 \text { " T-T x 8" Dia. w/ } \\
3(\text { ea) - Polypropylene element, } 20 " \text { long, } 1.5 \mathrm{um} \text { pore } \\
\text { size }\end{array}$ \\
\hline \multirow[t]{2}{*}{ L-402 } & 1 & Recycle Water Bio-Filter Vessel & $\$ 2,892$ & 4.2 & $\$ 12,146$ & $\begin{array}{l}\text { 1(ea) - Filter housing, } 316 \mathrm{LSS}, 38 \text { " T-T x 8" Dia. w/ } \\
3 \text { (ea) - Polypropylene element, } 20 \text { " long, } 0.2 \text { um pore } \\
\text { size }\end{array}$ \\
\hline & & SUB-TOTAL & $\$ 91,000$ & & $\$ 384,000$ & \\
\hline
\end{tabular}


A N V I L C O R P O R A T I O N Final Technical Progress Report

PROJECT: PetroStar Valdez ULSD - BDS/HDS Combo ANVIL NO: AE1416

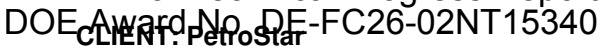

DATE: 6/17/05

REV NO.: 0

BDS EQUIPMENT - FACTORING SUMMARY

\begin{tabular}{|c|c|c|c|c|c|c|}
\hline $\begin{array}{l}\text { EQUIP. } \\
\text { ITEM NO. }\end{array}$ & $\begin{array}{l}\text { QUAN- } \\
\text { TITY }\end{array}$ & DESCRIPTION & $\begin{array}{l}\text { TOTAL } \\
\text { EQUIP. } \\
\text { COST, \$ }\end{array}$ & $\begin{array}{c}\text { FIELD } \\
\text { COST } \\
\text { MULTI- } \\
\text { PLIER }\end{array}$ & $\begin{array}{c}\text { TOTAL } \\
\text { FIELD } \\
\text { COST, \$ }\end{array}$ & NOTES \\
\hline \multirow[t]{3}{*}{ FREIGHT } & & Freight Allowance (7\% Equip Cost) & $\$ \$ \$ 300,000$ & & $\$ 300,000$ & \\
\hline & & SUB-TOTAL & $\$ 300,000$ & & $\$ 300,000$ & \\
\hline & & TOTAL & \begin{tabular}{|l|}
$4,581,643$ \\
\end{tabular} & & $\$ 14,140,000$ & \\
\hline & \multicolumn{4}{|c|}{ OWNER'S SERVICES \& COSTS (provided by owner) } & $\$$ & \\
\hline & \multicolumn{4}{|c|}{ INITIAL CHEMICAL CHARGE } & 76,000 & \\
\hline & \multicolumn{4}{|c|}{ ESCALATION (provided by owner) } & $\$$ & \\
\hline & \multicolumn{4}{|c|}{ UNADJUSTED COST ESTIMATE (UCE) } & $\$ 17,466,000$ & \\
\hline
\end{tabular}

${ }^{\star}$ Note: Equipment pricing by Pelorus. 
Final Technical Progress Report DOE Award No. DE-FC26-02NT15340
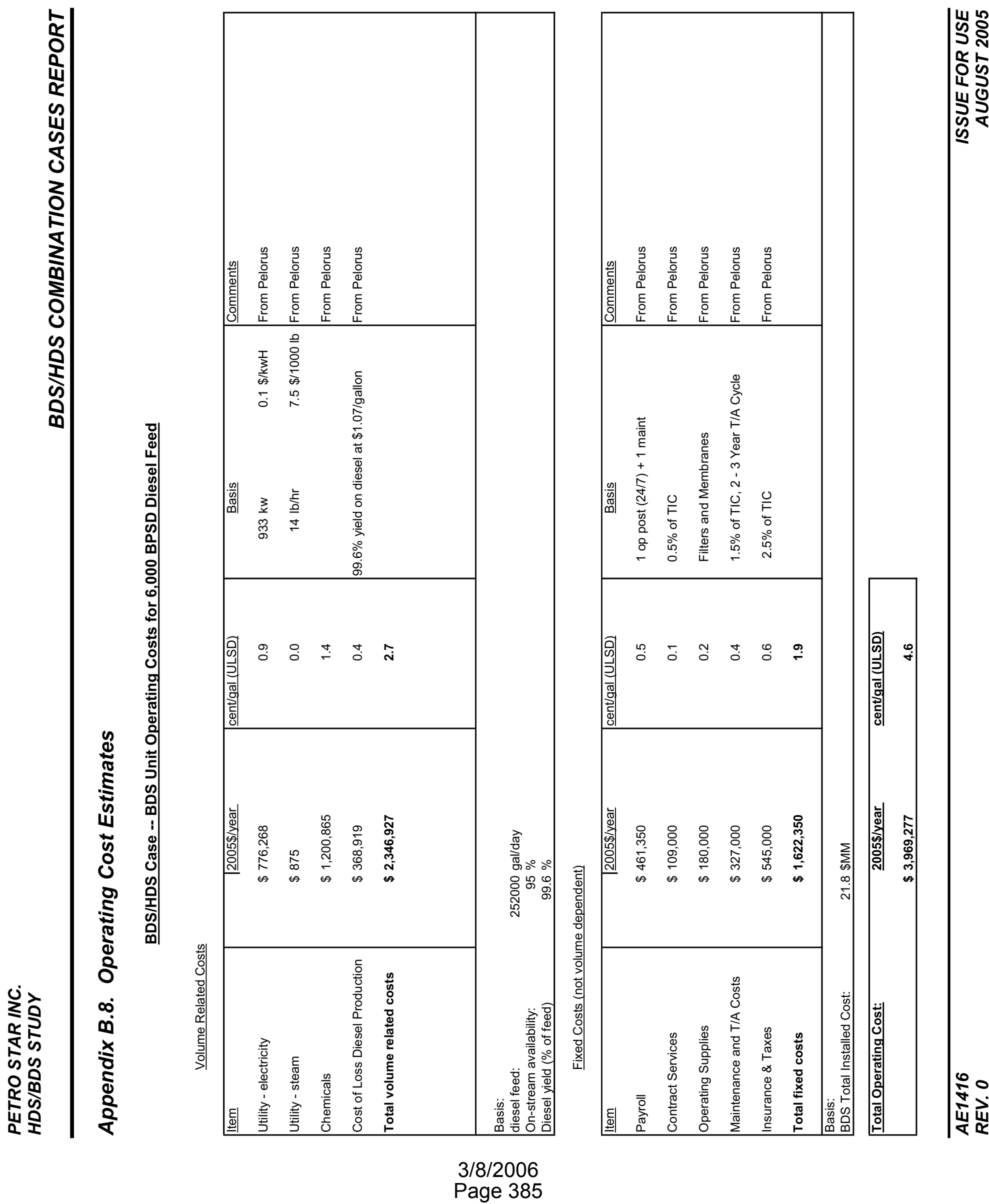
Final Technical Progress Report

DOE Award No. DE-FC26-02NT15340

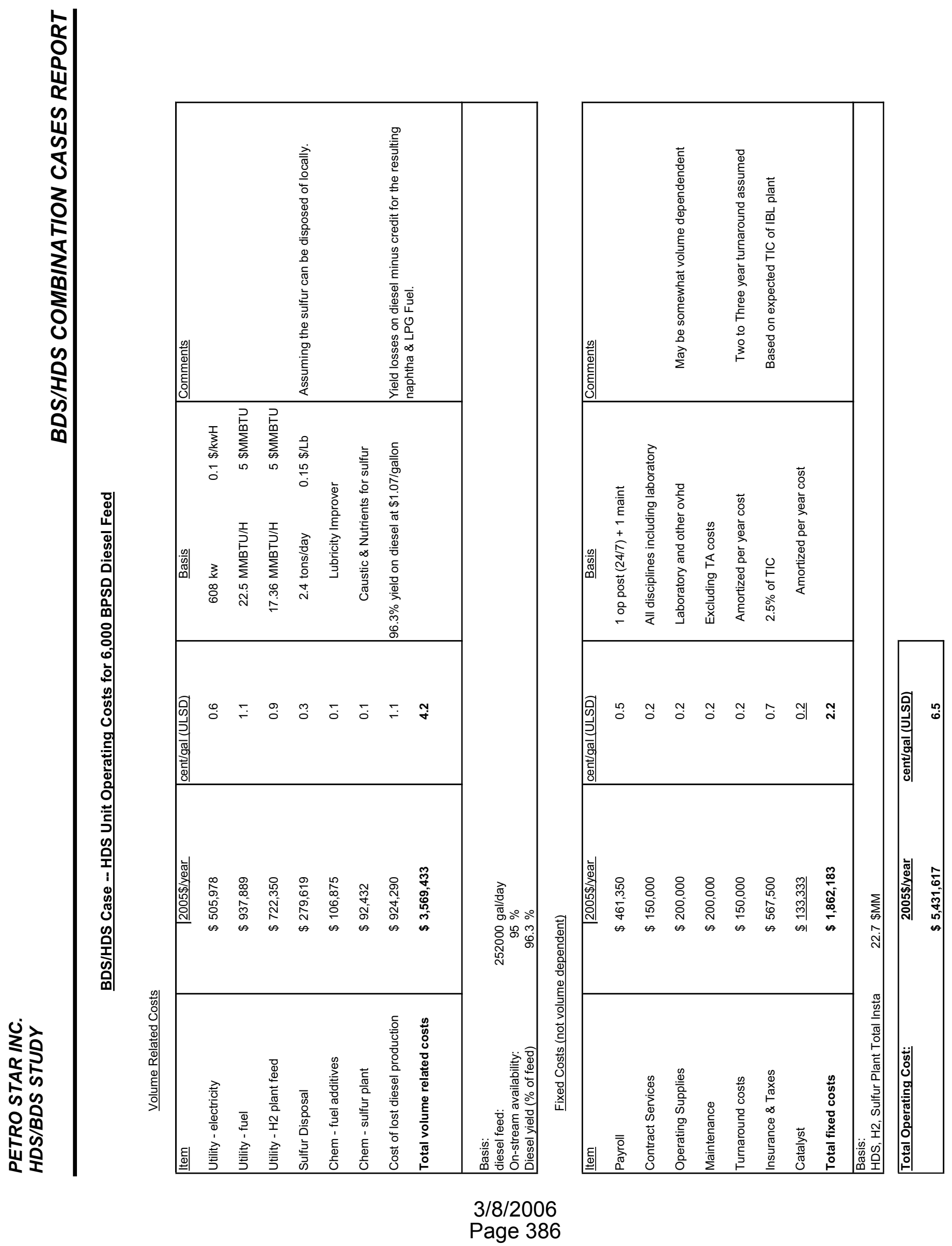

|

年定 
Appendix C.1 - HDS Unit Process Flow Diagrams and Material Balance 


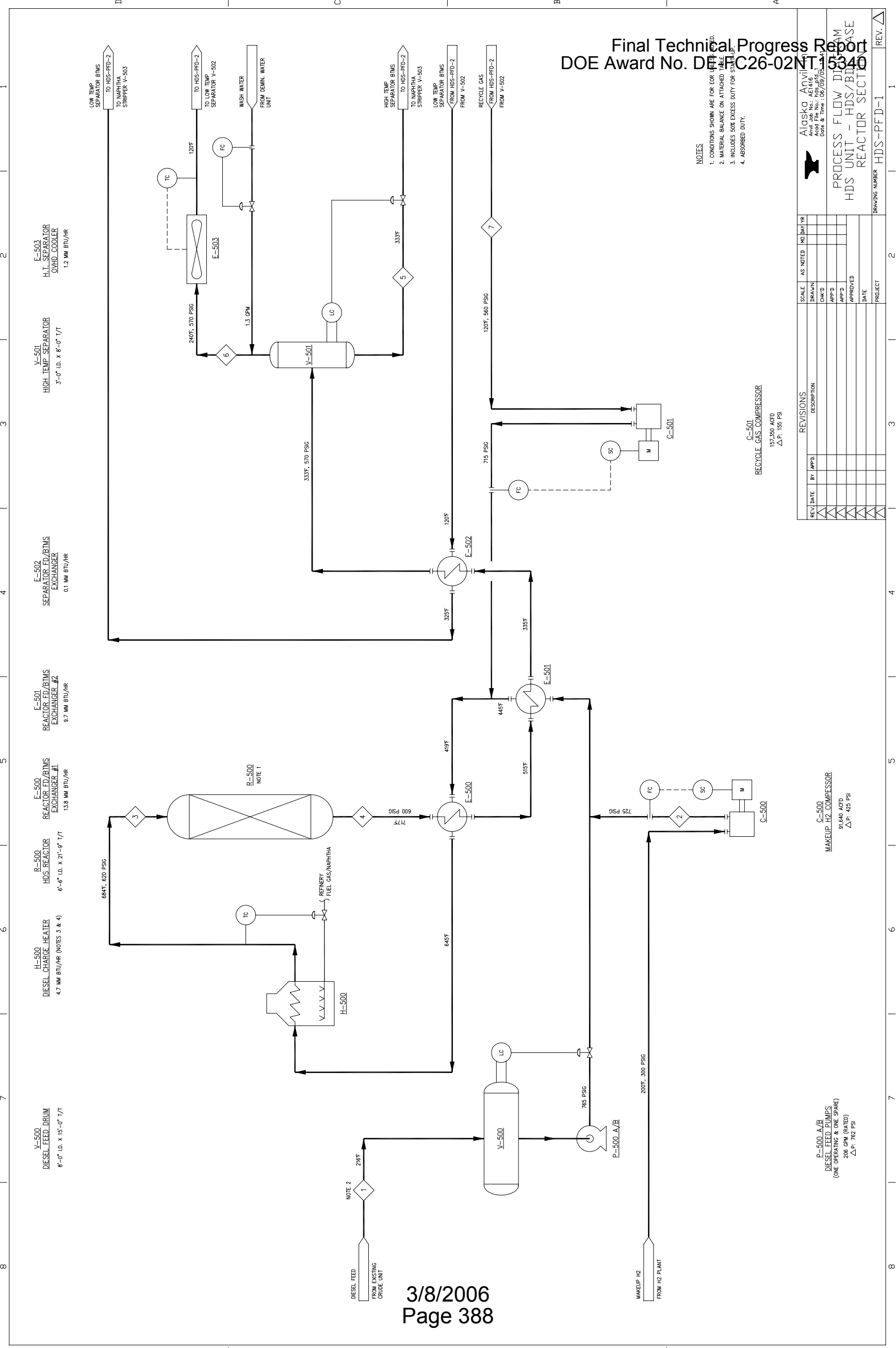


Final Technical Progress Report

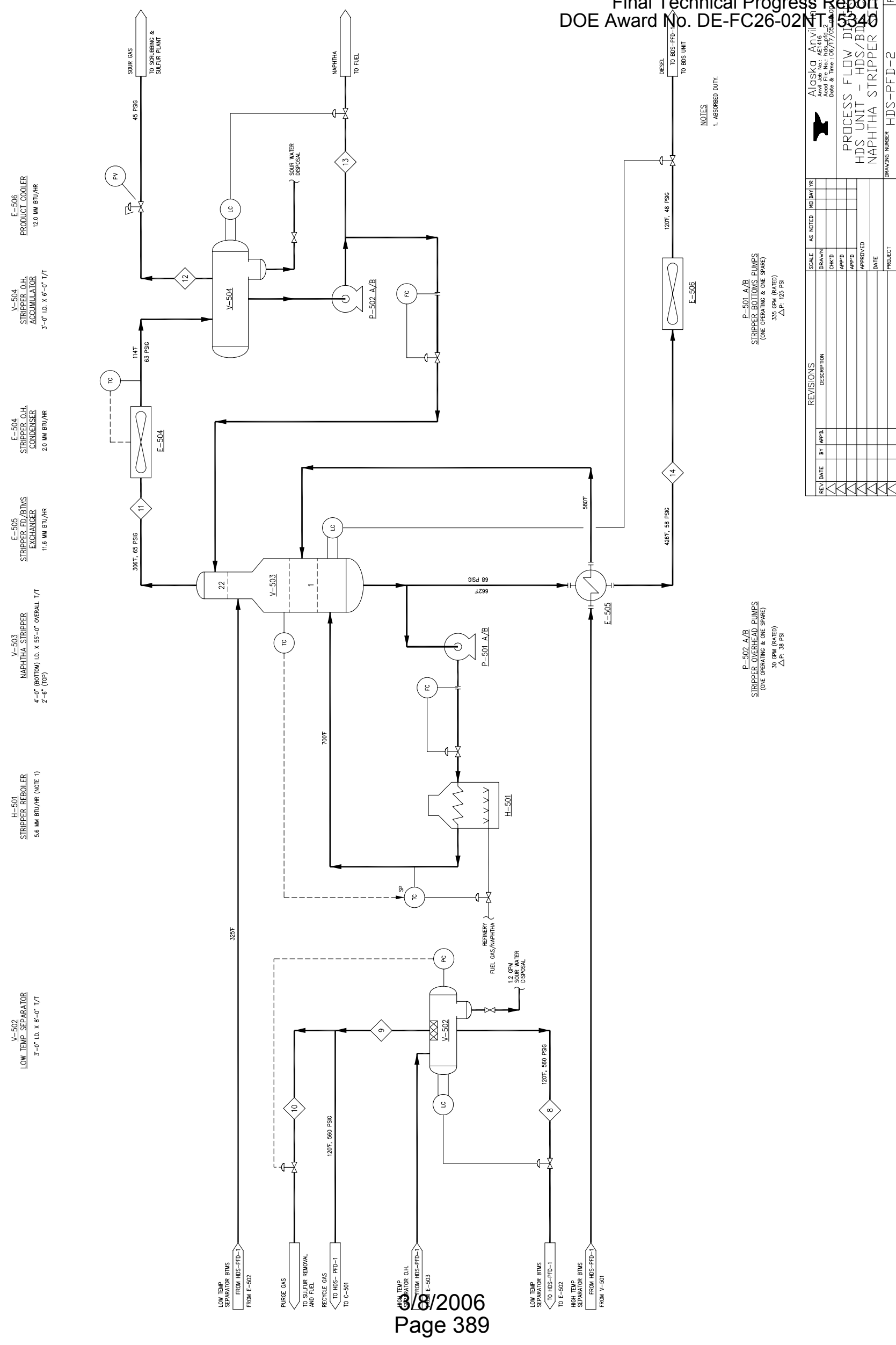




\section{Material Balance}

\section{HDS Unit EOR Material Balance -- HDS/BDS Case}

\begin{tabular}{|c|c|c|c|c|c|c|c|c|}
\hline Stream Number & & 1 & 2 & 3 & 4 & 5 & 6 & 7 \\
\hline Stream Description & & ANS Feed & Make-ıp H2 & Reactor Feed & Reactar Outlet & V-501 Liquid & $\begin{array}{c}\text { V-50t Vapor + } \\
\text { Wash Water }\end{array}$ & Recycle Gas \\
\hline Phase & & L[QUID & VAPOR & MIXED & MLXED & LIQUID & MIXED & VAPOF \\
\hline Mass Flaw Rate & LB/HA & 75427 & 346 & 79224 & 79224 & 74970 & 4883 & 3451 \\
\hline Temperature & $F$ & 216 & 413 & 684 & 717 & 333 & 240 & 120 \\
\hline Pressure & $\mathrm{PS}[\mathrm{G}$ & 3 & 725 & 620 & 600 & 570 & 570 & 560 \\
\hline Standard Lig Flaw & $\mathrm{BPO}$ & 6000 & $17 / \mathrm{a}$ & 4008 & 3160 & 6032 & 31 & $\mathrm{n} / \mathrm{a}$ \\
\hline Vapar Flaw & MSCFH & $\mathrm{nda}$ & 63.8 & 3286 & 307.7 & $\mathrm{n} / \mathrm{a}$ & 246.1 & 2269 \\
\hline Wt \% Vapor & & 0 & 100 & 37 & 50 & 0 & 92 & 100 \\
\hline MW & & 217.9 & 2.1 & 71.2 & 70.5 & 194.7 & 6.9 & 5.8 \\
\hline
\end{tabular}

\begin{tabular}{|c|c|c|c|c|c|c|c|c|}
\hline Stream Number & & 8 & 9 & 10 & $i 1$ & 12 & 13 & 14 \\
\hline Stream Description & & $\begin{array}{l}\text { V-502 HC } \\
\text { Liquid } \\
\end{array}$ & V-502 Vapor & Purge Gas & $\begin{array}{l}\text { Column Vepor } \\
\text { to Condenser }\end{array}$ & Sour Gas & Naphtha & $\begin{array}{c}\text { Diesel from } \\
\text { E-505 }\end{array}$ \\
\hline Phase & & L[GUID & VAPOR & VAPGA & VA.POR & VAPOR & LIOUID & L[OUID \\
\hline Mass Flow Rate & LB/HA & 695 & 3566 & 116 & 9898 & 636 & 1911 & 73115 \\
\hline Temperature & $F$ & 120 & 120 & 120 & 306 & 114 & 114 & 426 \\
\hline Pressure & $\mathrm{PSIG}$ & 560 & 560 & 560 & 65 & 63 & 104 & 58 \\
\hline Standard Liq Flow & BPD & 61 & $n / \mathrm{a}$ & $n$ & $n d a$ & no & 173 & 5830 \\
\hline Vapar Flaw & MSCFH & nia & 234.5 & 76 & 478 & 11.0 & $n / a$ & nia \\
\hline Wt \% Vapor & & 0 & 100 & 100 & 100 & 100 & 0 & 0 \\
\hline MW & & 1099 & 5.8 & 5.8 & 71.4 & 22.0 & 86.1 & 214.8 \\
\hline
\end{tabular}




\section{Appendix C.2 - HDS Unit Equipment List and Budgetary Equipment Datasheets}

\section{HDS Unit Equipment List}

\begin{tabular}{|c|c|c|c|c|c|}
\hline \multirow{2}{*}{ Item } & \multirow{2}{*}{ Service } & \multirow{2}{*}{ Description } & \multicolumn{2}{|l|}{ Design Conditions } & \multirow{2}{*}{ Metallurgy } \\
\hline & & & Pressure & Temp. & \\
\hline R-500 & HDS Reactor & $\begin{array}{l}6^{\prime}-6 " \text { ID } \times 21^{\prime}-9 " \text { T/T w/ } \\
\text { one bed of HR-526 Co } \\
\text { Mo catalyst. } \\
\end{array}$ & 670 PSIG/FV & $750^{\circ} \mathrm{F} /-20^{\circ} \mathrm{F}$ & $\begin{array}{l}\text { SA387 Gr. } 11 \mathrm{w} / 321 \mathrm{SS} \text { or } 347 \mathrm{SS} \\
\text { weld overlay. Internal trays are } 410 \\
\text { SS or } 321 \mathrm{SS}\end{array}$ \\
\hline V-500 & Diesel Feed Drum & $\begin{array}{l}\text { 8'-0" ID X 15'-0" T/T } \\
\text { (Horizontal) }\end{array}$ & 50 PSIG/FV & $275 /-20^{\circ} \mathrm{F}$ & Killed Carbon Steel \\
\hline V-501 & $\begin{array}{l}\text { High Temperature } \\
\text { Separator } \\
\end{array}$ & $\begin{array}{l}\text { 3'-0" ID X 8'-0" T/T } \\
\text { (Vertical) }\end{array}$ & $620 \mathrm{PSIG} / \mathrm{FV}$ & $380 /-20^{\circ} \mathrm{F}$ & Killed Carbon Steel w/0.15" CA \\
\hline V-502 & $\begin{array}{l}\text { Low Temperature } \\
\text { Separator }\end{array}$ & \begin{tabular}{|l|} 
3'-0" ID X 8'-0" T/T' \\
(Horizontal w/ Boot)
\end{tabular} & $610 \mathrm{PSIG} / \mathrm{FV}$ & $350 /-20^{\circ} \mathrm{F}$ & $\begin{array}{l}\text { Killed Carbon Steel w/ 0.1" CA } \\
(\mathrm{PWHT}) \text { and Monel Demister }\end{array}$ \\
\hline V-503 & Naphtha Stripper & $\begin{array}{l}\text { 2'-6” ID (Top), 4'-0" ID } \\
\text { (bottom) } \\
\text { X 55'-0"T/T (Overall) } \\
\text { w/ } 22 \text { valve trays }\end{array}$ & 125 PSIG/FV & $750 /-20^{\circ} \mathrm{F}$ & $\begin{array}{l}\text { Killed Carbon Steel w/ } 0.2 " \mathrm{CA} \text { and } \\
\text { Type } 410 \text { SS trays, supports, and } \\
\text { downcomers }\end{array}$ \\
\hline V-504 & $\begin{array}{l}\text { Stripper O. H. } \\
\text { Accumulator }\end{array}$ & $\begin{array}{l}\text { 3'-0" ID X 6'-0" T/T } \\
\text { (Horizontal w/Boot) }\end{array}$ & 125 PSIG/FV & $350 /-20^{\circ} \mathrm{F}$ & $\begin{array}{l}\text { Killed Carbon Steel w/0.125" CA } \\
\text { (PWHT) }\end{array}$ \\
\hline E-500 & $\begin{array}{l}\text { Reactor Fd/Btms } \\
\text { Exchanger \#1 }\end{array}$ & $\begin{array}{l}\text { 13.8 MM BTU/Hr, } \\
4500 \mathrm{FT}^{2} \text { w/ } 2 \text { shells, } \\
\text { TEMA type CEU }\end{array}$ & $\begin{array}{l}\text { Tubes: } 650 \text { PSIG } \\
\text { Shell: } 765 \text { PSIG }\end{array}$ & $\begin{array}{l}770^{\circ} \mathrm{F} \\
700^{\circ} \mathrm{F} /-20^{\circ} \mathrm{F}\end{array}$ & $\begin{array}{l}\text { Tubes: } 1 \frac{1 / 4}{\mathrm{Cr}}-1 / 2 \text { Mo tubes and } \\
\text { tube sheet and weld overlay } 316 \text { ss } \\
\text { for tube sheet } \\
\text { Shell: } 1 \frac{1 / 4}{\mathrm{Cr}}-1 / 2 \text { Mo clad w/ } 321 \\
\text { SS, baffles to be } 304 \text { SS }\end{array}$ \\
\hline E-501 & $\begin{array}{l}\text { Reactor Fd/Btms } \\
\text { Exchanger \#2 }\end{array}$ & $\begin{array}{l}\text { 9.7 MM BTU/Hr, } \\
2840 \mathrm{FT}^{2} \mathrm{w} / 2 \text { shells, } \\
\text { TEMA type CEU }\end{array}$ & $\begin{array}{l}\text { Tubes: } 640 \text { PSIG } \\
\text { Shell: } 775 \text { PSIG }\end{array}$ & $\begin{array}{l}540^{\circ} \mathrm{F} \\
470^{\circ} \mathrm{F} /-20^{\circ} \mathrm{F}\end{array}$ & 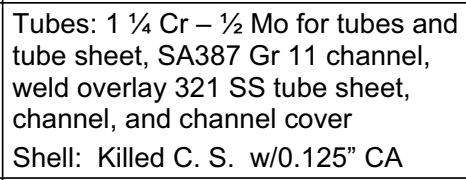 \\
\hline E-502 & $\begin{array}{l}\text { Separator } \\
\text { Fd/Btms } \\
\text { Exchanger }\end{array}$ & $\begin{array}{l}0.1 \mathrm{MM} \mathrm{BTU} / \mathrm{Hr}, \\
33 \mathrm{FT}^{2} \text {, double pipe. }\end{array}$ & $\begin{array}{l}\text { Tubes: } 630 \text { PSIG } \\
\text { Shell: } 610 \text { PSIG }\end{array}$ & $\begin{array}{l}360^{\circ} \mathrm{F} \\
360 /-20^{\circ} \mathrm{F}\end{array}$ & $\begin{array}{l}\text { Tubes: Carbon Steel } \\
\text { Shell: Carbon Steel }\end{array}$ \\
\hline$E-503$ & $\begin{array}{l}\text { H. T. Separator } \\
\text { Overhead Cooler }\end{array}$ & $\begin{array}{l}\text { 1.2 } \mathrm{MM} \mathrm{BTU} / \mathrm{Hr} \\
246 \mathrm{FT}^{2} \text { (Bare Tube) } \\
5240 \mathrm{FT}^{2} \text { Extended } \\
\text { Surface, } 5 \text { HP Fan } \\
\end{array}$ & Tubes: 620 PSIG & $350^{\circ} \mathrm{F}$ & $\begin{array}{l}\text { Seamless Carbon Steel tubes with } \\
\text { aluminum fins }\end{array}$ \\
\hline E-504 & $\begin{array}{l}\text { Stripper O. H. } \\
\text { Condenser }\end{array}$ & $\begin{array}{l}2.0 \mathrm{MM} \mathrm{BTU} / \mathrm{Hr} \\
371 \mathrm{FT}^{2} \text { (Bare Tube) } \\
7713 \mathrm{FT}^{2} \text { Extended } \\
\text { Surface, } 5 \text { HP Fan }\end{array}$ & Tubes: 125 PSIG & $350^{\circ} \mathrm{F}$ & $\begin{array}{l}\text { Seamless Carbon Steel tubes with } \\
\text { aluminum fins }\end{array}$ \\
\hline \begin{tabular}{|l|}
$E-505$ \\
\end{tabular} & $\begin{array}{l}\text { Stripper Fd/Btms } \\
\text { Exchanger }\end{array}$ & $\begin{array}{l}\text { 11.6 MM BTU/Hr } \\
3160 \mathrm{FT}^{2} \text { w/3 shells, } \\
\text { TEMA type CEU }\end{array}$ & $\begin{array}{l}\text { Tubes: } 620 \text { PSIG } \\
\text { Shell: } 100 \text { PSIG }\end{array}$ & $\begin{array}{l}630^{\circ} \mathrm{F} \\
710^{\circ} \mathrm{F} /-20^{\circ} \mathrm{F}\end{array}$ & $\begin{array}{l}\text { Tubes: } 18 \mathrm{Cr}-8 \mathrm{Ni} \text {, Channel is CS } \\
\text { w/ 0.25" CA, tube sheets are } 410 \mathrm{SS} \\
\text { Shell: CS w/0.25" CA, CS baffles }\end{array}$ \\
\hline E-506 & Product Cooler & $\begin{array}{l}\text { 12.0 } \mathrm{MM} \mathrm{BTU} / \mathrm{Hr} \\
2330 \mathrm{FT}^{2} \text { (Bare Tube) } \\
49860 \mathrm{FT}^{2} \text { Extended } \\
\text { Surface, } 20 \text { HP Fan } \\
\end{array}$ & Tubes: 100 PSIG & $450^{\circ} \mathrm{F}$ & $\begin{array}{l}\text { Seamless Carbon Steel tubes with } \\
\text { aluminum fins }\end{array}$ \\
\hline $\mathrm{H}-500$ & $\begin{array}{l}\text { Diesel Charge } \\
\text { Heater }\end{array}$ & $\begin{array}{l}\text { 4.7 MM BTU/Hr } \\
\text { Fired Heater, Convection } \\
\text { Section shared } \mathrm{w} / \mathrm{H}-501\end{array}$ & Tubes: 755 PSIG & $750^{\circ} \mathrm{F}$ & Tubes: $9 \mathrm{Cr}-1 \mathrm{Mo}$ w/ 0.1" CA \\
\hline $\mathrm{H}-501$ & Stripper Reboiler & \begin{tabular}{|l|} 
5.6 MM BTU/Hr \\
Fired Heater, Convection \\
Section shared $\mathrm{w} / \mathrm{H}-500$ \\
\end{tabular} & Tubes: 230 PSIG & $770^{\circ} \mathrm{F}$ & Tubes: $5 \mathrm{Cr}-1 / 2 \mathrm{Mo} \mathrm{w} / 0.1$ " CA \\
\hline
\end{tabular}




\begin{tabular}{|c|c|c|c|c|c|}
\hline \multirow{2}{*}{ Item } & \multirow{2}{*}{ Service } & \multirow{2}{*}{ Description } & \multicolumn{2}{|l|}{ Design Conditions } & \multirow{2}{*}{ Metallurgy } \\
\hline & & & Pressure & Temp. & \\
\hline C-500 & $\begin{array}{l}\text { Makeup Hydrogen } \\
\text { Compressor }\end{array}$ & $\begin{array}{l}\text { Capacity: } 91640 \text { ACFD } \\
\text { Motor Driven } \\
\text { Shaft HP: } 100 \text { BHP }\end{array}$ & $\begin{array}{l}\text { Suction: } 300 \text { PSIG } \\
\text { Disch: } 725 \text { PSIG }\end{array}$ & $\begin{array}{l}\text { Suction: } \\
200^{\circ} \mathrm{F}\end{array}$ & $\begin{array}{l}\text { Casing: killed C. S. } \\
\text { Internals: CS }\end{array}$ \\
\hline C-501 & $\begin{array}{l}\text { Recycle Gas } \\
\text { Compressor }\end{array}$ & $\begin{array}{l}\text { Capacity: } 157350 \text { ACFD } \\
\text { Motor Driven } \\
\text { Shaft HP: } 80 \text { BHP }\end{array}$ & $\begin{array}{l}\text { Suction: } 560 \text { PSIG } \\
\text { Disch: } 715 \text { PSIG }\end{array}$ & $\begin{array}{l}\text { Suction: } \\
120^{\circ} \mathrm{F}\end{array}$ & $\begin{array}{l}\text { Casing: killed C. S. } \\
\text { Internals: Stainless Steel }\end{array}$ \\
\hline $\begin{array}{l}P-500 \\
A / B\end{array}$ & $\begin{array}{l}\text { Diesel Feed Pump } \\
\text { (One Operating + } \\
\text { One Spare }\end{array}$ & $\begin{array}{l}\text { Rated Capacity: } 206 \text { GPM } \\
\text { Diff. Press: } 762 \text { PSI } \\
\text { Head: } 2182 \text { feet } \\
\text { Motor: } 150 \mathrm{HP}\end{array}$ & & & $\begin{array}{l}\text { Casing: killed C. S. w/ } 0.125 " \text { CA } \\
\text { Impeller: } 12 \% \text { chrome }\end{array}$ \\
\hline $\begin{array}{l}P-501 \\
\text { A/B }\end{array}$ & $\begin{array}{l}\text { Stripper Bottoms } \\
\text { Pump } \\
\text { (One Operating + } \\
\text { One Spare }\end{array}$ & $\begin{array}{l}\text { Rated Capacity: } 335 \text { GPM } \\
\text { Diff. Press: } 125 \text { PSI } \\
\text { Head: } 481 \text { feet } \\
\text { Motor: } 50 \text { HP }\end{array}$ & & & $\begin{array}{l}\text { Casing: killed C. S. w/ } 0.2 " \text { CA } \\
\text { Impeller: } 12 \% \text { chrome }\end{array}$ \\
\hline $\begin{array}{l}\mathrm{P}-502 \\
\mathrm{~A} / \mathrm{B}\end{array}$ & $\begin{array}{l}\text { Stripper Overhead } \\
\text { Pump } \\
\text { (One Operating + } \\
\text { One Spare }\end{array}$ & $\begin{array}{l}\text { Rated Capacity: } 30 \text { GPM } \\
\text { Diff. Press: } 38 \text { PSI } \\
\text { Head: } 120 \text { feet } \\
\text { Motor: } 5 \text { HP }\end{array}$ & & & $\begin{array}{l}\text { Casing: killed C. S. w/ } 0.125 " \text { CA } \\
\text { Impeller: } 12 \% \text { chrome }\end{array}$ \\
\hline
\end{tabular}




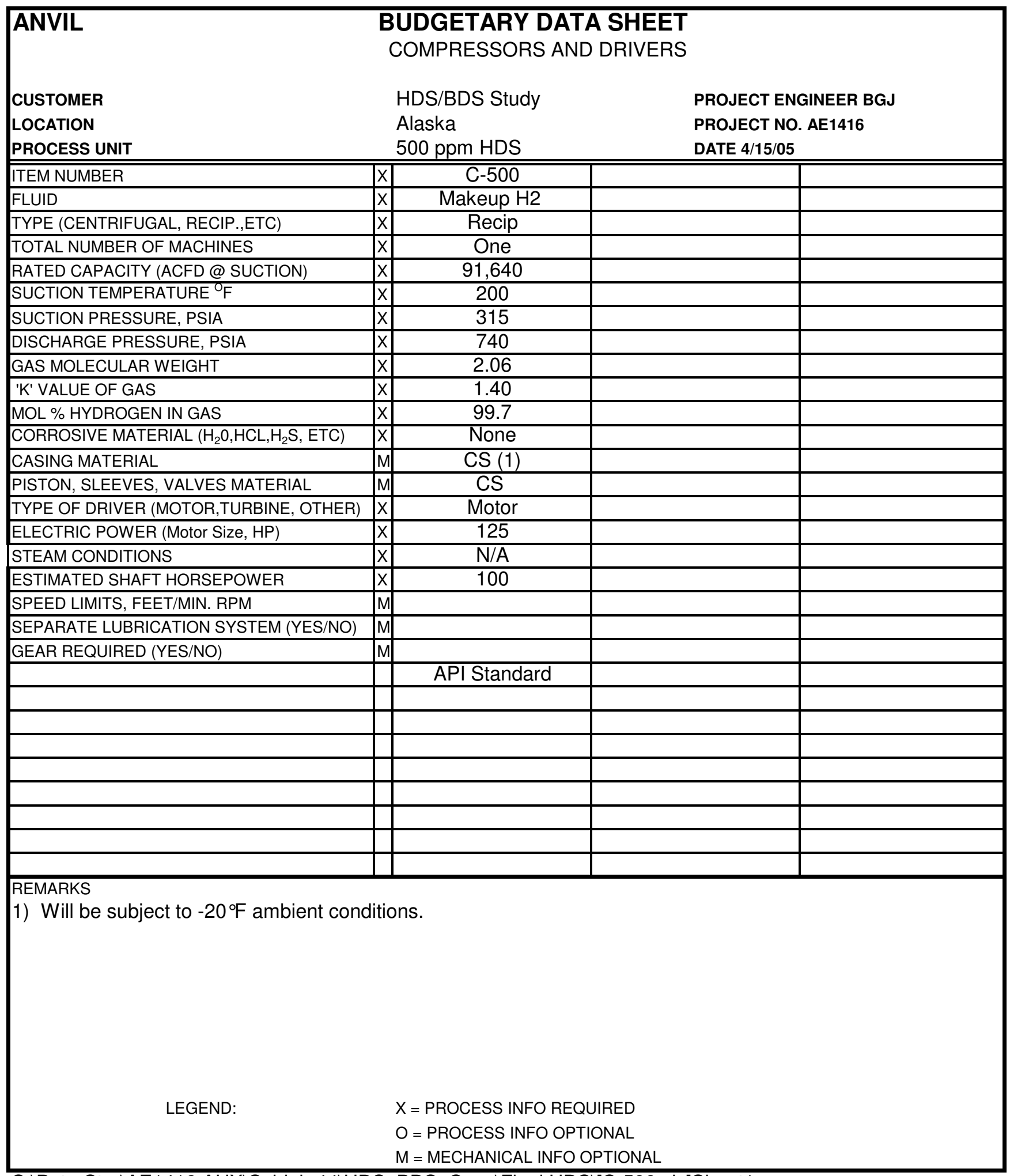

S:IPetroStar|AE1416.AUXISubjob 44IHDS_BDS_CaselFinal HDSI[C-500.xIs]Sheet1 


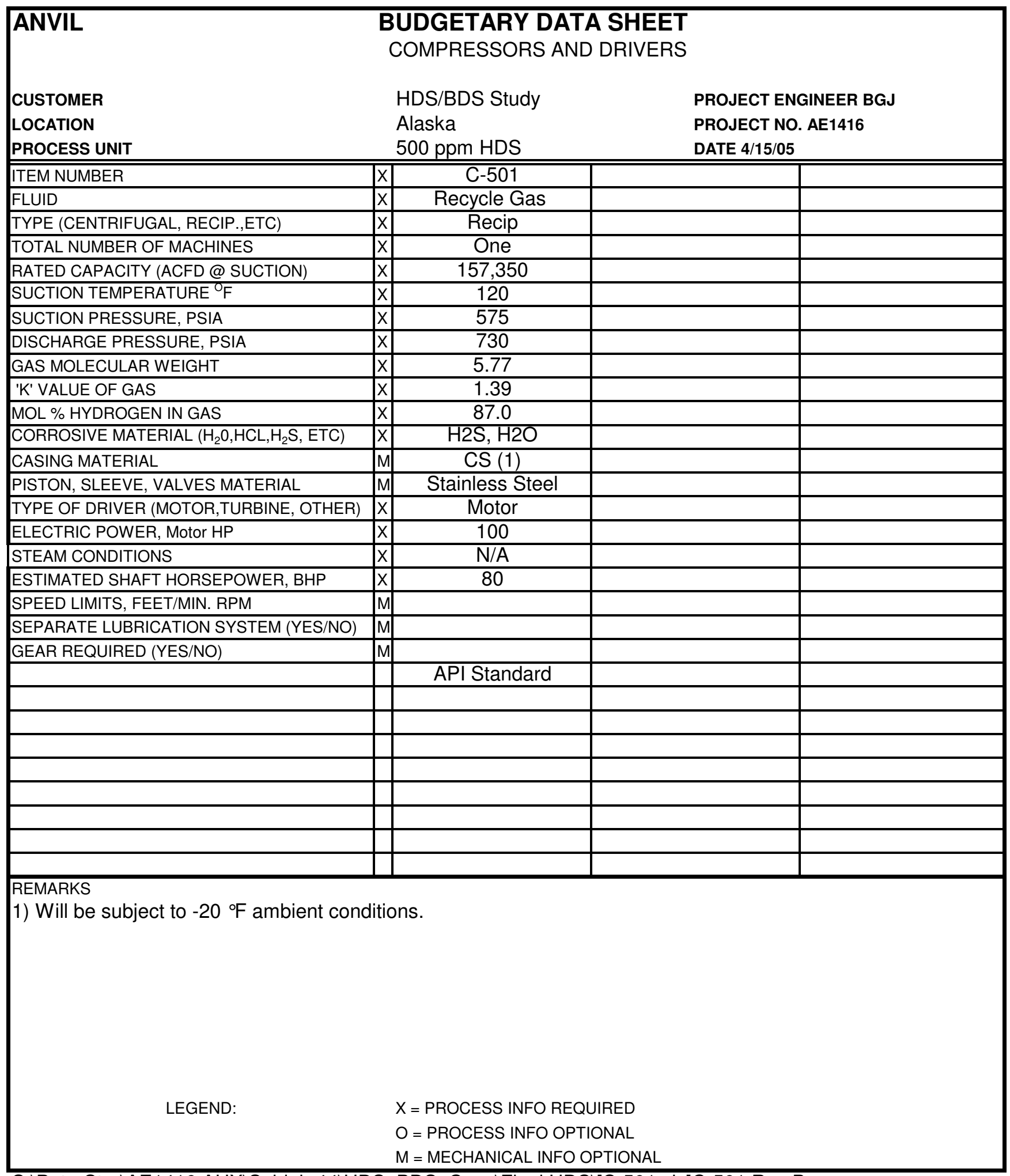

S:IPetroStar\AE1416.AUXISubjob 44IHDS_BDS_CaselFinal HDSI[C-501.xIs]C-501 Rev B 


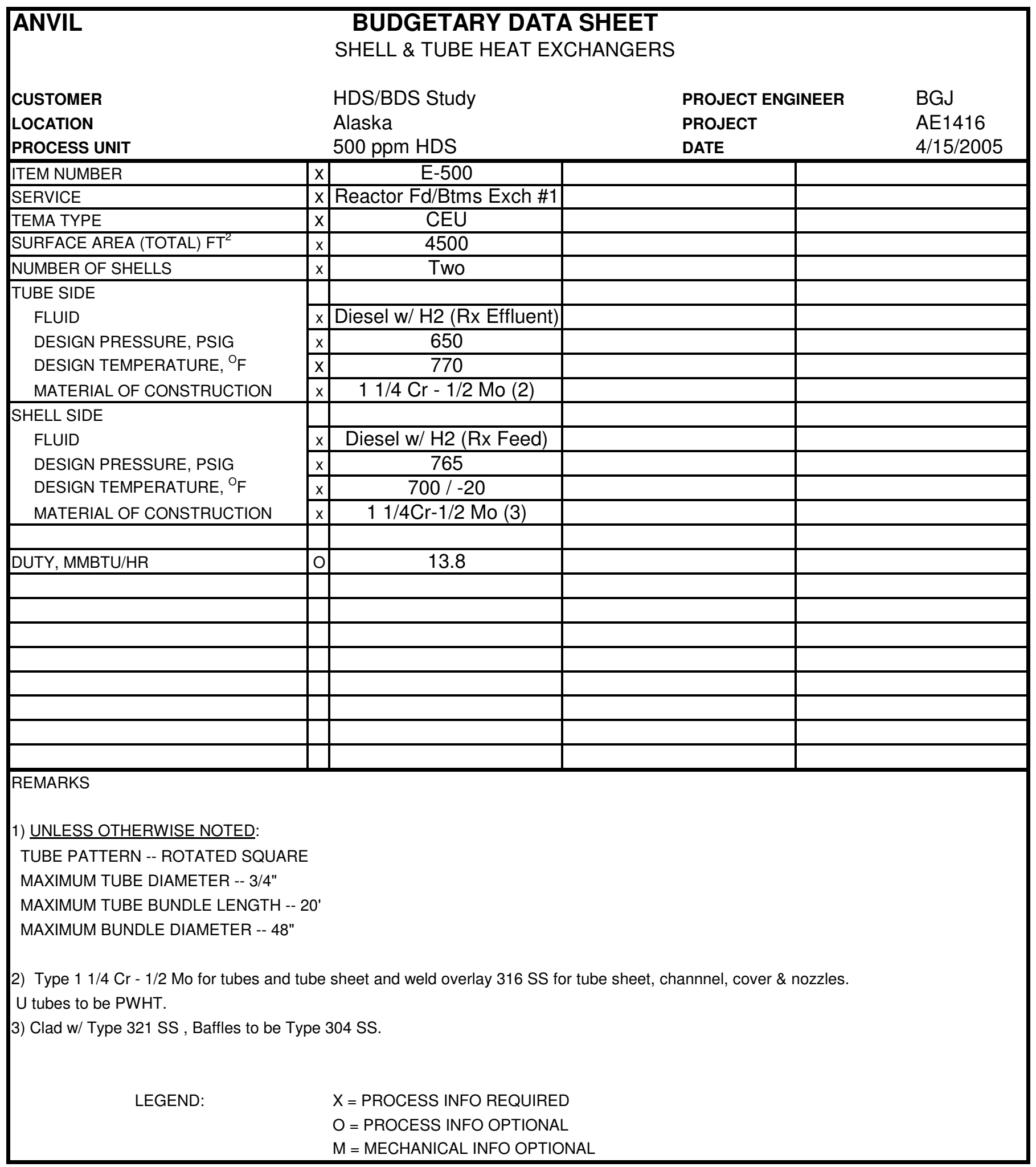

S:IPetroStarlAE1416.AUXISubjob 44|HDS_BDS_CaselFinal HDSI[E-503.xls]E-503 Rev B 


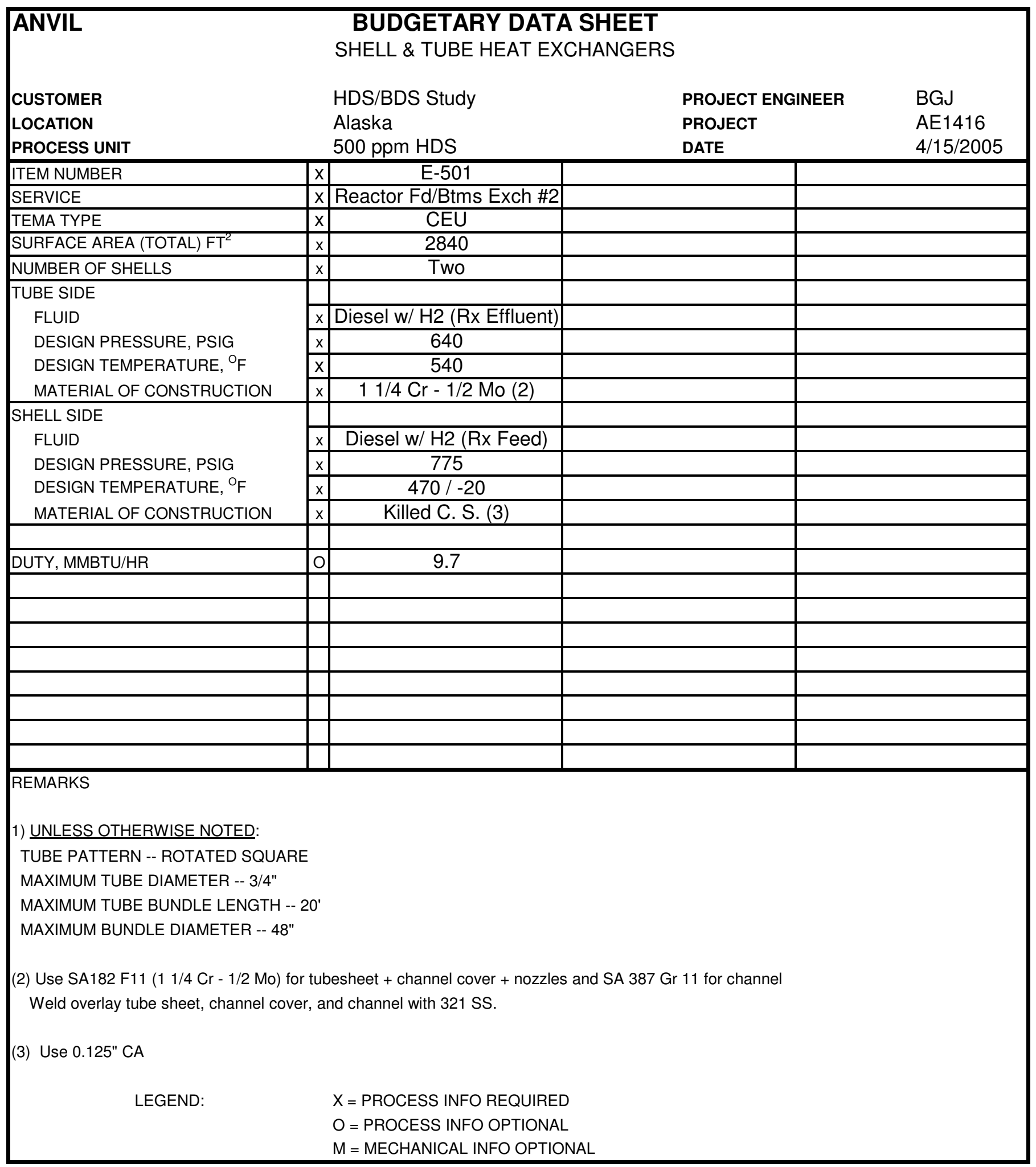

S:IPetroStarlAE1416.AUXISubjob 44|HDS_BDS_CaselFinal HDSI[E-503.xls]E-503 Rev B 


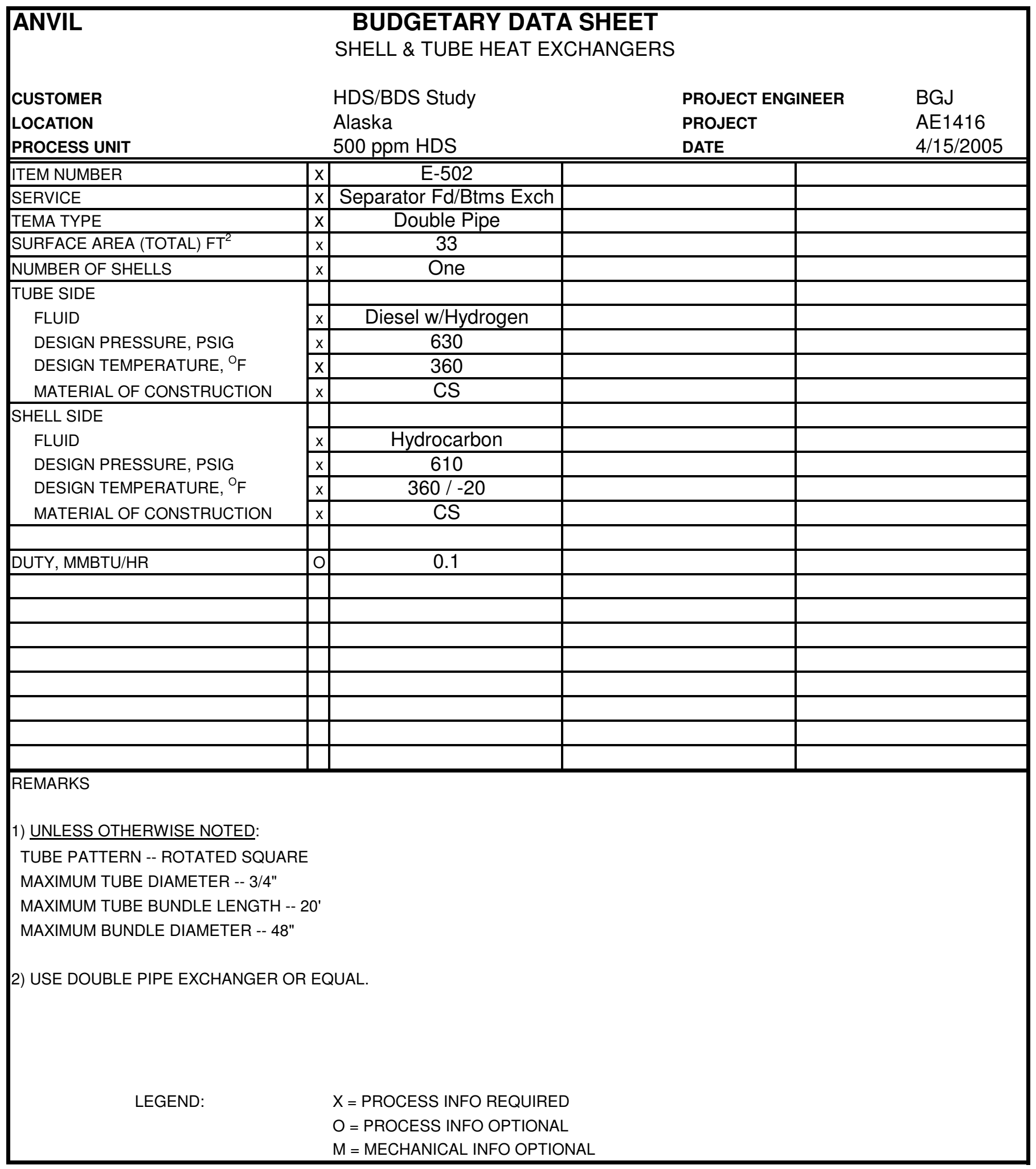

S:IPetroStarlAE1416.AUXISubjob 44|HDS_BDS_CaselFinal HDS|[E-503.xls]E-503 Rev B 


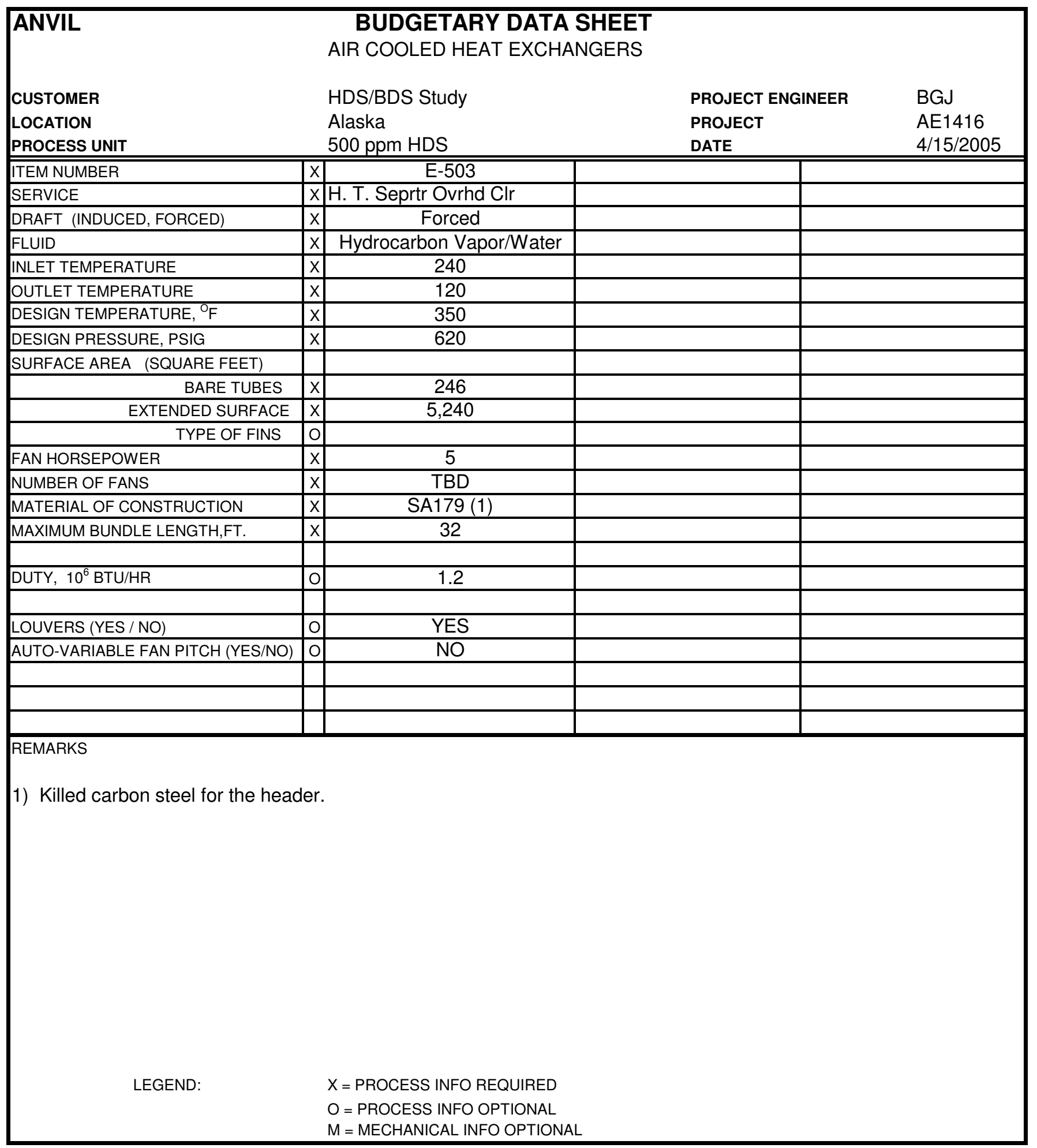




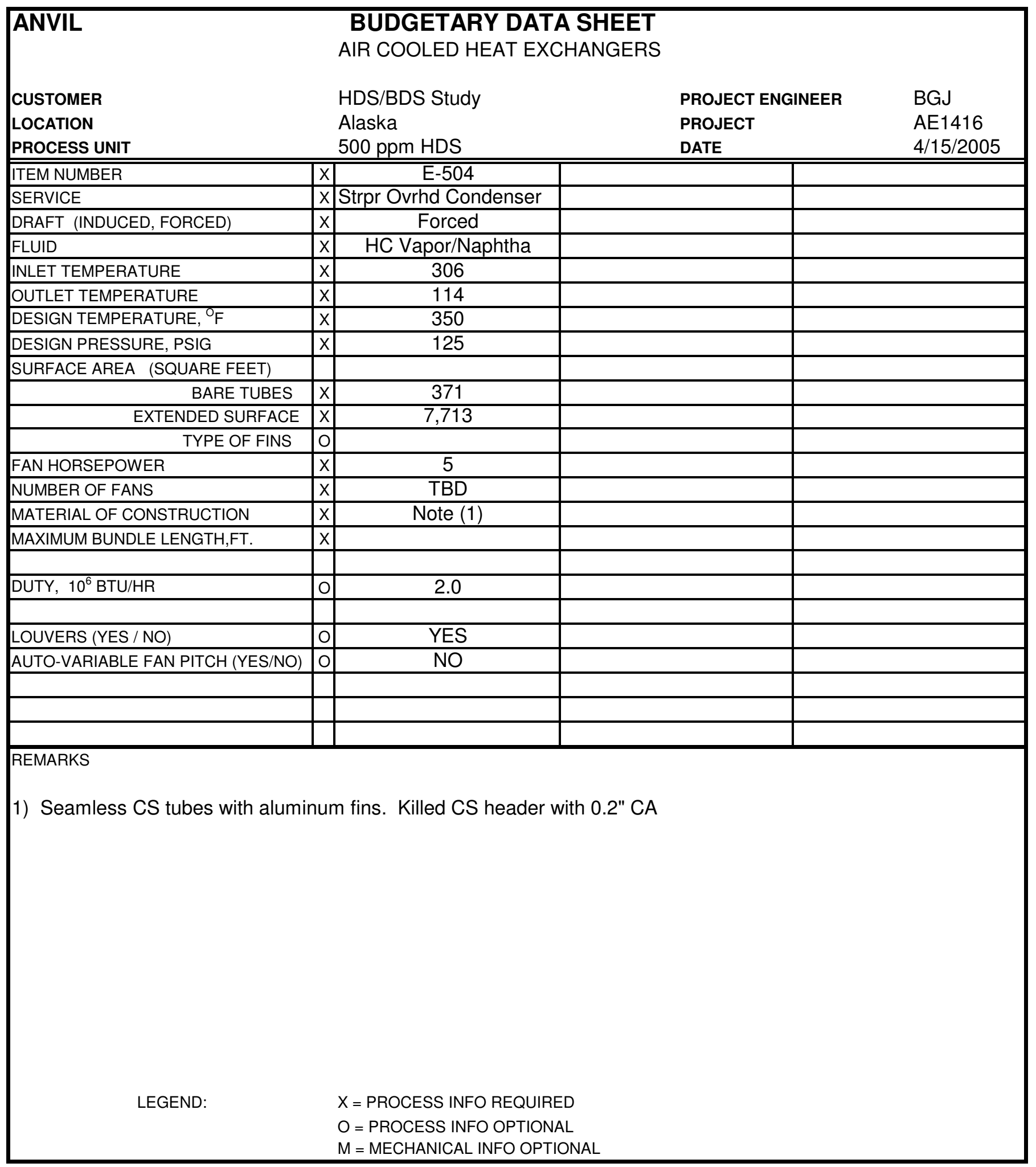

S:IPetroStar\AE1416.AUXISubjob 44\HDS_BDS_CaselFinal HDS\[E-504.xIs]E-504 Rev B 


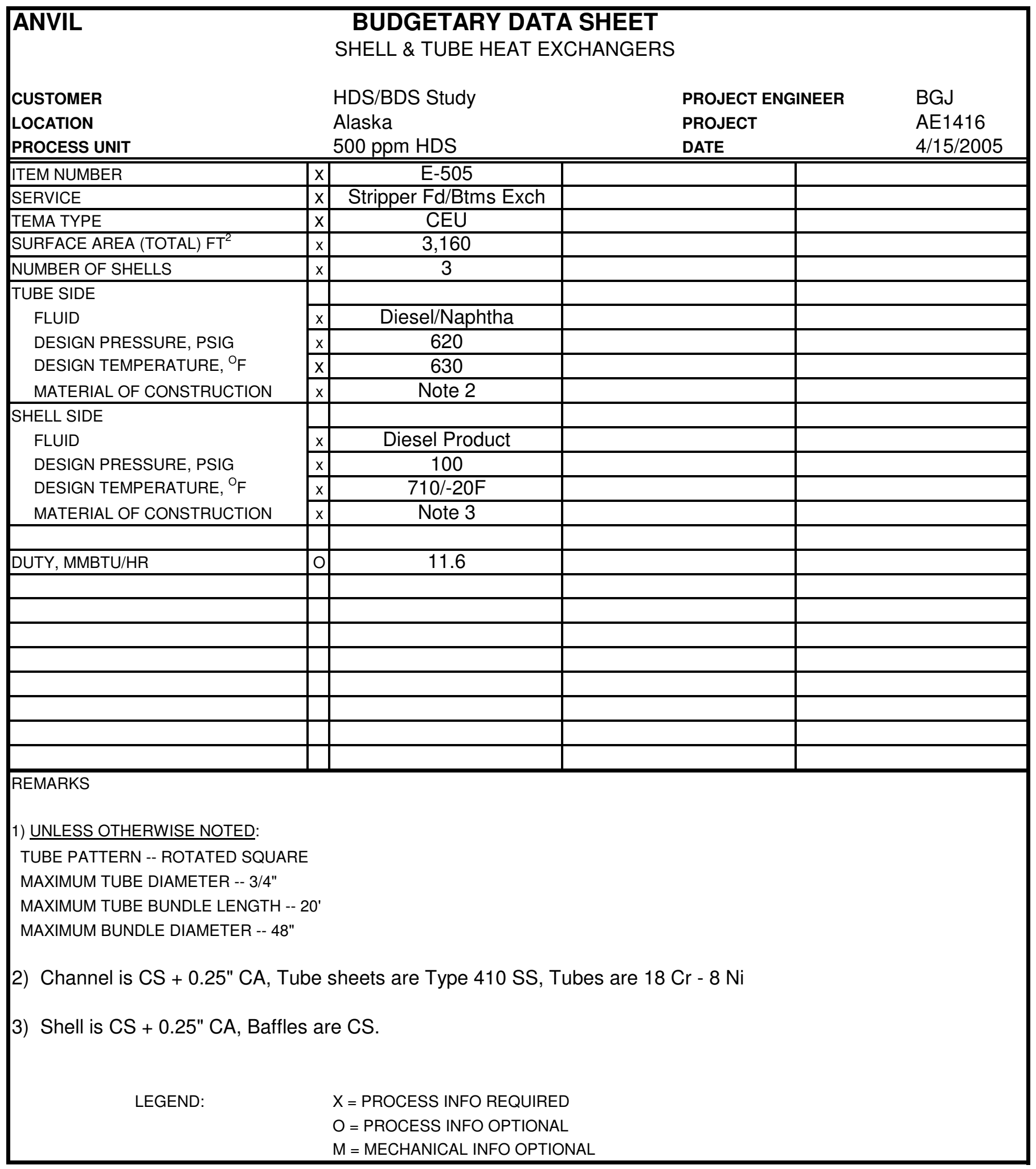




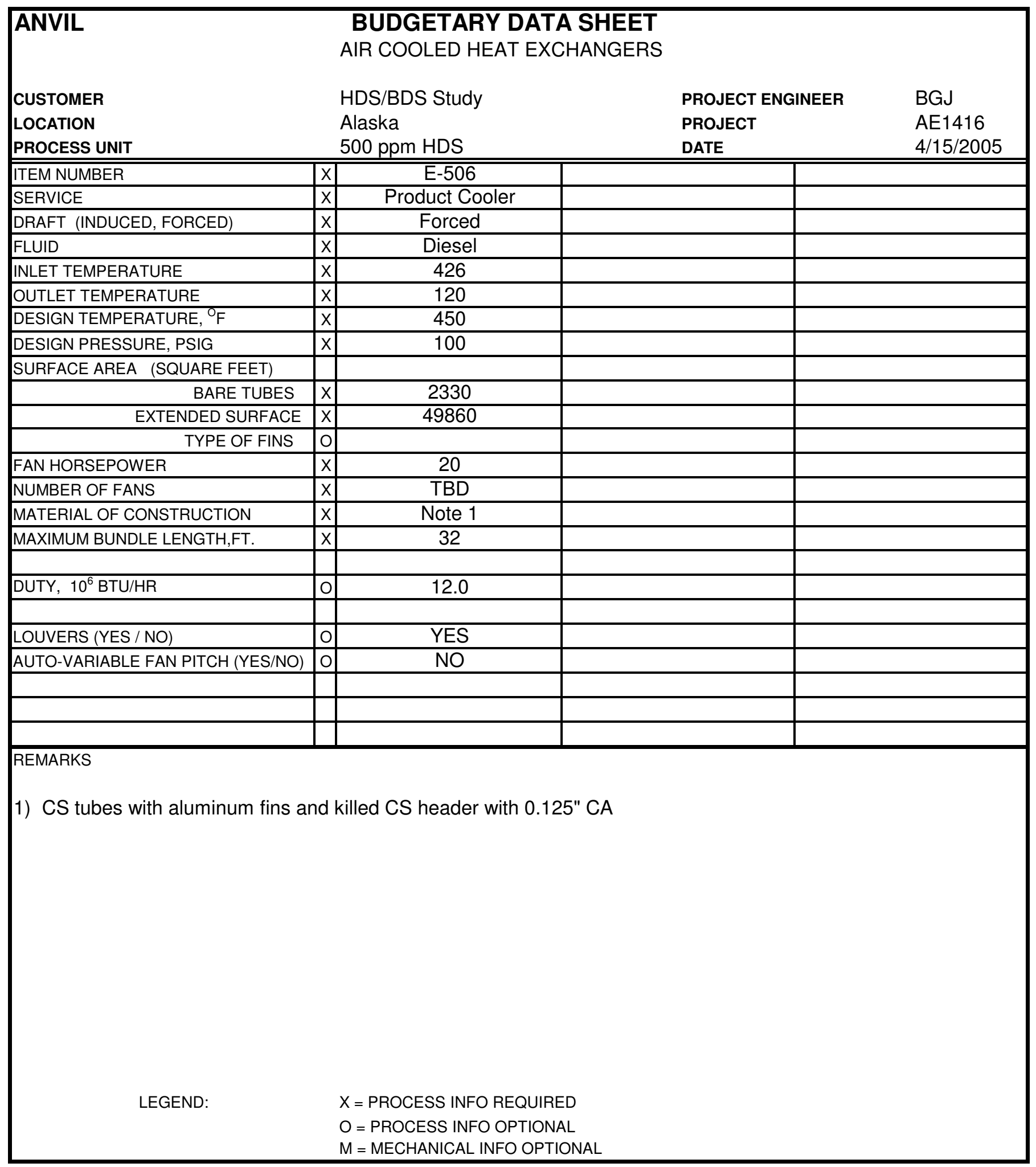

S:IPetroStar|AE1416.AUXISubjob 44|HDS_BDS_CaselFinal HDS|[E-506.xIs]E-506 Rev B 


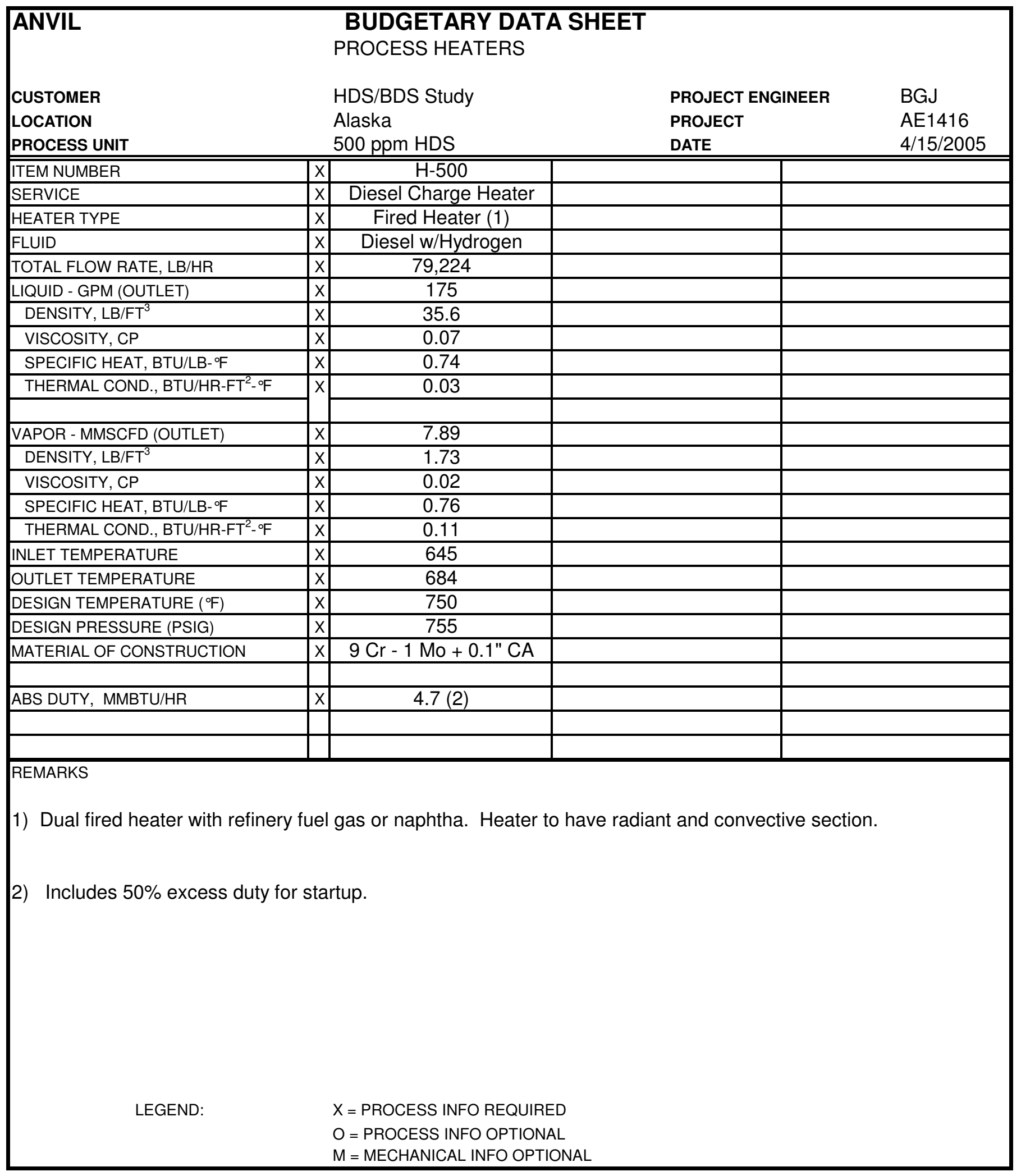




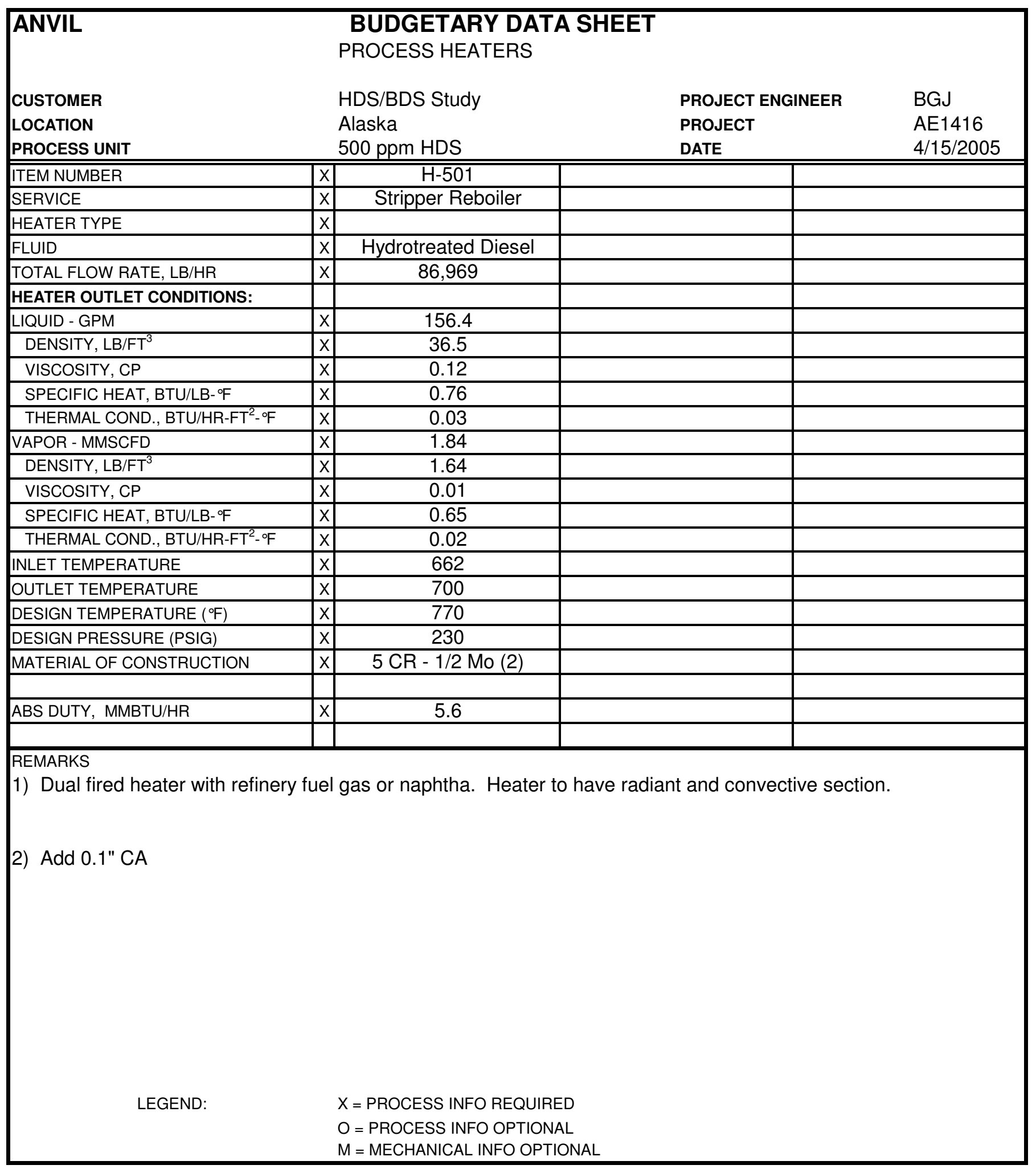




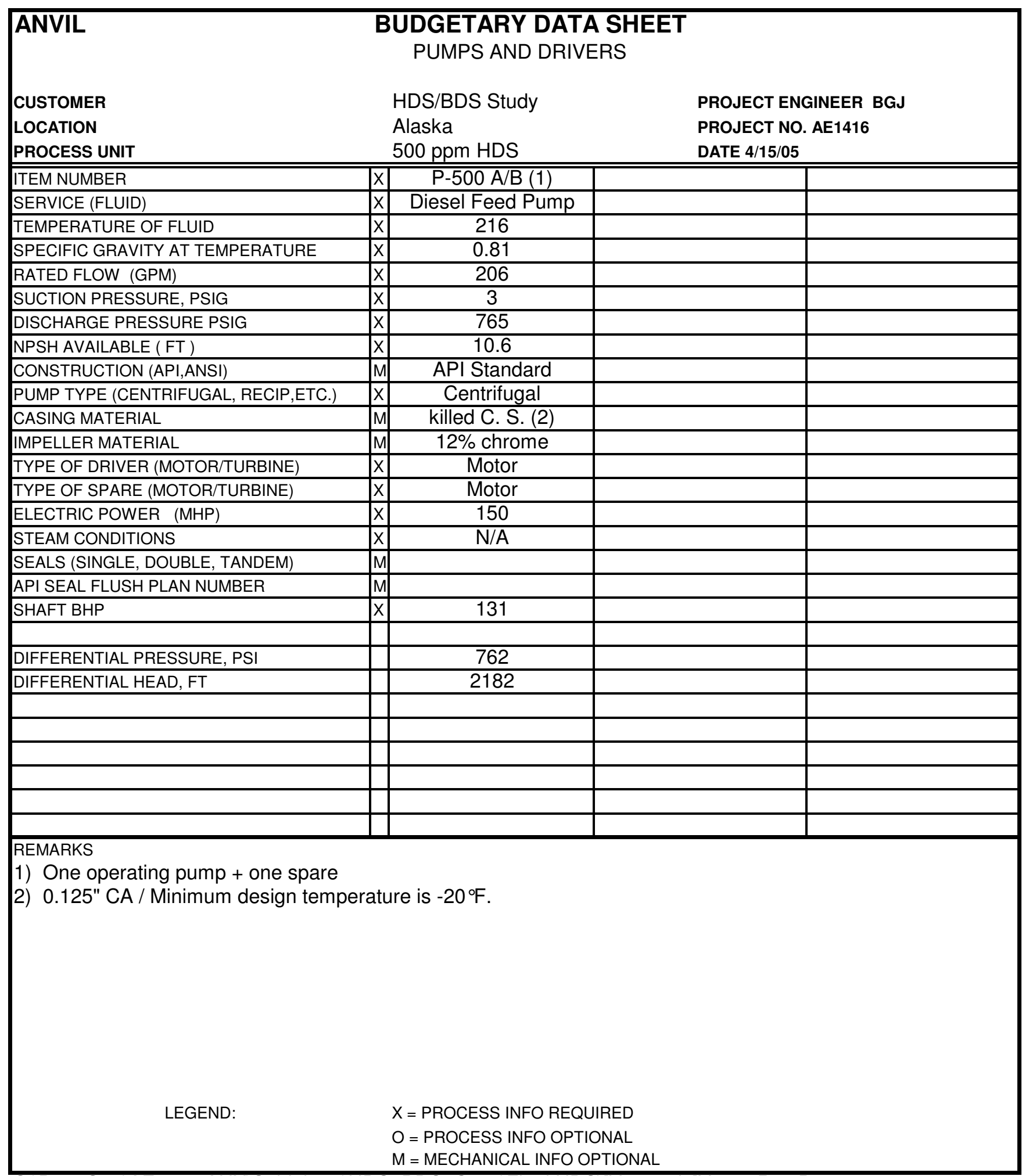

S:IPetroStar\AE1416.AUXISubjob 44|HDS_BDS_CaselFinal HDSI[V-504.xls]V-504 Rev B 


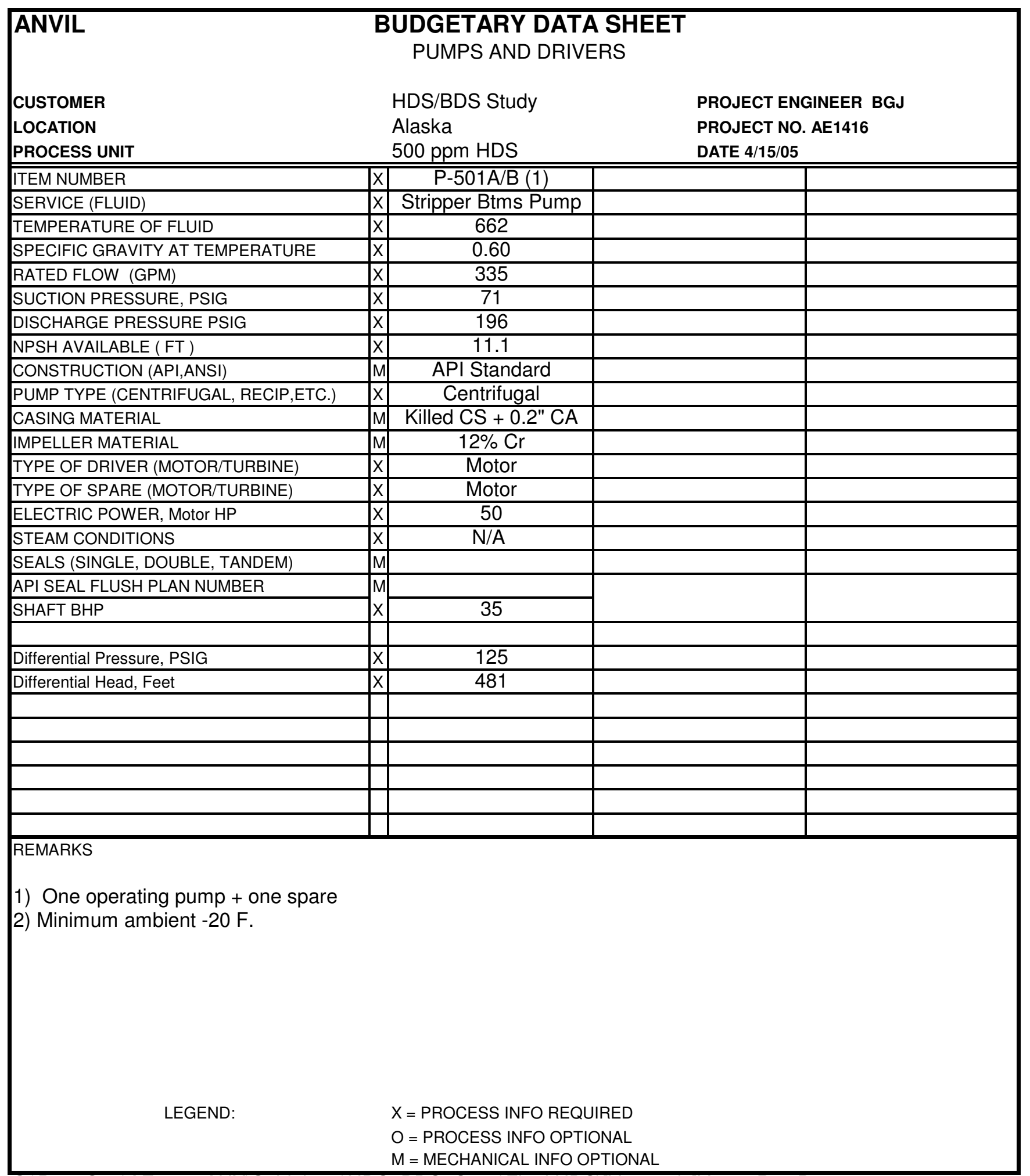

S:IPetroStar\AE1416.AUXISubjob 44|HDS_BDS_CaselFinal HDSI[V-504.xls]V-504 Rev B 


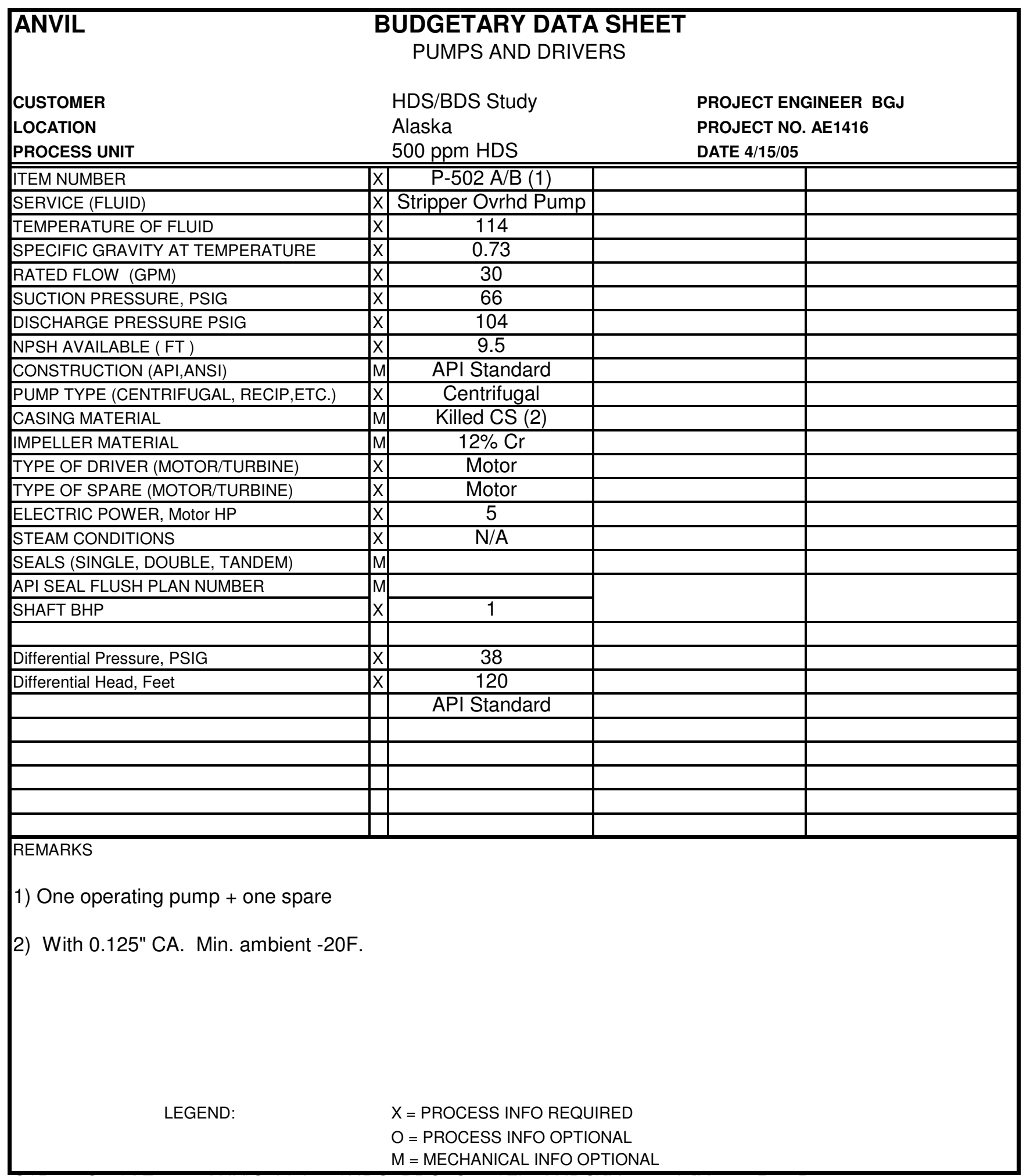

S:IPetroStar\AE1416.AUXISubjob 44|HDS_BDS_CaselFinal HDSI[V-504.xls]V-504 Rev B 


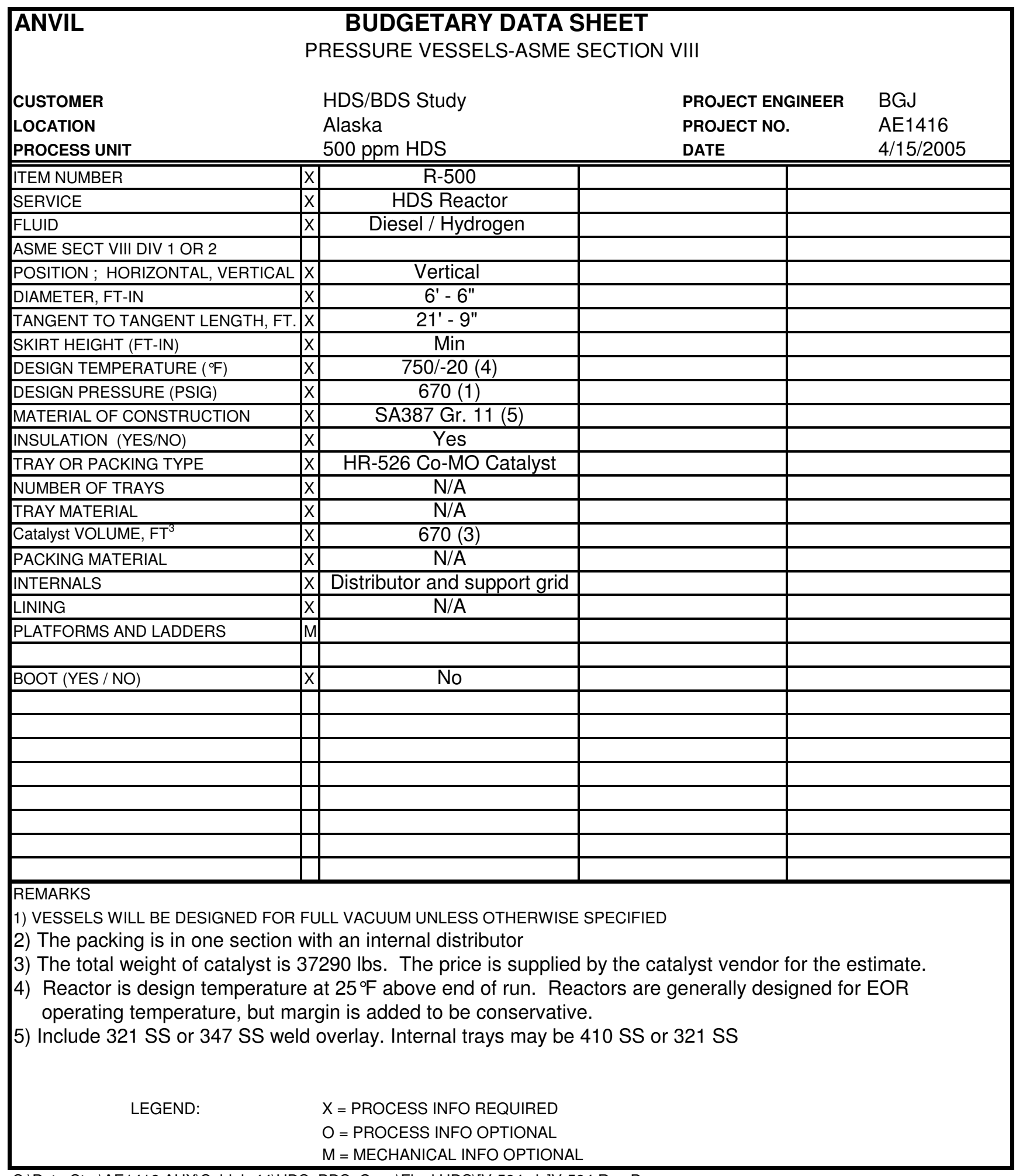

S:IPetroStarlAE1416.AUXISubjob 44|HDS_BDS_CaselFinal HDSI[V-504.xls]V-504 Rev B 


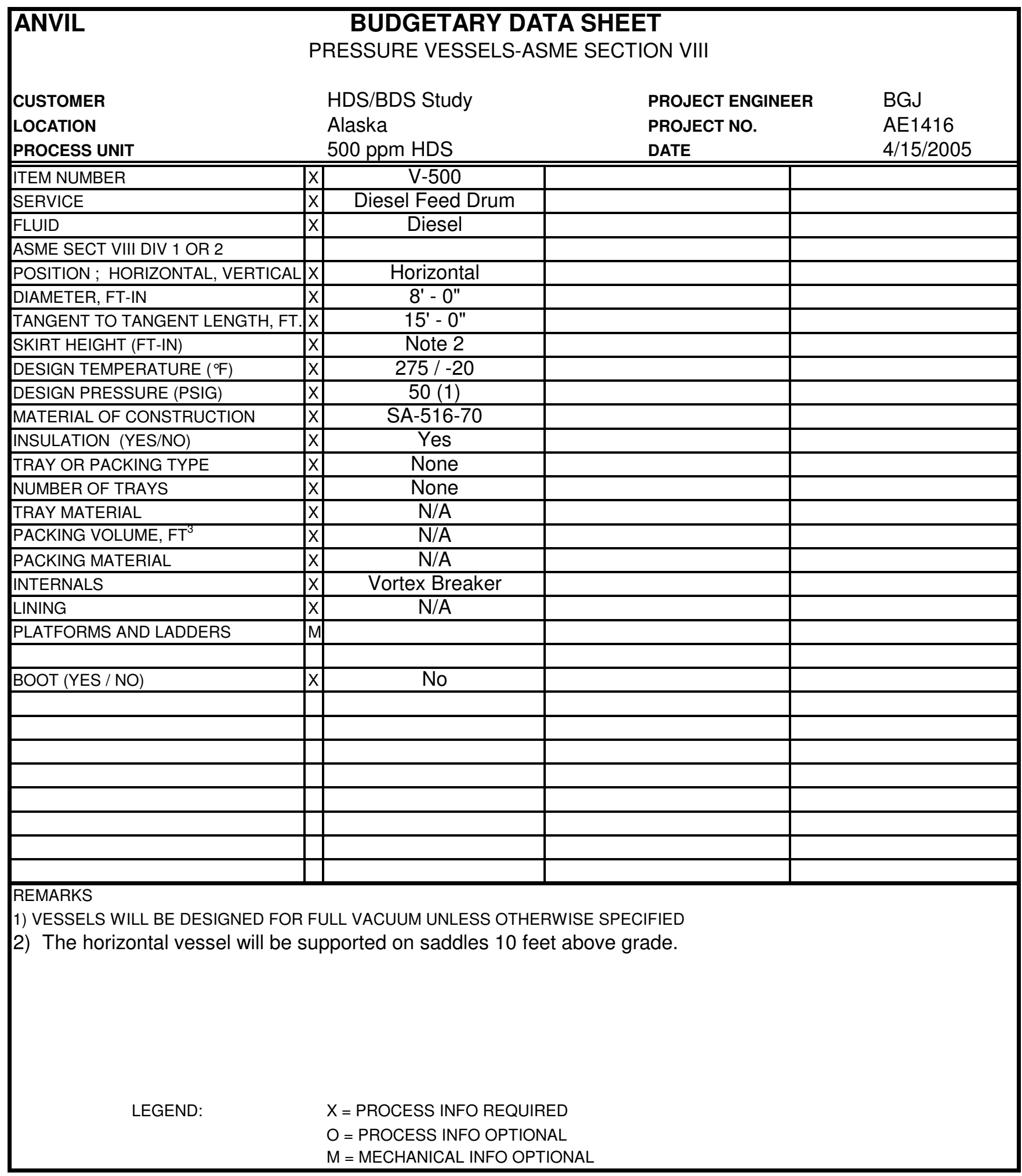

S:IPetroStar|AE1416.AUX|Subjob 44|HDS_BDS_CaselFinal HDS|[V-504.xIs]V-504 Rev B 


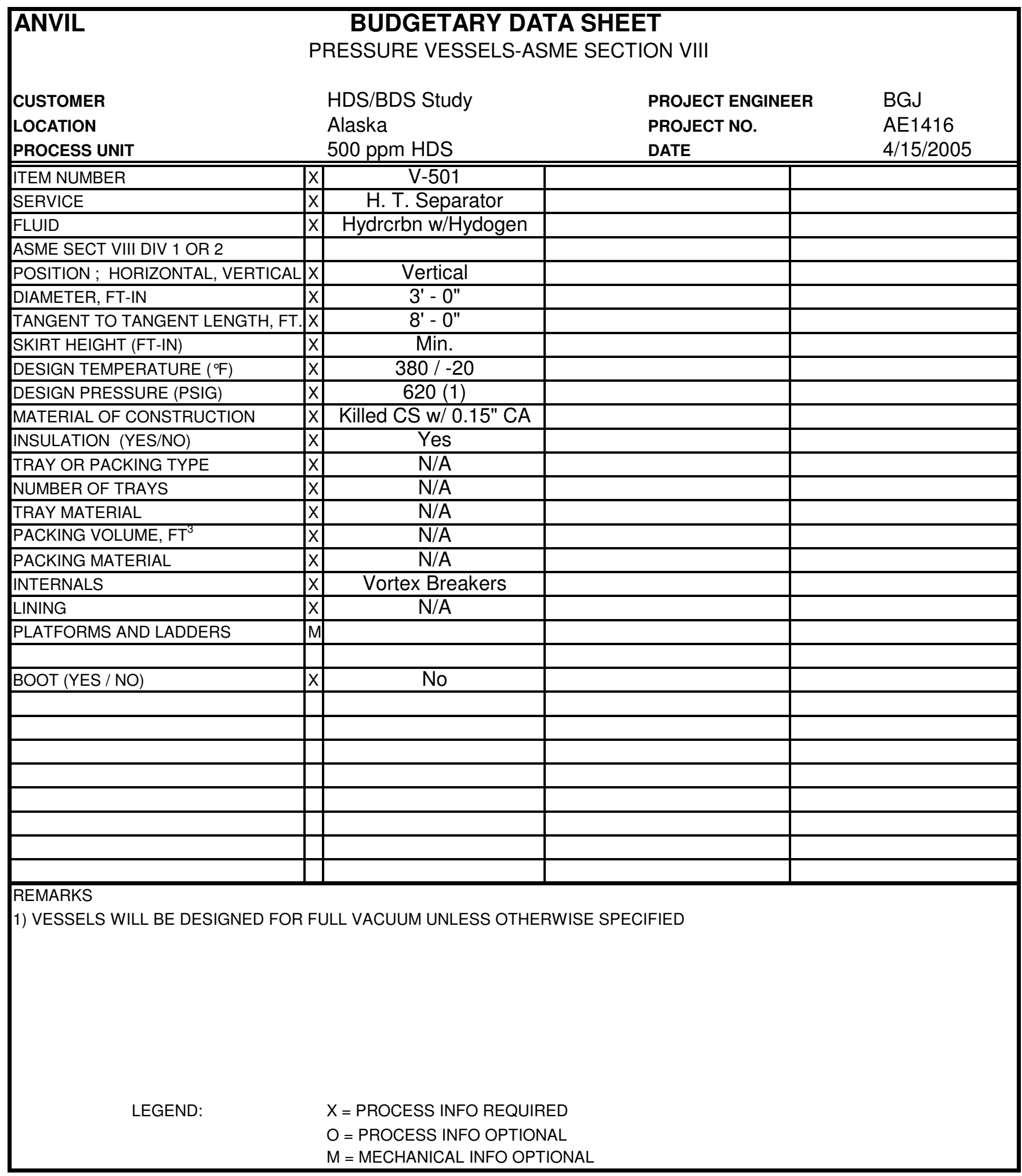

S:IPetroStar|AE1416.AUX|Subjob 44|HDS_BDS_CaselFinal HDS|[V-504.xIs]V-504 Rev B 


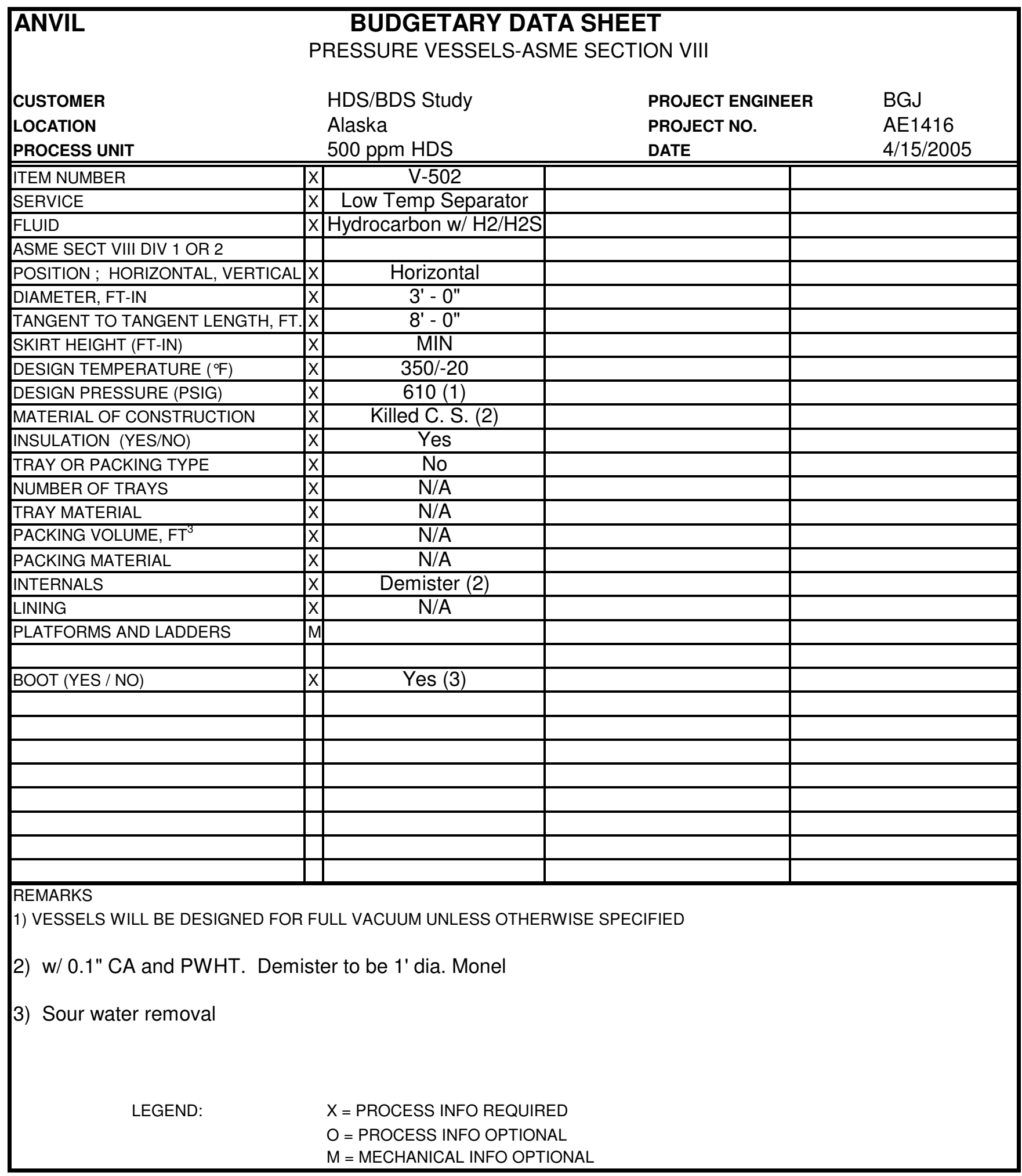

S:IPetroStar|AE1416.AUX|Subjob 44|HDS_BDS_CaselFinal HDS|[V-504.xIs]V-504 Rev B 


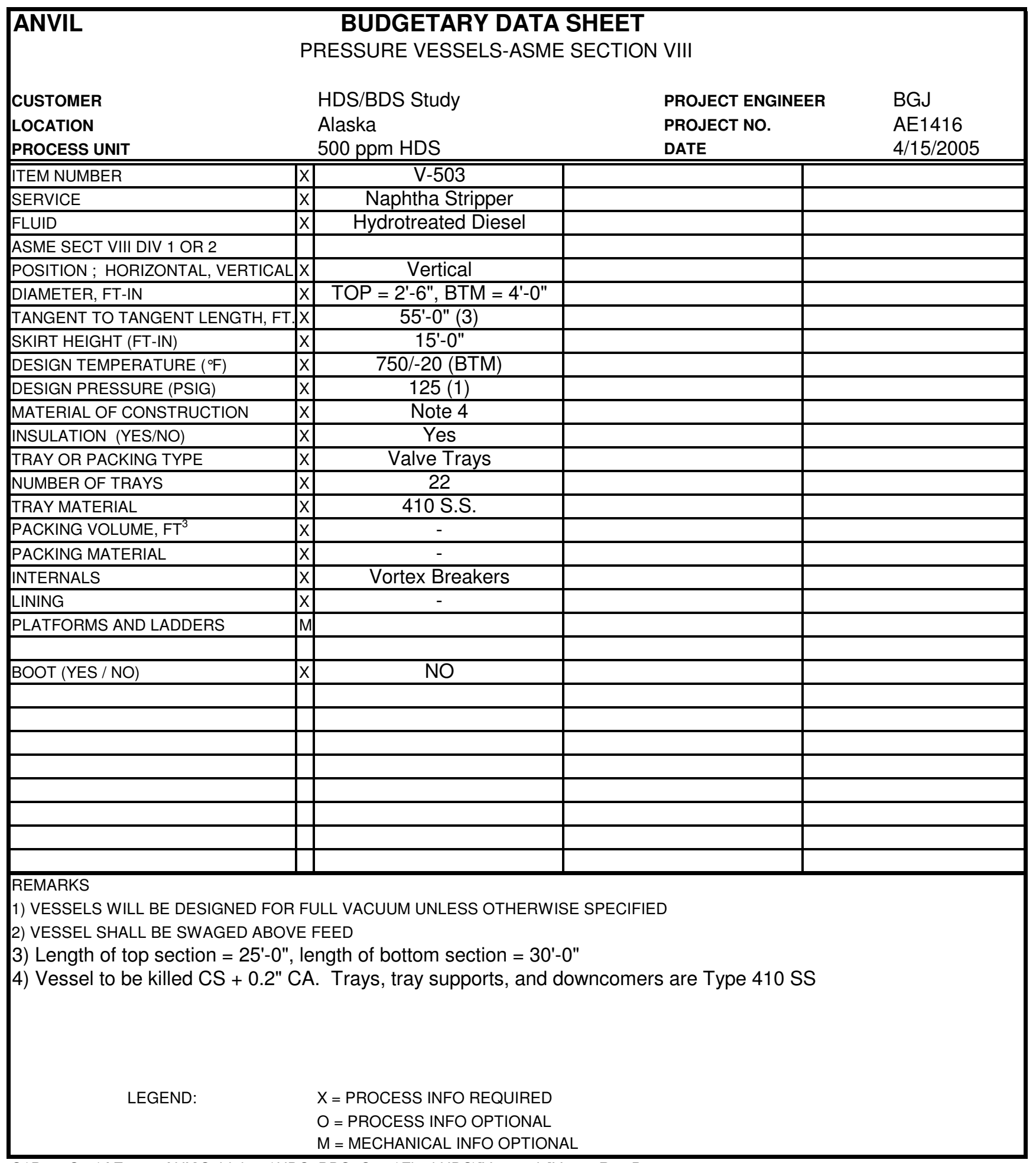




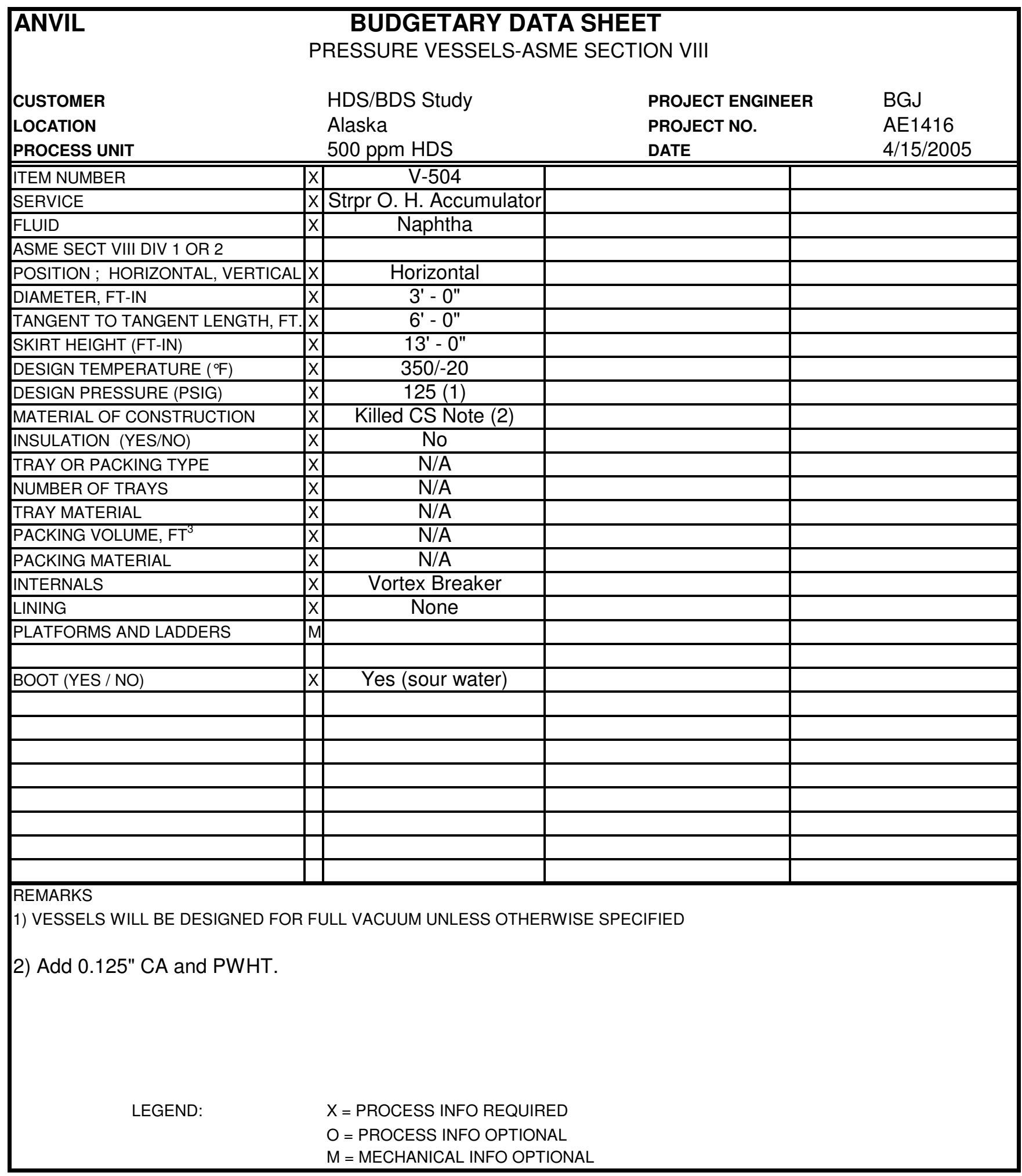

S:IPetroStar|AE1416.AUX|Subjob 44|HDS_BDS_CaselFinal HDS|[V-504.xIs]V-504 Rev B 
Appendix C.3 - BDS Unit Process Flow Diagrams and Material Balance 


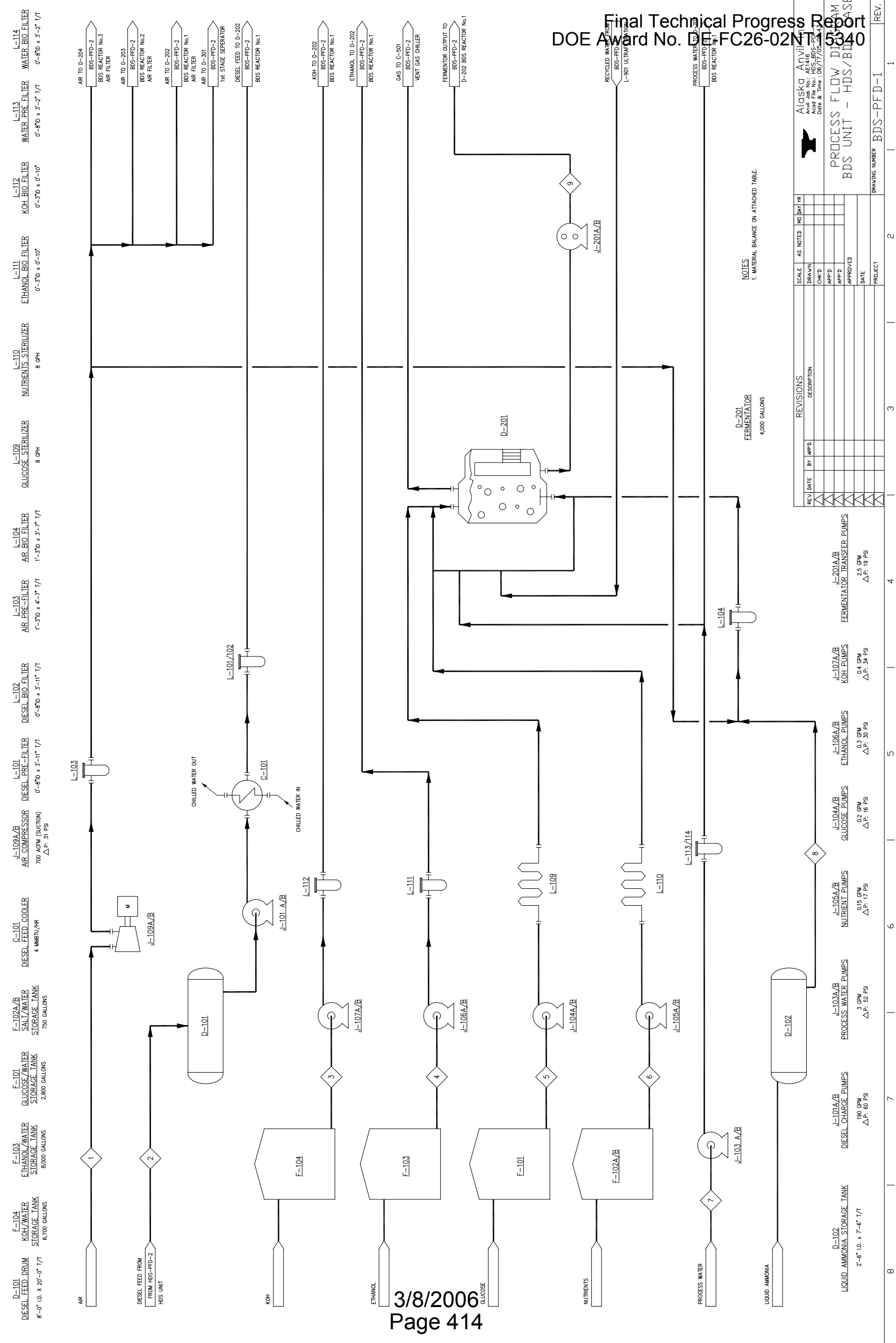




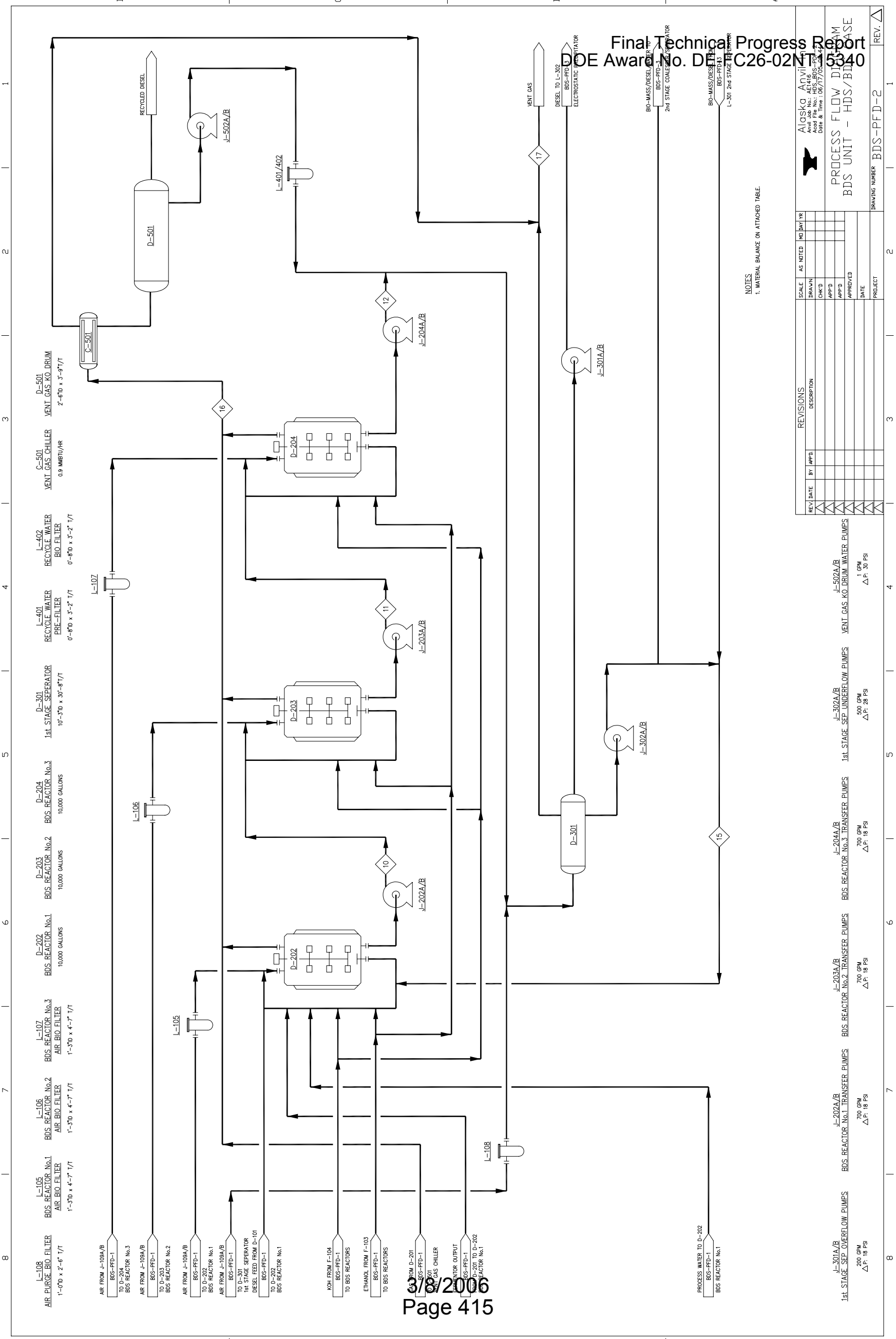




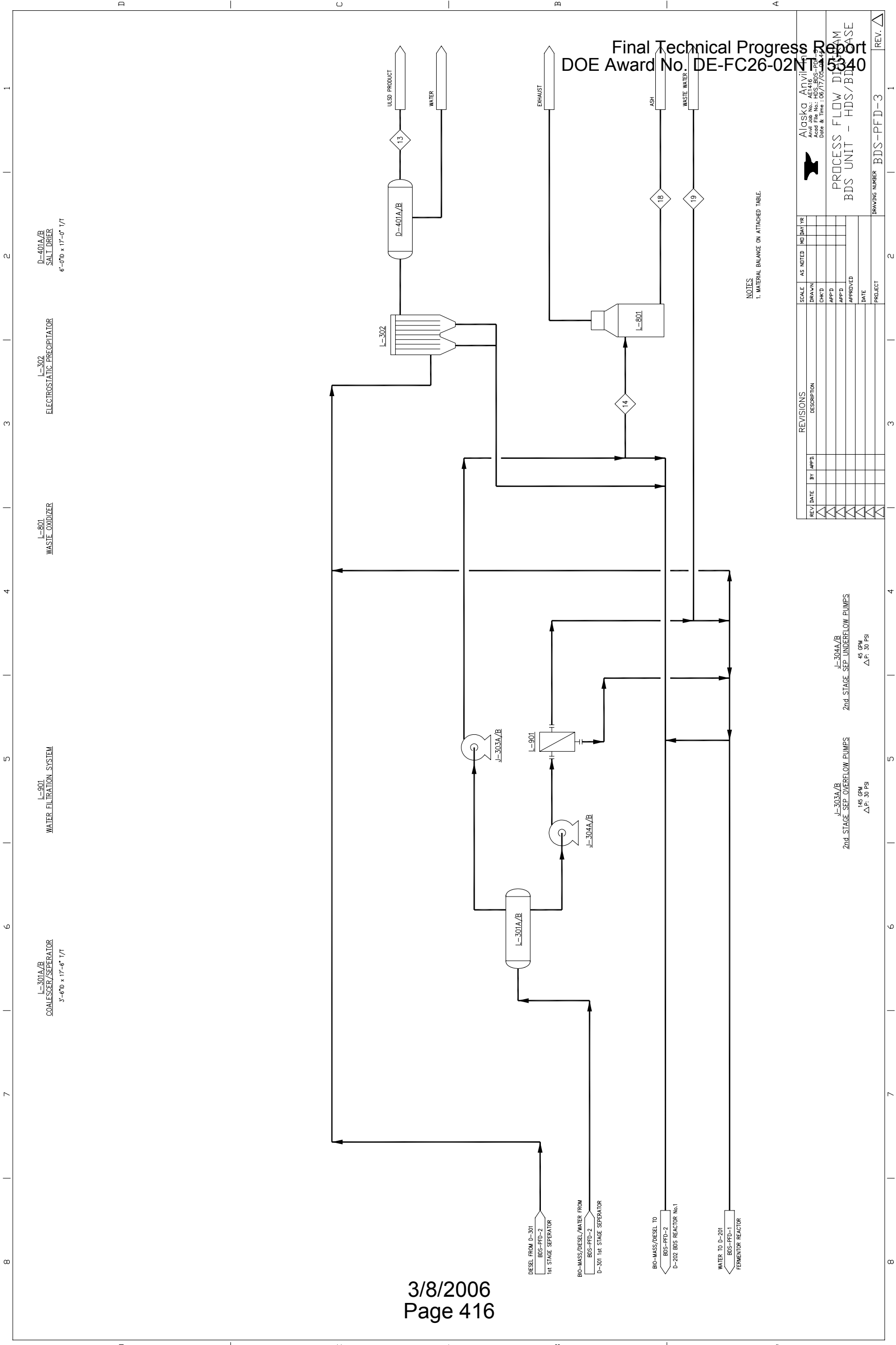




\section{Material Balance}

\section{BDS Unit Material Balance -- HDS/BDS Case}

\begin{tabular}{|l|l|c|c|c|c|c|c|c|}
\hline PFD Stream Number & & 1 & 2 & 3 & 4 & 5 & 6 & 7 \\
\hline Stream Number & & Air & Diesel & NaOH & Ethanol & Glucose & Nutrients & Process Water \\
\hline Stream Description & & Air Feed & Diesel Feed & NaOH Feed & Ethanol Feed & Glucose Feed & Nutrient Feed & $\begin{array}{c}\text { Process Water } \\
\text { Feed }\end{array}$ \\
\hline Phase & & VAPOR & LIOUID & LIGUID & LIOUID & LIGUID & LIGUID & LIOUID \\
\hline Hydrocarbon Mass Flo LB/HR & & 74483 & & & & & \\
\hline Aqueous Mass Flow & LB/HR & & & 200 & 100 & 75 & 60 & 1200 \\
\hline Biomass Mass Flow & LB/HR & & & & & & & \\
\hline Sulfur in HC & PPM & & 500 & & & & & \\
\hline Total Mass Flow Rate & LB/HR & 3000 & 74483 & 200 & 100 & 75 & 60 & 1200 \\
\hline Temperature & F & 68 & 176 & 68 & 68 & 68 & 68 & 68 \\
\hline Pressure & PSIG & 0 & 0 & 0 & 0 & 0 & 0 & 0 \\
\hline Standard Liq Flow & BPD & & 6000 & 9 & 7.5 & 4.3 & 4.1 & 82 \\
\hline Vapor Flow & MSCFH & 40 & & & & & & 0 \\
\hline Wt \% Vapor & & 100 & 0 & 0 & 0 & 0 & & 0 \\
\hline MW & & & & & & & & 0 \\
\hline
\end{tabular}

\begin{tabular}{|c|c|c|c|c|c|c|c|c|}
\hline PFD Stream Number & & 8 & 9 & 10 & 11 & 12 & 13 & 14 \\
\hline Stream Number & & Liquid Ammonia & $S-16$ & $S-17$ & S-18 & $S-19$ & S-27 & $5-31$ \\
\hline Stream Description & & \begin{tabular}{c|} 
Liquid \\
Ammonia Feed \\
\end{tabular} & $\begin{array}{c}\text { Fermentor } \\
\text { Output }\end{array}$ & R\#1 Output & R\#2 Output & R\#3 Output & Diesel Product & Biomass Purge \\
\hline Phase & & LIGUID & LIQUID & LIGUID & LIQUID & LIQUID & LIQUID & LIQUID \\
\hline Hydrocarbon Mass Flo & LB/HR & & & 126160 & 125966 & 125775 & 74271 & 195 \\
\hline Aqueous Mass Flow & LB/HR & 4.8 & 1007 & 140620 & 140771 & 140805 & 0 & 335 \\
\hline Biomass Mass Flow & LB/HR & & 74 & 30602 & 30602 & 30602 & 0 & 92 \\
\hline Sulfur in $\mathrm{HC}$ & PPM & & & 117 & 35 & 9 & 9 & 9 \\
\hline Mass Flow Rate & LB/HR & 4.8 & 1081 & 297382 & 297338 & 297182 & 74271 & 623 \\
\hline Temperature & $\mathrm{F}$ & -40 & 86 & 86 & 86 & 86 & 86 & 86 \\
\hline Pressure & PSIG & 210 & 33.1 & 36.4 & 36.4 & 26.8 & 5 & 5 \\
\hline Standard Liq Flow & $\mathrm{BPD}$ & 0.5 & 71 & 21295 & 21287 & 21274 & 5983 & 44 \\
\hline Vapor Flow & MSCFH & & & & & & & \\
\hline Wt \% Vapor & & 0 & 0 & 0 & 0 & 0 & 0 & 0 \\
\hline MW & & & & & & & & \\
\hline
\end{tabular}

\begin{tabular}{|l|l|c|c|c|c|c|c|c|}
\hline PFD Stream Number & & 15 & 16 & 17 & 18 & 19 & & \\
\hline Stream Number & & S-32 & S-38 & S-39 & Ash & Waste Water & & \\
\hline Stream Description & & $\begin{array}{c}\text { Reactor } \\
\text { Recvcle }\end{array}$ & $\begin{array}{c}\text { Reactor Air } \\
\text { Effluent }\end{array}$ & Gas Purge & $\begin{array}{c}\text { Incinerator } \\
\text { Waste }\end{array}$ & Waste Water & & \\
\hline Phase & & LIQUID & GAS & GAS & SOLID & LIQUID & & \\
\hline Hydrocarbon Mass Flo & LB/HR & 51876 & 567 & & & & & \\
\hline Aqueous Mass Flow & LB/HR & 138224 & 47 & & & 1372 & & \\
\hline Biomass Mass Flow & LB/HR & 30528 & & & & & & \\
\hline Sulfur in HC & PPM & 9 & 55 & & & & & \\
\hline Total Mass Flow Rate & LB/HR & 220628 & 3443 & 2844 & 14 & 1372 & & \\
\hline Temperature & F & 86 & 86 & 60 & 68 & 86 & & \\
\hline Pressure & PSIG & 37.4 & 10 & 1 & 0 & 10 & & \\
\hline Standard Liq Flow & BPD & 15148 & & & & 89 & & \\
\hline Vapor Flow & MSCFH & & 39 & 38 & & & & \\
\hline Wt \% Vapor & & 0 & 100 & 100 & 0 & 0 & & \\
\hline MW & & & & & & & & \\
\hline
\end{tabular}


Appendix C.4-BDS Unit Equipment List and Budgetary Equipment Datasheets 


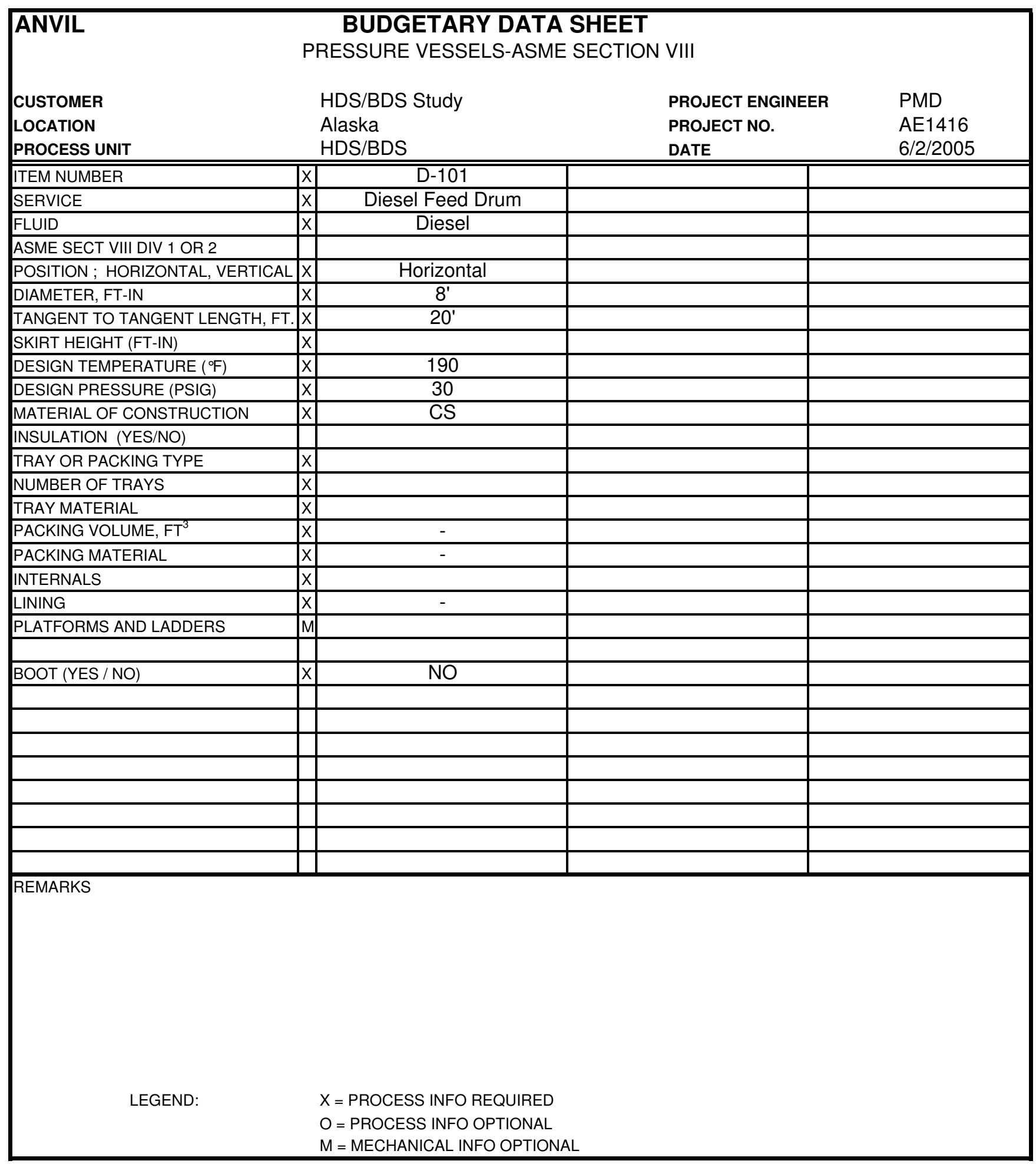




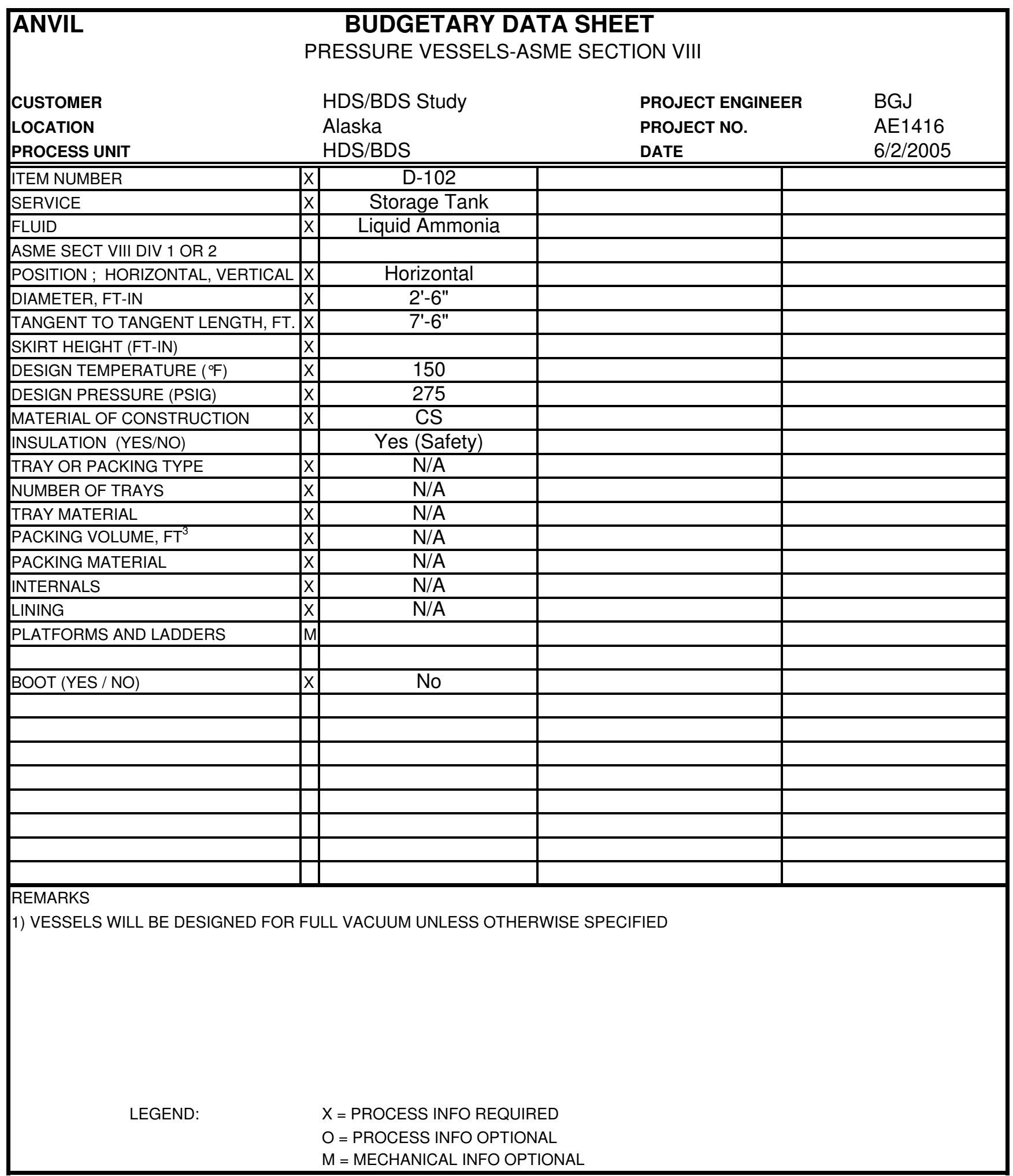




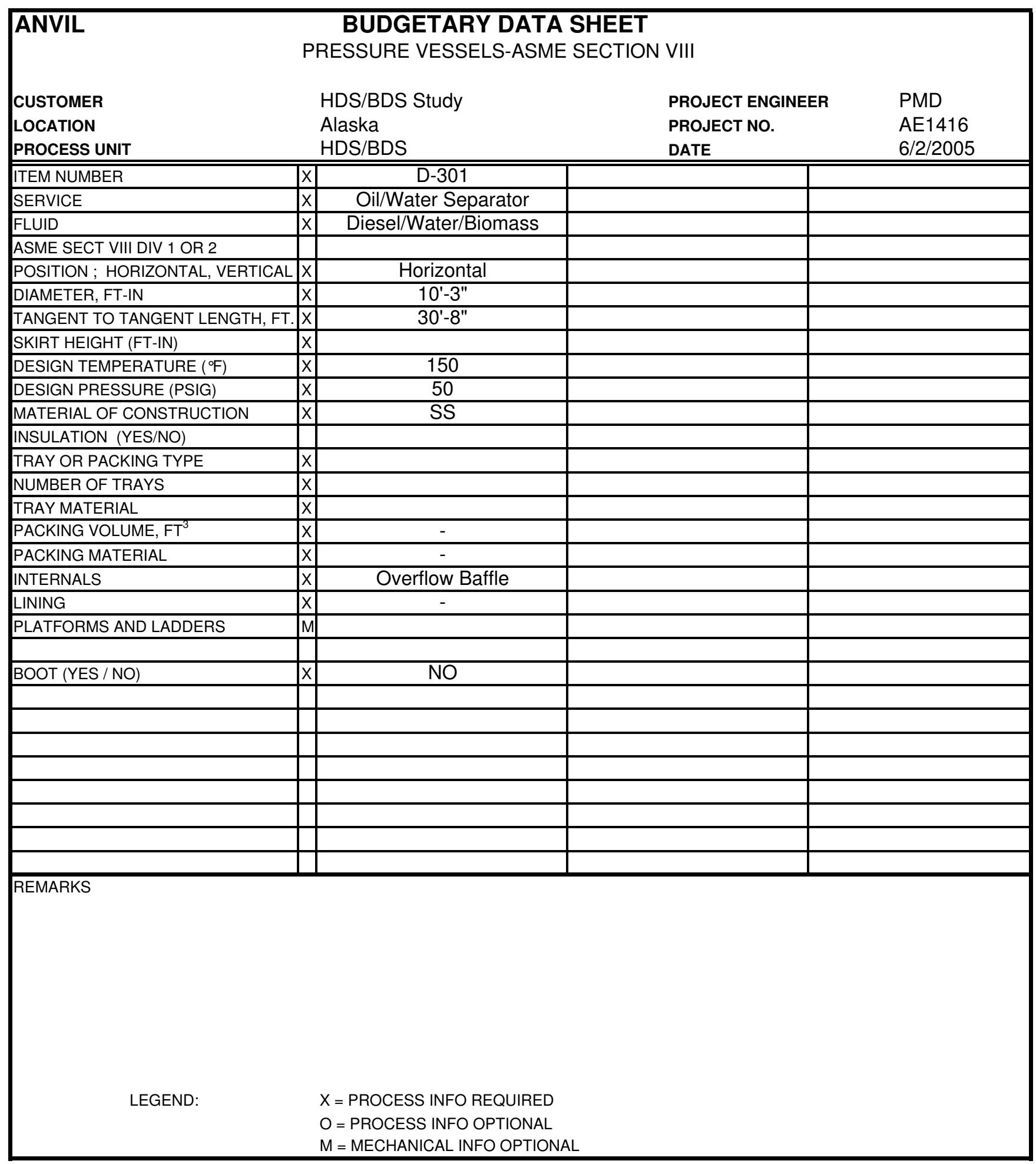




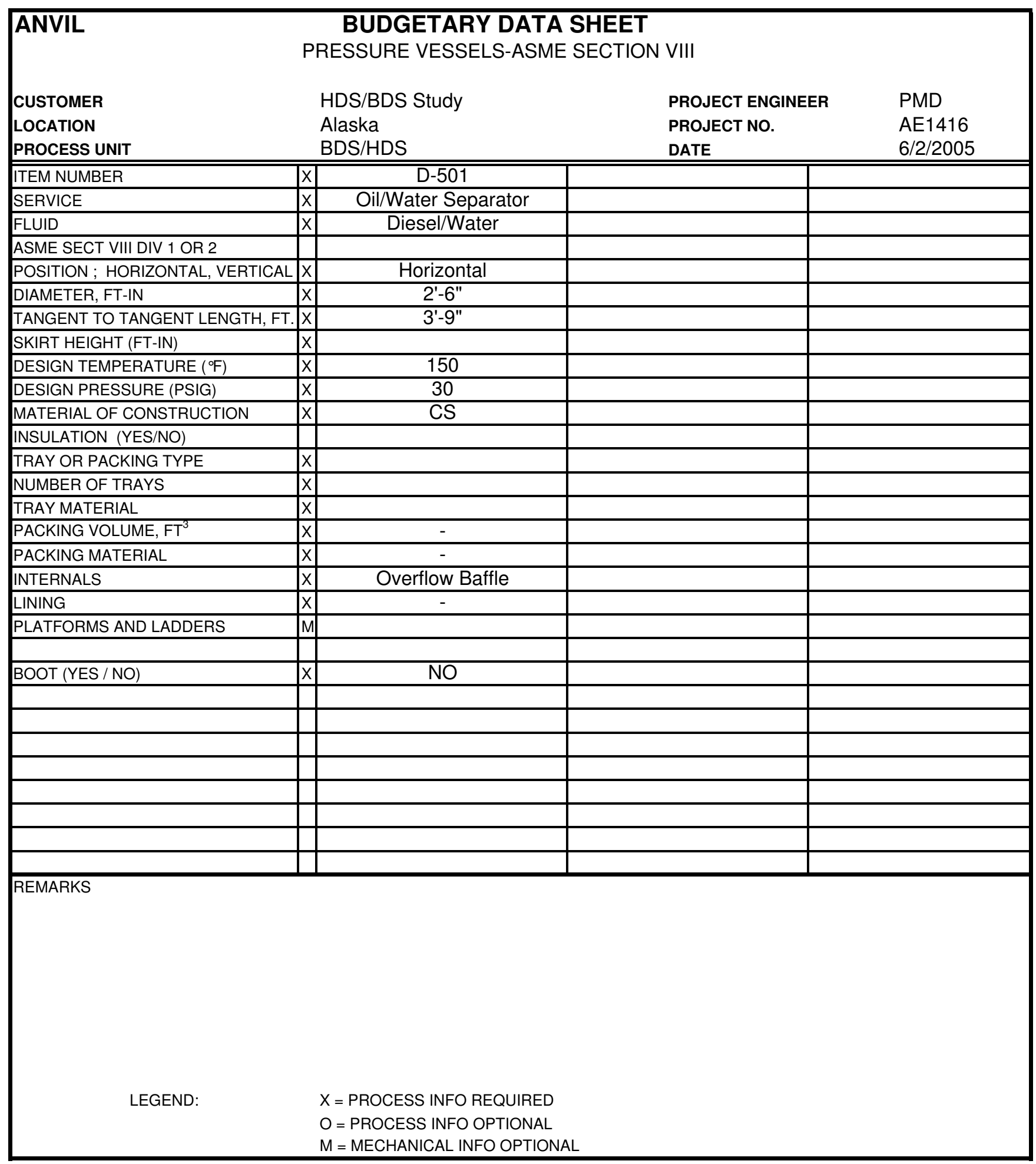




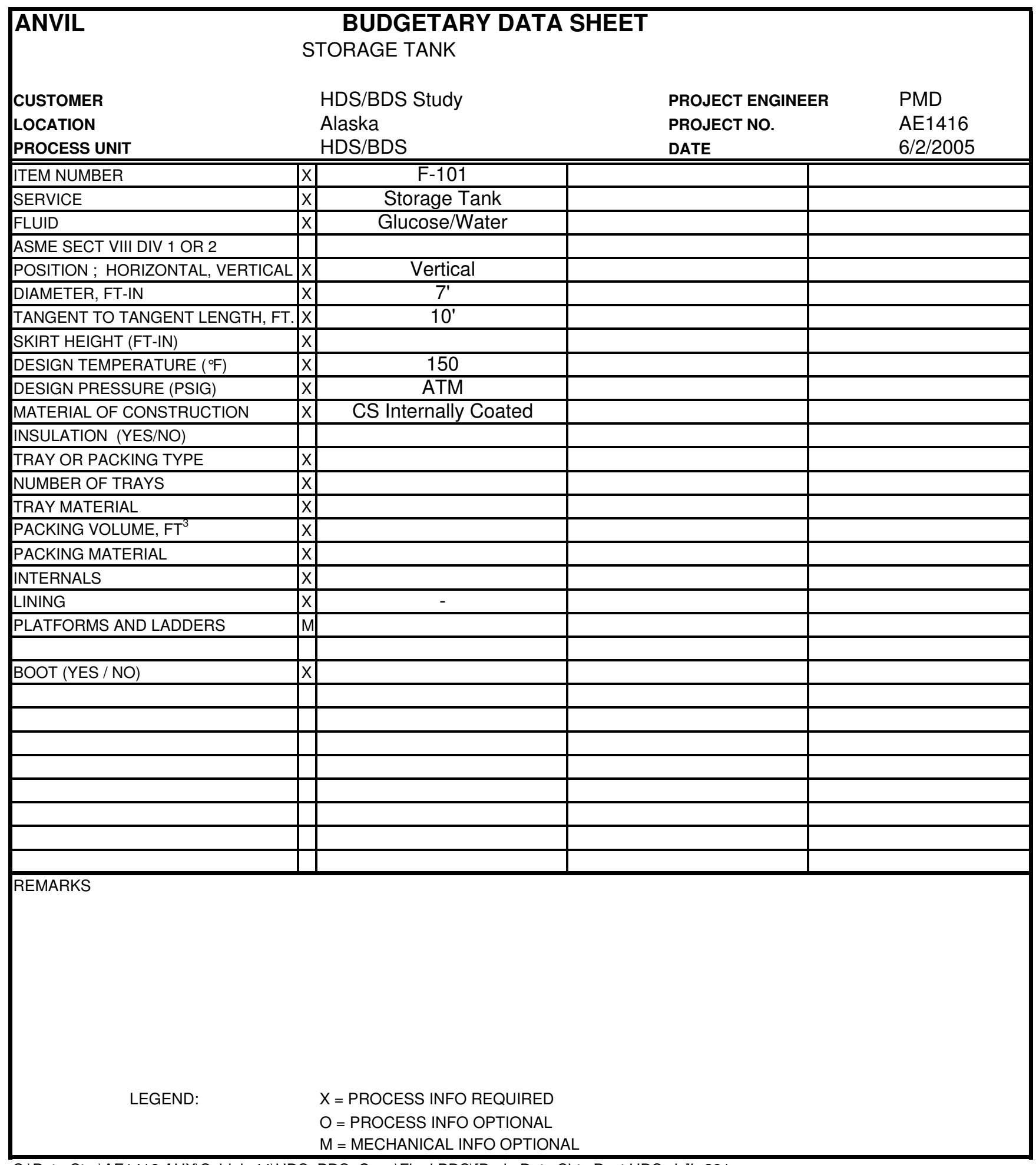




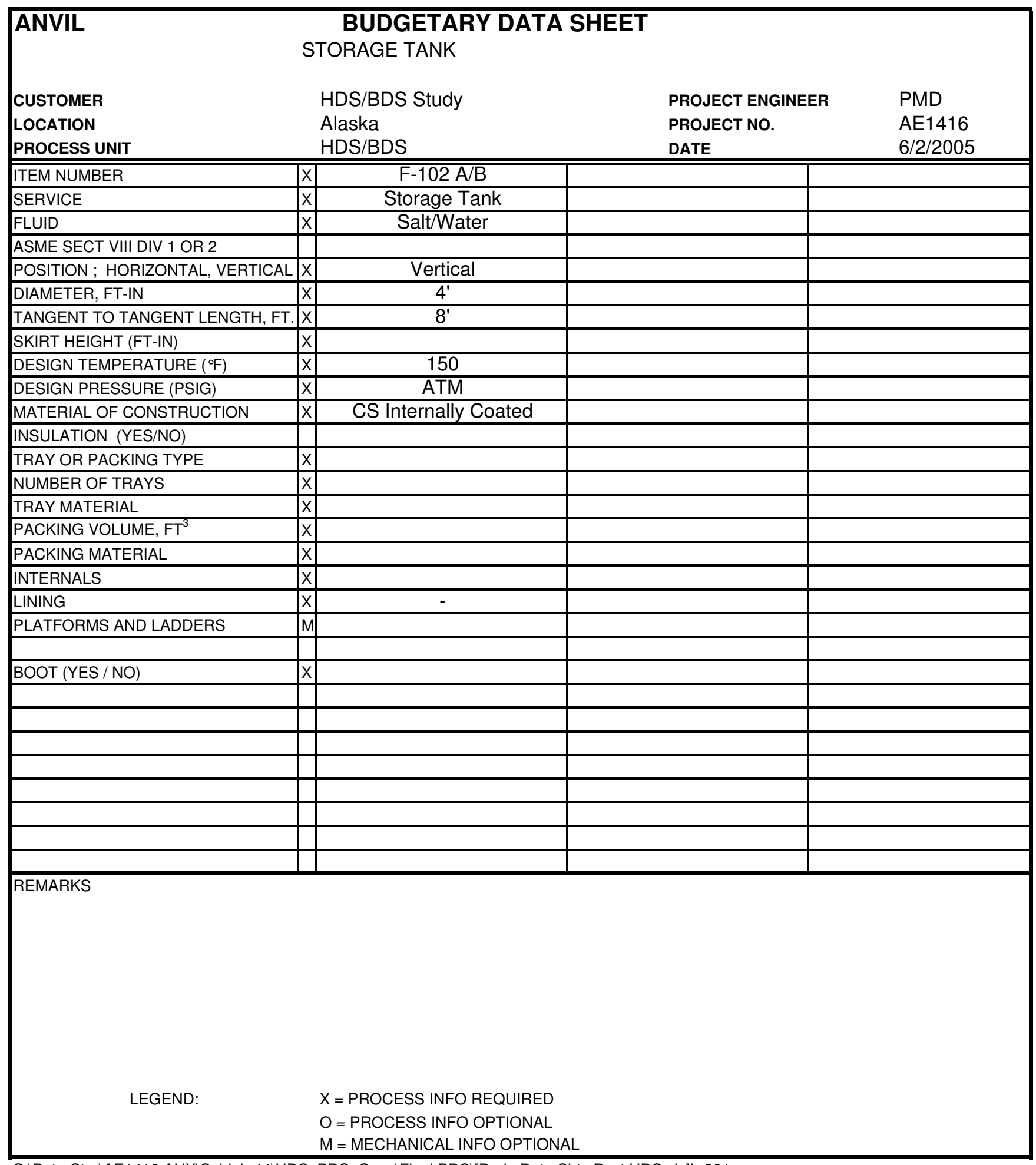




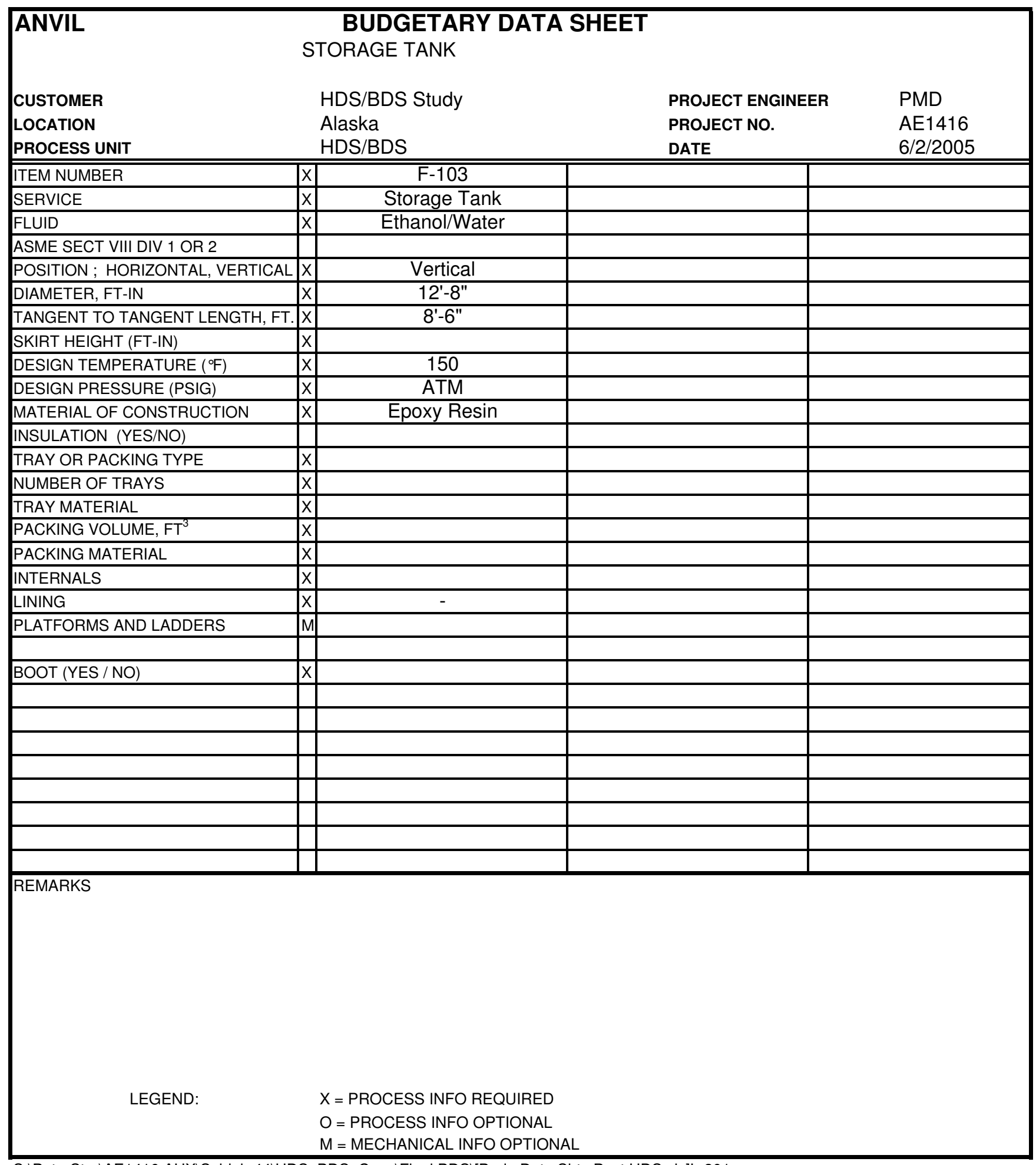




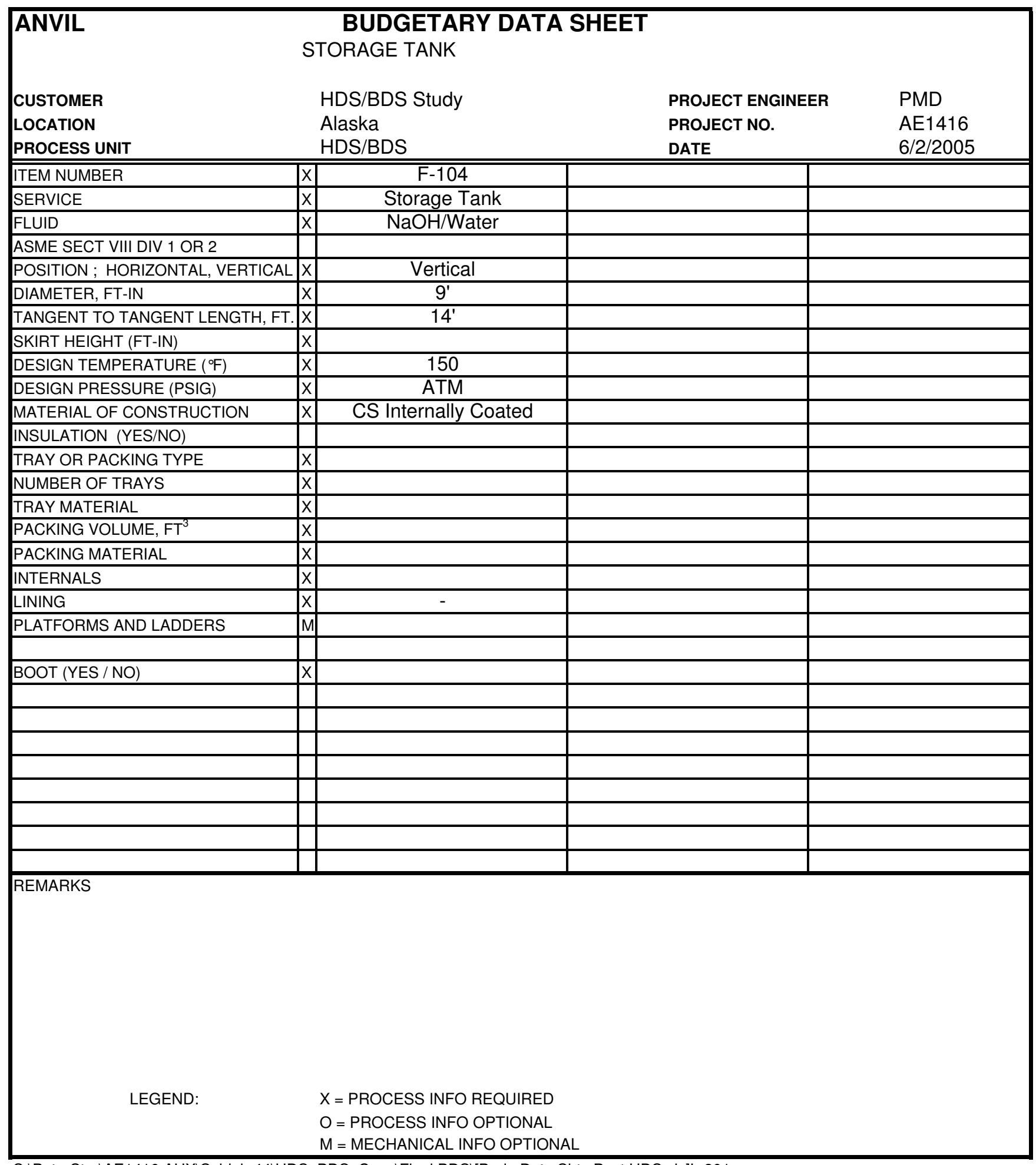




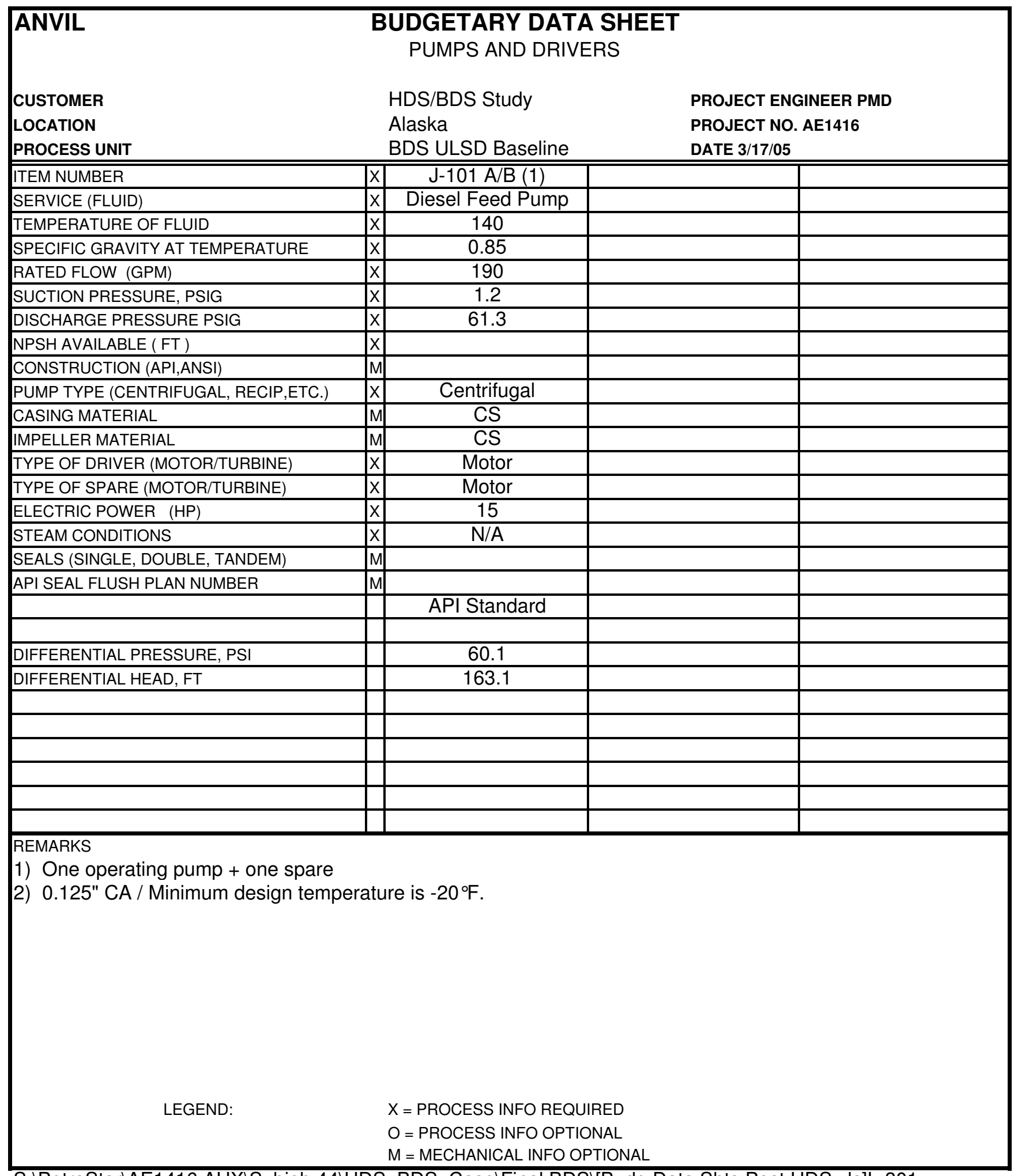

S:IPetroStar|AE1416.AUXISubjob 44IHDS_BDS_CaselFinal BDS|[Budg Data Shts Post HDS.xls]L-301 


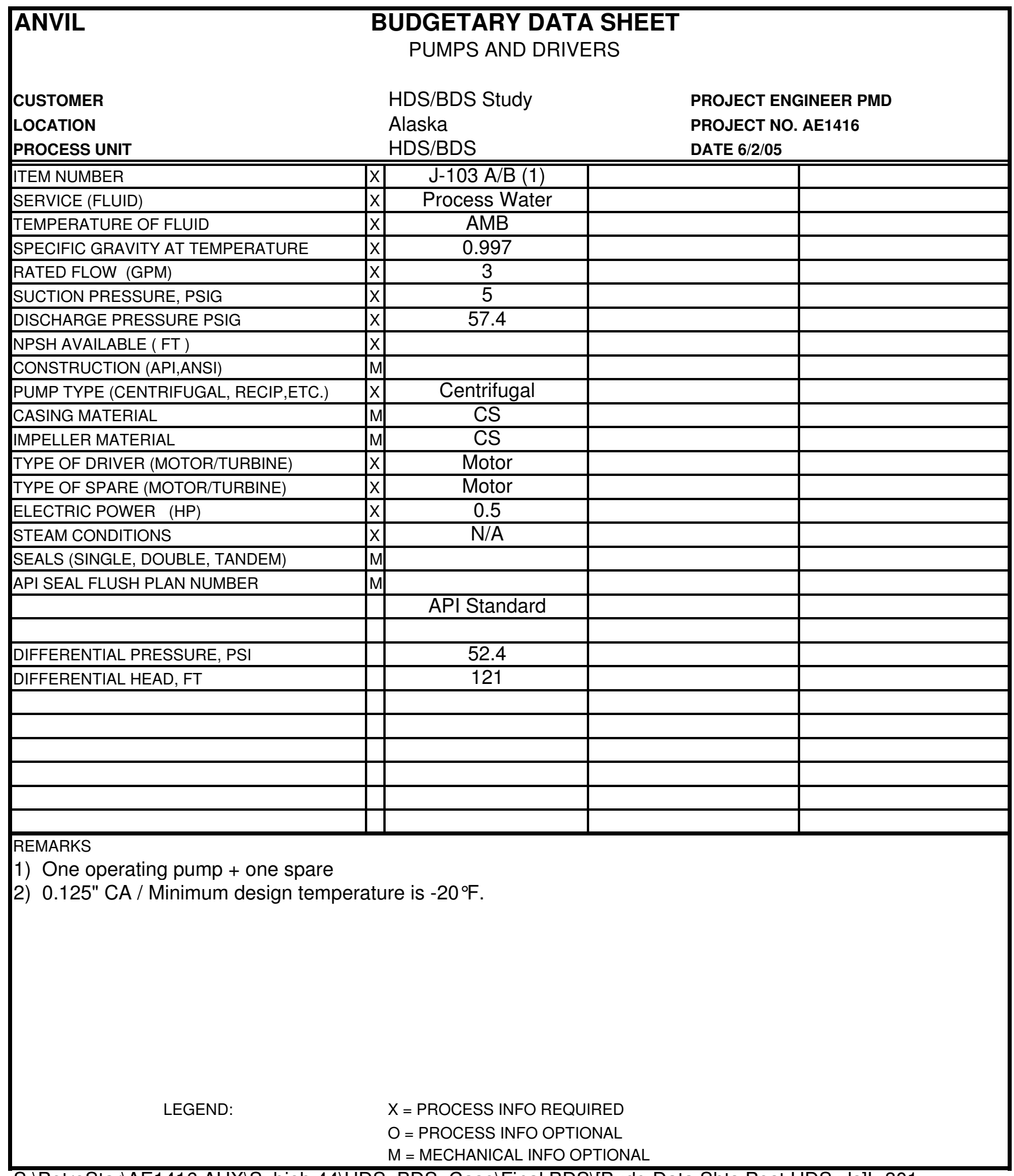

S:IPetroStar|AE1416.AUXISubjob 44IHDS_BDS_CaselFinal BDS|[Budg Data Shts Post HDS.xls]L-301 


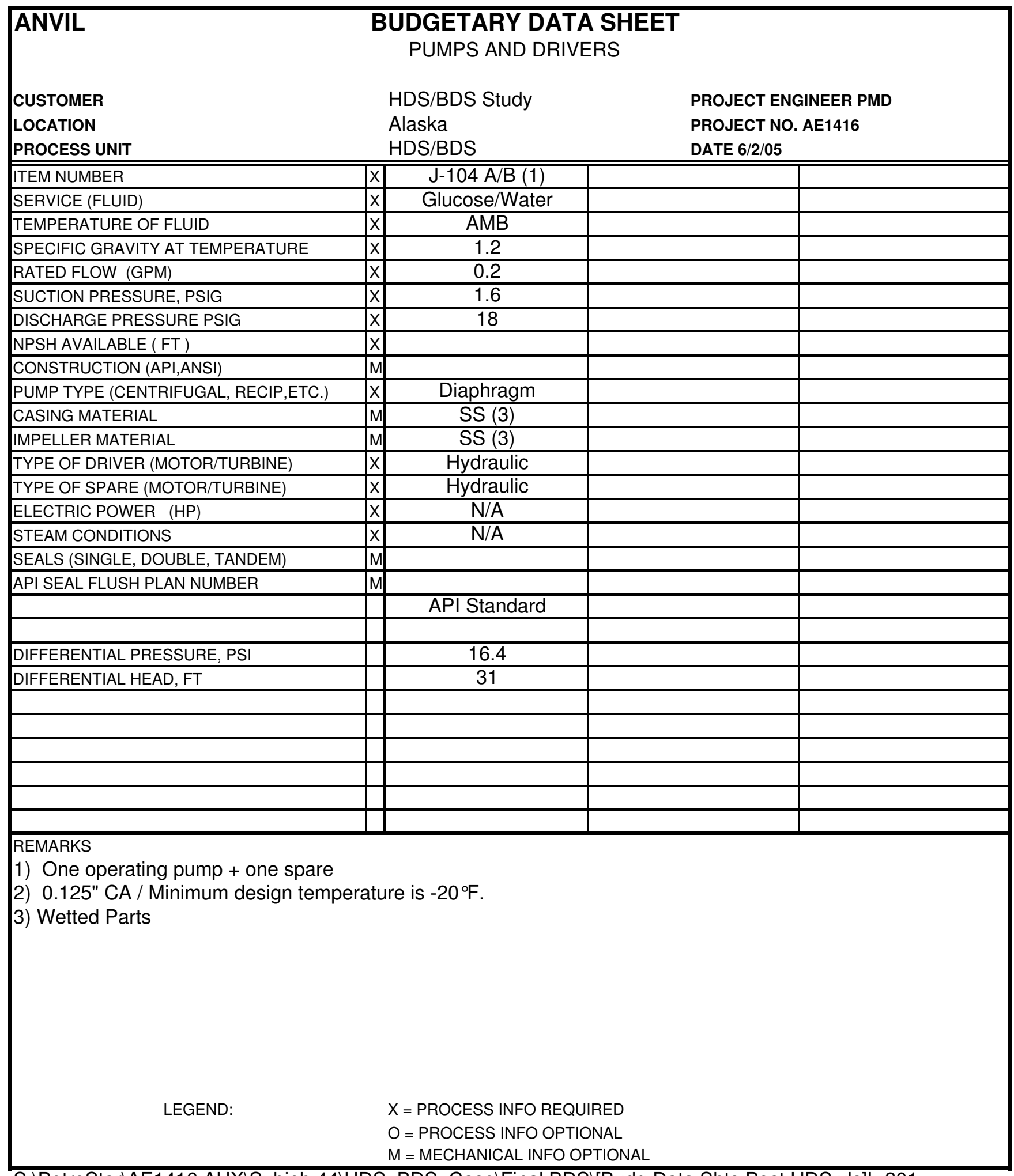

S:IPetroStar|AE1416.AUXISubjob 44IHDS_BDS_CaselFinal BDS|[Budg Data Shts Post HDS.xls]L-301 


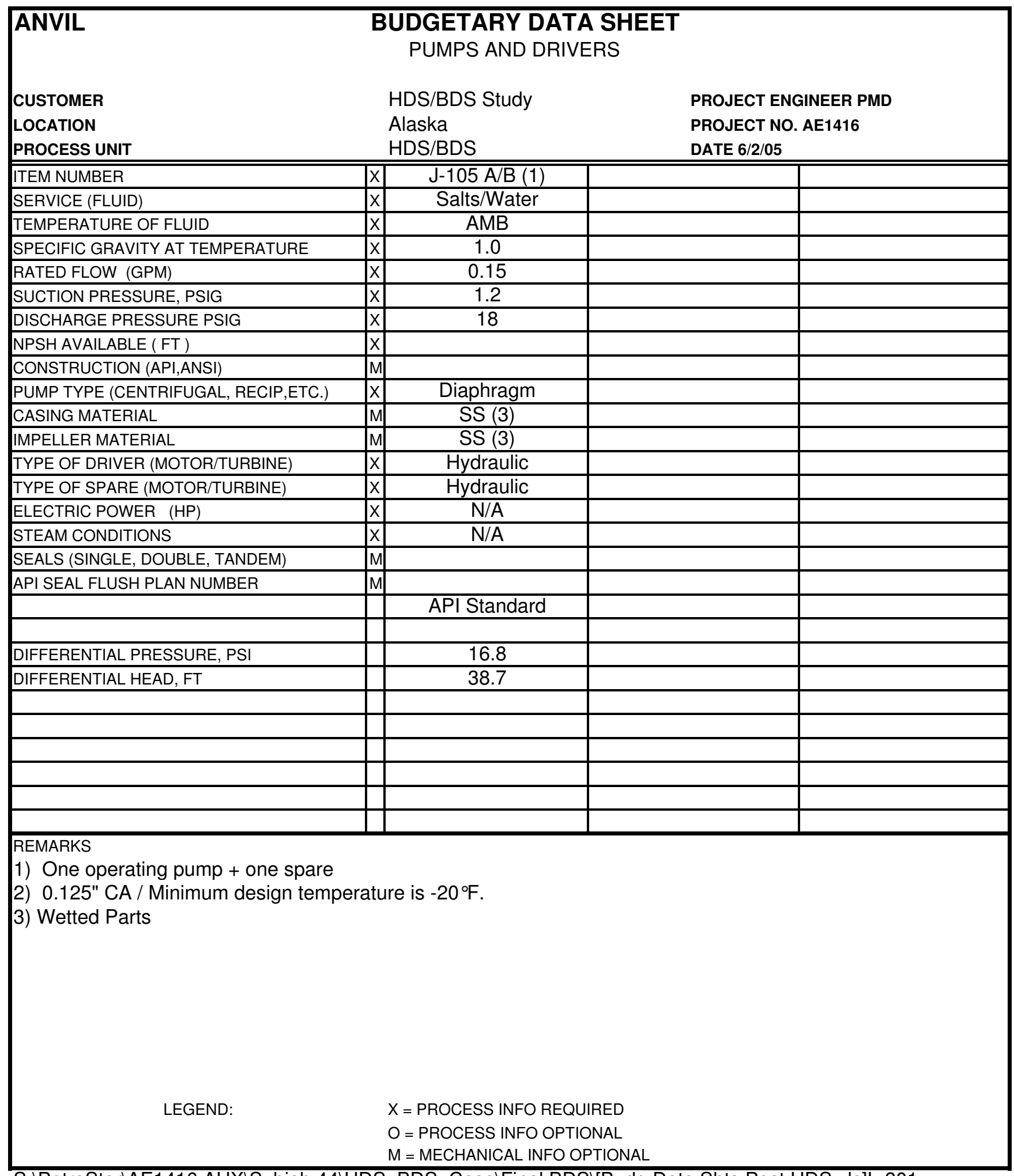

S:IPetroStar|AE1416.AUXISubjob 44IHDS_BDS_CaselFinal BDS|[Budg Data Shts Post HDS.xls]L-301 


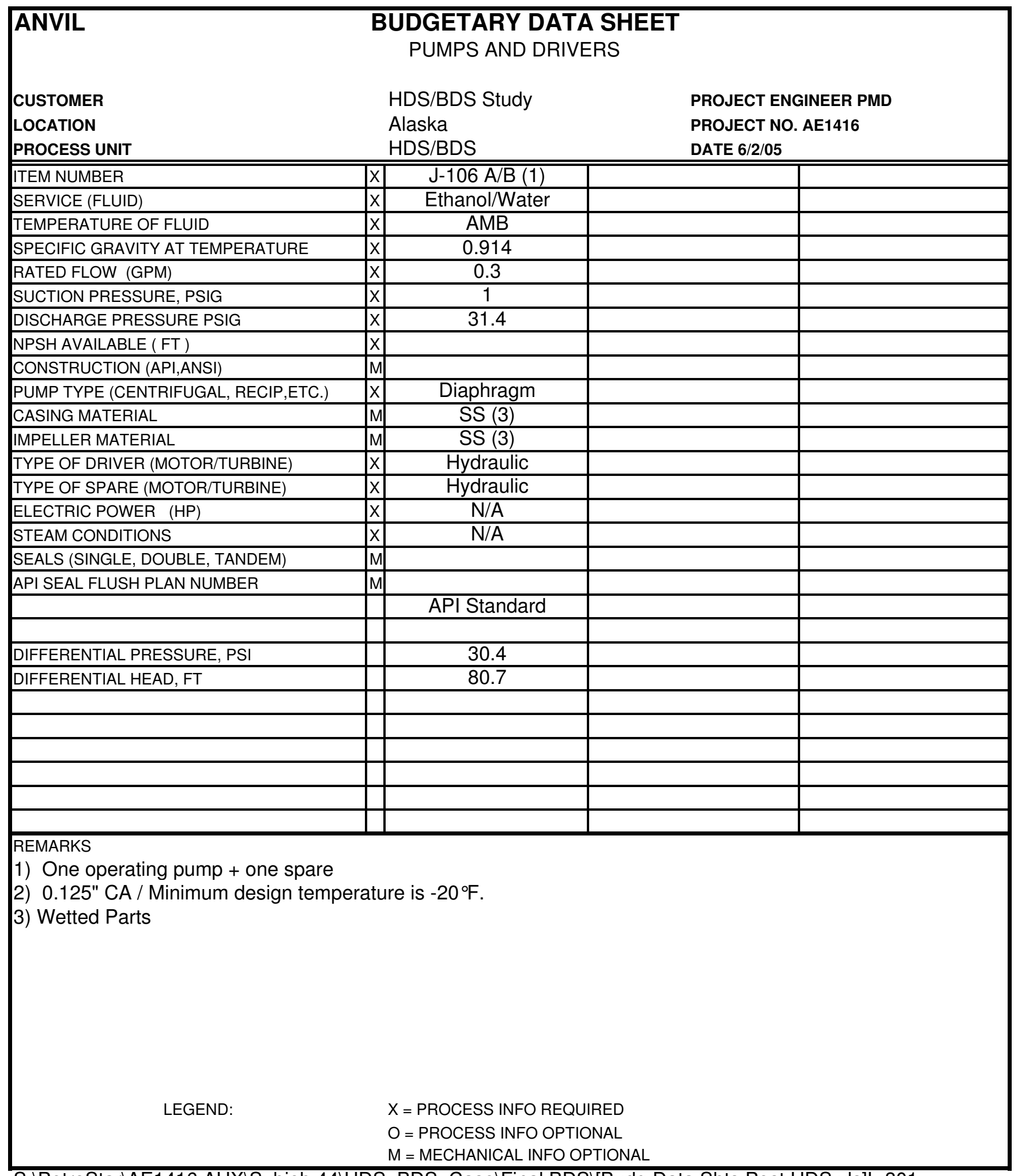

S:IPetroStar|AE1416.AUXISubjob 44IHDS_BDS_CaselFinal BDS|[Budg Data Shts Post HDS.xls]L-301 


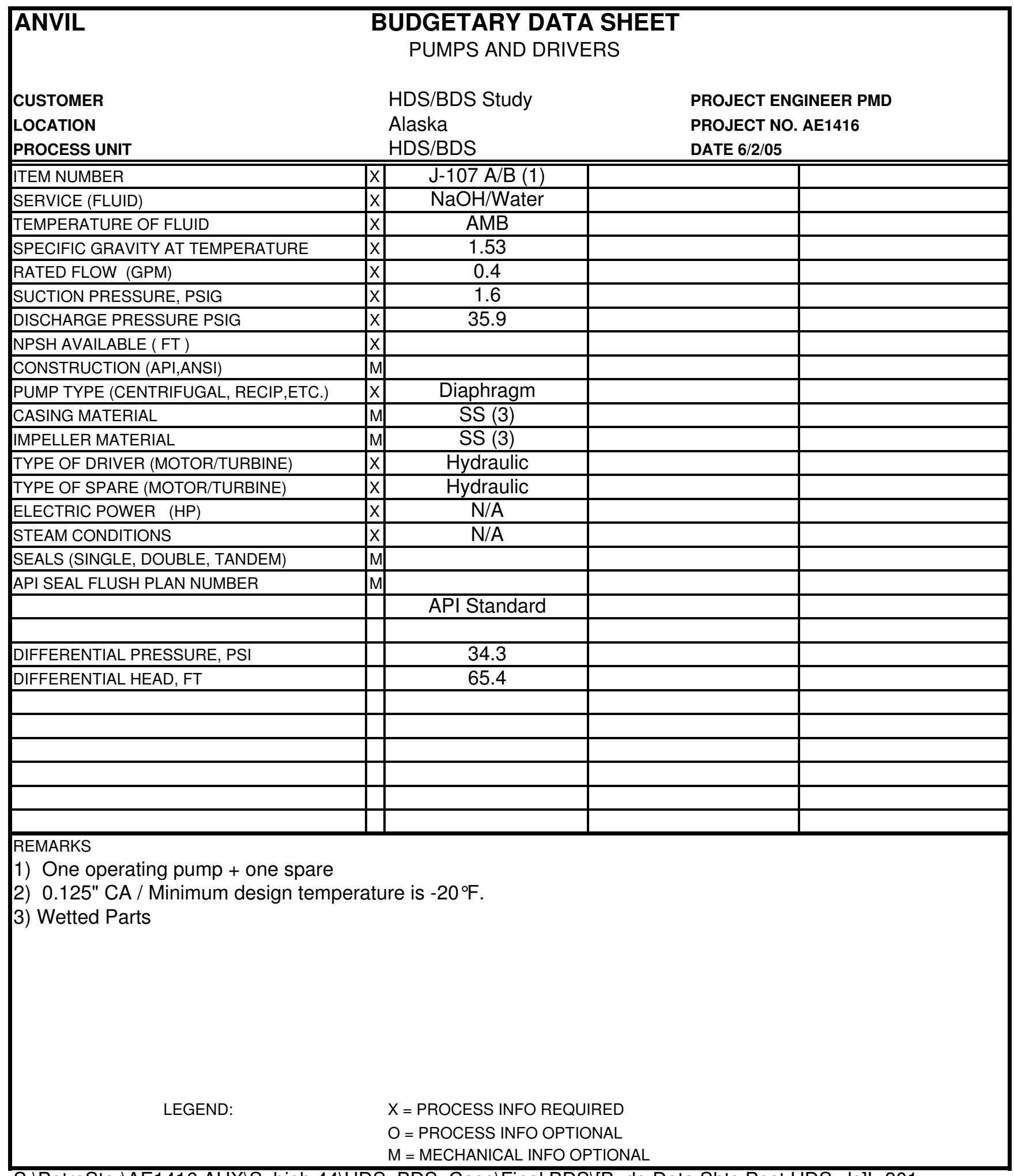

S:IPetroStar|AE1416.AUXISubjob 44IHDS_BDS_CaselFinal BDS|[Budg Data Shts Post HDS.xls]L-301 


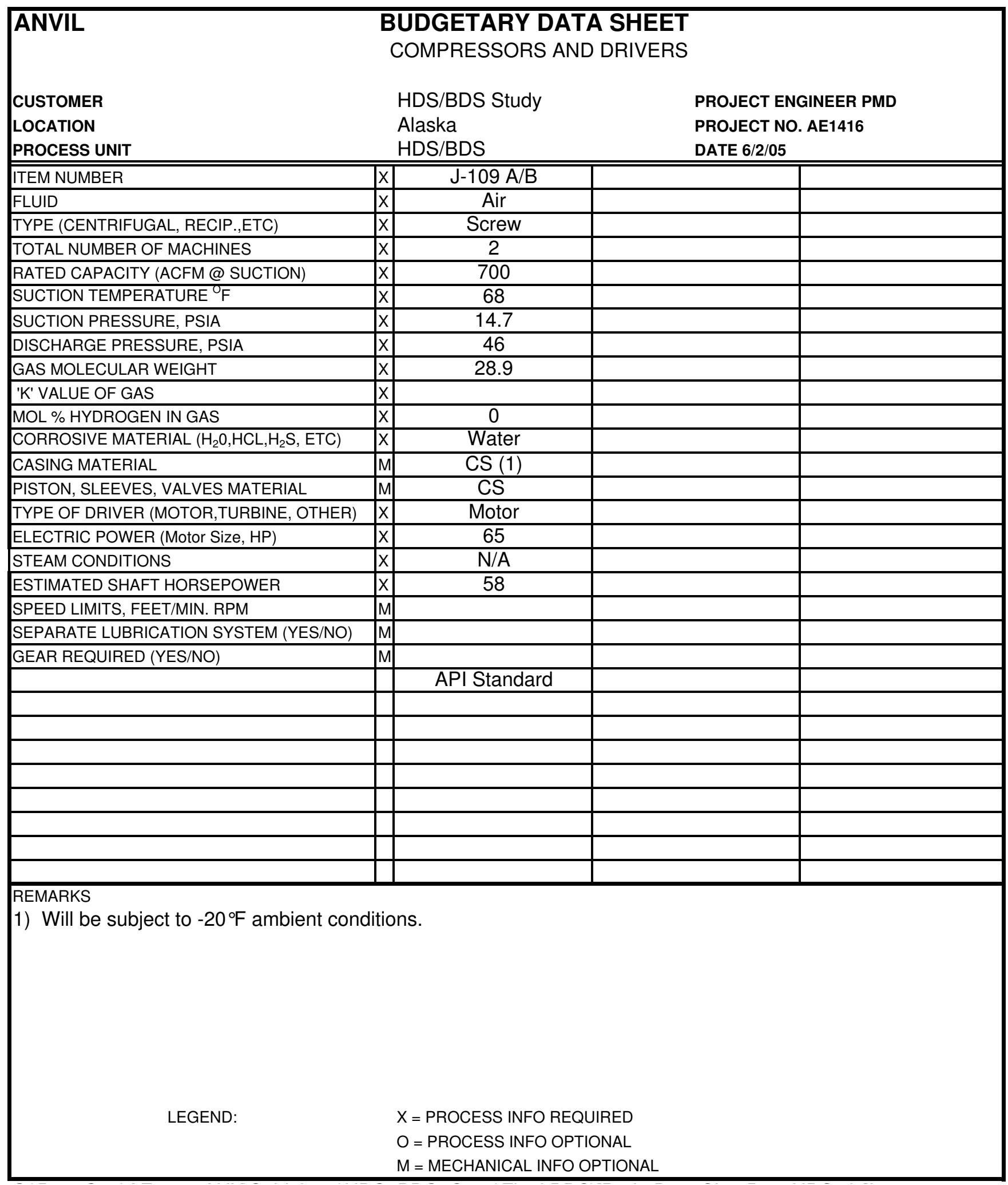




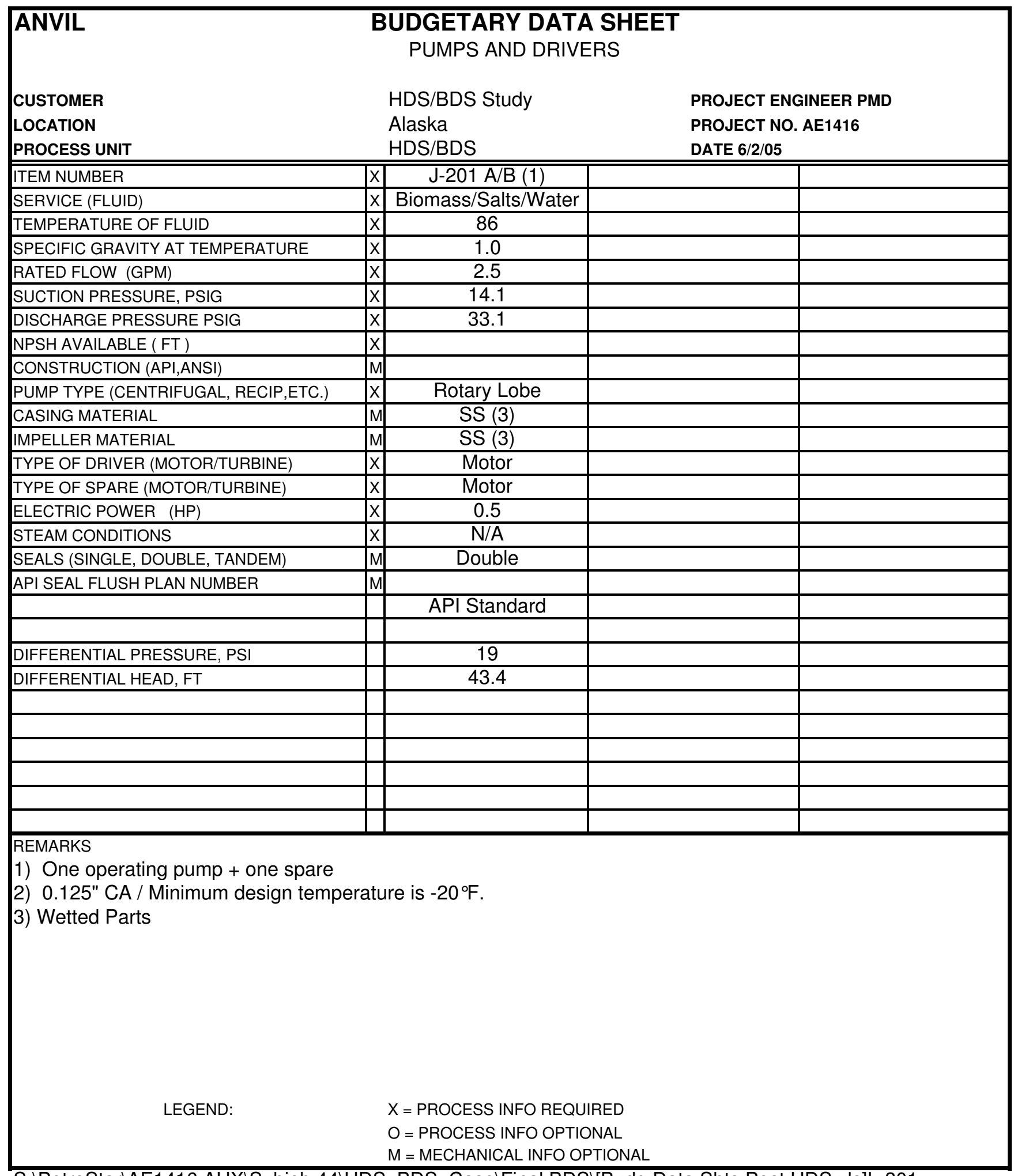

S:IPetroStar|AE1416.AUXISubjob 44IHDS_BDS_CaselFinal BDS|[Budg Data Shts Post HDS.xls]L-301 


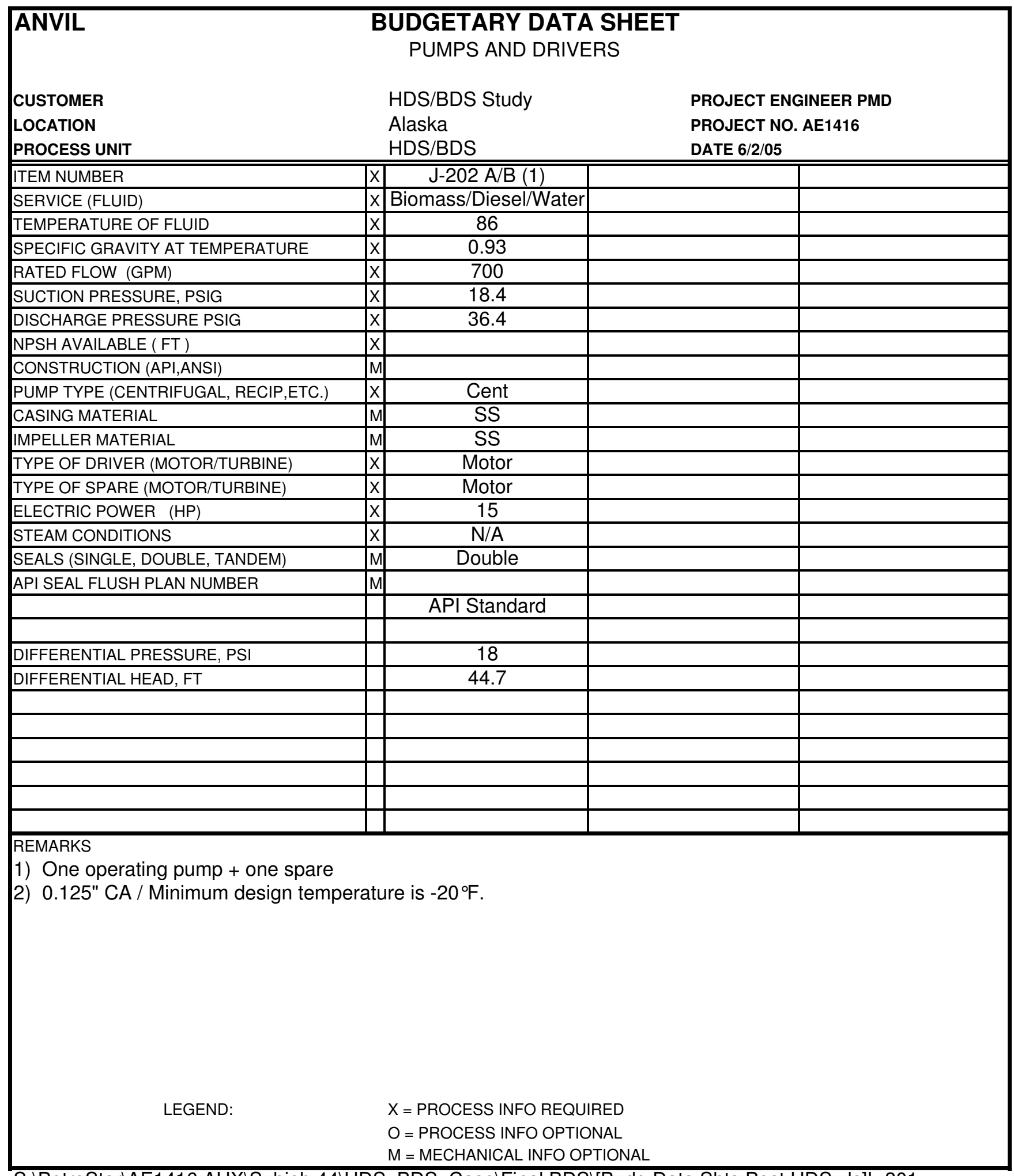

S:IPetroStar|AE1416.AUXISubjob 44IHDS_BDS_CaselFinal BDS|[Budg Data Shts Post HDS.xls]L-301 


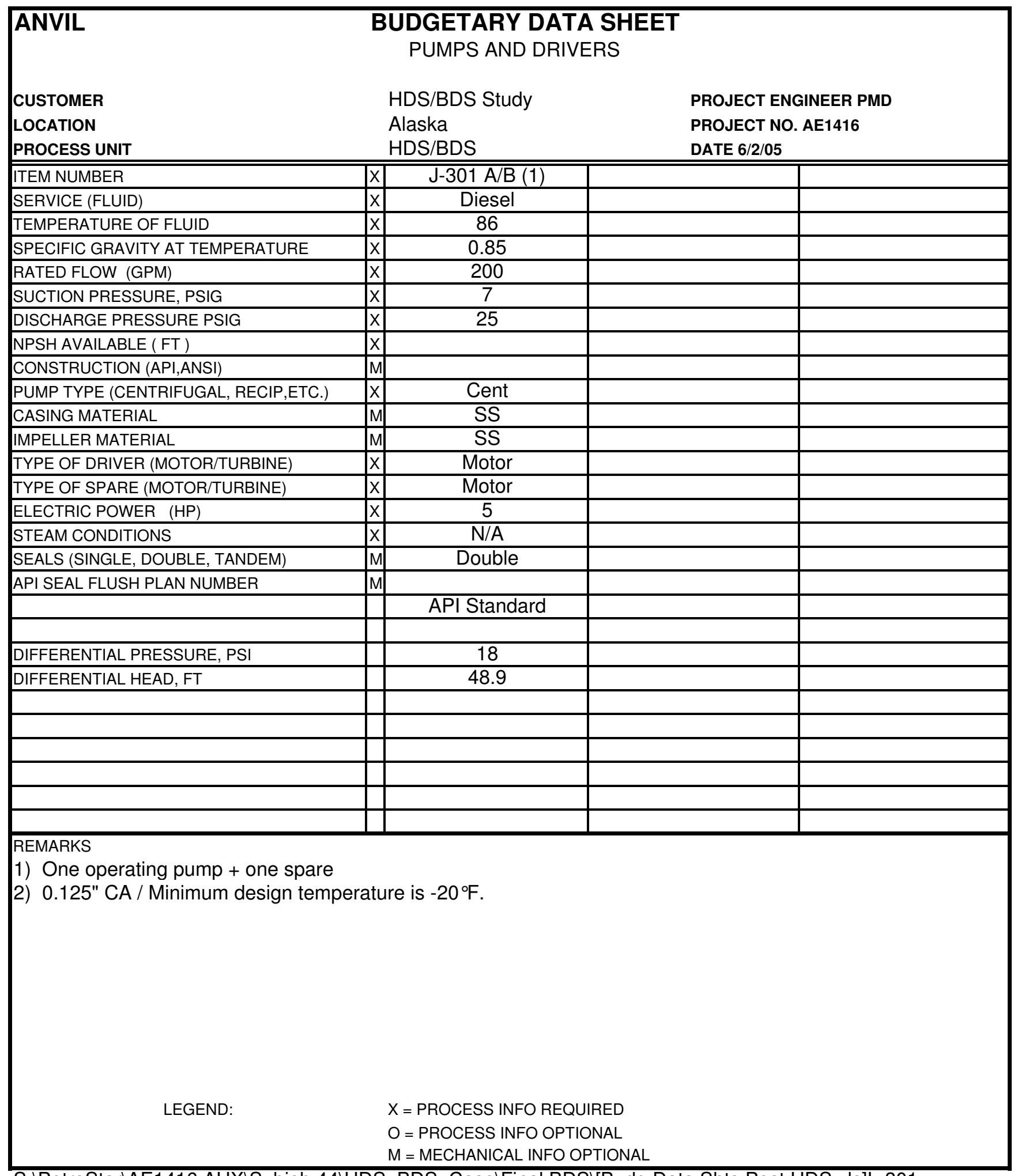

S:IPetroStar|AE1416.AUXISubjob 44IHDS_BDS_CaselFinal BDS|[Budg Data Shts Post HDS.xls]L-301 


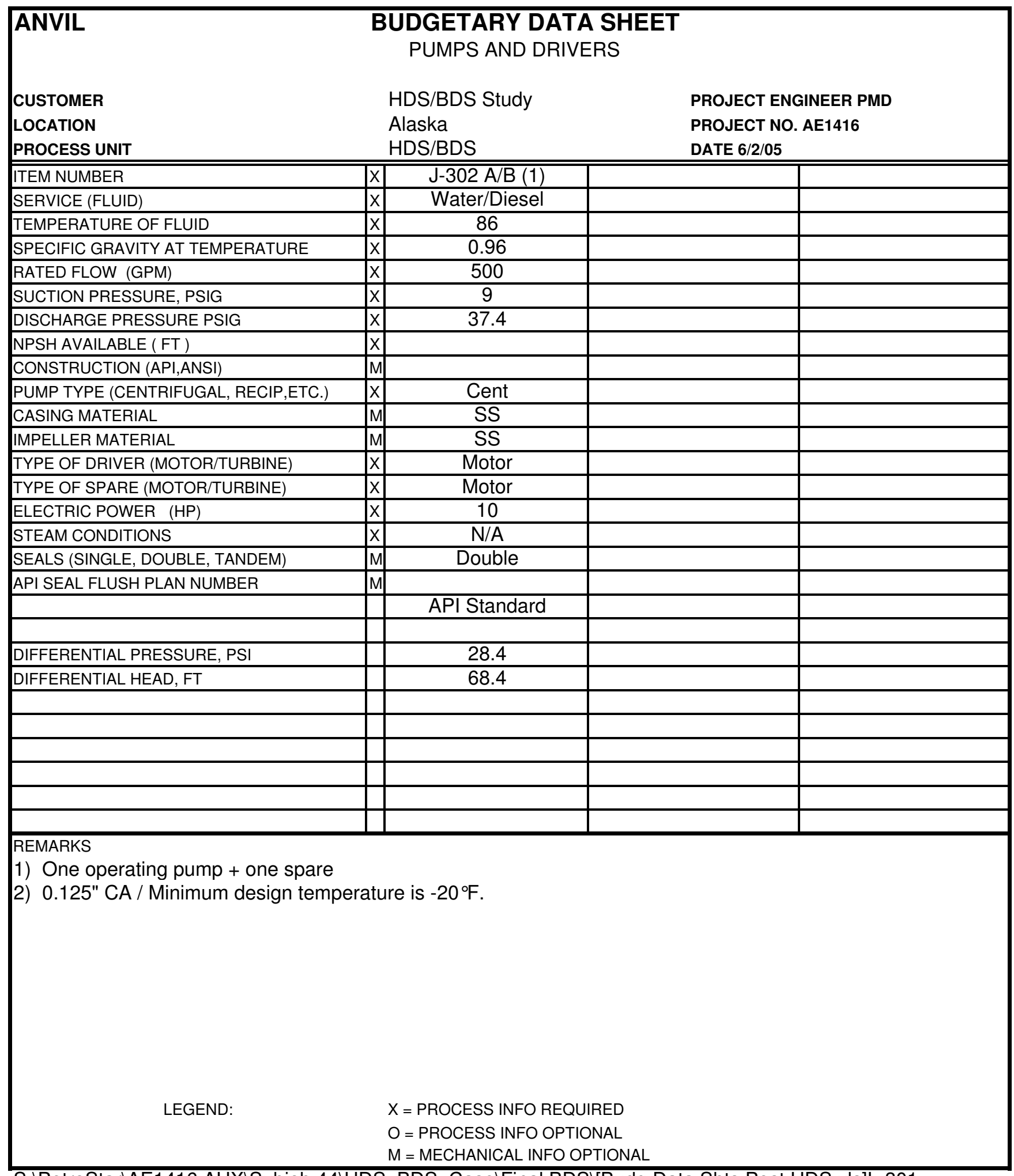

S:IPetroStar|AE1416.AUXISubjob 44IHDS_BDS_CaselFinal BDS|[Budg Data Shts Post HDS.xls]L-301 


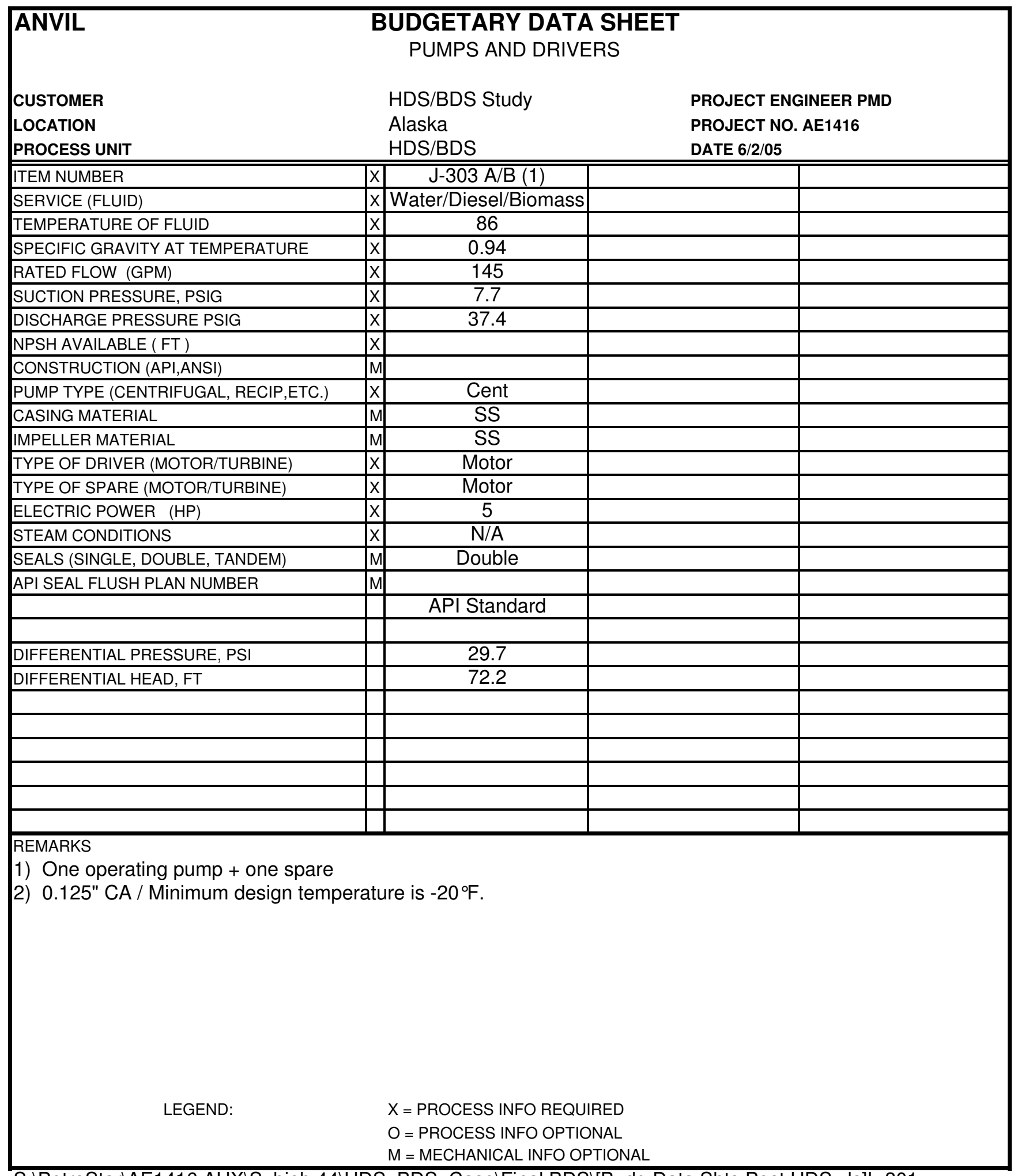

S:IPetroStar|AE1416.AUXISubjob 44IHDS_BDS_CaselFinal BDS|[Budg Data Shts Post HDS.xls]L-301 


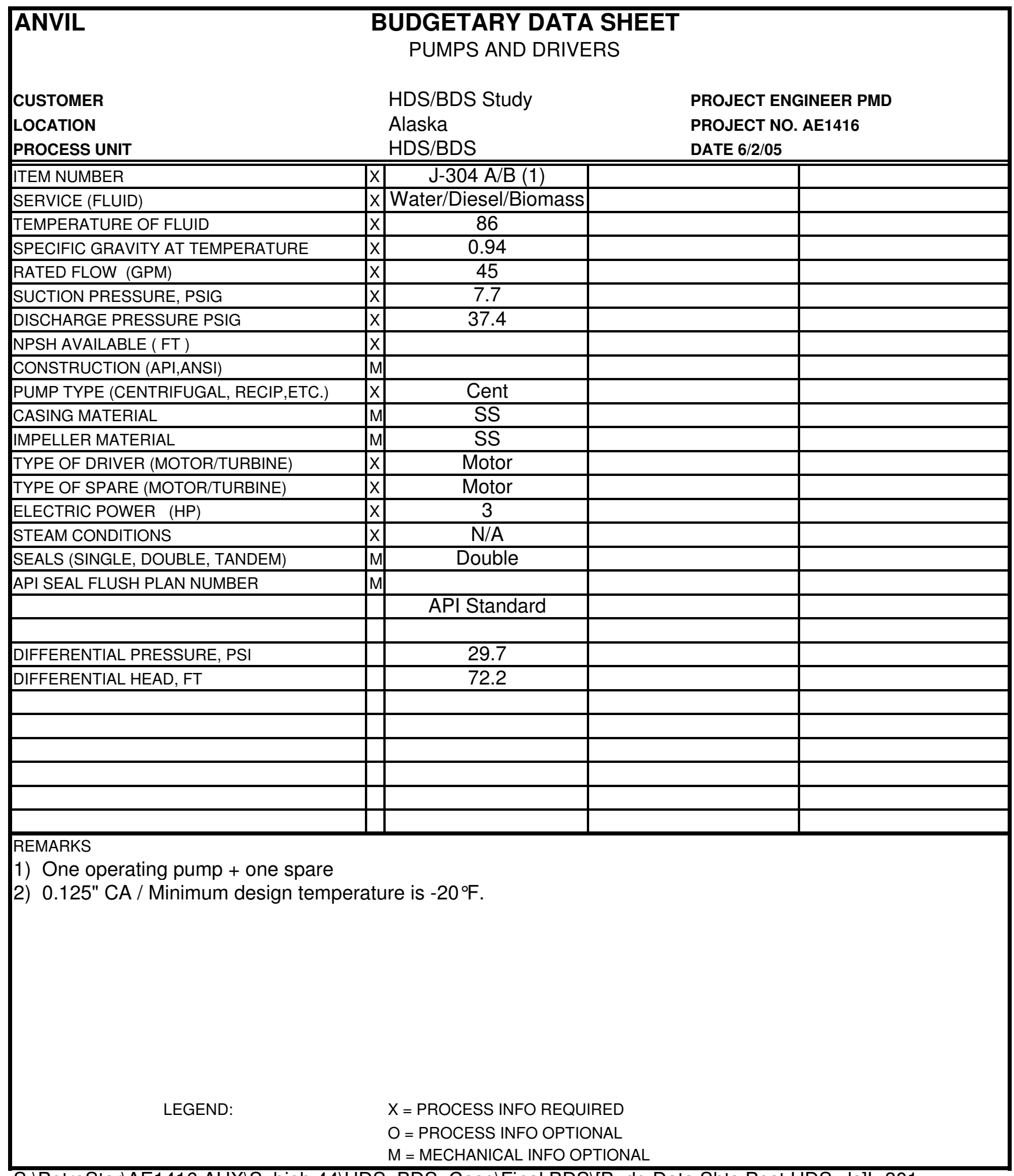

S:IPetroStar|AE1416.AUXISubjob 44IHDS_BDS_CaselFinal BDS|[Budg Data Shts Post HDS.xls]L-301 


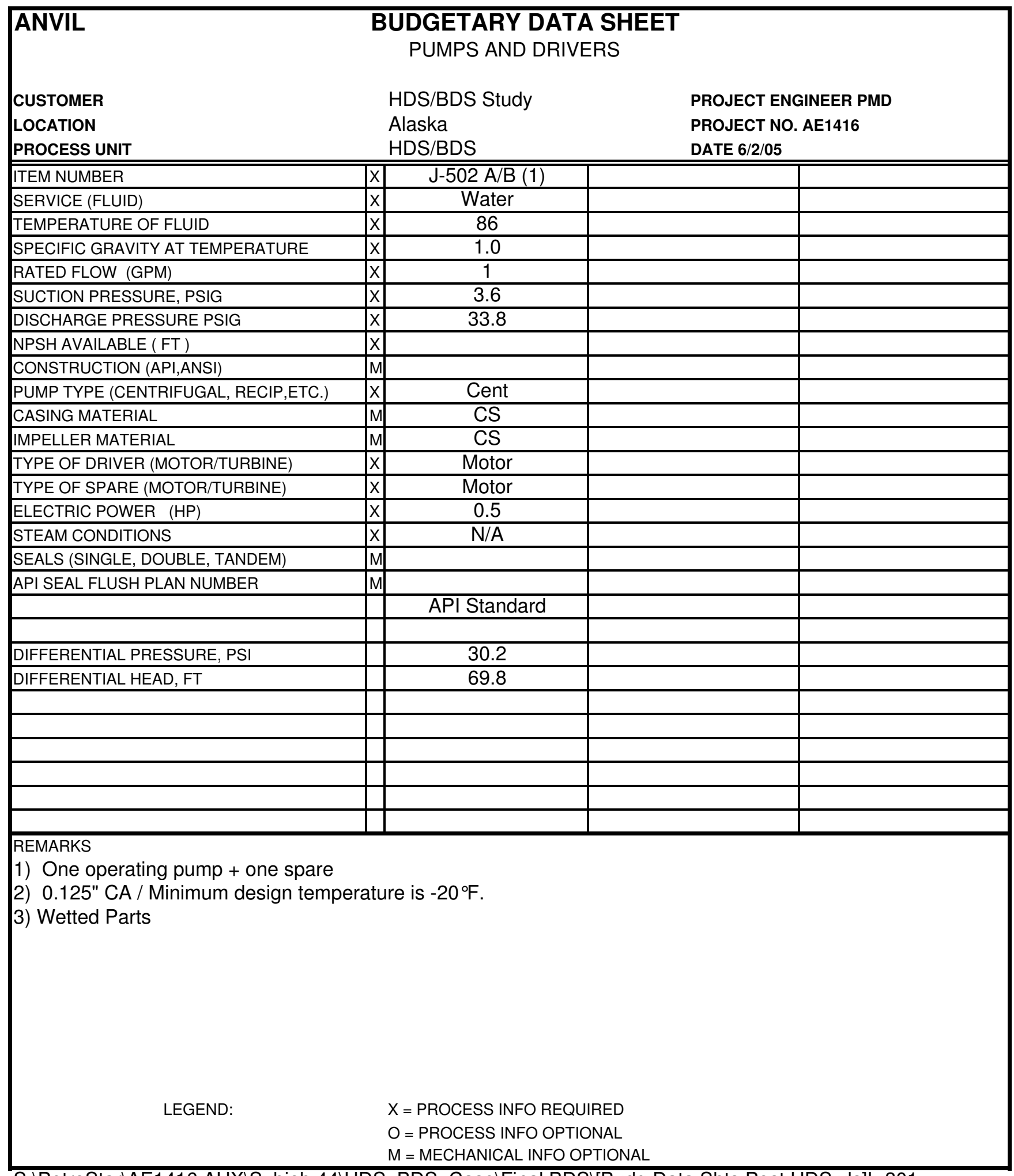

S:IPetroStar|AE1416.AUXISubjob 44IHDS_BDS_CaselFinal BDS|[Budg Data Shts Post HDS.xls]L-301 


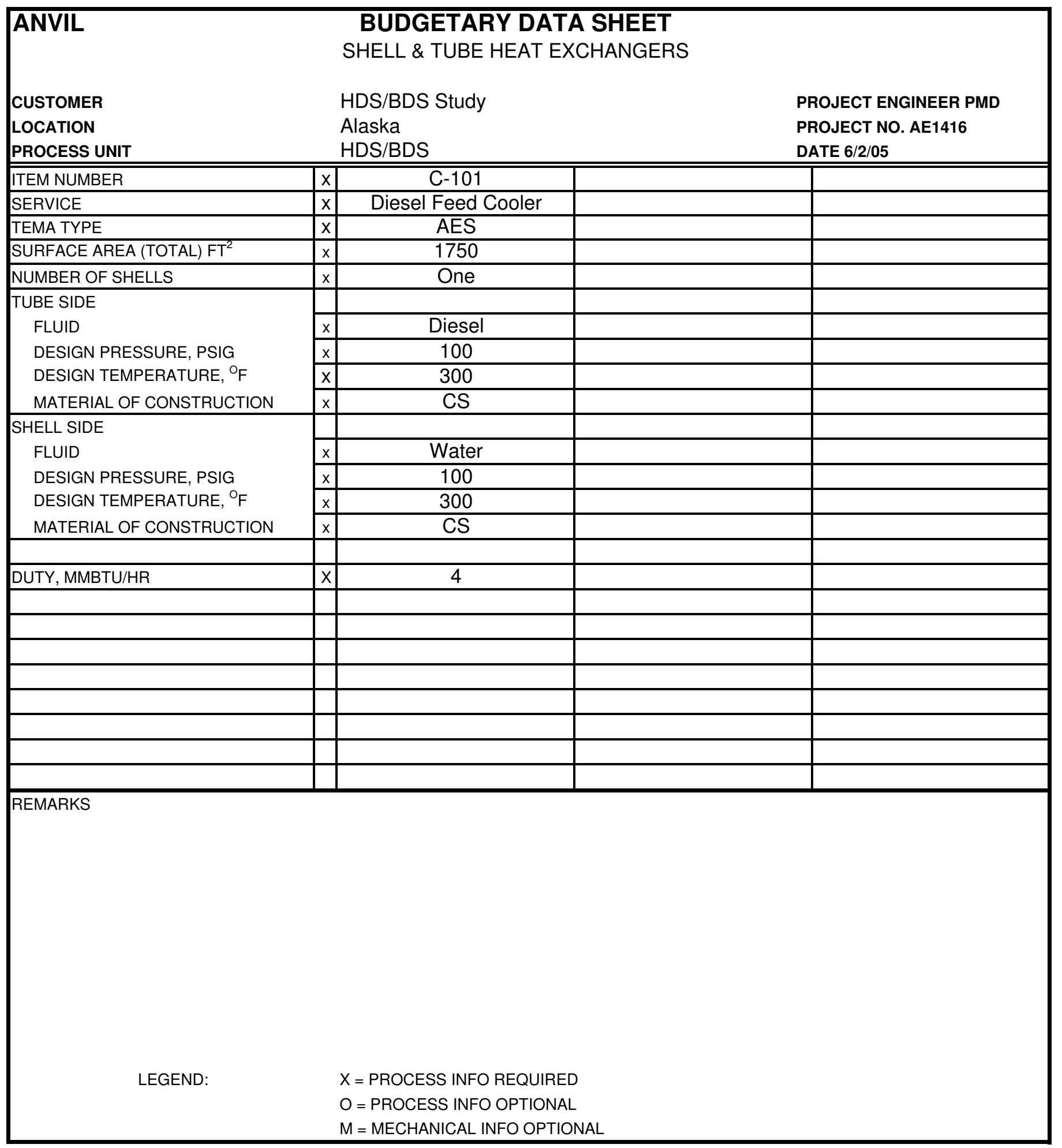




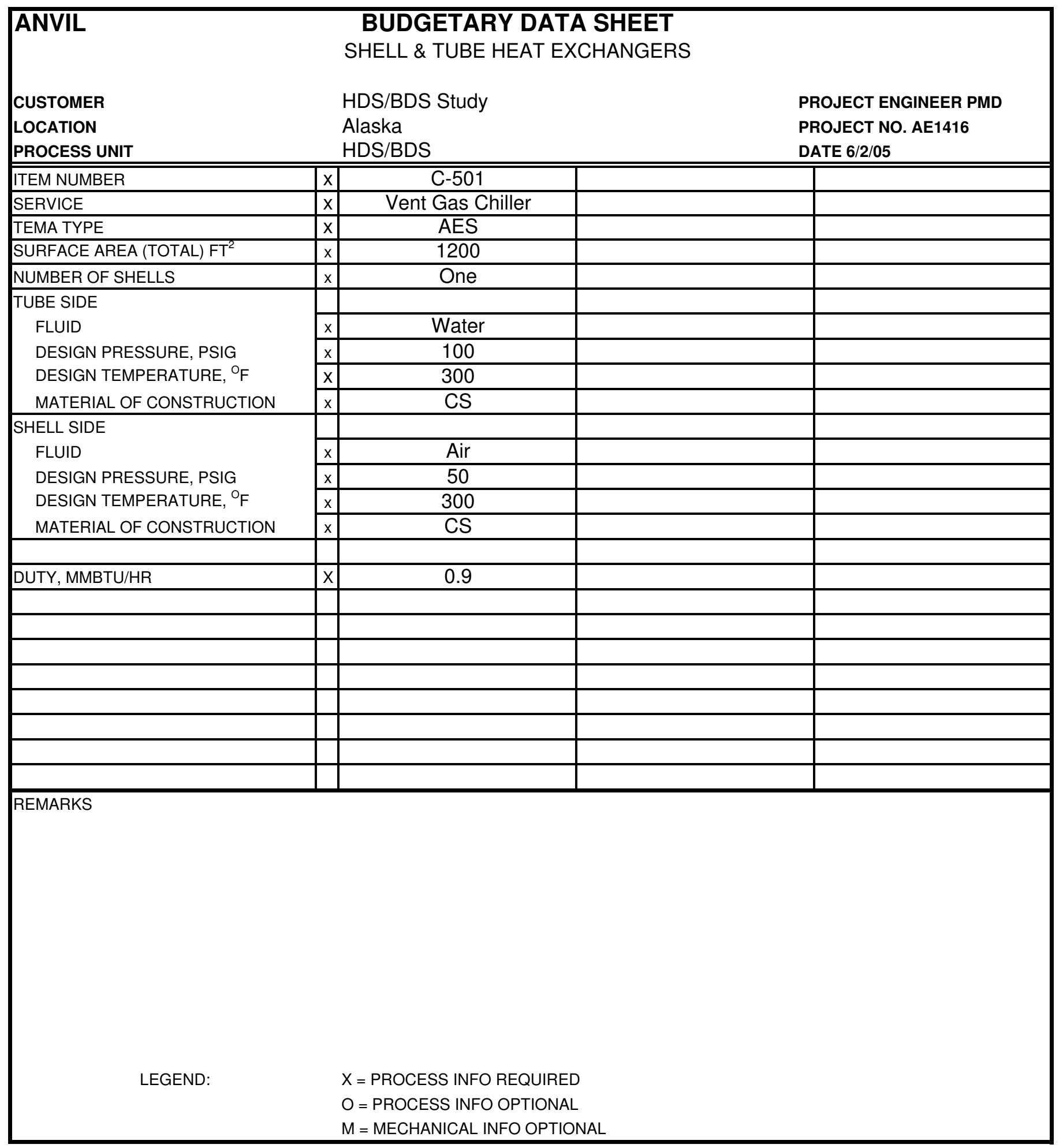




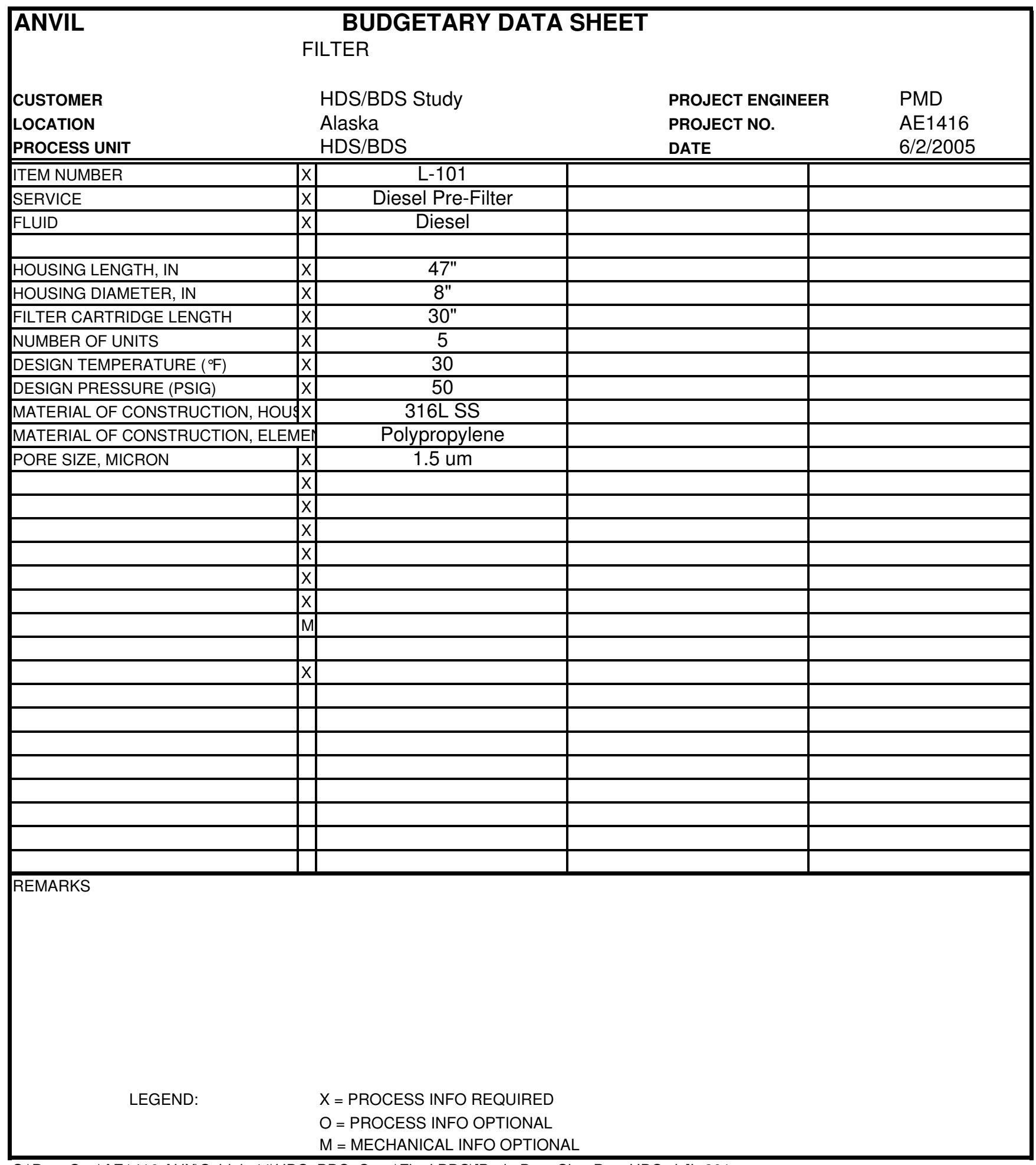




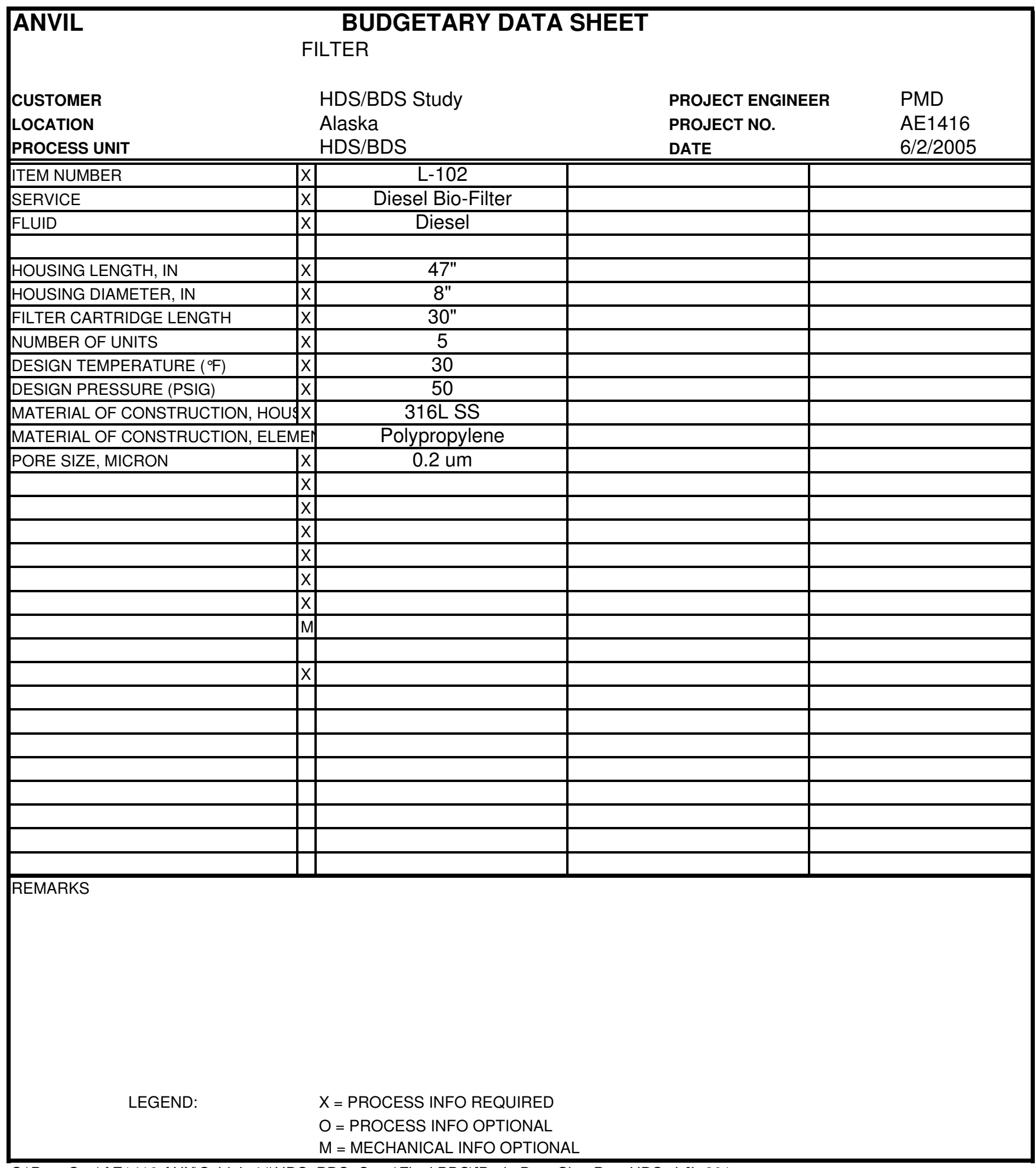




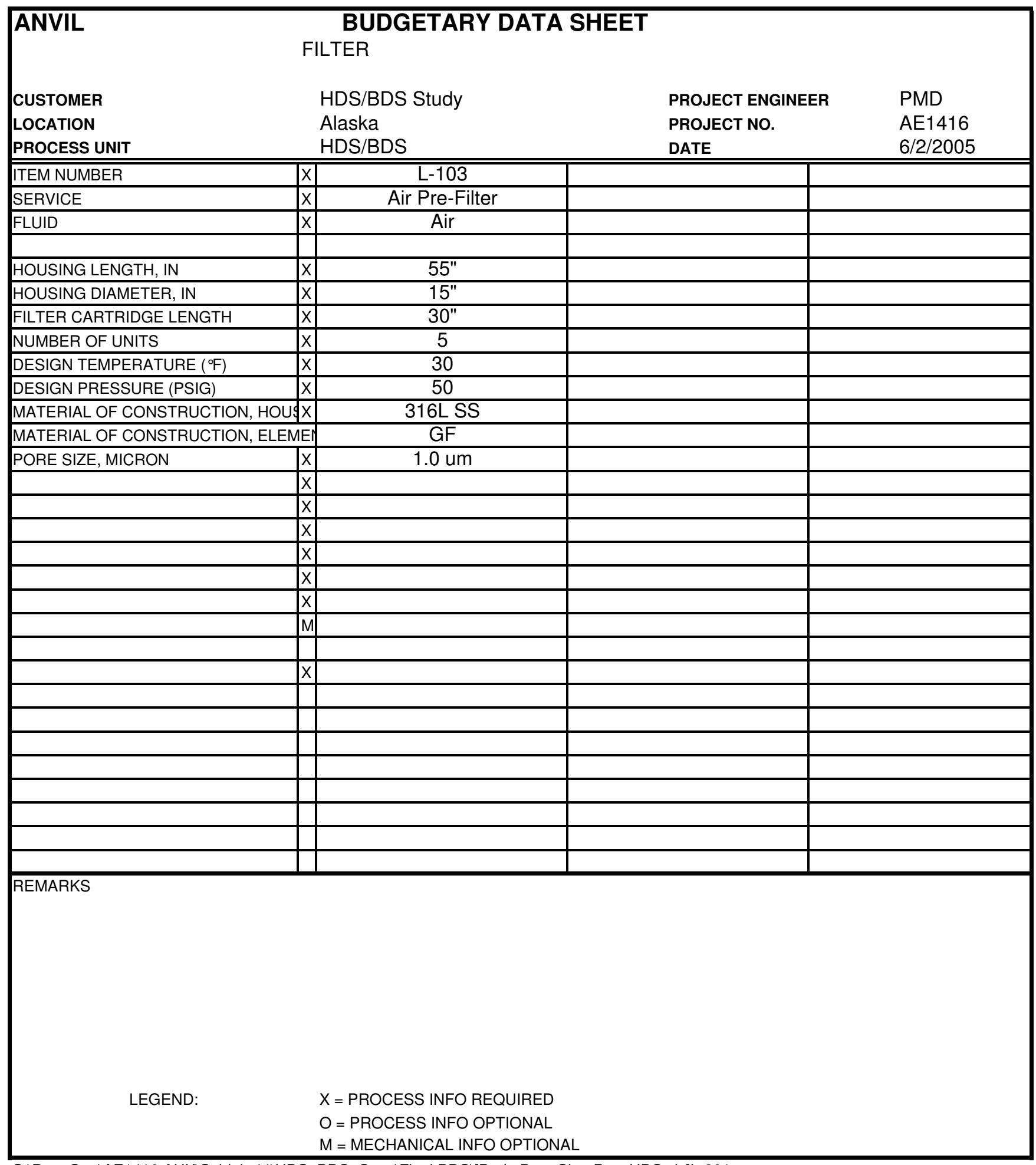




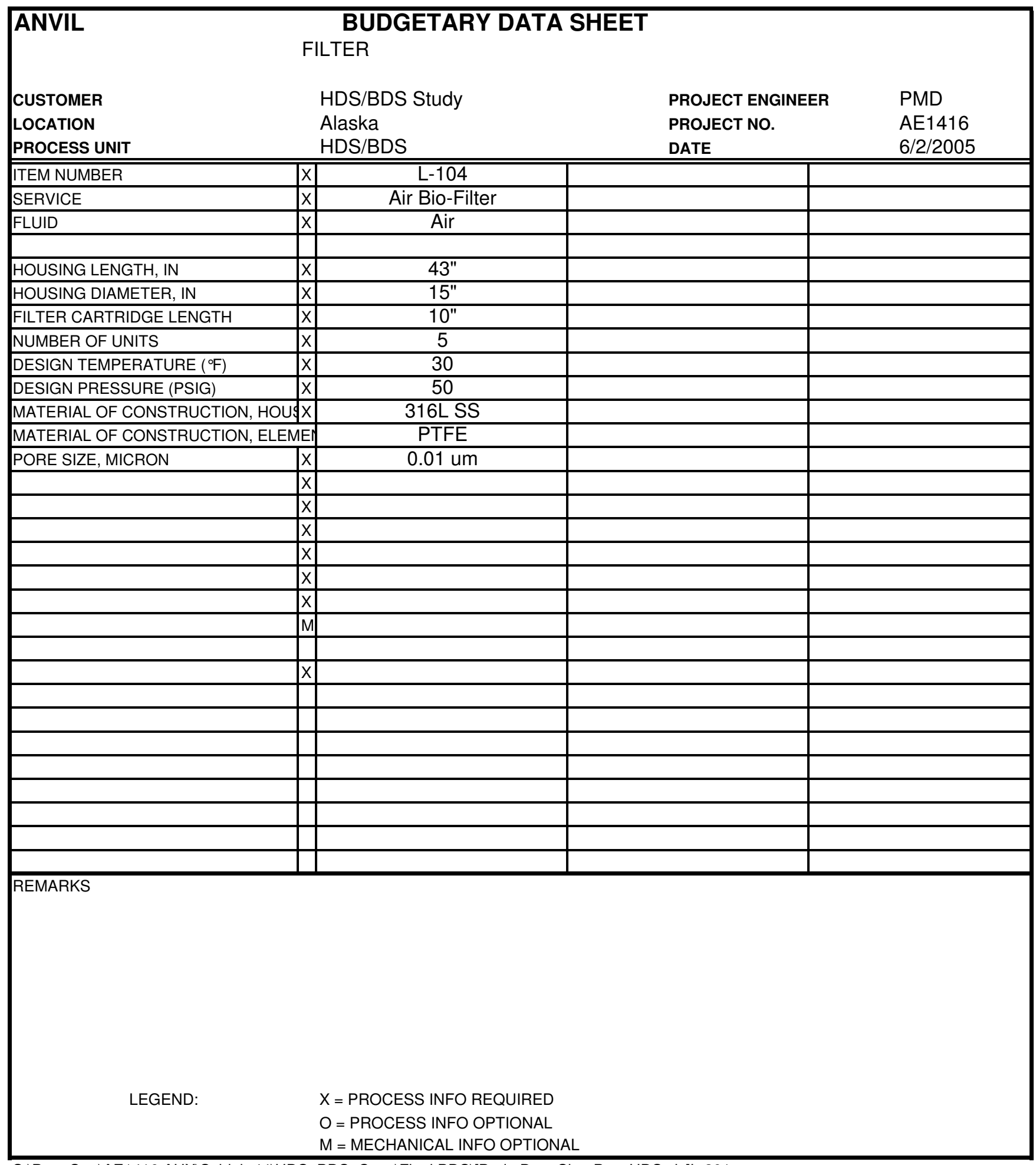




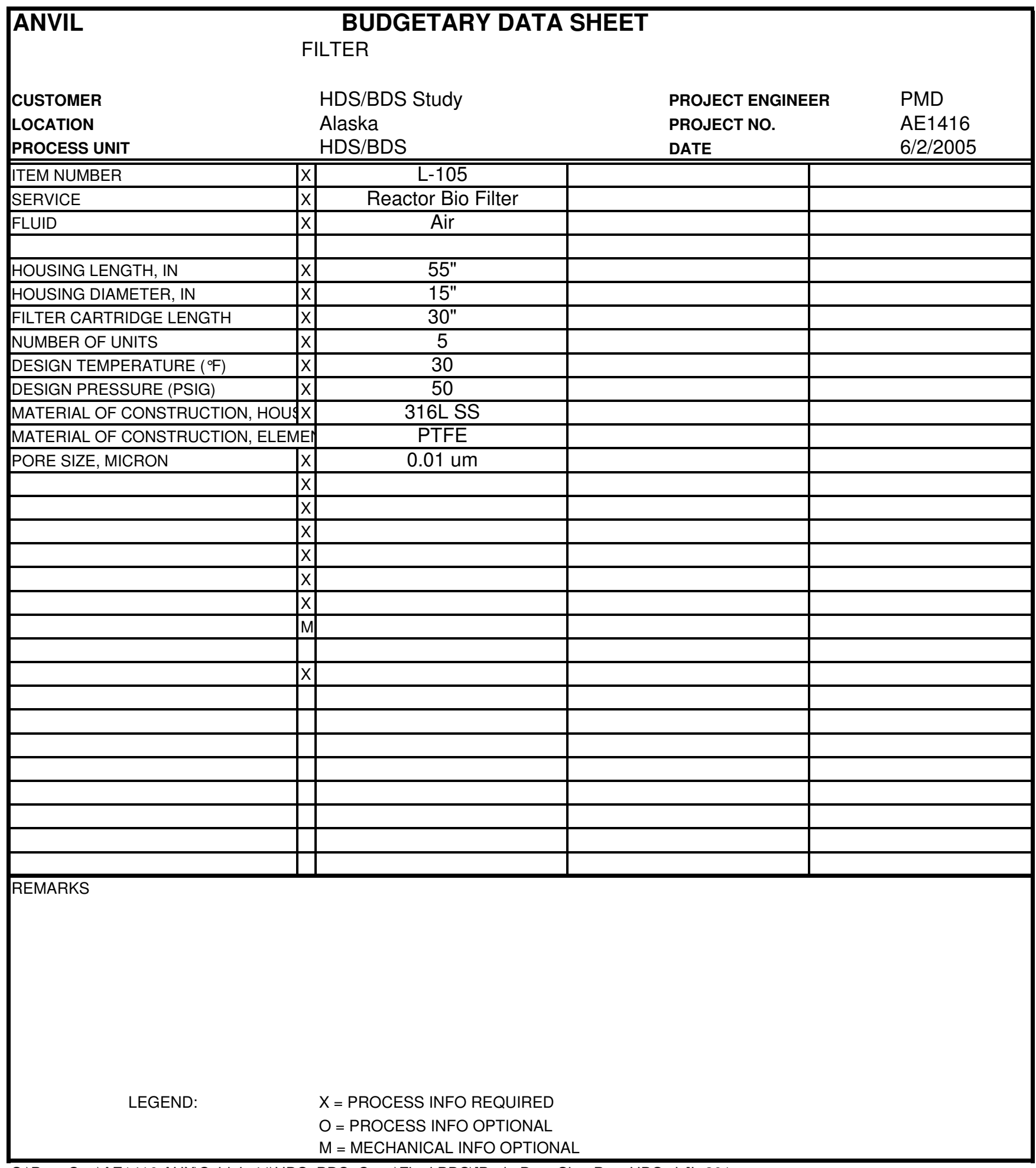




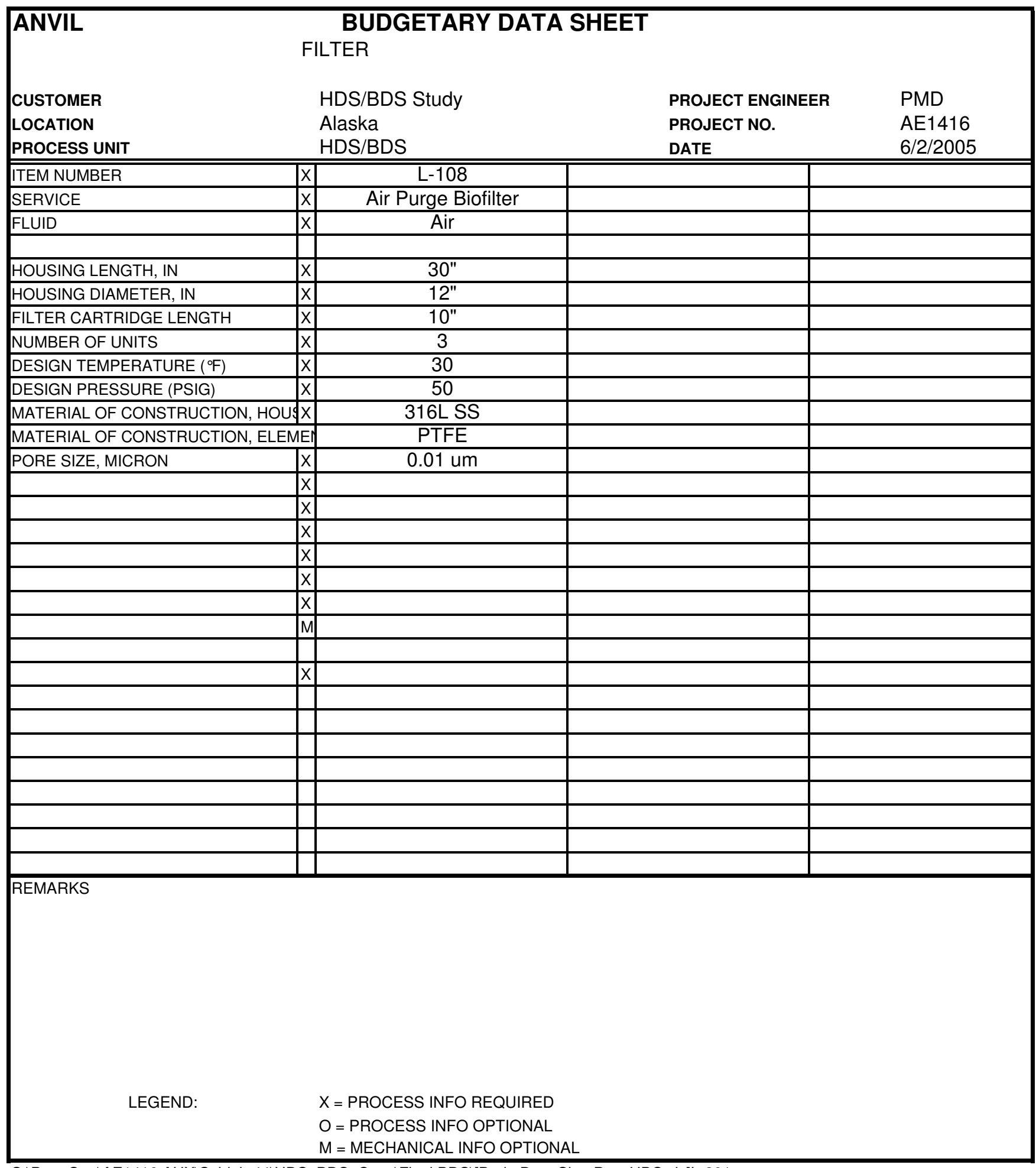




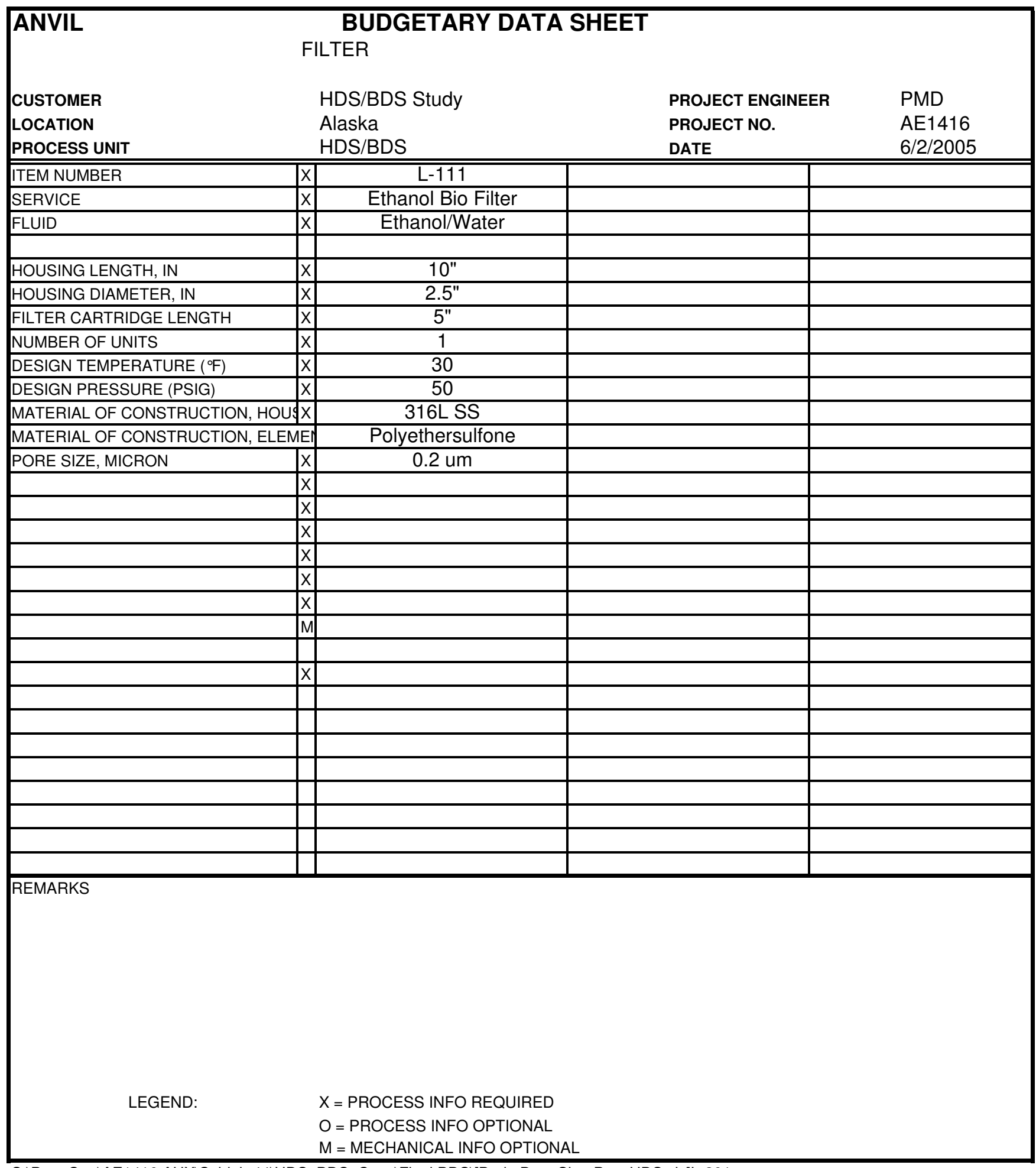




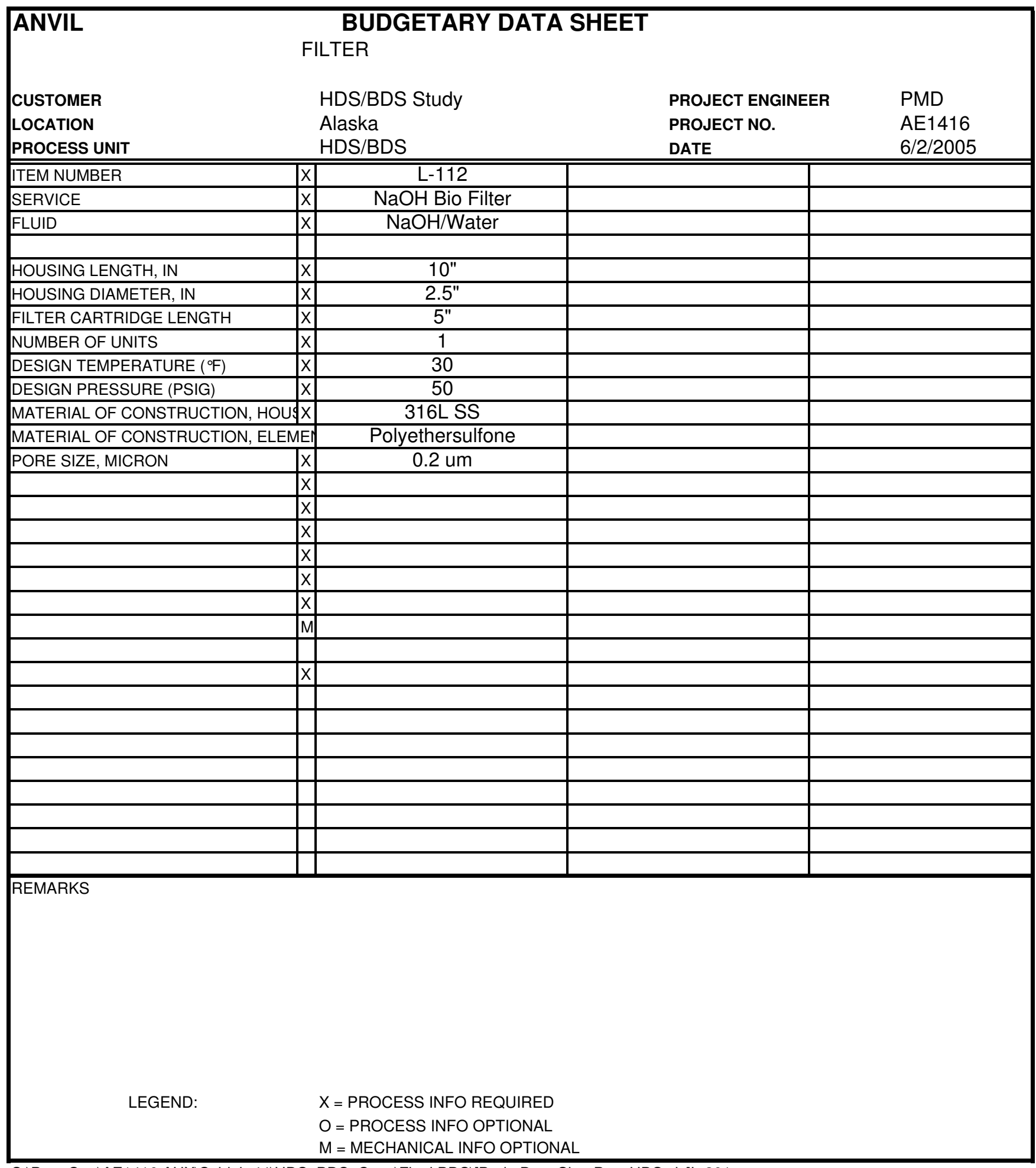




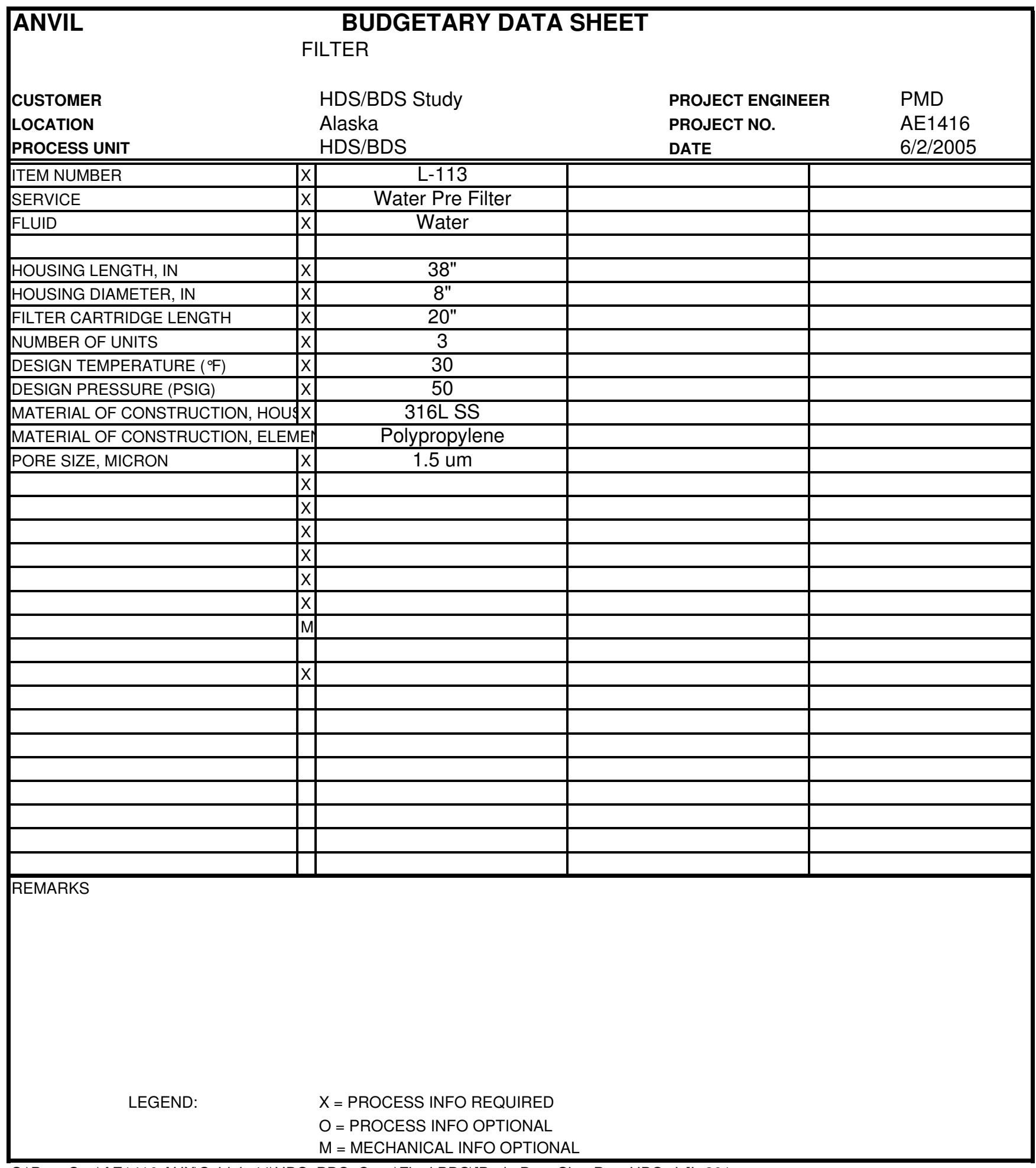




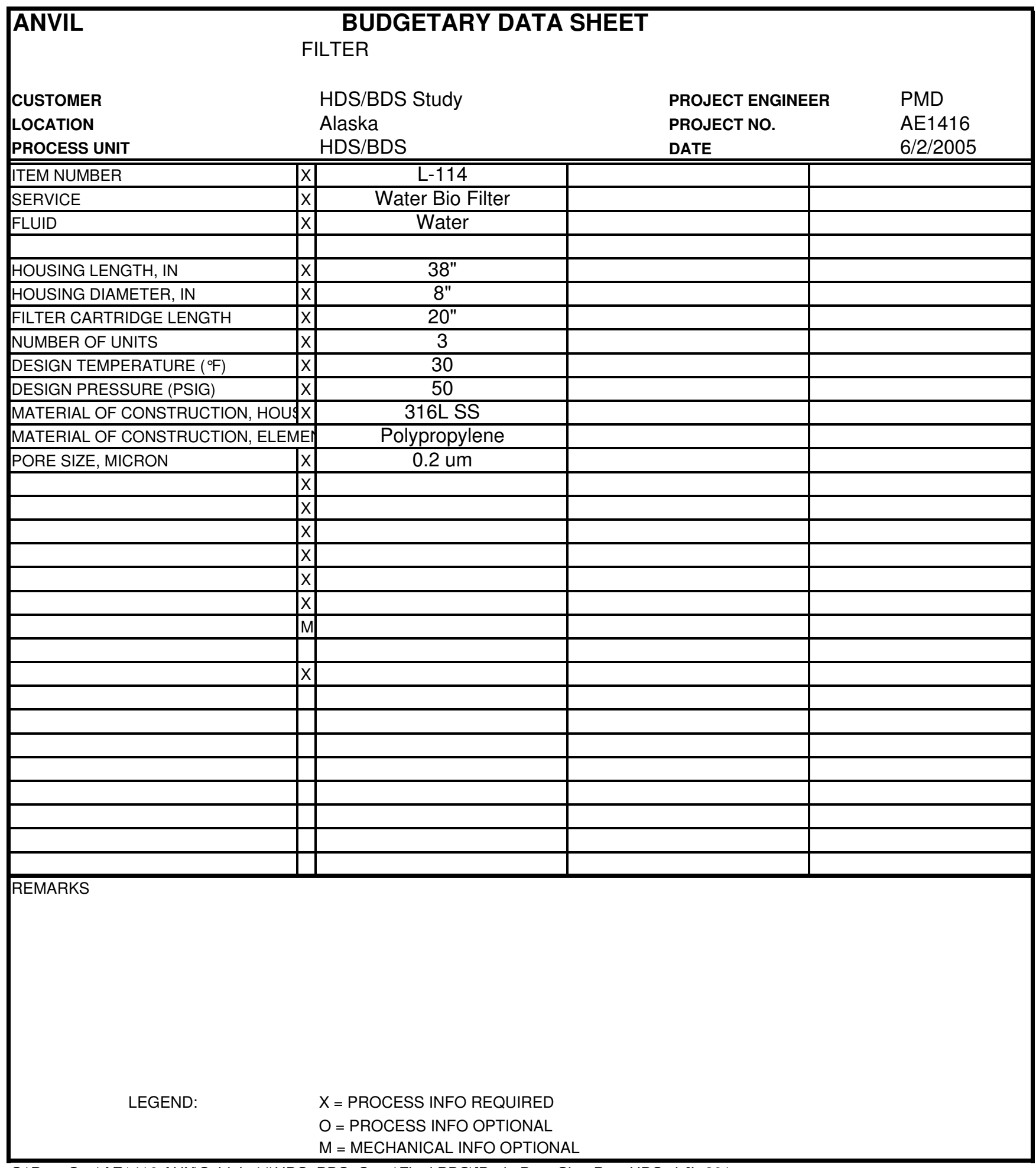




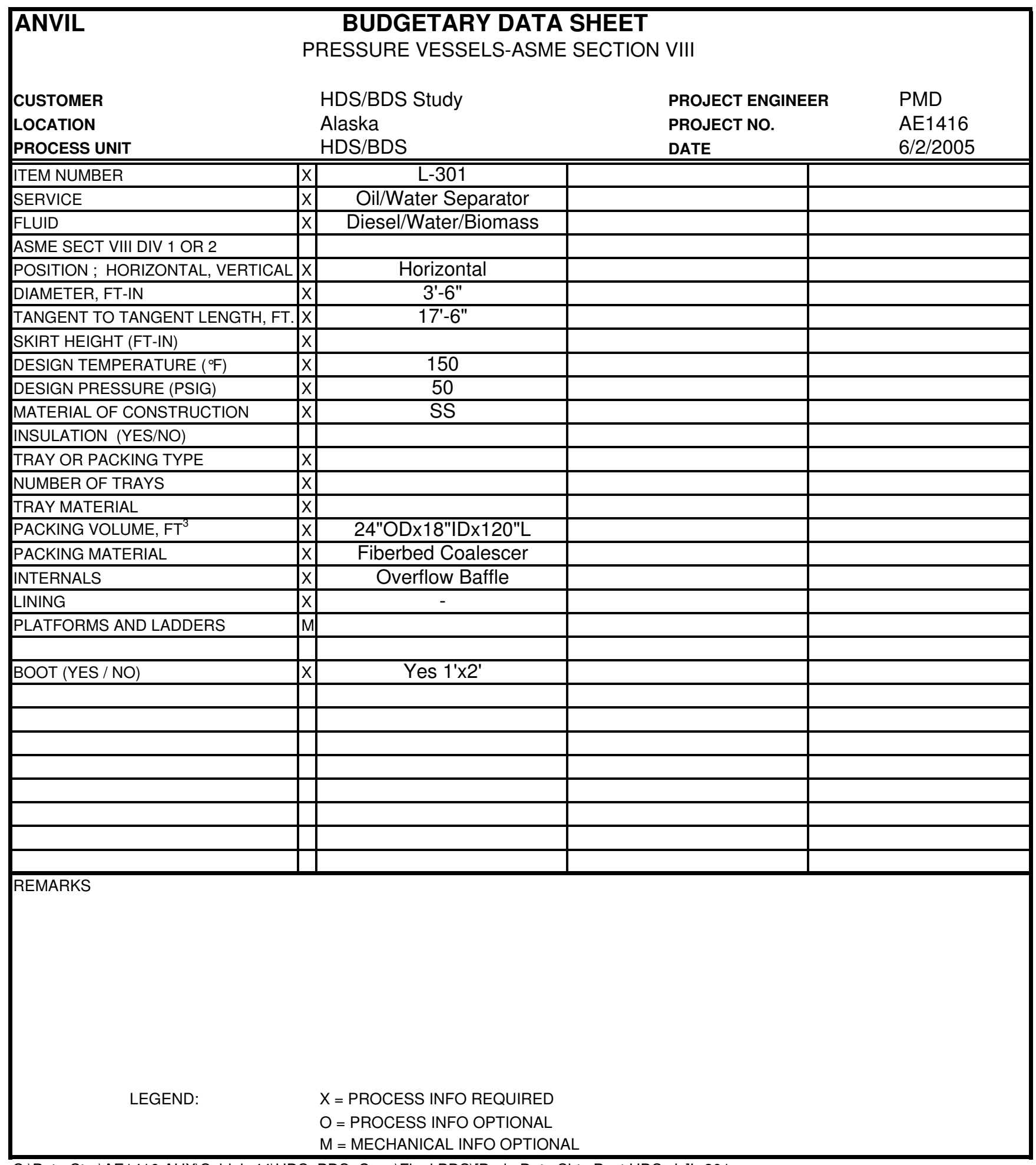




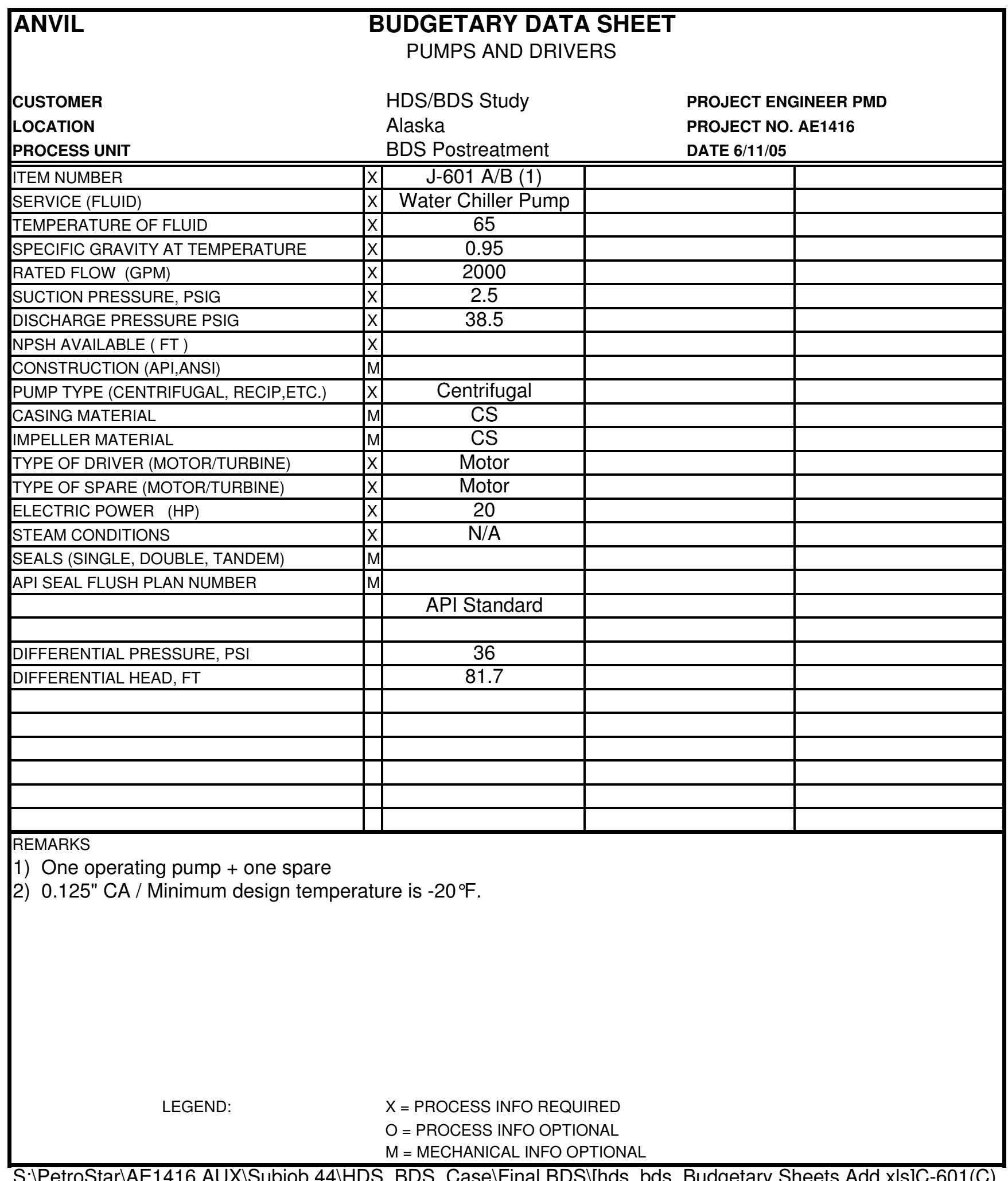




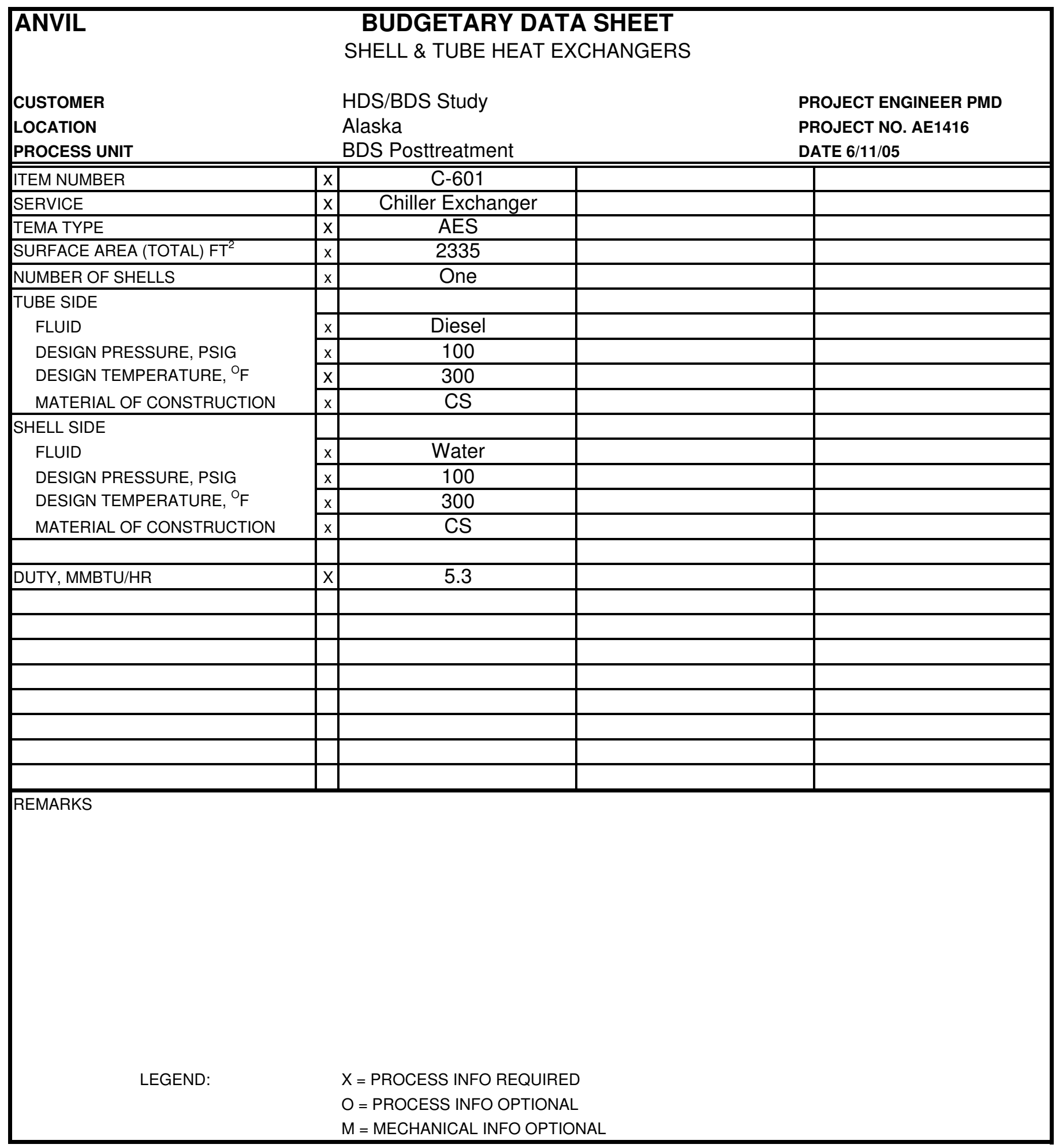




\section{Appendix C.5 - Utility Requirements}

Utility Requirements

HDS/BDS Case

6000 BPD Feed Rate, 10 ppmw Sulfur ULSD Product

\begin{tabular}{|c|c|c|c|c|c|}
\hline IBL HDS & $\begin{array}{c}\text { Power } \\
\text { kW }\end{array}$ & $\begin{array}{c}\text { Fuel Gas } \\
\text { MMBTU/hr }\end{array}$ & $\begin{array}{c}\text { Process Water } \\
\text { lb/hr }\end{array}$ & $\begin{array}{l}\text { BFW } \\
\text { lb/hr }\end{array}$ & \\
\hline Feed Gas Compressor & $\overline{55}$ & & & & \\
\hline H2 Makeup Compressor & 75 & & & & \\
\hline Recycle Gas Compressor & 60 & & & & \\
\hline Charge Pump & 100 & & & & \\
\hline Stripper Reflux Pump & 1 & & & & \\
\hline Stripper Btms Circ & 26 & & & & \\
\hline Effluent Air Cooler & 4 & & & & \\
\hline Prod Stripper Condenser & 4 & & & & \\
\hline Prod Rundown Cooler & 15 & & & & \\
\hline Wash Water Injection Pump & 1 & & & & \\
\hline Charge Heater & & 5.9 & & & \\
\hline Prod Stripper Reboiler & & 7.0 & & & \\
\hline Wash Water Injection Pump & & & 630 & & \\
\hline \multicolumn{6}{|l|}{ OBL HDS } \\
\hline $\mathrm{H} 2$ Plant & 47 & 24.7 & & 4166 & \\
\hline Sulfur Recovery & 96 & & & & \\
\hline Other OBL Allowance & 118 & & & & \\
\hline TOTAL HDS and OBL & 602 & 37.6 & 630 & 4166 & \\
\hline BDS & $\begin{array}{c}\text { Power } \\
\mathbf{k W}\end{array}$ & $\begin{array}{c}\text { Fuel Gas } \\
\text { MMBTU/hr }\end{array}$ & $\begin{array}{c}\text { Process Water } \\
\text { lb/hr }\end{array}$ & $\begin{array}{l}\text { BFW } \\
\text { lb/hr }\end{array}$ & $\begin{array}{c}\text { Steam } \\
\text { lb/hr }\end{array}$ \\
\hline Total Plant & $\overline{586}$ & & $\overline{1200}$ & & 8 \\
\hline TOTAL BDS & 586 & & 1200 & & 8 \\
\hline
\end{tabular}


Appendix C.6 - Cost Estimate Basis and Cost Estimate 


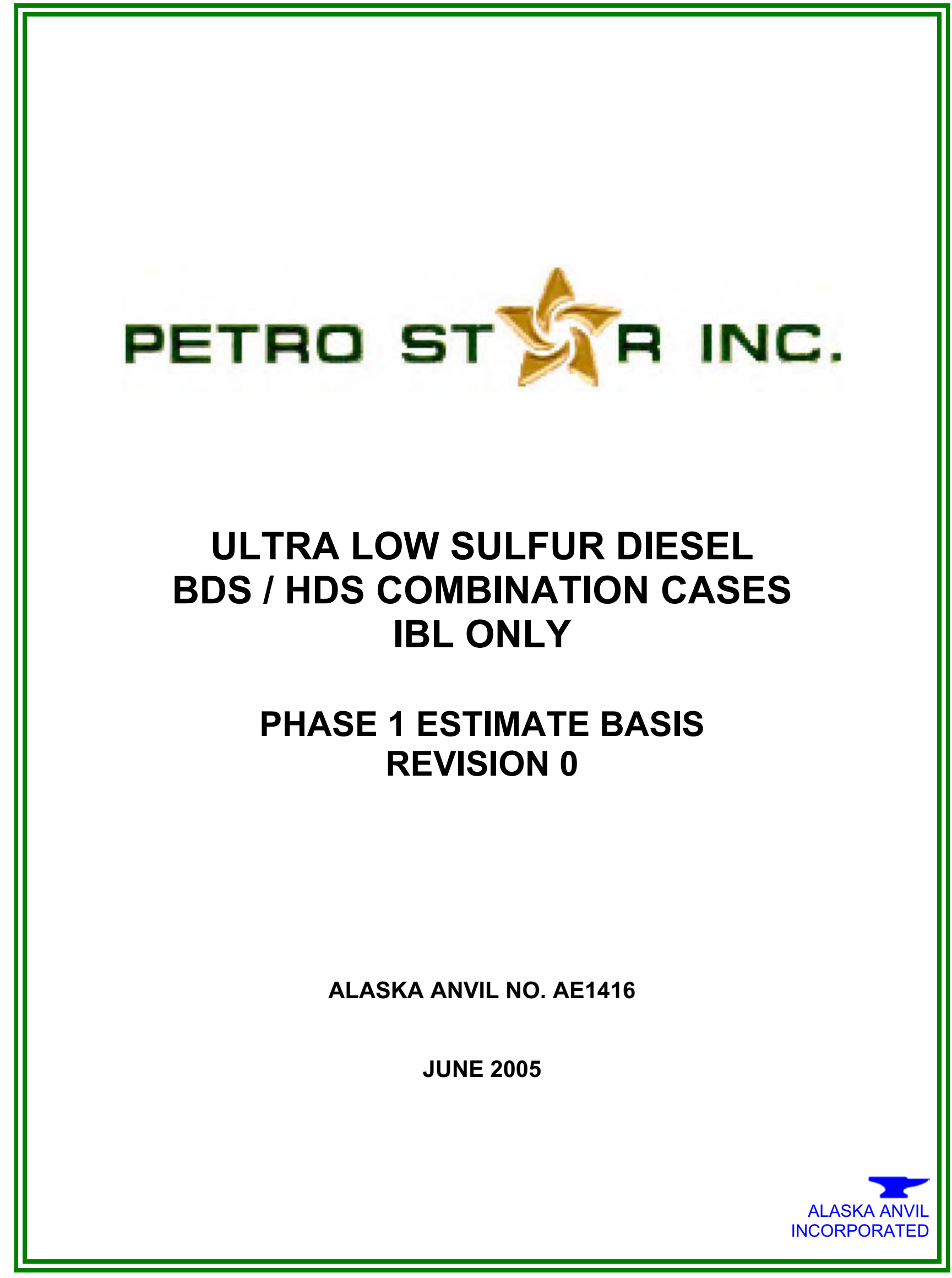




\section{ESTIMATE BASIS GOAL}

This Estimate Basis identifies information, qualifications, exceptions, and assumptions used in developing the cost estimate.

\section{ESTIMATE BASIS PURPOSE}

During the estimate review process, the project team uses the Estimate Basis for the following purposes:

- As a checklist of items to consider during estimate preparation.

- To document what is included and not included in the cost estimate.

- To assess cost risks of estimate components.

- As part of the decision support package for assessing the BDS process feasibility.

\section{GENERAL INFORMATION}

- The purpose of the project estimate is to determine if the ULSD BDS process is economically viable as a standalone process or in combination with an HDS Unit. These three (3) estimate scenarios address the BDS / HDS Combination cases.

- $\quad$ Estimate type:

- The estimate was developed using equipment based factored estimates for Inside Battery Limits (IBL) costs.

- There is a separate equipment-factoring summary for the BDS, HDS, and Pre Frac Equipment (Pre Frac Case Only)

- Most of the equipment pricing was derived from the ICARUS estimating program. Pricing for BDS equipment marked with an asterisk (*) was provided by Pelorus.

- The cost of the Hydrogen and Sulfur Units were factored off licensor quotes obtained for the standalone HDS case.

- Outside Battery Limits (OBL) costs have been excluded from this estimate.

- $\quad$ The project will be installed in a brownfield location within the Valdez Alaska Refinery.

\section{PROCESS BASIS}

\section{Facility Data}

- $\quad$ Facility type - Ultra Low Sulfur Diesel Treating Complex, which includes:

- Diesel Biodesulfurization Unit

- Diesel Hydrotreating Unit

- Hydrogen Production Unit

- Sulfur Unit

- Diesel Splitter (Pre Frac Case Only) 


\section{Design Basis}

Product specification - Feed 6,000 bpd of untreated diesel to produce 10-ppmw sulfur maximum ultra low sulfur diesel.

\section{COST BASIS}

\section{Labor, Indirects, Equipment, and Bulk Materials}

- Included in the equipment factor.

\section{Project Services}

- $\quad$ Estimated based on 15 percent of TIC for the BDS, HDS, and Pre Frac units; engineering costs for the Hydrogen and Sulfur Units was included in the licensor pricing.

\section{Owner Services}

Not included in the TIC cost. Historically, owner services will cost from 5 to 7 percent of TIC, not including licensing, royalties, or catalyst.

\section{Escalation}

Project is based on 2005 costs. No escalation is included.

\section{Location Factor}

All costs for this estimate have been developed from a U.S. Gulf Coast (USGC) basis. No location factor is included.

\section{Other Costs}

- Catalyst and chemical initial charge has been added as an additional line item.

- CEMS, air preheating, and burner management allowances have been added to the fired heater costs.

\section{ASSUMPTIONS}

- $\quad$ Process licensing and royalty costs are not included.

- Assumes fully installed pump spares, but no warehouse spares. 
Final Technical Progress Report DOE Award No. DE-FC26-02NT15340

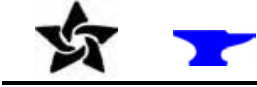

CLIENT: PetroStar

PROJECT: PetroStar Valdez ULSD - HDS/BDS Combo STAGE: Phase 1

\section{PROJECT DESCRIPTION:}

Install Ultra Low Sulfur Diesel Complex.

PROJECT COST ESTIMATE SUMMARY

COST ESTIMATE STRUCTURE
COST ESTIMATE PARAMETERS
Estimate Classification
Estimating Method
COST ESTIMATE SUMMARY
Expected Cost (\$MM)
High Range (\$MM)
Low Range (\$MM)

PROJECT COST ESTIMATE ANALYSIS

Total Project Expected Cost Component Analysis

CLIENT PROJECT NO.:

ANVIL PROJECT NO.: AE1416

REV NO.: 0

ANVIL PE: L. Nace

Date: 6/17/05

PROJECT RISKS:

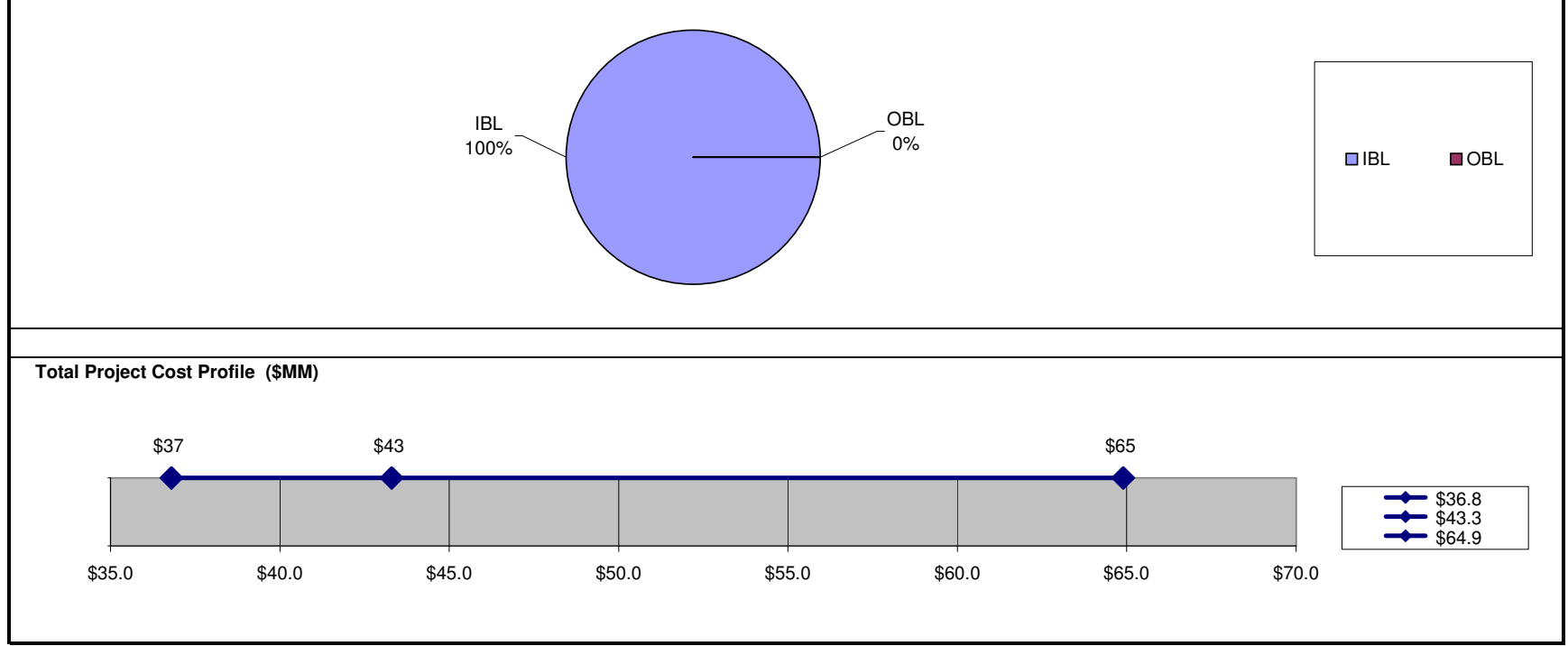

\begin{tabular}{|lr|}
\hline \multicolumn{2}{|c|}{$\begin{array}{c}\text { TOTAL PROJECT } \\
\text { COST - Valdez }\end{array}$} \\
\hline \multicolumn{2}{|c|}{ Phase 1 } \\
\hline \multicolumn{2}{|c|}{ Factored/ROM } \\
\hline & \\
\hline$\$$ & \\
\hline & 64.3 \\
\hline$\$$ & 36.8 \\
\hline$\$$ &
\end{tabular}

PROJECT SCHEDULE ESTIMATE ANALYSIS

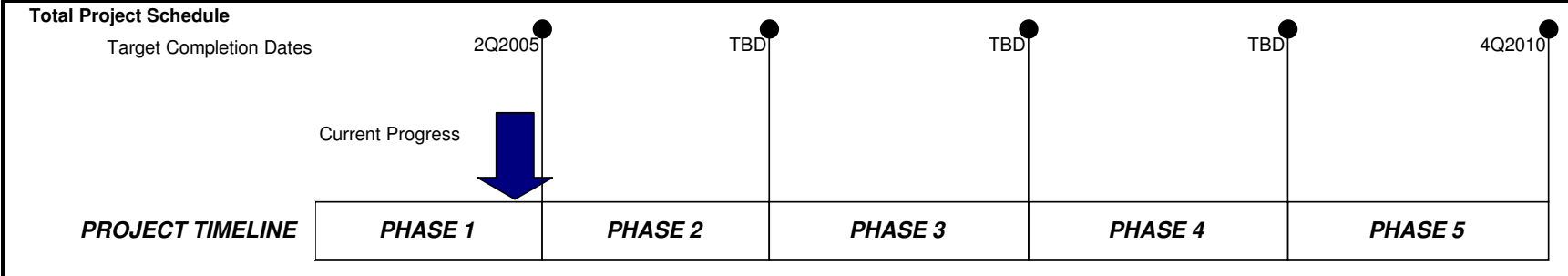


Petro Star Ultra Low Sulfur Diesel (6000 BPD) Project - Valdez Refinery

IBL Impact Matrix - 2005\$ - Total Installed Costs USGC

Scenario: HDS/BDS Combo

\begin{tabular}{|l|l|l|l|l||c|}
\hline $\begin{array}{c}\text { IBL } \\
\text { Component }\end{array}$ & \multicolumn{1}{c|}{ HDS } & \multicolumn{1}{c|}{ BDS } & Hydrogen & \multicolumn{1}{c|}{ Sulfur } & Total Cost \\
\hline & Unit & Unit & Unit & Unit & \$MM \\
\hline & $\begin{array}{l}\text { Diesel } \\
\text { Hydrotreater, } \\
\text { feed rate of } \\
\text { 6000 BPD, } \\
\text { producing LSD }\end{array}$ & $\begin{array}{l}\text { Biodesulfurizat } \\
\text { ion Unit, feed } \\
\text { rate of 6000 } \\
\text { BPD, } \\
\text { producing } \\
\text { ULSD (10 } \\
\text { ppmw S) }\end{array}$ & $\begin{array}{l}\text { H2 Production } \\
\text { Plant. }\end{array}$ & $\begin{array}{l}\text { Thiopaq } \\
\text { process to be } \\
\text { used for 3.4 } \\
\text { T/D. Will also } \\
\text { need sulfur } \\
\text { storage / } \\
\text { handling }\end{array}$ & \\
\hline
\end{tabular}


PROJECT: PetroStar Valdez ULSD - HDS/BDS Combo ANVIL NO: AE1416
CLIENT: PetroStar

DATE: 6/17/05

REV NO.: 0

HDS EQUIPMENT - FACTORING SUMMARY

\begin{tabular}{|c|c|c|c|c|c|c|}
\hline $\begin{array}{l}\text { EQUIPMENT } \\
\text { ITEM NO. } \\
\end{array}$ & $\begin{array}{l}\text { QUAN- } \\
\text { TITY }\end{array}$ & DESCRIPTION & $\begin{array}{l}\text { TOTAL } \\
\text { EQUIP. } \\
\text { COST, \$ } \\
\end{array}$ & $\begin{array}{l}* \text { FIELD } \\
\text { COST } \\
\text { MULTI- } \\
\text { PLIER } \\
\end{array}$ & $\begin{array}{l}\text { TOTAL } \\
\text { FIELD } \\
\text { COST, } \$ \\
\end{array}$ & NOTES \\
\hline COMPRESSORS & & & & & & \multirow{2}{*}{$\begin{array}{l}\text { Capacity: } 64 \text { CFM, Motor Driven, Shaft HP: } 100 \text { BHP, Motor } \\
\text { Size: } 125 \text { HP, Casing: killed C. S., Internals: CS } \\
\text { Capacity: } 109 \text { CFM, Motor Driven, Shaft HP: } 80 \text { BHP, Motor } \\
\text { Size: } 100 \text { HP, Casing: killed C. S., Internals: Stainless Steel }\end{array}$} \\
\hline C-500 & 1 & Makeup Hydrogen Compressor & $\$ 182,000$ & 2.8 & $\$ 509,600$ & \\
\hline \multirow[t]{2}{*}{ C-501 } & \multirow[t]{2}{*}{1} & Recycle Gas Compressor & $\$ 176,100$ & 2.8 & $\$ 493,080$ & \multirow[t]{2}{*}{$\begin{array}{l}\text { Capacity: } 109 \text { CFM, Motor Driven, Shaft HP: } 80 \text { BHP, Motor } \\
\text { Size: } 100 \text { HP, Casing: killed C. S., Internals: Stainless Steel }\end{array}$} \\
\hline & & SUB-TOTAL & $\$ 358,000$ & & $\$ 1,003,000$ & \\
\hline \multicolumn{7}{|l|}{ EXCHANGERS } \\
\hline E-500 & 1 & Reactor Fd/Btms Exchanger \#1 & $\$ 203,700$ & 4.0 & $\$ 814,800$ & $\begin{array}{l}\text { 13.8 MM BTU/Hr, } 4500 \text { FT2 (total) w/2 shells, TEMA type } \\
\text { CEU, Tubes: } 11 / 4 \mathrm{Cr}-1 / 2 \text { Mo tubes and tube sheet and weld } \\
\text { overlay } 316 \text { ss for tube sheet Shell: } 11 / 4 \mathrm{Cr}-1 / 2 \text { Mo clad w/ } \\
321 \text { SS, baffles to be } 304 \mathrm{SS}\end{array}$ \\
\hline E-501 & 1 & Reactor Fd/Btms Exchanger \#2 & $\$ 119,800$ & 4.0 & $\$ 479,200$ & $\begin{array}{l}\text { 9.7 MM BTU/Hr, } 2840 \text { FT2 (total) w/2 shells, TEMA type } \\
\text { CEU, Tubes: } 11 / 4 \mathrm{Cr}-1 / 2 \text { Mo for tubes and tube sheet, } \\
\text { SA387 Gr } 11 \text { channel, weld overlay } 321 \text { SS tube sheet, } \\
\text { channel, and channel cover, Shell: Killed C. S. w/ } 0.125 " \\
\text { CA }\end{array}$ \\
\hline E-502 & 1 & Separator Fd/Btms Exchanger & $\$ 8,200$ & 4.0 & $\$ 32,800$ & $\begin{array}{l}0.1 \mathrm{MM} \text { BTU/Hr, } 33 \text { FT2, double pipe, Tubes: Carbon Steel, } \\
\text { Shell: Carbon Steel }\end{array}$ \\
\hline \multirow[t]{2}{*}{ E-505 } & \multirow[t]{2}{*}{1} & \multirow[t]{2}{*}{ Stripper Fd/Btms Exchanger } & $\$ 144,400$ & 4.0 & $\$ 577,600$ & \multirow[t]{2}{*}{$\begin{array}{l}\text { 11.6 MM BTU/Hr, } 3,160 \mathrm{FT} 2 \text { (total) w/3 shells, TEMA type } \\
\text { CEU, Tubes: } 18 \mathrm{Cr}-8 \mathrm{Ni} \text {, Channel is CS w/ } 0.25 \text { " CA, tube } \\
\text { sheets are } 410 \mathrm{SS} \text {, Shell: CS w/0.25" CA, CS baffles }\end{array}$} \\
\hline & & & $\$ 476,000$ & & $\$ 1,904,000$ & \\
\hline \multicolumn{7}{|l|}{ AIR COOLERS } \\
\hline E-503 & 1 & H. T. Separator Overhead Cooler & $\$ 40,100$ & 4.0 & $\$ 160,400$ & \multirow{4}{*}{$\begin{array}{l}\text { 1.2 MM BTU/Hr, } 246 \text { FT2 Bare Tube, 5,240 FT2 Extended, } \\
\text { Shaft HP: } 5 \text { BHP, Seamless Carbon Steel tubes with } \\
\text { aluminum fins. } \\
\text { 2.0 MM BTU/Hr, } 371 \text { FT2 Bare Tube, 7,713 FT2 Extended, } \\
\text { Shaft HP: } 5 \text { BHP, Seamless Carbon Steel tubes with } \\
\text { aluminum fins. } \\
\text { 12.0 MM BTU/Hr, 2,330 FT2 (Bare Tube), 49,860 FT2 } \\
\text { Extended, Shaft HP: } 20 \text { BHP, Seamless Carbon Steel tubes } \\
\text { with aluminum fins. }\end{array}$} \\
\hline E-504 & 1 & Stripper O. H. Condenser & $\$ 37,200$ & 4.0 & $\$ 148,800$ & \\
\hline \multirow[t]{2}{*}{ E-506 } & \multirow[t]{2}{*}{1} & \multirow[t]{2}{*}{ Product Cooler } & $\$ 81,000$ & 4.0 & $\$ 324,000$ & \\
\hline & & & $\$ 158,000$ & & $\$ 633,000$ & \\
\hline \multicolumn{7}{|l|}{ FURNACES } \\
\hline $\mathrm{H}-500$ & 1 & Diesel Charge Heater & $\$ 277,300$ & 2.5 & $\$ 679,385$ & \multirow[t]{4}{*}{$\begin{array}{l}\text { 4.7 MM BTU/Hr, Fired Heater, Convection Section shared } \\
\text { w/ H-501, Tubes: } 9 \mathrm{Cr}-1 \mathrm{Mo} \text { w/ 0.1" CA }\end{array}$} \\
\hline $\mathrm{H}-500$ & 1 & CEMS & $\$ 100,000$ & 1.5 & $\$ 150,000$ & \\
\hline $\mathrm{H}-500$ & 1 & Preheating & $\$ 40,000$ & 1.0 & $\$ 40,000$ & \\
\hline $\mathrm{H}-500$ & 1 & Burner Management & $\$ 30,000$ & 1.0 & $\$ 30,000$ & \\
\hline $\mathrm{H}-501$ & 1 & Stripper Reboiler & $\$ 288,300$ & 2.5 & $\$ 706,335$ & \multirow{5}{*}{$\begin{array}{l}\text { 5.6 MM BTU/Hr, Fired Heater, Convection Section shared } \\
\text { w/ H-500, Tubes: } 5 \mathrm{Cr}-1 / 2 \mathrm{Mo} \text { w/ } 0.1 \text { " CA } \\
\text { Continuous Emission Monitoring System }\end{array}$} \\
\hline $\mathrm{H}-501$ & 1 & CEMS & $\$ 100,000$ & 1.5 & $\$ 150,000$ & \\
\hline $\mathrm{H}-501$ & 1 & Preheating & $\$ 40,000$ & 1.0 & $\$ 40,000$ & \\
\hline \multirow[t]{2}{*}{$\mathrm{H}-501$} & \multirow[t]{2}{*}{1} & \multirow[t]{2}{*}{ Burner Management } & $\$ 30,000$ & 1.0 & $\$ 30,000$ & \\
\hline & & & $\$ 906,000$ & & $\$ 1,826,000$ & \\
\hline \multicolumn{7}{|l|}{ PUMPS } \\
\hline$\overline{P-500} \mathrm{~A} / \mathrm{B}$ & 2 & Diesel Feed Pump & $\$ 232,000$ & 5.0 & $\$ 1,160,000$ & $\begin{array}{l}\text { API Centrifugal Pump, 206GPM, } 0.81 \text { SG, } 2182 \text { Ft. Head, } \\
\text { CS Case, } 12 \% \text { CR Impeller, 150HP Motor }\end{array}$ \\
\hline P-501 A/B & 2 & Stripper Bottoms Pump & $\$ 54,400$ & 5.0 & $\$ 272,000$ & $\begin{array}{l}\text { API Centrifugal Pump, 335GPM, } 0.60 \text { SG, } 481 \text { Ft. Head, CS } \\
\text { Case, } 12 \% \text { CR Impeller, 50HP Motor }\end{array}$ \\
\hline \multirow[t]{2}{*}{$P-502 A / B$} & 2 & Stripper Overhead Pump & $\$ 44,400$ & 5.0 & $\$ 222,000$ & \multirow[t]{2}{*}{$\begin{array}{l}\text { API Centrifugal Pump, 30GPM, } 0.73 \text { SG, } 120 \text { Ft. Head, CS } \\
\text { Case, } 12 \% \text { CR Impeller, 5HP Motor }\end{array}$} \\
\hline & & SUB-TOTAL & $\$ 331,000$ & & $\$ 1,654,000$ & \\
\hline
\end{tabular}


Final Technical Progress Report

ANVIL CORPORATION

OE Award No. DE-FC26-02NT15340

PROJECT: PetroStar Valdez ULSD - HDS/BDS Combo ANVIL NO: AE1416

CLIENT: PetroStar

DATE: $6 / 17 / 05$

REV NO.: 0

HDS EQUIPMENT - FACTORING SUMMARY

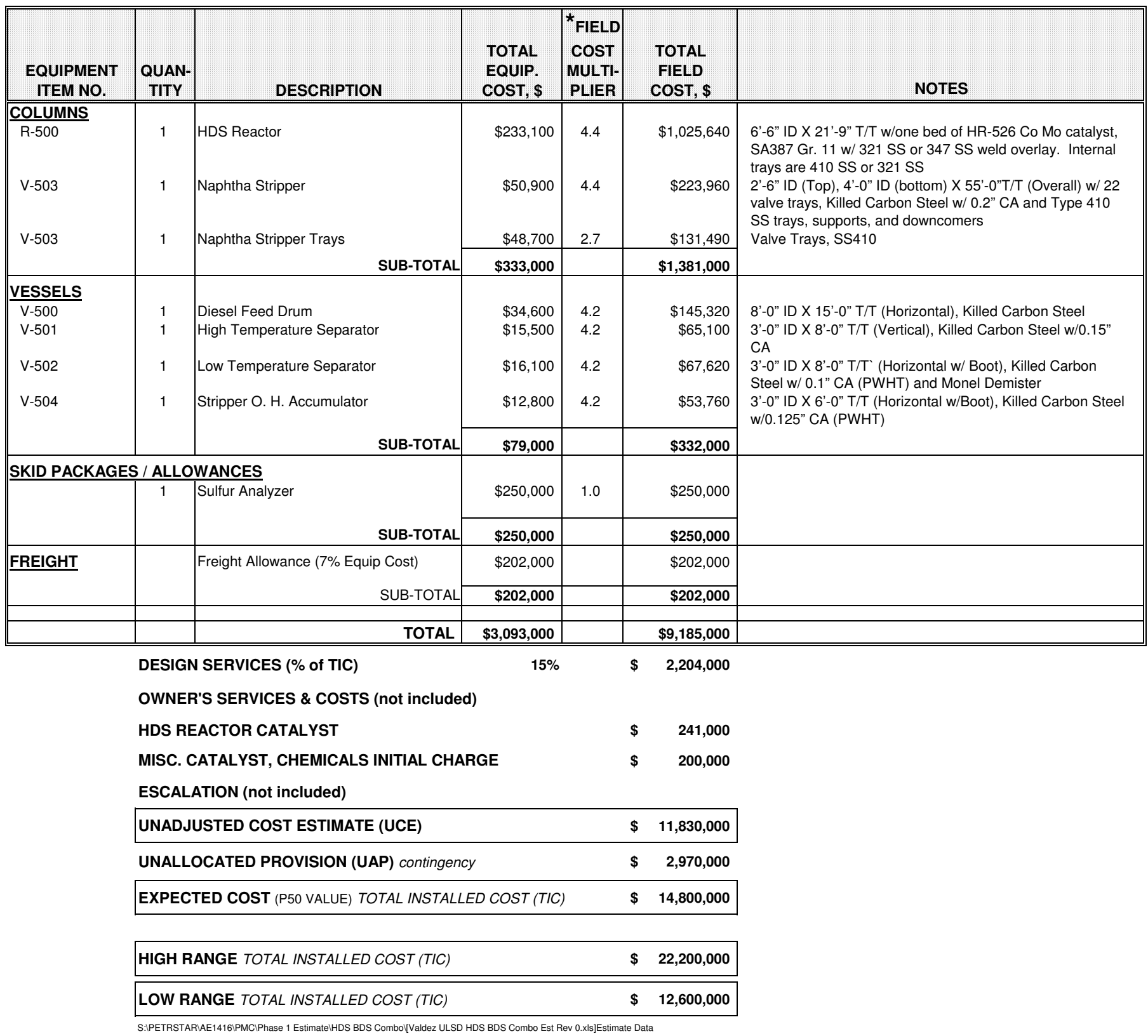

*Note: Field Cost Multiplier includes the following bulk material and installation labor: Civil, Concrete, Structural, Piping, Electrical, Instrumentation, Insulation, Fireproofing, Painting, and Testing. 
BDS EQUIPMENT - FACTORING SUMMARY

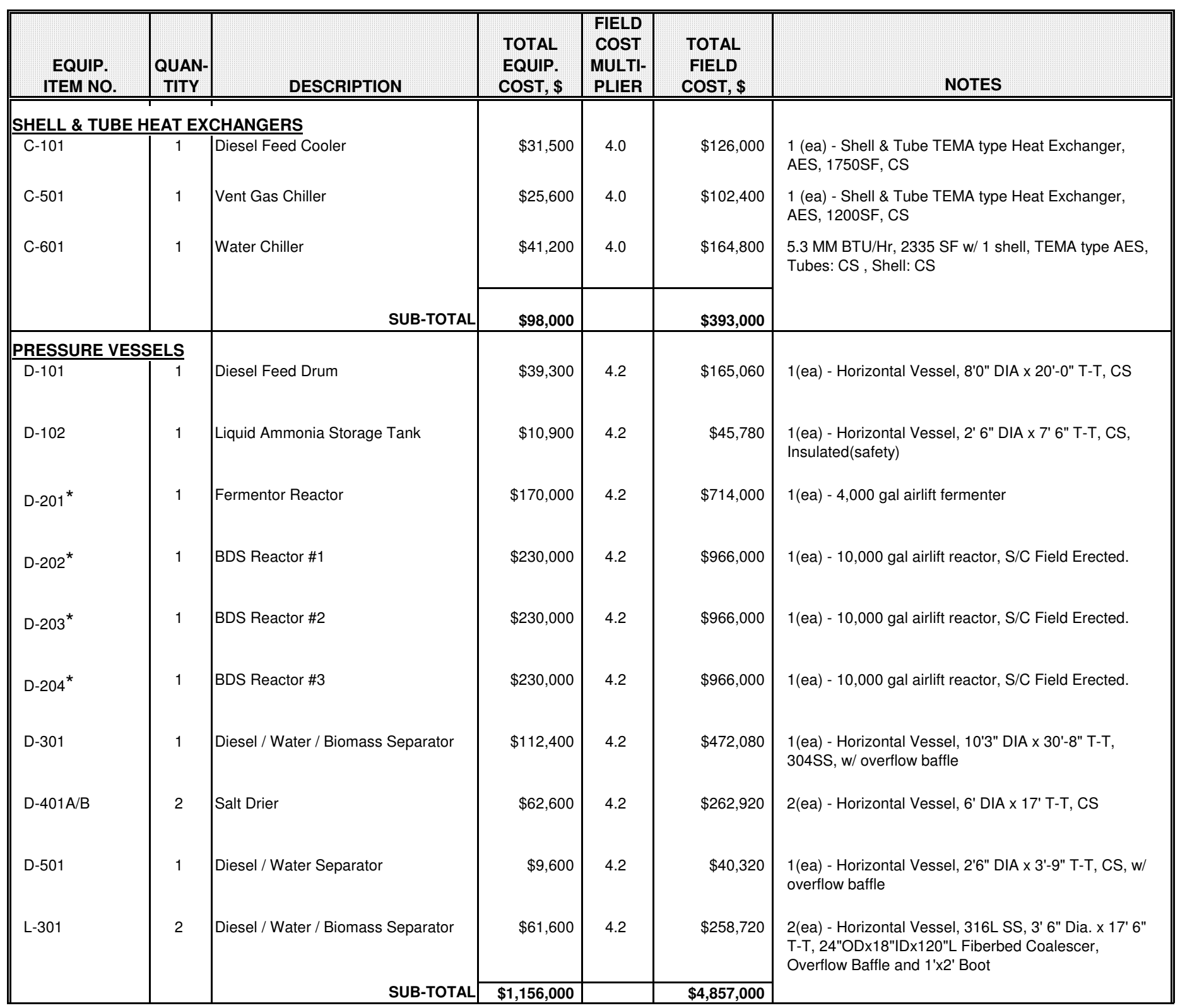


BDS EQUIPMENT - FACTORING SUMMARY

\begin{tabular}{|c|c|c|c|c|c|c|}
\hline $\begin{array}{l}\text { EQUIP. } \\
\text { ITEM NO. }\end{array}$ & $\begin{array}{l}\text { QUAN- } \\
\text { TITY }\end{array}$ & DESCRIPTION & $\begin{array}{l}\text { TOTAL } \\
\text { EQUIP. } \\
\text { COST, \$ }\end{array}$ & $\begin{array}{c}\text { FIELD } \\
\text { COST } \\
\text { MULTI- } \\
\text { PLIER } \\
\end{array}$ & $\begin{array}{l}\text { TOTAL } \\
\text { FIELD } \\
\text { COST, \$ }\end{array}$ & NOTES \\
\hline \multicolumn{7}{|c|}{ PACKAGED SKIDS/SYSTEMS } \\
\hline D-205* & 1 & Seed Tank A & $\$ 94,000$ & 2.5 & $\$ 235,000$ & Packaged Seed Fermenter System \\
\hline D-206* & 1 & Seed Tank B & $\$ 328,000$ & 2.5 & $\$ 820,000$ & Packaged Seed Fermenter System \\
\hline $\mathrm{J}-109 \mathrm{~A} / \mathrm{B}$ & 2 & Air Compressor & $\$ 266,000$ & 2.5 & $\$ 665,000$ & $\begin{array}{l}2(\text { ea) - Air Compressor, Screw, } 700 \text { ACFM, CS } \\
\text { body/internals, 65HP Motor Driven }\end{array}$ \\
\hline$L-109^{*}$ & 1 & Glucose Sterilizer & $\$ 33,000$ & 2.5 & $\$ 82,500$ & $\begin{array}{l}1(\text { ea) - Packaged } 8 \text { gph continuous steam sterilizer } \\
\text { system complete w/ preheater, heater, cooler, \& } \\
\text { holding coil }\end{array}$ \\
\hline L-110* & 1 & Nutrients Sterilizer & $\$ 33,000$ & 2.5 & $\$ 82,500$ & $\begin{array}{l}1(\mathrm{ea}) \text { - Packaged } 8 \mathrm{gph} \text { continuous steam sterilizer } \\
\text { system complete } \mathrm{w} / \text { preheater, heater, cooler, \& } \\
\text { holding coil }\end{array}$ \\
\hline L-302* & 1 & Electrostatic Precipitator & $\$ 448,000$ & 2.5 & $\$ 1,120,000$ & 1(ea) - Packaged ESP Unit \\
\hline L-601* & 1 & Water Chiller & $\$ 401,000$ & 2.5 & $\$ 1,002,500$ & $\begin{array}{l}\text { 1(ea) - Packaged propane refirigeration unit complete } \\
\text { w/ compressor, driver, lube oil system, KO drum \& all } \\
\text { necessary instr. \& controls. }\end{array}$ \\
\hline $\mathrm{L}-801^{*}$ & 1 & Waste Oxidizer & $\$ 280,000$ & 2.5 & $\$ 700,000$ & $\begin{array}{l}1 \text { (ea) - Packaged horizontal thermal oxidizer w/ high } \\
\text { intensity burner, refractory lined chamber \& stack. }\end{array}$ \\
\hline \multirow[t]{2}{*}{$\mathrm{L}-901^{*}$} & 1 & Water Filtration System & $\$ 212,000$ & 2.5 & $\$ 530,000$ & $\begin{array}{l}1 \text { (ea) - Cross flow filtration system for water } \\
\text { purification }\end{array}$ \\
\hline & \multicolumn{2}{|r|}{ SUB-TOTAL } & $\$ 2,095,000$ & & $\$ 5,238,000$ & \\
\hline \multicolumn{7}{|c|}{ ATMOSPHERE STORAGE TANKS } \\
\hline $\mathrm{F}-101$ & 1 & 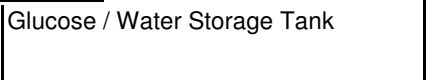 & $\$ 15,400$ & 2.9 & $\$ 44,660$ & $\begin{array}{l}\text { 1(ea) - Atm. Storage Tank, Flat Roof, Flat Bottom, 7' } \\
\text { DIA x 10' T-T, Epoxy Resin coated CS }\end{array}$ \\
\hline $\mathrm{F}-102 \mathrm{~A}$ & 1 & Salt / Water Storage Tank & $\$ 10,200$ & 2.9 & $\$ 29,580$ & $\begin{array}{l}\text { 1(ea) - Atm. Storage Tank, Flat Roof, Flat Bottom, 4' } \\
\text { DIA x 8' T-T, Epoxy Resin coated CS }\end{array}$ \\
\hline F-102B & 1 & Salt / Water Storage Tank & $\$ 10,200$ & 2.9 & $\$ 29,580$ & $\begin{array}{l}\text { 1(ea) - Atm. Storage Tank, Flat Roof, Flat Bottom, 4' } \\
\text { DIA x 8' T-T, Epoxy Resin coated CS }\end{array}$ \\
\hline F-103 & 1 & Ethanol / Water Storage Tank & $\$ 25,200$ & 2.9 & $\$ 73,080$ & $\begin{array}{l}\text { 1(ea) - Atm. Storage Tank, Flat Roof, Flat Bottom, } 12 \\
\text { 8"' DIA x 8' 6"' T-T, Epoxy Resin coated CS }\end{array}$ \\
\hline \multirow[t]{2}{*}{ F-104 } & 1 & $\mathrm{NaOH} /$ Water Storage Tank & $\$ 23,100$ & 2.9 & $\$ 66,990$ & $\begin{array}{l}\text { 1(ea) - Atm. Storage Tank, Flat Roof, Flat Bottom, 9' } \\
\text { DIA x 14' T-T, Epoxy Resin coated CS }\end{array}$ \\
\hline & & SUB-TOTAL- & $\$ 84,000$ & & $\$ 244,000$ & \\
\hline
\end{tabular}


BDS EQUIPMENT - FACTORING SUMMARY

\begin{tabular}{|c|c|c|c|c|c|c|}
\hline $\begin{array}{l}\text { EQUIP. } \\
\text { ITEM NO. }\end{array}$ & $\begin{array}{c}\text { QUAN- } \\
\text { TITY }\end{array}$ & DESCRIPTION & $\begin{array}{l}\text { TOTAL } \\
\text { EQUIP. } \\
\text { COST, \$ }\end{array}$ & $\begin{array}{c}\text { FIELD } \\
\text { COST } \\
\text { MULTI- } \\
\text { PLIER } \\
\end{array}$ & $\begin{array}{l}\text { TOTAL } \\
\text { FIELD } \\
\text { COST, \$ }\end{array}$ & NOTES \\
\hline PUMPS & & & & & & \\
\hline$\overline{J-101 A} / B$ & 2 & Diesel Charge Pump & $\$ 55,000$ & 4.5 & $\$ 247,500$ & $\begin{array}{l}\text { 2(ea) - API Centrifugal Pump, } 190 \mathrm{gpm}, 163 \mathrm{ft} \text {. Head, } \\
15 \mathrm{HP} \text { Motor driven, CS, } 1 \text { operating, } 1 \text { installed spare }\end{array}$ \\
\hline$J-103 A / B$ & 2 & Process Water Pump & $\$ 6,400$ & 4.5 & $\$ 28,800$ & $\begin{array}{l}\text { 2(ea) - Centrifugal Pump, } 3 \mathrm{gpm}, 121 \mathrm{ft} \text {. Head, .5HP } \\
\text { Motor driven, CS, } 1 \text { operating, } 1 \text { installed spare }\end{array}$ \\
\hline$J-104 A / B$ & 2 & Glucose Pump & $\$ 11,400$ & 4.5 & $\$ 51,300$ & $\begin{array}{l}\text { 2(ea) - Diaphragm Pump, } 0.2 \mathrm{gpm}, 31 \mathrm{ft} \text {. Head, } \\
\text { Hydraulic, } 304 \mathrm{SS}, 1 \text { operating, } 1 \text { installed spare }\end{array}$ \\
\hline$J-105 A / B$ & 2 & Nutrients Pump & $\$ 10,600$ & 4.5 & $\$ 47,700$ & $\begin{array}{l}\text { 2(ea) - Diaphragm Pump, } 0.15 \mathrm{gpm}, 39 \mathrm{ft} \text {. Head, } \\
\text { Hydraulic, } 304 \mathrm{SS}, 1 \text { operating, } 1 \text { installed spare }\end{array}$ \\
\hline $\mathrm{J}-106 \mathrm{~A} / \mathrm{B}$ & 2 & Ethanol Pump & $\$ 12,200$ & 4.5 & $\$ 54,900$ & $\begin{array}{l}\text { 2(ea) - Diaphragm Pump, } .3 \mathrm{gpm}, 81 \mathrm{ft} . \text { Head, } \\
\text { Hydraulic, } 304 \mathrm{SS}, 1 \text { operating, } 1 \text { installed spare }\end{array}$ \\
\hline $\mathrm{J}-107 \mathrm{~A} / \mathrm{B}$ & 2 & Potassium Hydroxide Pump & $\$ 12,600$ & 4.5 & $\$ 56,700$ & $\begin{array}{l}\text { 2(ea) - Diaphragm Pump, . } 4 \mathrm{gpm}, 65 \mathrm{ft} . \text { Head, } \\
\text { Hydraulic, 304SS, } 1 \text { operating, } 1 \text { installed spare }\end{array}$ \\
\hline $\mathrm{J}-201 \mathrm{~A} / \mathrm{B}$ & 2 & Fermentor Transfer Pump & $\$ 11,200$ & 4.5 & $\$ 50,400$ & $\begin{array}{l}\text { 2(ea) - Rotary Lobe Pump, } 2.5 \mathrm{gpm}, 43 \mathrm{ft} \mathrm{Head,} 0.5 \mathrm{HP} \\
\text { Motor, 304SS, } 1 \text { operating, } 1 \text { installed spare }\end{array}$ \\
\hline $\mathrm{J}-202 \mathrm{~A} / \mathrm{B}$ & 2 & BDS Reactor No1 Transfer Pump & $\$ 21,400$ & 4.5 & $\$ 96,300$ & $\begin{array}{l}\text { 2(ea) - Centrifugal Pump, } 700 \mathrm{gpm}, 45 \mathrm{ft} . \text { Head, } 15 \mathrm{HP} \\
\text { Motor driven, } 304 \mathrm{SS}, 1 \text { operating, } 1 \text { installed spare }\end{array}$ \\
\hline $\mathrm{J}-203 \mathrm{~A} / \mathrm{B}$ & 2 & BDS Reactor No2 Transfer Pump & $\$ 21,400$ & 4.5 & $\$ 96,300$ & $\begin{array}{l}\text { 2(ea) - Centrifugal Pump, } 700 \mathrm{gpm}, 45 \mathrm{ft} \text {. Head, } 15 \mathrm{HP} \\
\text { Motor driven, } 304 \mathrm{SS}, 1 \text { operating, } 1 \text { installed spare }\end{array}$ \\
\hline $\mathrm{J}-204 \mathrm{~A} / \mathrm{B}$ & 2 & BDS Reactor No3 Transfer Pump & $\$ 21,400$ & 4.5 & $\$ 96,300$ & $\begin{array}{l}\text { 2(ea) - Centrifugal Pump, } 700 \mathrm{gpm}, 45 \mathrm{ft} . \text { Head, } 15 \mathrm{HP} \\
\text { Motor driven, } 304 \mathrm{SS}, 1 \text { operating, } 1 \text { installed spare }\end{array}$ \\
\hline $\mathrm{J}-301 \mathrm{~A} / \mathrm{B}$ & 2 & 1st Stg Sep Overflow Pump & $\$ 47,600$ & 4.5 & $\$ 214,200$ & $\begin{array}{l}\text { 2(ea) - API Centrifugal Pump, } 200 \mathrm{gpm}, 49 \mathrm{ft} \text {. Head, } \\
\text { 5HP Motor driven, 304SS, } 1 \text { operating, } 1 \text { installed } \\
\text { spare }\end{array}$ \\
\hline$J-302 A / B$ & 2 & 1st Stg Sep Underflow Pump & $\$ 18,400$ & 4.5 & $\$ 82,800$ & $\begin{array}{l}\text { 2(ea) - Centrifugal Pump, } 500 \mathrm{gpm}, 68 \mathrm{ft} \text {. Head, } 10 \mathrm{HP} \\
\text { Motor driven, } 304 \mathrm{SS}, 1 \text { operating, } 1 \text { installed spare }\end{array}$ \\
\hline $\mathrm{J}-303 \mathrm{~A} / \mathrm{B}$ & 2 & 2nd Stg Sep Overflow Pump & $\$ 11,800$ & 4.5 & $\$ 53,100$ & $\begin{array}{l}\text { 2(ea) - Centrifugal Pump, } 145 \mathrm{gpm}, 72 \mathrm{ft} \text {. Head, } 5 \mathrm{HP} \\
\text { Motor driven, 304SS, } 1 \text { operating, } 1 \text { installed spare }\end{array}$ \\
\hline$J-304 A / B$ & 2 & 2nd Stg Sep Underflow Pump & $\$ 10,200$ & 4.5 & $\$ 45,900$ & $\begin{array}{l}\text { 2(ea) - Centrifugal Pump, } 45 \mathrm{gpm}, 72 \mathrm{ft} \text {. Head, } 3 \mathrm{HP} \\
\text { Motor driven, } 304 \mathrm{SS}, 1 \text { operating, } 1 \text { installed spare }\end{array}$ \\
\hline $\mathrm{J}-502 \mathrm{~A} / \mathrm{B}$ & 2 & Vent Gas KO Drum Wtr Pump & $\$ 8,600$ & 4.5 & $\$ 38,700$ & $\begin{array}{l}\text { 2(ea) - Centrifugal Pump, } 1 \mathrm{gpm}, 70 \mathrm{ft} \text {. Head, } 0.5 \mathrm{HP} \\
\text { Motor driven, CS, } 1 \text { operating, } 1 \text { installed spare }\end{array}$ \\
\hline \multirow[t]{2}{*}{$J-601$ A/B } & 2 & Chilled Water Pump & $\$ 19,800$ & 4.5 & $\$ 89,100$ & $\begin{array}{l}\text { Centrifugal Pump, 2000GPM, 0.95 SG, } 81.7 \text { Ft. Head, } \\
\text { CS Case, 12\%CR Impeller, 20HP Motor }\end{array}$ \\
\hline & & SUB-TOTAL & $\$ 300,000$ & & $\$ 1,350,000$ & \\
\hline
\end{tabular}


BDS EQUIPMENT - FACTORING SUMMARY

\begin{tabular}{|c|c|c|c|c|c|c|}
\hline $\begin{array}{l}\text { EQUIP. } \\
\text { ITEM NO. }\end{array}$ & $\begin{array}{l}\text { QUAN- } \\
\text { TITY }\end{array}$ & DESCRIPTION & $\begin{array}{l}\text { TOTAL } \\
\text { EQUIP. } \\
\text { COST, \$ }\end{array}$ & $\begin{array}{c}\text { FIELD } \\
\text { COST } \\
\text { MULTI- } \\
\text { PLIER } \\
\end{array}$ & $\begin{array}{l}\text { TOTAL } \\
\text { FIELD } \\
\text { COST, \$ }\end{array}$ & NOTES \\
\hline \multicolumn{7}{|l|}{ FILTERS } \\
\hline L-101 & 1 & Diesel Pre-Filter Vessel & $\$ 5,210$ & 4.2 & $\$ 21,882$ & $\begin{array}{l}\text { 1(ea) - Filter housing, } 316 \mathrm{LSS}, 47 " \mathrm{~T}-\mathrm{T} \times 8 \text { × Dia. w/ } \\
5(\text { ea) - Polypropylene element, } 30 " \text { long, } 1.5 \mathrm{um} \text { pore } \\
\text { size }\end{array}$ \\
\hline L-102 & 1 & Diesel Pre-Filter Vessel & $\$ 5,280$ & 4.2 & $\$ 22,176$ & $\begin{array}{l}\text { 1(ea) - Filter housing, } 316 \mathrm{LSS}, 47 " \text { T-T x } 8 \text { " Dia. w/ } \\
5(\text { ea) - Polypropylene element, } 30 " \text { long, } 0.2 \text { um pore } \\
\text { size }\end{array}$ \\
\hline L-103 & 1 & Air Pre-Filter Vessel & $\$ 11,400$ & 4.2 & $\$ 47,880$ & $\begin{array}{l}\text { 1(ea) - Filter housing, 316L SS, 55" T-T x 15" Dia. w/ } \\
5(\mathrm{ea}) \text { - GF element, 30" long, } 1.0 u m \text { pore size }\end{array}$ \\
\hline L-104 & 1 & Air Bio-Filter Vessel & $\$ 11,125$ & 4.2 & $\$ 46,725$ & $\begin{array}{l}\text { 1(ea) - Filter housing, } 316 \mathrm{~L} \text { SS, } 43 \text { " T-T x 15" Dia. w/ } 5 \\
\text { (ea) - PTFE element, } 10 " \text { long, } 0.01 \text { um pore size }\end{array}$ \\
\hline L-105 & 1 & BDS Reactor \#1 Air Bio Filter Vessel & $\$ 12,420$ & 4.2 & $\$ 52,164$ & $\begin{array}{l}\text { 1(ea) - Filter housing, } 316 \mathrm{~L} \text { SS, } 55^{\prime \prime} \text { T-T x 15" Dia. w/ } \\
5(\text { ea) - PTFE element, } 30 \text { long, } 0.01 \text { um pore size }\end{array}$ \\
\hline L-106 & 1 & BDS Reactor \#2 Air Bio Filter Vessel & $\$ 12,420$ & 4.2 & $\$ 52,164$ & $\begin{array}{l}\text { 1(ea) - Filter housing, 316L SS, 55" T-T x 15" Dia. w/ } \\
5(\mathrm{ea}) \text { - PTFE element, 30" long, } 0.01 \text { um pore size }\end{array}$ \\
\hline L-107 & 1 & BDS Reactor \#3 Air Bio Filter Vessel & $\$ 12,420$ & 4.2 & $\$ 52,164$ & $\begin{array}{l}\text { 1(ea) - Filter housing, 316L SS, 55" T-T x 15" Dia. w/ } \\
5(\text { ea) - PTFE element, 30" long, 0.01um pore size }\end{array}$ \\
\hline L-108 & 1 & Air Purge Bio-Filter Vessel & $\$ 7,011$ & 4.2 & $\$ 29,446$ & $\begin{array}{l}\text { 1(ea) - Filter housing, 316L SS, 30" T-T x 12" Dia. w/ } \\
\text { 3(ea) - PTFE element, } 10 " \text { long, 0.01um pore size }\end{array}$ \\
\hline L-111 & 1 & Ethanol Bio-Filter Vessel & $\$ 1,308$ & 4.2 & $\$ 5,494$ & $\begin{array}{l}\text { 1(ea) - Filter housing, } 316 \mathrm{~L} \mathrm{SS}, 10 " \text { T-T x } 2.5 " \text { Dia. w/ } \\
1 \text { (ea) - Polyethersulfone element, } 5 \text { "long, } 0.2 \text { um pore } \\
\text { size }\end{array}$ \\
\hline L-112 & 1 & $\mathrm{NAaOH}$ Bio-Filter Vessel & $\$ 1,308$ & 4.2 & $\$ 5,494$ & $\begin{array}{l}\text { 1(ea) - Filter housing, } 316 \mathrm{~L} \mathrm{SS}, 10 " \text { " T-T x } 2.5 \text { " Dia. w/ } \\
1 \text { (ea) - Polyethersulfone element, } 5 \text { " long, } 0.2 \mathrm{um} \text { pore } \\
\text { size }\end{array}$ \\
\hline L-113 & 1 & Water Pre-Filter Vessel & $\$ 2,850$ & 4.2 & $\$ 11,970$ & $\begin{array}{l}\text { 1(ea) - Filter housing, } 316 \mathrm{LSS}, 38 \text { " T-T x 8" Dia. w/ } \\
3(\text { ea) - Polypropylene element, } 20 " \text { long, } 1.5 \mathrm{um} \text { pore } \\
\text { size }\end{array}$ \\
\hline L-114 & 1 & Water Bio-Filter Vessel & $\$ 2,892$ & 4.2 & $\$ 12,146$ & $\begin{array}{l}\text { 1(ea) - Filter housing, } 316 \mathrm{LSS}, 38 \text { " T-T x 8" Dia. w/ } \\
3(\text { ea) - Polypropylene element, } 20 " \text { long, } 0.2 \mathrm{um} \text { pore } \\
\text { size }\end{array}$ \\
\hline L-401 & 1 & Recycle Water Pre-Filter Vessel & $\$ 2,850$ & 4.2 & $\$ 11,970$ & $\begin{array}{l}\text { 1(ea) - Filter housing, } 316 \mathrm{~L} \text { SS, 38" T-T x 8" Dia. w/ } \\
3(e a) \text { - Polypropylene element, 20" long, 1.5um pore } \\
\text { size }\end{array}$ \\
\hline \multirow[t]{2}{*}{ L-402 } & 1 & Recycle Water Bio-Filter Vessel & $\$ 2,892$ & 4.2 & $\$ 12,146$ & \multirow[t]{2}{*}{$\begin{array}{l}\text { 1(ea) - Filter housing, 316L SS, 38" T-T x 8" Dia. w/ } \\
3(\text { ea) - Polypropylene element, 20" long, 0.2um pore } \\
\text { size }\end{array}$} \\
\hline & & SUB-TOTAL & $\$ 91,000$ & & $\$ 384,000$ & \\
\hline \multirow[t]{3}{*}{ FREIGHT } & & Freight Allowance (7\% Equip Cost) & $\$ 268,000$ & & $\$ 268,000$ & \\
\hline & & SUB-TOTAL & $\$ 268,000$ & & $\$ 268,000$ & \\
\hline & & TOTAL & $\$ 4,092,593$ & & $\$ 12,730,000$ & \\
\hline
\end{tabular}


A N V I L C ORPORATION Final Technical Progress Report

PROJECT: PetroStar Valdez ULSD - HDS/BDS Combo ANVIL NO: AE1416

DOE CLY L

DATE: 6/17/05

REV NO.: 0

BDS EQUIPMENT - FACTORING SUMMARY

\begin{tabular}{||c|c|c|c|c|c|c||}
\hline \hline & & TOTAL & FIELD & COST & TOTAL & \\
EQUIP. & QUAN- & & TOTA & FQUIP. & MULTI- & FIELD \\
ITEM NO. & TITY & DESCRIPTION & COST, \$ & PLIER & COST, \$ & NOTES \\
\hline \hline
\end{tabular}

$\begin{array}{llll}\text { DESIGN SERVICES } & 15 \% & \$ 2,930,000\end{array}$

OWNER'S SERVICES \& COSTS (provided by owner) \$ -

INITIAL CHEMICAL CHARGE $\quad \$ \quad 45,000$

ESCALATION (provided by owner) $\$$ -

\begin{tabular}{|ll}
\hline UNADJUSTED COST ESTIMATE (UCE) & $\$ 15,705,000$ \\
\hline
\end{tabular}

CONTINGENCY $\quad \$ \quad 3,895,000$

EXPECTED COST (P50 VALUE) TOTAL INSTALLED COST (TIC) $\$ \mathbf{1 9 , 6 0 0 , 0 0 0}$

S:IPETRSTARIAE1416iPMCIPhase 1 EstimateliHDS BDS Combo:[Valdez ULSD HDS BDS Combo Est Rev 0.xis]Estimate Data

\footnotetext{
* Note: Equipment pricing by Pelorus.
} 
Final Technical Progress Report

DOE Award No. DE-FC26-02NT15340

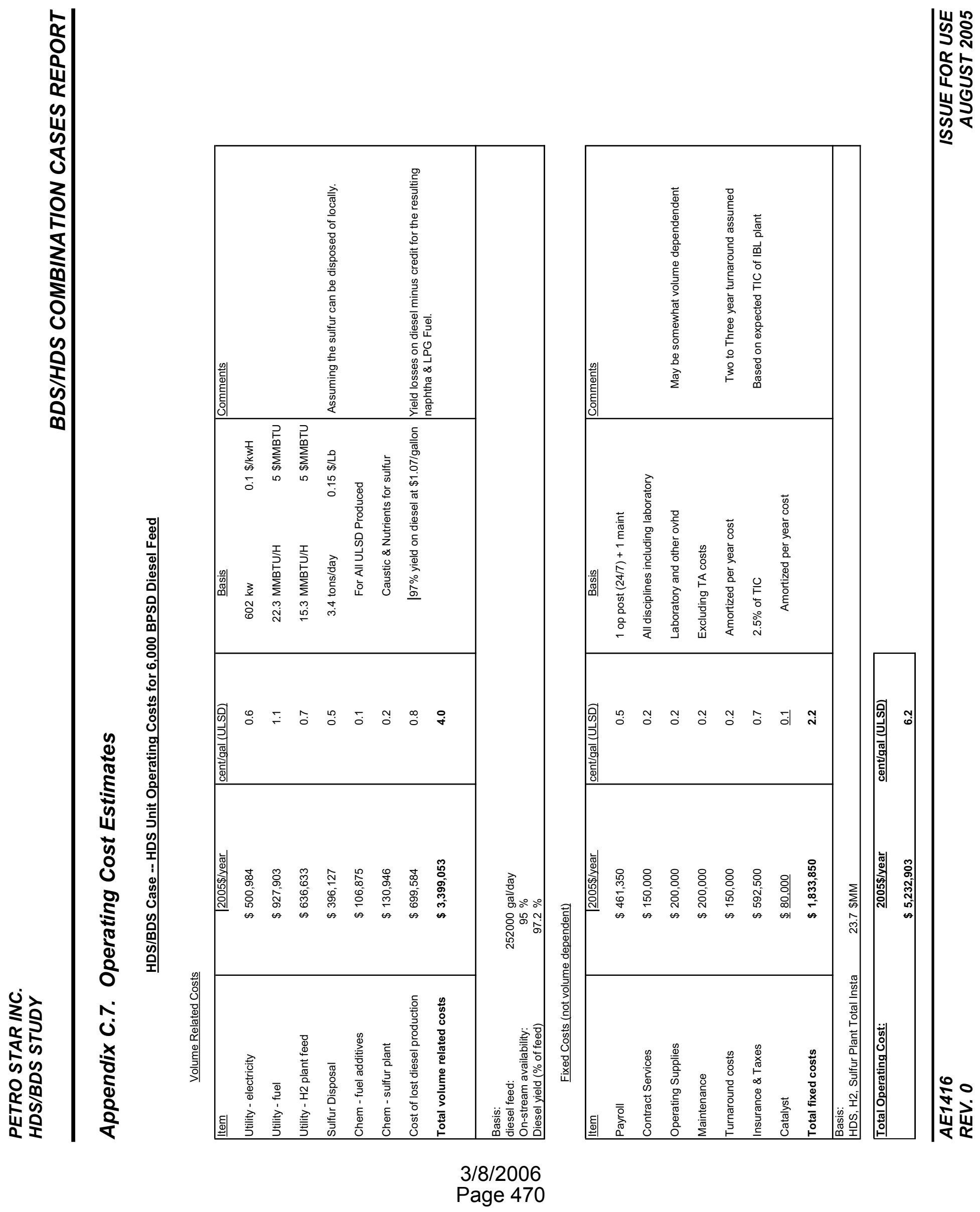


Final Technical Progress Report DOE Award No. DE-FC26-02NT15340
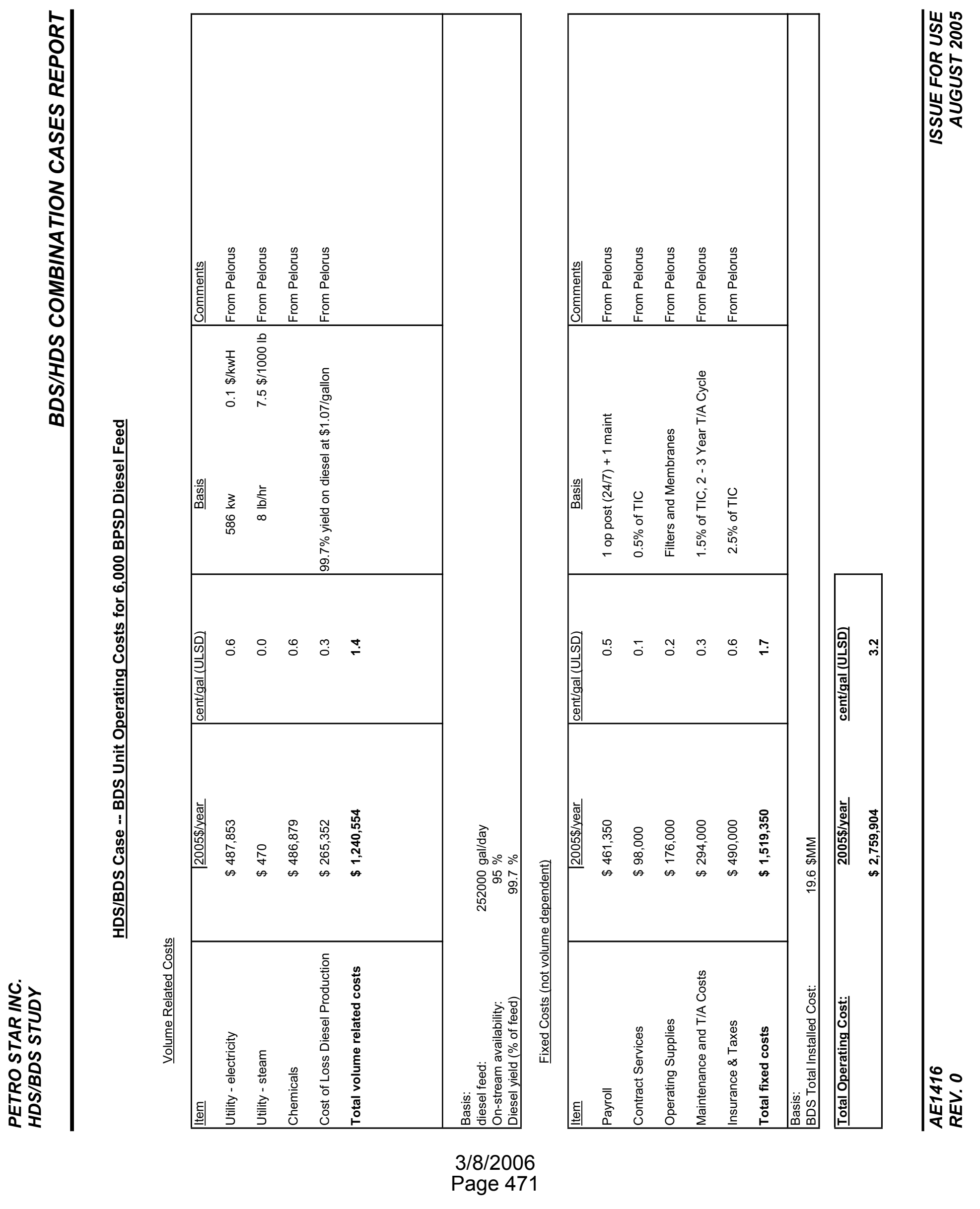
Appendix D.1 - Diesel Splitter Process Flow Diagram and Material Balance 


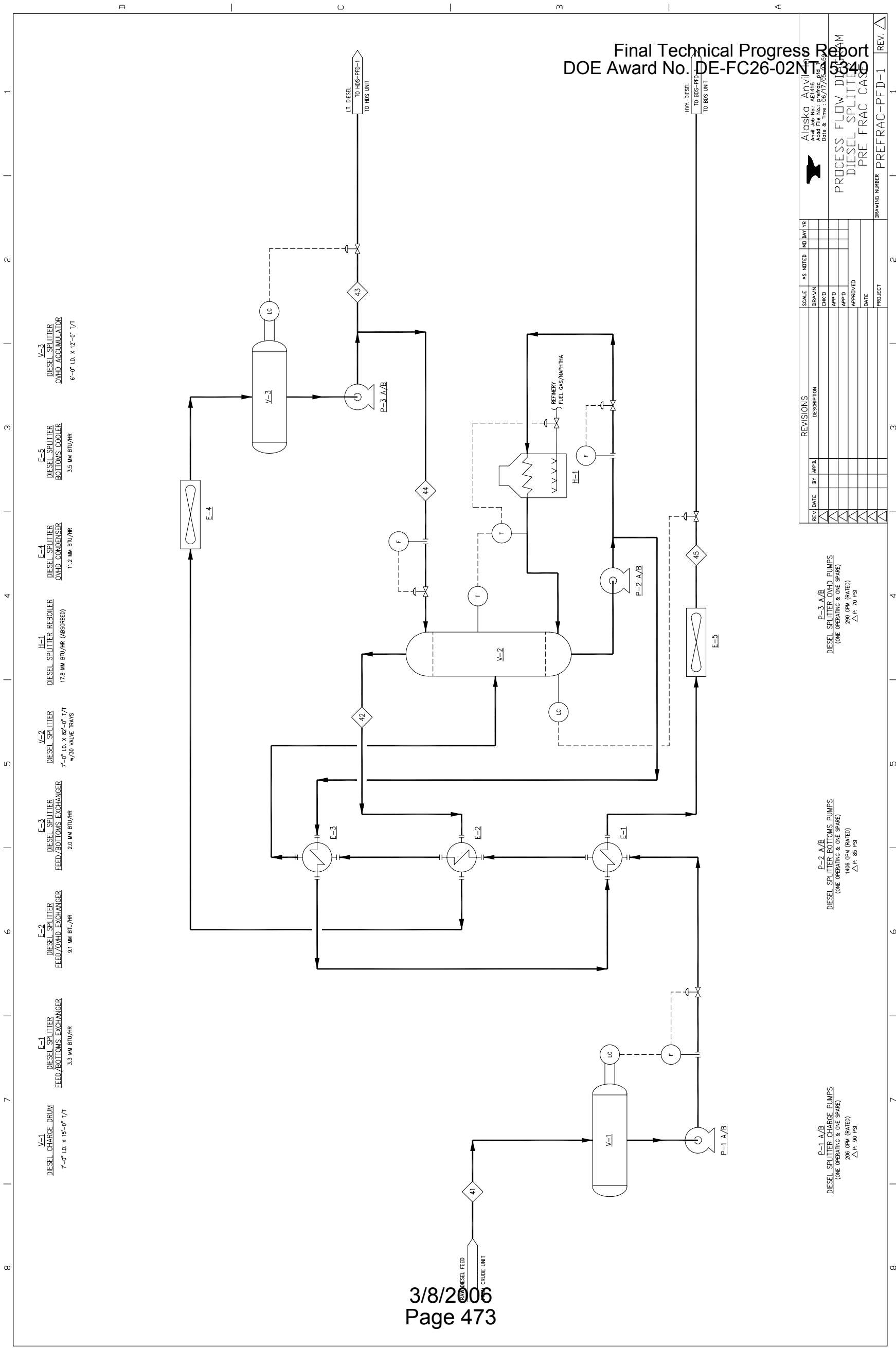




\section{Material Balance}

\section{Diesel Splitter Material Balance -- Pre Frac Case}

\begin{tabular}{|c|c|c|c|c|c|c|}
\hline Stream Number & & 41 & 42 & 43 & 44 & 45 \\
\hline Stream Descriptian & & ANS Feed & $\begin{array}{c}\text { Diesel Splitter } \\
\text { OVHD } \\
\end{array}$ & Light Diesel & $\begin{array}{c}\text { Diesel Splitter } \\
\text { Reflux } \\
\end{array}$ & Hesvy Diesel \\
\hline Phase & & LIOUID & VAPOR & LIOUID & LlOUID & LlOULD \\
\hline Mass Flow Rate & LB/HF & 75960 & 95923 & 47961 & 47961 & 28004 \\
\hline Temperature & $F$ & 216 & 559 & 380 & 380 & 120 \\
\hline Pressure & $\mathrm{PSG}$ & 20 & 10 & 72 & 72 & 68 \\
\hline Standard Liq Flow & BPD & 6021 & $n / \mathrm{a}$ & 3852 & 3852 & 2169 \\
\hline Vapor Flow & MSCFH & $\mathrm{n} / \mathrm{a}$ & 183.2 & $n / a$ & $n / a$ & $n / a$ \\
\hline Wt \% Vapor & & 0 & 100 & 0 & 0 & 0 \\
\hline $\mathrm{MW}$ & & 216.7 & 198.7 & 198.7 & 198.7 & 2566 \\
\hline
\end{tabular}




\section{Appendix D.2 - Diesel Splitter Equipment List and Budgetary Equipment Datasheets}

\section{Diesel Splitter Equipment List}

\begin{tabular}{|c|c|c|c|c|}
\hline Item & Service & Description & $\begin{array}{l}\text { Design Conditions } \\
\text { Pressure Temp. }\end{array}$ & Metallurgy \\
\hline E-1 & $\begin{array}{l}\text { Diesel Splitter Feed/B ottoms } \\
\text { Exchanger }\end{array}$ & $\begin{array}{l}\text { 3.3 MM BTU/Hr, } \\
\text { 450 FT2 w/1 shell, TEMA } \\
\text { type AEU }\end{array}$ & $\begin{array}{l}\text { Tubes: } 125 \text { PSIG, } 680^{\circ} \mathrm{F} \\
\text { Shell: } 120 \mathrm{PSIG}, 400^{\circ} \mathrm{F} /- \\
20^{\circ} \mathrm{F}\end{array}$ & $\begin{array}{l}\text { Tubes: } 1 \frac{1 / 4 \mathrm{Cr}-1 / 2 \mathrm{Mo}}{\text { Shell: } 1 \text { 1/4 } \mathrm{Cr}-1 / 2 \mathrm{Mo}} \\
\text { Sol }\end{array}$ \\
\hline $\mathrm{E}-2$ & $\begin{array}{l}\text { Diesel Splitter Feed/ } \\
\text { Overhead }\end{array}$ & $\begin{array}{l}\text { 9.1 MM BTU/Hr, } \\
\text { 1640 FT2 w/1 shell, } \\
\text { TEMA type AEU }\end{array}$ & $\begin{array}{l}\text { Tubes: } 120 \text { PSIG, } 550^{\circ} \mathrm{F} \\
\text { Shell: } 75 \text { PSIG, } 610^{\circ} \mathrm{F} /- \\
20^{\circ} \mathrm{F}\end{array}$ & $\begin{array}{l}\text { Tubes: } 1 \text { 1/4 Cr-1/2 Mo } \\
\text { Shell: } 1 \text { 1/4 Cr-1/2 Mo }\end{array}$ \\
\hline$\overline{\mathrm{E}-3}$ & $\begin{array}{l}\text { Diesel Splitter Feed / } \\
\text { Bottoms }\end{array}$ & $\begin{array}{l}2.0 \mathrm{MM} \text { BTU/Hr, } \\
620 \mathrm{FT} 2 \text { w/1 shell, TEMA } \\
\text { type AEU }\end{array}$ & $\begin{array}{l}\text { Tubes: } 125 \text { PSIG, } 750^{\circ} \mathrm{F} \\
\text { Shell: } 120 \mathrm{PSIG}, 600^{\circ} \mathrm{F} /- \\
20^{\circ} \mathrm{F}\end{array}$ & $\begin{array}{l}\text { Tubes: } 1 \text { 1/4 } \mathrm{Cr}-1 / 2 \mathrm{Mo} \\
\text { Shell: } 1 \text { 1/4 Cr-1/2 Mo }\end{array}$ \\
\hline E-4 & $\begin{array}{l}\text { Diesel Splitter Ovhd } \\
\text { Condenser }\end{array}$ & $\begin{array}{l}11,2 \text { MM BTU/Hr } \\
603 \text { FT2 Bare Tube } \\
12800 \text { FT2 Extended } \\
\text { Shaft HP: } 15 \text { B HP } \\
\end{array}$ & Tubes: 75 PSIG, $610^{\circ} \mathrm{F}$ & $\begin{array}{l}\text { Seamless Carbon Steel tubes } \\
\text { with aluminum fins }\end{array}$ \\
\hline E-5 & $\begin{array}{l}\text { Diesel Splitter Bottoms } \\
\text { Cooler }\end{array}$ & $\begin{array}{l}\text { 3.5 MM BTU/Hr } \\
729 \text { FT2 Bare Tube } \\
\text { 15500 FT2 Extended } \\
\text { Shaft HP: } 15 \text { B HP } \\
\end{array}$ & Tubes: 125 PSIG, $450^{\circ} \mathrm{F}$ & $\begin{array}{l}\text { Seamless Carbon Steel tubes } \\
\text { with aluminum fins }\end{array}$ \\
\hline H-1 & Diesel Splitter Reboiler & $\begin{array}{l}\text { 17.8 MM BTU/Hr } \\
\text { Fired Heater }\end{array}$ & Tubes: 150 PSIG, $750^{\circ} \mathrm{F}$ & Tubes: $9 \mathrm{Cr}-1 \mathrm{Mo}$ w/ 0.1 " CA \\
\hline P-1A/B & Diesel Splitter Charge Pump & $\begin{array}{l}\text { Rated Capacity: } 206 \text { GPM } \\
\text { Diff. Press: 90 PSI } \\
\text { Head: } 257 \text { feet } \\
\text { Motor: } 20 \mathrm{HP} \\
\end{array}$ & & $\begin{array}{l}\text { Casing: killed C. S. w/0.125' CA } \\
\text { Impeller: } 12 \% \text { chrome }\end{array}$ \\
\hline$P-2 \mathrm{~A} / \mathrm{B}$ & $\begin{array}{l}\text { Diesel Splitter Bottoms } \\
\text { Pump }\end{array}$ & $\begin{array}{l}\text { Rated Capacity: } 1406 \\
\text { GPM } \\
\text { Diff. Press: 85 PSI } \\
\text { Head: } 305 \text { feet } \\
\text { Motor: } 125 \text { HP } \\
\end{array}$ & & $\begin{array}{l}\text { Casing: killed C. S. w/0.125' CA } \\
\text { Impeller: } 12 \% \text { chrome }\end{array}$ \\
\hline $\mathbf{P - 3 A / B}$ & Diesel Splitter Reflux Pump & $\begin{array}{l}\text { Rated Capacity: } 290 \text { GPM } \\
\text { Diff. Press: } 70 \text { PSI } \\
\text { Head: } 188 \text { feet } \\
\text { Motor: } 20 \mathrm{HP} \\
\end{array}$ & & $\begin{array}{l}\text { Casing: killed C. S. w/ } 0.125^{\prime} \mathrm{CA} \\
\text { Impeller: } 12 \% \text { chrome }\end{array}$ \\
\hline V-1 & Charge Drum & $\begin{array}{l}7^{\prime}-0^{\prime} \text { ID X } 15^{\prime}-0^{\prime \prime} \mathrm{T} / \mathrm{T} \\
\text { (Horizontal) }\end{array}$ & 50 PSIG/FV $275 /-20^{\circ} \mathrm{F}$ & Killed Carbon Steel w/0.2' CA \\
\hline V-2 & Diesel Splitter Tower & $\begin{array}{l}7^{\prime}-6 " \text { ID X 82'-0" T/T } \\
\text { w/ } 30 \text { valve trays }\end{array}$ & $\begin{array}{l}75 \mathrm{PSIG} / \mathrm{FV} \quad 610^{\circ} \mathrm{F} \\
\text { (top) and } 750^{\circ} \mathrm{F} \\
\text { (bottom) }\end{array}$ & $\begin{array}{l}\text { Killed Carbon Steel } w / 0.2 \text { "' CA } \\
\text { and Type } 410 \text { SS trays, supports, } \\
\text { and downcomers }\end{array}$ \\
\hline V-3 & $\begin{array}{l}\text { Diesel Splitter Overhead } \\
\text { Accumulator }\end{array}$ & $\begin{array}{l}\text { 6'-0' ID X 12'-0" } \mathrm{T} / \mathrm{T}^{\prime} \\
\text { (Horizontal) }\end{array}$ & $75 \mathrm{PSIG} / \mathrm{FV} \quad 610 /-20^{\circ} \mathrm{F}$ & Killed Carbon Steel w/ 0.2' CA \\
\hline
\end{tabular}




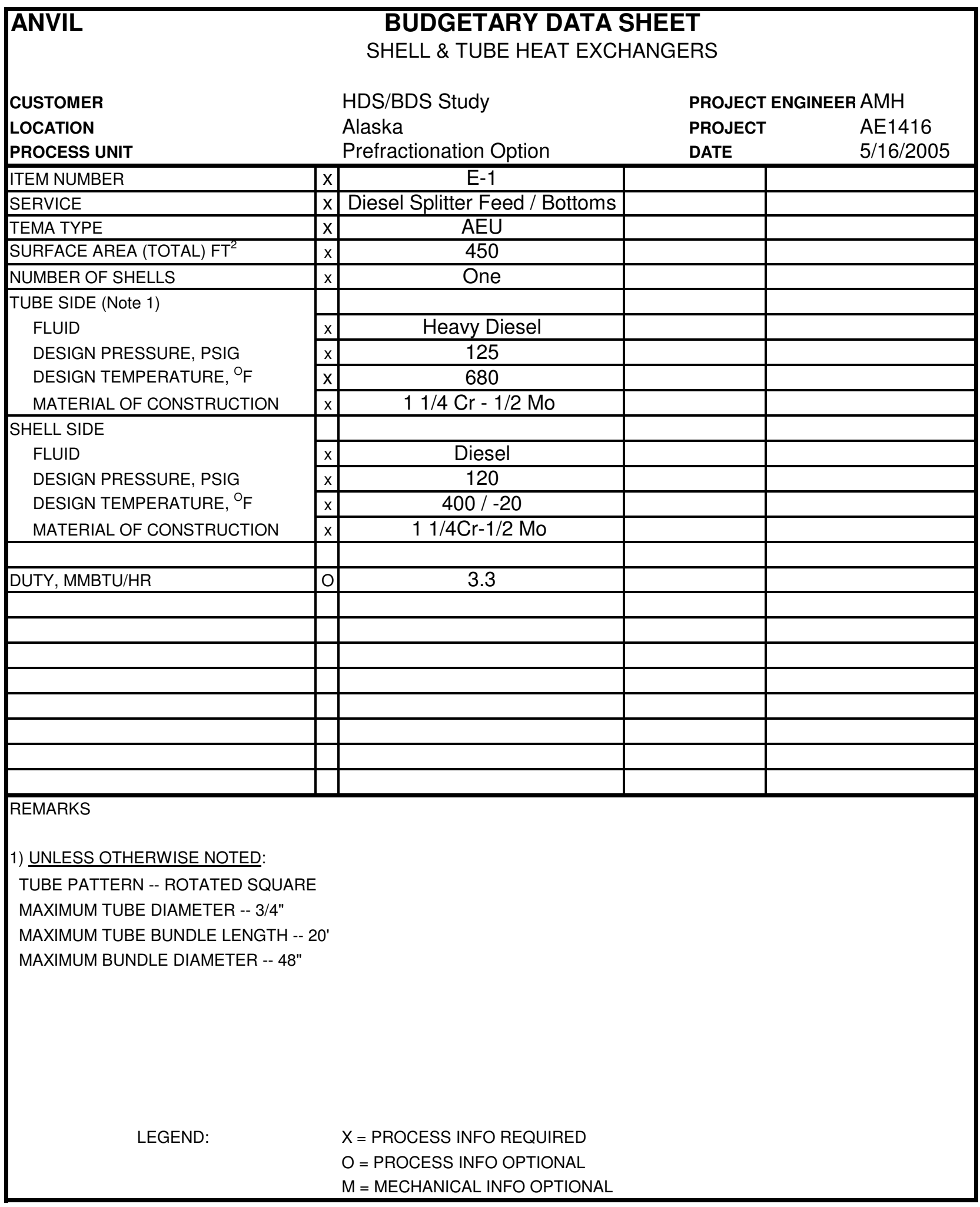

S:IPetroStar\AE1416.AUXIsubjob 45\Final Pre_Frac Case Diesel Splitterl[Budgetary Data Sheets.xIs]P-3 AB 


\begin{tabular}{|c|c|c|c|c|}
\hline \multirow[t]{2}{*}{ ANVIL } & \multicolumn{3}{|c|}{$\begin{array}{l}\text { BUDGETARY DATA SHEET } \\
\text { SHELL \& TUBE HEAT EXCHANGERS }\end{array}$} & \\
\hline & \multirow{3}{*}{\multicolumn{2}{|c|}{$\begin{array}{l}\text { HDS/BDS Study } \\
\text { Alaska } \\
\text { Prefractionation Option }\end{array}$}} & PROJECT ENGINEER AMH & \\
\hline LOCATION & & & PROJECT & AE1416 \\
\hline PROCESS UNIT & & & DATE & $5 / 16 / 2005$ \\
\hline ITEM NUMBER & $\mathrm{x}$ & $\mathrm{E}-2$ & & \\
\hline SERVICE & $\bar{x}$ & Diesel Splitter Feed / Overhead & & \\
\hline TEMA TYPE & $\bar{x}$ & AEU & & \\
\hline SURFACE AREA (TOTAL) FT ${ }^{2}$ & $\mathrm{x}$ & 1640 & & \\
\hline NUMBER OF SHELLS & $\mathrm{x}$ & One & & \\
\hline \multicolumn{5}{|l|}{ TUBE SIDE (Note 1) } \\
\hline \multirow{4}{*}{$\begin{array}{l}\text { FLUID } \\
\text { DESIGN PRESSURE, PSIG } \\
\text { DESIGN TEMPERATURE, }{ }^{\circ} \mathrm{F} \\
\text { MATERIAL OF CONSTRUCTION }\end{array}$} & $\mathrm{x}$ & Diesel Feed & & \\
\hline & $x$ & 120 & & \\
\hline & $\mathrm{x}$ & 550 & & \\
\hline & $\mathrm{x}$ & $11 / 4 \mathrm{Cr}-1 / 2 \mathrm{Mo}$ & & \\
\hline \multicolumn{5}{|l|}{ SHELL SIDE } \\
\hline \multirow{4}{*}{$\begin{array}{l}\text { FLUID } \\
\text { DESIGN PRESSURE, PSIG } \\
\text { DESIGN TEMPERATURE, }{ }^{\circ} \mathrm{F} \\
\text { MATERIAL OF CONSTRUCTION }\end{array}$} & $\mathrm{x}$ & Light Diesel & & \\
\hline & $\mathrm{x}$ & 75 & & \\
\hline & $\mathrm{x}$ & $610 /-20$ & & \\
\hline & $\mathrm{x}$ & $11 / 4 \mathrm{Cr}-1 / 2 \mathrm{Mo}$ & & \\
\hline \multirow{3}{*}{ DUTY, MMBTU/HR } & 0 & 91 & & \\
\hline & & & & \\
\hline & & & & \\
\hline \multirow{2}{*}{\multicolumn{5}{|c|}{ 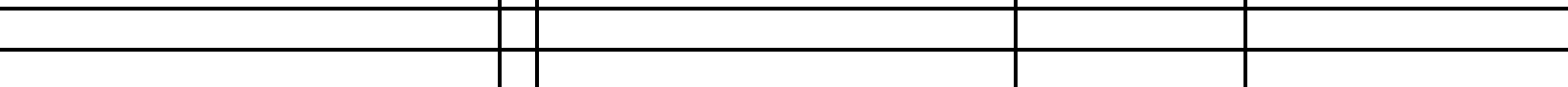 }} \\
\hline & & & & \\
\hline \multicolumn{5}{|c|}{\begin{tabular}{l|l|l} 
& & \\
\end{tabular}} \\
\hline \multicolumn{2}{|c|}{\begin{tabular}{c|c}
+2 &
\end{tabular}} & & & \\
\hline \multicolumn{5}{|l|}{ REMARKS } \\
\hline \multirow{5}{*}{\multicolumn{5}{|c|}{$\begin{array}{l}\text { 1) UNLESS OTHERWISE NOTED: } \\
\text { TUBE PATTERN -- ROTATED SQUARE } \\
\text { MAXIMUM TUBE DIAMETER -- 3/4" } \\
\text { MAXIMUM TUBE BUNDLE LENGTH -- 20' } \\
\text { MAXIMUM BUNDLE DIAMETER -- 48" }\end{array}$}} \\
\hline & & & & \\
\hline & & & & \\
\hline & & & & \\
\hline & & & & \\
\hline LEGEND: & & $X=$ PROCESS INFO REQUIRED & & \\
\hline & & O = PROCESS INFO OPTIONAL & & \\
\hline & & $M=$ MECHANICAL INFO OPTIONAL & & \\
\hline
\end{tabular}

S:IPetroStar\AE1416.AUX|subjob 45\Final Pre_Frac Case Diesel Splitterl[Budgetary Data Sheets.xIs]P-3 AB 


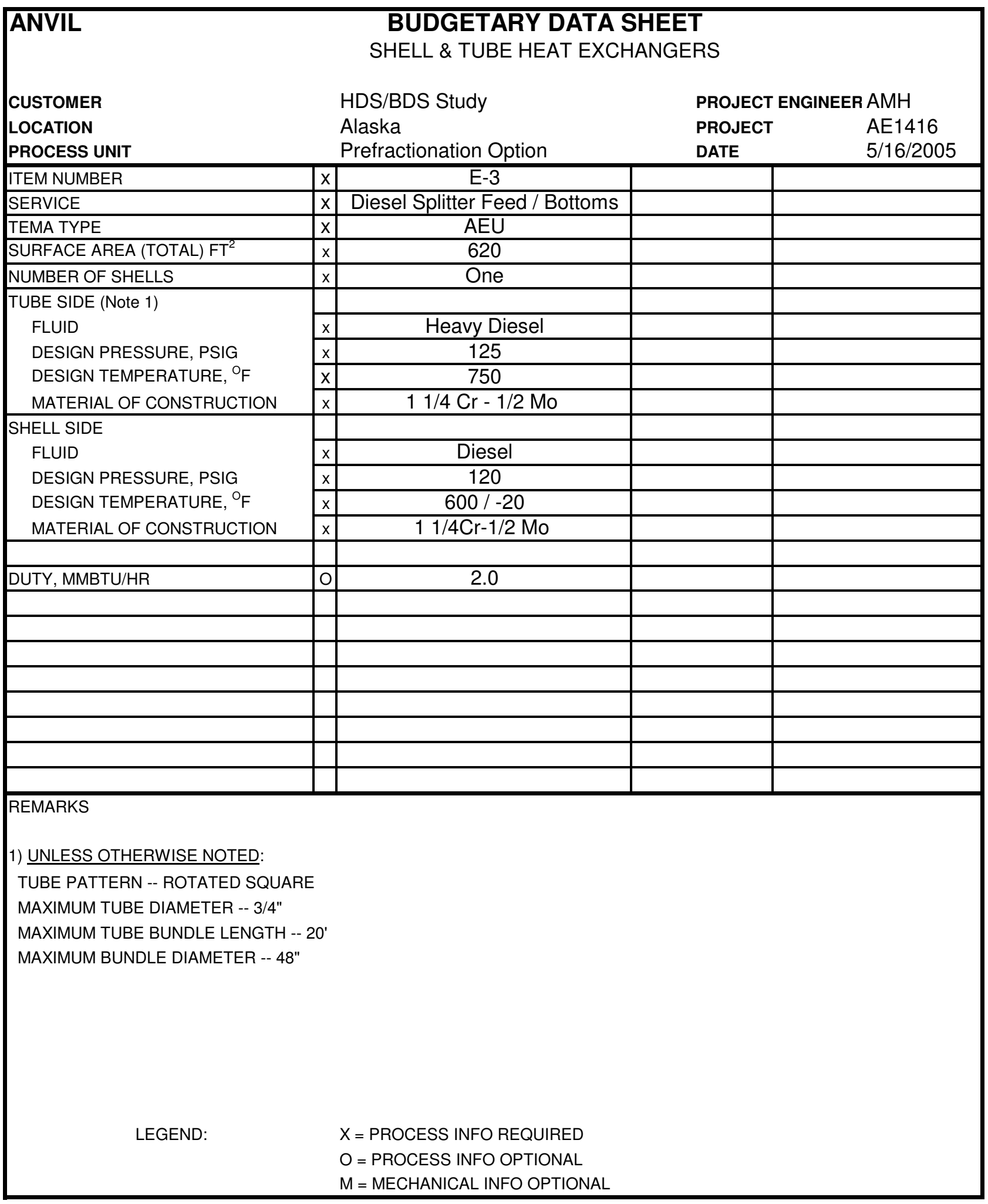

S:IPetroStar\AE1416.AUXIsubjob 45\Final Pre_Frac Case Diesel Splitterl[Budgetary Data Sheets.xIs]P-3 AB 


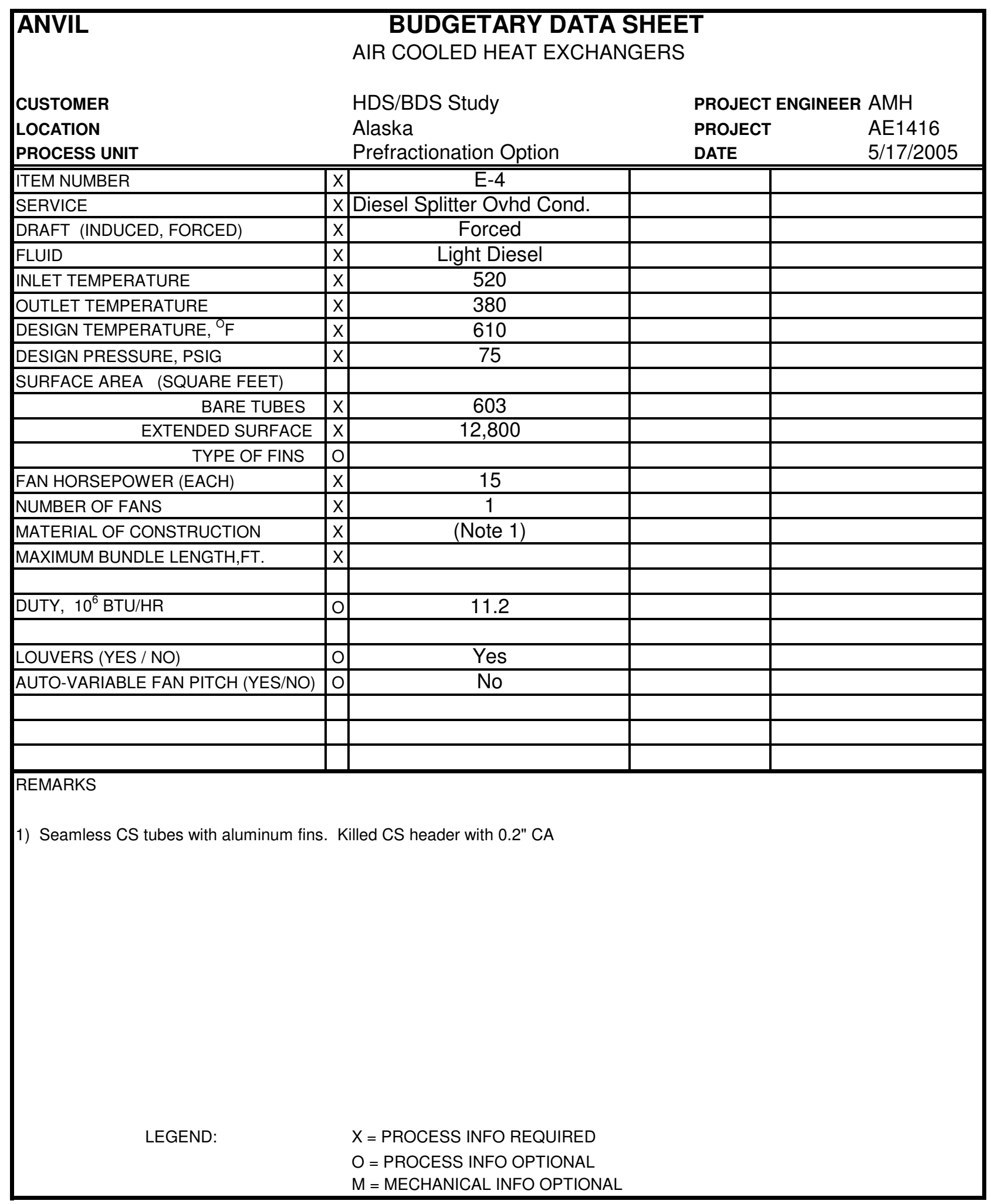

S:IPetroStar\AE1416.AUX|subjob 45\Final Pre_Frac Case Diesel Splitter|[Budgetary Data Sheets.xls]E-4 


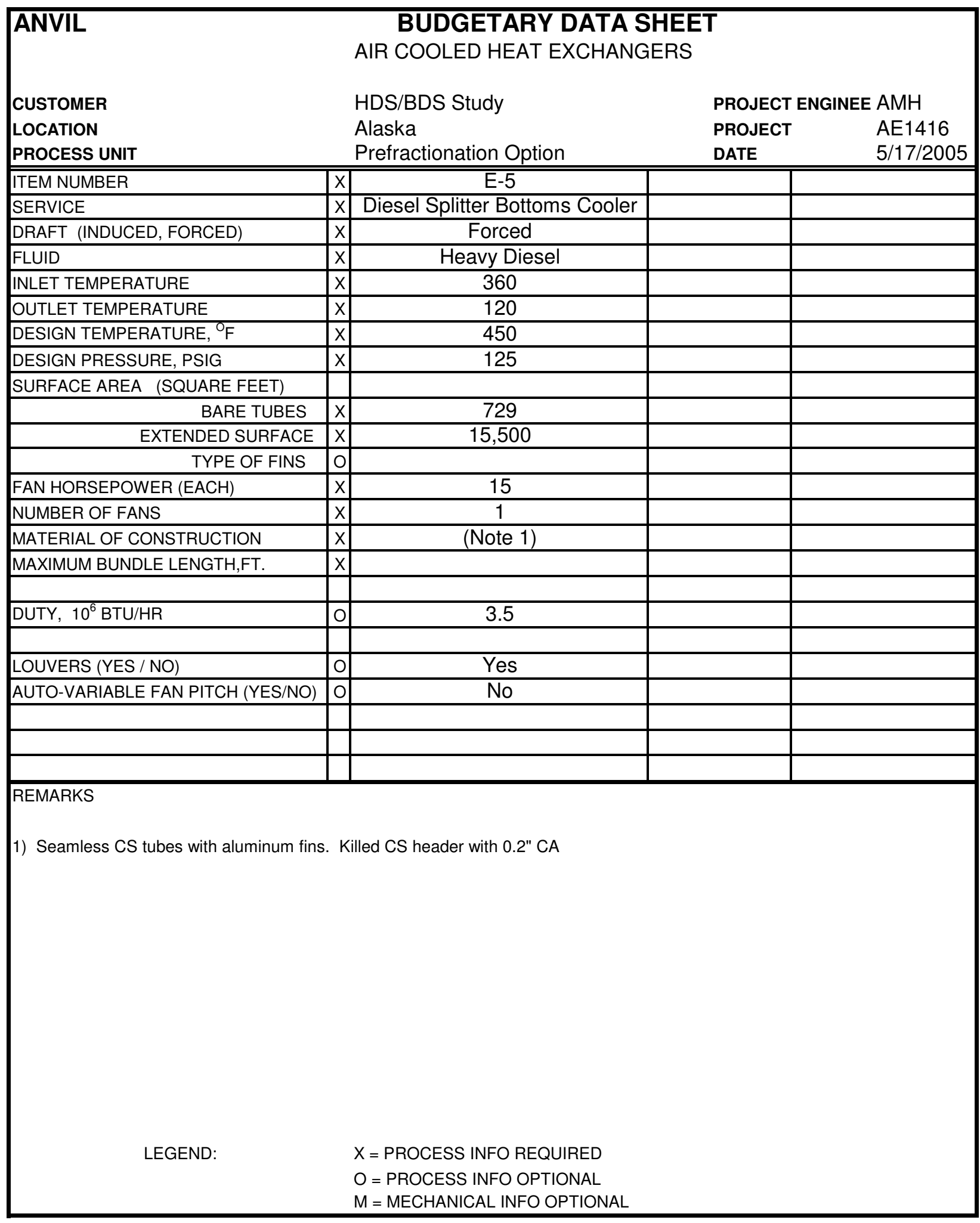

S:IPetroStar\AE1416.AUX|subjob 45\Final Pre_Frac Case Diesel Splitter|[Budgetary Data Sheets.xls]E-5 


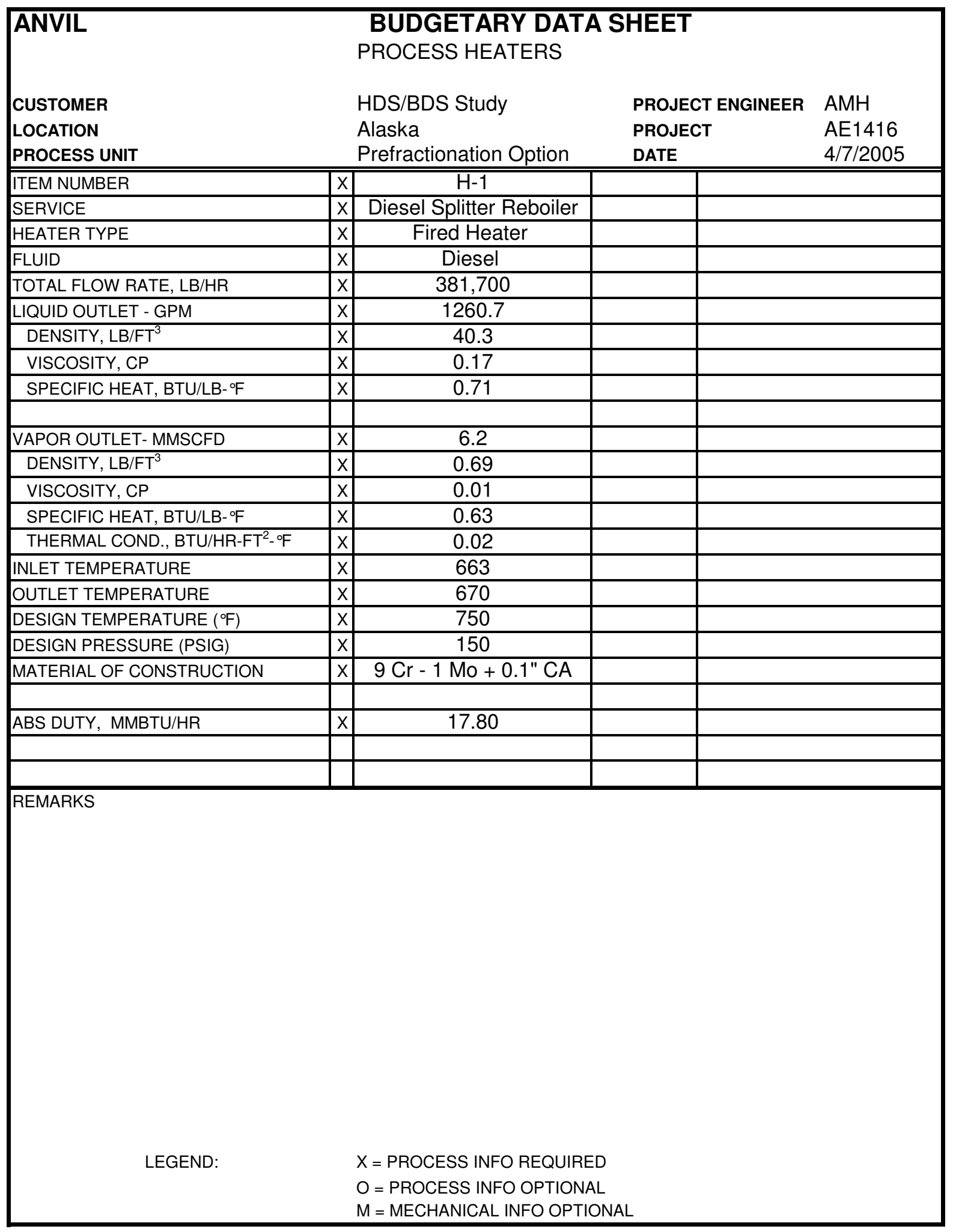




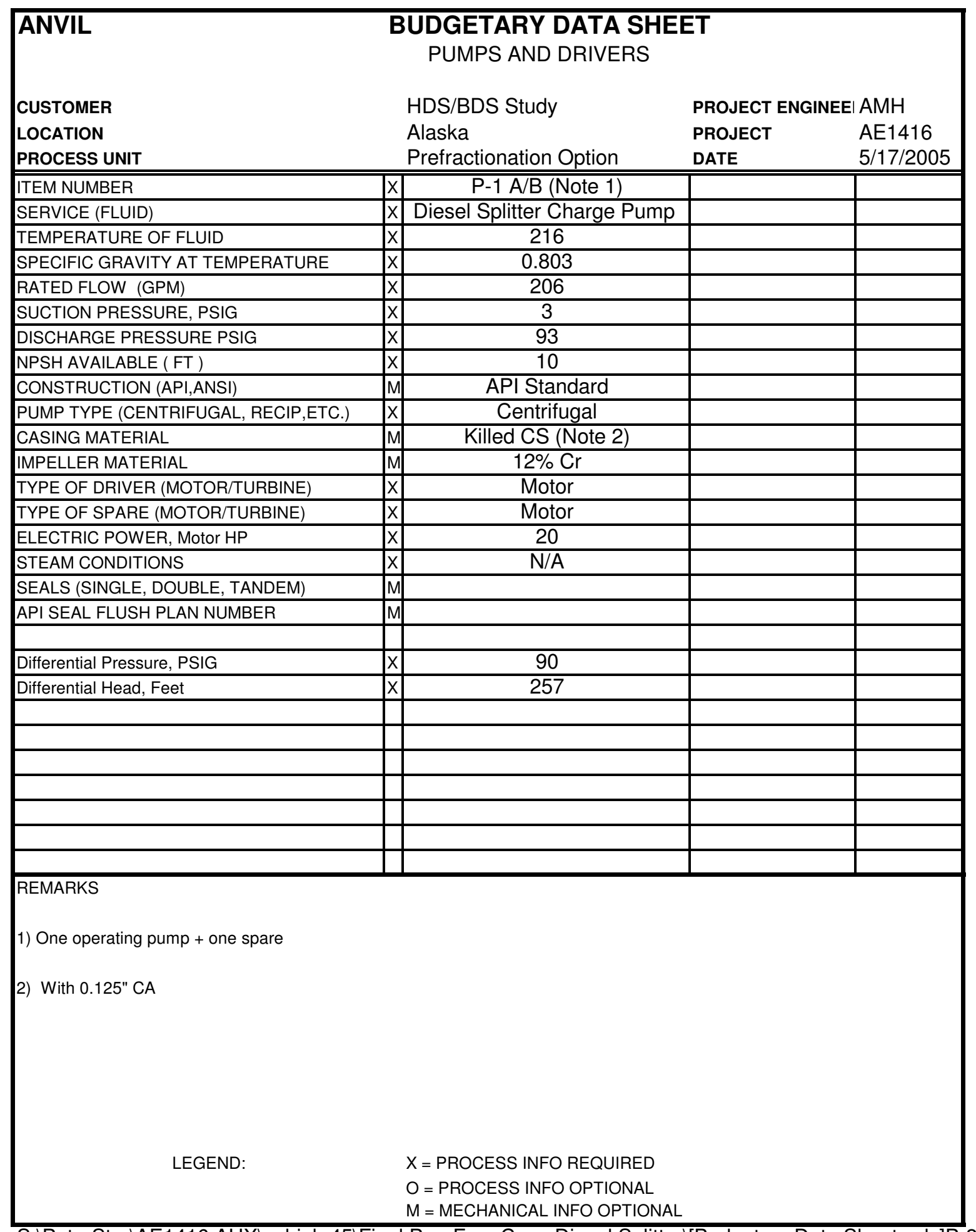

S:IPetroStar|AE1416.AUXIsubjob 45IFinal Pre_Frac Case Diesel Splitter|[Budgetary Data Sheets.XIs]P-3 AB 


\begin{tabular}{|c|c|c|c|c|}
\hline ANVIL & & $\begin{array}{l}\text { UDGETARY DATA SHEET } \\
\text { PUMPS AND DRIVERS }\end{array}$ & & \\
\hline CUSTOMER & & HDS/BDS Study & PROJECT ENGINEER & $\mathrm{AMH}$ \\
\hline LOCATION & & Alaska & PROJECT & $\mathrm{AE} 1416$ \\
\hline PROCESS UNIT & & Prefractionation Option & DATE & $5 / 17 / 2005$ \\
\hline ITEM NUMBER & $\overline{\mathrm{T} x}$ & P-2 A/B (Note 1) & & \\
\hline SERVICE (FLUID) & $x$ & Diesel Splitter Btms Pump & & \\
\hline TEMPERATURE OF FLUID & $x$ & 660 & & \\
\hline SPECIFIC GRAVITY AT TEMPERATURE & $x$ & 0.642 & & \\
\hline RATED FLOW (GPM) & $x$ & 1406 & & \\
\hline SUCTION PRESSURE, PSIG & $x$ & 12 & & \\
\hline DISCHARGE PRESSURE PSIG & $x$ & 97 & & \\
\hline NPSH AVAILABLE ( FT) & $x$ & 11 & & \\
\hline CONSTRUCTION (API,ANSI) & $\mathrm{M}$ & API Standard & & \\
\hline PUMP TYPE (CENTRIFUGAL, RECIP,ETC.) & $x$ & Centrifugal & & \\
\hline CASING MATERIAL & $M$ & Killed CS (Note 2) & & \\
\hline IMPELLER MATERIAL & $\mathrm{M}$ & $12 \% \mathrm{Cr}$ & & \\
\hline TYPE OF DRIVER (MOTOR/TURBINE) & $x$ & Motor & & \\
\hline TYPE OF SPARE (MOTOR/TURBINE) & $x$ & Motor & & \\
\hline ELECTRIC POWER, Motor HP & $x$ & 125 & & \\
\hline STEAM CONDITIONS & $x$ & $\mathrm{~N} / \mathrm{A}$ & & \\
\hline SEALS (SINGLE, DOUBLE, TANDEM) & $M$ & & & \\
\hline \multicolumn{5}{|l|}{ API SEAL FLUSH PLAN NUMBER } \\
\hline & & & & \\
\hline Differential Pressure, PSIG & $x$ & 85 & & \\
\hline \multirow[t]{3}{*}{ onterental meda, reet } & $x$ & 305 & & \\
\hline & & & & \\
\hline & & & & \\
\hline & & & & \\
\hline & & & & \\
\hline & & & & \\
\hline & & & & \\
\hline \multirow{3}{*}{\multicolumn{5}{|c|}{$\begin{array}{l}\text { REMARKS } \\
\text { 1) One operating pump + one spare } \\
\text { 2) With } 0.125 \text { " CA }\end{array}$}} \\
\hline & & & & \\
\hline & & & & \\
\hline LEGEND: & & $X=$ PROCESS INFO REQUIRED & & \\
\hline & & $\begin{array}{l}\mathrm{O}=\text { PROCESS INFO OPTIONAL } \\
\mathrm{M}=\text { MECHANICAL INFO OPTIONAL }\end{array}$ & & \\
\hline
\end{tabular}

S:IPetroStar|AE1416.AUXIsubjob 45IFinal Pre_Frac Case Diesel Splitter[[Budgetary Data Sheets.XIs]P-3 AB 


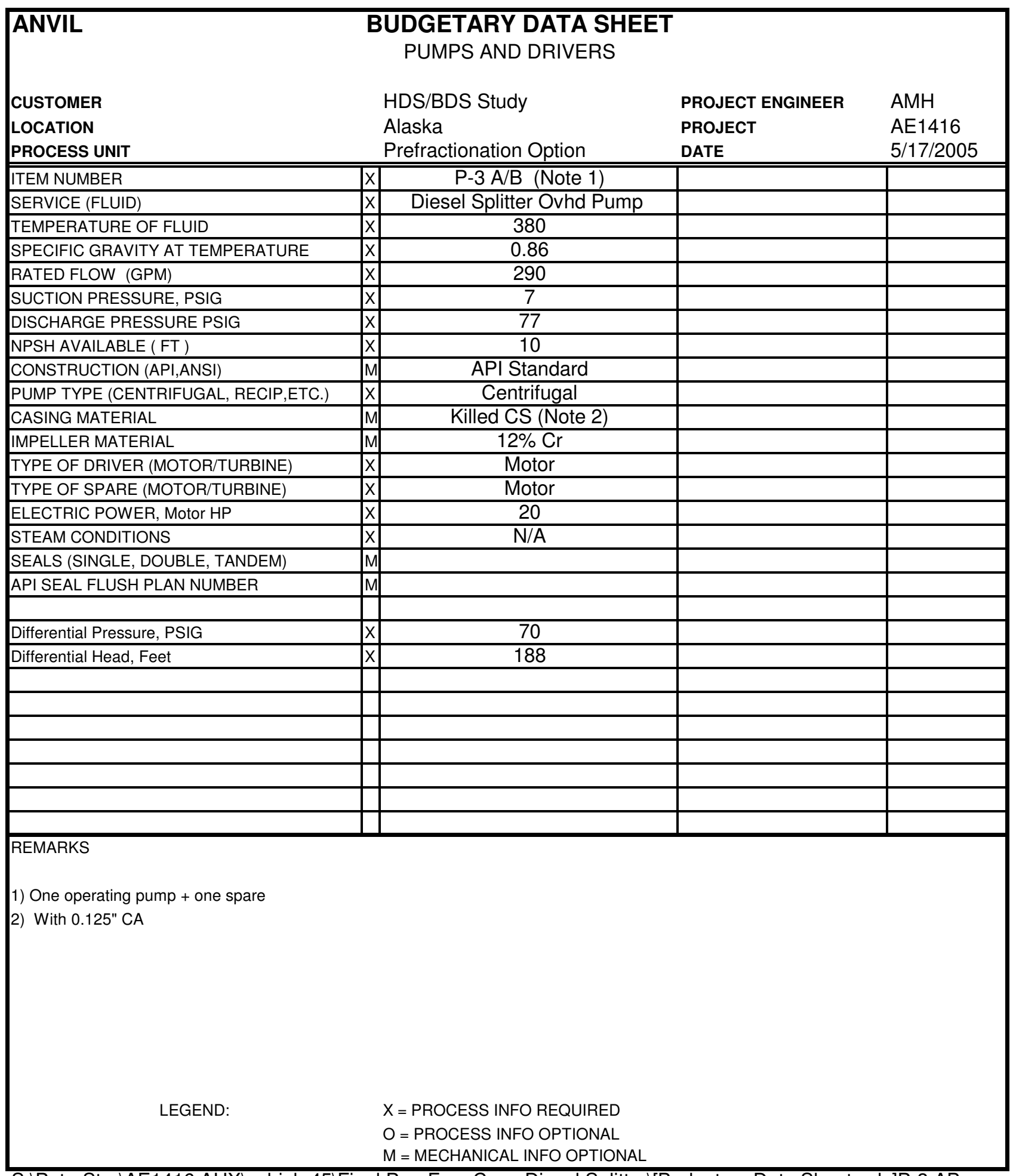

S:IPetroStar\AE1416.AUXIsubjob 45IFinal Pre_Frac Case Diesel Splitter|[Budgetary Data Sheets.xls]P-3 AB 


\begin{tabular}{|c|c|c|c|}
\hline \multicolumn{4}{|c|}{\begin{tabular}{|c} 
ANVIL \\
PRESSURE VESSELS-ASME SECTION VIII
\end{tabular}} \\
\hline $\begin{array}{l}\text { CUSTOMER } \\
\text { LOCATION } \\
\text { PROCESS UNIT } \\
\end{array}$ & $\begin{array}{l}\text { HDS/BDS Study } \\
\text { Alaska } \\
\text { Prefractionation Option }\end{array}$ & $\begin{array}{l}\text { PROJECT ENGINEER } \\
\text { PROJECT NO. } \\
\text { DATE }\end{array}$ & $\begin{array}{l}\text { AMH } \\
\text { AE1416 } \\
5 / 17 / 2005\end{array}$ \\
\hline ITEM NUMBER & $\overline{\mathrm{V}-1}$ & & \\
\hline SERVICE & Charge Drum & & \\
\hline FLUID & Diesel Feed & & \\
\hline \multicolumn{4}{|l|}{ ASME SECT VIII DIV 1 OR 2} \\
\hline POSITION ; HORIZONTAL, VERTICAL & Horizontal & & \\
\hline \begin{tabular}{|l|} 
DIAMETER, FT-IN \\
\end{tabular} & 7'-0" & & \\
\hline TANGENT TO TANGENT LENGTH, FT. & $15^{\prime}-0^{\prime \prime}$ & & \\
\hline SKIRT HEIGHT (FT-IN) & (Note 2) & & \\
\hline DESIGN TEMPERATURE ( $\left.{ }^{\circ} \mathrm{F}\right)$ & $275 /-20$ & & \\
\hline DESIGN PRESSURE (PSIG) & 50 (Note 1) & & \\
\hline MATERIAL OF CONSTRUCTION & (Note 3) & & \\
\hline INSULATION (YES/NO) & Yes & & \\
\hline TRAY OR PACKING TYPE & None & & \\
\hline NUMBER OF TRAYS & None & & \\
\hline TRAY MATERIAL & $\mathrm{N} / \mathrm{A}$ & & \\
\hline PACKING VOLUME, $\mathrm{FT}^{3}$ & $\mathrm{~N} / \mathrm{A}$ & & \\
\hline PACKING MATERIAL & $\mathrm{N} / \mathrm{A}$ & & \\
\hline INTERNALS & Vortex Breaker & & \\
\hline LINING & $\mathrm{N} / \mathrm{A}$ & & \\
\hline \multicolumn{4}{|l|}{ PLATFORMS AND LADDERS } \\
\hline $\mathrm{BOOT}(\mathrm{YES} / \mathrm{NO})$ & No & & \\
\hline \multicolumn{4}{|l|}{ 政) } \\
\hline & & & \\
\hline & & & \\
\hline & & & \\
\hline & & & \\
\hline & & & \\
\hline \multicolumn{4}{|c|}{$\begin{array}{l}\text { REMARKS } \\
\text { 1) Vessels will be designed for Full Vacuum unless otherwise specified. } \\
\text { 2) The horizontal vessel will be supported on saddles } 10 \text { feet above grade. } \\
\text { 3) Vessel to be killed CS + 0.2" CA. }\end{array}$} \\
\hline LEGEND: & $\begin{array}{l}X=\text { PROCESS INFO REQUIF } \\
O=\text { PROCESS INFO OPTIOI } \\
M=\text { MECHANICAL INFO OP }\end{array}$ & $\begin{array}{l}\text { RED } \\
\text { NAL } \\
\text { TIONAL }\end{array}$ & \\
\hline
\end{tabular}

S:IPetroStarlAE1416.AUX|subjob 45|Final Pre_Frac Case Diesel Splitter|[Budgetary Data Sheets.xls]P-3 AB 


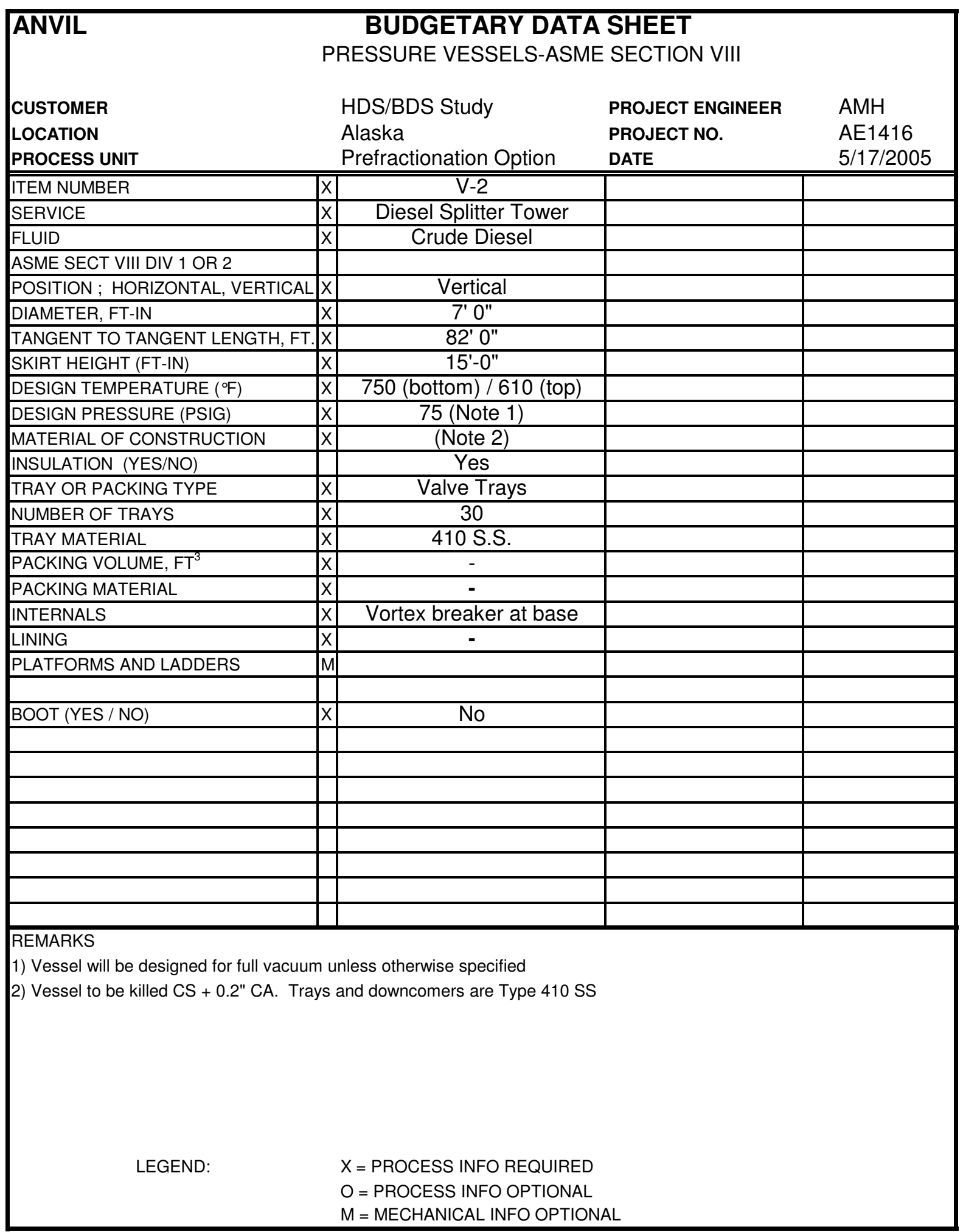

S:IPetroStar\AE1416.AUX|subjob 45\Final Pre_Frac Case Diesel Splitter|[Budgetary Data Sheets.xls]P-3 AB 


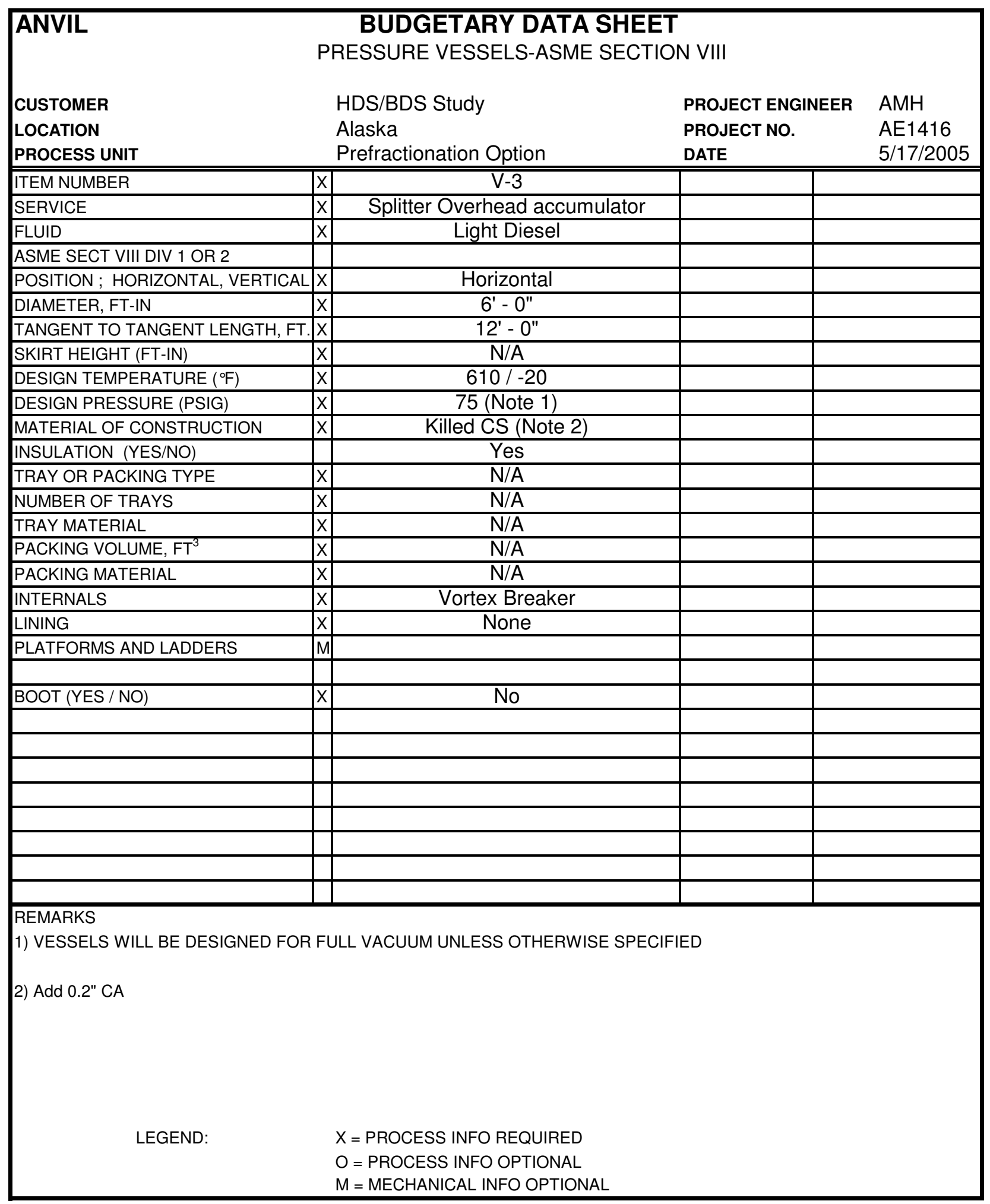

S:IPetroStar|AE1416.AUX|subjob 45|Final Pre_Frac Case Diesel Splitter|[Budgetary Data Sheets.xls]P-3 AB 


\section{Appendix D.3 - Sulfur Speciation in Diesel Splitter Products}

\begin{tabular}{|c|c|c|c|}
\hline \multicolumn{4}{|c|}{$\begin{array}{l}\text { Light Diesel Sulfur Speciation (Feed to HDS) } \\
\text { (Ratioed to 5,000 ppm*) }\end{array}$} \\
\hline Component & ppm wt sulfur & Component & ppm wt sulfur \\
\hline Hydrogen sulfide & $<1$ & 2-Ethyl thiophene & $<1$ \\
\hline Carbonyl sulfide & $<1$ & 2,5-Dimethyl thiophene & $<1$ \\
\hline Methyl mercaptan & $<1$ & 3-Ethyl thiophene & $<1$ \\
\hline Ethyl mercaptan & $<1$ & 2,4\&2,-Dimethyl thiophene & $<1$ \\
\hline Dimethyl sulfide & $<1$ & 3,4-Dimethyl thiophene & $<1$ \\
\hline Carbon disulfide & $<1$ & Methyl Ethyl thiophenes & $<1$ \\
\hline Isopropyl mercaptan & $<1$ & Trimethyl thiophenes & $<1$ \\
\hline Ethyl sulfide & $<1$ & Tetramethyl thiophenes & $<1$ \\
\hline tert-Butyl mercaptan & $<1$ & Benzothiophene & $<1$ \\
\hline N-Propyl mercaptan & $<1$ & Methyl benzothiophene & $<1$ \\
\hline Ethyl Methyl Sulfide & $<1$ & Dimethyl benzothiophene & 46 \\
\hline Thiophene & $<1$ & Trimethyl benzothiophene & 187 \\
\hline sec-Butyl Mercaptan & $<1$ & Tetramethyl Benzothiophene & 558 \\
\hline Isobutyl mercaptan & $<1$ & Dibenzothiophene & 162 \\
\hline Ethyl sulfide & $<1$ & 4-Methyl benzothiophene & 4 \\
\hline MN-butyl mercaptan & $<1$ & 3-Methyl DBZT+2-methyl DBZT & $<1$ \\
\hline Dimethyl disulfide & $<1$ & 1-Methyl dibenzothiophene & $<1$ \\
\hline 2-Methyl thiophene & $<1$ & 4,6 Dimethyl dibenzothiophene & $<1$ \\
\hline 3-Methyl thiophene & $<1$ & Dimethyl dibenzothiophene & $<1$ \\
\hline Tetra-hydro thiophene & $<1$ & Trimethyl dibenzothiophene & $<1$ \\
\hline Ethyl methyl disulfide & $<1$ & Tetramethyl dibenzothiophene & $<1$ \\
\hline 2-Methyl-tetra-hydro-thiophene & $<1$ & Unidentified volatile sulfur & 3,283 \\
\hline
\end{tabular}




\begin{tabular}{|l|c|l|c|}
\hline \multicolumn{4}{|c|}{$\begin{array}{c}\text { Heavy Diesel Sulfur Speciation (Feed to BDS) } \\
\text { (Ratioed to 5,000 ppm*) }\end{array}$} \\
\hline Component & ppm wt sulfur & Component & ppm wt sulfur \\
\hline Hydrogen sulfide & $<1$ & 2-Ethyl thiophene & $<1$ \\
\hline Carbonyl sulfide & $<1$ & 2,5-Dimethyl thiophene & $<1$ \\
\hline Methyl mercaptan & $<1$ & 3-Ethyl thiophene & $<1$ \\
\hline Ethyl mercaptan & $<1$ & 2,4\&2,-Dimethyl thiophene & $<1$ \\
\hline Dimethyl sulfide & $<1$ & 3,4-Dimethyl thiophene & $<1$ \\
\hline Carbon disulfide & $<1$ & Methyl Ethyl thiophenes & $<1$ \\
\hline Isopropyl mercaptan & $<1$ & Trimethyl thiophenes & $<1$ \\
\hline Ethyl sulfide & $<1$ & Tetramethyl thiophenes & $<1$ \\
\hline tert-Butyl mercaptan & $<1$ & Benzothiophene & $<1$ \\
\hline N-Propyl mercaptan & $<1$ & Methyl benzothiophene & $<1$ \\
\hline Ethyl Methyl Sulfide & $<1$ & Dimethyl benzothiophene & $<1$ \\
\hline Thiophene & $<1$ & Trimethyl benzothiophene & $<1$ \\
\hline sec-Butyl Mercaptan & $<1$ & Tetramethyl Benzothiophene & 344 \\
\hline Isobutyl mercaptan & $<1$ & Dibenzothiophene & 576 \\
\hline Ethyl sulfide & $<1$ & 4-Methyl benzothiophene & 401 \\
\hline MN-butyl mercaptan & $<1$ & 3-Methyl DBZT+2-methyl DBZT & 258 \\
\hline Dimethyl disulfide & $<1$ & 1-Methyl dibenzothiophene & 265 \\
\hline 2-Methyl thiophene & $<1$ & 4,6 Dimethyl dibenzothiophene & 268 \\
\hline 3-Methyl thiophene & $<1$ & Dimethyl dibenzothiophene & 968 \\
\hline Tetra-hydro thiophene & $<1$ & Trimethyl dibenzothiophene & 205 \\
\hline Ethyl methyl disulfide & $<1$ & Tetramethyl dibenzothiophene & 10 \\
\hline 2-Methyl-tetra-hydro-thiophene & $<1$ & Unidentified volatile sulfur & 3,283 \\
\hline
\end{tabular}


Appendix D.4 - HDS Unit Process Flow Diagrams and Material Balance 
Final Technical Progress Report

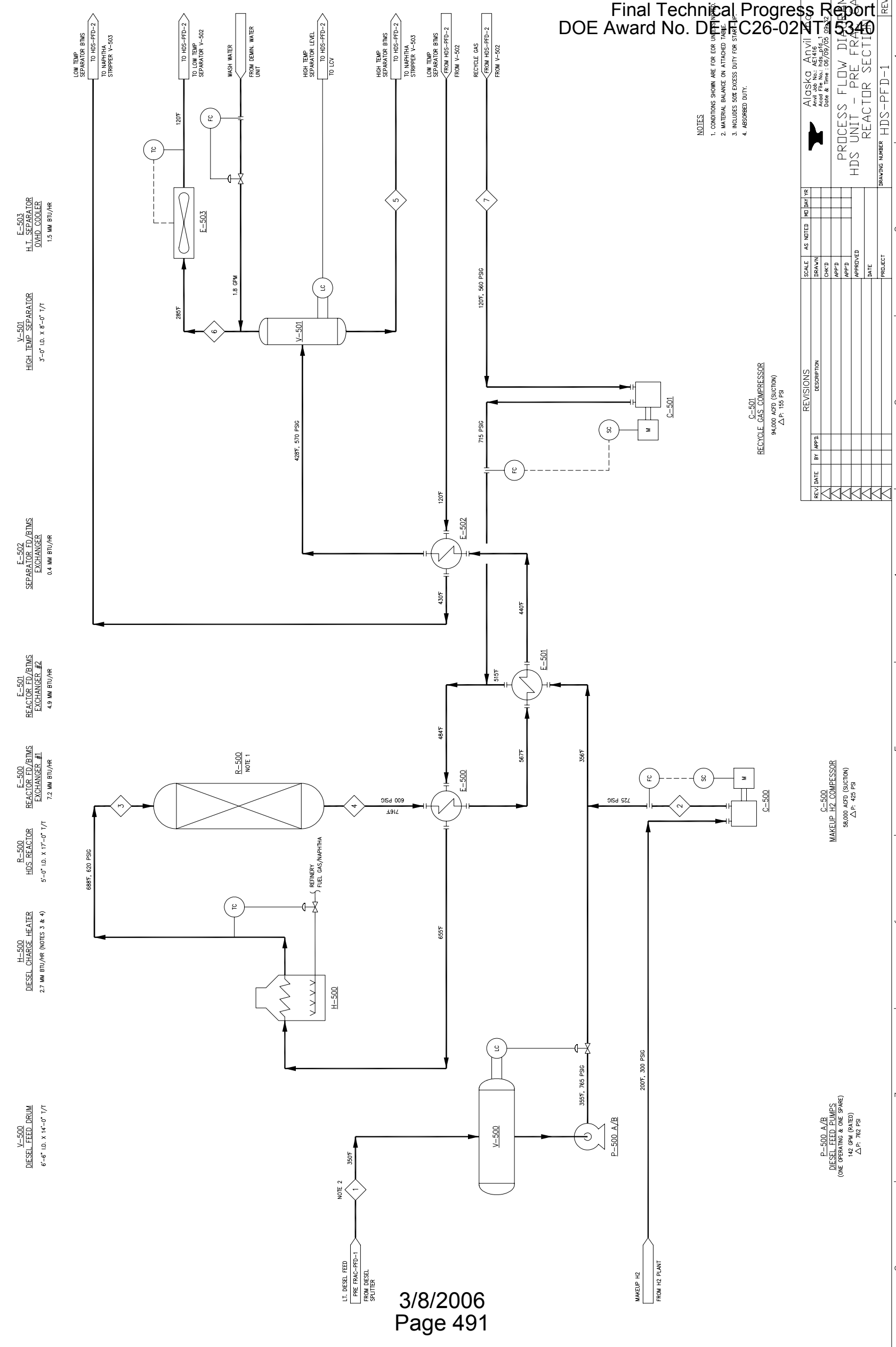


Final Technical Progress Report

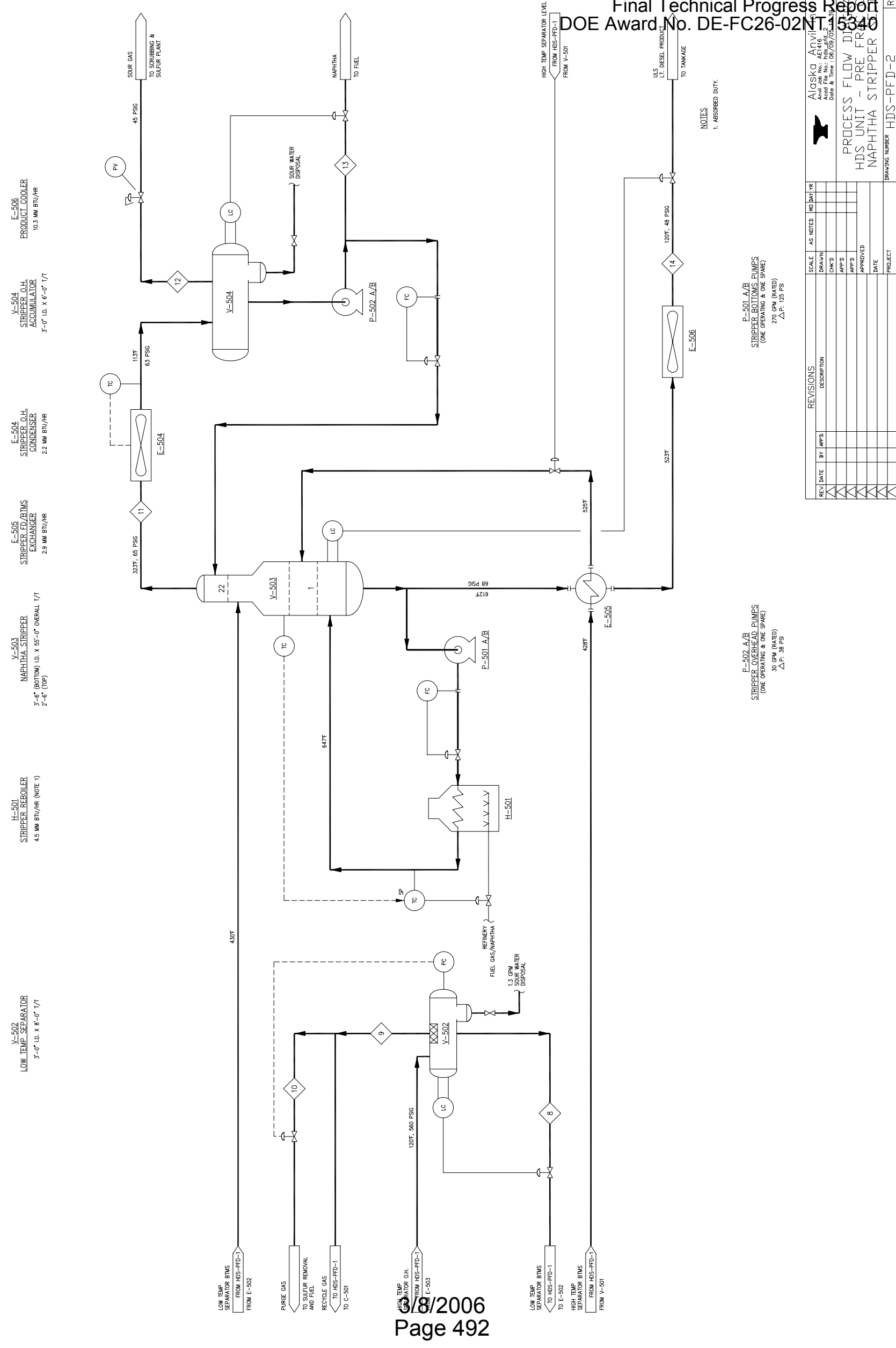




\section{Material Balance}

\section{HDS Unit EOR Material Balance -- Pre Frac Case}

\begin{tabular}{|c|c|c|c|c|c|c|c|c|}
\hline Stream Number & & 1 & 2 & 3 & 4 & 5 & 6 & 7 \\
\hline Stream Description & & Lt. Diesel Feed & Make-ıp $\mathrm{H}_{2}$ & Reactor Feed & Feactar Outlet & V-501 Liquid & $\begin{array}{l}\text { V-50t Vapor + } \\
\text { Wash Water }\end{array}$ & Recycle Gas \\
\hline Phase & & L[QUID & VAPOF & MIXED & MLXED & LIQUID & MIXED & VAPOF \\
\hline Mass Flaw Rate & LB/HA & 48196 & 216 & 50320 & 50321 & 46071 & 5140 & 1908 \\
\hline Temperature & $F$ & 350 & 442 & 608 & 716 & 428 & 295 & 120 \\
\hline Pressure & $\mathrm{PSIG}$ & 3 & 725 & 620 & 600 & 570 & 570 & 560 \\
\hline Standard Lig Flaw & $\mathrm{BPO}$ & 3870 & $\mathrm{n} / \mathrm{a}$ & 2066 & 1131 & 3753 & 129 & $\mathrm{nda}$ \\
\hline Vapor Flaw & MSCFH & $\mathrm{nla}$ & 39.8 & 214.9 & 217.6 & $\mathrm{n} / \mathrm{a}$ & 157.2 & 135.1 \\
\hline Wt \% Vapor & & 0 & 100 & 49 & 72 & 0 & 69 & 100 \\
\hline MW & & 197.9 & 2.1 & 71.4 & 77.3 & 174.3 & 11.8 & 5.4 \\
\hline
\end{tabular}

\begin{tabular}{|c|c|c|c|c|c|c|c|c|}
\hline Stream Number & & 8 & 9 & 10 & 11 & 12 & 13 & 14 \\
\hline Stream Description & & $\begin{array}{c}\text { V-502 HC } \\
\text { Liquid }\end{array}$ & $\mathrm{V}-502 \mathrm{Vapor}$ & Purge Gas & $\begin{array}{l}\text { Column Vepor } \\
\text { to Condenser }\end{array}$ & Sour Gas & Nephtha & Lt. Diesel \\
\hline Phase & & L[GUID & VAPQP & VAPQR & VAPOP & VAPOP & LIOLID & L[GUID \\
\hline Mass Flow Rate & LB/HA & 2285 & 1971 & 62 & 9710 & 379 & 2127 & 45843 \\
\hline Temperature & $F$ & 120 & 120 & 120 & 323 & 113 & 113 & 120 \\
\hline Pressure & $\mathrm{PS}[\mathrm{G}$ & 560 & 560 & 560 & 65 & 63 & 104 & 48 \\
\hline Standard Liq Flaw & $\mathrm{BPD}$ & 136 & $\mathrm{n} / \mathrm{a}$ & $r / \mathrm{a}$ & $\mathrm{n} / \mathrm{a}$ & $n / a$ & 190 & 3699 \\
\hline Vapor Flaw & MSCFH & nia & 139.5 & 4.4 & 47.9 & 7.9 & $\mathrm{n} / \mathrm{a}$ & $\mathrm{nla}$ \\
\hline Wt \% Vepor & & 0 & 100 & 100 & 100 & 100 & 0 & 0 \\
\hline MW & & 115.5 & 5.4 & 5.4 & 770 & 18.2 & 89.6 & 191.7 \\
\hline
\end{tabular}




\section{Appendix D.5 - HDS Unit Equipment List and Budgetary Equipment Datasheets}

\section{HDS Unit Equipment List}

\begin{tabular}{|c|c|c|c|c|c|}
\hline \multirow{2}{*}{ Item } & \multirow{2}{*}{ Service } & \multirow{2}{*}{ Description } & \multicolumn{2}{|l|}{ Design Conditions } & \multirow{2}{*}{ Metallurgy } \\
\hline & & & Pressure & Temp. & \\
\hline R-500 & HDS Reactor & $\begin{array}{l}5^{\prime}-0 " \text { ID X 17'-0" T/T w/ } \\
\text { one bed of HR-526 Co } \\
\text { Mo catalyst. } \\
\end{array}$ & 670 PSIG/FV & $750^{\circ} \mathrm{F} /-20^{\circ} \mathrm{F}$ & $\begin{array}{l}\text { SA387 Gr. } 11 \mathrm{w} / 321 \mathrm{SS} \text { or } 347 \mathrm{SS} \\
\text { weld overlay. Internal trays are } 410 \\
\text { SS or } 321 \mathrm{SS}\end{array}$ \\
\hline V-500 & Diesel Feed Drum & $\begin{array}{l}\text { 6'-6” ID X 14'-0" T/T } \\
\text { (Horizontal) }\end{array}$ & 50 PSIG/FV & $450 /-20^{\circ} \mathrm{F}$ & Killed Carbon Steel \\
\hline V-501 & $\begin{array}{l}\text { High Temperature } \\
\text { Separator } \\
\end{array}$ & $\begin{array}{l}\text { 3'-0" ID X 8'-0" T/T } \\
\text { (Vertical) }\end{array}$ & $620 \mathrm{PSIG} / \mathrm{FV}$ & $465 /-20^{\circ} \mathrm{F}$ & Killed Carbon Steel w/0.15" CA \\
\hline V-502 & $\begin{array}{l}\text { Low Temperature } \\
\text { Separator }\end{array}$ & \begin{tabular}{|l|} 
3'-0" ID X 8'-0" T/T \\
(Horizontal w/ Boot) \\
\end{tabular} & $610 \mathrm{PSIG} / \mathrm{FV}$ & $465 /-20^{\circ} \mathrm{F}$ & $\begin{array}{l}\text { Killed Carbon Steel w/ 0.1" CA } \\
(\mathrm{PWHT}) \text { and Monel Demister }\end{array}$ \\
\hline V-503 & Naphtha Stripper & $\begin{array}{l}\text { 2'-6” ID (Top), 3'-6" ID } \\
\text { (bottom) } \\
\text { X 55'-0"T/T (Overall) } \\
\text { w/ } 22 \text { valve trays } \\
\end{array}$ & 125 PSIG/FV & $700 /-20^{\circ} \mathrm{F}$ & $\begin{array}{l}\text { Killed Carbon Steel w/ } 0.2 " \mathrm{CA} \text { and } \\
\text { Type } 410 \text { SS trays, supports, and } \\
\text { downcomers }\end{array}$ \\
\hline V-504 & $\begin{array}{l}\text { Stripper O. H. } \\
\text { Accumulator }\end{array}$ & $\begin{array}{l}\text { 3'-0" ID X 6'-0" T/T } \\
\text { (Horizontal w/Boot) }\end{array}$ & 125 PSIG/FV & $375 /-20^{\circ} \mathrm{F}$ & $\begin{array}{l}\text { Killed Carbon Steel w/0.125" CA } \\
\text { (PWHT) }\end{array}$ \\
\hline E-500 & $\begin{array}{l}\text { Reactor Fd/Btms } \\
\text { Exchanger \#1 }\end{array}$ & $\begin{array}{l}\text { 7.2 MM BTU/Hr, } \\
1870 \mathrm{FT}^{2} \text { w/ } 2 \text { shells, } \\
\text { TEMA type CEU }\end{array}$ & $\begin{array}{l}\text { Tubes: } 650 \text { PSIG } \\
\text { Shell: } 765 \text { PSIG }\end{array}$ & $\begin{array}{l}770^{\circ} \mathrm{F} \\
705^{\circ} \mathrm{F} /-20^{\circ} \mathrm{F}\end{array}$ & $\begin{array}{l}\text { Tubes: } 1 \frac{1 / 4}{\mathrm{Cr}}-1 / 2 \text { Mo tubes and } \\
\text { tube sheet and weld overlay } 316 \text { ss } \\
\text { for tube sheet } \\
\text { Shell: } 1 \frac{1 / 4}{\mathrm{Cr}}-1 / 2 \text { Mo clad w/ } 321 \\
\text { SS, baffles to be } 304 \text { SS }\end{array}$ \\
\hline E-501 & $\begin{array}{l}\text { Reactor Fd/Btms } \\
\text { Exchanger \#2 }\end{array}$ & $\begin{array}{l}\text { 4.9 } \mathrm{MM} \mathrm{BTU} / \mathrm{Hr} \text {, } \\
1735 \mathrm{FT}^{2} \text { w/2 shells, } \\
\text { TEMA type CEU }\end{array}$ & $\begin{array}{l}\text { Tubes: } 640 \text { PSIG } \\
\text { Shell: } 775 \text { PSIG }\end{array}$ & $\begin{array}{l}600^{\circ} \mathrm{F} \\
540^{\circ} \mathrm{F} /-20^{\circ} \mathrm{F}\end{array}$ & 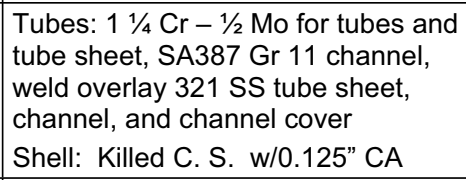 \\
\hline E-502 & $\begin{array}{l}\text { Separator } \\
\text { Fd/Btms } \\
\text { Exchanger }\end{array}$ & $\begin{array}{l}0.4 \mathrm{MM} \mathrm{BTU} / \mathrm{Hr}, \\
85 \mathrm{FT}^{2} \text {, double pipe. }\end{array}$ & $\begin{array}{l}\text { Tubes: } 630 \text { PSIG } \\
\text { Shell: } 610 \text { PSIG }\end{array}$ & $\begin{array}{l}465^{\circ} \mathrm{F} \\
465 /-20^{\circ} \mathrm{F}\end{array}$ & $\begin{array}{l}\text { Tubes: Carbon Steel } \\
\text { Shell: Carbon Steel }\end{array}$ \\
\hline$E-503$ & $\begin{array}{l}\text { H. T. Separator } \\
\text { Overhead Cooler }\end{array}$ & $\begin{array}{l}1.5 \mathrm{MM} \mathrm{BTU} / \mathrm{Hr} \\
318 \mathrm{FT}^{2} \text { (Bare Tube) } \\
6829 \mathrm{FT}^{2} \text { Extended } \\
\text { Surface, } 5 \text { HP Fan } \\
\end{array}$ & Tubes: 620 PSIG & $465^{\circ} \mathrm{F}$ & $\begin{array}{l}\text { Seamless Carbon Steel tubes with } \\
\text { aluminum fins }\end{array}$ \\
\hline E-504 & $\begin{array}{l}\text { Stripper O. H. } \\
\text { Condenser }\end{array}$ & $\begin{array}{l}\text { 2.2 } \mathrm{MM} \mathrm{BTU} / \mathrm{Hr} \\
406 \mathrm{FT}^{2} \text { (Bare Tube) } \\
8733 \mathrm{FT}^{2} \text { Extended } \\
\text { Surface, } 5 \text { HP Fan }\end{array}$ & Tubes: 125 PSIG & $375^{\circ} \mathrm{F}$ & $\begin{array}{l}\text { Seamless Carbon Steel tubes with } \\
\text { aluminum fins }\end{array}$ \\
\hline \begin{tabular}{|l|}
$E-505$ \\
\end{tabular} & $\begin{array}{l}\text { Stripper Fd/Btms } \\
\text { Exchanger }\end{array}$ & $\begin{array}{l}2.9 \mathrm{MM} \mathrm{BTU} / \mathrm{Hr} \\
830 \mathrm{FT}^{2} \text { w/1 shell, } \\
\text { TEMA type CEU }\end{array}$ & $\begin{array}{l}\text { Tubes: } 620 \text { PSIG } \\
\text { Shell: } 100 \text { PSIG }\end{array}$ & $\begin{array}{l}575^{\circ} \mathrm{F} \\
670^{\circ} \mathrm{F} /-20^{\circ} \mathrm{F}\end{array}$ & $\begin{array}{l}\text { Tubes: } 18 \mathrm{Cr}-8 \mathrm{Ni} \text {, Channel is CS } \\
\text { w/ 0.25" CA, tube sheets are } 410 \mathrm{SS} \\
\text { Shell: CS w/0.25" CA, CS baffles }\end{array}$ \\
\hline E-506 & Product Cooler & $\begin{array}{l}\text { 10.3 } \mathrm{MM} \text { BTU/Hr } \\
1463 \mathrm{FT}^{2} \text { (Bare Tube) } \\
31640 \mathrm{FT}^{2} \text { Extended } \\
\text { Surface, 20 HP Fan } \\
\end{array}$ & Tubes: 100 PSIG & $550^{\circ} \mathrm{F}$ & $\begin{array}{l}\text { Seamless Carbon Steel tubes with } \\
\text { aluminum fins }\end{array}$ \\
\hline $\mathrm{H}-500$ & $\begin{array}{l}\text { Diesel Charge } \\
\text { Heater }\end{array}$ & $\begin{array}{l}\text { 2.7 MM BTU/Hr } \\
\text { Fired Heater, Convection } \\
\text { Section shared } \mathrm{w} / \mathrm{H}-501\end{array}$ & Tubes: 755 PSIG & $760^{\circ} \mathrm{F}$ & Tubes: $9 \mathrm{Cr}-1 \mathrm{Mo}$ w/ 0.1" CA \\
\hline $\mathrm{H}-501$ & Stripper Reboiler & $\begin{array}{l}\text { 4.5 MM BTU/Hr } \\
\text { Fired Heater, Convection } \\
\text { Section shared } \mathrm{w} / \mathrm{H}-500 \\
\end{array}$ & Tubes: 230 PSIG & $710^{\circ} \mathrm{F}$ & Tubes: $5 \mathrm{Cr}-1 / 2 \mathrm{Mo} \mathrm{w} / 0.1$ " CA \\
\hline
\end{tabular}




\begin{tabular}{|c|c|c|c|c|c|}
\hline \multirow{2}{*}{ Item } & \multirow{2}{*}{ Service } & \multirow{2}{*}{ Description } & \multicolumn{2}{|l|}{ Design Conditions } & \multirow{2}{*}{ Metallurgy } \\
\hline & & & Pressure & Temp. & \\
\hline C-500 & $\begin{array}{l}\text { Makeup Hydrogen } \\
\text { Compressor }\end{array}$ & $\begin{array}{l}\text { Capacity: } 57312 \text { ACFD } \\
\text { Motor Driven } \\
\text { Shaft HP: } 71 \text { BHP }\end{array}$ & $\begin{array}{l}\text { Suction: } 300 \text { PSIG } \\
\text { Disch: } 725 \text { PSIG }\end{array}$ & $\begin{array}{l}\text { Suction: } \\
200^{\circ} \mathrm{F}\end{array}$ & $\begin{array}{l}\text { Casing: killed C. S. } \\
\text { Internals: CS }\end{array}$ \\
\hline C-501 & $\begin{array}{l}\text { Recycle Gas } \\
\text { Compressor }\end{array}$ & $\begin{array}{l}\text { Capacity: } 93744 \text { ACFD } \\
\text { Motor Driven } \\
\text { Shaft HP: } 54 \text { BHP }\end{array}$ & $\begin{array}{l}\text { Suction: } 560 \text { PSIG } \\
\text { Disch: } 715 \text { PSIG }\end{array}$ & $\begin{array}{l}\text { Suction: } \\
120^{\circ} \mathrm{F}\end{array}$ & $\begin{array}{l}\text { Casing: killed C. S. } \\
\text { Internals: Stainless Steel }\end{array}$ \\
\hline $\begin{array}{l}P-500 \\
A / B\end{array}$ & $\begin{array}{l}\text { Diesel Feed Pump } \\
\text { (One Operating + } \\
\text { One Spare }\end{array}$ & $\begin{array}{l}\text { Rated Capacity: } 142 \text { GPM } \\
\text { Diff. Press: } 762 \text { PSI } \\
\text { Head: } 2363 \text { feet } \\
\text { Motor: } 125 \text { HP }\end{array}$ & & & $\begin{array}{l}\text { Casing: killed C. S. w/ } 0.125 " \text { CA } \\
\text { Impeller: } 12 \% \text { chrome }\end{array}$ \\
\hline $\begin{array}{l}P-501 \\
A / B\end{array}$ & $\begin{array}{l}\text { Stripper Bottoms } \\
\text { Pump } \\
\text { (One Operating + } \\
\text { One Spare }\end{array}$ & $\begin{array}{l}\text { Rated Capacity: } 270 \text { GPM } \\
\text { Diff. Press: } 125 \text { PSI } \\
\text { Head: } 476 \text { feet } \\
\text { Motor: } 40 \text { HP }\end{array}$ & & & $\begin{array}{l}\text { Casing: killed C. S. w/ } 0.2 " \text { CA } \\
\text { Impeller: } 12 \% \text { chrome }\end{array}$ \\
\hline $\begin{array}{l}\mathrm{P}-502 \\
\mathrm{~A} / \mathrm{B}\end{array}$ & $\begin{array}{l}\text { Stripper Overhead } \\
\text { Pump } \\
\text { (One Operating + } \\
\text { One Spare }\end{array}$ & $\begin{array}{l}\text { Rated Capacity: } 30 \text { GPM } \\
\text { Diff. Press: } 38 \text { PSI } \\
\text { Head: } 119 \text { feet } \\
\text { Motor: } 5 \text { HP }\end{array}$ & & & $\begin{array}{l}\text { Casing: killed C. S. w/ } 0.125 \text { " CA } \\
\text { Impeller: } 12 \% \text { chrome }\end{array}$ \\
\hline
\end{tabular}




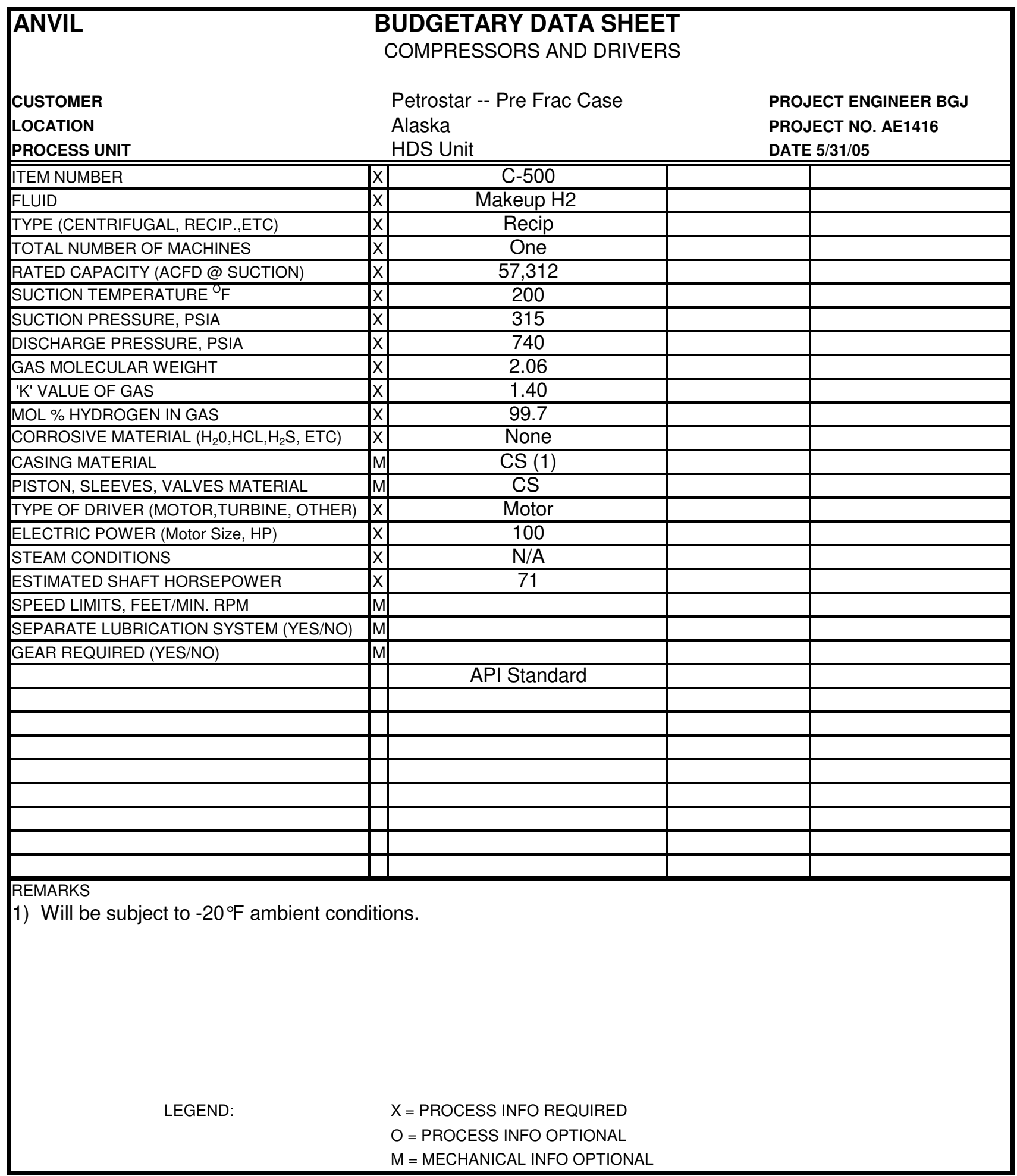

S:IPetroStar\AE1416.AUXIsubjob 45\Final Pre_Frac Case HDSI[V-504.xIs]V-504 Rev B 


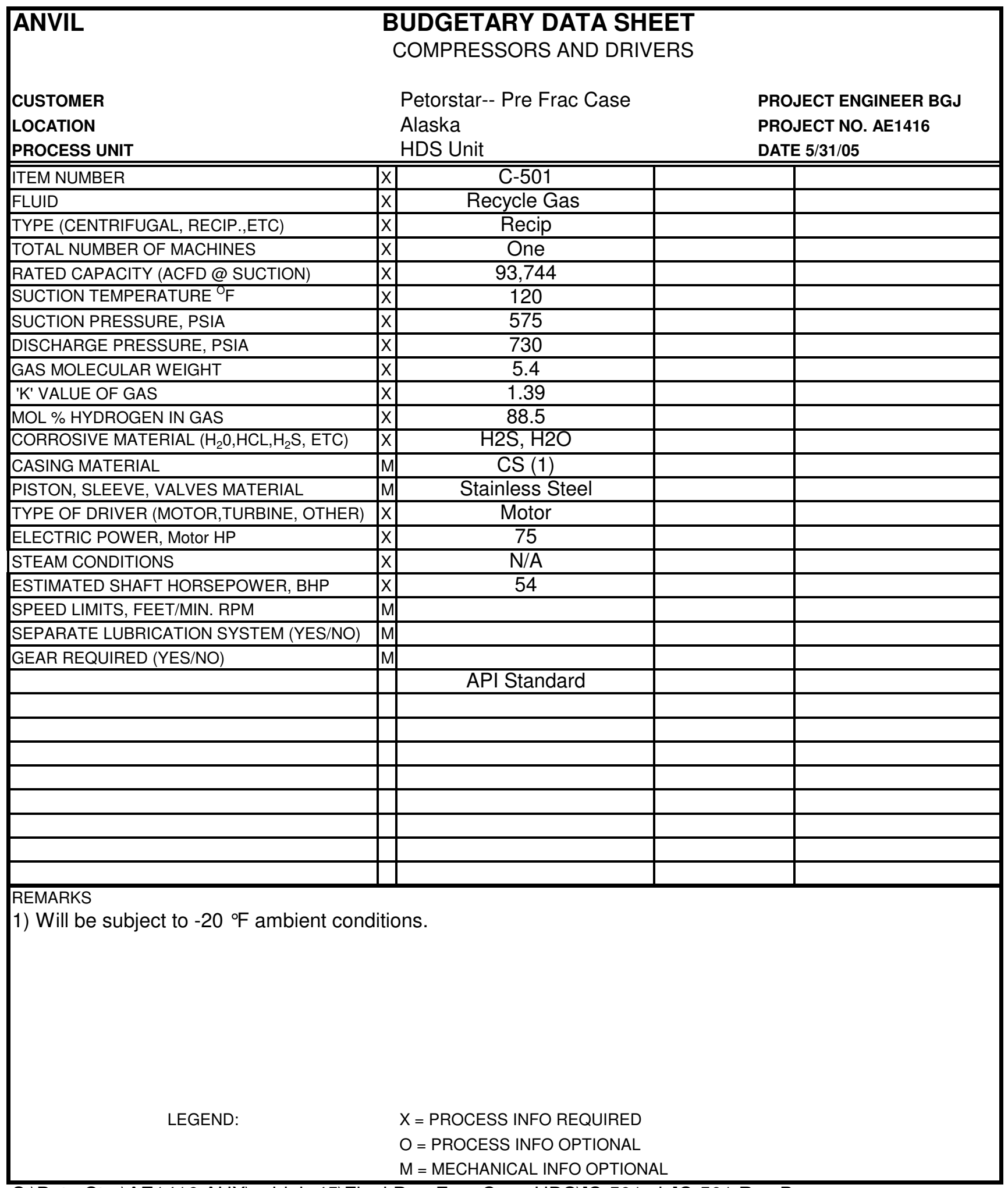

S:IPetroStar\AE1416.AUX|subjob 45|Final Pre_Frac Case HDS\[C-501.xls]C-501 Rev B 


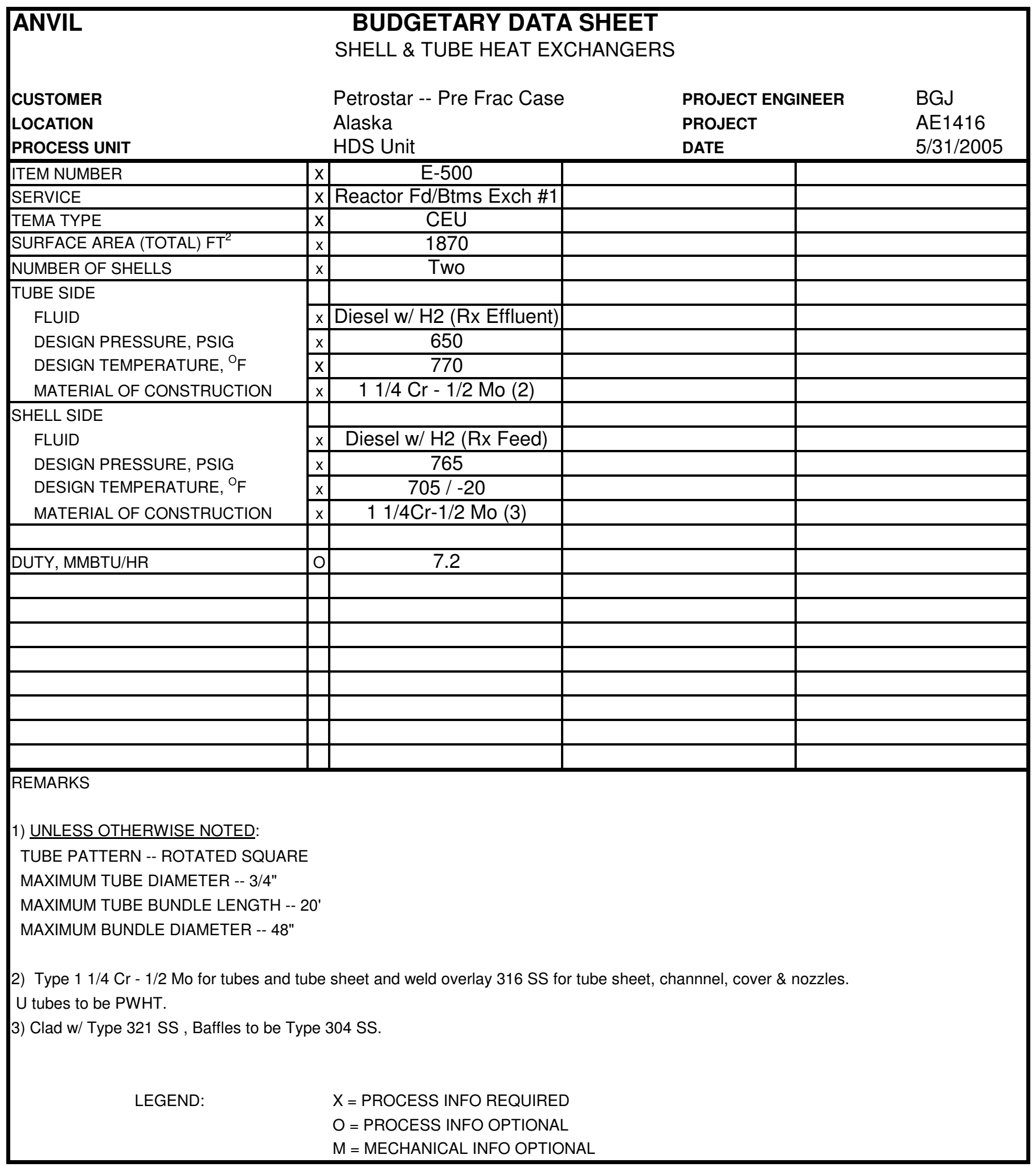

S:IPetroStar|AE1416.AUX|subjob 45|Final Pre_Frac Case HDSI[H-501.xls]Sheet1 


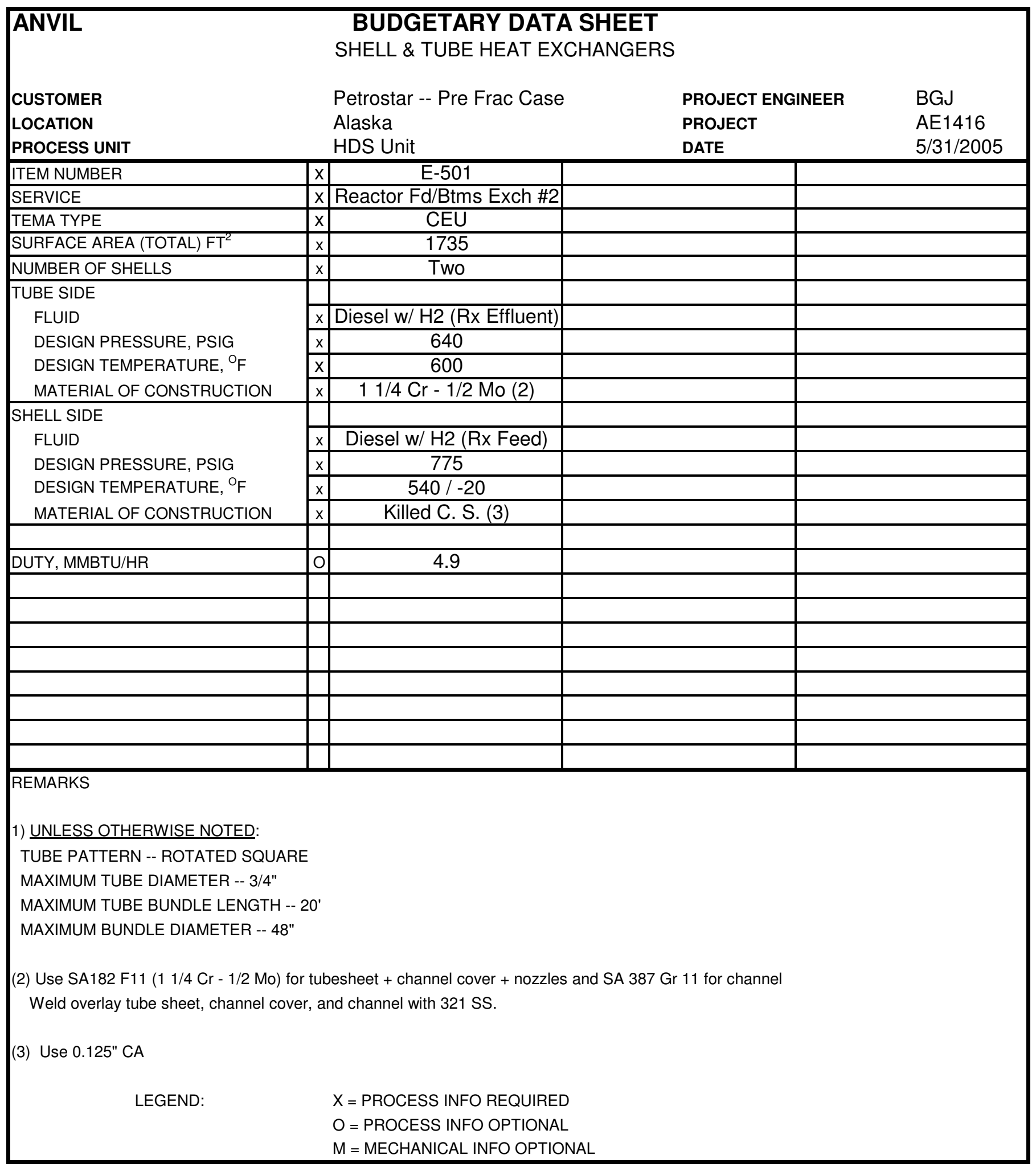

S:IPetroStar|AE1416.AUX|subjob 45|Final Pre_Frac Case HDSI[H-501.xls]Sheet1 


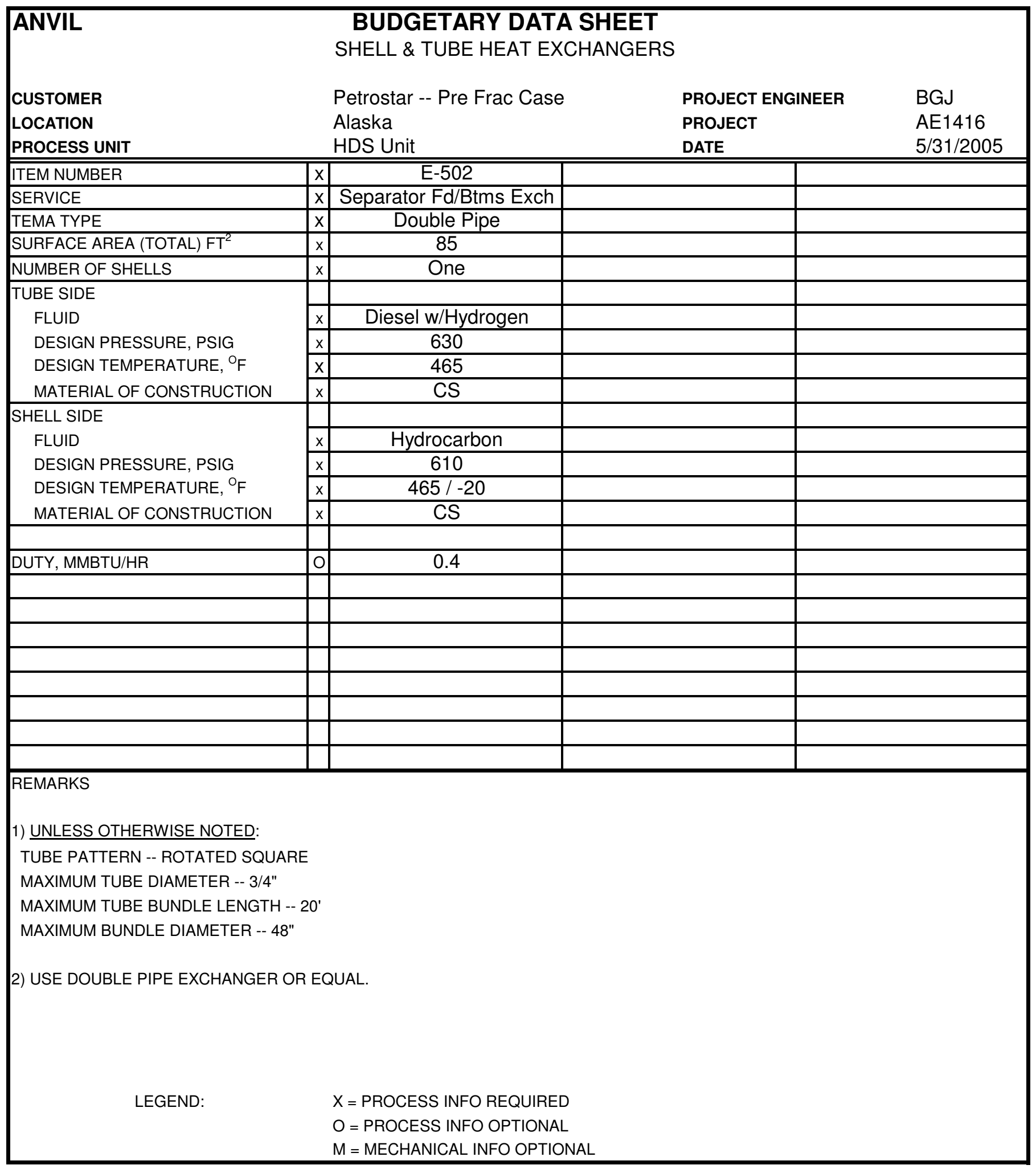




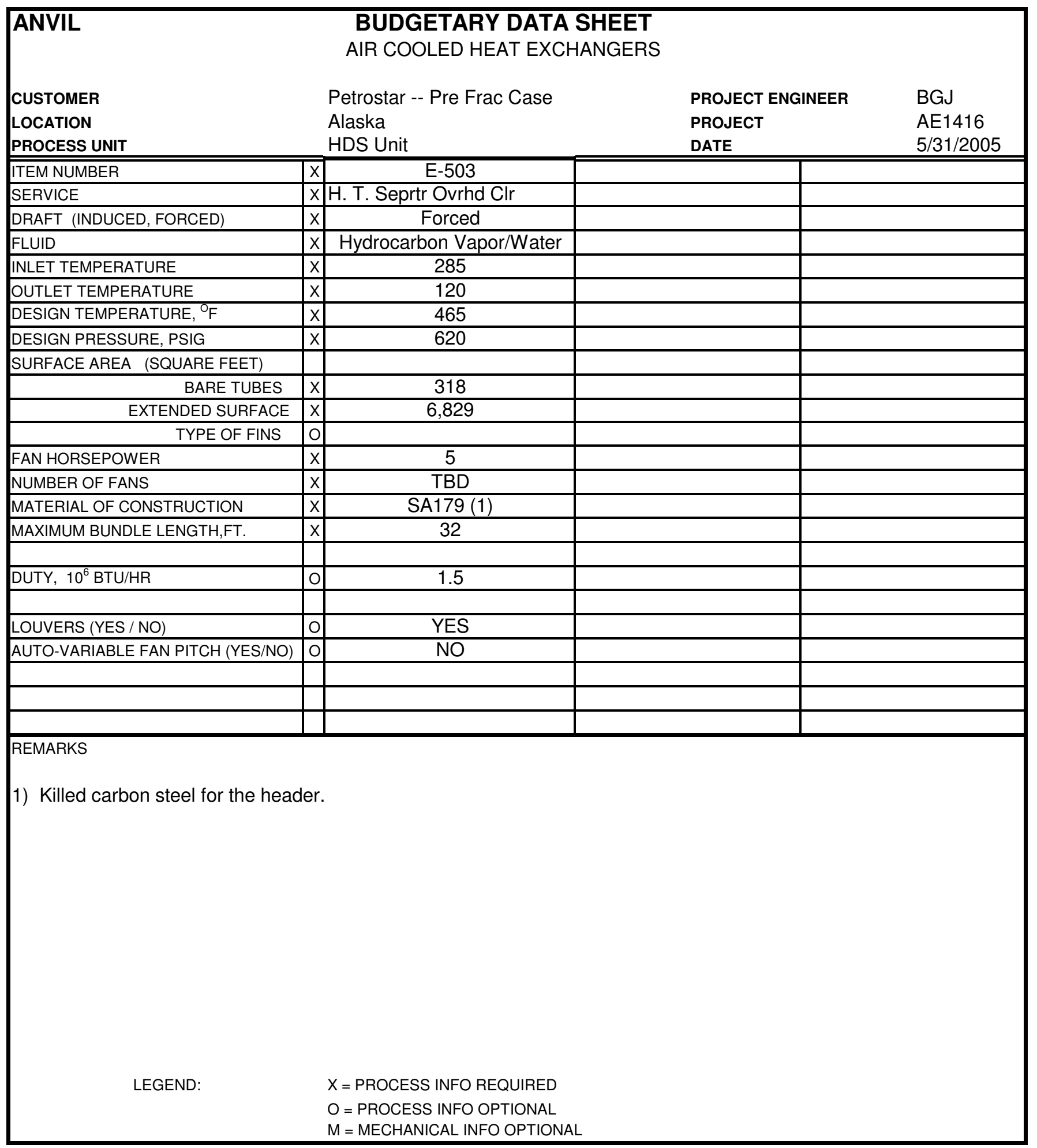




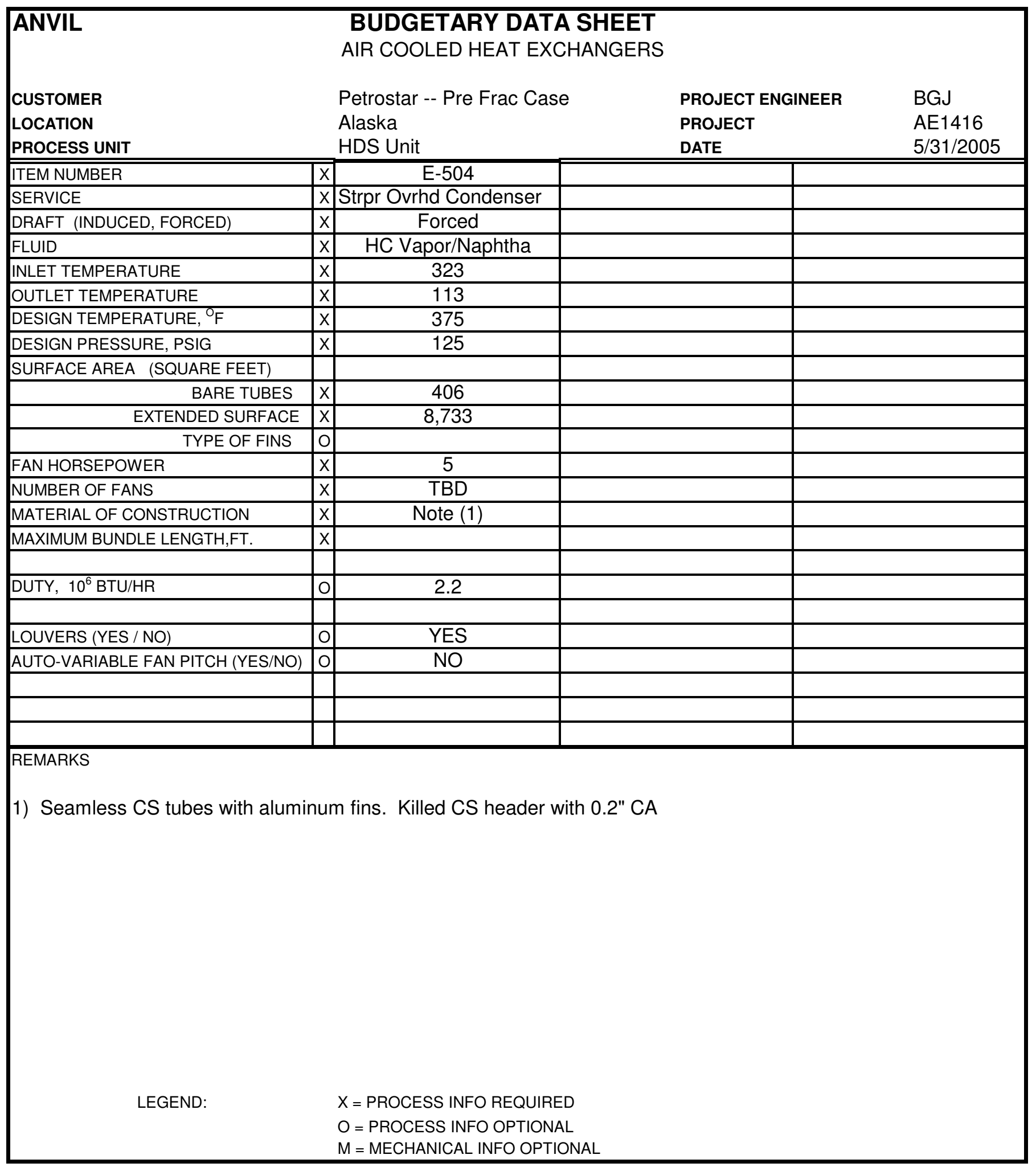

S:IPetroStar|AE1416.AUXIsubjob 45IFinal Pre_Frac Case HDSI[E-504.xls]E-504 Rev B 


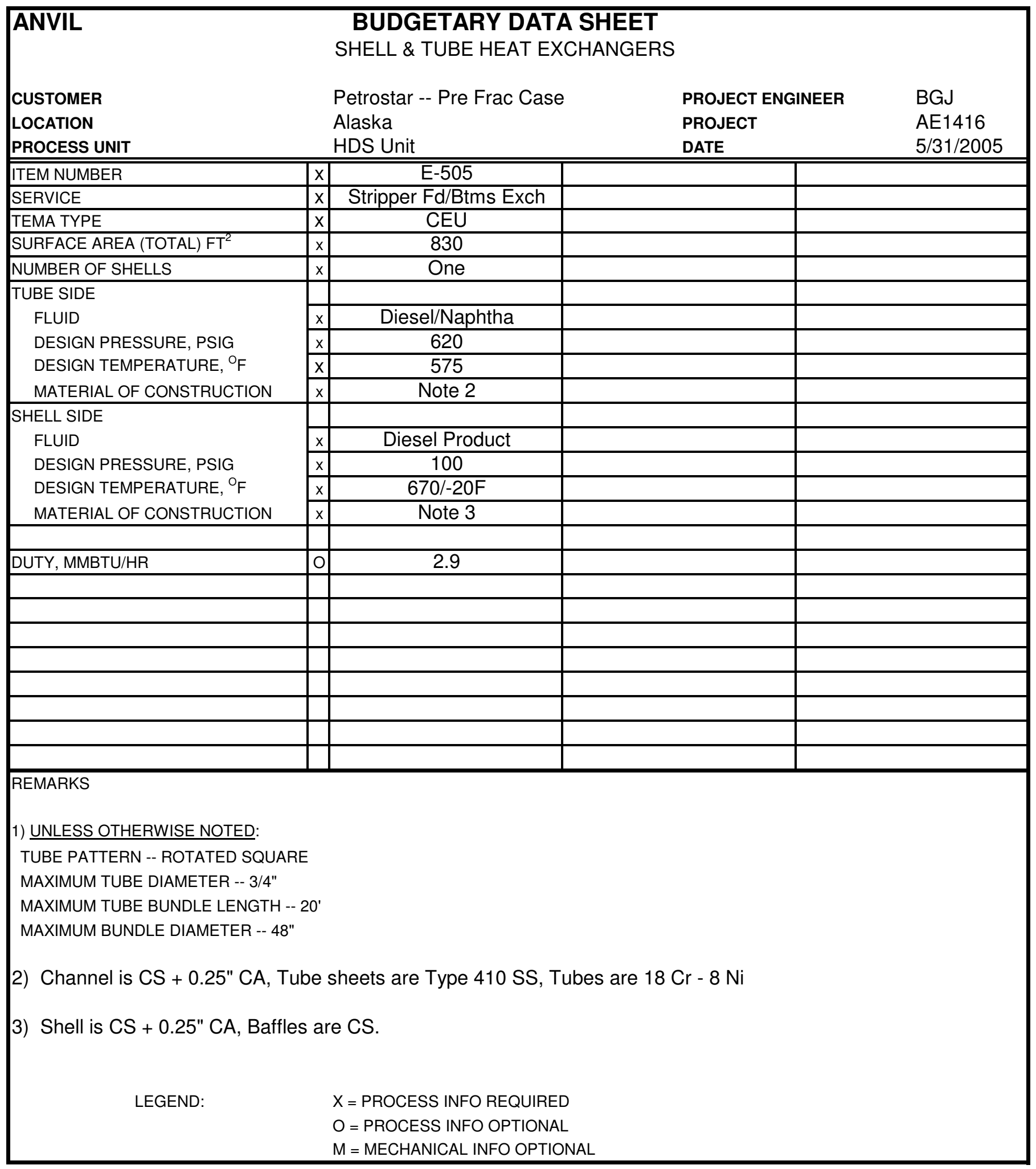

S:IPetroStar\AE1416.AUX|subjob 45\Final Pre_Frac Case HDS\[H-501.xIs]Sheet1 


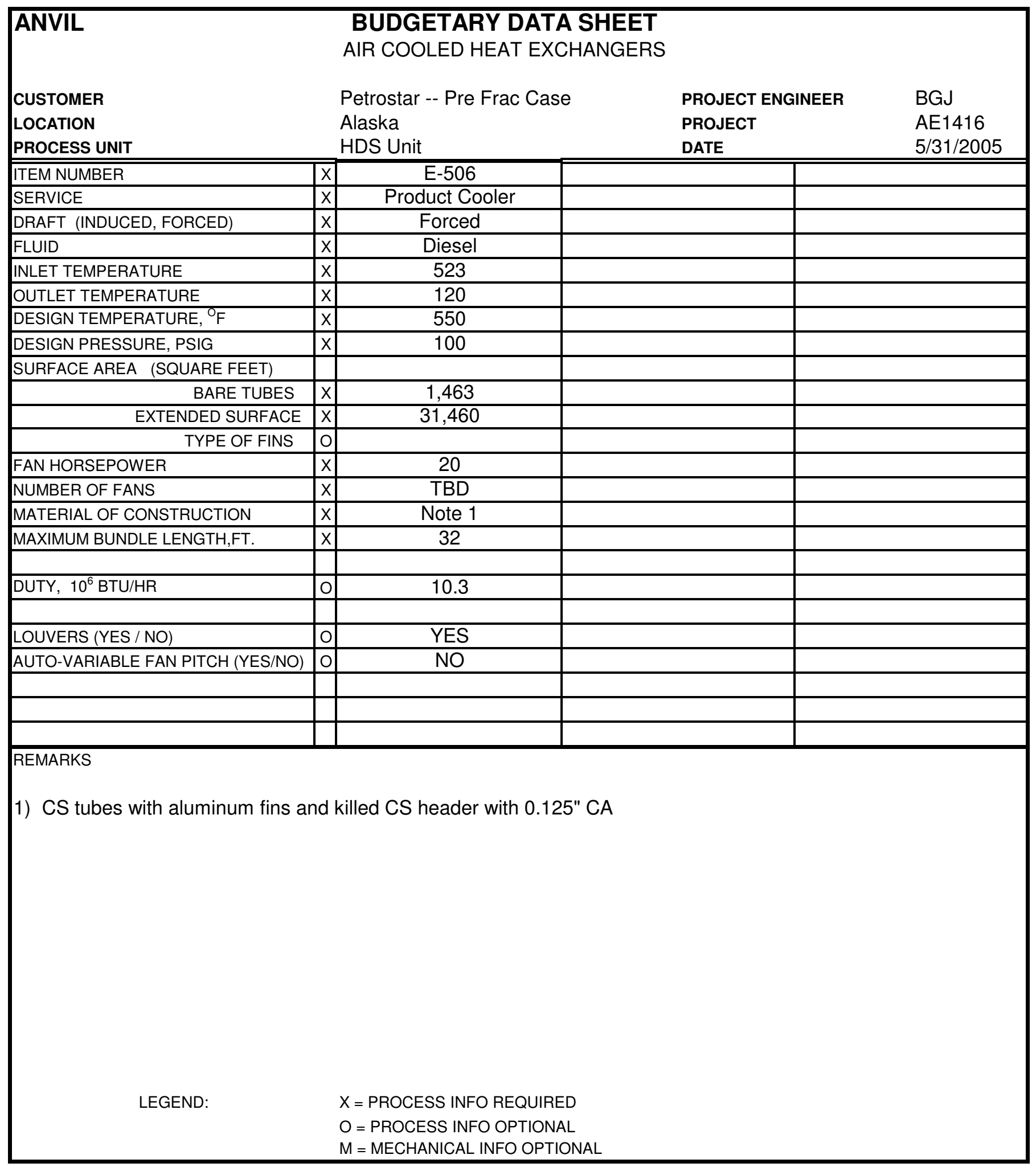

S:IPetroStar|AE1416.AUXIsubjob 45IFinal Pre_Frac Case HDSI[E-506.xls]E-506 Rev B 


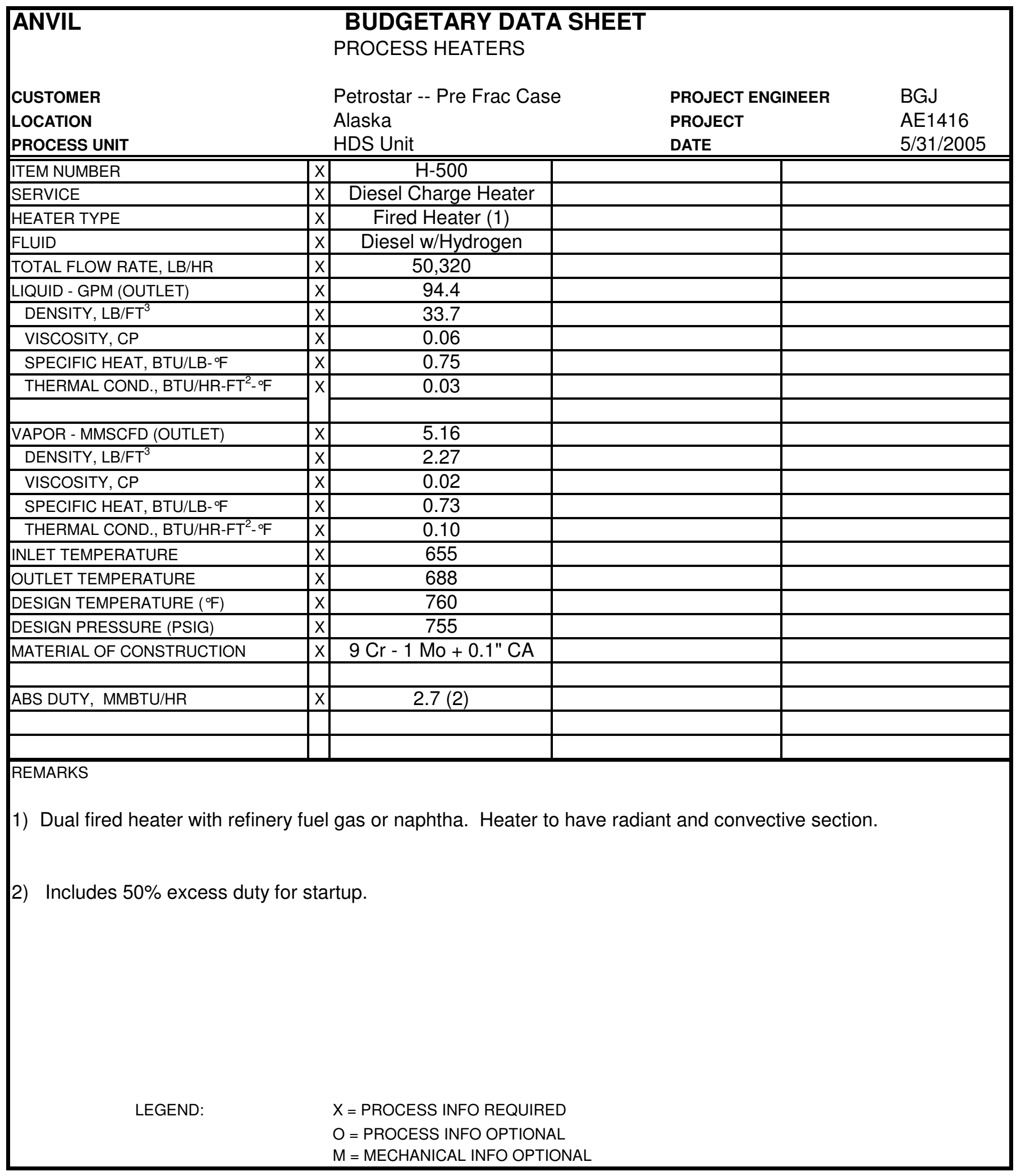




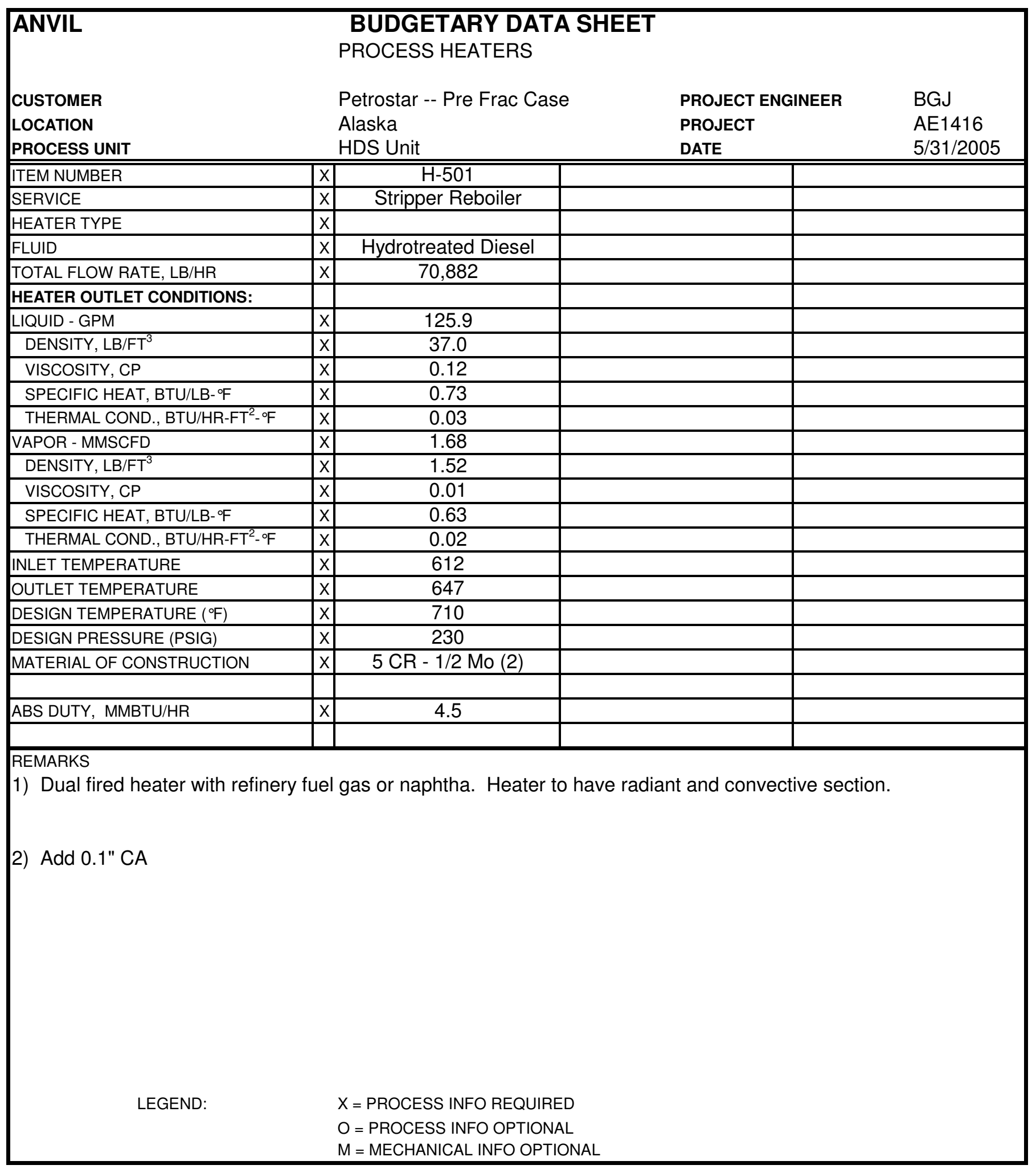




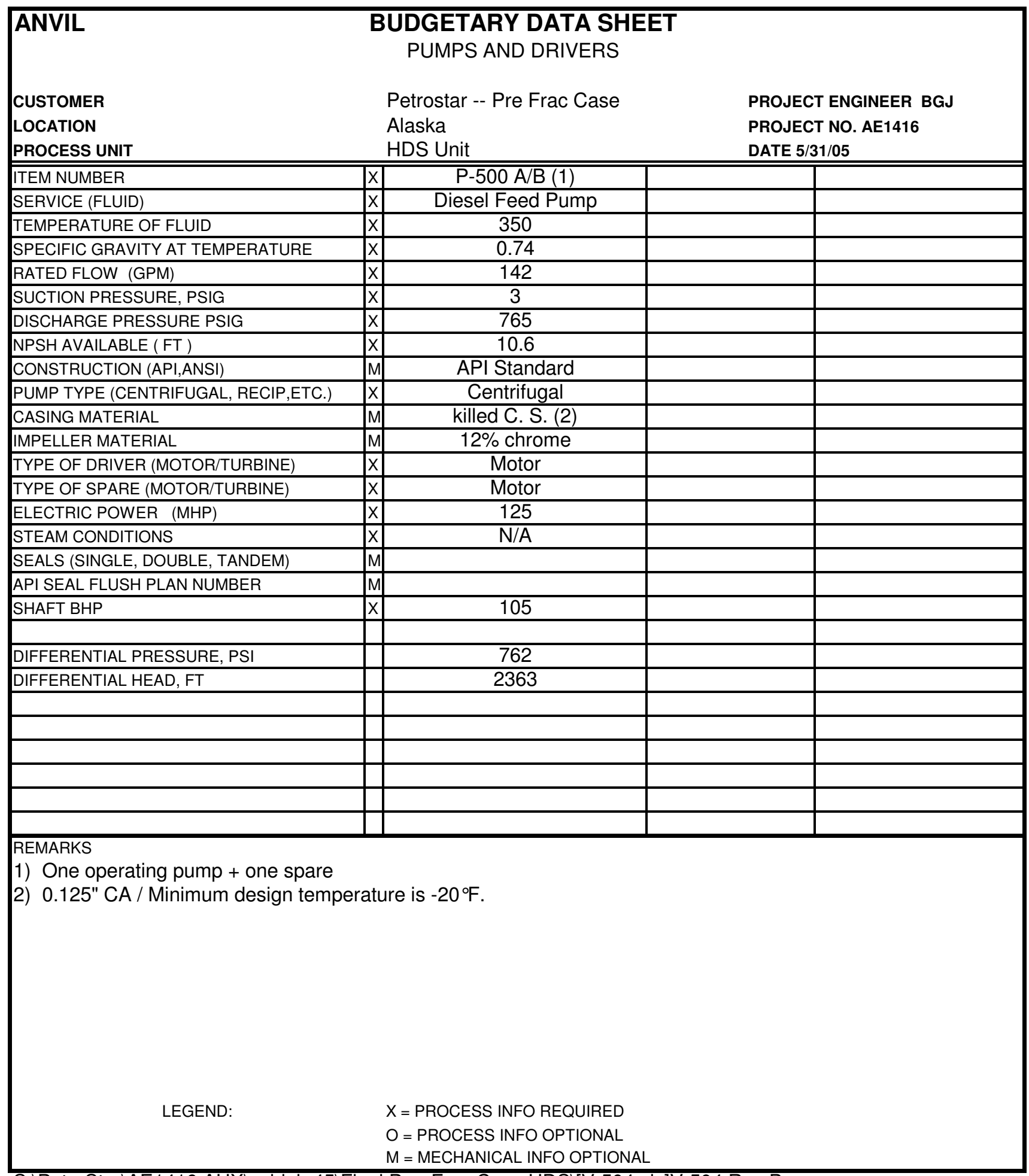

S:PetroStar|AE1416.AUXIsubjob 45|Final Pre_Frac Case HDS|[V-504.xls]V-504 Rev B 


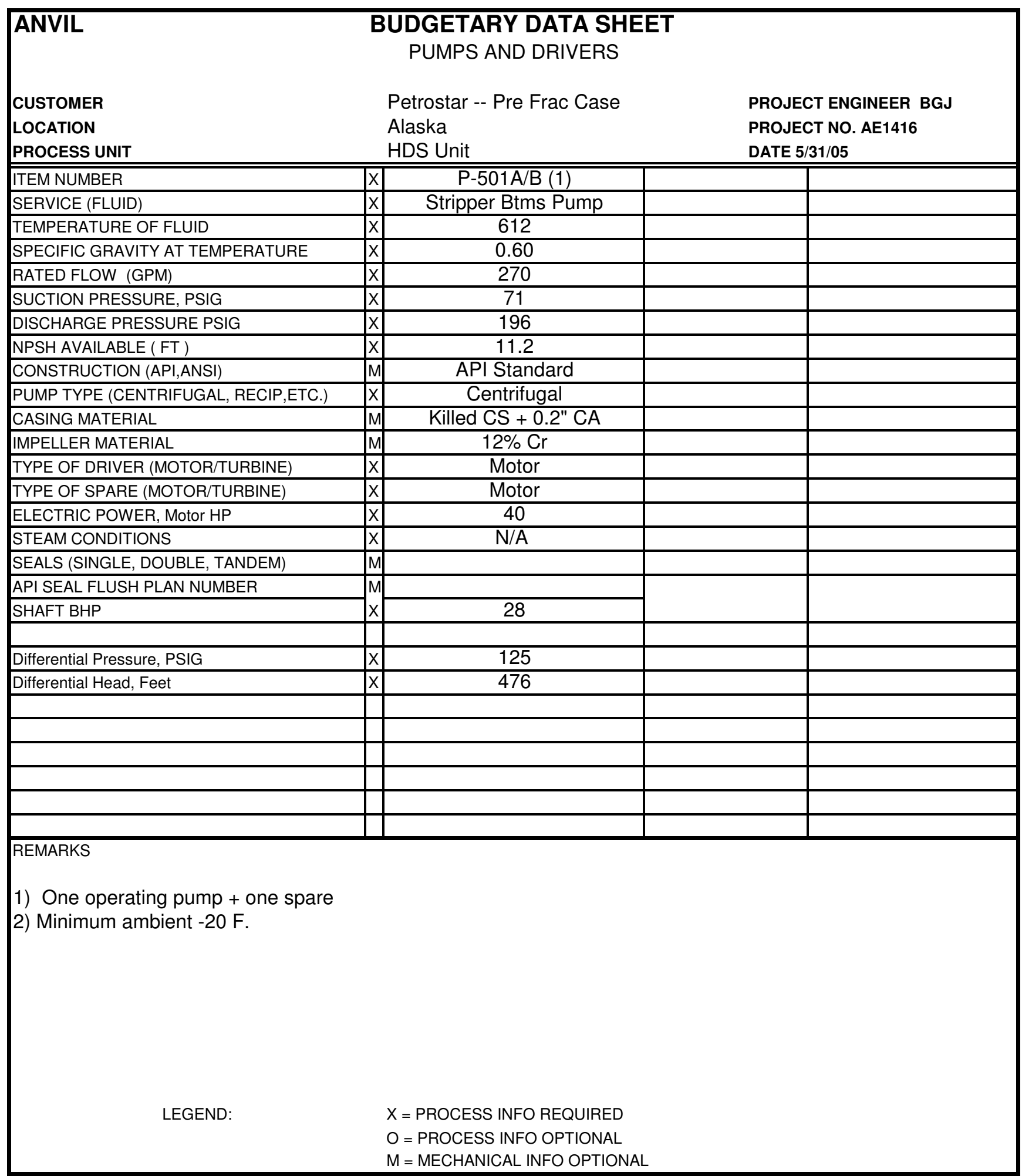

S:IPetroStar\AE1416.AUX|subjob 45\Final Pre_Frac Case HDS|[V-504.xls]V-504 Rev B 


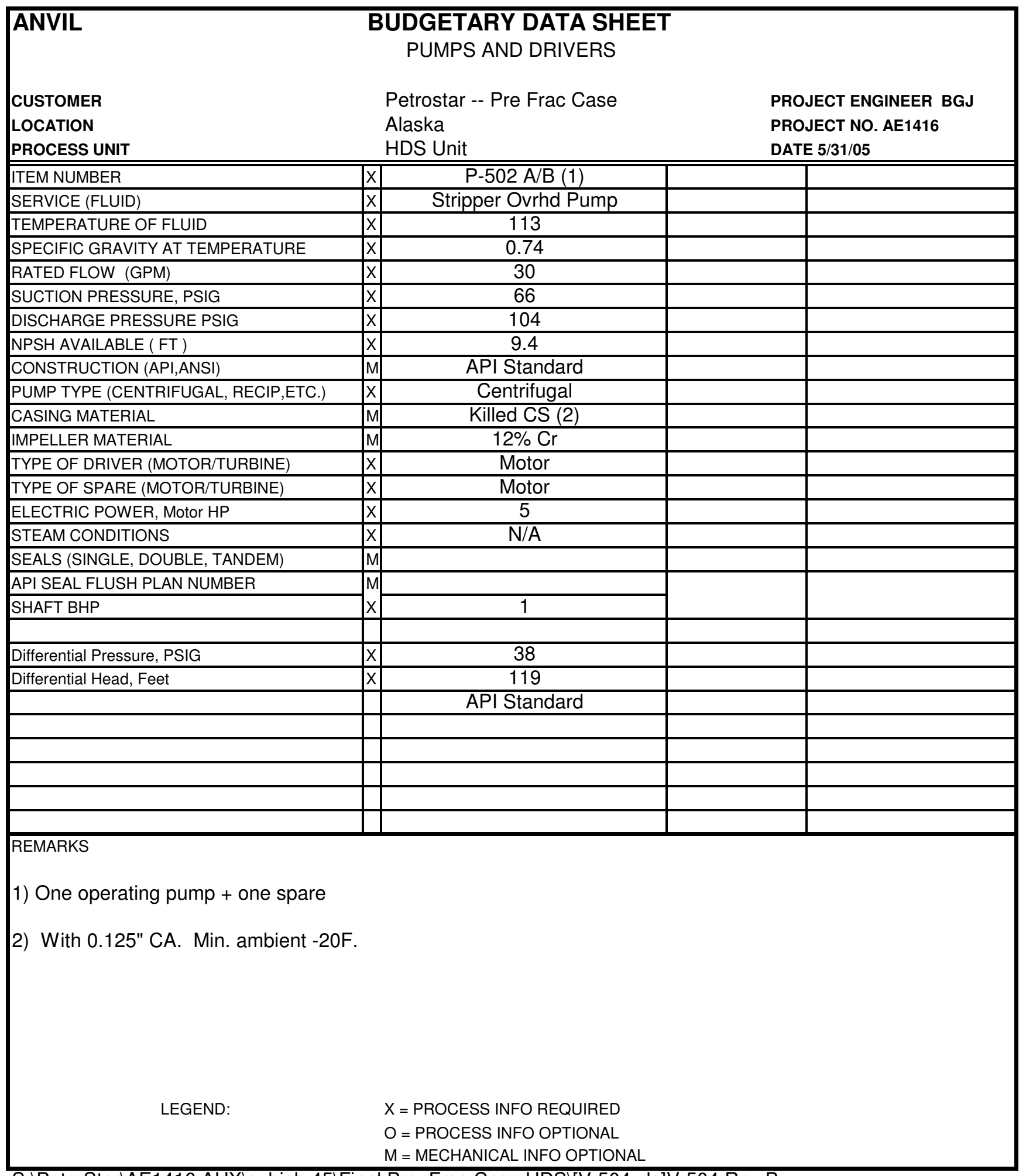

S:IPetroStar\AE1416.AUX|subjob 45\Final Pre_Frac Case HDS|[V-504.xls]V-504 Rev B 


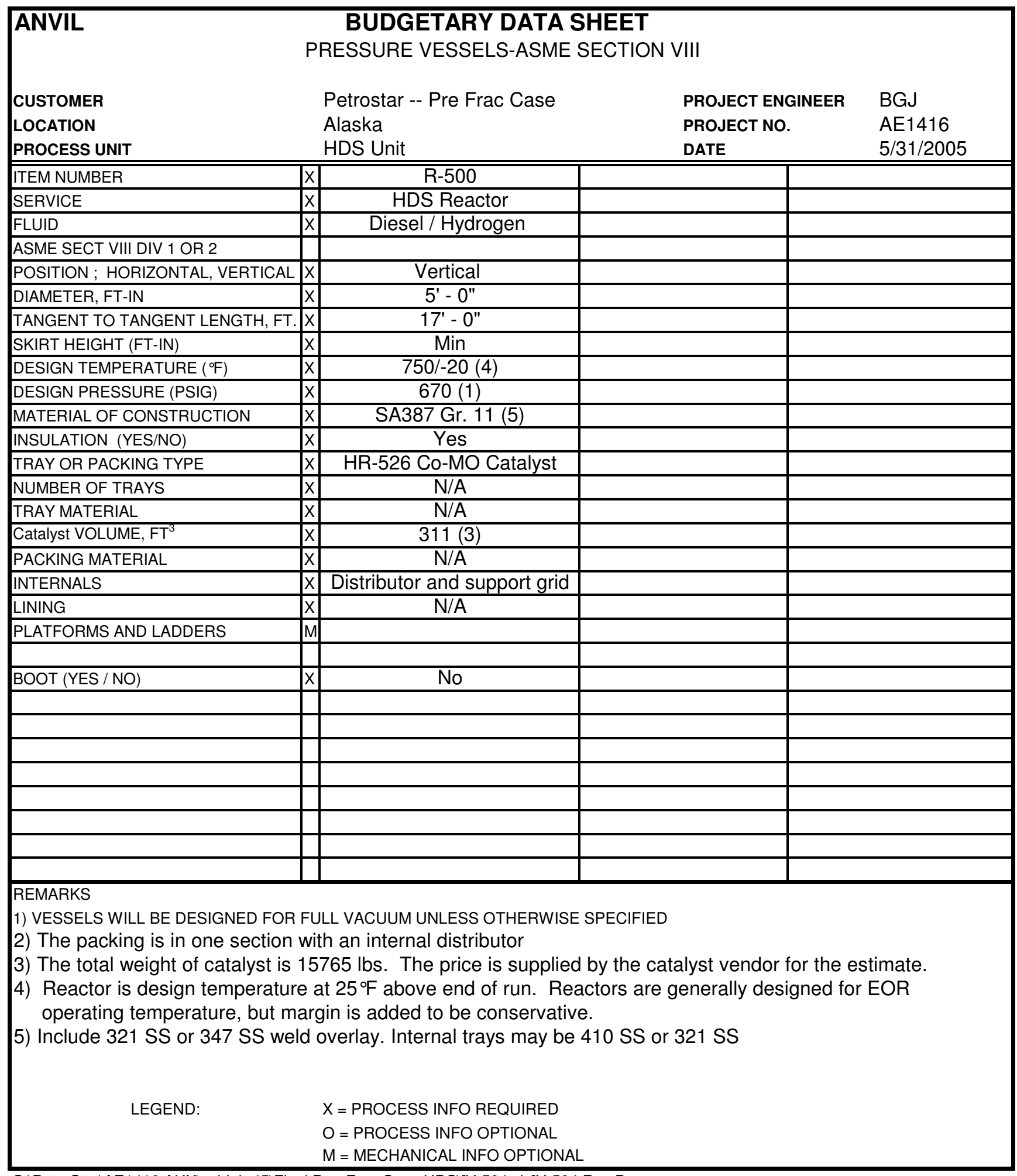

S:IPetroStar|AE1416.AUXIsubjob 45|Final Pre_Frac Case HDSI[V-504.XIs]V-504 Rev B 


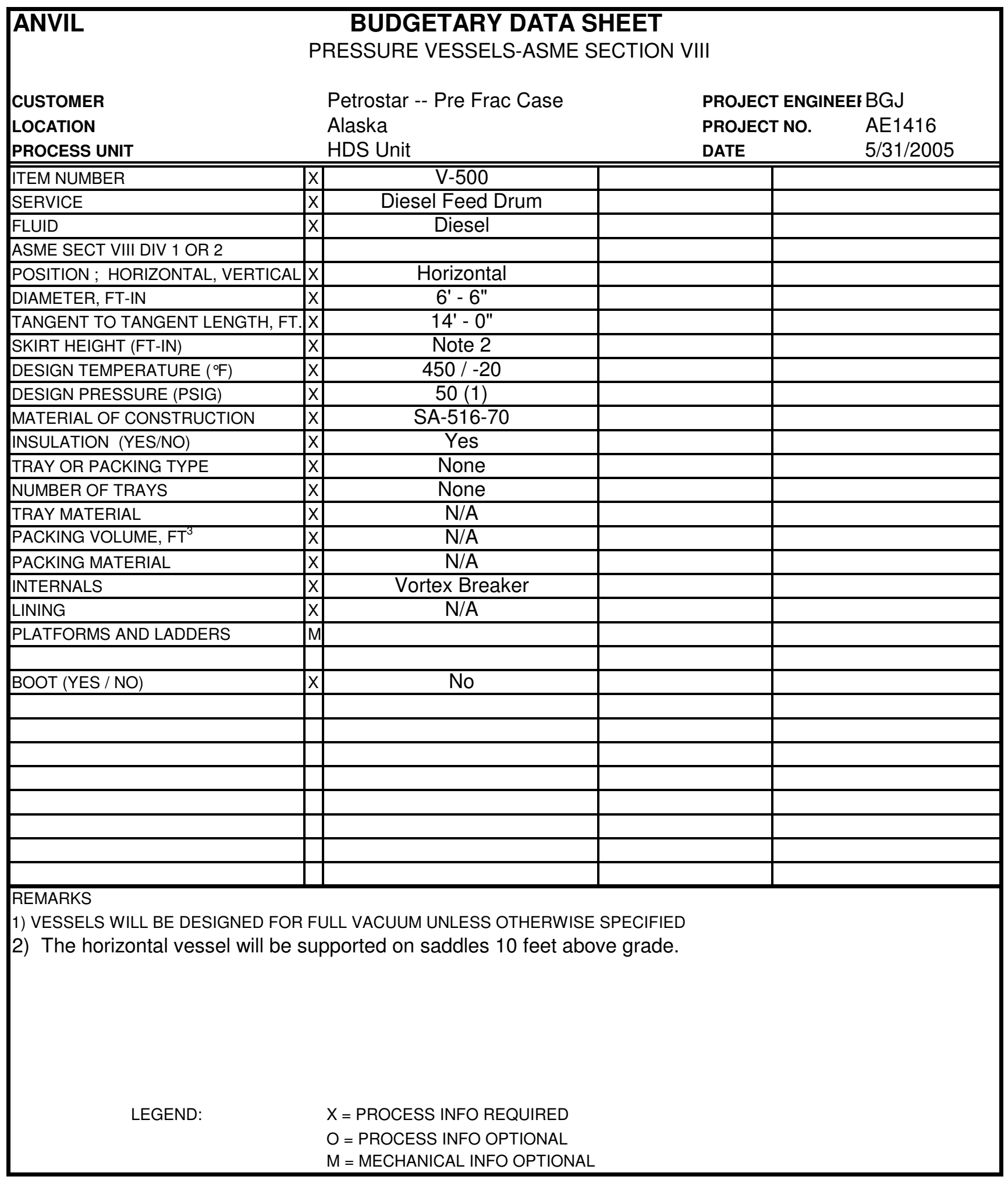

S:IPetroStar|AE1416.AUX|subjob 45|Final Pre_Frac Case HDSI[V-504.xls]V-504 Rev B 


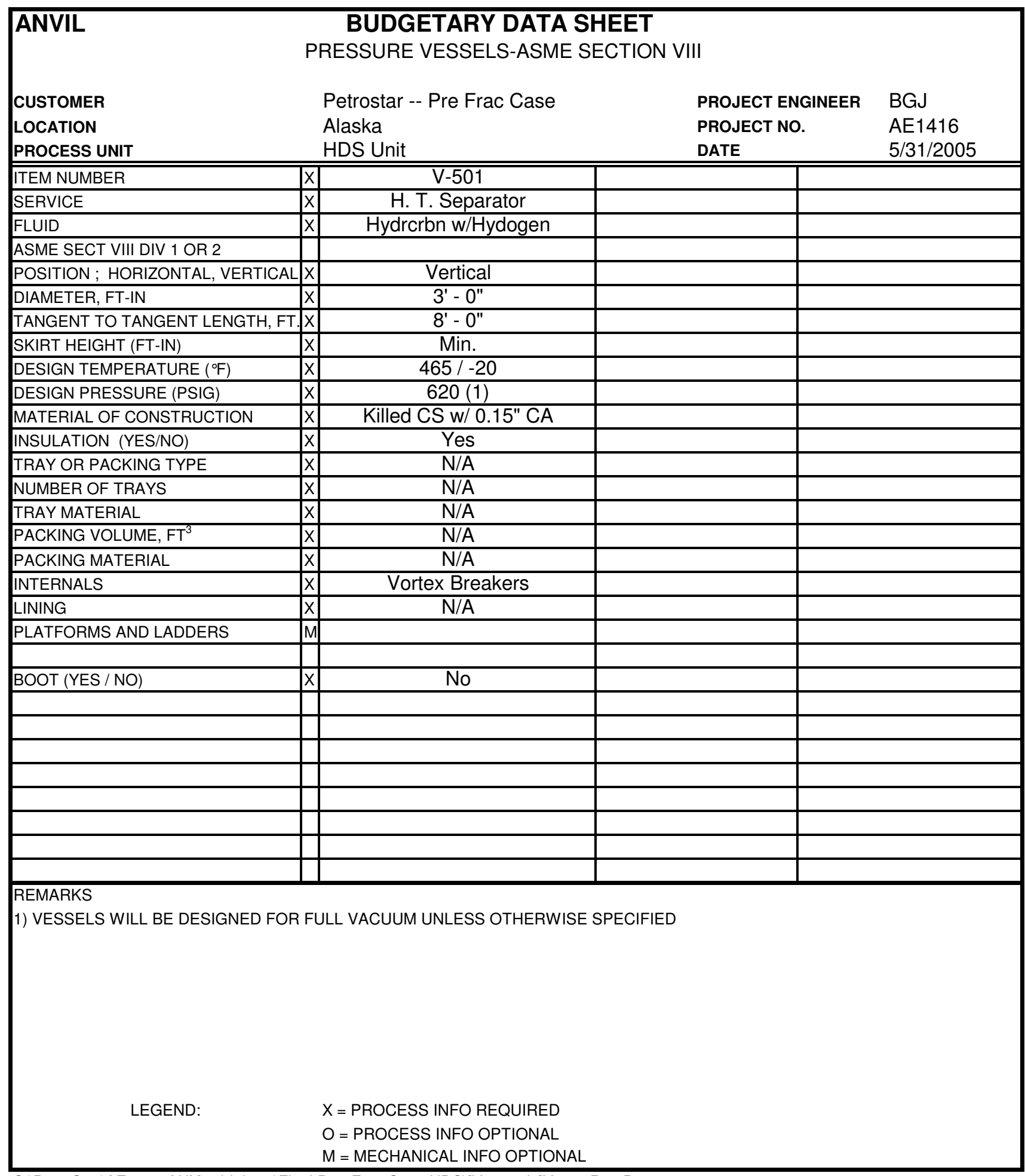




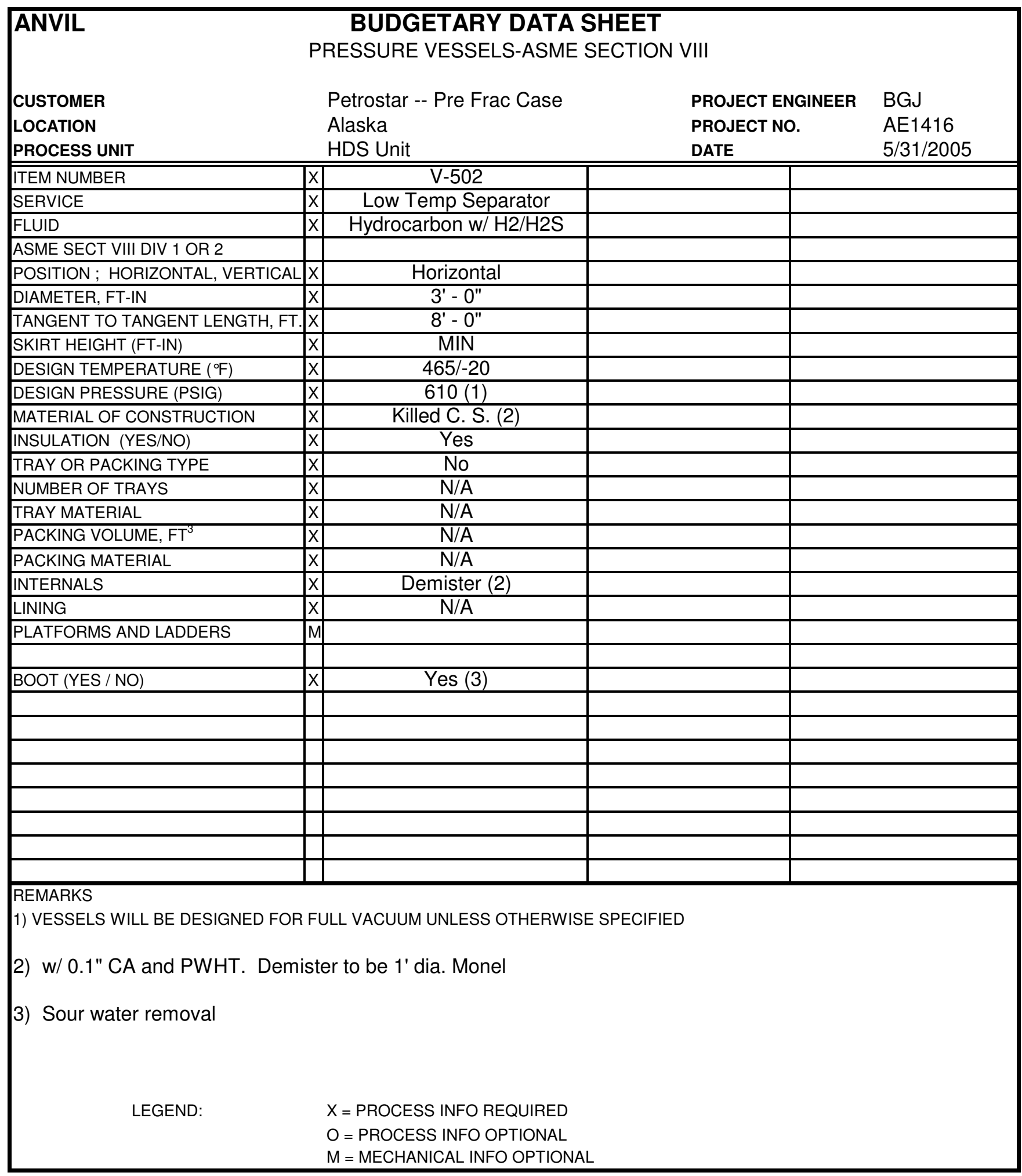

S:IPetroStar|AE1416.AUX|subjob 45|Final Pre_Frac Case HDSI[C-501.xIs]C-501 Rev B 


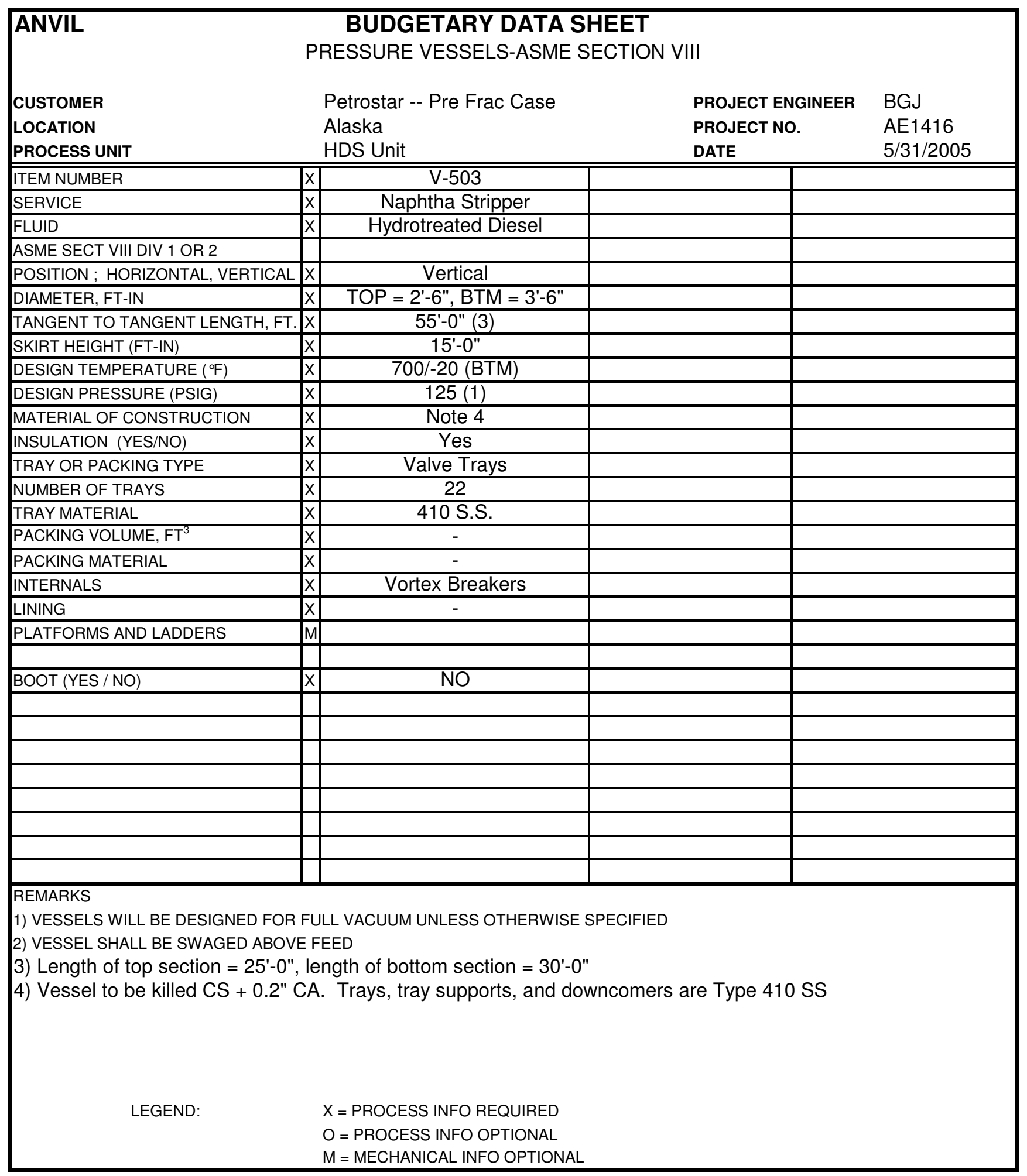




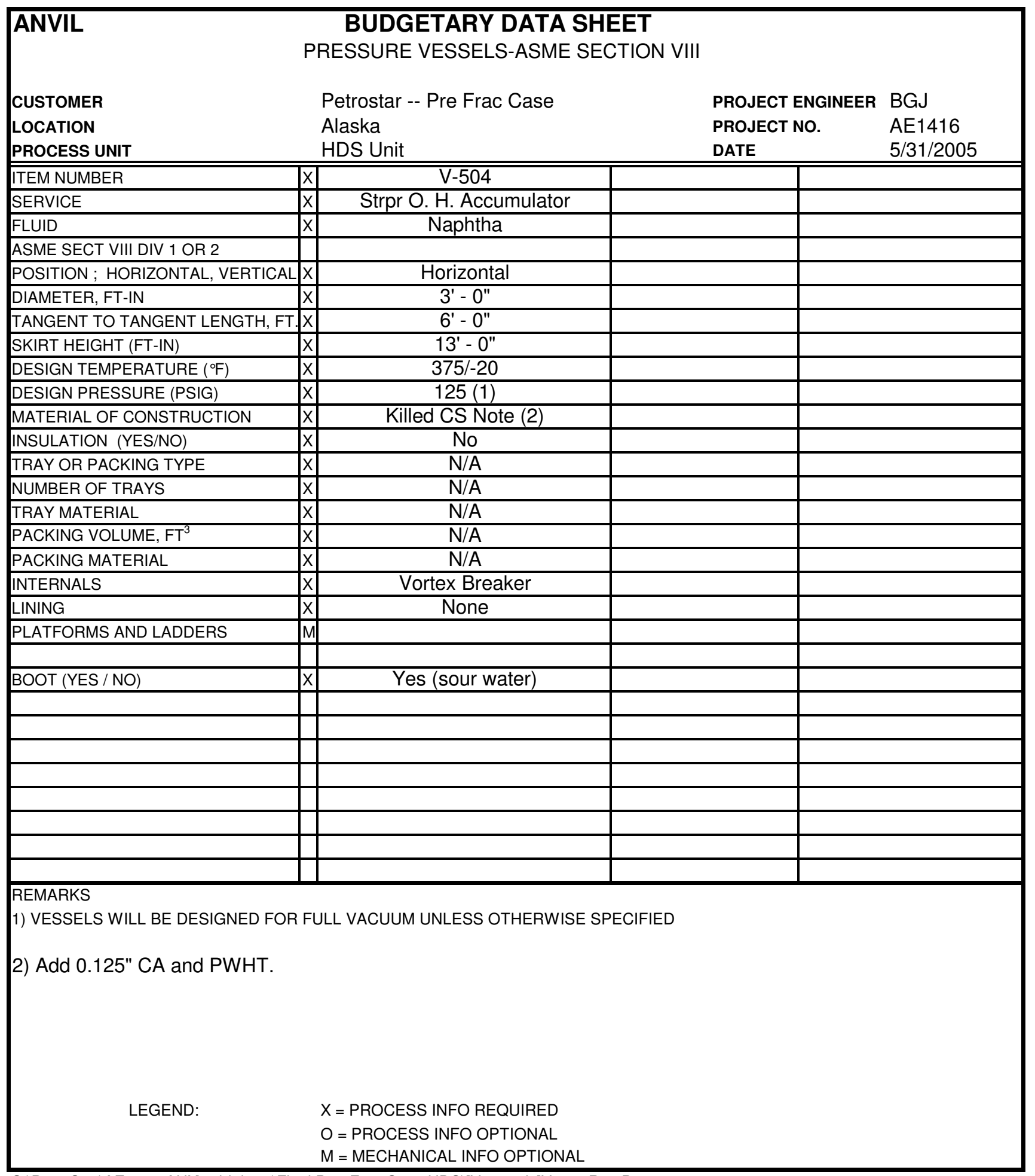


Appendix D.6 - BDS Unit Process Flow Diagrams and Material Balance 


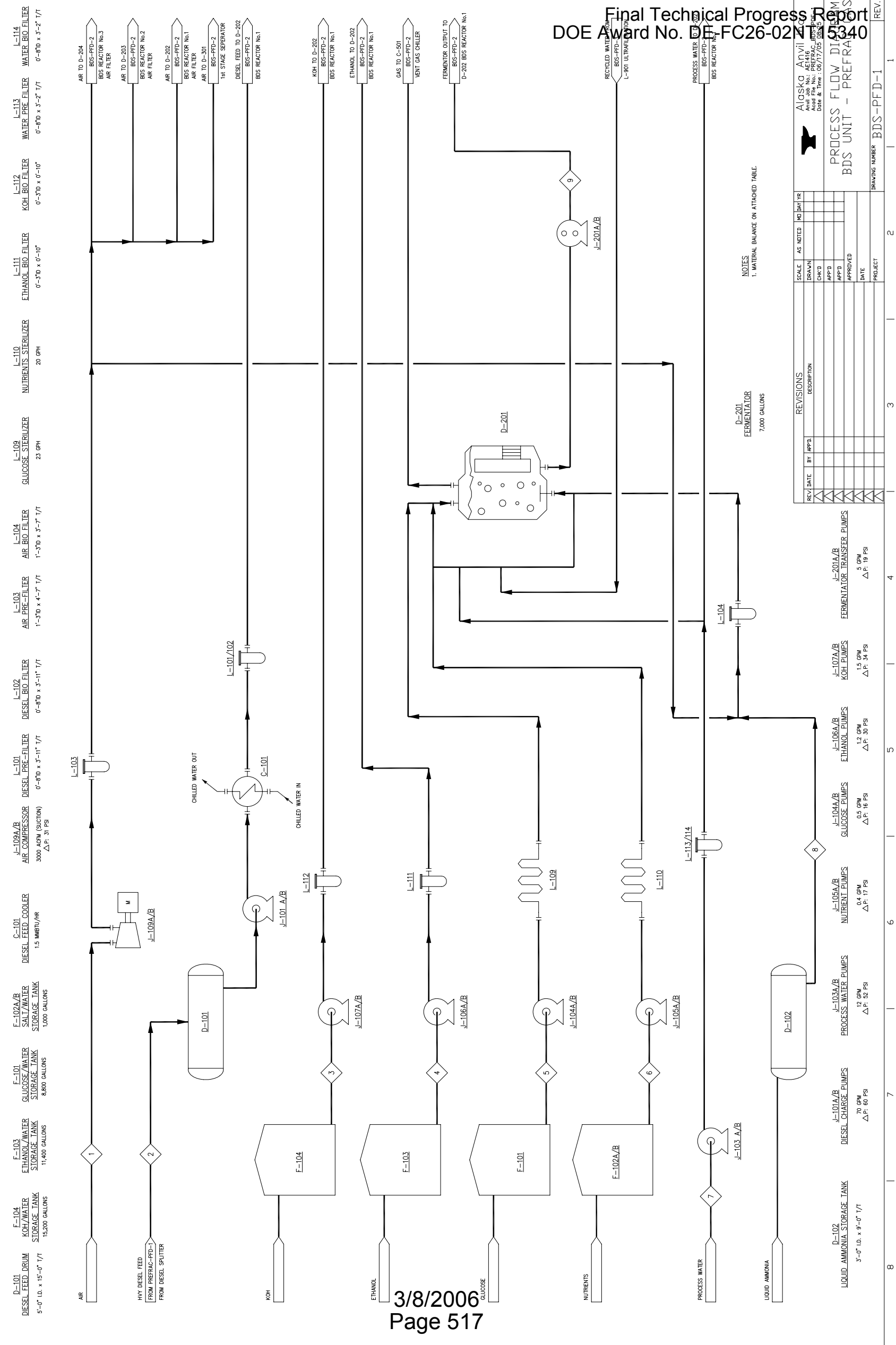




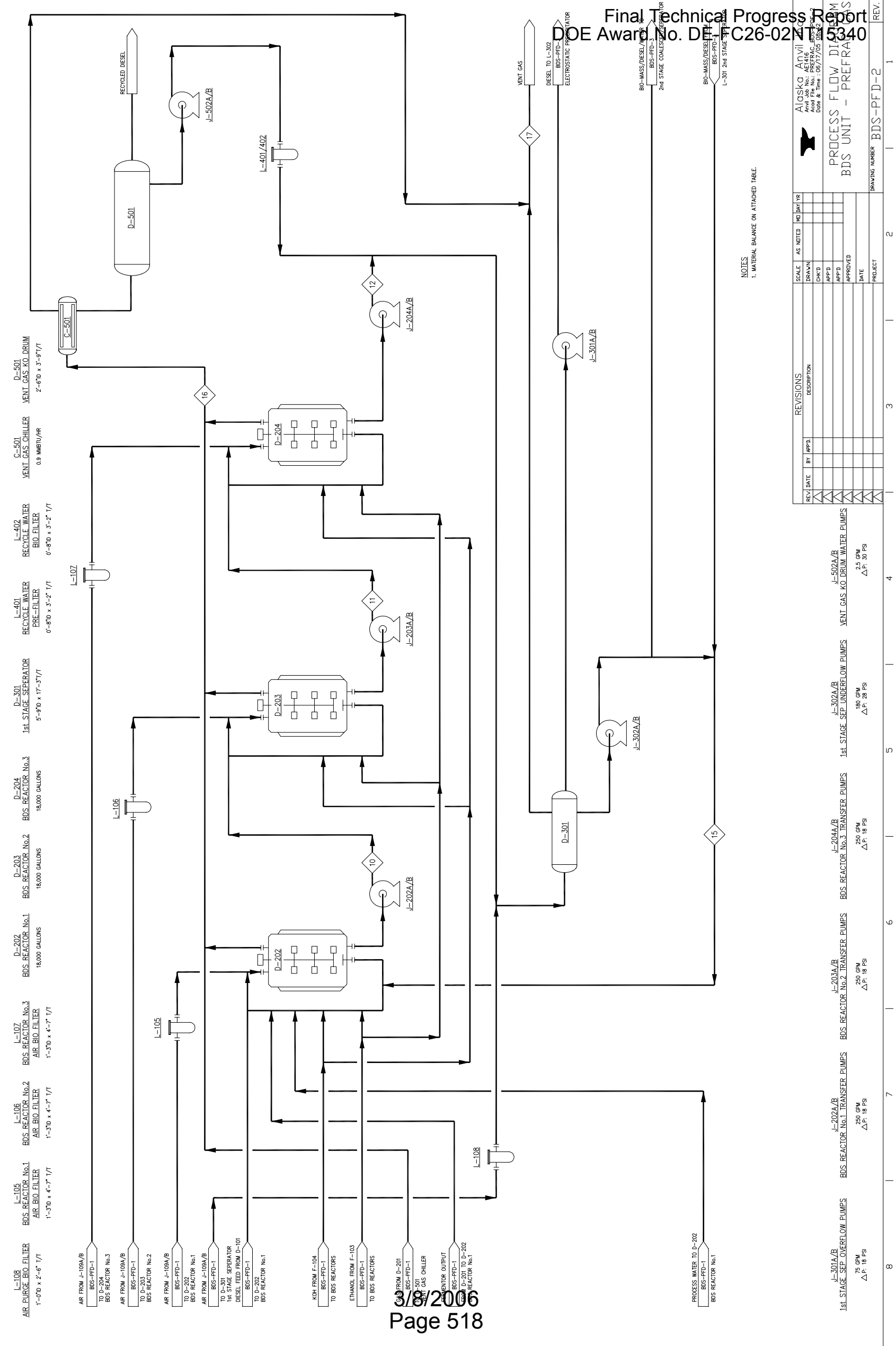


Final Technical Progress Report

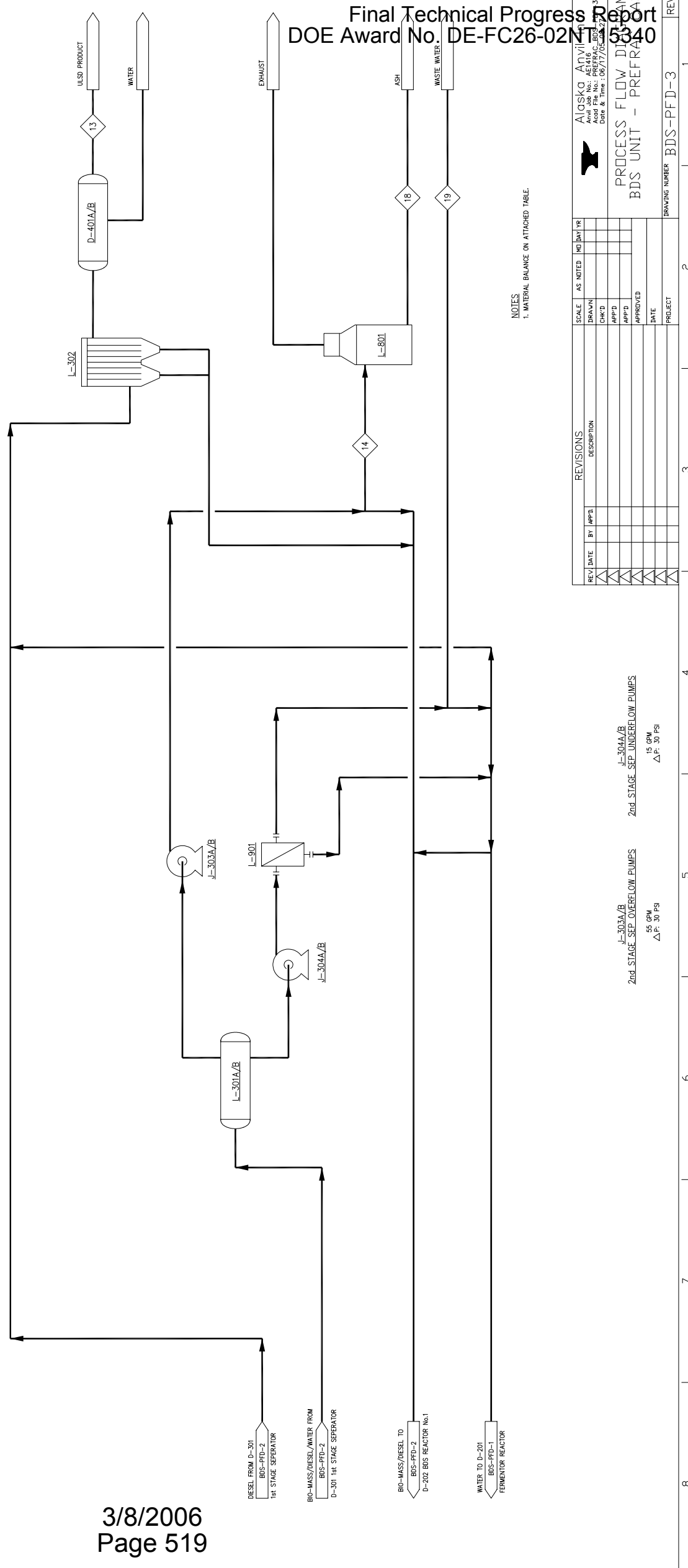




\section{Material Balance}

\section{BDS Unit Material Balance -- Prefractionation Case}

\begin{tabular}{|l|l|c|c|c|c|c|c|c|}
\hline PFD Stream Number & & 1 & 2 & 3 & 4 & 5 & 6 & 7 \\
\hline Stream Number & & Air & Diesel & NaOH & Ethanol & Glucose & Nutrients & Process Water \\
\hline Stream Description & & Air Feed & Diesel Feed & NaOH Feed & Ethanol Feed & Glucose Feed & Nutrient Feed & $\begin{array}{c}\text { Process Water } \\
\text { Feed }\end{array}$ \\
\hline Phase & & VAPOR & LIGUID & LIGUID & LIOUID & LIGUID & LIGUID & LIOUID \\
\hline Hydrocarbon Mass Flo LB/HR & & 26566 & & & & & \\
\hline Aqueous Mass Flow & LB/HR & & & 860 & 420 & 205 & 167 & 5000 \\
\hline Biomass Mass Flow & LB/HR & & & & & & & \\
\hline Sulfur in HC & PPM & & 6311 & & & & & \\
\hline Total Mass Flow Rate & LB/HR & 12000 & 26566 & 860 & 420 & 205 & 167 & 5000 \\
\hline Temperature & F & 68 & 176 & 68 & 68 & 68 & 68 & 68 \\
\hline Pressure & PSIG & 0 & 0 & 0 & 0 & 0 & 0 & 0 \\
\hline Standard Liq Flow & BPD & & 2140 & 39 & 31 & 12 & 11 & 344 \\
\hline Vapor Flow & MSCFH & 162 & & & & & & 0 \\
\hline Wt \% Vapor & & 100 & 0 & 0 & 0 & 0 & & 0 \\
\hline MW & & & & & & & & \\
\hline
\end{tabular}

\begin{tabular}{|c|c|c|c|c|c|c|c|c|}
\hline PFD Stream Number & & 8 & 9 & 10 & 11 & 12 & 13 & 14 \\
\hline Stream Number & & Liquid Ammonia & $S-16$ & $S-17$ & S-18 & $S-19$ & S-27 & $5-31$ \\
\hline Stream Description & & \begin{tabular}{c|} 
Liquid \\
Ammonia Feed \\
\end{tabular} & $\begin{array}{c}\text { Fermentor } \\
\text { Output }\end{array}$ & R\#1 Output & R\#2 Output & R\#3 Output & Diesel Product & Biomass Purge \\
\hline Phase & & LIGUID & LIQUID & LIQUID & LIQUID & LIQUID & LIQUID & LIQUID \\
\hline Hydrocarbon Mass Flo & LB/HR & & & 44379 & 44284 & 44213 & 26108 & 384 \\
\hline Aqueous Mass Flow & LB/HR & 14.7 & 1662 & 52895 & 53135 & 53175 & 0 & 710 \\
\hline Biomass Mass Flow & LB/HR & & 205 & 12143 & 12143 & 12143 & 0 & 205 \\
\hline Sulfur in $\mathrm{HC}$ & PPM & & & 1728 & 257 & 10 & 10 & 10 \\
\hline Mass Flow Rate & LB/HR & 14.7 & 1867 & 109417 & 109562 & 109531 & 26108 & 1300 \\
\hline Temperature & $\mathrm{F}$ & -40 & 86 & 86 & 86 & 86 & 86 & 86 \\
\hline Pressure & PSIG & 210 & 33.1 & 36.4 & 36.4 & 26.8 & 5 & 5 \\
\hline Standard Liq Flow & $\mathrm{BPD}$ & 1.4 & 128 & 7806 & 7806 & 7806 & 2103 & 90 \\
\hline Vapor Flow & MSCFH & & & & & & & \\
\hline Wt \% Vapor & & 0 & 0 & 0 & 0 & 0 & 0 & 0 \\
\hline MW & & & & & & & & \\
\hline
\end{tabular}

\begin{tabular}{|l|l|c|c|c|c|c|c|c|}
\hline PFD Stream Number & & 15 & 16 & 17 & 18 & 19 & & \\
\hline Stream Number & & S-32 & S-38 & S-39 & Ash & Waste Water & & \\
\hline Stream Description & & $\begin{array}{c}\text { Reactor } \\
\text { Recvcle }\end{array}$ & $\begin{array}{c}\text { Reactor Air } \\
\text { Effluent }\end{array}$ & Gas Purge & $\begin{array}{c}\text { Incinerator } \\
\text { Waste }\end{array}$ & Waste Water & & \\
\hline Phase & & LIQUID & GAS & GAS & SOLID & LIQUID & & \\
\hline Hydrocarbon Mass Flo & LB/HR & 11938 & 199 & & & & & \\
\hline Aqueous Mass Flow & LB/HR & 45764 & 183 & & & 6480 & & \\
\hline Biomass Mass Flow & LB/HR & 11938 & & & & & & \\
\hline Sulfur in HC & PPM & 10 & 666 & & & & & \\
\hline Total Mass Flow Rate & LB/HR & 75622 & 11620 & 11297 & 30 & 6480 & & \\
\hline Temperature & F & 86 & 86 & 60 & 68 & 86 & & \\
\hline Pressure & PSIG & 37.4 & 10 & 1 & 0 & 10 & & \\
\hline Standard Liq Flow & BPD & 5190 & & & & 420 & & \\
\hline Vapor Flow & MSCFH & & 154 & 149 & & & & \\
\hline Wt \% Vapor & & 0 & 100 & 100 & 0 & 0 & & \\
\hline MW & & & & & & & & \\
\hline
\end{tabular}


Appendix D.7 - BDS Unit Equipment List and Budgetary Equipment Datasheets 


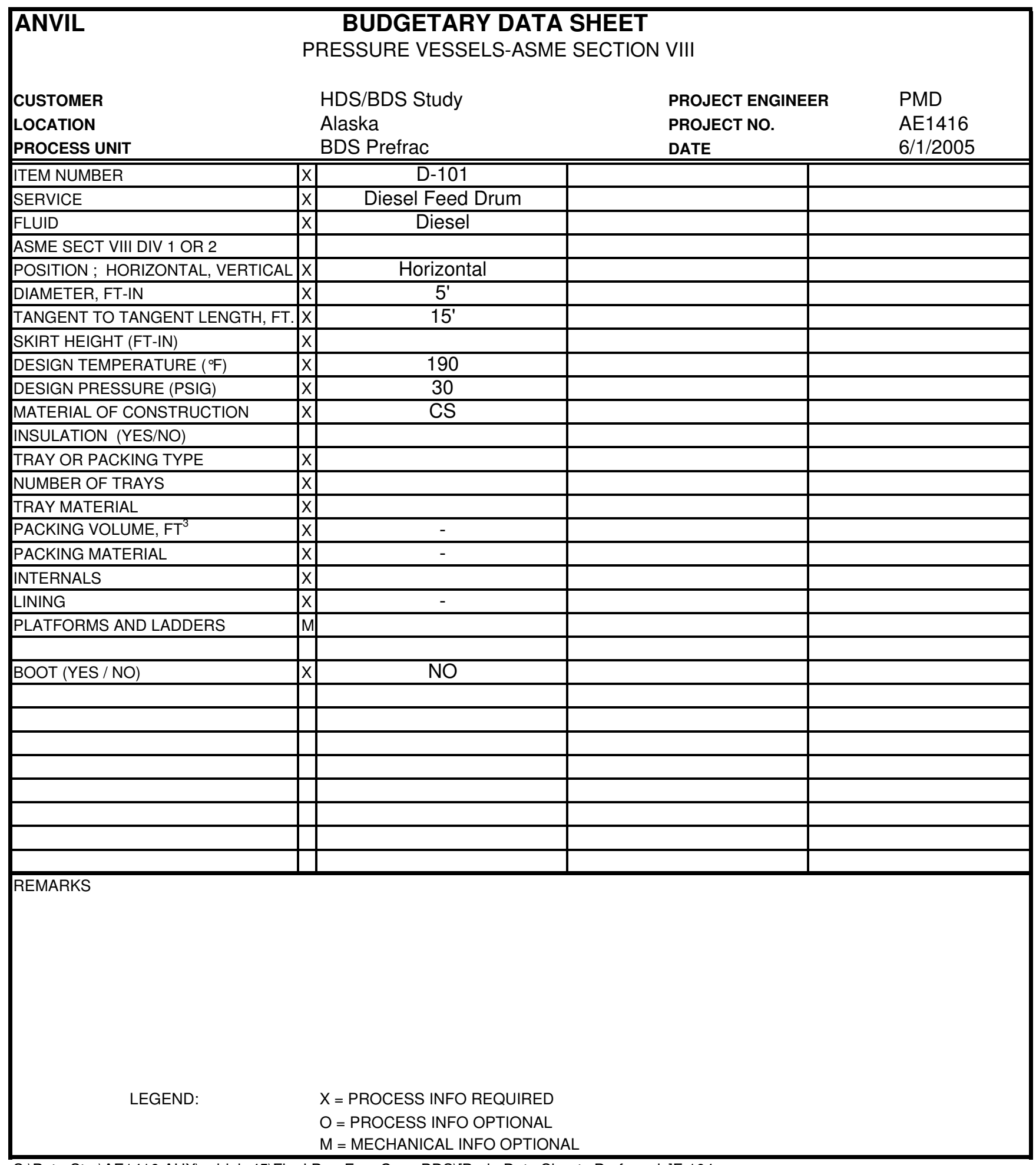




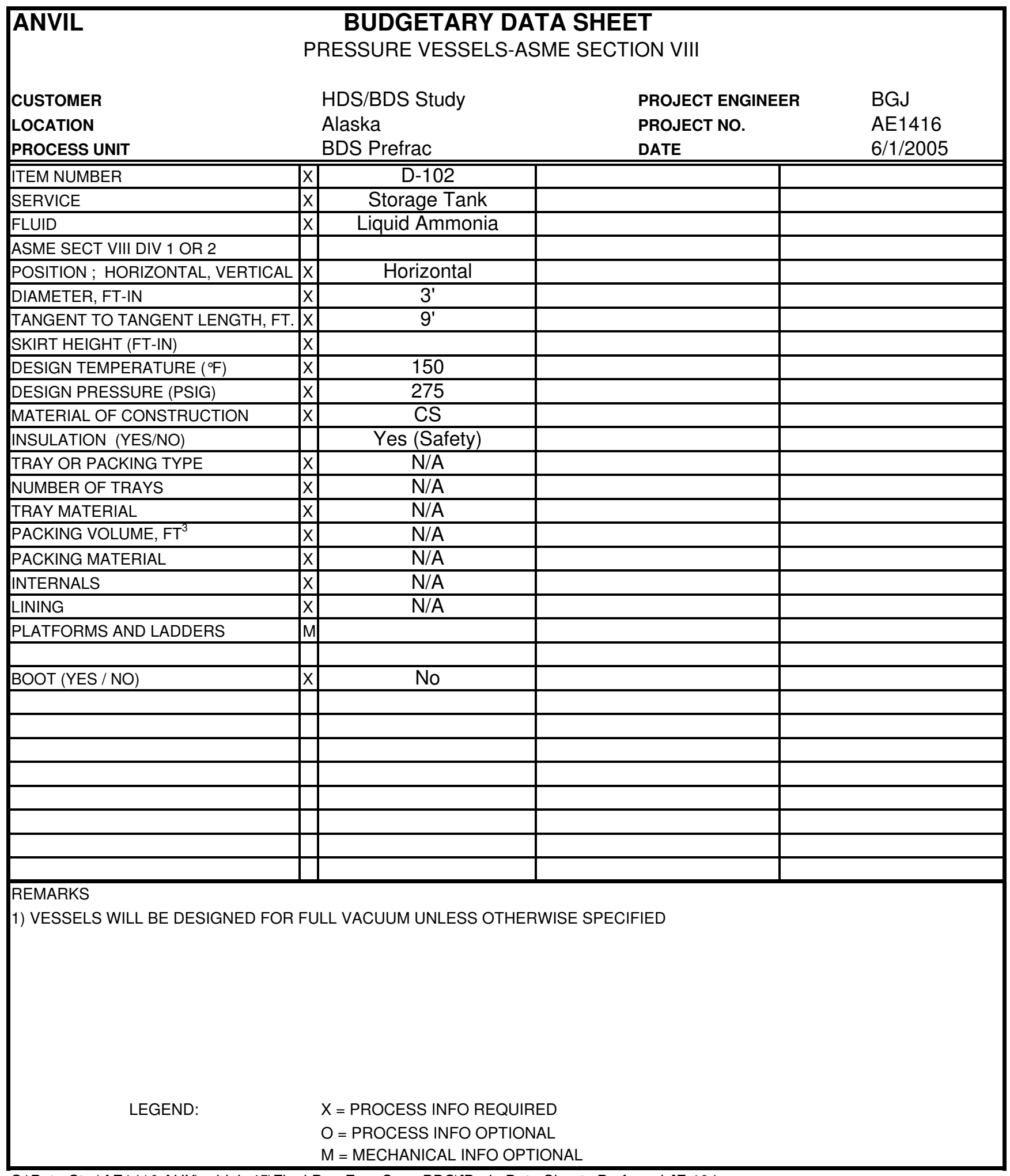




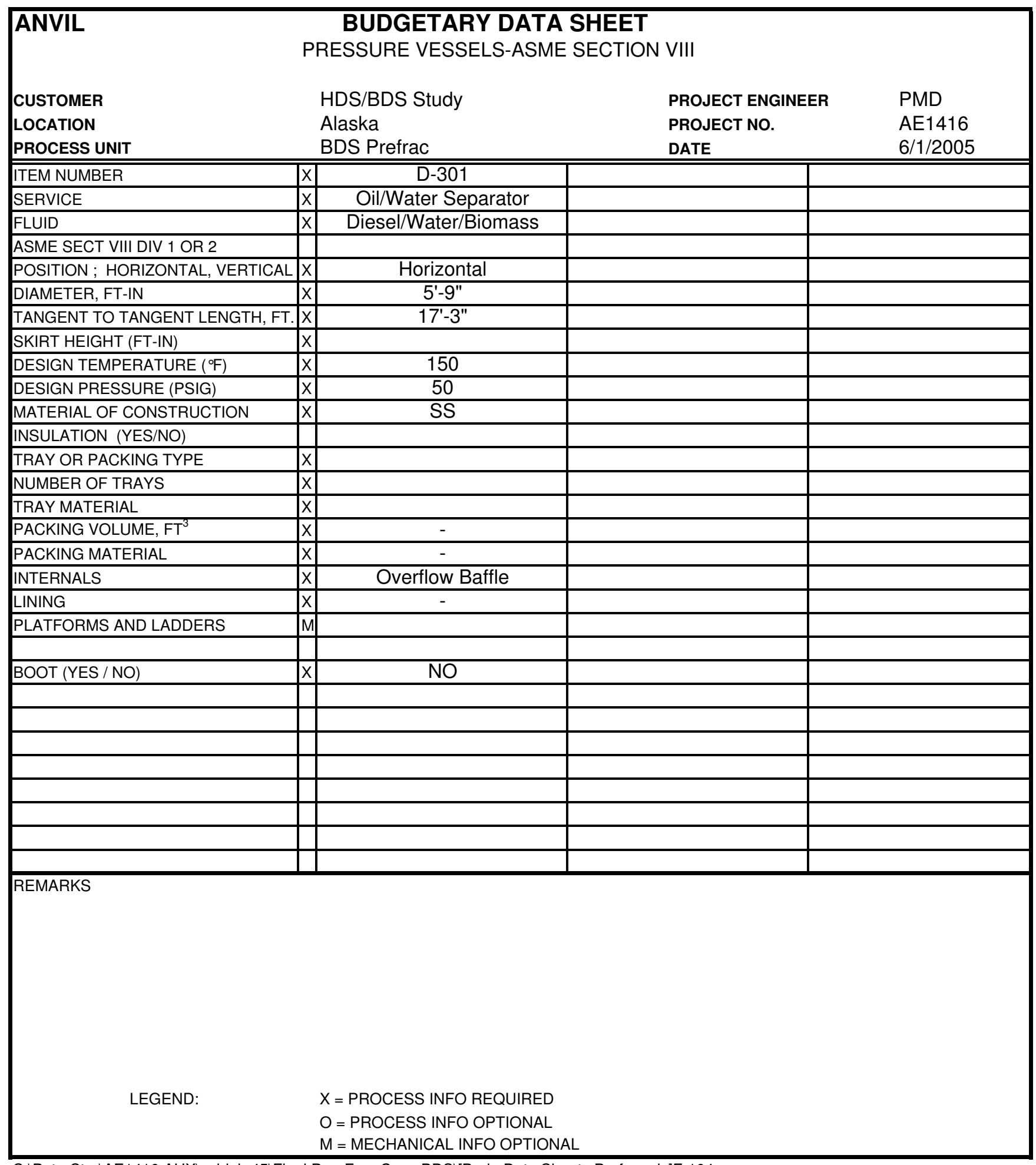




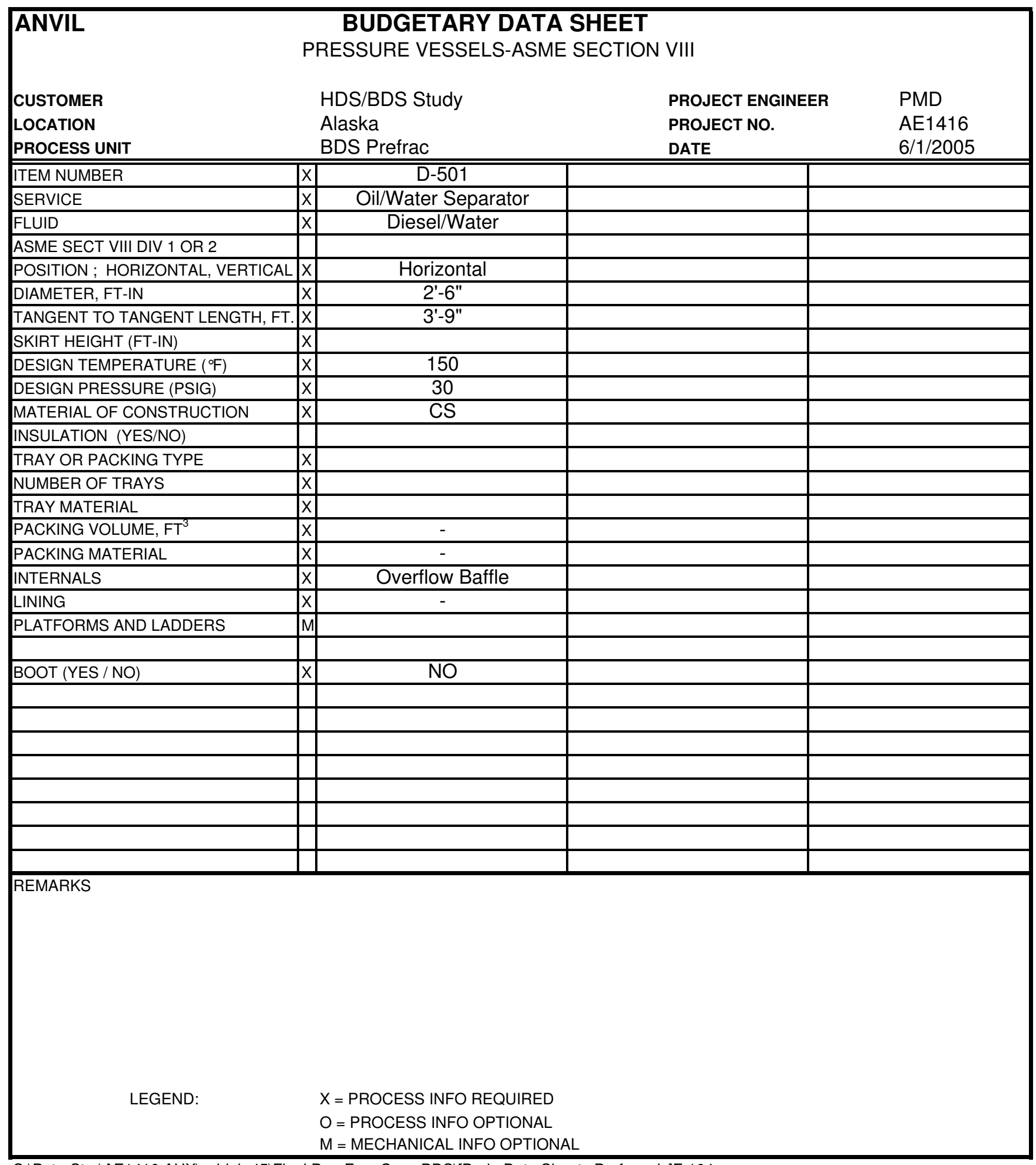




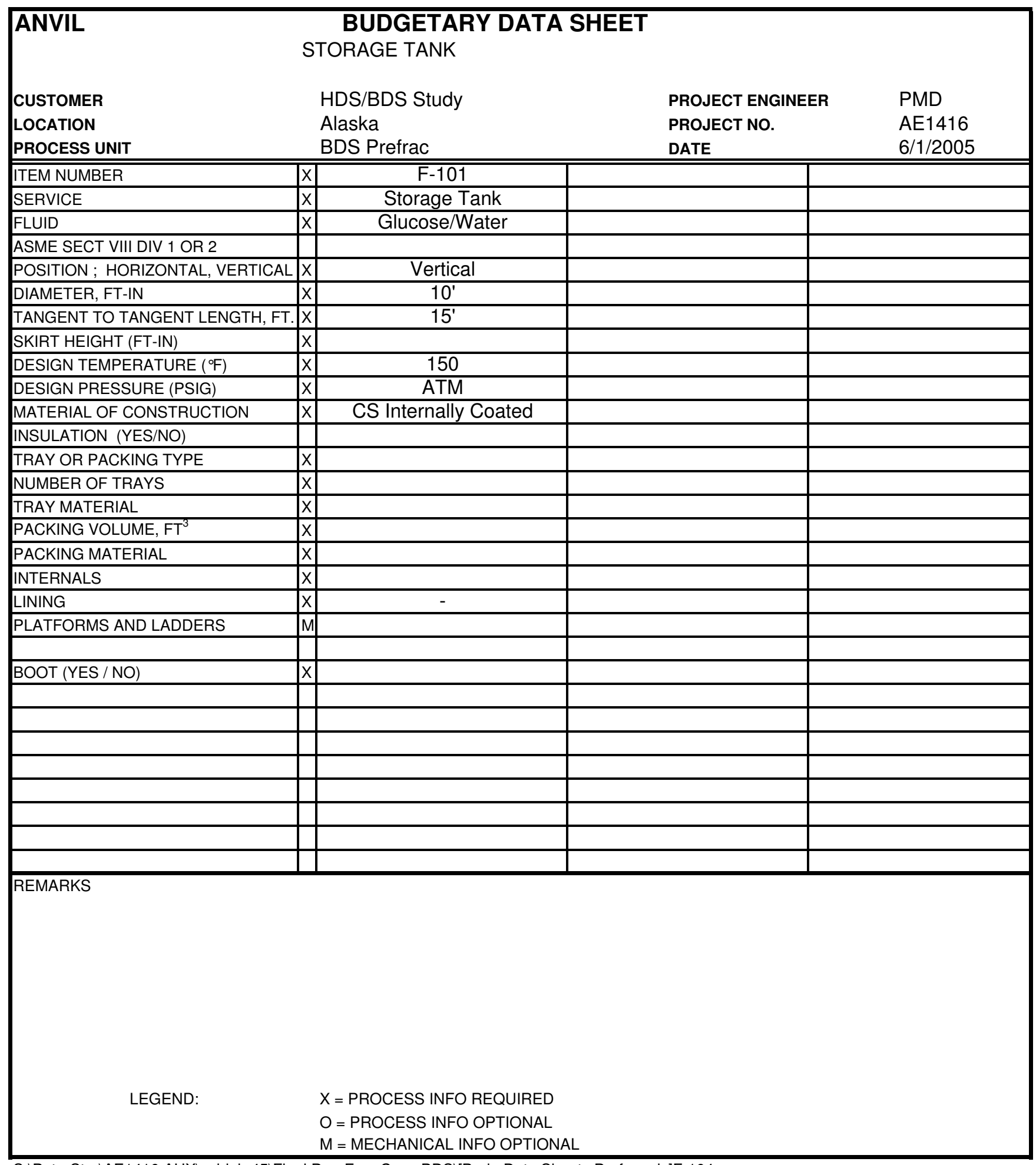




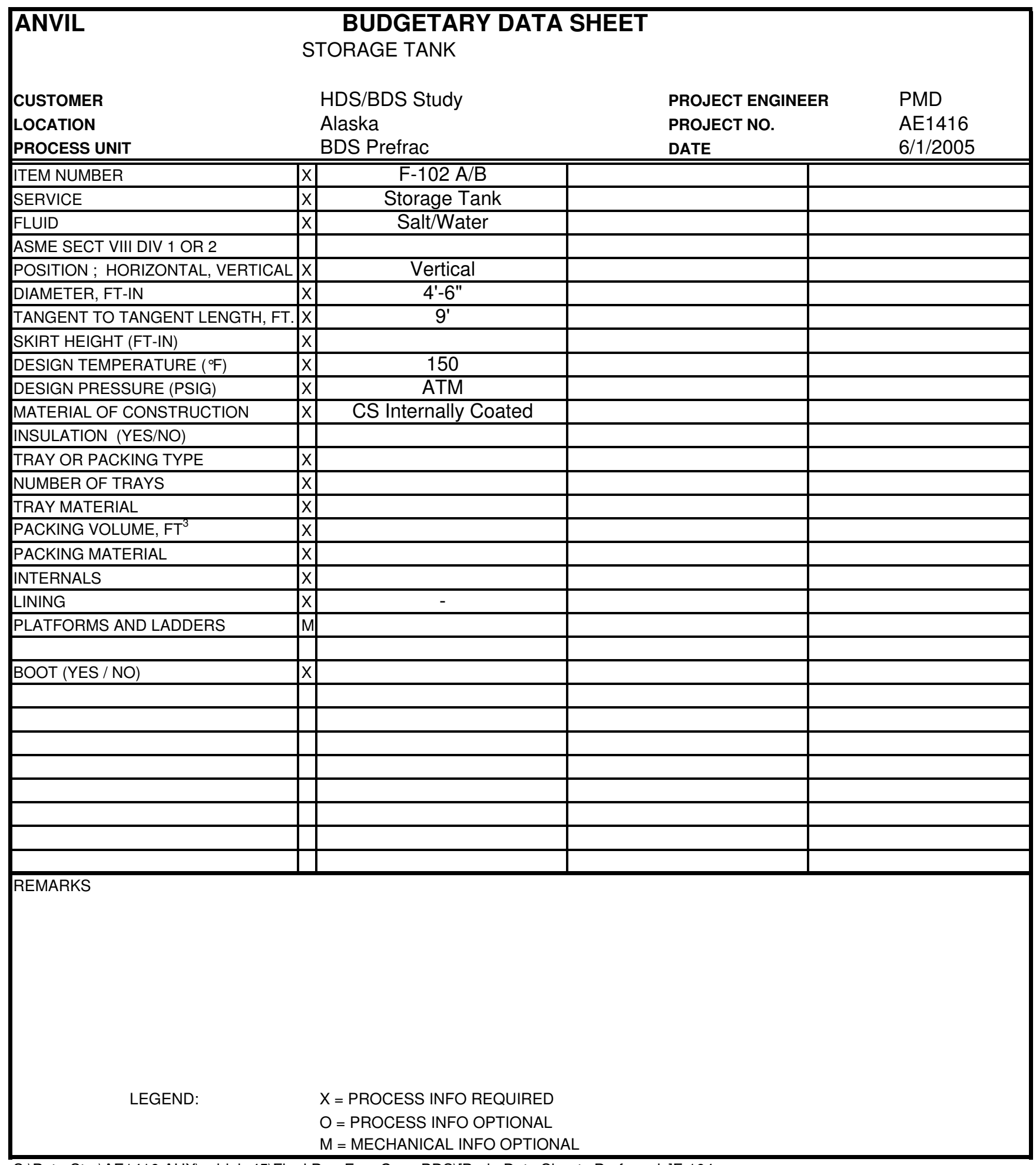




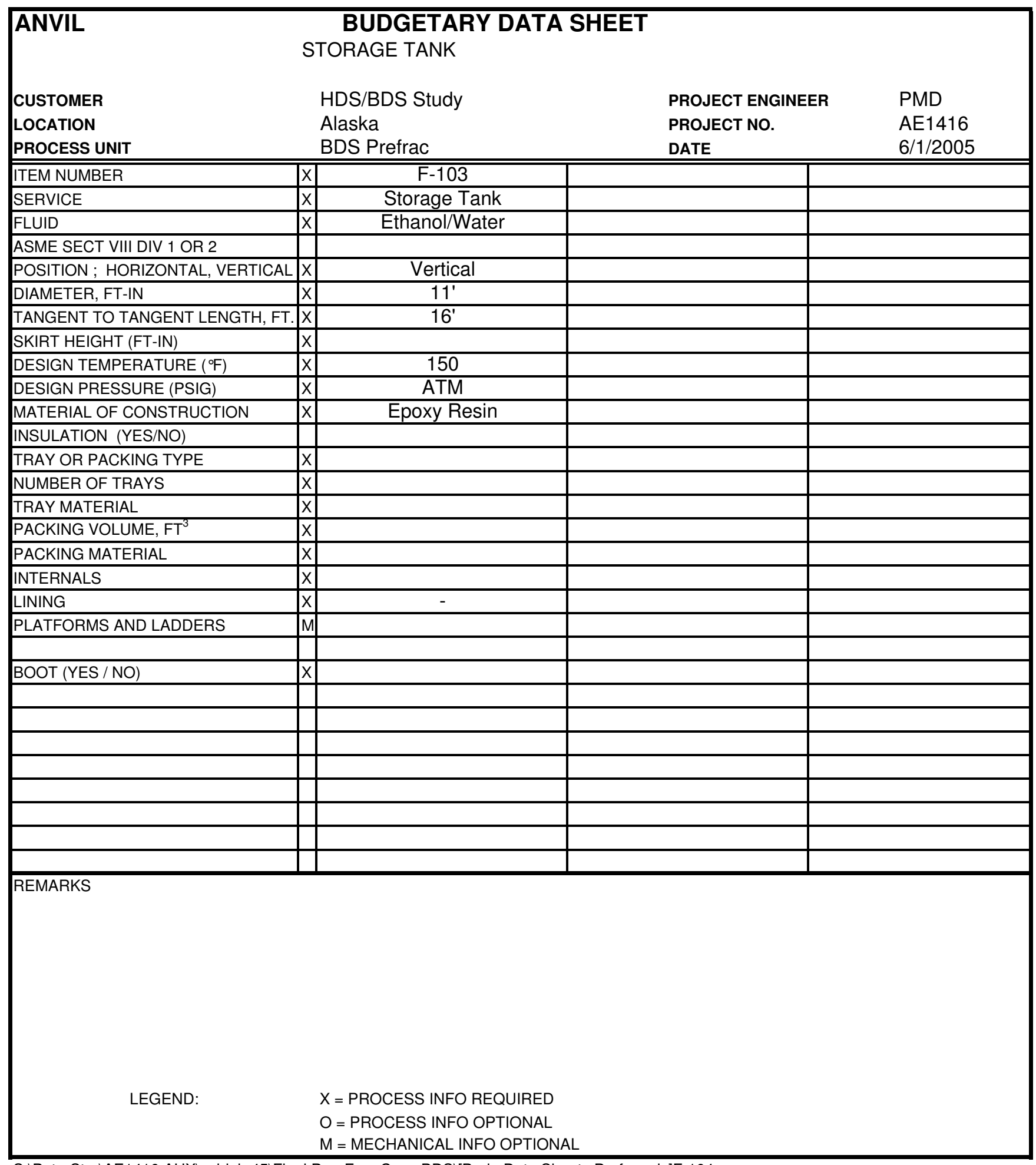




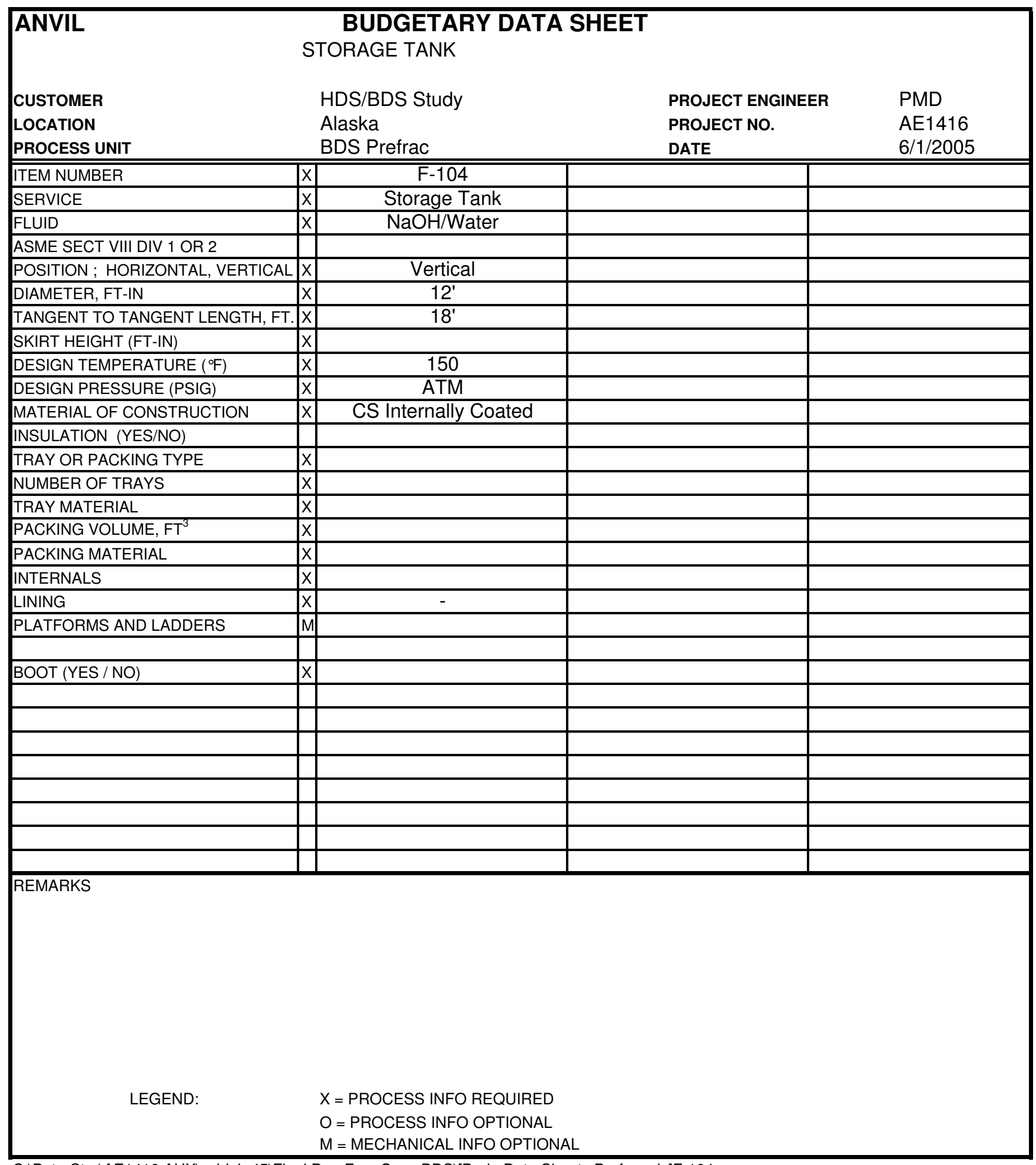




\begin{tabular}{|c|c|c|c|c|}
\hline ANVIL & & $\begin{array}{l}\text { UDGETARY DAT } \\
\text { PUMPS AND DRI }\end{array}$ & $\begin{array}{l}\text { A SHEET } \\
\text { ERS }\end{array}$ & \\
\hline CUSTOMER & & HDS/BDS Study & & PROJECT ENGINEER PMD \\
\hline LOCATION & & Alaska & & PROJECT NO. AE1416 \\
\hline PROCESS UNIT & & BDS Prefrac & & DATE 6/1/05 \\
\hline ITEM NUMBER & $\mathrm{x}$ & $\mathrm{J}-101 \mathrm{~A} / \mathrm{B}(1)$ & & \\
\hline SERVICE (FLUID) & $\mathrm{x}$ & Diesel Feed Pump & & \\
\hline TEMPERATURE OF FLUID & $\mathrm{x}$ & 140 & & \\
\hline SPECIFIC GRAVITY AT TEMPERATURE & $\mathrm{x}$ & 0.85 & & \\
\hline RATED FLOW (GPM) & $\mathrm{x}$ & 70 & & \\
\hline SUCTION PRESSURE, PSIG & $\mathrm{x}$ & 1.2 & & \\
\hline DISCHARGE PRESSURE PSIG & $\mathrm{x}$ & 61.3 & & \\
\hline NPSH AVAILABLE ( FT ) & $\mathrm{x}$ & & & \\
\hline CONSTRUCTION (API,ANSI) & $\mathrm{M}$ & & & \\
\hline PUMP TYPE (CENTRIFUGAL, RECIP,ETC.) & $\mathrm{x}$ & Centrifugal & & \\
\hline CASING MATERIAL & $\mathrm{M}$ & CS & & \\
\hline IMPELLER MATERIAL & $\mathrm{M}$ & $\mathrm{CS}$ & & \\
\hline TYPE OF DRIVER (MOTOR/TURBINE) & $\mathrm{x}$ & Motor & & \\
\hline TYPE OF SPARE (MOTOR/TURBINE) & $\mathrm{x}$ & Motor & & \\
\hline ELECTRIC POWER (HP) & $\mathrm{x}$ & 5 & & \\
\hline STEAM CONDITIONS & $\mathrm{x}$ & $\mathrm{N} / \mathrm{A}$ & & \\
\hline SEALS (SINGLE, DOUBLE, TANDEM) & $\mathrm{M}$ & & & \\
\hline API SEAL FLUSH PLAN NUMBER & $\mathrm{M}$ & & & \\
\hline & & API Standard & & \\
\hline DIFFERENTIAL PRESSURE, PSI & & 60.1 & & \\
\hline DIFFERENTIAL HEAD, FT & & 163.1 & & \\
\hline & & & & \\
\hline & & & & \\
\hline & & & & \\
\hline & & & & \\
\hline $\begin{array}{l}\text { REMARKS } \\
\text { 1) One operating pump + one spare } \\
\text { 2) } 0.125 " \mathrm{CA} / \text { Minimum design temp }\end{array}$ & atur & re is $-20^{\circ} \mathrm{F}$. & & \\
\hline LEGEND: & & $\begin{array}{l}X=\text { PROCESS INFO REC } \\
O=\text { PROCESS INFO OPT } \\
M=\text { MECHANICAL INFO }\end{array}$ & $\begin{array}{l}\text { IRED } \\
\text { DNAL } \\
\text { DTIONAL }\end{array}$ & \\
\hline
\end{tabular}

S:IPetroStar\AE1416.AUXIsubjob 45|Final Pre_Frac Case BDS\[Budg Data Sheets Prefrac.xls]F-104 


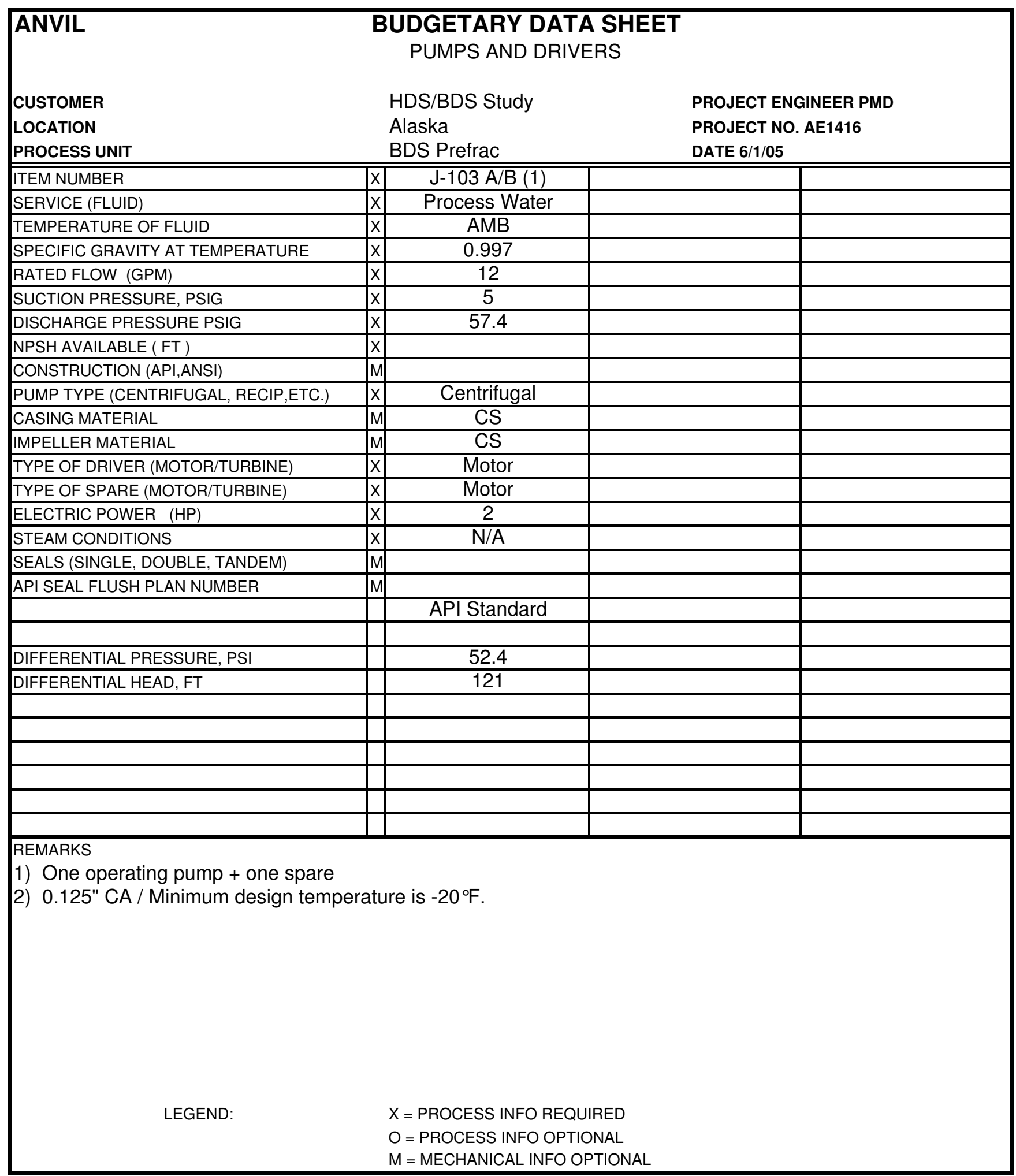

S:IPetroStar\AE1416.AUXIsubjob 45|Final Pre_Frac Case BDS\[Budg Data Sheets Prefrac.xls]F-104 


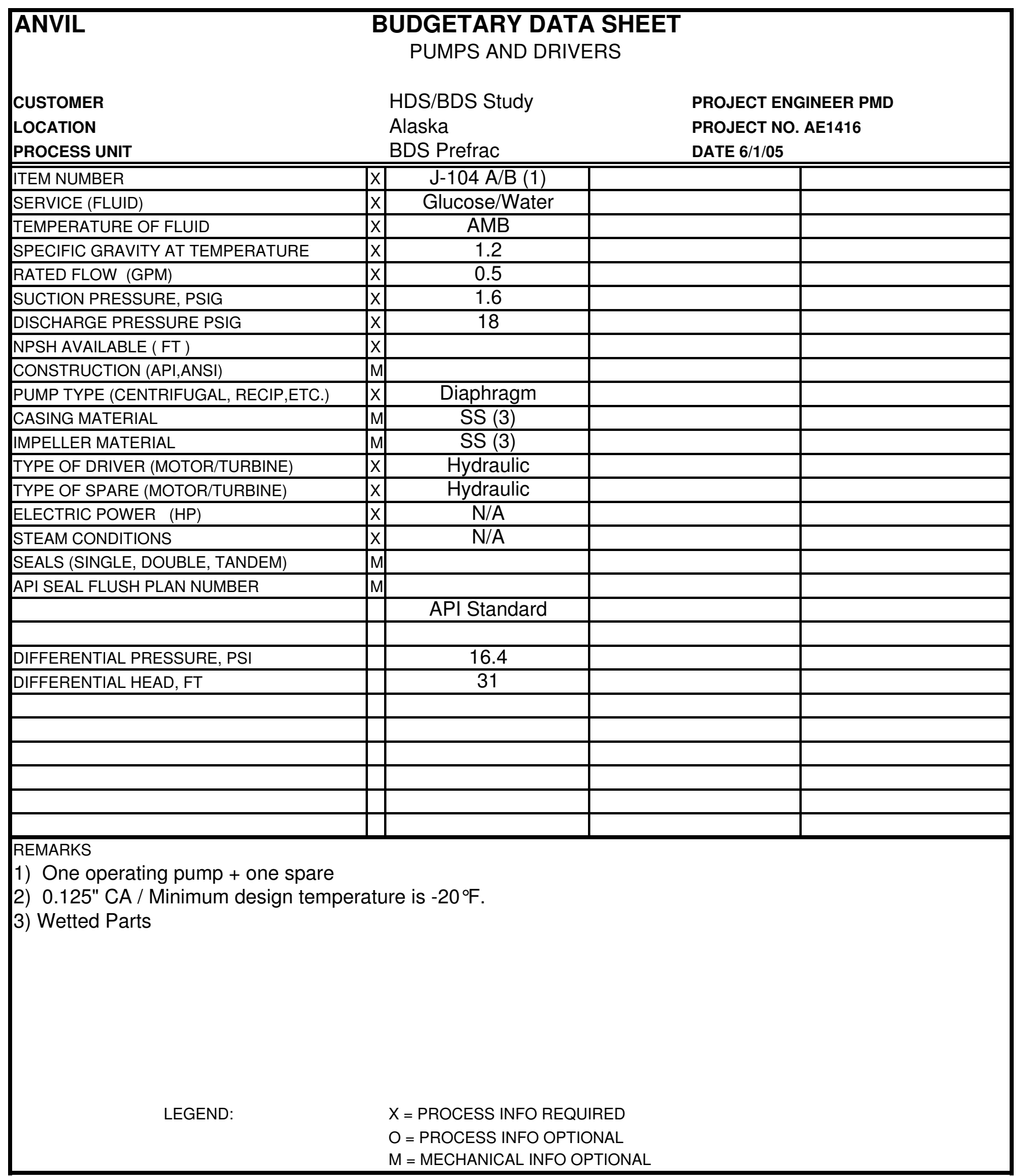

S:IPetroStar\AE1416.AUXIsubjob 45|Final Pre_Frac Case BDS\[Budg Data Sheets Prefrac.xls]F-104 


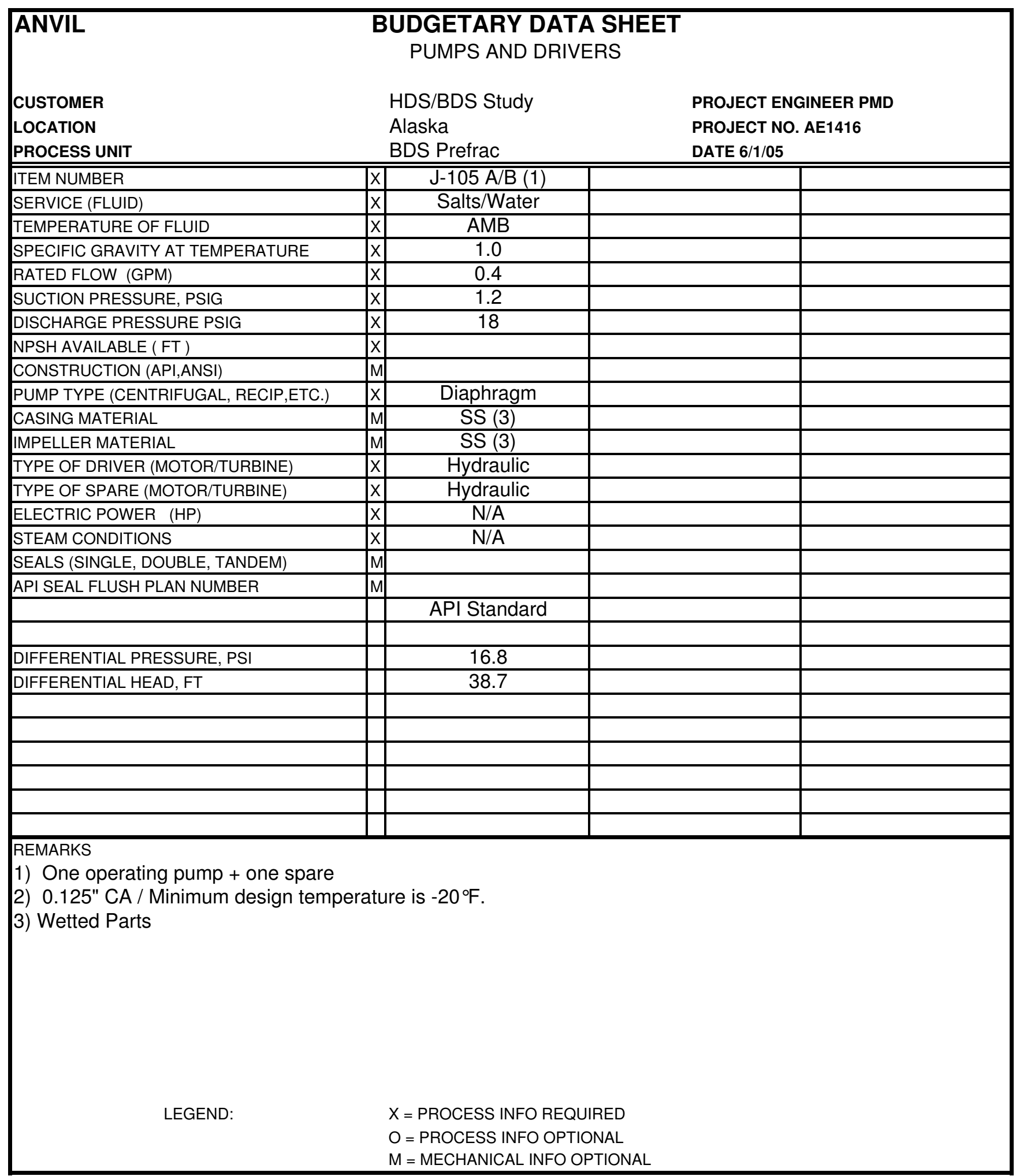

S:IPetroStar\AE1416.AUXIsubjob 45|Final Pre_Frac Case BDS\[Budg Data Sheets Prefrac.xls]F-104 


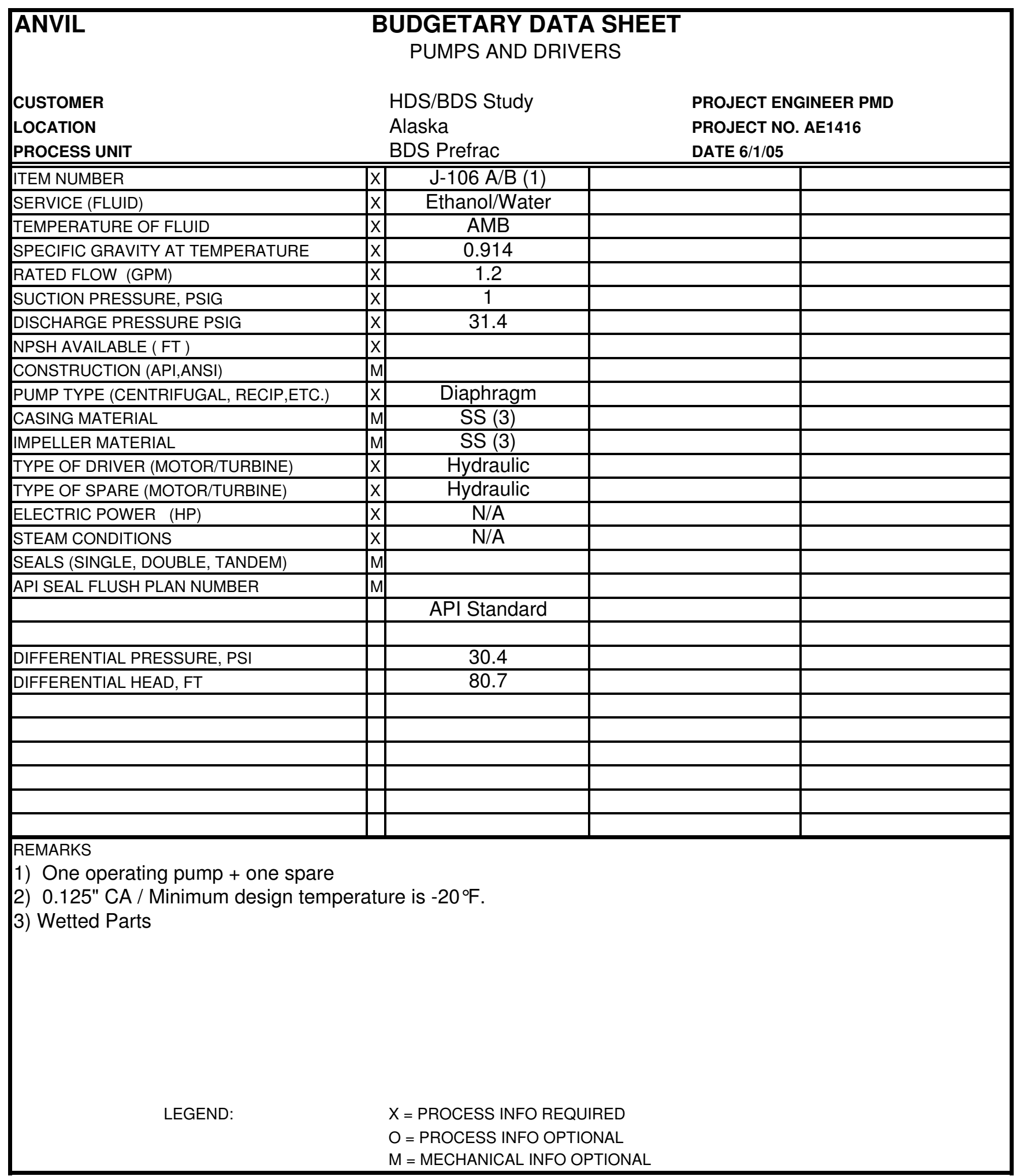

S:IPetroStar\AE1416.AUXIsubjob 45|Final Pre_Frac Case BDS\[Budg Data Sheets Prefrac.xls]F-104 


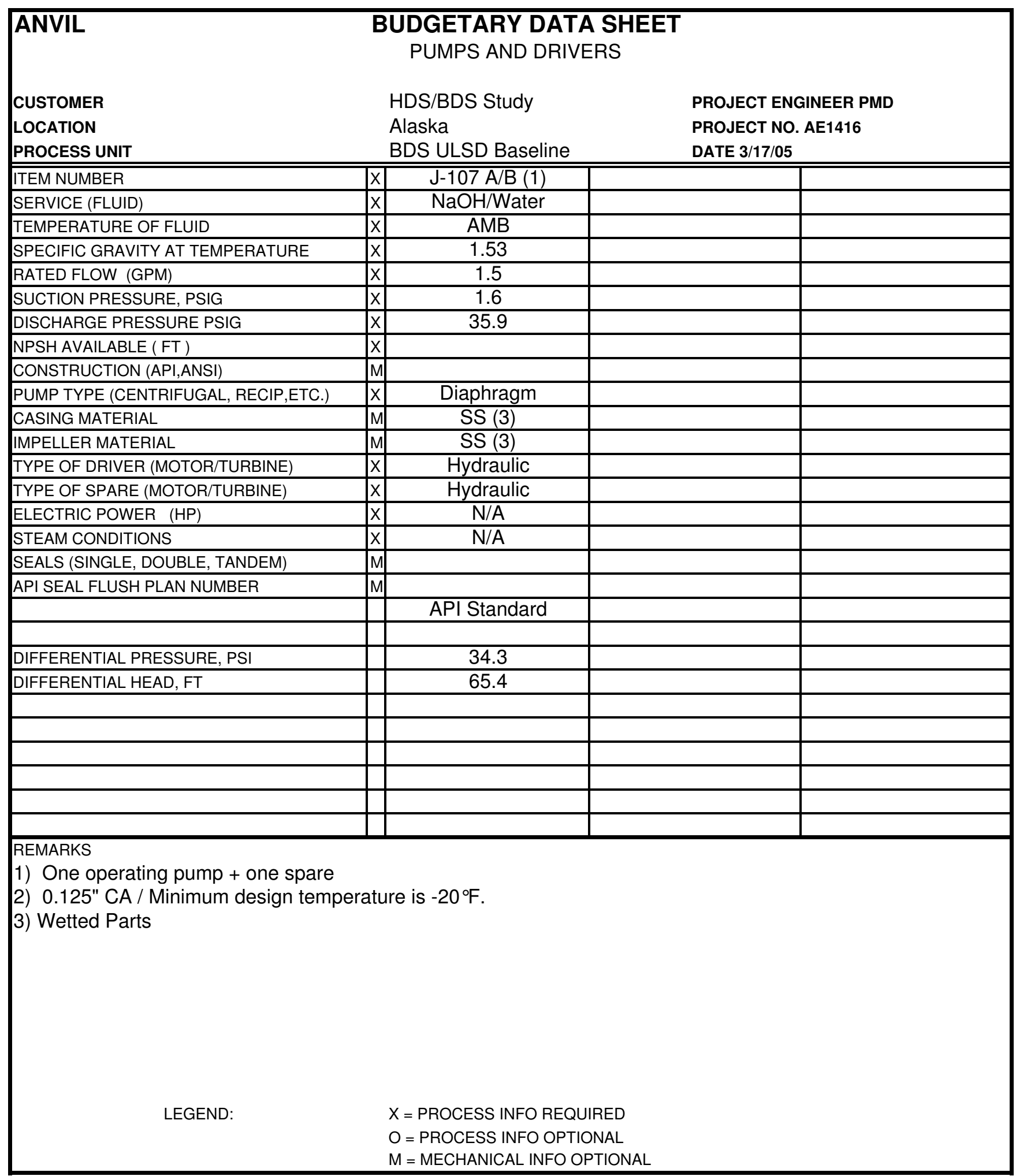

S:IPetroStar\AE1416.AUXIsubjob 45|Final Pre_Frac Case BDS\[Budg Data Sheets Prefrac.xls]F-104 


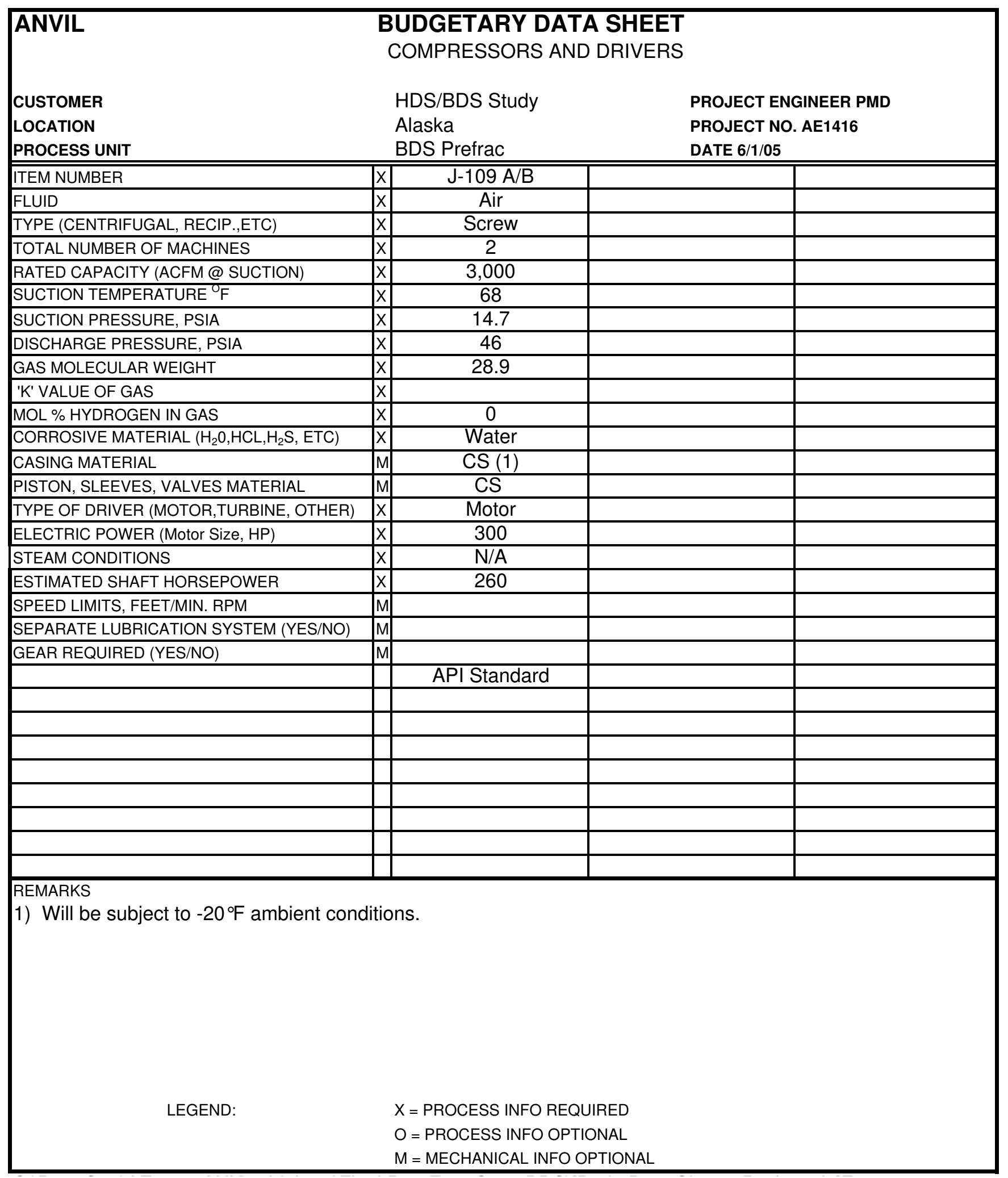




\begin{tabular}{|c|c|c|c|c|}
\hline ANVIL & & $\begin{array}{l}\text { UDGETARY DATA } \\
\text { PUMPS AND DRIVE }\end{array}$ & $\begin{array}{l}\text { SHEET } \\
\text { RS }\end{array}$ & \\
\hline CUSTOMER & & HDS/BDS Study & & PROJECT ENGINEER PMD \\
\hline LOCATION & & Alaska & & PROJECT NO. AE1416 \\
\hline PROCESS UNIT & & BDS Prefrac & & DATE 6/1/05 \\
\hline ITEM NUMBER & $\mathrm{X}$ & J-201 A/B (1) & & \\
\hline SERVICE (FLUID) & $x$ & Biomass/Salts/Water & & \\
\hline TEMPERATURE OF FLUID & $x$ & 86 & & \\
\hline SPECIFIC GRAVITY AT TEMPERATURE & $x$ & 1.0 & & \\
\hline RATED FLOW (GPM) & $x$ & 5 & & \\
\hline SUCTION PRESSURE, PSIG & $x$ & 14.1 & & \\
\hline DISCHARGE PRESSURE PSIG & $\mathrm{x}$ & 33.1 & & \\
\hline NPSH AVAILABLE ( FT ) & $\mathrm{x}$ & & & \\
\hline CONSTRUCTION (API,ANSI) & $\mathrm{M}$ & & & \\
\hline PUMP TYPE (CENTRIFUGAL, RECIP,ETC.) & $\mathrm{x}$ & Rotary Lobe & & \\
\hline CASING MATERIAL & $\mathrm{M}$ & SS (3) & & \\
\hline IMPELLER MATERIAL & $\mathrm{M}$ & SS (3) & & \\
\hline TYPE OF DRIVER (MOTOR/TURBINE) & $x$ & Motor & & \\
\hline TYPE OF SPARE (MOTOR/TURBINE) & $x$ & Motor & & \\
\hline ELECTRIC POWER (HP) & $x$ & 0.5 & & \\
\hline STEAM CONDITIONS & $x$ & $\mathrm{~N} / \mathrm{A}$ & & \\
\hline SEALS (SINGLE, DOUBLE, TANDEM) & $\mathrm{M}$ & Double & & \\
\hline API SEAL FLUSH PLAN NUMBER & $\mathrm{M}$ & & & \\
\hline & & API Standard & & \\
\hline DIFFERENTIAL PRESSURE, PSI & & 19 & & \\
\hline DIFFERENTIAL HEAD, FT & & 43.4 & & \\
\hline & & & & \\
\hline & & & & \\
\hline & & & & \\
\hline & & & & \\
\hline $\begin{array}{l}\text { REMARKS } \\
\text { 1) One operating pump + one spare } \\
\text { 2) } 0.125^{\prime \prime} \text { CA / Minimum design temp } \\
\text { 3) Wetted Parts }\end{array}$ & Ir & $\mathrm{re}$ is $-20^{\circ} \mathrm{F}$. & & \\
\hline LEGEND: & & $\begin{array}{l}X=\text { PROCESS INFO REQUI } \\
O=\text { PROCESS INFO OPTIO } \\
M=\text { MECHANICAL INFO OP }\end{array}$ & $\begin{array}{l}\text { RED } \\
\text { NAL } \\
\text { TIONAL }\end{array}$ & \\
\hline
\end{tabular}

S:IPetroStar\AE1416.AUXIsubjob 45|Final Pre_Frac Case BDS\[Budg Data Sheets Prefrac.xls]F-104 


\begin{tabular}{|c|c|c|c|c|}
\hline ANVIL & & $\begin{array}{l}\text { UDGETARY DATA } \\
\text { PUMPS AND DRIVE }\end{array}$ & $\begin{array}{l}\text { SHEET } \\
\text { RS }\end{array}$ & \\
\hline CUSTOMER & & HDS/BDS Study & & PROJECT ENGINEER PMD \\
\hline LOCATION & & Alaska & & PROJECT NO. AE1416 \\
\hline PROCESS UNIT & & BDS Prefrac & & DATE 6/1/05 \\
\hline ITEM NUMBER & $\mathrm{x}$ & $\mathrm{J}-202 \mathrm{~A} / \mathrm{B}(1)$ & & \\
\hline SERVICE (FLUID) & $x$ & Biomass/Diesel/Water & & \\
\hline TEMPERATURE OF FLUID & $\mathrm{x}$ & 86 & & \\
\hline SPECIFIC GRAVITY AT TEMPERATURE & $\mathrm{x}$ & 0.93 & & \\
\hline RATED FLOW (GPM) & $x$ & 250 & & \\
\hline SUCTION PRESSURE, PSIG & $x$ & 18.4 & & \\
\hline DISCHARGE PRESSURE PSIG & $\mathrm{x}$ & 36.4 & & \\
\hline NPSH AVAILABLE ( FT ) & $\mathrm{x}$ & & & \\
\hline CONSTRUCTION (API,ANSI) & $\mathrm{M}$ & & & \\
\hline PUMP TYPE (CENTRIFUGAL, RECIP,ETC.) & $\mathrm{x}$ & Cent & & \\
\hline CASING MATERIAL & $\mathrm{M}$ & SS & & \\
\hline IMPELLER MATERIAL & $\mathrm{M}$ & SS & & \\
\hline TYPE OF DRIVER (MOTOR/TURBINE) & $\mathrm{x}$ & Motor & & \\
\hline TYPE OF SPARE (MOTOR/TURBINE) & $\mathrm{x}$ & Motor & & \\
\hline ELECTRIC POWER (HP) & $\mathrm{x}$ & 5 & & \\
\hline STEAM CONDITIONS & $\mathrm{x}$ & $\mathrm{N} / \mathrm{A}$ & & \\
\hline SEALS (SINGLE, DOUBLE, TANDEM) & $\mathrm{M}$ & Double & & \\
\hline API SEAL FLUSH PLAN NUMBER & $\mathrm{M}$ & & & \\
\hline & & API Standard & & \\
\hline DIFFERENTIAL PRESSURE, PSI & & 18 & & \\
\hline DIFFERENTIAL HEAD, FT & & 44.7 & & \\
\hline & & & & \\
\hline & & & & \\
\hline & & & & \\
\hline & & & & \\
\hline $\begin{array}{l}\text { REMARKS } \\
\text { 1) One operating pump + one spare } \\
\text { 2) } 0.125 " \mathrm{CA} / \text { Minimum design temp }\end{array}$ & tu & re is $-20^{\circ} \mathrm{F}$. & & \\
\hline LEGEND: & & $\begin{array}{l}X=\text { PROCESS INFO REQUIF } \\
O=\text { PROCESS INFO OPTIOI } \\
M=\text { MECHANICAL INFO OP }\end{array}$ & $\begin{array}{l}\text { RED } \\
\text { NAL } \\
\text { TIONAL }\end{array}$ & \\
\hline
\end{tabular}

S:IPetroStar\AE1416.AUXIsubjob 45|Final Pre_Frac Case BDS\[Budg Data Sheets Prefrac.xls]F-104 


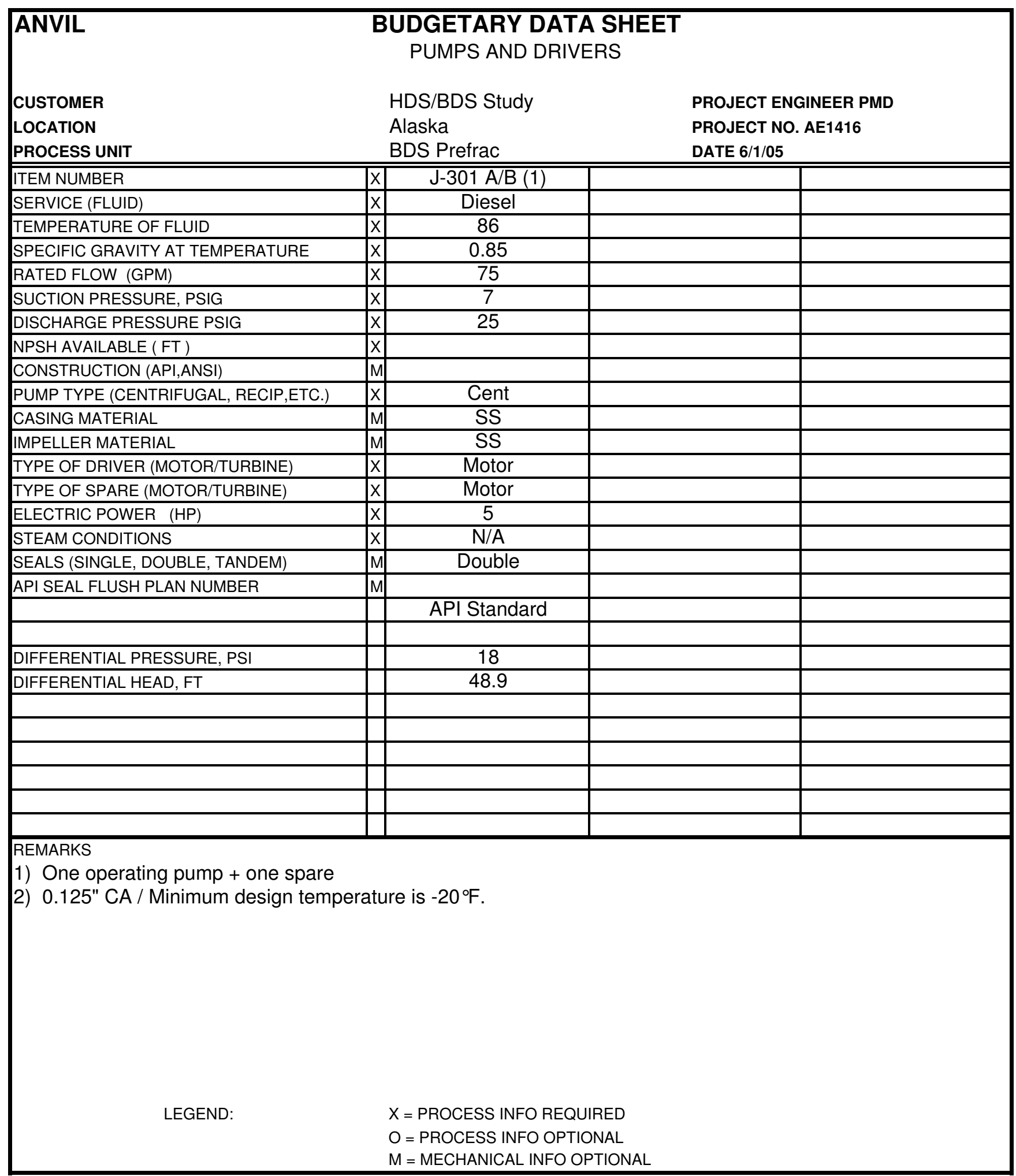

S:IPetroStar\AE1416.AUXIsubjob 45|Final Pre_Frac Case BDS\[Budg Data Sheets Prefrac.xls]F-104 


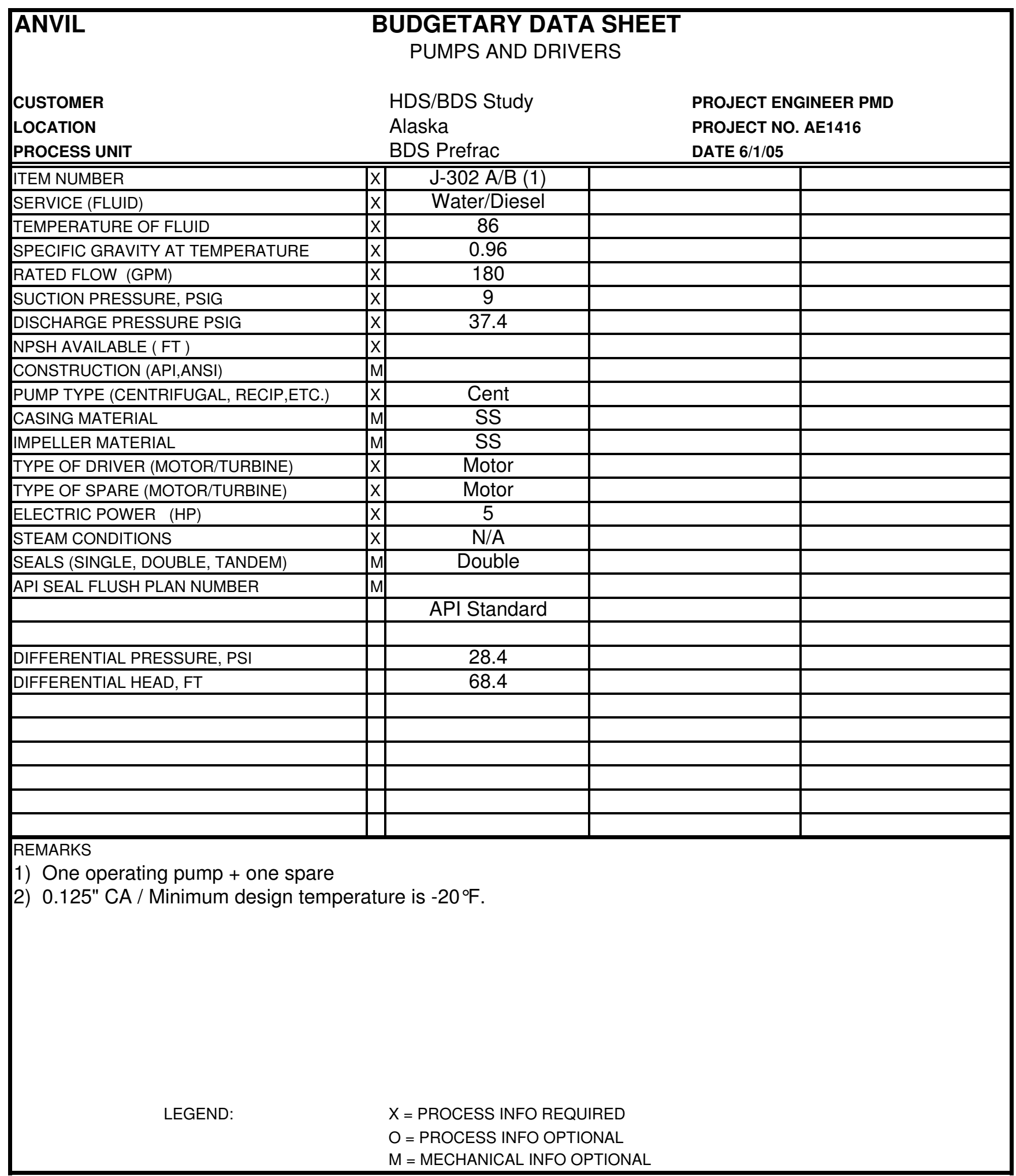

S:IPetroStar\AE1416.AUXIsubjob 45|Final Pre_Frac Case BDS\[Budg Data Sheets Prefrac.xls]F-104 


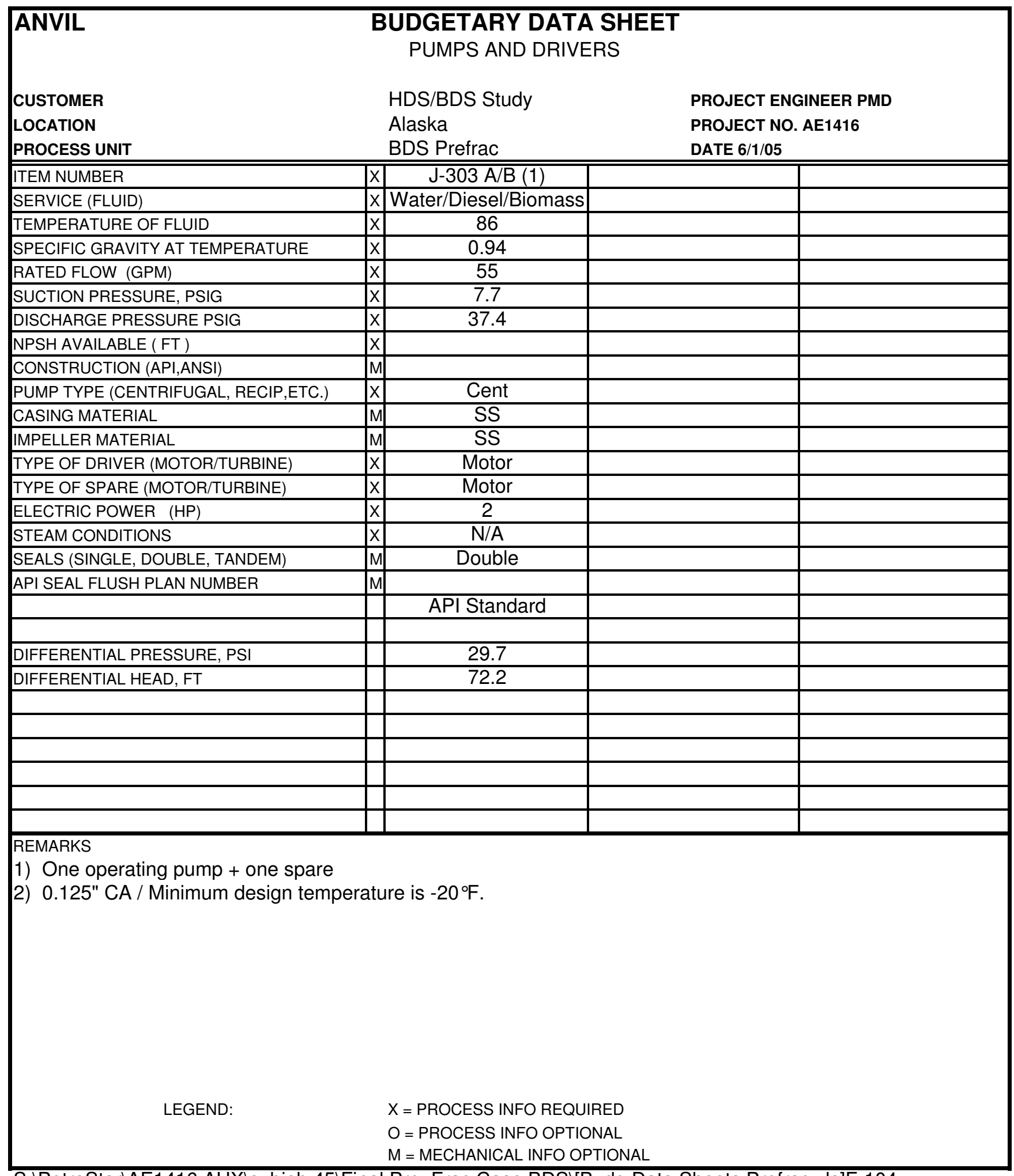

S:IPetroStar\AE1416.AUXIsubjob 45|Final Pre_Frac Case BDS\[Budg Data Sheets Prefrac.xls]F-104 


\begin{tabular}{|c|c|c|c|c|}
\hline ANVIL & & $\begin{array}{l}\text { UDGETARY DATA } \\
\text { PUMPS AND DRIVE }\end{array}$ & $\begin{array}{l}\text { SHEET } \\
\text { RS }\end{array}$ & \\
\hline CUSTOMER & & HDS/BDS Study & & PROJECT ENGINEER PMD \\
\hline LOCATION & & Alaska & & PROJECT NO. AE1416 \\
\hline PROCESS UNIT & & BDS Prefrac & & DATE 6/1/05 \\
\hline ITEM NUMBER & $\mathrm{x}$ & J-304 A/B (1) & & \\
\hline SERVICE (FLUID) & $x$ & Water/Diesel/Biomass & & \\
\hline TEMPERATURE OF FLUID & $x$ & 86 & & \\
\hline SPECIFIC GRAVITY AT TEMPERATURE & $x$ & 0.94 & & \\
\hline RATED FLOW (GPM) & $x$ & 15 & & \\
\hline SUCTION PRESSURE, PSIG & $x$ & 7.7 & & \\
\hline DISCHARGE PRESSURE PSIG & $\mathrm{x}$ & 37.4 & & \\
\hline NPSH AVAILABLE ( FT ) & $\mathrm{x}$ & & & \\
\hline CONSTRUCTION (API,ANSI) & $\mathrm{M}$ & & & \\
\hline PUMP TYPE (CENTRIFUGAL, RECIP,ETC.) & $\mathrm{x}$ & Cent & & \\
\hline CASING MATERIAL & $\mathrm{M}$ & SS & & \\
\hline IMPELLER MATERIAL & $\mathrm{M}$ & SS & & \\
\hline TYPE OF DRIVER (MOTOR/TURBINE) & $x$ & Motor & & \\
\hline TYPE OF SPARE (MOTOR/TURBINE) & $x$ & Motor & & \\
\hline ELECTRIC POWER (HP) & $x$ & 1.5 & & \\
\hline STEAM CONDITIONS & $x$ & $\mathrm{~N} / \mathrm{A}$ & & \\
\hline SEALS (SINGLE, DOUBLE, TANDEM) & $\mathrm{M}$ & Double & & \\
\hline API SEAL FLUSH PLAN NUMBER & $\mathrm{M}$ & & & \\
\hline & & API Standard & & \\
\hline DIFFERENTIAL PRESSURE, PSI & & 29.7 & & \\
\hline DIFFERENTIAL HEAD, FT & & 72.2 & & \\
\hline & & & & \\
\hline & & & & \\
\hline & & & & \\
\hline & & & & \\
\hline $\begin{array}{l}\text { REMARKS } \\
\text { 1) One operating pump + one spare } \\
\text { 2) } 0.125 " \mathrm{CA} / \text { Minimum design temp }\end{array}$ & atur & re is $-20^{\circ} \mathrm{F}$. & & \\
\hline LEGEND: & & $\begin{array}{l}X=\text { PROCESS INFO REQUI } \\
O=\text { PROCESS INFO OPTIO } \\
M=\text { MECHANICAL INFO OP }\end{array}$ & $\begin{array}{l}\text { RED } \\
\text { NAL } \\
\text { TIONAL }\end{array}$ & \\
\hline
\end{tabular}

S:IPetroStar\AE1416.AUXIsubjob 45|Final Pre_Frac Case BDS\[Budg Data Sheets Prefrac.xls]F-104 


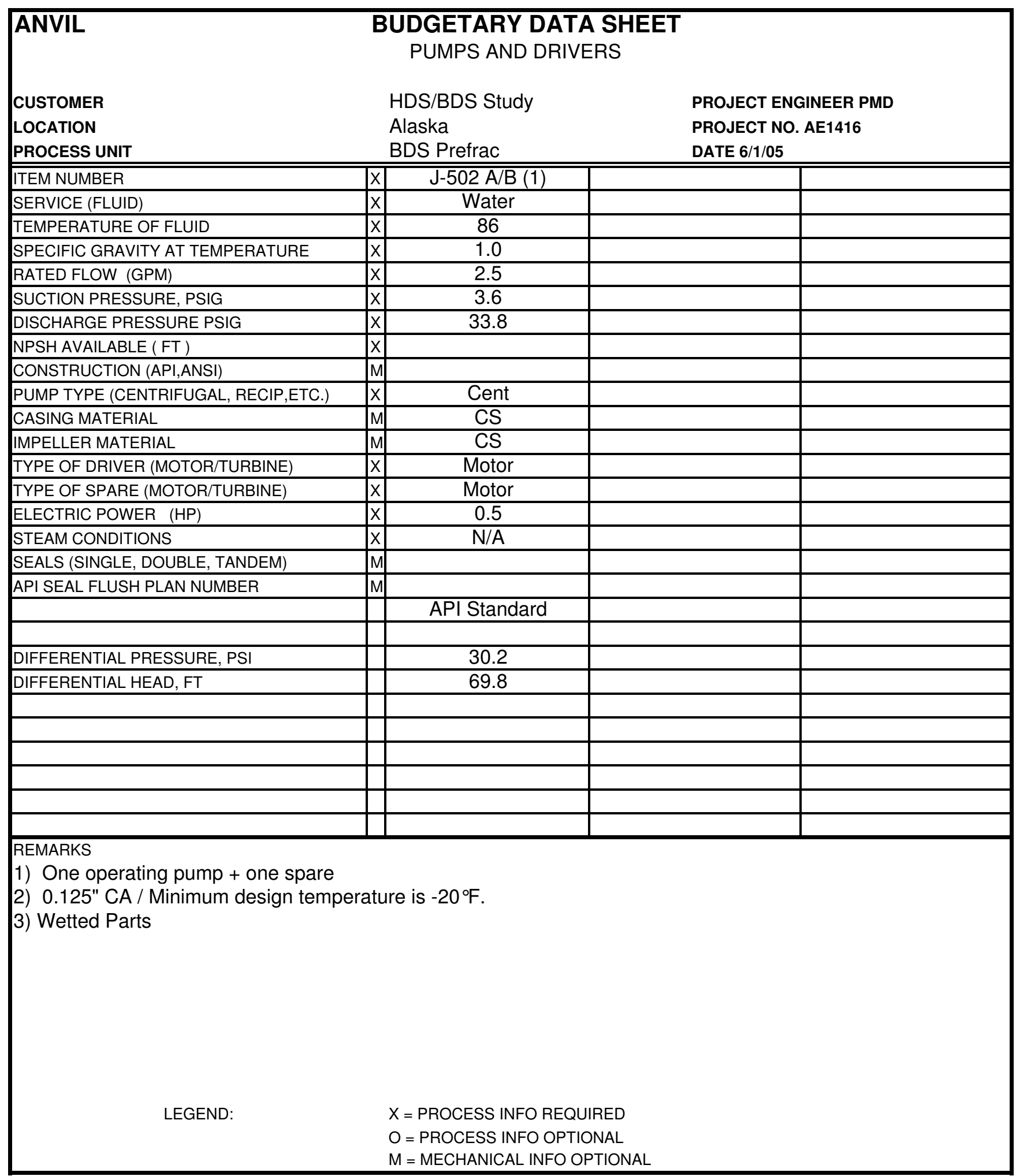

S:IPetroStar\AE1416.AUXIsubjob 45|Final Pre_Frac Case BDS\[Budg Data Sheets Prefrac.xls]F-104 


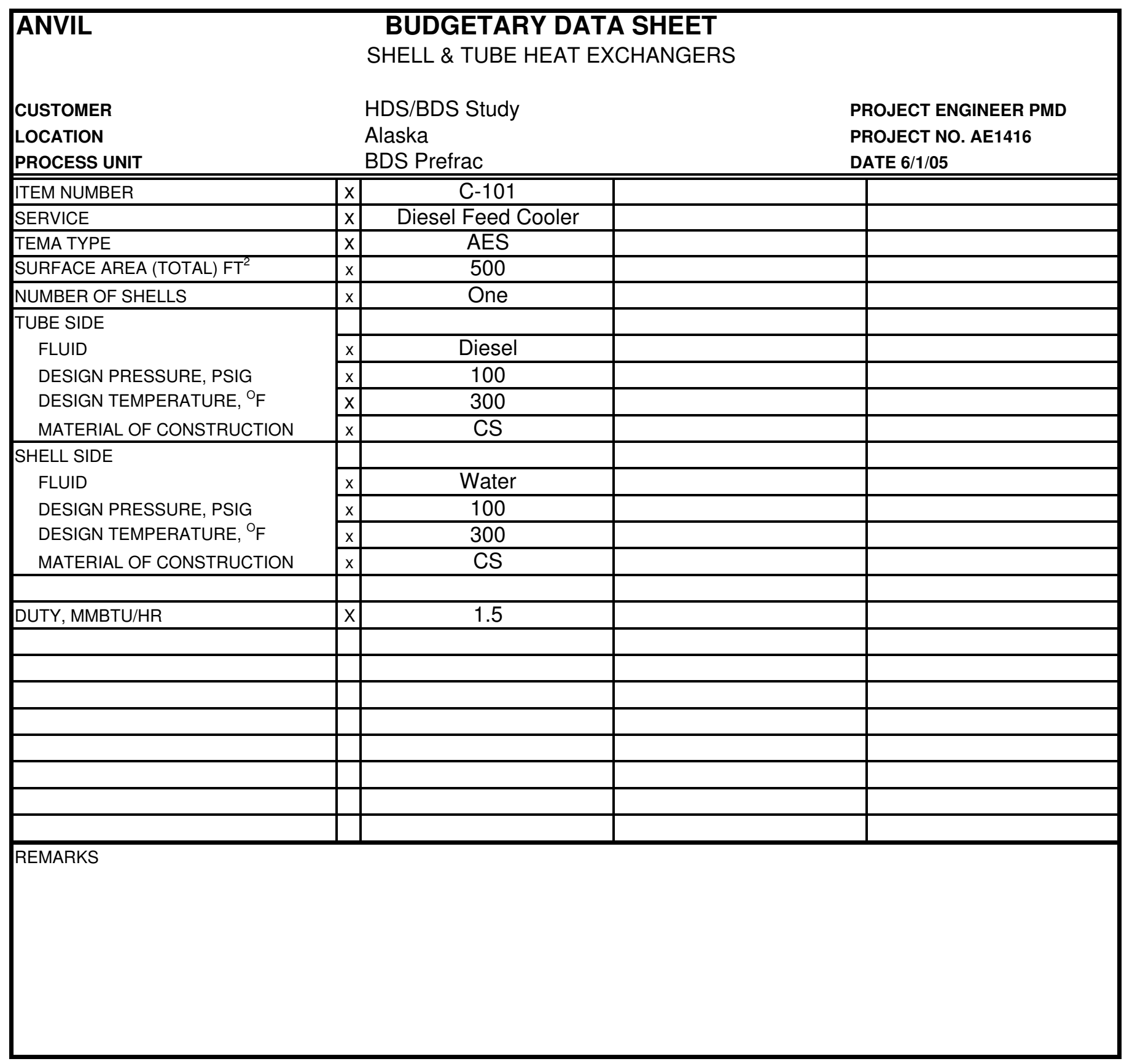




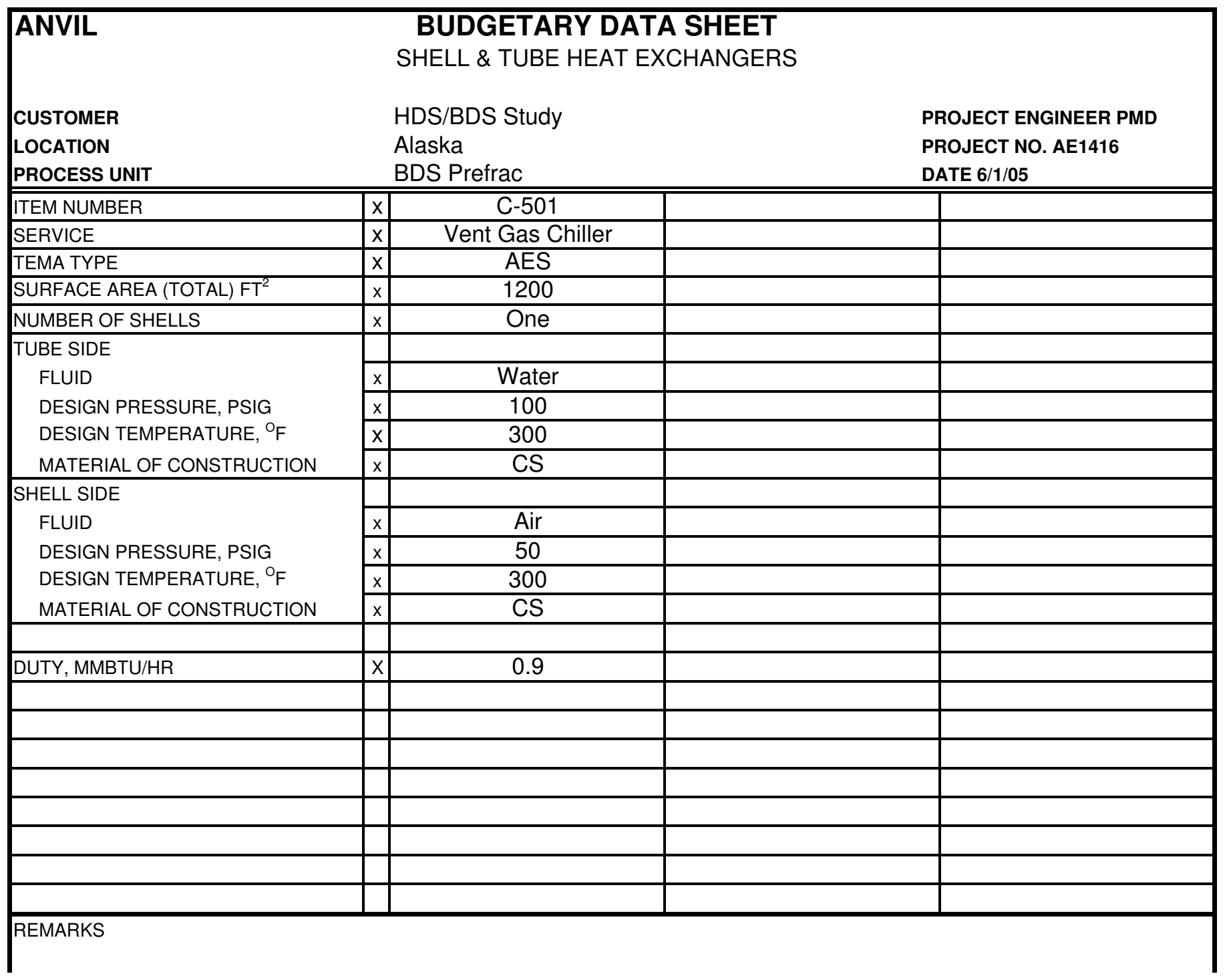




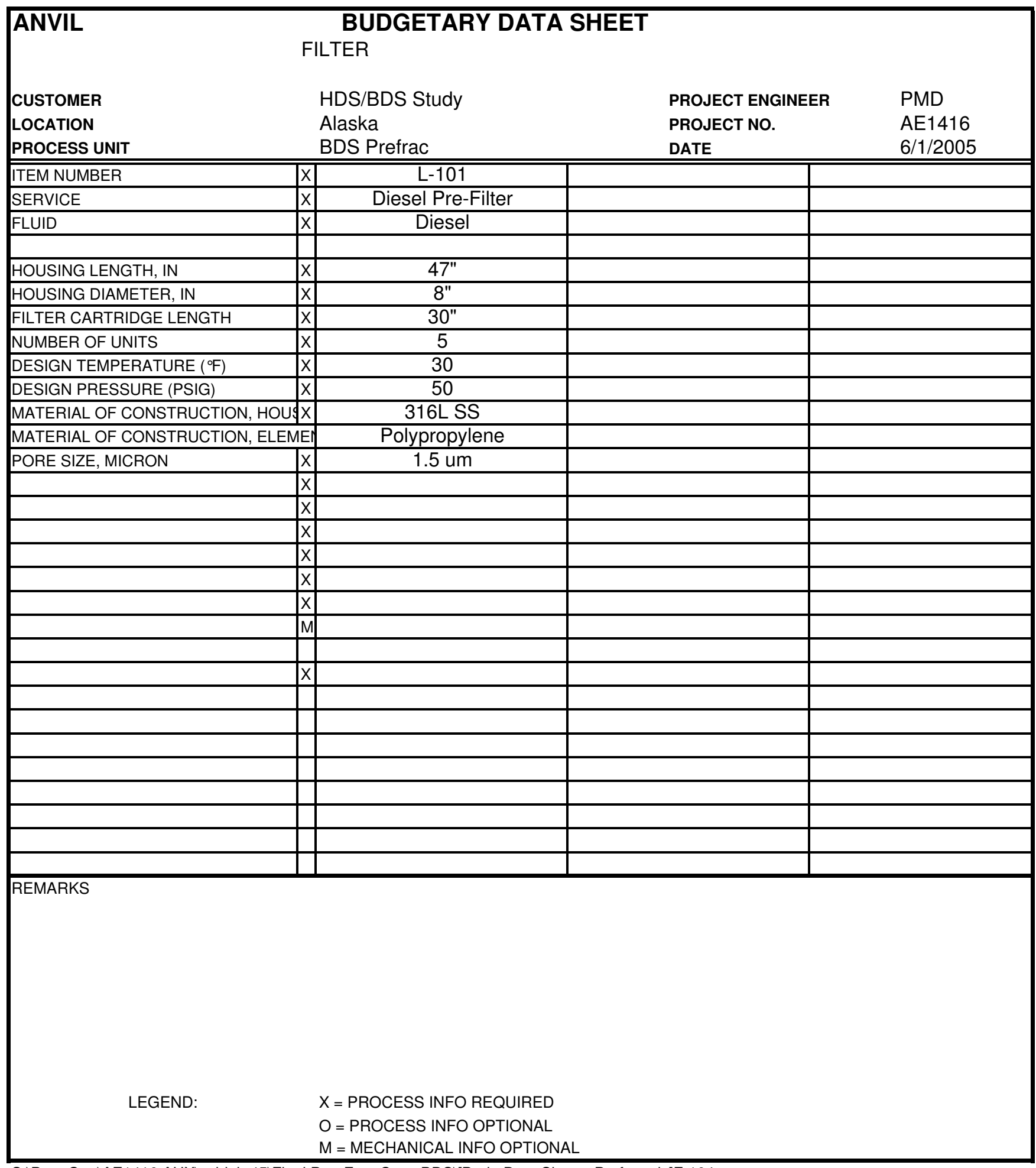




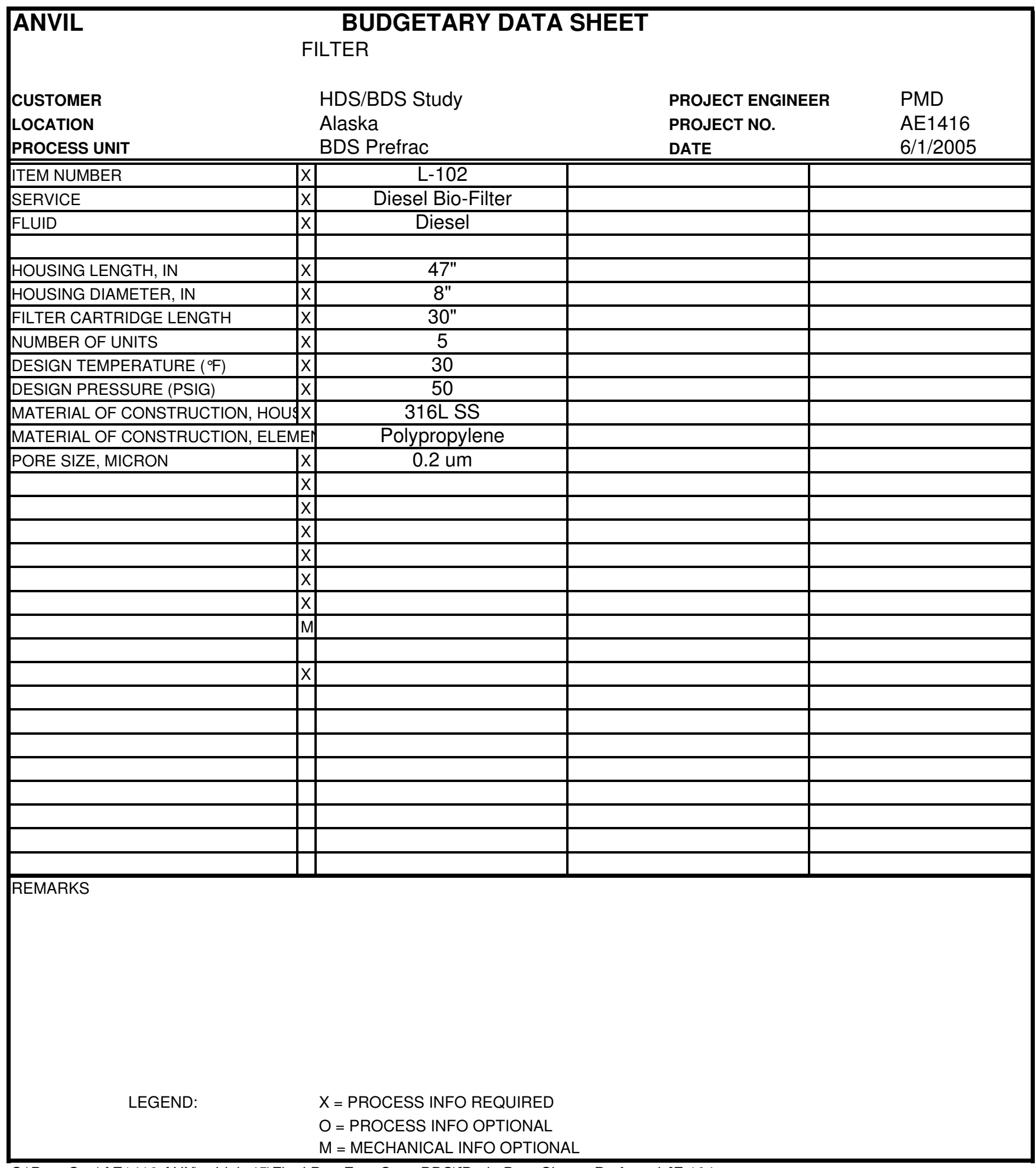




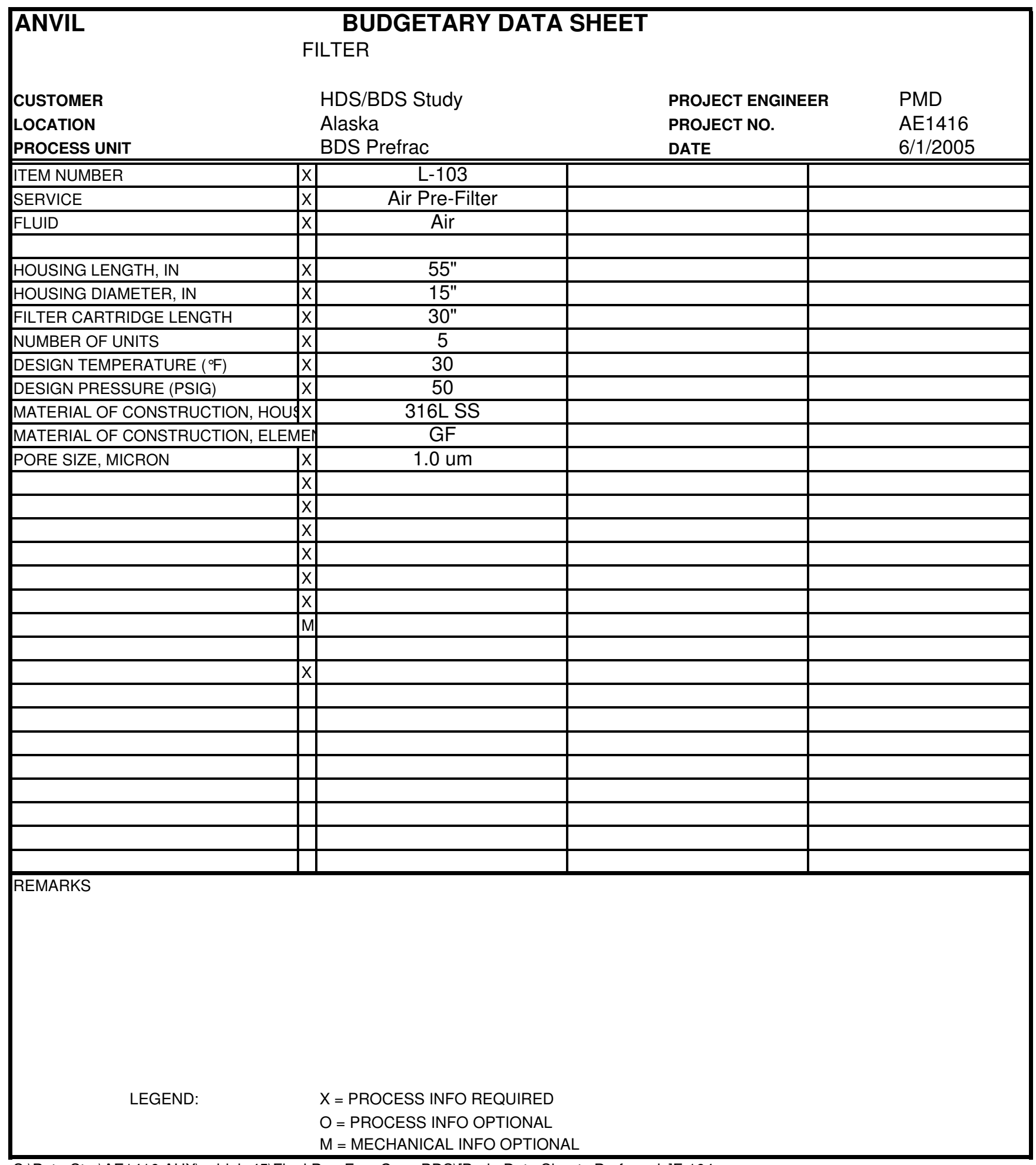




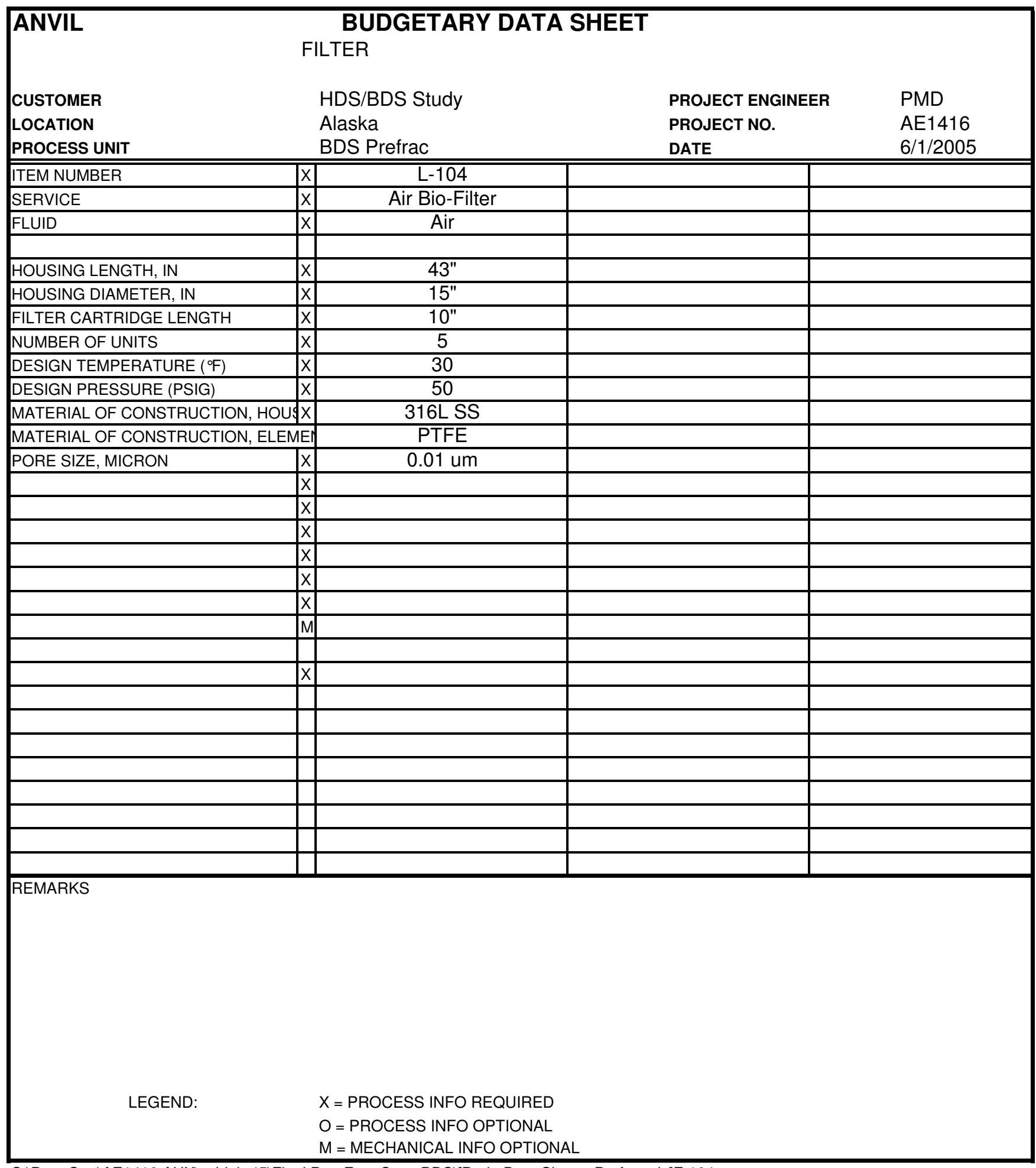




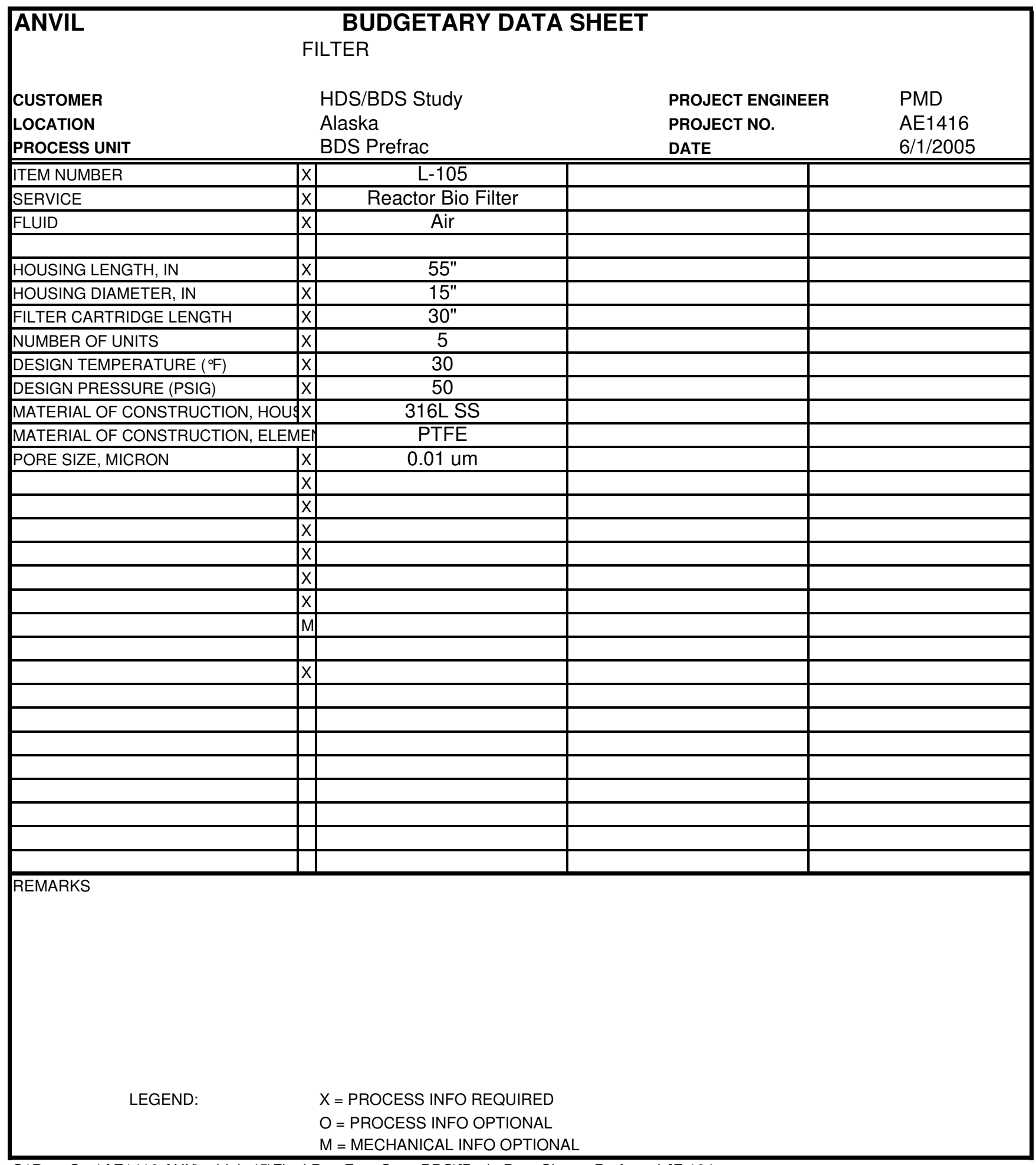




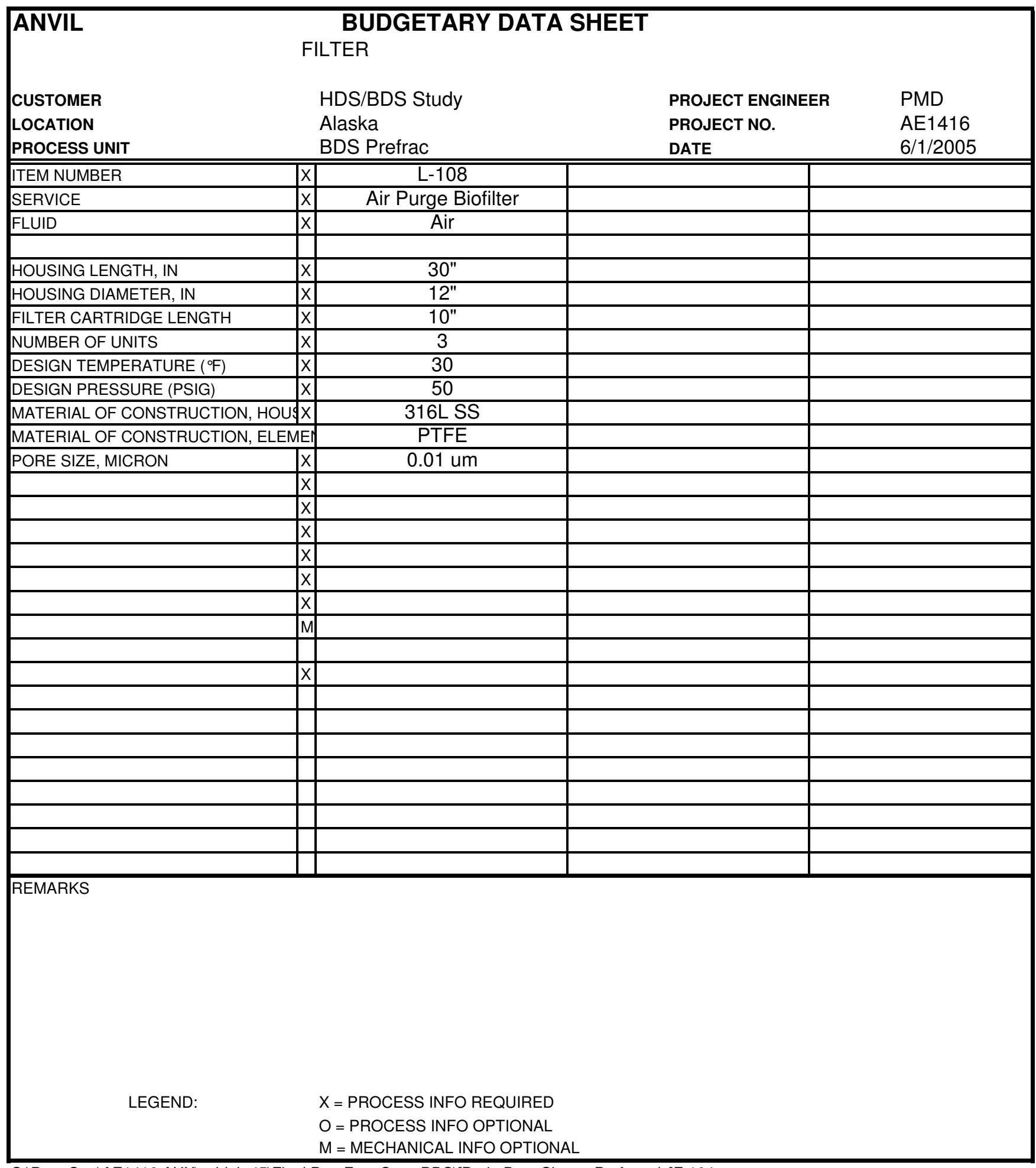




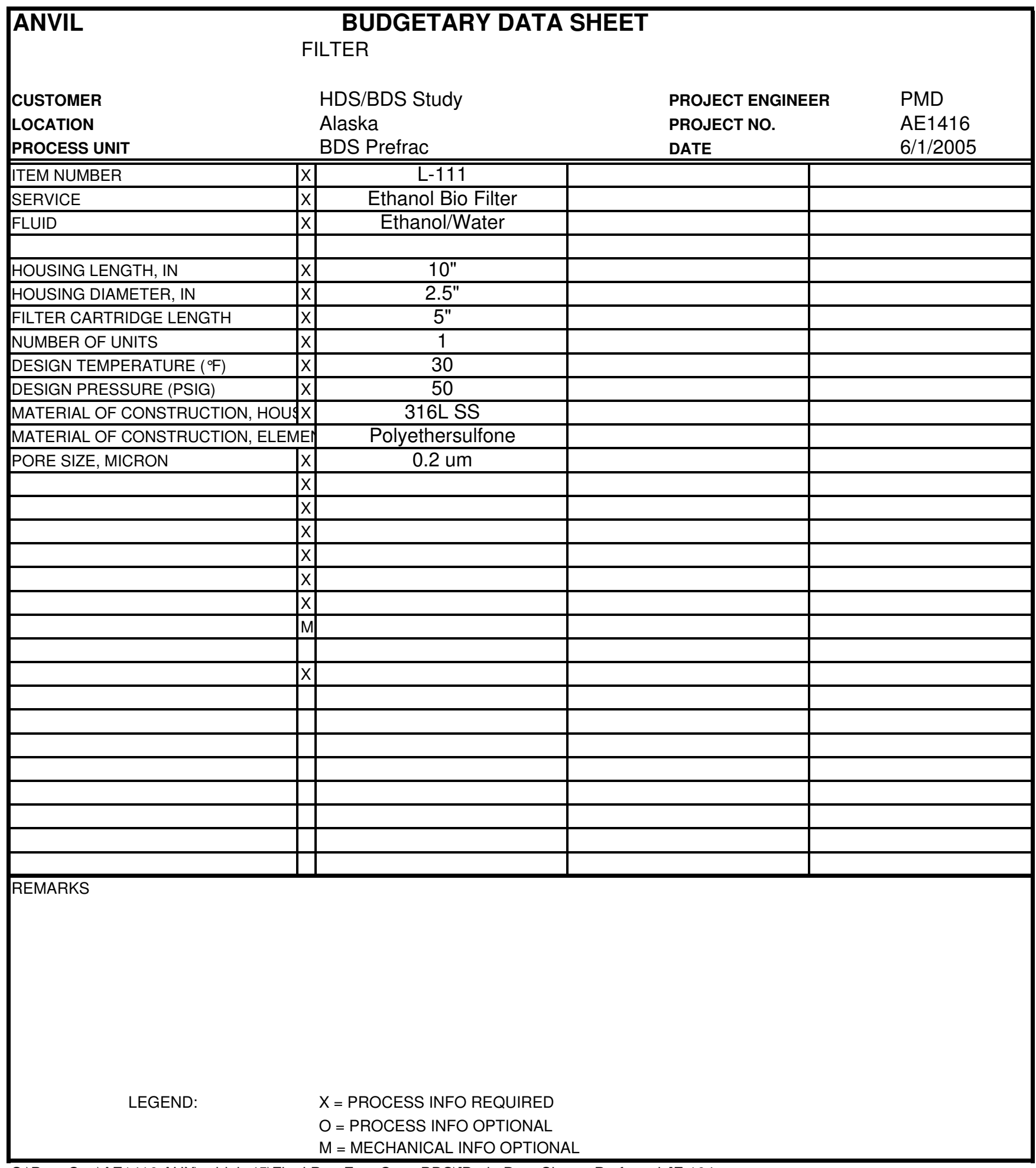




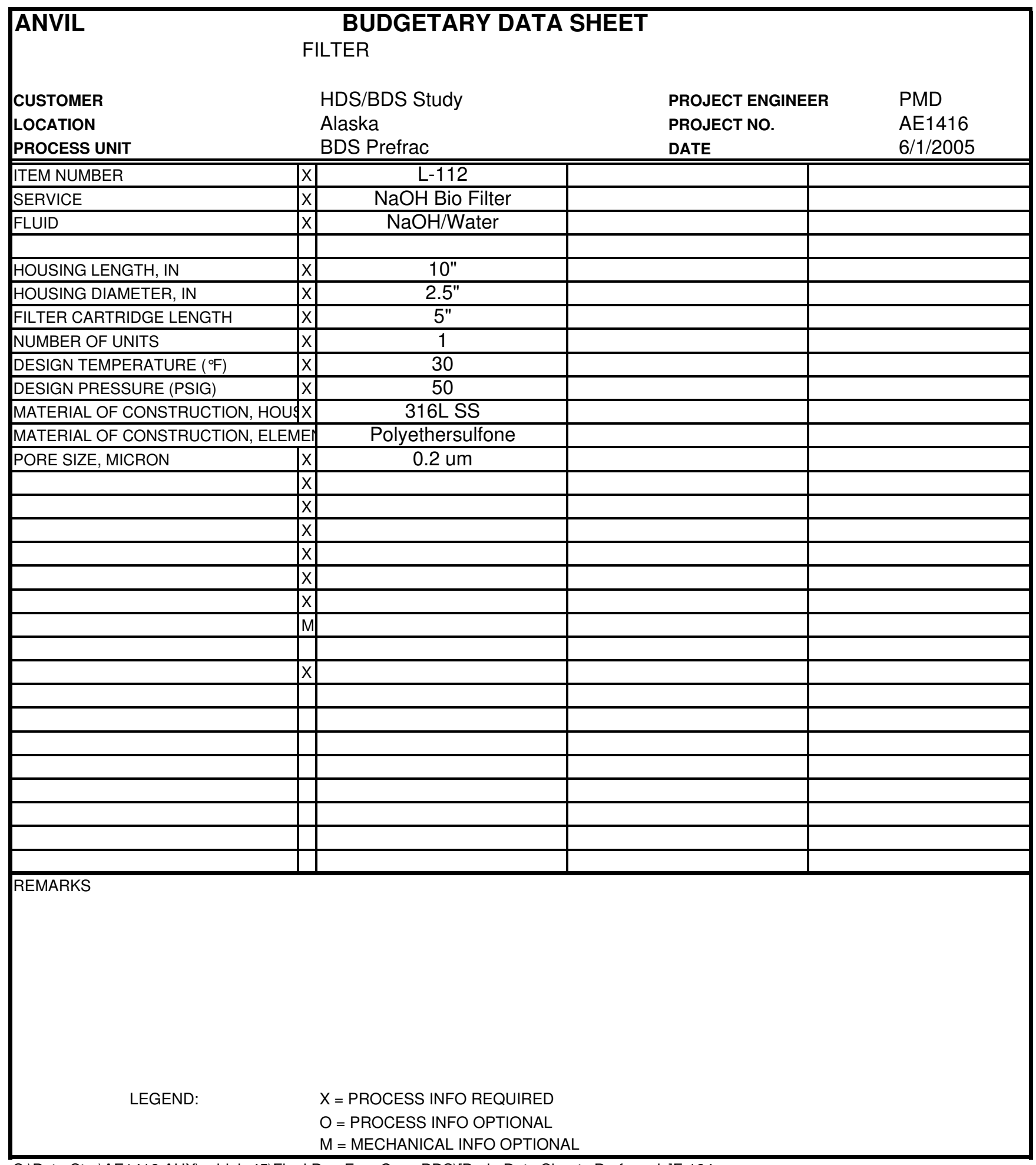




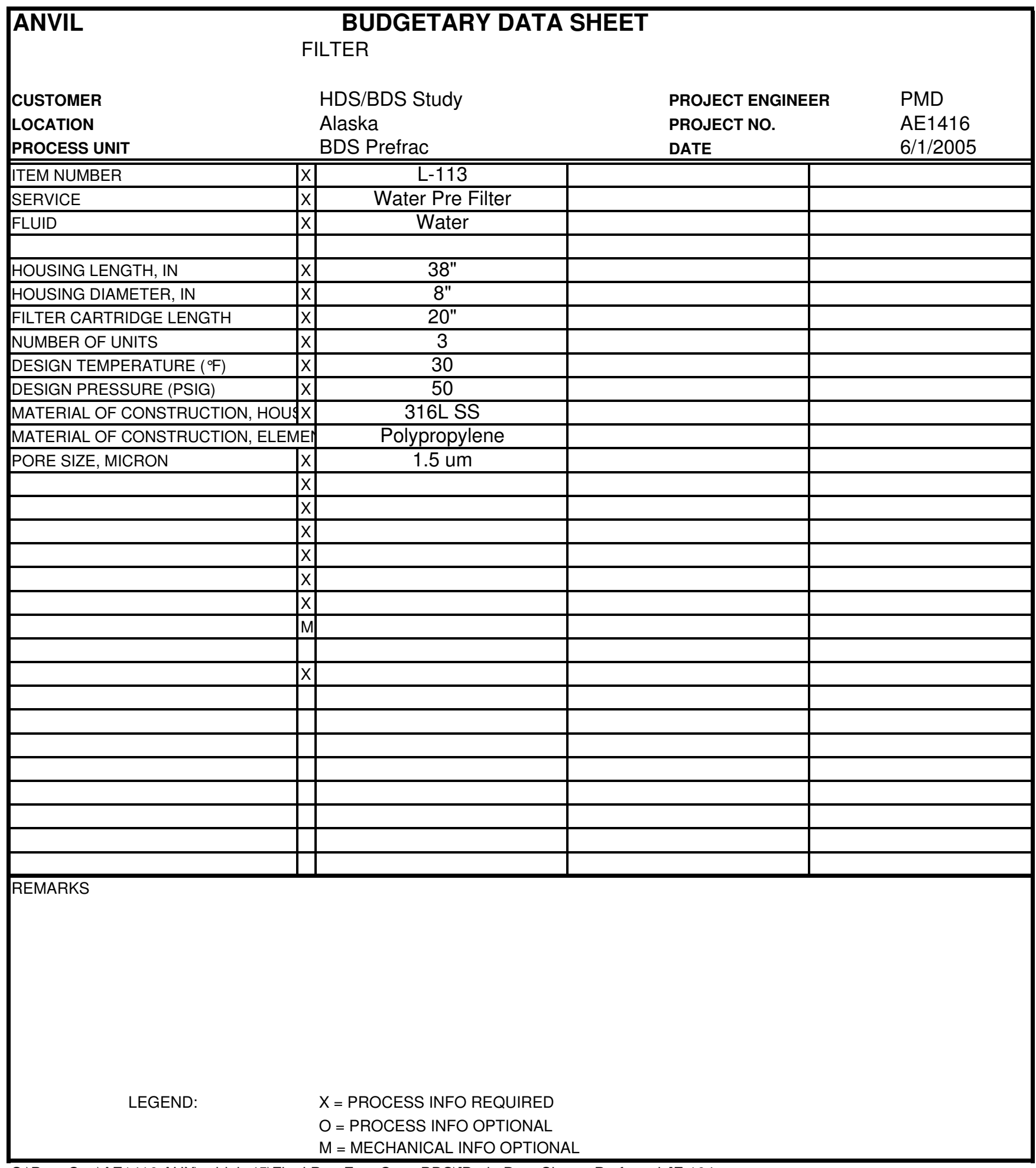




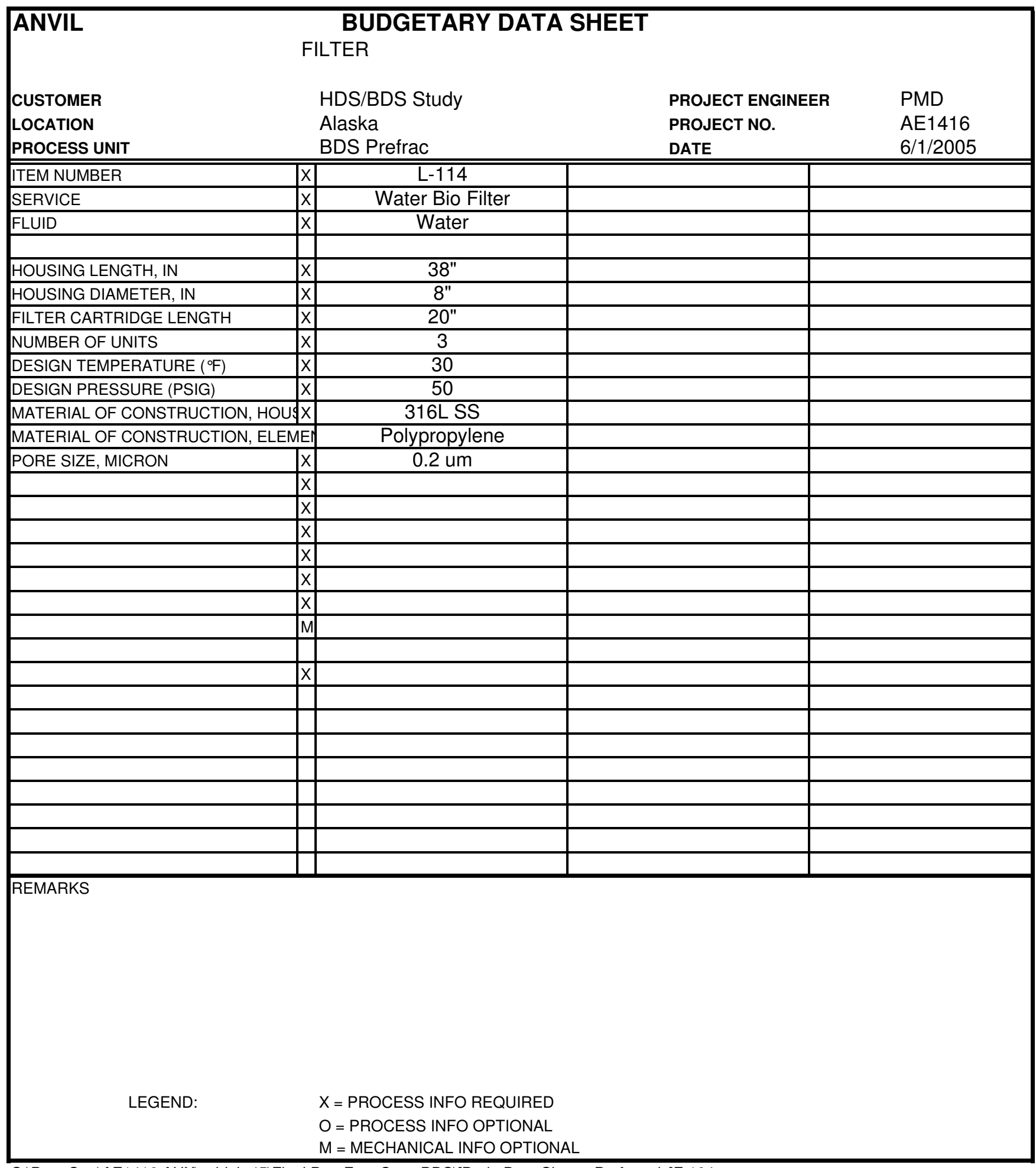




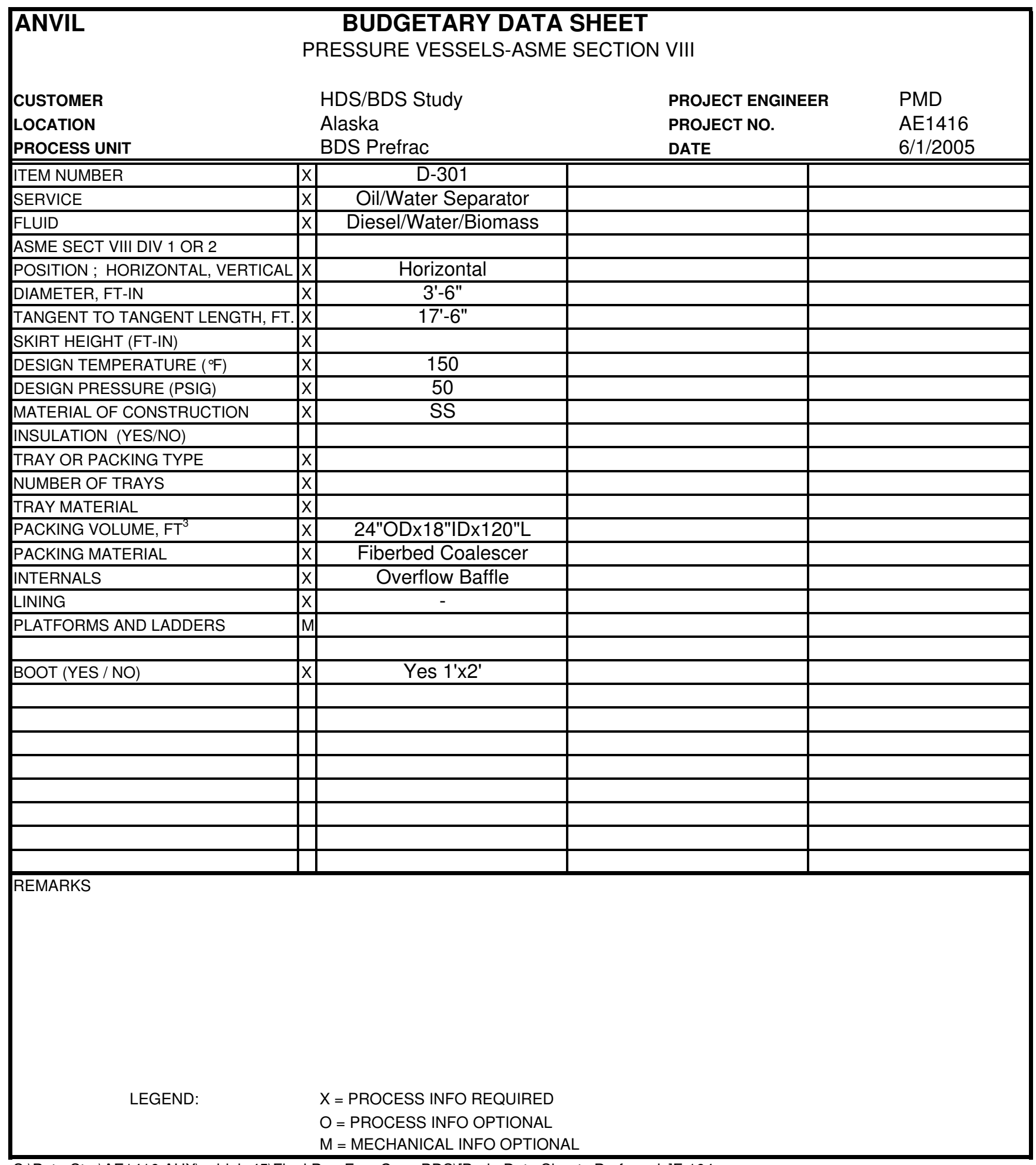




\begin{tabular}{|c|c|c|c|c|}
\hline ANVIL & & $\begin{array}{l}\text { UDGETARY DAT } \\
\text { PUMPS AND DRIV }\end{array}$ & $\begin{array}{l}\text { A SHEET } \\
\text { ERS }\end{array}$ & \\
\hline CUSTOMER & & HDS/BDS Study & & PROJECT ENGINEER PMD \\
\hline LOCATION & & Alaska & & PROJECT NO. AE1416 \\
\hline PROCESS UNIT & & BDS Prefractionation & & DATE 6/11/05 \\
\hline ITEM NUMBER & $\mathrm{x}$ & J-601 A/B (1) & & \\
\hline SERVICE (FLUID) & $\mathrm{x}$ & Diesel Feed Pump & & \\
\hline TEMPERATURE OF FLUID & $\mathrm{x}$ & 65 & & \\
\hline SPECIFIC GRAVITY AT TEMPERATURE & $\mathrm{x}$ & 0.95 & & \\
\hline RATED FLOW (GPM) & $\mathrm{x}$ & 2000 & & \\
\hline SUCTION PRESSURE, PSIG & $\mathrm{x}$ & 2.5 & & \\
\hline DISCHARGE PRESSURE PSIG & $\mathrm{x}$ & 38.5 & & \\
\hline NPSH AVAILABLE ( FT ) & $\mathrm{x}$ & & & \\
\hline CONSTRUCTION (API,ANSI) & $\mathrm{M}$ & & & \\
\hline PUMP TYPE (CENTRIFUGAL, RECIP,ETC.) & $\mathrm{x}$ & Centrifugal & & \\
\hline CASING MATERIAL & $\mathrm{M}$ & CS & & \\
\hline IMPELLER MATERIAL & $\mathrm{M}$ & CS & & \\
\hline TYPE OF DRIVER (MOTOR/TURBINE) & $\mathrm{x}$ & Motor & & \\
\hline TYPE OF SPARE (MOTOR/TURBINE) & $\mathrm{x}$ & Motor & & \\
\hline ELECTRIC POWER (HP) & $\mathrm{x}$ & 20 & & \\
\hline STEAM CONDITIONS & $\mathrm{x}$ & $\mathrm{N} / \mathrm{A}$ & & \\
\hline SEALS (SINGLE, DOUBLE, TANDEM) & $\mathrm{M}$ & & & \\
\hline API SEAL FLUSH PLAN NUMBER & $\mathrm{M}$ & & & \\
\hline & & API Standard & & \\
\hline DIFFERENTIAL PRESSURE, PSI & & 36 & & \\
\hline DIFFERENTIAL HEAD, FT & & 81.7 & & \\
\hline & & & & \\
\hline & & & & \\
\hline & & & & \\
\hline & & & & \\
\hline $\begin{array}{l}\text { REMARKS } \\
\text { 1) One operating pump + one spare } \\
\text { 2) } 0.125 " \mathrm{CA} / \text { Minimum design temp }\end{array}$ & atur & e is $-20^{\circ} \mathrm{F}$. & & \\
\hline LEGEND: & & $\begin{array}{l}x=\text { PROCESS INFO REQL } \\
0=\text { PROCESS INFO OPTI } \\
u=\text { MECHANICAL INFO O }\end{array}$ & $\begin{array}{l}\text { IRED } \\
\text { DNAL } \\
\text { DTIONAL }\end{array}$ & \\
\hline
\end{tabular}

S:IPetroStar\AE1416.AUX|subjob 45\Final Pre_Frac Case BDS\[pre Frac_Budgetary Sheets Add.xIs]C-601(D) 


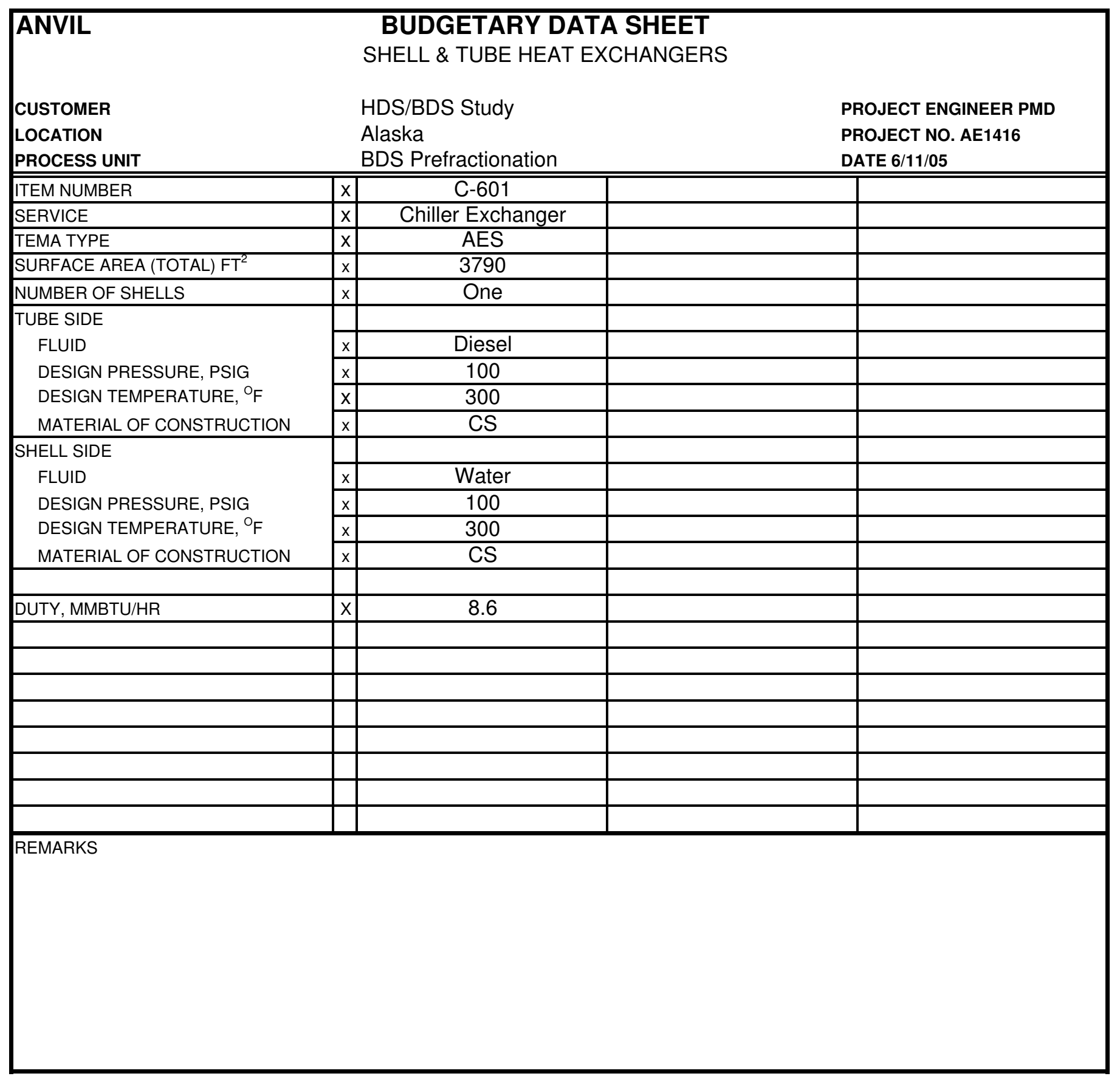




\section{Appendix D.8 - Utility Requirements}

Utility Requirements

Pre Frac Case

6000 BPD Feed Rate, 10 ppmw Sulfur ULSD Product

\begin{tabular}{|c|c|c|c|c|}
\hline & $\begin{array}{c}\text { Power } \\
\text { kW }\end{array}$ & $\begin{array}{c}\text { Fuel Gas } \\
\text { MMBTU/Hr }\end{array}$ & $\begin{array}{c}\text { Process Water } \\
\text { lb/hr }\end{array}$ & $\begin{array}{l}\text { BFW } \\
\text { lb/hr }\end{array}$ \\
\hline \multicolumn{5}{|l|}{ IBL DIESEL SPLITTER } \\
\hline Charge pump & 11 & & & \\
\hline Splitter reflux/prod pump & 12 & & & \\
\hline Splitter Btms Circ & 70 & & & \\
\hline Splitter Ovhd Cond Airfin & 8 & & & \\
\hline Prod rundown cooler & 10 & & & \\
\hline Splitter Reboiler & & 22.2 & & \\
\hline TOTAL DIESEL SPLITTER & 111 & 22.2 & & \\
\hline
\end{tabular}

\section{IBL HDS}

Feed Gas Compressor

H2 Makeup Compressor

Recycle Gas Compressor

$\begin{array}{cccc}\text { Power } & \text { Fuel Gas } & \text { Process Water } & \text { BFW } \\ \frac{\mathrm{kW}}{38} & \underline{\mathrm{MMBTU} / \mathrm{hr}} & \underline{\mathrm{lb} / \mathrm{hr}} & \underline{\mathbf{l b} / \mathrm{hr}}\end{array}$

Charge Pump

Stripper Reflux Pump

Stripper Btms Circ

Effluent Air Cooler

Prod Stripper Condenser

Prod Rundown Cooler

Wash Water Injection Pump

Charge Heater

38

55

40

80

Prod Stripper Reboiler

Wash Water Injection Pump

5.6

21

4

4

15

1

OBL

H2 Plant

Sulfur Recovery

Other OBL Allowance

TOTAL HDS AND OBL

890

2777

\begin{tabular}{|c|c|c|c|c|c|}
\hline BDS & $\begin{array}{c}\text { Power } \\
\text { kW }\end{array}$ & $\begin{array}{c}\text { Fuel Gas } \\
\text { MMBTU/hr }\end{array}$ & $\begin{array}{l}\text { Process Water } \\
\underline{\underline{\mathrm{lb}} / \mathrm{hr}}\end{array}$ & $\begin{array}{l}\text { BFW } \\
\text { lb/hr }\end{array}$ & $\begin{array}{l}\text { Steam } \\
\text { lb/hr }\end{array}$ \\
\hline Total Plant & 917 & & 5000 & & 21 \\
\hline TOTAL BDS & 917 & & 5000 & & 21 \\
\hline
\end{tabular}


Appendix D.9 - Cost Estimate Basis and Cost Estimate 


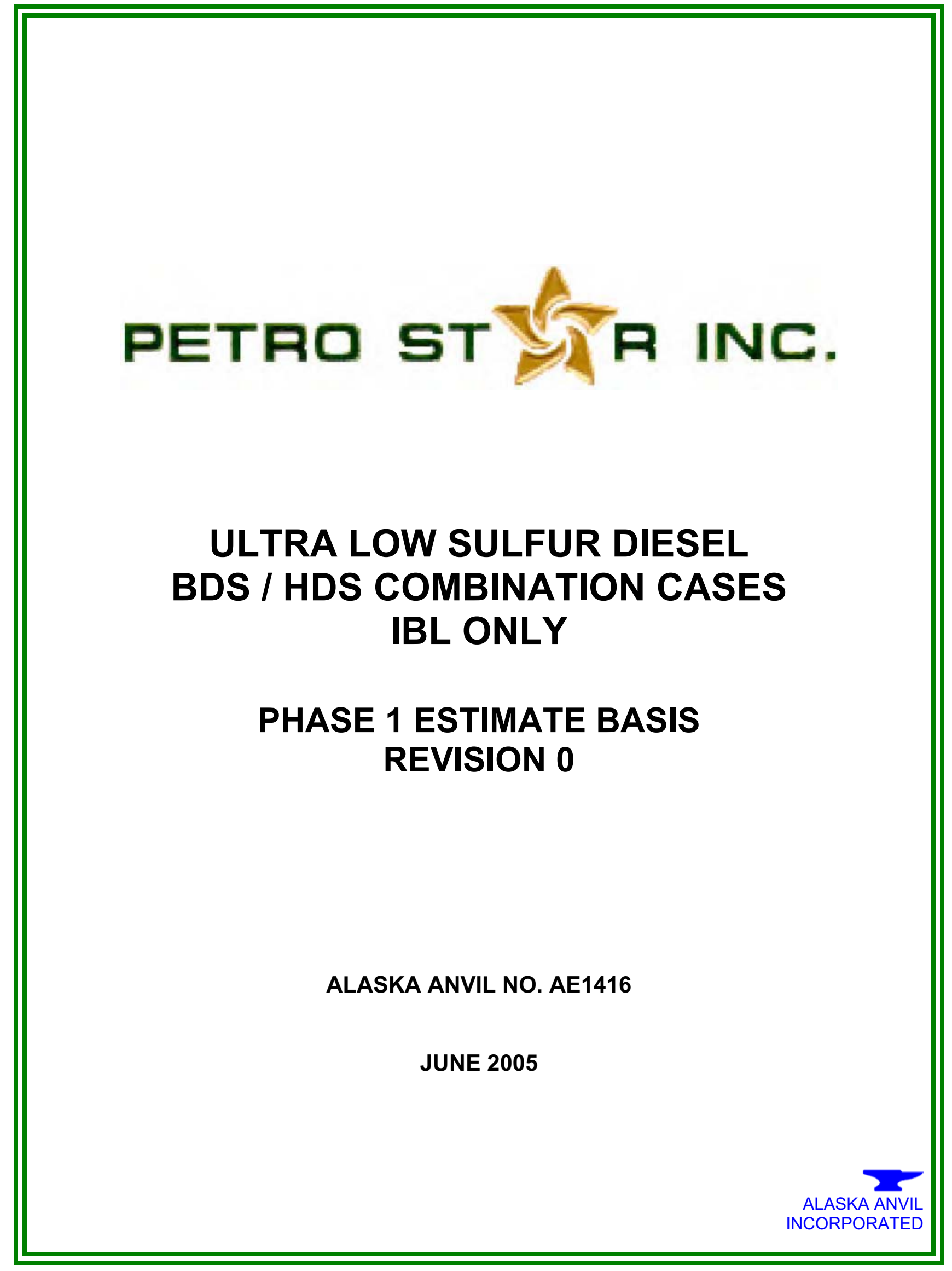




\section{ESTIMATE BASIS GOAL}

This Estimate Basis identifies information, qualifications, exceptions, and assumptions used in developing the cost estimate.

\section{ESTIMATE BASIS PURPOSE}

During the estimate review process, the project team uses the Estimate Basis for the following purposes:

- As a checklist of items to consider during estimate preparation.

- To document what is included and not included in the cost estimate.

- To assess cost risks of estimate components.

- As part of the decision support package for assessing the BDS process feasibility.

\section{GENERAL INFORMATION}

- The purpose of the project estimate is to determine if the ULSD BDS process is economically viable as a standalone process or in combination with an HDS Unit. These three (3) estimate scenarios address the BDS / HDS Combination cases.

- $\quad$ Estimate type:

- The estimate was developed using equipment based factored estimates for Inside Battery Limits (IBL) costs.

- There is a separate equipment-factoring summary for the BDS, HDS, and Pre Frac Equipment (Pre Frac Case Only)

- Most of the equipment pricing was derived from the ICARUS estimating program. Pricing for BDS equipment marked with an asterisk $\left(^{*}\right)$ was provided by Pelorus.

- The cost of the Hydrogen and Sulfur Units were factored off licensor quotes obtained for the standalone HDS case.

- Outside Battery Limits (OBL) costs have been excluded from this estimate.

- $\quad$ The project will be installed in a brownfield location within the Valdez Alaska Refinery.

\section{PROCESS BASIS}

\section{Facility Data}

- $\quad$ Facility type - Ultra Low Sulfur Diesel Treating Complex, which includes:

- Diesel Biodesulfurization Unit

- Diesel Hydrotreating Unit

- Hydrogen Production Unit

- Sulfur Unit

- Diesel Splitter (Pre Frac Case Only) 


\section{Design Basis}

Product specification - Feed 6,000 bpd of untreated diesel to produce 10-ppmw sulfur maximum ultra low sulfur diesel.

\section{COST BASIS}

\section{Labor, Indirects, Equipment, and Bulk Materials}

- Included in the equipment factor.

\section{Project Services}

- $\quad$ Estimated based on 15 percent of TIC for the BDS, HDS, and Pre Frac units; engineering costs for the Hydrogen and Sulfur Units was included in the licensor pricing.

\section{Owner Services}

Not included in the TIC cost. Historically, owner services will cost from 5 to 7 percent of TIC, not including licensing, royalties, or catalyst.

\section{Escalation}

Project is based on 2005 costs. No escalation is included.

\section{Location Factor}

All costs for this estimate have been developed from a U.S. Gulf Coast (USGC) basis. No location factor is included.

\section{Other Costs}

- Catalyst and chemical initial charge has been added as an additional line item.

- CEMS, air preheating, and burner management allowances have been added to the fired heater costs.

\section{ASSUMPTIONS}

- $\quad$ Process licensing and royalty costs are not included.

- Assumes fully installed pump spares, but no warehouse spares. 
Final Technical Progress Report DOE Award No. DE-FC26-02NT15340

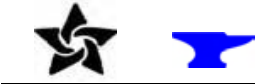

CLIENT: PetroStar

PROJECT: PetroStar Valdez ULSD - Pre Frac Scenario STAGE: Phase 1

\section{PROJECT COST \& SCHEDULE ESTIMATE SUMMARY}

CLIENT PROJECT NO.:

ANVIL PROJECT NO.: AE1416

REV NO.: 0
CLIENT PE: J. Boltz

ANVIL PE: L. Nace

Date: 6/17/05

\begin{tabular}{|l|l|}
\hline PROJECT DESCRIPTION: & PROJECT RISKS: \\
Install Ultra Low Sulfur Diesel Complex. & \\
\hline
\end{tabular}

PROJECT COST ESTIMATE SUMMARY

\begin{tabular}{|c|c|}
\hline COST ESTIMATE STRUCTURE & $\begin{array}{c}\text { TOTAL PROJECT } \\
\text { COST - Valdez }\end{array}$ \\
\hline \multicolumn{2}{|l|}{ COST ESTIMATE PARAMETERS } \\
\hline Estimate Classification & \\
\hline Estimating Method & \\
\hline \multicolumn{2}{|l|}{ COST ESTIMATE SUMMARY } \\
\hline Expected Cost (\$MM) & $\$$ \\
\hline High Range (\$MM) & $\$$ \\
\hline Low Range (\$MM) & $\$$ \\
\hline
\end{tabular}

PROJECT COST ESTIMATE ANAL YSIS

Total Project Expected Cost Component Analysis

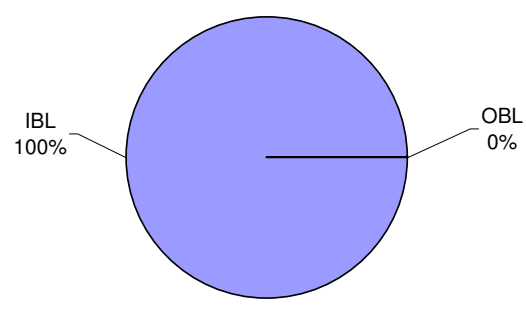

$\square I B L \quad \square O B L$

Total Project Cost Profile (\$MM)

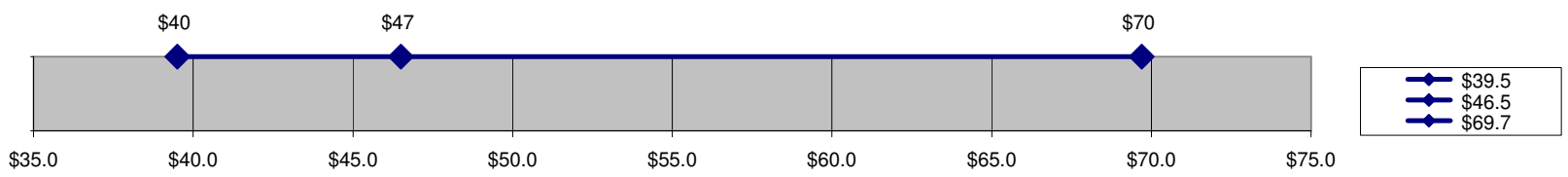

PROJECT SCHEDULE ESTIMATE ANALYSIS

\begin{tabular}{|c|c|c|c|c|}
\hline $\begin{array}{l}\text { Total Project Schedule } \\
\text { Target Completion Dates }\end{array}$ & & \\
\hline \\
PROJECT TIMELINE
\end{tabular}


Petro Star Ultra Low Sulfur Diesel (6000 BPD) Project - Valdez Refinery

IBL Impact Matrix - 2005\$ - Total Installed Costs USGC

Scenario: Pre Frac

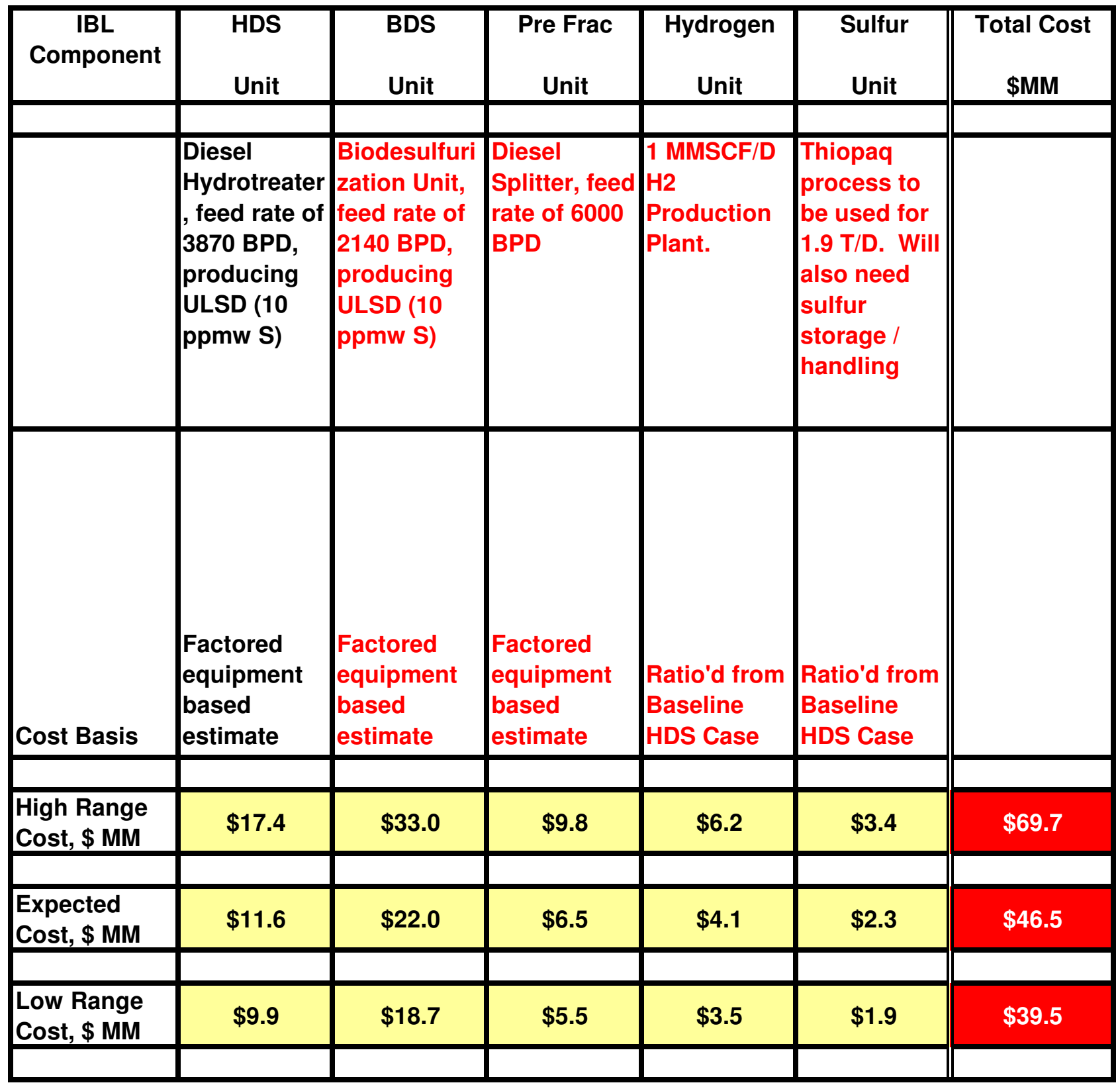


PROJECT: PetroStar Valdez ULSD - Pre Frac Scenario ANVIL NO: AE1416
CLIENT: PetroStar

DATE: 6/17/05

REV NO.: 0

HDS EQUIPMENT - FACTORING SUMMARY

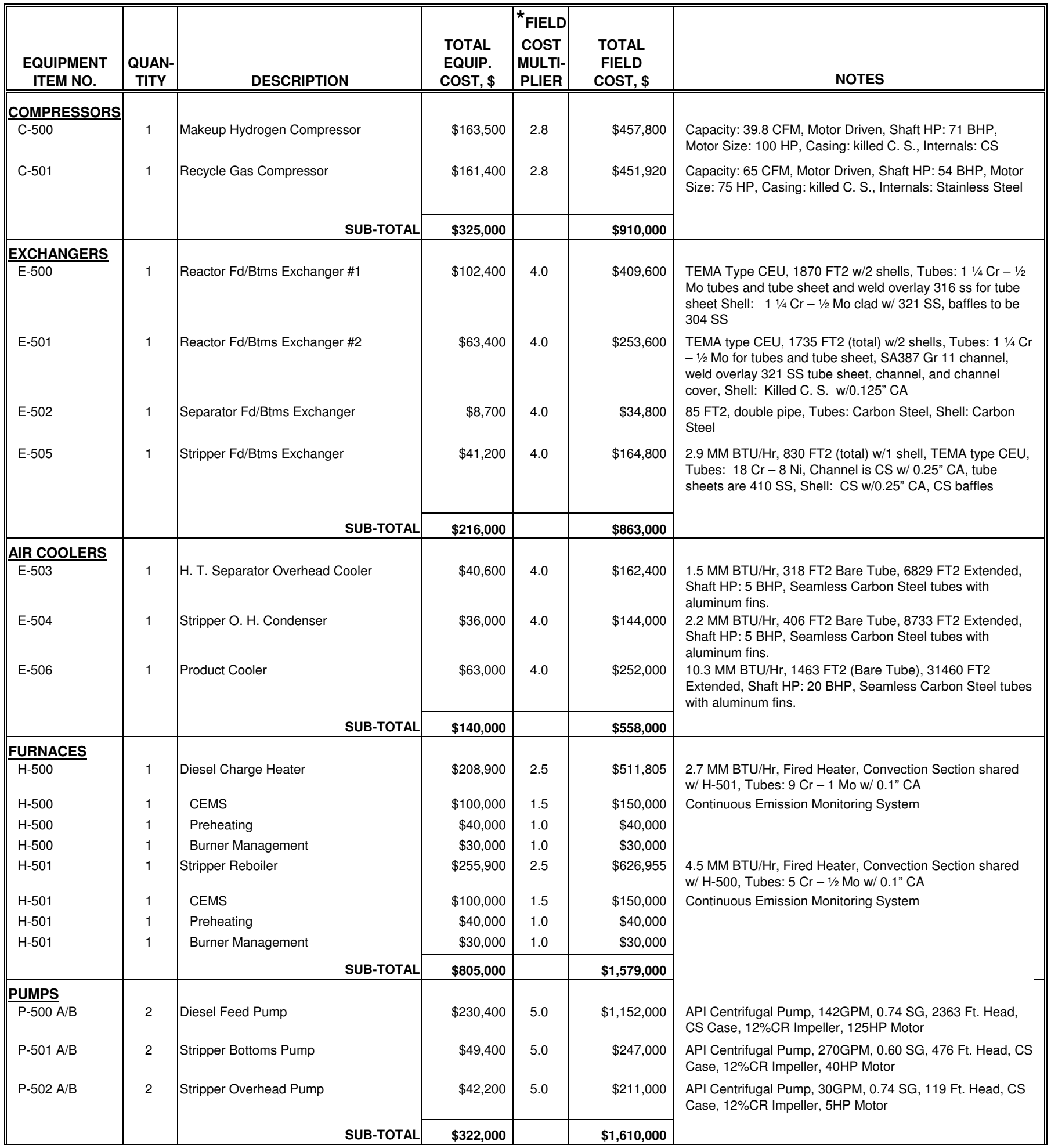


Final Technical Progress Report

ANVIL CORPORATION

OE Award No. DE-FC26-02NT15340

PROJECT: PetroStar Valdez ULSD - Pre Frac Scenario ANVIL NO: AE1416

CLIENT: PetroStar

DATE: $6 / 17 / 05$

REV NO.: 0

HDS EQUIPMENT - FACTORING SUMMARY

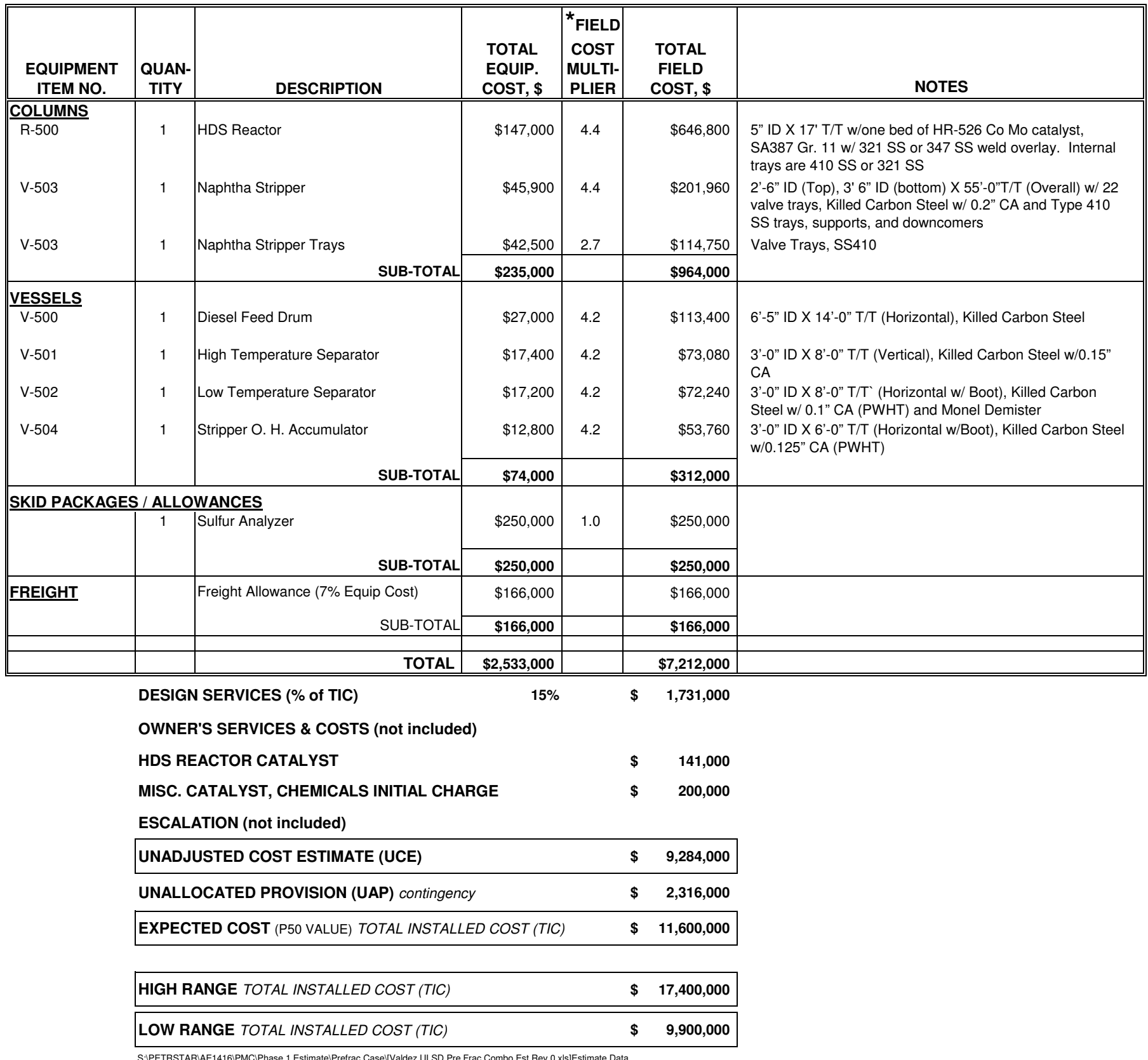

* Note: Field Cost Multiplier includes the following bulk material and installation labor: Civil, Concrete, Structural, Piping, Electrical, Instrumentation, Insulation, Fireproofing, Painting, and Testing. 
BDS EQUIPMENT - FACTORING SUMMARY

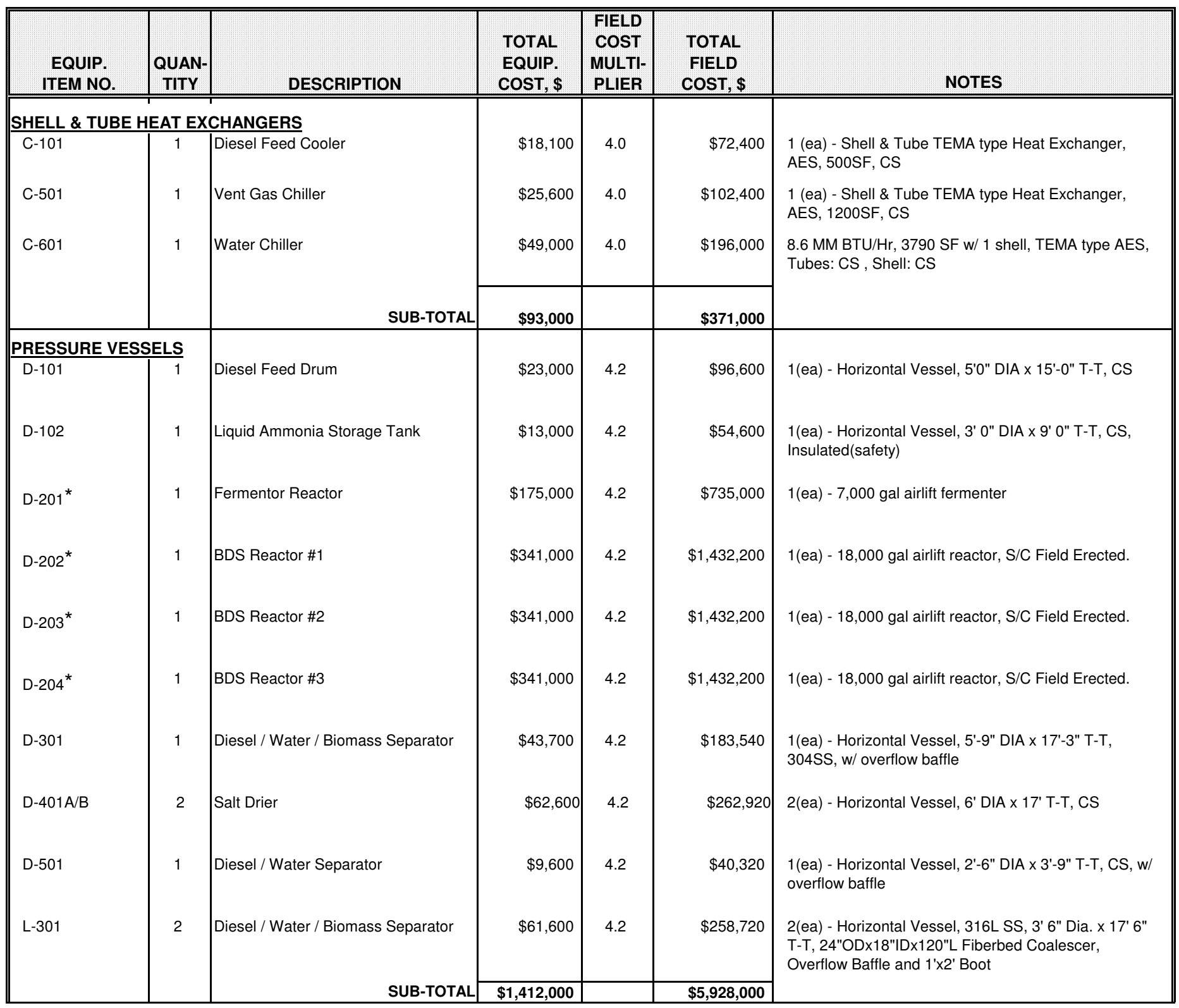


BDS EQUIPMENT - FACTORING SUMMARY

\begin{tabular}{|c|c|c|c|c|c|c|}
\hline $\begin{array}{l}\text { EQUIP. } \\
\text { ITEM NO. }\end{array}$ & $\begin{array}{l}\text { QUAN- } \\
\text { TITY }\end{array}$ & DESCRIPTION & $\begin{array}{l}\text { TOTAL } \\
\text { EQUIP. } \\
\text { COST, \$ }\end{array}$ & $\begin{array}{c}\text { FIELD } \\
\text { COST } \\
\text { MULTI- } \\
\text { PLIER } \\
\end{array}$ & $\begin{array}{l}\text { TOTAL } \\
\text { FIELD } \\
\text { COST, \$ }\end{array}$ & NOTES \\
\hline \multicolumn{7}{|c|}{ PACKAGED SKIDS/SYSTEMS } \\
\hline D-205* & 1 & 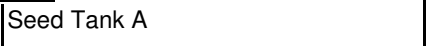 & $\$ 94,000$ & 2.5 & $\$ 235,000$ & Packaged Seed Fermenter System \\
\hline D-206* & 1 & Seed Tank B & $\$ 328,000$ & 2.5 & $\$ 820,000$ & Packaged Seed Fermenter System \\
\hline $\mathrm{J}-109 \mathrm{~A} / \mathrm{B}$ & 2 & Air Compressor & $\$ 428,800$ & 2.5 & $\$ 1,072,000$ & $\begin{array}{l}\text { 2(ea) - Air Compressor, Screw, } 3000 \text { ACFM, CS } \\
\text { body/internals, 300HP Motor Driven }\end{array}$ \\
\hline $\mathrm{L}-109^{*}$ & 1 & Glucose Sterilizer & $\$ 60,000$ & 2.5 & $\$ 150,000$ & $\begin{array}{l}\text { 1(ea) - Packaged } 23 \text { gph continuous steam sterilizer } \\
\text { system complete w/ preheater, heater, cooler, \& } \\
\text { holding coil }\end{array}$ \\
\hline L-110* & 1 & Nutrients Sterilizer & $\$ 60,000$ & 2.5 & $\$ 150,000$ & $\begin{array}{l}1(\text { ea) - Packaged } 20 \text { gph continuous steam sterilizer } \\
\text { system complete w/ preheater, heater, cooler, \& } \\
\text { holding coil }\end{array}$ \\
\hline L-302* & 1 & Electrostatic Precipitator & $\$ 254,000$ & 2.5 & $\$ 635,000$ & 1(ea) - Packaged ESP Unit \\
\hline $\mathrm{L}-601^{*}$ & 1 & Water Chiller & $\$ 533,000$ & 2.5 & $\$ 1,332,500$ & $\begin{array}{l}\text { 1(ea) - Packaged propane refirigeration unit complete } \\
\text { w/ compressor, driver, lube oil system, KO drum \& all } \\
\text { necessary instr. \& controls. }\end{array}$ \\
\hline L-801* & 1 & Waste Oxidizer & $\$ 436,000$ & 2.5 & $\$ 1,090,000$ & $\begin{array}{l}1 \text { (ea) - Packaged horizontal thermal oxidizer w/ high } \\
\text { intensity burner, refractory lined chamber \& stack. }\end{array}$ \\
\hline \multirow[t]{2}{*}{$\mathrm{L}-901^{*}$} & 1 & Water Filtration System & $\$ 90,000$ & 2.5 & $\$ 225,000$ & $\begin{array}{l}1(e a) \text { - Cross flow filtration system for water } \\
\text { purification }\end{array}$ \\
\hline & \multicolumn{2}{|r|}{ SUB-TOTAL } & $\$ 2,284,000$ & & $\$ 5,710,000$ & \\
\hline \multicolumn{7}{|c|}{ ATMOSPHERE STORAGE TANKS } \\
\hline $\mathrm{F}-101$ & 1 & Glucose / Water Storage Tank & $\$ 25,500$ & 2.9 & $\$ 73,950$ & $\begin{array}{l}\text { 1(ea) - Atm. Storage Tank, Flat Roof, Flat Bottom, 10' } \\
\text { DIA x 15' T-T, Epoxy Resin coated CS }\end{array}$ \\
\hline$F-102 A$ & 1 & Salt / Water Storage Tank & $\$ 11,100$ & 2.9 & $\$ 32,190$ & $\begin{array}{l}\text { 1(ea) - Atm. Storage Tank, Flat Roof, Flat Bottom, 4'- } \\
\text { 6" DIA x 9' T-T, Epoxy Resin coated CS }\end{array}$ \\
\hline $\mathrm{F}-102 \mathrm{~B}$ & 1 & Salt / Water Storage Tank & $\$ 10,200$ & 2.9 & $\$ 29,580$ & $\begin{array}{l}\text { 1(ea) - Atm. Storage Tank, Flat Roof, Flat Bottom, 4'- } \\
\text { 6" DIA x 9' T-T, Epoxy Resin coated CS }\end{array}$ \\
\hline $\mathrm{F}-103$ & 1 & Ethanol / Water Storage Tank & $\$ 28,200$ & 2.9 & $\$ 81,780$ & $\begin{array}{l}\text { 1(ea) - Atm. Storage Tank, Flat Roof, Flat Bottom, 11- } \\
\text { 0" DIA x 16' 0" T-T, Epoxy Resin coated CS }\end{array}$ \\
\hline \multirow[t]{2}{*}{ F-104 } & 1 & $\mathrm{NaOH}$ / Water Storage Tank & $\$ 32,800$ & 2.9 & $\$ 95,120$ & $\begin{array}{l}\text { 1(ea) - Atm. Storage Tank, Flat Roof, Flat Bottom, 12'- } \\
\text { 0" DIA x } 18^{\prime} \text { T-T, Epoxy Resin coated CS }\end{array}$ \\
\hline & & SUB-TOTAL & $\$ 108,000$ & & $\$ 313,000$ & \\
\hline
\end{tabular}


BDS EQUIPMENT - FACTORING SUMMARY

\begin{tabular}{|c|c|c|c|c|c|c|}
\hline $\begin{array}{l}\text { EQUIP. } \\
\text { ITEM NO. }\end{array}$ & $\begin{array}{c}\text { QUAN- } \\
\text { TITY }\end{array}$ & DESCRIPTION & $\begin{array}{l}\text { TOTAL } \\
\text { EQUIP. } \\
\text { COST, \$ }\end{array}$ & $\begin{array}{c}\text { FIELD } \\
\text { COST } \\
\text { MULTI- } \\
\text { PLIER } \\
\end{array}$ & $\begin{array}{l}\text { TOTAL } \\
\text { FIELD } \\
\text { COST, \$ }\end{array}$ & NOTES \\
\hline PUMPS & & & & & & \\
\hline$\overline{J-101 A} / B$ & 2 & Diesel Charge Pump & $\$ 48,800$ & 4.5 & $\$ 219,600$ & $\begin{array}{l}\text { 2(ea) - API Centrifugal Pump, } 70 \mathrm{gpm}, 163 \mathrm{ft} \text {. Head, } \\
5 \mathrm{HP} \text { Motor driven, CS, } 1 \text { operating, } 1 \text { installed spare }\end{array}$ \\
\hline $\mathrm{J}-103 \mathrm{~A} / \mathrm{B}$ & 2 & Process Water Pump & $\$ 6,400$ & 4.5 & $\$ 28,800$ & $\begin{array}{l}\text { 2(ea) - Centrifugal Pump, } 12 \mathrm{gpm}, 121 \mathrm{ft} \text {. Head, } 2 \mathrm{HP} \\
\text { Motor driven, CS, } 1 \text { operating, } 1 \text { installed spare }\end{array}$ \\
\hline$J-104 A / B$ & 2 & Glucose Pump & $\$ 13,000$ & 4.5 & $\$ 58,500$ & $\begin{array}{l}\text { 2(ea) - Diaphragm Pump, } 0.5 \mathrm{gpm}, 31 \mathrm{ft} \text {. Head, } \\
\text { Hydraulic driven, } 304 \mathrm{SS}, 1 \text { operating, } 1 \text { installed spare }\end{array}$ \\
\hline$J-105 A / B$ & 2 & Nutrients Pump & $\$ 12,600$ & 4.5 & $\$ 56,700$ & $\begin{array}{l}\text { 2(ea) - Diaphragm Pump, } 0.4 \mathrm{gpm}, 39 \mathrm{ft} \text {. Head, } \\
\text { Hydraulic driven, 304SS, } 1 \text { operating, } 1 \text { installed spare }\end{array}$ \\
\hline $\mathrm{J}-106 \mathrm{~A} / \mathrm{B}$ & 2 & Ethanol Pump & $\$ 14,600$ & 4.5 & $\$ 65,700$ & $\begin{array}{l}\text { 2(ea) - Diaphragm Pump, } 1.2 \mathrm{gpm}, 81 \mathrm{ft} \text {. Head, } \\
\text { Hydraulic driven, } 304 \mathrm{SS}, 1 \text { operating, } 1 \text { installed spare }\end{array}$ \\
\hline $\mathrm{J}-107 \mathrm{~A} / \mathrm{B}$ & 2 & Potassium Hydroxide Pump & $\$ 15,000$ & 4.5 & $\$ 67,500$ & $\begin{array}{l}\text { 2(ea) - Diaphragm Pump, } 1.5 \mathrm{gpm}, 65 \mathrm{ft} \text {. Head, } \\
\text { Hydraulic driven, } 304 \mathrm{SS}, 1 \text { operating, } 1 \text { installed spare }\end{array}$ \\
\hline $\mathrm{J}-201 \mathrm{~A} / \mathrm{B}$ & 2 & Fermentor Transfer Pump & $\$ 12,000$ & 4.5 & $\$ 54,000$ & $\begin{array}{l}\text { 2(ea) - Rotary Lobe Pump, } 5 \mathrm{gpm}, 43 \mathrm{ft} \mathrm{Head,} 0.5 \mathrm{HP} \\
\text { Motor, 304SS, } 1 \text { operating, } 1 \text { installed spare }\end{array}$ \\
\hline $\mathrm{J}-202 \mathrm{~A} / \mathrm{B}$ & 2 & BDS Reactor No1 Transfer Pump & $\$ 13,600$ & 4.5 & $\$ 61,200$ & $\begin{array}{l}\text { 2(ea) - Centrifugal Pump, } 250 \mathrm{gpm}, 45 \mathrm{ft} \text {. Head, } 5 \mathrm{HP} \\
\text { Motor driven, 304SS, } 1 \text { operating, } 1 \text { installed spare }\end{array}$ \\
\hline $\mathrm{J}-203 \mathrm{~A} / \mathrm{B}$ & 2 & BDS Reactor No2 Transfer Pump & $\$ 13,600$ & 4.5 & $\$ 61,200$ & $\begin{array}{l}\text { 2(ea) - Centrifugal Pump, } 250 \mathrm{gpm}, 45 \mathrm{ft} \text {. Head, } 15 \mathrm{HP} \\
\text { Motor driven, 304SS, } 1 \text { operating, } 1 \text { installed spare }\end{array}$ \\
\hline $\mathrm{J}-204 \mathrm{~A} / \mathrm{B}$ & 2 & BDS Reactor No3 Transfer Pump & $\$ 13,600$ & 4.5 & $\$ 61,200$ & $\begin{array}{l}\text { 2(ea) - Centrifugal Pump, } 250 \mathrm{gpm}, 45 \mathrm{ft} \text {. Head, } 15 \mathrm{HP} \\
\text { Motor driven, } 304 \mathrm{SS}, 1 \text { operating, } 1 \text { installed spare }\end{array}$ \\
\hline $\mathrm{J}-301 \mathrm{~A} / \mathrm{B}$ & 2 & 1st Stg Sep Overflow Pump & $\$ 46,200$ & 4.5 & $\$ 207,900$ & $\begin{array}{l}\text { 2(ea) - API Centrifugal Pump, } 75 \mathrm{gpm}, 49 \mathrm{ft} \text {. Head, } \\
\text { 5HP Motor driven, 304SS, } 1 \text { operating, } 1 \text { installed } \\
\text { spare }\end{array}$ \\
\hline$J-302 A / B$ & 2 & 1st Stg Sep Underflow Pump & $\$ 12,600$ & 4.5 & $\$ 56,700$ & $\begin{array}{l}\text { 2(ea) - Centrifugal Pump, } 180 \mathrm{gpm}, 68 \mathrm{ft} \text {. Head, } 5 \mathrm{HP} \\
\text { Motor driven, 304SS, } 1 \text { operating, } 1 \text { installed spare }\end{array}$ \\
\hline$J-303 A / B$ & 2 & 2nd Stg Sep Overflow Pump & $\$ 10,200$ & 4.5 & $\$ 45,900$ & $\begin{array}{l}\text { 2(ea) - Centrifugal Pump, } 55 \mathrm{gpm}, 72 \mathrm{ft} \text {. Head, } 2 \mathrm{HP} \\
\text { Motor driven, } 304 \mathrm{SS}, 1 \text { operating, } 1 \text { installed spare }\end{array}$ \\
\hline$J-304 A / B$ & 2 & 2nd Stg Sep Underflow Pump & $\$ 8,600$ & 4.5 & $\$ 38,700$ & $\begin{array}{l}\text { 2(ea) - Centrifugal Pump, } 15 \mathrm{gpm}, 72 \mathrm{ft} \text {. Head, } 1.5 \mathrm{HP} \\
\text { Motor driven, } 304 \mathrm{SS}, 1 \text { operating, } 1 \text { installed spare }\end{array}$ \\
\hline $\mathrm{J}-502 \mathrm{~A} / \mathrm{B}$ & 2 & Vent Gas KO Drum Wtr Pump & $\$ 8,600$ & 4.5 & $\$ 38,700$ & $\begin{array}{l}\text { 2(ea) - Centrifugal Pump, } 2.5 \mathrm{gpm}, 70 \mathrm{ft} \text {. Head, } 0.5 \mathrm{HP} \\
\text { Motor driven, CS, } 1 \text { operating, } 1 \text { installed spare }\end{array}$ \\
\hline \multirow[t]{2}{*}{$J-601$ A/B } & 2 & Chilled Water Pump & $\$ 19,800$ & 4.5 & $\$ 89,100$ & $\begin{array}{l}\text { API Centrifugal Pump, 2000GPM, 0.95 SG, } 81.7 \text { Ft. } \\
\text { Head, CS Case, 12\%CR Impeller, 20HP Motor }\end{array}$ \\
\hline & & SUB-TOTAL & $\$ 269,000$ & & $\$ 1,211,000$ & \\
\hline
\end{tabular}


BDS EQUIPMENT - FACTORING SUMMARY

\begin{tabular}{|c|c|c|c|c|c|c|}
\hline $\begin{array}{l}\text { EQUIP. } \\
\text { ITEM NO. }\end{array}$ & $\begin{array}{c}\text { QUAN- } \\
\text { TITY } \\
\end{array}$ & DESCRIPTION & $\begin{array}{l}\text { TOTAL } \\
\text { EQUIP. } \\
\text { COST, \$ }\end{array}$ & $\begin{array}{c}\text { FIELD } \\
\text { COST } \\
\text { MULTI- } \\
\text { PLIER } \\
\end{array}$ & $\begin{array}{l}\text { TOTAL } \\
\text { FIELD } \\
\text { COST, \$ } \\
\end{array}$ & NOTES \\
\hline \multicolumn{7}{|l|}{ FILTERS } \\
\hline $\mathrm{L}-101$ & 1 & Diesel Pre-Filter Vessel & $\$ 5,210$ & 4.2 & $\$ 21,882$ & $\begin{array}{l}\text { 1(ea) - Filter housing, } 316 \mathrm{LSS}, 47 " \mathrm{~T}-\mathrm{T} \times 8 \text { × Dia. w/ } \\
5(\text { ea) - Polypropylene element, } 30 " \text { long, } 1.5 \mathrm{um} \text { pore } \\
\text { size }\end{array}$ \\
\hline L-102 & 1 & Diesel Pre-Filter Vessel & $\$ 5,280$ & 4.2 & $\$ 22,176$ & $\begin{array}{l}\text { 1(ea) - Filter housing, } 316 \mathrm{LSS}, 47 " \mathrm{~T}-\mathrm{T} \text { x } 8 \text { " Dia. w/ } \\
5(\mathrm{ea}) \text { - Polypropylene element, } 30 \text { "long, } 0.2 \mathrm{um} \text { pore } \\
\text { size }\end{array}$ \\
\hline L-103 & 1 & Air Pre-Filter Vessel & $\$ 11,400$ & 4.2 & $\$ 47,880$ & $\begin{array}{l}\text { 1(ea) - Filter housing, 316L SS, 55" T-T x 15" Dia. w/ } \\
5(\text { ea) - GF element, 30" long, } 1.0 u m \text { pore size }\end{array}$ \\
\hline L-104 & 1 & Air Bio-Filter Vessel & $\$ 11,125$ & 4.2 & $\$ 46,725$ & $\begin{array}{l}\text { 1(ea) - Filter housing, } 316 \mathrm{~L} \mathrm{SS}, 43 " \mathrm{~T}-\mathrm{T} \times 15^{\prime \prime} \text { Dia. w/ } 5 \\
\text { (ea) - PTFE element, } 10 " \text { long, } 0.01 \text { um pore size }\end{array}$ \\
\hline L-105 & 1 & BDS Reactor \#1 Air Bio Filter Vessel & $\$ 12,420$ & 4.2 & $\$ 52,164$ & $\begin{array}{l}\text { 1(ea) - Filter housing, 316L SS, 55" T-T x 15" Dia. w/ } \\
5(\mathrm{ea}) \text { - PTFE element, 30" long, 0.01um pore size }\end{array}$ \\
\hline L-106 & 1 & BDS Reactor \#2 Air Bio Filter Vessel & $\$ 12,420$ & 4.2 & $\$ 52,164$ & $\begin{array}{l}\text { 1(ea) - Filter housing, 316L SS, 55" T-T x 15" Dia. w/ } \\
5 \text { (ea) - PTFE element, } 30 " \text { long, } 0.01 \text { um pore size }\end{array}$ \\
\hline L-107 & 1 & BDS Reactor \#3 Air Bio Filter Vessel & $\$ 12,420$ & 4.2 & $\$ 52,164$ & $\begin{array}{l}\text { 1(ea) - Filter housing, } 316 \mathrm{~L} \text { SS, } 55 " \mathrm{~T}-\mathrm{T} \times 15 \text { " Dia. w/ } \\
5 \text { (ea) - PTFE element, } 30 \text { "long, } 0.01 \text { um pore size }\end{array}$ \\
\hline L-108 & 1 & Air Purge Bio-Filter Vessel & $\$ 7,011$ & 4.2 & $\$ 29,446$ & $\begin{array}{l}\text { 1(ea) - Filter housing, 316L SS, 30" T-T x 12" Dia. w/ } \\
\text { 3(ea) - PTFE element, } 10 " \text { long, 0.01um pore size }\end{array}$ \\
\hline L-111 & 1 & Ethanol Bio-Filter Vessel & $\$ 1,308$ & 4.2 & $\$ 5,494$ & $\begin{array}{l}\text { 1(ea) - Filter housing, } 316 \mathrm{~L} \mathrm{SS}, 10 " \mathrm{~T}-\mathrm{T} \times 2.5 \text { " Dia. w/ } \\
1 \text { (ea) - Polyethersulfone element, } 5 \text { " long, } 0.2 \mathrm{um} \text { pore } \\
\text { size }\end{array}$ \\
\hline L-112 & 1 & $\mathrm{NAaOH}$ Bio-Filter Vessel & $\$ 1,308$ & 4.2 & $\$ 5,494$ & $\begin{array}{l}\text { 1(ea) - Filter housing, } 316 \mathrm{~L} \mathrm{SS}, 10 " \text { " T-T x } 2.5 \text { " Dia. w/ } \\
1 \text { (ea) - Polyethersulfone element, } 5 \text { " long, } 0.2 \text { um pore } \\
\text { size }\end{array}$ \\
\hline L-113 & 1 & Water Pre-Filter Vessel & $\$ 2,850$ & 4.2 & $\$ 11,970$ & $\begin{array}{l}\text { 1(ea) - Filter housing, } 316 \mathrm{~L} \text { SS, 38" T-T x 8" Dia. w/ } \\
3(\text { ea) - Polypropylene element, } 20 " \text { long, } 1.5 \mathrm{um} \text { pore } \\
\text { size }\end{array}$ \\
\hline L-114 & 1 & Water Bio-Filter Vessel & $\$ 2,892$ & 4.2 & $\$ 12,146$ & $\begin{array}{l}\text { 1(ea) - Filter housing, } 316 \mathrm{~L} \mathrm{SS}, 38 \text { " T-T x 8" Dia. w/ } \\
3(\text { ea) - Polypropylene element, 20" long, 0.2um pore } \\
\text { size }\end{array}$ \\
\hline L-401 & 1 & Recycle Water Pre-Filter Vessel & $\$ 2,850$ & 4.2 & $\$ 11,970$ & $\begin{array}{l}\text { 1(ea) - Filter housing, } 316 \mathrm{LSS}, 38 \text { " T-T x 8" Dia. w/ } \\
3(\text { ea) - Polypropylene element, } 20 \text { "long, } 1.5 \mathrm{um} \text { pore } \\
\text { size }\end{array}$ \\
\hline \multirow[t]{2}{*}{ L-402 } & 1 & Recycle Water Bio-Filter Vessel & $\$ 2,892$ & 4.2 & $\$ 12,146$ & \multirow[t]{2}{*}{$\begin{array}{l}\text { 1(ea) - Filter housing, } 316 \mathrm{LSS}, 38 \text { " T-T x 8" Dia. w/ } \\
3(\text { ea) - Polypropylene element, } 20 " \text { long, } 0.2 \text { um pore } \\
\text { size }\end{array}$} \\
\hline & & SUB-TOTAL & $\$ 91,000$ & & $\$ 384,000$ & \\
\hline \multirow[t]{3}{*}{ FREIGHT } & & Freight Allowance (7\% Equip Cost) & $\$ 298,000$ & & $\$ 298,000$ & \\
\hline & & SUB-TOTAL & $\$ 298,000$ & & $\$ 298,000$ & \\
\hline & & TOTAL & $\$ 4,554,693$ & & $\$ 14,210,000$ & \\
\hline
\end{tabular}


A N V I L C ORPORATION Final Technical Progress Report

PROJECT: PetroStar Valdez ULSD - Pre Frac Scenario ANVIL NO: AE1416
DOE_Al
DATE: 6/17/05
REV NO.: 0

BDS EQUIPMENT - FACTORING SUMMARY

\begin{tabular}{||c|c|c|c|c|c|c||}
\hline \hline & & TOTAL & FIELD & COST & TOTAL & \\
EQUIP. & QUAN- & & TOTA & EQUIP. & MULTI- & FIELD \\
ITEM NO. & TITY & DESCRIPTION & COST, \$ & PLIER & COST, \$ & NOTES \\
\hline \hline
\end{tabular}

\begin{tabular}{lccc|} 
DESIGN SERVICES & $15 \%$ & $\$ 3,270,000$ \\
OWNER'S SERVICES \& COSTS (provided by owner) & $\$$ & - \\
INITIAL CHEMICAL CHARGE & $\$$ & 103,000 \\
ESCALATION (provided by owner) & $\$$ & - \\
\hline UNADJUSTED COST ESTIMATE (UCE) & $\$ 17,583,000$ \\
\hline CONTINGENCY & $\$$ & $4,417,000$ \\
\hline EXPECTED COST (P5O VALUE) TOTAL INSTALLED COST (TIC) & $\$ 22,000,000$ \\
\hline
\end{tabular}

S:PEETRSTARIAE1416iPMCIPhase 1 Estimate|Prefrac Case[[Valdez ULSD Pre Frac Combo Est Rev 0.xis]Estimate Data

${ }^{\star}$ Note: Equipment pricing by Pelorus. 
Final Technical Progress Report

OE Award No. DE-FC26-02NT15340

PROJECT: PetroStar Valdez ULSD - Pre Frac Scenario ANVIL NO: AE1416

CLIENT: PetroStar

DATE: 6/17/05

REV NO.: 0

PRE FRAC EQUIPMENT - FACTORING SUMMARY

\begin{tabular}{|c|c|c|c|c|c|c|}
\hline $\begin{array}{l}\text { EQUIPMENT } \\
\text { ITEM NO. } \\
\end{array}$ & $\begin{array}{l}\text { QUAN- } \\
\text { TITY }\end{array}$ & DESCRIPTION & $\begin{array}{l}\text { TOTAL } \\
\text { EQUIP. } \\
\text { COST, \$ } \\
\end{array}$ & $\begin{array}{l}* \text { FIELD } \\
\text { COST } \\
\text { MULTI- } \\
\text { PLIER } \\
\end{array}$ & $\begin{array}{l}\text { TOTAL } \\
\text { FIELD } \\
\text { COST, \$ } \\
\end{array}$ & NOTES \\
\hline \multicolumn{7}{|l|}{ EXCHANGERS } \\
\hline $\mathrm{E}-1$ & 1 & Diesel Splitter Feed / Bottoms & $\$ 21,900$ & 4.0 & $\$ 87,600$ & $\begin{array}{l}\text { 3.3 MM BTU/Hr, } 450 \text { SF w/ } 1 \text { shell, TEMA type AEU, Tubes: } \\
11 / 4 \mathrm{Cr}-1 / 2 \mathrm{Mo} \text {, Shell: } 11 / 4 \mathrm{Cr}-1 / 2 \mathrm{Mo}\end{array}$ \\
\hline $\mathrm{E}-2$ & 1 & Diesel Splitter Feed / Overhead & $\$ 38,500$ & 4.0 & $\$ 154,000$ & $\begin{array}{l}\text { 9.1 MM BTU/Hr, } 1640 \text { SF w/ } 1 \text { shell, TEMA type AEU, } \\
\text { Tubes: } 11 / 4 \mathrm{Cr}-1 / 2 \mathrm{Mo} \text {, Shell: } 11 / 4 \mathrm{Cr}-1 / 2 \mathrm{Mo}\end{array}$ \\
\hline \multirow[t]{2}{*}{ E-3 } & 1 & Diesel Splitter Feed / Bottoms & $\$ 24,200$ & 4.0 & $\$ 96,800$ & \multirow[t]{2}{*}{$\begin{array}{l}\text { 2.0 MM BTU/Hr, } 620 \text { SF w/ } 1 \text { shell, TEMA type AEU, Tubes: } \\
11 / 4 \mathrm{Cr}-1 / 2 \mathrm{Mo} \text {, Shell: } 11 / 4 \mathrm{Cr}-1 / 2 \mathrm{Mo}\end{array}$} \\
\hline & & SUB-TOTAL & $\$ 85,000$ & & $\$ 338,000$ & \\
\hline \multicolumn{7}{|l|}{ AIR COOLERS } \\
\hline $\mathrm{E}-4$ & 1 & Diesel Splitter Ovhd Cond. & $\$ 45,500$ & 4.0 & $\$ 182,000$ & \multirow{3}{*}{$\begin{array}{l}\text { 11.2 MM BTU/Hr, } 603 \text { SF Bare Tube, } 12,800 \text { SF Extended, } \\
\text { 15 HP, Seamless Carbon Steel tubes with aluminum fins. } \\
\text { 3.5 MM BTU/Hr, } 729 \text { SF Bare Tube, 15,500 SF Extended, } \\
\text { 15 HP, Seamless Carbon Steel tubes with aluminum fins. }\end{array}$} \\
\hline \multirow[t]{2}{*}{ E-5 } & \multirow[t]{2}{*}{1} & \multirow[t]{2}{*}{ Diesel Splitter Bottoms Cooler } & $\$ 43,500$ & 4.0 & $\$ 174,000$ & \\
\hline & & & $\$ 89,000$ & & $\$ 356,000$ & \\
\hline \multirow{3}{*}{ FURNACES } & & & & & & \multirow{3}{*}{$\begin{array}{l}\text { 17.8 MM BTU/Hr, Vertical Fired Heater, Tubes: } 9 \mathrm{Cr}-1 \mathrm{Mo} \\
\text { w/ 0.1" CA }\end{array}$} \\
\hline & $\begin{array}{l}1 \\
1 \\
1 \\
1\end{array}$ & $\begin{array}{l}\text { Diesel Splitter Reboiler } \\
\text { CEMS } \\
\text { Preheating } \\
\text { Burner Management }\end{array}$ & $\begin{array}{r}\$ 507,700 \\
\$ 100,000 \\
\$ 40,000 \\
\$ 30,000\end{array}$ & $\begin{array}{l}2.5 \\
1.5 \\
1.0 \\
1.0\end{array}$ & $\begin{array}{r}\$ 1,243,865 \\
\$ 150,000 \\
\$ 40,000 \\
\$ 30,000\end{array}$ & \\
\hline & 1 & Burner Management & $\$ 678,000$ & & $\$ 1,464,000$ & \\
\hline \multicolumn{7}{|l|}{ PUMPS } \\
\hline$P-1 A / B$ & 2 & Diesel Splitter Charge Pump & $\$ 39,400$ & 5.0 & $\$ 197,000$ & $\begin{array}{l}\text { API Centrifugal Pump, 206GPM, } 0.80 \text { SG, } 257 \text { Ft. Head, CS } \\
\text { Case, 12\%CR Impeller, 20HP Motor }\end{array}$ \\
\hline P-2 A/B & 2 & Diesel Splitter Bottoms Pump & $\$ 67,000$ & 5.0 & $\$ 335,000$ & $\begin{array}{l}\text { API Centrifugal Pump, 1406GPM, } 0.642 \text { SG, } 305 \text { Ft. Head, } \\
\text { CS Case, } 12 \% \text { CR Impeller, 125HP Motor }\end{array}$ \\
\hline \multirow[t]{2}{*}{ P-3 A/B } & \multirow[t]{2}{*}{2} & \multirow[t]{2}{*}{ Diesel Splitter Ovhd Pump } & $\$ 61,000$ & 5.0 & $\$ 305,000$ & \multirow[t]{2}{*}{$\begin{array}{l}\text { API Centrifugal Pump, 290GPM, } 0.86 \text { SG, } 188 \text { Ft. Head, CS } \\
\text { Case, } 12 \% \text { CR Impeller, 20HP Motor }\end{array}$} \\
\hline & & & $\$ 167,000$ & & $\$ 837,000$ & \\
\hline \multicolumn{7}{|l|}{ COLUMNS } \\
\hline V-2 & 1 & Diesel Splitter Tower & $\$ 107,300$ & 4.4 & $\$ 472,120$ & \multirow{3}{*}{$\begin{array}{l}\text { 7'-0" Dia. X } 82 \text { '-0" T/T w/ } 30 \text { valve trays, Carbon Steel w/ } \\
0.2 " \text { CA and Type } 410 \text { SS trays, supports, and downcomers } \\
\text { Valve Trays, SS } 410\end{array}$} \\
\hline \multirow[t]{2}{*}{$\mathrm{V}-2$} & \multirow[t]{2}{*}{1} & \multirow[t]{2}{*}{ Diesel Splitter Tower Trays } & $\$ 110,400$ & 2.7 & $\$ 298,080$ & \\
\hline & & & $\$ 218,000$ & & $\$ 770,000$ & \\
\hline \multicolumn{7}{|l|}{ VESSELS } \\
\hline $\mathrm{V}-1$ & 1 & Diesel Feed Charge Drum & $\$ 34,300$ & 4.2 & $\$ 144,060$ & \multirow{3}{*}{$\begin{array}{l}\text { 7'-0" ID X 15'-0" T/T Horizontal Vessel, } 50 \text { PSIG, Full } \\
\text { Vacuum, Carbon Steel w/ 0.2" CA } \\
\text { 6'-0" ID X 12'-0" T/T Horizontal Vessel, } 75 \text { PSIG, Full } \\
\text { Vacuum, Carbon Steel w/ 0.2" CA }\end{array}$} \\
\hline \multirow[t]{2}{*}{ V-3 } & \multirow[t]{2}{*}{1} & \multirow[t]{2}{*}{ Splitter Overhead accumulator } & $\$ 27,000$ & 4.2 & $\$ 113,400$ & \\
\hline & & & $\$ 61,000$ & & $\$ 257,000$ & \\
\hline \multirow[t]{3}{*}{ FREIGHT } & & \multirow{2}{*}{\begin{tabular}{|r|} 
Freight Allowance (7\% Equip Cost) \\
SUB-TOTAL
\end{tabular}} & $\$ 91,000$ & & $\$ 91,000$ & \\
\hline & & & $\$ 91,000$ & & $\$ 91,000$ & \\
\hline & & TOTAL & $\$ 1,389,000$ & & $\$ 4,113,000$ & \\
\hline
\end{tabular}


Final Technical Progress Report

ANVIL CORPORATION

OE Award No. DE-FC26-02NT15340

PROJECT: PetroStar Valdez ULSD - Pre Frac Scenario ANVIL NO: AE1416

CLIENT: PetroStar

DATE: $6 / 17 / 05$

REV NO.: 0

PRE FRAC EQUIPMENT - FACTORING SUMMARY

\begin{tabular}{|c|c|c|c|c|c|c|}
\hline \hline & & & * FIELD & \\
EQUIPMENT & QUAN- & & TOTAL & COST & TOTAL \\
ITEM NO. & TITY & DESCRIPTION & EQUIP. & MULTI- & FIELD & \\
COST, \$ & PLIER & COST, \$ & \\
\hline \hline
\end{tabular}

DESIGN SERVICES (\% of TIC)

$15 \%$

$\$ 967,000$

OWNER'S SERVICES \& COSTS (not included)

MISC. CATALYST, CHEMICALS INITIAL CHARGE $\quad \$ \quad 100,000$

ESCALATION (not included)

\begin{tabular}{|lcc|}
\hline UNADJUSTED COST ESTIMATE (UCE) & $\mathbf{\$}$ & $\mathbf{5 , 1 8 0 , 0 0 0}$ \\
\hline UNALLOCATED PROVISION (UAP) contingency & $\$$ & $\mathbf{1 , 3 2 0 , 0 0 0}$ \\
\hline EXPECTED COST (P5O VALUE) TOTAL INSTALLED COST (TIC) & $\$ \mathbf{6 , 5 0 0 , 0 0 0}$ \\
\hline
\end{tabular}

\begin{tabular}{|lcr|}
\hline HIGH RANGE TOTAL INSTALLED COST (TIC) & $\mathbf{\$}$ & $\mathbf{9 , 8 0 0 , 0 0 0}$ \\
\hline LOW RANGE TOTAL INSTALLED COST $(T I C)$ & $\mathbf{\$}$ & $\mathbf{5 , 5 0 0 , 0 0 0}$ \\
\hline
\end{tabular}

S:PETRSTARIAE1416iPMCIPhase 1 EstimatelPrefrac Case[IValdez ULSD Pre Frac Combo Est Rev 0.xls]Estimate Data

* Note: Field Cost Multiplier includes the following bulk material and installation labor: Civil, Concrete, Structural, Piping, Electrical, Instrumentation, Insulation, Fireproofing, Painting, and Testing. 
Final Technical Progress Report DOE Award No. DE-FC26-02NT15340
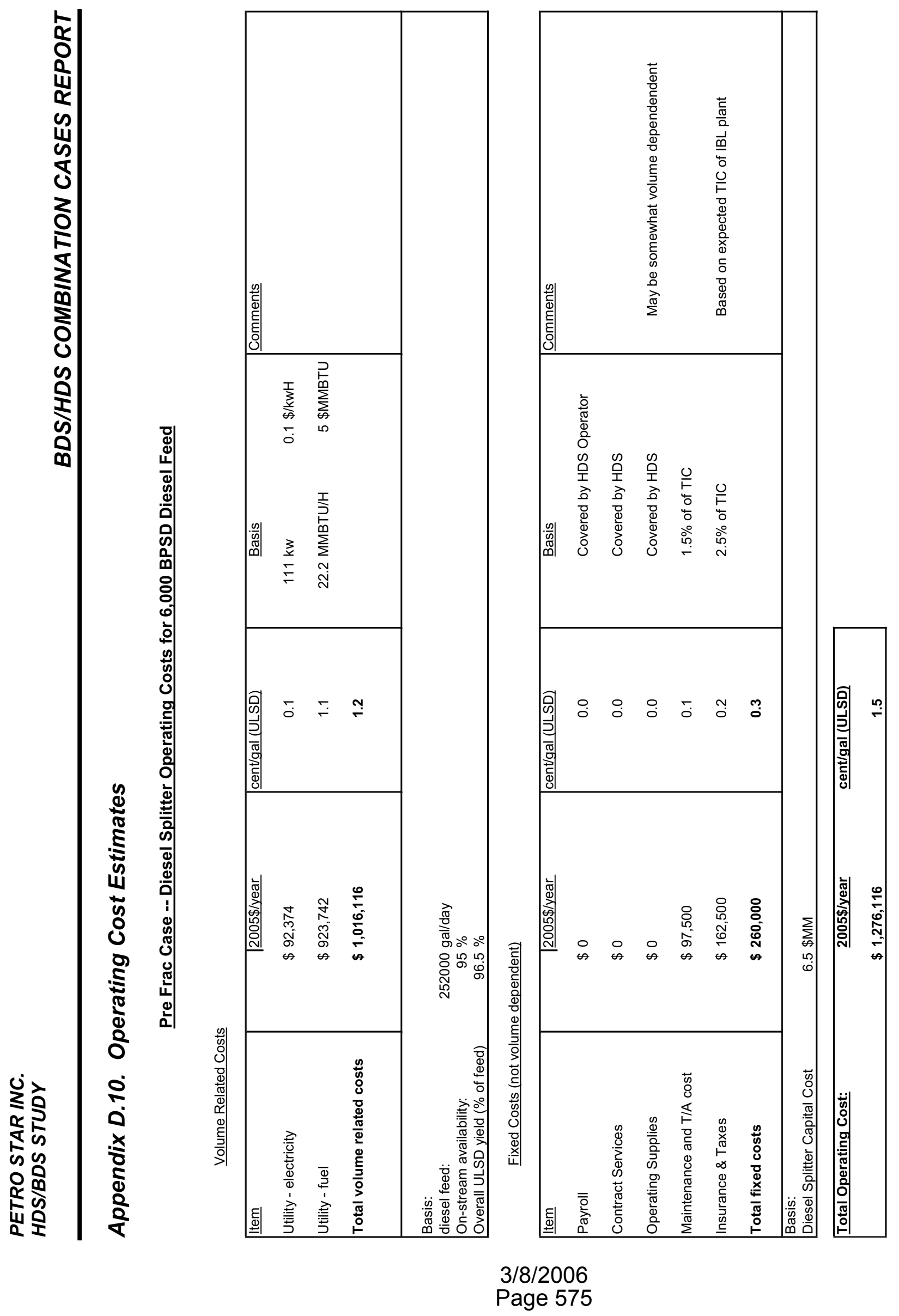


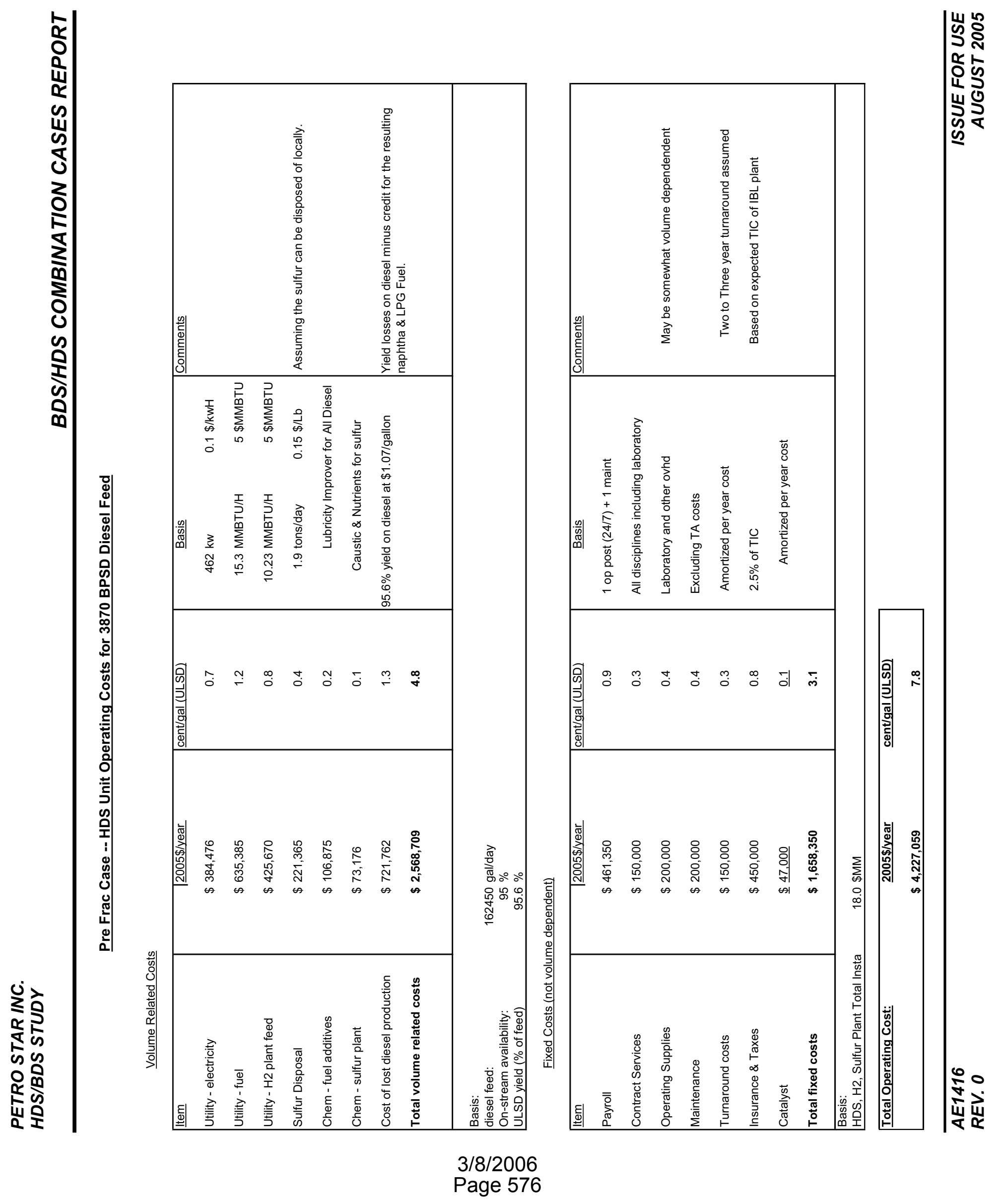


Final Technical Progress Report DOE Award No. DE-FC26-02NT15340

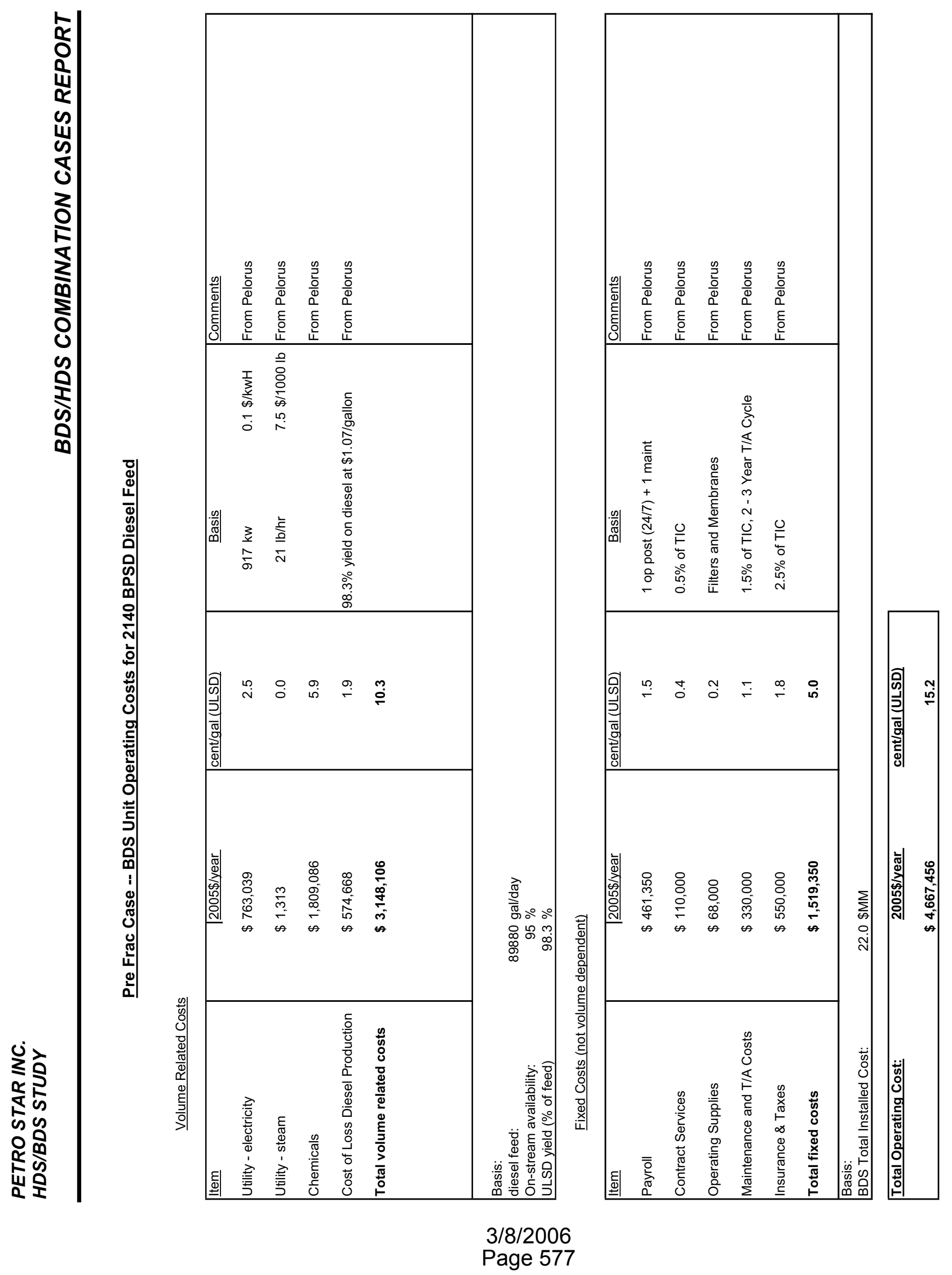

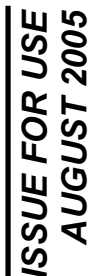

年定 